\title{
Estimates of Emergency Operating Capacity in U.S. Manufacturing and Nonmanufacturing Industries
}

Volume 2 - Summary Reports for 4-Digit

SIC Manufacturing Industries

D. B. Belzer

D. E. Serot

M. A. Kellogg

March 1991

Prepared for

the Federal Emergency Management Agency under a Related Services Agreement with the U.S. Department of Energy under Contract DE-AC06-76RLO 1830

Pacific Northwest Laboratory Operated for the U.S. Department of Energy by Battelle Memorial Institute 


\title{
DISCLAIMER
}

This report was prepared as an account of work sponsored by an agency of the United States Government. Neither the United States Government nor any agency thereof, nor Battelle Memorial Institute, nor any of theit emplayees, makes any warranty, expressed or implied, or assumes any legal liability or responsibility for the accuracy, completeness, or usefulness of any information, apparatus, product, or process disclosed, or represents that its use would not infringe privately owned rights. Reference herein to any specific commercial product, process, or service by trade name, trademark, manufacturer, or otherwise does not necessarily constitute or imply its endorsernent, recommendation, or favoring by the United States Government or any agency thereof, or Battelle Memorial Institute. The views and opinions of authors expressed herein do not necessarily state or reflect those of the United States Government or any agency thereof.

\author{
PACIFIC NORTHWEST LABORATORY \\ operated by \\ BATTELLE MEMORIAL INSTITUTE \\ for the \\ UNITED STATES DEPARTMENT OF ENERGY \\ under Contract DE-ACO6-76RLO 1830
}

Printed in the United Slates of America

Available to DOE and DOE contractors from the Office of Scientific and Technical Information, P.O. Box 62, Oak Ridge, TN 37831; prices available from (615) 576-8401. FTS 626-840T.

Available to the public from the National Technical Information Service, U.S. Department of Commerce, 5285 Port Royal Rd., Springfield, VA 22161. 
PNL-766l Vol. 2

UC -000

\section{ESTIMATES OF EMERGENCY OPERATING CAPACITY IN U.S. MANUFACTURING AND NONMANUFACTURING INDUSTRIES}

Volume 2 - Summary Reports for 4-Digit SIC Manufacturing Industries

D. B. Belzer

D. E. Serot $(a)$

M. A. Kellogg (b)

March 1991

Prepared for the Federal Emergency Management Agency under a Related Services Agreement with the U.S. Department of Energy under Contract DE-ACO6-76RLO 1830

Pacific Northwest Laboratory Richland, Washington 99352

(a) D/E/S Research, Richl and, Washington

(b) ERCE, Portland, Oregon 



\section{SUMMARY}

To develop integrated policies for mobilization preparedness, planners require estimates of available productive capacity during national emergency conditions. This report is the second of a two-volume report that develops estimates for emergency operating capacity (EOC) for 446 manufacturing industries at the 4-digit Standard Industrial Classification (SIC) leve] of aggregation and for 24 key nonmanufacturing sectors.

This volume presents tabular and graphical results of the historical analysis and the projections of EOC for the 446 four-digit SIC manufacturing sectors. To aid potential users in understanding and exploiting interrelationships among the various data and forecast elements, a one-page format of tabular results and graphics was developed for each 4-digit SIC industry. Key data series for each sector include 1) industry output, 2) implied capacity output--obtained by dividing output by an annual capacity utilization rate, 3) capital stock, and 4) predicted capacity. Although these one-page summary reports provide the sole presentation vehicle for the manufacturing EOC estimates, computer files were also developed that include only the estimates of emergency capacity and other selected data items.

Volume 1, Methodologies and Data Sources, lays out the general concepts and methods used to develop these emergency operating estimates. The procedure for estimating the manufacturing EOC basically follows that used in a previous study for the Federal Emergency Management Agency (FEMA) in 1984. The key data input is the set of historical capacity utilization measures collected by the Bureau of the Census in its Survey of Plant Capacity. These utilization measures are used in conjunction with output measures to develop estimates of "practical" capacity by 4-digit SIC industry. Data collected in the Survey of Plant Capacity on weekly plant hours are used to estimate the additional output that may be expected should the plant operate seven days per week, 24 hours per day. The resulting emergency capacity est imates are adjusted to account for required maintenance and the loss of productivity from greater reliance on shift work. 



\section{CONTENTS}

SUMMARY .............................

1.0 INTRODUCTION $\ldots \ldots \ldots \ldots \ldots \ldots \ldots$

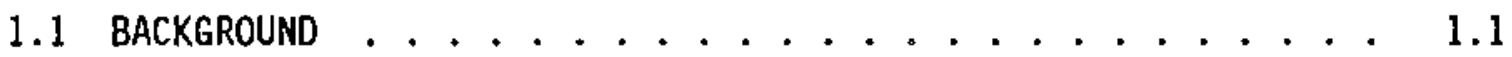

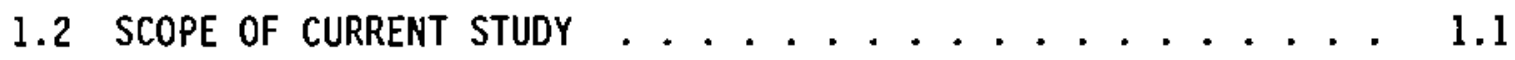

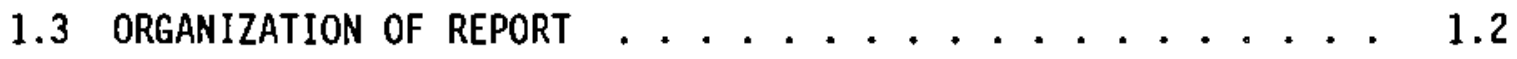

2.0 DESCRIPTION OF INDUSTRY REPORTS . . . . . . . . . . . 2.1

2.1 HISTORICAL AND FORECAST VALUES ................. 2.1

2.2 AUXILIARY INFORMATION $\ldots \ldots \ldots . \ldots . \ldots . \ldots . \ldots$

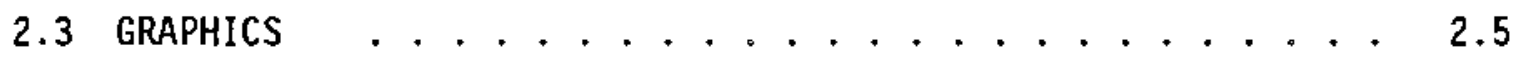

3.0 INDUSTRY REPORTS $\ldots \ldots \ldots \ldots \ldots \ldots \ldots . \ldots \ldots$

\section{TABLES}

2.1 Sample of Format - Bolts, Nuts, Rivets, and Washers . . . . . 2.2 
.

. 

below.

For informational purposes, the contents of Volume 1 are reproduced CONTENTS OF VOLUME 1

SUMMARY $\ldots \ldots \ldots \ldots \ldots \ldots \ldots \ldots \ldots \ldots$

1.0 INTRODUCTION . . . . . . . . . . . . . . . 1.1

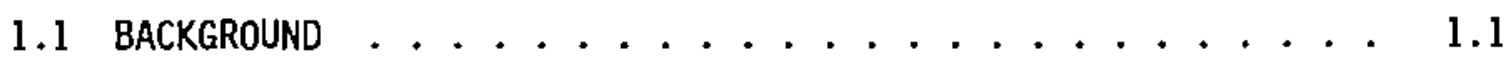

1.2 SCOPE OF CURRENT STUDY ......................... $1 . \ldots$

1.3 ORGANIZATION OF REPORT $\ldots \ldots \ldots \ldots \ldots \ldots . \ldots \ldots$

2.0 CONCEPTS AND GENERAL APPROACH $\ldots \ldots \ldots . \ldots \ldots$

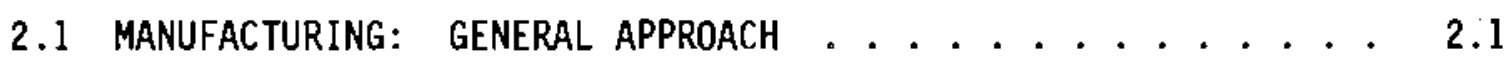

2.1.1 Definitions of Capacity . . . . . . . . 2.2

2.1.2 Concepts and Proposed Method ............ 2.3

2.2 NONMANUFACTURING: GENERAL APPROACH . . . . . . 2.4

3.0 ESTIMATION OF CAPACITY IN MANUFACTURING: CONCEPTS

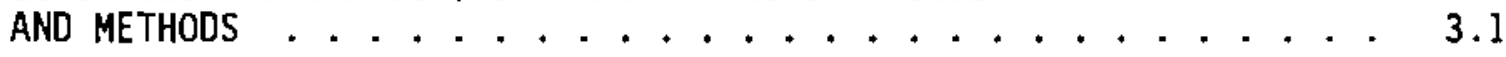

3.1 DESCRIPTION OF CENSUS SURVEY APPROACH . . . . . . . . 3.1

3.1.1 Problems and Modifications to Methods ...... 3.2

3.2 STEPS TO IMPLEMENT THE CENSUS SURVEY APPROACH $\ldots \ldots \ldots$

3.2.1 Step 1: Imputations of Missing Values for Fourth
Quarter Practical Utilization Rates . . . . . 3.4

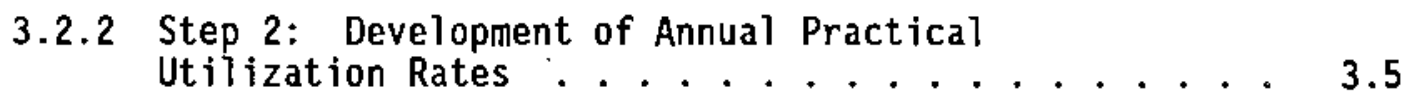

3.2.3 Step 3: Estimates of lmplied Practical Capacity by 4 -Digit SIC Industry .......... 3.7

3.2.4 Step 4: Capital Stocks and Investment . . . . . 3.8

3.2.5 Step 5: Forecasting Capacity by 4-Digit SIC Industry. . . . . . . . . . . 3.8 
3.2.6 Step 6: Forecast of Emergency Capacity: Using Shift Factors ............ 3.10

3.2.7 Time-Phased Measures of Emergency Capacity . . . . . 3.11

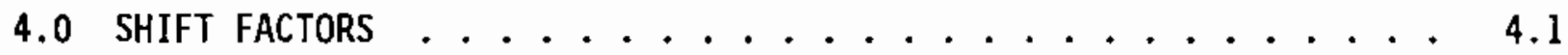

4.1 PLANT AND EQUIPMENT CONSTRAINTS TO CONTINUOUS

24-HOUR PRODUCTION .................... 4.2

4.1.1 Informal Manufacturing Survey ......... 4.2

4.1 .2 Survey Results ............... 4.3

4.2 LABOR PRODUCTIVITY AND SHIFT WORK . . . . . . . 4.5

4.2.1 Shift Work and Individual .......... 4.5

4.2.2 Quantifying Productivity Effects of Shift Work . . . 4.7

4.3 DERIVATION OF SHIFT FACTORS FROM CENSUS DATA . . . . . 4.10

4.3.1 Census Bureau Data .............. 4.11

4.3.2 Shift Factor Estimation ........... 4.12

4.3.3 Output and Hours Worked ........... 4.14

4.4 REFERENCES .......................... 4.17

5.0 EMERGENCY OPERATING CAPACITY ESTIMATES: MANUFACTURING . . . . 5.1

5.1 HISTORICAL AND FORECAST VALUES ................ 5.1

5.2 AUXILIARY INFORMATION . . . . . . . . . . 5.3

5.3 GRAPHICS . . . . . . . . . . . . . . 5.5

6.0 ESTIMATION CAPACITY IN NONMANUFACTURING INDUSTRIES:

CONCEPTS AND METHODS ...................... 6.1

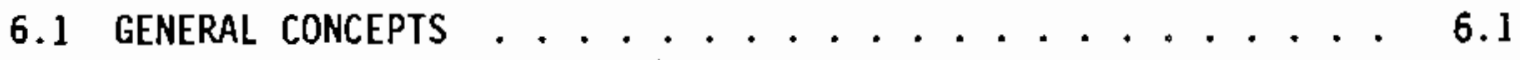

6.1 .1 Defining Nonmanufacturing Industries ...... 6.1

6.1 .2 Recent Trends in Nonmanufacturing Industries .... 6.2

6.1.3 Technology, Productivity, and Measurement Problems in the Nonmanufacturing Sector........ 6.4 
6.2 METHODS FOR MEASURING EMERGENCY OPERATING CAPACITY

IN NONMANUFACTURING INOUSTRIES

6.2 .1 Measures of Physical Capacity ........ 6.7

6.2.2 Production Frontiers and Emergency Dperating Capacity ................ 6.7

6.3 REFERENCES .......................... 6.9

7.0 EMERGENCY OPERATING CAPACITY ESTIMATES: NONMANUFACTURING . . . 7.1

7.1 SUMMARY OF INDUSTRY SPECIFIC METHODOLOGIES . . . . . . 7.1

7.1 .1 Mining . . . . . . . . . . . 7.2

7.1 .2 Transportation Industries ......... 7.4

7.1 .3 Public Utilities .............. 7.5

7.1 .4 Communications . . . . . . . . . . 7.6

7.1 .5 Banking ................ 7.6

7.1 .6 Hotels and Lodging Places . . . . . . . 7.7

7.1.7 Computer and Data Processing Services . . . . . 7.7

7.1.8 Automotive Repair Shops and Services . . . . . 7.7

7.1 .9 U.S. Postat Service ............. . 7.7

7.1 .10 Doctors and Dentists............ . 7.8

7.1 .11 Hospitals . . . . . . . . . . . 7.8

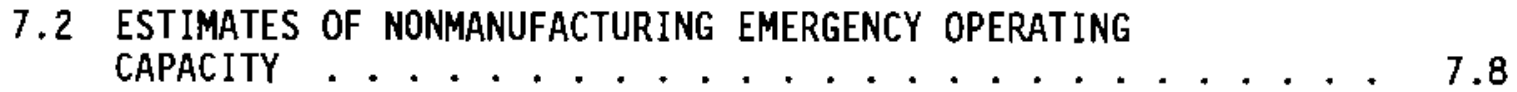

8.0 PRODUCTION FUNCTION ANALYSIS . . . . . . . . . . 8.1

8.1 APPLICATION OF THE FARE-GOSSKOPF-KOKKELLENBERG

METHODOLOGY TO ESTIMATING EMERGENCY DPERATING CAPACITY

FOR MANUFACTURING INDUSTRIES $\ldots \ldots \ldots . \ldots . \ldots . \ldots$

8.2 A COST FRONTIER METHODOLOGY FOR ESTIMATING EMERGENCY OPERATING CAPACITY .................... 8.4

8.3 REFERENCES . . . . . . . . . . . . . . 8.19 
APPENDIX A - INDUSTRY CONCORDANCES . . . . ........ A.l

APPENDIX B - IMPUTATIONS FOR MISSING PRACTICAL CAPACITY ESTIMATES .......................... B.

APPENDIX C - INDUSTRY SPECIFIC METHODOLOGIES FOR NONMANUFACTURING ......................

APPENDIX D - PRODUCTION FUNCTIONS: TECHNICAL NOTES . . . . . . D.1 


\subsection{INTRODUCTION}

Development of integrated mobilization preparedness policies requires planning estimates of available productive capacity during national energency conditions. Such estimates must be developed in a manner that allows evaluation of current trends in capacity and the consideration of uncertainties in various data inputs and in engineering assumptions.

This study, conducted by Pacific Northwest Laboratory (PNL), developed estimates of emergency operating capacity (EOC) for 446 manufacturing industries at the 4-digit Standard Industrial Classification (SIC) level of aggregation and for 24 key non-manufacturing sectors.

\subsection{BACKGROUND}

In 1983, the Federal Emergency Management Agency (FEMA) contracted for the development of estimates of EOC in the manufacturing, mining, and utilities industries. The overal] study (conducted during 1984) that produced these estimates also included detail on the potential for increasing labor force utilization and on investment lead time for manufacturing industries. PNL was responsible for the EOC estimation task in the study.

The EOC estimates from the 1984 study were provided for approximately 180 manufacturing sectors, 7 mining sectors, and the electric utility industry. A principal use of these estimates within FEMA has been in the Resolution of Capacity Shortfalls (ROCS) model. This model has been used in trade investigation studies and mobilization planning exercises. The EOC estimates were also used by other government agencies and consulting firms in studies related to mobilization and planning.

\subsection{SCOPE OF CURRENT STUDY}

In addition to updating the estimates of EOC from the 1984 study, the current study also extends the earlier work in several important ways. First, in manufacturing, the industry disaggregation extends to the 4-digit SIC level of aggregation. Based upon the industry production database provided by the Office of Business Analysis in the Department of Commerce, 446 manufacturing sectors were analyzed. The 4-digit disaggregation allows more targeted ana1yses by FEMA and other agencies and allows maximum flexibility for generating more aggregate models.

Second, more intensive analys is was performed relating to the potentia] of increasing operating hours within various manufacturing sectors. Two issues were examined: 1) the loss of worker productivity resulting from operating multiple shifts, and 2) approximate maintenance requirements by typical manufacturing establishments. 
Third, some exploratory work was performed to investigate the potential contribution of "frontier" production function analysis to the estimation of emergency capacity. Several variants of this technique, which has gained more recognition in the academic literature in the past decade, were tested and comparative analysis performed.

Finally, this report extends the previous work in a major way in providing estimates of EOC for key non-manufacturing sectors. Key sectors in transportation, communications, and services were analyzed to develop estimates of EOC.

\subsection{ORGANIZATION OF REPORT}

The report is presented in two volumes. Volume 1 of the report presents the overall methodology and concepts. It also includes full documentation and results of the EOCs in non-manufacturing. This second volume, Sumary Reports for 4-Digit SIC Manufacturing Industries, contains one-page summary reports of EOC estimates and related data for each 4-digit manufacturing sector. Chapter 2 of this volume provides a detailed description of the one-page summary reports that were produced for each 4-digit manufacturing sectors. Chapter 3 comprises the summary reports. 


\subsection{DESCRIPTION OF INDUSTRY REPORTS}

The industry data and forecasts developed in this study may be valuable in a wide range of future analytical studies related to industrial mobilization issues. To aid potential users in understanding and exploiting interrelationships among the various data and forecast elements, a one-page format of tabular results and graphics was developed for each 4-digit SIC industry. Although these one-page summary reports provide the sole presentation vehicle for the manufacturing EOC estimates, computer files were al so developed that include only the estimates of emergency capacity and other selected data items.

This section describes the presentation format used for the manufacturing $E O C$, the results for one industry serve as an example.

\subsection{HISTORICAL AND FORECAST VALUES}

As an example of this reporting format, Table 2.1 shows the one-page summary results for SIC 3452, Bolts, Nuts, Rivets, and Washers. Although many of the items in the table are self-explanatory, it may be useful to summarize the data sources and methodology of the previous section as the various components of the table are discussed.

The first four columns relate only to the historical period covering 1974-1988. The output measure through 1986, as discussed in Volume 1, is from the Office of Business Analysis (OBA) industry database. Federal Reserve Board (FRB) indexes of production were used to extrapolate 1987 and 1988 estimates. The quality of this extrapolation varies from sector to sector, depending upon the match between the FRB measure and the 4-digit SIC.

The 04 CU Rate is the fourth quarter utilization rate as published by the Bureau of Census in the Survey of Plant Capacity (SPC). Values of the utilization rate that have been imputed are denoted with an asterisk. In the case of Bolts and Nuts, no imputations were required.

The Ann. CU Rate is the practical utilization rate converted to an annual basis. The annualization relied upon a regression interpolation procedure using FRB capacity utilization rates.

The fourth column, Implied Prac. Capac., is the implied industry capacity based on the annualized practical capacity estimate. It is simply the output in Column 1 divided by the utilization rate in Column 3 .

The last five columns relate to the forecast of future practical and emergency capacity. Column 5, Gross Invest., shows gross investment in equipment in 1982 constant dollars from the OBA capital stocks database. 
TABLE 2.1. Sample of Format - Bolts, Nuts, Rivets, and Washers Ho. 297 sIC 3452 Bolts, nuts, rivets, and wabers MILLIOHS OF 1982 DOLLARS (EXCL. CJ RATES)

\begin{tabular}{|c|c|c|c|c|c|c|c|}
\hline & Q & ar. & $\begin{array}{l}\text { Impl ied } \\
\text { Prec. }\end{array}$ & $\begin{array}{l}\text { Gross } \\
\text { ln- }\end{array}$ & $\begin{array}{l}\text { Net } \\
\text { Cop. }\end{array}$ & $\begin{array}{l}\text { Pred. } \\
\text { Prac. }\end{array}$ & Emergency tespoci ty \\
\hline & Rate & Rate & ceope. & veat. & stk. & Copos. & \\
\hline
\end{tabular}

\begin{tabular}{|c|c|c|c|c|c|c|c|c|c|c|}
\hline 1974 & 4,752 & 72 & 74.8 & 6,353 & 262.3 & 1,608 & 7,013 & & & \\
\hline 1975 & 3,499 & 45 & 50.7 & 6,903 & 125.5 & 1,680 & 6,903 & & & \\
\hline 1976 & 4,029 & 58 & 53.3 & 7,554 & 149.6 & 1,700 & 7,028 & & & \\
\hline 1977 & 4,525 & 58 & 58.0 & 7,790 & 206.5 & 1,832 & 7,577 & & & \\
\hline 1978 & 4,925 & 67 & 63.0 & 7,013 & 159.3 & 1, 037 & 7,595 & & & \\
\hline 1979 & 5,120 & 72 & 70.8 & 7,233 & 54.8 & 1,745 & 7,213 & & & \\
\hline 1980 & 4,715 & 64 & 65.4 & 7,204 & 195.0 & 1,787 & 7,391 & & & \\
\hline 1981 & 4,605 & 61 & 63.4 & 7,260 & 169.9 & 1,790 & 7,436 & & & \\
\hline 1982 & 3,672 & 50 & 53.1 & 6,922 & 42.8 & 1,687 & 6,976 & & & \\
\hline 1983 & 3,953 & 64 & 58.0 & 6,820 & 103.8 & 1,635 & 6,760 & & & \\
\hline 1984 & 4,478 & 75 & 71.8 & 6,236 & 105.9 & 1,588 & 6,562 & & & \\
\hline 1985 & 4,625 & 70 & 72.2 & 6,400 & 142.5 & 1,575 & $6,5 \uparrow 1$ & & & \\
\hline 1986 & 4,610 & 64 & 65.9 & 6,992 & 137.4 & 1,560 & 6,448 & & & \\
\hline 1987 & 4,692 & $\overline{73}$ & 68.7 & 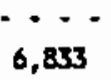 & 155.7 & $\begin{array}{l}\cdots \\
1,563\end{array}$ & $\cdots$ & & $\cdots \cdot$ & $=-\cdot$ \\
\hline 1988 & 6,800 & 7 & 74.8 & 6,419 & 198.3 & 9,600 & 6,646 & 8,444 & 9,102 & 9,272 \\
\hline 1989 & & & & & 205.0 & 1,655 & 6,843 & 8,684 & 9,370 & 9,545 \\
\hline 1990 & & & & & 209.3 & 1,702 & 7,037 & 8,941 & 9,637 & 9,817 \\
\hline 1991 & & & & & 218.7 & 1,74 & 7,252 & 9.214 & 9,931 & 10,116 \\
\hline 1992 & & & & & 213.5 & 1,796 & 7,426 & 9,432 & 10,167 & 10,357 \\
\hline
\end{tabular}

Cepacity-Copitol stock Regression: Code 0 RSO 0.590 Coefficients: Const $\cdot 16.6$ stock 4.1

Ave. Copocity/Stock $(74-86): 4.1$ Ave. Ueekly Hours (High) 113.0 Shift Factor 1.39 Ave Weekly Hours (LOW) 103.6 Shift Factor 1.52 Note: Cutput, Imvestment, and Capital stock extrapolated for 1997 and 1988.
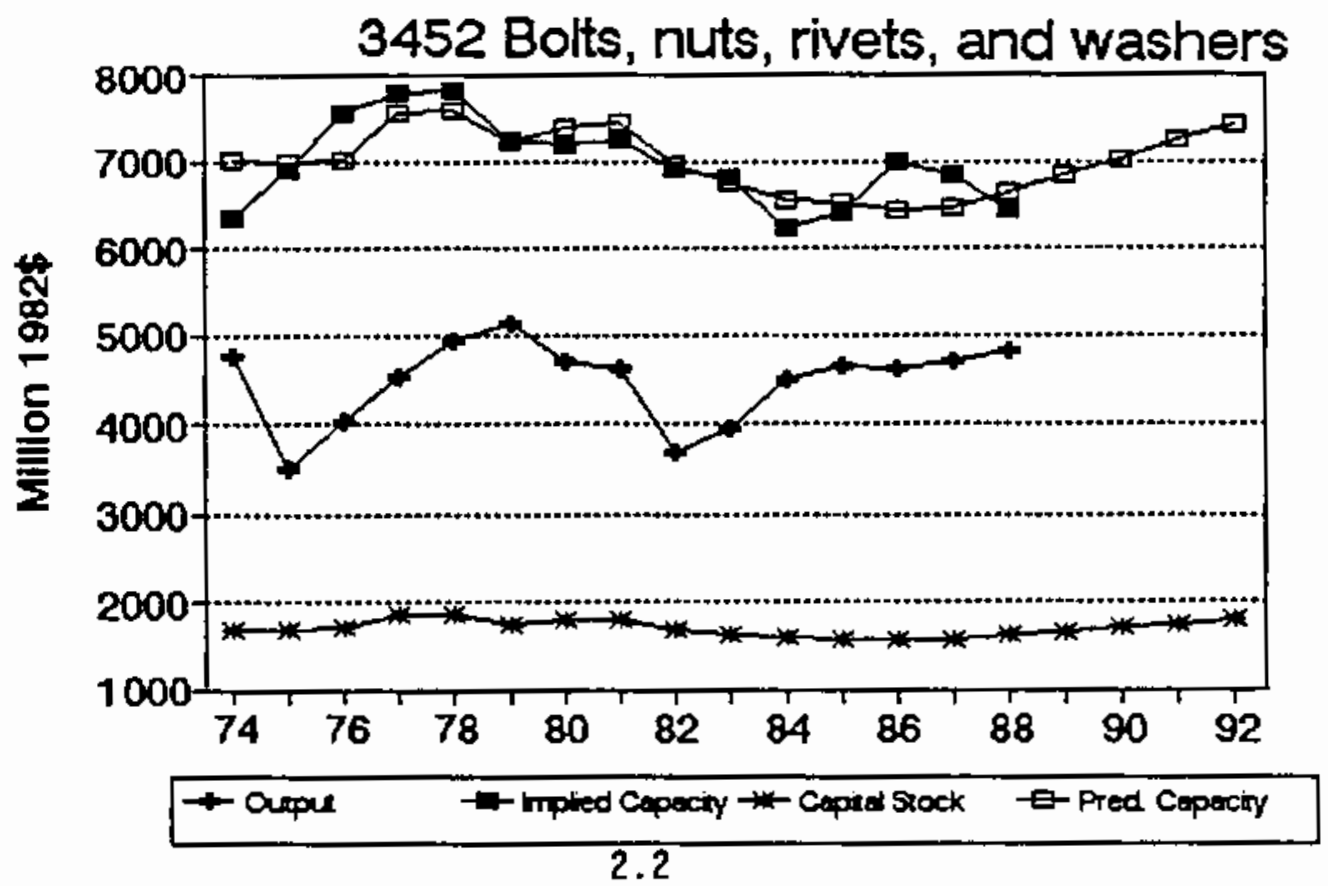
Historical data were available only through 1986. Investment was extrapolated through 1992 based upon the INFORUM Outlook for June 1990.

Column 6, Net. Cap. Stk., presents the net equipment stock from the OBA capital stocks database. As for investment, these values are expressed in 1982 constant dollars. Values from the OBA database itself run only through 1986. The capital stock estimates for 1987-1992 are based on a perpetual inventory method starting with the 1986 capital stock and the forecasts of gross investment. The depreciation rate used to generate the stock series was derived from analysis of the implied rates in the OBA database.

The predicted capacity in Column 7 results from the smoothing regression, using net capital stock as the explanatory variable, described in chapter 3 of Volume 1. The regression was over the period 1974-1986. The coefficients of this regression were used to forecast practical capacity over the period 1987 through 1992.

The last three columns show the emergency capacity estimates for three different time frames: 1) after 3 months, 2) after 6 months, and 3) greater than 6 months. The longest time frame, "greater than 6 months", is derived by multiplying the projected practical capacity by the shift factor. For Bolts, Nuts, Rivets and Washers, the shift factor used was 1.42 . The intermediate time frame estimates, for 3 and 6 months, were developed from the Census Bureau information at the 2-digit SIC level described in Section 4.2.7 of Volume 1 .

\subsection{AUXILIARY INFORMATION}

Below the tabular portion of the tables is auxiliary information that may be valuable to the user. The first two lines, starting with "CapacityCapacity Stock Regression," provides some information relating to the smoothing regression. The first value is a code that indicates the quality and type of smoothing regression that was performed. The codes can be interpreted as follows:

Code

0
Regression Quality

Good fit, predicted value for either 1985 or 1986 was within approximately 5 percent of implied practical capacity

(a) INFORUM--Interindustry Forecasting Model--University of Maryland. The INFORUM Model provides annual equipment investment forecasts for 57 sectors in the U.S. economy. 
Code

1

$-1$

$-2$
Regression Quality

Sat isfactory fit, predicted value for 1985 and 1986 was more than 5 percent from implied practical capacity, but regression was judged satisfactory on inspection.

Apparent break in series, perhaps from change in SPC sample. Capital stock is used in smoothing regression but constant adjustment is added to better fit last several years of data

Negative coefficient on capital stock observed in initial regression. Time trend replaces capital stock for smoothing regression

The quality of fit in the final regression equation is measured by the $R^{2}$ (RSQ) shown after the code value. On the next line the regression coefficients are shown. In the case of Bolts, Nuts, Rivets, and Washers, the smoothing regression results are:

$$
Q^{D C}=-16.6+4.1 * \text { Stock, } \quad 1974-1986 \quad R^{2}=0.590
$$

If a constant adjustment or dummy variable is added to the regression, an additional term would be shown on this line. For example, a regression that added a dumny variable for 1983 through 1986 would show a term: D(8386). A time trend regression, corresponding to a code of -2 above, would substitute the word "Time" for "Stock."

At the end of line two is the average capacjty-capital stock ratio over the period 1974-1986. This number can be compared with the marginal capacitycapital stock as represented by the regression coefficient on the net stock. In this instance, the values are the same (to two significant digits), indicating that over the historical period, implied capacity has been roughly proportional to net stock.

The third line under the tabular data relates to the estimation of shift factors. As discussed in Section 4.3 of Volume 1, two estimates of the shift factor were computed to account for year-to-year variation. The "high" estimate of recent average weekly hours is shown first, followed by the shift factor based on this estimate. The "low" estimate of weekly hours is shown next, followed by the shift factor based on this number of hours. 


\subsection{GRAPHICS}

Four key data series are plotted over the period 1974-1992: equipment stock $(\star)$, output $(+)$, implied practical capacity from the SPC (solid rectangle), and the predicted value of practical capacity from the smoothing regression of Equation (3.1) in Volume 1 (outlined rectangle). All series are plotted in terms of millions of 1982 constant dollars. The outputs and implied capacity are plotted through 1988, although the regression in Equation (3.1) was estimated only through 1986. The outputs for 1987 and 1988 are estimated with an unknown level of error because they have been extrapolated from 1986 levels through the use of FRB production indexes.

The fitted values of capacity provide a clear picture of how well the regression formulation of Equation (3.1) has provided a smoothed or averaged series of practical capacity over the 1974-1986 period. In the case of Bolts, Nuts, Rivets, and Washers, the pattern suggests a definite decline in capacity over the first half of the 1980s. The investment forecast suggests some increase in capacity output into the early 1990s, although still not exceeding levels of the late 1970s.

(a) Again, the correspondence between the 4-digit SIC sectors and the FRB indexes is shown in Appendix A of Volume 1 . 


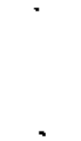


3.0 INDUSTRY REPORTS 


\section{NO. 1 SIC 2011 Meatpacking plants}

MILLIONS OF 1982 DOLLARS (EXCL. CU RATES)

$\begin{array}{lccccccc} & \text { Q4 } & \text { Ann. Implied Gross } & \text { Het } & \text { Pred. } & \ldots \ldots & \ldots \\ \text { Gross } & \text { CU } & \text { CU } & \text { Prac. } & \text { In- } & \text { Cap. } & \text { Prac. } & \text { Emergency Capacity } \\ \text { Output Rate } & \text { Rate } & \text { Capac. } & \text { vest. } & \text { Stk. } & \text { Capac. } & 3 \text { mon. } 6 \text { mon. }>6 \text { mon. }\end{array}$

\begin{tabular}{|c|c|c|c|c|c|c|c|c|c|c|}
\hline 1974 & 43,402 & 84 & 87.1 & 49,850 & 189.9 & 2,146 & 50,152 & & & \\
\hline 1975 & 40,798 & 86 & 83.8 & 48,696 & 365.1 & 2,319 & 50,750 & & & \\
\hline 1976 & 46,784 & 87 & 86.3 & 54,202 & 147.2 & 2,273 & 51,348 & & & \\
\hline 1977 & 44,390 & 86 & 86.9 & 51,093 & 211.8 & 2,290 & 51,945 & & & \\
\hline 1978 & 44,403 & 85 & 85.9 & 51,709 & 239.3 & 2,326 & 52,543 & & & \\
\hline 1979 & 44,099 & 81 & 82.6 & 53,548 & 147.9 & 2,271 & 53,140 & & & \\
\hline 1980 & 44,508 & 85 & 84.0 & 52,985 & 158.0 & 2,229 & 53,738 & & & \\
\hline 1981 & 46,101 & 84 & 85.1 & 54,156 & 107.0 & 2,145 & 54,335 & & & \\
\hline 1982 & $44,82 t$ & $\pi 7$ & 79.4 & 56,484 & 342.8 & 2,272 & 54,933 & & & \\
\hline 1983 & 44,429 & 77 & 76.7 & 57,895 & 116.9 & 2,178 & 55,531 & & & \\
\hline 1984 & 44,327 & 78 & 78.3 & 56,595 & 212.7 & 2,175 & 56,128 & & & \\
\hline 1985 & 45,275 & 80 & 79.3 & 57,083 & 139.0 & 2,098 & 56,726 & & & \\
\hline 1986 & 44,384 & 82 & 81.7 & 54,297 & 160.2 & 2,045 & 57,323 & & & \\
\hline-- & $\because-$ & $\cdots$ & $\cdots$ & $\therefore$ & $\cdots$ & $-\cdots$ & $\because \cdots$ & $=\cdot$ & --- & --- \\
\hline 1987 & 44,732 & 80 & 80.9 & 55,291 & 199.3 & 2,032 & 57,921 & & & \\
\hline 1988 & 45,189 & 81 & 80.6 & 56,090 & 206.3 & 2,028 & 58,518 & 100,800 & 108,679 & 110,307 \\
\hline 1989 & & & & & 212.9 & 2,031 & 59,116 & 101,829 & 109,789 & 111,433 \\
\hline 1990 & & & & & 217.0 & 2,037 & 59,713 & 102,858 & 110,899 & 112,560 \\
\hline 1991 & & & & & 218.1 & 2,045 & 60,311 & 103,888 & 112,008 & 113,686 \\
\hline 1992 & & & & & 217.6 & 2,050 & 60,909 & 104,917 & 113,118 & 114,813 \\
\hline
\end{tabular}

Capecity-Capital Stock Regression: Code -2 RSO 0.670

Coefficients: Const 49554.9 Time 597.6

Ave. Capacity/Stock $(74-86): 24.3$ Ave. Weekly Hours (High) 80.0 Shift Factor 1.88 Ave Heekly Hours (Low) 74.9 Shift factor 2.01 Note: output, Investment, and Capital stock extrapolated for 1987 and 1988.

\section{Meatpacking plants}

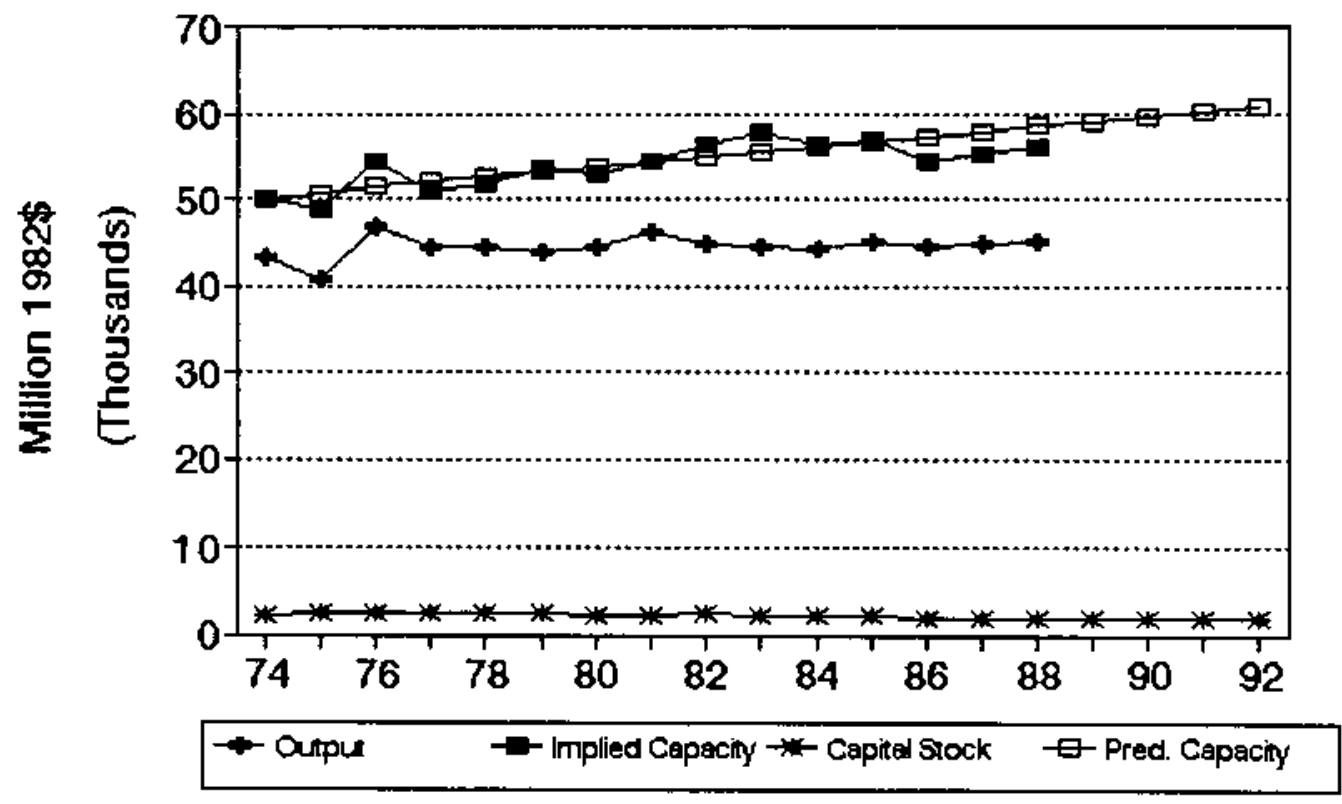




\section{No. 2 sic 2013 sausages and other prepared meats}

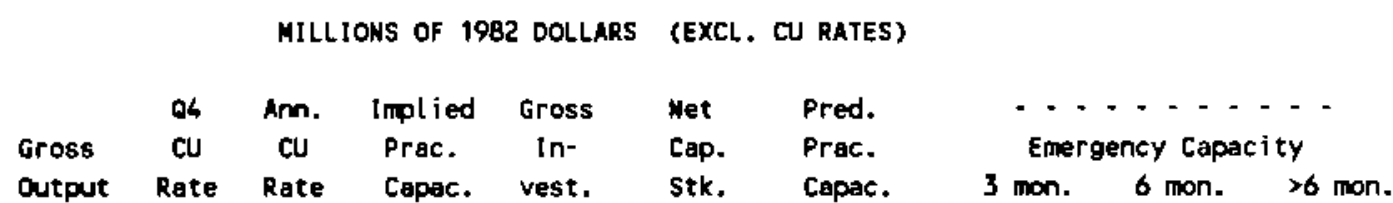

\begin{tabular}{|c|c|c|c|c|c|c|c|c|c|c|}
\hline 1974 & 9,645 & 61 & 61.6 & 15,670 & 123.8 & 938 & 15,021 & & & \\
\hline 1975 & 9,160 & 67 & 64.5 & 14,206 & 104.1 & 980 & 15,298 & & & \\
\hline 1976 & 10,374 & 63 & 64.5 & 16,097 & 101.5 & 1,019 & 15,555 & & & \\
\hline 1977 & 12,683 & 75 & 70.6 & 17,965 & 266.8 & 1,215 & 16,846 & & & \\
\hline 1978 & 11,784 & 74 & 74.5 & 15,821 & 110.4 & 1,253 & 17,090 & & & \\
\hline 1979 & 11,538 & 73 & 73.3 & 15,731 & 52.5 & 1,231 & 16,950 & & & \\
\hline 1980 & 12,282 & 68 & 70.0 & 17,551 & 78.9 & 1,235 & 16,975 & & & \\
\hline 1981 & 11,839 & 70 & 69.4 & 17,059 & 52.5 & 1,211 & 16,817 & & & \\
\hline 1982 & 12,325 & 71 & 70.6 & 17,463 & 217.2 & 1,337 & 17,646 & & & \\
\hline 1983 & 13,128 & 72 & 71.6 & 18,344 & 106.7 & 1,347 & 17,710 & & & \\
\hline 1984 & 13,163 & 69 & 70.3 & 18,729 & $(3.4)$ & 1,252 & 18,994 & & & \\
\hline 1985 & 13,521 & 71 & 70.3 & 19,243 & 142.9 & 1,296 & 19,285 & & & \\
\hline 1986 & 14,007 & 70 & 70.5 & 19,874 & 145.8 & 1,339 & 19,568 & & & \\
\hline-- & --- & - & $-\cdots$ & $\cdots$ & $\because$ & $\cdots \cdot$ & $\cdots \cdot$ & $=--$ & --- & --- \\
\hline 1987 & 14,273 & 66 & 67.5 & 21,138 & 140.1 & 1,377 & 19,816 & & & \\
\hline 1988 & 14,630 & 69 & 67.9 & 21,559 & 164.9 & 1,436 & 20,208 & 25,659 & 27,221 & 27,544 \\
\hline 1989 & & & & & 188.3 & 1,515 & 20,725 & 26,314 & 27,917 & 28,248 \\
\hline 1990 & & & & & 202.6 & 1,602 & 21,296 & 27,039 & 28,686 & 29,026 \\
\hline 1991 & & & & & 206.4 & 1,685 & 21,848 & 27,741 & 29,430 & $29,7 \pi 9$ \\
\hline 1992 & & & & & 204.5 & 1,761 & 22,346 & 28,373 & 30,101 & 30,458 \\
\hline
\end{tabular}

Capecity-Capital Stock Regression: Code -1 RSO 0.780

Coefficients: Const 8846.0 stock 6.6 D(84-86) 1908.9

Ave. Capacity/Stock $(74-86): 14.3$

Ave. Weekly Hours (High) 110.7 Shift Factor 1.36 Ave Heekly Hours (LOW) 109.3 Shift factor 1.38 Note: Output, Investment, and Capital Stock extrapolated for 1987 and 1988.

2013 Sausages and other prepared meats

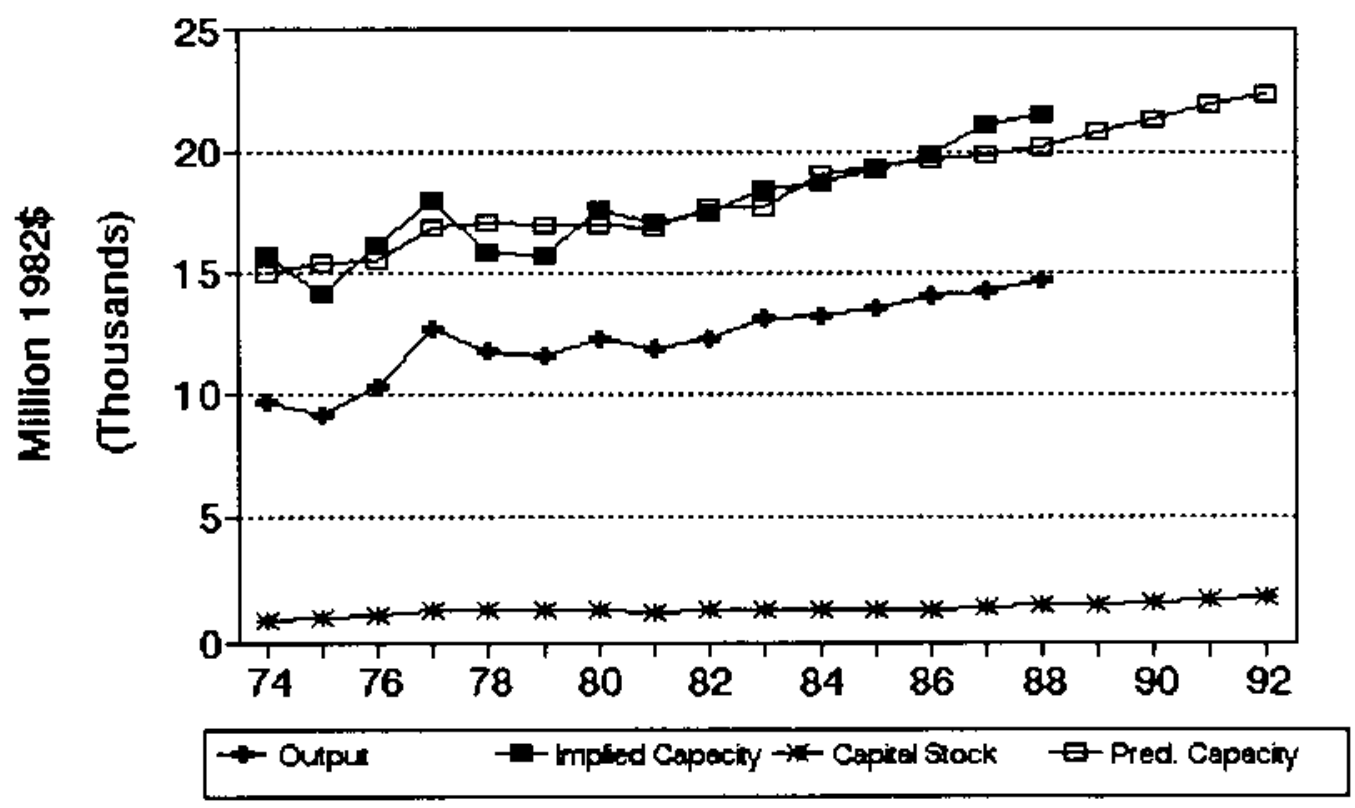




\section{No. 3 sIC 2016 Poultry dressing plants}

\section{MILLIONS OF 1982 DOLLARS (EXCL. CU RATES)}

$\begin{array}{lccccccc} & \text { Q4 } & \text { Ann. } & \text { Implied Gross } & \text { Wet } & \text { Pred. } & \ldots & \ldots \\ \text { Gross } & \text { CU } & \text { CU } & \text { Prac. } & \text { In- } & \text { Cap. } & \text { Prac. } & \text { Emergency Capacity } \\ \text { Output } & \text { Rate } & \text { Rate } & \text { Capac. } & \text { vest. } & \text { Stk. } & \text { Capac. } & 3 \text { mon. } 6 \text { mon. }>6 \text { mon. }\end{array}$

\begin{tabular}{|c|c|c|c|c|c|c|c|c|c|c|}
\hline 1974 & 5,274 & 86 & 90.7 & 5,816 & 104.7 & 695 & 5,303 & & & \\
\hline 1975 & 4,993 & 92 & 87.5 & 5,709 & 47.2 & 687 & 5,194 & & & \\
\hline 1976 & 5,831 & 90 & 90.3 & 6,457 & 112.2 & 740 & 5,979 & & & \\
\hline 1977 & 5,961 & 87 & 88.9 & 6,706 & 180.6 & 854 & 7,668 & & & \\
\hline 1978 & 6,404 & 87 & 87.7 & 7,299 & 165.1 & 948 & 9,055 & & & \\
\hline 1979 & 7,101 & 75 & 79.2 & 8,961 & 103.8 & 979 & 9,519 & & & \\
\hline 1980 & 7,642 & 80 & 78.8 & 9,696 & 165.6 & 1,066 & 10,790 & & & \\
\hline 1981 & 8,490 & $B 2$ & 82.4 & 10,307 & 106.5 & 1,090 & 11,159 & & & \\
\hline 1982 & 8,973 & 74 & 76.6 & 11,714 & 121.4 & 1,123 & 11,643 & & & \\
\hline 1983 & 9,224 & $\pi$ & 72.4 & 12,741 & 90.8 & 1,121 & 11,621 & & & \\
\hline 1984 & 8,735 & 82 & 79.3 & 11,047 & 15.4 & 1,048 & 10,535 & & & \\
\hline 1985 & 9,303 & 68 & 73.3 & 12,692 & 122.7 & 1,079 & $10, \infty 91$ & & & \\
\hline 1986 & 9,565 & 83 & 78.1 & 12,251 & 158.6 & 1,142 & 11,934 & & & \\
\hline - - - & $\cdots \cdot \cdot$ & - & -- & $\cdots$ & $\cdot-$ & - - - - & $\cdot \cdot-$ & 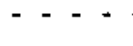 & 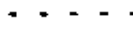 & --- \\
\hline 1987 & 9,829 & 83 & 83.2 & 11,808 & 125.1 & 1,171 & 12,362 & & & \\
\hline 1988 & 10,182 & 88 & 86.0 & 11,838 & 138.3 & 1.211 & 12,950 & 22,135 & 23,828 & 24,178 \\
\hline 1989 & & & & & 150.6 & 1,260 & 13,672 & 23,368 & 25,456 & 25,525 \\
\hline 1990 & & & & & 158.2 & 1,312 & 14,445 & 24,690 & 26,579 & 26,970 \\
\hline 1991 & & & & & 160.3 & 1,362 & 15,186 & 25,952 & 27,938 & 28,348 \\
\hline 1992 & & & & & 159.3 & 1,406 & $\uparrow 5,845$ & 27,083 & 29,155 & 29,583 \\
\hline
\end{tabular}

Capacity-Capital Stock Regression: Code 0 RSO 0.870 Coefficients: Const -4991.4 stock 14.8 Ave. Weekly Hours (High) 80.8 Shift Factor 1.87 Ave Weekly Hours (Low) 7.4 Shift Factor 2.00 Note: Output, Investment, and Capital Stock extrapolated for 1987 and 1988.

\section{Poultry dressing plants}

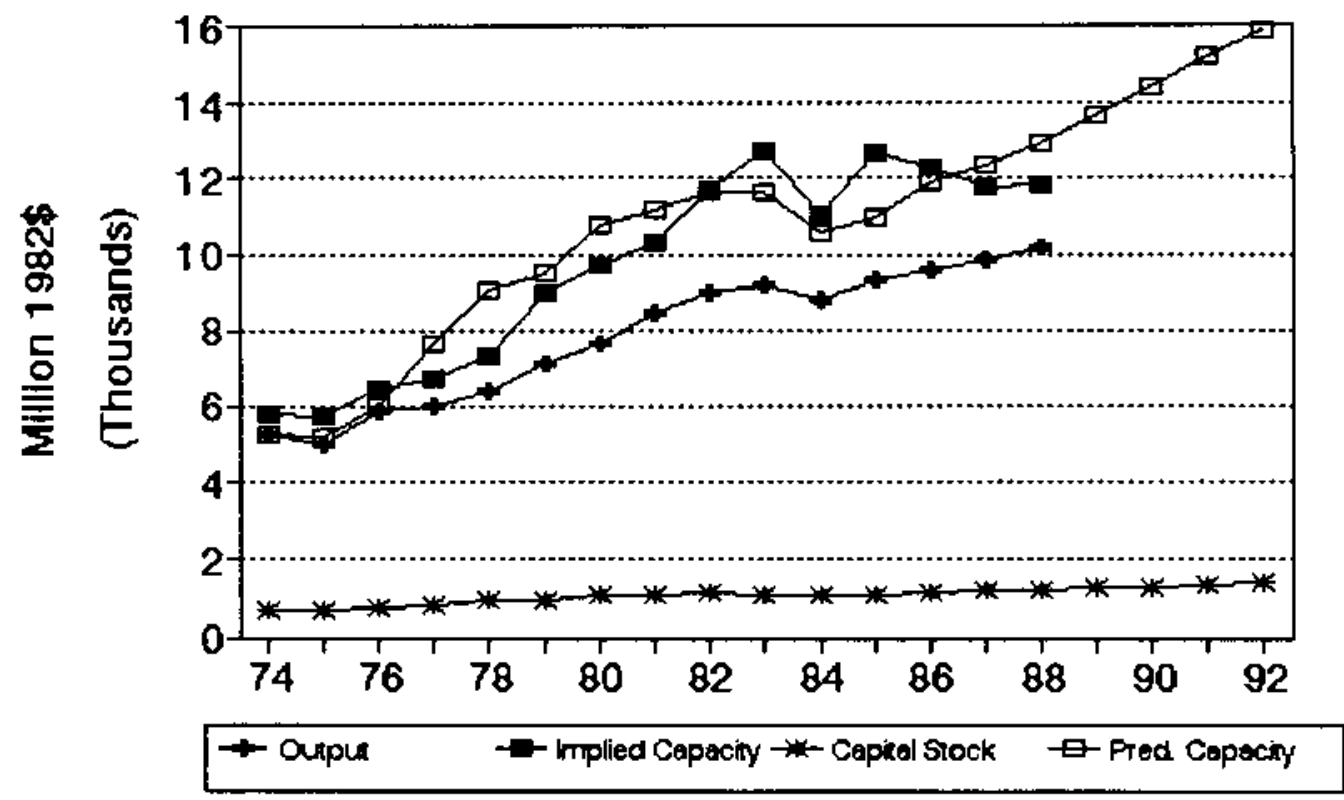




\section{No. 4 sIC 2017 Poultry and ogg processing}

MILLIONS OF 1982 DOLLARS (EXCL. CU RATES)

$\begin{array}{llllllll} & \text { Q4 } & \text { Amn. Implied Gross } & \text { Met } & \text { Pred. } & \ldots \ldots \\ \text { Gross } & \text { CU } & \text { CU } & \text { Prac. } & \text { In. } & \text { Cap. } & \text { Prac. } & \text { Emergency Capecity } \\ \text { Output } & \text { Rete } & \text { Rate } & \text { Capac. } & \text { vest. } & \text { 5tk. } & \text { Capac. } & 3 \text { mon. } 6 \text { mon. }>6 \text { mon. }\end{array}$

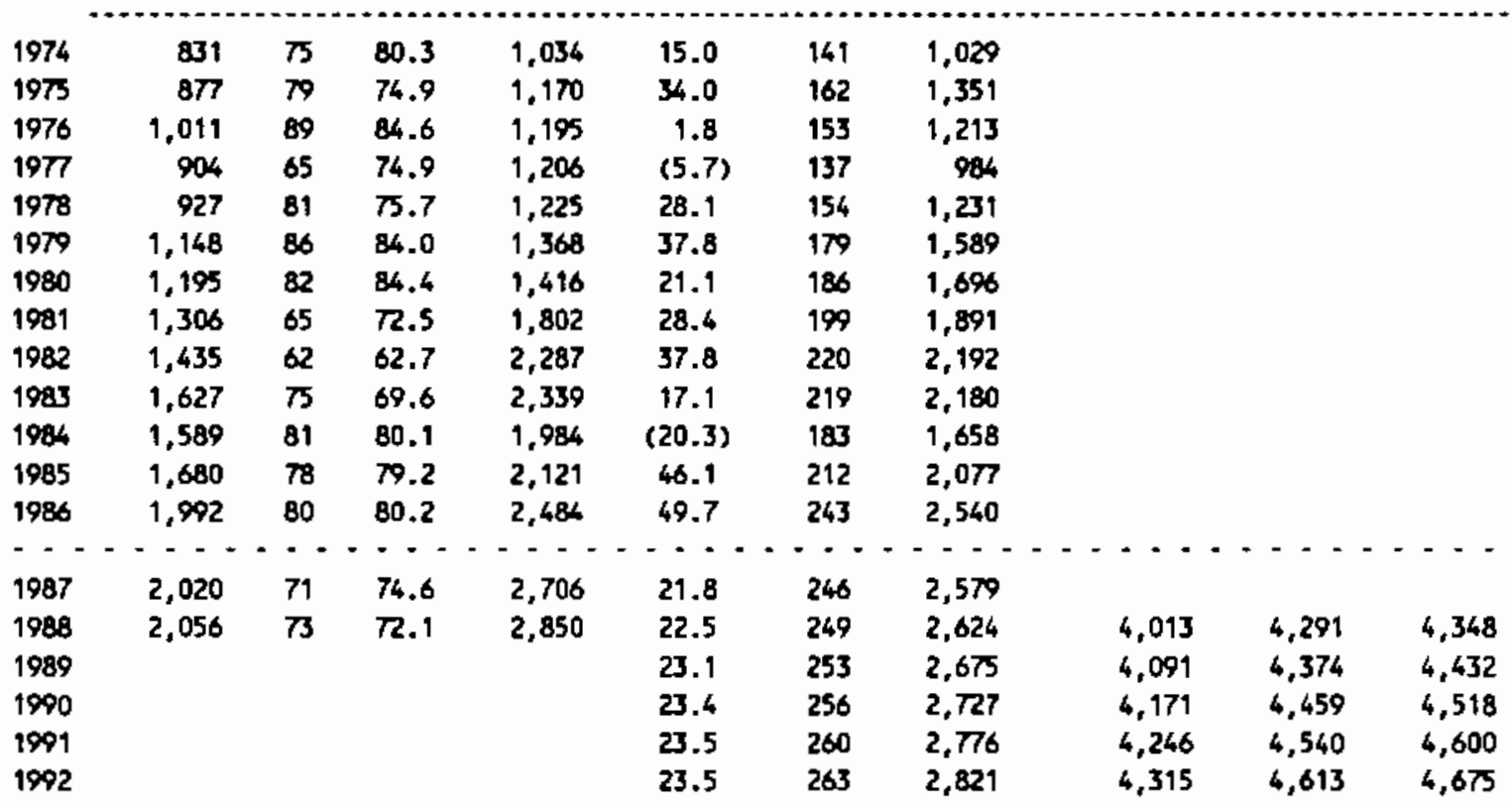

Capacity-Cepital Stock Regression: Code 0 RSO 0.890

Coefficients: Const -1032.8 stock 14.7

Ave. Capacity/5tack $(74-86): 9.1$ Ave. Weekly Hours (High) 91.0 Shift Factor 1.66 Ave Weekly Hours (LOW) 90.2 Shift factor 1.67 Note: Output, Investment, and Capital stock extrapolated for 1987 and 1988.

\section{Poultry and egg processing}

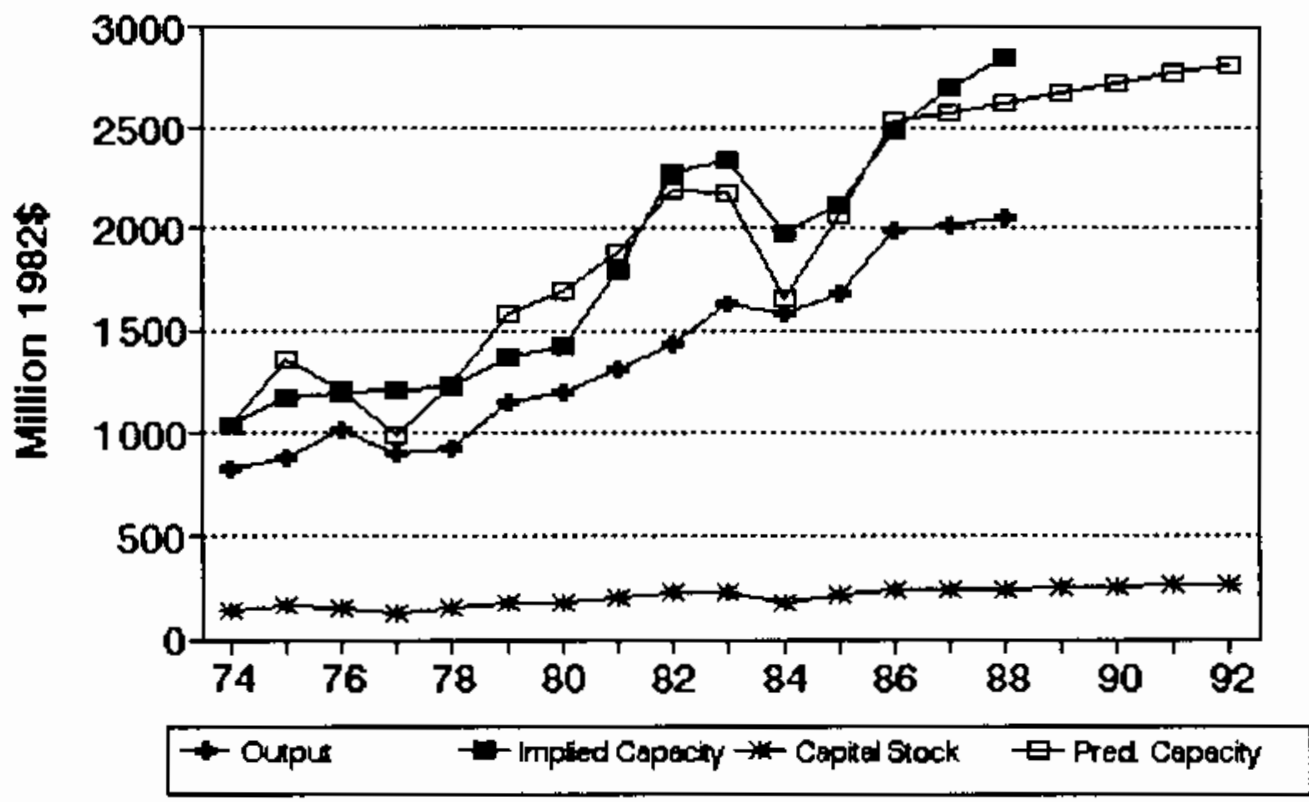


HO. 5 SIC 2021 Creamery butter

MILLIOWS OF 1982 DOLLARS (EXCL. CU RATES)

$\begin{array}{lccccccc} & \text { Q4 } & \text { Amn. Implied Gross } & \text { Net } & \text { Pred. } & \ldots & \ldots \\ \text { Gross } & \text { CU } & \text { CU } & \text { Prac. } & \text { In- } & \text { Cap. } & \text { Prac. } & \text { Emergency Capacity } \\ \text { Output Rate } & \text { Rate } & \text { Capac. } & \text { vest. } & \text { Stk. } & \text { Capac. } & 3 \text { mon. } 6 \text { mon. }>6 \text { mon. }\end{array}$

\begin{tabular}{|c|c|c|c|c|c|c|c|c|c|c|}
\hline 1974 & 1,763 & 85 * & 84.6 & 2,083 & 4.5 & 86 & 1,965 & & & \\
\hline 1975 & 1,804 & $80 *$ & 81.6 & 2,210 & 27.9 & 102 & 2,113 & & & \\
\hline 1976 & 1,772 & 86 * & 83.8 & 2,114 & 8.2 & 97 & 2,068 & & & \\
\hline 1977 & 1,337 & 87 & 86.7 & 1,542 & $(34.6)$ & 54 & 1,662 & & & \\
\hline 1978 & 1,398 & $\pi$ & 80.8 & 1,732 & 20.4 & 64 & 1,734 & & & \\
\hline 1979 & 1,262 & 88 & 83.9 & 1,504 & 5.6 & 59 & 1,707 & & & \\
\hline 1980 & 1,237 & 94 & 91.8 & 1,348 & 9.4 & 57 & 1,688 & & & \\
\hline 1981 & 1,270 & 92 & 92.8 & 1,369 & 8.9 & 54 & 1,662 & & & \\
\hline 1982 & 1,701 & 100 & 97.0 & 1,754 & 5.6 & 48 & 1,604 & & & \\
\hline 1983 & 1,735 & $91 \star$ & 94.4 & 1,837 & 5.7 & 42 & 1,546 & & & \\
\hline 1984 & 1,543 & $81 *$ & 84.6 & 1,823 & 13.2 & 42 & 1,547 & & & \\
\hline 1985 & 1,582 & 72 & 75.3 & 2,102 & 23.7 & 50 & 2,140 & & & \\
\hline 1986 & 1,499 & 72 & 72.0 & 2,082 & 4.3 & 40 & 2,044 & & & \\
\hline $\begin{array}{c}-\cdot- \\
1987\end{array}$ & - - - . & $\because-$ & $\therefore$ & $\cdots$ & $=--$ & $\cdots$ & - - - - & $\cdots-$ & $-\cdots$ & -- \\
\hline 1987 & 1,497 & 69 & 70.1 & 2,135 & 8.1 & 35 & $\begin{array}{l}1,993 \\
1,970\end{array}$ & 1.968 & 2,035 & 2,049 \\
\hline $\begin{array}{l}1900 \\
1989\end{array}$ & & 14 & rc. 1 & & 10.2 & 31 & 1,964 & 1,962 & 2,028 & 2,042 \\
\hline 1990 & & & & & 10.8 & 32 & 1,966 & 1,966 & 2,030 & 2,044 \\
\hline 1991 & & & & & 11.0 & 32 & 1,968 & 1,966 & 2,033 & 2,047 \\
\hline 1992 & & & & & 10.9 & 32 & 1,970 & 1,967 & 2,034 & 2,048 \\
\hline
\end{tabular}

Capacity-Capital Stock Regression: Code -1 RSQ 0.560 Coefficients: Const 1150.4 Stock 9.4 D(85-86) 517.0

Ave. Capacity/stock $(74-86): 29.5$ Ave. Weekly Hours (High) 146.8 Shift Factor 1.04 Ave Heekly Hours (LOW) 143.3 Shift Factor 1.06 - Inputed Note: Output, Investment, and Capital Stock extrapolated for 1987 and 1988.

\section{Creamery butter}

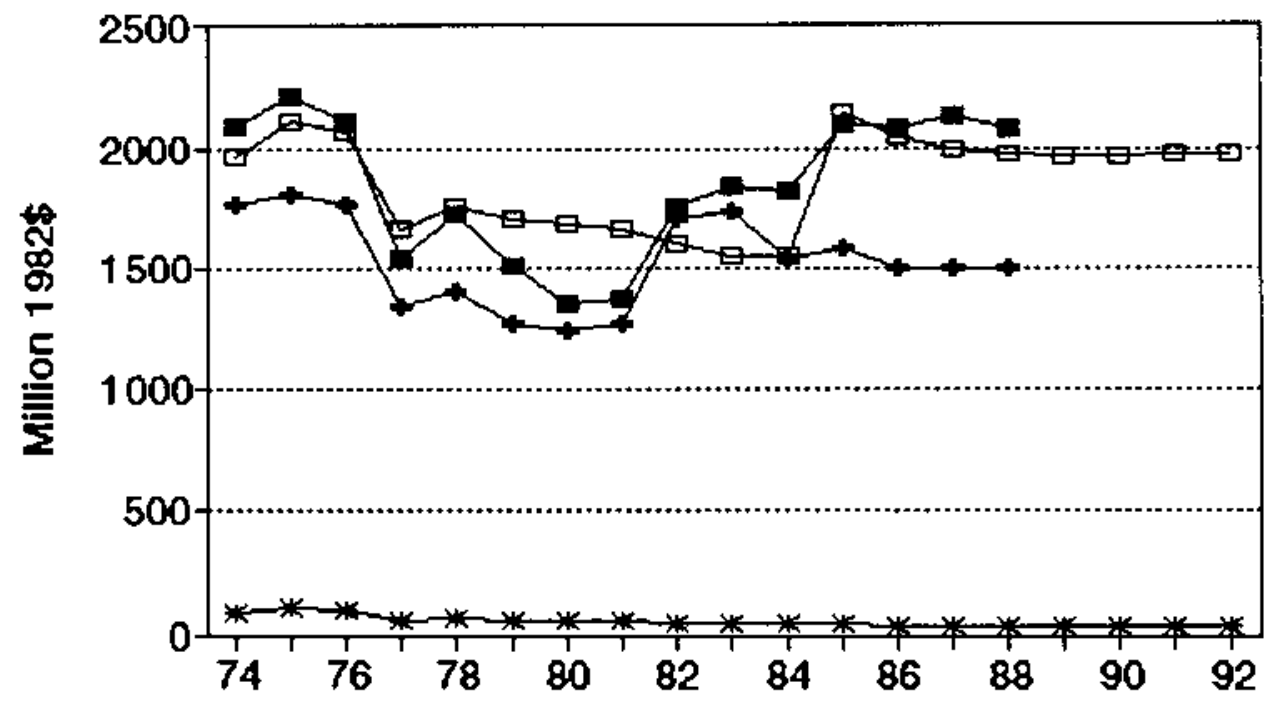

$\rightarrow$ - Oupur $\rightarrow$-mplied Cepacity $\rightarrow$ - Cepite Sock $\quad \square-$ Pred. Cepacity


No. 6 SIC 2022 Cheese, natural and processed

MILLIONS OF 1982 DOLLARS (EXCL. CU RATES)

$\begin{array}{lccccccc} & 04 & \text { Ann. Implied Gross } & \text { Net } & \text { Pred. } & -\ldots \\ \text { Gross } & \text { CU } & \text { CU } & \text { Prac. } & \text { In- } & \text { Cap. } & \text { Prac. } & \text { Emergency Capacity } \\ \text { Output Rate } & \text { Rate } & \text { Capac. } & \text { vest. } & \text { Stk. } & \text { Capac. } & 3 \text { mon. } 6 \text { mon. }>6 \text { mon. }\end{array}$

\begin{tabular}{|c|c|c|c|c|c|c|c|c|c|c|}
\hline 1974 & 8,231 & 63 & 63.0 & 13,065 & 135.8 & 689 & 12,681 & & & \\
\hline 1975 & 8,502 & 77 & 71.8 & 11,849 & 126.6 & 763 & 13,064 & & & \\
\hline 1976 & 9,065 & 65 & 69.5 & 13,043 & 103.0 & 811 & 13,313 & & & \\
\hline 1977 & 8,851 & 60 & 61.9 & 14,305 & 0.2 & 760 & 13,050 & & & \\
\hline 1978 & 8,742 & 66 & 63.8 & 13,713 & 71.8 & 780 & 13,152 & & & \\
\hline 1979 & 8,936 & 73 & 70.4 & 12,698 & 87.0 & 813 & 13,326 & & & \\
\hline 1980 & 9,726 & 76 & 74.9 & 12,990 & 128.1 & 884 & 13,692 & & & \\
\hline 1981 & 10,639 & 74 & 74.8 & 14,233 & 138.6 & 959 & 14,084 & & & \\
\hline 1982 & 10,797 & 73 & 73.4 & 14,714 & 109.1 & 1,003 & 14,311 & & & \\
\hline 1983 & 10,845 & 73 & 73.0 & 14,856 & 88.1 & 1,022 & 14,610 & & & \\
\hline 1984 & 10,889 & Bi & 78.0 & 13,960 & 79.8 & 1,029 & 14,465 & & & \\
\hline 1985 & 11,476 & 80 & 80.4 & 16,278 & 104.7 & 1,056 & $14,5 \mathrm{~B} 7$ & & & \\
\hline 1986 & 12,376 & 83 & 81.9 & 15,115 & 104.9 & 1,079 & 14,704 & & & \\
\hline-- & --- & $\cdot$ & $\cdots$ & $\cdots \cdot$ & $\cdots$ & --- & $--\cdot$ & 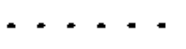 & $-\cdot-$ & --- \\
\hline 1987 & 12,557 & 81 & 81.8 & 15,361 & 98.5 & 1,097 & 14,797 & & & \\
\hline 1988 & 12,798 & 81 & 81.0 & 15,800 & 98.5 & 1,113 & 14,882 & 17,082 & 17,689 & 17,814 \\
\hline 1989 & & & & & 98.6 & 1,129 & 14,962 & 17,173 & 17.783 & 17,909 \\
\hline 1990 & & & & & 98.6 & 1,143 & 15,035 & 17,257 & 17,871 & 17,997 \\
\hline 1991 & & & & & 98.6 & 1,156 & 15,104 & 17,336 & 17,952 & 18,079 \\
\hline 1992 & & & & & 98.7 & 1,168 & 15,167 & 17,409 & 18,027 & 18,155 \\
\hline
\end{tabular}

Capacity-Capital Stock Regression: Code 0 RSO 0.520

Coefficients: Const 9105.8 Stock $\mathbf{5 . 2}$

Ave. Capacity/Stock $(74-86) ; 15.4$

Ave. Weekly Hours (High) 126.8 Shift Factor 1.20 Ave Weekly Hours (LOW) 124.1 Shift factor 1.22 Wote: Output, Investment, and Capital Stock extrapolated for 1987 and 1988.

\section{Cheese, natural and processed}

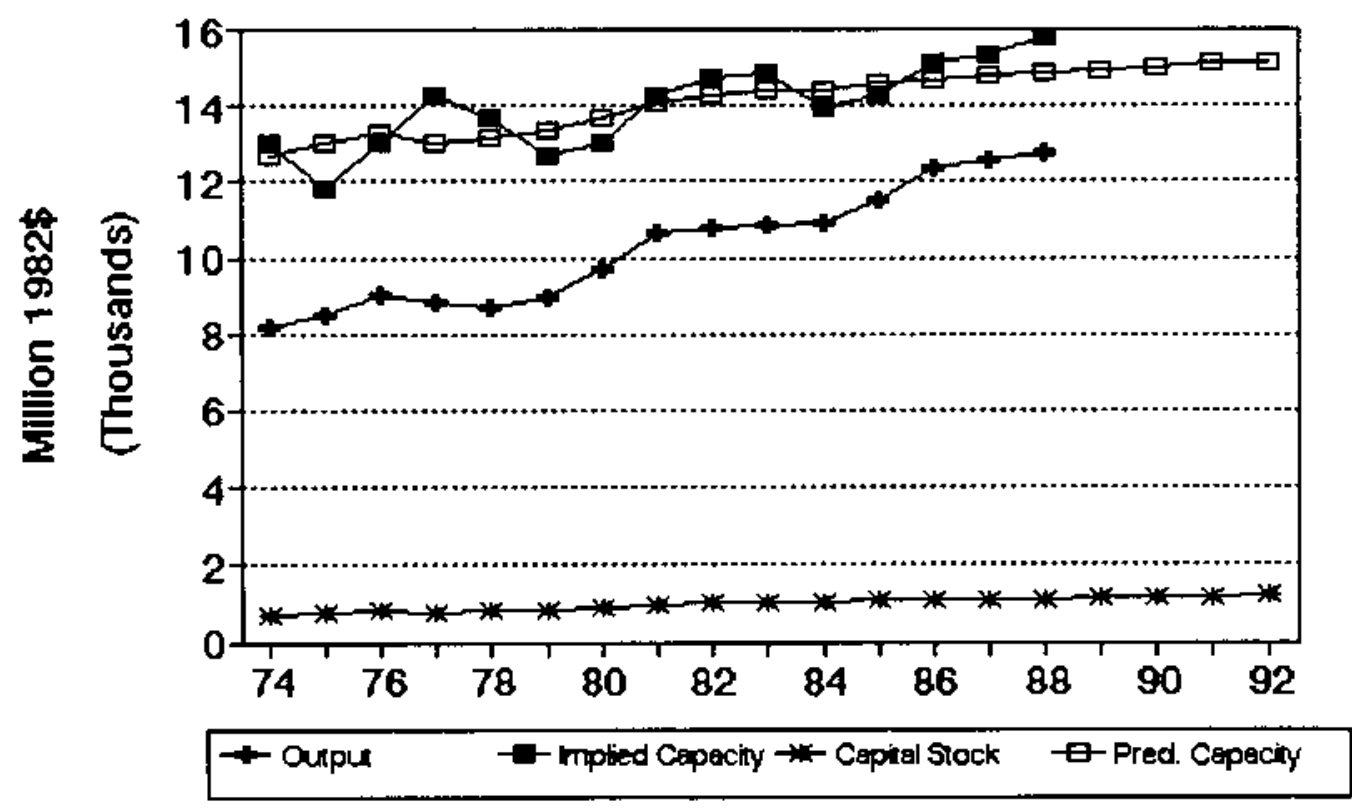




\section{No. 7 sIC 2023 Condensed and evaporated milk}

\section{MILLIONS OF 1982 DOLLARS (EXCL. CU RATES)}

$\begin{array}{lccccccc} & 04 & \text { Ann. Implied Gross } & \text { Net } & \text { Pred. } & \ldots & \ldots \ldots \\ \text { Gross } & \text { CU } & \text { CU } & \text { Prac. } & \text { In- } & \text { Cap. } & \text { Prac. } & \text { Emergency Capacity } \\ \text { Output } & \text { Rate } & \text { Rate } & \text { Capac. } & \text { vest. } & \text { Stk. } & \text { Capac. } & 3 \text { mon. } 6 \text { mon. }>6 \text { mon. }\end{array}$

\begin{tabular}{|c|c|c|c|c|c|c|c|c|c|c|}
\hline 1974 & 3,814 & 74 & 74.0 & 5,154 & 56.4 & 421 & 5,396 & & & \\
\hline 1975 & 3,855 & 62 & 66.5 & 5,797 & 81.4 & 460 & 5,713 & & & \\
\hline 1976 & 3,711 & 78 & 72.0 & 5,155 & 27.7 & 448 & 5,610 & & & \\
\hline 1977 & 4,591 & 80 & 79.3 & 5,792 & 110.5 & 516 & 6,167 & & & \\
\hline 1978 & 4,528 & 56 & 65.0 & 6,965 & 37.7 & 512 & 6,140 & & & \\
\hline 1970 & 4,311 & 65 & 61.6 & 6,995 & 93.9 & 562 & 6,542 & & & \\
\hline 1980 & 4,415 & 64 & 66.4 & 6,858 & 64.6 & 582 & 6,709 & & & \\
\hline 1981 & 4,852 & 68 & 66.5 & 7,296 & 112.3 & 648 & 7,250 & & & \\
\hline 1982 & 4,748 & 71 & 69.9 & 6,795 & 16.5 & 621 & 7,023 & & & \\
\hline 1983 & 5,753 & 74 & 72.9 & $7,8 \%$ & 99.7 & 672 & 7,445 & & & \\
\hline 1984 & 5,143 & 74 & 74.0 & 6,950 & $(15.7)$ & 612 & 6,955 & & & \\
\hline 1985 & 5,381 & 79 & 77.1 & 6,978 & 138.9 & 699 & 7,666 & & & \\
\hline 1986 & 5,898 & 72 & 74.6 & 7,904 & 85.5 & 730 & 7,917 & & & \\
\hline - - - & $\cdots$ & $\because$ & $\because \cdot$ & - - - - & $-\cdots$ & $\cdots$ & $\cdots \cdot$ & ----- & $\cdots \cdot$ & 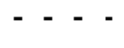 \\
\hline 1987 & 5,997 & 67 & 68.9 & 8,707 & 77.8 & 753 & 8,108 & & & \\
\hline 1988 & 6,127 & 74 & 71.4 & 8,585 & 89.6 & 787 & 8,382 & 9,513 & 9,992 & 10,092 \\
\hline 1989 & & & & & 100.7 & 829 & B, 725 & 9,903 & 10,402 & 10,505 \\
\hline 1990 & & & & & 107.6 & 875 & 9,099 & 10,327 & 10,848 & 10,956 \\
\hline 1991 & & & & & 109.4 & 919 & 9,460 & 10,737 & 11.278 & 11,390 \\
\hline 1992 & & & & & 108.5 & 959 & 9,787 & 11,108 & 11,668 & 11,783 \\
\hline
\end{tabular}

Capecity-Capital Stock Regression: Code 0 RSQ 0.790 Coefficients: Const 1852.5 stock 8.2

Ave. Copecity/Stock (74-86): 11.6 Ave. Weekly Hours (High) 126.0 Shift Factor 1.20 Ave Weekly Hours (LOW) 124.2 Shift Factor 1.22 Note: Output, Investment, and Capital Stock extrapolated for 1987 and 1988.

\section{Condensed and evaporated milk}

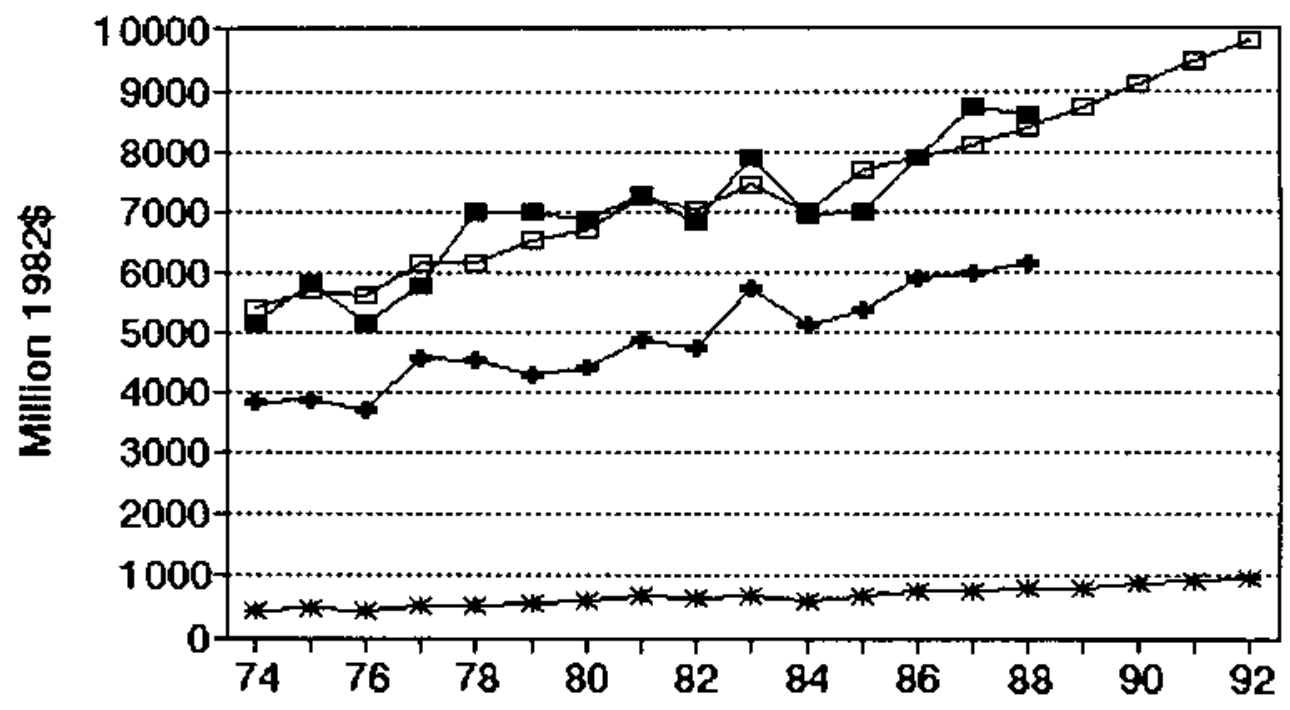

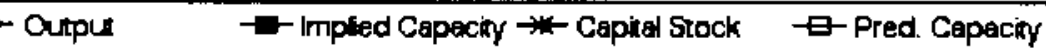


No. 8 sIC 2024 Ice crean and frozen desserta

MILLIONS OF 1982 DOLLARS (EXCL. CU RATES)

$\begin{array}{lccccccc} & \text { Q4 } & \text { Amn. } & \text { Implied Gross } & \text { Net } & \text { Pred. } & \ldots \\ \text { Gross } & \text { CU } & \text { CU } & \text { Prac. } & \text { In- } & \text { Cap. } & \text { Prac. } & \text { Emergency Capacity } \\ \text { Output Rate } & \text { Rate } & \text { Capac, } & \text { vest. } & \text { Stk. } & \text { Capac. } & 3 \text { mon. } 6 \text { mon. }>6 \text { mon. }\end{array}$

\begin{tabular}{|c|c|c|c|c|c|c|c|c|c|c|}
\hline 1974 & 2,865 & 57 & 57.0 & 5,026 & $(28.7)$ & 551 & 5,761 & & & \\
\hline 1975 & 2,970 & 59 & 58.3 & 5,099 & 40.7 & 536 & 5,938 & & & \\
\hline 1976 & 2,946 & 45 & 50.3 & 5,864 & 49.3 & 520 & 6,021 & & & \\
\hline 1977 & 3,012 & 53 & 50.0 & 6,023 & 102.4 & 570 & 5,543 & & & \\
\hline 1978 & 2,926 & 48 & 49.9 & 5,867 & 50.9 & 561 & 5,644 & & & \\
\hline 1979 & 2,986 & 46 & 46.8 & 6,387 & 28.9 & 533 & 5,973 & & & \\
\hline 1980 & 2,958 & 45 & 45.4 & 6,518 & 48.7 & 524 & 6,070 & & & \\
\hline 1981 & 2,965 & 48 & 46.9 & 6,324 & 56.0 & 522 & 6,094 & & & \\
\hline 1982 & 2,856 & 54 & 51.8 & 5,519 & 137.1 & 593 & 5,273 & & & \\
\hline 1983 & 2,915 & 59 & 57.1 & 5,103 & 51.0 & 581 & 5,413 & & & \\
\hline 1984 & 3,300 & 54 & 55.9 & 5,905 & 16.1 & 540 & 5,791 & & & \\
\hline 1985 & 3,293 & 57 & 55.9 & 5,893 & 46.9 & 534 & 5,861 & & & \\
\hline 1986 & 3,216 & 60 & 58.9 & 5,463 & 73.9 & 556 & 5,609 & & & \\
\hline 1987 & 3,238 & 62 & $\begin{array}{l}-\cdots \\
61.3\end{array}$ & D,286 & $\begin{array}{l}\cdots \\
58.4\end{array}$ & $\because 561$ & $\because \cdots$ & $\cdots$ & -- & - \\
\hline 1988 & 3,266 & 54 & 57.0 & 5,729 & 65.4 & 572 & 5,423 & 7,155 & 7,706 & 7,820 \\
\hline 1989 & & & & & 71.9 & 589 & 5,228 & 6,898 & 7,420 & 7,539 \\
\hline 1990 & & & & & 75.9 & 608 & 5,005 & 6,603 & 7,112 & 7,217 \\
\hline 1991 & & & & & 77.0 & 626 & 4,791 & 6,321 & 6,808 & 6,908 \\
\hline 1992 & & & & & 76.5 & 642 & 4,604 & 6,074 & 6,542 & 6,639 \\
\hline
\end{tabular}

Capacity-Capital Stock Regression: Code -1 RSO 0.270

Coefficients: Const 12185.4 Stock -11.7 o( $84-86)-92.1$ Ave. Capacity/Stock $(74-86): 10.5$ Ave. Weekly Hours (High) 104.6 Shift Factor 1.44 Ave Weekly Hours (Low) 99.6 Shift Factor 1.51 Note: Output, Investment, and Capital stock extrapolated for 1987 and 1988.

\section{4 lce cream and frozen desserts}

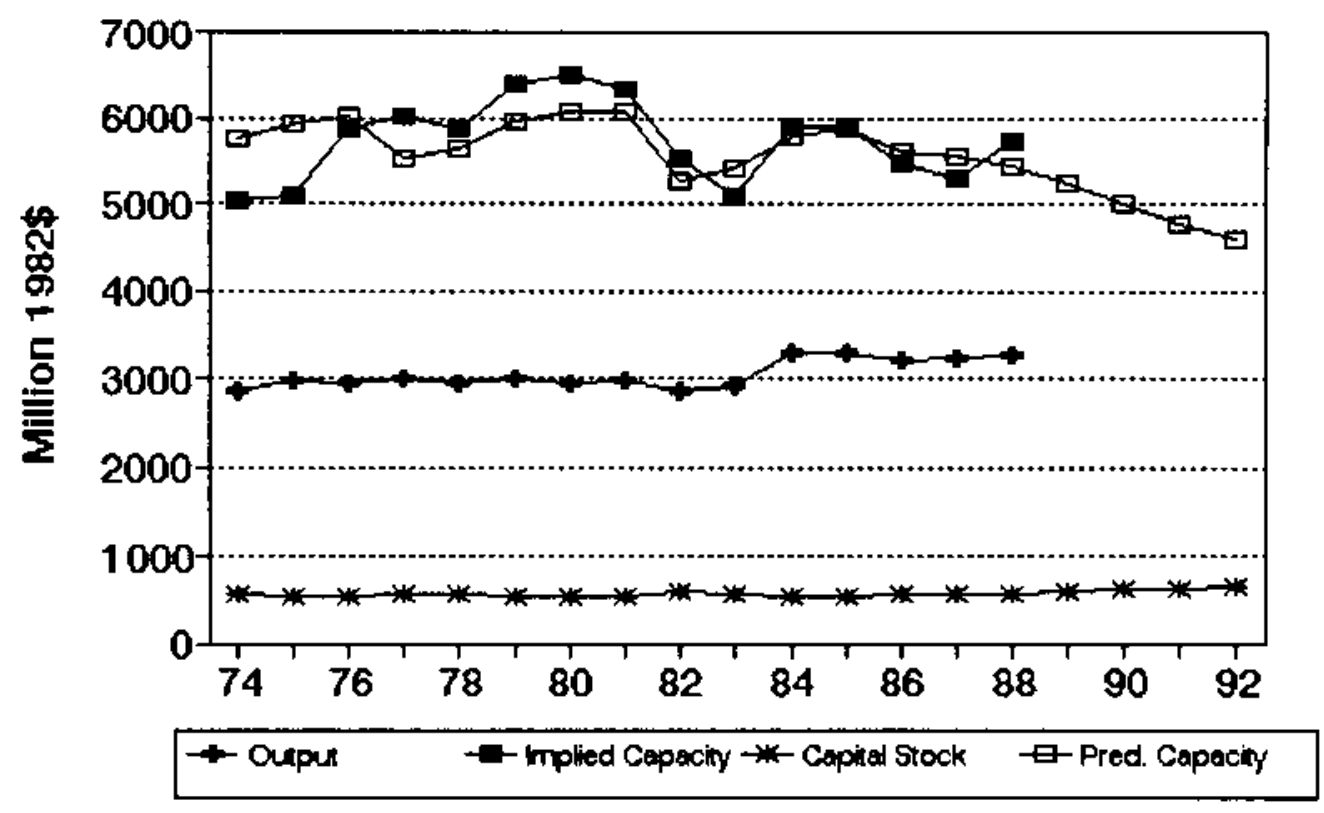


No. 9 sIC 2026 Fluid milk

MILLIONS OF 1982 DOLLARS (EXCL. CU RATES)

$\begin{array}{lccccccc} & 04 & \text { Am. Implied Gross } & \text { Net } & \text { Pred. } & -\ldots \ldots \ldots \\ \text { Gross } & \text { CU } & \text { CU } & \text { Prac. } & \text { In- } & \text { Cap. } & \text { Prac. } & \text { Emergency Capacity } \\ \text { Output } & \text { Rate } & \text { Rate } & \text { Capac. } & \text { vest. } & \text { StK. } & \text { Capec. } & 3 \text { mon. } 6 \text { mon. }>6 \text { mon. }\end{array}$

\begin{tabular}{|c|c|c|c|c|c|c|c|c|c|c|}
\hline 1974 & 18,471 & 71 & 71.8 & 25,734 & 323.7 & 2,566 & 26,096 & & & \\
\hline 1975 & 18,904 & 79 & 75.6 & 25,000 & 212.4 & 2,518 & 26,041 & & & \\
\hline 1976 & 19,260 & 71 & 73.9 & 26,063 & 130.8 & 2,401 & 25,907 & & & \\
\hline 1977 & 19,479 & 74 & 73.0 & 26,683 & 294.4 & 2,448 & 25,961 & & & \\
\hline 1978 & 19,283 & $\pi$ & 73.5 & 26,233 & 280.9 & 2,482 & 26,001 & & & \\
\hline 1979 & 18,223 & 72 & 72.3 & 25,191 & 18.1 & 2,278 & 25,766 & & & \\
\hline 1980 & 18,400 & 72 & 72.1 & 25,510 & 243.9 & 2,299 & 25,790 & & & \\
\hline 1981 & 17,817 & 71 & 71.6 & 24,897 & 210.6 & 2,286 & $25, \pi 75$ & & & \\
\hline 1982 & 18,714 & 64 & 66.6 & 28,119 & 276.5 & 2,332 & 25,827 & & & \\
\hline 1983 & 18,707 & 77 & 72.0 & 25,969 & 146.2 & 2,253 & 25,738 & & & \\
\hline 1984 & 19,039 & 72 & 74.1 & 25,700 & 200.5 & 2,229 & 25,710 & & & \\
\hline 1985 & 19,230 & 76 & 74.5 & 25,806 & 185.6 & 2,194 & 25,669 & & & \\
\hline 1986 & 19,043 & 76 & 76.1 & 25,011 & 188.6 & 2,164 & 25,635 & & & \\
\hline . . & $\begin{array}{l}--- \\
19,149\end{array}$ & 80 & $\because \overline{78.5}$ & $\begin{array}{l}--- \\
24,379\end{array}$ & $\ddot{220.4}$ & 2,166 & 25,637 & $--\cdot$ & ---- & $\cdots$ \\
\hline 1988 & 19,288 & 81 & 80.6 & 23,928 & 228.8 & 2,177 & 25,649 & 32,781 & 34,693 & 35,088 \\
\hline 1989 & & & & & 236.6 & 2,194 & 25,669 & 32,807 & 34,720 & 35,115 \\
\hline 1990 & & & & & 241.5 & 2,214 & 25,692 & 32,836 & 34,751 & 35,147 \\
\hline 1991 & & & & & 242.7 & 2,234 & 25,715 & 32,865 & 34,782 & 35,178 \\
\hline 1992 & & & & & 242.1 & 2,250 & 25,734 & 32,890 & 34,808 & 35,204 \\
\hline
\end{tabular}

Capecity-Capital Stock Regression: Code 0 RSO 0.030 Coefficients: Const 23148.5 stock 1.2

Ave. Copecity/stock $(74-86): 11.0$ Ave. Heekly Hours (High) 110.3 Shift Factor 1.37 Ave Heekly Hours (Low) 108.2 Shift Factor 1.39 Note: Output, Investment, and Capital Stock extrapolated for 1987 and 1988.

\section{Fluid milk}

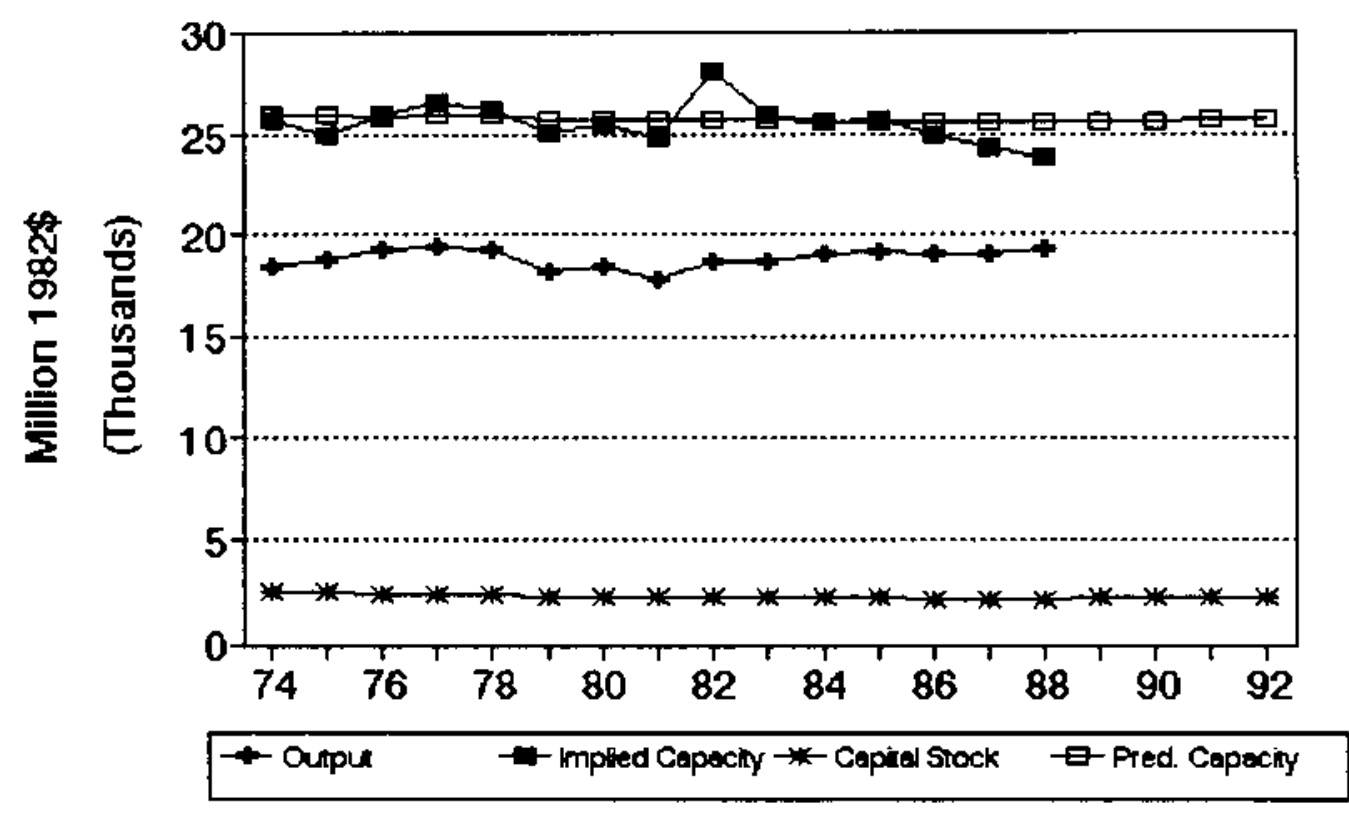


No. 10 SIC 2032 canned apecialties

MILLIONS OF 1982 DOLLARS (EXCL. CU RATES)

$\begin{array}{lcccclcc} & Q 4 & \text { Ann. } & \text { Implied Gross } & \text { Met } & \text { Pred. } & \ldots \ldots \ldots \\ \text { Gross } & \text { CU } & \text { CU } & \text { Prac. } & \text { In- } & \text { Cap. } & \text { Prac. } & \text { Energency Capacity } \\ \text { Output Rate } & \text { Rate } & \text { Capac. } & \text { vest. } & \text { Stk. } & \text { Capac. } & 3 \text { mon. } 6 \text { mon. }>6 \text { mon. }\end{array}$

\begin{tabular}{|c|c|c|c|c|c|c|c|c|c|c|}
\hline 1974 & 4,075 & 70 & 70.0 & 5,821 & 47.9 & 848 & 5,489 & & & \\
\hline 1975 & 4,112 & 71 & 70.6 & 5,822 & 79.8 & 851 & 5,501 & & & \\
\hline 1976 & 4,219 & 80 & 76.6 & 5,506 & 50.0 & 825 & 5,407 & & & \\
\hline 1977 & 3,985 & $\pi$ & 79.4 & 5,020 & 52.8 & 803 & 5,326 & & & \\
\hline 1978 & 4,204 & 83 & 81.5 & 5,158 & 78.7 & 807 & 5,340 & & & \\
\hline 1979 & 3,980 & $\pi$ & 78.0 & 5,103 & 83.3 & 815 & 5,371 & & & \\
\hline 1980 & 3,953 & 74 & 74.4 & 5,315 & 30.8 & 774 & 5,224 & & & \\
\hline 1981 & 4,008 & B2 & 79.0 & 5,074 & 84.6 & 783 & 5,255 & & & \\
\hline 1982 & 4,182 & $\pi 9$ & Bo.1 & 5,219 & 122.6 & 832 & 5,430 & & & \\
\hline 1983 & 4,249 & 80 & 79.6 & 5,336 & 72.8 & 829 & 5,421 & & & \\
\hline 1984 & 4,521 & 75 & 76.9 & 5,881 & 47.3 & 805 & 5,334 & & & \\
\hline 1985 & 4,193 & 77 & 76.3 & 5,490 & 83.1 & 817 & 5,370 & & & \\
\hline 1986 & 4,196 & $\pi$ & 78.3 & 5,362 & 144.9 & 891 & 5,642 & & & \\
\hline$-\cdot$ & $-\cdots$ & - - & $\cdots$ & $\cdots$ & $-\cdot$ & $\cdots$ & $\therefore$ & $\cdot-$ & $\cdots$ & $-\cdot$ \\
\hline 1987 & 4,205 & 83 & 81.5 & 5,159 & 89.7 & 906 & 5,697 & & & \\
\hline 1988 & 4,217 & 81 & 81.8 & 5,158 & 91.0 & 921 & 5,752 & 8,299 & 8,877 & 8,996 \\
\hline 1989 & & & & & 92.6 & 937 & 5,807 & 8,378 & 8,962 & 9,003 \\
\hline 1990 & & & & & 93.7 & 952 & 5,863 & 8,458 & 9,048 & 9,169 \\
\hline 1991 & & & & & 95.0 & 967 & 5,918 & 8,538 & 9.133 & 9,256 \\
\hline 1992 & & & & & 96.3 & 983 & 5,974 & 8,619 & 9.219 & 9,343 \\
\hline
\end{tabular}

Capacity-Capital Stock Regression: Code 0 RSO 0.140

Coefficients: Const 2439.0 stock 3.6

Ave. Capacity/Stock $(74-86): 6.6$ Ave. Heekly Hours (High) 96.5 Shift Factor 1.56 Ave Heekly Hours (Low) 92.9 Shift factor 1.62 Mote: Output, Investment, and Capital stock extrapolated for 1987 and 1988.

\section{Canned specialties}

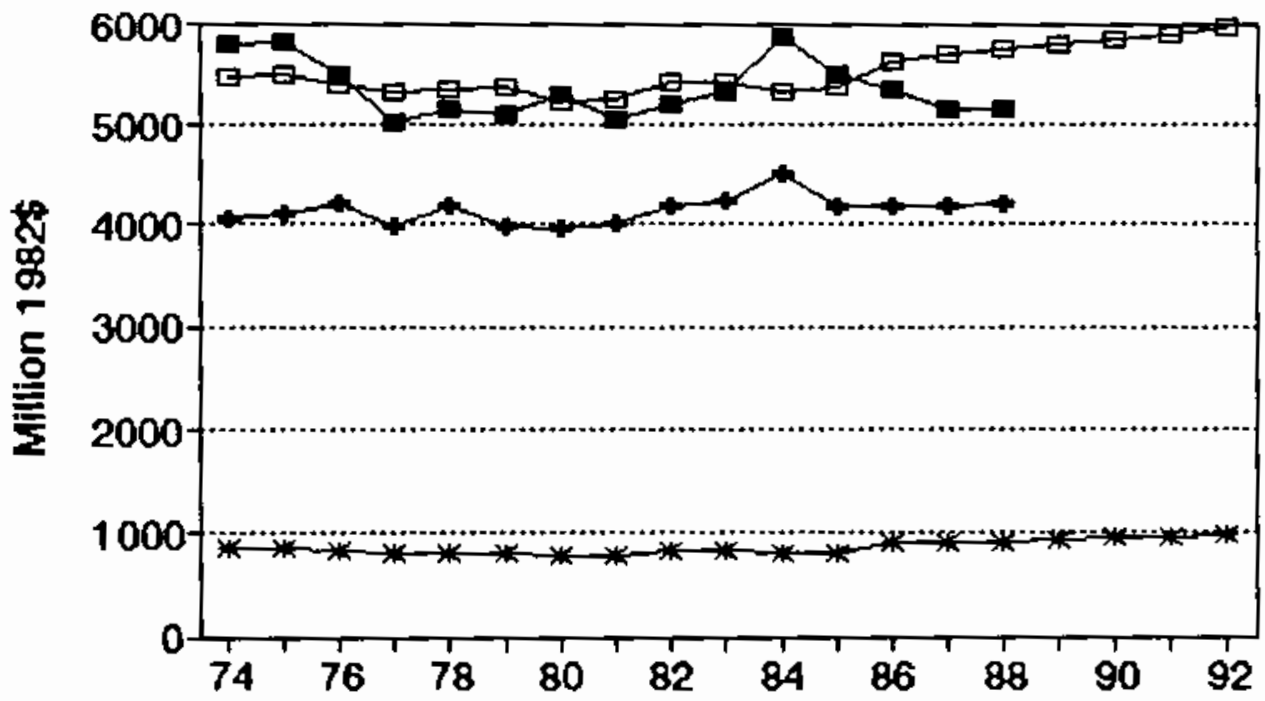

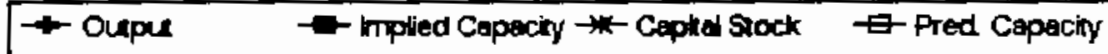




\section{11 sIc 2033 canned fruits and vegetables}

MILLIONS OF 1982 DOLLARS (EXCL. CU RATES)

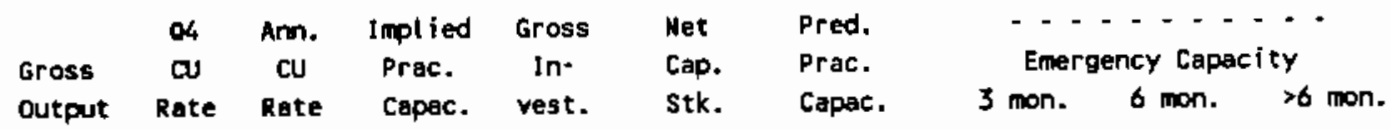

\begin{tabular}{|c|c|c|c|c|c|c|c|c|c|c|}
\hline 1974 & 9,840 & 56 & 56.0 & 17,571 & 167.3 & 2,023 & 14,521 & & & \\
\hline 1975 & 9,771 & 71 & 65.4 & 14,946 & 278.1 & 2,125 & 14,521 & & & \\
\hline 1976 & 9,993 & 69 & 69.8 & 14,327 & 171.5 & 2,115 & 14,521 & & & \\
\hline 1977 & 9,827 & 56 & 60.9 & 16,142 & 191.3 & 2,121 & 14,521 & & & \\
\hline 1978 & 9,500 & 61 & 59.1 & 16,067 & 222.6 & 2,155 & 14,521 & & & \\
\hline 1979 & 10,342 & 75 & 69.8 & 14,827 & 150.9 & 2,116 & 14,521 & & & \\
\hline 1980 & 9,959 & $\pi$ & $\pi .0$ & 13,279 & 233.8 & 2,157 & 14,521 & & & \\
\hline 1981 & 9,602 & 74 & 74.4 & 12,910 & 242.3 & 2,199 & 14,521 & & & \\
\hline 1982 & 9,170 & 72 & 72.8 & 12,605 & 188.1 & 2,186 & 14,521 & & & \\
\hline 1983 & 8,818 & 67 & 68.9 & 12,803 & 159.8 & 2,148 & 14,521 & & & \\
\hline 1984 & 10,187 & 76 & 72.6 & 14,026 & 119.8 & 2,072 & 15,223 & & & \\
\hline 1985 & 10,199 & 69 & 71.6 & 14,239 & 150.7 & 2,030 & 15,287 & & & \\
\hline 1986 & 10,334 & 54 & 59.6 & 17,332 & 155.1 & 1,991 & 15,351 & & & \\
\hline$-\cdots$ & --- & -- & -- & $-\cdot-$ & $\cdots$ & $-\cdots$ & $\because--$ & ----- & $-\quad--$ & $-\cdots$ \\
\hline 1987 & 10,362 & 60 & 57.8 & 17,943 & 146.2 & 1,945 & 15,415 & & & \\
\hline 1988 & 10,399 & $\pi$ & 70.6 & 14,725 & 140.2 & 1,898 & 15,479 & 21,452 & 23,018 & 23,342 \\
\hline 1989 & & & & & 134.2 & 1,849 & 15,542 & 21,541 & 23,113 & 23,438 \\
\hline 1990 & & & & & 128.2 & 1,799 & 15,606 & 21,629 & 23,208 & 23,534 \\
\hline 1991 & & & & & 122.2 & 1,748 & 15,670 & 21,718 & 23,303 & 23,631 \\
\hline 1992 & & & & & 116.2 & 1,696 & 15,734 & 21,806 & 23,398 & 23,727 \\
\hline
\end{tabular}

Capacity-Capital stock Regression: Code - 2 RSO 0.040

Coefficients: Const 14521.2 Time 63.8

Ave. Capacity/Stock $(74-86): 7.0$ Ave. Weekly Hours (High) 100.0 Shift factor 1.51 Ave Heekly Hours (Low) 99.2 Shift factor 1.52 Note; Output, Investment, and Capital stock extrapolated for 1987 and 1988.

\section{Canned fruits and vegetables}

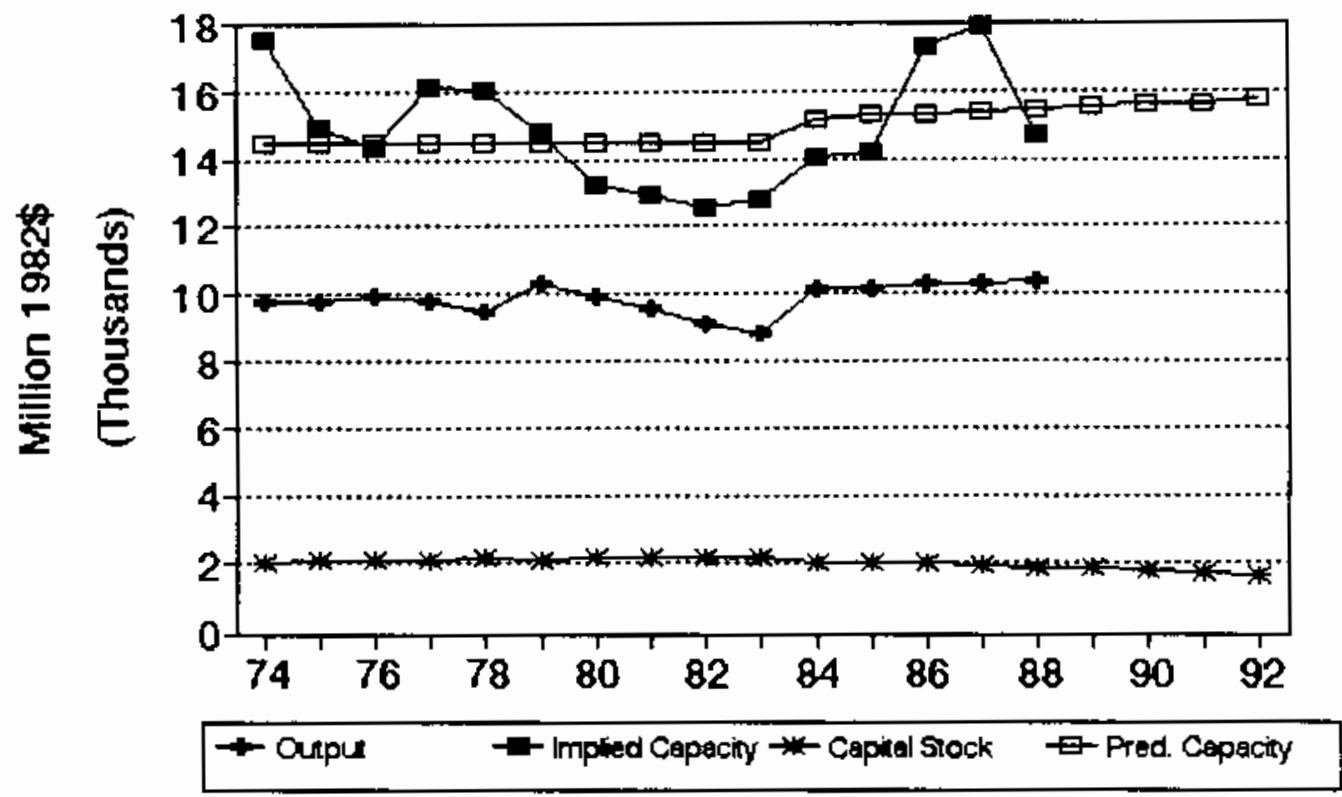


No. 12 sIC 2034 Dehydrated fruits, vegetables

MILLIOWS OF 1982 DOLLARS (EXCL. CU RATES)

$\begin{array}{lccccccc} & \text { Q4 } & \text { Ann. } & \text { Implied } & \text { Gross } & \text { Net } & \text { Pred. } & \ldots . \ldots . . . \\ \text { Gross } & \text { CU } & \text { CU } & \text { Prac. } & \text { In- } & \text { Cap. } & \text { Prac. } & \text { Emergency Capacity } \\ \text { Output } & \text { Rate } & \text { Rate } & \text { Capac. } & \text { vest. } & \text { Stk. } & \text { Capac. } & 3 \text { mon. } 6 \text { mon. }>6 \text { mon. }\end{array}$

\begin{tabular}{|c|c|c|c|c|c|c|c|c|c|c|}
\hline 1974 & 1,634 & 61 & 61.0 & 2,678 & 42.8 & 350 & 2,571 & & & \\
\hline 1975 & 1,737 & 62 & 61.6 & 2,819 & 61.1 & 382 & 2,571 & & & \\
\hline 1976 & 1,823 & 73 & 68.9 & 2,646 & 47.8 & 401 & 2,571 & & & \\
\hline 1977 & 1,689 & 80 & 77.4 & 2,183 & 30.4 & 403 & 2,426 & & & \\
\hline 1978 & 1,745 & 65 & 70.6 & 2,470 & 56.8 & 428 & 2,390 & & & \\
\hline 1979 & 1,351 & 72 & 69.4 & 1,948 & $\$ 9.1$ & 490 & 2,353 & & & \\
\hline 1980 & 1,758 & 80 & 77.0 & 2,283 & 23.3 & 477 & 2,317 & & & \\
\hline 1981 & 1,684 & 86 & 83.8 & 2,011 & 29.6 & 671 & 2,281 & & & \\
\hline 1982 & 1,852 & 88 & 87.3 & 2,123 & 53.2 & 485 & 2,244 & & & \\
\hline 1993 & 1,623 & 63 & 72.4 & 2,242 & (28.7) & 421 & 2,208 & & & \\
\hline 1984 & 1,529 & $66^{\circ}$ & 65.1 & 2,348 & 46.2 & 431 & 2,172 & & & \\
\hline 1985 & 1,850 & 78 & 73.6 & 2,512 & 83.5 & 474 & 2,136 & & & \\
\hline 1986 & 1,749 & 88 & 84.3 & 2,076 & 34.2 & 467 & 2,099 & & & \\
\hline$\because \cdot$ & $\because=$ & $-\cdot$ & 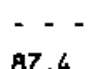 & 2011 & $\begin{array}{l}--- \\
538\end{array}$ & 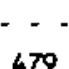 & $\cdots$ & $\because=$ & $\cdots$ & -- \\
\hline $\begin{array}{l}1988 \\
1988\end{array}$ & 1,767 & $B 7=$ & $\begin{array}{l}87.4 \\
86.8\end{array}$ & $\begin{array}{l}2,017 \\
2,037\end{array}$ & $\begin{array}{l}35.8 \\
61.8\end{array}$ & $\begin{array}{l}479 \\
499\end{array}$ & $\begin{array}{l}2,003 \\
2,027\end{array}$ & 2,650 & $2, \pi / 5$ & 2,801 \\
\hline 1989 & & & & & 69.3 & 525 & 1,990 & 2,602 & 2,725 & 2,751 \\
\hline 1990 & & & & & 73.9 & 553 & 1,954 & 2,555 & 2,676 & 2,700 \\
\hline 1991 & & & & & 75.1 & 579 & 1,918 & 2,508 & 2,626 & 2,650 \\
\hline 1992 & & & & & 74.5 & 603 & 1,881 & 2,460 & 2,576 & 2,600 \\
\hline
\end{tabular}

Capacity-Capital stock Regression: Code -2 RSQ 0.370

Coefficients: Const 2571.2 Time $\mathbf{- 3 6 . 3}$

Ave. Capacity/Stock $(74-86): 5.3$

Ave. Weekly Hours (High) 109.2 Shift Factor 1.38 Ave Neekly Hours (Low) 106.5 Shift factor 1.42

* Imputed Note: Output, Investment, and Capital Stock extrapolated for 1987 and 1988.

\section{Dehydrated fruits, vegetables}

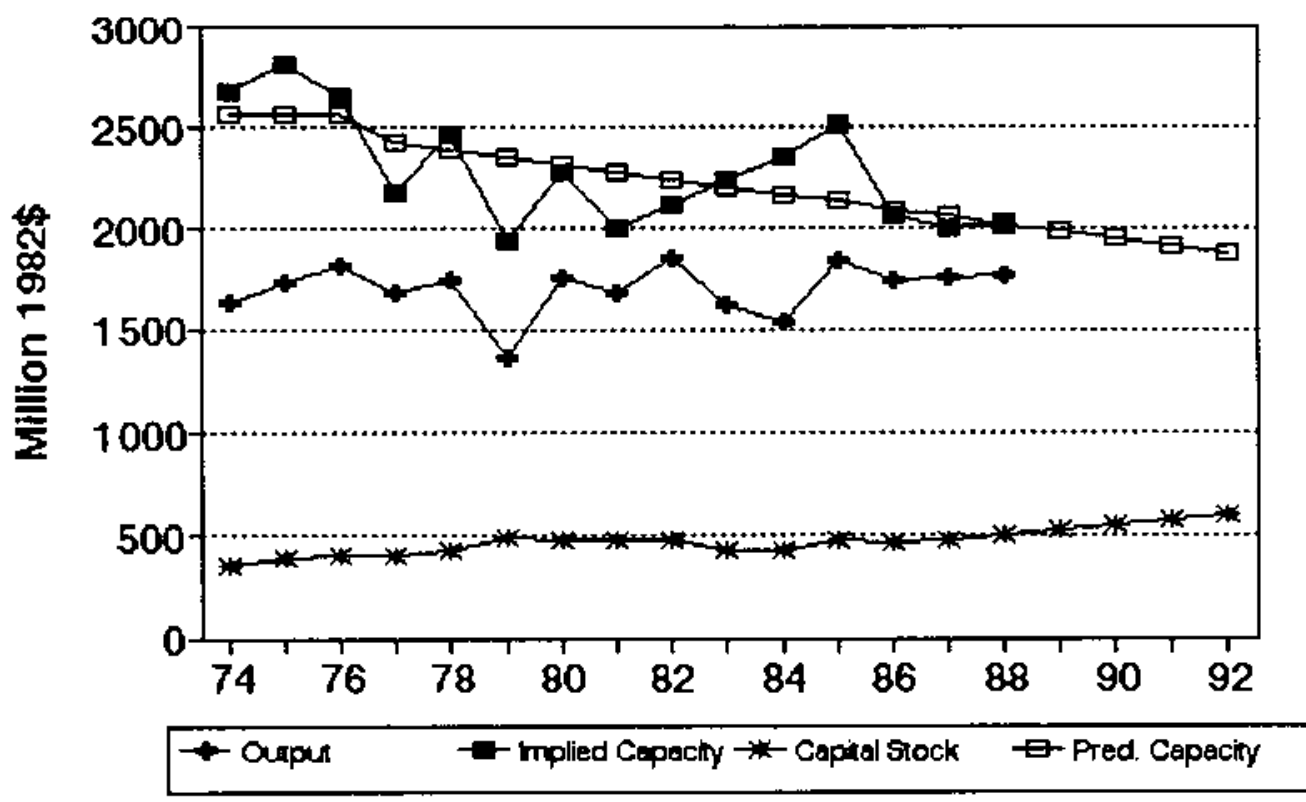




\section{NO. 13 sIC 2035 Pickles, sauces, and salad dressing}

MILLIOWS OF 1982 DOLLARS (EXCL. CU RATES)

$\begin{array}{lccccccc} & \text { Q4 } & \text { Am. } & \text { Implied Gross } & \text { Net } & \text { Pred. } & \ldots \ldots \ldots & \ldots \ldots \\ \text { Gross } & \text { CU } & \text { CU } & \text { Prac. } & \text { In- } & \text { Cap. } & \text { Prac. } & \text { Emergency Capacity } \\ \text { Output } & \text { Rate } & \text { Rate } & \text { Capac. } & \text { vest. } & \text { Stk. } & \text { Capac. } & 3 \text { mon. } 6 \text { mon. }>6 \text { mon. }\end{array}$

\begin{tabular}{|c|c|c|c|c|c|c|c|c|c|c|}
\hline 1974 & 2,363 & 54 & 54.0 & 4,376 & 69.5 & 441 & 4,146 & & & \\
\hline 1975 & 2,421 & 72 & 65.3 & 3,710 & 20.5 & 428 & 4,010 & & & \\
\hline 1976 & 2,386 & 74 & 73.3 & 3,257 & 29.2 & 426 & 3,959 & & & \\
\hline 1977 & 4,151 & 53 & 60.9 & 6,820 & 201.1 & 577 & 5,592 & & & \\
\hline 1978 & 4,261 & 59 & 56.8 & 7,500 & T2.1 & 602 & 5,852 & & & \\
\hline 1979 & 4,232 & 63 & 61.5 & 6,881 & 80.7 & 633 & 6,184 & & & \\
\hline 1980 & 4,287 & 78 & 72.4 & 5,923 & 60.6 & 644 & 6,296 & & & \\
\hline 1981 & 3,968 & 83 & 81.1 & 4.891 & 55.9 & 648 & 6,347 & & & \\
\hline 1982 & 4,285 & 78 & 79.9 & 5,364 & 56.1 & 652 & 6,386 & & & \\
\hline 1983 & 4,512 & 69 & 72.6 & 6,234 & 34.9 & 634 & 6,190 & & & \\
\hline 1984 & 4,384 & 67 & 67.8 & 6,471 & 74.6 & 654 & 6,407 & & & \\
\hline 1985 & 4,538 & 66 & 66.4 & 6,837 & 110.0 & 708 & 6,982 & & & \\
\hline 1986 & 4,812 & 60 & 62.3 & 7,730 & 121.6 & 771 & 7,651 & & & \\
\hline$-\cdot$ & $-\cdot-$ & - & $\cdots \cdot$ & --- & $-\cdot-$ & $\cdots \cdot$ & $-\cdot-$ & $\cdots \cdot \cdots$ & --- & $\cdots \cdot$ \\
\hline 1987 & 4,917 & 59 & 59.4 & 8,282 & 101.6 & 813 & 8,097 & & & \\
\hline 1988 & 5,058 & 70 & 65.9 & 7.678 & 126.9 & 877 & 8,776 & 12,670 & 13,749 & 13,971 \\
\hline 1989 & & & & & 150.6 & 960 & 9,655 & 13,938 & 15,125 & 15,370 \\
\hline 1990 & & & & & 165.2 & 1,051 & 10,620 & 15,332 & 16,637 & 16,907 \\
\hline 1991 & & & & & 169.1 & 1,139 & 11,552 & 16,677 & 18,097 & 18,390 \\
\hline 1992 & & & & & 167.1 & 1,218 & 12,391 & 17,889 & 19,412 & 19,726 \\
\hline
\end{tabular}

Capacity-Capital Stock Regression: code 0 RSO 0.640

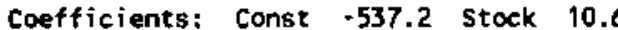

Ave. Capecity/Stock $(74-86): 9.7$ Ave. Heekly Hours (High) 94.8 Shift Factor 1.59 Ave Ueekly Hours (Low) 83.5 Shift Factor 1.81 Note; Output, Investment, and Capital stock extrapolated for 1987 and 1988.

\section{Pickles, sauces, and salad dressin}

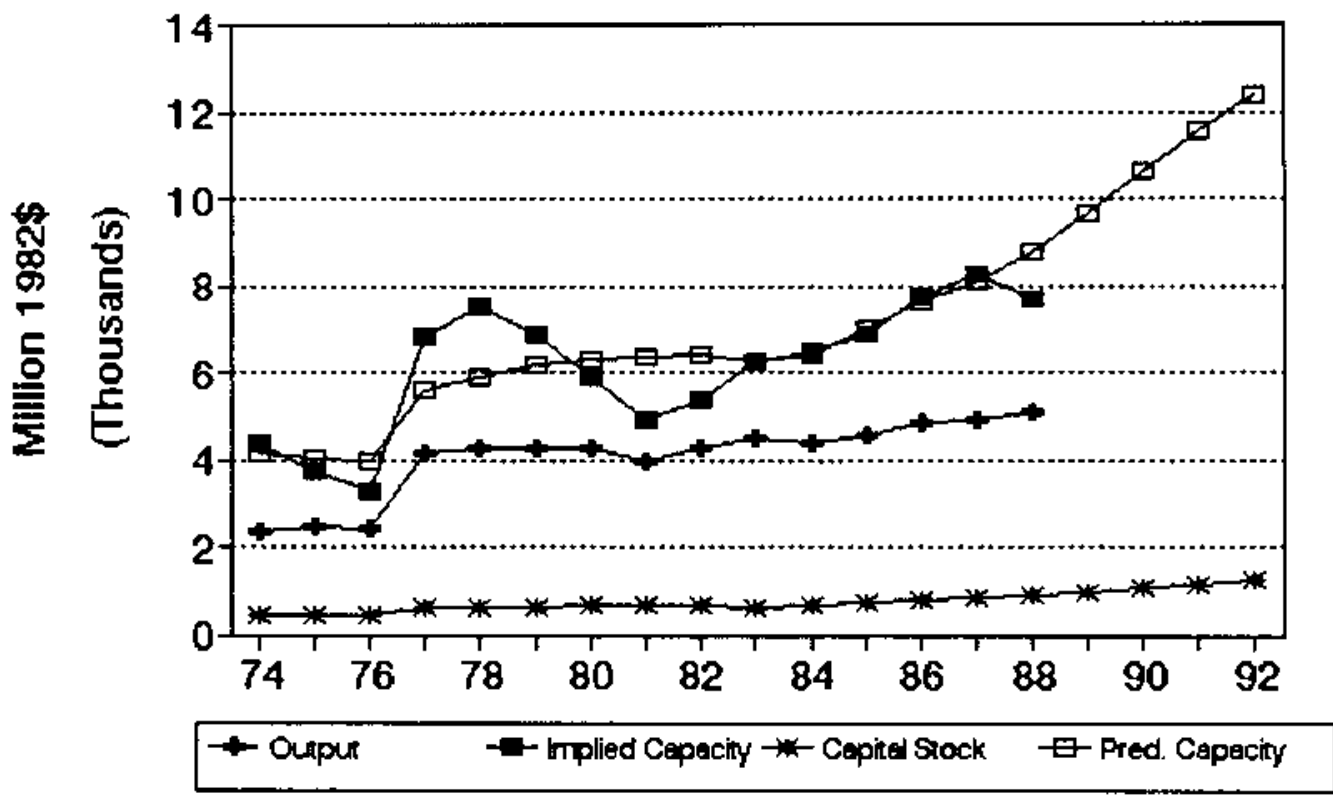




\section{No. 14 SIC 2037 Frozen fruita and vegetables}

\begin{tabular}{|c|c|c|c|c|c|c|c|c|c|}
\hline \multirow{3}{*}{$\begin{array}{l}\text { Gross } \\
\text { Output }\end{array}$} & \multicolumn{4}{|c|}{ MILLIDNS OF 1982 DOLLARS } & \multicolumn{2}{|c|}{ (EXCL. CU RATES) } & \multirow{2}{*}{\multicolumn{3}{|c|}{ Emergency Capacity }} \\
\hline & $\begin{array}{l}04 \\
\omega\end{array}$ & $\begin{array}{c}\text { Ann. } \\
\omega\end{array}$ & $\begin{array}{l}\text { Impl ied } \\
\text { Prac. }\end{array}$ & $\begin{array}{c}\text { Gross } \\
\text { In- }\end{array}$ & $\begin{array}{l}\text { Het } \\
\text { Cap. }\end{array}$ & $\begin{array}{l}\text { Pred. } \\
\text { Prac. }\end{array}$ & & & \\
\hline & Rate & & Capac. & & Stk. & Capac. & 3 mon. & 6 mon. & $>6$ mon. \\
\hline 4,662 & 9 & 100.0 & 4,662 & 177.7 & 1,075 & 7,189 & & & \\
\hline 4,655 & 62 & 74.4 & 6,258 & 90.7 & 1,090 & 7,224 & & & \\
\hline 4,846 & 48 & 53.1 & 9,119 & 56.0 & 1,070 & 7,178 & & & \\
\hline 5,012 & 75 & 65.2 & 7,688 & 185.9 & 1,171 & 7,407 & & & \\
\hline 5,463 & 86 & 82.3 & 6,635 & 98.2 & 1,184 & 7,437 & & & \\
\hline 5.156 & 58 & 68.3 & 7,556 & 68.8 & 1,168 & 7,400 & & & \\
\hline 5,171 & 60 & 59.6 & 8,674 & 137.1 & 1,214 & 7,505 & & & \\
\hline 5,118 & 58 & 59.3 & 8,634 & 136.1 & 1,254 & 7,594 & & & \\
\hline 5,522 & 58 & 57.8 & 9,549 & 278.7 & 1,424 & 7,979 & & & \\
\hline 5,515 & 80 & 71.4 & 7,724 & 188.1 & 1,494 & 8,139 & & & \\
\hline 5,537 & 66 & 72.0 & 7,693 & 133.0 & 1,508 & 8,169 & & & \\
\hline 5,207 & 71 & 69.2 & 7,525 & 139.7 & 1,526 & 8,212 & & & \\
\hline 5,519 & 67 & 68.9 & 8,006 & 159.6 & 1,560 & 8,287 & & & \\
\hline 5,572 & $\cdots$ & 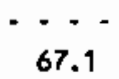 & $\begin{array}{c}\cdots, 300 \\
8,-\cdots\end{array}$ & $\cdots$ & 1,607 & 8,394 & $\cdots \cdot-$ & $--\cdot$ & $\cdots$ \\
\hline 5,642 & 73 & 70.7 & 7,983 & 176.1 & 1,655 & 8,502 & 9,326 & 9,848 & 9,955 \\
\hline & & & & 180.1 & 1,702 & 8,610 & 9.444 & 9,973 & 10,082 \\
\hline & & & & 184.2 & 1.750 & 8,718 & 9,563 & 10,098 & 10,209 \\
\hline & & & & 188.2 & 1,798 & 8,827 & 9,683 & 10,225 & 10,337 \\
\hline & & & & 192.2 & 1,867 & 8,937 & 9,803 & 10,351 & 10,465 \\
\hline
\end{tabular}

Capacity-Capitat Stock Regression: Code O RSO 0.110

Coefficients: Const 4756.5 Stock 2.3

Ave. Capacity/Stock $(74-86): 6.0$ Ave. Weekly Hours (High) 129.6 Shift Factor 1.17 Ave Ueekly Hours (Low) 126.6 Shift Factor 1.20 Note: Output, Investment, and Capital Stock extrapolated for 1987 and 1988.

\section{Frozen fruits and vegetables}

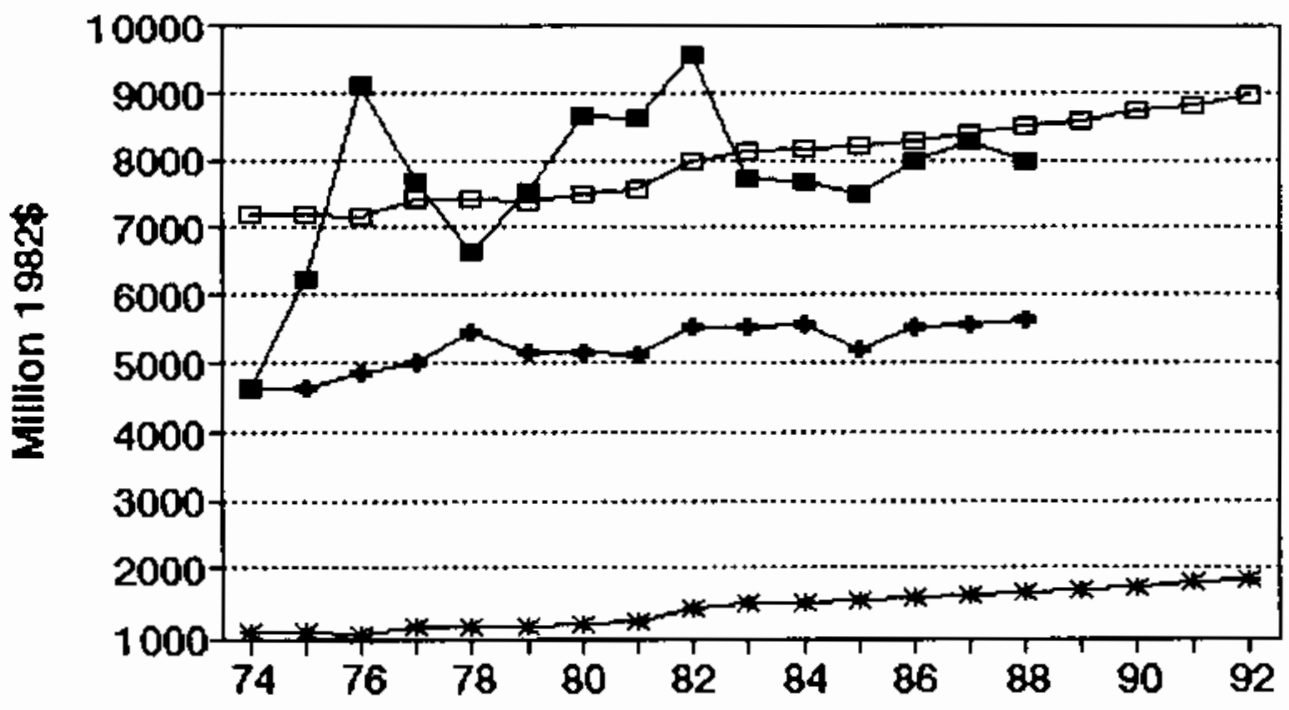

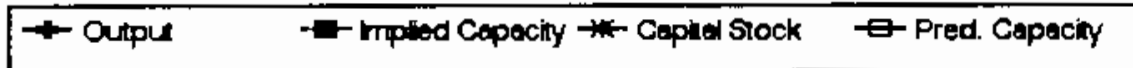




\section{yo. 15 SIC 2038 Fromen specialties}

MILLIONS OF 1982 DOLLARS (EXCL. CU RATES)

$\begin{array}{lccccccc} & \text { Q4 Ann. Implied Gross } & \text { Net } & \text { Pred. } & \ldots \ldots \ldots \\ \text { Gross } & \text { CU } & \text { CU } & \text { Prac. } & \text { In- } & \text { Cap. } & \text { Prac. } & \text { Emergency Capacity } \\ \text { Output } & \text { Rate } & \text { Rate } & \text { Capac. } & \text { vest. } & \text { Stk. } & \text { Capac. } & 3 \text { mon. } 6 \text { mon. }>6 \text { mon. }\end{array}$

\begin{tabular}{|c|c|c|c|c|c|c|c|c|c|c|}
\hline 1974 & 5,137 & 84 & 84.9 & 6,048 & 128.7 & 835 & 6.823 & & & \\
\hline 1975 & 4,725 & 66 & 72.4 & 6,531 & 80.8 & 852 & 6.866 & & & \\
\hline 1976 & 5,188 & 71 & 69.0 & 7,514 & 89.4 & 876 & 6,925 & & & \\
\hline 1977 & 5,419 & 73 & 73.6 & 7,360 & 152.3 & 953 & 7,118 & & & \\
\hline 1978 & 5,815 & $\pi$ & 73.9 & 7.870 & 152.0 & 1,022 & 7,294 & & & \\
\hline 1979 & 5,363 & 74 & 73.6 & 7,287 & 110.3 & 1,052 & 7,368 & & & \\
\hline 1980 & 5,056 & 70 & 71.6 & 7,059 & 90.0 & 1,048 & 7,359 & & & \\
\hline 1981 & 4,753 & 64 & 66.4 & 7,156 & 50.5 & 1,008 & 7,259 & & & \\
\hline 1982 & 5,091 & 73 & 69.6 & 7,317 & 127.4 & 1,042 & 7,345 & & & \\
\hline 1983 & 5,438 & 67 & 69.2 & 7,860 & 156.3 & 1,099 & 7,487 & & & \\
\hline 1984 & 5,769 & 74 & 71.6 & 8,062 & 122.1 & 1,124 & 7,550 & & & \\
\hline 1985 & 5,761 & 75 & 74.6 & 7,719 & 206.8 & 1,227 & 7,811 & & & \\
\hline 1986 & 5,772 & 80 & 78.3 & 7,373 & 162.6 & 1,284 & 7,853 & & & \\
\hline$-\cdots$ & $\because \cdot-$ & - & $-\cdots$ & $\because \cdots$ & $-\cdots$ & $\cdots$ & $\cdots$ & ---- & $\cdot \cdot \cdot$ & $-\cdots$ \\
\hline 1987 & 5,832 & 75 & 76.9 & 7,582 & 145.1 & 1,322 & 8,048 & & & \\
\hline 1988 & 5,911 & 82 & 79.4 & 7,449 & 164.5 & 1,376 & 8,184 & 11,620 & 12,429 & 12,596 \\
\hline 1989 & & & & & 182.6 & 1,443 & 8,355 & 11,862 & 12,688 & 12,858 \\
\hline 1990 & & & & & 193.8 & 1,517 & 8,539 & 12,124 & 12,968 & 13,142 \\
\hline 1991 & & & & & 196.7 & 1,587 & 8,716 & 12,375 & 13,236 & 13,414 \\
\hline 1992 & & & & & 195.3 & 1,649 & 8,874 & 12,599 & 13,476 & 13,657 \\
\hline
\end{tabular}

Capacity-Capital Stock Regression: Code 0 RSO 0.380

Coefficients: Const 4718.5 Stock 2.5

Ave. Capacity/Stock $(74 \cdot 86): 7.1$ Ave. Heekly Hours (High) 98.0 Shift Factor 1.54 Ave Heekly Hours (LOW) 95.9 Shift factor 1.57 Note: Output, Investment, and Capital Stock extrapolated for 1987 and 1988.

\section{Frozen specialties}

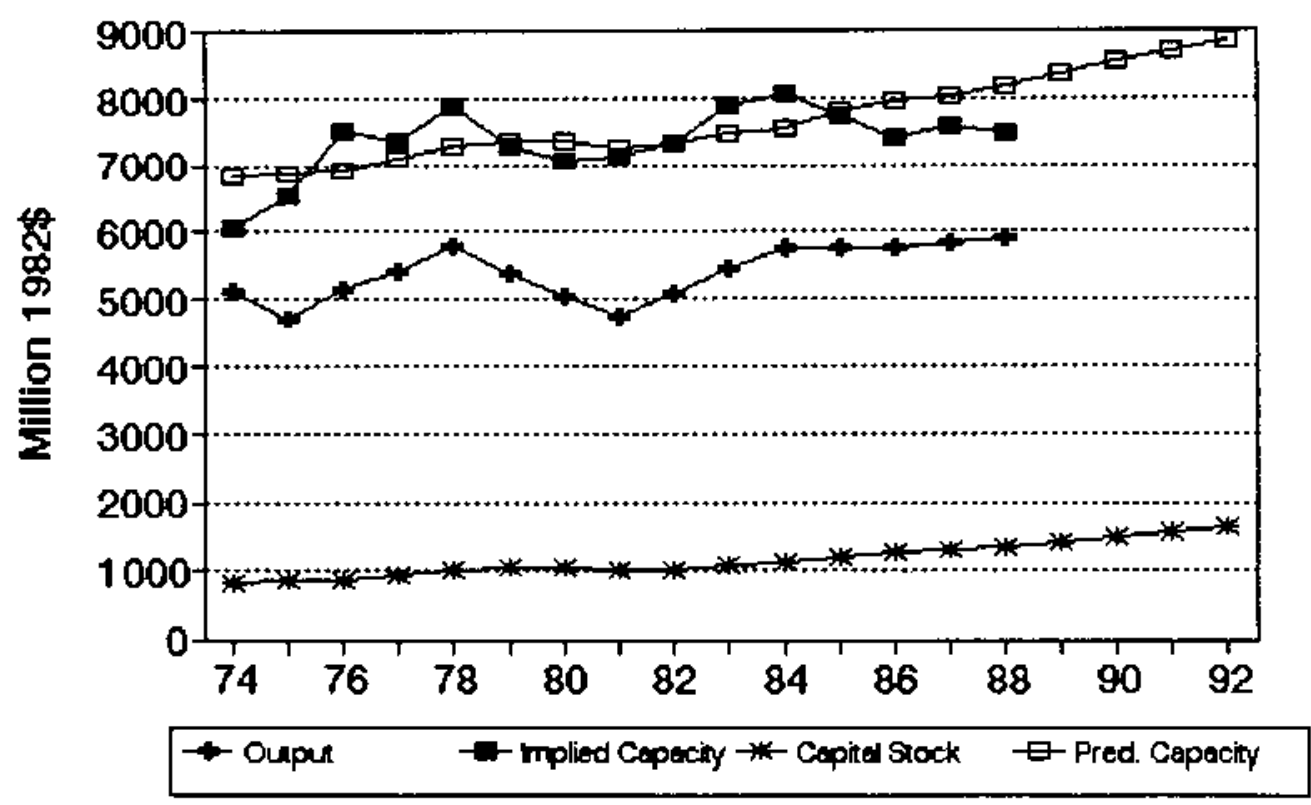


No. 16 SIC 2041 Flour, other grain mill products

MILLIONS OF 1982 DOLLARS (EXCL. CU RATES)

$\begin{array}{lccccccc} & \text { Q4 } & \text { Ann. Implied Gross } & \text { Net } & \text { Pred. } & \ldots \\ \text { Gross } & \text { CU } & \text { CU } & \text { Prac. } & \text { In- } & \text { Cap. } & \text { Proc. } & \text { Emergency Capacity } \\ \text { Output Rate } & \text { Rate } & \text { Capac. } & \text { vest. } & \text { Stk. } & \text { Capac. } & 3 \text { mon. } 6 \text { mon. }>6 \text { mon. }\end{array}$

\begin{tabular}{|c|c|c|c|c|c|c|c|c|c|c|}
\hline 1974 & 4,814 & 96 & 96.0 & 5,015 & 91.0 & 646 & 5,598 & & & \\
\hline 1975 & 4,929 & 89. & 91.4 & 5,393 & 106.9 & 687 & 6,349 & & & \\
\hline 1976 & 5,039 & 79 & 82.6 & 6,100 & 53.0 & 676 & 6,139 & & & \\
\hline 1977 & 5,286 & 78 & 78.4 & 6,745 & 56.9 & $\infty 69$ & 6.007 & & & \\
\hline 1978 & 4,792 & 72 & 74.3 & 6,453 & 79.5 & 683 & 6,263 & & & \\
\hline 1979 & 4,576 & 81 & 77.6 & 5,895 & 43.6 & 664 & 5,928 & & & \\
\hline 1980 & 4,786 & 85 & 83.5 & 5,732 & 67.8 & 668 & 5,987 & & & \\
\hline 1981 & 5,040 & 82 & 83.1 & 6,064 & 65.8 & 668 & 5,999 & & & \\
\hline 1982 & 4,929 & 72 & 75.8 & 6,507 & 116.4 & 714 & 6,844 & & & \\
\hline 1993 & 5,115 & 79 & 76.4 & 6,697 & 30.2 & 676 & 6,150 & & & \\
\hline 1984 & 5,256 & 79 & 79.0 & 6,653 & 93.5 & 698 & 6,548 & & & \\
\hline 1985 & 5,372 & 81 & 80.3 & 6,694 & 67.0 & 694 & 6,476 & & & \\
\hline 1986 & 5,545 & 86 & 84.1 & 6,592 & 58.3 & 682 & 6,250 & & & \\
\hline$\because-$ & $-\cdots$ & $\cdots$ & $\because-$ & 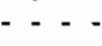 & $-\cdots$ & $\because \cdot$ & $\therefore-$ & $\because$ & $-\cdots$ & -- \\
\hline 1987 & 5,596 & 85 & 85.4 & 0,555 & 73.4 & 685 & 6,311 & & & \\
\hline 1988 & 5,663 & 90 & 88.1 & 6,427 & 73.9 & 689 & 6,373 & 6,270 & 6,355 & 6,373 \\
\hline 1989 & & & & & 74.3 & 692 & 6,437 & 6,332 & 6,419 & 6,437 \\
\hline 1990 & & & & & 74.5 & 695 & 6,498 & 6,392 & 6,480 & 6,498 \\
\hline 1991 & & & & & 74.6 & 698 & 6,554 & 6,448 & 6,536 & 6,554 \\
\hline 1992 & & & & & 74.5 & 701 & 6,604 & 6,497 & 6,586 & 6,604 \\
\hline
\end{tabular}

Capacity-Capital Stock Regression: Code 0 RSO 0.330 Coefficients: Const -6301.7 stock 18.4

Ave. Capacity/stock $(74-86): 9.1$ Ave. Weekly Hours (High) 160.8 Shift Factor 1.00 Ave Veekly Hours (Lou) 158.1 Shift Factor 1.02 - Imputed Note: Output, Imvestment, and Capital Stock extrapolated for 1987 and 1988.

\section{Flour, other grain mill products}

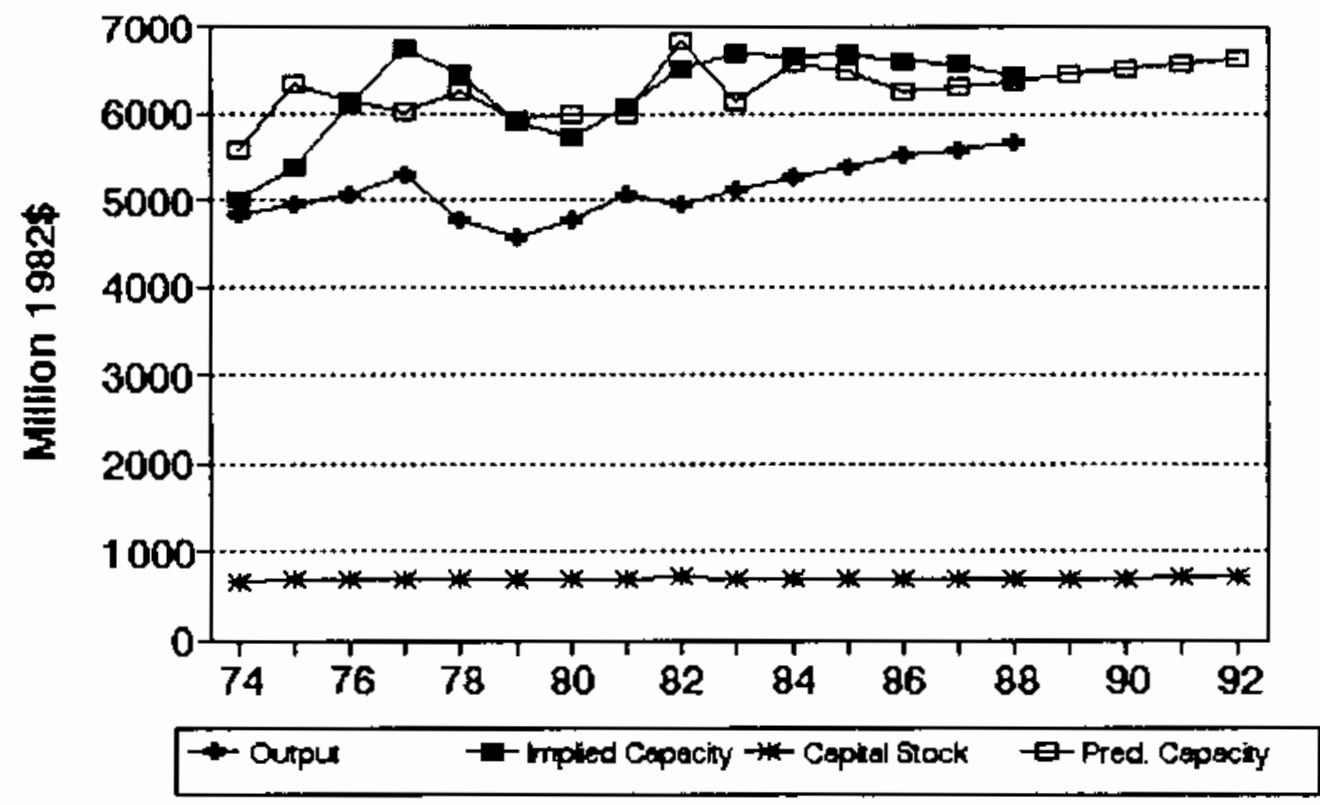




\section{No. 17 sIC 2043 Cereal breakfast foods}

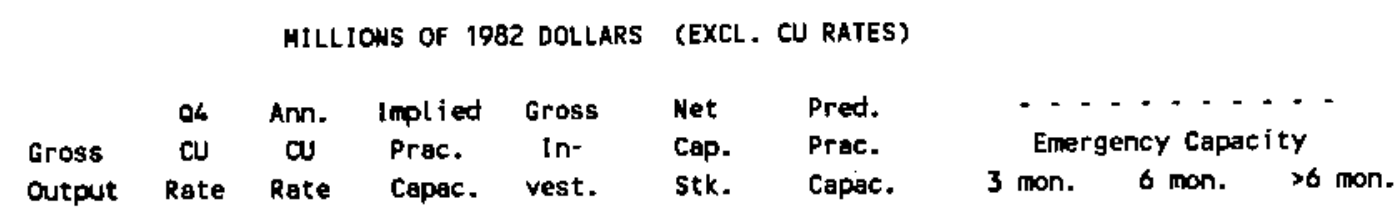

\begin{tabular}{|c|c|c|c|c|c|c|c|c|c|c|}
\hline 1974 & 3,234 & $90 *$ & 90.2 & 3,584 & 121.7 & 648 & 4,032 & & & \\
\hline 1975 & 3,403 & 84 & 86.3 & 3,942 & 90.8 & 687 & 4,150 & & & \\
\hline 1976 & 3,609 & 85 & 84.6 & 4,264 & 127.3 & 757 & 4,360 & & & \\
\hline 1977 & 3,909 & 85 & 85.0 & 4,599 & 168.3 & 863 & 4,681 & & & \\
\hline 1978 & 3,842 & 51 & 63.8 & 6,026 & 100.9 & 896 & 4,781 & & & \\
\hline 1979 & 4,036 & 79 & 68.5 & 5,892 & 129.1 & 956 & 4,962 & & & \\
\hline 1980 & 3,953 & 85 & 82.8 & 4,776 & 141.4 & 1,028 & 5,181 & & & \\
\hline 1981 & 3,993 & 81 & 82.5 & 4,840 & 116.6 & 1,072 & 5,313 & & & \\
\hline 1982 & 4,128 & 83 & 82.3 & 5,019 & 95.5 & 1,096 & 5,386 & & & \\
\hline 1983 & 4,444 & 81 & 81.8 & 5,437 & 188.2 & 1,204 & 5,714 & & & \\
\hline 1984 & 4,851 & 79 & 79.8 & 6,082 & 156.0 & 1,276 & 5,930 & & & \\
\hline 1985 & 5,109 & 82 & 80.9 & 6,317 & 200.7 & 1,386 & 6,262 & & & \\
\hline 1986 & 5,203 & 77 & 78.9 & 6,596 & 217.3 & 1,504 & 6,622 & & & \\
\hline - - - & $\cdots \cdot-$ & -- & $\cdots$ & $-\cdot-$ & $\cdots-$ & $-\cdot-\cdot$ & - - - - & $\cdots \cdots$ & $\cdots$ & $-\cdots$ \\
\hline 1987 & 5,330 & 84 & 81.4 & 6,550 & 165.6 & 1,572 & 6,824 & & & \\
\hline 1988 & 5,500 & 87 & 85.9 & 6,405 & 190.7 & 1,659 & 7,090 & 7,597 & 7,894 & 7,955 \\
\hline 1989 & & & & & 214.3 & 1,765 & 7,410 & 7,939 & 8,249 & 8,313 \\
\hline 1990 & & & & & 228.7 & 1,878 & 7,752 & 8,306 & 8,630 & 8,698 \\
\hline 1991 & & & & & 232.6 & 1,988 & 8,083 & 8,661 & 9,000 & 9,070 \\
\hline 1992 & & & & & 230.7 & 2,088 & $B, 38 B$ & 8,987 & 9,338 & 9,411 \\
\hline
\end{tabular}

Capacity-Capital Stock Regression: Code 0 RSA 0.700 Coefficients: Const 2071.5 stock 3.0

Ave. Capacity/Stock $(74-86): 5.0$ Ave. Heekly Hours (High) 135.6 Shift Factor 1.12 Ave Heekly Hours (LoW) 133.2 Shift Factor 1.14 * Imputed Note: Output, Investment, and Capital Stock extropolated for 1987 and 1988.

\section{Cereal breakfast foods}

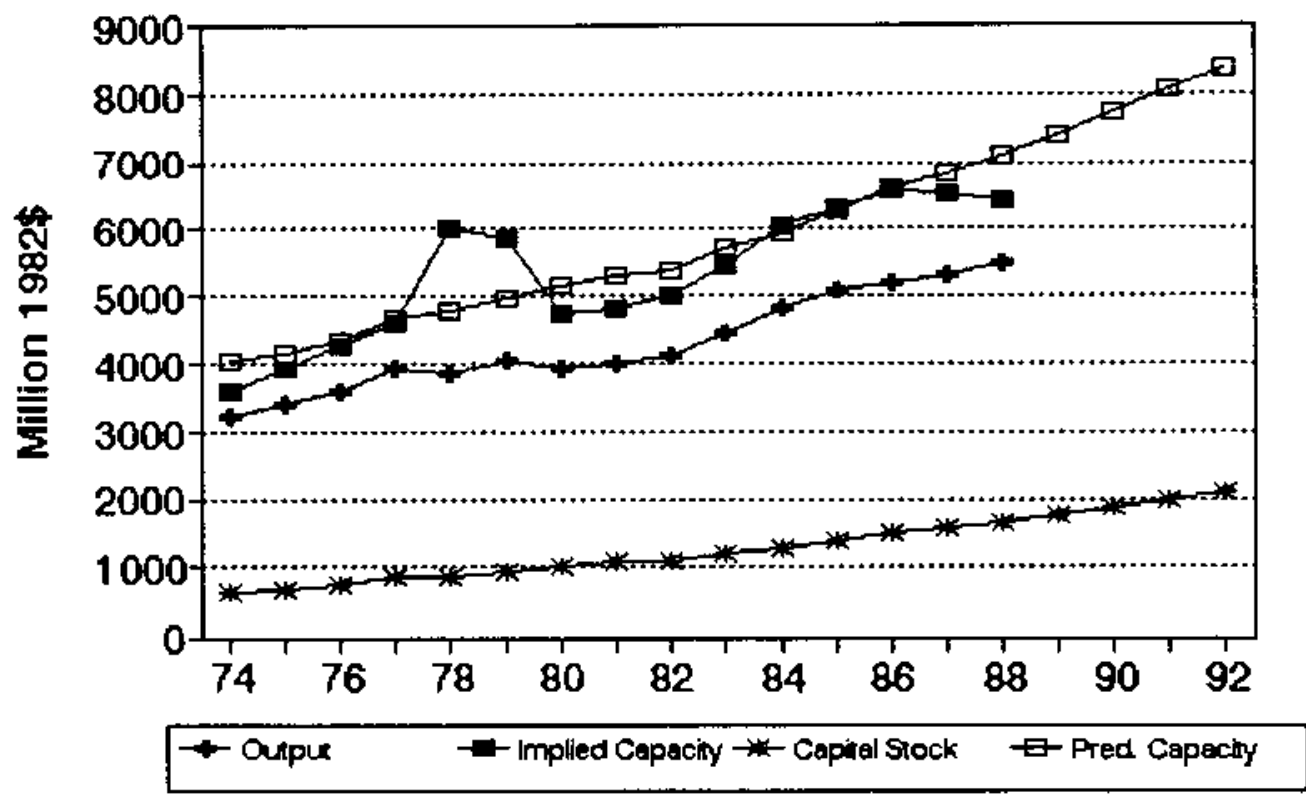




\section{No. 18 SIC 2044 Rice Dilling}

MILLIONS OF 1982 DOLLARS (EXCL. CU RATES)

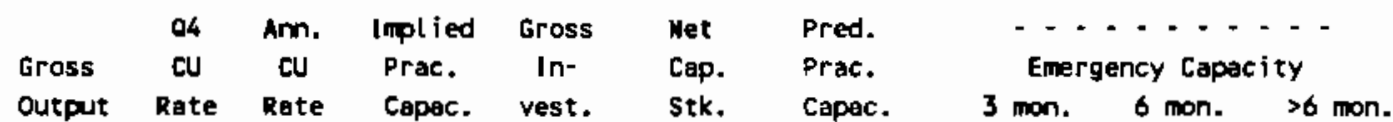

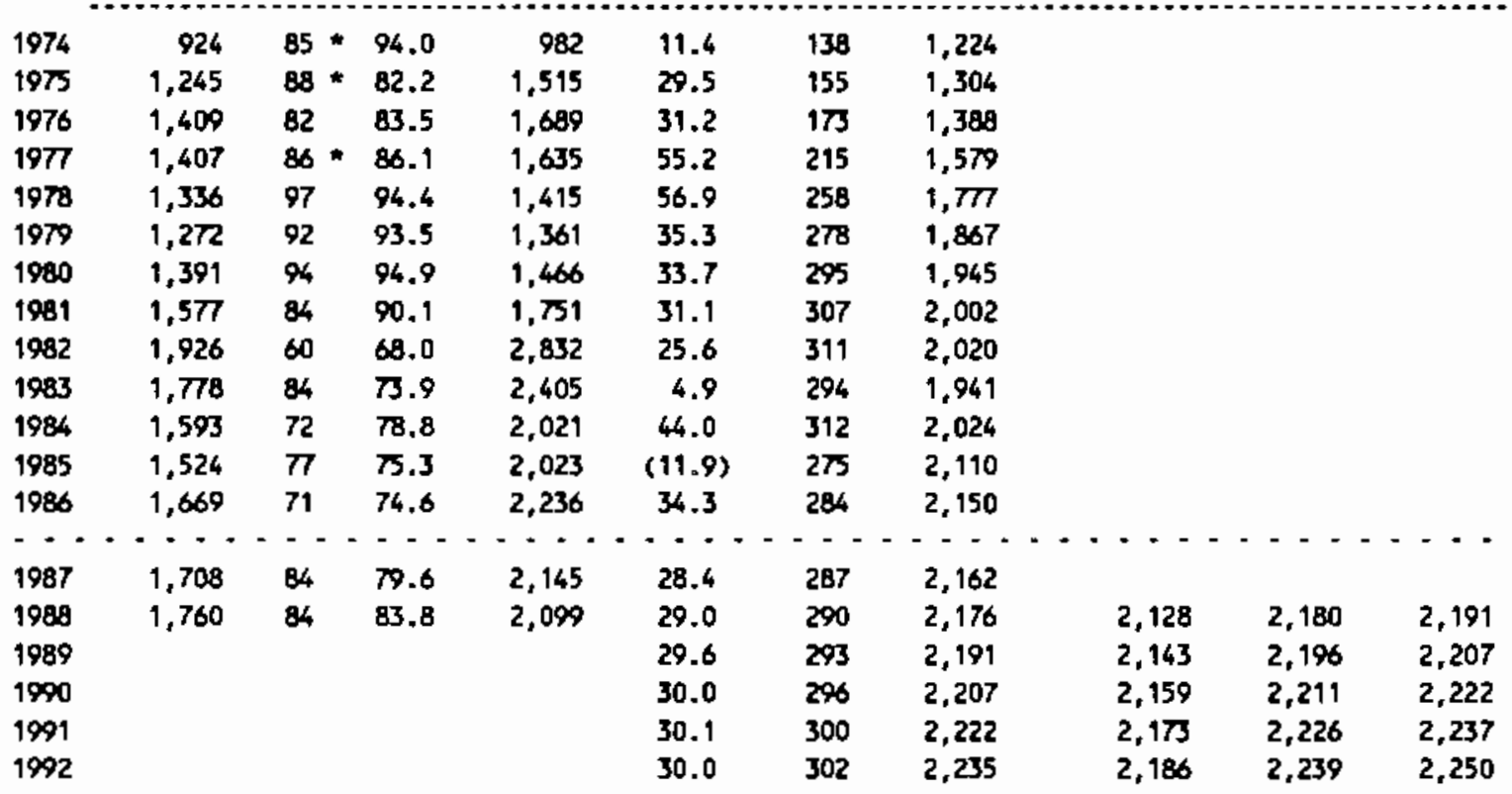

Capecity-Capital Stock Regression: Code - 1 RSO 0.410

Coefficients: Const 594.0 stock 4.6 D(B5-86) 255.3

Ave. Capacity/Stock $(74-86): 7.1$ Ave. Weekly Hours (High) 166.8 Shift Factor 1.01 Ave Weekly Hours (Lo) 157.2 Shift Factor 1.07 - Imputed Note: Output, Investment, and Capital stock extrapoleted for 1987 and 1988.

\section{Rice milling}

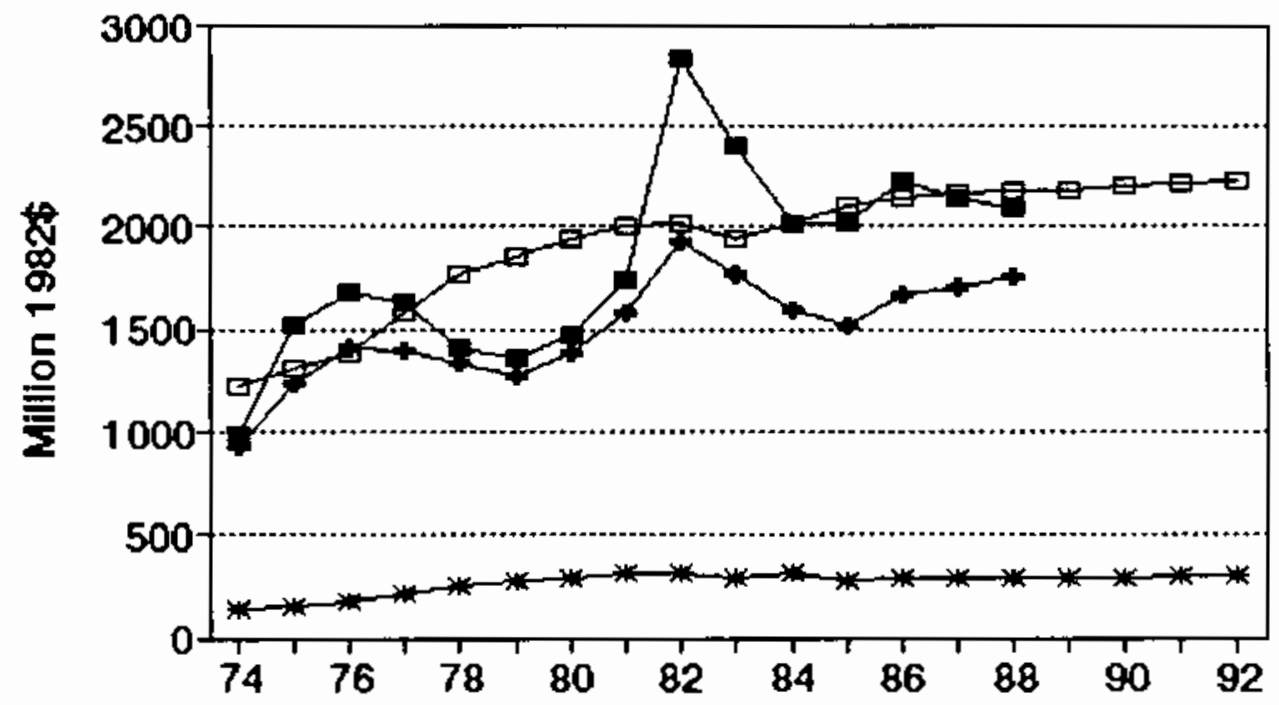

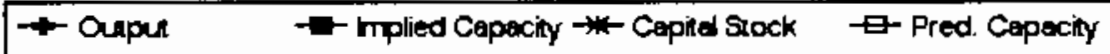


No. 19 sIC 2045 Blended and prepared flour

MILLIONS DF 1982 DOLLARS (EXCL. CU RATES)

$\begin{array}{lccccccc} & \alpha & \text { Ann. Implied } & \text { Gross } & \text { Net } & \text { Pred. } & \ldots \ldots \ldots \\ \text { Gross } & \text { CU } & \text { CU } & \text { Prac. } & \text { In- } & \text { Cap. } & \text { Prac. } & \text { Emergency Capacity } \\ \text { Output } & \text { Rate } & \text { Rate } & \text { Capac. } & \text { vest. } & \text { Stk. } & \text { Capac. } & 3 \text { mon. } 6 \text { mon. }>6 \text { mon. }\end{array}$

\begin{tabular}{|c|c|c|c|c|c|c|c|c|c|c|}
\hline 1974 & 1,674 & 82 & 89.4 & 1,872 & 19.3 & 225 & 1,808 & & & \\
\hline 1975 & 1,593 & 90 & 83.4 & 1,911 & 29.4 & 232 & 1,868 & & & \\
\hline 1976 & 1,634 & 82 & 84.4 & 1,935 & 13.5 & 224 & 1,799 & & & \\
\hline 1977 & 1,409 & 80 & 81.9 & 1,721 & $(0.3)$ & 204 & 1,623 & & & \\
\hline 1978 & 1,424 & 87 & 85.5 & 1,666 & 38.1 & 221 & 1,771 & & & \\
\hline 1979 & 1,410 & 78 & 81.0 & 1,740 & 5.8 & 207 & 1,652 & & & \\
\hline 1980 & 1,404 & 83 & 82.3 & 1,706 & 35.0 & 220 & 1,770 & & & \\
\hline 1981 & 1,367 & 82 & 84.3 & 1,622 & 24.4 & 222 & 1,787 & & & \\
\hline 1982 & 1,439 & 74 & 76.3 & 1,885 & 24.6 & 225 & 1,806 & & & \\
\hline 1983 & 1,518 & 77 & 73.2 & 2,018 & 92.4 & 292 & 2,392 & & & \\
\hline 1984 & 1,633 & 52 & 63.1 & 2,588 & 78.3 & 345 & 2,869 & & & \\
\hline 1985 & 1,611 & 51 & 51.5 & 3,129 & 23.8 & 343 & 2,835 & & & \\
\hline 1986 & 1,672 & 51 & 51.8 & 3,229 & 52.4 & 369 & 3,062 & & & \\
\hline $\begin{array}{l}--- \\
1987\end{array}$ & $\cdots \cdot$ & - & $\cdots$ & $\because--$ & $-\cdots$ & $\cdots$ & - - & $-\cdot$ & $\cdots-$ & -- \\
\hline $\begin{array}{l}1987 \\
1988\end{array}$ & 1,690 & 58 & 55.7 & 3,036 & 57.5 & 400 & 3,328 & & & \\
\hline 1988 & 1,713 & 58 & 57.9 & 2,958 & 60.7 & 432 & 3,603 & 4,269 & 4.631 & 4,706 \\
\hline 1989 & & & & & 63.9 & 464 & 3,886 & 4,604 & 4,995 & 5,075 \\
\hline 1990 & & & & & 67.2 & 498 & 4,177 & 4,948 & 5,368 & 5,455 \\
\hline 1991 & & & & & 70.4 & 532 & 4,474 & 5,301 & 5,750 & 5,843 \\
\hline 1992 & & & & & 73.7 & 567 & 4,778 & 5,661 & 6,141 & 6,240 \\
\hline
\end{tabular}

Capecity-Capital Stock Regression: Code 0 RSO 0.880

Coefficients: Const -143.9 Stock 8.7

Ave. Capecity/stock $(74-86): 8.1$ Ave. Heekly Hours (High) 115.7 Shift Factor 1.31 Ave Heekly Hours (Low) 112.6 Shift factor 1.34 Note: output, Investment, and Capital Stock extrapolated for 1987 and 1988.

\section{Blended and prepared flour}

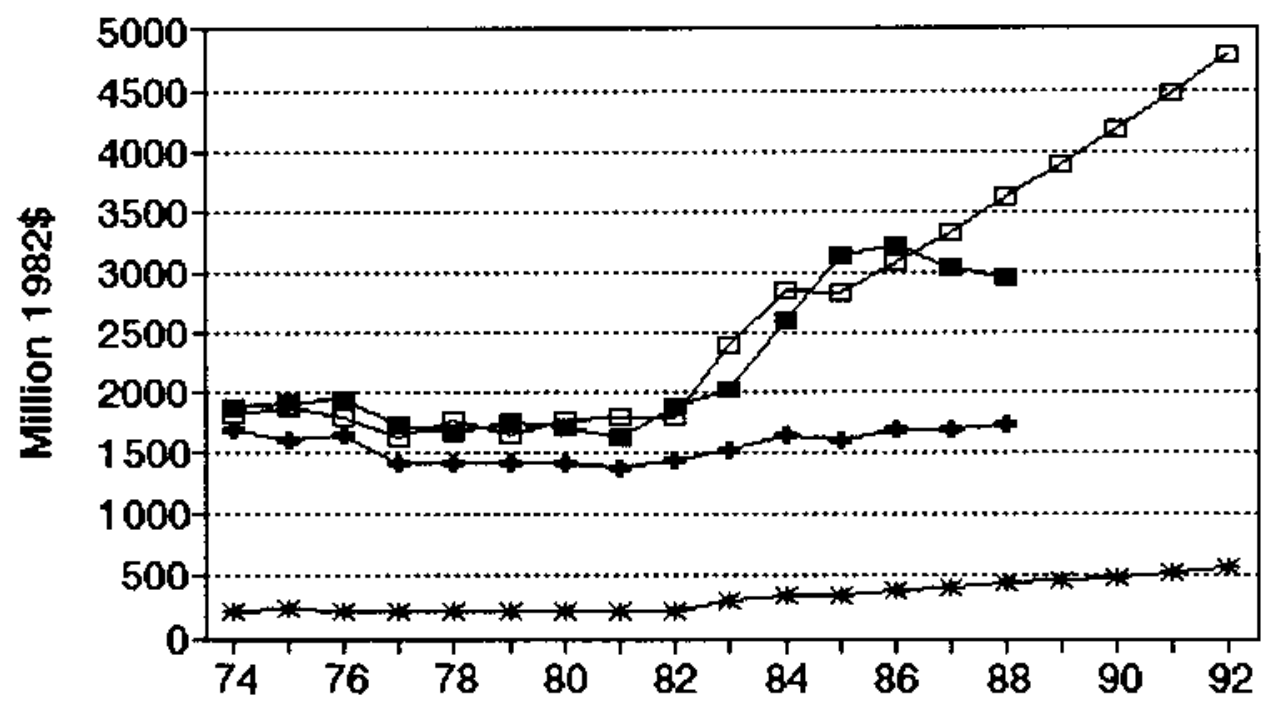

- Oupe $\quad-$ - mplied Capecity to- Cepiel Stock $\square$-Pred Cepecty 
No. 20 sIc 2046 wet corn milling

MILLIONS OF 1982 DOLLARS (EXCL. CU RATES)

$\begin{array}{lccccccc} & \text { Q4 } & \text { Ann. } & \text { Implied Gross } & \text { Net } & \text { Pred. } & \ldots . . \\ \text { Gross } & \text { CU } & \text { CU } & \text { Prac. } & \text { In- } & \text { Cap. } & \text { Prac. } & \text { Energency Capacity } \\ \text { Output Rate } & \text { Rate } & \text { Capac. } & \text { vest. } & \text { Stk. } & \text { Capac. } & 3 \text { mon. } 6 \text { mon. }>6 \text { mon. }\end{array}$

\begin{tabular}{|c|c|c|c|c|c|c|c|c|c|c|}
\hline 1974 & 2,104 & 99 & 100.0 & 2,104 & 155.1 & 1,509 & 1,800 & & & \\
\hline 1975 & 2,126 & 92 & 94.1 & 2,260 & 216.4 & 1,613 & 2,117 & & & \\
\hline 1976 & 2,386 & 88 & 89.4 & 2,669 & 220.9 & 1,720 & 2,444 & & & \\
\hline 1977 & 2,730 & 93 & 91.3 & 2,990 & 363.1 & 1,963 & 3,186 & & & \\
\hline 1978 & 2,814 & 94 & 93.8 & 2,909 & 282.1 & 2,116 & 3,652 & & & \\
\hline 1979 & 2,795 & 91 & 92.1 & 3,036 & 50.5 & 2,035 & 3,406 & & & \\
\hline 1980 & 3,107 & 92 & 91.8 & 3,384 & 210.3 & 2,105 & 3,619 & & & \\
\hline 1981 & 3,495 & 86 & 88.5 & 3,850 & 439.6 & 2,398 & 4,515 & & & \\
\hline 1982 & 3,279 & 90 & 88.4 & 3,709 & 181.6 & 2,413 & 4,558 & & & \\
\hline 1983 & 3,541 & 92 & 91.1 & 3,885 & $(31.8)$ & 2,217 & 3,962 & & & \\
\hline 1984 & 4,060 & 94 & 93.5 & 4,341 & 249.9 & 2,287 & 4,175 & & & \\
\hline 1985 & 5,781 & 84 & 87.8 & 6,587 & 323.8 & 2,430 & 4,610 & & & \\
\hline 1986 & 4,510 & 92 & 89.2 & 5,058 & 297.5 & 2,534 & 4,928 & & & \\
\hline$\cdots$ & $--\cdot$ & 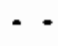 & $\cdots$ & $-\cdots$ & $\cdots$ & $-\cdots$ & $\cdots$ & -- & $\cdots$ & $-\cdot-$ \\
\hline 1987 & 4,692 & 93 & 92.7 & 5,062 & 308.4 & 2,651 & 5,285 & & & \\
\hline 1988 & 4,938 & 85 & 88.0 & 5,612 & 382.2 & 2,833 & 5,841 & 5,744 & 5,859 & 5,882 \\
\hline 1989 & & & & & 451.3 & 3,071 & 6,566 & 6,457 & 6,586 & 6,612 \\
\hline 1990 & & & & & 495.8 & 3,333 & 7,366 & 7,244 & 7,388 & 7,418 \\
\hline 1991 & & & & & 505.2 & 3,587 & 8,140 & 8,005 & 8,165 & 8,197 \\
\hline 1992 & & & & & 499.5 & 3,815 & 8,839 & 8,692 & 8,865 & 8,901 \\
\hline
\end{tabular}

Capacity-Capitel Stock Regression: Code 0 RSO 0.670

Coefficients: Const -2804.4 stock 3.1

Ave. Capacity/Stock (74-86): 1.7

Ave. Weekly Hours (High) 166.8 Shift Factor 1.01 Ave Weekly Hours (Low) 164.4 Shift Factor 1.02 Note: Output, Investment, and Capital Stock extrapolated for 1987 and 1988.

\section{Wet corn milling}

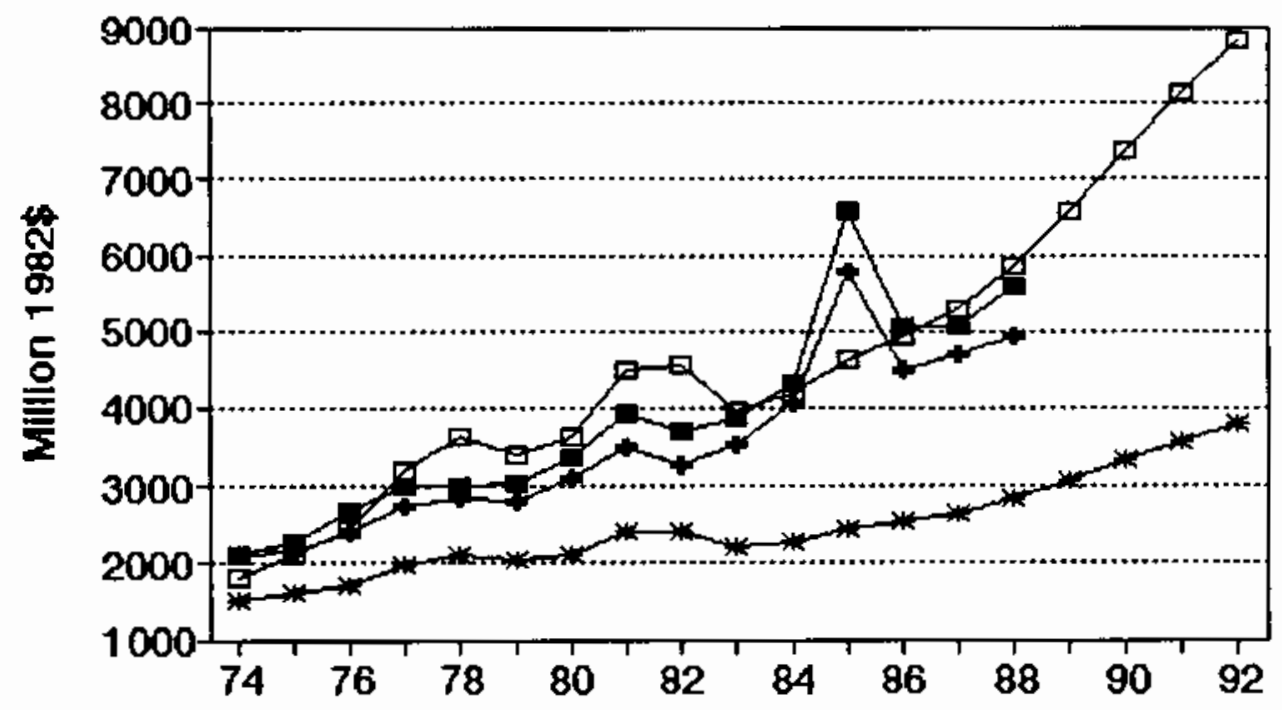

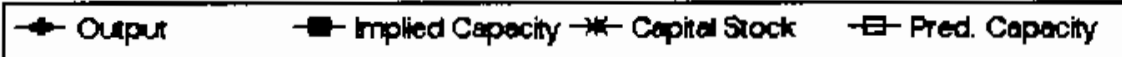




\section{No. 21 sIc 2047 Dog, cat, and other pet food}

MILLIONS OF 1982 DOLLARS (EXCL. CU RATES)

$\begin{array}{lccccccc} & Q 4 & \text { Ann. } & \text { Implied Gross } & \text { Het } & \text { Pred. } & \ldots \ldots \ldots \\ \text { Gross } & \text { CU } & \text { CU } & \text { Prac. } & \text { In- } & \text { Cap. } & \text { Prac. } & \text { Emergency Capacity } \\ \text { Dutput } & \text { Rate } & \text { Rate } & \text { Capac. } & \text { vest. } & \text { Stk. } & \text { Capac. } & 3 \text { mon. } 6 \text { mon. }>6 \text { mon. }\end{array}$

\begin{tabular}{|c|c|c|c|c|c|c|c|c|c|c|}
\hline 1974 & 2,821 & 81 & 81.0 & 3,483 & 155.3 & 816 & 4,963 & & & \\
\hline 1975 & 3,007 & 61 & 68.5 & 4,390 & 167.5 & 911 & 5,770 & & & \\
\hline 1976 & 3,441 & 48 & 52.9 & 6,508 & 50.8 & 885 & 5,551 & & & \\
\hline 1977 & 3,719 & 65 & 58.6 & 6,364 & 131.6 & 934 & 5,971 & & & \\
\hline 1978 & 3,863 & 76 & 71.9 & 5,375 & 108.6 & 958 & 6,176 & & & \\
\hline 1979 & 3,666 & 68 & 71.0 & 5,166 & 2.6 & 882 & 5,524 & & & \\
\hline 1980 & 4,026 & 68 & 68.0 & 5,920 & 99.3 & 899 & 5,668 & & & \\
\hline 1981 & 3,929 & 7 & 73.6 & 5,337 & 17.6 & 838 & 5,150 & & & \\
\hline 1982 & 4,417 & 72 & 73.9 & 5,979 & 171.1 & 917 & 5,827 & & & \\
\hline 1983 & 4,576 & 65 & 67.6 & 6,767 & 48.8 & $B 75$ & 5.463 & & & \\
\hline 1984 & 4,564 & 79 & 73.8 & 6,188 & 85.7 & 866 & 5,392 & & & \\
\hline 1985 & 5,472 & 72 & 74.6 & 7,333 & 81.0 & 854 & 7,751 & & & \\
\hline 1986 & 5,905 & 72 & 72.0 & 8,201 & 95.8 & 858 & 7,783 & & & \\
\hline$\cdots$ & $-\cdot-$ & -- & -- & & $\because-$ & -- & $\cdots$ & $-\cdot-$ & $--\cdot$ & $\cdots$ \\
\hline 1987 & 6,106 & 71 & 71.4 & 8,555 & 103.3 & 869 & 7,874 & & & \\
\hline 1988 & 6,378 & 71 & 71.0 & 8,983 & 105.9 & 881 & 7,977 & 8,779 & 9.119 & 9,189 \\
\hline 1989 & & & & & 108.3 & 894 & 8,089 & 8,903 & 9.248 & 9,319 \\
\hline 1990 & & & & & 109.8 & 907 & 8,202 & 9,027 & 9,377 & 9,449 \\
\hline 1991 & & & & & 110.2 & 919 & 8,306 & 9,141 & 9,496 & 9,569 \\
\hline 1992 & & & & & 110.0 & 930 & 8,397 & 9,242 & 9,600 & 9.674 \\
\hline
\end{tabular}

Capacity-Capital Stock Regression: Code -1 RSQ 0.510

Coefficients: Const -2005.1 Stock 8.5 D(85-86) 2461.3

Ave. Capacity/Stock $(74-86): 6.7$ Ave. Weekly Hours (High) 131.9 Shift Factor 1.15 Ave Weekly Hours (Low) 126.2 Shift Factor 1.20 Note: Dutput, Investment, and Cepital Stock extrapolated for 1987 and 1988.

\section{Dog, cat, and other pet food}

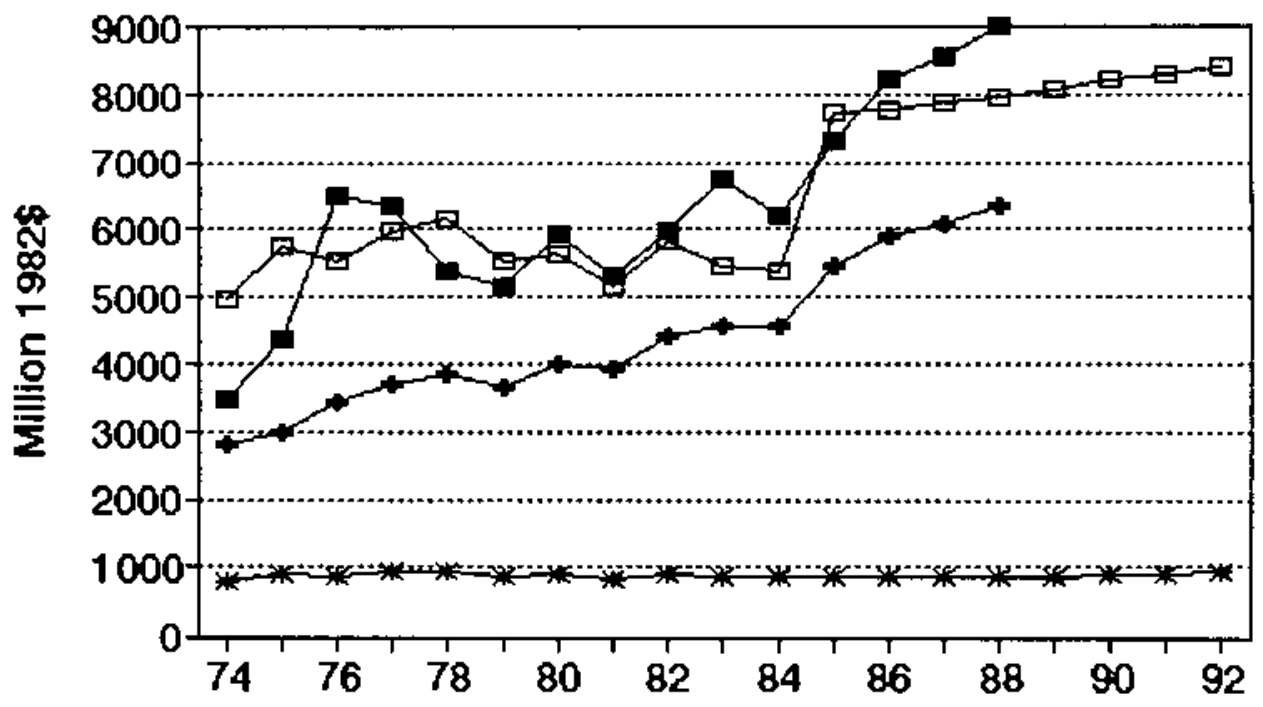

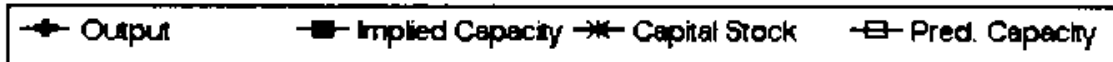


xo. 22 SIC 2048 Prepared feeda, n.e.c.

MILLIONS OF 1982 DOLLARS (EXCL. CU RATES)

$\begin{array}{lccccccc} & \alpha 4 & \text { Ann. Implied } & \text { Gross } & \text { Net } & \text { Pred. } & -\ldots . \ldots \\ \text { Gross } & \boldsymbol{C U} & \mathrm{CU} & \text { Prac. } & \text { In- } & \text { Cap. } & \text { Prac. } & \text { Emergency Capacity } \\ \text { Output } & \text { Rate } & \text { Rate } & \text { Capac. } & \text { vest. } & \text { Stk. } & \text { Capac. } & 3 \text { mon. } 6 \text { mon. }>6 \text { mon. }\end{array}$

\begin{tabular}{|c|c|c|c|c|c|c|c|c|c|c|}
\hline 1974 & 8,248 & 42 & 43.8 & 18,815 & 105.4 & 1,373 & 15,047 & & & \\
\hline 1975 & 8,209 & 73 & 60.2 & 13,631 & 161.4 & 1,427 & 14,477 & & & \\
\hline 1976 & 8,233 & $\pi$ & 73.9 & 11,136 & 85.2 & 1,403 & 14,723 & & & \\
\hline 197 & 8,908 & 73 & 74.3 & 11,994 & 101.2 & 1,390 & 14,772 & & & \\
\hline 1978 & 9,256 & 68 & 70.4 & 13,154 & 128.3 & 1,418 & 14,572 & & & \\
\hline 1979 & 9,506 & 79 & 74.8 & 12,707 & 136.5 & 1,443 & 14,303 & & & \\
\hline 1980 & 9,678 & 68 & 72.6 & 13,327 & 160.6 & 1,488 & 13,824 & & & \\
\hline 1981 & 10,234 & 68 & 68.7 & 14,869 & 131.1 & 1,498 & 13,714 & & & \\
\hline 1982 & 11,269 & 67 & 67.1 & 16,787 & 82.6 & 1,459 & 14,134 & & & \\
\hline 1983 & 10,704 & 67 & 66.7 & 16,042 & 117.2 & 1,448 & 14,253 & & & \\
\hline 1984 & 10,796 & 62 & 64.6 & 16,713 & $(14.2)$ & 1,316 & 15,648 & & & \\
\hline 1985 & 10,772 & 65 & 63.9 & 16.847 & 109.9 & 1,306 & 15,760 & & & \\
\hline 1986 & 10,033 & 65 & 65.5 & 15,324 & 86.1 & 1,270 & 16,136 & & & \\
\hline$\cdots$ & $\ldots$ & $\because$ & $\cdots$ & $\cdots$ & $\cdots$ & $-\cdots$ & $-\cdot-$ & $--\cdot-$ & $\cdots$ & . . \\
\hline 1987 & 10,163 & 67 & 66.4 & 15,304 & 88.7 & 1,239 & 16,461 & & & \\
\hline 1988 & 10,334 & 70 & 68.8 & 15,018 & 86.8 & 1,210 & 16,776 & 26,322 & 28,588 & 29,056 \\
\hline 1989 & & & & & 85.0 & 1,181 & 17,081 & 26,801 & 29,107 & 29,584 \\
\hline 1990 & & & & & 83.1 & 1,153 & 17,377 & 27,265 & 29,612 & 30,096 \\
\hline 1991 & & & & & 81.2 & 1,126 & 17,665 & 27,717 & 30,102 & 30,595 \\
\hline 1992 & & & & & 79.4 & 1,100 & 17,945 & 28,157 & 30,581 & 31,081 \\
\hline
\end{tabular}

Cepacity-Capital Stock Regression: Code 0 RSO 0.110

Coefficients: Const 29608.2 Stock -10.6

Ave. Capacity/stock $(74-86): 10.5$

Ave. Weekly Hours (High) 87.1 Shift factor 1.73 Ave Weekly Hours (LOW) 85.5 Shift Factor 1.76

Note: Output, Investment, and Capital Stock extrapolated for 1987 and 1988.

2048 Prepared feeds, n.e.c.

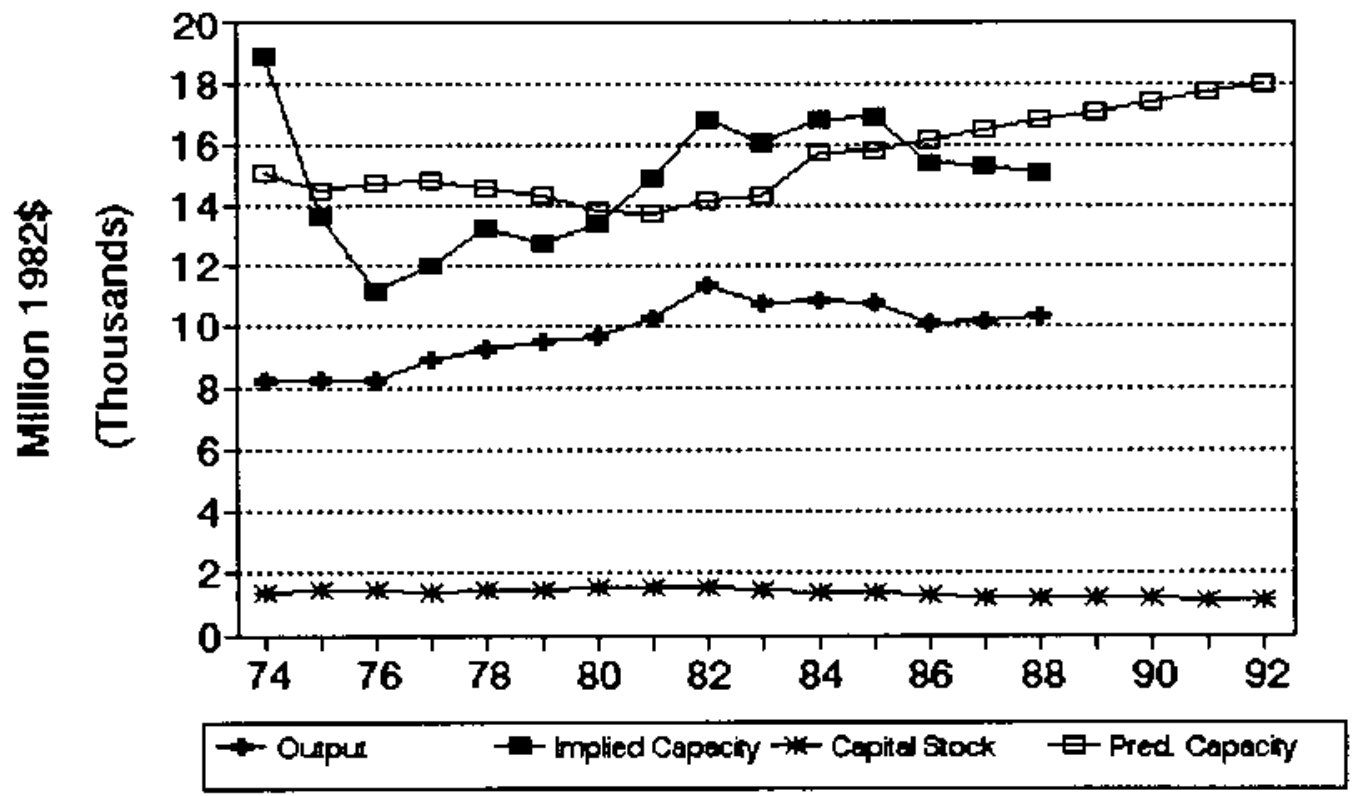


No. 23 SIC 2051 Bread, cake, and related products

MILLIONS OF 1982 DOLLARS (EXCL. CU RATES)

$\begin{array}{lccccccc} & \text { Q4 } & \text { Ann. } & \text { Implied } & \text { Gross } & \text { Net } & \text { Pred. } & \ldots \ldots \\ \text { Gross } & \text { CU } & \text { CU } & \text { Prac. } & \text { In } & \text { Cap. } & \text { Prac. } & \text { Energency Capacity } \\ \text { Output } & \text { Rate } & \text { Rate } & \text { Capac. } & \text { vest. } & \text { Stk. } & \text { Capac. } & 3 \text { mon. } 6 \text { mon. }>6 \text { man. }\end{array}$

\begin{tabular}{|c|c|c|c|c|c|c|c|c|c|c|}
\hline 1974 & 13,812 & 79 & 80.6 & 17,131 & 407.2 & 3,164 & 18,633 & & & \\
\hline 1975 & 13,936 & 74 & 75.1 & 18,550 & 378.8 & 3,240 & 18,984 & & & \\
\hline 1976 & 14,477 & 76 & $\pi .1$ & 19,280 & 353.9 & 3,299 & 19,257 & & & \\
\hline 1977 & 13,802 & 76 & 76.3 & 18,100 & 158.4 & 3,179 & 18.701 & & & \\
\hline 1978 & 13,541 & 68 & 71.3 & 19,006 & 325.0 & 3,225 & 18,912 & & & \\
\hline 1979 & 13,377 & 69 & 68.6 & 19,509 & 202.6 & 3,155 & 18,588 & & & \\
\hline 1980 & 13,074 & 69 & 69.2 & 18,885 & 294.1 & 3,168 & 18,651 & & & \\
\hline 1981 & 12,963 & 67 & 68.1 & 19,040 & 214.0 & 3,098 & 18,329 & & & \\
\hline 1982 & 13,151 & 66 & 66.3 & 19,848 & 390.2 & 3,187 & 18,739 & & & \\
\hline 1983 & 12,792 & 64 & 64.6 & 19,792 & 107.6 & 3,001 & 17,880 & & & \\
\hline 1984 & 12,662 & 78 & 73.1 & 17,325 & 287.4 & 2,995 & 17,849 & & & \\
\hline 1985 & 12,737 & $\pi$ & 76.2 & 16,732 & 323.4 & 3,018 & 17.957 & & & \\
\hline 1986 & 13,167 & 77 & 76.5 & 17,210 & 296.4 & 3,016 & 17,950 & & & \\
\hline$-\cdots$ & $--\cdot$ & -- & $-\cdot$ & --- & $-\cdots$ & $\cdots$ & $\ldots$ & $--\cdot-$ & $\cdot-\cdot$ &.-- \\
\hline 1987 & 13,092 & 76 & 76.5 & 17,123 & 315.7 & 3,033 & 18,028 & & & \\
\hline 1988 & 12,993 & $\pi$ & 73.4 & 17,245 & 337.1 & 3,070 & 18,196 & 19,441 & 20,355 & 20,544 \\
\hline 1989 & & & & & 357.1 & 3,123 & 18,441 & 19,703 & 20,628 & 20,820 \\
\hline 1990 & & & & & 369.4 & 3,183 & 18,718 & 19,999 & 20,938 & 21,133 \\
\hline 1991 & & & & & 372.6 & 3,240 & 18,983 & 20.282 & 21,235 & 21,432 \\
\hline 1992 & & & & & 371.0 & 3,290 & 19,214 & 20,528 & 21,493 & 21,692 \\
\hline
\end{tabular}

Capacity-Capital Stock Regression: Code 0 RSQ 0.180

Coefficients: Const 4011.4 stock 4.6

Ave, Capacity/Stock $(74-86): 5.9$

Ave. Heekly Hours (High) 134.8 Shift factor 1.13 Ave Heekly Hours (Low) 126.6 Shift Factor 1.20 Note: Output, Investment, and Copital stock extrapolated for 1987 and 1988.

\section{Bread, cake, and related products}

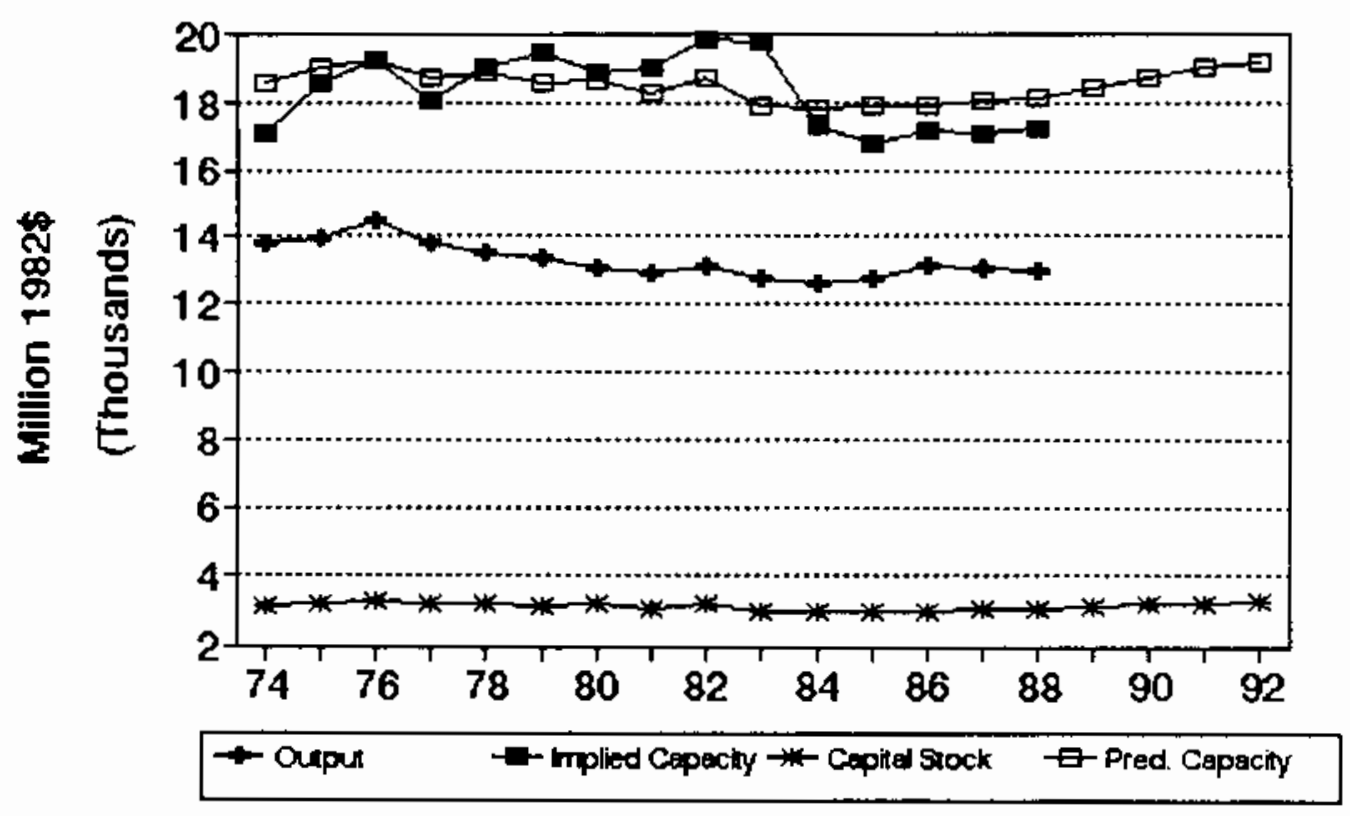




\section{Ho. 24 SIC 2052 Cooktes and crackers}

Millions of 1982 DOLLARS (EXCL. CU RATES)

$\begin{array}{lccccccc} & \alpha 4 & \text { Ann. Implied Gross } & \text { Net } & \text { Pred. } & -\ldots \ldots \\ \text { Gross } & \text { CU } & \text { CU } & \text { Prac. } & \text { In- } & \text { Cap. } & \text { Prac. } & \text { Emergency Capacity } \\ \text { Dutput } & \text { Rate } & \text { Rate } & \text { Capac. } & \text { vest. } & \text { Stk. } & \text { Capac. } & 3 \text { mon. } 6 \text { mon. }>6 \text { mon. }\end{array}$

\begin{tabular}{|c|c|c|c|c|c|c|c|c|c|c|}
\hline 1974 & 4,242 & 79 & 80.3 & 5,285 & 49.4 & 723 & 5,012 & & & \\
\hline 1975 & 4,176 & 83 & 80.9 & 5.163 & 160.5 & 807 & 5,352 & & & \\
\hline 1976 & 4,215 & 82 & 82.3 & 5,125 & 89.4 & 819 & 5,401 & & & \\
\hline 1977 & 4,261 & 79 & 80.3 & 5,303 & 137.5 & 878 & 5,635 & & & \\
\hline 1978 & 4,364 & 75 & 76.7 & 5,689 & 114.8 & 913 & 5,776 & & & \\
\hline 1979 & 4,611 & 75 & 75.0 & 6,152 & 114.6 & 947 & 5,915 & & & \\
\hline 1980 & 4,490 & 78 & 77.1 & 5,837 & 96.0 & 963 & 5,976 & & & \\
\hline 1981 & 4.564 & $\pi$ & $\pi .7$ & 5,876 & 73.6 & 958 & 5,957 & & & \\
\hline 1982 & 4,679 & 77 & 76.9 & 6,085 & 120.9 & 995 & 6,106 & & & \\
\hline 1983 & 4,734 & 68 & 71.3 & 6,669 & 99.4 & 1,009 & 6,161 & & & \\
\hline 1984 & 5,222 & $\pi$ & 72.7 & $7,1 \mathrm{gs}$ & 225.4 & 1,142 & 6,685 & & & \\
\hline 1985 & 5,577 & 76 & 75.7 & 7,372 & 190.6 & 1,236 & 7,076 & & & \\
\hline 1986 & 5,126 & 78 & 77.5 & 6,619 & 156.4 & 1,292 & 7,300 & & & \\
\hline$-\cdots$ & 5201 & $\because 4$ & $=-$ & $\cdots$ & $\begin{array}{l}-- \\
1574\end{array}$ & 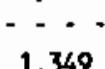 & 7530 & $\cdots-\cdots$ & $\cdots$ & $-\cdot-$ \\
\hline 1988 & 5,300 & 71 & 72.1 & 7,350 & 188.2 & 1,433 & 7,864 & 8,759 & 9,249 & 9,351 \\
\hline 1989 & & & & & 217.0 & 1,539 & 8,289 & 9.232 & 9,749 & 9,856 \\
\hline 1990 & & & & & 234.7 & 1,654 & 8,752 & 9,748 & 10,293 & 10,406 \\
\hline 1991 & & & & & 239.5 & 1,765 & 9.198 & 10,245 & 10,818 & 10,936 \\
\hline 1992 & & & & & 237.1 & 1,865 & 9,600 & 10,692 & 11,291 & 11,414 \\
\hline
\end{tabular}

Capacity-Capital stock Regression: Code 0 RSO 0.790

Coefficients: Const 2111.6 stock 4.0

Ave. Capacity/stock $(74-86): 6.2$ Ave. Weekly Hours (High) 127.6 Shift Factor 1.19 Ave Weekly Hours (Low) 125.0 Shift Factor 1.21 Mote: Output, Investment, and Capital Stock extrapolated for 1987 and 1988.

\section{Cookies and crackers}

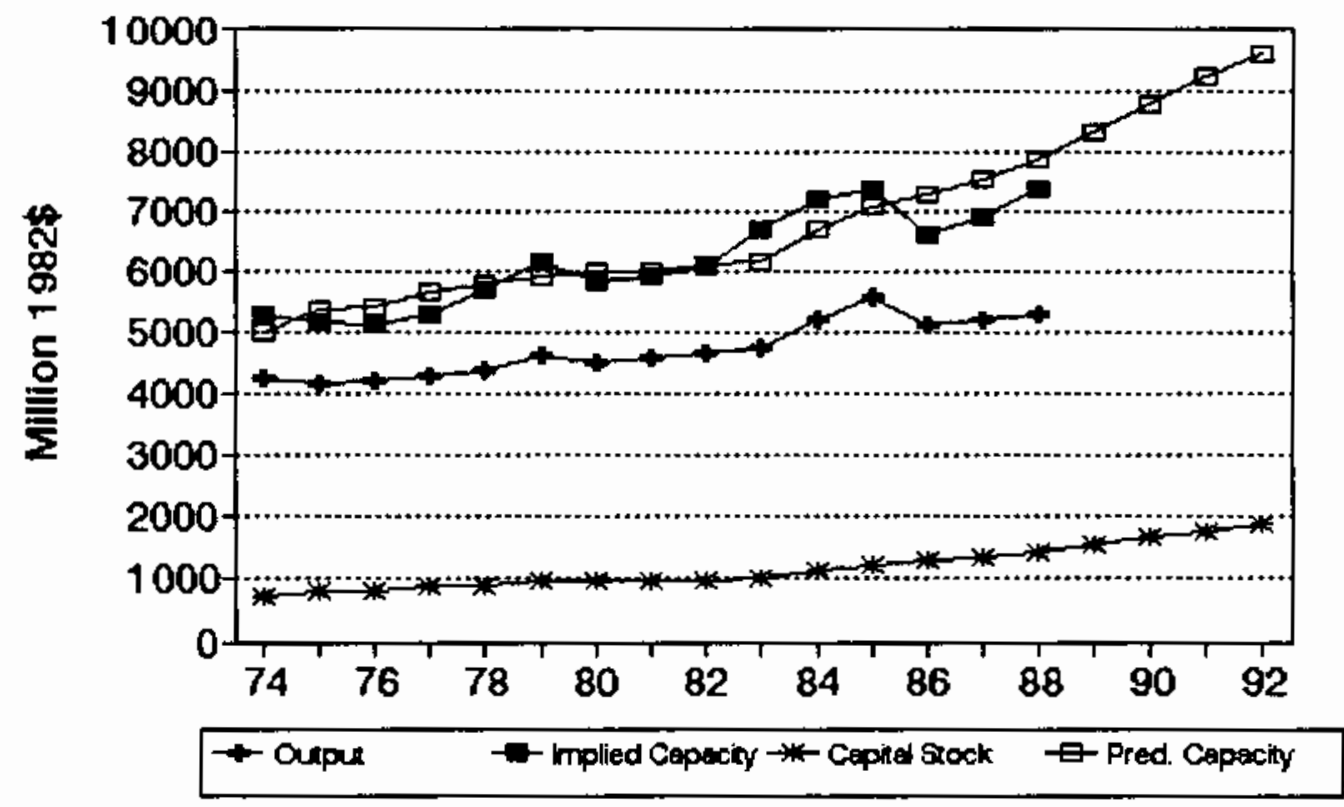




\section{No. 25 SIC 2061 Raw cade sugar}

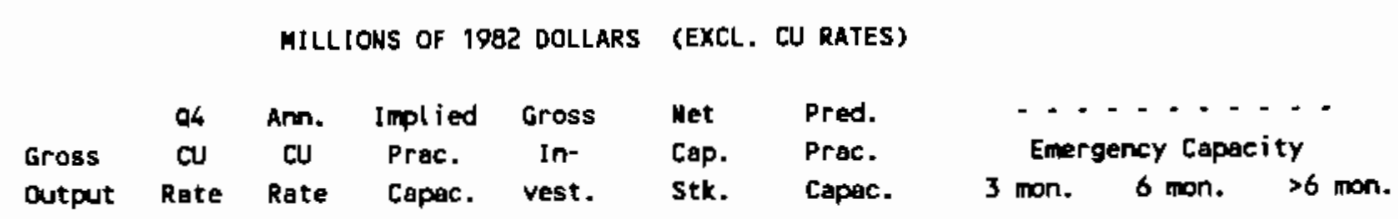

\begin{tabular}{|c|c|c|c|c|c|c|c|c|c|c|}
\hline 1976 & 967 & $67 *$ & 67.0 & 1,443 & 1.3 & 784 & 1,439 & & & \\
\hline 1975 & 857 & 71 & 69.3 & 1,236 & 107.6 & 835 & 1,424 & & & \\
\hline 1976 & 1,009 & 75 & 73.5 & 1,373 & 115.4 & 891 & 1,407 & & & \\
\hline 1977 & 1,330 & $\pi 2 *$ & 73.2 & 1,818 & 54.2 & 882 & 1,410 & & & \\
\hline 1978 & 1,039 & $\pi$ & 72.8 & 1,428 & 48.5 & 866 & 1,415 & & & \\
\hline 1979 & 1,057 & $\pi$ & 73.6 & 1,399 & 111.9 & 904 & 1,403 & & & \\
\hline 1980 & 1,082 & 83 & 80.8 & 1,340 & 104.1 & 933 & 1,395 & & & \\
\hline 1981 & 1,473 & 93 & 89.3 & 1,652 & 142.2 & 995 & 1,376 & & & \\
\hline 1982 & 1,035 & $\varphi$ & 96.8 & 1,070 & 57.6 & 973 & 1,383 & & & \\
\hline 1983 & 1,136 & 88 & 92.1 & 1,234 & 53.7 & 943 & 1,392 & & & \\
\hline 1984 & 1,097 & 78 & 84.8 & 1,341 & (22.7) & 844 & 1,421 & & & \\
\hline 1985 & 1,196 & 74 & $\pi .5$ & 1,583 & 98.1 & 859 & 1,417 & & & \\
\hline 1986 & 1,121 & 84 & 80.3 & 1,397 & 37.1 & 813 & 1,431 & & & \\
\hline$\cdots$ & $-\cdots$ & $-\cdots$ & $\cdots$ & $\because-\cdot$ & $\cdots$ & $\cdots$ & - - - & --- & 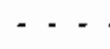 & $\because-$ \\
\hline 1987 & 1,132 & 88 & 86.5 & 1,309 & 60.0 & 792 & 1,437 & & & \\
\hline 1988 & 1,147 & 91 & 89.9 & 1,277 & 58.8 & $T 72$ & 1,443 & 1,402 & 1,438 & 1,446 \\
\hline 1989 & & & & & 57.6 & 753 & 1,448 & 1,408 & 1,444 & 1,451 \\
\hline 1990 & & & & & 56.3 & 734 & 1,454 & 1,413 & 1,449 & 1,457 \\
\hline 1991 & & & & & 55.1 & 716 & 1,459 & 1,418 & 1,455 & 1,462 \\
\hline 1992 & & & & & 53.9 & 699 & 1,464 & 1,423 & 1,460 & 1,467 \\
\hline
\end{tabular}

Capacity-Capital stock Regression: Code 0 RSO 0.010

Coefficients: Const 1671.5 stock -0.3

Ave. Capacity/Stock $(74-86): 1.6$ Ave. Heekly Hours (High) 165.6 Shift Factor 1.00 Ave Weekly Hours (Low) 165.2 Shift Factor 1.00 * Imputed Note: Output, Imestment, and Capital Stock extrapolated for 1987 and 1988.

2061 Raw cane sugar

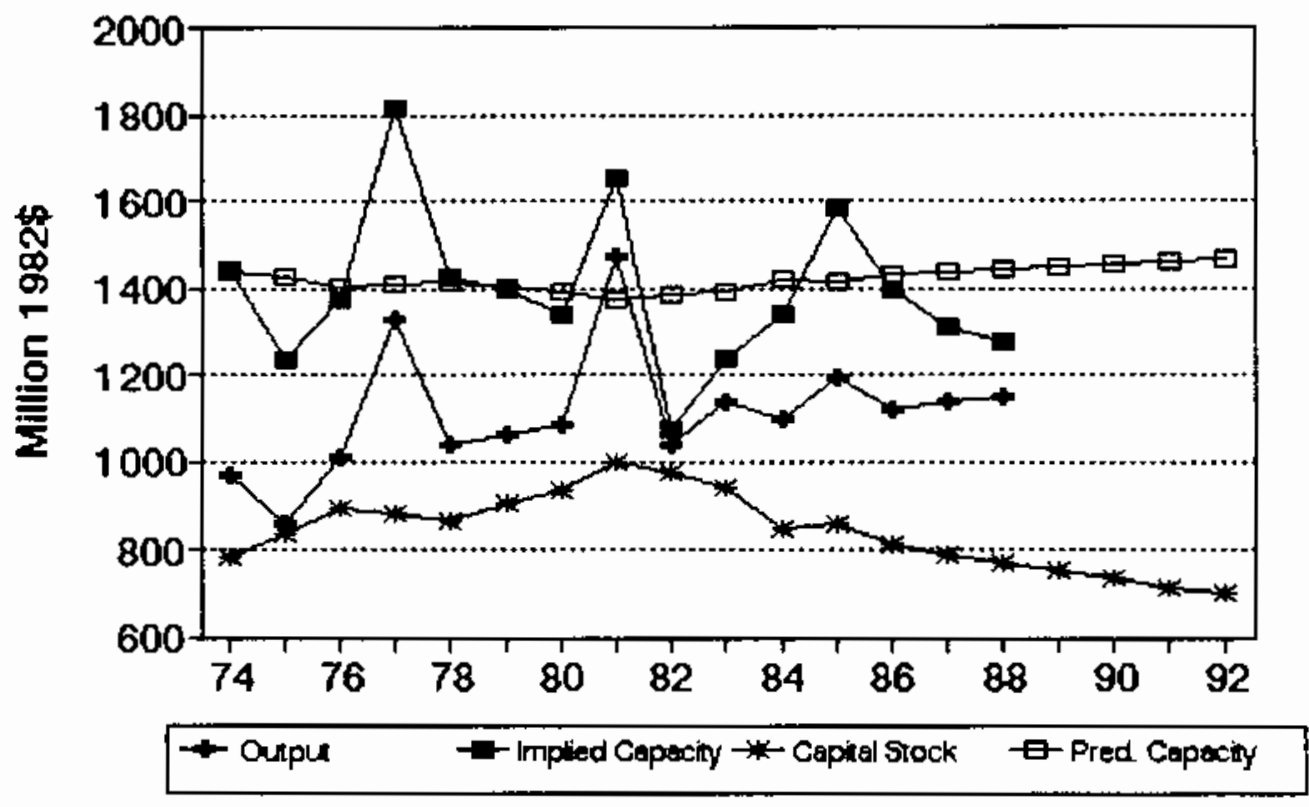




\section{NO. 26 sIC 2062 Cane sugar refining}

MILLIONS OF 1982 DOLLARS (EXCL. CU RATES)

$\begin{array}{lccccccc} & \alpha & \text { Ann. } & \text { Implied Gross } & \text { Net } & \text { Pred. } & \ldots \\ \text { Gross } & \text { a } & \text { CU } & \text { Prac. } & \text { In- } & \text { Cap. } & \text { Prac. } & \text { Emergency Capacity } \\ \text { Output } & \text { Rate } & \text { Rate } & \text { Capac. } & \text { vest. } & \text { Stk. } & \text { Capac. } & 3 \text { mon. } 6 \text { mon. }\end{array}$

\begin{tabular}{|c|c|c|c|c|c|c|c|c|c|c|}
\hline 1976 & 4,354 & 85 & 85.7 & 5,079 & 79.8 & 712 & 4,578 & & & \\
\hline 1975 & 3,157 & 82 & 82.8 & 3,814 & 101.9 & 738 & 4.672 & & & \\
\hline 1976 & 4,096 & 87 & 85.0 & 4,816 & 66.8 & 730 & 4,641 & & & \\
\hline 1977 & 4,012 & 78 & 81.5 & 4,922 & 33.2 & 695 & 4,517 & & & \\
\hline 1978 & 3,319 & 79 & 78.7 & 4,216 & $(21.0)$ & 613 & 4,224 & & & \\
\hline 1979 & 3.577 & 81 & 80.2 & 4,459 & 55.7 & 609 & 4,209 & & & \\
\hline 1980 & 3,137 & 84 & 83.0 & 3,780 & 86.6 & 632 & 4,292 & & & \\
\hline 1981 & 3,644 & 78 & 80.4 & 6,532 & 6.7 & 581 & 4,109 & & & \\
\hline 1982 & 3,050 & 79 & 78.6 & 3,882 & 9.7 & 540 & 3,962 & & & \\
\hline 1983 & 2,912 & 73 & 75.2 & 3,872 & 38.2 & 529 & 3,920 & & & \\
\hline 1984 & 2,857 & $79 *$ & 76.7 & 3,724 & 64.0 & 543 & 3,972 & & & \\
\hline 1985 & 2,524 & 86 & 83.3 & 3,031 & 12.7 & 509 & 2.969 & & & \\
\hline 1986 & 2,698 & 89 & 88.0 & 2,839 & 27.0 & 490 & 2,901 & & & \\
\hline$-\cdots$ & $\cdots$ & $\cdots$ & $--\cdot$ & $\cdots$ & --- & $\cdots$ & $\cdots$ & $-\cdots$ & $\cdots$ & -- \\
\hline 1987 & 2,446 & 83 & 85.3 & 2,868 & 18.8 & 463 & 2,805 & & & \\
\hline 1988 & 2,379 & 86 & 84.9 & 2,804 & 15.6 & 436 & 2,706 & 3,031 & 3,123 & 3,162 \\
\hline 1989 & & & & & 12.4 & 408 & 2,605 & 2,917 & 3,006 & 3,025 \\
\hline 1990 & & & & & 9.1 & 379 & 2,502 & 2,802 & 2,887 & 2,905 \\
\hline 1991 & & & & & 5.9 & 349 & 2,397 & 2,684 & 2,766 & 2,783 \\
\hline 1992 & & & & & 2.7 & 320 & 2,290 & 2,564 & 2,642 & 2,658 \\
\hline
\end{tabular}

Capacity-Capital Stock Regression: Code - $t$ RSQ 0.690 Coefficients: Const 2024.0 stock 3.6 D(85-86) -880.6

Ave. Capecity/Stock $(74-86): 6.7$ Ave. Heekly Hours (High) 130.8 Shift Factor 1.16 Ave Weekly Hours (Low) 129.6 Shift factor 1.17 - Imputed Note: Output, Imvestment, and Capital stock extrapolated for 1987 and 1988.

\section{Cane sugar refining}

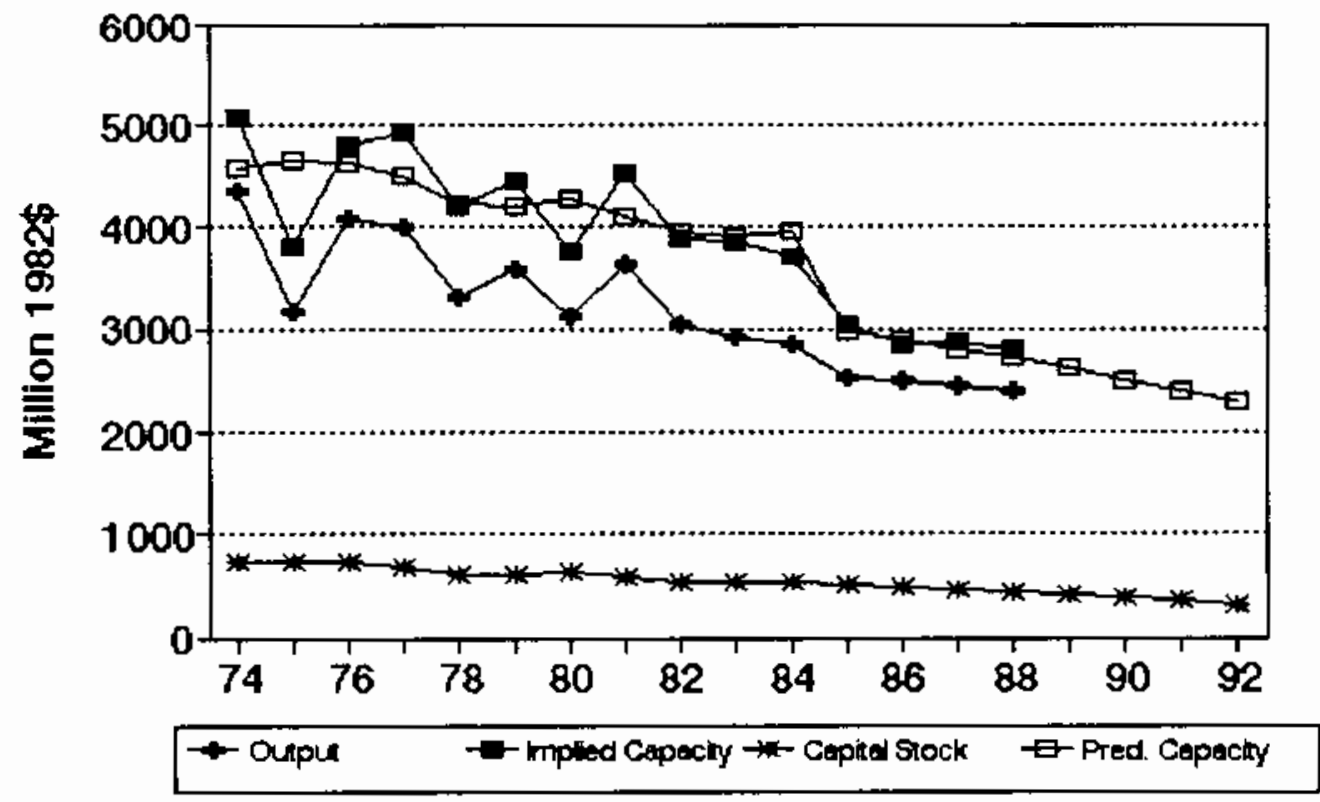




\section{No. 27 sIC 2063 Beet sugar}

MILLIONS OF 1982 DOLLARS (EXCL. CU RATES)

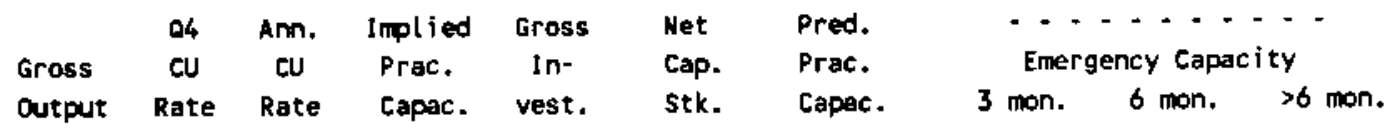

\begin{tabular}{|c|c|c|c|c|c|c|c|c|c|c|}
\hline 1974 & 1,554 & 100 & 100.0 & 1,556 & 101.9 & 973 & 1,873 & & & \\
\hline 1975 & 1,750 & 100 & 97.7 & 1,792 & 145.9 & 1,028 & 1,931 & & & \\
\hline 1976 & 2,435 & 100 & 99.5 & 2,446 & 178.7 & 1,117 & 2,026 & & & \\
\hline 1977 & 2,042 & 100 & 100.0 & 2,042 & 86.3 & 1,107 & 2,014 & & & \\
\hline 1978 & 1,859 & 100 & 100.0 & 1.859 & $(1.4)$ & 1,018 & 1,920 & & & \\
\hline 1979 & 1,809 & 93 & 95.4 & 1,896 & 47.9 & 980 & 1,880 & & & \\
\hline 1980 & 1,391 & 98 & 96.9 & 1,435 & 74.5 & 967 & 1,867 & & & \\
\hline 1981 & 1,635 & 82 & 89.0 & 1,838 & 59.5 & 936 & 1,834 & & & \\
\hline 1982 & 1,551 & 87 & 84.8 & 1,829 & 7.3 & 856 & 1,750 & & & \\
\hline 1983 & 1,540 & 93 & 90.3 & 1,706 & 58.3 & 824 & 1,716 & & & \\
\hline 1984 & 1,779 & 93 & 94.1 & 1,890 & 70.5 & 804 & 1,696 & & & \\
\hline 1985 & 1,829 & $\infty$ & 96.9 & 1,888 & 188.4 & 893 & 1,789 & & & \\
\hline 1986 & 1,831 & 92 & 95.4 & 1,919 & 107.5 & 903 & 1,799 & & & \\
\hline 1987 & - - - & $\because$ & $\begin{array}{ll}- & - \\
0 & -\end{array}$ & … & $\begin{array}{ll}\cdots \\
1310 & 0\end{array}$ & - . & 1,833 & $-\cdots$ & $\cdots$ & $\cdots$ \\
\hline $\begin{array}{l}7987 \\
1988\end{array}$ & $\begin{array}{l}1,821 \\
1,808\end{array}$ & $\begin{array}{l}90 \\
97\end{array}$ & $\begin{array}{l}74.8 \\
96.5\end{array}$ & $\begin{array}{l}1,9<2 \\
1,873\end{array}$ & 169.0 & 1,001 & 1,903 & 1,889 & 1,900 & 1,903 \\
\hline 1989 & & & & & 204.5 & 1,096 & 2,002 & 1,988 & 2,000 & 2,002 \\
\hline 1990 & & & & & 226.4 & 1,202 & 2,113 & 2,098 & 2,111 & 2,113 \\
\hline 1991 & & & & & 232.2 & 1,302 & 2,219 & 2,203 & 2,216 & 2,219 \\
\hline 1992 & & & & & 229.3 & 1,389 & 2,309 & 2,293 & 2,307 & 2,309 \\
\hline
\end{tabular}

Capacity-Capital stock Regression: Code 0 RSQ 0.190

Coefficients: Const 851.8 stock 1.1

Ave. Capacity/Stock $(74-86): 1.9$ Ave. Heekly Hours (High) 168.0 Shift Factor 1.00 Ave Yeekly Hours (Low) 168.0 Shift Factor 1.00 Note: Output, Investment, and Capital Stock extrapolated for 1987 and 1988.

\section{Beet sugar}

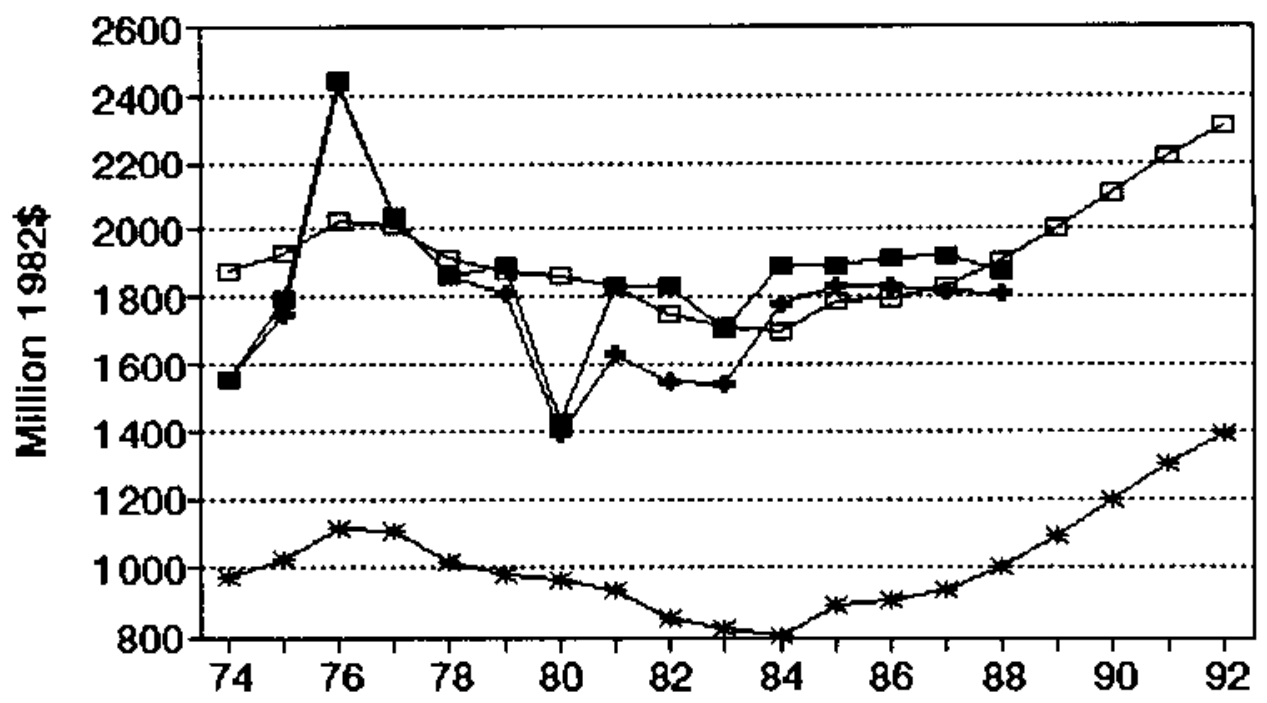

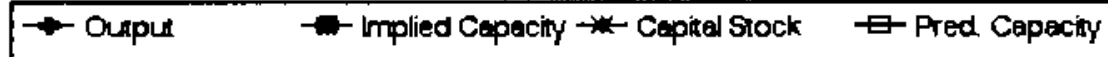




\section{No. 28 sIC 2065 confectionery products}

\section{MILLIONS OF 1982 DOLLARS (EXCL. CU RATES)}

$\begin{array}{lccccccc} & 04 & \text { Ann. Implied Gross } & \text { Net } & \text { Pred. } & -\ldots \ldots \ldots \\ \text { Gross } & \text { CU } & \text { CU } & \text { Prac. } & \text { In- } & \text { Cap. } & \text { Prac. } & \text { Emergency Capacity } \\ \text { Output } & \text { Rate } & \text { Rate } & \text { Capac. } & \text { vest. } & \text { Stk. } & \text { Capac. } & 3 \text { mon. } 6 \text { mon. }>6 \text { mon. }\end{array}$

\begin{tabular}{|c|c|c|c|c|c|c|c|c|c|c|}
\hline 1976 & 5,174 & 55 & 55.0 & 9,408 & 163.0 & 1,109 & 7,414 & & & \\
\hline 1975 & 4,584 & 76 & 68.1 & 6,728 & 126.1 & 1,138 & 7,531 & & & \\
\hline 1976 & 4,710 & 70 & 72.3 & 6,520 & 106.8 & 1,146 & 7,564 & & & \\
\hline 1977 & 5,414 & 69 & 69.4 & 7,805 & 155.5 & 1,199 & 7,779 & & & \\
\hline 1978 & 5,714 & 73 & 71.5 & 7,991 & 149.1 & 1,242 & 7,956 & & & \\
\hline $197 \%$ & 5,682 & 78 & 76.1 & 7,464 & 171.5 & 1,299 & 8,185 & & & \\
\hline 1980 & 6,200 & $\pi$ & 76.1 & 8,145 & 211.1 & 1,392 & 8,562 & & & \\
\hline 1981 & 6,570 & 76 & 75.6 & 8,688 & 53.9 & 1,333 & 8,321 & & & \\
\hline 1982 & 6,945 & 78 & 77.3 & 8,990 & 281.9 & 1,489 & 8,958 & & & \\
\hline 1983 & 6,361 & 74 & $\pi .5$ & 8,425 & 171.7 & 1,536 & 9,147 & & & \\
\hline 1984 & 6,324 & 65 & 68.4 & 9,248 & 38.6 & 1,458 & 8,831 & & & \\
\hline 1985 & 6,385 & 64 & 64.4 & 9,918 & 209.1 & 1,550 & 9,202 & & & \\
\hline 1986 & 6,414 & 68 & 66.5 & 9,645 & 202.6 & 1,629 & 9,526 & & & \\
\hline : & 6,521 & $\begin{array}{l}-- \\
71\end{array}$ & 69.9 & 9,333 & $\begin{array}{l}--- \\
159.3\end{array}$ & 1,665 & 9,672 & --- & $\cdots$ & $-\cdot-$ \\
\hline 1988 & 6,664 & 73 & 72.3 & 9,223 & 164.7 & 1,704 & 9,829 & 11,860 & 12,596 & 12,748 \\
\hline 1989 & & & & & 169.7 & 1,745 & 9,994 & 12,059 & 12,808 & 12,962 \\
\hline 1990 & & & & & 172.8 & 1,785 & 10,159 & 12,259 & 13,019 & 13,17 \\
\hline 1991 & & & & & 173.6 & 1,824 & 10,316 & 12,447 & 13,220 & 13,379 \\
\hline 1992 & & & & & 173.2 & 1,859 & 10,458 & 12,619 & 13,402 & 13,564 \\
\hline
\end{tabular}

Capecity-Capital stock Regression: Code 0 RSO 0.440

Coefficients: Const 2913.0 Stock 4.1

Ave. Capacity/Stock (74-86): 6.2 Ave. Weekly Hours (High) 116.6 Shift Factor 1.30 Ave Heekly Hours (Low) 108.7 Shift Factor 1.39 Note: Output, Investment, and Capital Stock extrapolated for 1987 and 1988.

\section{Confectionery products}

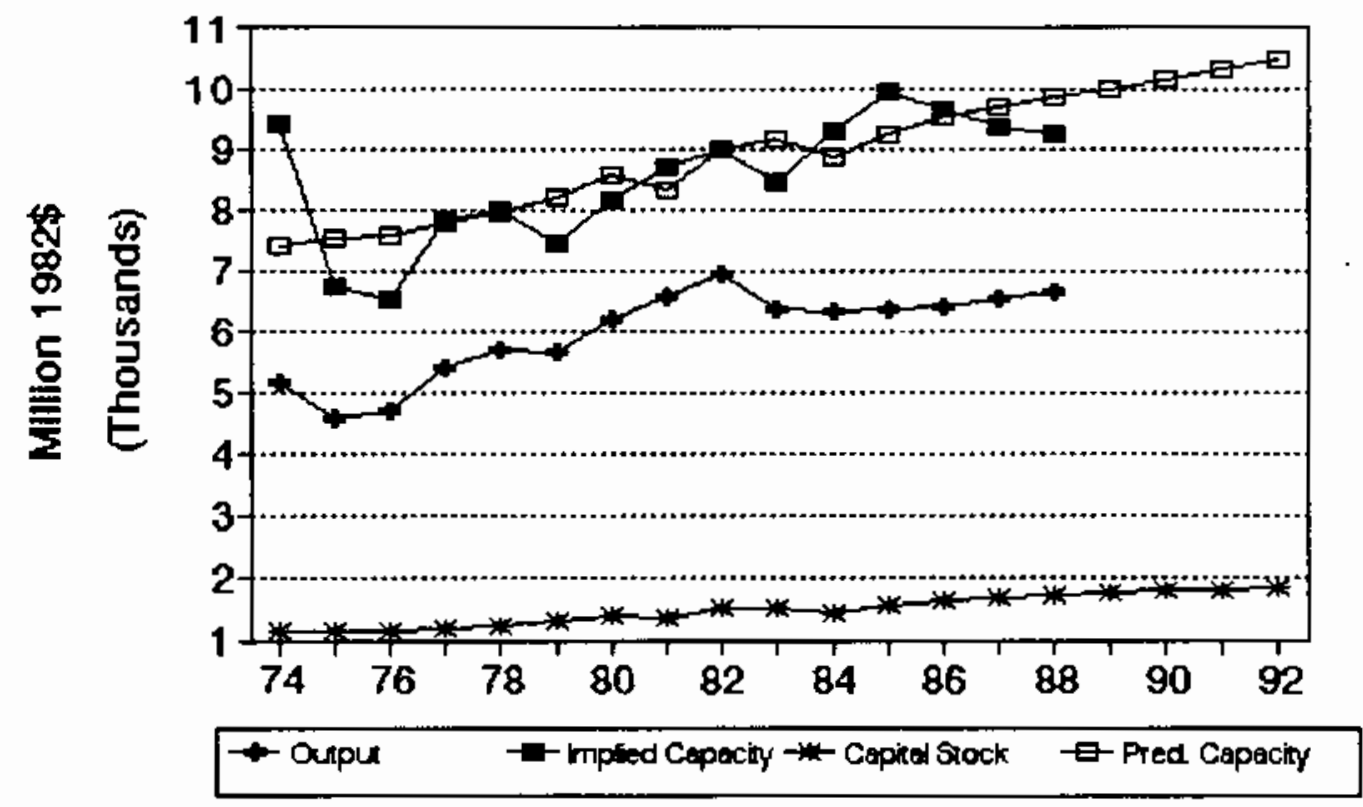




\section{No. 29 SIC 2066 Chocolate and cocoa product:}

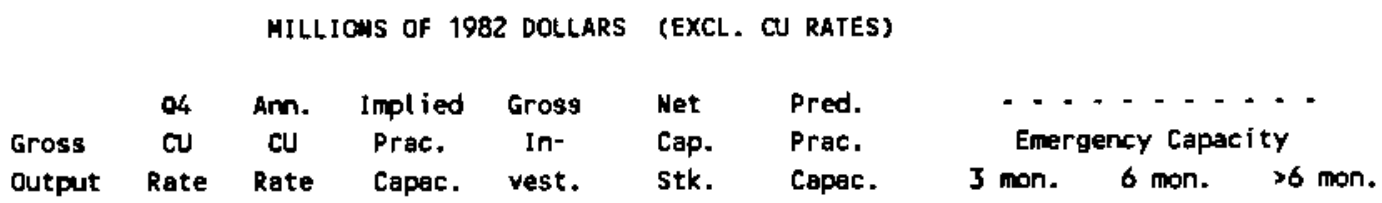

\begin{tabular}{|c|c|c|c|c|c|c|c|c|c|c|}
\hline 1974 & 1,821 & 70 & 70.6 & 2,580 & 27.9 & 383 & 2,504 & & & \\
\hline 1975 & 1,667 & 76 & 73.5 & 2,269 & 23.6 & 376 & 2,478 & & & \\
\hline 1976 & 1,731 & 74 & 74.7 & 2,318 & 25.4 & 371 & 2,460 & & & \\
\hline 1977 & 1,973 & 79 & 77.2 & 2,554 & 86.3 & 422 & 2,643 & & & \\
\hline 1978 & 2,093 & $n$ & 7.9 & 2,688 & 43.7 & 430 & 2,671 & & & \\
\hline 1979 & 2,053 & 82 & 80.1 & 2,563 & 7.3 & 404 & 2,579 & & & \\
\hline 1980 & 2,028 & $\pi 9$ & 80.2 & 2,528 & 39.2 & 410 & 2,599 & & & \\
\hline 1981 & 2,242 & 80 & 79.8 & 2,810 & 49.8 & 425 & 2,652 & & & \\
\hline 1982 & 2,251 & $\pi 9$ & 79.3 & 2,838 & 39.5 & 430 & 2,671 & & & \\
\hline 1983 & 2,068 & 68 & 72.1 & 2,869 & 51.4 & 447 & 2,734 & & & \\
\hline 1984 & 2,176 & 73 & 71.3 & 3,054 & 67.7 & 480 & 2,849 & & & \\
\hline 1985 & 2,231 & 73 & 73.0 & 3,056 & 7.7 & 522 & 3,000 & & & \\
\hline 1986 & 2,173 & $\pi$ & 76.9 & 2,827 & 69.6 & 554 & 3,114 & & & \\
\hline$\cdots$ & $-\cdot-$ & - & -- & $\cdots$ & -- & $\cdots$ & $\cdots \cdot$ & $\cdots \cdot--$ & --- & $-\cdots$ \\
\hline 1987 & 2,212 & 79 & 79.0 & 2,798 & 61.7 & 578 & 3,200 & & & \\
\hline 1988 & 2,263 & 80 & 79.6 & 2,842 & 74.4 & 613 & 3,326 & 3,253 & 3,393 & 3,422 \\
\hline 1989 & & & & & 86.3 & 658 & 3,485 & 3,409 & 3,556 & 3,586 \\
\hline 1990 & & & & & 93.6 & 707 & 3,660 & 3,580 & 3,734 & 3,766 \\
\hline 1991 & & & & & 95.6 & 735 & 3,830 & 3,746 & 3,908 & 3,941 \\
\hline 1992 & & & & & 94.6 & 798 & 3,985 & 3,898 & 4,066 & 4,100 \\
\hline
\end{tabular}

Capacity-Capital Stock Regression: Code 0 RSQ 0.620

Coefficients: Const 1137.3 stock 3.6

Ave. Capacity/Stock (74-86): 6.2 Ave. Weekly Hours (High) 156.0 Shift Factor 1.03 Ave Meekly Hours (LOW) 139.8 Shift Factor 1.14 Note: Output, Investment, and Cepital Stock extrapolated for 1987 and 1988.

\section{Chocolate and cocoa products}

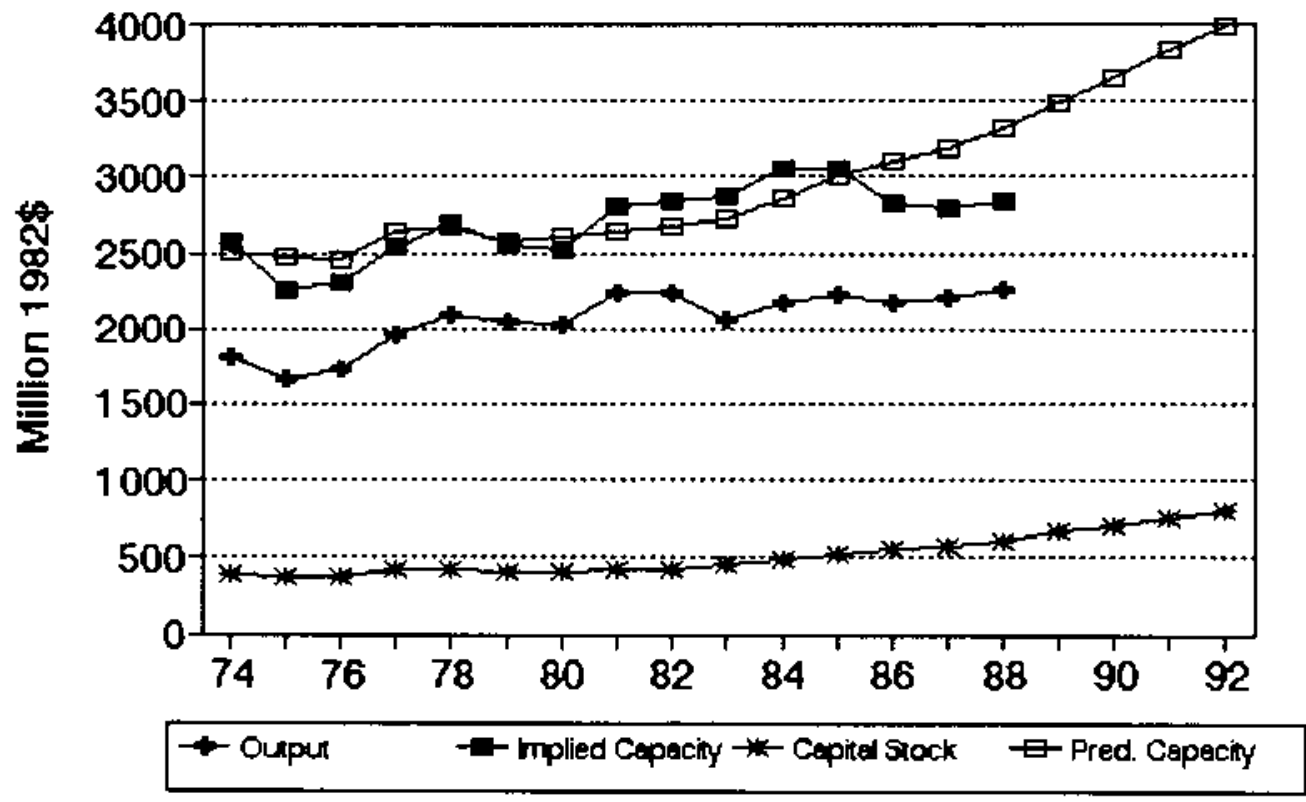




\title{
HO. 30 sIC 2067 Chewing gum
}

\author{
MILLIONS OF 1982 DOLLARS (EXCL. CU RATES)
}

$\begin{array}{lccccccc} & \text { Q4 Amn. } & \text { Implied Gross } & \text { Net } & \text { Pred. } & \ldots \ldots \ldots \\ \text { Gross } & \text { CU } & \text { CU } & \text { Prac. } & \text { In- } & \text { Cap. } & \text { Prac. } & \text { Emergency Capacity } \\ \text { Output } & \text { Rate } & \text { Rate } & \text { Capac. } & \text { vest. } & \text { Stk. } & \text { Capac. } & 3 \text { mon. } 6 \text { mon. }>6 \text { mon. }\end{array}$

\begin{tabular}{|c|c|c|c|c|c|c|c|c|c|c|}
\hline 1974 & 937 & 97 & 100.0 & 937 & 0.1 & 210 & 977 & & & \\
\hline 1975 & 764 & $86 *$ & B7.4 & 874 & 23.5 & 218 & 1,036 & & & \\
\hline 1976 & 823 & 74 & 78.0 & 1,055 & 33.8 & 234 & 1,167 & & & \\
\hline 1977 & 866 & 62 & 67.1 & 1.290 & 37.0 & 252 & 1,307 & & & \\
\hline 1978 & 890 & 57 & 59.4 & 1,514 & 28.1 & 260 & 1,368 & & & \\
\hline 1979 & 955 & 60 & 58.8 & 1,624 & 50.5 & 287 & 1,583 & & & \\
\hline 1980 & $7 r 0$ & 53 & 56.1 & 1,373 & 17.6 & 280 & 1,529 & & & \\
\hline 1981 & 791 & 54 & 54.4 & 1,454 & $(26.8)$ & 233 & 1,155 & & & \\
\hline 1982 & 912 & 50 & 51.2 & 1,780 & 52.3 & 259 & 1,813 & & & \\
\hline 1983 & 902 & 54 & 52.2 & 1,728 & 21.3 & 254 & 1,770 & & & \\
\hline 1984 & 948 & 54 & 54.7 & 1,733 & 15.3 & 243 & 1,684 & & & \\
\hline 1985 & 915 & 63 & 59.7 & 1,532 & 1.1 & 221 & 1,510 & & & \\
\hline 1986 & 916 & 61 & 62.3 & 1,470 & 17.3 & 215 & 1,465 & & & \\
\hline$=-$ & $\cdots$ & $\cdots$ & $-\cdots$ & $\because-$ & $\cdots$ & $\therefore$ & $\because-$ & $-\cdots$ & $-\cdots$ & $-\cdots$ \\
\hline 1987 & 910 & 65 & 63.7 & 1,430 & 15.4 & 208 & 1,407 & & & \\
\hline 1988 & 903 & 79 & 73.6 & 1,227 & 14.7 & 201 & 1,349 & 1,507 & 1,592 & 1,610 \\
\hline 1989 & & & & & 13.9 & 194 & 1,292 & 1,442 & 1,524 & 1,541 \\
\hline 1990 & & & & & 13.2 & 186 & 1,234 & 1,378 & 1,657 & 1,473 \\
\hline 1991 & & & & & 12.4 & 179 & 1,177 & 1,315 & 1,389 & 1,404 \\
\hline 1992 & & & & & 11.7 & 172 & 1,120 & 1,251 & 1,322 & 1,336 \\
\hline
\end{tabular}

Capacity-Capital Stock Regression: code -1 RSO 0.830

Coefficients: Const $\quad-690.9$ stock 7.9 D(82-86) 447.7

Ave. Capacity/stock $(74-86): 5.8$ Ave. Weekly Hours (High) 127.2 Shift Factor 1.19 Ave Heekly Hours (Low) 120.6 Shift Factor 1.26 - Imputed Note: Output, Imestment, and Capital stock extrapolated for 1987 and 1988.

2067 Chewing gum

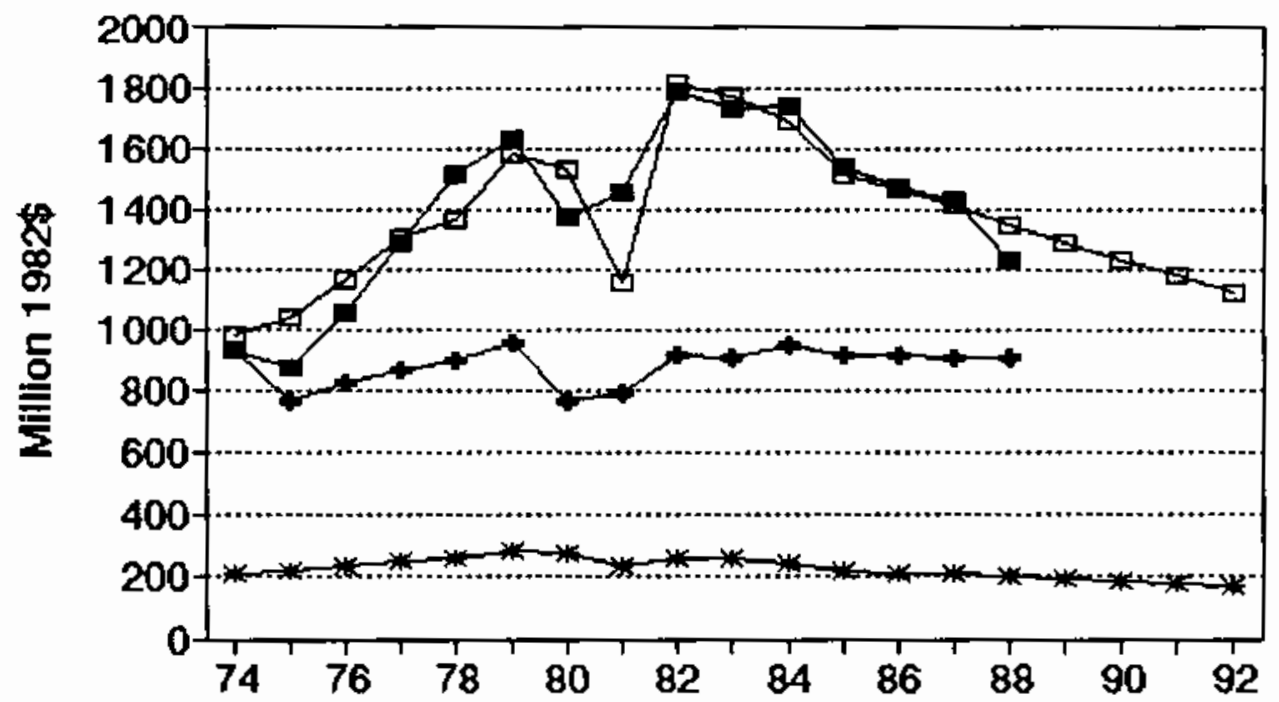

- Oupue t-tmpied Cepeciny to-Cepitel Stock E-Pred Capecity


NO. 31 SIC 2074 cottonseed oil mills

MILLIONS OF 1982 DOLLARS (EXCL. CU RATES)

$\begin{array}{lccccccc} & 04 & \text { Ann. Implied } & \text { Gross } & \text { Het } & \text { Pred. } & \ldots \ldots & \ldots . . \ldots \\ \text { Gross } & \text { CU } & \text { CU } & \text { Prac. } & \text { In. } & \text { Cap. } & \text { Prac. } & \text { Emergency Capacity } \\ \text { Dutput } & \text { Rate } & \text { Rate } & \text { Capac. } & \text { vest. } & \text { Stk. } & \text { Capac. } & 3 \text { mon. } 6 \text { mon. }>6 \text { mon. }\end{array}$

\begin{tabular}{|c|c|c|c|c|c|c|c|c|c|c|}
\hline 1974 & 787 & $71 *$ & 81.9 & 962 & 13.9 & 229 & 889 & & & \\
\hline 1975 & 981 & 88 & 76.2 & 1,288 & 31.8 & 236 & 904 & & & \\
\hline 1976 & 685 & 92 & 89.4 & 766 & 23.9 & 235 & 902 & & & \\
\hline 1977 & 724 & 94 & 95.3 & 760 & 39.7 & 249 & 933 & & & \\
\hline 1978 & 878 & 100 & 99.8 & 890 & 42.7 & 268 & 972 & & & \\
\hline 1979 & 776 & 100 & 99.6 & 779 & 0.7 & 247 & 928 & & & \\
\hline 1980 & 905 & 100 & 100.0 & 905 & 28.8 & 255 & 944 & & & \\
\hline 1981 & 908 & 81 & 91.3 & 995 & 36.8 & 272 & 980 & & & \\
\hline 1982 & 883 & 66 & 70.5 & 1,253 & 44.8 & 297 & 1,034 & & & \\
\hline 1983 & 659 & 74 & 69.9 & 942 & 9.8 & 286 & 1,011 & & & \\
\hline 1984 & $\pi T 2$ & 83 & 82.6 & 934 & 39.3 & 303 & 1,047 & & & \\
\hline 1985 & 1,002 & 89 & 87.1 & 1,151 & 20.2 & 299 & 1,038 & & & \\
\hline 1986 & 706 & 58 & 71.5 & 987 & 16.0 & 291 & 1,020 & & & \\
\hline$\therefore-$ & --- & $-\cdots$ & $\cdots$ & - - - & - - - & $\cdots$ & $\because \cdots$ & $\cdots$ & $-\cdots$ & $\cdots$ \\
\hline 1987 & 718 & $\pi$ & 69.3 & 1,037 & 30.2 & 296 & 1,033 & & & \\
\hline 1988 & 735 & 90 & 84.0 & 875 & 33.6 & 305 & 1,051 & 1,011 & 1,051 & 1,059 \\
\hline 1989 & & & & & 36.7 & 316 & 1,075 & 1,034 & 1,074 & 1,083 \\
\hline 1990 & & & & & 38.6 & 328 & 1,101 & 1,059 & 1,100 & 1,109 \\
\hline 1991 & & & & & 39.2 & 340 & 1,126 & 1,083 & 1,125 & 1,134 \\
\hline 1992 & & & & & 38.9 & 350 & 1,148 & 1,104 & 1,147 & 1,156 \\
\hline
\end{tabular}

Capacity-Capital Stock Regression: Code 0 RSO 0.110

Coefficients: Const 401.0 stock 2.1

Ave. Capacity/Stock $(74-86): 3.6$

Ave. Weekly Hours (High) 157.2 Shift Factor 1.01 Ave Heekly Hours (Low) 146.4 Shift Factor 1.08

* Imputed Note: Output, Imvestment, and Capital Stock extrapolated for 1987 and 1988.

\section{Cottonseed oil mills}
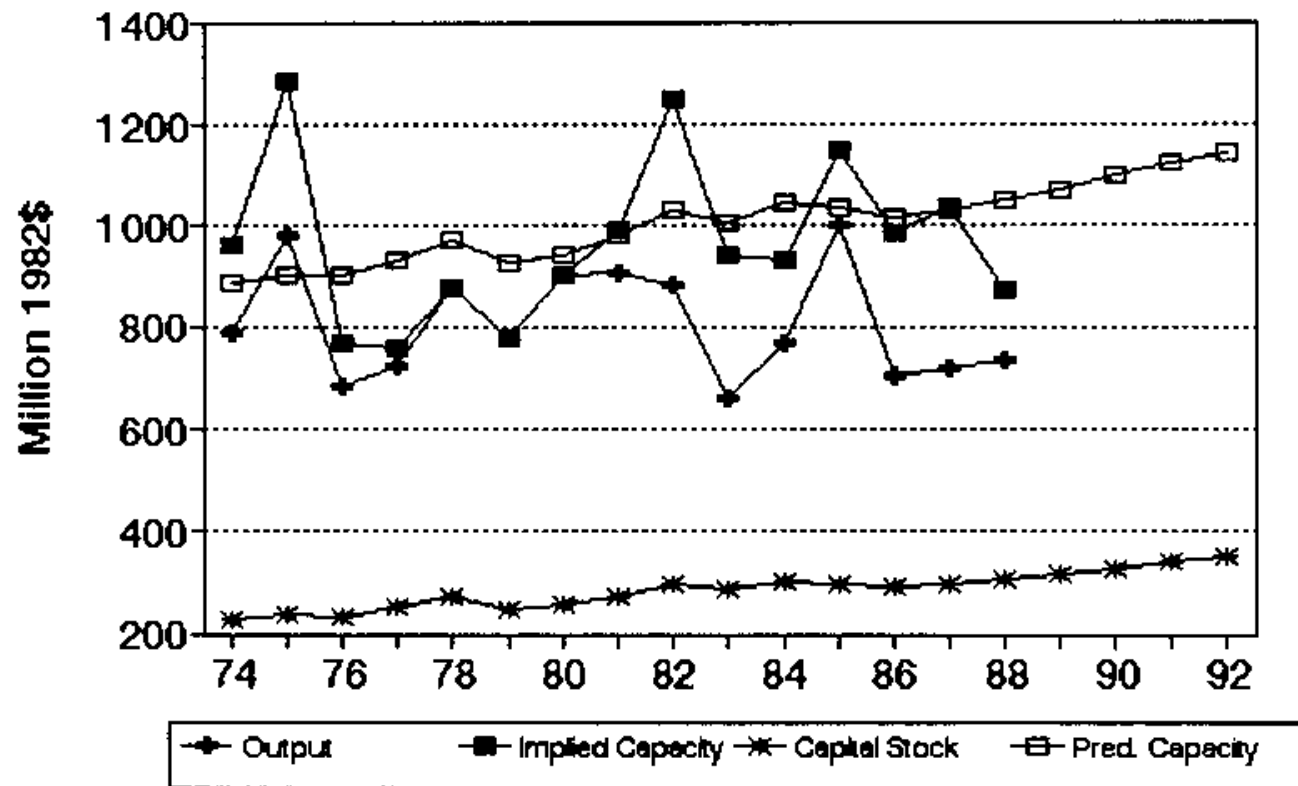
No. 32 sIc 2075 Soybean oil aills

MILLIONS OF 1982 DOLLARS (EXCL. CU RATES)

$\begin{array}{lccccccc} & 04 & \text { Arn. Implied Gross } & \text { Het } & \text { Pred. } & \ldots \\ \text { Gross } & \text { CU } & \text { CU } & \text { Prac. } & \text { In- } & \text { Cap. } & \text { Prac. } & \text { Emergency Capacity } \\ \text { Output Rate } & \text { Rate } & \text { Capac. } & \text { vest. } & \text { Stk. } & \text { Capac. } & 3 \text { mon. } 6 \text { mon. }>6 \text { mon. }\end{array}$

\begin{tabular}{|c|c|c|c|c|c|c|c|c|c|c|}
\hline 1974 & 7,048 & 7 & 77.2 & 9,130 & 161.8 & 1,019 & 8,665 & & & \\
\hline 1975 & 6,859 & $82 *$ & 79.9 & 8,584 & 165.1 & 1,118 & 9,026 & & & \\
\hline 1976 & 7,783 & $\pi$ & 80.0 & 9,736 & 114.2 & 1,159 & 9,178 & & & \\
\hline 1977 & 6,046 & 82 & 80.9 & 8,218 & 124.6 & 1,204 & 9,344 & & & \\
\hline 1978 & 7,773 & $\pi$ & 77.6 & 10,016 & 185.4 & 1,307 & 9,720 & & & \\
\hline 1979 & 7,996 & 92 & 85.6 & 9,338 & $(17,3)$ & 1,210 & 9,364 & & & \\
\hline 1980 & 8,595 & 91 & 91.4 & 9,406 & 125.0 & 1,251 & 9,515 & & & \\
\hline 1981 & 8,047 & 81 & 84.8 & 9,495 & 157.8 & 1,318 & 9,759 & & & \\
\hline 1982 & 8,587 & 80 & 80.4 & 10,684 & 170.7 & 1,381 & 9,992 & & & \\
\hline 1983 & 7,694 & 85 & 83.1 & 9,256 & 88.0 & 1,338 & 9,833 & & & \\
\hline 1984 & 8,642 & 85 & 85.0 & 10,167 & 57.9 & 1,283 & 9,634 & & & \\
\hline 1985 & 9,582 & 81 & 82.5 & 11,614 & 75.5 & 1,245 & 11,914 & & & \\
\hline 1986 & 10,170 & 86 & 84.1 & 12,089 & 82.4 & 1,212 & 11,790 & & & \\
\hline$-\cdots$ & $-\cdots$ & $\because$ & $\cdots$ & $\cdots$ & $-\cdots$ & $\because-$ & $\cdots$ & --2 & --- & $\cdots-$ \\
\hline 1987 & 10,581 & 88 & 87.3 & $\uparrow 2,128$ & 113.1 & 1,211 & 11,789 & & & \\
\hline 1988 & 11,139 & 85 & 86.1 & 12,934 & 113.4 & 1,212 & 11.790 & 11,765 & 11,854 & 11,872 \\
\hline 1989 & & & & & 113.7 & 1,212 & 11,791 & 11,767 & 11,855 & 11,874 \\
\hline 1990 & & & & & 113.8 & 1,212 & 11,793 & 11,768 & 11,857 & 11,875 \\
\hline 1991 & & & & & 113.9 & 1,213 & 11,795 & 11,770 & 11,859 & 11.877 \\
\hline 1992 & & & & & 113.9 & 1,213 & 11,796 & $11, \pi R$ & 11,861 & 11,879 \\
\hline
\end{tabular}

Capacity-Capital Stack Regression: Code -1 RSo 0.770

Coefficients: Const 4933.6 Stock $3.7 \mathrm{D}(85-86) 2419.2 \quad$ Ave. Capacity/Stock (74-86): 8.0 Ave. Ueekly Hours (High) 166.8 Shift factor 1.01 Ave veekly Hours (Low) 164.2 Shift factor 1.02 * Imputed Mote: Output, Imestment, and Capital Stock extrapolated for 1987 and 1988.

\section{Soybean oil mills}

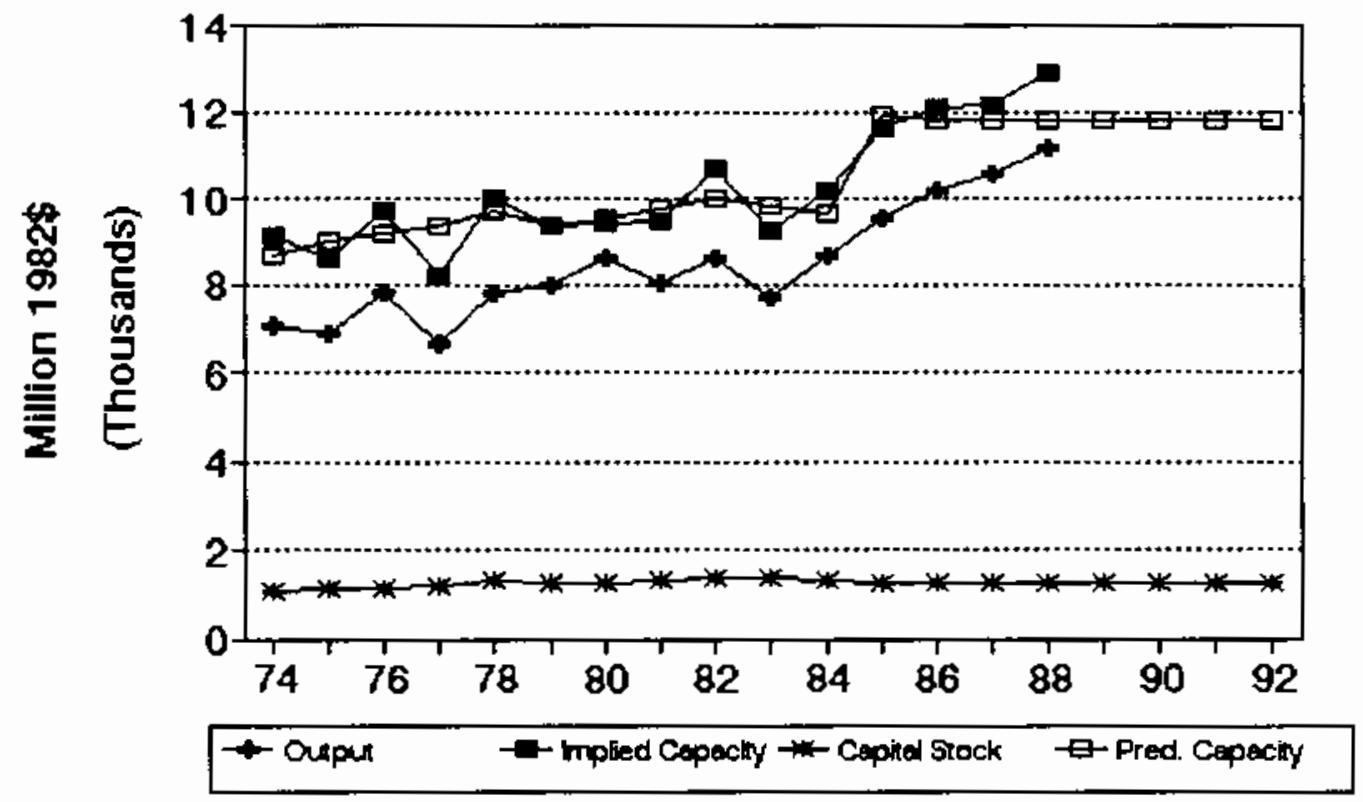




\section{No. 33 sIc 2076 Vegetable oil mills, nec}

MILLIONS OF 1982 DOLLARS (EXCL. CU RATES)

\begin{tabular}{|c|c|c|c|c|c|c|c|}
\hline & $\begin{array}{l}04 \\
\mathrm{CU}\end{array}$ & $\begin{array}{l}\text { Ann. } \\
\text { CU }\end{array}$ & $\begin{array}{l}\text { Implied } \\
\text { Prac. }\end{array}$ & $\begin{array}{l}\text { Gross } \\
\ln -\end{array}$ & $\begin{array}{l}\text { Net } \\
\text { Cap. }\end{array}$ & $\begin{array}{l}\text { Pred. } \\
\text { Prac. }\end{array}$ & $\begin{array}{l}- \text { - - - } \\
\text { Emergency Capacity }\end{array}$ \\
\hline & Rate & Rate & Cepec. & vest. & stk. & Capac. & \\
\hline
\end{tabular}

\begin{tabular}{|c|c|c|c|c|c|c|c|c|c|c|}
\hline 1976 & 615 & $84 *$ & 85.2 & 488 & 19.3 & 84 & 553 & & & \\
\hline 1975 & 668 & $80 *$ & 80.4 & 831 & 19.4 & 94 & 572 & & & \\
\hline 1976 & 697 & $82 *$ & 81.0 & 862 & 14.2 & 100 & 585 & & & \\
\hline 1977 & 322 & $82 \star$ & 82.4 & 391 & $(23.7)$ & 72 & 528 & & & \\
\hline 1978 & 297 & $86=$ & 83.5 & 356 & 7.1 & 74 & 532 & & & \\
\hline 1979 & 311 & 81 & 82.0 & 379 & 25.3 & 93 & 570 & & & \\
\hline 1980 & 367 & 67 & 72.5 & 507 & 15.6 & 102 & 580 & & & \\
\hline 1981 & 500 & 69 & 68.6 & 729 & 5.6 & 102 & 588 & & & \\
\hline 1982 & 562 & 81 & 76.4 & 735 & 22.2 & 117 & 617 & & & \\
\hline 1983 & 600 & $80 *$ & 80.3 & 747 & 43.1 & 153 & 689 & & & \\
\hline 1984 & 468 & $80 *$ & 80.5 & 581 & 16.4 & 162 & 708 & & & \\
\hline 1985 & 515 & $78 *$ & 79.0 & 652 & 32.1 & 185 & 755 & & & \\
\hline 1986 & 609 & $80 *$ & 79.3 & 767 & 1.2 & 177 & 738 & & & \\
\hline$\cdots$ & ---- & -- & - - - & -- & $--\cdot$ & $-\cdots$ & $\because-$ & - & --- & -- \\
\hline 1987 & 610 & $79 *$ & 79.4 & 768 & 19.6 & 188 & 760 & & & \\
\hline 1988 & 612 & $76 *$ & 7.0 & 795 & 20.1 & 199 & 781 & 761 & 782 & 787 \\
\hline 1989 & & & & & 20.6 & 209 & 803 & 782 & 804 & 808 \\
\hline 1990 & & & & & 21.2 & 220 & 824 & 803 & 825 & 830 \\
\hline 1991 & & & & & 21.7 & 231 & 845 & 824 & 847 & 851 \\
\hline 1992 & & & & & 22.2 & 241 & 867 & 844 & 868 & 873 \\
\hline
\end{tabular}

Capacity-Capital Stock Regression: Code 0 RSO 0.190 Coefficients: Conșt 384.2 stock 2.0

Ave. Capecity/Stock $(74-86): 5.3$ Ave. Heekly Hours (High) 166.8 Shift factor 1.01 Ave Weekly Hours (LOW) 164.2 Shift factor 1.02 * Imputed Note: Output, Investment, and Capital Stock extrapolated for 1987 and 1988.

\section{Vegetable oil mills, nec}

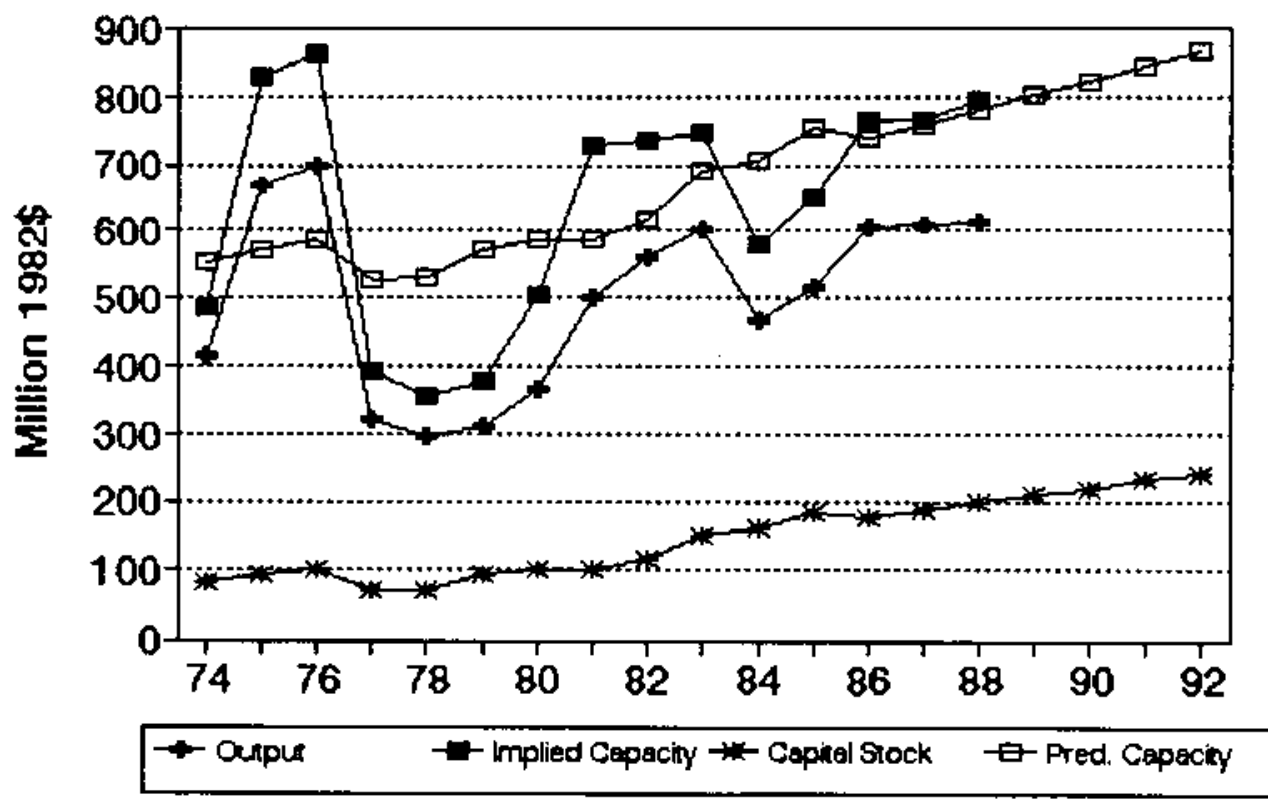




\section{No. 34 sIC 2077 Animal and atrine fats and oils}

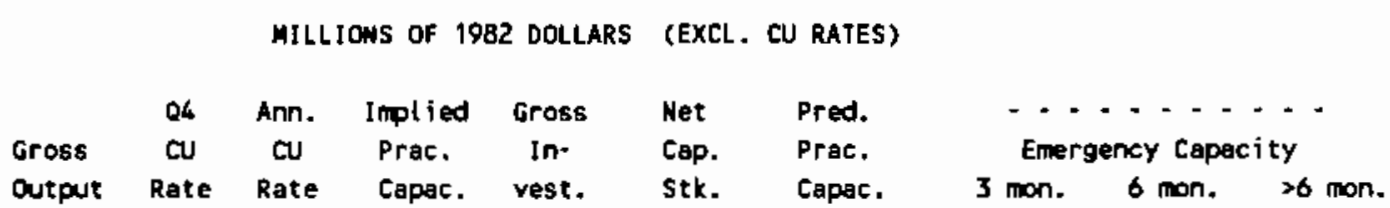

\begin{tabular}{|c|c|c|c|c|c|c|c|c|c|c|}
\hline 1974 & 1,280 & 74 & 81.6 & 1,568 & $(66.0)$ & 461 & 1,549 & & & \\
\hline 1975 & 1,305 & $\pi$ & 71.0 & 1,838 & 116.0 & 532 & 1,688 & & & \\
\hline 1976 & 1,402 & Bo & 7.4 & 1,813 & 63.0 & 547 & 1,826 & & & \\
\hline 1977 & 1,594 & 76 & 78.7 & 2,024 & 85.1 & 581 & 1,965 & & & \\
\hline 1978 & 1,666 & 72 & 74.6 & 2,231 & 98.3 & 625 & 2,103 & & & \\
\hline 1979 & 1,511 & 79 & 76.2 & 1,983 & 102.4 & 666 & 2,242 & & & \\
\hline 1980 & 1,650 & 89 & 86.7 & 1,903 & $\pi .7$ & 675 & 2,380 & & & \\
\hline 1981 & 1,692 & 75 & 82.2 & 2,059 & 59.6 & 670 & 2,519 & & & \\
\hline 1992 & 1,744 & 53 & 60.4 & 2,887 & 44.1 & 648 & 2,657 & & & \\
\hline 1983 & 1,710 & 53 & 52.5 & 3,256 & 24.6 & 611 & 2,796 & & & \\
\hline 1984 & 1,981 & 53 & 54.4 & 3,641 & 64.2 & 613 & 2,934 & & & \\
\hline 1985 & 2,004 & 73 & 65.8 & 3,048 & $(9.8)$ & 547 & 3,073 & & & \\
\hline 1986 & 2,019 & 74 & 74.9 & 2,694 & 29.7 & 522 & 3,211 & & & \\
\hline - & 2,061 & - & 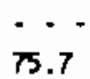 & 2,723 & $\begin{array}{l}\cdots \\
30.0\end{array}$ & 497 & 3,350 & $\cdots$ & $-\cdots$ & - - \\
\hline 1988 & 2,116 & 95 & 87.6 & 2,417 & 26.3 & 472 & 3,488 & 4,742 & 5,114 & 5,190 \\
\hline 1989 & & & & & 22.6 & 446 & 3,627 & 4,930 & 5,317 & 5,397 \\
\hline 1990 & & & & & 19.0 & 418 & 3,765 & 5,118 & 5,520 & 5,603 \\
\hline 1991 & & & & & 15.3 & 390 & 3,904 & 5,306 & 5,723 & 5,809 \\
\hline 1992 & & & & & 11.6 & 361 & 4,042 & 5,495 & 5,926 & 6,015 \\
\hline
\end{tabular}

Capecity-Capital Stock Regression: Code -2 RSO 0.690

Coefficients: Const 1410.9 Time 138.5

Ave. Capacity/Stock $(74-86): 4.0$ Ave. Weekly Hours (High) 101.4 Shift Factor 1.49 Ave Heekly Hours (LOW) 96.5 Shift Factor 1.56 Note: Output, Investment, and Capital Stock extrapolated for 1987 and 1988.

\section{Animal and marine fats and oils}

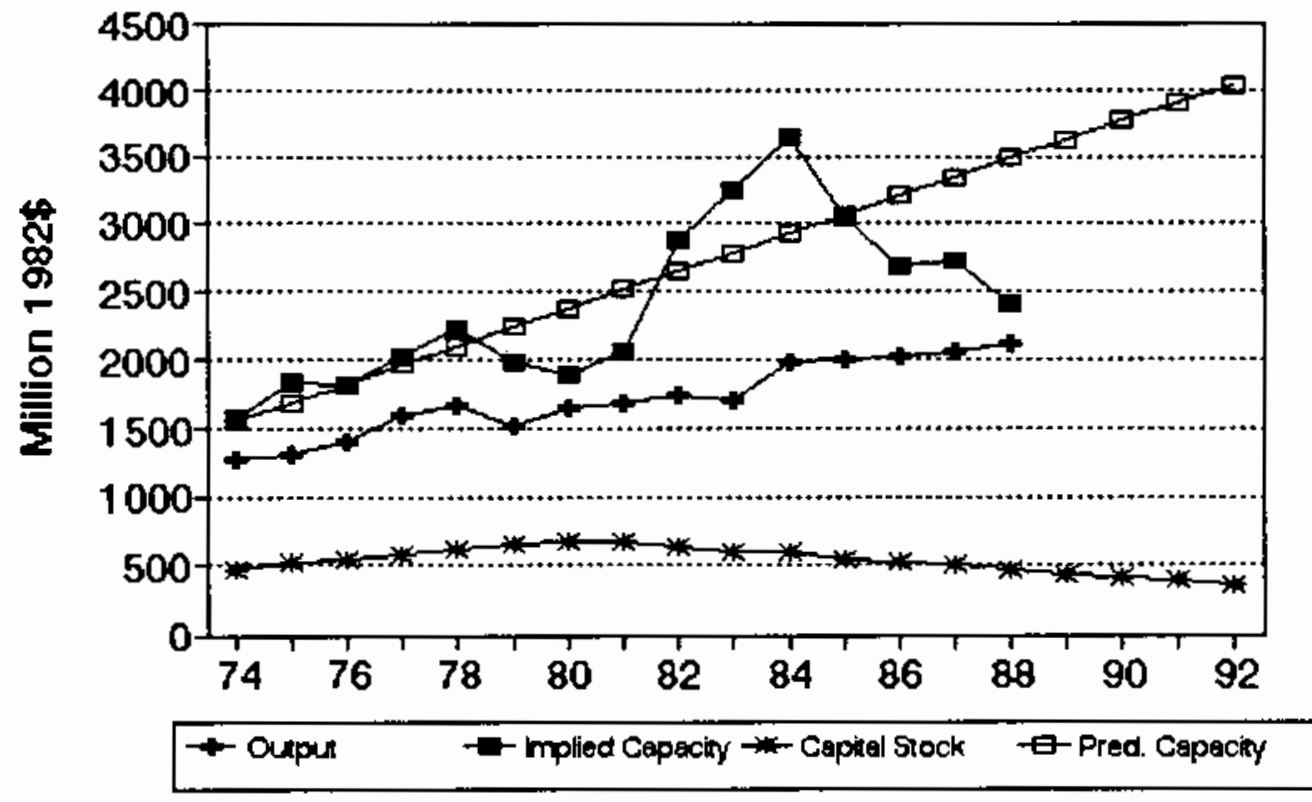


No. 35 sIC 2079 shortening and cooking oils

\section{MILLIONS OF 1982 DOLLARS (EXCL. CU RATES)}

$\begin{array}{lccccccc} & 04 & \text { Amn. } & \text { Implied Gross } & \text { Net } & \text { Pred. } & \ldots \ldots \ldots \\ \text { Gross } & \text { CU } & \text { CU } & \text { Prac. } & \text { In- } & \text { Cap. } & \text { Prac. } & \text { Emergency Capacity } \\ \text { Output } & \text { Rate } & \text { Rate } & \text { Capac. } & \text { vest. } & \text { \$tk. } & \text { Capac. } & 3 \text { mon. } 6 \text { mon. }>6 \text { mon. }\end{array}$

\begin{tabular}{|c|c|c|c|c|c|c|c|c|c|c|}
\hline 1974 & 4,106 & 80 & 80.7 & 5,091 & $(1.3)$ & 695 & 4,667 & & & \\
\hline 1975 & 4,333 & 94 & 88.4 & 4,900 & 140.4 & 767 & 5,405 & & & \\
\hline 1976 & 4,500 & 86 & 88.9 & 5,060 & 45.6 & 745 & 5,180 & & & \\
\hline 1977 & 4.704 & 86 & 86.1 & 5,462 & 130.4 & 803 & 5,772 & & & \\
\hline 1978 & 5,364 & 77 & 80.5 & 6,665 & 97.5 & 828 & 6,023 & & & \\
\hline 1979 & 5,455 & 84 & 81.4 & 6,706 & 105.1 & 859 & 6,341 & & & \\
\hline 1980 & 5,073 & 76 & 79.1 & 6,414 & 52.8 & 839 & 6,133 & & & \\
\hline 1981 & 5,024 & 78 & 77.4 & 6,491 & 126.2 & 888 & 6,639 & & & \\
\hline 1982 & 4,915 & 81 & 79.8 & 6,158 & 24.3 & 840 & 6,152 & & & \\
\hline 1983 & 4,347 & 86 & 84.1 & 5,171 & 50.2 & 819 & 5,933 & & & \\
\hline 1984 & 4,398 & 84 & 84.9 & 5,180 & 18.0 & 768 & 5,413 & & & \\
\hline 1985 & 4,513 & 81 & 82.1 & 5,494 & 88.8 & 786 & 5,598 & & & \\
\hline 1986 & 4.715 & 77 & 78.6 & $5, \infty$ & 65.5 & 780 & 5,534 & & & \\
\hline$\cdots$ & $\cdots$ & - & $-\cdot$ & $\cdots$ & $\cdots$ & $-\cdots$ & $\cdots$ & $-\cdots$ & $=\cdot$ & $-\cdot-$ \\
\hline 1987 & 4,765 & 77 & 77.0 & 6,185 & 78.1 & 787 & 5,607 & & & \\
\hline 1988 & 4,830 & 82 & 80.1 & 6,029 & 85.4 & 801 & 5,747 & 6,217 & 6,414 & 6,454 \\
\hline 1989 & & & & & 92.2 & 820 & 5,945 & 6,431 & 6,634 & 6,676 \\
\hline 1990 & & & & & 96.4 & 842 & 6,168 & 6,672 & 6,883 & 6,926 \\
\hline 1991 & & & & & 97.6 & 863 & 6,382 & 6,903 & 7,121 & 7,166 \\
\hline 1992 & & & & & 97.0 & 881 & 6,570 & 7,107 & 7,332 & 7,378 \\
\hline
\end{tabular}

Capecity-Capital Stock Regression: Code 0 RSO 0.620

Coefficients: Const -2427.3 stock 10.2

Ave. Capacity/stock $(74-86): 7.2$ Ave. Weekly Hours (High) 135.4 Shift factor 1.12 Ave Weekly Hours (Low) 128.0 Shift Factor 1.19 Note: Output, Imvestment, and Cspital Stock extrapolated for 1987 and 1988.

\section{Shortening and cooking oils}
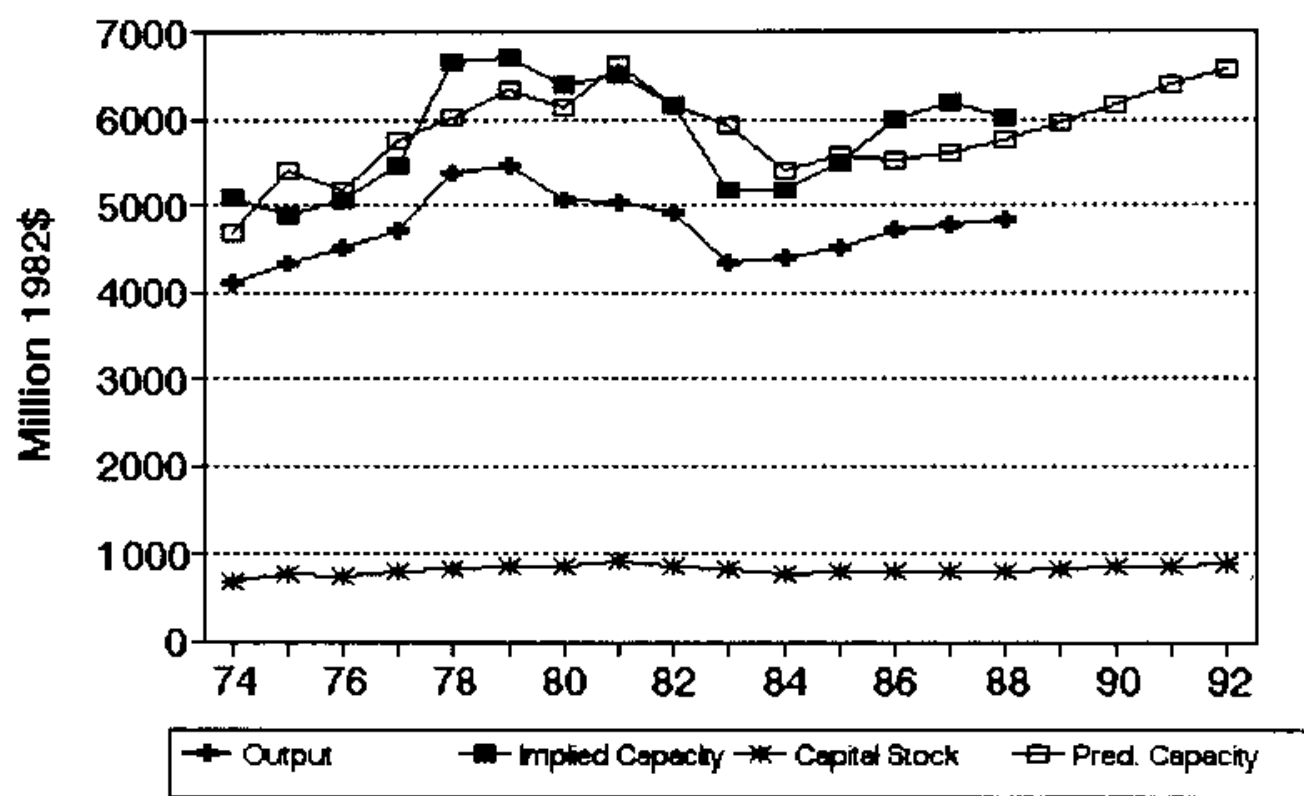


\section{No. 36 sIC 2082 Malt beverages}

\section{MILLIOWS OF 1982 DOLLARS (EXCL. CU RATES)}

$\begin{array}{lccccccc} & \text { Q4 } & \text { Am. Implied Gross } & \text { Net } & \text { Pred. } & \ldots \ldots \ldots \\ \text { Gross } & \text { CU } & \text { CU } & \text { Prac. } & \text { In- } & \text { Cap. } & \text { Prac. } & \text { Emergency Capacity } \\ \text { Output } & R a t e & \text { Rate } & \text { Capac. } & \text { vest. } & \text { Stk. } & \text { Capac. } & 3 \text { mon. } 6 \text { mon. }>6 \text { mon. }\end{array}$

\begin{tabular}{|c|c|c|c|c|c|c|c|c|c|c|}
\hline 1974 & 8,127 & 70 & 70.0 & 11,610 & 630.1 & 3,971 & 10,555 & & & \\
\hline 1975 & 7,906 & 80 & 76.3 & 10,369 & 558.0 & 4,223 & 11,106 & & & \\
\hline 1976 & 8,321 & $\pi$ & 78.1 & 10,651 & 623.0 & 4,531 & 11,79 & & & \\
\hline 1977 & 9,081 & 73 & 74.5 & 12,190 & 622.6 & 4,815 & 12,401 & & & \\
\hline 1978 & 9,980 & 70 & 71.1 & 14,032 & 729.0 & 5,179 & 13,196 & & & \\
\hline 1979 & 9,688 & $\pi$ & 71.9 & $13,4 \pi$ & 647.9 & 5,450 & 13,789 & & & \\
\hline 1980 & 10,194 & $\pi$ & 74.3 & 13,729 & 610.0 & 5,662 & 14,253 & & & \\
\hline 1981 & 10,551 & 86 & 81.9 & 12,886 & 330.1 & 5,578 & 14,069 & & & \\
\hline 1992 & 11,228 & 71 & 76.6 & 14,654 & 713.9 & 5,822 & 14,603 & & & \\
\hline 1983 & 11,139 & 76 & 74.1 & $15,02 B$ & 96.8 & 5,459 & 13,809 & & & \\
\hline 1984 & 10,877 & 76 & 76.0 & 14,312 & 733.7 & 5,685 & 14,304 & & & \\
\hline 1985 & 10,938 & 78 & 77.3 & 14,159 & 303.1 & 5,466 & 13,824 & & & \\
\hline 1986 & 11,128 & 79 & 78.6 & 14,153 & 408.2 & 5,346 & 13,561 & & & \\
\hline $\begin{array}{l}--- \\
1987\end{array}$ & $\begin{array}{l}\cdots \\
11,411\end{array}$ & 79 & 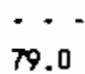 & $\begin{array}{l}\cdots, 444 \\
14,4\end{array}$ & $\begin{array}{l}--. \\
550.1\end{array}$ & $\begin{array}{c}\cdots \\
5,376\end{array}$ & $\cdots-$ & $\cdot \cdot-$ & $-\cdot-\cdot$ & $-\cdots$ \\
\hline 1988 & 11,789 & 79 & 79.0 & 14,922 & 565.1 & 5,619 & 13,720 & 13,614 & 13,873 & 13,926 \\
\hline 1989 & & & & & 579.2 & 5,471 & 13,835 & 13,728 & 13,989 & 14,043 \\
\hline 1990 & & & & & 587.8 & 5,527 & 13,958 & 13,849 & 14,113 & 14,167 \\
\hline 1991 & & & & & 590.2 & 5,580 & 14,073 & 13,964 & 14,229 & 14.284 \\
\hline 1992 & & & & & 589.0 & 5,626 & 14,175 & 14,065 & 14,332 & 14,387 \\
\hline
\end{tabular}

Capecity-Capital Stock Regression: Code 0 RSO 0.730 Coefficients: Const 1868.8 stock 2.2

Ave. Capacity/stock $(74-86): 2.5$ Ave. Weekly Hours (High) 100.7 Shift Factor 1.01 he Weekly Hours (Low) 156.6 Shift Factor 1.04 Note: Output, Investment, and Capital stock extrapolated for 1987 and 1988.

\section{Malt beverages}

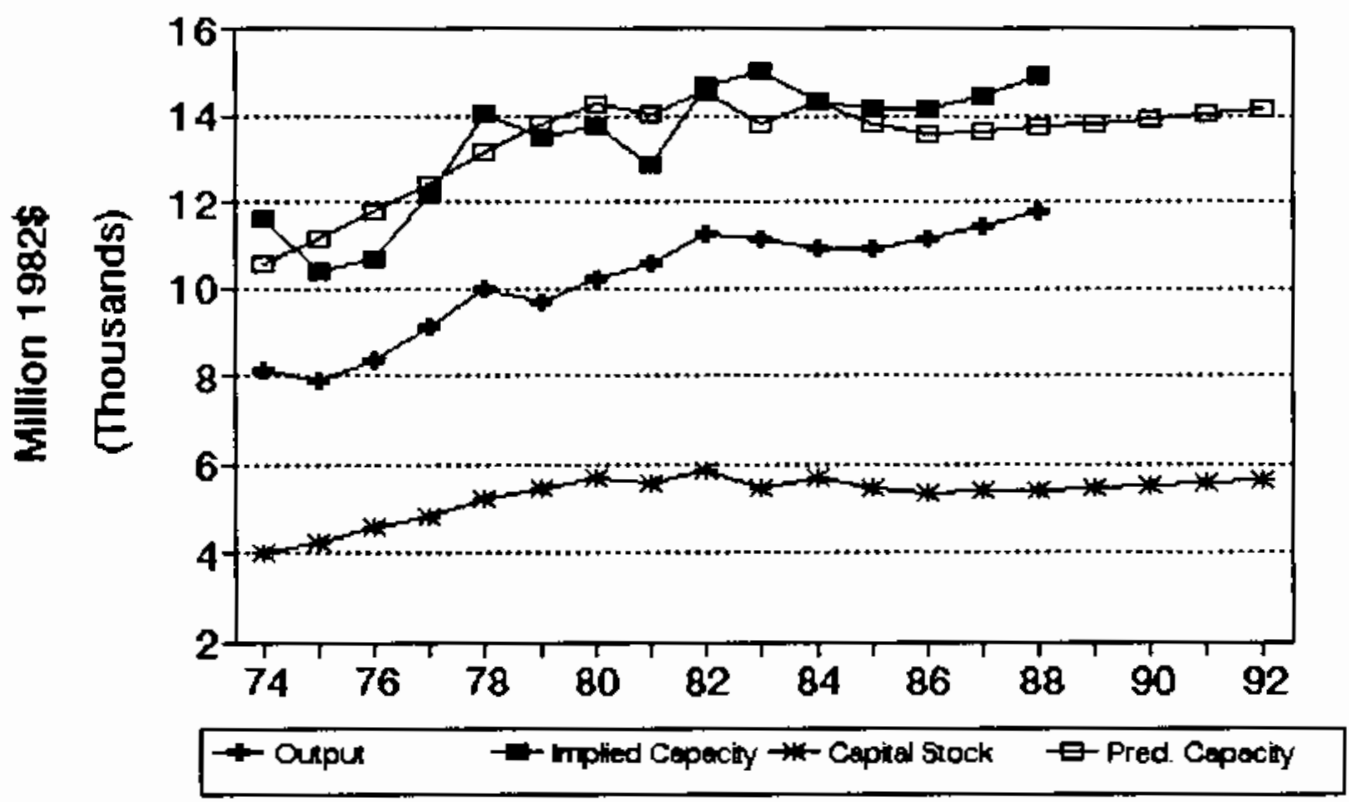


No. 37 sIC 2083 Malt

MILLIONS OF 1982 DOLLARS (EXCL. CU RATES)

$\begin{array}{lccccccc} & \text { Q4 } & \text { Ann. } & \text { Implied Gross } & \text { Net } & \text { Pred. } & \ldots & \ldots . \ldots \\ \text { Gross } & \text { CU } & \text { CU } & \text { Prac. } & \text { In- } & \text { Cap. } & \text { Prac. } & \text { Emergency Capacity } \\ \text { Output } & \text { Rate } & \text { Rate } & \text { Capac. } & \text { vest. } & \text { Stk. } & \text { Capac. } & 3 \text { mon. } 6 \text { mon. }>6 \text { mon. }\end{array}$

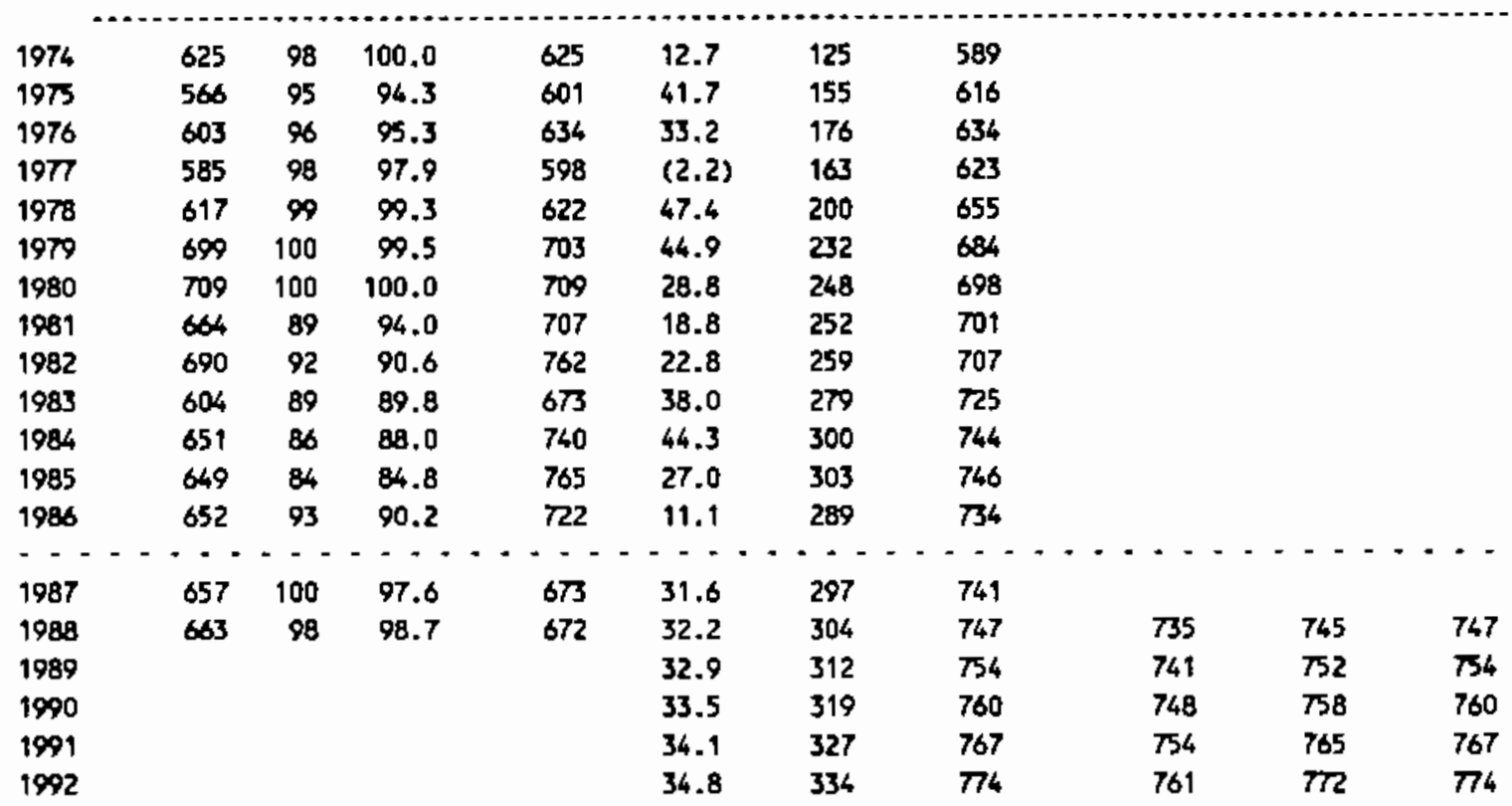

Capacity-Capital stock Regression: Code 0 RSO 0.770

Coefficients: Const 480.2 stock 0.9

Ave. Capecity/Stock $(74-86): 3.0$ Ave. Weekly Hours (High) 168.0 Shift Factor 1.00 Ave Ueekly Hours (Low) 160.0 Shift factor 1.05 Note: Output, investment, and Capital stock extrapolated for 1987 and 1988.

\section{Malt}
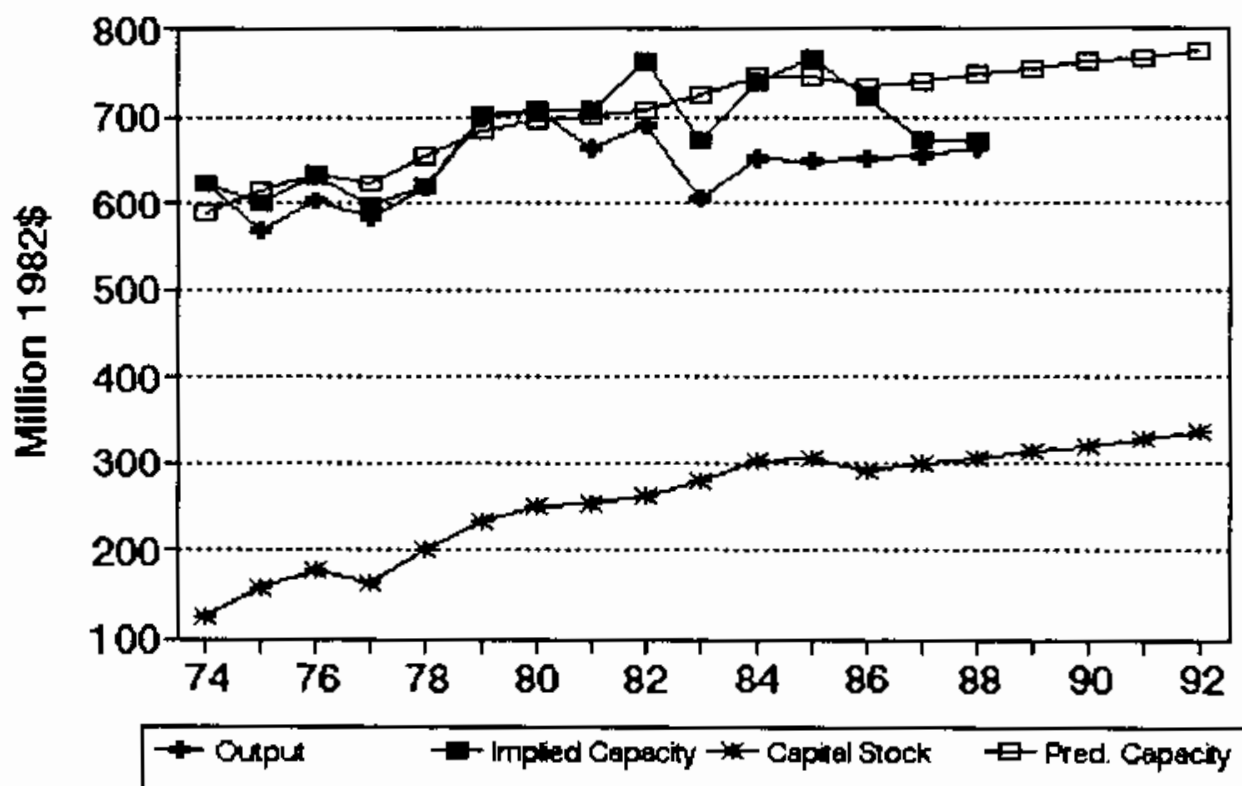


\section{No. 38 SIC 2084 wines, brandy, and brandy spirits}

\section{MILLIONS OF 1982 DOLLARS (EXCL. CU RATES)}

$\begin{array}{lccccccc} & \text { Q4 } & \text { Ann. Implied Gross } & \text { Net } & \text { Pred. } & \ldots & \ldots \\ \text { Gross } & \text { CU } & \text { CU } & \text { Prac. } & \text { In- } & \text { Cap. } & \text { Prac. } & \text { Emergency Capacity } \\ \text { Output } & \text { Rate } & \text { Rate } & \text { Capac. } & \text { vest. } & \text { Stk. } & \text { Capac. } & 3 \text { mon. } 6 \text { mon. }>6 \text { mon. }\end{array}$

\begin{tabular}{|c|c|c|c|c|c|c|c|c|c|c|}
\hline 1974 & 2,350 & 71 & 71.0 & 3,310 & 111.2 & 523 & 3,168 & & & \\
\hline $19 \pi 5$ & 1,999 & 65 & 67.3 & 2,972 & 140.7 & 582 & 3,379 & & & \\
\hline 1976 & $1, \infty 97$ & 58 & 60.6 & 3,294 & 63.4 & 565 & 3,317 & & & \\
\hline 1977 & 2,253 & 66 & 63.0 & 3,576 & 87.7 & 571 & 3,340 & & & \\
\hline 1978 & 2,303 & 50 & 56.0 & 4,273 & 160.8 & 645 & 3,600 & & & \\
\hline 1979 & 2,695 & 79 & 68.1 & 3,956 & 134.3 & 692 & 3,767 & & & \\
\hline 1980 & 2,662 & 89 & 85.3 & 3,123 & 96.4 & 705 & 3,813 & & & \\
\hline 1981 & 2,648 & 75 & 80.3 & 3,300 & 93.5 & 720 & 3,864 & & & \\
\hline 1982 & 3,004 & 66 & 69.6 & 4,329 & 162.3 & 804 & 4,161 & & & \\
\hline 1983 & 2,681 & 54 & 58.5 & 4,583 & 93.9 & 822 & 4,224 & & & \\
\hline 1984 & 2,583 & 71 & 64.6 & 3,997 & 65.0 & 814 & 4,196 & & & \\
\hline 1985 & 2,749 & 67 & 68.5 & 4,013 & 98.6 & 839 & 4,286 & & & \\
\hline 1986 & 3,003 & 62 & 63.9 & 4,701 & 81.9 & 847 & 4,313 & & & \\
\hline$\because 987$ & 3,050 & 61 & $\begin{array}{l}\cdots \\
61.4\end{array}$ & 4,970 & $\begin{array}{r}--- \\
82.1\end{array}$ & $\begin{array}{l}-- \\
855\end{array}$ & $\begin{array}{l}\cdots, 342 \\
4,--\end{array}$ & -- & -- & - - - \\
\hline 1988 & 3,113 & 67 & 64.8 & 4,808 & 78.0 & 859 & 4,354 & 5,731 & 6,102 & 6,178 \\
\hline 1989 & & & & & 74.0 & 858 & 4,351 & 5,726 & 6,097 & 6,174 \\
\hline 1990 & & & & & 69.9 & 853 & 4,333 & 5,704 & 6,073 & 6,149 \\
\hline 1991 & & & & & 65.8 & 844 & 4,303 & 5,664 & 6,030 & 6,106 \\
\hline 1992 & & & & & 61.7 & 832 & 4,261 & 5,608 & 5,971 & 6,046 \\
\hline
\end{tabular}

Capacity-Capital Stock Regression: Code 0 RSO 0.510 Coefficients: Const 1323.9 stock 3.5 Ave. Weekly Hours (High) 106.3 Shift Factor 1.42 Ave Weekly Hours (LOW) 99.2 Shift Factor 1.52 Note: Output, Investment, and Capital stock extrapolated for 1987 and 1988.

\section{Wines, brandy, and brandy spirits}
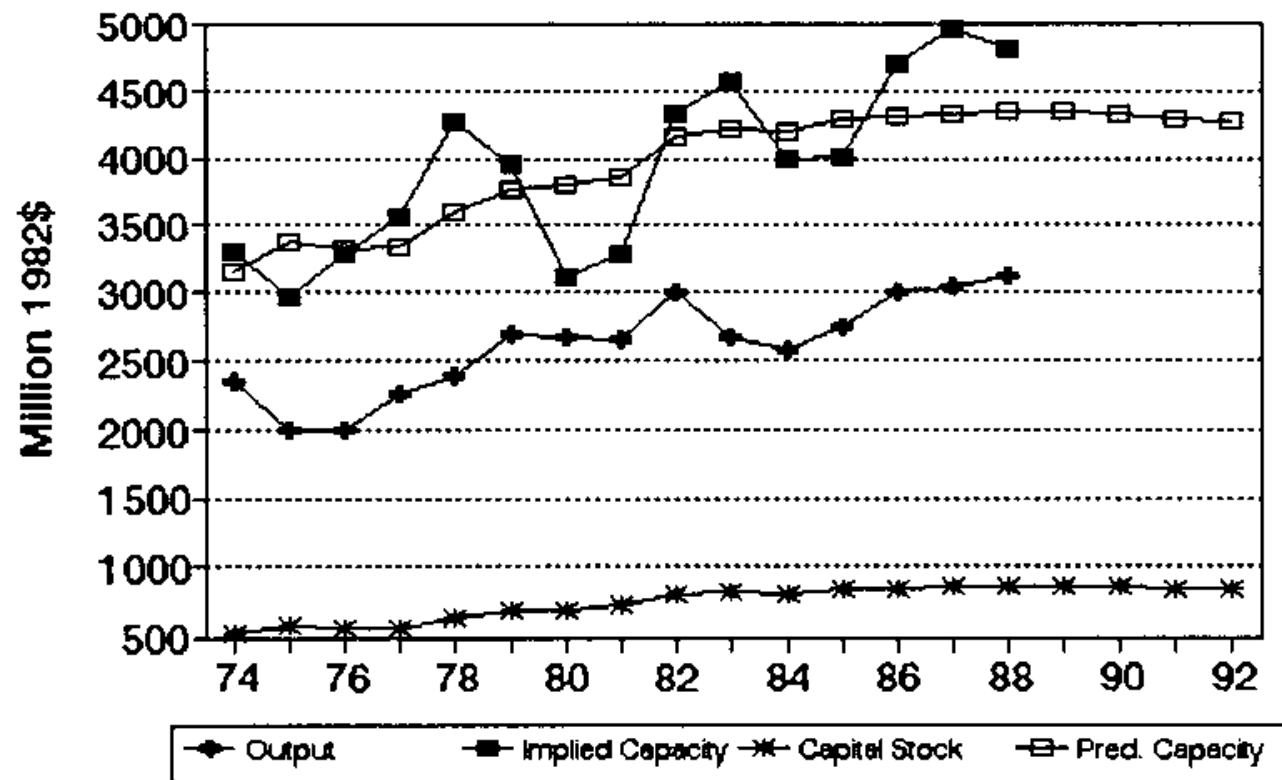


\section{No. 39 SIC 2085 Distilled liquor, except brandy}

MILLIONS OF 1982 DOLLARS (EXCL. CI RATES)

$\begin{array}{lccccccc} & \alpha 4 & \text { Ann. Implied Gross } & \text { Ket } & \text { Pred. } & \ldots \ldots \ldots \\ \text { Gross } & \text { CU } & \text { CU } & \text { Prac. } & \text { In- } & \text { Cap. } & \text { Prac. } & \text { Emergency Capacity } \\ \text { Output Rate } & \text { Rate } & \text { Capac. } & \text { vest. } & \text { Stk. } & \text { Capac. } & 3 \text { mon. } 6 \text { mon. }>6 \text { mon. }\end{array}$

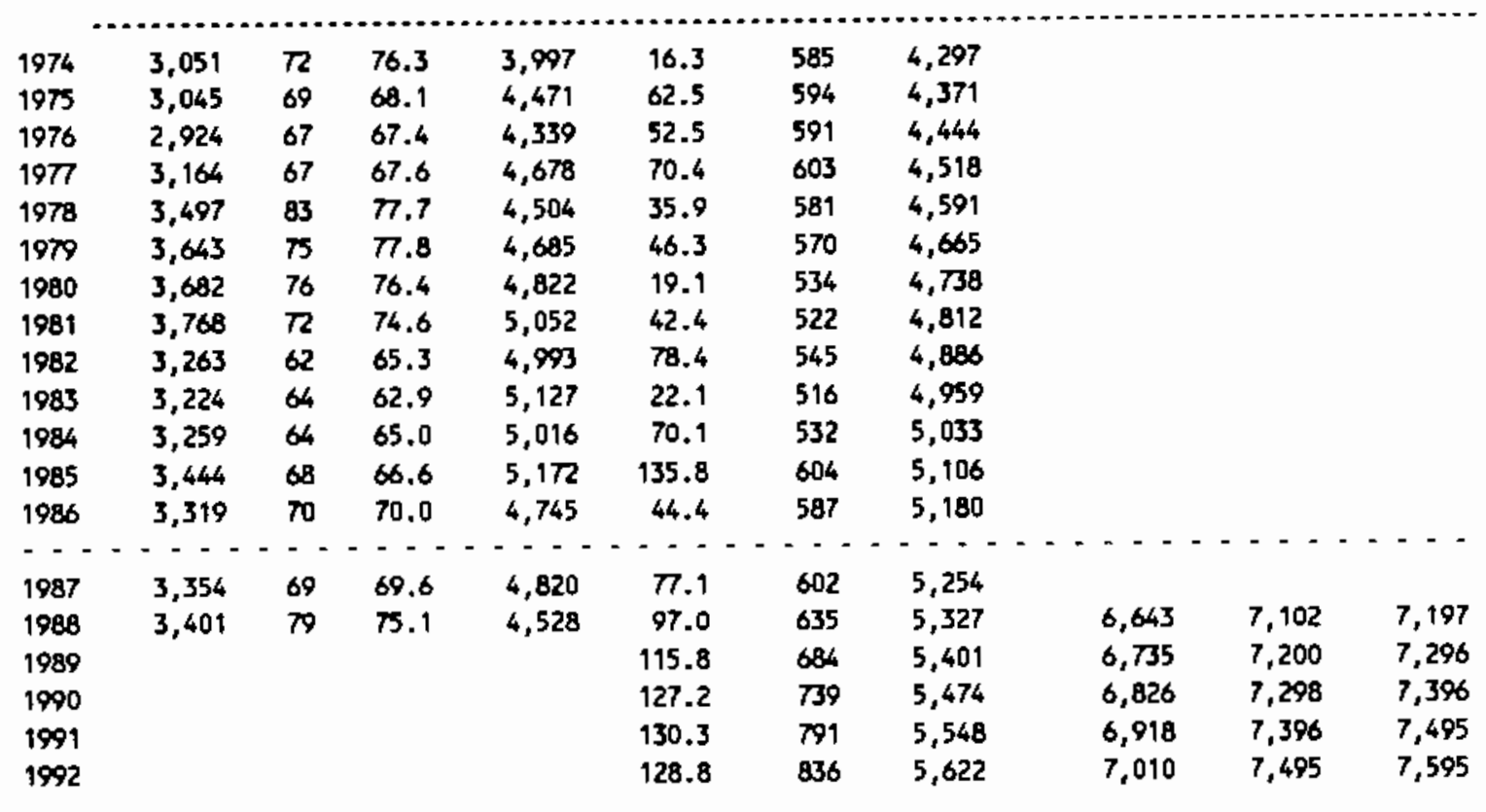

Capacity-Capital Stock Regression: Code -2 RSO 0.690

Coefficients: Const 4223.3 Time 73.6 Ave. Capacity/Stock $(74-86): 8.4$ Ave. Weekly Hours (High) 111.7 Shift Factor 1.35 Ave Weekly Hours (Low) 91.7 Shift Factor 1.64 Note: output, Imvestment, and Capital Stock extrapolated for 1987 and 1988.

\section{Distilled liquor, except brandy}

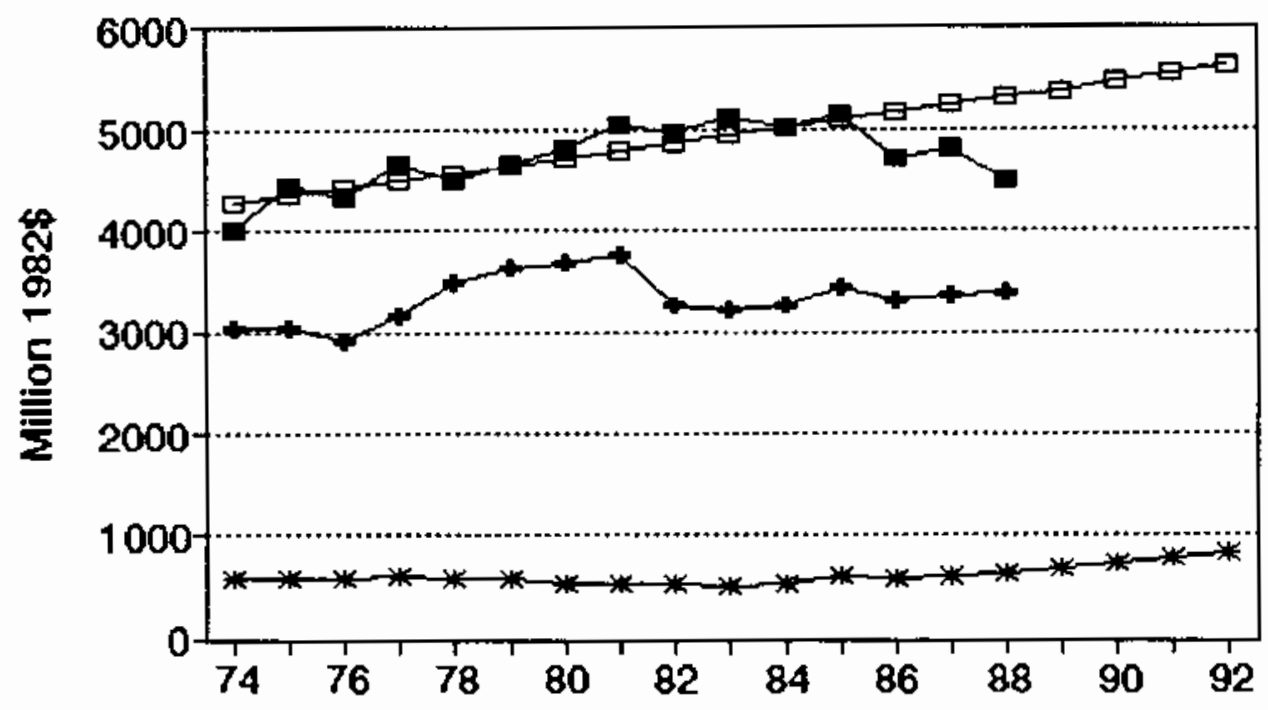

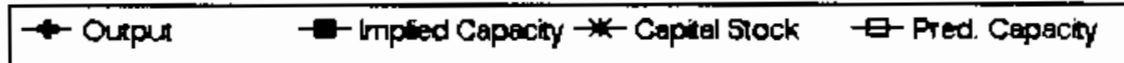




\title{
HO. 40 BIC 2086 Bottled and canned soft drinks
}

\author{
MILLIONS OF 1982 DOLLARS (EXCL. CU RATES)
}

$\begin{array}{lccccccc} & \text { Q4 } & \text { Ann. Implied Gross } & \text { Net } & \text { Pred. } & -\ldots & \ldots \\ \text { Gross } & \text { CU } & \text { CU } & \text { Prac. } & \text { In- } & \text { Cap. } & \text { Prac. } & \text { Emergency Capacity } \\ \text { Output } & \text { Rate } & \text { Rate } & \text { Capac. } & \text { vest. } & \text { Stk. } & \text { Capac. } & 3 \text { mon. } 6 \text { mon. }>6 \text { mon. }\end{array}$

\begin{tabular}{|c|c|c|c|c|c|c|c|c|c|c|}
\hline 1974 & 14,372 & 72 & 80.0 & 17,973 & 107.7 & 3,118 & 19,725 & & & \\
\hline 1975 & 14,773 & 61 & 61.6 & 23,984 & 458.9 & 3,301 & 21,061 & & & \\
\hline 1976 & 14,966 & 67 & 64.0 & 23,368 & 312.0 & 3,335 & 21,316 & & & \\
\hline 1977 & 16,161 & 76 & 73.8 & 21,907 & 669.7 & 3,705 & 24,019 & & & \\
\hline 1978 & 17,234 & 68 & 7.2 & 23,868 & 512.2 & 3,905 & 25,484 & & & \\
\hline 1979 & 17,628 & 68 & 67.8 & 26,018 & 396.6 & 3,993 & 26,132 & & & \\
\hline 1980 & 16,981 & 59 & 63.4 & 26,768 & 578.5 & 4,251 & 28,019 & & & \\
\hline 1981 & 16,045 & 55 & 58.1 & 27,626 & 380.9 & 4,305 & 28,413 & & & \\
\hline 1922 & 16,836 & 50 & 51.3 & 32,792 & 652.5 & 4,603 & 30,593 & & & \\
\hline 1983 & 16,870 & 55 & 52.5 & 32,119 & 415.5 & 4,653 & 30,964 & & & \\
\hline 1984 & 16,935 & 55 & 56.6 & 29,928 & 126.3 & 4,431 & 29,335 & & & \\
\hline 1985 & 17,970 & 64 & 60.8 & 29,546 & 487.3 & 4,552 & 30,222 & & & \\
\hline 1986 & 18,814 & 62 & 63.9 & 29,424 & 354.6 & 4,527 & 30,039 & & & \\
\hline $\begin{array}{l}--- \\
1987\end{array}$ & $=-\cdot$ & -- & $\because \cdots$ & $\begin{array}{l}\cdots \\
28.044\end{array}$ & $\begin{array}{l}\because \cdot- \\
410.5\end{array}$ & $\begin{array}{l}\cdots-\cdot \\
4.566\end{array}$ & $\begin{array}{l}\cdots \\
30.321\end{array}$ & $-\cdot \cdot$ & --- & $\cdots$ \\
\hline 1988 & 19,166 & 69 & 68.1 & 28,143 & 407.9 & 4,598 & 30,561 & 42,652 & 45,980 & 46,667 \\
\hline 1989 & & & & & 405.2 & 4,626 & 30,762 & 42,933 & 46,282 & 46,974 \\
\hline 1990 & & & & & 402.6 & 4,649 & 30,928 & 43,163 & 46,531 & 47,227 \\
\hline 1991 & & & & & 400.0 & 4,667 & 31,060 & 43,348 & 46,730 & 47,429 \\
\hline 1992 & & & & & 397.3 & 4,681 & 31,163 & 43,491 & 46,884 & 47,585 \\
\hline
\end{tabular}

Capacity-Capitat Stock Regression: Code 0 RSO 0.850

Coefficients: Const -3097.6 stock 7.3

Ave. Capacity/Stock $(74-86): 0.6$ Ave. Heekly Hours (High) 98.8 Shift factor 1.53 Ave Ueekly Hours (Low) 97.3 Shift Factor 1.55 Mote: Output, Investment, and Capital stock extrapolated for 1987 and 1988.

\section{Bottled and canned soft drinks}

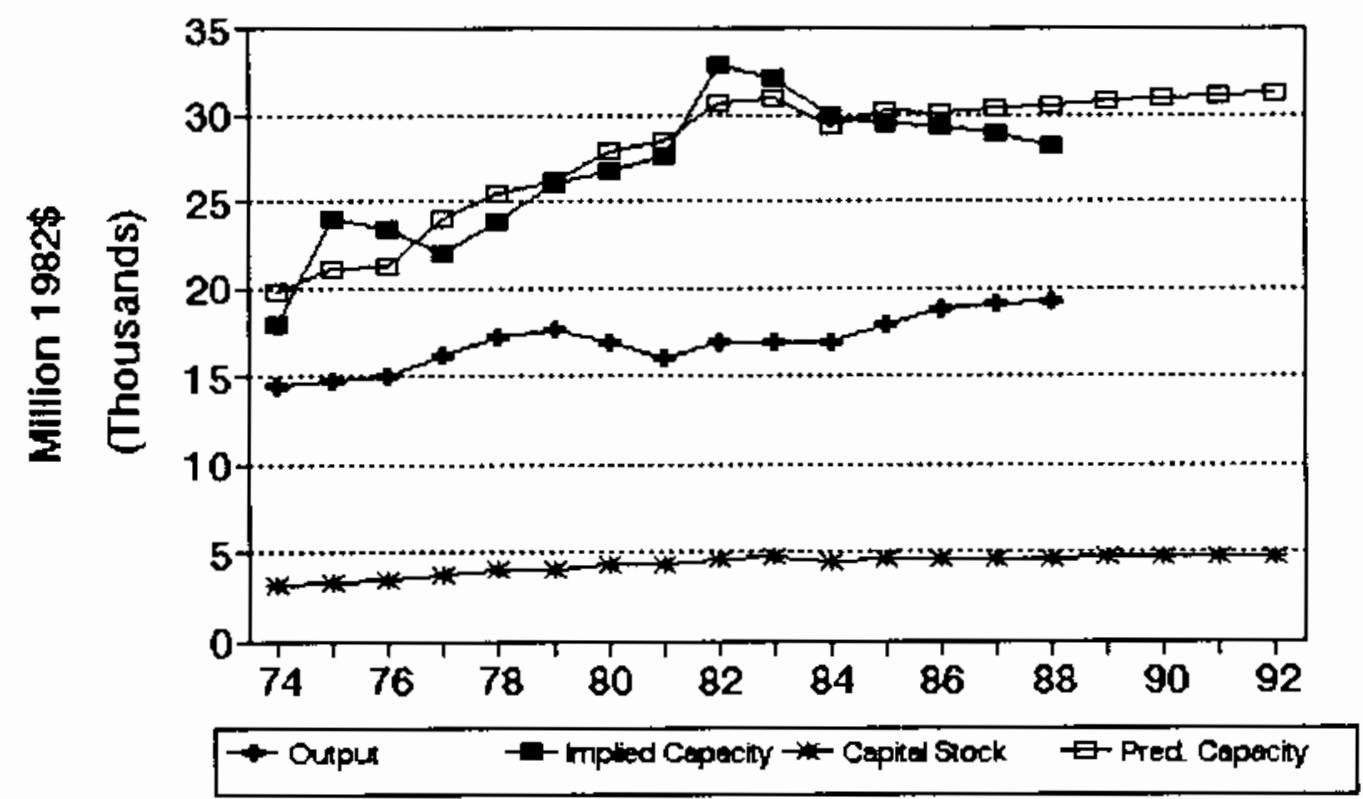




\section{No. 41 sIC 2087 Flavoring extracts and sirups, n.e.c.}

MILLIONS OF 1982 DOLLARS (EXCL. CU RATES)

$\begin{array}{lccccccc} & \text { Q4 } & \text { Ann. } & \text { Implied Gross } & \text { Net } & \text { Pred. } & \ldots \ldots \ldots \\ \text { Gross } & \text { CU } & \text { CU } & \text { Prac. } & \text { In- } & \text { Cap. } & \text { Prac. } & \text { Energency Capacity } \\ \text { Output Rate Rate } & \text { Capac. } & \text { vest. } & \text { Stk. } & \text { Capac. } & 3 \text { mon. } 6 \text { mon. }>6 \text { mon. }\end{array}$

\begin{tabular}{|c|c|c|c|c|c|c|c|c|c|c|}
\hline 1974 & 3,050 & 37 & 37.0 & 8,264 & 56.1 & 413 & 7,033 & & & \\
\hline 1975 & 2,657 & 38 & 37.6 & 7,061 & $(29.9)$ & 355 & 7,018 & & & \\
\hline 1976 & 3,213 & 47 & 43.6 & 7,365 & 7.3 & 395 & 7,002 & & & \\
\hline 1977 & 3,469 & 53 & 50.8 & 6,797 & 27.2 & 389 & 6,986 & & & \\
\hline 1978 & 3,924 & 58 & 56.1 & 6,991 & $\pi .1$ & 428 & 6,971 & & & \\
\hline 1979 & 3,750 & 64 & 61.8 & 6,072 & 52.0 & 442 & 6,955 & & & \\
\hline 1980 & 3,802 & 64 & 64.0 & 5,941 & 15.9 & 422 & 6,939 & & & \\
\hline 1991 & 3,822 & 66 & 65.3 & 5,857 & 126.9 & 505 & 6,824 & & & \\
\hline 1982 & 4,232 & 61 & 62.9 & 6,731 & $(13.4)$ & 452 & 6,908 & & & \\
\hline 1983 & 4,088 & 59 & 59.8 & 6,841 & 17.7 & 430 & 6,893 & & & \\
\hline 1984 & 4,283 & 59 & 59.0 & 7,259 & 88.4 & $4 \pi$ & 6,877 & & & \\
\hline 1985 & 4,491 & 62 & 60.9 & 7,377 & 68.4 & 497 & 6,861 & & & \\
\hline 1986 & 4,568 & 58 & 59.5 & 7,677 & 42.1 & 492 & 6,846 & & & \\
\hline-- & $-\cdots$ & $\because$ & -- & $\cdots$ & $=$. & -- & $-\cdots$ & $\cdot \cdot \cdot$ & $-\cdots$ & -- \\
\hline 1987 & 4,661 & 49 & 52.4 & 8,900 & 51.3 & 497 & 6,830 & & & \\
\hline 1988 & 4,786 & 49 & 49.0 & 9,767 & 51.6 & 502 & 6,814 & 8,671 & 9,222 & 9,336 \\
\hline 1989 & & & & & 51.9 & 507 & 6,799 & 8,651 & 9,201 & 9,314 \\
\hline 1990 & & & & & 52.3 & 512 & 6,783 & 8,632 & 9,180 & 9.293 \\
\hline 1991 & & & & & 52.6 & 516 & 6,767 & 8,612 & 9,158 & 9,271 \\
\hline 1992 & & & & & 52.9 & 520 & 6,752 & 8,592 & 9,137 & 9,250 \\
\hline
\end{tabular}

Capacity-Capital Stock Regression: Code -2 RSQ 0.010

Coefficients: Const 7048.8 Time -15.6

Ave. Capacity/Stock $(74-86): 15.8$ Ave. Weekly Hours (High) $\$ 10.1$ Shift Factor 1.37 Ave Heekly Hours (Low) 90.2 Shift Factor 1.67 Note: Output, Imvestment, and Capital Stock extrapolated for 1987 and 1988.

\section{Flavoring extracts and sirups, n.e}

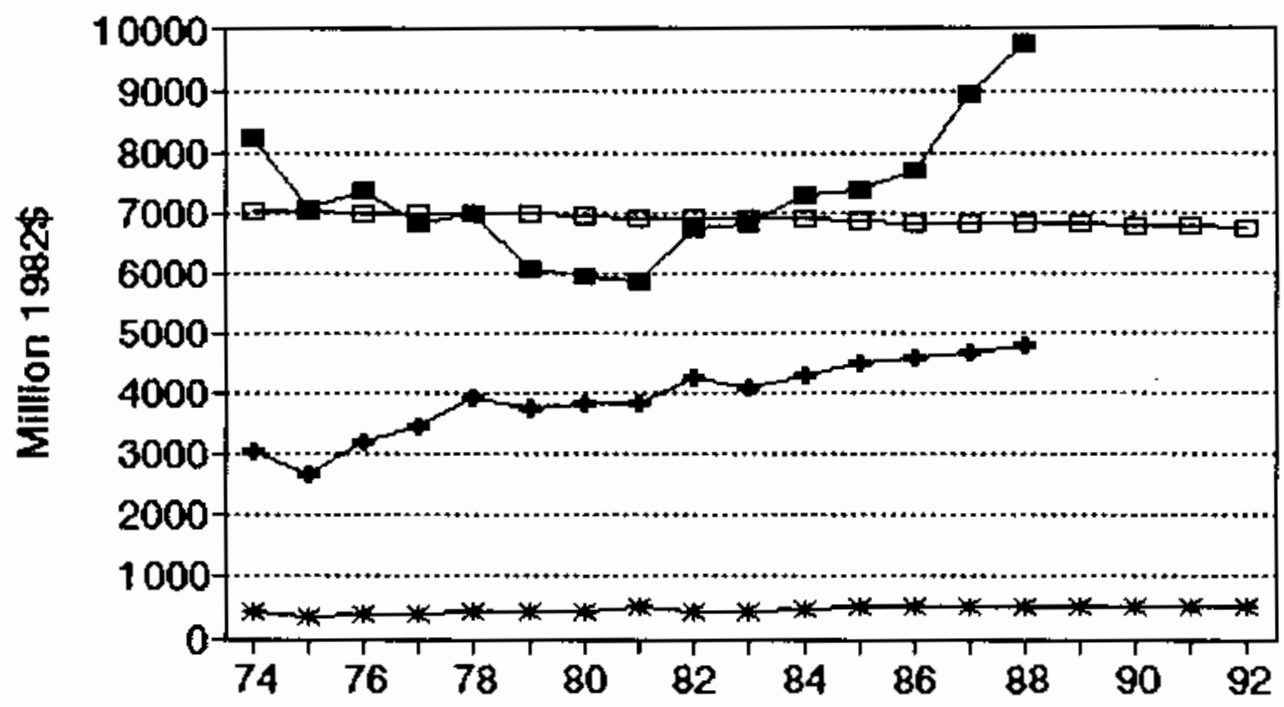

- Oupu $\rightarrow$ - Impled Capaciy - Capited Srock $\rightarrow$ - Pred. Cepacity




\section{No. 42 SIC 2091 Canned and cured beafooda}

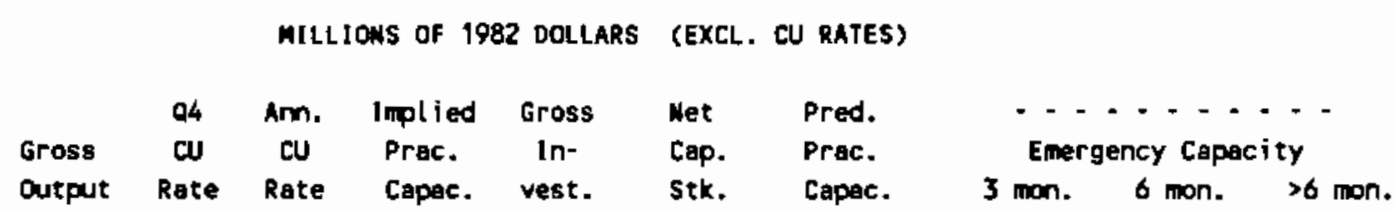

\begin{tabular}{|c|c|c|c|c|c|c|c|c|c|c|}
\hline 1974 & 1,928 & 67 & 67.0 & 2,877 & 57.1 & 250 & 3,345 & & & \\
\hline 1975 & 1,743 & 52 & 57.6 & 3,024 & $(28.0)$ & 208 & 3,212 & & & \\
\hline 1976 & 2,304 & 60 & 57.0 & 4,042 & 30.6 & 224 & 3,262 & & & \\
\hline 1977 & 2,164 & 71 & 66.9 & 3,236 & 34.9 & 242 & 3,320 & & & \\
\hline 1978 & 2,283 & 56 & 60.4 & 3,782 & 33.3 & 258 & 3,369 & & & \\
\hline 1979 & 2,370 & 63 & 59.6 & 3,974 & 61.2 & 298 & 3,492 & & & \\
\hline 1980 & 2,152 & 76 & 71.1 & 3,026 & 27.1 & 302 & 3,504 & & & \\
\hline 1981 & 2,630 & 71 & 72.9 & 3,608 & 34.6 & 312 & 3,535 & & & \\
\hline 1982 & 1,757 & 55 & 61.0 & 2,880 & $(19.6)$ & 269 & 3,404 & & & \\
\hline 1983 & 1,931 & 54 & 54.4 & 3,551 & 7.6 & 254 & 3,356 & & & \\
\hline 1984 & 1,704 & $61 *$ & 58.5 & 2,915 & $(61.6)$ & 177 & 3,117 & & & \\
\hline 1985 & 572 & $68 *$ & 65.7 & BT2 & $(22.4)$ & 142 & 845 & & & \\
\hline 1986 & 580 & 76 * & 73.0 & 795 & 4.8 & 135 & 822 & & & \\
\hline$-\cdot$ & $-\cdots$ & -- & $\cdots$ & $\cdots$ & $-\cdots$ & $=-$ & $\cdots$ & - & -- & -- \\
\hline 1987 & 565 & 86 & 82.2 & 687 & $(20.9)$ & 102 & 719 & & & \\
\hline 1988 & 545 & 71 & 76.6 & 711 & $(25.6)$ & 67 & 611 & 670 & 688 & 691 \\
\hline 1989 & & & & & (30.3) & 31 & 499 & 546 & 561 & 564 \\
\hline 1990 & & & & & $(35.0)$ & (7) & 381 & 418 & 429 & 431 \\
\hline 1991 & & & & & (39.7) & (46) & 260 & 285 & 292 & 294 \\
\hline 1992 & & & & & $(44.4)$ & (87) & 135 & 167 & 151 & 152 \\
\hline
\end{tabular}

Capacity-Capital Stock Regression: Code -1 RSO 0.860

Coefficients: Const 2566.5 stock 3.1 D(85-86) -2162.8 Ave. Capacity/Stock (74-86): 12.6 Ave. Weekly Hours (High) 134.5 Shift Factor 1.13 Ave Yeekly Hours (LOW) 136.5 Shift factor 1.13 - Imputed Note: Output, Imestment, and Capital Stock extrapolated for 1987 and 1988.

\section{Canned and cured seafoods}

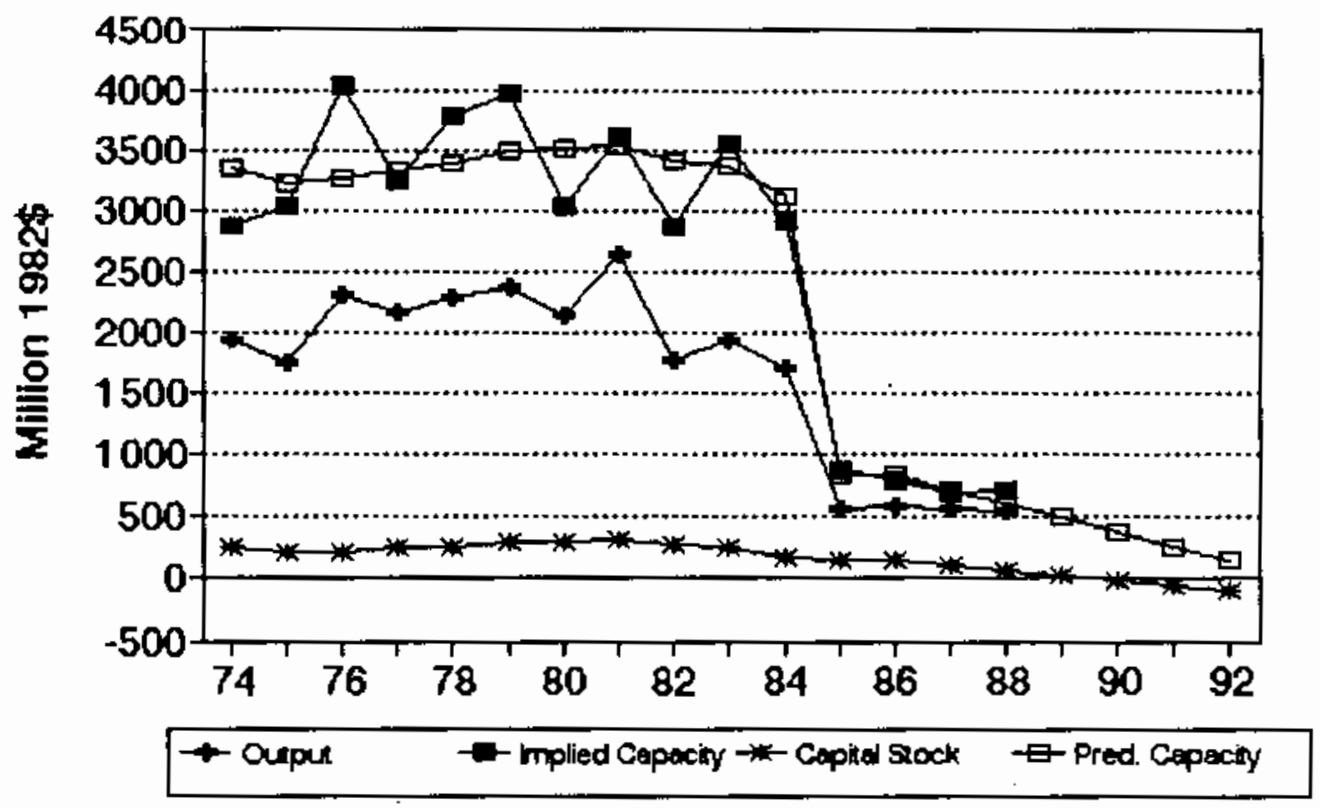




\title{
No. 43 SIC 2092 Fresh or frozen packaged fish
}

\author{
MILLIONS OF 1982 DOLLARS (EXCL. CU RATES)
}

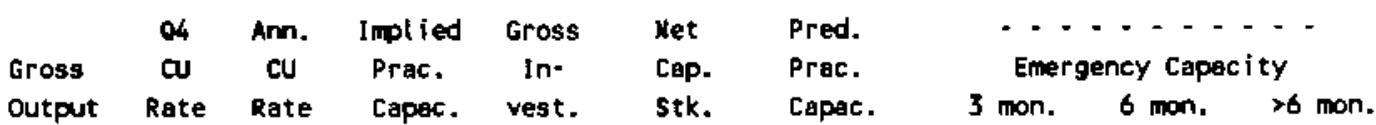

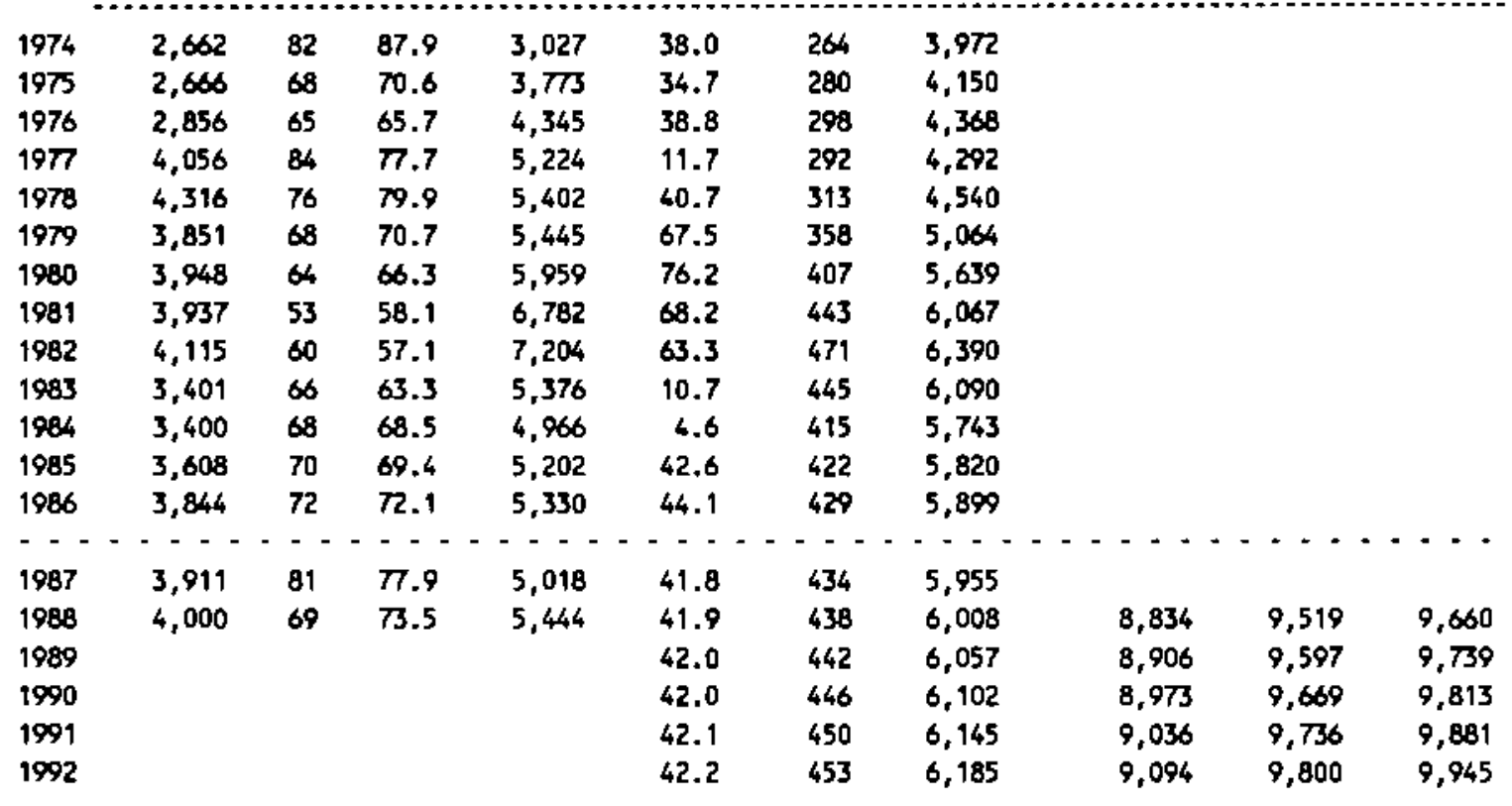

Capacity-Capital Stock Regression: Code 0 RSO 0.600 Coefficients: Const 870.7 stock 11.7

Ave. Capacity/Stock $(74-86): 14.1$ Ave. Weekly Hours (High) 93.8 Shift Factor 1.61 Ave Weekly Hours (Low) 87.9 Shift Factor 1.72 Note: Output, Investment, and Capital stock extrapoleted for 1987 and 1988.

\section{Fresh or frozen packaged fish}

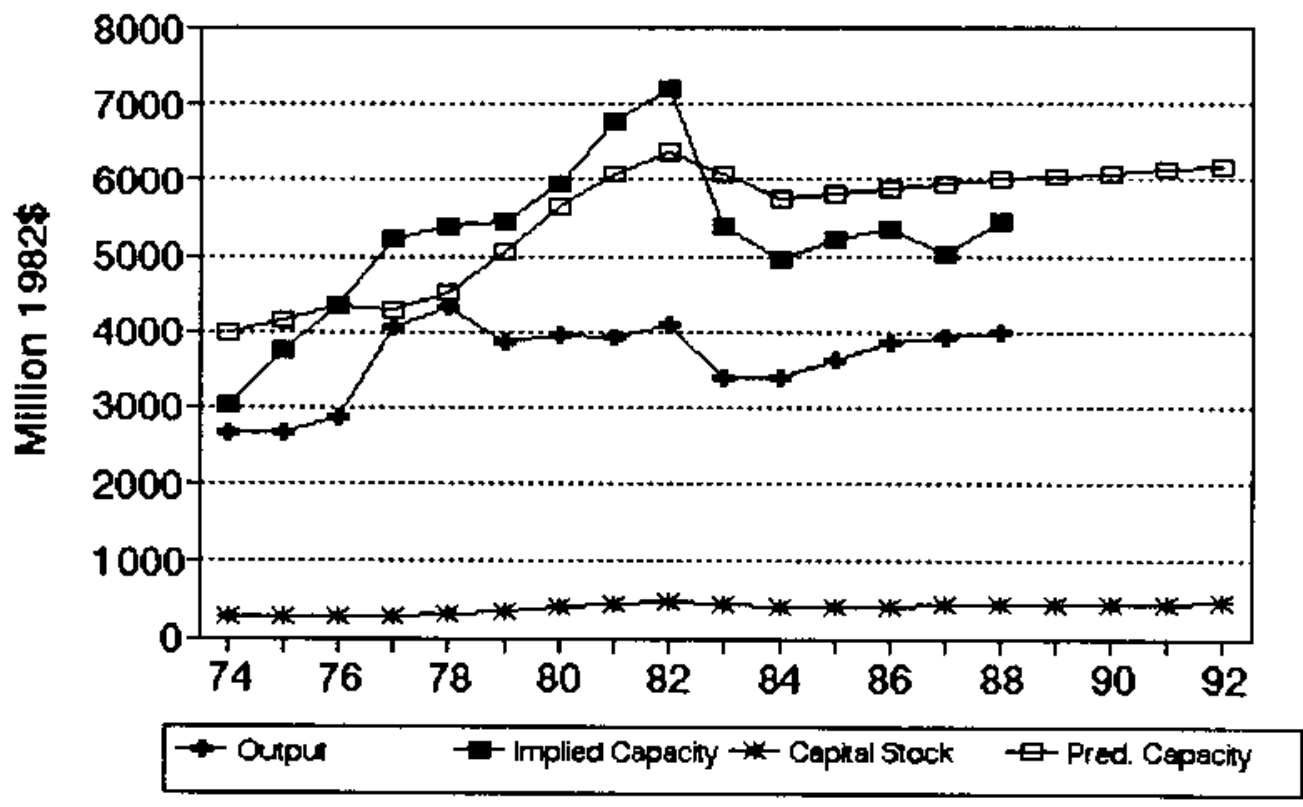


No. 44 SIC 2095 Ronsted coffee

MILLIONS OF 1982 DOLLARS (EXCL, CU RATES)

$\begin{array}{lccccccc} & \text { Q4 } & \text { Ann. } & \text { Implied } & \text { Gross } & \text { Het } & \text { Pred. } & \ldots \ldots \ldots \\ \text { Gross } & \text { CU } & \text { CU } & \text { Prac. } & \text { In- } & \text { Cap. } & \text { Prac. } & \text { Emergency Capacity } \\ \text { Output } & \text { Rate } & \text { Rate } & \text { Capac. } & \text { vest. } & \text { Stk. } & \text { Capac. } & 3 \text { mon. } 6 \text { mon. }\end{array}$

\begin{tabular}{|c|c|c|c|c|c|c|c|c|c|c|}
\hline 1974 & 5,656 & 95 & 95.0 & 5,854 & 68.3 & 814 & 7,999 & & & \\
\hline 1975 & 6,046 & 97 & 96.3 & 6,282 & 85.3 & 822 & 8,010 & & & \\
\hline 1976 & 6,079 & 83 & 88.3 & 6,888 & 78.1 & 821 & 8,008 & & & \\
\hline 1977 & 4,351 & 69 & 74.3 & 5,860 & 126.5 & 862 & 8,060 & & & \\
\hline 1978 & 5,558 & 45 & 54.0 & 10,293 & 45.4 & 825 & 8,013 & & & \\
\hline 1979 & 5,723 & 67 & 58.8 & 9,741 & 111.1 & 848 & 8,043 & & & \\
\hline 1980 & 5,771 & 71 & 69.5 & 8,304 & 68.5 & 831 & 8,021 & & & \\
\hline 1981 & 5,916 & 64 & 66.6 & 8,880 & 107.5 & 854 & 8,040 & & & \\
\hline 1982 & 5,847 & 66 & 65.3 & 8.961 & 68.6 & 839 & 8,031 & & & \\
\hline 1983 & 5,808 & 67 & 66.6 & 8,717 & 87.2 & 845 & 8,038 & & & \\
\hline 1984 & 6,026 & 70 & 68.9 & 8,749 & 114.1 & 878 & 8,080 & & & \\
\hline 1985 & 6,250 & 74 & 72.5 & 8,621 & 170.3 & 964 & 8,188 & & & \\
\hline 1988 & 5,536 & 73 & 73.4 & 7,545 & 140.0 & 1,019 & 8,257 & & & \\
\hline$\cdots$ & $-\cdot-$ & -- & $-\cdot$ & $\cdots \cdot$ & $\cdots$ & $\because-$ & $\therefore-$ & . . & $\cdots-$ & $-\cdot-$ \\
\hline 1987 & 5,535 & 69 & 70.5 & 7,851 & 119.7 & 1,052 & 8,298 & & & \\
\hline 1988 & 5,533 & 69 & 69.0 & 8,019 & 137.4 & 1,100 & 8,357 & 10,471 & 11,171 & 11,316 \\
\hline 1989 & & & & & 154.0 & 1,160 & 8,433 & 10,566 & 11,272 & 11,418 \\
\hline 1990 & & & & & 164.2 & 1,225 & 8,514 & 10,668 & 11,381 & 11,528 \\
\hline 1991 & & & & & 167.0 & 1,288 & 8,592 & 10,766 & 11,486 & 11.634 \\
\hline 1992 & & & & & 165.6 & 1,343 & 8,662 & 10,853 & 11,579 & 11,729 \\
\hline
\end{tabular}

Capacity-Capital Stock Regression: Code 0 RSa 0.000 Cpefficients: Const 6980.3 stock 1.3 Ave. Capacity/Stock (74-86):9.3 Ave. Weekly Hours (High) 111.5 Shift Factor 1.35 Ave Weekly Hours (LOW) 108.8 Shift Factor 1.39 Wote: Output, Investment, and Capital Stock extrapolated for 1987 and 1988.

\section{Roasted coffee}

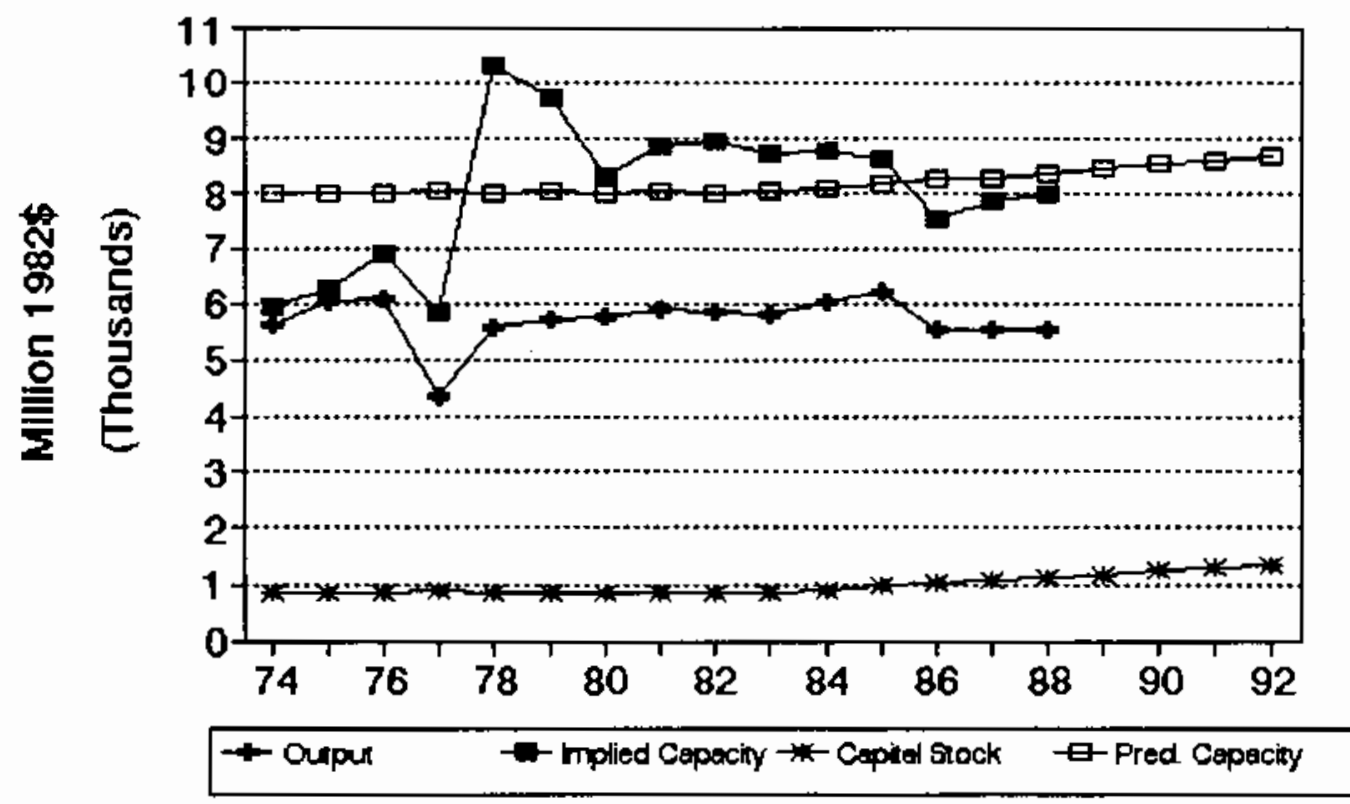




\section{No. 45 SIC 2097 Manufactured ice}

MILLIONS OF 1982 DOLLARS (EXCL. CU RATES)

\begin{tabular}{|c|c|c|c|c|c|c|c|}
\hline & $\begin{array}{l}\text { Q4 } \\
\text { CU }\end{array}$ & Am. & $\begin{array}{l}\text { Implied } \\
\text { Prac. }\end{array}$ & $\begin{array}{l}\text { Gross } \\
\text { In- }\end{array}$ & $\begin{array}{l}\text { Net } \\
\text { Cap. }\end{array}$ & $\begin{array}{l}\text { Pred. } \\
\text { Prac. }\end{array}$ & $\begin{array}{c}\cdots \\
\text { Emergency Capacity }\end{array}$ \\
\hline thut & Rate & Rate & Capac. & vest. & stk. & Capac. & \\
\hline
\end{tabular}

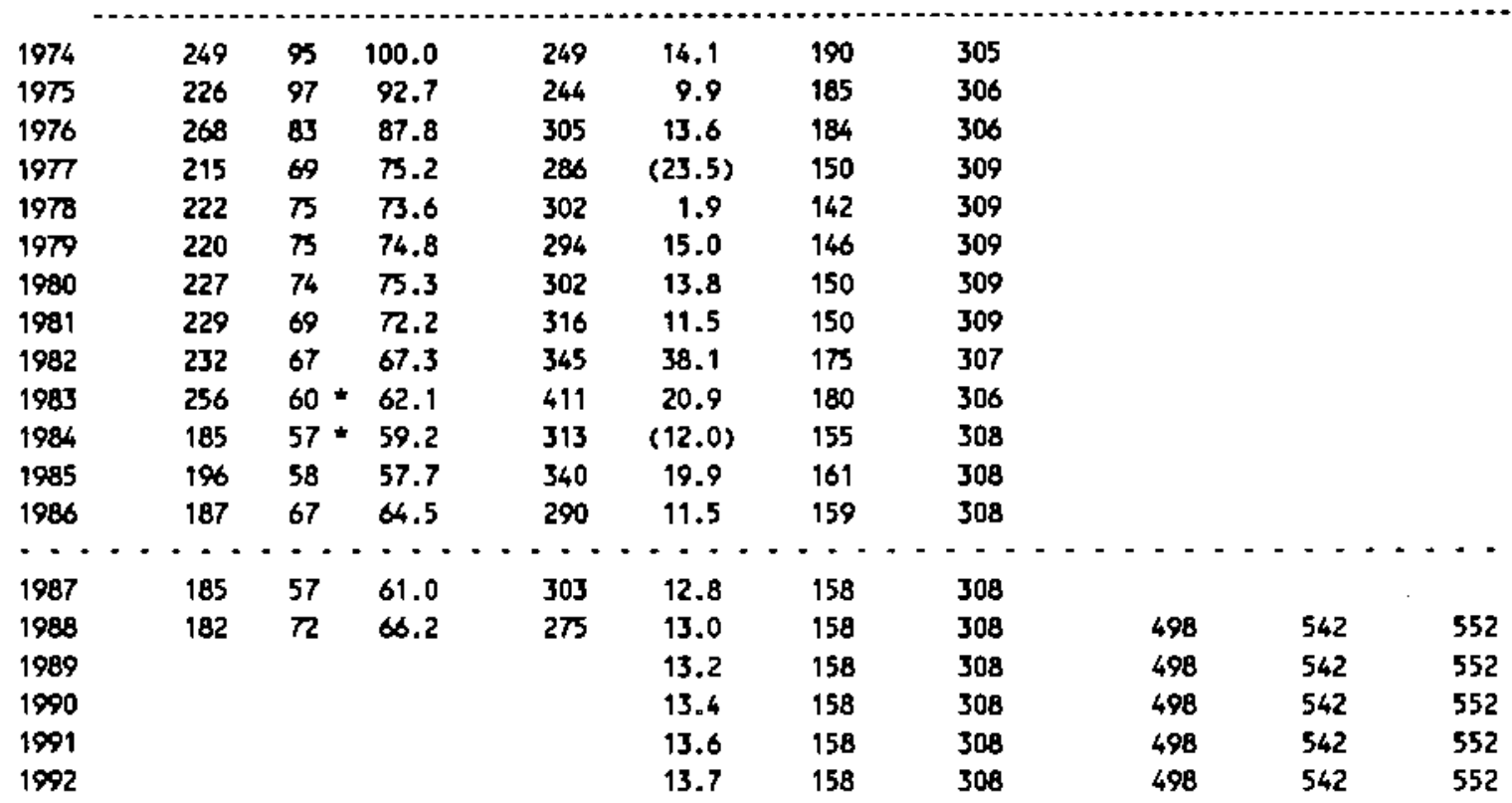

Capacity-Capital stock Regression: Code 0 RSO 0.000

Coefficients: Const 321.9 Stock -0.1

Ave. Capacity/Stock $(74-86): 1.9$ Ave. Weekly Hours (High) 84.2 Shift Factor 1.79 Ave Weekly Hours (Low) 78.4 Shift Factor 1.92 * Imputed Note; Output, Investment, and Capital stock extrapolated for 1987 and 1988.

2097 Manufactured ice

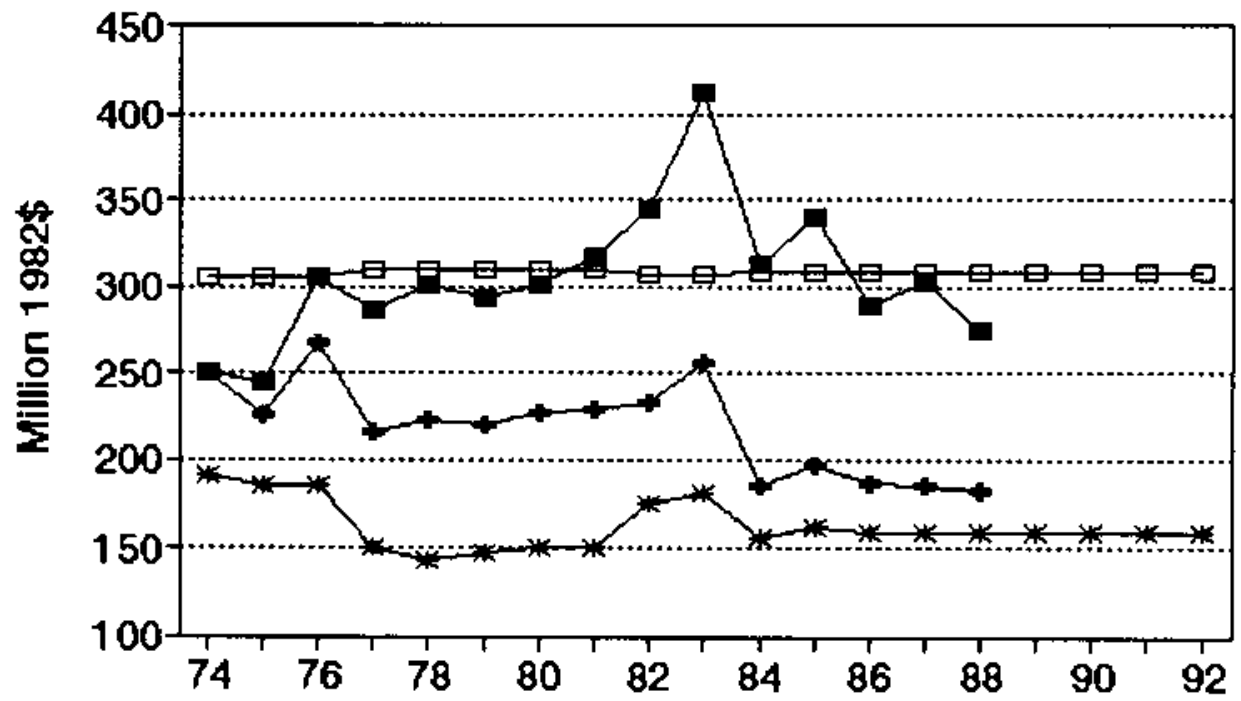

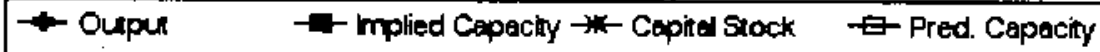




\section{No. 46 SIC 2098 Macaroni and apaghetti}

MILLIONS OF 1982 DOLLARS (EXCL. CU RATES)

$\begin{array}{lccccccc} & 04 & \text { Am. Implied Gross } & \text { Net } & \text { Pred. } & \ldots \\ \text { Gross } & \text { CU } & \text { CU } & \text { Prac. } & \text { In- } & \text { Cap. } & \text { Prac. } & \text { Emergency Capacity } \\ \text { Output } & \text { Rate } & \text { Rate } & \text { Capac. } & \text { vest. } & \text { Stk. } & \text { Capac. } & 3 \text { mon. } 6 \text { mon. }>6 \text { mon. }\end{array}$

\begin{tabular}{|c|c|c|c|c|c|c|c|c|c|c|}
\hline 1974 & 849 & 73 & 73.0 & 1,164 & 41.0 & 182 & 1,214 & & & \\
\hline 1975 & 826 & 73 & 73.0 & 1,132 & 59.2 & 220 & 1,246 & & & \\
\hline 1976 & 897 & 95 & 86.8 & 1,035 & 12.1 & 213 & 1,240 & & & \\
\hline 1977 & 1,023 & 63 & $\pi .0$ & 1,365 & 28.7 & 221 & 1,266 & & & \\
\hline 1078 & 1,046 & 55 & 58.0 & 1,804 & 32.1 & 231 & 1,256 & & & \\
\hline 1979 & 917 & 90 & 76.9 & 1,193 & 4.4 & 217 & 1,243 & & & \\
\hline 1980 & 926 & 84 & 86.3 & 1,071 & 53.8 & 253 & 1,273 & & & \\
\hline 1981 & 959 & 86 & 85.3 & 1,125 & 45.4 & 279 & $1,2 \%$ & & & \\
\hline 1982 & 1,074 & 84 & 84.8 & 1,268 & 68.3 & 327 & 1,334 & & & \\
\hline 1983 & 1,104 & 83 & 83.4 & 1,326 & 33.2 & 337 & 1,343 & & & \\
\hline 1984 & 1,063 & 83 & 83.0 & 1,280 & (57.8) & 262 & 1,280 & & & \\
\hline 1995 & 1,163 & 83 & 83.0 & 1,401 & 58.2 & 298 & 1,310 & & & \\
\hline 1986 & 1,222 & 87 & 85.5 & 1,429 & 25.0 & 300 & 1,312 & & & \\
\hline $\begin{array}{l}\cdots \\
1987\end{array}$ & 1,239 & 92 & $\begin{array}{l}-\cdots \\
90.1\end{array}$ & $\cdots$ & 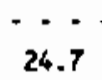 & 302 & 1,314 & $\cdots$ & -- & $\cdots-$ \\
\hline 1988 & 1,261 & 95 & 93.9 & 1,344 & 24.0 & 304 & 1,315 & 1,404 & 1,426 & 1,428 \\
\hline 1989 & & & & & 23.2 & 304 & 1,315 & 1,404 & 1,424 & 1,429 \\
\hline 1990 & & & & & 22.5 & 304 & 1,315 & 1,404 & 1,424 & 1,428 \\
\hline 1991 & & & & & 21.8 & 303 & 1,314 & 1,403 & 1,423 & 1,428 \\
\hline 1992 & & & & & 21.1 & 302 & 1,313 & 1,402 & 1,422 & 1,426 \\
\hline
\end{tabular}

Capacity-Capital stock Regression: code 0 RSO 0.040

Coefficients: Const 1063.1 stock 0.8

Ave. Capacity/Stock $(74-86): 5.0$ Ave. Weekly Hours (High) 140.3 Shift Factor 1.09 Ave Yeekly Hours (LOW) 138.7 Shift Factor 1.10 Note: Output, Investment, and Capital stock extrapolated for 1987 and 1989.

\section{Macaroni and spaghetti}

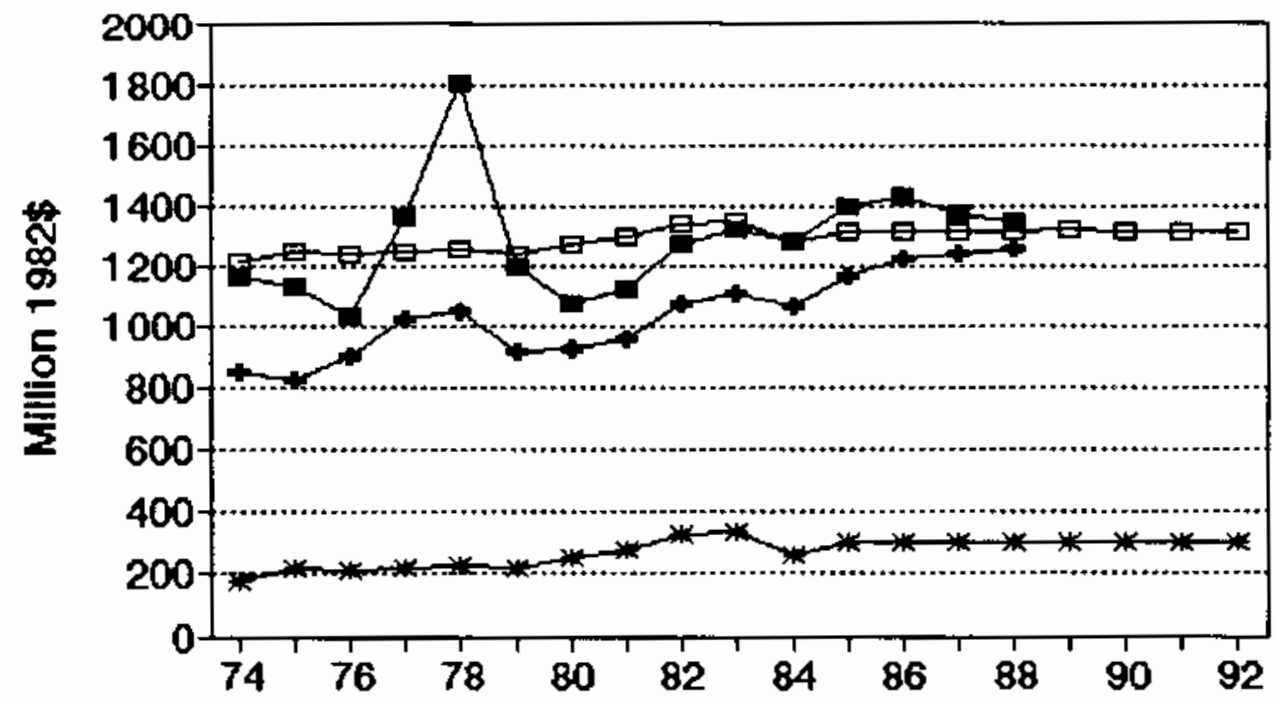

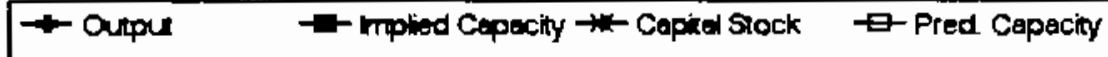


No. 47 BIC 2099 Food preparations, nec

MILLIONS OF 1982 dollars (EXCL. CU RATES)

$\begin{array}{lccccccc} & \text { Q4 } & \text { Ann. Implied Gross } & \text { Net } & \text { Pred. } & \ldots \ldots \\ \text { Gross } & \text { CU } & \text { CU } & \text { Prac. } & \text { In- } & \text { Cap. } & \text { Prac. } & \text { Emergency Capacity } \\ \text { Output } & \text { Rate } & \text { Rate } & \text { Capac. } & \text { vest. } & \text { Stk. } & \text { Capac. } & 3 \text { mon. } 6 \text { mon. }>6 \text { mon. }\end{array}$

\begin{tabular}{|c|c|c|c|c|c|c|c|c|c|c|}
\hline 1974 & 6,987 & 59 & 59.0 & 11,842 & 76.7 & 1,613 & 11,310 & & & \\
\hline 1975 & 7,035 & 63 & 61.5 & 11,439 & 304.5 & $t, 790$ & 12,807 & & & \\
\hline 1976 & 8,271 & 53 & 56.8 & 14,573 & 147.3 & 1,788 & 12,876 & & & \\
\hline 1977 & 7,496 & 53 & 53.0 & 14,143 & 136.1 & 1,784 & 12,847 & & & \\
\hline 1978 & 8,413 & 51 & 51.8 & 16,257 & 203.1 & 1,844 & 13,378 & & & \\
\hline 1979 & 8,072 & 79 & 68.5 & 11,784 & 214.3 & 1,908 & 13,951 & & & \\
\hline 1980 & 8,745 & 76 & 77.1 & 11,339 & 178.9 & 1,935 & 14,192 & & & \\
\hline 1981 & 8,602 & $\pi 0$ & 72.3 & 11,906 & 204.8 & 1,978 & 14,580 & & & \\
\hline 1982 & 11,083 & 68 & 68.8 & 16,121 & 274.1 & 2,077 & 15,470 & & & \\
\hline 1983 & 11,047 & 65 & 66.1 & 16,706 & 254.5 & 2,146 & 16,082 & & & \\
\hline 1984 & 10,740 & 65 & 65.0 & 16,523 & 202.2 & 2,167 & 16,271 & & & \\
\hline 1985 & 11,188 & 61 & 62.5 & 17,901 & 294.4 & 2,276 & 17,249 & & & \\
\hline 1986 & 11,439 & 61 & 61.0 & $18, \pi 53$ & 306.3 & 2,391 & 18,276 & & & \\
\hline--- & $-\cdot-$ & $\because$ & $\cdot \cdot$ & $-\cdot-$ & -- & $\cdots$ & $\cdots \cdot$ & --- & $=\cdot \cdot$ & --- \\
\hline 1987 & 11,652 & 65 & 63.5 & 18,350 & 227.0 & 2,425 & 18,581 & & & \\
\hline 1988 & 11,935 & 72 & 69.4 & 17,203 & 238.2 & 2,467 & 18,961 & 22,841 & 24,386 & 24,706 \\
\hline 1969 & & & & & 248.7 & 2,517 & 19,404 & 23,375 & 24,957 & 25,283 \\
\hline 1990 & & & & & 255.1 & 2,569 & 19,869 & 23,936 & 25,555 & 25,889 \\
\hline 1991 & & & & & 256.8 & 2,618 & 20,312 & 24,469 & 26,125 & 26,467 \\
\hline 1992 & & & & & 256.0 & 2,663 & 20,712 & 24,951 & 26,639 & 26,988 \\
\hline
\end{tabular}

Capacity-Capital Stock Regression: Code D RSQ 0.570

Coefficients: Const -3120.7 stock 9.0

Ave. Capacity/Stock $(74-86): 7.4$ Ave. Weekly Hours (High) 116.0 Shift Factor 1.30 Ave Neekly Hours (Low) 109.1 Shift factor 1.38 Note: Output, Imestment, and Capital Stock extrapolated for 1987 and 1988.

\section{Food preparations, nec}

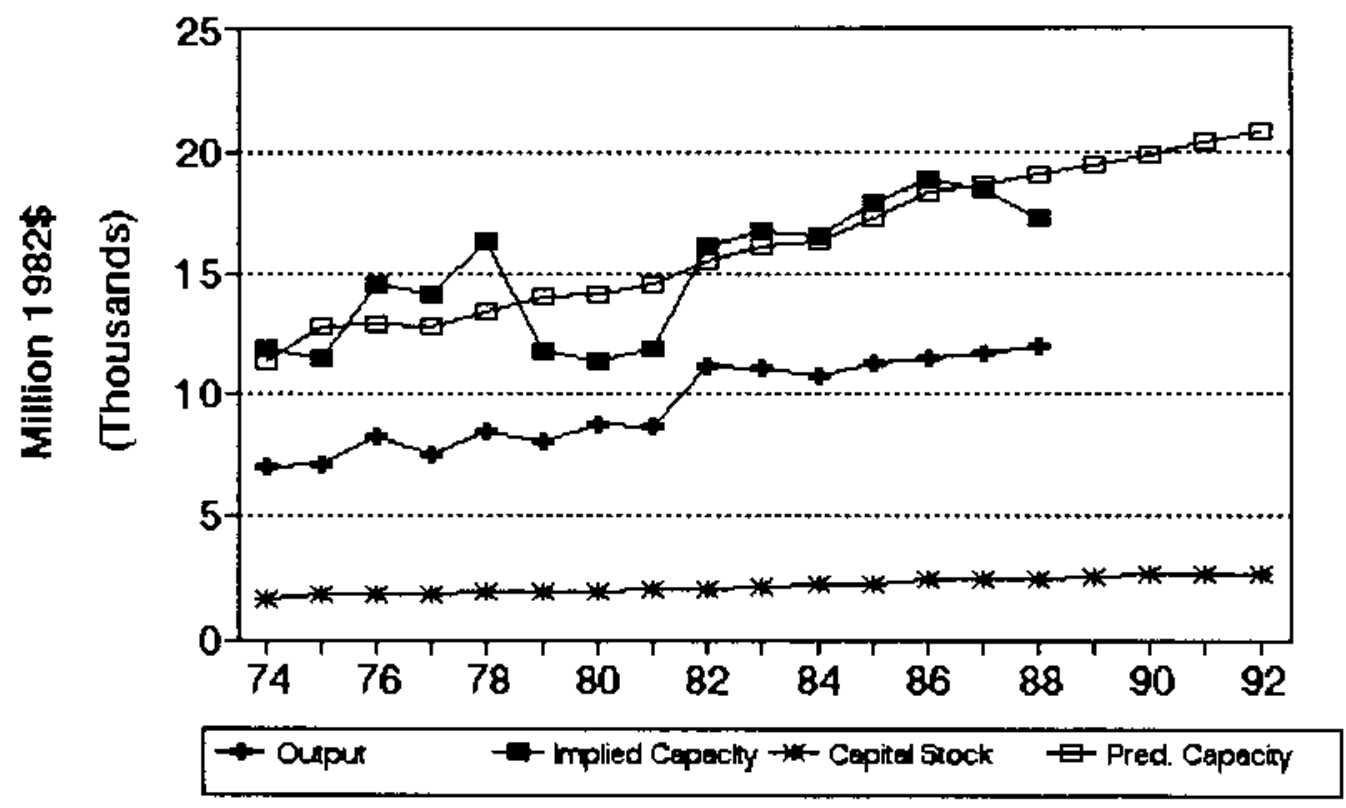




\section{No. 48 SIC 2111 Cigarettes}

MILLIONS OF 1982 DOLLARS (EXCL. CU RATES)

$\begin{array}{lccccccc} & 04 & \text { Ann. } & \text { Implied } & \text { Gross } & \text { Net } & \text { Pred. } & -\ldots \ldots \\ \text { Gross } & \text { CU } & \text { CU } & \text { Prac. } & \text { In- } & \text { Cap. } & \text { Prac. } & \text { Emergency Capacity } \\ \text { Output Rate } & \text { Rate } & \text { Capac. } & \text { vest. } & \text { Stk. } & \text { Capac. } & 3 \text { mon. } 6 \text { mon. }>6 \text { mon. }\end{array}$

\begin{tabular}{|c|c|c|c|c|c|c|c|c|c|c|}
\hline 1974 & 11,545 & 83 & as. 0 & 13,900 & 216.3 & 1,329 & 15,202 & & & \\
\hline 1975 & 12,061 & 84 & 83.6 & 14,422 & 153.4 & 1,373 & 15,150 & & & \\
\hline 1976 & 12,291 & 78 & 80.3 & 15,316 & 150.0 & 1,408 & 15,110 & & & \\
\hline 197 & 11,677 & 74 & 75.5 & 15,467 & 140.7 & 1,425 & 15,000 & & & \\
\hline 1978 & 11.633 & $\pi$ & 74.6 & 15,580 & 214.2 & 1,510 & 14,989 & & & \\
\hline 1979 & $11, \infty 61$ & $\pi$ & 76.3 & 15,293 & 165.5 & 1,543 & 14,950 & & & \\
\hline 1980 & 12,073 & 82 & 80.1 & 15,068 & 271.9 & 1.673 & 14,794 & & & \\
\hline 1981 & 12,956 & 88 & 85.8 & 15,109 & 401.7 & 1,928 & 14,495 & & & \\
\hline 1982 & 12,324 & 86 & 86.8 & 14,206 & 400.8 & 2,171 & 14,209 & & & \\
\hline 1983 & 11,150 & $80 *$ & 82.2 & 13,570 & 441.1 & 2,441 & 13,890 & & & \\
\hline 1984 & 11,084 & $80 *$ & 79.9 & 13,873 & 450.1 & 2,707 & 13,576 & & & \\
\hline 1985 & 10,967 & 81 & 80.6 & 13,608 & 382.1 & 2,893 & 13,357 & & & \\
\hline 1986 & 10,567 & 87 & 04.8 & 12,469 & 439.7 & 3,122 & 13,087 & & & \\
\hline$\ldots$ & --- & $\cdots$ & $\because-$ & $\because \cdots$ & -- & $-\cdots$ & $\cdots$ & $-\cdot=$ & - - - & $-\cdots$ \\
\hline 1987 & 10,732 & 80 & 82.6 & 12,989 & 294,1 & 3,205 & 12,989 & & & \\
\hline 1988 & 10,951 & 86 & 83.8 & 13,075 & 296.2 & 3,284 & 12,895 & 14,924 & 15,487 & 15,603 \\
\hline 1989 & & & & & 298.1 & 3,360 & 12,805 & 14,820 & 15,379 & 15,494 \\
\hline 1990 & & & & & 299.3 & 3,432 & 12,720 & 14.722 & 15,277 & 15,392 \\
\hline 1991 & & & & & 299.6 & 3,500 & 12,641 & 14,629 & 15,181 & 15,295 \\
\hline 1992 & & & & & 299.4 & 3,562 & 12,567 & 14,544 & 15,092 & 15,206 \\
\hline
\end{tabular}

Capacity-Capital Stock Regression: Code 0 hso 0.630

Coefficients: Const 16771.0 stock -1.2

Ave. Capacity/Stock $(74-86): 7.4$ Ave. Weekly Hours (High) 125.3 Shift Factor 1.21 Ave Heekly Hours (LOW) 117.7 Shift Factor 1.28 * Imputed Note: Output, Investment, and Capital Stock extrapolated for 1987 and 1988.

\section{Cigarettes}

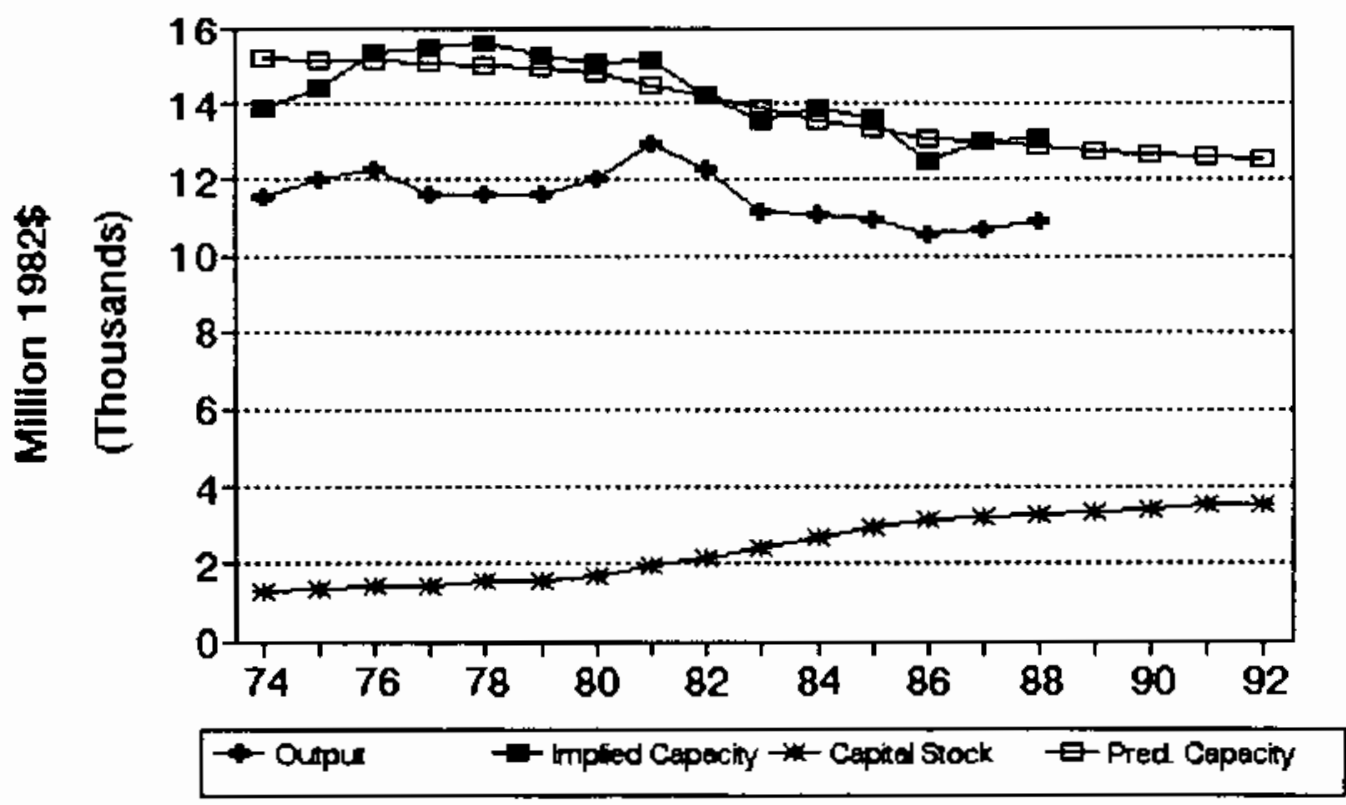




\title{
No. 50 sIC 2131 Chewing and smoking tobacco
}

\author{
MILLIONS OF 1982 DOLLARS (EXCL. CU RATES)
}

$\begin{array}{lccccccc} & \text { Q } & \text { Ann. Implied Gross } & \text { Net } & \text { Pred. } & \ldots \ldots \ldots \\ \text { Gross } & \text { d } & \text { CU } & \text { Prac. } & \text { In- } & \text { Cap. } & \text { Prac. } & \text { Emergency Capacity } \\ \text { Output Rate } & \text { Rate } & \text { Capac. } & \text { vest. } & \text { Stk. } & \text { Capac. } & 3 \text { mon. } 6 \text { mon. }>6 \text { mon. }\end{array}$

\begin{tabular}{|c|c|c|c|c|c|c|c|c|c|c|}
\hline 1974 & 490 & $99 *$ & 98.9 & 496 & $(6.5)$ & 94 & 695 & & & \\
\hline 1975 & 558 & 9 & 99.0 & 564 & 4.7 & 91 & 691 & & & \\
\hline 1976 & 582 & $96 *$ & 97.0 & 600 & 0.8 & 85 & 682 & & & \\
\hline 1977 & 654 & $96 *$ & 94.8 & 690 & 15.2 & 91 & 691 & & & \\
\hline 1978 & 690 & 95 & 94.7 & 728 & 9.8 & 92 & 692 & & & \\
\hline 1979 & 710 & 95 & 95.0 & 747 & 7.5 & 90 & 600 & & & \\
\hline 1980 & 694 & $\infty$ & 97.5 & 711 & 5.3 & $\mathbf{8 8}$ & 686 & & & \\
\hline 1981 & 670 & 96 & 97.1 & 690 & 26.8 & 106 & 713 & & & \\
\hline 1992 & 658 & 86 & 89.8 & 733 & 4.8 & 102 & 707 & & & \\
\hline 1983 & 661 & 87 & 86.6 & 763 & 14.6 & 107 & 715 & & & \\
\hline 1984 & 692 & 67 & 74.5 & 929 & $(10.4)$ & 89 & 688 & & & \\
\hline 1985 & 772 & $68 \div$ & 67.3 & 1,146 & 8.6 & 89 & 1,138 & & & \\
\hline 1986 & 787 & $71 \star$ & 69.9 & 1,127 & 5.9 & 87 & 1,135 & & & \\
\hline - . & $\begin{array}{c}-.-- \\
812\end{array}$ & 68 & 69.3 & $\begin{array}{l}\cdots \\
1,172\end{array}$ & $\begin{array}{c}--- \\
4.5\end{array}$ & $-\cdots$ & $\because \cdots$ & $-\cdot$ & -- & - - \\
\hline 1988 & 845 & $71 *$ & 70.1 & 1,207 & 3.9 & 80 & 1,124 & 1,933 & 2,088 & 2,119 \\
\hline 1989 & & & & & 3.6 & 76 & 1,118 & 1,923 & 2,077 & 2,108 \\
\hline 1990 & & & & & 2.8 & 72 & 1,112 & 1,913 & 2,065 & 2,097 \\
\hline 1991 & & & & & 2.3 & 67 & 1,105 & 1,901 & 2,053 & 2,084 \\
\hline 1992 & & & & & 1.7 & 63 & 1,099 & 1,890 & 2,041 & 2,072 \\
\hline
\end{tabular}

Capacity-Capital stock Regression: Code -1 RSO 0.720

Coefficients: Const 555.8 stock 1.5 D(85-86) 449.7

Ave. Capacity/Stock $(74-86): 8.2$

Ave. Weekly Hours (High) 80.0 Shift Factor 1.89 Ave Weekly Hours (LOW) 66.5 Shift Factor 2.27

* Imputed Note: Output, Imvestment, and Capital Stock extrapolated for 1987 and 1988.

\section{Chewing and smoking tobacco}

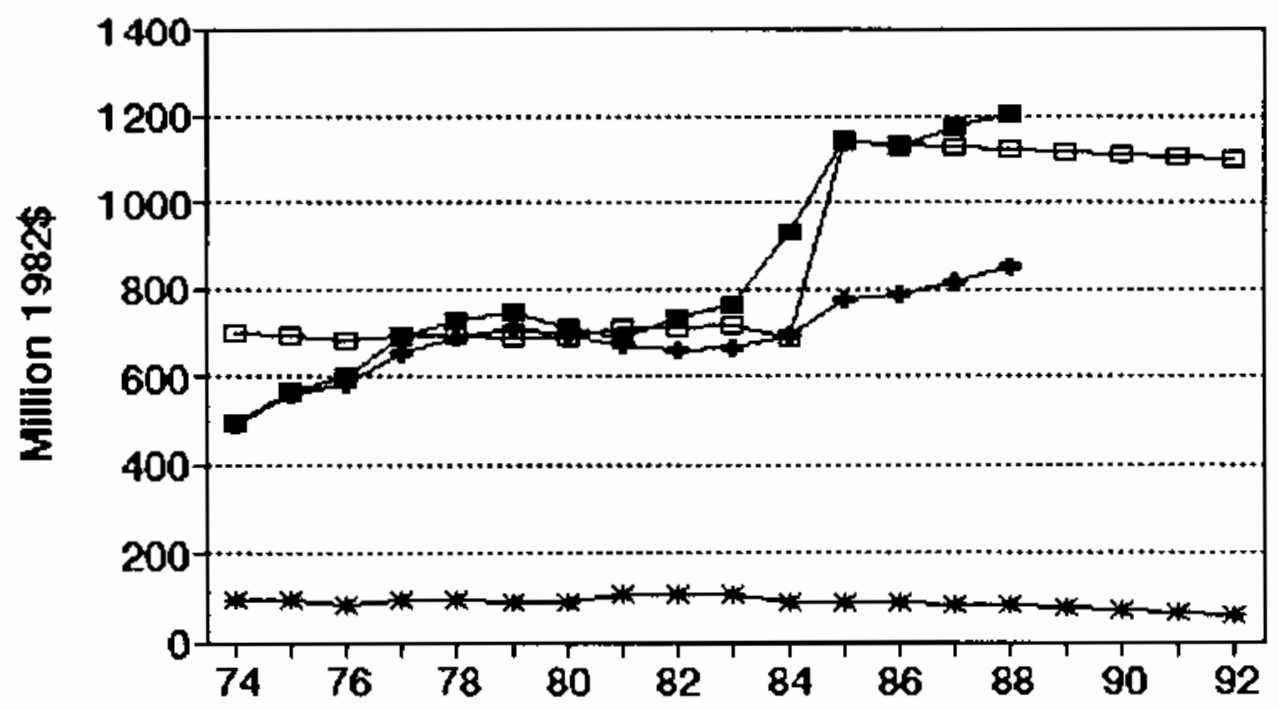

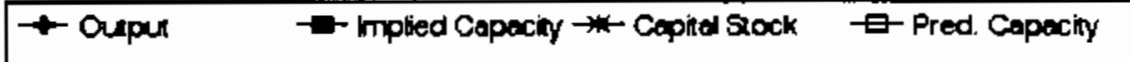




\section{No. 51 SIC 2141 Tobacco bteming and redrying}

MILLIONS OF 1982 DOLLARS (EXCL. CU RATES)

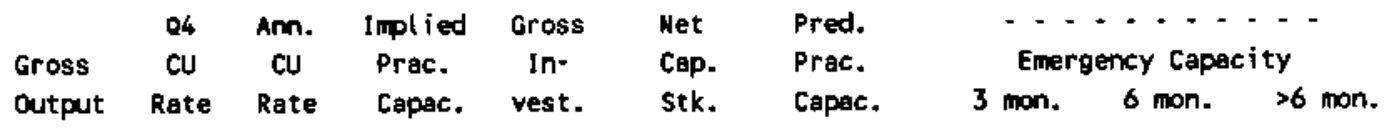

\begin{tabular}{|c|c|c|c|c|c|c|c|c|c|c|}
\hline 1974 & 3,782 & 59 & 59.8 & 6,326 & 7.3 & 333 & 6,207 & & & \\
\hline 1975 & 3,574 & 69 & 63.9 & 5,596 & 12.1 & 315 & 5,977 & & & \\
\hline 1976 & 3.636 & 47 & 55.4 & 6,559 & 28.6 & 312 & 5,746 & & & \\
\hline 1977 & 3,166 & 72 & 62.8 & 5,009 & 73.9 & 353 & 5,518 & & & \\
\hline 1978 & 3,176 & 71 & 71.5 & 4,442 & 39.8 & 358 & 5,289 & & & \\
\hline 1979 & 2,744 & 45 & 54.7 & 5,019 & 4.6 & 331 & 5,059 & & & \\
\hline 1980 & 2,854 & 59 & 53.7 & 5,313 & 26.0 & 323 & 4,829 & & & \\
\hline 1981 & 2,846 & 63 & 61.6 & 6,623 & 26.3 & 316 & 4,600 & & & \\
\hline 1982 & 3,100 & 61 & 61.5 & 5,038 & 49.5 & 332 & 4,370 & & & \\
\hline 1983 & 2,557 & 57 & 58.5 & 4,374 & 69.3 & 366 & 4,140 & & & \\
\hline 1986 & 2,394 & 62 & 60.5 & 3,957 & 36.8 & 364 & 3,911 & & & \\
\hline 1985 & 2,367 & 72 & 67.9 & 3,487 & 3.1 & 331 & 3,681 & & & \\
\hline 1986 & 2,108 & 68 & 69.4 & 3,038 & 31.6 & 327 & 3,452 & & & \\
\hline-- & $--\cdot$ & -- & $\cdots$ & $\cdots \cdot$ & $\cdots$ & $\cdots$ & $-\quad--$ & $\cdots$ & $\cdot \cdot$ & --- \\
\hline 1987 & 2,070 & 82 & 76.9 & 2,706 & 31.5 & 322 & 3,222 & & & \\
\hline 1988 & 2,042 & 83 & 82.6 & 2,472 & 31.0 & 318 & 2,992 & 3,395 & 3,587 & 3,627 \\
\hline 1989 & & & & & 30.5 & 313 & 2,763 & 3,135 & 3,312 & 3,349 \\
\hline 1990 & & & & & 30.0 & 309 & 2,533 & 2,876 & 3,037 & 3,070 \\
\hline 1991 & & & & & 29.5 & 306 & 2,304 & 2,614 & 2,761 & 2,792 \\
\hline 1992 & & & & & 29.0 & 300 & 2,074 & 2,353 & 2,486 & 2,516 \\
\hline
\end{tabular}

Capacity-Capital Stock Regression: Code -2 RSO 0.780

Coefficients: Const 6436.5 Time -229.6

Ave. Capacity/Stock (74-86): 14.4 Ave. Weekly Hours (High) 125.1 Shift Factor 1.21 Ave Weekly Hours (Low) 120.1 Shift factor 1.26 Note: Dutput, Investment, and Capital Stock extrapolated for 1987 and 1988.

\section{Tobacco stemming and redrying}

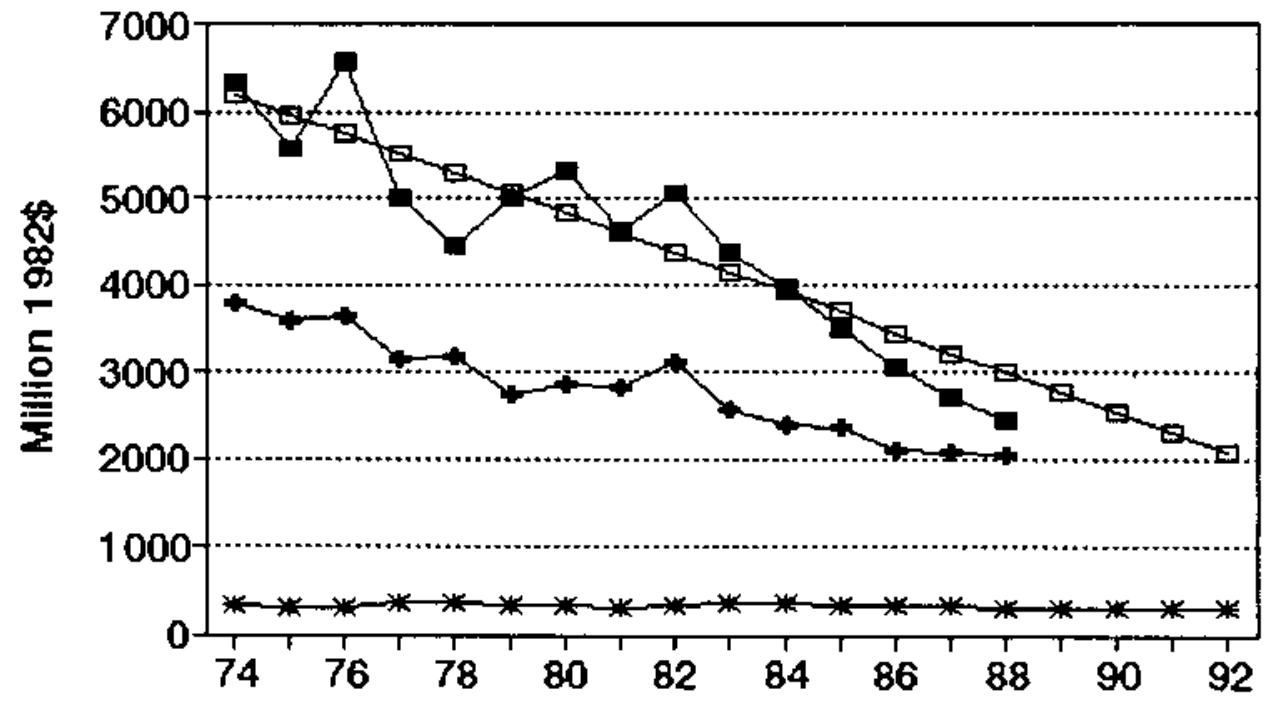

\begin{tabular}{|c|c|c|}
\hline+ - Oupun & $\Rightarrow$ - Implied Capachy $\rightarrow$ - Capital Stock & E-Pred Copociny \\
\hline
\end{tabular}


No. 52 sIc 2211 Heaving ailla, cotton

MILLIONS OF 1982 doLlarS (EXCL. CU RATES)

$\begin{array}{lccccccc} & Q 4 & \text { Am. Implied Gross } & \text { Wet } & \text { Pred. } & -\ldots \ldots \ldots \\ \text { Gross } & \text { cU } & \text { CU } & \text { Prac. } & \text { In- } & \text { Cap. } & \text { Prac. } & \text { Energency Capacity } \\ \text { Output } & \text { Rate } & \text { Rate } & \text { Capac. } & \text { vest. } & \text { Stk. } & \text { Capac. } & 3 \text { mon. } 6 \text { mon. }>6 \text { mon. }\end{array}$

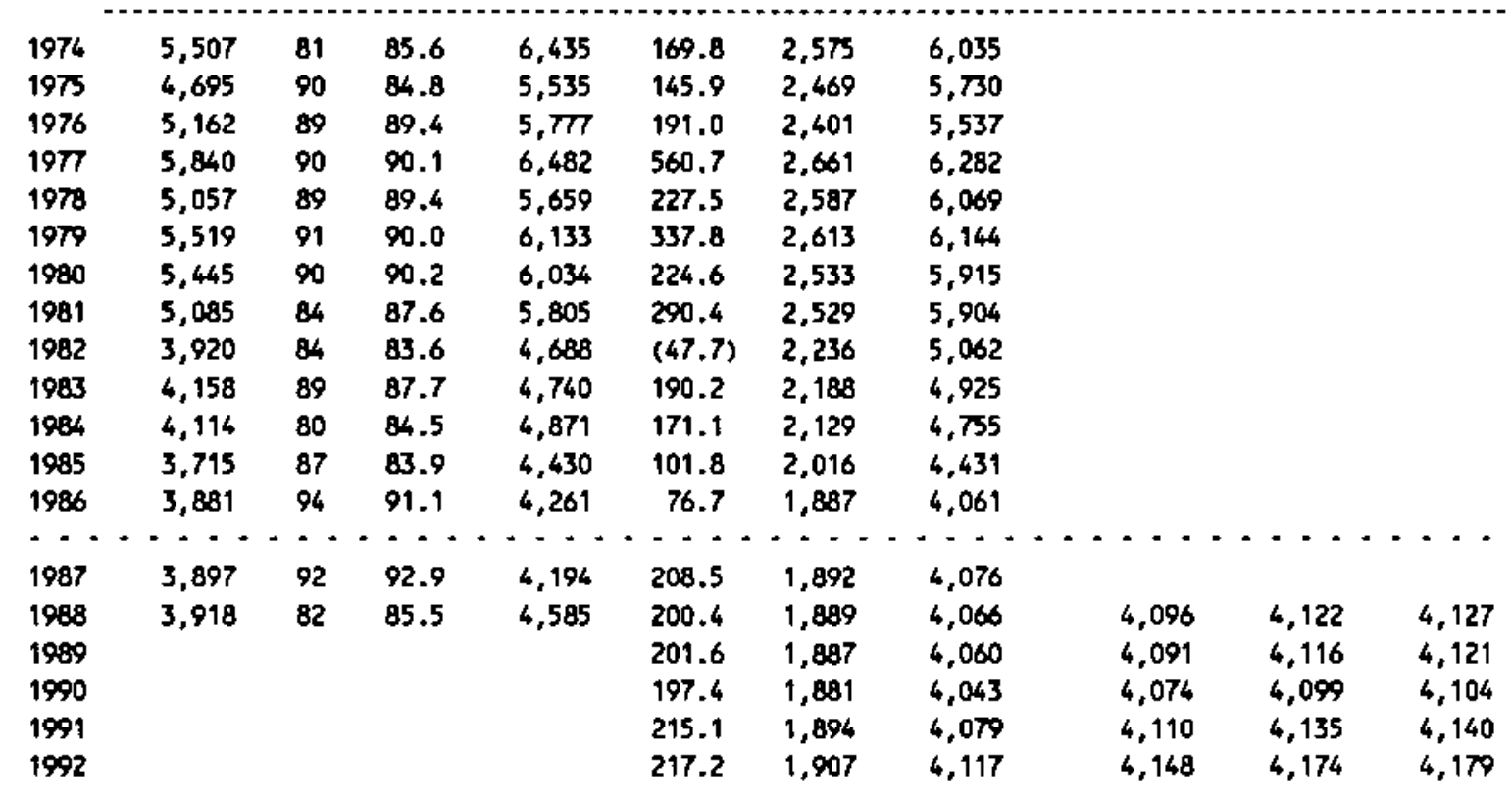

Capacity-Copital stock Regression: Code 0 RSO 0.900 Coefficients: Const -1359.4 stock 2.9

Ave. Capacity/Stock $(74-86): 2.3$ Ave. Weekly Hours (High) 153.6 Shift factor 1.01 Ave Veekly Hours (LOW) 151.2 Shift Factor 1.03 Note: Output, Imvestment, and Capital Stock extrapolated for 1987 and 1988.

\section{Weaving mills, cotton}

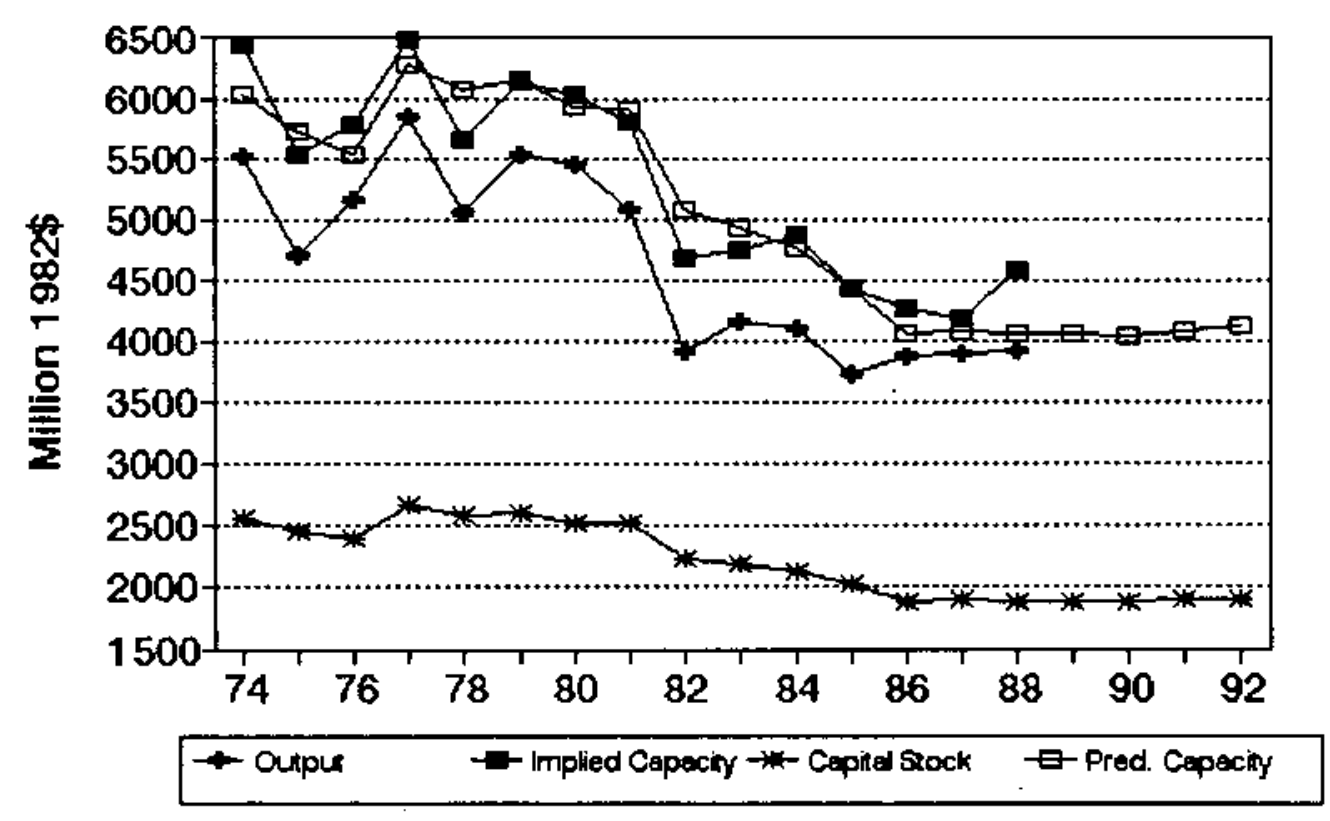


No. 53 sIC 2221 Weaving mills, synthetic

MILLIONS OF 1982 DOLLARS (EXCL. CU RATES)

$\begin{array}{lccccccc} & 04 & \text { Ann. } & \text { Implied Gross } & \text { Net } & \text { Pred. } & \ldots \ldots \ldots \\ \text { Gross } & \text { d } & \text { CU } & \text { Prac. } & \text { In- } & \text { Cap. } & \text { Prac. } & \text { Emergency Capacity } \\ \text { Output } & \text { Rate } & \text { Rate } & \text { Capac. } & \text { vest. } & \text { Stk. } & \text { Capac. } & 3 \text { mon. } 6 \text { mon. }\end{array}$

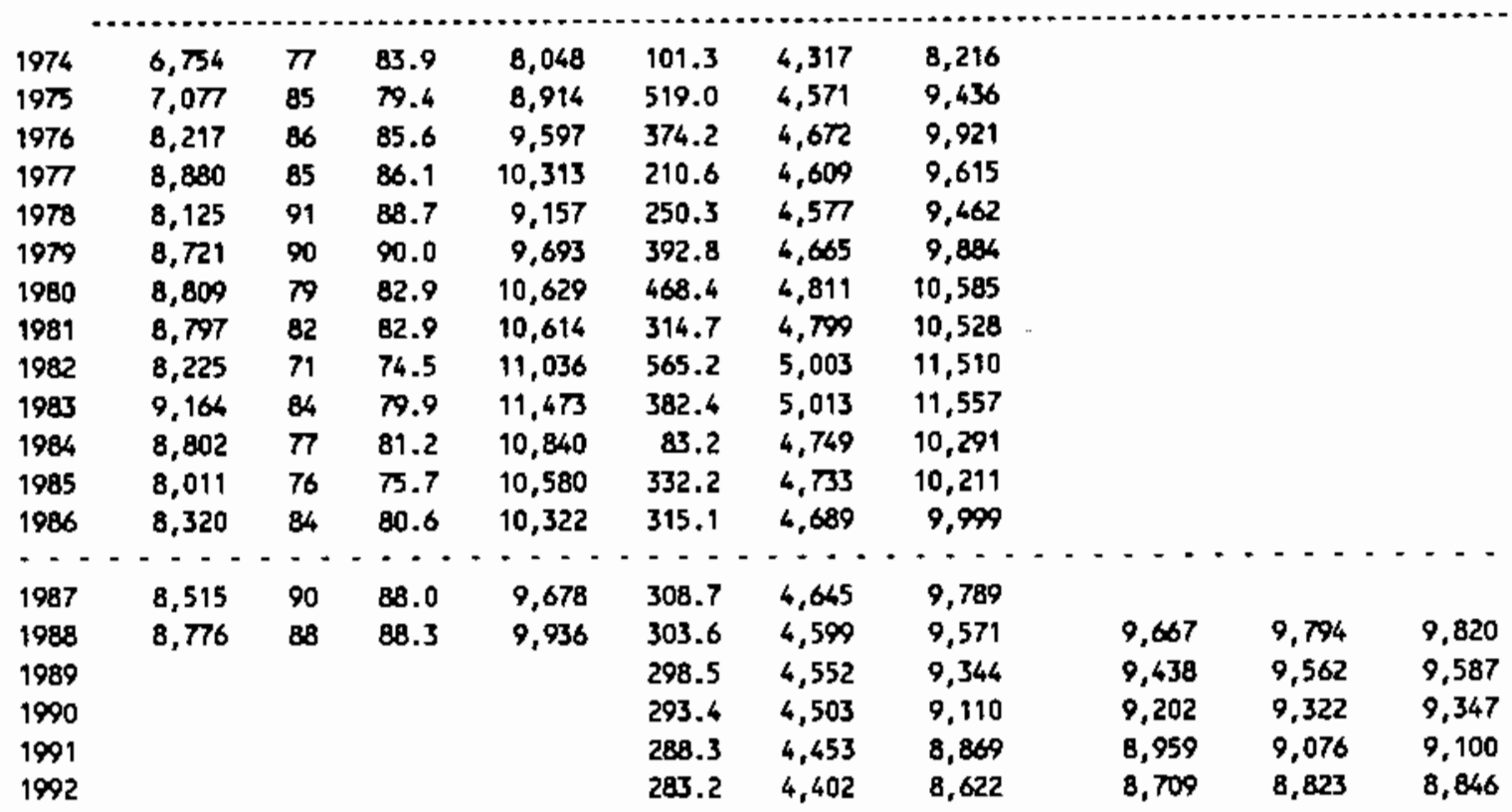

Capacity-Capital Stock Regression: Code 0 RSO 0.840

Coefficients: Const -12501.8 stock 4.8

Ave. Capecity/Stock (74-86): 2.1

Ave. Weekly Hours (High) 151.9 Shift Factor 1.03 Ave Heekly Hours (Low) 141.3 shift Factor 1.10 Mote: Output, Investment, and Capital Stock extrapolated for 1987 and 1988.

\section{Weaving mills, synthetic}

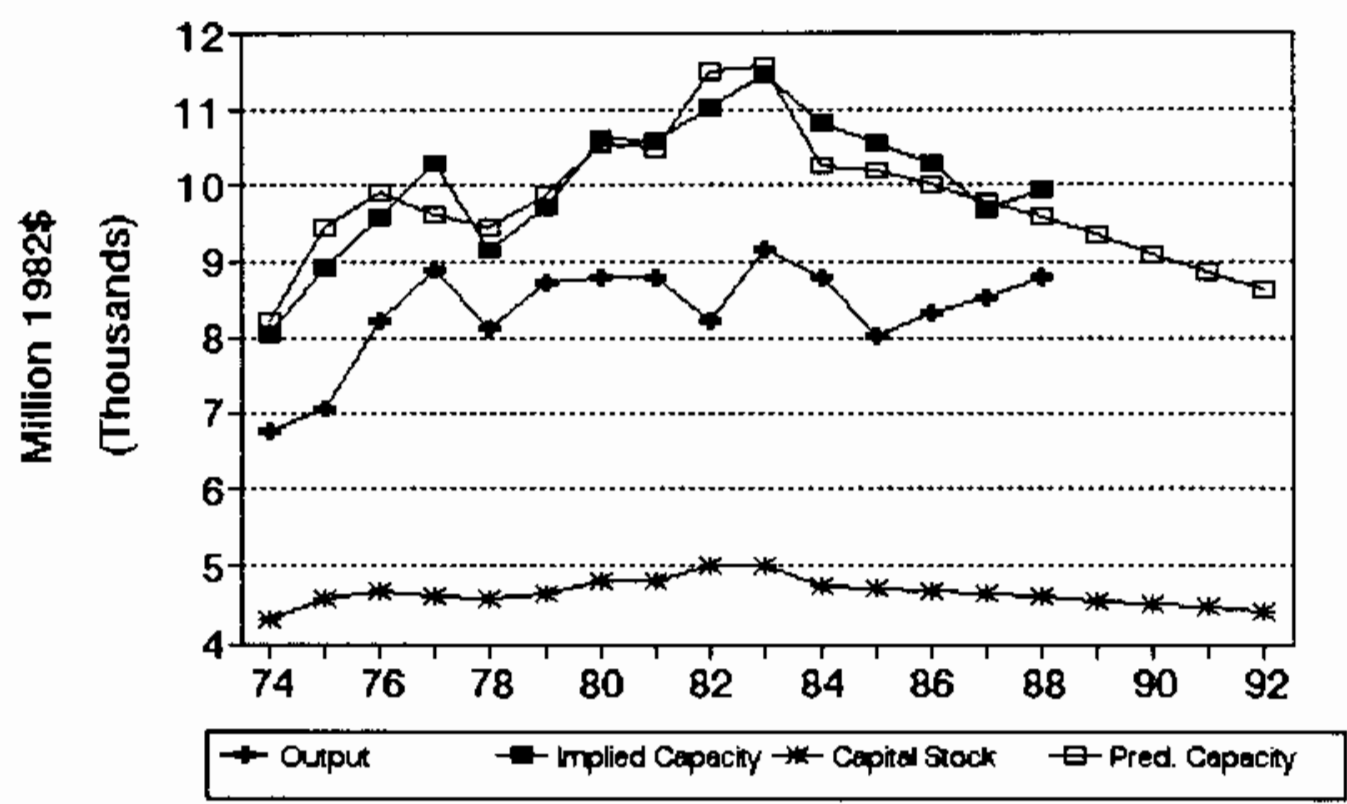




\section{No. 54 BIC 2231 Weaving and finiahing wills, wool}

MILLIOAS OF 1982 DOLLARS (EXCL. CU RATES)

$\begin{array}{lccccccc} & 04 & \text { Am. } & \text { Implied } & \text { Gross } & \text { Net } & \text { Pred. } & \ldots \\ \text { Gross } & \text { CU } & \text { CU } & \text { Prac. } & \text { In- } & \text { Cap. } & \text { Prac. } & \text { Emergency Capacity } \\ \text { Output } & \text { Rate } & \text { Rate } & \text { Capac. } & \text { vest. } & \text { Stk. } & \text { Capac. } & 3 \text { mon. } 6 \text { mon. }\end{array}$

\begin{tabular}{|c|c|c|c|c|c|c|c|c|c|c|}
\hline 1974 & 876 & 64 & 68.6 & 1,279 & 45.2 & 370 & 1,207 & & & \\
\hline 1975 & 752 & 73 & 67.9 & 1,108 & (61.7) & 301 & 1,161 & & & \\
\hline 1976 & 845 & 81 & 78.0 & 1,063 & 58.5 & 328 & 1,179 & & & \\
\hline 1977 & 790 & 61 & 69.1 & 1,143 & $(49.0)$ & 255 & 1,130 & & & \\
\hline 1978 & $\pi$ & 62 & 61.6 & 1,258 & 44.0 & 271 & 1,141 & & & \\
\hline 1979 & 769 & $70 *$ & 66.5 & 1,158 & 42.7 & 282 & 1,148 & & & \\
\hline 1980 & 752 & 77 & 74.1 & 1,015 & 15.2 & 268 & 1,138 & & & \\
\hline 1981 & 866 & 77 & 78.5 & 1,104 & 19.2 & 253 & 1,129 & & & \\
\hline 1982 & 750 & 63 & 67.8 & 1,106 & 45.0 & 264 & 1,136 & & & \\
\hline 1983 & 894 & 91 & 80.9 & 1,105 & 17.0 & 247 & 1,125 & & & \\
\hline 1984 & 994 & 72 & 80.3 & 1,237 & 28.5 & 243 & 1,123 & & & \\
\hline 1985 & 886 & 67 & 68.4 & 1,294 & 20.3 & 233 & 1,116 & & & \\
\hline 1986 & 763 & 89 & 80.4 & 949 & 15.2 & 220 & 1,107 & & & \\
\hline-- & $-\cdots$ & $\cdots$ & $\because \cdot$ & $\cdots \cdot$ & $\cdot-\cdot$ & -- & $-\cdots$ & $\cdots$ & $\cdots$ & -- \\
\hline 1987 & 767 & 75 & 80.5 & 954 & 30.2 & 222 & 1,108 & & & \\
\hline 1988 & 773 & 79 & 77.2 & 1,001 & 31.8 & 225 & $t, 110$ & 1,133 & 1,184 & 1,194 \\
\hline 1989 & & & & & 33.4 & 229 & 1,113 & 1,136 & 1,187 & 1,198 \\
\hline 1990 & & & & & 35.0 & 234 & 1,116 & 1,139 & 1,191 & 1,201 \\
\hline 1991 & & & & & 36.6 & 240 & 1,121 & 1,164 & 1,195 & 1,206 \\
\hline 1992 & & & & & 38.3 & 267 & 1,125 & 1,148 & 1,200 & 1,211 \\
\hline
\end{tabular}

Capacity-Capital stock Regression: Code 1 RSQ 0.070

Coefficients: Const 960.7 stock $0.7 \quad$ Ave. Capacity/Stock $(74-86): 4.2$ Ave. Heekly Hours (High) 141.6 Shift Factor 1.08 Ave Heekly Hours (Low) 138.0 Shift Factor 1.10 * Imputed Hote: Output, Investment, and Capital stock extrapolated for 1987 and 1988.

\section{Weaving and finishing mills, wool}

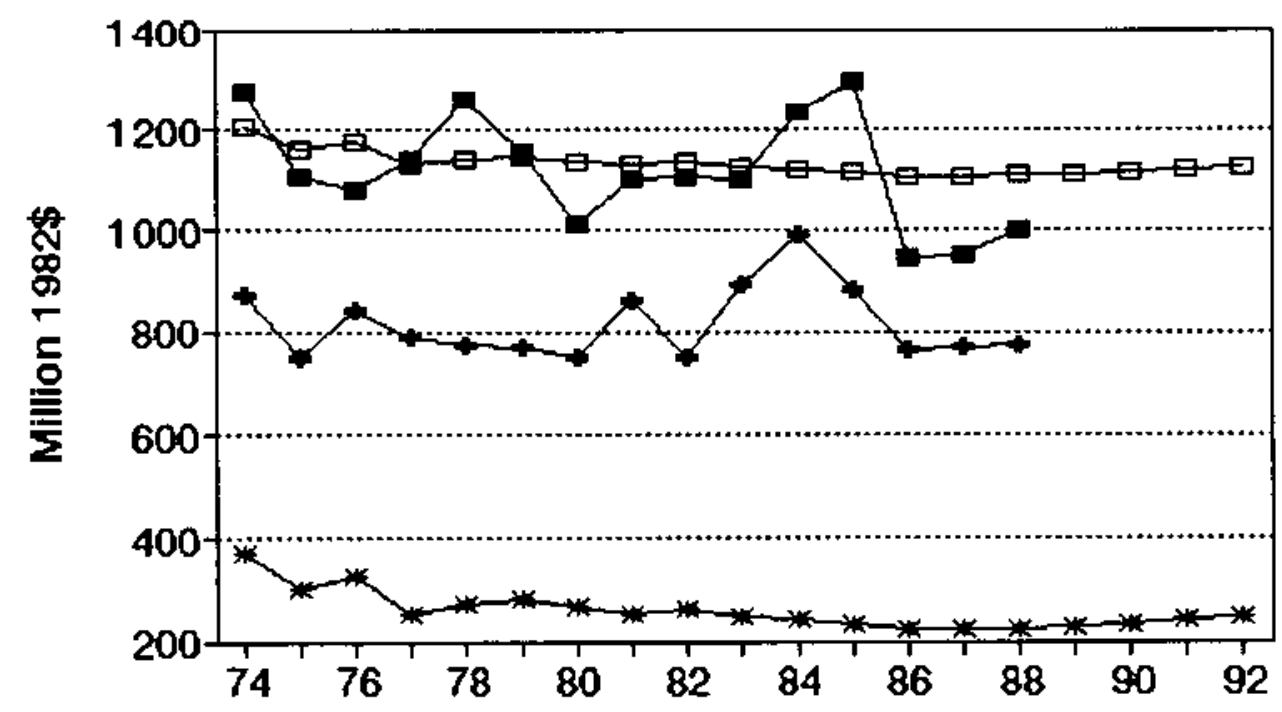

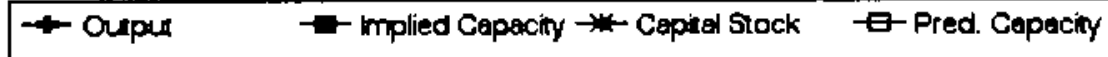


no. 55 sIc 2241 Narrow fabric nillB

MILLIONS OF 1982 DOLLARS (EXCL. CU RATES)

$\begin{array}{lccccccc} & \text { Q4 } & \text { Ann. Implied Gross } & \text { Net } & \text { Pred. } & \ldots \\ \text { Gross } & \text { CU } & \text { CU } & \text { Prac. } & \text { In- } & \text { Cap. } & \text { Prac. } & \text { Emergency Capacity } \\ \text { Output } & \text { Rate } & \text { Rate } & \text { Capac. } & \text { vest. } & \text { Stk. } & \text { Capac. } & 3 \text { mon. } 6 \text { mon. }>6 \text { mon. }\end{array}$

\begin{tabular}{|c|c|c|c|c|c|c|c|c|c|c|}
\hline 1974 & 923 & 65 & 68.6 & 1,345 & 57.4 & 413 & 1,184 & & & \\
\hline 1975 & 759 & 66 & 64.3 & 1,180 & $(17.7)$ & 367 & 1,237 & & & \\
\hline 1976 & 902 & 90 & B1.1 & 1,112 & $(6.6)$ & 334 & 1,276 & & & \\
\hline 1977 & 875 & 84 & 86.7 & 1,008 & 25.3 & 332 & 1,278 & & & \\
\hline 1978 & 870 & 86 & 85.2 & 1,021 & 12.9 & 318 & 1,295 & & & \\
\hline 1979 & 942 & $\pi$ & 77.0 & 1,223 & 39.8 & 329 & 1,282 & & & \\
\hline 1980 & B78 & 64 & 66.9 & 1,312 & 15.7 & 316 & 1,297 & & & \\
\hline 1981 & 958 & 64 & 65.0 & 1,474 & 27.4 & 314 & 1,300 & & & \\
\hline 1982 & 851 & 66 & 65.0 & 1,310 & $(26.4)$ & 263 & 1,359 & & & \\
\hline 1983 & 924 & 85 & 78.3 & 1,181 & 12.4 & 252 & 1,373 & & & \\
\hline 1984 & 950 & 76 & 80.4 & 1,182 & 64.8 & 290 & 1,328 & & & \\
\hline 1985 & 973 & 45 & 56.4 & 1,725 & 28.1 & 290 & 1,328 & & & \\
\hline 1986 & 1,048 & 67 & 58.5 & 1,791 & 26.1 & 290 & 1,328 & & & \\
\hline$\because-$ & $--\cdot$ & $\cdots$ & -- & $-\cdots$ & $\cdots-$ & $\cdots$ & $\cdots-$ & $\cdots \cdot \cdot$ & $--\cdot$ & $=--$ \\
\hline 1987 & 1,044 & 75 & 72.1 & 1,447 & 25.5 & 288 & 1,330 & & & \\
\hline 1988 & 1,038 & 7 & 74.8 & 1,388 & 22.4 & 283 & 1,335 & 1,398 & 1,449 & 1,460 \\
\hline 1989 & & & & & 22.8 & 280 & 1,340 & 1,403 & 1,454 & 1,464 \\
\hline 1990 & & & & & 21.2 & 275 & 1,346 & 1,409 & 1,460 & 1,471 \\
\hline 1991 & & & & & 28.1 & 277 & 1,343 & 1,406 & 1,457 & 1,468 \\
\hline 1992 & & & & & $2 B .9$ & 280 & 1,339 & 1,402 & 1,453 & 1,464 \\
\hline
\end{tabular}

Capecity-Capital stock Regression: Code 2 RSO 0.040

Coefficients: Const 1668.3 stock -1.2

Ave. Capacity/Stock $(74-86): 4.1$ Ave. Weekly Hours (High) 139.4 Shift Factor 1.09 Ave Weekly Hours (Low) 131.1 Shift Factor 1.16 Note: Output, Investment, and Capital stock extrapolated for 1987 and 1988.

\section{Narrow fabric mills}

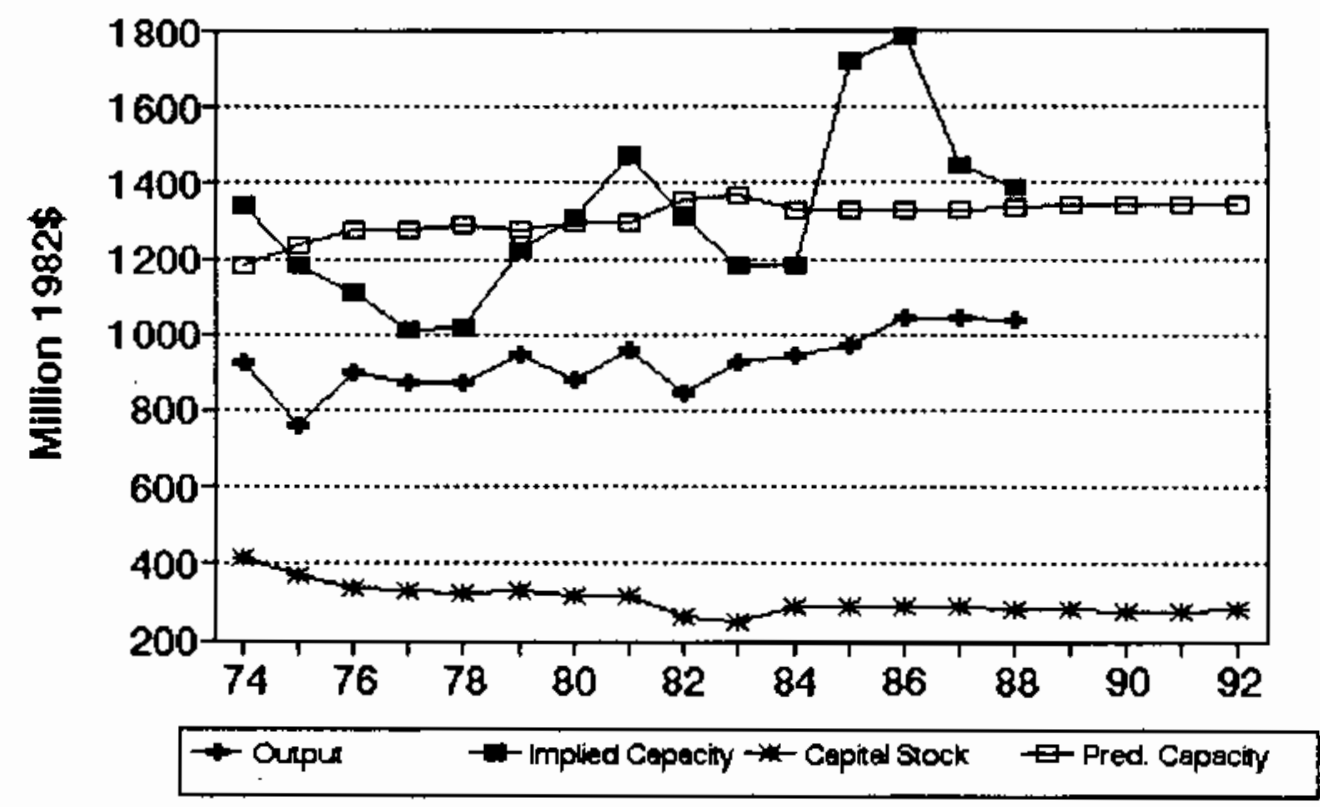


No. 56 sIc 2251 Women's hosiery, except socks

MILLIONS OF 1982 DOLLARS (EXCL. CU RATES)

$\begin{array}{lccccccc} & 04 & \text { Ann. } & \text { Implied Gross } & \text { Het } & \text { Pred. } & \ldots . . . . . . . \\ \text { Gross } & \text { CU } & \text { CU } & \text { Prac. } & \text { In- } & \text { Cap. } & \text { Prac. } & \text { Emergency Capacity } \\ \text { Output } & \text { Rate } & \text { Rate } & \text { Capac. } & \text { vest. } & \text { Stk. } & \text { Capac. } & 3 \text { mon. } 6 \text { mon. }>6 \text { mon. }\end{array}$

\begin{tabular}{|c|c|c|c|c|c|c|c|c|c|c|}
\hline 1974 & 878 & 74 & 75.8 & 1,158 & $(58.4)$ & 536 & 1,178 & & & \\
\hline 1975 & 876 & 61 & 65.3 & 1,342 & (11.9) & 475 & 1,256 & & & \\
\hline 1976 & 771 & 67 & 64.8 & 1,191 & $(8.2)$ & 421 & 1,334 & & & \\
\hline 1977 & 1,039 & 78 & 74.0 & 1,404 & 23.7 & 398 & 1,411 & & & \\
\hline 1978 & 1,255 & 85 & 82.4 & 1,523 & 27.5 & 379 & 1,489 & & & \\
\hline 1979 & 1,417 & 78 & 80.5 & 1,759 & (16.8) & 322 & 1,567 & & & \\
\hline 1980 & 1,318 & 79 & 78.6 & 1.677 & 23.3 & 307 & 1,644 & & & \\
\hline 1981 & 1,341 & 81 & 80.8 & 1,659 & 22.5 & 291 & 1,722 & & & \\
\hline 1982 & 1,391 & 81 & 80.8 & 1,720 & 25.5 & 279 & 1,800 & & & \\
\hline 1983 & 1,511 & 88 & 85.6 & 1,765 & 30.9 & 273 & 1,878 & & & \\
\hline 1984 & 1,571 & $\pi$ & 81.6 & 1,925 & 38.2 & 274 & 1,955 & & & \\
\hline 1985 & 1,486 & 70 & 72.4 & 2,051 & 32.6 & 272 & 2,033 & & & \\
\hline 1986 & 1,596 & 74 & 72.4 & 2,205 & 24.5 & 204 & 2,111 & & & \\
\hline $\begin{array}{l}-\cdots \\
1987\end{array}$ & $\begin{array}{l}\cdots, 623 \\
1, \cdots\end{array}$ & $\begin{array}{l}-- \\
85\end{array}$ & $\begin{array}{l}-7 \\
80.9\end{array}$ & 2,005 & $\begin{array}{r}-7 \\
45.4\end{array}$ & $2 \pi$ & $\begin{array}{c}\cdots \\
2,188\end{array}$ & $-\cdot$ & -- & $-\cdots$ \\
\hline 1988 & 1,658 & 82 & 83.0 & 1,998 & 50.0 & 292 & 2,266 & 2,899 & 3,075 & 3,111 \\
\hline 1989 & & & & & 54.6 & 310 & 2,344 & 2,999 & 3,180 & 3,218 \\
\hline 1990 & & & & & 59.2 & 331 & 2,421 & 3,098 & 3,286 & 3,325 \\
\hline 1991 & & & & & 63.8 & 354 & 2,499 & 3,197 & 3,391 & 3,431 \\
\hline 1992 & & & & & 68.5 & 378 & 2,577 & 3,297 & 3,497 & 3,538 \\
\hline
\end{tabular}

Capacity-Capital Stock Regression: Code -2 RSO 0.920

Coefficients: Const 1100.6 Time 7.7

Ave. Capacity/Stock $(74-86): 4.8$

Ave. Weekly Hours (High) 109.9 Shift factor 1.37 Ave Weekly Hours (Low) 99.5 Shift Factor 1.52 Note: Output, Imvestment, and Capital stock extrapolated for 1987 and 1988.

\section{Women's hosiery, except socks}

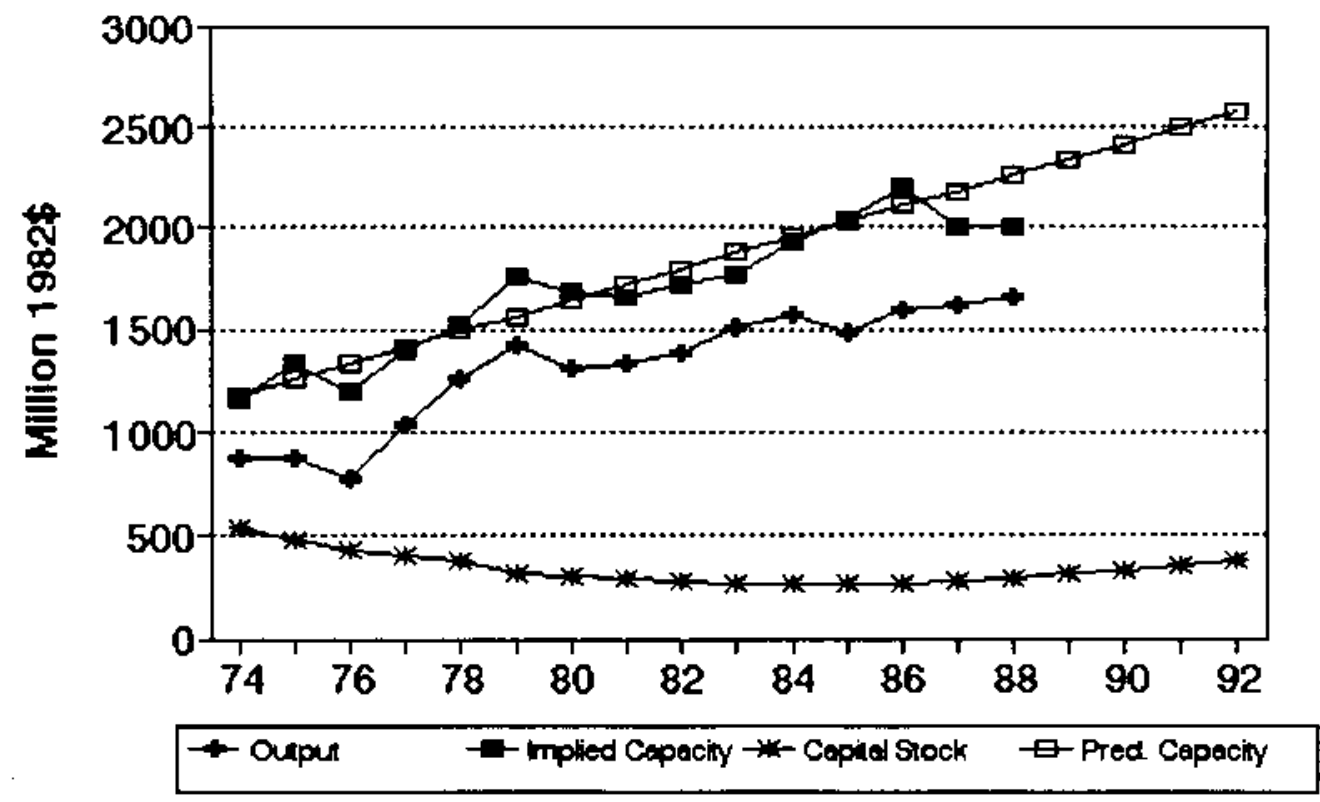


No. 57 sIC 2252 Hosiery, n.e.c.

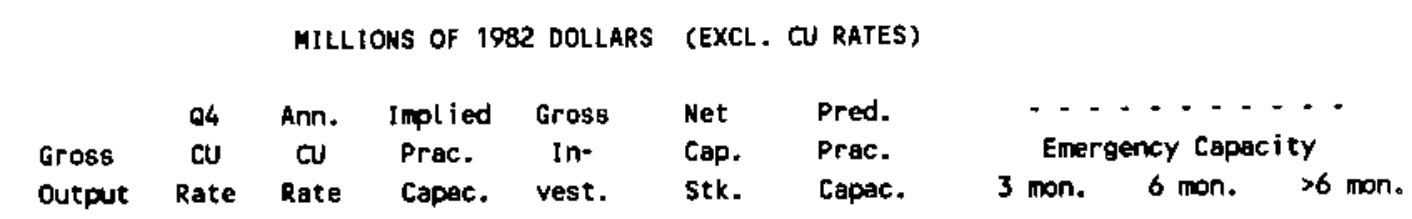

\begin{tabular}{|c|c|c|c|c|c|c|c|c|c|c|}
\hline 1974 & 1,000 & 63 & 70.3 & 1,422 & (12.1) & 300 & 1,425 & & & \\
\hline 1975 & 1,100 & 81 & 71.2 & 1,546 & 27.0 & 299 & 1,469 & & & \\
\hline 1976 & 1,190 & 73 & 75.9 & 1,567 & 16.2 & 288 & 1,513 & & & \\
\hline 1977 & 1,329 & 98 & 89.3 & 1,489 & 35.7 & 294 & 1,558 & & & \\
\hline 1978 & 1,403 & 81 & 87.3 & 1,607 & 27.9 & 292 & 1,602 & & & \\
\hline 1979 & 1,311 & 92 & 87.4 & 1,500 & $(32.9)$ & 235 & 1,646 & & & \\
\hline 1980 & 1,374 & 83 & 86.1 & 1,597 & 23.4 & 234 & 1,691 & & & \\
\hline 1981 & 1,356 & 74 & 79.7 & 1,700 & 26.6 & 235 & 1,735 & & & \\
\hline 1982 & 1,471 & 75 & 73.9 & 1,989 & 32.9 & 242 & 1,779 & & & \\
\hline 1983 & 1,586 & 87 & 83.6 & 1,890 & 25.6 & 243 & 1,824 & & & \\
\hline 1984 & 1,459 & 73 & 80.2 & 1,819 & 44.5 & 261 & 1,868 & & & \\
\hline 1985 & 1,423 & 75 & 73.4 & 1,938 & 49.1 & 281 & 1,912 & & & \\
\hline 1986 & 1,480 & 80 & 77.7 & 1,906 & 28.7 & 282 & 1,957 & & & \\
\hline$-\cdot$ & $\cdots \cdot$ & -- & -- & $\cdots$ & $\cdots-$ & $\cdots$ & --- & $\cdot-$ & $-=$ & $\cdots$ \\
\hline 1987 & 1,496 & 72 & 75.3 & 1,987 & 38.4 & 292 & 2,001 & & & \\
\hline 1988 & 1,516 & 81 & 7.2 & 1,963 & 40.5 & 304 & 2,045 & 2,516 & 2,658 & 2,687 \\
\hline 1989 & & & & & 42.6 & 316 & 2,090 & 2,571 & 2,716 & 2,746 \\
\hline 1990 & & & & & 44.7 & 329 & 2,134 & 2,625 & 2,773 & 2,804 \\
\hline 1991 & & & & & 46.8 & 343 & 2,178 & 2,680 & 2,831 & 2,862 \\
\hline 1992 & & & & & 48.9 & 358 & 2,223 & 2,735 & 2,889 & 2,920 \\
\hline
\end{tabular}

Capacity-Capital Stock Regression: Code -2 RSQ 0.780

Coefficients: Const 1380.3 Time 44.3

Ave. Capacity/Stock $(74-86): 6.3$

Ave. Weekly Hours (High) 115.0 Shift Factor 1.31 Ave Meekly Hours (LOW) 101.7 Shift Factor 1.48 Note: Output, Investment, and Capital Stock extrapolated for 1987 and 1988.

2252 Hosiery, n.e.c.

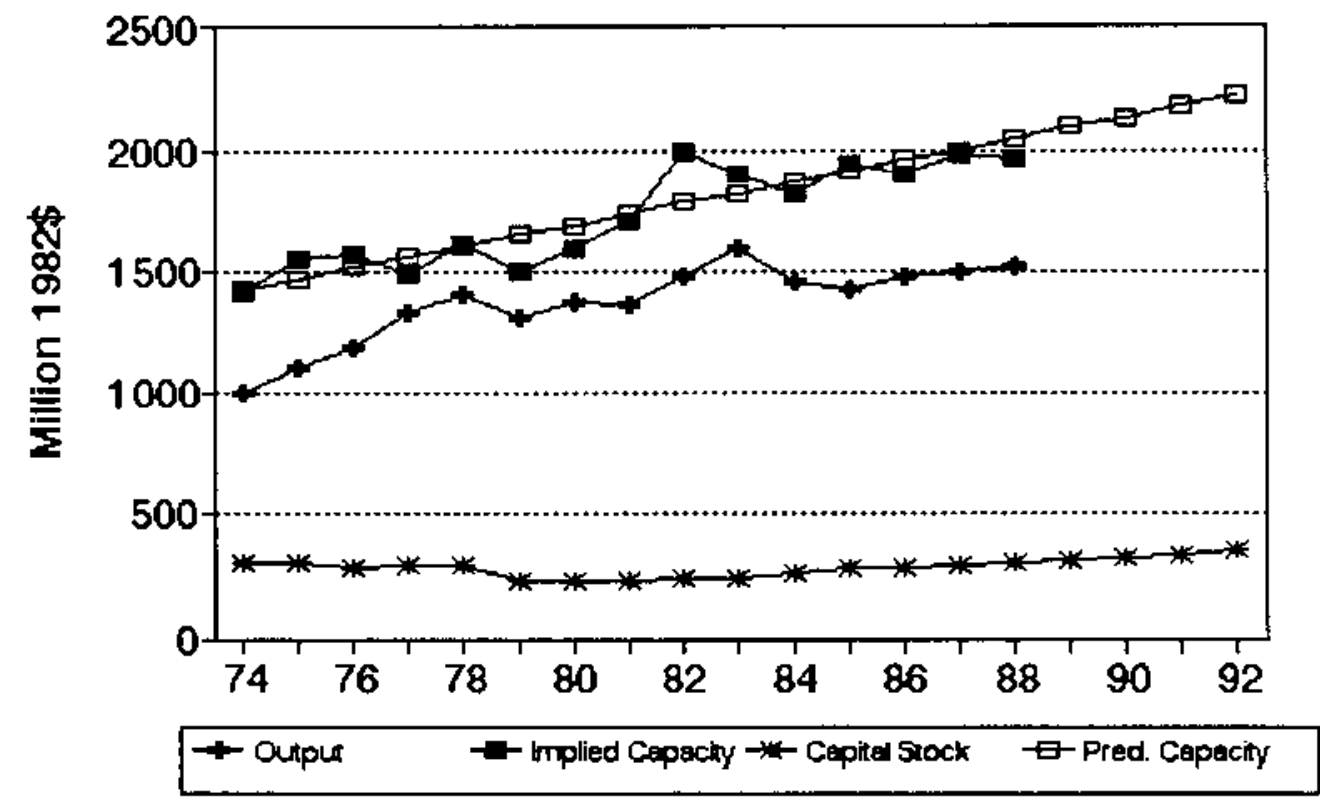


No. 58 SIC 2253 Knit outerwear n11l:

MILLIONS OF 1982 DOLLARS (EXCL. CU RATES)

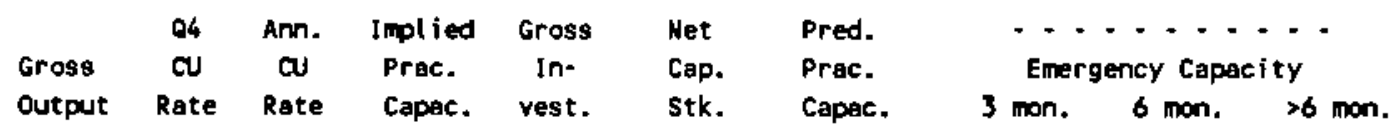

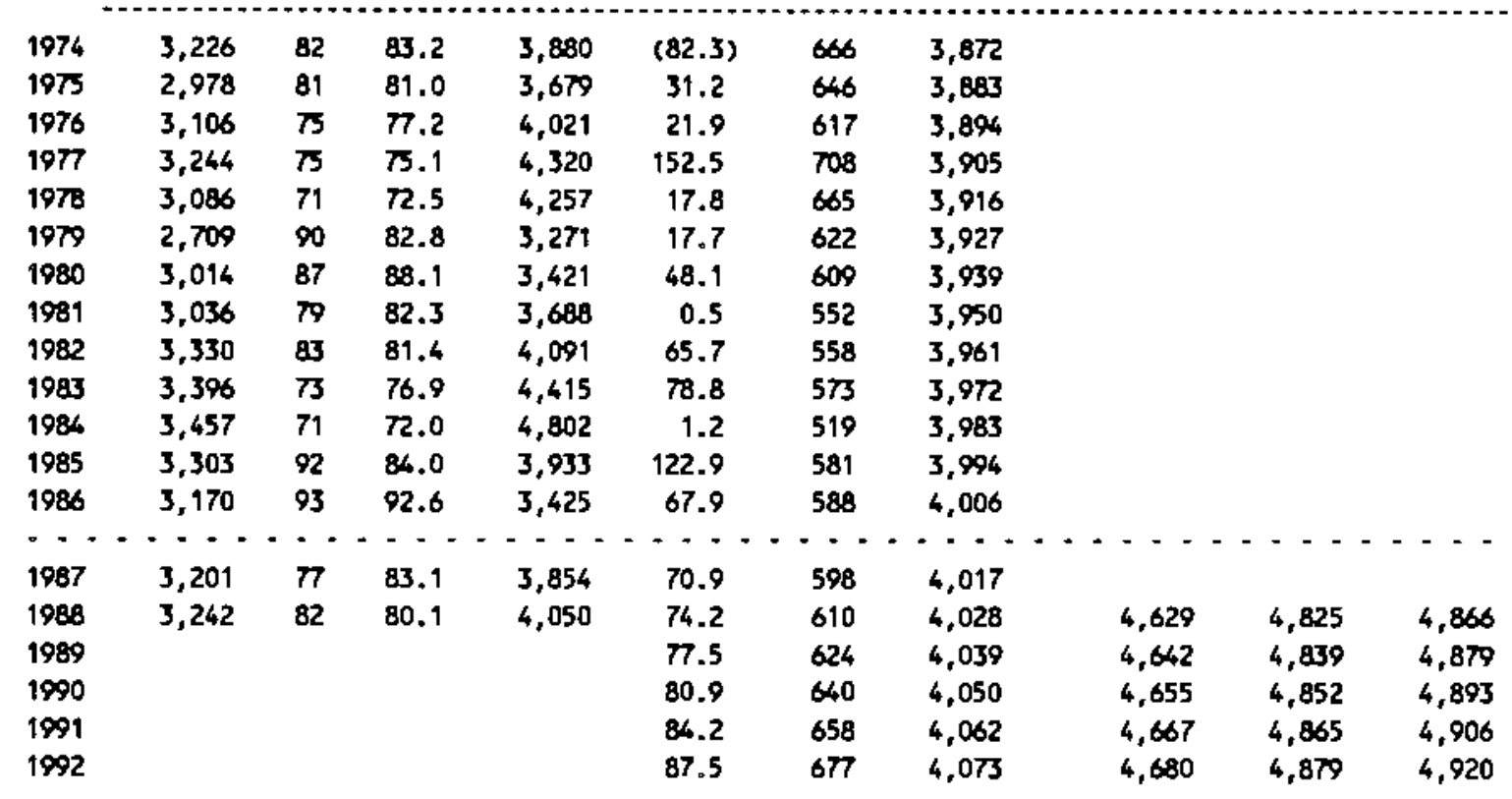

Capacity-Capital Stock Regression: Code -2 RSO 0.010

Coefficients: Const 3860.3 Time 11.2

Ave. Capacity/Stock $(74-86): 6.5$ Ave. Weekly Hours (High) 125.5 Shift Factor 1.21 Ave Weekly Hours (LOW) 105.2 Shift Factor 1.43 Mote: Output, Investment, and Capital stock extrapolated for 1987 and 1988.

\section{Knit outerwear mills}

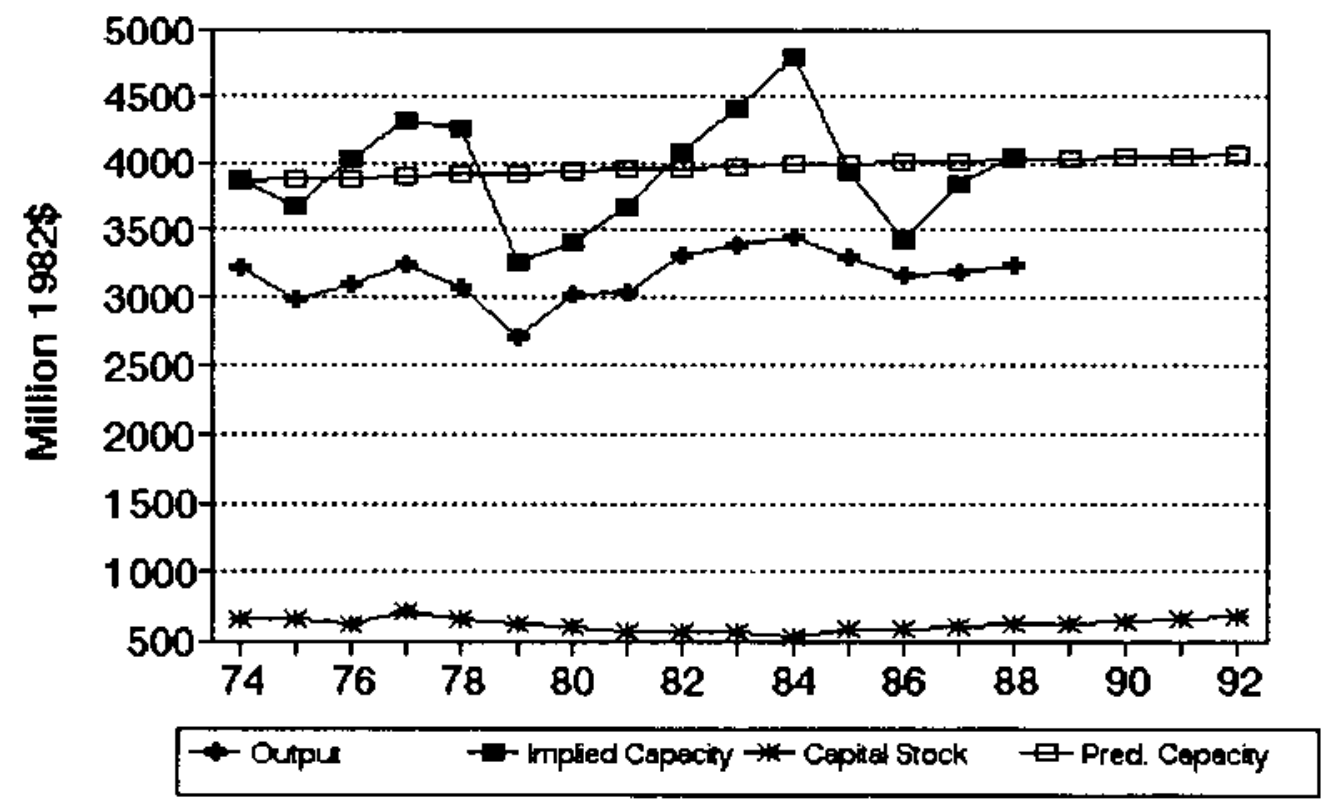




\section{No. 59 sIC 2254 knit underwear mills}

MILLIONS OF 1982 DOLLARS (EXCL. CU RATES)

$\begin{array}{lccccccc} & \text { a4 } & \text { Ann. Implied Gross } & \text { Net } & \text { Pred. } & \ldots \ldots \ldots \\ \text { Gross } & \text { CU } & \text { CU } & \text { Prac. } & \text { In- } & \text { Cap. } & \text { Prac. } & \text { Emergency Capacity } \\ \text { Output } & \text { Rate } & \text { Rate } & \text { Capac. } & \text { vest. } & \text { \$tk. } & \text { Capac. } & 3 \text { mon. } 6 \text { mon. }>6 \text { mon. }\end{array}$

\begin{tabular}{|c|c|c|c|c|c|c|c|c|c|c|}
\hline 1974 & 1,106 & 92 & 94.3 & 1.173 & 6.6 & 160 & 1,104 & & & \\
\hline 1975 & 1,076 & 87 & 88.1 & 1,222 & 22.3 & 166 & 1,154 & & & \\
\hline 1976 & 1,093 & 90 & 88.9 & 1,230 & 20.2 & 170 & 1,184 & & & \\
\hline 1977 & 937 & 89 & 89.6 & 1,046 & 7.9 & 162 & 1,121 & & & \\
\hline 1978 & 1,085 & 80 & 83.4 & 1,301 & 28.9 & 174 & 1,222 & & & \\
\hline 1979 & 1,060 & 68 & 72.4 & 1,464 & 49.7 & 206 & 1,477 & & & \\
\hline 1980 & 1,030 & 76 & $\pi .0$ & 1,411 & 5.7 & 194 & 1,382 & & & \\
\hline 1981 & 927 & 71 & $\pi .6$ & 1,263 & 6.6 & 184 & 1,303 & & & \\
\hline 1982 & 771 & 72 & 71.5 & 1,078 & (8.5) & 161 & 1,116 & & & \\
\hline 1983 & 814 & 86 & 81.0 & 1,006 & $(0.9)$ & 147 & 1,000 & & & \\
\hline 1984 & 1,015 & 100 & 95.3 & 1,065 & 47.1 & 177 & 1,265 & & & \\
\hline 1985 & 1,118 & 79 & 86.7 & 1,290 & 25.6 & 185 & 1,310 & & & \\
\hline 1986 & 1,190 & 83 & 81.4 & 1,462 & 28.0 & 196 & 1,393 & & & \\
\hline$\therefore$ & $\cdots$ & -- & $--\overline{1}$ & $\because \cdots$ & - - - - & $\cdots$ & $-7-$ & $\cdots-$ & $-\cdots$ & $-\cdots$ \\
\hline 1987 & 1,205 & 96 & 91.2 & 1,321 & 22.1 & 199 & 1,425 & & & \\
\hline 1988 & 1,225 & 89 & 91.5 & 1,339 & 26.7 & 208 & 1,492 & 1,918 & 2,016 & 2,036 \\
\hline 1989 & & & & & 31.5 & 220 & 1,591 & 2,045 & 2,150 & 2,172 \\
\hline 1990 & & & & & 26.9 & 227 & 1,646 & 2,113 & 2,221 & 2,244 \\
\hline 1991 & & & & & 28.5 & 234 & 1,706 & 2,191 & 2,303 & 2,326 \\
\hline 1992 & & & & & 23.8 & 236 & 1,721 & 2,213 & 2,326 & 2,349 \\
\hline
\end{tabular}

Capecity-Capital Stock Regression: Code 0 RSO 0.770

Coefficients: Const -188.7 Stock 8.1

Ave. Capecity/Stock $(74-86): 7.0$ Ave. Weekly Hours (High) 110.5 Shift Factor 1.37 Ave Weekly Hours (Low) 102.3 Shift factor 1.48 Note: Output, Imvestment, and Capital Stock extrapolated for 1987 and 1988.

\section{Knit underwear mills}

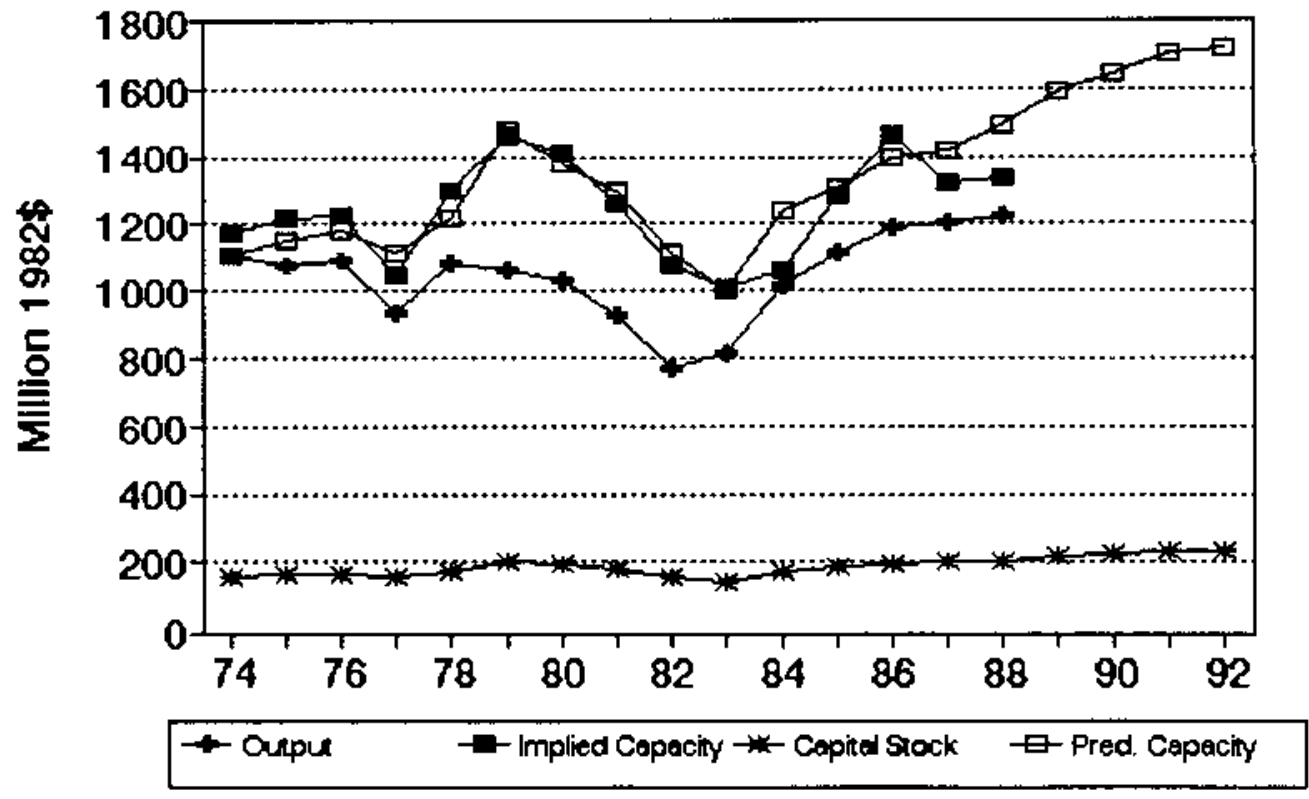


yo. 60 sic 2257 Circular knit fabric nills

MILLIONS OF 1982 DOLLARS (EXCL. CU RATES)

$\begin{array}{lccccccc} & \text { Q4 } & \text { Am. } & \text { Implied } & \text { Gross } & \text { Net } & \text { Pred. } & \ldots \\ \text { Gross } & \text { CU } & \text { CU } & \text { Prac. } & \text { In- } & \text { Cap. } & \text { Prac. } & \text { Emergency Capacity } \\ \text { Output } & \text { Rate } & \text { Rate } & \text { Capac. } & \text { vest. } & \text { Stk. } & \text { Capac. } & 3 \text { mon. } 6 \text { mon. }>6 \text { mon. }\end{array}$

\begin{tabular}{|c|c|c|c|c|c|c|c|c|c|c|}
\hline 1974 & 3,271 & 69 & 78.5 & 4,165 & $(26.0)$ & 2,154 & 4,737 & & & \\
\hline 1975 & 3,365 & 85 & $\pi .2$ & 4,472 & 71.4 & 2,120 & 4,691 & & & \\
\hline 1976 & 3,307 & $\pi$ & 78.7 & 4,204 & $(84.5)$ & 1,929 & 4,432 & & & \\
\hline 1977 & 3,778 & 72 & 74.1 & 5,099 & 36.4 & 1,854 & 4,330 & & & \\
\hline 1978 & 3,442 & 81 & 77.6 & 4,434 & $(61.0)$ & 1,683 & 4,097 & & & \\
\hline 1979 & 3,482 & 83 & 81.7 & 4,261 & 32.0 & 1,600 & 3,985 & & & \\
\hline 1980 & 3,158 & 81 & 81.5 & 3,876 & 60.6 & 1,543 & 3,908 & & & \\
\hline 1981 & 2,784 & 67 & 74.8 & 3,723 & 8.0 & 1,435 & 3,762 & & & \\
\hline 1982 & 2,507 & 66 & 65.6 & 3,820 & $(118.3)$ & 1,218 & 3,467 & & & \\
\hline 1983 & 2,657 & 80 & 7.8 & 3,505 & 13.5 & 1,133 & 3,350 & & & \\
\hline 1984 & 2,281 & $80 \star$ & 82.3 & 2,773 & (33.7) & 1,008 & 3,181 & & & \\
\hline 1985 & 2,190 & 81 & 79.6 & 2,752 & 74.4 & 986 & 3,151 & & & \\
\hline 1986 & 2,572 & 86 & 83.6 & 3,079 & 35.0 & 927 & 3,071 & & & \\
\hline 1987 & 2,650 & 82 & 83.9 & 3,161 & 44.7 & - . & 3,006 & $\cdots$ & -- & $-\cdots$ \\
\hline 1988 & 2,755 & 88 & 85.2 & 3,233 & 74.4 & 866 & 2,989 & 3,820 & 3,970 & 4,002 \\
\hline 1989 & & & & & 105.1 & 885 & 3,014 & 3,852 & 4,004 & 4,036 \\
\hline 1990 & & & & & 75.6 & 872 & 2,997 & 3,830 & 3,982 & 4,013 \\
\hline 1991 & & & & & 85.4 & 871 & 2,995 & 3.828 & 3,979 & 4,010 \\
\hline 1992 & & & & & 55.9 & 840 & 2,953 & 3,774 & 3,923 & 3.954 \\
\hline
\end{tabular}

Capacity-Capital stock Regression: Code 0 RSQ 0.710

Coefficients: Const 1812.3 Stock 1.4

Ave. Capacity/Stock $(74-86): 2.6$ Ave. Heekly Hours (High) 112.8 Shift Factor 1.34 Ave Heekly Hours (LOW) 110.0 Shift Factor 1.37 * Imputed Note: Output, Investment, and Capital stock extrapolated for 1987 and 1988.

\section{Circular knit fabric mills}

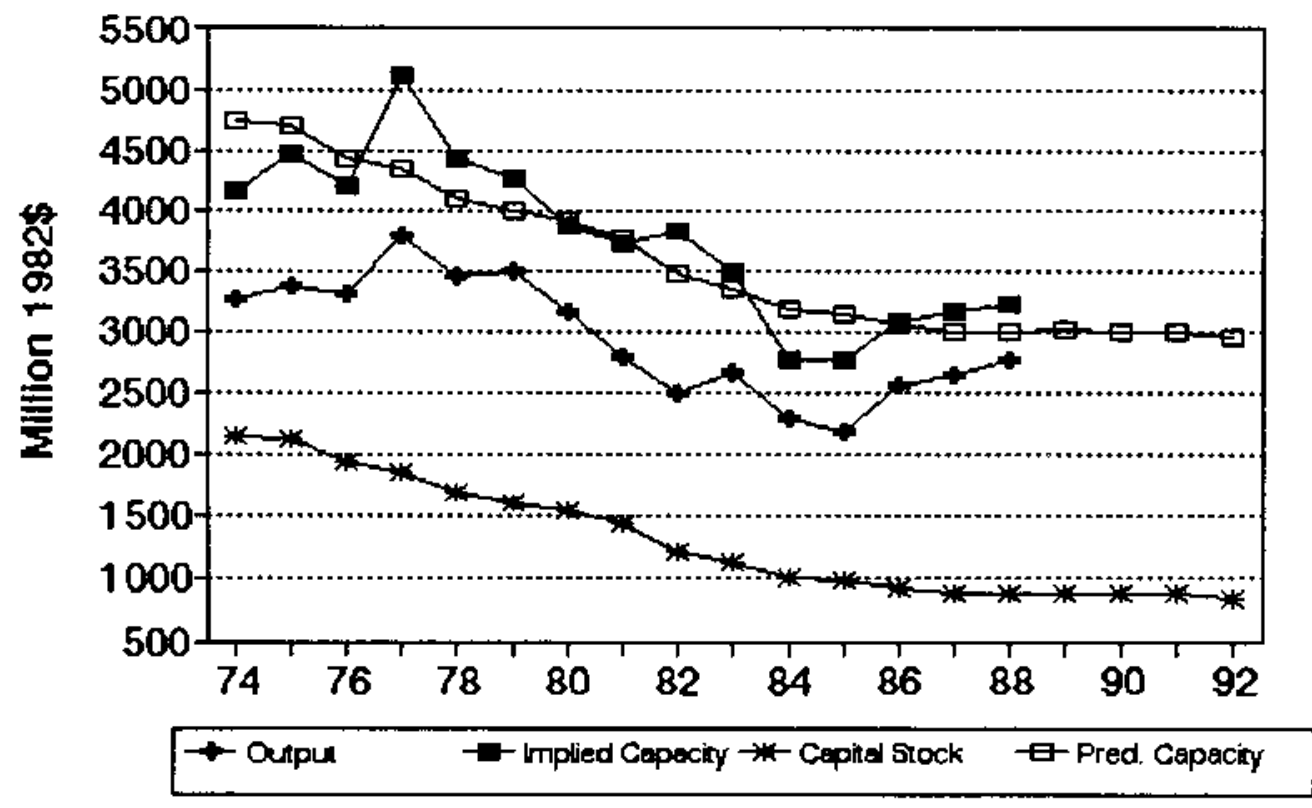




\section{No. 61 sIC 2258 Warp knit fabric ailla}

MILLIOWS OF 1982 DOLLARS (EXCL. CU RATES)

\begin{tabular}{|c|c|c|c|c|c|c|c|}
\hline & $\begin{array}{l}\text { O4 } \\
\text { CU }\end{array}$ & $\begin{array}{c}\text { Ann. } \\
\text { du }\end{array}$ & $\begin{array}{l}\text { Implied } \\
\text { Prac. }\end{array}$ & $\begin{array}{l}\text { Gross } \\
\text { In- }\end{array}$ & $\begin{array}{l}\text { Net } \\
\text { Cap. }\end{array}$ & $\begin{array}{l}\text { Pred. } \\
\text { Prac. }\end{array}$ & $\begin{array}{c}- \\
\text { Energency Capacity }\end{array}$ \\
\hline & & & & mest & stk & Свpec. & \\
\hline
\end{tabular}

\begin{tabular}{|c|c|c|c|c|c|c|c|c|c|c|}
\hline 1974 & 1,402 & 84 & 84.0 & 1,660 & $(21.9)$ & 589 & 2,145 & & & \\
\hline 1975 & 1,504 & 83 & 83.4 & 1,804 & $(54.2)$ & 514 & 1,722 & & & \\
\hline 1976 & 1,813 & $\pi$ & 79.3 & 2,288 & 187.0 & 689 & 2,592 & & & \\
\hline 1977 & 1,862 & 81 & 79.5 & 2,342 & (3.3) & 636 & 2,385 & & & \\
\hline 1978 & 2,046 & 84 & 82.9 & 2,460 & 29.5 & 629 & 2,366 & & & \\
\hline 1970 & 1,975 & 73 & 77.1 & 2,560 & 65.5 & 654 & 2,511 & & & \\
\hline 1980 & 1,840 & 63 & 66.8 & 2,756 & 35.3 & 648 & 2,473 & & & \\
\hline 1981 & 1,734 & 66 & 64.9 & 2,673 & 26.3 & 632 & 2,383 & & & \\
\hline 1982 & 1,440 & 66 & 64.8 & 2,224 & 40.6 & 626 & 2,353 & & & \\
\hline 1983 & 1,787 & 80 & 74.0 & 2,415 & 35.7 & 613 & 2,280 & & & \\
\hline 1984 & 1,609 & 83 & 81.9 & 1,965 & (17.3) & 553 & 1,943 & & & \\
\hline 1985 & 1,587 & 66 & 72.4 & 2,193 & 40.3 & 550 & 2,390 & & & \\
\hline 1986 & 1,434 & 50 & 56.0 & 2,560 & 40.4 & 546 & 2,364 & & & \\
\hline$\cdots$ & $\because \cdot$ & $\cdots$ & $\cdots$ & $\because \cdot$ & $\cdots$ & $\because$ & $\cdots$ & -- & $-\cdot$ & -- \\
\hline 1987 & 1,466 & 52 & 51.3 & 2,861 & 39.3 & 541 & 2,338 & & & \\
\hline 1988 & 1,510 & $51 *$ & 51.3 & 2,942 & 43.7 & 541 & 2,339 & 2,218 & 2,318 & 2,339 \\
\hline 1989 & & & & & 48.1 & 546 & 2,364 & 2,242 & 2,343 & 2,364 \\
\hline 1990 & & & & & 43.8 & 546 & 2,364 & 2,241 & 2,343 & 2,364 \\
\hline 1991 & & & & & 45.3 & 547 & 2,371 & 2,249 & 2,350 & 2,371 \\
\hline 1992 & & & & & 41.0 & 544 & 2,354 & 2,232 & 2,333 & 2,354 \\
\hline
\end{tabular}

Capacity-Capital Stock Regression: Code -1 RSO 0.520

Coefficients: Const -1160.4 5tock $5.6 \quad$ D(85-86) 463.1

Ave. Capacity/stock (74-86): 3.8 Ave. Heekly Hours (High) 156.0 Shift Factor 1.00 Ave Heekly Hours (LOW) 151.1 Shift Factor 1.03 - Imputed Note: Output, Investment, and Capital stock extrapolated for 1987 and 1988.

\section{Warp knit fabric mills}

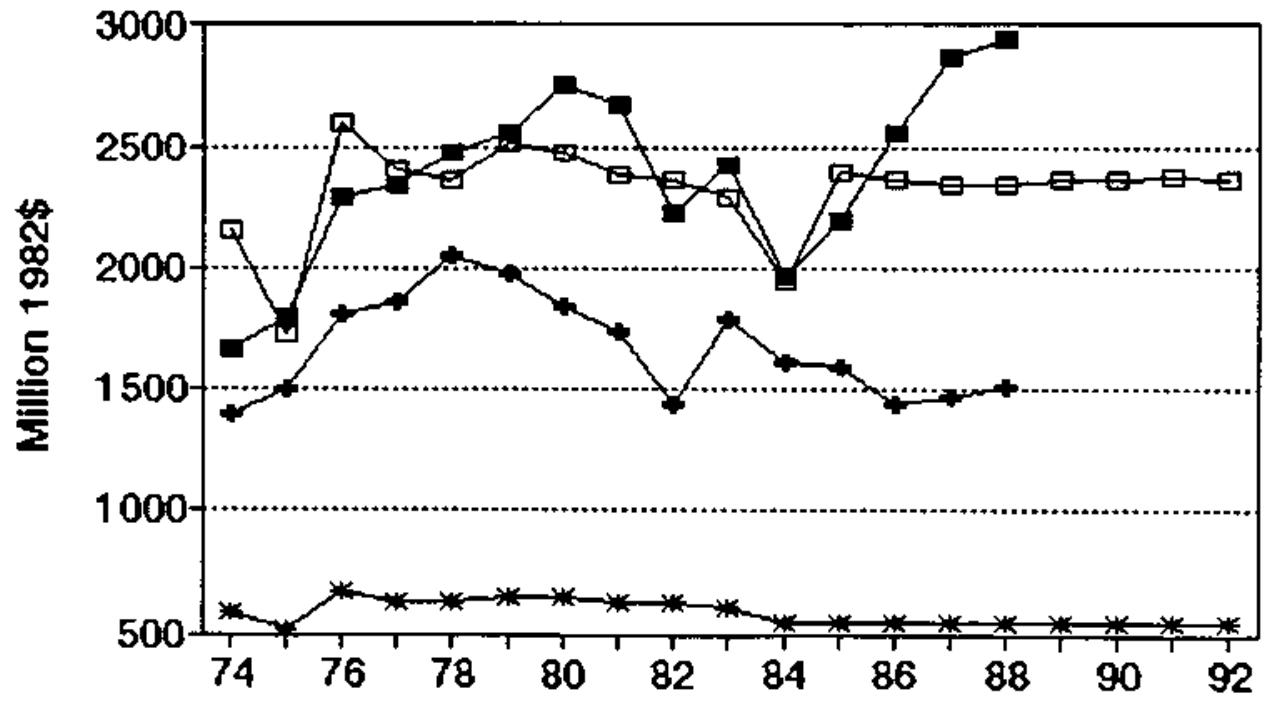

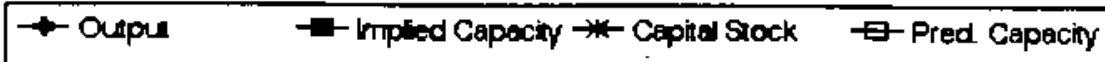




\section{No. 62 SIC 2259 rnitting mills, nec}

MILLIONS OF 1982 DOLLARS (EXCL. O RATES)

$\begin{array}{lccccccc} & \text { Q4 } & \text { Ann. } & \text { Implied Gross } & \text { Het } & \text { Pred. } & -\ldots & \ldots \\ \text { Gross } & \text { CU } & \text { CU } & \text { Prac. } & \text { In- } & \text { Cap. } & \text { Prac. } & \text { Emergency Capacity } \\ \text { Output } & \text { Rate } & \text { Rate } & \text { Capac. } & \text { vest. } & \text { Stk. } & \text { Capac. } & 3 \text { mon. } 6 \text { mon. }>6 \text { mon. }\end{array}$

\begin{tabular}{|c|c|c|c|c|c|c|c|c|c|c|}
\hline 1974 & 192 & 64 & 68.4 & 281 & 6.0 & 45 & 215 & & & \\
\hline 1975 & 193 & 49 & 53.4 & 362 & 140.1 & 174 & 372 & & & \\
\hline 1976 & 155 & 52 & 50.7 & 306 & $(121.7)$ & 49 & 220 & & & \\
\hline 1977 & 133 & 55 & 54.2 & 246 & (15.1) & 32 & 200 & & & \\
\hline 1978 & 149 & 60 & 58.2 & 256 & 6.7 & 37 & 205 & & & \\
\hline 1979 & 137 & 80 & $\pi .3$ & 190 & 6.4 & 41 & 210 & & & \\
\hline 1980 & 124 & 77 & 78.0 & 159 & 2.2 & 40 & 209 & & & \\
\hline 1981 & 102 & 63 & 69.5 & 146 & $(0.1)$ & 37 & 205 & & & \\
\hline 1982 & 117 & 63 & 62.7 & 186 & 9.6 & 42 & 212 & & & \\
\hline 1983 & 132 & 70 & 67.9 & 195 & 3.0 & 41 & 210 & & & \\
\hline 1984 & 105 & $59 *$ & 64.0 & 164 & $(7.7)$ & 30 & 197 & & & \\
\hline 1985 & 107 & 70 & 65.4 & 163 & 2.2 & 29 & 196 & & & \\
\hline 1986 & 136 & 71 & 70.3 & 193 & 2.3 & 28 & 195 & & & \\
\hline$\cdots$ & -- & $\cdot$ & $\cdots$ & $-\cdots$ & 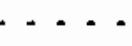 & $\cdots-$ & -- & $\because$ & -- & - \\
\hline 1987 & 139 & 70 & 70.7 & 197 & (4.6) & 21 & 186 & & & \\
\hline 1928 & 144 & 71 & 70.6 & 204 & $(5.6)$ & 13 & 176 & 203 & 211 & 213 \\
\hline 1989 & & & & & $(6.6)$ & 5 & 166 & 191 & 199 & 201 \\
\hline 1990 & & & & & $(7.6)$ & (3) & 156 & 180 & 187 & 189 \\
\hline 1991 & & & & & $(8.6)$ & (12) & 146 & 168 & 175 & 177 \\
\hline 1992 & & & & & $(9.6)$ & (20) & 136 & 156 & 163 & 164 \\
\hline
\end{tabular}

Capacity-Capital Stock Regression: Code 0 RSO 0.500

Coefficients: Const 160.3 stock 1.2 Ave. Capacity/Stock (74-86): 4.6 Ave. Weekly Hours (High) 125.5 Shift Factor 1.21 Ave Heekly Hours (Low) 105.2 Shift Factor 1.43 - Imputed Note: Output, Investment, and Capital Stock extrapolated for 1987 and 1988.

2259 Knitting mills, nec

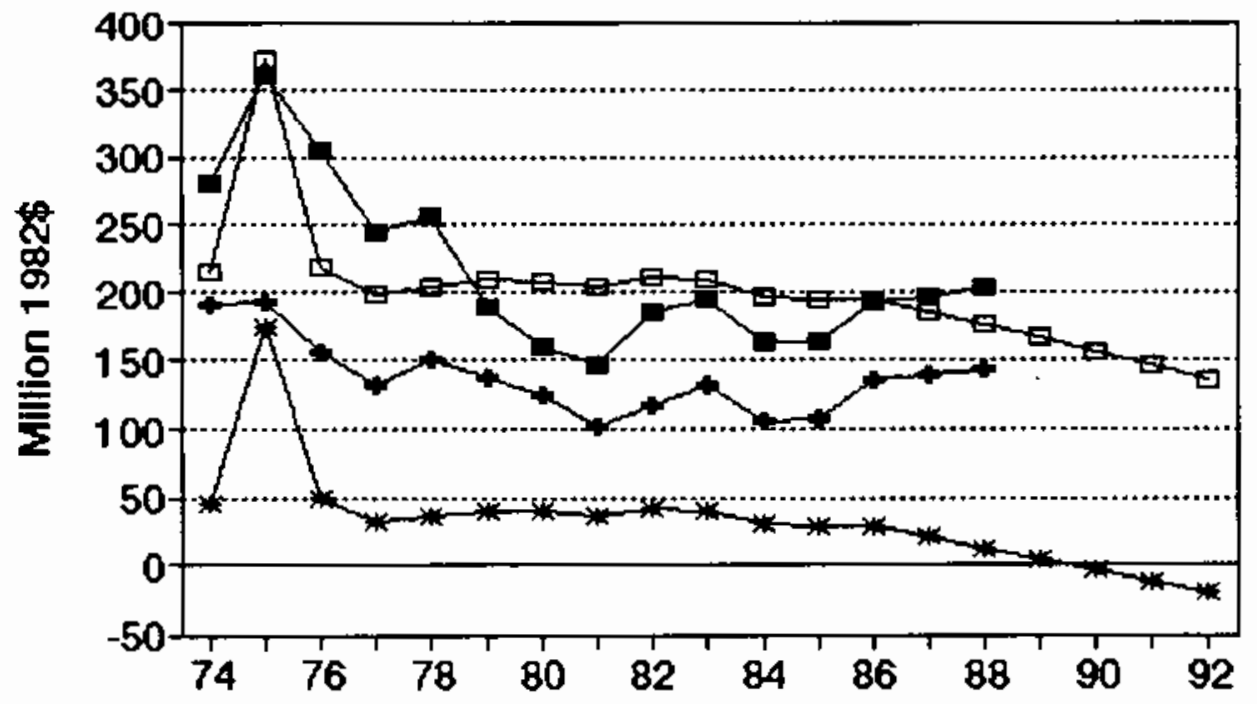

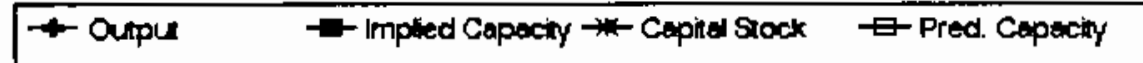


No. 63 sIC 2261 Finishing plants, cotton

MILLIONS OF 1982 DOLLARS (EXCL. CU RATES)

$\begin{array}{lccccccc} & \text { O4 } & \text { Am. Implied Gross } & \text { Net } & \text { Pred. } & \ldots \ldots \ldots & \ldots \\ \text { Gross } & \text { a } & \text { CU } & \text { Prac. } & \text { In- } & \text { Cap. } & \text { Prac. } & \text { Emergency Capacity } \\ \text { Output Rate Rate } & \text { Capac. } & \text { vest. } & \text { Stk. } & \text { Capac. } & 3 \text { mon. } 6 \text { mon. }>6 \text { mon. }\end{array}$

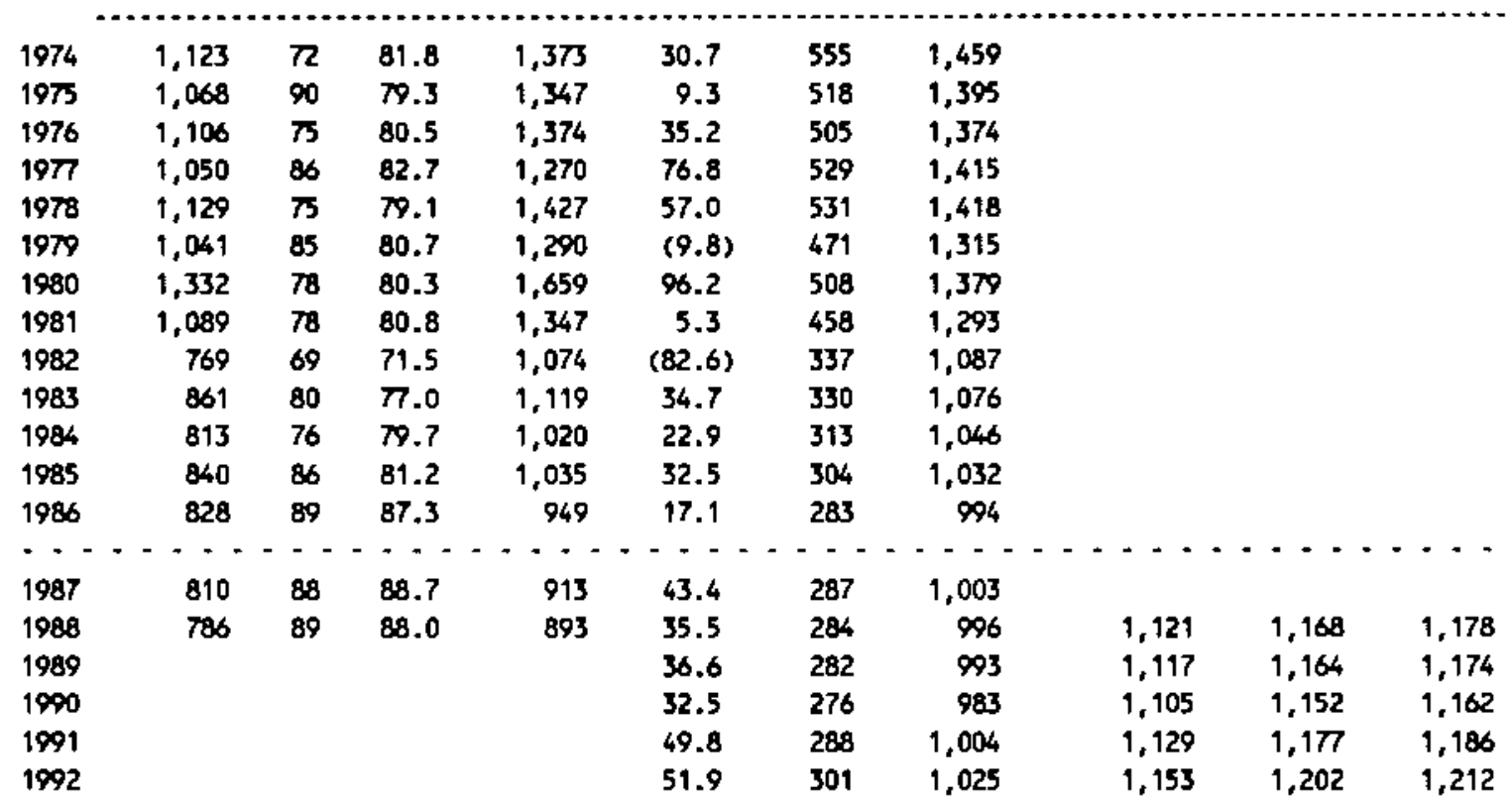

Capacity-Capital Stock Regression: Code 0 RSO 0.760

Coefficients: Const 512.7 Stock $1.7 \quad$ Ave. Capacity/Stock $(74-86): 2.9$ Ave. Weekly Hours (High) 128.4 Shift Factor 1.18 Ave Heekly Hours (Low) 124.8 Shift Factor 1.22 Note: Output, Imvestment, and Capital stock extrapolated for 1987 and 1988.

2261 Finishing plants, cotton
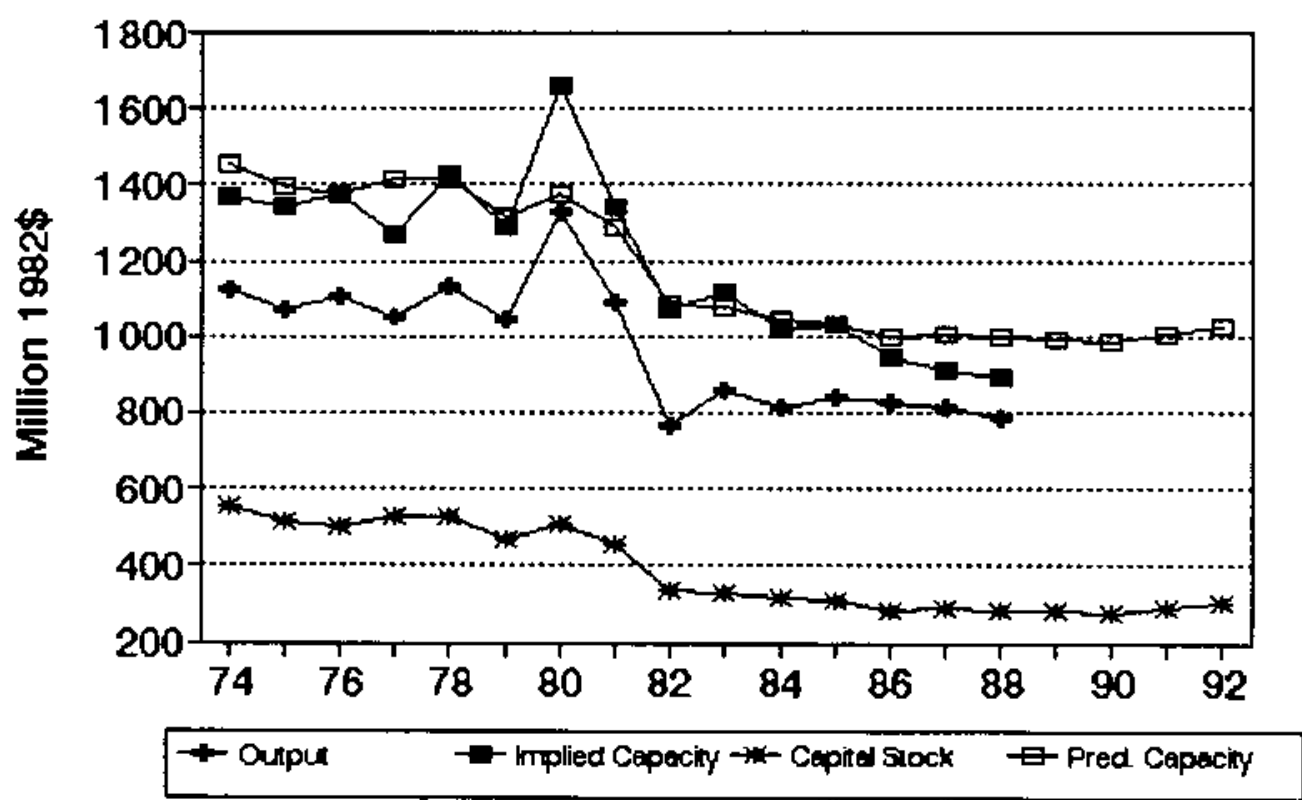


\title{
No. 64 sIC 2262 Finishing plants, synthetics
}

\author{
MILLIOWS OF 1982 dOLLARS (EXCL, CU RATES)
}

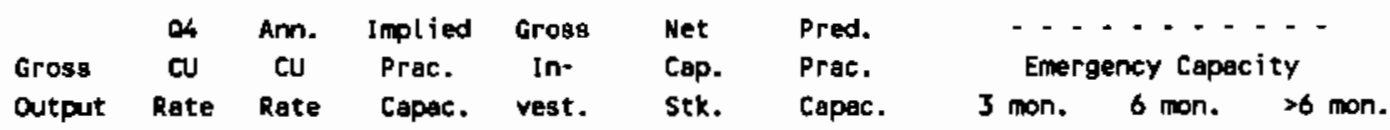

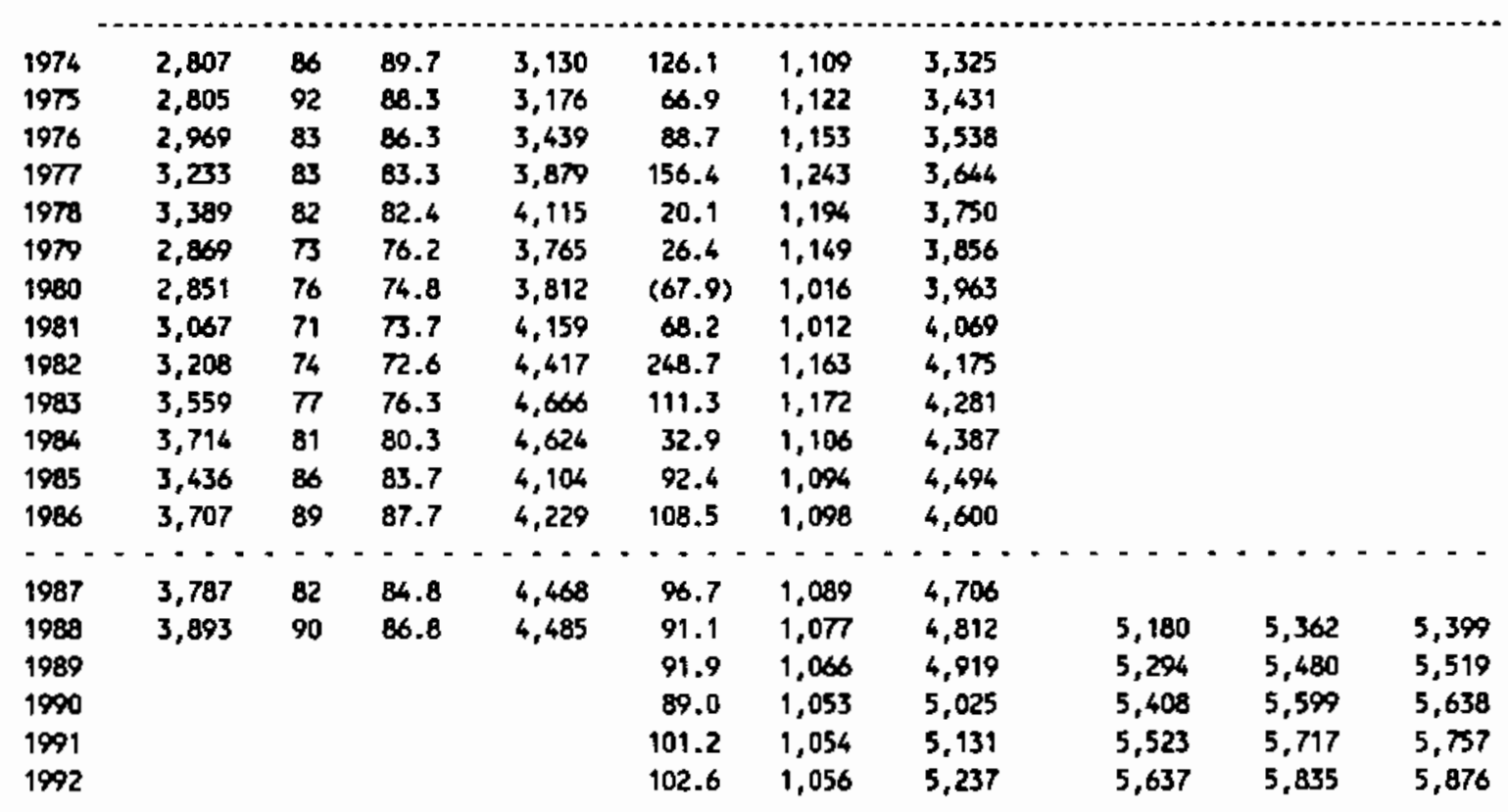

Capacity-Capital stock Regression: Code -2 RSO 0.700

Coefficients: const 3218.9 Time 106.2

Ave. Capacity/Stock $(74-86): 3.5$

Ave. Weekly Hours (High) 135.6 Shift Factor 1.12 Ave Heekly Hours (Low) 133.2 Shift Factor 1.14 Note: Output, Investment, and Capital stock extrapolated for 1987 and 1988.

2262 Finishing plants, synthetics

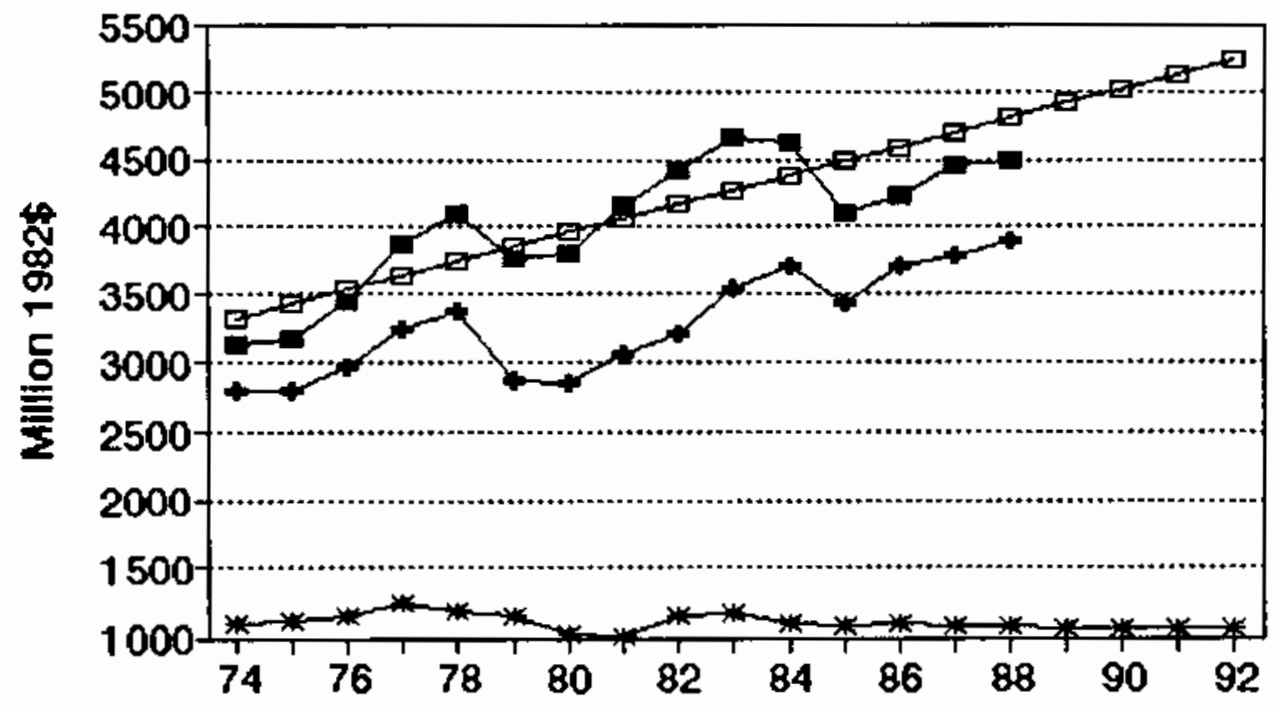

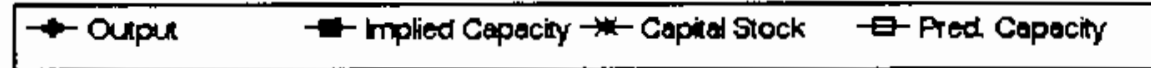




\section{No. 65 SIC 2269 Pintshing plants, nec}

MILLIONS OF 1982 DOLLARS (EXCL. CU RATES)

$\begin{array}{lccccccc} & \alpha 4 & \text { Ann. } & \text { Implied } & \text { Gross } & \text { Net } & \text { Pred. } & \ldots \ldots \ldots \\ \text { Gross } & \text { dU } & \text { CU } & \text { Prac. } & \text { In- } & \text { Cap. } & \text { Prac. } & \text { Emergency Capacity } \\ \text { Output } & \text { Rate } & \text { Rate } & \text { Capac. } & \text { vest. } & \text { Stk. } & \text { Capac. } & 3 \text { mon. } 6 \text { mon. }>6 \text { mon. }\end{array}$

\begin{tabular}{|c|c|c|c|c|c|c|c|c|c|c|}
\hline 1974 & 828 & 59 & 59.0 & 1,404 & $(13.5)$ & 365 & 1,842 & & & \\
\hline 1975 & 700 & 47 & 51.5 & 1,376 & 17.2 & 363 & 1,827 & & & \\
\hline 1976 & 887 & 45 & 45.8 & 1,938 & 1.3 & 344 & 1,707 & & & \\
\hline 1977 & 1,138 & 46 & 45.6 & 2,494 & 54.9 & 373 & 1,904 & & & \\
\hline 1978 & 1,157 & 60 & 54.8 & 2,114 & 6.5 & 358 & 1,792 & & & \\
\hline 1979 & 832 & 78 & 71.3 & 1,167 & $(21.0)$ & 315 & 1,520 & & & \\
\hline 1980 & 732 & 59 & 66.1 & 1,137 & $(13.0)$ & 282 & 1,307 & & & \\
\hline 1981 & 727 & 68 & 64.6 & 1,125 & 5.2 & 265 & 1,205 & & & \\
\hline 1982 & 1,064 & 74 & 71.8 & 1,483 & 101.6 & 332 & 1,632 & & & \\
\hline 1983 & 1,184 & 69 & 70.9 & 1,670 & 20.9 & 317 & 1,531 & & & \\
\hline 1984 & 1,063 & 69 & 69.0 & 1,540 & $(23.9)$ & 262 & 1,181 & & & \\
\hline 1985 & 944 & 69 & 69.0 & 1,368 & 13.5 & 245 & 1,391 & & & \\
\hline 1986 & 892 & 66 & 67.1 & 1,329 & 16.6 & 232 & 1,306 & & & \\
\hline-- & --- & - & -- & $\cdots$ & $\cdots$ & $\cdots$ & $-\cdots$ & -- & -- & $-\cdots$ \\
\hline 1987 & 897 & 57 & 60.4 & 1,488 & 12.5 & 215 & 1,199 & & & \\
\hline 1988 & 904 & 68 & 63.9 & 1,415 & 12.0 & 200 & 1,102 & 1,077 & 1,102 & 1,107 \\
\hline 1989 & & & & & 11.6 & 186 & 1,015 & 992 & 1,014 & 1,019 \\
\hline 1990 & & & & & 11.1 & 173 & 936 & 915 & 935 & 940 \\
\hline 1991 & & & & & 10.7 & 162 & 864 & 844 & 863 & 867 \\
\hline 1992 & & & & & 10.2 & 152 & 798 & 780 & 798 & 802 \\
\hline
\end{tabular}

Capecity-Capital Stock Regression: Code -1 RSO 0.390

Coefficients: Const -487.9 stock $6.4 \quad \mathrm{D}(85-86) \quad 317.8$

Ave. Capacity/Stock $(74-86): 5.0$ Ave. Weekly Hours (High) 153.0 Shift Factor 1.00 Ave Weekly Hours (Low) 151.6 Shift Factor 1.01 Note: Output, Investment, and Capital stock extrapolated for 1997 and 1988.

\section{Finishing plants, nec}

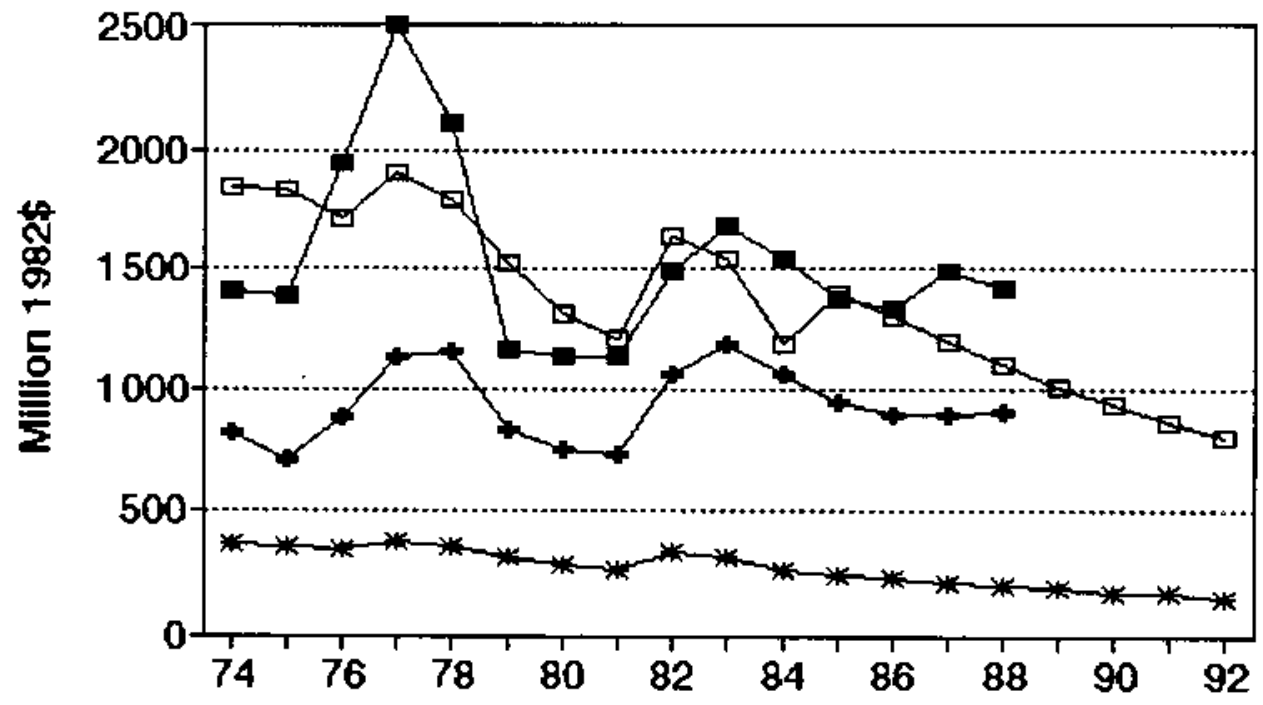

*- Ouput $\rightarrow$ - mplied Cepeciry $\rightarrow$ - Cepited Stock $\quad \square$ Pred Cepeciry 


\section{Ho. 66 BIC 2271 Woven carpet and rugs}

MILLIONS OF 1982 DOLLARS (EXCL. CU RATES)

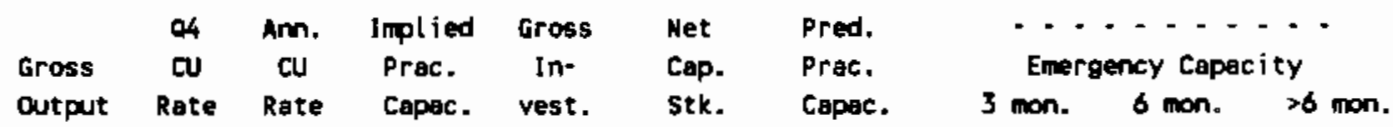

\begin{tabular}{|c|c|c|c|c|c|c|c|c|c|c|}
\hline 1974 & 302 & 58 & 69.7 & 434 & 16.1 & 133 & 446 & & & \\
\hline 1975 & 237 & 58 & 54.5 & 435 & 8.1 & 125 & 432 & & & \\
\hline 1976 & 284 & 78 & 70.8 & 401 & 3.7 & 113 & 415 & & & \\
\hline 1977 & 162 & 80 & 80.6 & 201 & $(49.1)$ & 57 & 326 & & & \\
\hline 1978 & 187 & 70 & 73.7 & 254 & 0.5 & 52 & 316 & & & \\
\hline 1979 & 262 & $67 *$ & 67.5 & 387 & 29.6 & 71 & 347 & & & \\
\hline 1980 & 224 & $58 \star$ & 61.1 & 366 & 6.5 & 67 & 341 & & & \\
\hline 1981 & 218 & $56 *$ & 59.6 & 366 & $(0.9)$ & 57 & 325 & & & \\
\hline 1982 & 215 & $54 *$ & 53.5 & 402 & 12.1 & 59 & 329 & & & \\
\hline 1983 & 199 & 71 & 65.6 & 303 & 2.9 & 53 & 318 & & & \\
\hline 1984 & 278 & 71 & 73.9 & 376 & 17.6 & 60 & 329 & & & \\
\hline 1985 & 367 & $\pi$ & 70.4 & 521 & 8.0 & 58 & 497 & & & \\
\hline 1986 & 349 & 77 & 74.4 & 469 & 6.6 & 55 & 493 & & & \\
\hline $\begin{array}{l}-- \\
1987\end{array}$ & $=-$ & $\begin{array}{l}-\cdots \\
81\end{array}$ & 79.9 & $\because 33$ & $\begin{array}{c}-\cdots \\
7.5\end{array}$ & $\because 5$ & $\begin{array}{l}--- \\
490\end{array}$ & $\cdots$ & - & $\cdots$ \\
\hline 1988 & 342 & 70 & 73.4 & 466 & 7.7 & 52 & 488 & 502 & 525 & 530 \\
\hline 1989 & & & & & 7.9 & 51 & 487 & 501 & 524 & 528 \\
\hline 1990 & & & & & 8.2 & 51 & 486 & 500 & 523 & 527 \\
\hline 1991 & & & & & 8.4 & 50 & 486 & 500 & 522 & 527 \\
\hline 1992 & & & & & 8.6 & 51 & 486 & 500 & 523 & 527 \\
\hline
\end{tabular}

Capacity-Capital Stock Regression: Code - 1 RSO 0.630

Coefficients: Const 234.1 stock 1.6 o(85-86) 171.3

Ave. Capacity/stock $(74-86): 5.1$ Ave. Weekly Hours (High) 140.4 Shift Factor 1.09 Ave Weekly Hours (Low) 138.0 Shift Factor 1.10 * Imputed Note: Output, Investment, and Capital Stock extrapolated for 1987 and 1988.

\section{Woven carpet and rugs}

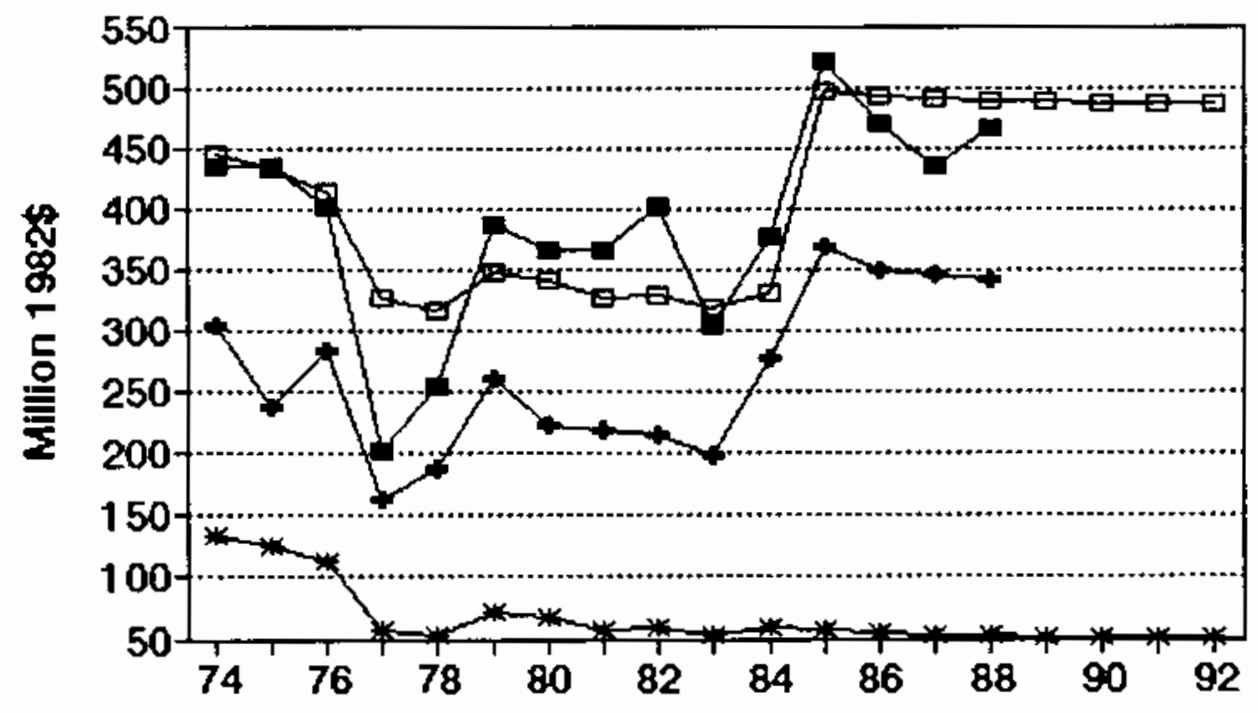


No. 67 SIC 2272 Tufted carpet and rugs

MILLIONS OF 1982 DOLLARS (EXCL, CU RATES)

$\begin{array}{lccccccc} & \text { a4 } & \text { Ann. Implied Gross } & \text { Net } & \text { Pred. } & \ldots \ldots \ldots \\ \text { Gross } & \text { CU } & \text { CU } & \text { Prac. } & \text { In. } & \text { Cap. } & \text { Prac. } & \text { Energency Capacity } \\ \text { Output } & \text { Rate } & \text { Rate } & \text { Capac. } & \text { vest. } & \text { Stk. } & \text { Capac. } & 3 \text { mon. } 6 \text { mon. }>6 \text { mon. }\end{array}$

\begin{tabular}{|c|c|c|c|c|c|c|c|c|c|c|}
\hline 1974 & 4,344 & 58 & 70.1 & 6,193 & 43.4 & 1,106 & 6,776 & & & \\
\hline 1975 & 4,080 & 58 & 56.6 & 7,507 & 106.7 & 1,144 & 7,116 & & & \\
\hline 1976 & 4,683 & 79 & 71.4 & 6,557 & 12.9 & 1,087 & 7,452 & & & \\
\hline 1977 & 6,020 & 80 & 81.1 & 7,427 & 258.1 & 1,257 & 7,790 & & & \\
\hline 1978 & 6,207 & 74 & 76.2 & 8,167 & 84.4 & 1,251 & 8,128 & & & \\
\hline 1979 & 6,894 & 70 & 70.9 & 9,728 & 47.1 & 1,207 & 8,467 & & & \\
\hline 1980 & 6,102 & 60 & 63.4 & 9,628 & 65.4 & 1,179 & 8,805 & & & \\
\hline 1981 & 5,685 & 58 & 61.9 & 9,191 & 57.6 & 1,142 & 9,143 & & & \\
\hline 1982 & 5,467 & 56 & 55.8 & 9,793 & $(24.8)$ & 1,029 & 9,481 & & & \\
\hline 1983 & 6,677 & 72 & 67.3 & 9,919 & 75.5 & 1,012 & 9,819 & & & \\
\hline 1984 & 7,262 & 70 & 73.7 & 9,854 & 138.7 & 1,054 & 10,157 & & & \\
\hline 1985 & 7,205 & 72 & 70.0 & 10,291 & 71.9 & 1,031 & 10,495 & & & \\
\hline 1986 & 7,610 & 77 & 76.6 & 10,225 & 85.4 & 1,022 & 10,833 & & & \\
\hline$\cdots$ & $\cdots \cdot$ & $\cdot$ & $\cdots$ & --- & -- & $\therefore-$ & --- & --- & --- & $-\cdot-$ \\
\hline 1987 & 7,758 & 82 & 80.6 & 9,631 & 96.7 & 1,025 & 11,171 & & & \\
\hline 1988 & 7.953 & 77 & 78.1 & 10,181 & 88.2 & 1,019 & 11,509 & 11,963 & 12,531 & 12,649 \\
\hline 1989 & & & & & 89.4 & 1,015 & 11,847 & 12,315 & 12,899 & 13,020 \\
\hline 1990 & & & & & 85.0 & 1,006 & 12,185 & 12,666 & 13,267 & 13,392 \\
\hline 1991 & & & & & 103.6 & 1,017 & 12,526 & 13,017 & 13,636 & 13,763 \\
\hline 1992 & & & & & 105.8 & 1,029 & 12,862 & 13,369 & 14,006 & 14,135 \\
\hline
\end{tabular}

Capecity-Capital Stock Regression: Cade -2 R5O 0.830 Coefficients: Const 6438.0 Time 338.1

Ave. Capacity/Stock (74-86): 7.9 Ave. Weekly Hours (High) 138.6 Shift Factor 1.10 Ave Weekly Hours (Low) 136.8 Shift Factor 1.11 Note: Output, Investment, and Capital Stock extrapolated for 1987 and 1988.

\section{Tufted carpet and rugs}

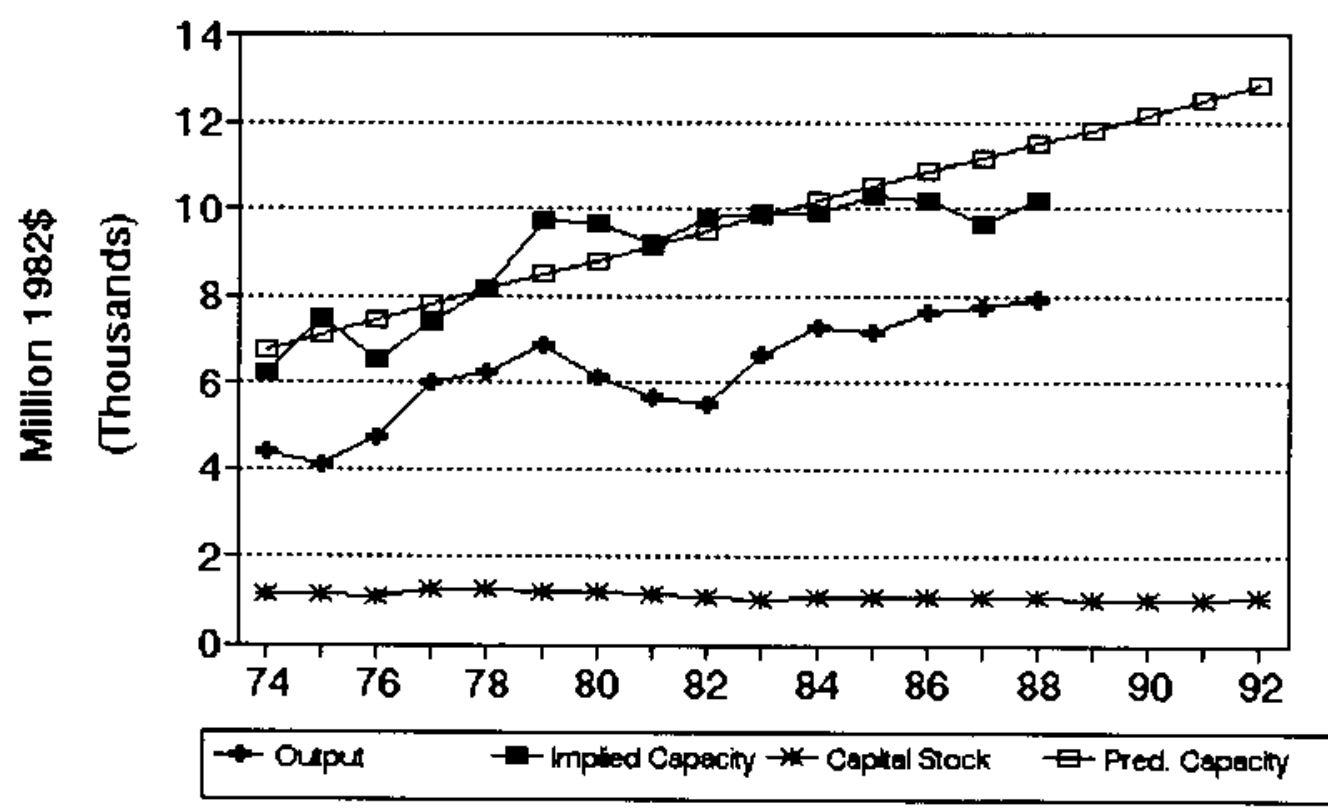


Ho. 68 sIC 2279 carpet and rugs, n.e.c.

MILLIONS OF 1982 DOLLARS (EXCL. CU RATES)

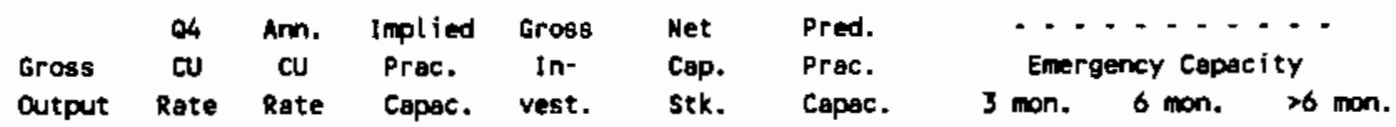

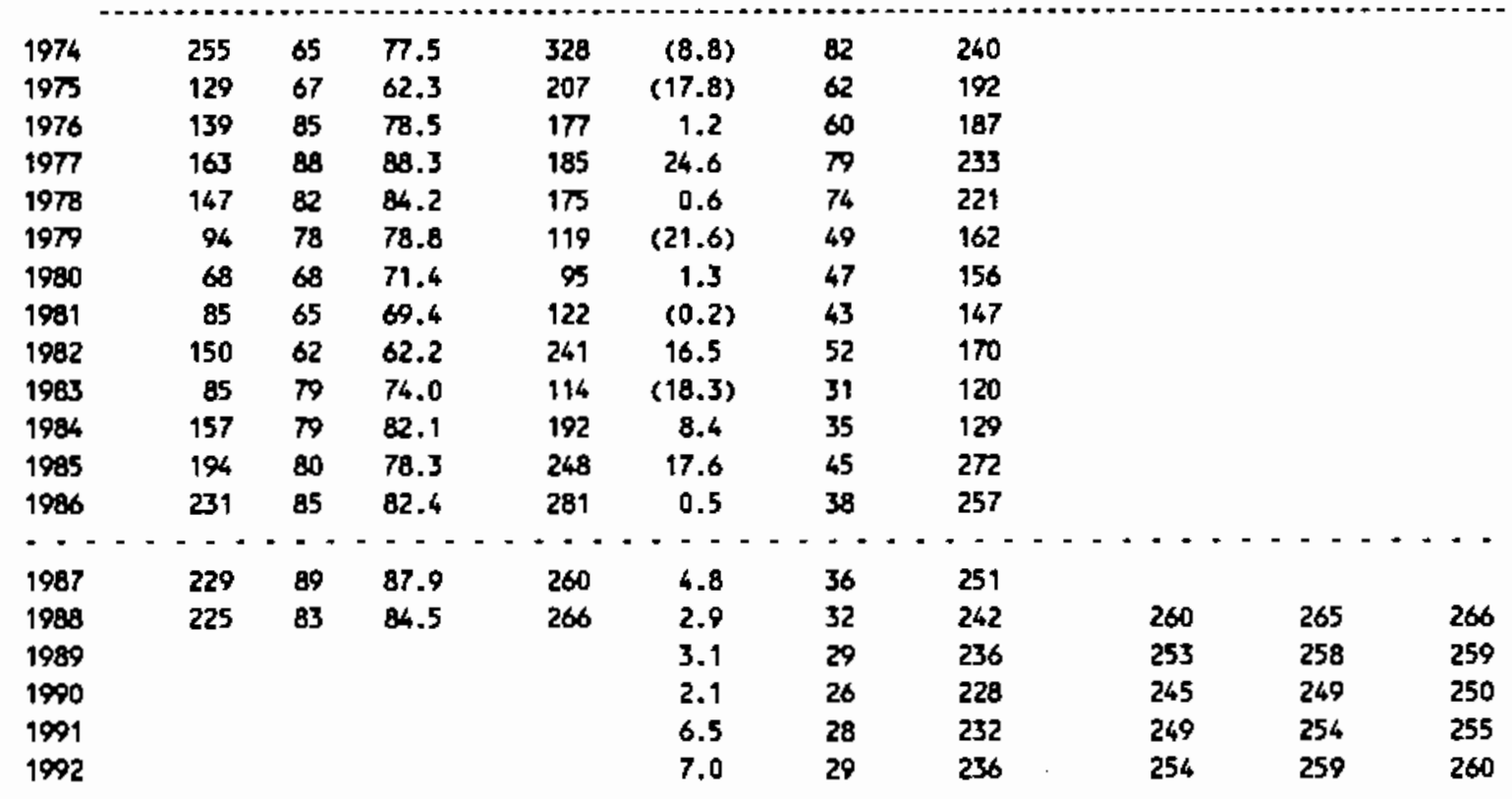

Capacity-Capital stock Regression: Code - 1 RSO 0.500

Coefficients: Const 45.1 stock 2.4 D(85-86) 121.1

Ave. Capacity/Stock $(74-86): 3.6$ Ave. Weekly Hours (High) 138.6 shift factor 1.10 Ave Heekly Hours (LOW) 136.8 Shift Factor 1.11 Mote: Output, Investment, and Capital Stock extrapolated for 1987 and 1983.

2279 Carpet and rugs, n.e.c.

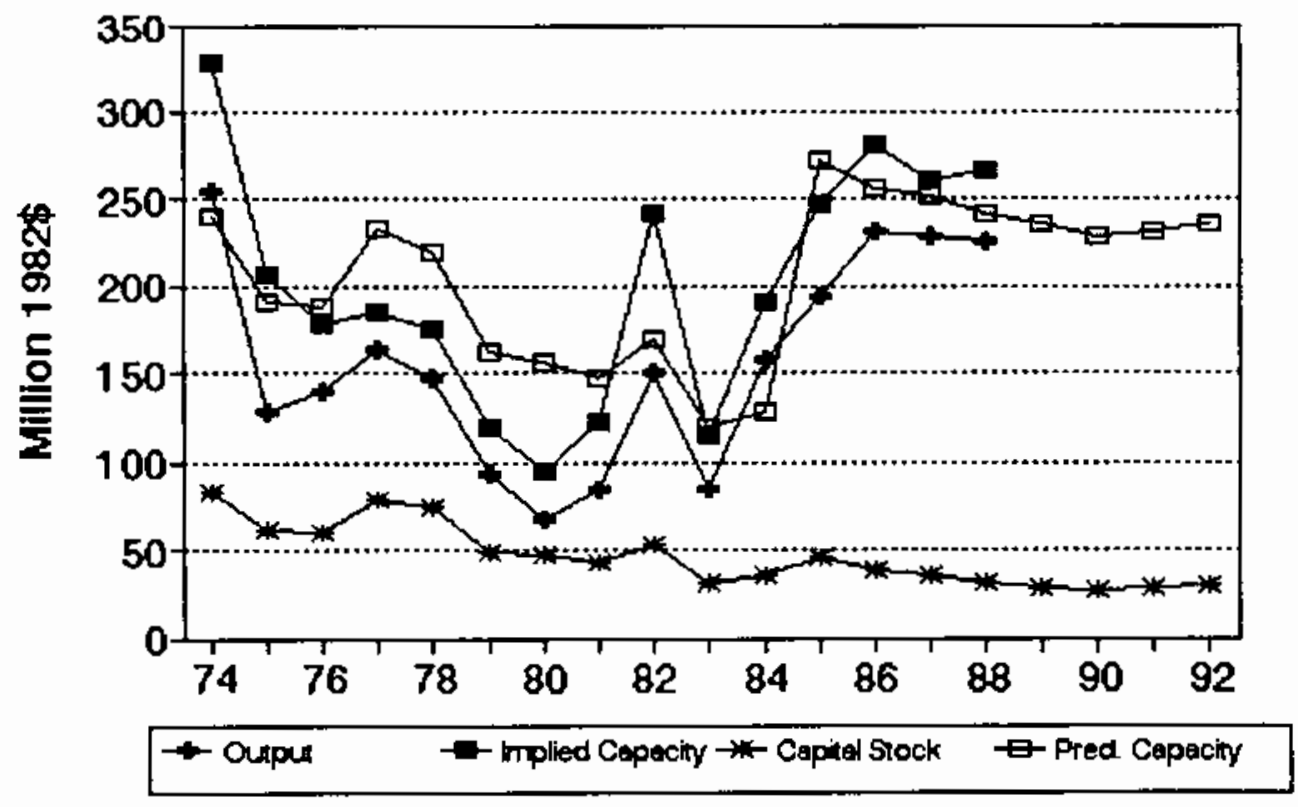




\section{No. 69 SIC 2281 Yarn mills, except wool}

MILLIONS OF 1982 DOLLARS (EXCL. CU RATES)

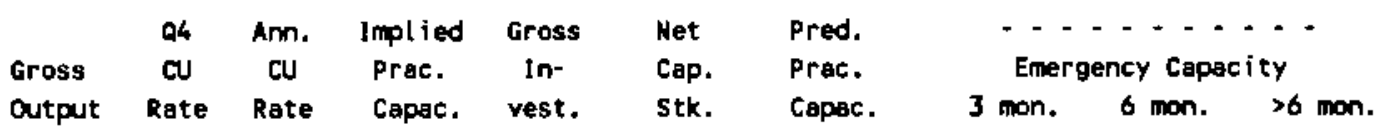

\begin{tabular}{|c|c|c|c|c|c|c|c|c|c|c|}
\hline 1974 & 4,286 & 68 & 82.0 & 5,224 & 304.0 & 2,590 & 5,414 & & & \\
\hline 1975 & 4,142 & 81 & 71.2 & 5,822 & 149.4 & 2,568 & 5,518 & & & \\
\hline 1976 & 4,482 & 84 & 82.9 & 5,404 & 98.3 & 2,489 & 5,622 & & & \\
\hline 197 & 5,004 & 87 & 87.4 & 5,728 & 177.5 & 2,481 & 5,726 & & & \\
\hline 1978 & 5,166 & 85 & 85.7 & 6,028 & 268.1 & 2,555 & 5,830 & & & \\
\hline 1979 & 4,917 & 87 & 85.5 & 5,751 & $(35.0)$ & 2,341 & 5,933 & & & \\
\hline 1980 & 4,605 & 82 & 83.5 & 5,516 & 230.3 & 2,378 & 6,037 & & & \\
\hline 1981 & 4,522 & 59 & 70.9 & 6,378 & 166.9 & 2,351 & 6,141 & & & \\
\hline 1982 & 4,335 & 67 & 63.1 & 6,876 & 103.9 & 2,263 & 6,245 & & & \\
\hline 1983 & 5,052 & 82 & 78.0 & 6,478 & 224.7 & 2,292 & 6,349 & & & \\
\hline 1984 & 5,157 & 73 & 79.5 & 6,491 & 203.1 & 2,298 & 6,452 & & & \\
\hline 1985 & 4,905 & 83 & 7.8 & 6,305 & 140.7 & 2,250 & 6,556 & & & \\
\hline 1986 & 5,573 & 89 & 86.0 & 6,484 & 172.9 & 2,233 & 6,660 & & & \\
\hline-- & ---- & - & -- & --- & -- & $\cdots$ & $\cdots$ & $\cdots$ & -- & -- \\
\hline 1987 & 5,560 & 84 & 86.4 & 6,437 & 196.2 & 2,241 & 6,764 & & & \\
\hline 1988 & 5,542 & 93 & 88.9 & 6,233 & 183.7 & 2,235 & 6,868 & 6,715 & 6,881 & 6,916 \\
\hline 1989 & & & & & 185.4 & 2,232 & 6,972 & 6,817 & 6,986 & 7,020 \\
\hline 1990 & & & & & 179.0 & 2,223 & 7,075 & 6,918 & 7,090 & 7,125 \\
\hline 1991 & & & & & 206.4 & 2,241 & 7,179 & 7,020 & 7,194 & 7,229 \\
\hline 1992 & & & & & 209.6 & 2,262 & 7,283 & 7,121 & 7,298 & 7,334 \\
\hline
\end{tabular}

Capecity-Capital Stock Regression: Code -2 RSO 0.640

Coefficients: Const 5310.4 time 103.8

Ave. Capscity/Stock $(74-86): 2.5$ Ave. Weekly Hours (High) 157.2 Shift Factor 1.01 Ave Heekly Hours (Low) 152.4 Shift Factor 1.04 Note: Output, Investment, and Capital Stock extrapolated for 1987 and 1988.

\section{Yarn mills, except wool}

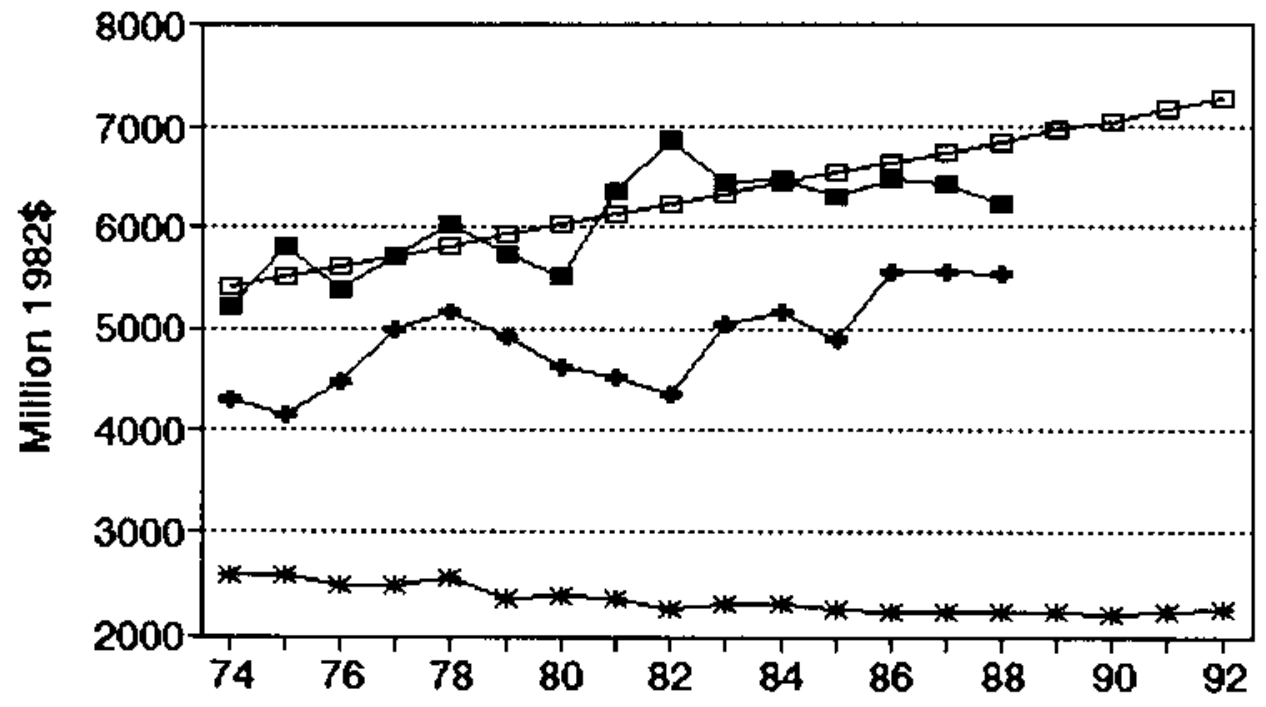

- Oxpur $\rightarrow-$ mplled Capeciny $\rightarrow$ Capial Stock $\square$ - Pred. Cepacity 


\section{No. 70 sIc 2282 Throwing and winding nills}

MILLIONS OF 1982 DOLLARS (EXCL. CU RATES)

$\begin{array}{lccccccc} & 04 & \text { Ann. Implied } & \text { Gross } & \text { Net } & \text { Pred. } & \ldots & \ldots . . \\ \text { Gross } & \text { CU } & \text { CU } & \text { Prac. } & \text { In- } & \text { Cap. } & \text { Prac. } & \text { Emergency Capacity } \\ \text { Output } & \text { Rate } & \text { Rate } & \text { Capac. } & \text { vest. } & \text { Stk. } & \text { Capac. } & 3 \text { mon. } 6 \text { mon. }\end{array}$

\begin{tabular}{|c|c|c|c|c|c|c|c|c|c|c|}
\hline 1974 & $1, \pi / 2$ & 54 & 71.5 & 2,481 & 85.7 & 1,037 & 3,391 & & & \\
\hline 1975 & 1,932 & 73 & 59.7 & 3,237 & 66.8 & 1,057 & 3,436 & & & \\
\hline 1976 & 2,520 & 60 & 64.7 & 3,894 & 104.6 & 1,108 & 3,553 & & & \\
\hline 1977 & 2,240 & 63 & 63.4 & 3,533 & 61.4 & 1,111 & 3,559 & & & \\
\hline 1978 & 2,273 & 72 & 68.6 & 3,312 & 7.9 & 1,059 & 3,439 & & & \\
\hline 1979 & 2,303 & 71 & 70.5 & 3,266 & $(47.0)$ & 955 & 3,204 & & & \\
\hline 1980 & 2,178 & 55 & 60.4 & 3,604 & 12.3 & 909 & 3,099 & & & \\
\hline 1981 & 2,006 & 53 & 57.9 & 3,468 & 7.5 & 920 & 3,125 & & & \\
\hline 1982 & 1.954 & 59 & 55.5 & 3,520 & 50.9 & 899 & 3,076 & & & \\
\hline 1993 & 2,321 & 74 & 70.6 & 3,288 & 124.1 & 937 & 3,163 & & & \\
\hline 1984 & 2,075 & 80 & 82.4 & 2,520 & (106.1) & 766 & 2,769 & & & \\
\hline 1985 & 2,208 & 92 & 85.3 & 2,588 & 42.1 & 737 & 2,708 & & & \\
\hline 1986 & 1,998 & 78 & 82.3 & 2,427 & 26.9 & 695 & 2,614 & & & \\
\hline 1987 & 1,989 & 92 & $\ddot{87.3}$ & $\cdots$ & - * & $\underset{703}{\cdots}$ & 2,631 & --- & -- & -1 \\
\hline 1988 & 1,976 & 77 & 81.5 & 2,426 & 61.7 & 697 & 2,618 & 2,587 & 2,673 & 2,691 \\
\hline 1989 & & & & & 63.5 & 694 & 2,610 & 2,579 & 2,665 & 2,683 \\
\hline 1990 & & & & & 56.9 & 684 & 2,588 & 2,557 & 2,643 & 2,661 \\
\hline 1991 & & & & & 84.8 & 703 & 2,632 & 2,600 & 2,687 & 2,705 \\
\hline 1992 & & & & & 88.1 & 724 & 2,678 & 2,646 & 2,735 & 2,753 \\
\hline
\end{tabular}

Capacity-Capital Stock Regression: Code 0 RSO 0.410

Coefficients: Const 1034.8 stock 2.3

Ave, Capaci ty/Stock $(74-86): 3.4$ Ave. Ueekly Hours (High) 163.2 Shift Factor 1.03 Ave Weekly Hours (Low) 157.2 Shift Factor 1.07 Note: Output, Imvestment, and Capital Stock extrapolated for 1987 and 1988.

\section{Throwing and winding mills}

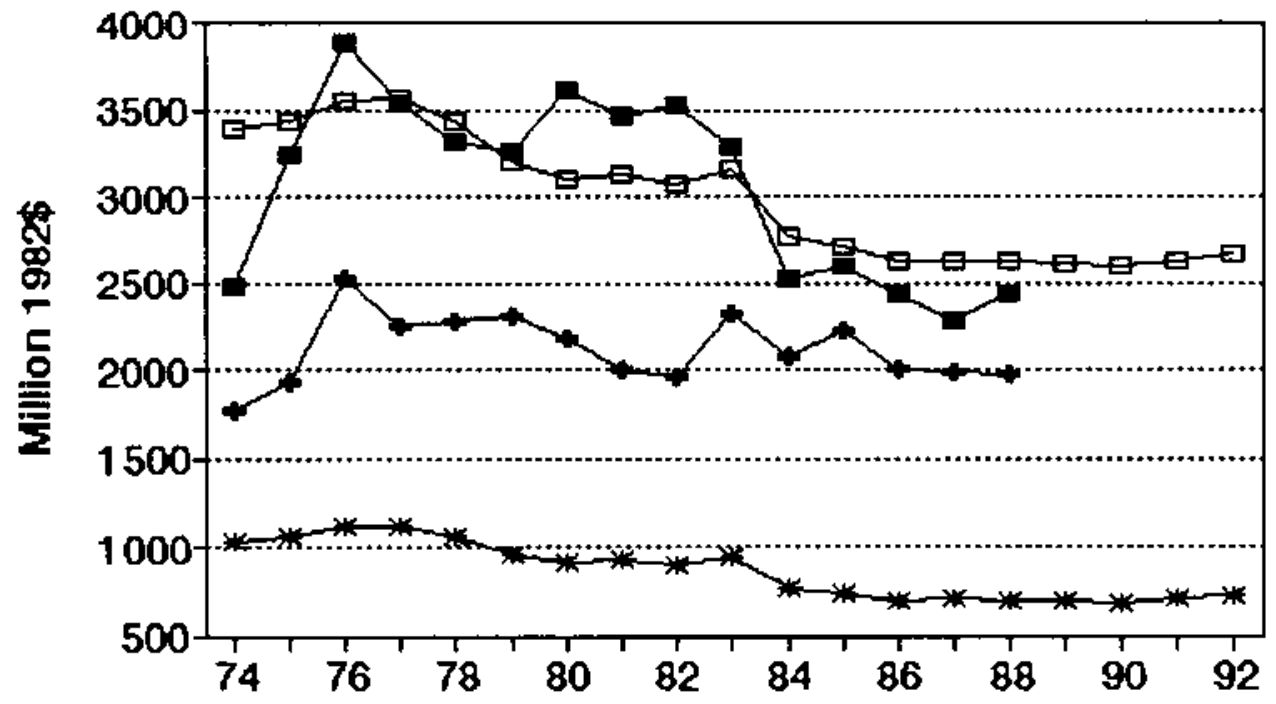

- Outpu t-Implied Cepecily $\rightarrow$ - Coptel Stock E-Pred. Cepeciny 


\section{พo. 71 sIC 2283 Wool yarp aills}

MILLIONS OF 1982 DOLLARS (EXCL. CU RATES)

$\begin{array}{lccccccc} & 04 & \text { Amn. } & \text { Implied } & \text { Gross } & \text { Het } & \text { Pred. } & \ldots \ldots . . . \\ \text { Gross } & \text { CU } & \text { CU } & \text { Prac. } & \text { In- } & \text { Cap. } & \text { Prac. } & \text { Emergency Capacity } \\ \text { Dutput } & \text { Rate } & \text { Rate } & \text { Capac. } & \text { vest. } & 5 t k . & \text { Capac. } & 3 \text { mon. } 6 \text { mon. }>6 \text { mon. }\end{array}$

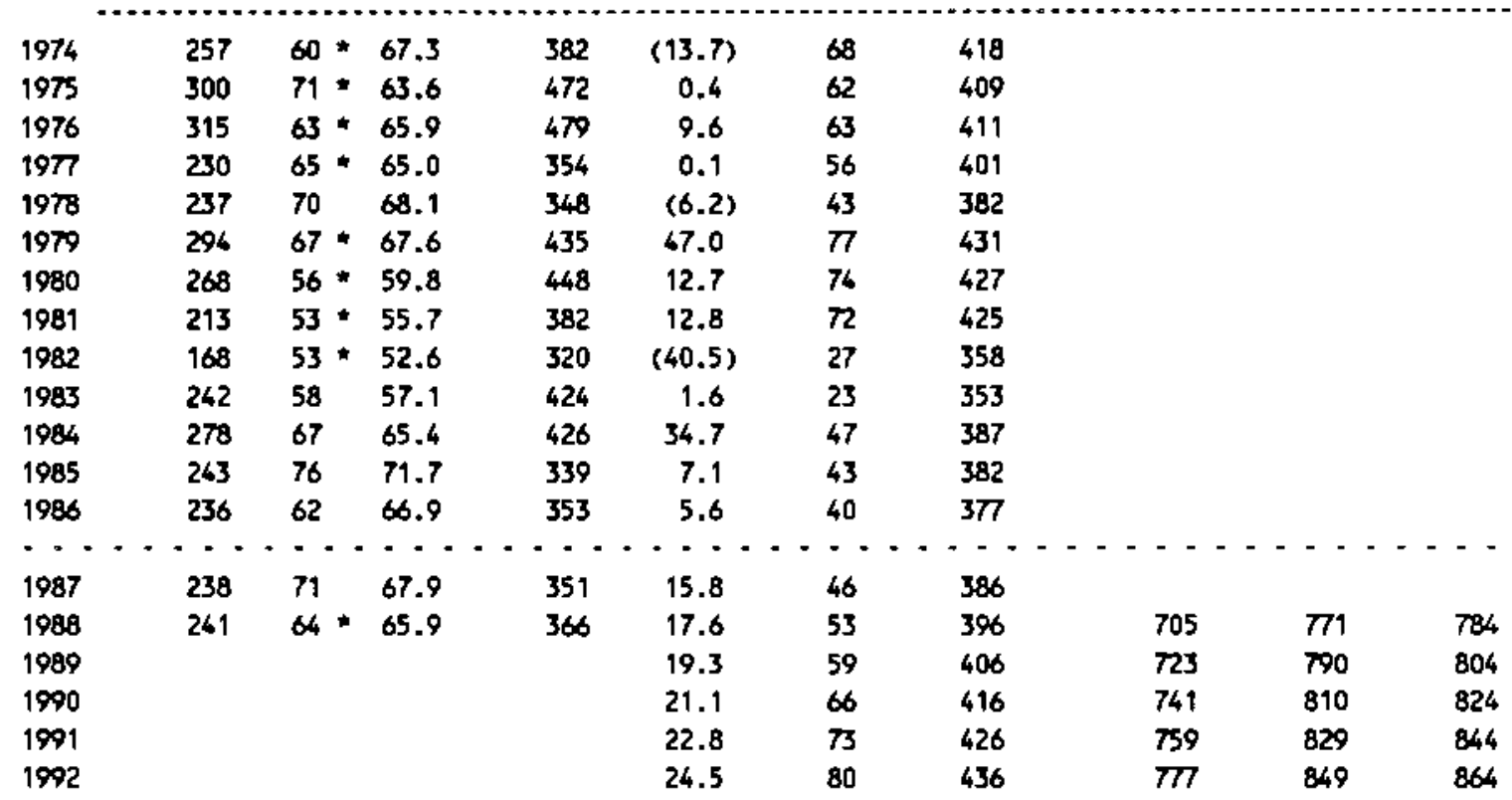

Capacity-Capital Stock Regression: Code 1 R5Q 0.240 Coefficients: Const 319.1 stock 1.5

Ave. Capacity/Stock (74-86): 7.4 Ave. Weekly Hours (High) 76.1 Shift Factor 1.98 Ave Yeekly Hours (LOW) 76.1 Shift Factor 1.98 * Imputed Note: Output, Investment, and Capital Stock extrapolated for 1987 and 1988.

\section{Wool yarn mills}

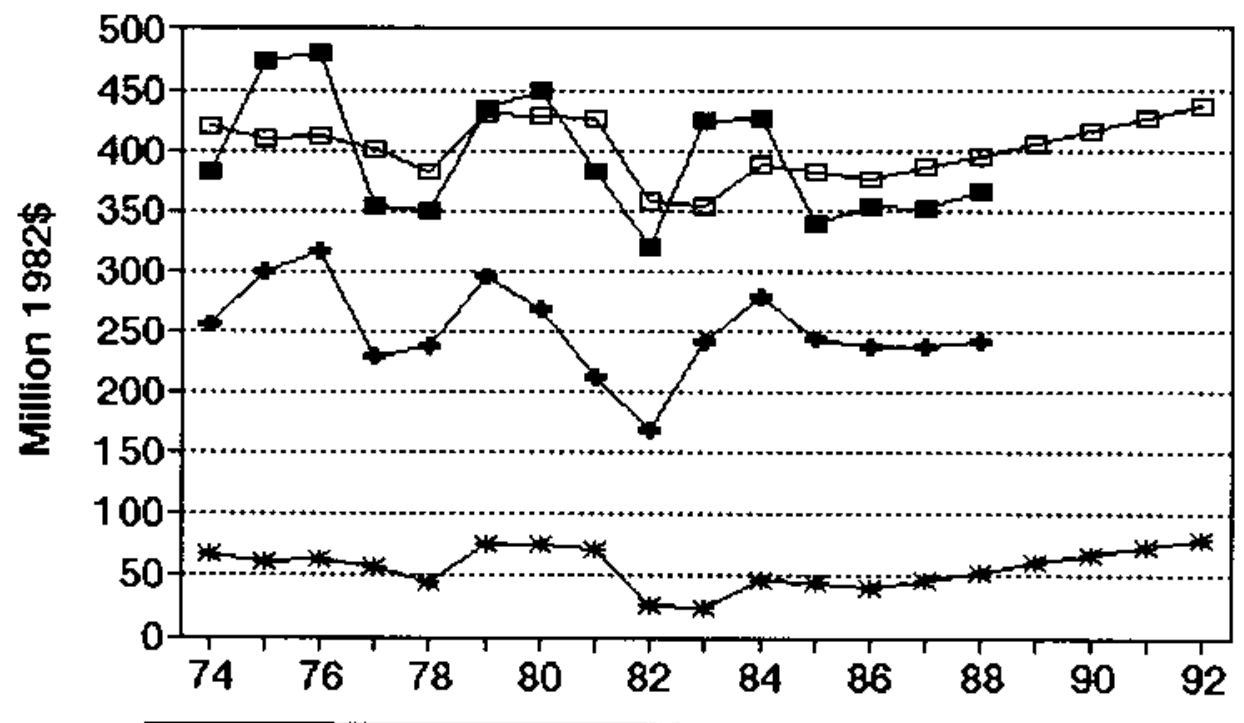

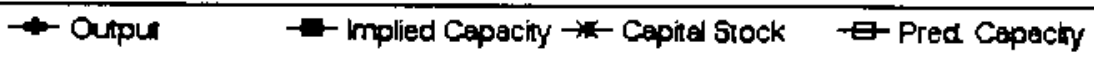


No. 72 SIC 2284 Thread wills

MILLIONS OF 1982 DOLLARS (EXCL. CU RATES)

$\begin{array}{lccccccc} & \text { Q4 } & \text { Am. } & \text { Implied } & \text { Gross } & \text { Net } & \text { Pred. } & \ldots . . . \\ \text { Gross } & \text { CU } & \text { CU } & \text { Prac. } & \text { In- } & \text { Cap. } & \text { Prac. } & \text { Emergency Capacity } \\ \text { Output } & \text { Rate } & \text { Rate } & \text { Capac. } & \text { vest. } & \text { Stk. } & \text { Capac. } & 3 \text { mon. } 6 \text { mon. }>6 \text { mon. }\end{array}$

\begin{tabular}{|c|c|c|c|c|c|c|c|c|c|c|}
\hline 1974 & 715 & 79 & 82.5 & 867 & 14.3 & 254 & 895 & & & \\
\hline 1975 & 637 & 92 & 85.7 & 743 & 8.1 & 244 & 866 & & & \\
\hline 1976 & 699 & 78 & as.2 & 840 & 8.9 & 233 & 836 & & & \\
\hline 1977 & $\pi 78$ & 81 & 80.2 & 971 & 60.2 & 270 & 942 & & & \\
\hline 1978 & 771 & 74 & 76.6 & 1,006 & 22.8 & 268 & 938 & & & \\
\hline 1979 & 780 & 82 & 78.8 & 990 & 29.2 & 272 & 948 & & & \\
\hline 1980 & 756 & 85 & 83.8 & 903 & 19.1 & 266 & 930 & & & \\
\hline 1981 & 758 & 86 & 86.7 & 874 & 3.5 & 246 & ars & & & \\
\hline 1982 & 588 & 63 & 71.3 & 825 & 4.8 & 229 & 824 & & & \\
\hline 1983 & 593 & 78 & 72.7 & 816 & 19.9 & 230 & 826 & & & \\
\hline 1984 & 495 & $\infty 6$ & 71.2 & 695 & $(30.4)$ & 184 & 693 & & & \\
\hline 1985 & 485 & 71 & 68.8 & 706 & 10.7 & 180 & 681 & & & \\
\hline 1986 & 493 & 73 & 72.1 & 684 & 9.6 & 173 & $\$ 67$ & & & \\
\hline$\cdots$ & -- & - & - - & $\cdots$ & -- & $\cdots$ & $-\cdots$ & $\cdots$ & -- & -- \\
\hline 1987 & 500 & 84 & 80.0 & 625 & 3.6 & 164 & 635 & & & \\
\hline 1988 & 509 & 88 & 86.3 & 590 & 2.3 & 153 & 602 & 647 & 666 & 671 \\
\hline 1989 & & & & & 1.0 & 141 & 569 & 611 & 629 & 633 \\
\hline 1990 & & & & & $(0,2)$ & 129 & 534 & 573 & 591 & 594 \\
\hline 1991 & & & & & $(1.5)$ & 117 & 498 & 535 & 551 & 555 \\
\hline 1992 & & & & & (2.8) & 105 & 462 & 496 & 511 & 514 \\
\hline
\end{tabular}

Capacity-Capital Stock Regression: Code 0 Rso 0.830

Coefficients: Const 158.6 stock 2.9

Ave. Capacity/Stock $(74-86): 3.6$ Ave. Weekly Hours (High) 136.8 Shift Factor 1.11 Ave Weekly Hours (Low) 133.2 Shift Factor 1.14 Mote: Output, Investment, and Capital Stock extrapolated for 1987 and 1988.

\section{Thread mills}

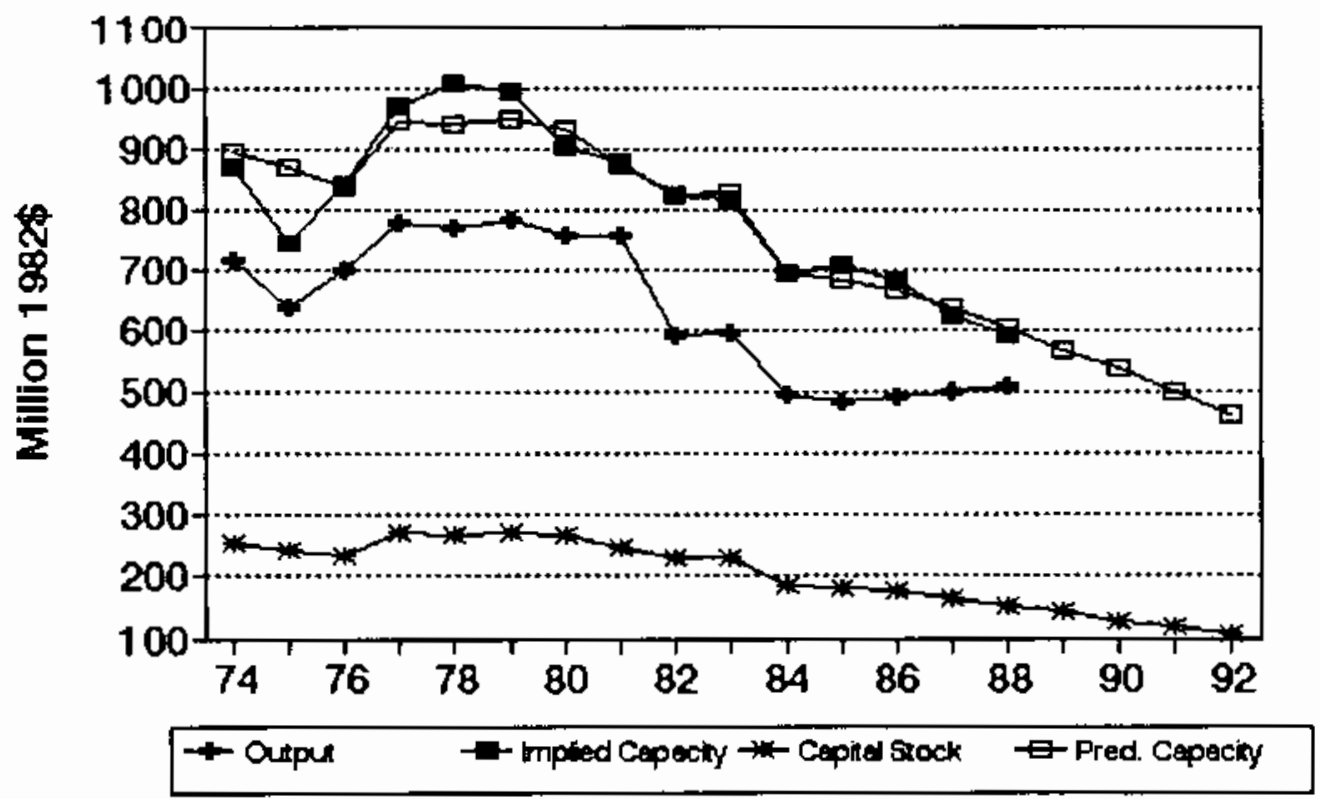


No. 73 SIC 2291 Pelt goods, except woven felts and hats MILLIONS OF 1982 DOLLARS (EXCL. CU RATES)

$\begin{array}{lccccccc} & \text { Q4 } & \text { Ann. } & \text { Implied Gross } & \text { Net } & \text { Pred. } & \ldots \ldots \ldots \\ \text { Gross } & \text { CU } & \text { CU } & \text { Prac. } & \text { In- } & \text { Cap. } & \text { Prac. } & \text { Emergency Capacity } \\ \text { Output } & \text { Rate } & \text { Rate } & \text { Capac. } & \text { vest. } & \text { Stk. } & \text { Capac. } & 3 \text { mon. } 6 \text { mon. }>6 \text { mon. }\end{array}$

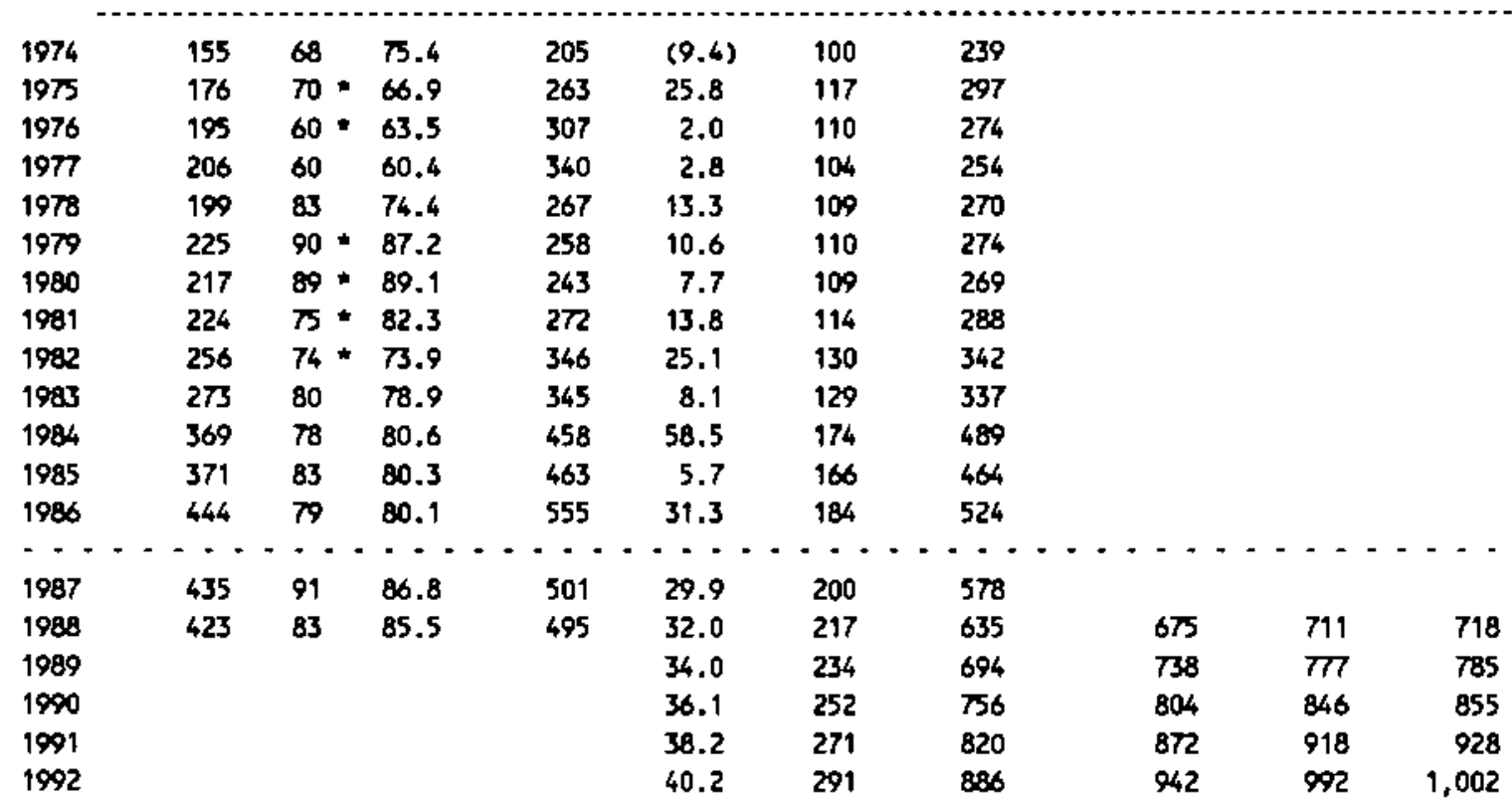

Capecity-Capital Stock Regression: Code 0 RSO 0.890

Coefficients: Const -100.0 stock 3.4

Ave. Capacity/Stock $(74-86): 2.6$ Ave. Weekly Hours (High) 134.5 Shift Factor 1.13 Ave Weekly Hours (Low) 122.3 Shift factor 1.24 - Inputed Note: Output, Investment, and Capital Stock extrapolated for 1987 and 1988.

\section{Felt goods, except woven felts and}

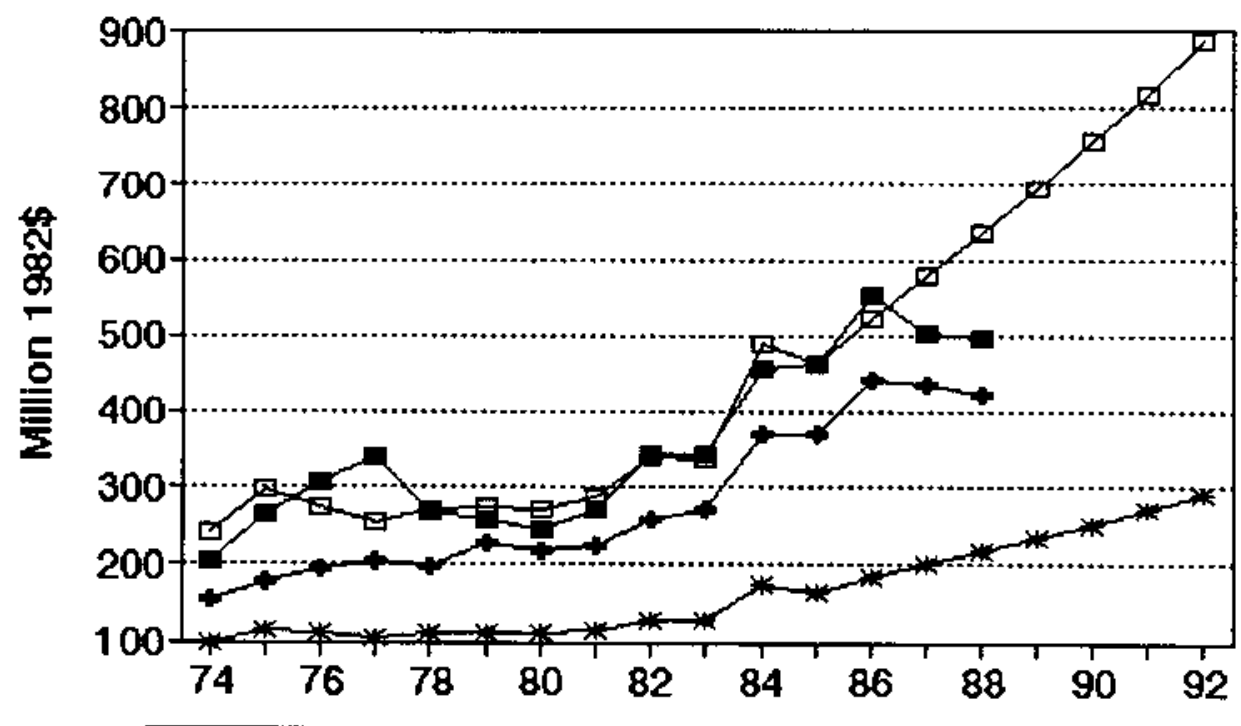

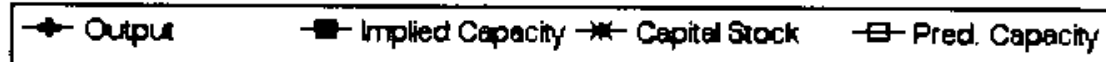




\section{No. 74 SIC 2292 Lace gooda}

MILLIONS OF 1982 DOLLARS (EXCL. CU RATES)

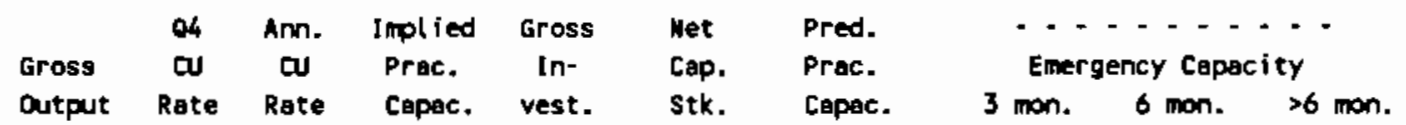

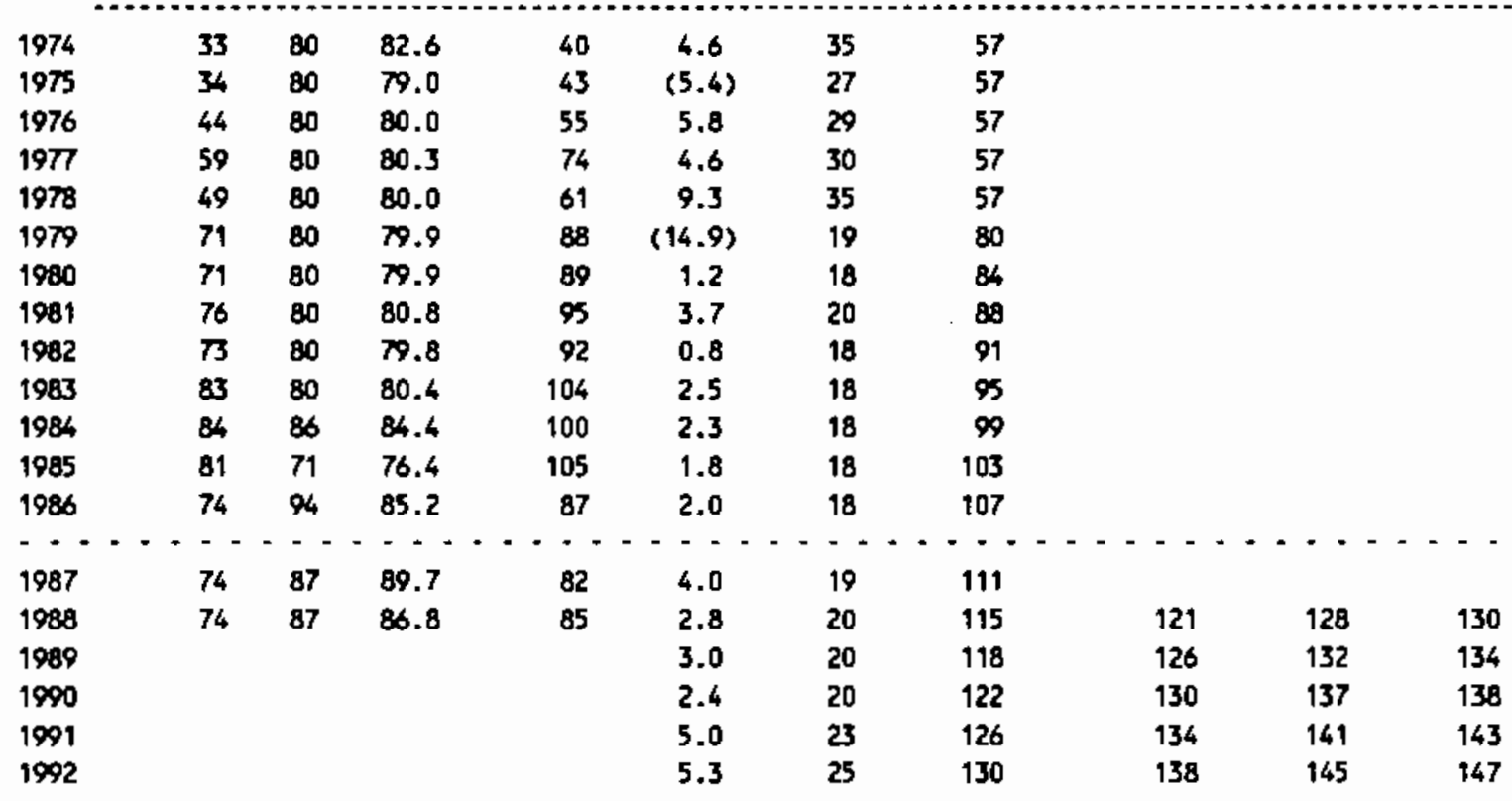

Capacity-Capital 5tock Regression: Code -2 RSO 0.770

Coefficients: Const 56.7 Time 3.9

Aye. Capacity/Stock $(74-86): 3.4$ Ave. Weekly Hours (High) 134.5 Shift Factor 1.13 Ave Weekly Hours (Low) 122.3 Shift Factor 1.24 Note: Output, Imvestment, and Capital stock extrapolated for 1987 and 1988.

\section{Lace goods}

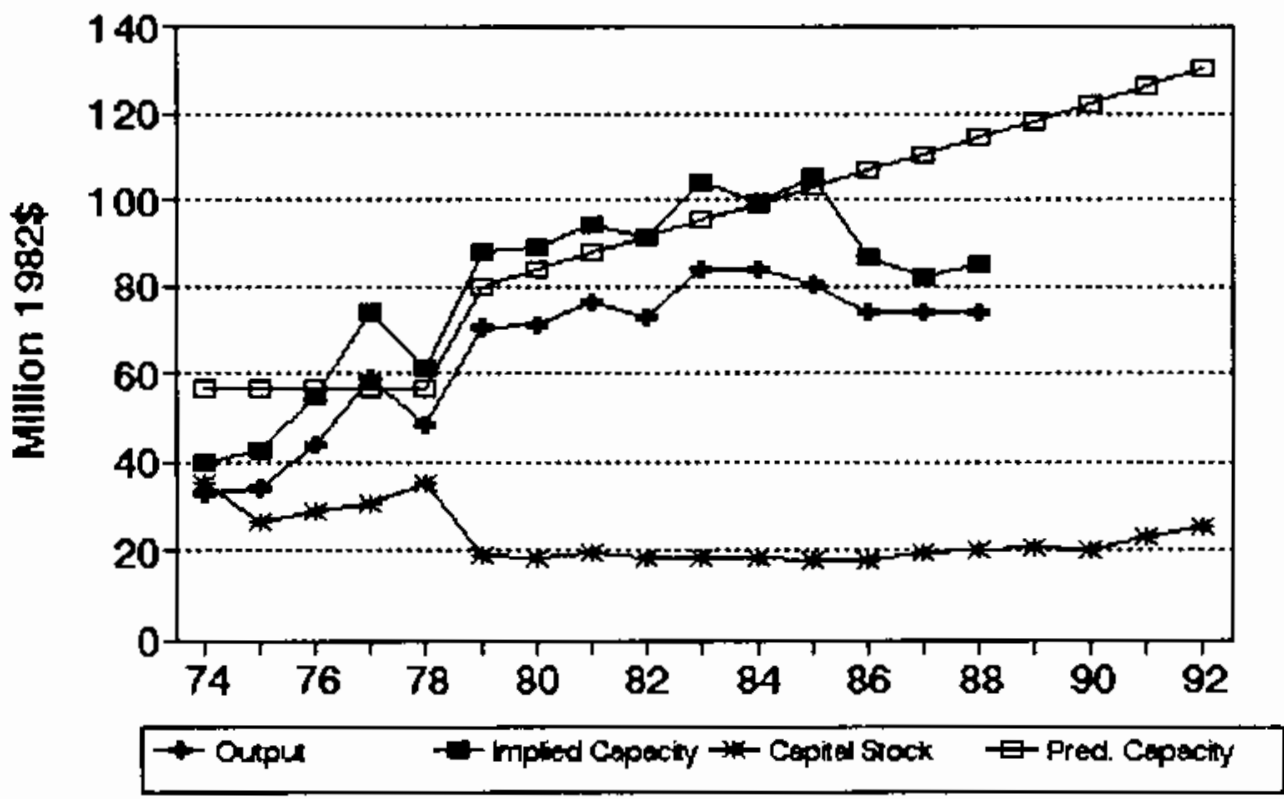




\section{No. 75 sIC 2293 padding and upholstery filling}

MILLIONS OF 1982 DOLLARS (EXCL. CU RATES)

$\begin{array}{lccccccc} & \text { Q4 } & \text { Amn. Implied Gross } & \text { Wet } & \text { Pred. } & -\ldots \ldots \ldots \\ \text { Gross } & \text { CU } & \text { CU } & \text { Prac. } & \text { In- } & \text { Cap. } & \text { Prac. } & \text { Emergency Capacity } \\ \text { Output Rate } & \text { Rate } & \text { Capac. } & \text { vest. } & \text { Stk. } & \text { Capac. } & 3 \text { mon. } 6 \text { mon. }>6 \text { mon. }\end{array}$

\begin{tabular}{|c|c|c|c|c|c|c|c|c|c|c|}
\hline 1974 & 234 & 52 & 73.5 & 318 & 1.7 & 82 & 480 & & & \\
\hline 1975 & 254 & 48 & 44.5 & 569 & 5.8 & 81 & 479 & & & \\
\hline 1976 & 256 & 70 & 62.3 & 412 & 12.5 & 86 & 484 & & & \\
\hline 1977 & 304 & $67 \star$ & 70.6 & 431 & 3.8 & 83 & 481 & & & \\
\hline 1978 & 341 & $64 \div$ & 65.4 & 521 & 16.1 & 91 & 488 & & & \\
\hline 1979 & 349 & 62 & 62.0 & 563 & 17.0 & 99 & 495 & & & \\
\hline 1980 & 266 & 63 & 62.3 & 427 & B.4 & 98 & 494 & & & \\
\hline 1981 & 285 & 50 & 59.7 & 477 & 3.2 & 92 & 489 & & & \\
\hline 1982 & 283 & 49 & 48.0 & 589 & 4.9 & 87 & 485 & & & \\
\hline 1983 & 296 & $57 *$ & 56.2 & 527 & 2.0 & 80 & 479 & & & \\
\hline 1984 & 346 & 64 & 65.6 & 527 & $(4.5)$ & 68 & 468 & & & \\
\hline 1985 & 365 & 90 & 77.7 & 470 & 11.1 & 71 & 470 & & & \\
\hline 1986 & 368 & 85 & 85.7 & 429 & 8.4 & 71 & 470 & & & \\
\hline - - - & $\cdots$ & $\because$ & $\because$ & $\because$ & $\because-$ & - & -7 & -- & $-\cdot$ & -- \\
\hline 1987 & 369 & 88 & 87.6 & 421 & 5.8 & 68 & 467 & & & $\operatorname{con}$ \\
\hline 1988 & 370 & 97 & 92.5 & 401 & 5.6 & 66 & 465 & 574 & 603 & 609 \\
\hline 1989 & & & & & 5.5 & 63 & 463 & 572 & 600 & 606 \\
\hline 1990 & & & & & 5.4 & 61 & 461 & 569 & 598 & 604 \\
\hline 1991 & & & & & 5.2 & 59 & 459 & 567 & 595 & 601 \\
\hline 1992 & & & & & 5.1 & 57 & 458 & 565 & 593 & 599 \\
\hline
\end{tabular}

Capacity-Capital Stock Regression: Code 0 RSQ 0.010 Coefficients: Const 406.8 stock 0.9

Ave. Capacity/stock (74-86): 5.7 Ave. Heekly Hours (High) 115.5 Shift Factor 1.31 Ave Heekly Hours (Low) 86.6 Shift Factor 1.74 * Imputed Note: Output, Investment, and Capital stock extrapolated for 1987 and 1988.

\section{Paddings and upholstery filling}

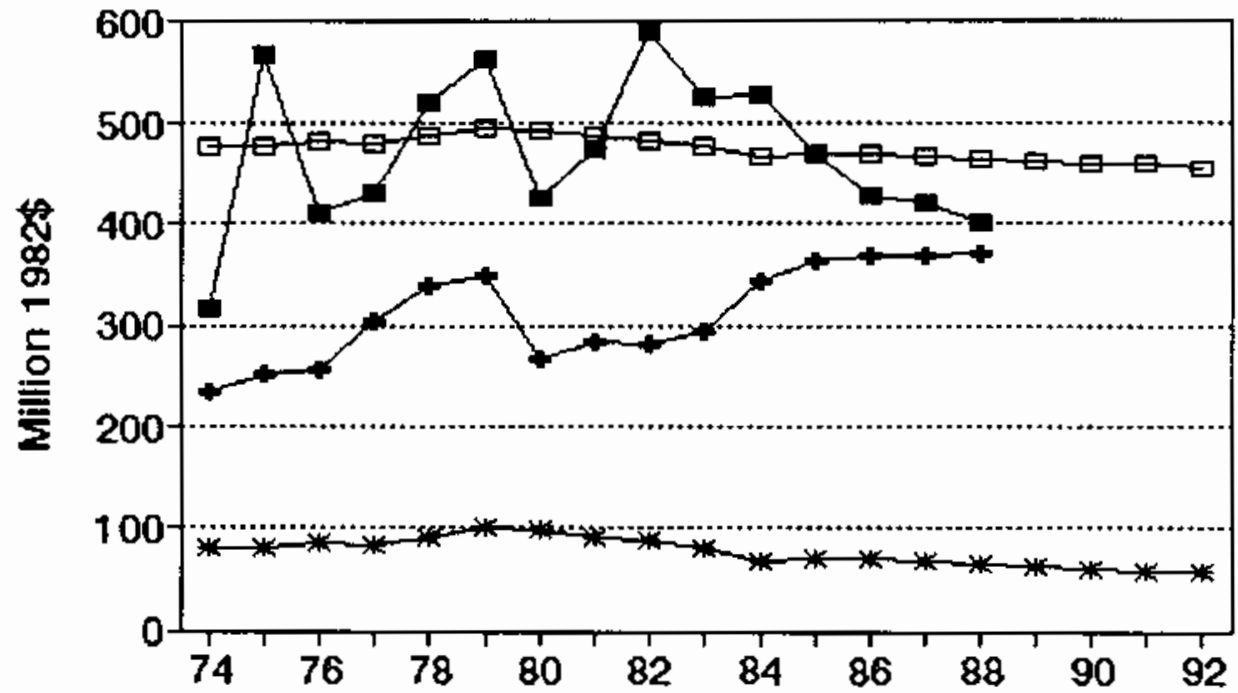

4- Oupu $\quad \rightarrow$ - Implied Capaciny $\rightarrow$ - Cepited Srock $\quad$-Pred. Cepecity




\section{No. 76 sIC 2294 processed textile waste}

MILLIOWS OF 1982 DOLLARS (EXCL. CU RATES)

$\begin{array}{lccccccc} & \text { Q4 } & \text { Ann. Implied Gross } & \text { Net } & \text { Pred. } & \ldots \\ \text { Gross } & \text { CU } & \text { CU } & \text { Prac. } & \text { In- } & \text { Cap. } & \text { Prac. } & \text { Emergency Capacity } \\ \text { Output } & \text { Rate } & \text { Rate } & \text { Capac. } & \text { vest. } & \text { Stk. } & \text { Capac. } & 3 \text { mon. } 6 \text { mon. }>6 \text { mon. }\end{array}$

\begin{tabular}{|c|c|c|c|c|c|c|c|c|c|c|}
\hline 1976 & 309 & 90 & 90.0 & 343 & 9.1 & 50 & 358 & & & \\
\hline 1975 & 180 & $88 *$ & 89.0 & 203 & 1.7 & 48 & 352 & & & \\
\hline 1976 & 235 & $87 *$ & 87.8 & 268 & 8.0 & 52 & 361 & & & \\
\hline 1977 & 242 & $86 *$ & 86.7 & 270 & 0.7 & 49 & 354 & & & \\
\hline 1978 & 288 & $85 *$ & 85.6 & 336 & 1.9 & 47 & 349 & & & \\
\hline 1979 & 318 & 84 & 84.5 & 376 & 11.5 & 54 & 365 & & & \\
\hline 1980 & 295 & 79 & 80.9 & 365 & 6.5 & 55 & 368 & & & \\
\hline 1981 & 293 & 78 & 78.4 & 374 & 5.3 & 55 & 367 & & & \\
\hline 1992 & 302 & 57 & 64.9 & 465 & 2.5 & 51 & 360 & & & \\
\hline 1983 & 344 & 70 & 65.1 & 528 & $(0.2)$ & 46 & 347 & & & \\
\hline 1984 & 359 & 91 & 83.1 & 432 & 17.0 & 56 & 372 & & & \\
\hline 1985 & 291 & $\pi$ & 81.0 & 359 & 12.2 & 62 & 384 & & & \\
\hline 1986 & 309 & $\pi$ & 76.3 & 405 & 10.6 & 65 & 393 & & & \\
\hline$\because \dot{ }$ & 309 & $\begin{array}{l}-\cdot \\
60\end{array}$ & $\begin{array}{l}-- \\
66.4\end{array}$ & 466 & $\begin{array}{l}--- \\
11.7\end{array}$ & $\begin{array}{l}- \\
70\end{array}$ & 404 & - & -- & -- \\
\hline 1988 & 310 & 82 & 73.8 & 420 & 12.5 & $\pi$ & 416 & 520 & 550 & 556 \\
\hline 1989 & & & & & 13.3 & 80 & 428 & 535 & 566 & 572 \\
\hline 1990 & & & & & 14.2 & 86 & 441 & 551 & 583 & 589 \\
\hline 1991 & & & & & 15.0 & 91 & 454 & 568 & 601 & 607 \\
\hline 1992 & & & & & 15.8 & 97 & 468 & 585 & 619 & 626 \\
\hline
\end{tabular}

Capacity-Capital Stock Regression: Code 0 RSO 0.030

Coefficients: Const 239.3 stock 2.4

Ave. Cepacity/Stock $(74-86): 6.9$ Ave. Weekly Hours (High) 112.8 Shift Factor 1.34 Ave Weekly Hours (Low) 111.7 Shift Factor 1.35 * Imputed Note: Output, Investment, and Capital Stock extrapolated for 1987 and 1928.

2294 processed textile waste

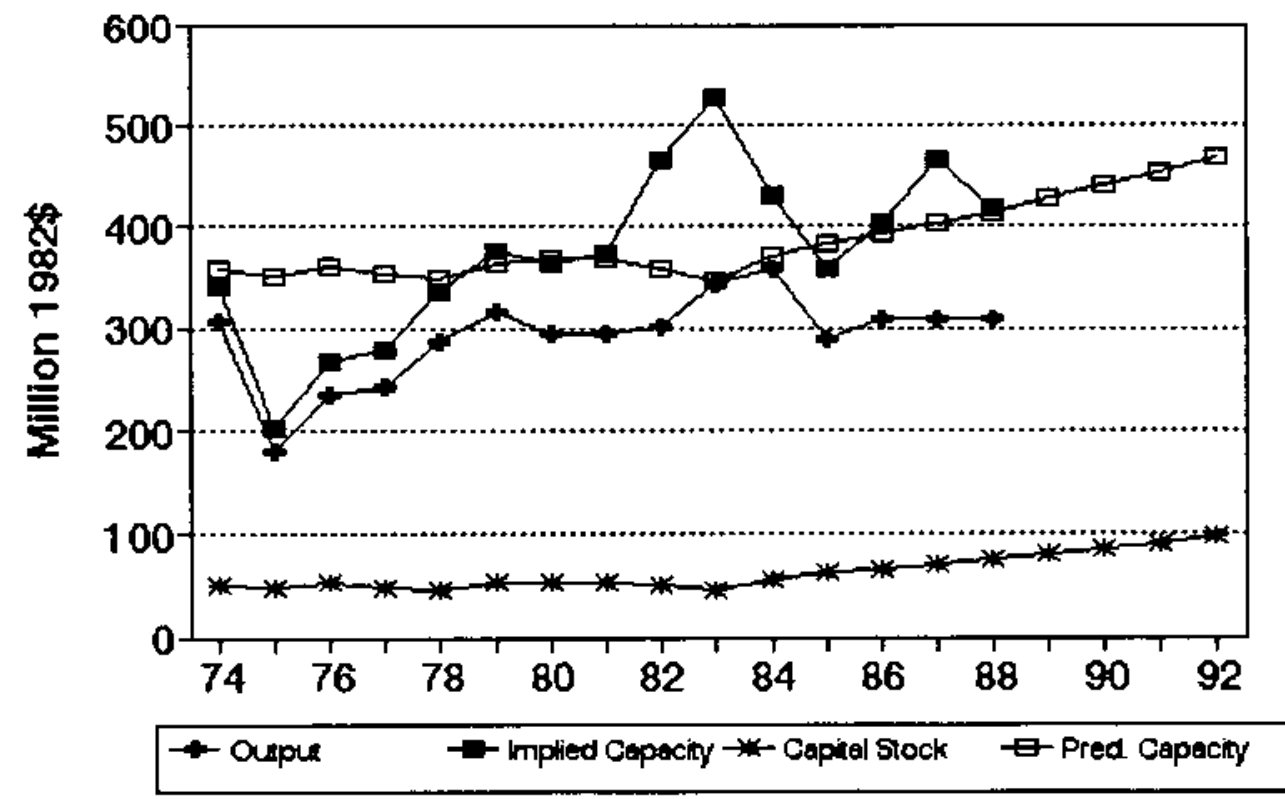


No. 77 sIc 2295 coated fabrics, not rubberized

MILLIONS OF 1982 DOLLARS (EXCL. CU RATES)

\begin{tabular}{|c|c|c|c|c|c|c|c|}
\hline & $\begin{array}{l}04 \\
\text { cu }\end{array}$ & $\begin{array}{l}\text { Ann. } \\
\text { Cu }\end{array}$ & $\begin{array}{l}\text { Inplied } \\
\text { Prac. }\end{array}$ & $\begin{array}{l}\text { Gross } \\
\text { In- }\end{array}$ & $\begin{array}{l}\text { Net } \\
\text { Cap. }\end{array}$ & $\begin{array}{l}\text { Pred. } \\
\text { Prac. }\end{array}$ & Emergency Cepacity \\
\hline & Rate & Rate & Canac & vest & stk & Capar. & \\
\hline
\end{tabular}

\begin{tabular}{|c|c|c|c|c|c|c|c|c|c|c|}
\hline 1974 & 1,309 & 48 & 50.1 & 2,611 & 96.0 & 569 & 2,475 & & & \\
\hline 1975 & 1,227 & 59 & 53.9 & 2,275 & 63.1 & 590 & 2,533 & & & \\
\hline 1976 & 1,456 & 47 & 51.5 & 2,829 & 50.9 & 596 & 2,548 & & & \\
\hline 1977 & 1,258 & 53 & 50.9 & 2,469 & $(64.6)$ & 493 & 2,279 & & & \\
\hline 1978 & 1,101 & 68 & 62.4 & 1,766 & 8.6 & 467 & 2,201 & & & \\
\hline 1979 & 1.165 & 61 & 63.5 & 1,836 & (11.1) & 423 & 2,083 & & & \\
\hline 1980 & 1,015 & 56 & 57.8 & 1,757 & $(31.9)$ & 363 & 1,921 & & & \\
\hline 1981 & 1,014 & 54 & 55.4 & 1,830 & 13.8 & 347 & 1,879 & & & \\
\hline 1982 & 1,137 & 60 & 57.6 & 1,976 & 61.0 & 373 & 1,947 & & & \\
\hline 1983 & 1,141 & 62 & 61.6 & 1,853 & 37.6 & 372 & 1,945 & & & \\
\hline 1984 & 1,326 & 62 & 62.7 & 2,116 & 55.9 & 384 & 1,978 & & & \\
\hline 1985 & 1,201 & 59 & 59.9 & 2,007 & 27.7 & 369 & 1,938 & & & \\
\hline 1986 & 1,162 & 46 & 50.8 & 2,288 & 24.9 & 353 & 1,893 & & & \\
\hline--- & $-\cdot \cdot$ & - & $-\cdot$ & $\cdots \cdot$ & --- & $\cdots$ & $\cdots$ & -- & $-\cdots$ & $\cdots$ \\
\hline 1987 & 1,161 & 56 & 52.3 & 2,218 & 37.2 & 349 & 1,893 & & & \\
\hline 1988 & 1,159 & 57 & 56.5 & 2,052 & 32.0 & 340 & 1,859 & 1,949 & 2,061 & 2,084 \\
\hline 1989 & & & & & 32.7 & 333 & 1,840 & 1,929 & 2,040 & 2,063 \\
\hline 1990 & & & & & 30.0 & 324 & 1,817 & 1,904 & 2,014 & 2,036 \\
\hline 1991 & & & & & 41.5 & 328 & 1,827 & 1,915 & 2,025 & 2,048 \\
\hline 1992 & & & & & 42.9 & 332 & 1,839 & 1,928 & 2,039 & 2,062 \\
\hline
\end{tabular}

Capacity-Capital Stock Regression: Code 0 RSQ 0.540

Coefficients: Const 943.5 stock $2.7 \quad$ Ave. Capacity/Stock (74-86): 4.8 Ave. Yeekly Hours (High) 135.7 Shift Factor 1.12 Ave Heekly Hours (Low) 124.9 Shift Factor 1.21 Note: Output, Imvestment, and Capital stock extrapolated for 1987 and 1988.

\section{Coated fabrics, not rubberized}

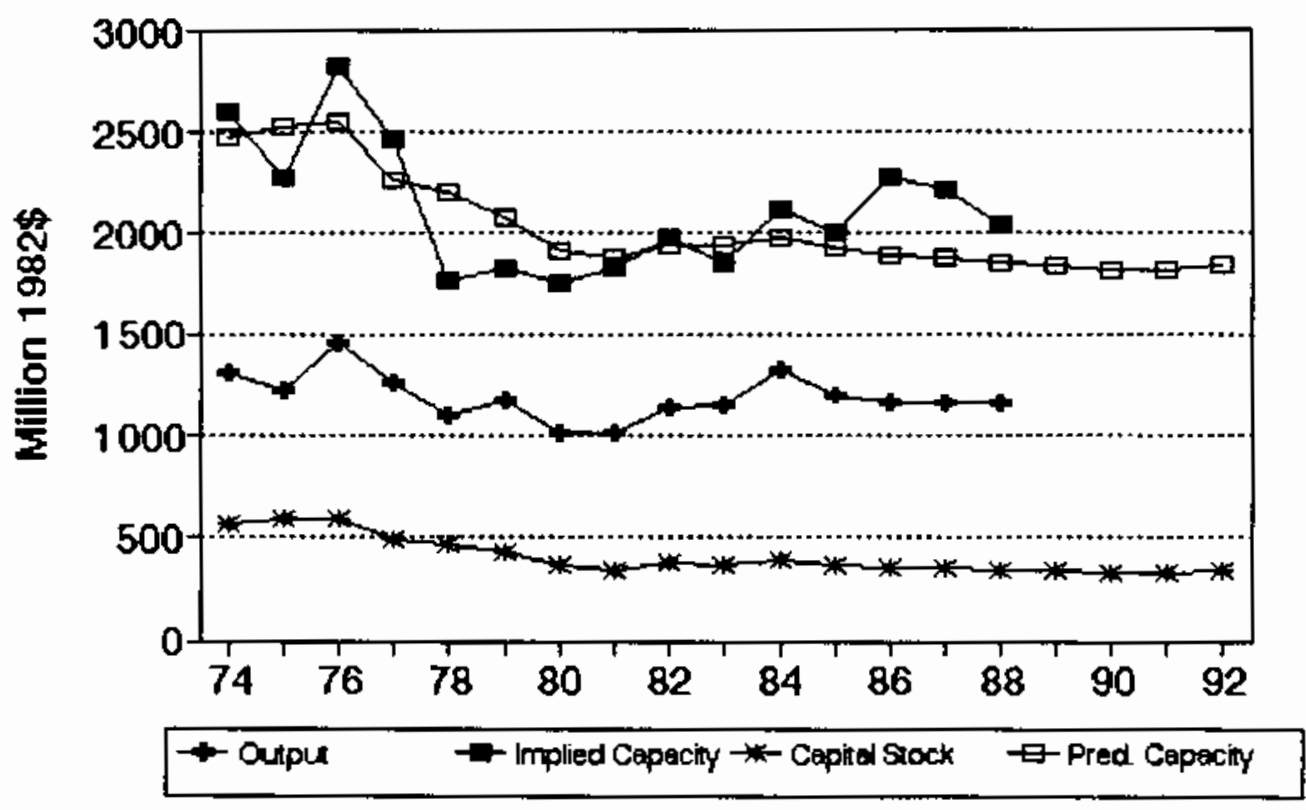


No. 78 SIC 2296 Tire cord and fabric

\section{MILLIONS OF 1982 DOLLARS (EXCL. CU RATES)}

$\begin{array}{lccccccc} & \text { Q4 } & \text { Ann. Implied Gross } & \text { Net } & \text { Pred. } & -\ldots & \ldots \\ \text { Gross } & \text { CU } & \text { CU } & \text { Prac. } & \text { In- } & \text { Cap. } & \text { Prac. } & \text { Emergency Capacity } \\ \text { Output } & \text { Rate } & \text { Rate } & \text { Capac. } & \text { vest. } & \text { Stk. } & \text { Capec. } & 3 \text { mon. } 6 \text { mon. }>6 \text { mon. }\end{array}$

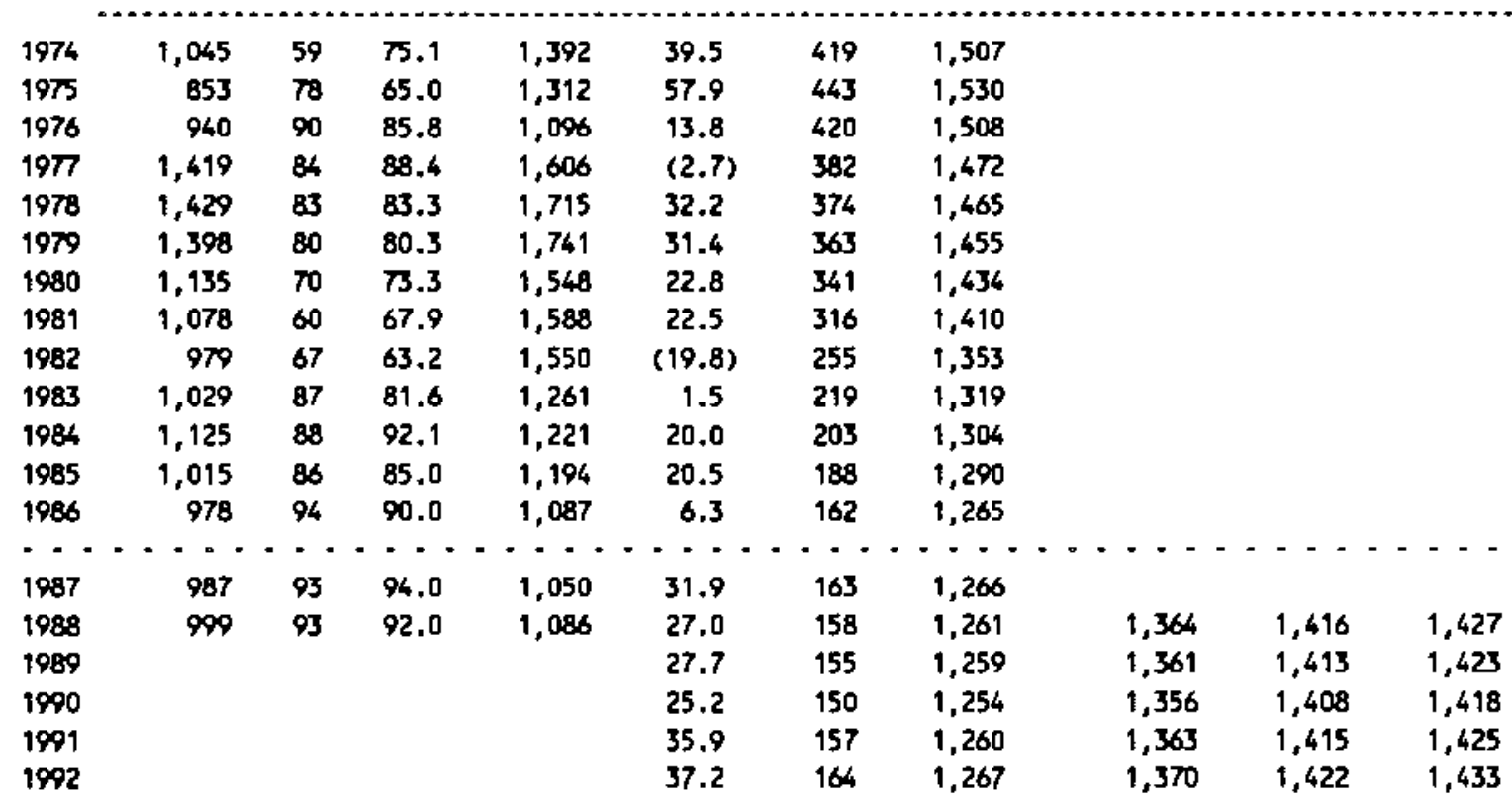

Capecity-Capital Stock Regression: Code i RSO 0.160

Coefficients: Const 1112.6 stock 0.9

Ave. Capacity/5tock $(74-86): 4.5$ Ave. Yeekly Hours (High) 134.4 Shift Factor 1.13 Ave Heekly Hours (LOW) 133.2 Shift Factor 1.14 Wote: Output, Investment, and Capital Stock extrapolated for 1987 and 1988.

2296 Tire cord and fabric

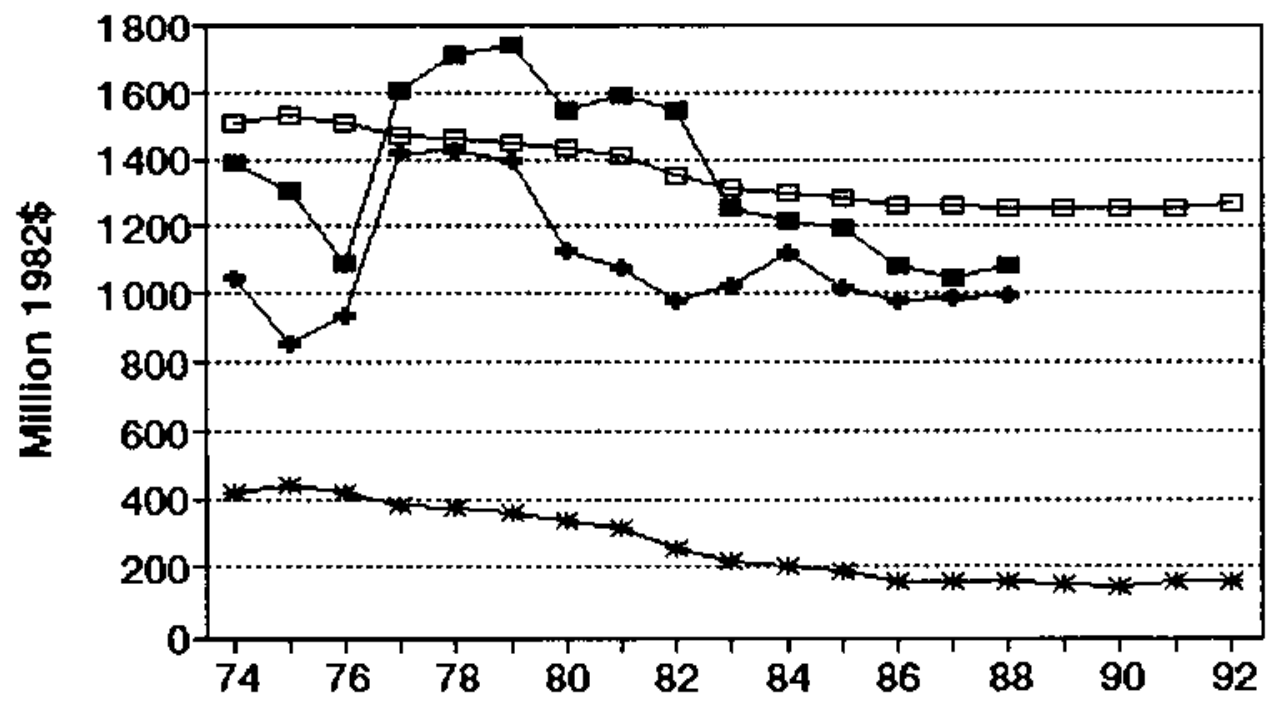

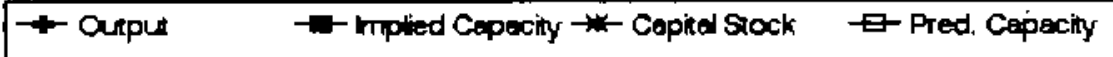




\section{No. 79 sIc 2297 Non woven fabrics}

MILLIONS OF 1982 DOLLARS (EXCl. CU RATES)

$\begin{array}{lccccccc} & \text { Q4 } & \text { Am. Implied Gross } & \text { Net } & \text { Pred. } & \ldots \ldots . . . \\ \text { Gross } & \text { CU } & \text { CU } & \text { Prac. } & \text { In- } & \text { Cap. } & \text { Prac. } & \text { Emergency Capacity } \\ \text { Output } & \text { Rate } & \text { Rate } & \text { Capac. } & \text { vest. } & \text { Stk. } & \text { Capac. } & 3 \text { mon. } 6 \text { mon. }>6 \text { mon. }\end{array}$

\begin{tabular}{|c|c|c|c|c|c|c|c|c|c|c|}
\hline 1974 & 639 & 68 & 69.9 & 914 & 33.2 & 371 & 1,020 & & & \\
\hline 1975 & 693 & 83 & 76.5 & 906 & 59.3 & 409 & 1,120 & & & \\
\hline 1976 & 793 & $T 2$ & 76.1 & 1,042 & 53.7 & 438 & 1,201 & & & \\
\hline 1977 & 1,021 & 64 & 67.2 & 1,520 & 55.4 & 467 & 1,277 & & & \\
\hline 1978 & 1,322 & 83 & 75.9 & 1,743 & 123.5 & 557 & 1,521 & & & \\
\hline 1979 & 1,336 & 88 & 86.0 & 1,554 & 11.1 & 537 & 1,466 & & & \\
\hline 1980 & 1,390 & 87 & 87.3 & 1,592 & 53.7 & 555 & 1,516 & & & \\
\hline 1981 & 1,446 & 81 & 83.9 & 1,723 & 99.4 & 615 & 1,677 & & & \\
\hline 1982 & 1,273 & 67 & 72.1 & 1,767 & 76.4 & 647 & 1,763 & & & \\
\hline 1983 & 1,338 & 75 & 72.2 & 1,853 & 85.0 & 686 & 1,870 & & & \\
\hline 1984 & 1,406 & 59 & 65.4 & 2,149 & 160.1 & 795 & 2,163 & & & \\
\hline 1985 & 1,327 & 62 & 60.7 & 2,187 & 69.9 & 811 & 2,207 & & & \\
\hline 1986 & 1,383 & 69 & 66.3 & 2,088 & 67.2 & 821 & 2,234 & & & \\
\hline$\cdots$ & $\cdots$ & - & -- & $\therefore-$ & $\cdot-$ & $\cdots \cdot$ & $\cdots$ & $-\cdots$ & -- & $-\cdot-$ \\
\hline 1987 & 1,367 & 76 & $73: 4$ & 1,861 & 102.3 & B68 & 2,360 & & & \\
\hline 1988 & 1,345 & 70 & 72.1 & 1,865 & 106.5 & 915 & 2,488 & 2,427 & 2,581 & 2,612 \\
\hline 1989 & & & & & 110.6 & 964 & 2,619 & 2,555 & 2,717 & $2, \pi 50$ \\
\hline 1990 & & & & & 114.8 & 1,013 & 2,752 & 2,685 & 2,855 & 2,890 \\
\hline 1991 & & & & & 119.0 & 1,063 & 2,888 & 2,818 & 2,996 & 3,032 \\
\hline 1992 & & & & & 123.2 & 1,114 & 3,026 & 2,952 & 3,138 & 3,177 \\
\hline
\end{tabular}

Capacity-Capital stock Regression: Code 0 RSO 0.900 Coefficients: Const 16.6 stock 2.7

Ave. Capacity/Stock $(74-86): 2.7$ Ave. Heekly Hours (High) 159.6 Shift Factor 1.05 ave Weekly Hours (LOH) 150.6 Shift Factor 1.11 Note: Output, Investment, and Capital Stock extrapolated for 1987 and 1988.

\section{Non woven fabrics}

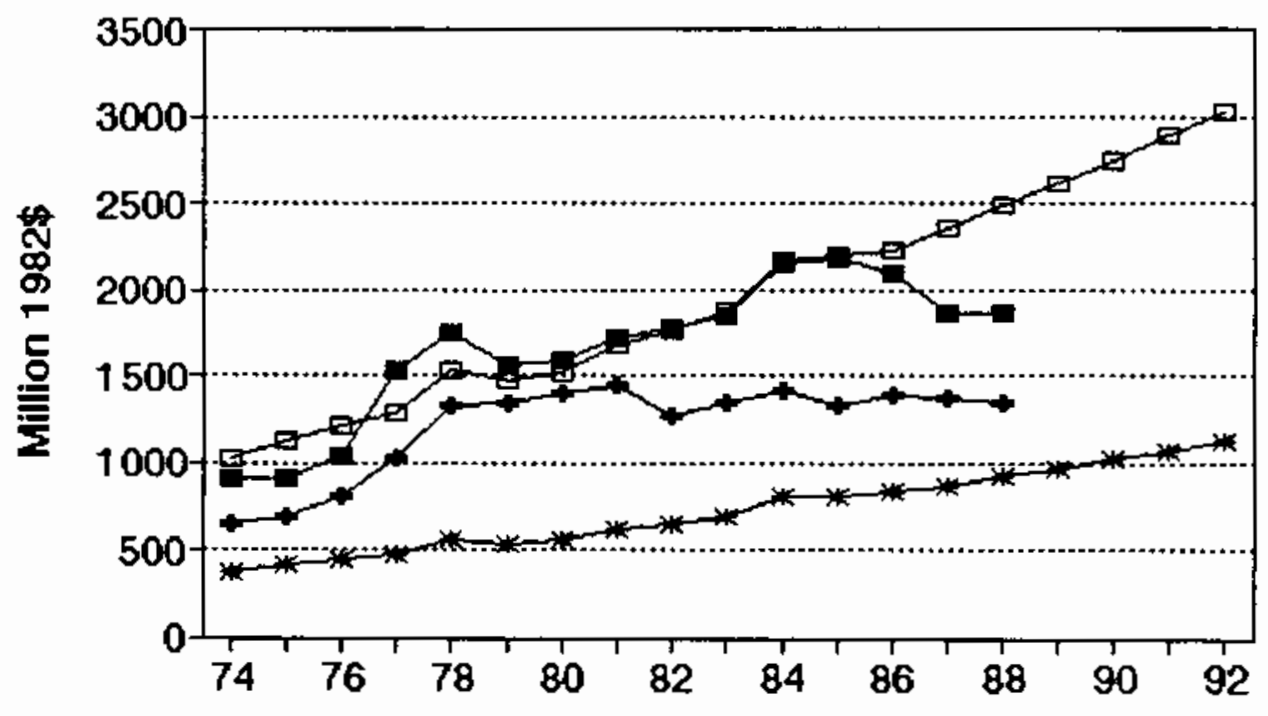

$\rightarrow$ - Oupur $\rightarrow$-mplied Capecing $\rightarrow$ - Capital Stock $\square$ - Pred. Cepecity 


\section{No. 80 sIc 2298 Cordage and twine}

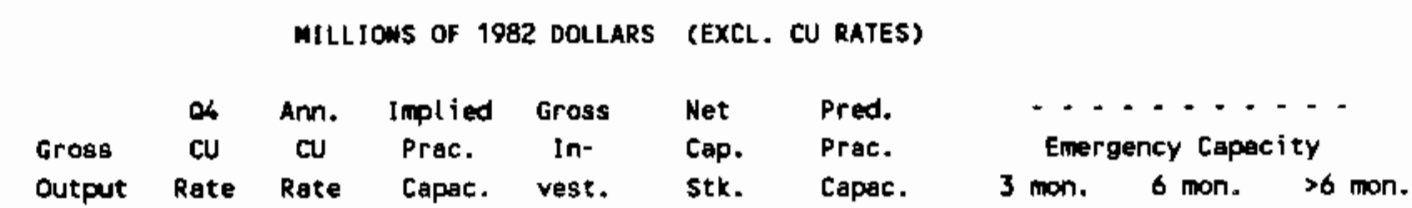

\begin{tabular}{|c|c|c|c|c|c|c|c|c|c|c|}
\hline 1974 & 528 & 65 & 65.5 & 805 & 8.7 & 157 & 765 & & & \\
\hline 1975 & 459 & 70 & 67.9 & 675 & 9.9 & 156 & 754 & & & \\
\hline 1976 & 445 & 60 & 63.7 & 698 & 11.2 & 155 & 751 & & & \\
\hline 1977 & 492 & 60 & 60.0 & 819 & 9.7 & 153 & 733 & & & \\
\hline 1978 & 512 & 76 & 70.0 & 731 & 9.1 & 150 & 712 & & & \\
\hline 1979 & 507 & 81 & 79.1 & 640 & 9.2 & 167 & 688 & & & \\
\hline 1980 & 366 & 78 & 79.1 & 463 & $(2.3)$ & 136 & 589 & & & \\
\hline $198 t$ & 367 & 68 & 71.9 & 510 & 6.2 & 129 & 554 & & & \\
\hline 1982 & 371 & 67 & 67.3 & 551 & 6.1 & 125 & 520 & & & \\
\hline 1983 & 416 & 50 & 56.5 & 736 & 19.7 & 133 & 583 & & & \\
\hline 1984 & 420 & 53 & 52.0 & 807 & 36.3 & 155 & 750 & & & \\
\hline 1985 & 405 & 67 & 61.7 & 657 & 4.1 & 147 & OBS & & & \\
\hline 1986 & 406 & 62 & 63.9 & 636 & 7.2 & 141 & 646 & & & \\
\hline$\cdots$ & $\cdots \cdot$ & -- & -- & $-\cdot$ & $\cdots \cdot$ & $\cdots$ & $=-$ & $\cdots$ & 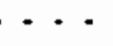 & $\cdots$ \\
\hline 1987 & 408 & 67 & 65.1 & 627 & 14.2 & 143 & 659 & & & \\
\hline 1988 & 412 & 64 & 65.1 & 632 & 12.6 & 143 & 660 & 945 & 1,021 & 1,036 \\
\hline 1989 & & & & & 12.8 & 144 & 662 & 948 & 1,024 & 1,040 \\
\hline 1990 & & & & & 12.0 & 143 & 657 & 942 & 1,017 & 1,033 \\
\hline 1991 & & & & & 15.5 & 166 & 680 & 974 & 1,052 & 1,068 \\
\hline 1992 & & & & & 15.9 & 149 & 704 & 1,008 & 1,089 & 1,106 \\
\hline
\end{tabular}

Capacity-Capital stock Regression: Code 0 RSO 0.560

Coefficients: Const $\mathbf{- 4 1 3 . 9}$ Stock $\mathbf{7 . 5}$

Ave. Capacity/Stock $(74-86): 4.6$ Ave. Weekly Hours (High) 96.0 Shift Factor 1.57 Ave Heekly Hours (Low) 85.6 Shift factor 1.76 Note: Output, Investment, and Capital Stock extrapolated for 1987 and 1988.

\section{Cordage and twine}

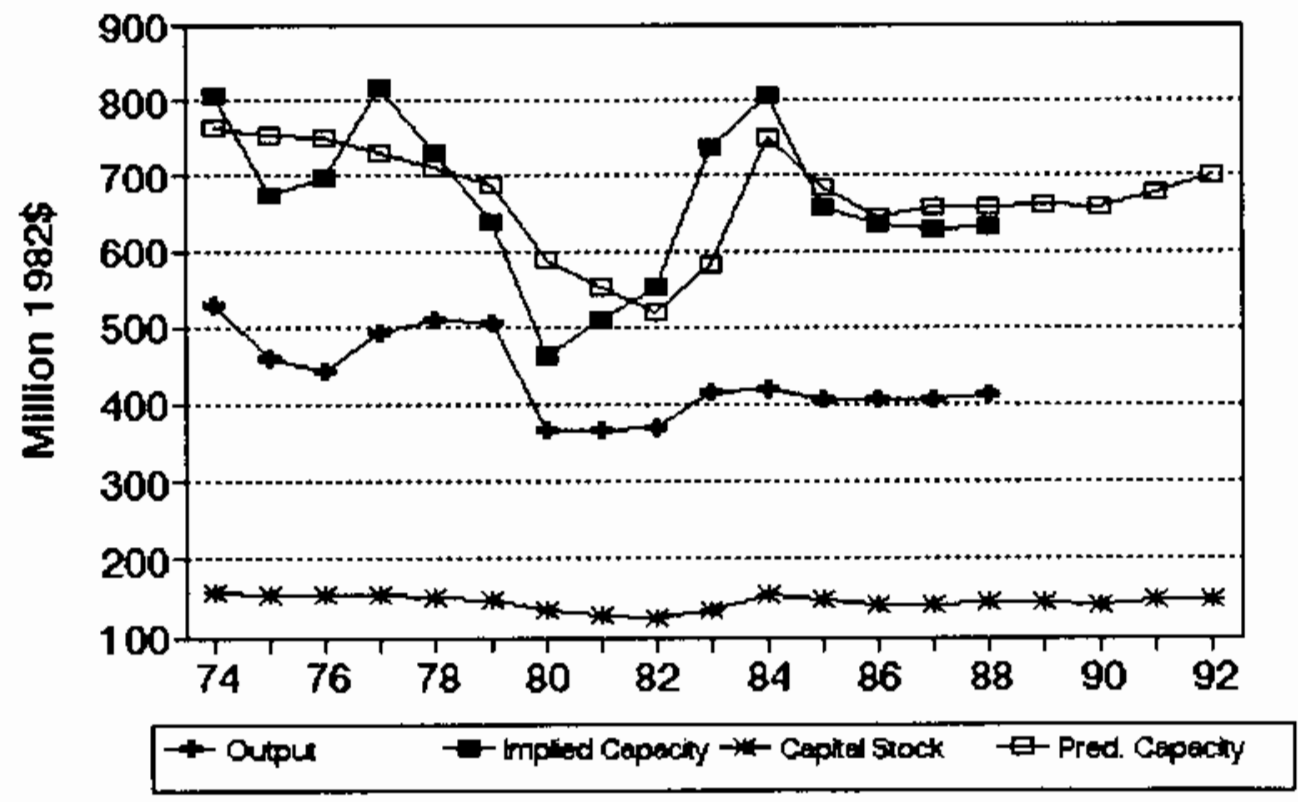


No. 81 sIC 2299 Textile goods, n.e.c. MILLIONS OF 1982 DOLLARS (EXCL. CU RATES)

\begin{tabular}{|c|c|c|c|c|c|c|c|}
\hline & $\begin{array}{l}\text { Q4 } \\
\mathrm{CU}\end{array}$ & Ann. & $\begin{array}{l}\text { Inplied } \\
\text { Prac. }\end{array}$ & $\begin{array}{c}\text { Gross } \\
\text { In- }\end{array}$ & $\begin{array}{l}\text { Net } \\
\text { Cep. }\end{array}$ & $\begin{array}{l}\text { Pred. } \\
\text { Prac. }\end{array}$ & Emergency Capecity \\
\hline itput & Rete & Rate & Сарас. & 105 & stk. & Capac. & \\
\hline
\end{tabular}

\begin{tabular}{|c|c|c|c|c|c|c|c|c|c|c|}
\hline 1974 & 210 & 83 & 85.0 & 247 & $(34.2)$ & 205 & 381 & & & \\
\hline 1975 & 213 & 82 & 81.6 & 261 & $(27.5)$ & 167 & 354 & & & \\
\hline 1976 & 290 & 78 & 79.5 & 365 & 27.4 & 182 & 365 & & & \\
\hline 1977 & 283 & 79 & 78.9 & 358 & $(3.8)$ & 166 & 354 & & & \\
\hline 1978 & 403 & 86 & 83.4 & 484 & 10.0 & 164 & 352 & & & \\
\hline 1979 & 327 & $88 *$ & 86.9 & 376 & 13.2 & 164 & 352 & & & \\
\hline 1980 & 330 & 87 * & 86.9 & 380 & 4.5 & 154 & 346 & & & \\
\hline 1981 & 380 & $82 *$ & 84.4 & 450 & 15.9 & 155 & 346 & & & \\
\hline 1982 & 287 & $82 *$ & 81.8 & 351 & $(14.2)$ & 128 & 328 & & & \\
\hline 1983 & 300 & 83 & $B 2.7$ & 364 & 2.0 & 118 & 320 & & & \\
\hline 1984 & 233 & 23 & 83.3 & 279 & $(9.2)$ & 99 & 307 & & & \\
\hline 1985 & 214 & 85 & 83.9 & 255 & 7.1 & 96 & 305 & & & \\
\hline 1986 & 200 & 83 & 83.8 & 239 & 3.1 & 89 & 300 & & & \\
\hline$\cdots$ & $-\cdot$ & $\cdots$ & $\cdots$ & $\cdots$ & $\cdots$ & $\cdots$ & $\cdots$ & $\cdots$ & $\cdots \cdot$ & $\cdots$ \\
\hline 1987 & 202 & $87 *$ & 85.7 & 236 & 1.6 & 81 & 294 & & & \\
\hline 1988 & 205 & $87 *$ & 86.9 & 236 & 1.5 & 73 & 289 & 418 & 448 & 454 \\
\hline 1989 & & & & & 1.5 & 67 & 285 & 411 & 441 & 447 \\
\hline 1990 & & & & & 1.4 & 61 & 281 & 406 & 435 & 441 \\
\hline 1991 & & & & & 1.8 & 56 & 277 & 401 & 430 & 436 \\
\hline 1992 & & & & & 1.8 & 52 & 276 & 396 & 425 & 431 \\
\hline
\end{tabular}

Capacity-Capital Stock Regression: Cade O RSQ 0.100 Coefficients: Const 238.4 stock 0.7

Ave. Capecity/Stock $(74-86): 2.3$ Ave. Weekly Hours (High) 96.0 Shift Factor 1.57 Ave Heekly Hours (Low) 85.6 Shift Factor 1.76 - Imputed Note: Output, Investment, and Capital Stock extrapolated for 1987 and 1988.

2299 Textile goods, n.e.c.

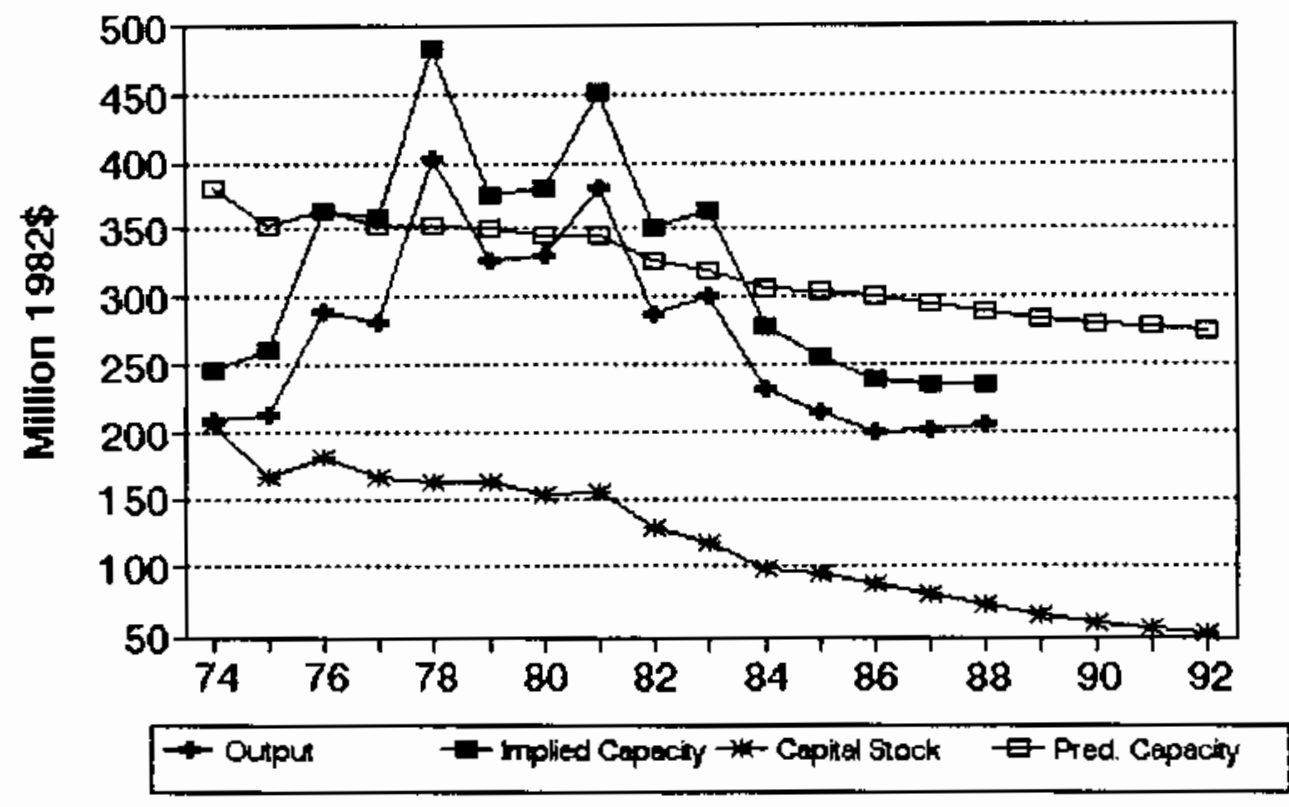




\section{No. 82 sIC 2311 Men's and boys' suits and coats}

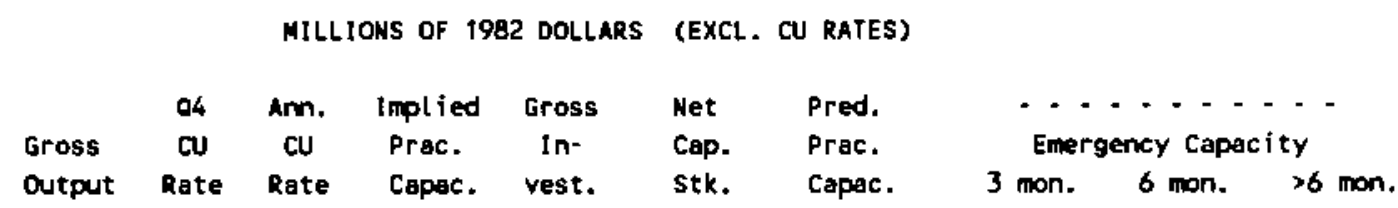

\begin{tabular}{|c|c|c|c|c|c|c|c|c|c|c|}
\hline 1974 & 3,970 & 75 & 76.4 & 5,198 & 25.1 & 326 & 5,054 & & & \\
\hline 1975 & 3,516 & 71 & 70.3 & 5,001 & 12.7 & 313 & 4,856 & & & \\
\hline 1976 & 3,633 & $T 2$ & 71.7 & 5,067 & 19.0 & 307 & 4,754 & & & \\
\hline 1977 & 3,938 & 82 & 78.7 & 5,003 & 27.7 & 309 & 4,788 & & & \\
\hline 1978 & 3,405 & 81 & 81.6 & 6,173 & 18.0 & 300 & 4,651 & & & \\
\hline 1979 & 3,240 & $\pi$ & 77.2 & 6,195 & 14.0 & 289 & 4,453 & & & \\
\hline 1990 & 3,308 & 79 & 77.4 & 4,272 & 6.2 & 270 & 4,136 & & & \\
\hline 1981 & 3,203 & 84 & 82.3 & 3,894 & 21.7 & 266 & 4,068 & & & \\
\hline 1982 & 3,045 & 79 & 80.5 & 3,784 & 27.1 & 266 & 4,060 & & & \\
\hline 1983 & 2,954 & 82 & 80.7 & 3,660 & 14.8 & 253 & 3,853 & & & \\
\hline 1984 & 3,039 & 86 & 85.2 & 3,566 & $(2.9)$ & 227 & 3,399 & & & \\
\hline 1985 & 3,057 & 90 & 87.8 & 3,481 & 28.2 & 229 & 3,447 & & & \\
\hline 1986 & 2,917 & 79 & 82.3 & 3,543 & 17.1 & 222 & 3,318 & & & \\
\hline$\cdots$ & $\cdots$ & $\because$ & $\cdots$ & $-\cdots$ & $-\cdots$ & -- & $\cdots$ & $\cdots$ & $\cdots$ & $\cdots$ \\
\hline 1987 & 2,875 & 89 & 85.0 & 3,381 & 19.5 & 217 & 3,232 & & & \\
\hline 1988 & 2,822 & 82 & 84.6 & 3,334 & 19.5 & 212 & 3,155 & 7,441 & 8,096 & 8,231 \\
\hline 1989 & & & & & 19.6 & 208 & 3,088 & 7,283 & 7,923 & 8,056 \\
\hline 1990 & & & & & 19.6 & 205 & 3,028 & 7,142 & 7,770 & 7,900 \\
\hline 1991 & & & & & 20.6 & 203 & 2,992 & 7,058 & 7,678 & 7,807 \\
\hline 1992 & & & & & 20.6 & 201 & 2,960 & 6,983 & 7,597 & 7,724 \\
\hline
\end{tabular}

Capecity-Capital Stock Regression: Code 0 RSO 0.850

Coefficients: Const -437.6 Stock 16.9

Ave. Capacity/stock $(74-86): 15.3$ Ave. Weekly Hours (High) 57.8 Shift Factor 2.61 Ave Weekly Hours (Lou) 47.9 Shift Factor 3.15 Note: Output, Investment, and Capital Stock extrapolated for 1987 and 1988.

\section{Men's and boys' suits and coats}

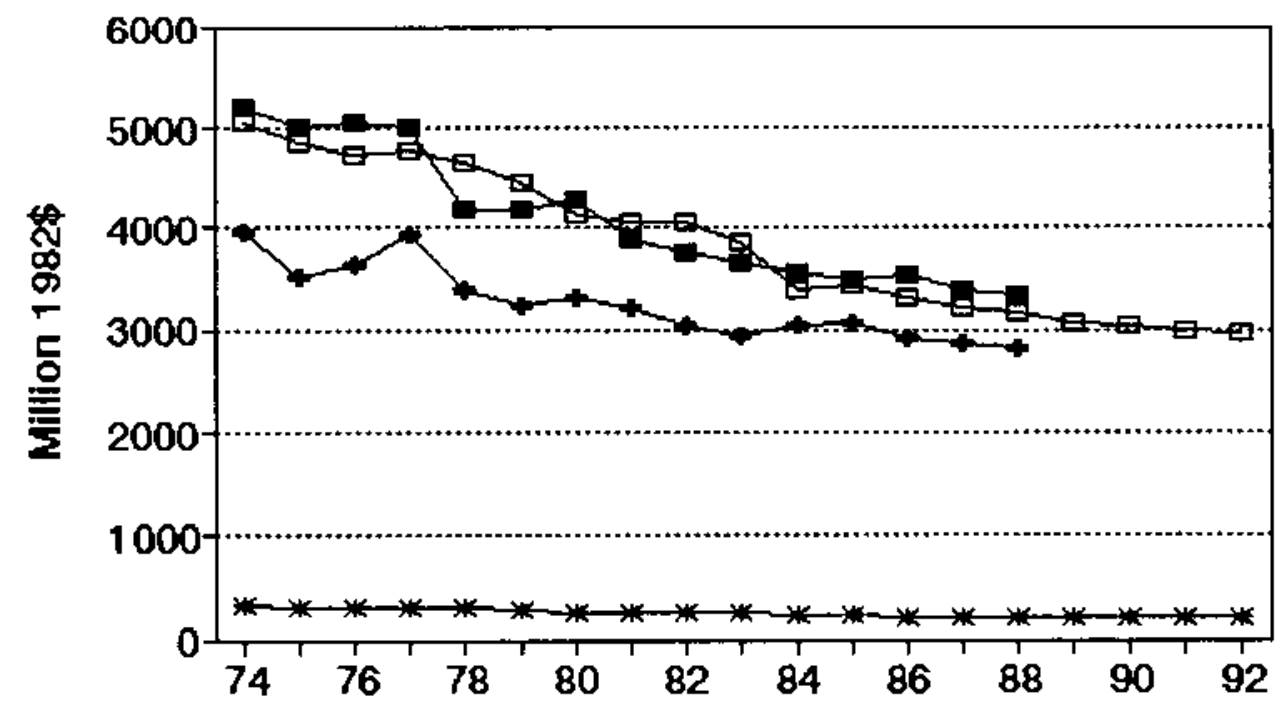

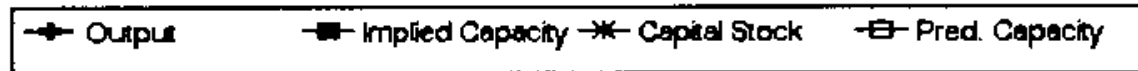


No. 83 sIC 2321 Men's and boy"' shirts and nightwear MILLIONS OF 1982 DOLLARS (EXCL. CU RATES)

\begin{tabular}{|c|c|c|c|c|c|c|c|}
\hline & $\begin{array}{l}04 \\
\text { av }\end{array}$ & Ann. & $\begin{array}{l}\text { Impl ied } \\
\text { Prac. }\end{array}$ & $\begin{array}{l}\text { Gross } \\
\text { In- }\end{array}$ & $\begin{array}{l}\text { Net } \\
\text { Cap. }\end{array}$ & $\begin{array}{l}\text { Pred. } \\
\text { Prac. }\end{array}$ & $\begin{array}{l}\cdots \\
\text { Energency Capacity }\end{array}$ \\
\hline & & & & vect & sti & Canec & \\
\hline
\end{tabular}

\begin{tabular}{|c|c|c|c|c|c|c|c|c|c|c|}
\hline 1974 & 3,690 & 92 & 92.0 & 4,011 & 26.3 & 364 & 4,231 & & & \\
\hline 1975 & 3,432 & 90 & 90.8 & 3,782 & 10.3 & 348 & 4,098 & & & \\
\hline 1976 & 3,942 & 83 & 85.6 & 4,604 & 20.4 & 343 & 4,051 & & & \\
\hline 1977 & 3,629 & 83 & 83.0 & 4,373 & 37.5 & 352 & 4,133 & & & \\
\hline 1978 & 3,809 & 88 & 86.1 & 4,422 & 25.8 & 356 & 4,162 & & & \\
\hline 1979 & 3,545 & 90 & 89.3 & 3,972 & 3.6 & 336 & 3,996 & & & \\
\hline 1980 & 3,608 & 93 & 91.9 & 3,927 & 26.8 & 339 & 4,020 & & & \\
\hline 1981 & 3,838 & 93 & 93.0 & 4,127 & 13.9 & 329 & 3,933 & & & \\
\hline 1982 & 3,428 & 94 & 93.6 & 3,661 & 17.3 & 322 & 3,869 & & & \\
\hline 1983 & 3,486 & 89 & 90.9 & 3,836 & 34.4 & 329 & 3,931 & & & \\
\hline 1984 & 3,710 & $88 \div$ & 88.6 & 4,188 & 42.0 & 342 & 4,042 & & & \\
\hline 1985 & 3,322 & $88 *$ & 87.9 & 3,780 & 37.1 & 348 & 4,095 & & & \\
\hline 1986 & 3,496 & 87 & 87.2 & 4,007 & 35.5 & 352 & 4,130 & & & \\
\hline-- & $\because \cdots$ & $\cdots$ & -- & $-\cdots$ & - - - & $\cdots$ & $\cdots$ & $---\cdot$ & --- & $-\cdots$ \\
\hline 1987 & 3,510 & 91 & 89.5 & 3,921 & 27.2 & 348 & 4.094 & & & \\
\hline 1988 & 3,528 & $91 *$ & 91,0 & 3,876 & 27.2 & 344 & 4,061 & 11,482 & 12,609 & 12,842 \\
\hline 1989 & & & & & 27.3 & 341 & 4,032 & 11,400 & 12,519 & 12,730 \\
\hline 1990 & & & & & 27.3 & 338 & 4,006 & 11,325 & 12,436 & 12,666 \\
\hline 1991 & & & & & 28.2 & 336 & 3,989 & 11,278 & 12,385 & 12,614 \\
\hline 1992 & & & & & 28.2 & 334 & 3,974 & 11,236 & 12,339 & 12,566 \\
\hline
\end{tabular}

Capacity-Capital Stock Regression: Code 0 RSe 0.140 Coefficients: Const 1098.6 stock 8.6 Ave. Capacity/Stock $(74-86): 11.8$ Ave. Weekly Hours (High) 47.7 Shift Factor 3.16 Ave Heekly Hours (Low) 47.7 Shift Factor 3.16 * Imputed Note: Output, Investment, and Capital Stock extrapolated for 1987 and 1988.

2321 Men's and boys' shirts and nightwe

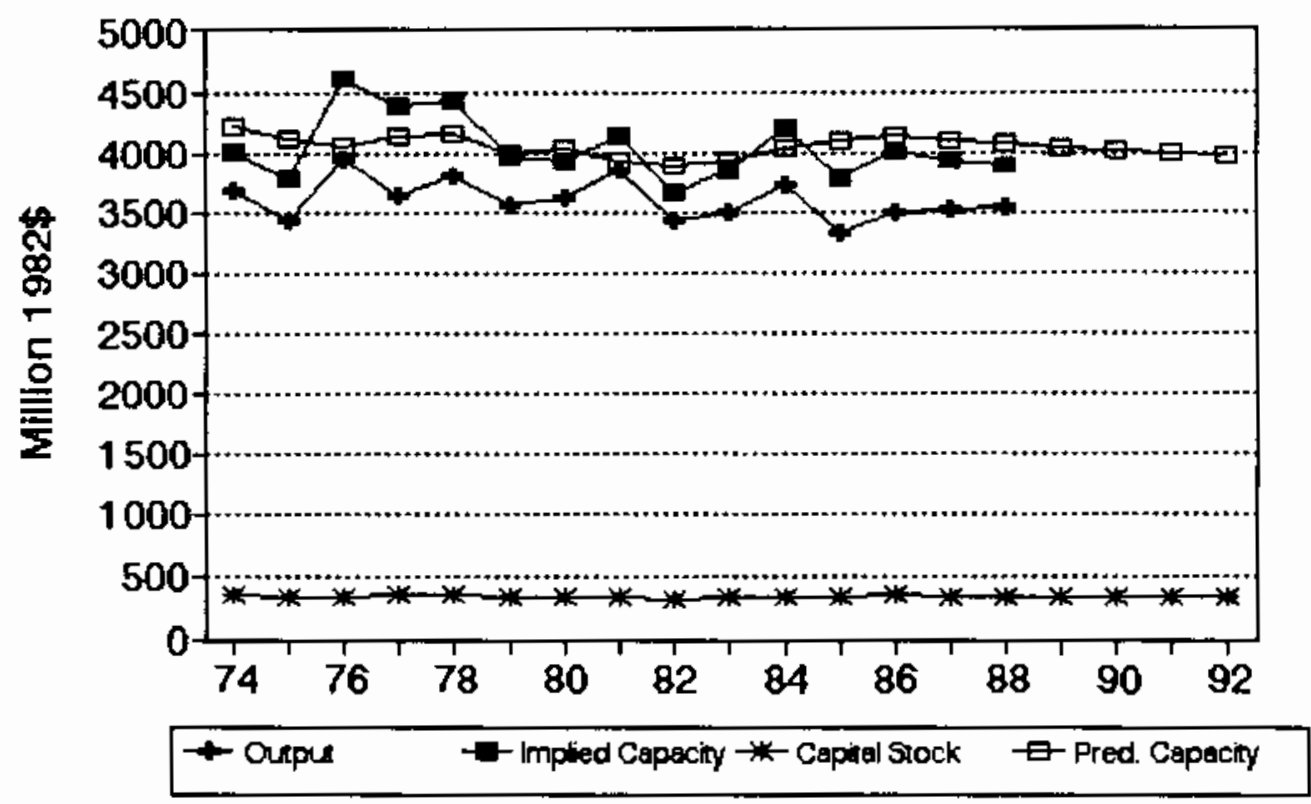


No. 84 SIC 2322 Men's and boys' underwear

MILLIONS OF 1982 DOLLARS (EXCL. CU RATES)

$\begin{array}{lccccccc} & 04 & \text { Ann. } & \text { Implied } & \text { Gross } & \text { Net } & \text { Pred. } & -\ldots \ldots \ldots \\ \text { Gross } & \text { CU } & \text { CU } & \text { Prac. } & \text { In- } & \text { Cap. } & \text { Prac. } & \text { Emergency Capecity } \\ \text { Output } & \text { Rate } & \text { Rate } & \text { Capac. } & \text { vest. } & \text { Stk. } & \text { Capac. } & 3 \text { mon. } 6 \text { mon. }>6 \text { mon. }\end{array}$

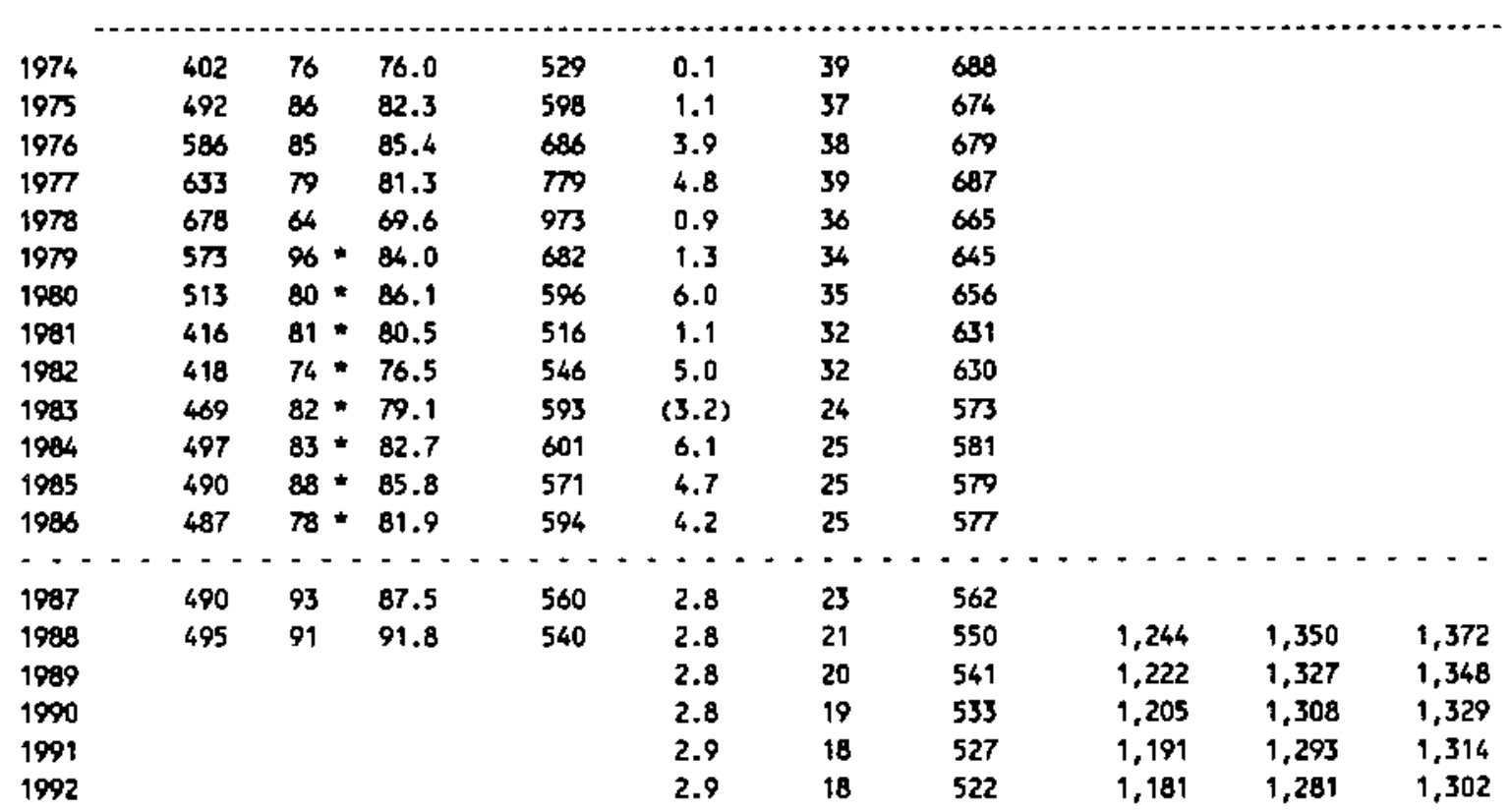

Capecity-Capital Stock Regression: Code 0 RSO 0.130

Coefficients: Const 383.6 stock $7.8 \quad$ Ave. Capacity/Stock $(74-86): 19.7$ Ave. Weekly Hours (High) 60.5 Shift Factor 2.49 Ave Heekly Hours (Low) 60.5 Shift Factor 2.49

- Imputed Note: Output, Investment, and Capital stock extropoloted for 1987 and 1988.

\section{Men's and boys' underwear}

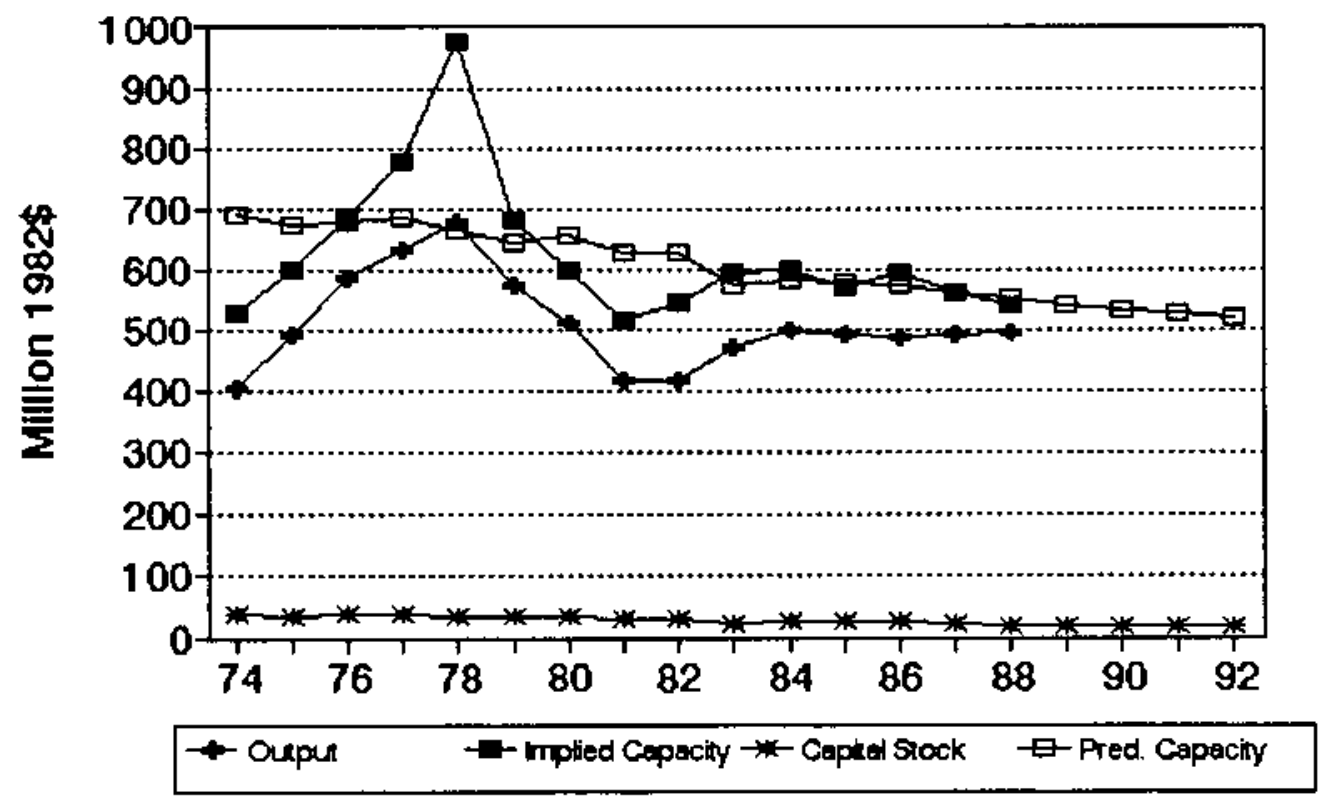




\section{No. 85 SIC 2323 Men's and boys' neckwear}

MILLIONS OF 1982 DOLLARS (EXCL. CU RATES)

$\begin{array}{lccccccc} & \text { Q4 } & \text { Anm. Implied Gross } & \text { Net } & \text { Pred. } & \ldots & \ldots \\ \text { Gross } & \text { CU } & \text { CU } & \text { Prac. } & \text { In- } & \text { Cap. } & \text { Prac. } & \text { Emergency Capacity } \\ \text { Output } & \text { Rate } & \text { Rate } & \text { Capac. } & \text { vest. } & \text { Stk. } & \text { Capec. } & 3 \text { mon. } 6 \text { mon. }>6 \text { mon. }\end{array}$

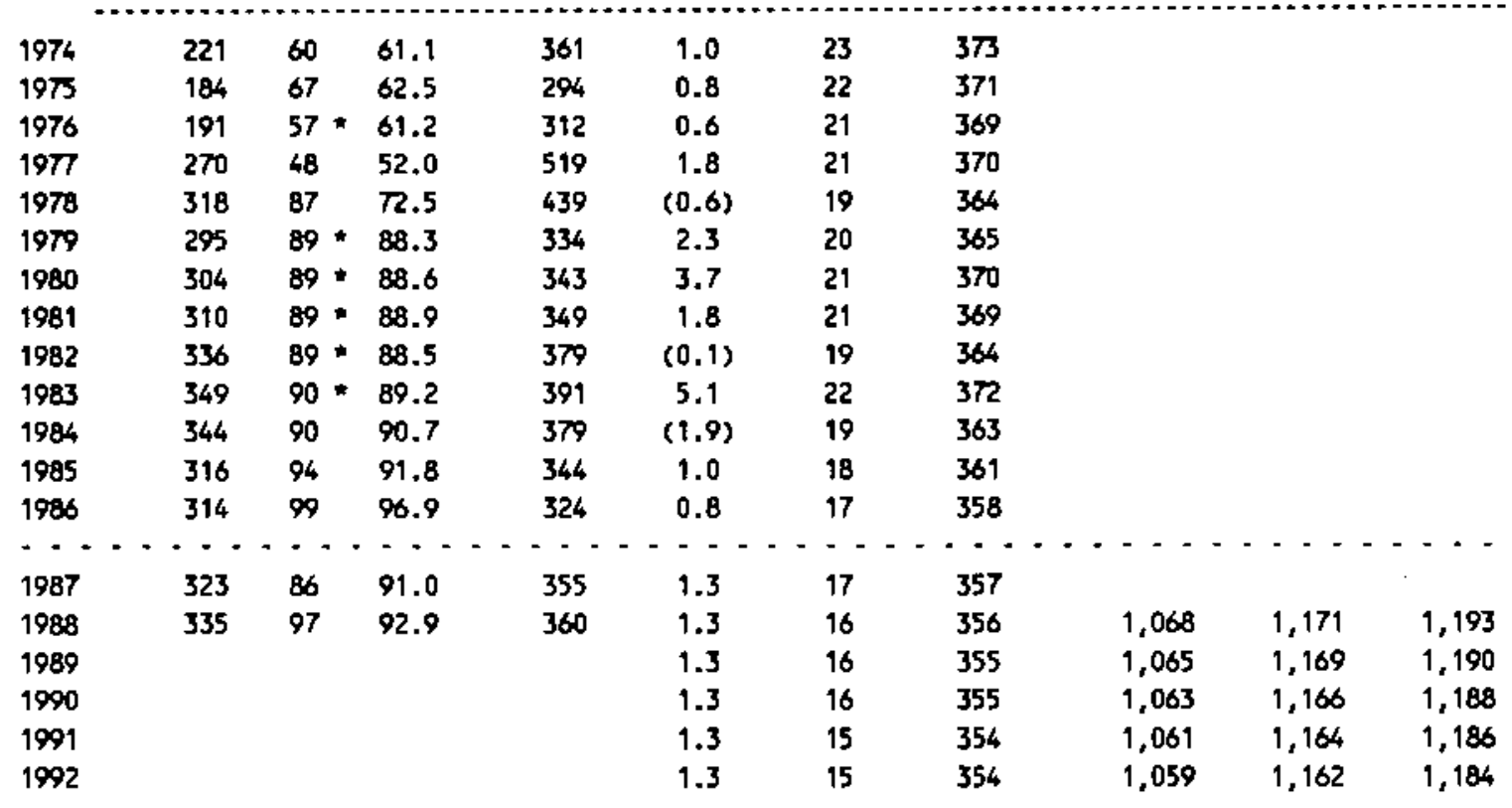

Capacity-Capital Stock Regression: Code 0 RSO 0.010

Coefficients: Const 313.8 Stock $2.6 \quad$ Ave. Capacity/Stock (74-86): 18.2 Ave, Ueekly Hours (High) 45.0 Shift Factor 3.35 Ave Heekly Hours (Low) 42.4 Shift factor 3.56 * Imputed Hote: Output, Investment, and Capital Stock extrapolated for 1987 and 1988.

\section{Men's and boys' neckwear}
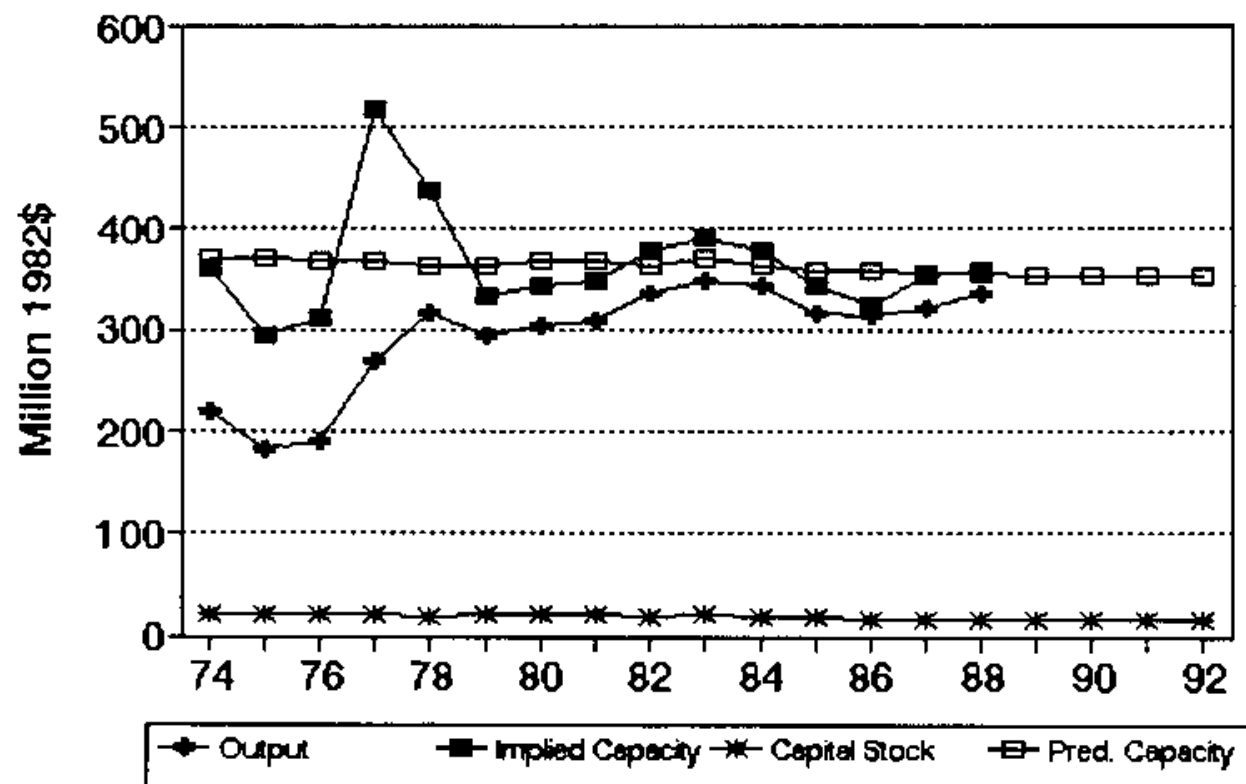


\section{No. 86 SIC 2327 Men's and boys' separate trousers}

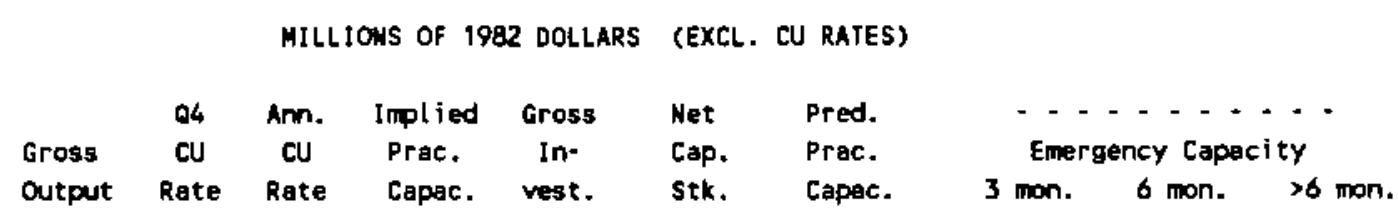

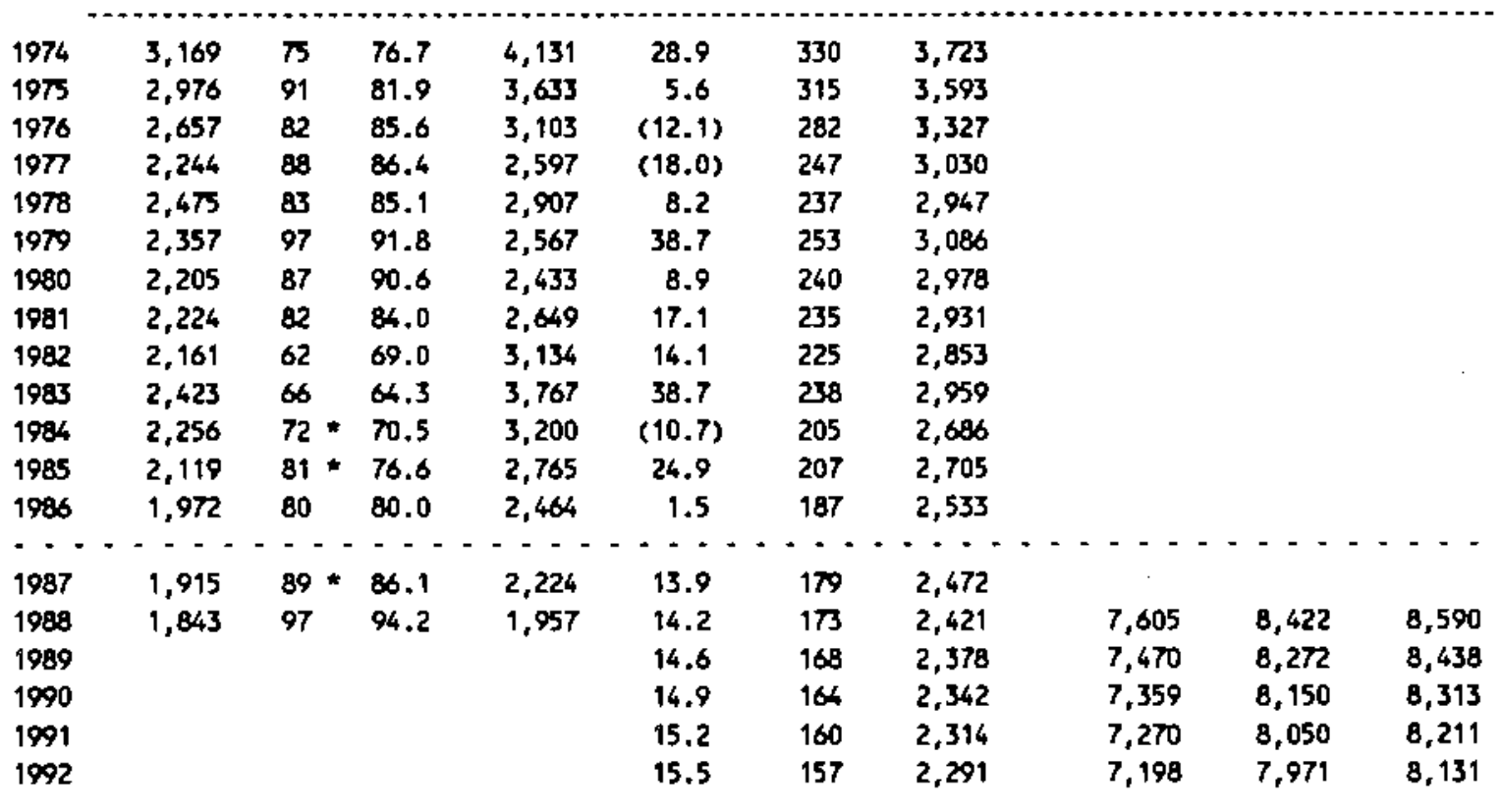

Capacity-Capital stock Regression: Code 0 RSa 0.410

Coefficients: Const 986.2 stock 8.3

Ave. Capacity/Stock $(74-86): 12.3$

Ave. Weekly Hours (High) 42.5 Shift Factor 3.55 Ave Heekly Hours (Low) 41.5 Shift Factor 3.63

* Inputed Note: Output, Imestment, and Capital Stock extrapolated for 1987 and 1988.

\section{Men's and boys' separate trousers}
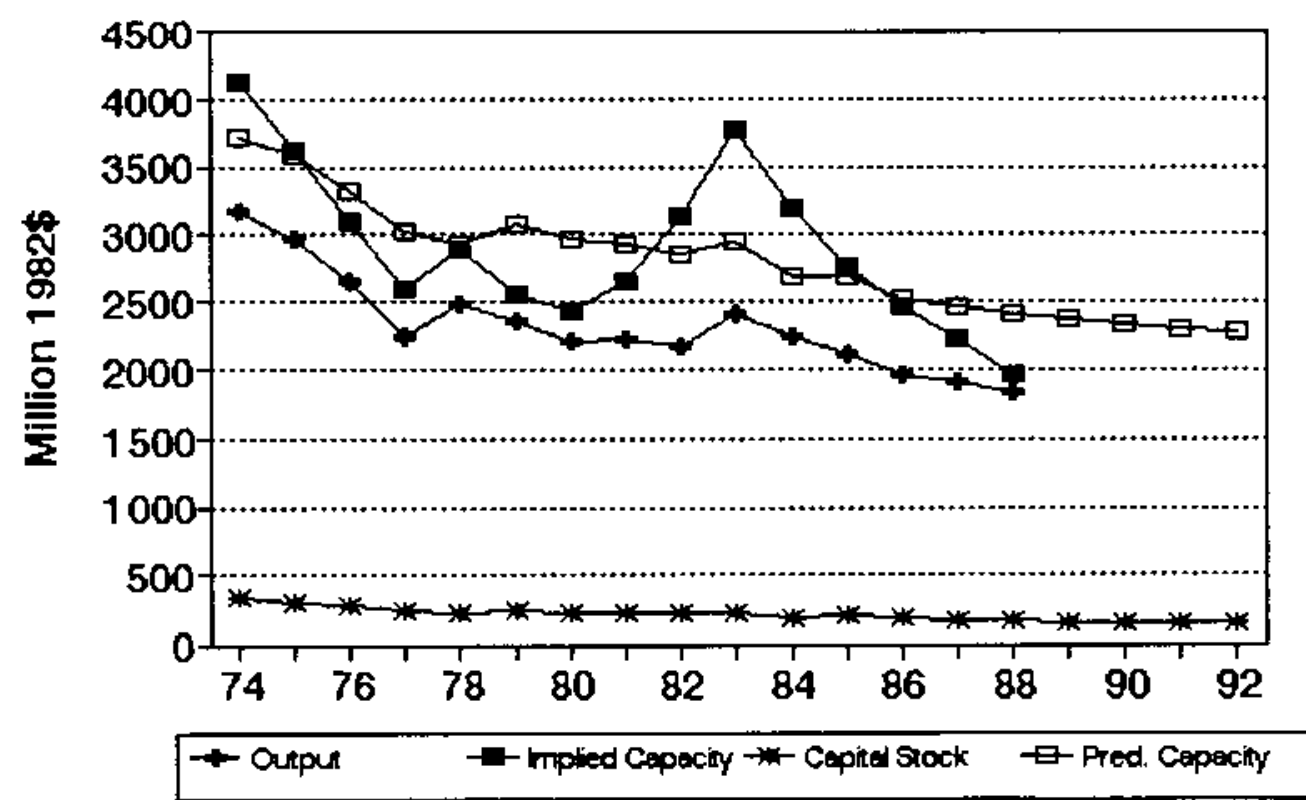


\section{No. 87 sIC 2328 Men's and boys' work clothing}

MILLIONS OF 1982 DOLLARS (EXCL. CU RATES)

$\begin{array}{lccccccc} & 04 & \text { Ann. Implied Gross } & \text { Net } & \text { Pred. } & \ldots \ldots \ldots \\ \text { Gross } & \text { CU } & \text { CU } & \text { Prac. } & \text { In. } & \text { Cap. } & \text { Prac. } & \text { Emergency Capacity } \\ \text { Output } & \text { Rate } & \text { Rate } & \text { Capac. } & \text { vest. } & 5 \text { Stk. } & \text { Capac. } & 3 \text { mon. } 6 \text { mon. }>6 \text { mon. }\end{array}$

\begin{tabular}{|c|c|c|c|c|c|c|c|c|c|c|}
\hline 1974 & 3,233 & 67 & 67.2 & 4,810 & 34.6 & 289 & 4,919 & & & \\
\hline 1975 & 3,818 & 83 & 76.6 & 4,983 & 25.8 & 294 & 4,967 & & & \\
\hline 1976 & 6,233 & 90 & 87.4 & 4,844 & 36.5 & 308 & 5,085 & & & \\
\hline 1977 & 4,812 & 89 & 89.5 & 5,378 & 74.5 & 357 & 5,495 & & & \\
\hline 1978 & 4,768 & 67 & $\pi .3$ & 6,332 & 32.9 & 363 & 5,546 & & & \\
\hline 1979 & 4,629 & 95 & 84.5 & 5,477 & 10.9 & 350 & 5,441 & & & \\
\hline 1980 & 6,639 & 85 & 88.7 & 5,228 & 65.0 & 391 & 5,784 & & & \\
\hline 1981 & 4,777 & 83 & 83.8 & 5,702 & $(15.4)$ & 353 & 5,461 & & & \\
\hline 1982 & 4,616 & 90 & 87.3 & 5,287 & 58.3 & 384 & 5,728 & & & \\
\hline 1983 & 4,968 & 83 & 85.6 & 5,804 & 20.3 & 377 & 5,669 & & & \\
\hline 1984 & 4,956 & 83 & 83.1 & 5,962 & 34.0 & 383 & 5,715 & & & \\
\hline 1985 & 4,850 & 86 & 84.8 & 5,722 & 39.3 & 391 & 5,787 & & & \\
\hline 1986 & 4,651 & $\pi$ & 79.1 & 5,890 & 35.3 & 394 & 5,814 & & & \\
\hline$\cdots$ & $\cdots$ & $\because$ & $\cdots \cdot$ & $\cdots \cdot$ & $\cdots$ & $\cdots$ & $-\cdots$ & $--\cdot$ & $\cdots \cdot$ & $\cdots$ \\
\hline 1987 & 4,809 & 87 & 82.5 & 5,828 & 36.4 & 399 & 5,855 & & & \\
\hline 1988 & 5,022 & 7 & 80.8 & 6,219 & 36.4 & 404 & 5,893 & 19,025 & 21,010 & 21,419 \\
\hline 1989 & & & & & 36.5 & 408 & 5,928 & 19,139 & 21,135 & 21,548 \\
\hline 1990 & & & & & 36.5 & 412 & 5,960 & 19,246 & 21,251 & 21,666 \\
\hline 1991 & & & & & 37.8 & 417 & 6,001 & 19,377 & 21,398 & 21,815 \\
\hline 1992 & & & & & 37.8 & 421 & 6,039 & 19,499 & 21,532 & 21,953 \\
\hline
\end{tabular}

Capacity-Cepital Stock Regression: Code 0 RSO 0.470

Coefficients: Const 2477.1 stock 8.5

Ave. Capacity/5tock $(74-86): 15.4$ Ave. Weekly Hours (High) 41.5 Shift Factor 3.63 Ave Heekly Hours (Low) 41.0 Shift Factor 3.68 Note: Output, Investment, and Capital Stock extrapolated for 1987 and 1988.

\section{Men's and boys' work clothing}

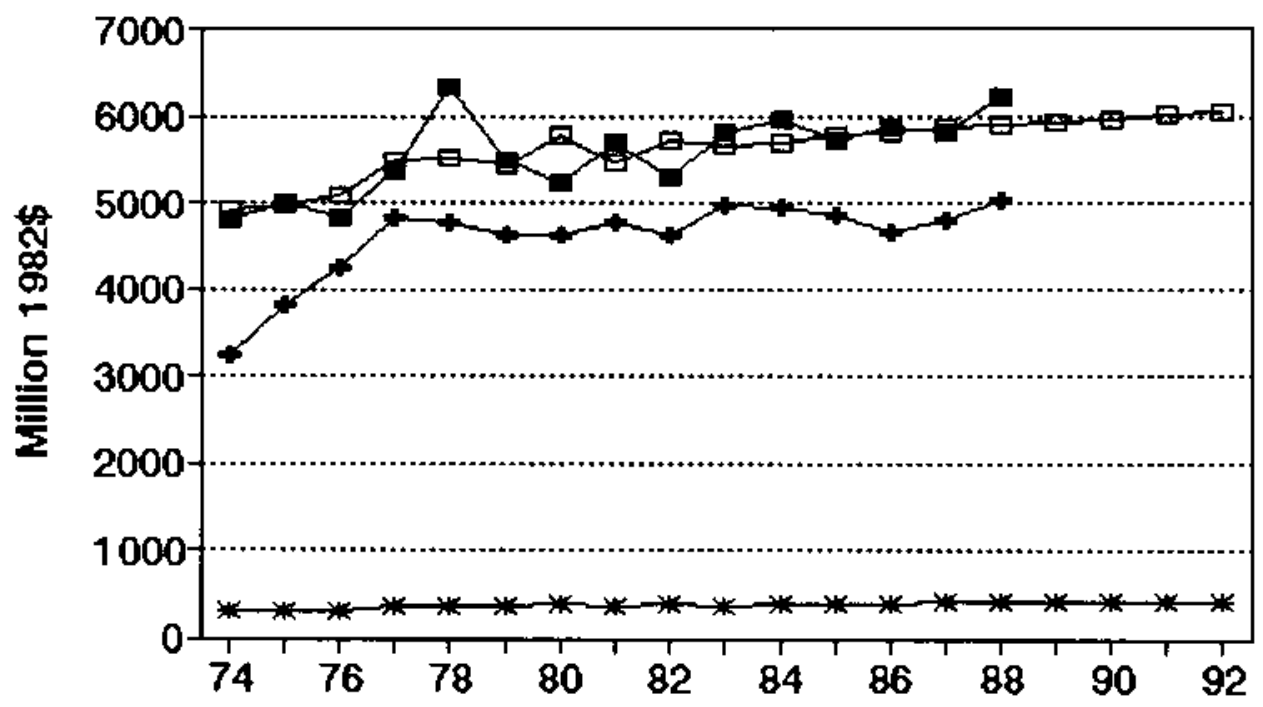

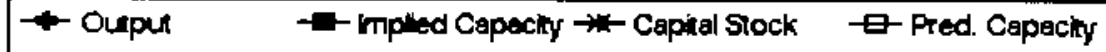


No. 88 SIC 2329 Men's and boys' clothing, n.e.c.

\begin{tabular}{|c|c|c|c|c|c|c|c|}
\hline & & MILL & ONS OF 19 & 2 DOLLA & (EXCL & U RATES) & \\
\hline Gross & $\begin{array}{l}a_{4} \\
\text { Cu }\end{array}$ & $\begin{array}{c}\text { Ann. } \\
\text { Cu }\end{array}$ & $\begin{array}{l}\text { Impl ied } \\
\text { Prac. }\end{array}$ & $\begin{array}{l}\text { Gross } \\
\text { In- }\end{array}$ & $\begin{array}{l}\text { Wet } \\
\text { Cep. }\end{array}$ & $\begin{array}{l}\text { Pred. } \\
\text { Prac. }\end{array}$ & $\begin{array}{l}- \\
\text { Emergency Capacity }\end{array}$ \\
\hline utput & Rate & Rate & Capac. & vest. & stk. & Capoc. & 3 mon. $\quad 6$ mon. $\quad>6$ mon. \\
\hline
\end{tabular}

\begin{tabular}{|c|c|c|c|c|c|c|c|c|c|c|}
\hline 1974 & 1,528 & 86 & 87.0 & 1,756 & 4.8 & 121 & 1,801 & & & \\
\hline 1975 & 1,489 & 7 & 78.8 & 1,890 & 12.2 & 124 & 1,865 & & & \\
\hline 1976 & 1,428 & 79 & 78.3 & 1,825 & 13.3 & 128 & 1,037 & & & \\
\hline 1977 & 1,436 & 78 & 78.7 & 1,823 & 19.0 & 137 & 2,109 & & & \\
\hline 1978 & 1,485 & 85 & 82.5 & 1,800 & 10.5 & 137 & 2,119 & & & \\
\hline 1979 & 1,517 & 92 & 89.4 & 1,697 & $(7.7)$ & 121 & 1,808 & & & \\
\hline 1980 & 1,514 & 66 & 75.7 & 2,000 & 14.0 & 126 & 1,897 & & & \\
\hline 1981 & 1,467 & 71 & 69.2 & 2,121 & 18.3 & 133 & 2,043 & & & \\
\hline 1982 & 1,699 & 68 & 68.9 & 2,466 & 24.7 & 146 & 2,278 & & & \\
\hline 1983 & 1,554 & $\pi$ & 70.4 & 2,207 & $(1.0)$ & 134 & 2,053 & & & \\
\hline 1984 & 1,683 & $\pi *$ & 74.5 & 2,259 & 4.5 & 128 & 1,935 & & & \\
\hline 1985 & 1,613 & 79 & 77.2 & 2,089 & 21.8 & 138 & 2,126 & & & \\
\hline 1986 & 1,812 & 83 & 81.4 & 2,226 & 15.1 & 141 & 2,187 & & & \\
\hline . . & $\cdots$ & $\ddot{85}$ & $\therefore 4$ & $\cdots$ & 11.3 & 140 & $\begin{array}{l}\cdots, 174 \\
2,17\end{array}$ & $-\cdot$ & $\cdots$ & $\cdots$ \\
\hline 1988 & 1,844 & $84=$ & 84.3 & 2,189 & 11.3 & 140 & 2,163 & 7,149 & 7,901 & 8,056 \\
\hline 1989 & & & & & 11.3 & 139 & 2,152 & 7,115 & 7,863 & 8,017 \\
\hline 1990 & & & & & 11.3 & 139 & 2,143 & 7,083 & 7,828 & 7.981 \\
\hline 1991 & & & & & 11.3 & 138 & 2,135 & 7,057 & 7,790 & 7,952 \\
\hline 1992 & & & & & 11.3 & 138 & 2,127 & 7,033 & $7,7 \pi 2$ & 7,925 \\
\hline
\end{tabular}

Capecity-Capital stock Regression: Code 0 RSO 0.410

Coefficients: Const -480.4 Stock 18.9

Ave. Capacity/Stock $(74-86): 15.3$ Ave. Heekly Hours (High) 40.5 Shift Factor 3.72 Ave Heekly Hours (Low) 40.5 Shift Factor 3.72 - Imputed Note: Output, Investment, and Capital stock extrapolated for 1987 and 1988.

\section{Men's and boys' clothing, n.e.c.}

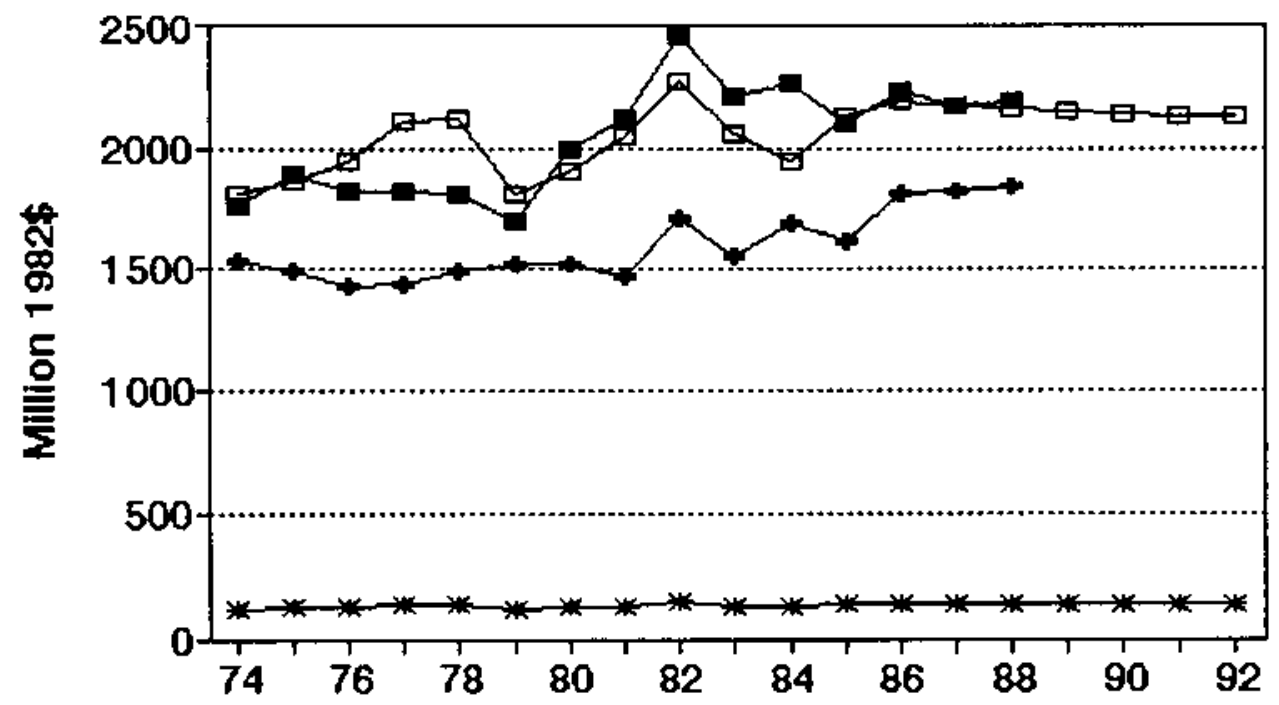

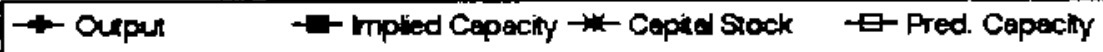




\title{
No, 89 sIC 2331 Woren's and misses' blouses and waists
}

\author{
MILLIONS OF 1982 DOLLARS (EXCL. CU RATES)
}

$\begin{array}{lccccccc} & \text { Q4 } & \text { Ann. Implied Gross } & \text { Het } & \text { Pred. } & \ldots & \ldots \\ \text { Gross } & \text { CU } & \text { CU } & \text { Prac. } & \text { In- } & \text { Cap. } & \text { Prac. } & \text { Emergency Capacity } \\ \text { Output Rate } & \text { Rate } & \text { Capac. } & \text { vest. } & \text { Stk. } & \text { Capac. } & 3 \text { mon. } 6 \text { mon. }>6 \text { mon. }\end{array}$

\begin{tabular}{|c|c|c|c|c|c|c|c|c|c|c|}
\hline 1974 & 2,320 & 75 & 75.8 & 3,062 & 6.0 & 179 & 3,369 & & & \\
\hline 1975 & 2,510 & 80 & 76.8 & 3,267 & $(0.6)$ & 167 & 3,262 & & & \\
\hline 1976 & 2,701 & 74 & 76.3 & 3,538 & 3.8 & 160 & 3,197 & & & \\
\hline 197 & 3,135 & 64 & 68.1 & 4,606 & 89.5 & 233 & 3.855 & & & \\
\hline 1978 & 3,280 & 84 & 76.6 & 4,281 & 18.5 & 235 & 3,873 & & & \\
\hline 1979 & 3,270 & 82 & 82.8 & 3,951 & $(4.9)$ & 215 & 3,697 & & & \\
\hline 1980 & 3,016 & 89 & 86.3 & 3,493 & 24.0 & 224 & 3,772 & & & \\
\hline 1981 & 3,165 & 87 & 87.8 & 3,605 & 18.4 & 226 & 3,794 & & & \\
\hline 1982 & 3,910 & 78 & 81.1 & 4,820 & 63.5 & 269 & 4,176 & & & \\
\hline 1983 & 3,645 & 93 & 87.2 & 4,180 & 14.3 & 262 & 4,117 & & & \\
\hline 1984 & 3,297 & 93 & 93.5 & 3,528 & 1.8 & 244 & 3,955 & & & \\
\hline 1985 & 3,048 & 93 & 92.6 & 3,292 & 18.3 & 242 & 3,934 & & & \\
\hline 1986 & 3,082 & 93 & 92.9 & 3,317 & 21.9 & 242 & 3,940 & & & \\
\hline$\cdots$ & $\cdots-$ & -- & $-\cdot$ & 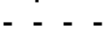 & $-\cdots$ & $-\cdots$ & $\cdots$ & $=-\cdot$ & --- & --- \\
\hline 1987 & 3,206 & 93 & 93.1 & 3,444 & 26.6 & 248 & 3,991 & & & \\
\hline 1988 & 3,373 & 93 & 93.0 & 3,629 & 27.5 & 254 & 4,045 & 13,362 & 14,777 & 15,069 \\
\hline 1989 & & & & & 28.4 & 261 & 4,104 & 13,554 & 14,990 & 15,286 \\
\hline 1990 & & & & & 29.4 & 267 & 4,165 & 13,758 & 15,215 & 15,516 \\
\hline 1991 & & & & & 30.3 & 275 & 4,230 & 13,972 & 15,451 & 15,737 \\
\hline 1992 & & & & & 31.2 & 282 & 4,298 & 14,195 & 15,698 & 16,008 \\
\hline
\end{tabular}

Capecity-Capital Stock Regression: Code 1 RSQ 0.310

Coefficients: Const 1762.7 stock 9.0

Ave. Capacity/Stock $(74-86): 16.9$ Ave. Weekly Hours (High) 40.5 Shift Factor 3.72 Ave Weekly Hours (Low) 40.5 Shift factor 3.72 Note: output, Investment, and Capital Stock extrapolated for 1987 and 1988.

\section{Women's and misses' blouses and wa}

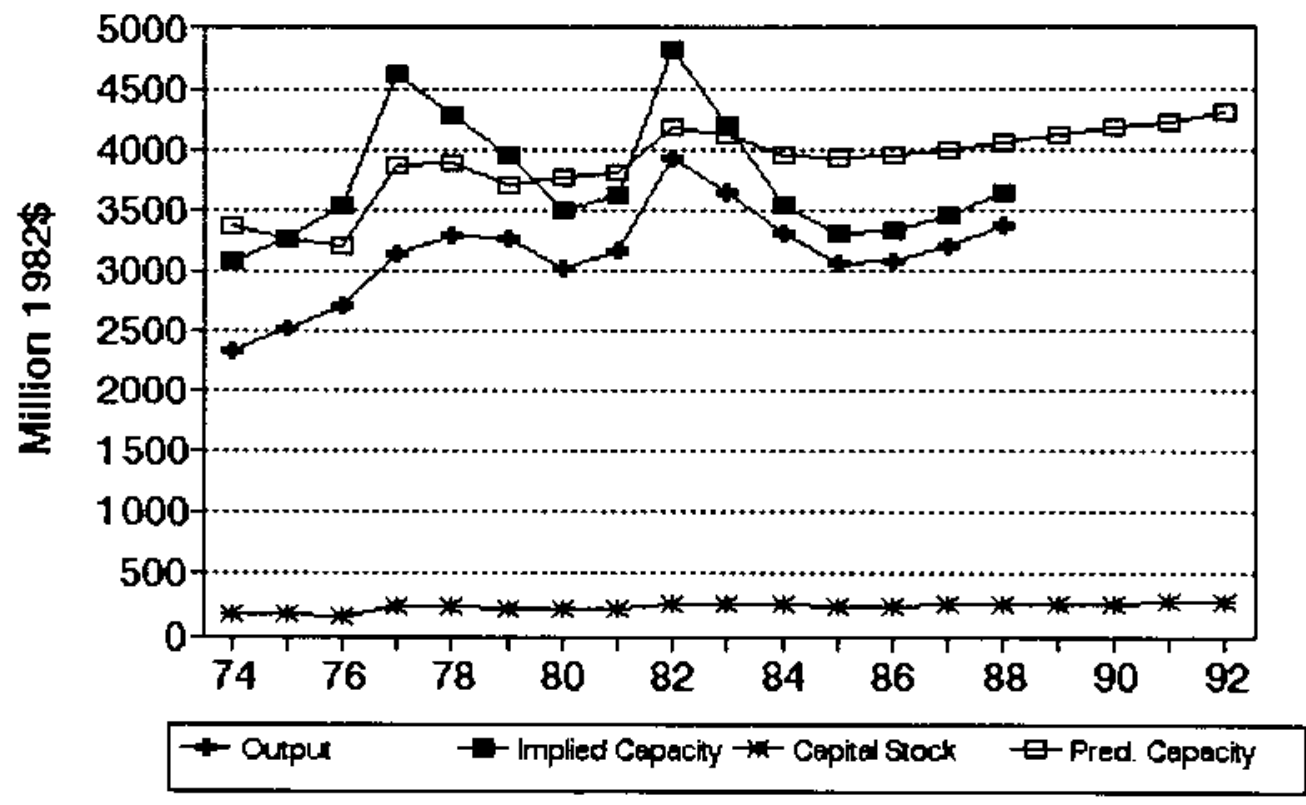




\section{NO. 90 sIC 2335 women'a and misags' dresses}

\section{MILLIONS OF 1982 DOLLARS (EXCL. CU RATES)}

$\begin{array}{lcccclcc} & 04 & \text { Ann. Implied Gross } & \text { Net } & \text { Pred. } & \ldots \ldots \\ \text { Gross } & \text { CU } & \text { CU } & \text { Prac. } & \text { In. } & \text { Cap. } & \text { Prac. } & \text { Emergency Capacity } \\ \text { Output Rate } & \text { Rate } & \text { Capac. } & \text { vest. } & \text { Stk. } & \text { Capac. } & 3 \text { mon. } 6 \text { mon. }>6 \text { mon. }\end{array}$

\begin{tabular}{|c|c|c|c|c|c|c|c|c|c|c|}
\hline 1974 & 5,188 & $\pi$ & 75.0 & 6,918 & $(94.6)$ & 649 & 5,685 & & & \\
\hline 1975 & 5,191 & 91 & 85.0 & 6,107 & 38.8 & 458 & 6,082 & & & \\
\hline 1976 & 5,374 & 84 & B6. 6 & 6,204 & 29.3 & 456 & 6,479 & & & \\
\hline 1977 & 5,343 & $9 B$ & $92 . B$ & 5,760 & 30.7 & 657 & 6,876 & & & \\
\hline 1978 & 5,440 & $\pi$ & 83.6 & 6,505 & 9.2 & 436 & 7,272 & & & \\
\hline 1979 & 4,727 & 67 & 70.0 & $6, \pi 53$ & $(1.7)$ & 405 & 7,669 & & & \\
\hline 1980 & 4,781 & 40 & 50.1 & 9,539 & 10.6 & 387 & 8,066 & & & \\
\hline 1981 & 4,683 & 56 & 50.0 & 9,367 & 60.1 & 416 & 8,463 & & & \\
\hline 1982 & 4,609 & $50 *$ & 52.5 & 8,770 & $(25.4)$ & 362 & 8,860 & & & \\
\hline 1983 & 4,822 & $56 *$ & 54.1 & 8,907 & 34.3 & 365 & 9,257 & & & \\
\hline 1984 & 4,909 & $54 \star$ & 54.8 & 8,963 & 10.7 & 345 & 9,653 & & & \\
\hline 1985 & 5,080 & $51 *$ & 52.2 & 9,726 & 20.0 & 335 & 10,050 & & & \\
\hline 1986 & 5,252 & $43 *$ & 46.4 & 11,333 & 12.8 & 318 & 10,447 & & & \\
\hline $\begin{array}{l}--\cdot \\
1987\end{array}$ & 5,228 & $\begin{array}{l}\cdots \\
44\end{array}$ & $\because 3.8$ & 11,944 & $\begin{array}{l}-. . \\
42.4\end{array}$ & 332 & 10.844 & $\cdots \cdot-$ & --- & --- \\
\hline 1988 & 5,195 & 52 & 49.2 & 10,563 & 47.8 & 349 & 11,241 & 11,817 & 12,755 & 12,949 \\
\hline 1989 & & & & & 53.1 & 370 & 11,638 & 12,234 & 13,206 & 13,406 \\
\hline 1990 & & & & & 58.5 & 395 & 12,034 & 12,652 & 13,656 & 13,864 \\
\hline 1991 & & & & & 63.9 & 423 & 12,431 & 13,069 & 14,106 & 14,321 \\
\hline 1992 & & & & & 69.2 & 453 & 12,828 & 13,486 & 14,557 & 14,778 \\
\hline
\end{tabular}

Capacity-Capital stock Regression: Code -2 RSO 0.770

Coefficients: Const 5288.3 Time 396.8

Ave. Capacity/Stock (74-86): 20.2

Ave. Weekly Hours (High) 131.9 Shift factor 1.15 Ave Weekly Hours (Low) 131.9 Shift Factor 1.15

* Imputed Note; Output, Imvestment, and Cepital Stock extrapolated for 1987 and 1988.

\section{Women's and misses' dresses}

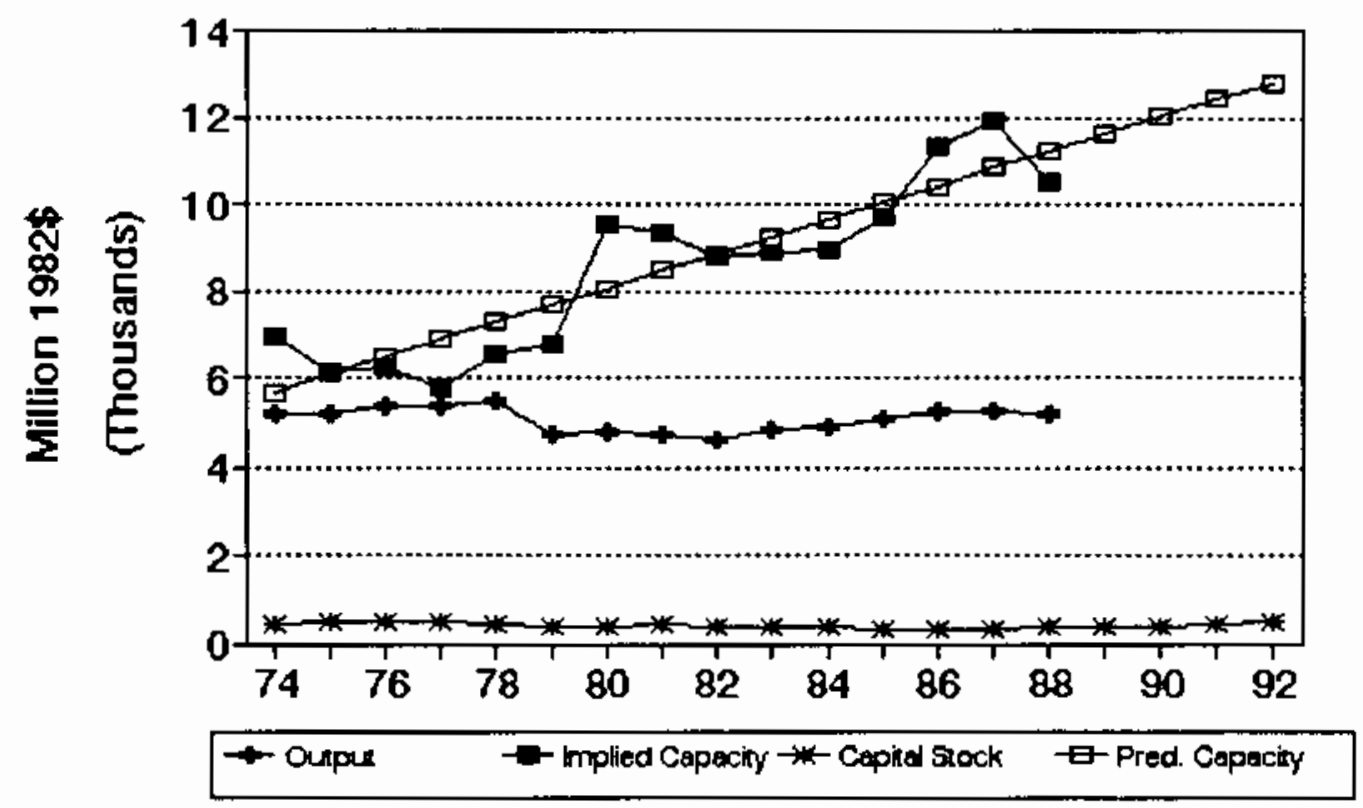


No. 91 SIC 2337 Wonen's and wisses' auits and coets

MILLIONS OF 1982 DOLLARS (EXCL, CU RATES)

$\begin{array}{lccccccc} & \text { Q4 } & \text { Ann. Implied Gross } & \text { Net } & \text { Pred. } & -\ldots . \ldots \\ \text { Gross } & \text { CU } & \text { CU } & \text { Prac. } & \text { In- } & \text { Cap. } & \text { Prac. } & \text { Emergency Capacity } \\ \text { Output } & \text { Rate } & \text { Rate } & \text { Capac. } & \text { vest. } & \text { Stk. } & \text { Capac. } & 3 \text { mon. } 6 \text { mon. }>6 \text { mon. }\end{array}$

\begin{tabular}{|c|c|c|c|c|c|c|c|c|c|c|}
\hline 1974 & 2,510 & $58 *$ & 60.1 & 4,178 & 7.7 & 231 & 5,695 & & & \\
\hline 1975 & 2,680 & $\pi$ & 65.7 & 4,077 & 12.7 & 227 & 5,639 & & & \\
\hline 1976 & 2,948 & $\pi$ & 7.2 & 3,817 & 8.7 & 218 & 5,540 & & & \\
\hline 1977 & 3,698 & 71 & 74.3 & 4,975 & 99.7 & 292 & 6,401 & & & \\
\hline 1978 & 3,618 & 81 & 77.6 & 4,662 & (34.1) & 237 & 5,764 & & & \\
\hline 1979 & 3,589 & 76 & 77.9 & 4,609 & 12.5 & 229 & 5,666 & & & \\
\hline 1980 & 3,644 & 77 & 76.5 & 4,764 & 36.5 & 242 & 5,817 & & & \\
\hline 1981 & 4,112 & 59 & 65.8 & 6,250 & 13.8 & 232 & 5,700 & & & \\
\hline 1982 & 4,928 & 52 & 54.1 & 9,114 & 95.5 & 295 & 6,427 & & & \\
\hline 1963 & 4,680 & $58 *$ & 55.5 & 8,431 & 25.6 & 287 & 6,334 & & & \\
\hline 1984 & 4,119 & $60 *$ & 60.1 & 6,849 & $(74.8)$ & 190 & 5,212 & & & \\
\hline 1985 & 3,661 & $62 *$ & 60.1 & 6,096 & 7.7 & 176 & 5,053 & & & \\
\hline 1986 & 3,868 & 60 & 60.4 & 6,406 & 16.8 & 170 & 4,979 & & & \\
\hline 1987 & $\begin{array}{c}-.- \\
4,010\end{array}$ & $\cdots$ & 68.4 & $\begin{array}{l}--- \\
5,866\end{array}$ & 17.3 & 166 & 4,937 & --- & $\cdot \cdot \cdot$ & $-\cdots$ \\
\hline 1988 & 4,202 & 60 & 64.9 & 6,478 & 17.3 & 163 & 4,900 & 14,179 & 15,593 & 15,885 \\
\hline 1989 & & & & & 17.3 & 160 & 4,868 & 14,086 & 15,490 & 15,780 \\
\hline 1990 & & & & & 17.3 & 157 & 4,639 & 14,006 & 15,400 & 15,689 \\
\hline 1991 & & & & & 17.6 & 156 & 4,818 & 13,942 & 15,332 & 15,620 \\
\hline 1992 & & & & & 17.6 & 154 & 4,799 & 13,888 & 15,273 & 15,559 \\
\hline
\end{tabular}

Capacity-Capital Stack Regression: Code 0 RSQ 0.080 Coefficients: Const 3018.2 Stock 11.6

Ave. Capacity/stack $(74-86): 24.5$ Ave. Heekly Hours (High) 46.5 Shift Factor 3.24 Ave Heekly Hours (Lou) 45.0 Shift factor 3.35 * Imputed Note: Output, Investment, and Capital Stock extrapolated for 1987 and 1988.

2337 Women's and misses' suits and coat

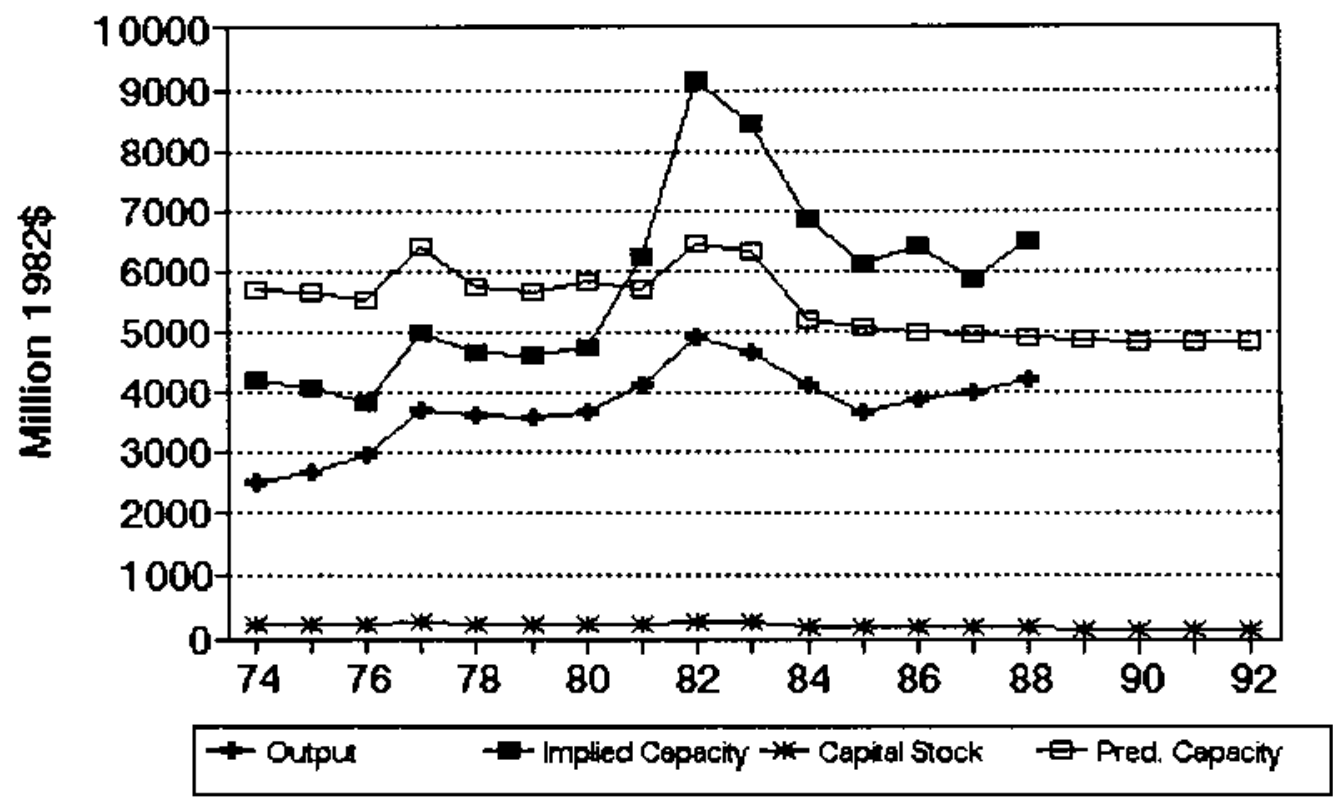


No. 92 sic 2339 Women's and misees' outerwear, n.e.c.

HILLIONS OF 1982 DOLLARS (EXCL. CU RATES)

$\begin{array}{lccccccc} & Q 4 & \text { Ann. Implied Gross } & \text { Net } & \text { Pred. } & \ldots \ldots \\ \text { Gross } & \text { CU } & \text { CU } & \text { Prac. } & \text { In- } & \text { Cap. } & \text { Prac, } & \text { Emergency Capacity } \\ \text { Output } & \text { Rate } & \text { Rate } & \text { Capac. } & \text { vest. } & \text { Stk. } & \text { Capac. } & 3 \text { mon. } 6 \text { mon. }>6 \text { mon. }\end{array}$

\begin{tabular}{|c|c|c|c|c|c|c|c|c|c|c|}
\hline 1974 & 3,502 & 72 & 72.7 & 4,816 & 32.0 & 261 & 5,512 & & & \\
\hline 1975 & 3,712 & 85 & 78.8 & 4,709 & 20.7 & 264 & 5,533 & & & \\
\hline 1976 & 3,786 & 46 & 60.9 & 6,223 & 23.5 & 270 & 5,565 & & & \\
\hline 1977 & 4,799 & 80 & 67.4 & 7,125 & 88.8 & 337 & 5,965 & & & \\
\hline 1978 & 4,036 & 80 & 80.1 & 6,161 & 17.7 & 333 & 5,943 & & & \\
\hline 1979 & 4,607 & 78 & 78.8 & 5,850 & 18.1 & 331 & 5,926 & & & \\
\hline 1980 & 5,267 & 86 & 83.0 & 6,349 & 42.9 & 351 & 6,044 & & & \\
\hline 1981 & 5,004 & 83 & 84.2 & 5,945 & 84.5 & 407 & 6,376 & & & \\
\hline 1982 & 4,827 & $\pi$ & 77.8 & 6,207 & 36.2 & 413 & 6,411 & & & \\
\hline 1983 & 4,868 & 84 & 80.5 & 6,047 & 46.7 & 427 & 6,495 & & & \\
\hline 1984 & 5,239 & 84 & 84.4 & 6,206 & $(14.3)$ & 383 & 6,235 & & & \\
\hline 1985 & 4,835 & 84 & 83.6 & 5,781 & 15.8 & 369 & 6,151 & & & \\
\hline 1986 & 4,890 & 63 & 70.8 & 6.908 & 34.8 & 372 & 6,171 & & & \\
\hline-- & $\cdots$ & $\because$ & $\because \cdot$ & $\cdots$ & --- & $\because-$ & $\because \cdots$ & $=\cdot-$ & --- & --- \\
\hline 1987 & 5,114 & $B 3$ & 75.6 & 6,767 & 31.0 & 372 & 0.172 & & & \\
\hline 1988 & 5,420 & 73 & 76.8 & 7,062 & 30.7 & 372 & 6,171 & 19,916 & 21,969 & 22,394 \\
\hline 1989 & & & & & 30.4 & 372 & 6,168 & 19,907 & 21,960 & 22,384 \\
\hline 1990 & & & & & 30.1 & 371 & 6,164 & 19,894 & 21,945 & 22,369 \\
\hline 1991 & & & & & 29.8 & 370 & 6,158 & 19,876 & 21,025 & 22,349 \\
\hline 1992 & & & & & 29.5 & 369 & 6,152 & 19,854 & 21,901 & 22,324 \\
\hline
\end{tabular}

Capacity-Capital Stock Regression: Code 0 RSO 0.240

Coefficients: Const 3970.7 stock 5.9

Ave. Capacity/Stock (74-86): 17.3

Ave. Weekly Hours (High) 41.6 Shift Factor 3.63 Ave Weekly Hours (Low) 40.9 Shift factor 3.69

Note: Output, Investment, and Capital stock extrapoloted for 1987 and 1988.

\section{Women's and misses' outerwear, n.e}

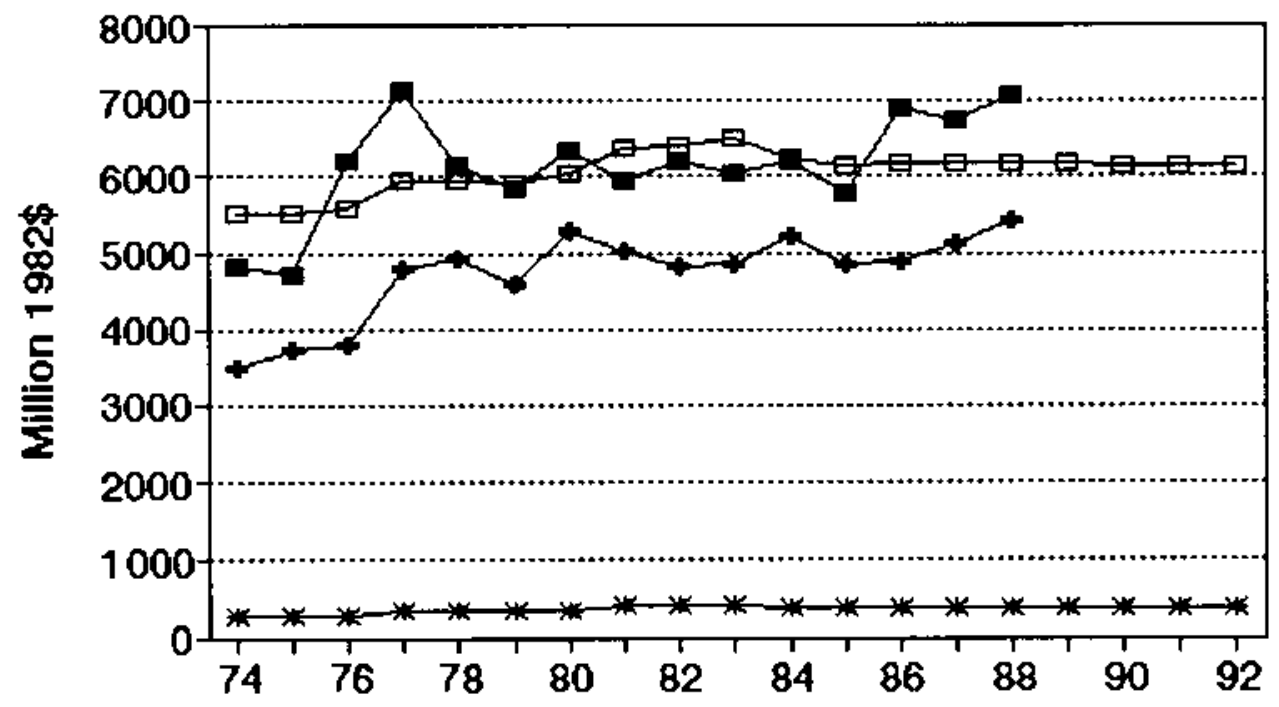

- Oupu $\quad-$ - Implied Cepacity $\rightarrow$ - Cepial Stock $\quad$ - Pred. Capacisy 


\section{No. 93 sIC 2341 Women's and children's underwear}

MILLIONS OF 1982 DOLLARS (EXCL. CU RATES)

$\begin{array}{lccccccc} & \text { Q4 } & \text { Am. } & \text { Implied } & \text { Gross } & \text { Net } & \text { Pred. } & \ldots \\ \text { Gross } & \text { CU } & \text { CU } & \text { Prac. } & \text { In- } & \text { Cap. } & \text { Prac. } & \text { Emergency Capacity } \\ \text { Output } & \text { Rate } & \text { Rate } & \text { Capac. } & \text { vest. } & \text { Stk. } & \text { Capac. } & 3 \text { mon. } 6 \text { mon. }>6 \text { mon. }\end{array}$

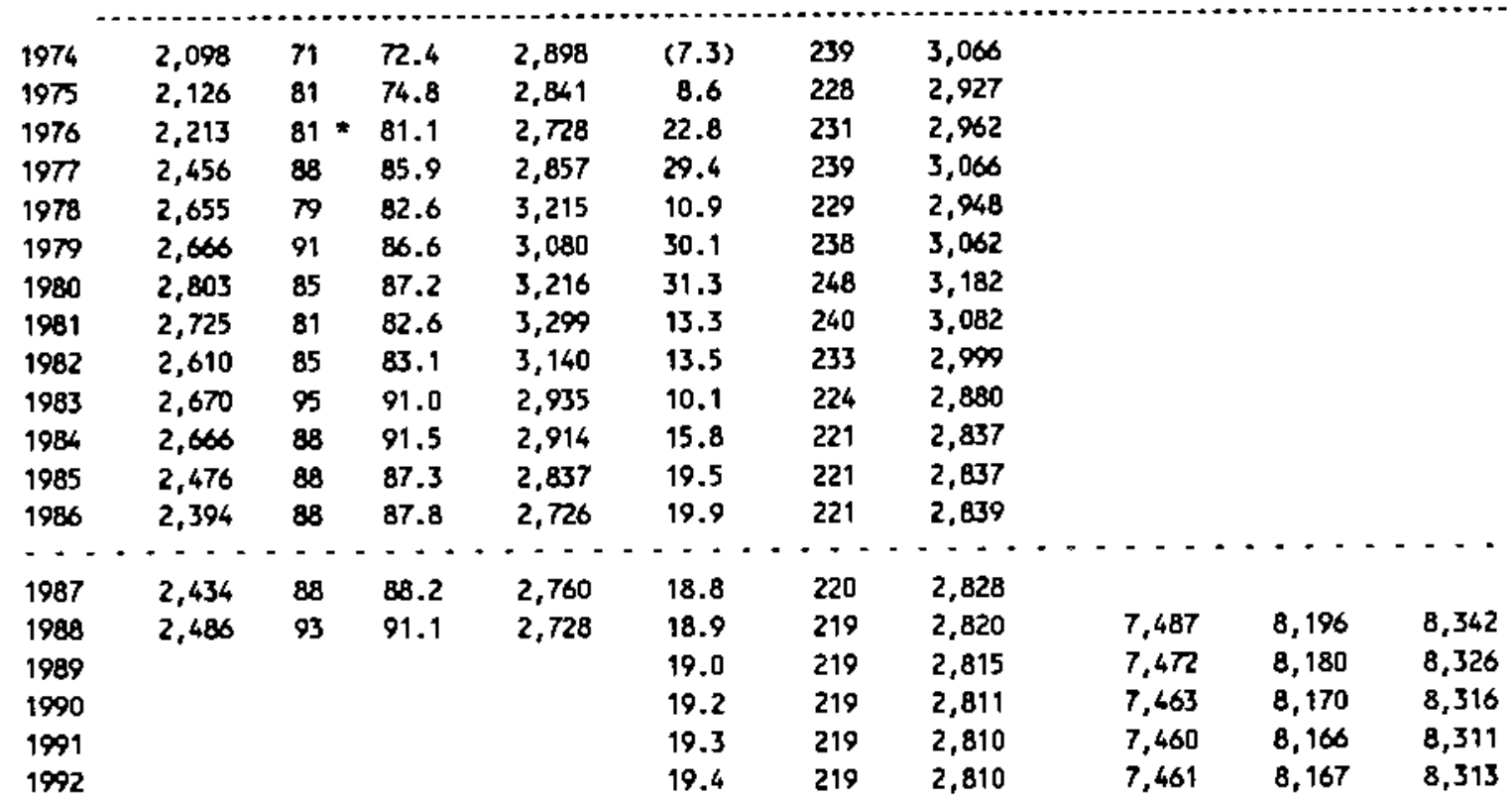

Capacity-Capital Stock Regression: code 0 RSO 0.330

Coefficients: Const 21.7 Stock $12.8 \quad$ Ave. Capacity/Stock $(74-86): 12.8$ Ave. Ueekly Hours (High) 51.0 Shift Factor 2.96 Ave Weekly Hours (Low) 42.7 Shift factor 3.53

- imputed Note: Output, Imvestment, and Capital Stock extrapolated for 1987 and 1988.

2341 Women's and children's underwear
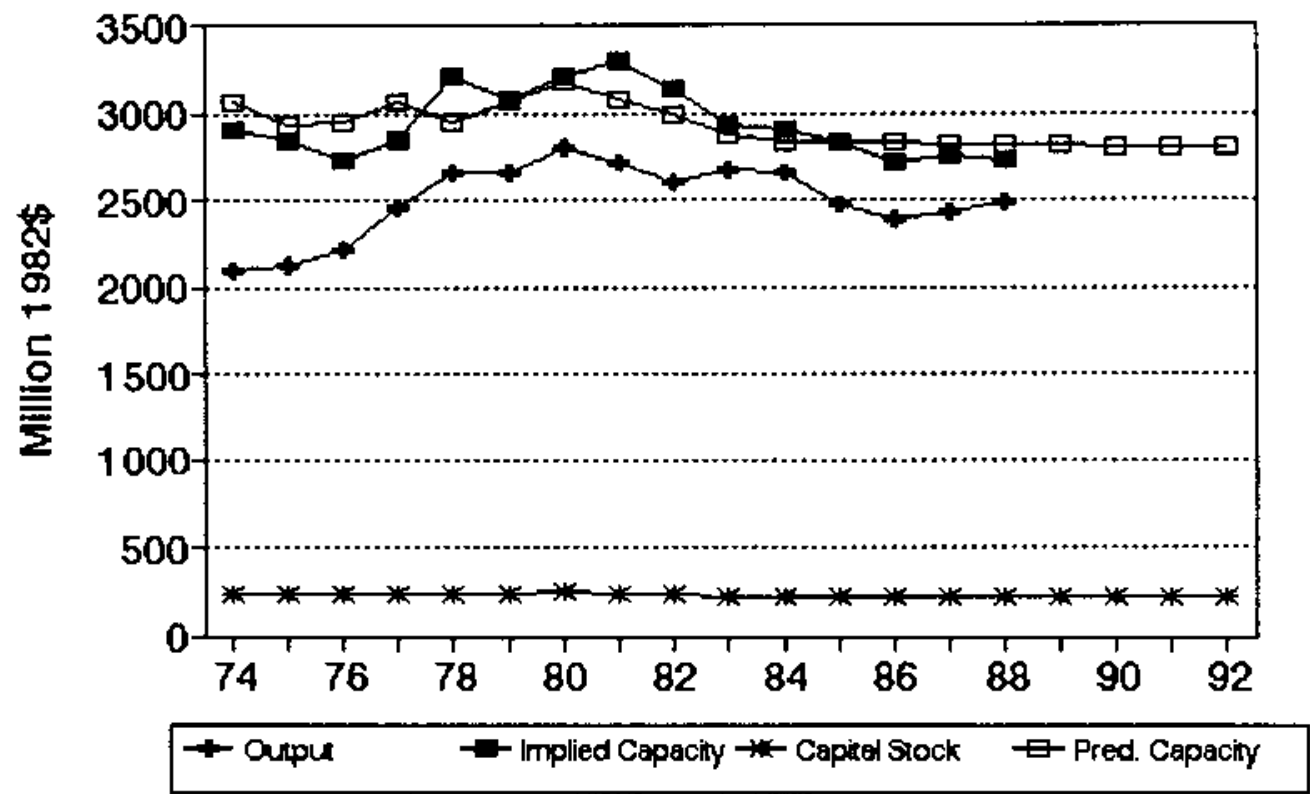


\section{NO. 94 SIC 2342 Brassieres and allied garmenta}

MILLIONS OF 1982 DOLLARS (EXCL. CU RATES)

$\begin{array}{lccccccc} & \text { Q4 } & \text { Ann. Implied Gross } & \text { Net } & \text { Pred. } & \ldots \\ \text { Gross } & \text { CU } & \text { CU } & \text { Prac. } & \text { In. } & \text { Cap. } & \text { Prac. } & \text { Emergency Capacity } \\ \text { Output } & \text { Rate } & \text { Rate } & \text { Capac. } & \text { vest. } & \text { Stk. } & \text { Capac. } & 3 \text { mon. } 6 \text { mon. }>6 \text { mon. }\end{array}$

\begin{tabular}{|c|c|c|c|c|c|c|c|c|c|c|}
\hline 1974 & 909 & 82 & 82.0 & 1,109 & 1.6 & 78 & 1,095 & & & \\
\hline 1975 & 865 & 81 & 81.4 & 1,063 & 2.5 & 73 & 1,096 & & & \\
\hline 1976 & 967 & 86 & 84.1 & 1,150 & 2.3 & 69 & 1,006 & & & \\
\hline 1977 & 890 & 79 & 81.9 & 1,087 & 6.1 & 67 & 1,097 & & & \\
\hline 1978 & 921 & 65 & 70.4 & 1,307 & 8.5 & 68 & 1,097 & & & \\
\hline 1979 & 802 & 84 & 76.9 & 1,043 & 4.4 & 65 & 1,097 & & & \\
\hline 1980 & 652 & 68 & 74.0 & 881 & 0.5 & 58 & 1,098 & & & \\
\hline 1981 & 682 & 83 & 77.4 & 881 & 4.4 & 56 & 1,009 & & & \\
\hline 1982 & 721 & 64 & 71.1 & 1,014 & 9.2 & 57 & 1,098 & & & \\
\hline 1983 & 771 & 77 & 72.1 & 1,069 & 3.9 & 54 & 1,099 & & & \\
\hline 1984 & 783 & 51 & 60.8 & 1,289 & 2.3 & 50 & 1,100 & & & \\
\hline 1985 & 718 & $66 \pm$ & 60.2 & 1,192 & 8.8 & 52 & 1,099 & & & \\
\hline 1986 & 819 & 71 & 69.0 & 1,186 & 5.2 & $5 t$ & 1,100 & & & \\
\hline $\begin{array}{l}--\cdot \\
1987\end{array}$ & $\begin{array}{l}--\cdot \\
800\end{array}$ & 81 & • & $\cdots$ & $\dot{*}-\overline{4}$ & 51 & 1,099 & $\cdots$ & $\cdot-$ & - - - \\
\hline 1988 & 776 & $87 *$ & 84.5 & 918 & 6.7 & 52 & 1,099 & 3,269 & 3,622 & 3,695 \\
\hline 1989 & & & & & 7.0 & 52 & 1,099 & 3,269 & 3,622 & 3,695 \\
\hline 1990 & & & & & 7.2 & 53 & 1,099 & 3,268 & 3,621 & 3,694 \\
\hline 1991 & & & & & 7.5 & 54 & $1,0 \infty 9$ & 3,268 & 3,621 & 3,694 \\
\hline 1992 & & & & & 7.7 & 55 & 1,009 & 3,267 & 3,620 & 3,693 \\
\hline
\end{tabular}

Capacity-Capital Stock Regression: Code 0 RSO 0.000

Coefficients: Const 1108.4 stock -0.2

Ave. Capacity/Stock $(74-86): 17.9$

Ave. Heekly Hours (High) 44.9 Shift Factor 3.36 Ave Weekly Hours (Low) 44.9 Shift Factor 3.36

* Imputed Note: Output, Imvestment, and Capital Stock extrapolated for 1987 and 1988.

\section{Brassieres and allied garments}

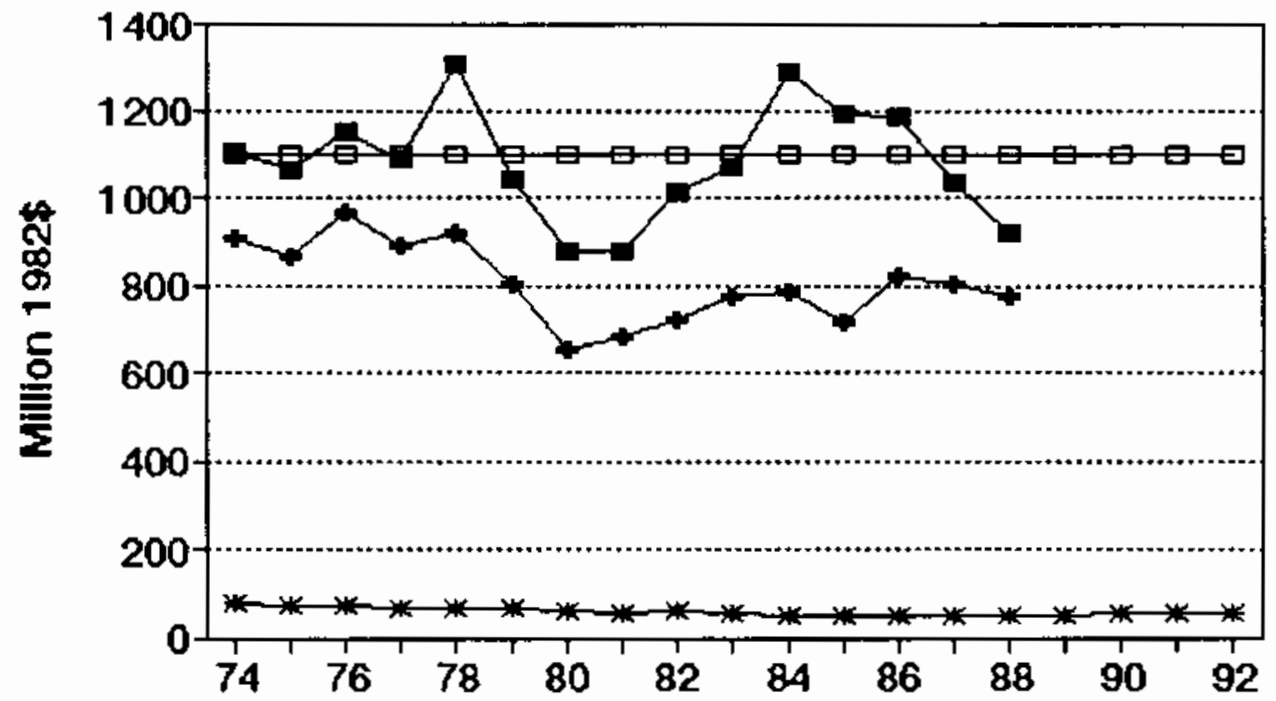

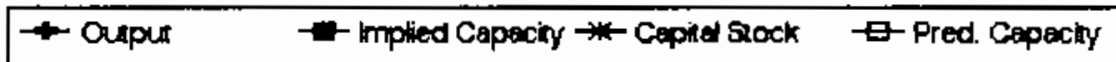




\section{No. 95 sIC 2351 Millinery}

\section{MILLIONS OF 1982 DOLLARS (EXCL. CU RATES)}

\begin{tabular}{|c|c|c|c|c|c|c|c|}
\hline & $\begin{array}{l}\text { Q4 } \\
\mathrm{CU}\end{array}$ & $\begin{array}{c}\text { Ann. } \\
\text { Cu }\end{array}$ & $\begin{array}{l}\text { Impl ied } \\
\text { Prac. }\end{array}$ & $\begin{array}{c}\text { Gross } \\
\text { In- }\end{array}$ & $\begin{array}{l}\text { Net } \\
\text { Cap. }\end{array}$ & $\begin{array}{l}\text { Pred. } \\
\text { Prac. }\end{array}$ & Energency Capacity \\
\hline & Rate & Oata & Caner & & th & actc & \\
\hline
\end{tabular}

\begin{tabular}{|c|c|c|c|c|c|c|c|c|c|c|}
\hline 1974 & 82 & 77 & 77.0 & 105 & $(0.3)$ & 3 & 126 & & & \\
\hline 1975 & 123 & $\pi$ & 7.0 & 159 & 1.3 & 4 & 126 & & & \\
\hline 1976 & 120 & $\pi$ & 7.0 & 156 & 0.3 & 3 & 126 & & & \\
\hline 1977 & 98 & $\pi$ & 77.0 & 127 & 12.0 & 13 & 136 & & & \\
\hline 1978 & 106 & $\pi$ & 77.0 & 138 & 2.0 & 12 & 136 & & & \\
\hline 1979 & 103 & $\pi$ & 7.0 & 134 & $(9.4)$ & 2 & 125 & & & \\
\hline 1980 & 90 & 77 & 77.0 & 117 & $(0.8)$ & 1 & 124 & & & \\
\hline 1981 & 87 & $\pi$ & 7.0 & 113 & 1.4 & 2 & 125 & & & \\
\hline 1982 & 100 & $\pi$ & 7.0 & 130 & 0.3 & 2 & 125 & & & \\
\hline 1983 & 94 & $\pi$ & 77.0 & 122 & 0.0 & 2 & 125 & & & \\
\hline 1984 & 88 & $\pi$ & $\pi .0$ & 115 & 0.1 & 2 & 124 & & & \\
\hline 1985 & 95 & 77 & 77.0 & 123 & 0.2 & 2 & 124 & & & \\
\hline 1986 & 83 & $\pi$ & 77.0 & 107 & 0.2 & 2 & 124 & & & \\
\hline - - - & $\because$ & $-\cdot$ & - - & $\because$ & $-7-$ & $\therefore$ & $\because-$ & - & --1 & $\cdots$ \\
\hline 1987 & 82 & 77 & 77.0 & 107 & 0.7 & 2 & 125 & & & \\
\hline 1988 & 82 & 77 & 77.0 & 106 & 0.7 & 3 & 125 & 195 & 212 & 215 \\
\hline 1989 & & & & & 0.7 & 3 & 126 & 196 & 212 & 216 \\
\hline 1990 & & & & & 0.7 & 4 & 126 & 197 & 213 & 216 \\
\hline 1991 & & & & & 1.0 & 4 & 127 & 198 & 214 & 218 \\
\hline 1992 & & & & & 1.0 & 5 & 128 & 199 & 215 & 219 \\
\hline
\end{tabular}

Capacity-Capital Stock Regression: Code 0 R50 0.060 Coefficients: Const 122.4 stock 1.1

Ave. Capecity/stock $(74-86): 33.2$ Ave. Weekly Hours (High) 88.0 Shift Factor 1.71 Ave Heekly Hours (Low) 86.3 Shift Factor 1.75 Note: Output, Investment, and Capital Stock extrapolated for 1987 and 1988.

\section{Millinery}

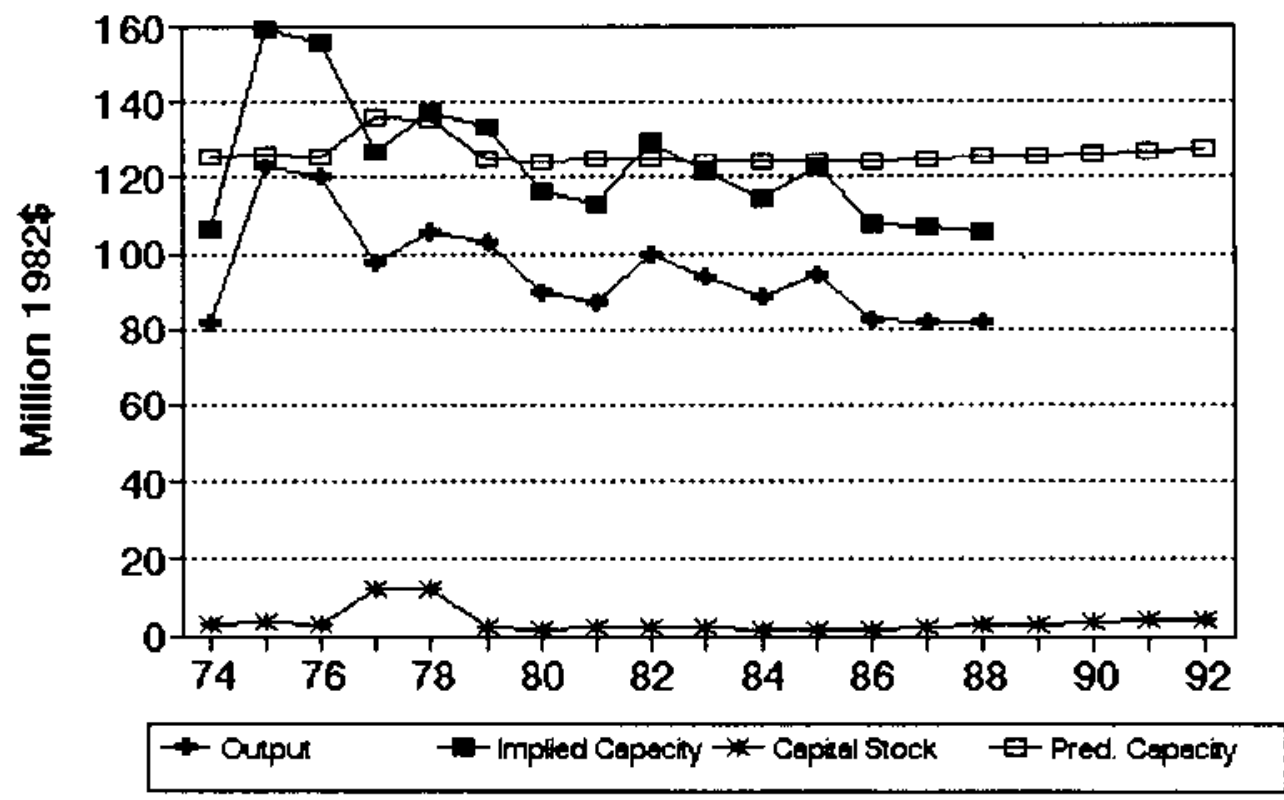




\section{No. 96 BIC 2352 Hats and caps except millinery}

MILLIONS OF 1982 DOLLARS (EXCL. CU RATES)

$\begin{array}{lccccccc} & \text { Q4 } & \text { Ann. Implied } & \text { Gross } & \text { Net } & \text { Pred. } & \ldots . . \\ \text { Gross } & \text { CU } & \text { CU } & \text { Prac. } & \text { In- } & \text { Cap. } & \text { Prac. } & \text { Energency Capacity } \\ \text { Output Rate Rate } & \text { Capac. } & \text { vest. } & \text { Stk. } & \text { Capac. } & 3 \text { mon. } 6 \text { mon. }>6 \text { mon. }\end{array}$

\begin{tabular}{|c|c|c|c|c|c|c|c|c|c|c|}
\hline 1974 & 373 & 84 & 84.0 & 445 & $(8.0)$ & 31 & 438 & & & \\
\hline 1975 & 371 & 77 & 79.6 & 466 & 10.1 & 37 & 532 & & & \\
\hline 1976 & 362 & 64 & 68.9 & 525 & 5.8 & 39 & 564 & & & \\
\hline 1977 & 388 & 71 & 68.4 & 567 & 4.6 & 40 & 578 & & & \\
\hline 1978 & 436 & 67 & 68.5 & 636 & 4.0 & 41 & 582 & & & \\
\hline 1979 & 448 & 88 & 80.1 & 560 & 9.3 & 46 & 663 & & & \\
\hline 1980 & 609 & 85 & 86.1 & 707 & 17.8 & 59 & 867 & & & \\
\hline 1981 & 559 & 73 & 77.5 & 722 & 10.1 & 65 & 950 & & & \\
\hline 1992 & 429 & 60 & 4.9 & 661 & $(6.4)$ & 55 & 796 & & & \\
\hline 1983 & 421 & 68 & 65.0 & 648 & 1.6 & 52 & 762 & & & \\
\hline 1984 & $4 \pi 7$ & 45 & 53.6 & 890 & 4.1 & 53 & 765 & & & \\
\hline 1985 & 463 & $39 *$ & 41.3 & 1,120 & 8.5 & 57 & 831 & & & \\
\hline 1986 & 483 & 41 & 40.3 & 1,198 & 3.4 & 56 & 815 & & & \\
\hline$\cdots$ & -- & $\cdots$ & 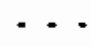 & $\because-$ & $-\cdots$ & $\cdot$ & $=-$ & $\cdots$ & $\cdots$ & -- \\
\hline 1987 & 496 & 40 & 40.4 & 1,229 & 5.6 & 57 & 835 & & & \\
\hline 1988 & 514 & $39 *$ & 39.5 & 1,302 & 5.7 & 59 & 855 & 1,326 & 1,442 & 1,465 \\
\hline 1989 & & & & & 5.7 & 60 & 874 & 1,356 & 1,474 & 1,498 \\
\hline 1990 & & & & & 5.8 & 61 & 893 & 1,385 & 1,505 & 1,530 \\
\hline 1991 & & & & & 5.8 & 62 & 911 & 1,413 & 1,536 & 1,561 \\
\hline 1992 & & & & & 5.9 & 63 & 928 & 1,440 & 1,565 & 1,591 \\
\hline
\end{tabular}

Capacity-Capital Stock Regression: Code 0 RSO 0.430

Coefficients: Const -31.0 stock 15.1

Ave. Capacity/Stock $(74-86): 14.5$

Ave. Heekly Hours (High) 88.0 Shift Factor 1.71 Ave Weekly Hours (LOW) 86.3 Shift Factor 1.75

* Imputed Note: Output, Investment, and Capital stock extrapolated for 1987 and 1988.

\section{Hats and caps except millinery}
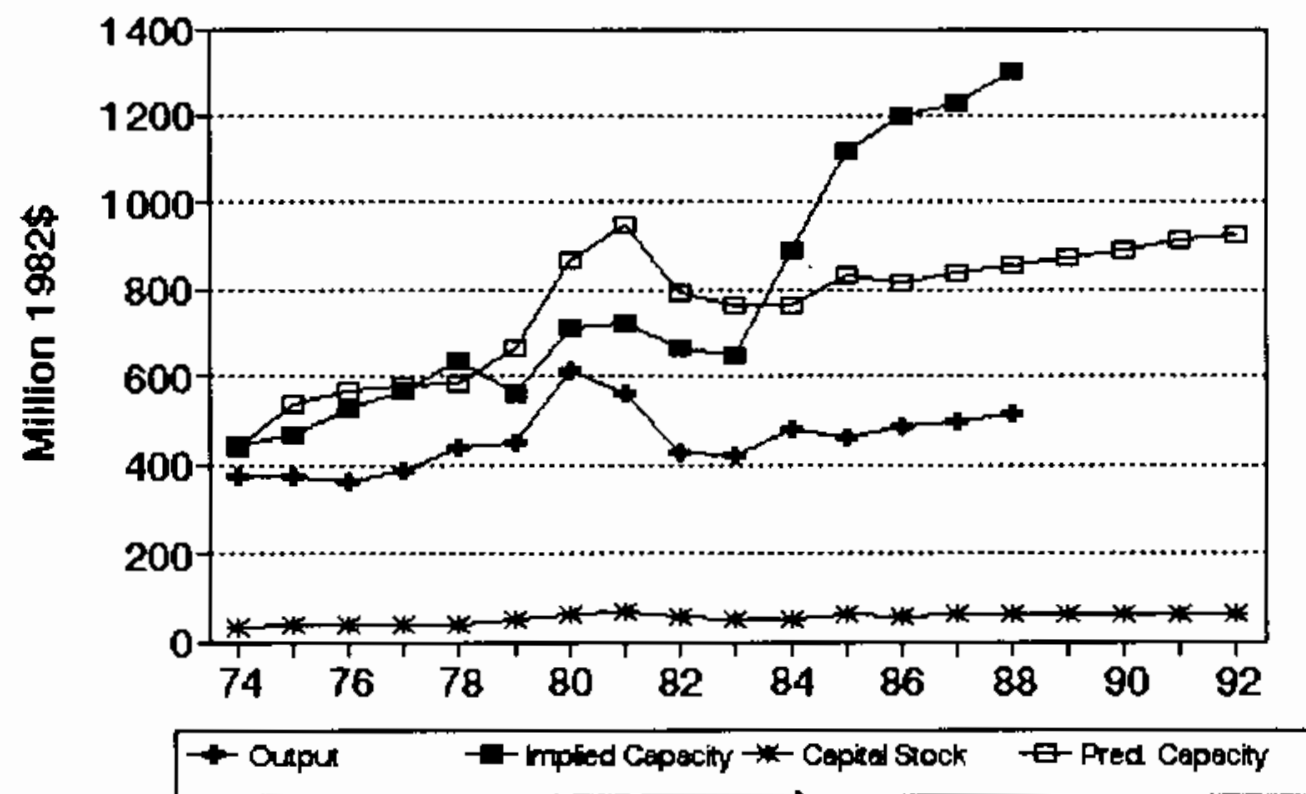


\section{No. 97 sIC 2361 Children's dresses and blouses}

\section{MILLIONS OF 1982 DOLLARS (EXCL. CU RATES)}

$\begin{array}{lccccccc} & Q 4 & \text { Ann. Implied } & \text { Gross } & \text { Met } & \text { Pred. } & -\ldots \ldots \ldots \\ \text { Gross } & \text { CU } & \text { CU } & \text { Prac. } & \text { In- } & \text { Cap. } & \text { Prac. } & \text { Emergency Capacity } \\ \text { Output Rate } & \text { Rate } & \text { Capac. } & \text { vest. } & \text { Stk. } & \text { Capac. } & 3 \text { mon. } 6 \text { mon. }>6 \text { mon. }\end{array}$

\begin{tabular}{|c|c|c|c|c|c|c|c|c|c|c|}
\hline 1974 & 847 & $62 *$ & 62.8 & 1,348 & 8.6 & 106 & 1,304 & & & \\
\hline 1975 & 797 & $60 \%$ & 59.5 & 1,339 & 8.4 & 107 & 1,303 & & & \\
\hline 1976 & 847 & $\pi$ & 70.6 & 1,200 & 7.6 & 106 & 1,303 & & & \\
\hline 1977 & 1,049 & 76 & 76.8 & 1,367 & 16.2 & 113 & 1,289 & & & \\
\hline 1978 & 1,092 & 75 & 75.5 & 1,446 & (27.1) & 80 & 1,359 & & & \\
\hline 1979 & 1,045 & 74 & 74.4 & 1,405 & 9.8 & 83 & 1,352 & & & \\
\hline 1980 & 1,064 & 93 & 85.8 & 1,239 & $(1.9)$ & 74 & 1,370 & & & \\
\hline 1981 & 1,150 & 84 & 87.4 & 1,315 & 1.5 & 70 & 1,380 & & & \\
\hline 1982 & 1,420 & 80 & 81.2 & 1,748 & 34.3 & 94 & 2,033 & & & \\
\hline 1983 & 1,445 & 61 & 68.2 & 2,120 & 4.7 & 89 & 2,044 & & & \\
\hline 1984 & 1,346 & 61 & 61.4 & 2,193 & $(1.2)$ & 79 & 2,065 & & & \\
\hline 1985 & 1,275 & 61 & 60.7 & 2,101 & 11.9 & 82 & 2,059 & & & \\
\hline 1986 & 1,277 & $61 *$ & 60.6 & 2,106 & 5.4 & 78 & 2,066 & & & \\
\hline$\cdots$ & $-\cdots$ & - - - & $-\cdot-$ & $-\cdots$ & -- & $\cdot$ & $\cdots \cdot$ & $-\cdot$ & -- & $\cdots$ \\
\hline 1987 & 1,315 & 61 & 60.9 & 2,160 & 7.9 & 77 & 2,068 & & & \\
\hline 1988 & 1,368 & $62 *$ & 61.3 & 2,230 & 7.9 & 76 & 2,070 & 6,866 & 7,645 & 7,806 \\
\hline 1989 & & & & & 7.9 & 76 & 2,071 & 6,870 & 7,650 & 7,811 \\
\hline 1990 & & & & & 7.9 & 75 & 2,072 & 6,874 & 7,654 & 7,815 \\
\hline 1991 & & & & & 8.9 & 76 & 2,071 & 6,870 & 7,650 & 7,811 \\
\hline 1992 & & & & & 8.9 & 76 & 2,070 & 6,867 & 7,647 & 7,808 \\
\hline
\end{tabular}

Capacity-Capital stock Regression: Code -1 RSQ 0.910

Coefficients: Const 1525.4 Stock -2.1 D(82-86) $704.1 \quad$ Ave. Capacity/Stock (74-86): 18.1 Ave. Weekly Hours (High) 40.0 Shift Factor 3.77 Ave Weekly Hours (LOW) 40.0 Shift Factor 3.77

* Imputed Note: Output, Investment, and Capital Stock extrapolated for 1987 and 1988.

\section{Children's dresses and blouses}

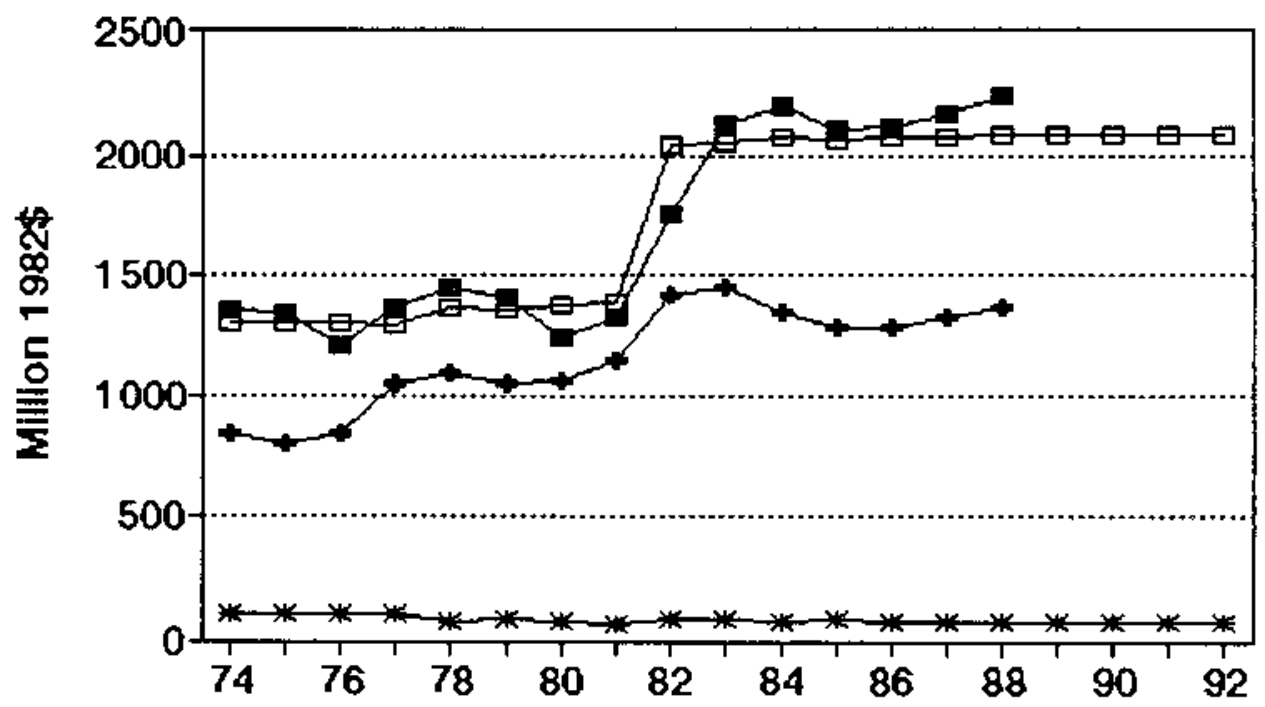

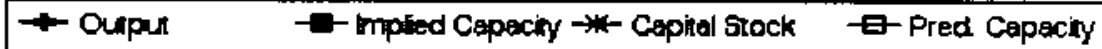




\section{No. 98 sIC 2363 Children's coats and suits}

MILLIONS OF 1982 DOLLARS (EXCL. CU RATES)

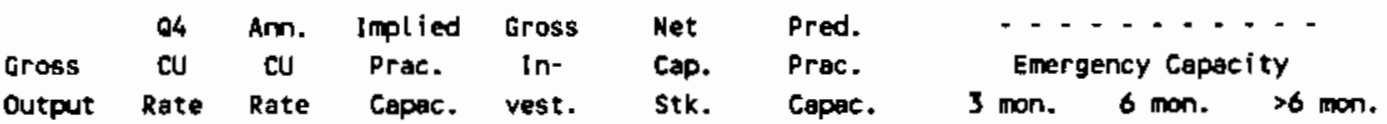

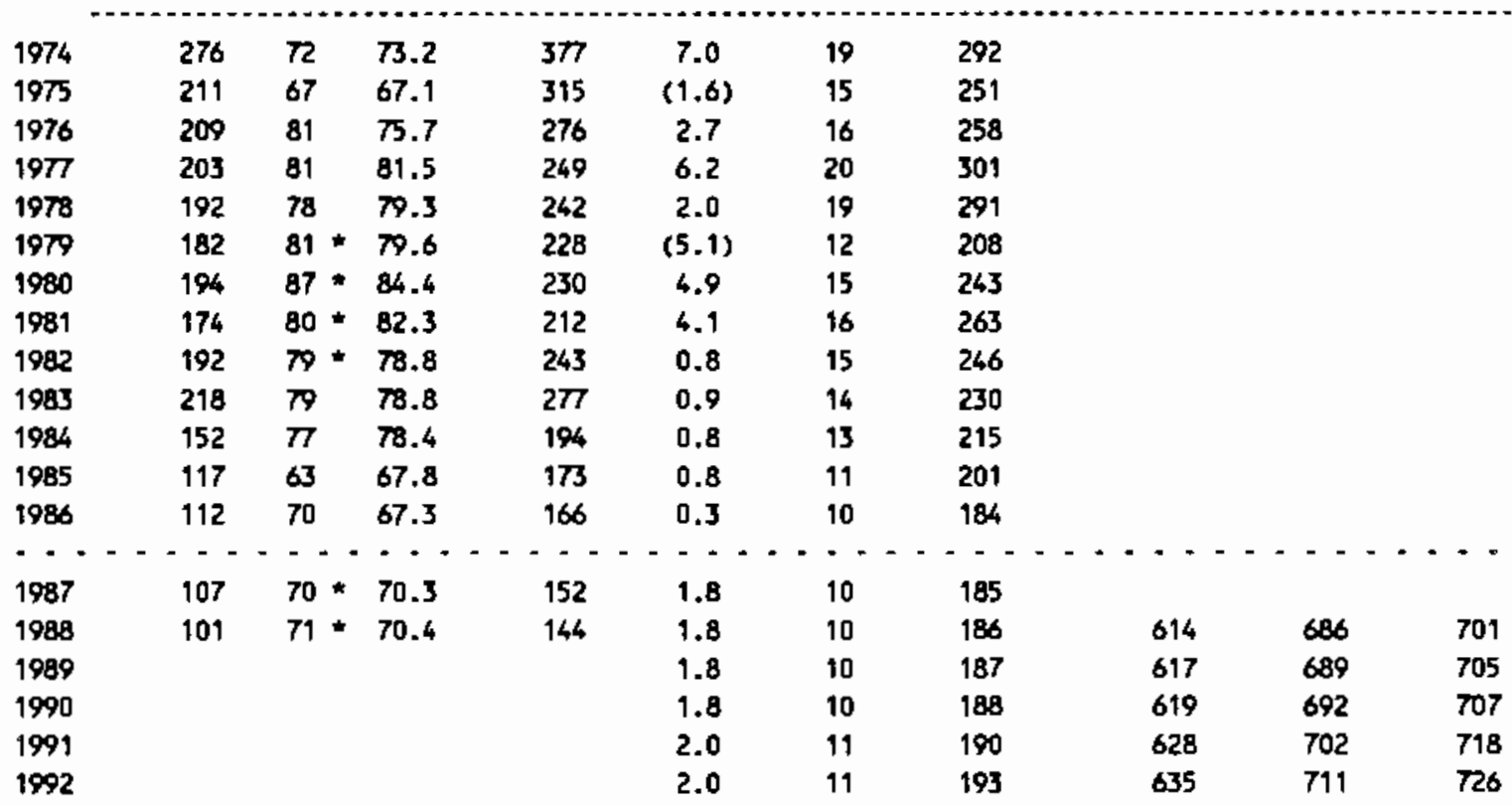

Capecity-Cepital stock Regression: Code 0 RSO 0.400 Coefficients: Const 61.3 stock 12.3 Ave. Weekly Hours (High) 40.0 shift Factor 3.77 Ave Yeekly Hours (Low) 40.0 shift Factor 3.77 - Inputed Note: Output, Imvestment, and Capital Stock extrapolated for 1987 and 1988.

\section{Children's coats and suits}

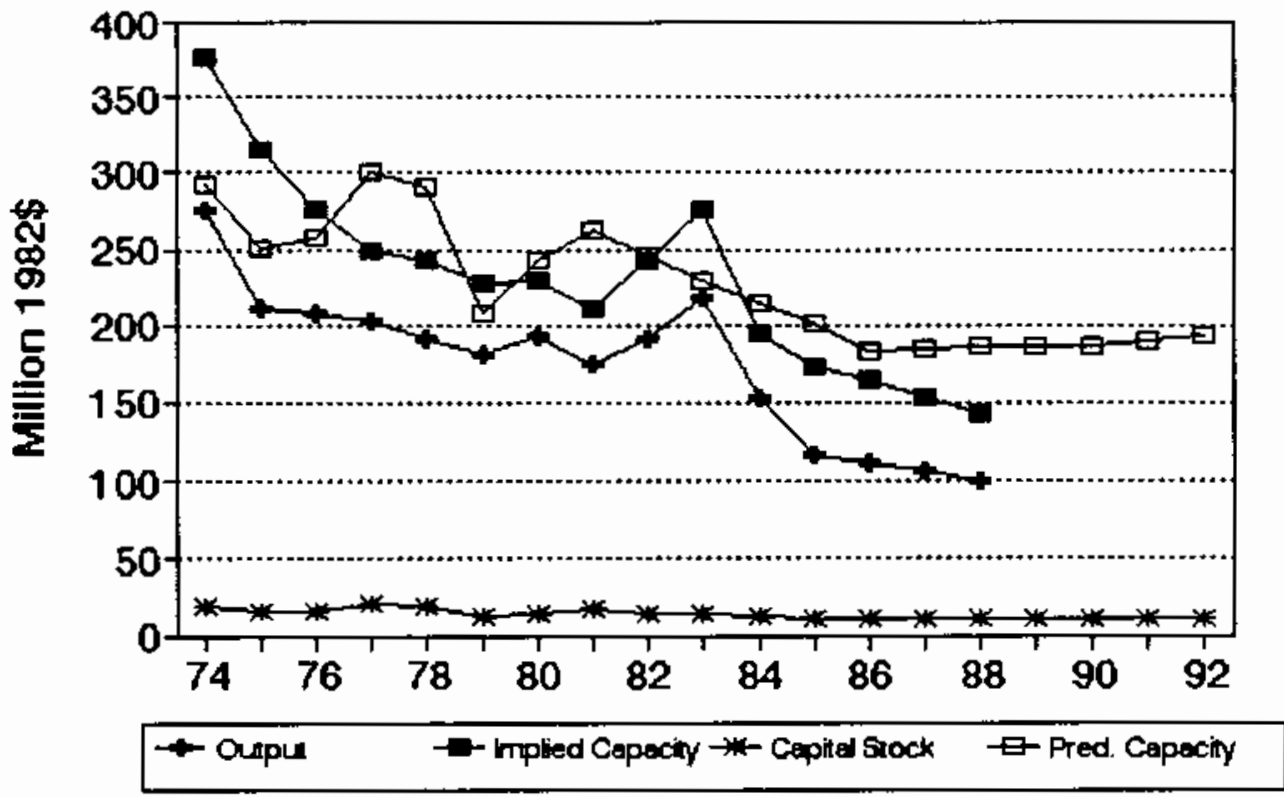


No. 99 sIC 2369 Children's outerwear, n.e.c.

MILLIONS OF 1982 DOLLARS (EXCL. CU RATES)

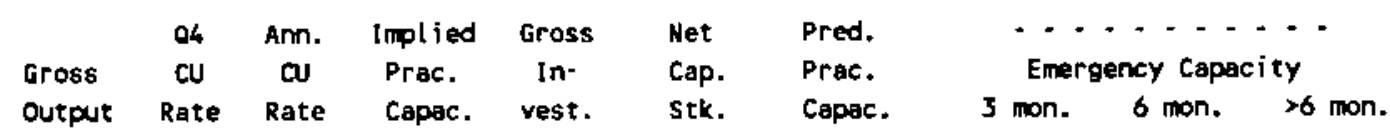

\begin{tabular}{|c|c|c|c|c|c|c|c|c|c|c|}
\hline 1974 & 866 & 80 & 84.1 & 1,030 & 4.4 & 76 & 1,126 & & & \\
\hline 1975 & 847 & 60 & 61.5 & 1,377 & 6.7 & 7 & 1,127 & & & \\
\hline 1976 & 906 & 83 & 74.2 & 1,220 & 4.7 & $\pi$ & 1,116 & & & \\
\hline 1977 & 937 & 85 & 85.7 & 1,093 & 17.2 & 84 & 1,169 & & & \\
\hline 1978 & 1,056 & 84 & 84.9 & 1,244 & 9.5 & 86 & 1,177 & & & \\
\hline 1979 & 862 & 93 & 89.8 & 960 & $(19.9)$ & 60 & 1,038 & & & \\
\hline 1980 & 1,030 & 98 & 95.9 & 1,074 & 17.0 & 71 & 1,097 & & & \\
\hline 1981 & 855 & $\pi$ & 83.7 & 1,021 & 8.7 & 73 & 1,108 & & & \\
\hline 1982 & 1,093 & 79 & 76.7 & 1,425 & 42.8 & 106 & 1,287 & & & \\
\hline 1983 & 1,060 & 100 & 91.4 & 1,159 & 10.3 & 106 & 1,288 & & & \\
\hline 1984 & 1,194 & 100 & 100.0 & 1,194 & 6.1 & 102 & 1,268 & & & \\
\hline 1985 & 1,284 & 100 & 98.1 & 1,310 & 21.6 & 113 & 1,328 & & & \\
\hline 1986 & 1,356 & $100 *$ & 99.7 & 1,361 & 13.2 & 115 & 1,340 & & & \\
\hline$\because$ & $\cdots$ & -- & $-\cdots$ & $\cdots$ & $\cdots$ & 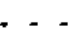 & $\cdots-$ & $\cdots$ & -- & $-\cdots$ \\
\hline 1987 & 1,392 & 100 & 100.0 & 1,392 & 18.4 & 123 & 1,381 & & & \\
\hline 1988 & 1,441 & 100 & 100.0 & 1,441 & 19.4 & 130 & 1,423 & 4,793 & 5,268 & 5,367 \\
\hline 1989 & & & & & 20.5 & 138 & 1,467 & 4,943 & 5,433 & 5,534 \\
\hline 1990 & & & & & 21.5 & 147 & 1,513 & 5,097 & 5,602 & 5,706 \\
\hline 1991 & & & & & 22.5 & 155 & 1,560 & 5,256 & 5,777 & 5,884 \\
\hline 1992 & & & & & 23.6 & 164 & 1,609 & 5,419 & 5,055 & 6,066 \\
\hline
\end{tabular}

Capacity-Capital Stock Regression: Code 0 RSO 0.440

Coefficients: Const 704.1 stock 5.5 Ave. Capacity/Stock $(74-86): 13.5$ Ave. Weekly Hours (High) 40.0 Shift Factor 3.77 Ave Weekly Hours (LOW) 40.0 Shift factor 3.77 - Imputed Note: Output, Investment, and Capital Stock extrapolated for 1987 and 1988.

\section{Children's outerwear, n.e.c.}
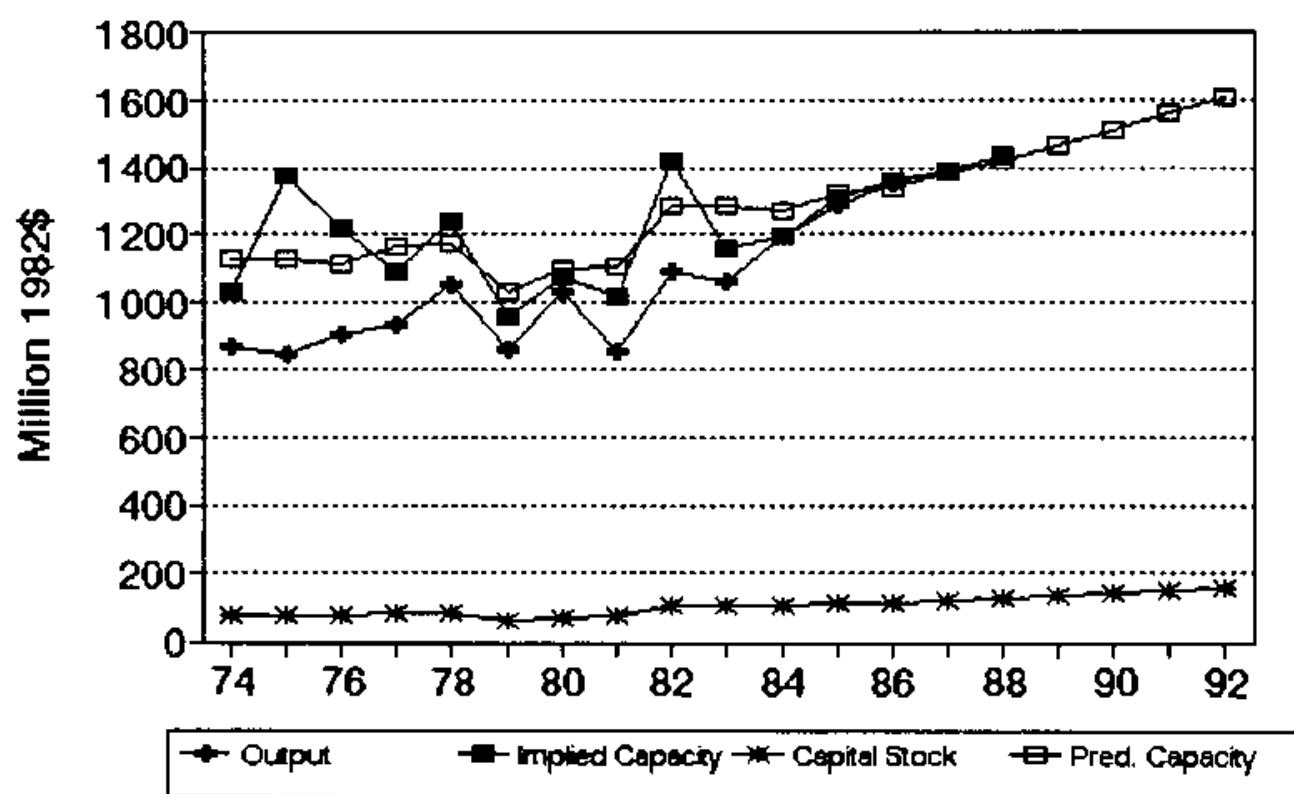


\section{หo. 100 sIC 2371 Fur gooda}

MILLIONS OF 1982 DOLLARS (EXCL. CU RATES)

$\begin{array}{lccccccc} & \text { Q } & \text { Ann. Implied Gross } & \text { Net } & \text { Pred. } & -\ldots \ldots \\ \text { Gross } & \text { CU } & \text { CU } & \text { Prac. } & \text { In- } & \text { Cap. } & \text { Prac. } & \text { Emergency Capacity } \\ \text { Qutput } & \text { Rate } & \text { Rate } & \text { Capac. } & \text { vest. } & \text { Stk. } & \text { Capac. } & 3 \text { mon. } 6 \text { mon. } 96 \text { mon. }\end{array}$

\begin{tabular}{|c|c|c|c|c|c|c|c|c|c|c|}
\hline 1974 & 523 & 64 & 65.2 & 803 & (5.1) & 24 & B61 & & & \\
\hline 1975 & 611 & 79 & 71.3 & 858 & $(2.6)$ & 19 & BSt & & & \\
\hline 1976 & 485 & 73 & $\pi .4$ & 643 & (3.6) & 14 & 861 & & & \\
\hline 1977 & 723 & 78 & 76.6 & 943 & $(1,1)$ & 12 & BS1 & & & \\
\hline 1978 & 719 & 78 & 78.2 & 919 & $(1.0)$ & 10 & 881 & & & \\
\hline 1979 & 781 & 80 & 79.3 & 985 & 5.3 & 14 & 861 & & & \\
\hline 1980 & 673 & 73 & 75.6 & 891 & 4.3 & 16 & 861 & & & \\
\hline 1981 & 613 & 73 & 73.1 & 838 & 3.2 & 17 & 861 & & & \\
\hline 1982 & 410 & 69 & 70.2 & 585 & 3.4 & 18 & 560 & & & \\
\hline 1983 & $38 B$ & 73 & 71.3 & 544 & 1.5 & 17 & 526 & & & \\
\hline 1984 & 343 & 73 & 73.6 & 465 & $(7.4)$ & 9 & 493 & & & \\
\hline 1985 & 327 & 73 & 72.4 & 451 & $(0.2)$ & 8 & 459 & & & \\
\hline 1986 & 302 & 70 & 71.0 & 425 & 0.2 & 7 & 426 & & & \\
\hline$-\cdot-$ & $\cdots$ & 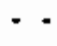 & $\cdots$ & $\cdots$ & -- & - & -- & $\cdots$ & $\because-$ & $-\cdots$ \\
\hline 1987 & 295 & 73 & 72.0 & 409 & 2.0 & 8 & 392 & & & \\
\hline 1988 & 285 & 76 & 74.9 & 381 & 2.3 & 10 & 359 & 1,197 & 1,326 & 1,353 \\
\hline 1989 & & & & & 2.7 & 11 & 325 & 1,085 & 1,202 & 1,226 \\
\hline 1990 & & & & & 3.0 & 13 & 292 & 973 & 1,078 & 1,100 \\
\hline 1991 & & & & & 3.4 & 15 & 258 & 862 & 955 & 974 \\
\hline 1992 & & & & & 3.7 & 17 & 225 & 750 & 831 & 847 \\
\hline
\end{tabular}

Capacity-Capital Stock Regression: Code -2 RSQ 0.840 Coefficients: Const 860.9 Time -33.5 Ave. Capacity/Stock (74-86): 50.9 Ave, Heekly Hours (High) 40.0 Shift Factor 3.77 Ave Neekly Hours (LOW) 40.0 Shift Factor 3.77 Note: Output, Investment, and Capital Stock extrapolated for 1987 and 1988.

\section{Fur goods}

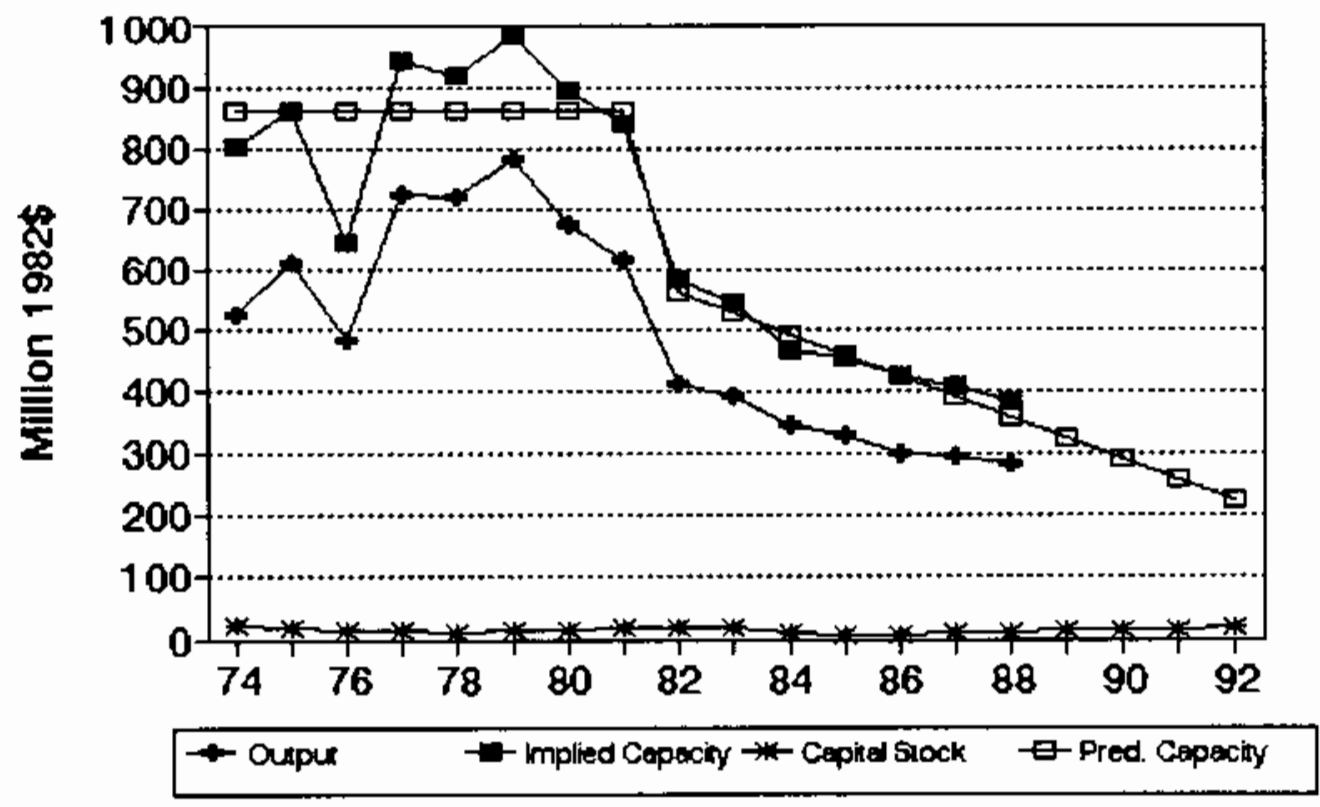




\section{No. 101 sIC 2381 Fabric dress and work gloves}

MILLIONS OF 1982 DOLLARS (EXCL. CU RATES)

$\begin{array}{lccccccc} & 04 & \text { Ann. Implied } & \text { Gross } & \text { Net } & \text { Pred. } & \ldots \ldots \\ \text { Gross } & \text { dU } & \text { CU } & \text { Prac. } & \text { In- } & \text { Cap. } & \text { Prac. } & \text { Emergency Capacity } \\ \text { Output } & \text { Rate } & \text { Rate } & \text { Capac. } & \text { vest. } & \text { 5tk. } & \text { Capac. } & 3 \text { mon. } 6 \text { mon. }>6 \text { mon. }\end{array}$

\begin{tabular}{|c|c|c|c|c|c|c|c|c|c|c|}
\hline 1974 & 480 & 59 & 59.7 & 805 & 7.9 & 40 & 627 & & & \\
\hline 1975 & 412 & 60 & 58.5 & 705 & 5.2 & 42 & 681 & & & \\
\hline 1976 & 450 & 75 & 69.3 & 648 & 5.8 & 44 & 752 & & & \\
\hline 1977 & 436 & 53 & 61.6 & 708 & 3.7 & 44 & 738 & & & \\
\hline 1978 & 473 & 61 & 58.1 & 815 & 4.7 & 45 & 802 & & & \\
\hline 1979 & 402 & 78 & 71.7 & 561 & $(6.1)$ & 36 & 506 & & & \\
\hline 1980 & 303 & 74 & 75.5 & 402 & $(0.1)$ & 34 & 423 & & & \\
\hline 1981 & 266 & 75 & 74.7 & 356 & 1.5 & 33 & 392 & & & \\
\hline 1982 & 219 & 68 & 70.4 & 311 & $(1.0)$ & 30 & 282 & & & \\
\hline 1983 & 229 & 6 & 66.7 & 343 & 7.0 & 34 & 416 & & & \\
\hline 1984 & 252 & 87 & 79.6 & 317 & 0.3 & 31 & 332 & & & \\
\hline 1985 & 224 & 81 & 82.8 & 271 & $(6.2)$ & 23 & 269 & & & \\
\hline 1986 & 172 & 78 & 79.0 & 217 & 0.4 & 21 & 219 & & & \\
\hline$\cdots$ & $\cdots+\cdots$ & -- & $-\cdot$ & $-\cdot-$ & --- & - & -- & $-\cdot$ & -- & - - \\
\hline 1987 & 165 & 77 & 77.5 & 213 & 2.1 & 22 & 225 & & & \\
\hline 1988 & 157 & 69 & 72.0 & 218 & 2.1 & 22 & 231 & 698 & 738 & 754 \\
\hline 1989 & & & & & 2.1 & 22 & 236 & 716 & 757 & 773 \\
\hline 1990 & & & & & 2.1 & 22 & 242 & 732 & 774 & 791 \\
\hline 1991 & & & & & 2.4 & 23 & 258 & 782 & $B 27$ & 845 \\
\hline 1992 & & & & & 2.4 & 23 & 274 & 828 & 875 & 895 \\
\hline
\end{tabular}

Capacity-Capital stock Regression: Code -1 RSO 0.900 Coefficients: Const -712.3 Stock $33.6 \quad \mathrm{D}(85-86) 212.5$ Ave. Capacity/Stock $(74-86): 14.2$ Ave. Weekly Hours (High) 48.1 Shift Factor 3.27 Ave Weekly hours (Low) 46.9 Shift Factor 3.36 Note: Output, Investment, and Capital stock extrapolated for 1987 and 1988.

\section{Fabric dress and work gloves}

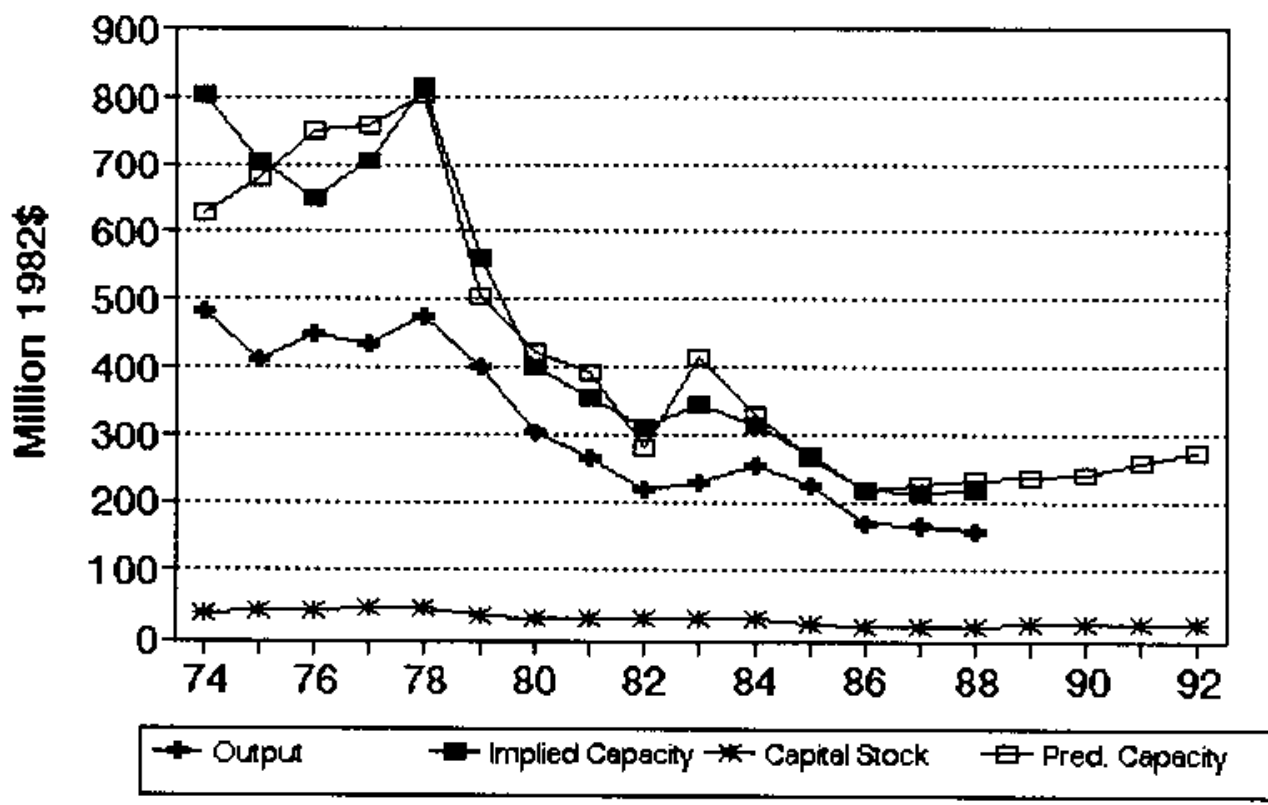




\section{No. 102 sIC 2384 Robes and dressing gown:}

MILLIONS OF 1982 DOLLARS (EXCL. CU RATES)

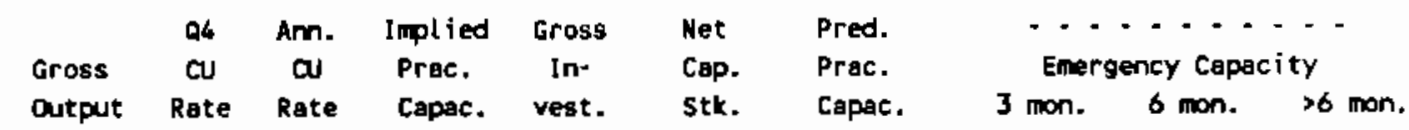

\begin{tabular}{|c|c|c|c|c|c|c|c|c|c|c|}
\hline 1974 & 309 & $94 *$ & 94.4 & 328 & 16.7 & 41 & 285 & & & \\
\hline 1975 & 278 & $87 *$ & 89.7 & 310 & $(6.9)$ & 32 & 319 & & & \\
\hline 1976 & 260 & 95. & 91.7 & 283 & 2.8 & 32 & 353 & & & \\
\hline 1977 & 365 & 96 & 95.5 & 383 & $(2.5)$ & 28 & 388 & & & \\
\hline 1978 & 403 & 89. & 91.5 & 440 & 6.9 & 32 & 422 & & & \\
\hline 1979 & 397 & 86 & 87.1 & 456 & 2.9 & 33 & 456 & & & \\
\hline 1980 & 483 & $\pi$ & 79.1 & $6+1$ & 10.4 & 40 & 491 & & & \\
\hline 1981 & 352 & 83 & 80.0 & 440 & $(2.8)$ & 35 & 525 & & & \\
\hline 1982 & 406 & 81 & 81.8 & 497 & 1.0 & 34 & 559 & & & \\
\hline 1983 & 411 & 60 & 67.9 & 605 & $(2.7)$ & 29 & 594 & & & \\
\hline 1984 & 416 & 60 & 60.0 & 693 & $(1.2)$ & 26 & 628 & & & \\
\hline 1985 & 386 & $60 *$ & 59.9 & 644 & 3.3 & 27 & 662 & & & \\
\hline 1986 & 415 & $60 *$ & 60.2 & 689 & 1.2 & 25 & 697 & & & \\
\hline $\begin{array}{l}--- \\
1987\end{array}$ & $\begin{array}{c}-\ldots \\
428\end{array}$ & 60 & 60. & $\because 714$ & $\begin{array}{l}--- \\
2.4\end{array}$ & -- & 731 & - & - & - - \\
\hline 1988 & 446 & $63 *$ & 61.9 & 721 & 2.4 & 26 & 765 & 735 & 757 & 765 \\
\hline 1989 & & & & & 2.4 & 26 & 799 & 768 & 790 & 799 \\
\hline 1990 & & & & & 2.4 & 26 & 834 & 801 & 824 & 834 \\
\hline 1991 & & & & & 2.6 & 26 & 268 & 834 & 858 & 268 \\
\hline 1992 & & & & & 2.6 & 27 & 902 & 867 & 892 & 902 \\
\hline
\end{tabular}

Capacity-Capital Stock Regression: Code $\quad-2$ RSO 0.850

Coefficients: Const 250.5 Tine 34.3 Ave. Capacity/Stock (74-86): 15.5 Ave. Ueekly Hours (High) 1983.0 shift factor 1.00 Ave Weekly Hours (Low) 1983.0 Shift factor 1.0 * Imputed Note: Output, Investment, and Capital Stock extrapolated for 1987 and 1988.

\section{Robes and dressing gowns}

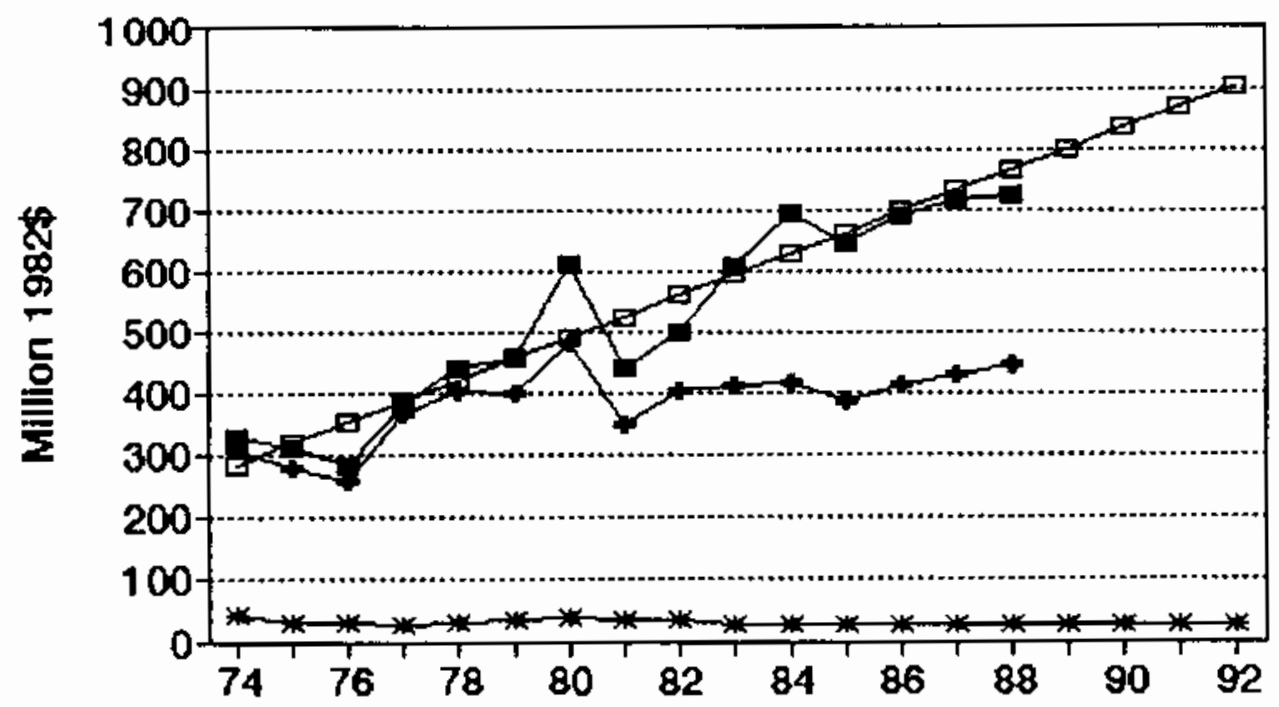

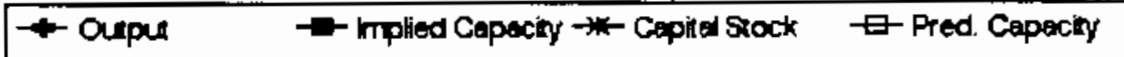




\section{No. 103 SIC 2385 Waterproof outergarments}

\section{MILLIONS OF 1982 DOLLARS (EXCL. CU RATES)}

$\begin{array}{lccccccc} & \text { Q4 } & \text { Am. Implied Gross } & \text { Net } & \text { Pred. } & \ldots \ldots \ldots \\ \text { Gross } & \text { CU } & \text { CU } & \text { Prac. } & \text { In- } & \text { Cap. } & \text { Prac. } & \text { Emergency Capacity } \\ \text { Output } & \text { Rate } & \text { Rate } & \text { Capac. } & \text { vest. } & \text { Stk. } & \text { Capac. } & 3 \text { mon. } 6 \text { mon. }>6 \text { mon. }\end{array}$

\begin{tabular}{|c|c|c|c|c|c|c|c|c|c|c|}
\hline 1974 & 490 & 94 & $\$ 4.0$ & 521 & 0.2 & 47 & 558 & & & \\
\hline 1973 & 423 & 82 & 86.5 & 489 & 0.4 & 43 & 549 & & & \\
\hline 1976 & 450 & $68 *$ & 73.5 & 612 & 2.5 & 42 & 546 & & & \\
\hline 1977 & 427 & 65 & 66.3 & 644 & 3.8 & 42 & 545 & & & \\
\hline 1978 & 428 & 78 & 73.1 & 586 & 5.9 & 43 & 549 & & & \\
\hline 1979 & 383 & 81 & 79.9 & 479 & $(4.9)$ & 35 & 529 & & & \\
\hline 1980 & 382 & 92 & 87.9 & 435 & 1.7 & 33 & 524 & & & \\
\hline 1981 & 390 & $86 *$ & 88.0 & 443 & $(3.4)$ & 27 & 510 & & & \\
\hline 1982 & 448 & $91 *$ & 88.8 & 504 & 9.7 & 32 & 524 & & & \\
\hline 1983 & 451 & 98 & 95.3 & $4 \pi 3$ & 7.3 & 35 & 530 & & & \\
\hline 1924 & 418 & 57 & 72.4 & 578 & (3.1) & 29 & 515 & & & \\
\hline 1985 & 363 & $60 *$ & 59.0 & 616 & 2.0 & 27 & 512 & & & \\
\hline 1986 & 317 & 62 & 61.3 & 517 & 1.2 & 25 & 507 & & & \\
\hline-- & -- & $\cdots$ & $-\cdots$ & $\cdots$ & -- & - & $-\cdot-$ & $\cdots$ & $\cdots$ & $\cdots$ \\
\hline 1987 & 310 & 53 & 56.4 & 549 & 2.1 & 24 & 504 & & & \\
\hline 1988 & 300 & $47 \pm$ & 49.3 & 608 & 2.1 & 23 & 502 & 1,819 & 1,931 & 1,977 \\
\hline 1989 & & & & & 2.1 & 22 & 500 & 1,812 & 1,924 & 1.969 \\
\hline 1990 & & & & & 2.1 & 22 & 498 & 1,805 & 1,917 & 1.962 \\
\hline 1991 & & & & & 2.1 & 21 & 497 & 1,800 & 1,911 & 1.956 \\
\hline 1992 & & & & & 2.1 & 20 & 495 & 1,795 & 1.906 & 1.951 \\
\hline
\end{tabular}

Capecity-Capital Stock Regression: Code 1 RSO 0.060 Coefficients: Const 447.2 stock 2.4 Ave. Weekly Hours (High) 40.0 Shift Factor 3.94 Ave Weekly Hours (LOW) 40.0 shift Factor 3.94 * Imputed Note: Output, Investment, and Capital Stock extrapolated for 1987 and 1988.

\section{Waterproof outergarments}

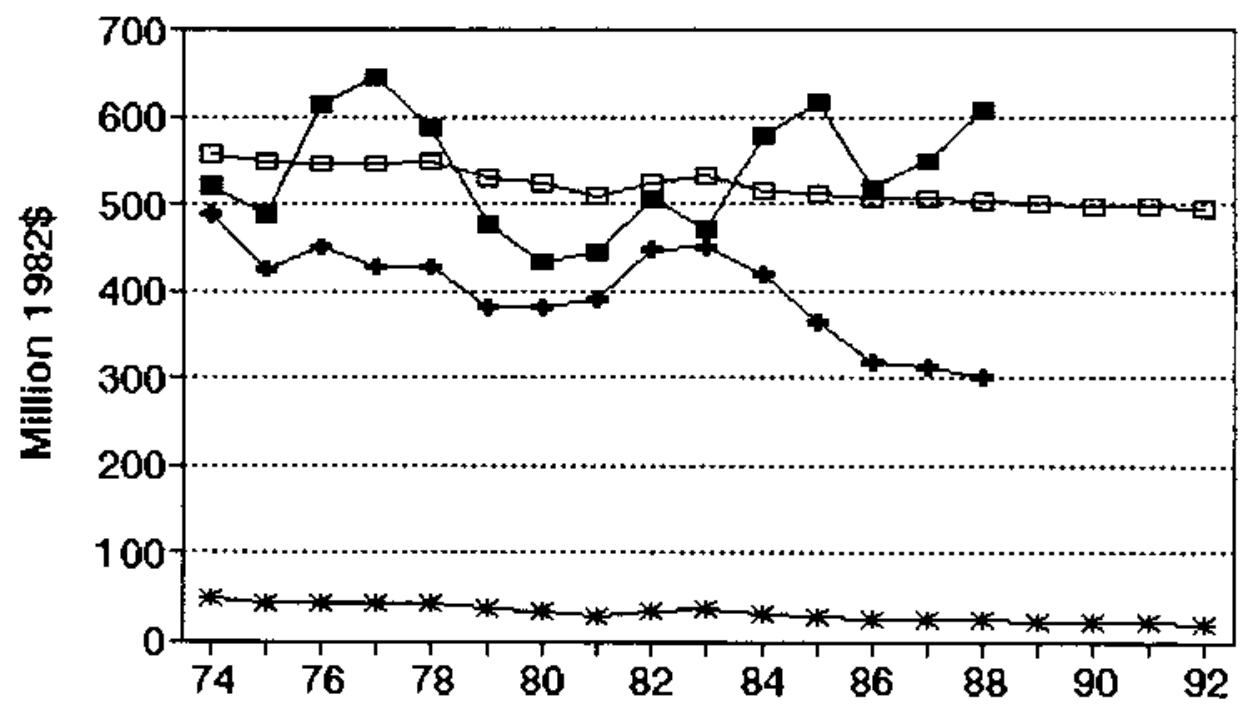

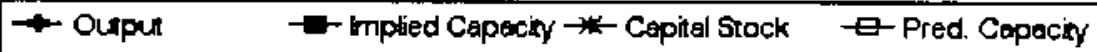




\section{No. 104 SIC 2386 Leather and sheep lined clothing}

MILLIONS OF 1982 DOLLARS (EXCL. CU RATES)

$\begin{array}{lccccccc} & 04 & \text { Ann. Implied Gross } & \text { Net } & \text { Pred. } & \ldots . . . \\ \text { Gross } & \text { CU } & \text { CU } & \text { Prac. } & \text { In- } & \text { Cap. } & \text { Prac. } & \text { Emergency Capacity } \\ \text { Output } & \text { Rate } & \text { Rate } & \text { Capac. } & \text { vest. } & \text { Stk. } & \text { Capac. } & 3 \text { mon. } 6 \text { mon. }>6 \text { mon. }\end{array}$

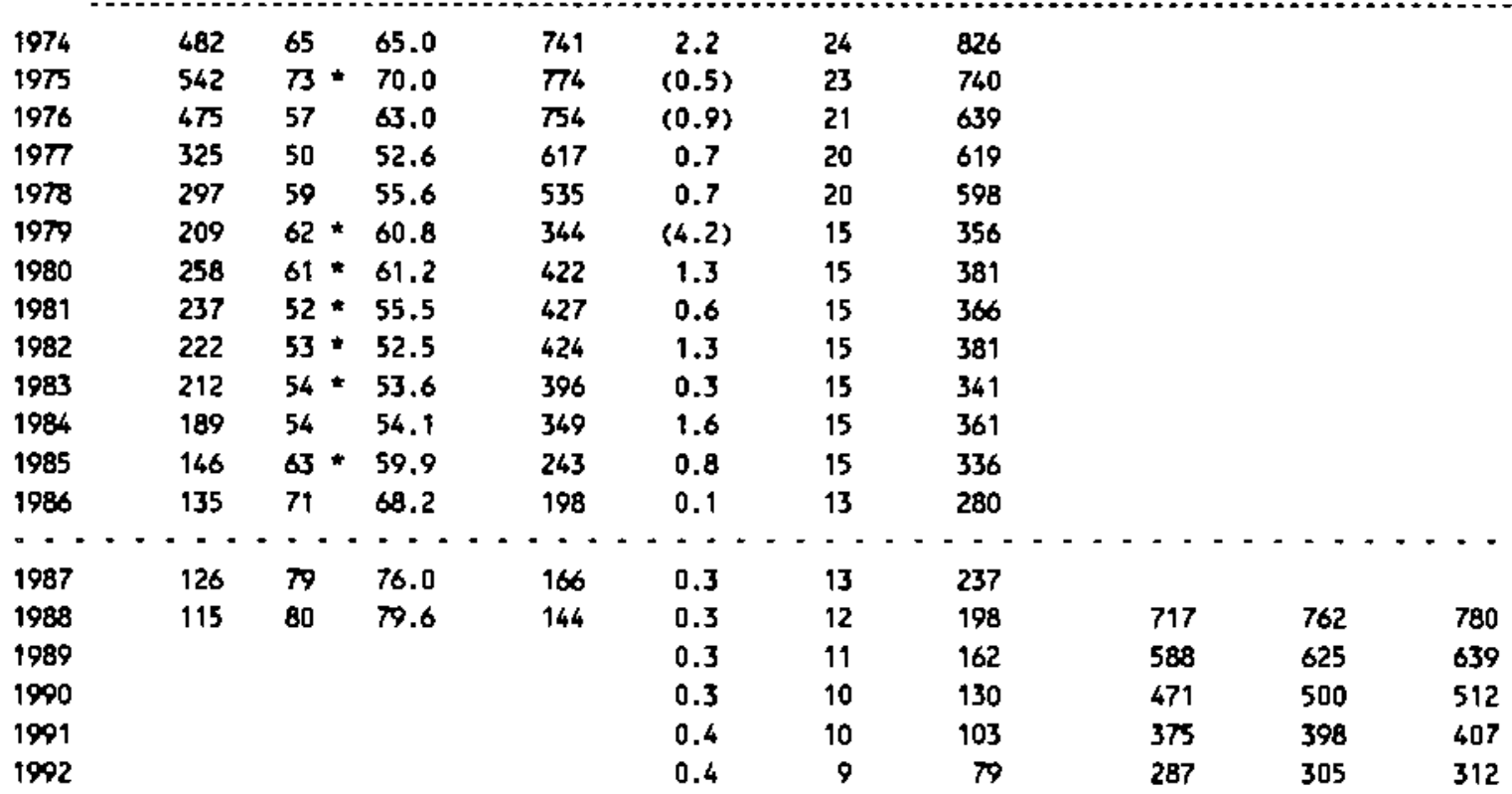

Capacity-Capital Stock Regression: Cade 1 RSQ 0.880 Coefficients: Const -396.0 stock $\mathbf{5 0 . 5}$ Ave. Weekly Hours (High) 40.0 Shift Factor 3.94 Ave Weekly Hours (LOW) 40.0 shift factor 3.94 - Imputed Note: Output, Investment, and Capital stock extrapolated for 1987 and 1988.

\section{Leather and sheep lined clothing}

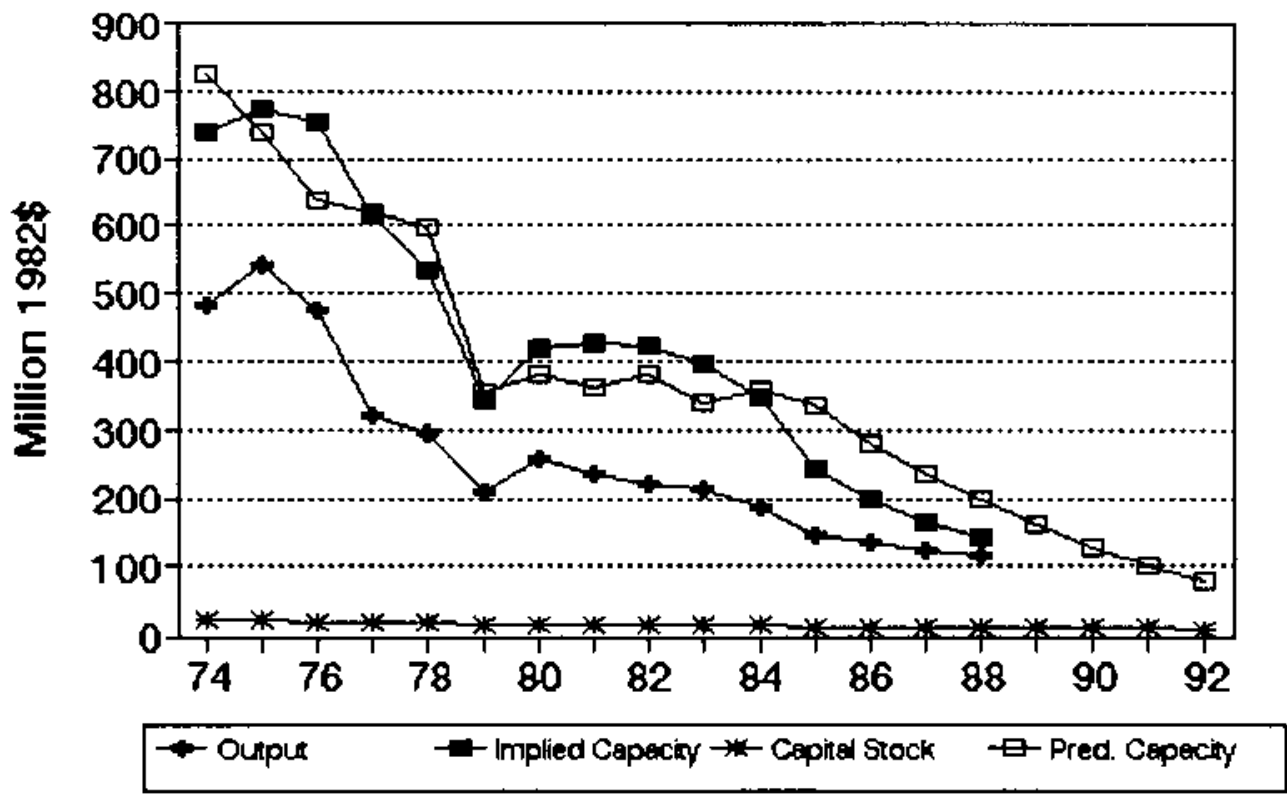


No. 105 BIC 2387 Apparel belts

MILLIONS OF 1982 DOLLARS (EXCL, CU RATES)

$\begin{array}{lccccccc} & \text { Q4 } & \text { Ann. } & \text { Implied Gross } & \text { Net } & \text { Pred. } & \ldots \ldots \ldots \\ \text { Gross } & \text { CU } & \text { CU } & \text { Prac. } & \text { In- } & \text { Cap. } & \text { Prac. } & \text { Emergency Capacity } \\ \text { Output } & \text { Rate } & \text { Rate } & \text { Capac. } & \text { vest. } & \text { Stk. } & \text { Capac. } & 3 \text { mon. } 6 \text { mon. }>6 \text { mon. }\end{array}$

\begin{tabular}{|c|c|c|c|c|c|c|c|c|c|c|}
\hline 1974 & 455 & 53 & 55.3 & 823 & (2.7) & 27 & 961 & & & \\
\hline 1975 & 448 & 69 & 58.8 & 762 & $(7.6)$ & 18 & 649 & & & \\
\hline 1976 & 461 & 57 & 62.0 & 744 & 3.6 & 20 & 711 & & & \\
\hline 1977 & 534 & 64 & 62.2 & 859 & 6.3 & 24 & 857 & & & \\
\hline 1978 & 591 & 66 & 65.6 & 900 & 4.4 & 26 & 926 & & & \\
\hline 1979 & 487 & 71 & 69.2 & 704 & 1.3 & 25 & 895 & & & \\
\hline 1980 & 474 & 73 & 72.1 & 658 & 1.7 & 24 & 874 & & & \\
\hline 1981 & 567 & 36 & 49.7 & 1,140 & 3.7 & 26 & 919 & & & \\
\hline 1982 & 550 & 41 & 38.8 & 1,416 & 7.8 & 31 & 1,092 & & & \\
\hline 1983 & 562 & 46 & 43.9 & 1,279 & 1.5 & 29 & 1,051 & & & \\
\hline 1984 & 541 & 54 & 51.9 & 1,041 & 6.0 & 33 & 1,162 & & & \\
\hline 1985 & 479 & $55 *$ & 53.8 & 889 & $(0.8)$ & 29 & 1,044 & & & \\
\hline 1986 & 513 & $54^{\star}$ & 53.9 & 950 & 2.3 & 29 & 1,027 & & & \\
\hline$\cdots$ & $-\cdots$ & - - - & -- & $\cdots$ & $\cdots$ & $-\cdot$ & $\cdots$ & $\cdots$ & $\cdots$ & $-\cdots$ \\
\hline 1987 & 521 & $57 \star$ & 55.9 & 931 & 4.1 & 30 & 1,077 & & & \\
\hline 1988 & 531 & 44 & 48.8 & 1,088 & 4.4 & 32 & 1,131 & 2,366 & 2,502 & 2,557 \\
\hline 1989 & & & & & 4.6 & 33 & 1,189 & 2,488 & 2,630 & 2,688 \\
\hline 1990 & & & & & 4.9 & 35 & 1,251 & 2,616 & 2,766 & 2,827 \\
\hline 1991 & & & & & 5.1 & 37 & 1,315 & 2,751 & 2,909 & 2,972 \\
\hline 1992 & & & & & 5.4 & 39 & 1,382 & 2,892 & 3,057 & 3,124 \\
\hline
\end{tabular}

Capacity-Capital stock Regression: Code 0 RSO 0.410

Coefficients: Const 31.2 5tock 34.7

Ave. Capacity/5tock $(74-86): 35.9$

Ave. Weekly Hours (High) 69.7 Shift factor 2.26 Ave Heekly Hours (Low) 69.7 Shift Factor 2.26

- Imputed Note: Output, Investment, and Capitat Stock extrapolated for 1987 and 1988.

\section{Apparel belts}

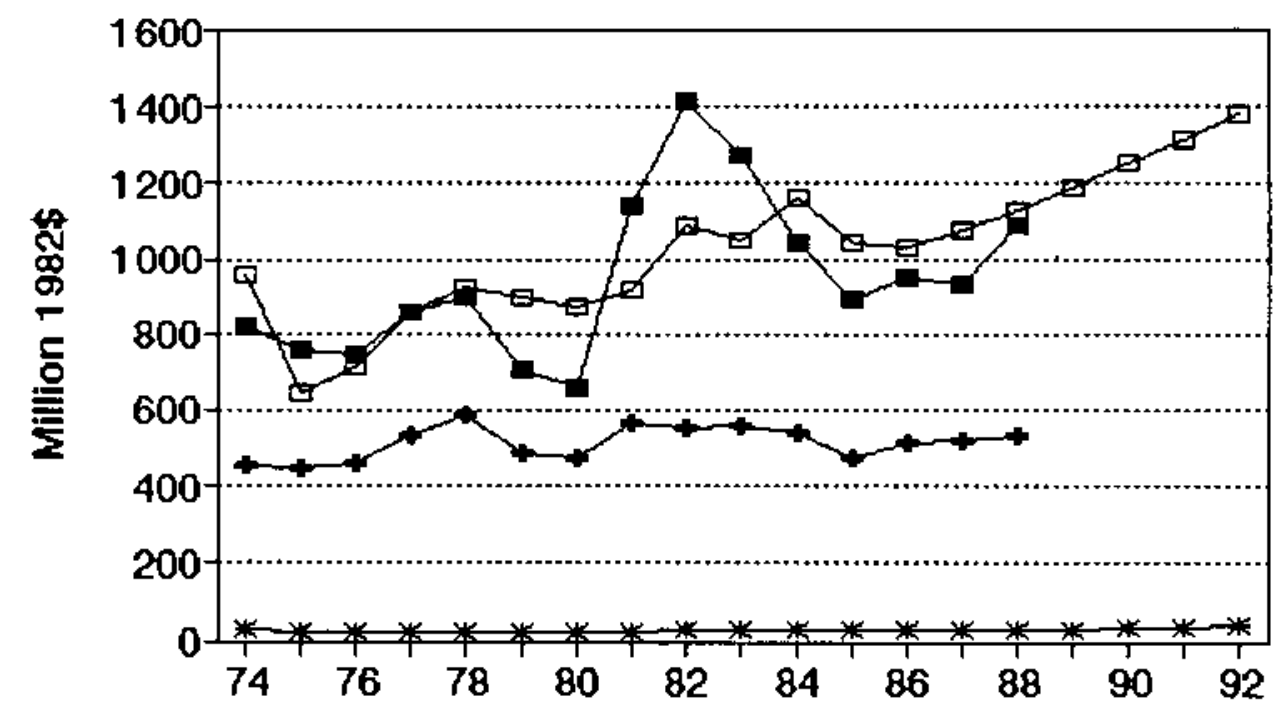

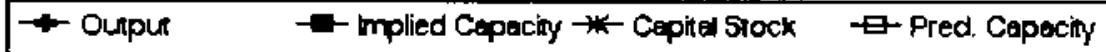


No. 106 sIC 2389 Apparel and accessories, n.e.c.

M!LLIONS OF 1982 DOLLARS (EXCL. CU RATES)

$\begin{array}{lccccccc} & a 4 & \text { Ann. Implied } & \text { Gross } & \text { Net } & \text { Pred. } & -\ldots & -\ldots \\ \text { Gross } & \text { CU } & \text { CU } & \text { Prac. } & \text { In- } & \text { Cap. } & \text { Prec. } & \text { Emergency Capacity } \\ \text { Output Rate Rate } & \text { Cepac. } & \text { vest. } & \text { Stk. } & \text { Capac. } & 3 \text { mon. } 6 \text { mon. }>6 \text { mon. }\end{array}$

\begin{tabular}{|c|c|c|c|c|c|c|c|c|c|c|}
\hline 1974 & 325 & 67 & 68.6 & 474 & 11.8 & 28 & 387 & & & \\
\hline 1975 & 262 & $83 *$ & 74.0 & 354 & 4.9 & 30 & 398 & & & \\
\hline 1976 & 308 & 72 & 76.2 & 404 & 6.1 & 33 & 414 & & & \\
\hline 1977 & 262 & 72 & 72.6 & 360 & $(6.0)$ & 24 & 365 & & & \\
\hline 1978 & 287 & 87 * & 81.9 & 350 & 6.7 & 28 & 387 & & & \\
\hline 1979 & 349 & 95 & 92.2 & 370 & 3.9 & 29 & 390 & & & \\
\hline 1980 & 379 & 84 & 88.0 & 431 & 4.7 & 30 & 396 & & & \\
\hline 1981 & 360 & 95 & 91.1 & 396 & 0.6 & 28 & 383 & & & \\
\hline 1982 & 274 & 71 & 79.4 & 345 & 2.4 & 27 & 378 & & & \\
\hline 1983 & 278 & 87 & 80.7 & 364 & $1 . t$ & 24 & 364 & & & \\
\hline 1984 & 338 & $\mathrm{B3}$ & 85.7 & 394 & 5.5 & 26 & 375 & & & \\
\hline 1985 & 307 & $80 *$ & 80.6 & 380 & 5.3 & 28 & 383 & & & \\
\hline 1986 & 294 & $\pi$ & 76.8 & 384 & 2.2 & 26 & 374 & & & \\
\hline-- & ---- & - & -- & $-\cdots$ & $-\cdots$ & - & $-\cdots$ & $-\cdot-$ & -- & $-\cdots$ \\
\hline 1987 & 301 & $\pi$ & 76.4 & 393 & 3.9 & 26 & 375 & & & \\
\hline 1988 & 309 & $67 \star$ & 71.0 & 635 & 3.9 & 26 & 376 & 1,091 & 1,149 & 1,172 \\
\hline 1989 & & & & & 3.9 & 26 & 377 & 1,093 & 1,151 & 1,175 \\
\hline 1990 & & & & & 3.9 & 27 & 377 & 1,095 & 1,153 & 1,177 \\
\hline 1991 & & & & & 4.1 & 27 & 379 & 1,099 & 1,158 & 1,181 \\
\hline 1992 & & & & & 4.1 & 27 & 380 & 1,103 & 1,161 & 1,185 \\
\hline
\end{tabular}

Capacity-Capital Stock Regression: Code 0 RSO 0.140

Coefficients: Const 225.4 stock 5.7

Ave. Capecity/Stock $(74-86): 13.8$ Ave. Heekly Hours (High) 50.5 Shift Factor 3.12 Ave Veekly Hours (Low) 50.5 Shift Factor 3.12 * Imputed Note: Output, Imvestment, and Capital Stock extrapolated for 1987 and 1988.

\section{Apparel and accessories, n.e.c.}

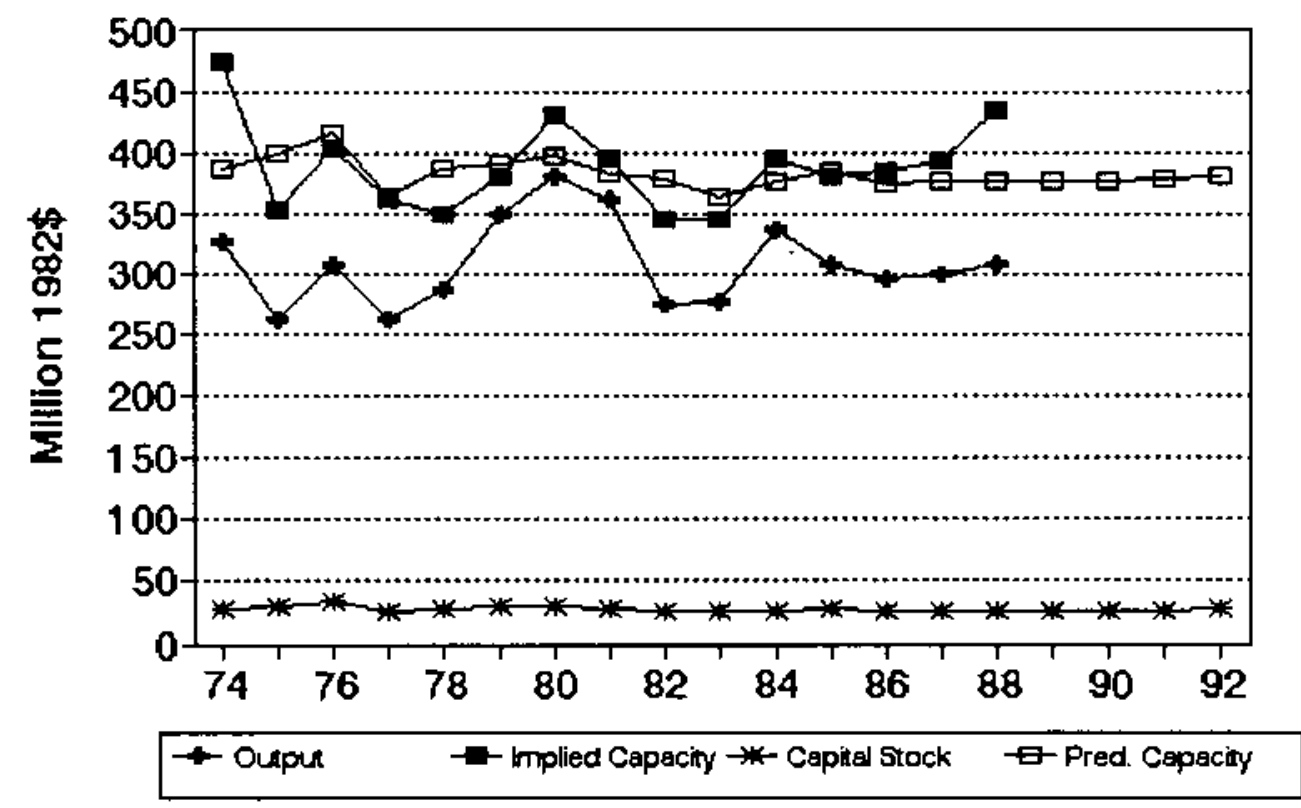


No. 107 sIC 2391 Curtains and draperies

MILLIONS OF 1982 DOLLARS (EXCL. CU RATES)

$\begin{array}{lccccccc} & \text { Q4 } & \text { Am. Implied Gross } & \text { Net } & \text { Pred. } & -\ldots \ldots \ldots \\ \text { Gross } & \text { CU } & \text { CU } & \text { Prac. } & \text { In- } & \text { Cap. } & \text { Prac. } & \text { Emergency Capacity } \\ \text { Output } & \text { Rate } & \text { Rate } & \text { Capac. } & \text { vest. } & \text { Stk. } & \text { Capac. } & 3 \text { mon. } 6 \text { mon. } 76 \text { non. }\end{array}$

\begin{tabular}{|c|c|c|c|c|c|c|c|c|c|c|}
\hline 1974 & 1,105 & $66 *$ & 68.2 & 1,620 & 5.9 & 107 & 1,525 & & & \\
\hline 1975 & 1,067 & 69 & 63.1 & 1,690 & 2.6 & 101 & 1,571 & & & \\
\hline 1976 & 1,146 & 73 & 71.7 & 1,598 & 2.0 & 95 & 1,618 & & & \\
\hline 1977 & 1,375 & B1 & 79.0 & 1,740 & 16.3 & 103 & 1,664 & & & \\
\hline 1978 & 1,340 & 77 & 78.9 & 1,698 & 5.3 & 99 & 1,710 & & & \\
\hline 1979 & 1,163 & 77 & 7.0 & 1,510 & 3.1 & 94 & 1,757 & & & \\
\hline 1980 & 1,170 & 70 & 72.5 & 1,614 & $(9.0)$ & 79 & 1,803 & & & \\
\hline 1981 & 1,172 & 57 & 62.0 & 1,892 & 9.6 & 81 & 1,850 & & & \\
\hline 1982 & 1,073 & 56 & 55.8 & $t, 921$ & 4.3 & $\pi$ & 1,896 & & & \\
\hline 1983 & 1,097 & 59 & 57.7 & 1,901 & 12.1 & 80 & 1,943 & & & \\
\hline 1984 & 1,267 & 69 & 66.4 & 1,908 & $(0.6)$ & 71 & 1,989 & & & \\
\hline 1985 & 1,339 & $65 *$ & 65.5 & 2,045 & 7.6 & 70 & 2,036 & & & \\
\hline 1986 & 1,496 & $65 *$ & 64.8 & 2,307 & 5.7 & 68 & 2,082 & & & \\
\hline 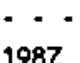 & $\cdots$ & $\begin{array}{l}-\cdots \\
71 \ldots\end{array}$ & 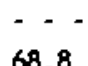 & - - - - & $-\cdots$ & -- & - - - & $\cdots$ & -- & - - - \\
\hline 1988 & $\begin{array}{l}1,304 \\
1,516\end{array}$ & 71 * & $\begin{array}{l}\infty .0 \\
71.1\end{array}$ & $\begin{array}{l}2,188 \\
2,133\end{array}$ & $\begin{array}{l}3.1 \\
5.1\end{array}$ & $\begin{array}{l}04 \\
61\end{array}$ & $\begin{array}{l}2,128 \\
2,175\end{array}$ & 2,113 & 2,157 & 2,175 \\
\hline 1989 & & & & & 5.1 & 59 & 2,221 & 2,158 & 2,203 & 2,221 \\
\hline 1990 & & & & & 5.1 & 57 & 2,268 & 2,203 & 2,249 & 2,268 \\
\hline 1991 & & & & & 5.2 & 55 & 2,314 & 2,248 & 2,295 & 2,314 \\
\hline 1992 & & & & & 5.2 & 53 & 2,361 & 2,293 & 2,341 & 2,361 \\
\hline
\end{tabular}

Capacity-Capital Stock Regression: Code -2 RSQ 0.680

Coefficients: Const 1478.2 Time 46.4 Ave. Capecity/Stock (74-86): 20.8 Ave. Heekly Hours (High) 1984.0 Shift Factor 1.00 Ave Heekly Hours (Low) 1984.0 Shift Factor 1.0 * Imputed Note: Output, Imestment, and Capital Stock extrapolated for 1987 and 1988.

\section{Curtains and draperies}

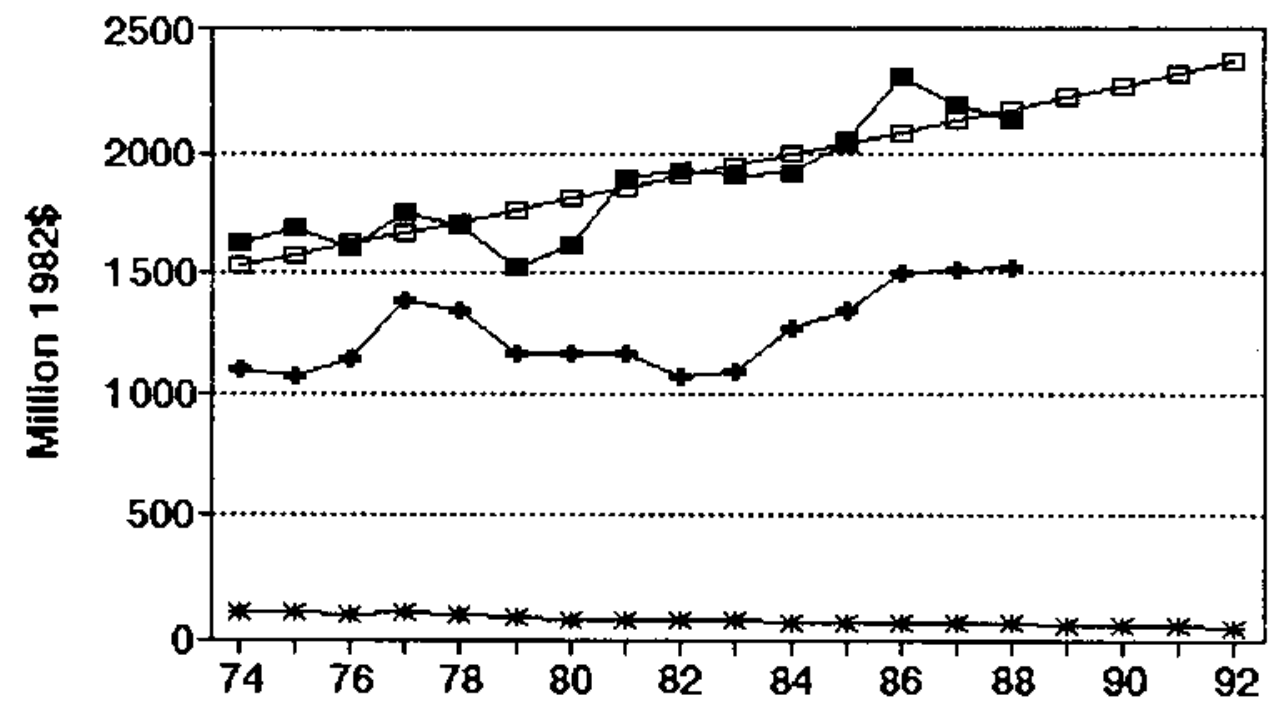

+- Oupu $\quad$ - Impled Copecin $\rightarrow$ - Cepinel Stock $\quad \square$ Pred Copecing 


\section{NO. 108 sIc 2392 EOuBe furnishings, n.e.c.}

MILLIONS OF 1982 DOLLARS (EXCL. CU RATES)

$\begin{array}{lccccccc} & 04 & \text { Ann. Implied } & \text { Gross } & \text { Net } & \text { Pred. } & -\ldots \ldots \ldots \\ \text { Gross } & \text { CU } & \text { CU } & \text { Prac. } & \text { In- } & \text { Cap. } & \text { Prac. } & \text { Emergency Capacity } \\ \text { Output } & \text { Rate } & \text { Rate } & \text { Capac. } & \text { vest. } & \text { Stk. } & \text { Capac. } & 3 \text { mon. } 6 \text { mon. }>6 \text { mon. }\end{array}$

\begin{tabular}{|c|c|c|c|c|c|c|c|c|c|c|}
\hline 1974 & 2,778 & 42 & 43.5 & 6,392 & 13.6 & 375 & 5,070 & & & \\
\hline 1973 & $2, \pi 78$ & 69 & 55.8 & 4,980 & 22.7 & 368 & 4,983 & & & \\
\hline 1976 & 2,976 & 68 & 68.6 & 4,339 & 35.9 & 372 & 5,025 & & & \\
\hline 1977 & 3,384 & 81 & 76.9 & 4,400 & 20.8 & 359 & 4,877 & & & \\
\hline 1978 & 3,426 & 71 & 75.1 & 4,562 & 19.9 & 345 & 4,713 & & & \\
\hline 1979 & 3,366 & 82 & 78.0 & 4,317 & $(3.0)$ & 311 & 4,319 & & & \\
\hline 1980 & 3,009 & 80 & 80.6 & 3,844 & $(3.4)$ & 278 & 3,942 & & & \\
\hline 1981 & 3,056 & 85 & 83.4 & 3,666 & 50.3 & 294 & 4,128 & & & \\
\hline 1982 & 3,289 & 90 & 87.5 & 3,760 & 45.5 & 303 & 4,228 & & & \\
\hline 1983 & 3,546 & 70 & 77.7 & 4,566 & 3.7 & 273 & 3,878 & & & \\
\hline 1984 & 3,730 & 88 & 82.5 & 4,524 & 62.5 & 299 & 4,185 & & & \\
\hline 1985 & 4,011 & 81 & 82.7 & 4,848 & 33.6 & 297 & 4,938 & & & \\
\hline 1986 & 4,089 & $81 \star$ & 81.2 & 5,035 & 34.8 & 298 & 4,946 & & & \\
\hline-- & --- & -- & $-\cdot-$ & $-\cdots$ & $-\cdots$ & -- & $-\cdots$ & -- & -- & -- \\
\hline 1987 & 4,144 & 91 * & 87.5 & 4,737 & 40.1 & 303 & 5,007 & & & \\
\hline 1988 & 4,216 & $92 *$ & 91.4 & 4,611 & 42.2 & 310 & 5,086 & 5,004 & 5.062 & 5,086 \\
\hline 1989 & & & & & 44.3 & 318 & 5.179 & 5,096 & 5,155 & 5,179 \\
\hline 1990 & & & & & 46.4 & 327 & 5,286 & 5,201 & 5,262 & 5,286 \\
\hline 1991 & & & & & 48.5 & 337 & 5,405 & 5,318 & 5,380 & 5,405 \\
\hline 1992 & & & & & 50.6 & 349 & 5,534 & 5,445 & 5,508 & 5,534 \\
\hline
\end{tabular}

Capacity-Capital stock Regression: Code -1 RSO 0.410 Coefficients: Const 719.6 stock 11.6 D(85-86) $774.8 \quad$ Ave. Capacity/Stock (74-86): 14.2 Ave. Weekly Hours (High) 1984.0 shift Factor 1.00 Ave Heekly Hours (Low) 1984.0 Shift Factor 1.0 - Imputed Note: Output, Investment, and Capital Stock extrapolated for 1987 and 1988.

\section{House fumishings, n.e.c.}

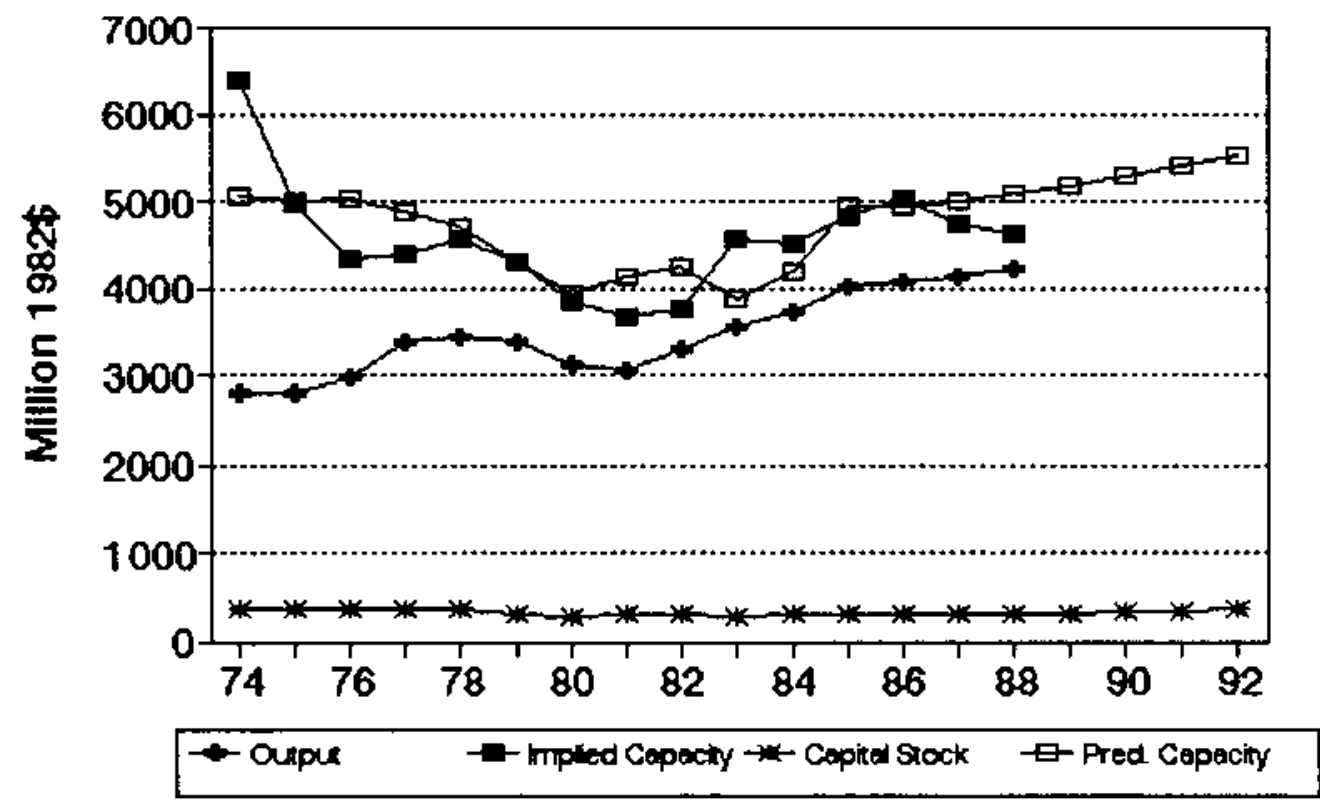


No. 109 SIC 2393 Textile bags

MILLIONS OF 1982 DOLLARS (EXCL. CU RATES)

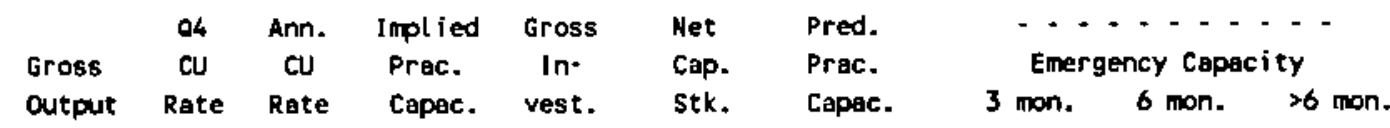

\begin{tabular}{|c|c|c|c|c|c|c|c|c|c|c|}
\hline 1974 & 471 & 45 & 45.2 & 1,041 & 3.6 & 65 & 872 & & & \\
\hline 1975 & 484 & 63 & 55.8 & 868 & 3.9 & 64 & 849 & & & \\
\hline 1976 & 479 & 68 & 66.1 & 725 & 5.9 & 65 & 826 & & & \\
\hline 1977 & 489 & 52 & 58.2 & 842 & 5.5 & 66 & 803 & & & \\
\hline 1978 & 531 & 57 & 55.2 & 962 & 0.3 & 61 & 780 & & & \\
\hline 1979 & 455 & 78 & 70.2 & 649 & 23.4 & 78 & 737 & & & \\
\hline 1980 & 407 & 72 & 74.2 & 548 & 19.1 & 90 & 734 & & & \\
\hline 1981 & 439 & 81 & 7.7 & 565 & 0.0 & 83 & 711 & & & \\
\hline 1982 & 431 & 72 & 75.3 & 572 & $(5.9)$ & 71 & 688 & & & \\
\hline 1983 & 449 & 72 & 72.2 & 623 & 9.6 & 74 & 665 & & & \\
\hline 1984 & 468 & 70 & 71.1 & 658 & $(5.9)$ & 62 & 642 & & & \\
\hline 1985 & 501 & 61 & 64.2 & 780 & 6.8 & 63 & 619 & & & \\
\hline 1986 & 493 & 74 & 69.1 & 714 & 2.8 & 59 & 596 & & & \\
\hline$\cdots$ & $\cdots$ & $\cdots$ & $-\cdot$ & $\cdots$ & $--\cdot$ & -- & $\therefore$ & -- & $-\cdot$ & $\cdots$ \\
\hline 1987 & 491 & 69 & 70.9 & 693 & 2.9 & 56 & 573 & & & \\
\hline 1988 & 489 & 69 * & 69.2 & 706 & 2.5 & 53 & 550 & 787 & 809 & 817 \\
\hline 1989 & & & & & 2.2 & 49 & 527 & 754 & $\pi / 5$ & 783 \\
\hline 1990 & & & & & 1.8 & 46 & 506 & 721 & 741 & 749 \\
\hline 1991 & & & & & 1.5 & 43 & 481 & 688 & 707 & 715 \\
\hline 1992 & & & & & 1.1 & 40 & 458 & 655 & 673 & 681 \\
\hline
\end{tabular}

Capacity-Capital Stock Regression: Code -2 RSQ 0.330

Coefficients: Const 895.4 Time $-23.0 \quad$ Ave. Capecity/Stock (74-86): 10.6 Ave. Weekly Hours (High) 106.0 Shift Factor 1.49 Ave Heekly Hours (Low) 102.0 Shift Factor 1.54 * Imputed Note: Output, Investment, and Capital Stock extrapolated for 1987 and 1988.

\section{Textile bags}

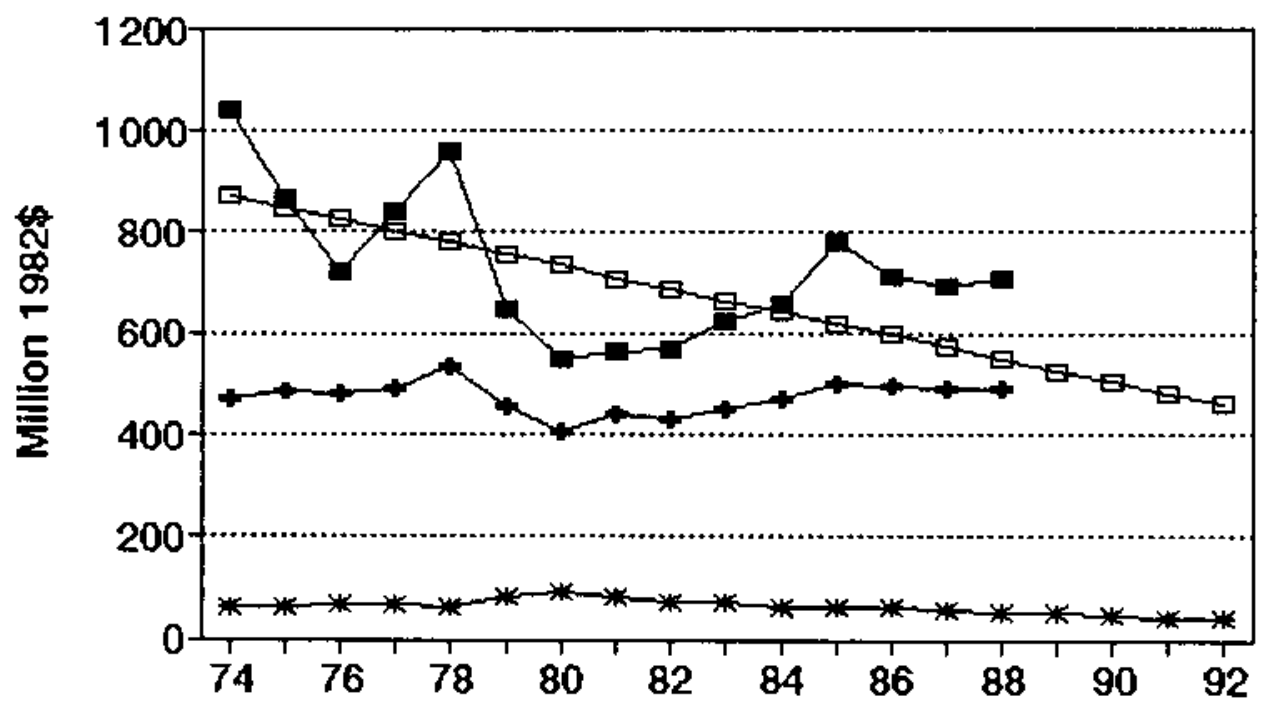

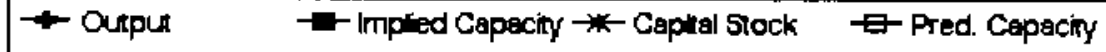


No. 110 sIC 2394 Canvas and related products

MILLIONS OF 1982 DOLLARS (EXCL. CU RATES)

$\begin{array}{lccccccc} & 04 & \text { Am. Implied } & \text { Gross } & \text { Net } & \text { Pred. } & \ldots & \ldots \\ \text { Gross } & \text { CU } & \text { CU } & \text { Prac. } & \text { In- } & \text { Cap. } & \text { Prac. } & \text { Emergency Capacity } \\ \text { Output } & \text { Rate } & \text { Rate } & \text { Capac. } & \text { vest. } & \text { Stk. } & \text { Capac. } & \text { I mon. } 6 \text { mon. }>6 \text { mon. }\end{array}$

\begin{tabular}{|c|c|c|c|c|c|c|c|c|c|c|}
\hline 1974 & 419 & 82 & 82.0 & 511 & 9.2 & 61 & 769 & & & \\
\hline 1975 & 463 & 85 & 83.9 & 551 & 6.6 & 61 & $7 r 9$ & & & \\
\hline 1976 & 470 & $\pi$ & 78.8 & 608 & 7.7 & 63 & 802 & & & \\
\hline 1977 & 756 & 82 & 79.4 & 952 & 21.9 & 77 & 1,023 & & & \\
\hline 1978 & 861 & 86 & 84.5 & 1,019 & 25.8 & 95 & 1,290 & & & \\
\hline 1979 & 713 & 51 & 64.1 & 1,112 & $(21.4)$ & 68 & 875 & & & \\
\hline 1980 & 602 & 61 & 57.3 & 1,052 & 5.3 & 67 & 868 & & & \\
\hline 1981 & 741 & 63 & 62.3 & 1,191 & 11.0 & 72 & 941 & & & \\
\hline 1982 & 748 & 65 & 64.3 & 1,165 & 11.6 & 77 & 1,014 & & & \\
\hline 1983 & 788 & 78 & 73.1 & 1,078 & 6.4 & 76 & 1,008 & & & \\
\hline 1984 & 933 & 68 & 71.8 & 1,300 & 14.4 & 84 & 1,118 & & & \\
\hline 1985 & 974 & 86 & 79.3 & 1,229 & 13.5 & 90 & 1,212 & & & \\
\hline 1986 & $\infty 1$ & 69 & 75.4 & 1,314 & 18.8 & 101 & 1,384 & & & \\
\hline$\cdots$ & $\cdots \cdot$ & $-\cdot$ & -- & --- & $-\cdot-$ & -- & $\cdots$ & $\cdots$ & $\cdots$ & -- \\
\hline 1987 & 1,021 & 67 & 67.8 & 1,506 & 10.2 & 103 & 1,420 & & & \\
\hline 1988 & 1,061 & 83 & 77.0 & 1,377 & 10.2 & 106 & 1,453 & 2,462 & 2,566 & 2,607 \\
\hline 1989 & & & & & 10.2 & 108 & 1,483 & 2,514 & 2,620 & 2,662 \\
\hline 1990 & & & & & 10.2 & 109 & 1,511 & 2,561 & 2,669 & 2,713 \\
\hline 1991 & & & & & 10.5 & 111 & 1,541 & 2,612 & 2,722 & 2,767 \\
\hline 1992 & & & & & 10.5 & 113 & 1,569 & 2,659 & 2,771 & 2,817 \\
\hline
\end{tabular}

Capacity-Capital Stock Regression: code 0 RSa 0.510

Coefficients: Const -156.6 Stock 15.2

Ave. Capacity/Stock (74-86): 13.2

Ave. Weekly Hours (High) 87.7 Shift Factor 1.70 Ave Weekly Hours (Low) 65.3 Shift factor 2.41

Note: Output, Investment, and Capital stock extrapolated for 1987 and 1988.

\section{Canvas and related products}

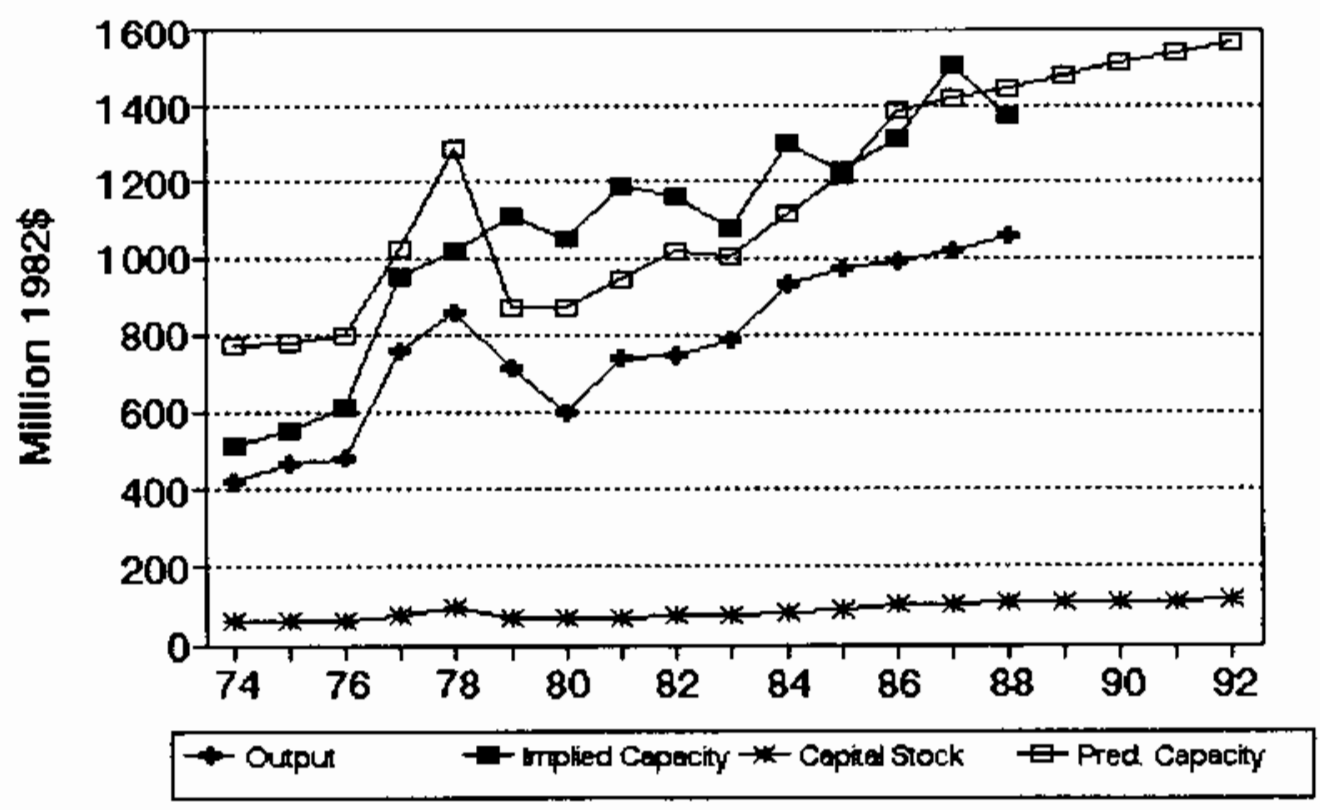


No. 111 sIC 2395 pleating and stitching

MILLIONS OF 1982 DOLLARS (EXCL. CU RATES)

$\begin{array}{lccccccc} & 04 & \text { Ann. } & \text { Implied Gross } & \text { Net } & \text { Pred. } & -\ldots \ldots \\ \text { Gross } & \text { CU } & \text { CU } & \text { Prac. } & \text { In- } & \text { Cap. } & \text { Prac. } & \text { Emergency Capacity } \\ \text { Output } & \text { Rate } & \text { Rate } & \text { Capac. } & \text { vest. } & \text { Stk. } & \text { Capac. } & 3 \text { mon. } 6 \text { mon. }>6 \text { mon. }\end{array}$

\begin{tabular}{|c|c|c|c|c|c|c|c|c|c|c|}
\hline 1974 & 474 & 49 & 50.7 & 934 & $\uparrow 1.7$ & 61 & 1,000 & & & \\
\hline 1975 & 524 & 79 & 64.1 & 817 & 9.3 & 64 & 1,022 & & & \\
\hline 1976 & 573 & 67 & 71.9 & 797 & 12.4 & $7 t$ & 1,060 & & & \\
\hline 1977 & 883 & 87 & 80.2 & 1,101 & 44.8 & 107 & 1,273 & & & \\
\hline 1978 & 952 & 74 & 79.3 & 1,201 & 11.5 & 109 & 1,289 & & & \\
\hline 1979 & 789 & $63 \star$ & 67.2 & 1,174 & $(25.4)$ & 79 & 1,106 & & & \\
\hline 1980 & 875 & 49 & 54.2 & 1,613 & 5.1 & 78 & 1,104 & & & \\
\hline 1981 & 860 & 69 & 61.8 & 1,392 & 7.6 & 80 & 1,115 & & & \\
\hline 1982 & 910 & $71 *$ & 69.5 & 1,309 & 22.5 & 95 & 1,205 & & & \\
\hline 1983 & 923 & 84 & 78.6 & 1,174 & 5.9 & 94 & 1,196 & & & \\
\hline 1984 & 868 & 84 & 85.4 & 1,018 & $(4.3)$ & 83 & 1,131 & & & \\
\hline 1985 & 776 & $74 \star$ & 76.6 & 1,013 & 0.1 & $\pi$ & 1,095 & & & \\
\hline 1986 & 867 & 74 & 73.8 & 1,176 & 11.0 & 81 & 1,122 & & & \\
\hline$\because$ & $\cdots-\cdot$ & -- & $--\cdot$ & $-\cdot-$ & $--\cdot$ & -- & $\cdots$ & $\cdots \cdot \cdots$ & $\cdots \cdots$ & $\cdots \cdot$ \\
\hline 1987 & 904 & $88 *$ & 83.0 & 1,089 & 9.5 & 84 & 1,139 & & & \\
\hline 1988 & 954 & $90 *$ & 89.3 & 1,068 & 9.5 & 87 & 1,156 & 1,969 & 2,044 & 2,074 \\
\hline 1989 & & & & & 9.6 & 89 & 1,171 & 1,095 & 2,071 & 2,102 \\
\hline 1990 & & & & & 9.6 & 92 & 1,185 & 2,019 & 2,096 & 2,127 \\
\hline 1991 & & & & & 10.6 & 95 & 1,204 & 2,052 & 2,130 & 2,162 \\
\hline 1992 & & & & & 10.6 & 98 & 1,222 & 2,082 & 2,161 & 2,193 \\
\hline
\end{tabular}

Capacity-Capital Stock Regression: Code 0 RSO 0.150

Coefficients: Const 638.5 stock 6.0

Ave. Capacity/Stock $(74-86): 13.7$ Ave. Heekly Hours (High) 87.7 Shift factor 1.79 Ave Heekly Hours (Low) 65.3 Shift Factor 2.41 * Imputed Note: Output, Investment, and Capital Stock extrapolated for 1987 and 1988.

\section{Pleating and stitching}

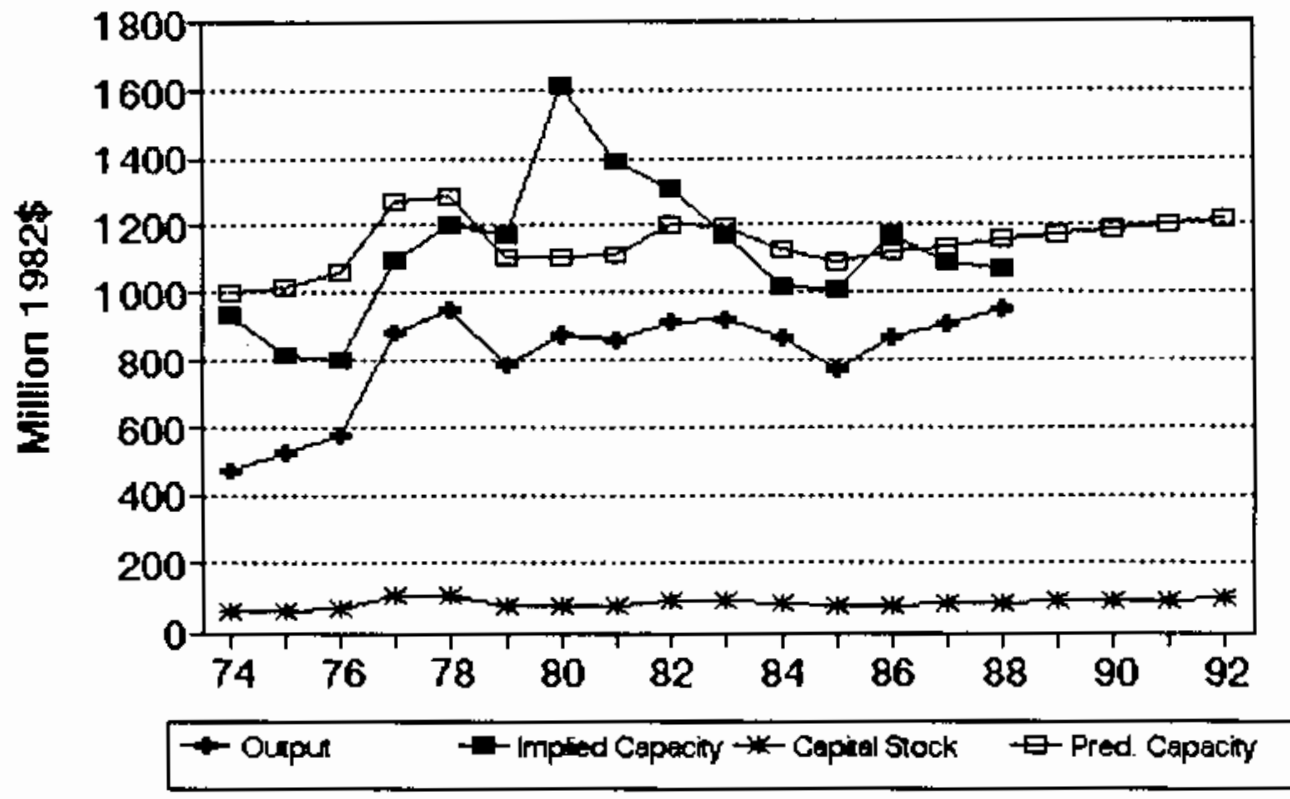




\section{No. 112 sIC 2396 Autonotive and apparel trimings}

MILLIONS OF 1982 DOLLARS (EXCL. CU RATES)

$\begin{array}{lccccccc} & \text { Q4 } & \text { Ann. Implied Gross } & \text { Net } & \text { Pred. } & \ldots . \ldots . . . \\ \text { Gross } & \text { CU } & \text { CU } & \text { Prac. } & \text { In- } & \text { Cap. } & \text { Prac. } & \text { Emergency Capacity } \\ \text { Output } & \text { Rate } & \text { Rate } & \text { Capac. } & \text { vest. } & \text { Stk. } & \text { Capac. } & 3 \text { mon. } 6 \text { mon. }>6 \text { mon. }\end{array}$

\begin{tabular}{|c|c|c|c|c|c|c|c|c|c|c|}
\hline 1974 & 1,262 & 68 & 71.2 & $1, \pi / 2$ & 37.0 & 298 & 3,000 & & & \\
\hline 1975 & 1,524 & 73 & 65.7 & 2,321 & $(25,9)$ & 253 & 2,389 & & & \\
\hline 1976 & 1,941 & 77 & $\pi 5.8$ & 2,562 & 25.7 & 259 & 2,472 & & & \\
\hline 1977 & 2,364 & 88 & 85.1 & 2,779 & 74.6 & 310 & 3,173 & & & \\
\hline $19 \pi$ & 2,418 & 84 & 86.0 & 2,812 & 23.5 & 308 & 3,151 & & & \\
\hline 1979 & 2,320 & 74 & 7.7 & 2,986 & 15.9 & 300 & 3,035 & & & \\
\hline 1980 & 2,065 & 73 & 73.2 & 2,821 & 55.0 & 329 & 3,430 & & & \\
\hline 1981 & 2,267 & 62 & 66.3 & 3,422 & (16.8) & 291 & 2,903 & & & \\
\hline 1982 & 2,120 & 61 & 60.7 & 3,490 & 34.9 & 302 & 3,061 & & & \\
\hline 1983 & 2,733 & 79 & 71.7 & 3,812 & 32.3 & 309 & 3,165 & & & \\
\hline 1984 & 3,007 & 75 & 78.1 & 3,851 & 42.3 & 325 & 3,376 & & & \\
\hline 1985 & 3,044 & 60 & 64.3 & 4,732 & 56.8 & 354 & 3,781 & & & \\
\hline 1986 & 3,030 & 85 & 7.3 & 4,023 & 77.4 & 402 & 4,448 & & & \\
\hline $\begin{array}{l}\cdots \\
1987\end{array}$ & $\begin{array}{l}\cdots \\
3,122\end{array}$ & 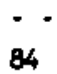 & $\begin{array}{l}\cdots \\
84.7\end{array}$ & $\cdots$ & 33.9 & $\because 06$ & $\begin{array}{l}-\cdots \\
4,497\end{array}$ & --- & --- & -- \\
\hline 1988 & 3,245 & 86 & 85.3 & 3,806 & 33.9 & 409 & 4,543 & 6,586 & 6,833 & 6,933 \\
\hline 1989 & & & & & 33.9 & 412 & 4,586 & 6,649 & 6,898 & 6,998 \\
\hline 1990 & & & & & 33.9 & 415 & 4,626 & 6,706 & 6,958 & 7,059 \\
\hline 1991 & & & & & 34.6 & 418 & 4,672 & 6,773 & 7,027 & 7,129 \\
\hline 1992 & & & & & 34.6 & 421 & 4,714 & 6,834 & 7,090 & 7,194 \\
\hline
\end{tabular}

Capacity-Capital Stock Regression: Code 1 RSO 0.440

Coefficients: Const -1125.1 stock 13.9

Ave. Capacity/Stock $(74-86): 10.2$ Ave, Heekly Hours (High) 103.2 Shift factor 1.53 Ave Weekly Hours (Low) 99.8 Shift Factor 1.58 Note: Output, Investment, and Capital Stock extrapolated for 1987 and 1988.

\section{Automotive and apparel trimmings}

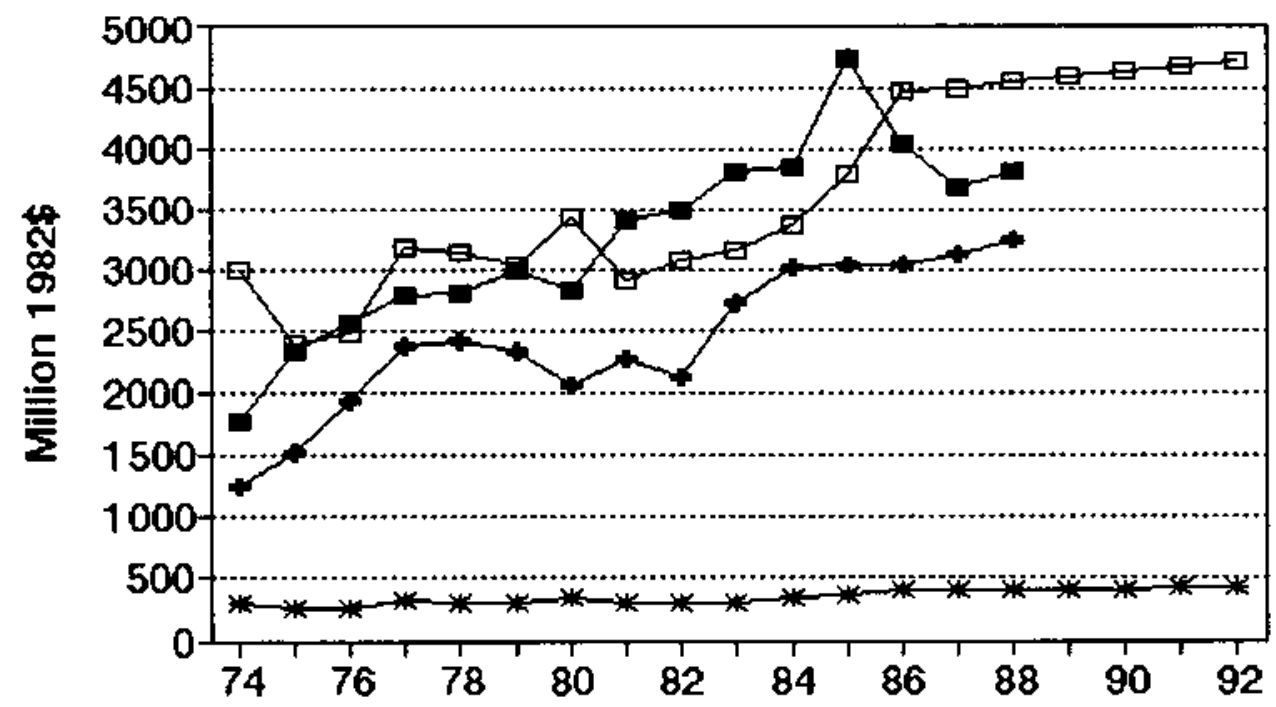

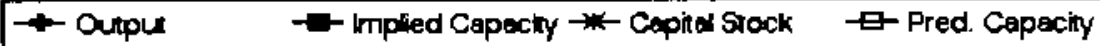




\section{No. 113 sIC 2397 schiffli machine embroideries}

MILLIONS OF 1982 DOLLARS (EXCL. CU RATES)

$\begin{array}{lccccccc} & \text { Q4 } & \text { Ann. Implied Gross } & \text { Net } & \text { Pred. } & \ldots \ldots \\ \text { Gross } & \text { CU } & \text { CU } & \text { Prac. } & \text { In- } & \text { Cap. } & \text { Prac. } & \text { Emergency Capacity } \\ \text { Output } & \text { Rate } & \text { Rate } & \text { Capac. } & \text { vest. } & \text { Stk. } & \text { Capac. } & 3 \text { mon. } 6 \text { mon. }>6 \text { mon. }\end{array}$

\begin{tabular}{|c|c|c|c|c|c|c|c|c|c|c|}
\hline 1974 & 195 & 63 & 64.8 & 300 & (31.3) & 63 & 349 & & & \\
\hline 1975 & 217 & 67 & 62.5 & 347 & $(4.7)$ & 52 & 346 & & & \\
\hline 1976 & 223 & 68 & 67.8 & 329 & 5.9 & 51 & 345 & & & \\
\hline 1977 & 222 & $\pi$ & 71.8 & 310 & $(0.5)$ & 45 & 344 & & & \\
\hline 1978 & 256 & 76 & 75.2 & 341 & 14.1 & 53 & 346 & & & \\
\hline 1979 & 168 & 74 & 74.8 & 224 & $(16.7)$ & 33 & 340 & & & \\
\hline 1980 & 259 & 69 & 70.8 & 366 & 6.4 & 36 & 341 & & & \\
\hline 1981 & 275 & 66 & 67.3 & 408 & 3.8 & 36 & 341 & & & \\
\hline 1982 & 306 & 68 & 66.8 & 457 & 18.2 & 50 & 345 & & & \\
\hline 1983 & 264 & 76 & 72.7 & 363 & $(5.9)$ & 40 & 342 & & & \\
\hline 1984 & 283 & 76 & 77.0 & 367 & 27.0 & 62 & 349 & & & \\
\hline 1985 & 241 & 71 & 72.0 & 335 & 4.7 & 61 & 348 & & & \\
\hline 1986 & 240 & 71 & 70.8 & 338 & 5.0 & 60 & 348 & & & \\
\hline$\therefore$ & $\cdots \cdots$ & -- & $-\cdots$ & -- & $\cdots \cdot$ & $\because$ & $\cdots$ & -- & -- & -- \\
\hline 1987 & 243 & 78 & 75.6 & 322 & 12.5 & 67 & 350 & & & \\
\hline 1988 & 248 & $m$ & 78.6 & 316 & 13.8 & 74 & 352 & 510 & 530 & 537 \\
\hline 1989 & & & & & 15.1 & 82 & 355 & 514 & 533 & 541 \\
\hline 1990 & & & & & 16.4 & 90 & 357 & 517 & 537 & 545 \\
\hline 1991 & & & & & 17.7 & $\infty$ & 360 & 521 & 541 & 549 \\
\hline 1992 & & & & & 19.0 & 109 & 363 & 525 & 545 & 553 \\
\hline
\end{tabular}

Capacity-Capital Stock Regression: Code 0 RSQ 0.000

Coefficients: Const 330.4 Stock 0.3 Ave. Capacity/Stock (74-86): 7.0 Ave. Heekly Hours (High) 103.2 Shift Factor 1.53 Ave Heekly Hours (LOW) 99.8 Shift Factor 1.58 Mote; Output, Investment, and Capital stock extrapolated for 1987 and 1988.

\section{Schiffli machine embroideries}

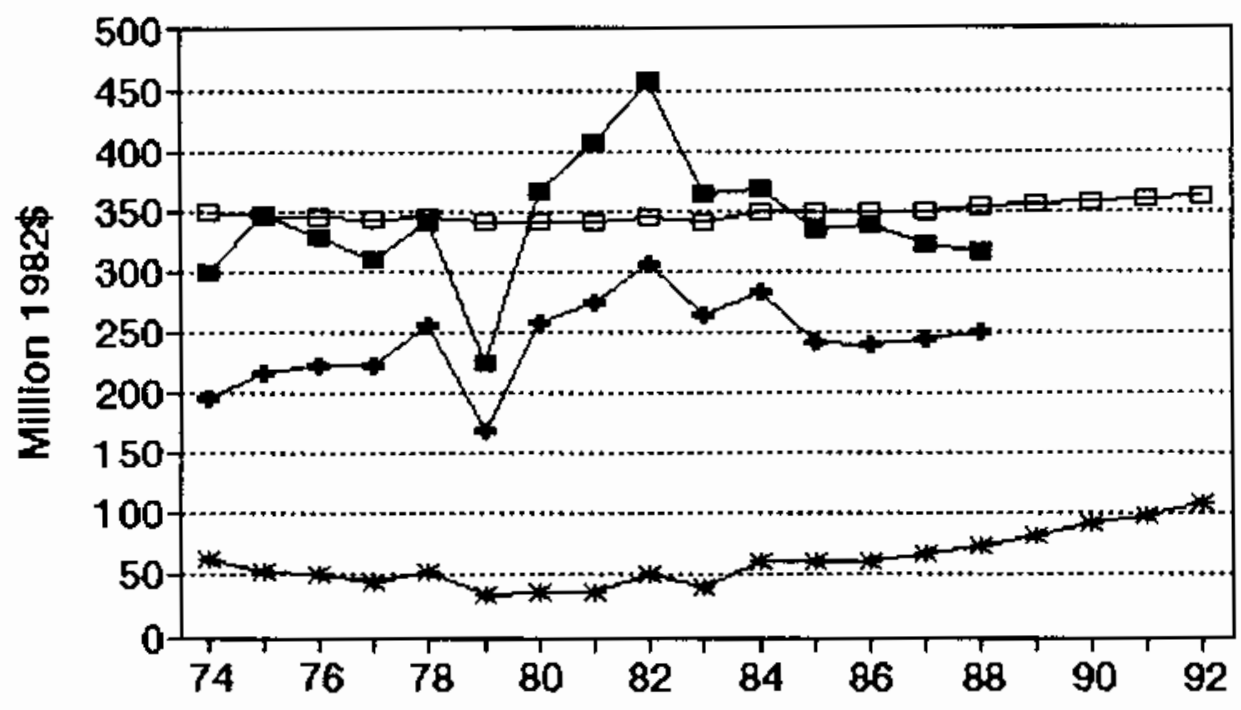

Oupur

-E mplied Cepacity $\rightarrow$ - Copited Sock

E Pred. Cepeciny 
No. 114 SIC 2399 Pabricated textile products, n.e.c.

MILLIONS OF 1982 DOLLARS (EXCL. CU RATES)

$\begin{array}{lccccccc} & \text { Q4 } & \text { Ann. Implied Gross } & \text { Net } & \text { Pred. } & \ldots \ldots \\ \text { Gross } & \text { CU } & \text { CU } & \text { Prac. } & \text { In- } & \text { Cap. } & \text { Prac. } & \text { Emergency Capacity } \\ \text { Output } & \text { Rate } & \text { Rate } & \text { Capac. } & \text { vest. } & \text { Stk. } & \text { Capac, } & 3 \text { mon. } 6 \text { mon. }>6 \text { mon. }\end{array}$

\begin{tabular}{|c|c|c|c|c|c|c|c|c|c|c|}
\hline 1974 & 1,632 & $55 *$ & 58.0 & 2,813 & 44.5 & 301 & 3,298 & & & \\
\hline 1975 & 1,606 & 61 & 53.7 & 2,990 & $(7.5)$ & 274 & 2,928 & & & \\
\hline 1976 & 1,658 & 65 & 63.7 & 2,601 & 13.4 & 266 & 2,823 & & & \\
\hline 1977 & 1,586 & 71 * & 70.0 & 2,266 & 6.5 & 252 & 2,627 & & & \\
\hline 1978 & 1,451 & 74 & 73.4 & 1,978 & $(8.8)$ & 224 & 2,242 & & & \\
\hline 1979 & 1,524 & 86 & 81.7 & 1,865 & 4.2 & 209 & 2,032 & & & \\
\hline 1980 & 1,396 & 63 & 71.4 & 1,955 & 32.6 & 219 & 2,176 & & & \\
\hline 1981 & 1,297 & 63 & 63.2 & 2,050 & 20.4 & 216 & 2,134 & & & \\
\hline 1982 & 1,488 & 62 & 61.7 & 2,413 & 32.2 & 221 & 2,203 & & & \\
\hline 1983 & 1,730 & 87 & 76.8 & 2,278 & 51.1 & 243 & 2,497 & & & \\
\hline 1984 & 2,157 & 75 & 81.4 & 2,650 & 25.8 & 240 & 2,457 & & & \\
\hline 1985 & 2,228 & 68 & 69.2 & 3,221 & 50.8 & 261 & 2,748 & & & \\
\hline 1986 & 2,047 & 47 & 54.6 & 3,752 & 23.0 & 255 & 2,667 & & & \\
\hline$\cdots$ & $\cdots \cdot$ & - & $-\cdots$ & $\cdots$ & --- & $-\cdots$ & $-\cdot-$ & $--\cdot$ & $-\cdots$ & $\cdots$ \\
\hline 1987 & 2,055 & 65 & 58.5 & 3,511 & 25.6 & 252 & 2,621 & & & \\
\hline 1988 & 2,064 & 79 & 73.8 & 2,797 & 25.6 & 269 & 2,580 & 7,412 & 7,808 & 7,967 \\
\hline 1989 & & & & & 25.7 & 246 & 2,545 & 7,311 & 7.701 & 7,859 \\
\hline 1990 & & & & & 25.7 & 244 & 2,514 & 7.222 & 7,607 & 7,762 \\
\hline 1991 & & & & & 27.0 & 263 & 2,504 & 7,195 & 7,578 & 7,733 \\
\hline 1992 & & & & & 27.0 & 243 & 2,496 & 7,171 & 7,553 & 7,707 \\
\hline
\end{tabular}

Capecity-Capital Stock Regression: Code 1 RSQ 0.440

Coefficients: Const -852.1 Stock 13.8

Ave. Capacity/Stock $(74-86): 10.3$. Ave. Weekly Hours (High) 51.0 Shift Factor 3.09 Ave Heekly Hours (Low) 46.9 Shift Factor 3.36 * Imputed Note: Output, Investment, and Capital Stock extrapolated for 1987 and 1988.

\section{Fabricated textile products, n.e.c}

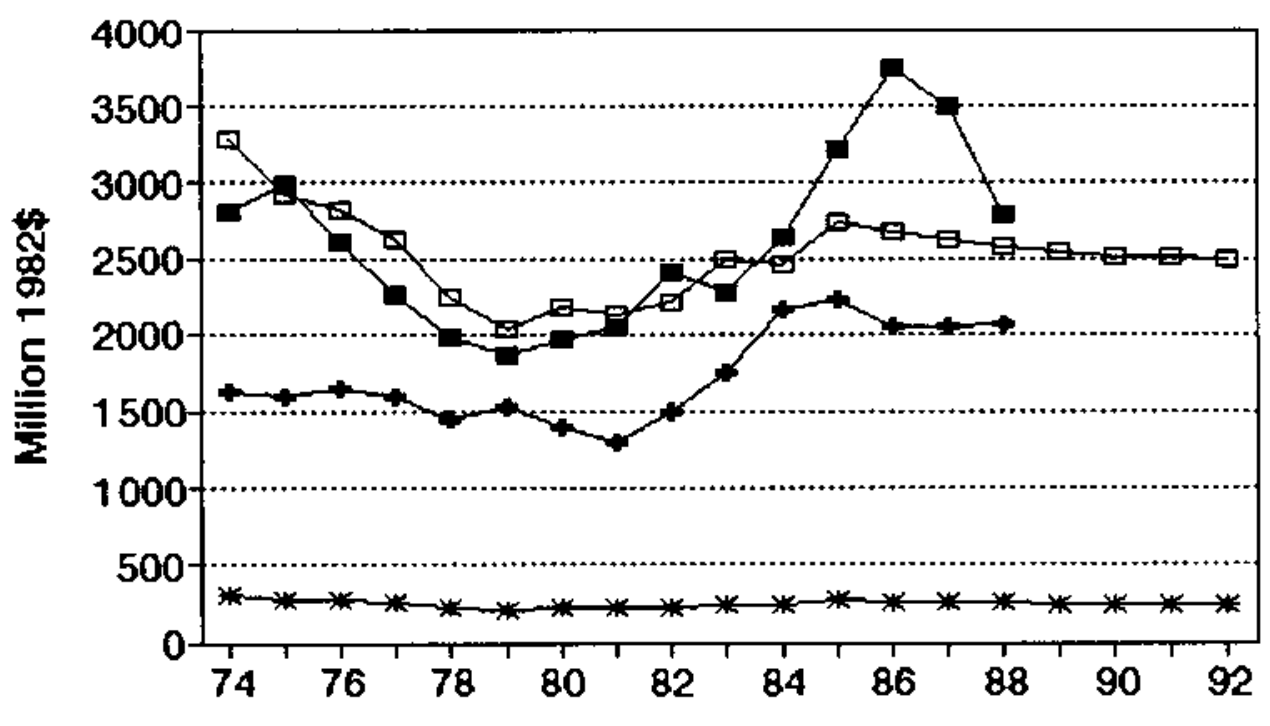

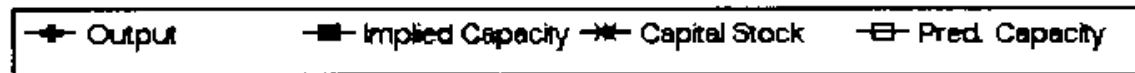




\section{No. 115 sIc 2411 Logging camps and logging contractors}

MILLIONS OF 1982 DOLLARS (EXCL. CU RATES)

$\begin{array}{lccccccc} & 04 & \text { Ann. } & \text { Implied Gross } & \text { Net } & \text { Pred. } & \ldots . \ldots . . . \\ \text { Gross } & \text { CU } & \text { cU } & \text { Prac. } & \text { In. } & \text { Cap. } & \text { Prac. } & \text { Emergency Capacity } \\ \text { Output } & \text { Rate } & \text { Rate } & \text { Capac. } & \text { vest. } & \text { Stk. } & \text { Capac. } & 3 \text { mon. } 6 \text { mon. }>6 \text { mon. }\end{array}$

\begin{tabular}{|c|c|c|c|c|c|c|c|c|c|c|}
\hline 1974 & 5,596 & 69 & 69.2 & 8,084 & 219.1 & 2,627 & 7,670 & & & \\
\hline 1975 & 6,149 & 71 & 70.2 & 8,763 & 719.6 & 3,081 & 7,915 & & & \\
\hline 1976 & 6,012 & 88 & 81.6 & 7,366 & 340.1 & 3,145 & 8,161 & & & \\
\hline 1977 & 7,138 & 90 & 89.3 & 7,994 & $9 \% 5.4$ & 3,798 & 8,406 & & & \\
\hline 1978 & 6,690 & 88 & 88.7 & 7,539 & 576.7 & 4,007 & 8,651 & & & \\
\hline 1979 & 6,676 & 72 & 78.0 & 8,561 & 281.0 & 3,930 & 8,897 & & & \\
\hline 1980 & 8,125 & 84 & 79.4 & 10,227 & 248.1 & 3,810 & 9,142 & & & \\
\hline 1981 & 7,656 & 76 & 79.1 & 9,682 & 238.5 & 3,667 & 9,388 & & & \\
\hline 1982 & 8,304 & 83 & 80.4 & 10,333 & 272.3 & 3,540 & 9,633 & & & \\
\hline 1983 & 7,677 & 89 & 86.8 & 8,843 & 89.1 & 3,230 & 9,878 & & & \\
\hline 1984 & 7,912 & 66 & 74.7 & 10,592 & 245.6 & 3,077 & 10,124 & & & \\
\hline 1985 & 7,556 & $\pi$ & 72.9 & 10,368 & 188.5 & 2,886 & 10,369 & & & \\
\hline 1986 & 7,426 & 67 & 70.8 & 10,496 & 244.3 & 2,770 & 10,615 & & & \\
\hline$\cdots$ & $\cdots$ & $\because$ & $\cdots$ & $--\cdot$ & $-\cdot$ & $\because \cdots$ & $\cdots$ & $\cdot \cdot \cdot$ & $-\cdot-$ & --- \\
\hline 1987 & 7.523 & 70 & 68.9 & 10,926 & 366.0 & 2,773 & 10,860 & & & \\
\hline 1988 & 7,651 & 86 & 80.0 & 9,563 & 461.5 & 2,872 & 11,105 & 34,244 & 36,210 & 37,003 \\
\hline 1989 & & & & & 480.3 & 2,976 & 11,351 & 35,000 & 37,010 & 37,821 \\
\hline 1990 & & & & & 447.8 & 3,034 & 11,596 & 35,757 & 37,811 & 38,638 \\
\hline 1991 & & & & & 475.7 & 3,113 & 11,842 & 36,514 & 38,611 & 39,456 \\
\hline 1992 & & & & & 559.5 & 3,265 & 12,087 & 37,271 & 39,411 & 40,273 \\
\hline
\end{tabular}

Capecity-Capital stock Regression: Code -2 RSO 0.640

Coefficients: Const 7424.4 Time 245.4

Ave. Capacity/stock $(74-86): 2.7$ Ave. Weekly Hours (High) 47.3 Shift Factor 3.33 Ave Weekly Hours (Low) 46.5 Shift factor 3.38 Note: Output, Investment, and Capital Stock extrapolated for 1987 and 1988.

\section{Logging camps and logging contract}

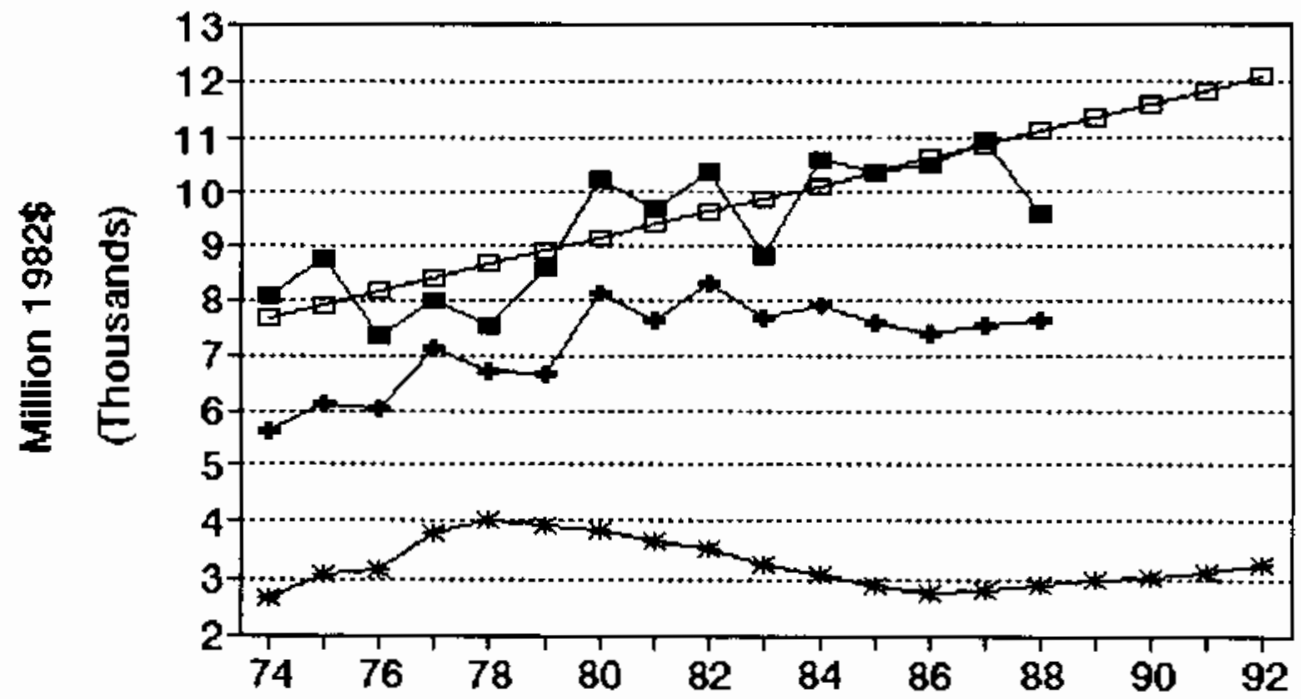

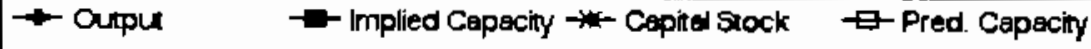


No. 116 sIC 2421 Sawm111s and planing mille, general MILLIONS OF 1982 DOLLARS (EXCL. CU RATES)

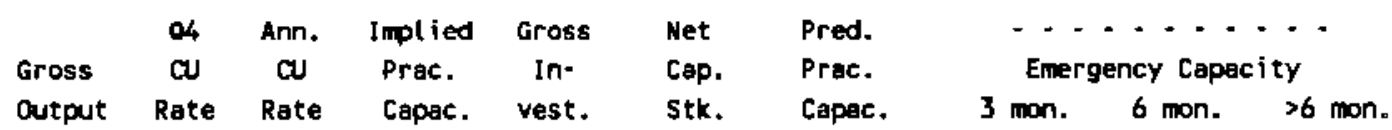

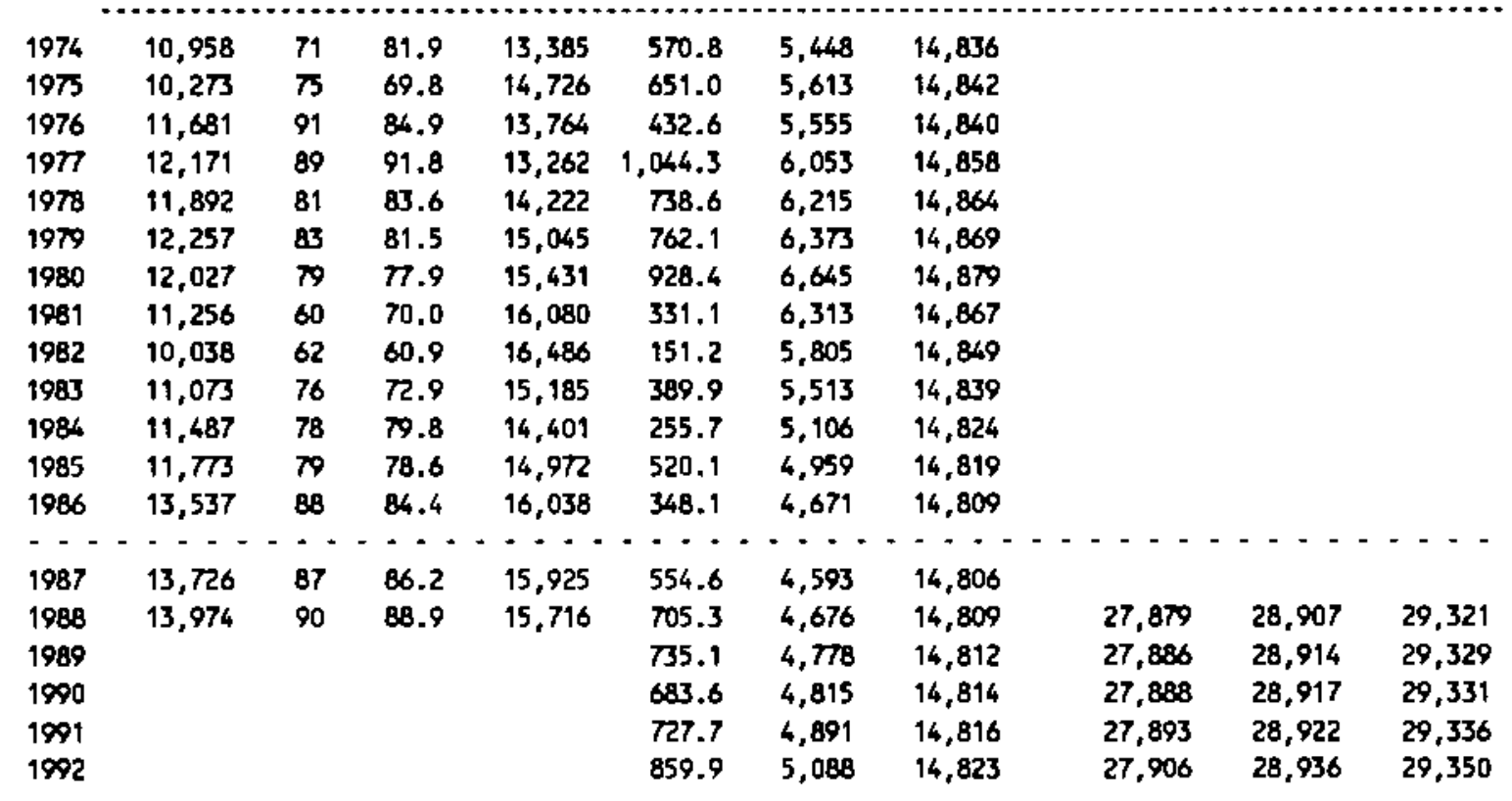

Capecity-Capital Stock Regression: Code 1 RSO 0.000 Coefficients: Const 14641.8 stock 0.0 Ave. Capacity/Stock $(74-86): 2.6$ Ave. Weekly Hours (High) 79.6 Shift factor 1.98 Ave Weekly Hours (Low) 73.3 Shift Factor 2.09 Note: Output, Imvestment, and Capital Stock extrapolated for 1987 and 1988.

2421 Sawmills and planing mills, genera

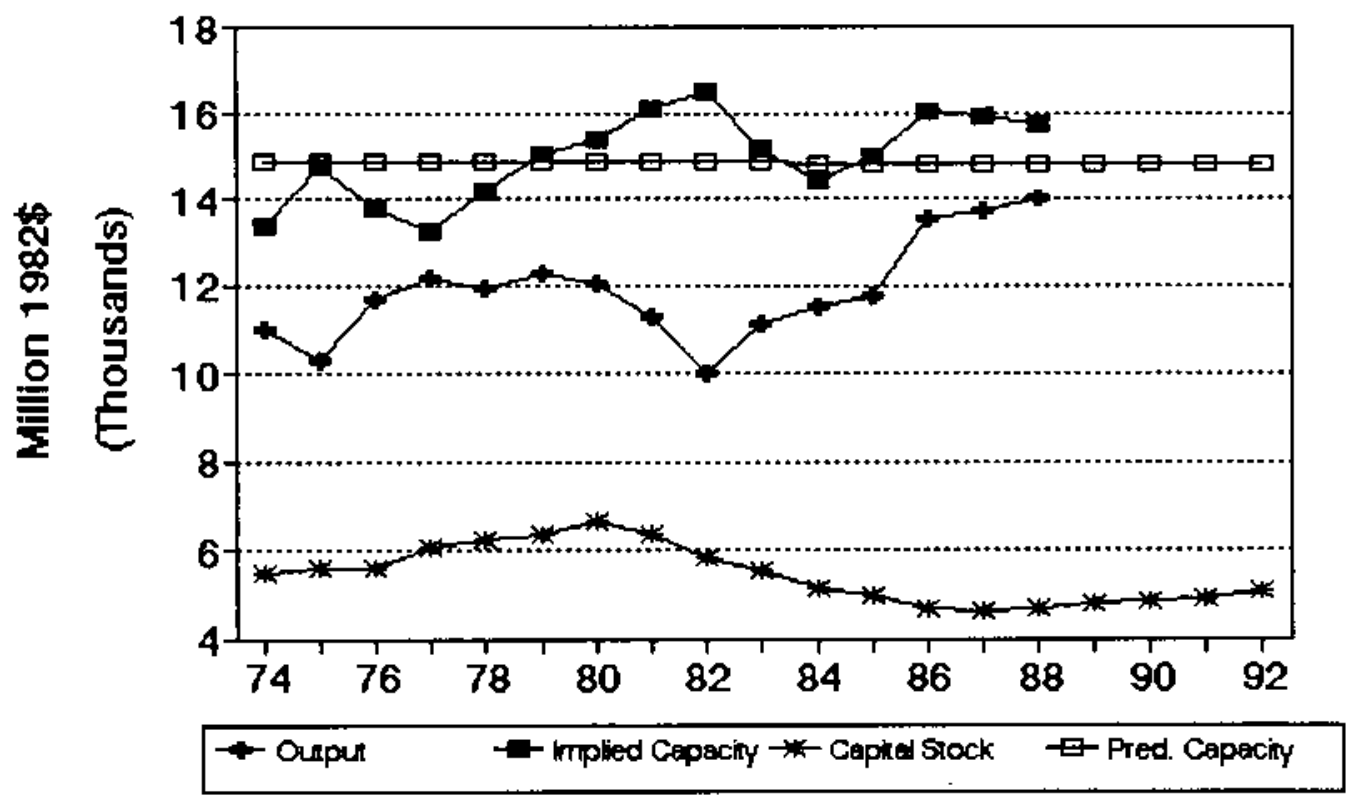




\section{No, 117 SIC 2426 Hardwood dimenaion and flooring}

MILLIONS OF 1982 DOLLARS (EXCL. CU RATES)

\begin{tabular}{|c|c|c|c|c|c|c|c|}
\hline & $\begin{array}{l}04 \\
\mathrm{CU}\end{array}$ & $\begin{array}{l}\text { Ann. } \\
\text { Cu }\end{array}$ & $\begin{array}{l}\text { Implied } \\
\text { Prac. }\end{array}$ & $\begin{array}{l}\text { Gross } \\
\text { In- }\end{array}$ & $\begin{array}{l}\text { Net } \\
\text { Cap. }\end{array}$ & $\begin{array}{l}\text { Pred. } \\
\text { Prac. }\end{array}$ & $\begin{array}{l}---1- \\
\text { Emergency Capacity }\end{array}$ \\
\hline$+n$ & Rate & Rate & & fest & & & \\
\hline
\end{tabular}

\begin{tabular}{|c|c|c|c|c|c|c|c|c|c|c|}
\hline 1974 & 1,040 & 59 & 62.1 & 1,673 & 29.2 & 350 & 1,468 & & & \\
\hline 1975 & 1,041 & 70 & 64.8 & 1,607 & 50.0 & 365 & $1,5 \%$ & & & \\
\hline 1976 & 1,116 & 59 & 63.2 & 1,765 & 32.6 & 362 & 1,564 & & & \\
\hline 1977 & 1,171 & 71 & 67.0 & 1,748 & 35.0 & 362 & 1,563 & & & \\
\hline 1978 & 1,249 & 67 & 68.4 & 1,827 & 55.0 & 379 & 1,712 & & & \\
\hline 1979 & 1,126 & 86 & 78.6 & 1,432 & 22.4 & 364 & 1,586 & & & \\
\hline 1980 & 990 & $84 *$ & 83.4 & 1,187 & 32.9 & 358 & 1,534 & & & \\
\hline 1981 & 1,003 & 81 & 83.2 & 1,205 & 16.9 & 335 & 1,340 & & & \\
\hline 1982 & 919 & 87 & 84.6 & 1,087 & 41.4 & 334 & 1,328 & & & \\
\hline 1983 & 1,029 & 90 & 89.9 & 1,144 & 33.6 & 323 & 1,234 & & & \\
\hline 1984 & 1,151 & 84 & 87.4 & 1,318 & 23.4 & 304 & 1,072 & & & \\
\hline 1985 & 1.245 & 89 & 87.1 & 1,428 & 43.2 & 304 & 1,489 & & & \\
\hline 1986 & 1,349 & 86 & 87.1 & 1,550 & 40.4 & 304 & 1,489 & & & \\
\hline-- & --- & -- & $\cdots$ & $\because \cdot$ & $\cdots$ & $\cdots$ & $\because \cdots$ & $\cdots$ & $\because \cdot$ & $\cdots$ \\
\hline 1987 & 1,363 & 87 & 86.2 & 1,582 & 35.5 & 297 & 1,436 & & & \\
\hline 1988 & 1,381 & 84 & 85.2 & 1,622 & 36.2 & 293 & 1,397 & 3,130 & 3,259 & 3,312 \\
\hline 1989 & & & & & 36.4 & 289 & 1,364 & 3,057 & 3,183 & 3,234 \\
\hline 1990 & & & & & 36.1 & 285 & 1,334 & 2,988 & 3,112 & 3,162 \\
\hline 1991 & & & & & 36.3 & 282 & 1,300 & 2,934 & 3,055 & 3,104 \\
\hline 1992 & & & & & 37.0 & 281 & 1,294 & 2,809 & 3,019 & 3,067 \\
\hline
\end{tabular}

Capacity-Capital Stock Regression: Code -1 RSO 0.460

Coefficients: Const -1498.3 stock 8.5 D(85-86), 417.0

Ave. Capacity/Stock $(74-86): 4.3$ Ave, Weekly Hours (High) 66.4 Shift Factor 2.37 Ave Weekly Hours (Low) 63.4 Shift factor 2.49

* Imputed Note: Output, Investment, and Capital Stock extrapoleted for 1987 and 1988.

\section{Hardwood dimension and flooring}

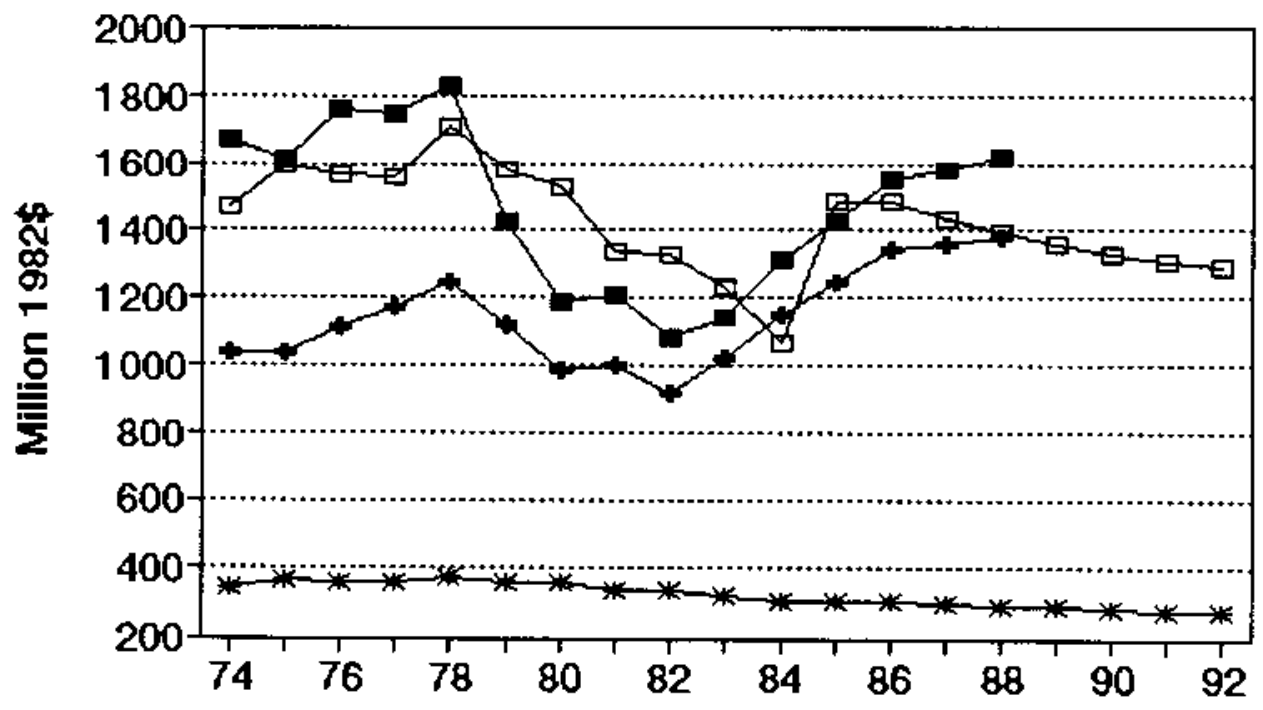

- Oupu $\quad-$ - Implied Capacity $\rightarrow$ - Cepite Stock $\quad \square$ Pred. Cepecity 
No. 118 SIC 2429 Special product samills, n.e.c. MILLIONS OF 1982 DOLLARS (EXCL. CU RATES)

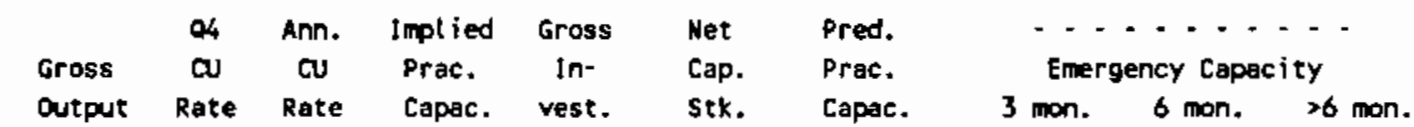

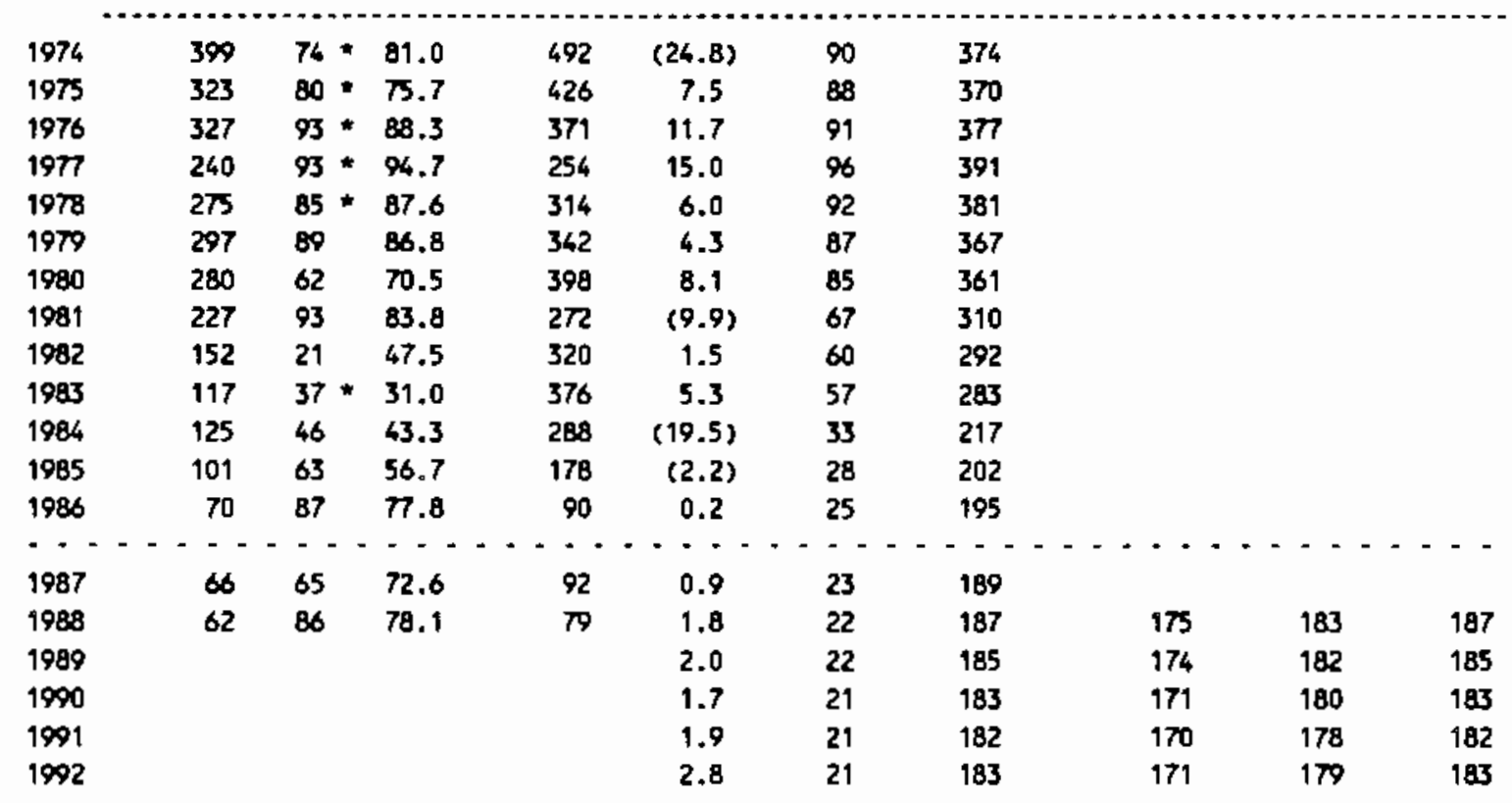

Copacity-Capital Stock Regression: Code 1 RSQ 0.470 Coefficients: Const 124.4 stock . 2.8

Ave. Capacity/Stock $(76-86): 4.6$ Ave. Heek(y Hours (High) 1984.0 shift Factor 1.00 Ave Weekly Hours (LOW) 1984.0 shift Factor 1.0 - Imputed Mote: Output, Investment, and Capital stock extrapolated for 1997 and 1988.

\section{Special product sawmills, n.e.c.}

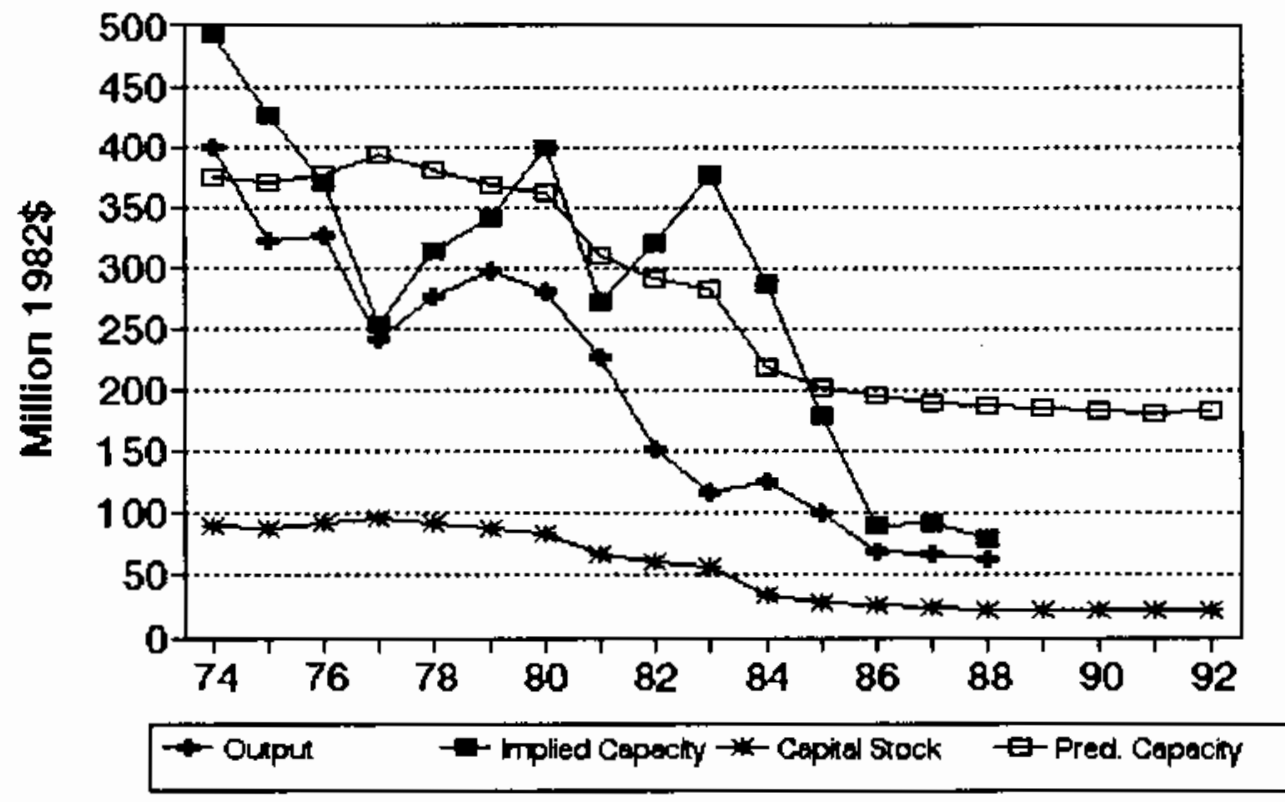




\section{No. 119 SIC 2431 Millwork}

MJLLIONS OF 1982 DOLLARS (EXCL. CU RATES)

$\begin{array}{lccccccc} & 04 & \text { Am. } & \text { Implied Gross } & \text { Net } & \text { Pred. } & -\ldots \ldots \\ \text { Gross } & \text { CU } & \text { CU } & \text { Prac. } & \text { In- } & \text { Cap. } & \text { Prac. } & \text { Emergency Capacity } \\ \text { Output } & \text { Rate } & \text { Rate } & \text { Capac. } & \text { vest. } & \text { Stk. } & \text { Capac. } & 3 \text { mon. } 6 \text { mon. }>6 \text { mon. }\end{array}$

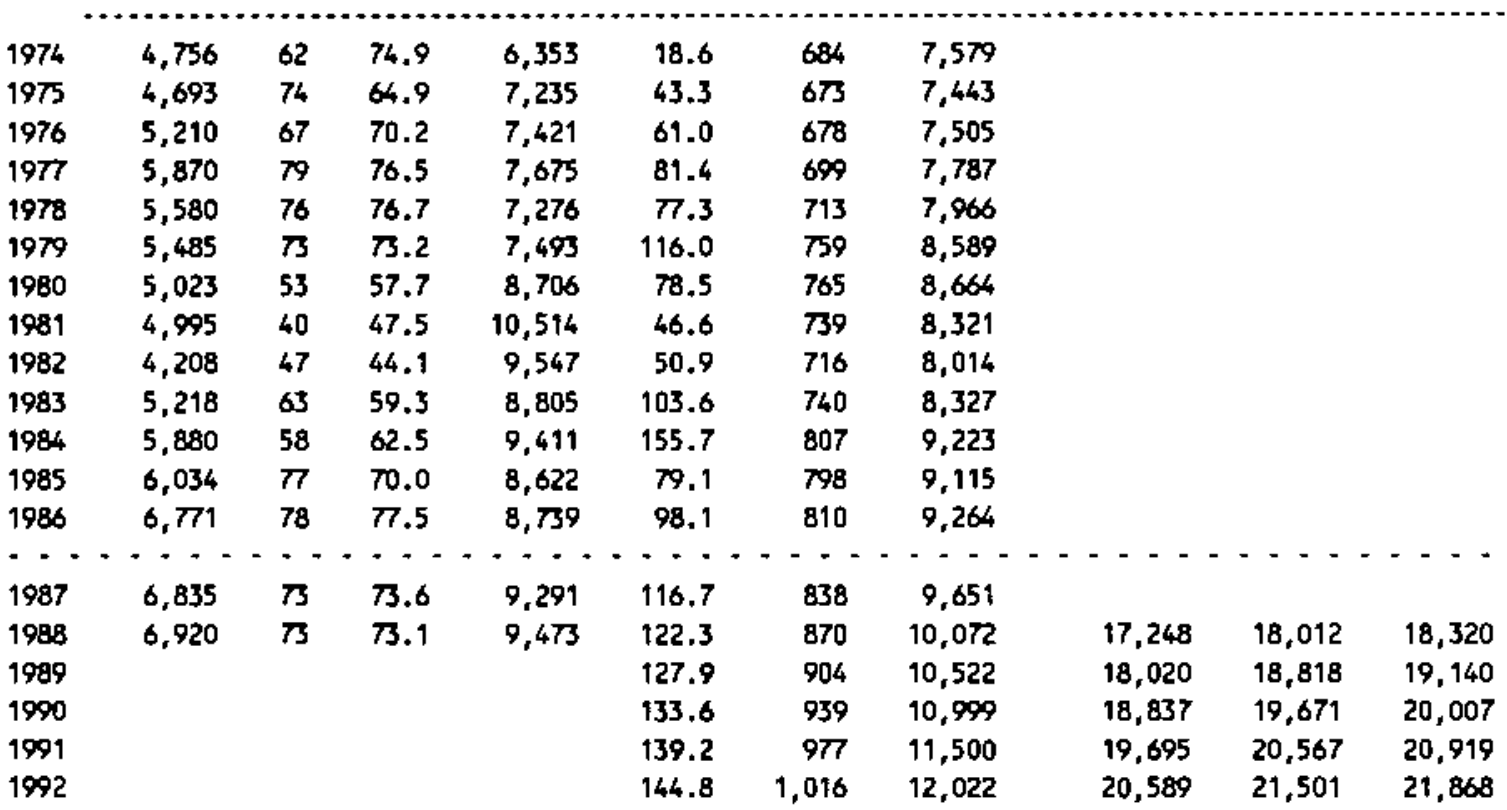

Capacity-Capital Stock Regression: Code 0 RSQ 0.310

Coefficients: Const -1564.9 stock 13.4

Ave. Capacity/Stock $(74-86): 11.3$ Ave. Weekly Hours (High) 86.6 Shift Factor 1.82 Ave Ueekly Hours (LOW) 81.4 shift Factor 1.93 Note: Output, Investment, and Capital Stock extrapolated for 1987 and 1988.

\section{Millwork}

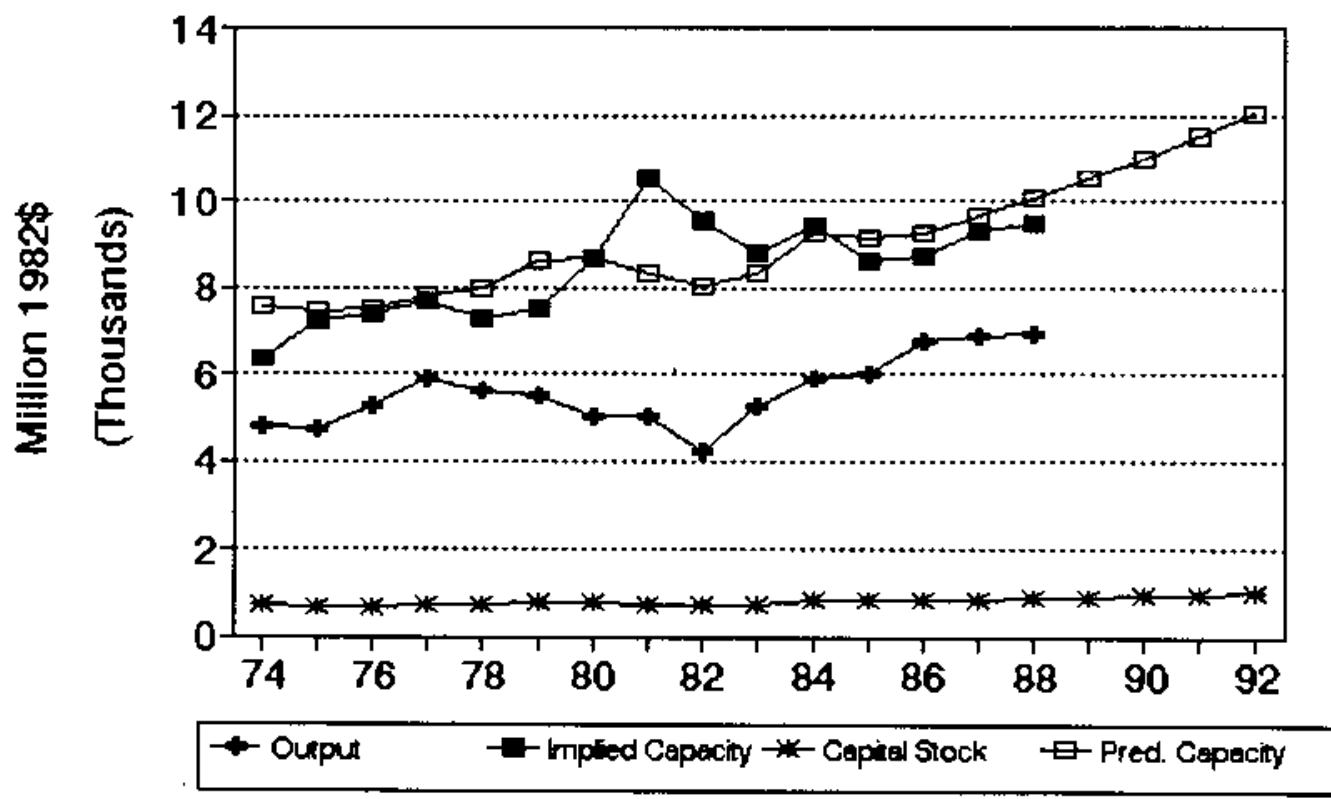




\section{No. 120 sIC 2434 Wood kitchen cabinet:}

MILLIOWS OF 1982 DOLLARS (EXCL. CU RATES)

$\begin{array}{lccccccc} & 04 & \text { Ann. } & \text { Implied Gross } & \text { Net } & \text { Pred. } & \ldots \\ \text { Gross } & \text { CU } & \text { CU } & \text { Prac. } & \text { In- } & \text { Cap. } & \text { Prac. } & \text { Emergency Capacity } \\ \text { Output } & \text { Rete } & \text { Rate } & \text { Capac. } & \text { vest. } & \text { 5tk. } & \text { Capec. } & 3 \text { mon. } 6 \text { mon. }>6 \text { mon. }\end{array}$

\begin{tabular}{|c|c|c|c|c|c|c|c|c|c|c|}
\hline 1974 & 1,586 & 60 & 69.9 & 2,269 & $(24.3)$ & 284 & 3,084 & & & \\
\hline 1975 & 1,391 & 45 & 47.6 & 2,922 & 15.2 & 277 & 3,044 & & & \\
\hline 1976 & 1,543 & 53 & 50.0 & 3,087 & 7.9 & 262 & 2,965 & & & \\
\hline 1977 & 2,311 & 62 & 59.9 & 3,858 & 72.8 & 307 & 3,208 & & & \\
\hline 1978 & 2,595 & 67 & 64.8 & 4,003 & 66.1 & 343 & 3,400 & & & \\
\hline 1979 & 2,582 & 80 & 74.4 & 3,470 & 76.6 & 386 & 3,634 & & & \\
\hline 1980 & 2,411 & 67 & 69.3 & 3,479 & 14.2 & 367 & 3,530 & & & \\
\hline 1981 & 2,271 & 63 & 67.7 & 3,355 & 45.1 & 377 & 3,584 & & & \\
\hline 1982 & 2,078 & 59 & 60.1 & 3,460 & 88. 1 & 422 & 3,827 & & & \\
\hline 1983 & 2,482 & 85 & 77.3 & 3,211 & 42.3 & 417 & 3,804 & & & \\
\hline 1984 & 2,968 & 78 & 83.5 & 3,554 & 24.6 & 396 & 3,687 & & & \\
\hline 1985 & 2,917 & 71 & 73.6 & 3,963 & 47.5 & 399 & 3,705 & & & \\
\hline 1986 & 3,106 & 65 & 67.2 & 4,621 & 58.3 & 413 & 3,781 & & & \\
\hline $\begin{array}{l}\cdots \\
1987\end{array}$ & $\cdots$ & $\begin{array}{l}-- \\
76\end{array}$ & $\begin{array}{l}-\cdots \\
70.9\end{array}$ & $\begin{array}{l}\cdots, 480 \\
4\end{array}$ & 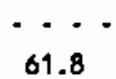 & $\begin{array}{l}--- \\
430\end{array}$ & $\begin{array}{l}\cdots \\
3,872\end{array}$ & $\cdots$ & $-\cdots$ & -- \\
\hline 1988 & 3,282 & 87 & 82.9 & 3,958 & 64.6 & 448 & 3,968 & 8,515 & 8,902 & 9,058 \\
\hline 1989 & & & & & 67.4 & 466 & 4,069 & 8,732 & 9,128 & 9,288 \\
\hline 1990 & & & & & 70.2 & 486 & 4,173 & 8,957 & 9,364 & 9,528 \\
\hline 1991 & & & & & 73.0 & 506 & 4,282 & 9,190 & 9,607 & 9,776 \\
\hline 1992 & & & & & $\pi .8$ & 527 & 4,394 & 9,430 & 9,858 & 10,031 \\
\hline
\end{tabular}

Capecity-Capital Stock Regression: Code 1 RSQ 0.290 Coefficients: Const 1546.8 stock 5.4 Ave. Weekly Hours (High) 69.0 Shift Factor 2.28 Ave Heekly Hours (Low) 60.0 Shift factor 2.63 Wote: Output, Investment, and Capital Stock extrapolated for 1987 and 1989.

\section{Wood kitchen cabinets}

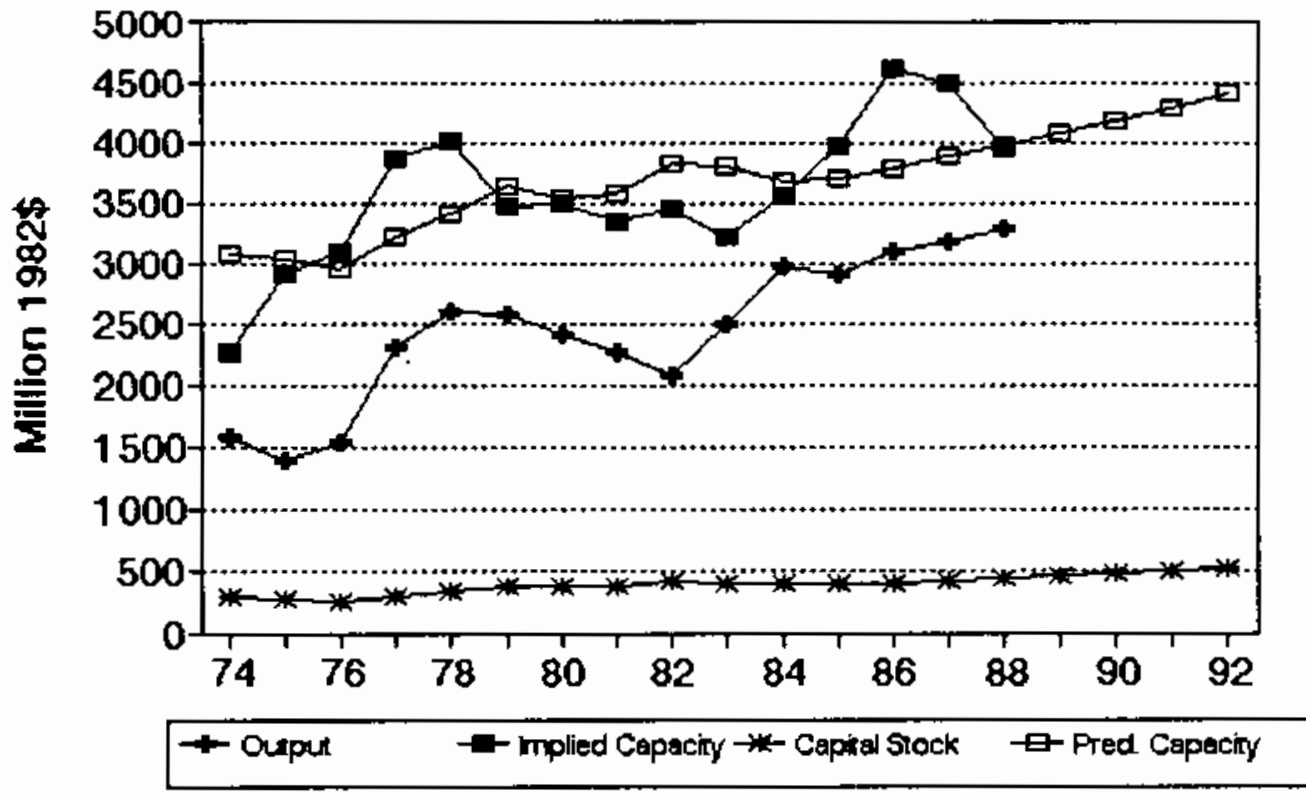




\section{No. 121 SIC 2435 Hardwood veneer and plywood}

MILLIONS OF 1982 DOLLARS (EXCL. CU RATES)

$\begin{array}{lccccccc} & Q 4 & \text { Ann. Implied } & \text { Gross } & \text { Het } & \text { Pred. } & \ldots \ldots \ldots \\ \text { Gross } & \text { CU } & \text { CU } & \text { Prac. } & \text { In- } & \text { Cap. } & \text { Prac. } & \text { Emergency Capacity } \\ \text { Output } & \text { Rate } & \text { Rate } & \text { Capec. } & \text { vest. } & \text { Stk. } & \text { Capac. } & 3 \text { mon. } 6 \text { mon. }>6 \text { mon. }\end{array}$

\begin{tabular}{|c|c|c|c|c|c|c|c|c|c|c|}
\hline 1974 & 1,384 & 62 & 62.0 & 2,232 & 20.3 & 400 & 1,711 & & & \\
\hline 1975 & 1,219 & 67 & 65.1 & 1,872 & 7.3 & 369 & 1,909 & & & \\
\hline 1976 & 1,297 & 83 & 77.0 & 1,685 & 7.1 & 339 & 2,105 & & & \\
\hline 1977 & 1,539 & 80 & 81.1 & 1,898 & 71.3 & 367 & 1,921 & & & \\
\hline 1978 & 1,513 & 69 & 73.1 & 2,069 & 25.2 & 350 & 2,034 & & & \\
\hline 1979 & 1,551 & 82 & 77.1 & 2,011 & 45.8 & 351 & 2,024 & & & \\
\hline 1980 & 1,455 & 72 & 75.8 & 1,920 & 65.3 & 369 & 1,908 & & & \\
\hline 1981 & 1,491 & 68 & 69.5 & 2,146 & 21.7 & 345 & 2,062 & & & \\
\hline 1982 & 1,296 & 82 & 76.8 & 1,689 & 2.3 & 305 & 2,319 & & & \\
\hline 1983 & 1,492 & 76 & 78.3 & 1,906 & 50.9 & 309 & 2,295 & & & \\
\hline 1984 & 1,553 & 52 & 61.0 & 2,545 & 20.1 & 286 & 2,446 & & & \\
\hline 1985 & 1,622 & 54 & 53.3 & 3,046 & 21.8 & 266 & 2,570 & & & \\
\hline 1986 & 1,713 & 61 & 58.4 & 2,934 & 26.1 & 254 & 2,651 & & & \\
\hline$\cdot \cdot$ & $\cdots \cdot$ & $\cdot-$ & $\cdots$ & $\cdots$ & --- & -- & $-\cdots$ & $\cdots$ & $\cdots-$ & $-\cdot-$ \\
\hline 1987 & 1,725 & 65 & 63.5 & 2,716 & 28.2 & 243 & 2,719 & & & \\
\hline 1988 & 1,740 & 67 & 66.3 & 2,626 & 31.0 & 237 & 2,760 & 4,722 & 4,942 & 5,031 \\
\hline 1989 & & & & & 31.5 & 232 & 2,791 & 4,774 & 4,997 & 5,087 \\
\hline 1990 & & & & & 30.6 & 227 & 2,823 & 4,829 & 5,055 & 5,146 \\
\hline 1991 & & & & & 31.4 & 224 & 2,845 & 4,867 & $5, \infty 94$ & 5,186 \\
\hline 1992 & & & & & 33.8 & 223 & 2,848 & 4,872 & 5,100 & 5,192 \\
\hline
\end{tabular}

Capacity-Capital Stock Regression: Code 1 RSQ 0.420 Coefficients: Const 4290.1 Stock -6.5

Ave. Capacity/Stock $(74-86): 6.5$ Ave. Weekly Hours (High) 86.4 Shift Factor 1.82 Ave Weekly Hours (Low) 85.1 Shift Factor 1.85 Note: Output, Investment, and Capital Stock extrapolated for 1987 and 1988.

\section{Hardwood veneer and plywood}

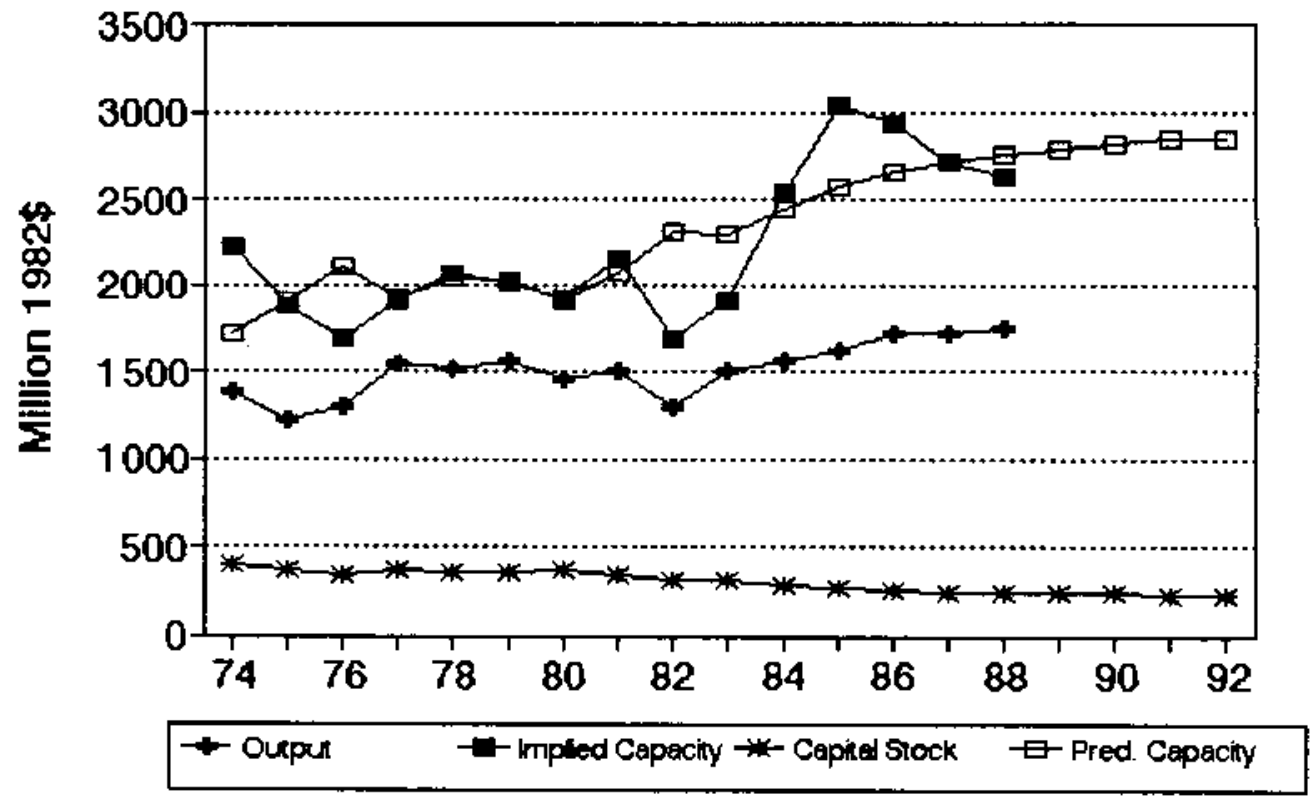


No. 122 SIC 2436 goftwood veneer and plywood

MILLIONS OF 1982 DOLLARS (EXCL. CU RATES)

$\begin{array}{lccccccc} & 04 & \text { Am. Implied } & \text { Gross } & \text { Net } & \text { Pred. } & -\ldots \ldots \ldots \\ \text { Gross } & \text { CU } & \text { CU } & \text { Prac. } & \text { In- } & \text { Cap. } & \text { Prac. } & \text { Emergency Capacity } \\ \text { Output Rate } & \text { Rate } & \text { Capac. } & \text { vest. } & \text { Stk. } & \text { Capac. } & 3 \text { mon. } 6 \text { mon. }\end{array}$

\begin{tabular}{|c|c|c|c|c|c|c|c|c|c|c|}
\hline 1974 & 3,130 & 70 & 7.7 & 4,028 & 195.9 & 1,744 & 4,219 & & & \\
\hline 1975 & 3,119 & 81 & 74.1 & 4,207 & 271.3 & 1,852 & 4,215 & & & \\
\hline 1976 & 3,625 & 87 & 84.8 & 4,276 & 286.1 & 1,957 & 4,211 & & & \\
\hline 1977 & 3,681 & 91 & 91.0 & 4.048 & 167.0 & 1,939 & 4,212 & & & \\
\hline 1978 & 3,835 & 91 & 90.7 & 4,229 & 271.5 & 2,025 & 4,209 & & & \\
\hline 1979 & 3,584 & 83 & 85.4 & 4,198 & 172.8 & 2,009 & 4,209 & & & \\
\hline 1980 & 3,340 & 80 & 79.2 & 4,217 & 298.1 & 2,108 & 4,205 & & & \\
\hline 1981 & 3,369 & 74 & 78.7 & 4,280 & 184.2 & 2,089 & 4,206 & & & \\
\hline 1982 & 3,204 & 79 & 76.8 & 4,171 & 79.2 & 1.961 & 4,211 & & & \\
\hline 1983 & 3,656 & 88 & 86.6 & 4,221 & 76.1 & 1,826 & 4,216 & & & \\
\hline 1984 & 3,763 & 83 & 87.0 & 4,325 & 233.9 & 1,829 & 4,216 & & & \\
\hline 1985 & 3,791 & 93 & 89.3 & 4,246 & 119.2 & 1,723 & 4,220 & & & \\
\hline 1986 & 3,995 & 92 & 92.3 & 4,330 & 111.0 & 1,617 & 4,224 & & & \\
\hline 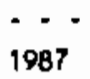 & 4,021 & 90 & 89.8 & $\begin{array}{l}\cdots, 478 \\
4,478\end{array}$ & : - & 1,601 & A,225 & $\cdots$ & - - & 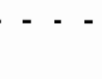 \\
\hline 1988 & 4,055 & 95 & 93.2 & 4,353 & 239.3 & 1,628 & 4,224 & 5,119 & 5,197 & 5,229 \\
\hline 1989 & & & & & 247.4 & 1,660 & 4,223 & 5,117 & 5,196 & 5,228 \\
\hline 1990 & & & & & 233.6 & 1,673 & 4,222 & 5,117 & 5,195 & 5,227 \\
\hline 1991 & & & & & 245.4 & 1,697 & 4,221 & 5,1 ใช & 5,194 & 5,226 \\
\hline 1992 & & & & & 281.4 & 1,754 & 4,219 & 5,113 & 5,192 & 5,223 \\
\hline
\end{tabular}

Capecity-Capital stock Regression: Code 1 RSQ 0.000 Coefficients: Const 4286.7 stock -0.0

Ave. Capocity/Stock $(74-86): 2.2$ Ave. Weekly Hours (High) 128.0 Shift Factor 1.24 Ave Weekly Hours (Low) 123.1 Shift Factor 1.28 Note: Output, Investment, and Capital Stock extrapolated for 1987 and 1988.

\section{Softwood veneer and plywood}

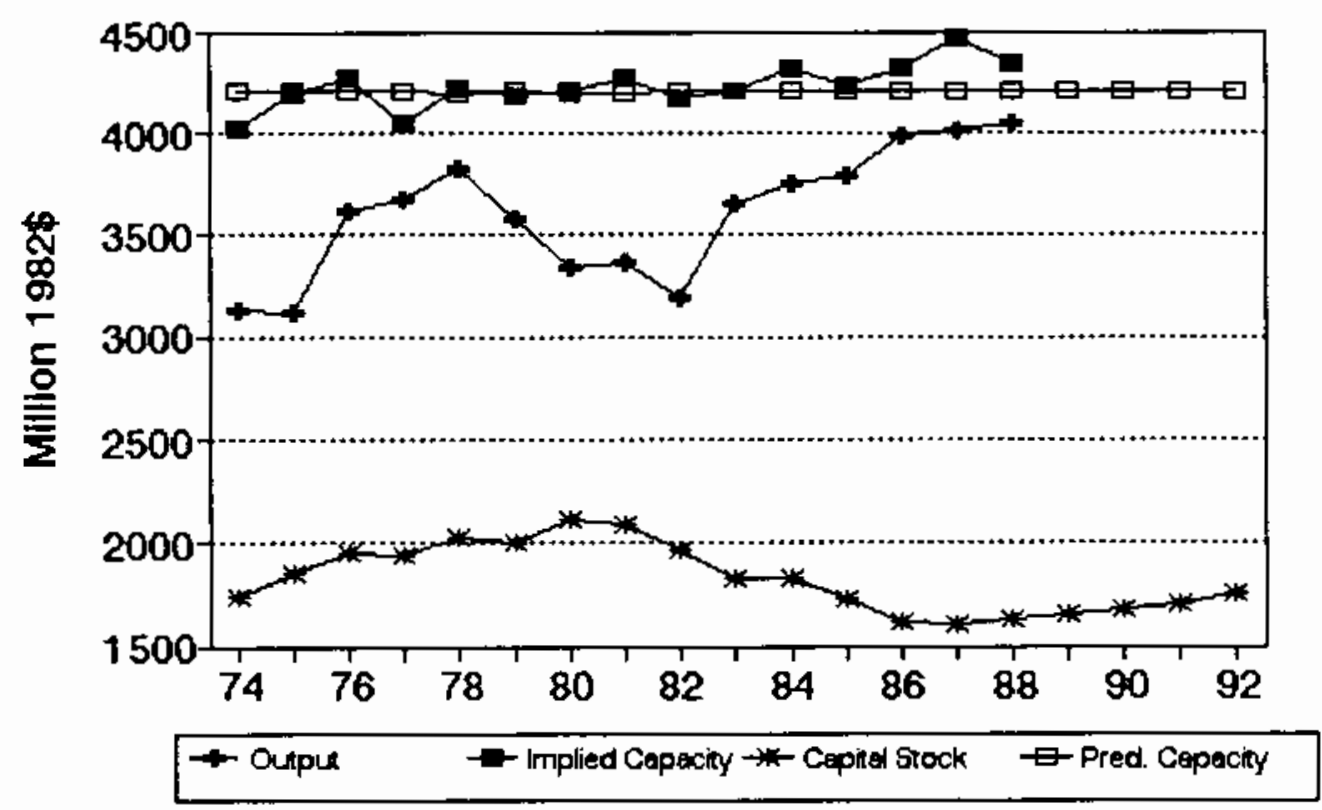




\section{No. 123 sIC 2439 structural wood members, n.e.c.}

MILLIONS OF 1982 DOLLARS (EXCL. CU RATES)

$\begin{array}{lccccccc} & \text { a4 } & \text { Ann. } & \text { Implied } & \text { Gross } & \text { Net } & \text { Pred. } & \ldots \\ \text { Gross } & \text { a } & \text { CU } & \text { Prac. } & \text { In- } & \text { Cap. } & \text { Prac. } & \text { Emergency Capacity } \\ \text { Output } & \text { Rate } & \text { Rate } & \text { Capac. } & \text { vest. } & \text { Stk. } & \text { Capac. } & 3 \text { mon. } 6 \text { mon. }>6 \text { mon. }\end{array}$

\begin{tabular}{|c|c|c|c|c|c|c|c|c|c|c|}
\hline 1974 & 707 & 78 & $B 1 . B$ & 865 & 9.1 & 144 & 1,147 & & & \\
\hline 1975 & 770 & 70 & 71.8 & 1,073 & 36.0 & 170 & 1,475 & & & \\
\hline 1976 & 940 & 56 & 61.4 & $1,53 t$ & $(1.3)$ & 158 & 1,325 & & & \\
\hline 1977 & 1,038 & 60 & 58.9 & 1,761 & 23.6 & 170 & 1,477 & & & \\
\hline 1978 & 1,209 & 64 & 62.4 & 1,937 & 42.5 & 198 & 1,852 & & & \\
\hline 1979 & 1,153 & 52 & 56.3 & 2,048 & 16.9 & 201 & 1,881 & & & \\
\hline 1980 & 955 & 41 & 44.6 & 2,143 & 14.1 & 199 & 1,858 & & & \\
\hline 1981 & 846 & 34 & 37.1 & 2,278 & 9.7 & 192 & 1,769 & & & \\
\hline 1982 & 856 & 59 & 49.6 & 1,727 & 28.1 & 200 & 1,875 & & & \\
\hline 1983 & 1,067 & 60 & 64.0 & 1,667 & 31.4 & 210 & 1,997 & & & \\
\hline 1984 & 1,146 & 70 & 69.3 & 1,654 & 7.1 & 195 & 1,806 & & & \\
\hline 1985 & 1,138 & 65 & 66.9 & 1,701 & 48.7 & 219 & 2,122 & & & \\
\hline 1986 & 1.232 & 45 & 52.5 & 2,344 & 26.2 & 221 & 2,147 & & & \\
\hline$-\quad-$ & --- & -- & -- & $-\ldots$ & $\cdots$ & $\cdots$ & $-\cdots$ & $\cdots$ & $\cdots$ & -- \\
\hline 1987 & 1,256 & 60 & 54.1 & 2,320 & 29.0 & 226 & 2,205 & & & \\
\hline 1988 & 1,289 & $\pi$ & 69.4 & 1,857 & 29.9 & 230 & 2,269 & 2,955 & 3,079 & 3,128 \\
\hline 1989 & & & & & 30.8 & 236 & 2,337 & 3,044 & 3,171 & 3,222 \\
\hline 1990 & & & & & 31.7 & 241 & 2,409 & 3,138 & 3,269 & 3,322 \\
\hline 1991 & & & & & 32.6 & 247 & 2,485 & 3,237 & 3,372 & 3,427 \\
\hline 1992 & & & & & 33.5 & 253 & 2,564 & 3,340 & 3,480 & 3,536 \\
\hline
\end{tabular}

Capecity-Capital Stock Regression: Code O RSO 0.500

Coefficients: Const -733.6 stock 13.0

Ave. Capacity/Stock $(74-86): 9.2$ Ave. Weekly Hours (High) 114.4 Shift Factor 1.38 Ave Yeekly Hours (Low) 106.3 Shift Factor 1.48 Note: Output, Investment, and Cepital stock extrapolated for 1987 and 1988.

2439 Structural wood members, n.e.c.

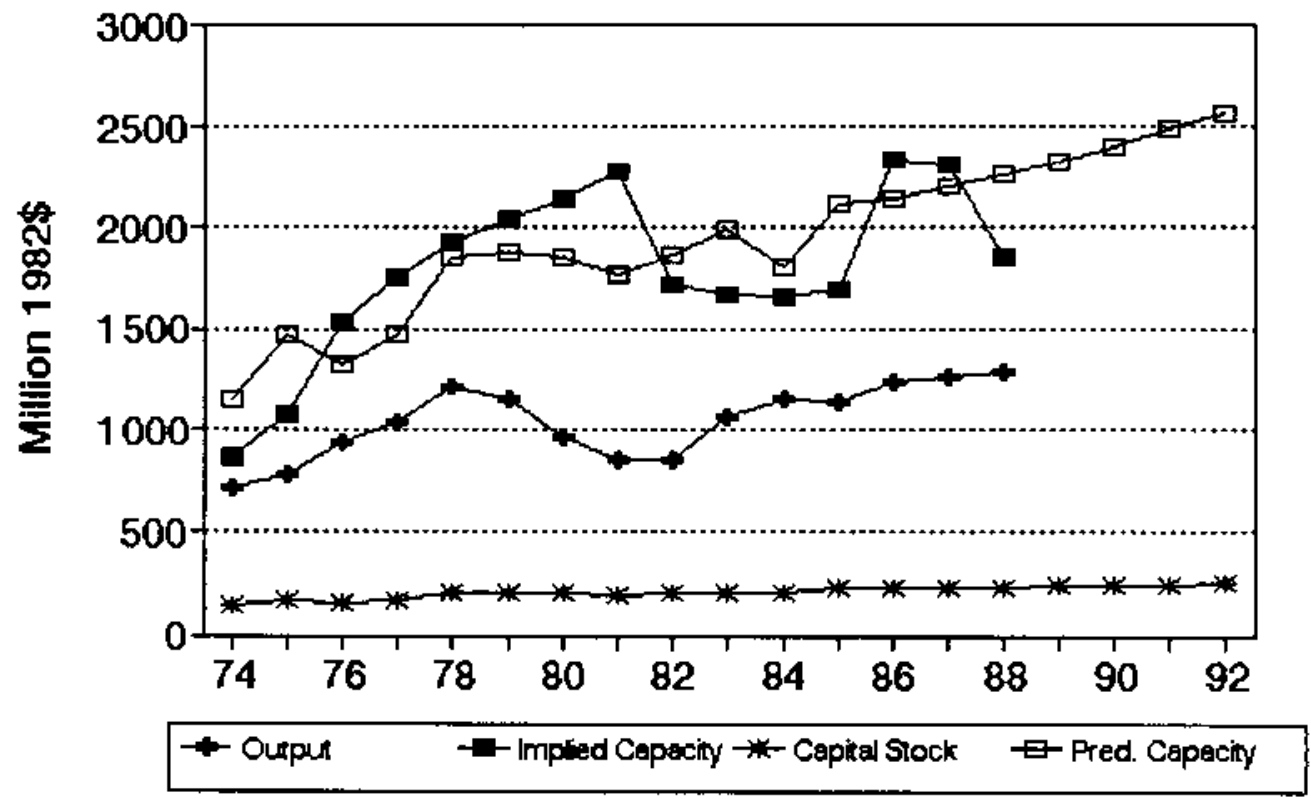




\section{No. 124 BIC 2441 Mailed wood boxes and shook}

MILLIONS OF 1982 DOLLARS (EXCL. CU RATES)

$\begin{array}{lccccccc} & 04 & \text { Ann. } & \text { Implied } & \text { Gross } & \text { Net } & \text { Pred. } & \ldots \ldots \\ \text { Gross } & \text { CU } & \text { CU } & \text { Prac. } & \text { In- } & \text { Cap. } & \text { Prac. } & \text { Emergency Capacity } \\ \text { Output } & \text { Rate } & \text { Rate } & \text { Capoc. } & \text { vest. } & \text { Stk. } & \text { Capac. } & 3 \text { mon. } 6 \text { mon. }\end{array}$

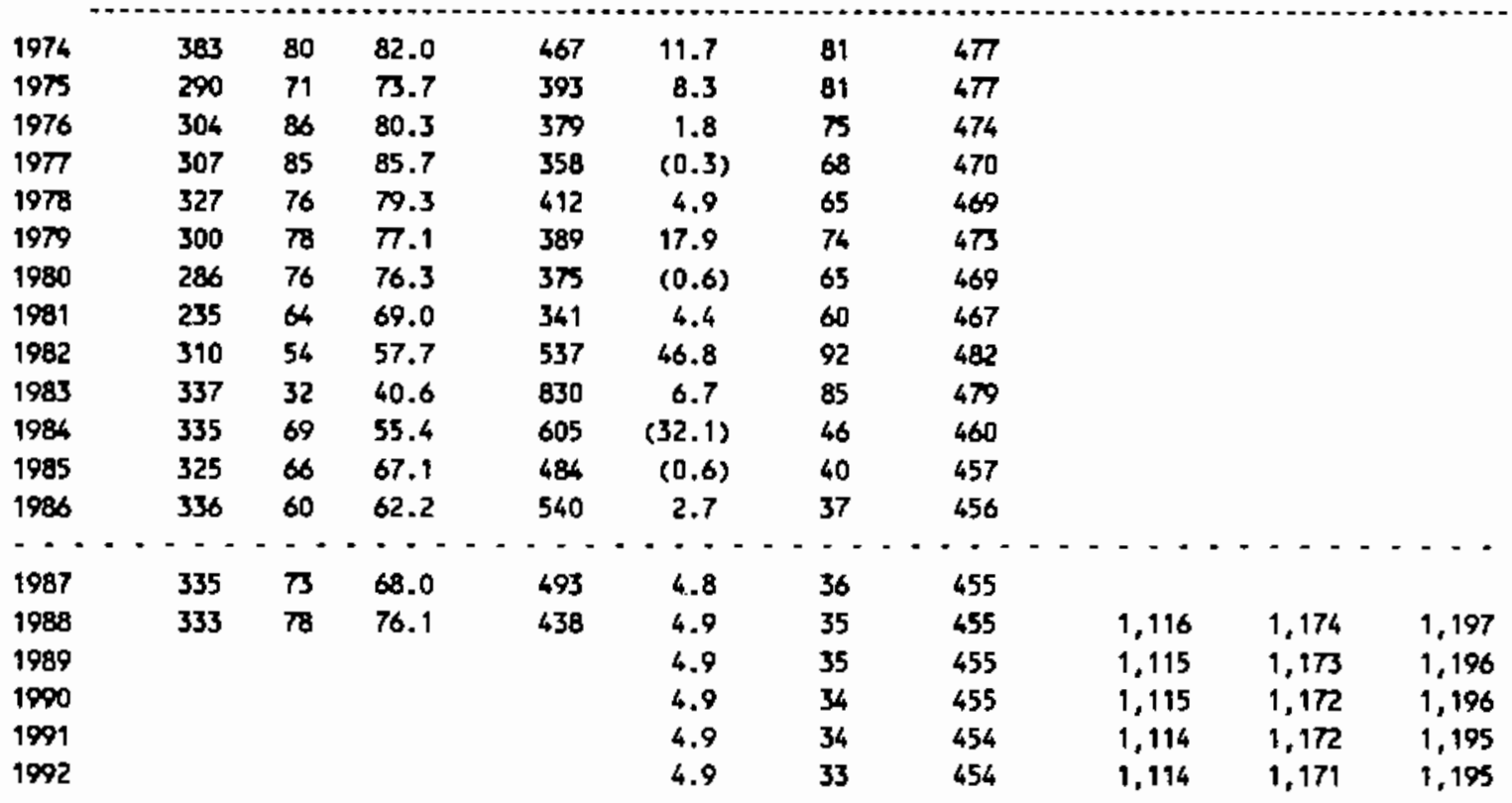

Capocity-Capital stock Regression: Code 0 RSO 0.000 Coefficients: Const 438.5 stock 0.5

Ave. Capocity/Stock $(74-86): 7.0$ Ave. Weekly Hours (High) 59.9 Shift Factor 2.63 Ave Ueekly Hours (Low) 51.0 Shift Factor 3.09 Note: Dutput, Investment, and Capital Stock extrapolated for 1987 and 1988.

\section{Nailed wood boxes and shook}

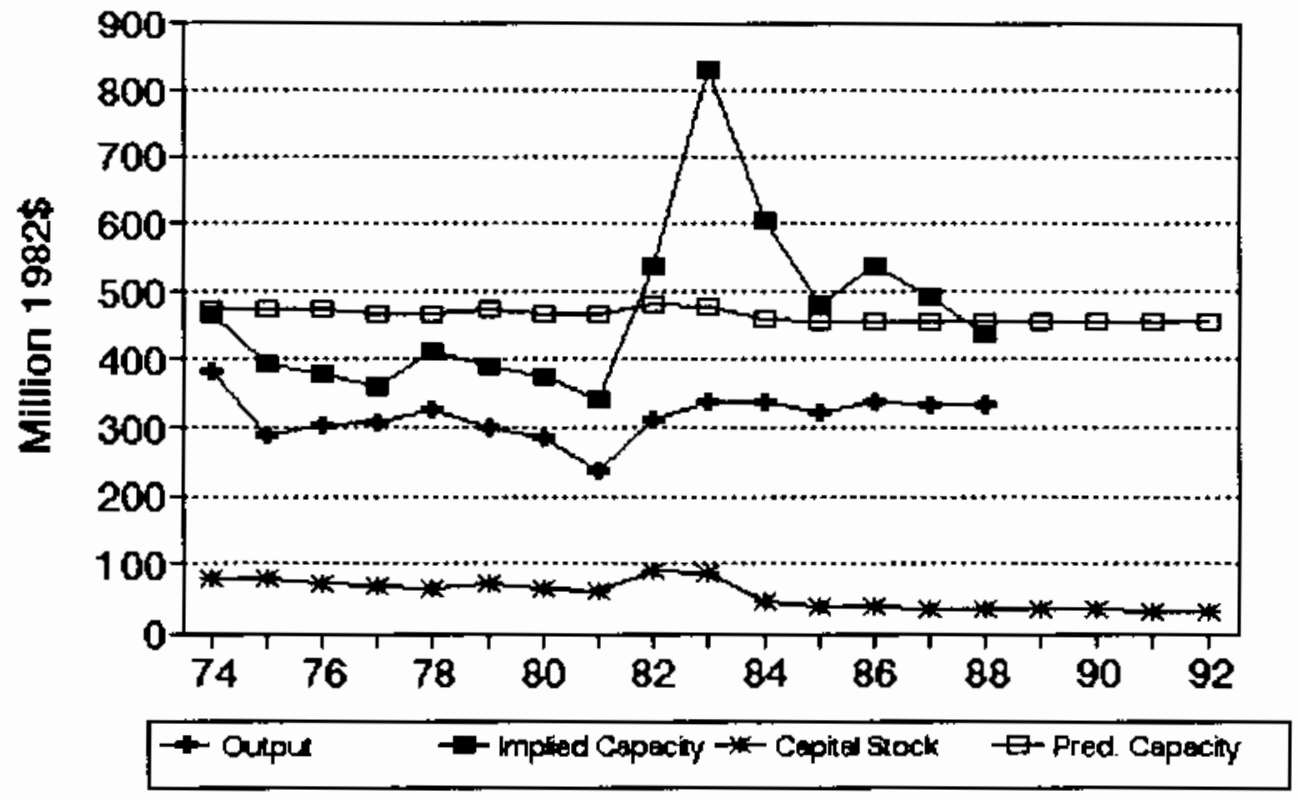




\title{
No. 125 SIC 2448 wood pallets and skids
}

\author{
MILLIONS OF 1982 DOLLARS (EXCL. CU RATES)
}

$\begin{array}{lccccccc} & 04 & \text { Ann. Implied Gross } & \text { Net } & \text { Pred. } & \ldots & \ldots \\ \text { Gross } & \text { CU } & \text { CU } & \text { Prac. } & \text { In- } & \text { Cap. } & \text { Prac. } & \text { Emergency Capacity } \\ \text { Output } & \text { Rate } & \text { Rate } & \text { Cepac. } & \text { vest. } & \text { Stk. } & \text { Capac. } & 3 \text { mon. } 6 \text { mon. }>6 \text { mon. }\end{array}$

\begin{tabular}{|c|c|c|c|c|c|c|c|c|c|c|}
\hline 1974 & 588 & 72 & 81.8 & 719 & 10.5 & 222 & 607 & & & \\
\hline 1975 & 611 & 58 & 60.2 & 1,016 & 53.4 & 255 & 1,141 & & & \\
\hline 1976 & 679 & 69 & 64.8 & 1,048 & 17.3 & 249 & 1,054 & & & \\
\hline 1977 & 860 & 75 & 74.2 & 1,160 & 25.4 & 252 & 1,095 & & & \\
\hline 1970 & 912 & 73 & 73.5 & 1,242 & 68.1 & 295 & 1,808 & & & \\
\hline 1979 & 869 & 84 & 79.2 & 1,097 & 11.3 & 281 & 1,583 & & & \\
\hline 1980 & 931 & 62 & 68.0 & 1,369 & 23.8 & 278 & 1,537 & & & \\
\hline 1981 & 934 & 58 & 61.9 & 1,508 & 30.6 & 280 & 1,565 & & & \\
\hline 1982 & 1,008 & 57 & 57.1 & 1,768 & 53.4 & 299 & 1,876 & & & \\
\hline 1983 & 1,022 & $44 *$ & 51.2 & 1,995 & 28.8 & 291 & 1,742 & & & \\
\hline 1984 & 1,259 & 42 & 44.2 & 2,849 & 40.0 & 292 & 1,763 & & & \\
\hline 1985 & 1,185 & $B 1$ & 66.5 & 1,781 & 28.4 & 283 & 1,795 & & & \\
\hline 1986 & 1,196 & 72 & 75.3 & 1.588 & 22.6 & 270 & 1,573 & & & \\
\hline$\div-$ & $-\cdot-$ & $\because$ & $\because-$ & $-\cdot-$ & $-\cdots$ & $\because$ & $-\cdot-$ & $\cdots$ & $--\cdot-$ & $\cdots$ \\
\hline 1987 & 1,247 & 90 & 82.3 & 1,515 & 32.9 & 267 & 1,526 & & & \\
\hline 1988 & 1,316 & 92 & 91.3 & 1,441 & 33.1 & 264 & 1,486 & 4,366 & 4,592 & 4,683 \\
\hline 1989 & & & & & 33.2 & 263 & 1,454 & 4,273 & 4,493 & 4,582 \\
\hline 1990 & & & & & 33.3 & 261 & 1,429 & 4,198 & 4,415 & 4,502 \\
\hline 1991 & & & & & 33.5 & 260 & 1,409 & 4,140 & 4,354 & 4,440 \\
\hline 1992 & & & & & 33.6 & 259 & 1,394 & 4,096 & 4,308 & 4,393 \\
\hline
\end{tabular}

Capacity-Capital Stock Regression: Code -1 RSQ 0.480

Coefficients: Const -3067.6 Stock 16.5 D(85-86) 180.7 Ave. Capacity/Stock (74-86): 5.4 Ave. Heekly Hours (High) 50.0 Shift Factor 3.15 Ave Meekly Hours (Loh) 48.0 Shift Factor 3.28 - Imputed Note: Qutput, Investment, and Capital Stack extrapolated for 1987 and 1988.

\section{Wood pallets and skids}
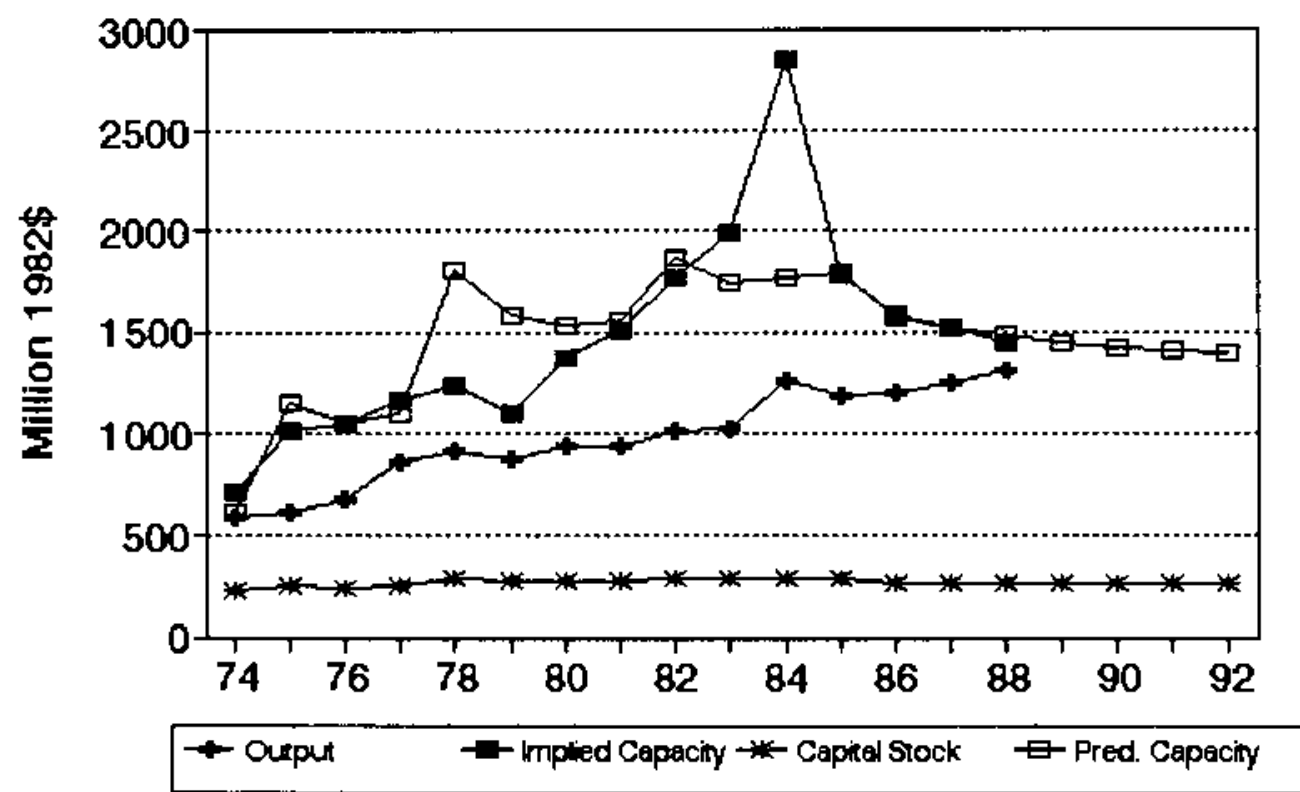


\section{No. 126 gIC 2449 Hood containers, n.e.c.}

MILLIONS OF 1982 DOLLARS (EXCL. CU RATES)

$\begin{array}{lccccccc} & 04 & \text { Am. Implied Gross } & \text { Net } & \text { Pred. } & \ldots & \ldots \\ \text { Gross } & \text { CU } & \text { CU } & \text { Prac. } & \text { In- } & \text { Cap. } & \text { Prac. } & \text { Emergency Capacity } \\ \text { Output Rate } & \text { Rate } & \text { Capac. } & \text { vest. } & \text { Stk. } & \text { Capac. } & 3 \text { mon. } 6 \text { mon. }>6 \text { mon. }\end{array}$

\begin{tabular}{|c|c|c|c|c|c|c|c|c|c|c|}
\hline 1974 & 494 & 82 & 82.0 & 602 & $(10.1)$ & 71 & 452 & & & \\
\hline 1975 & 480 & 73 & 76.4 & 629 & 10.7 & 75 & 469 & & & \\
\hline 1976 & 455 & 62 & 66.1 & 687 & 2.2 & 71 & 452 & & & \\
\hline 1977 & 345 & 72 & 68.3 & 505 & 18.0 & 81 & 490 & & & \\
\hline 1978 & 347 & 89 & 82.6 & 420 & 16.2 & 88 & 517 & & & \\
\hline 1979 & 303 & 95 & 92.8 & 327 & $(7.8)$ & 73 & 460 & & & \\
\hline 1980 & 342 & 100 & 98.1 & 349 & 10.0 & 74 & 465 & & & \\
\hline 1981 & 354 & 88 & 92.5 & 383 & 1.3 & 67 & 439 & & & \\
\hline 1982 & 313 & 80 & 83.0 & 377 & 8.9 & 67 & 439 & & & \\
\hline 1983 & 295 & 80 & 80.0 & 369 & 9.4 & 67 & 439 & & & \\
\hline 1984 & 262 & 73 & 75.6 & 346 & $(11.7)$ & 49 & 370 & & & \\
\hline 1985 & 254 & 66 & 68.6 & 370 & $(3.5)$ & 40 & 336 & & & \\
\hline 1986 & 213 & 80 & 74.8 & 285 & 1.8 & 36 & 323 & & & \\
\hline-- & $=-$ & - & $\cdots$ & $-\cdots$ & $\cdots-$ & - & - - - & $\cdot-$ & $-\cdot$ & - \\
\hline 1987 & 206 & 79 & 79.4 & 259 & 3.5 & 34 & 317 & & & \\
\hline 1988 & 197 & 88 & 84.6 & 233 & 3.8 & 33 & 312 & 451 & 470 & \\
\hline 1989 & & & & & 3.9 & 32 & 309 & 446 & 464 & 472 \\
\hline 1990 & & & & & 3.8 & 32 & 306 & 441 & 460 & 467 \\
\hline 1991 & & & & & 3.8 & 31 & 303 & 438 & 456 & 463 \\
\hline 1992 & & & & & 4.1 & 31 & 302 & 436 & 454 & 461 \\
\hline
\end{tabular}

Capacity-Capitat Stock Regression: Code 1 RSO 0.200

Coefficients: Const 187.5 Stock 3.8 Ave. Capacity/Stock $(74-86): 6.6$ Ave. Heekly Hours (High) 103.1 Shift Factor 1.53 Ave Heekly Hours (Low) 79.9 Shift Factor 1.97 Mote: Output, Investment, and Capital stock extrapolated for 1987 and 1988.

2449 Wood containers, n.e.c.

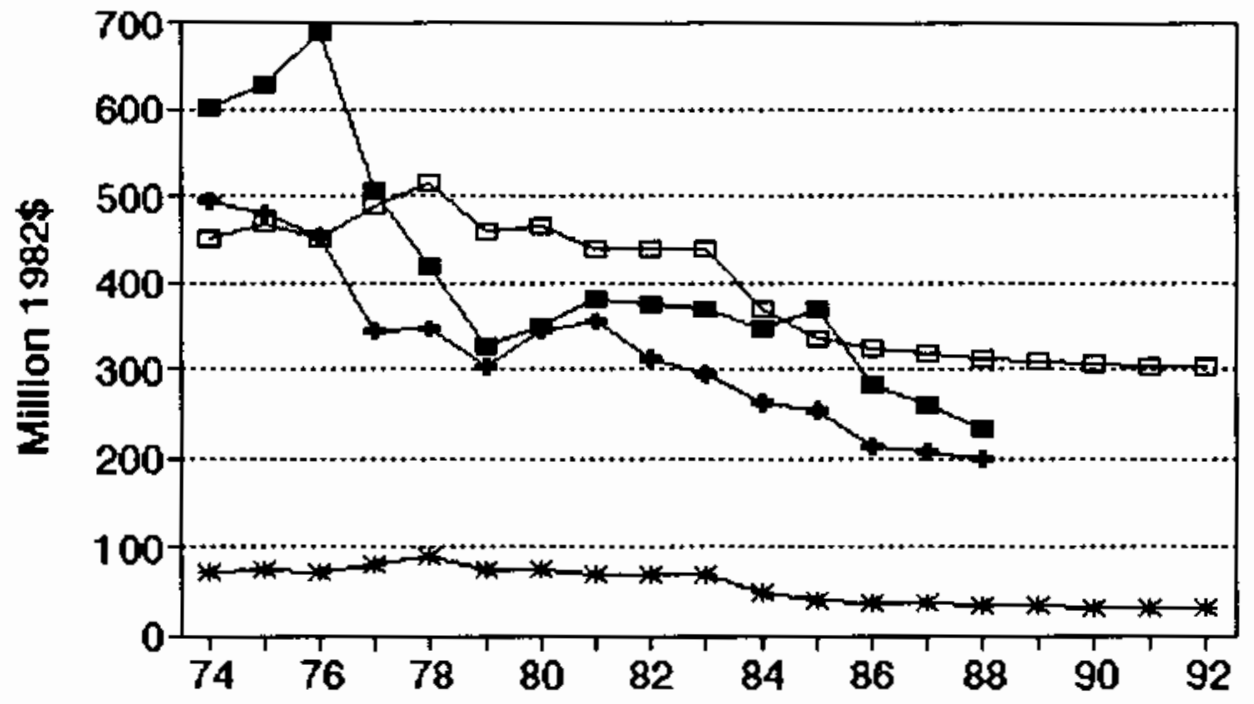

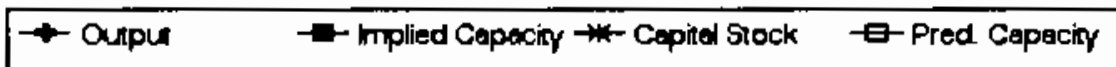




\section{Ho. 127 SIC 2451 Mobile homes}

MILLIONS OF 1982 DOLLARS (EXCL. CU RATES)

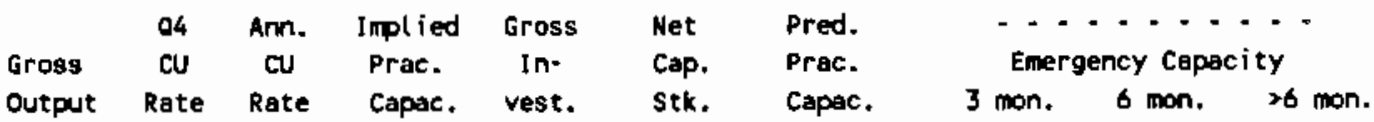

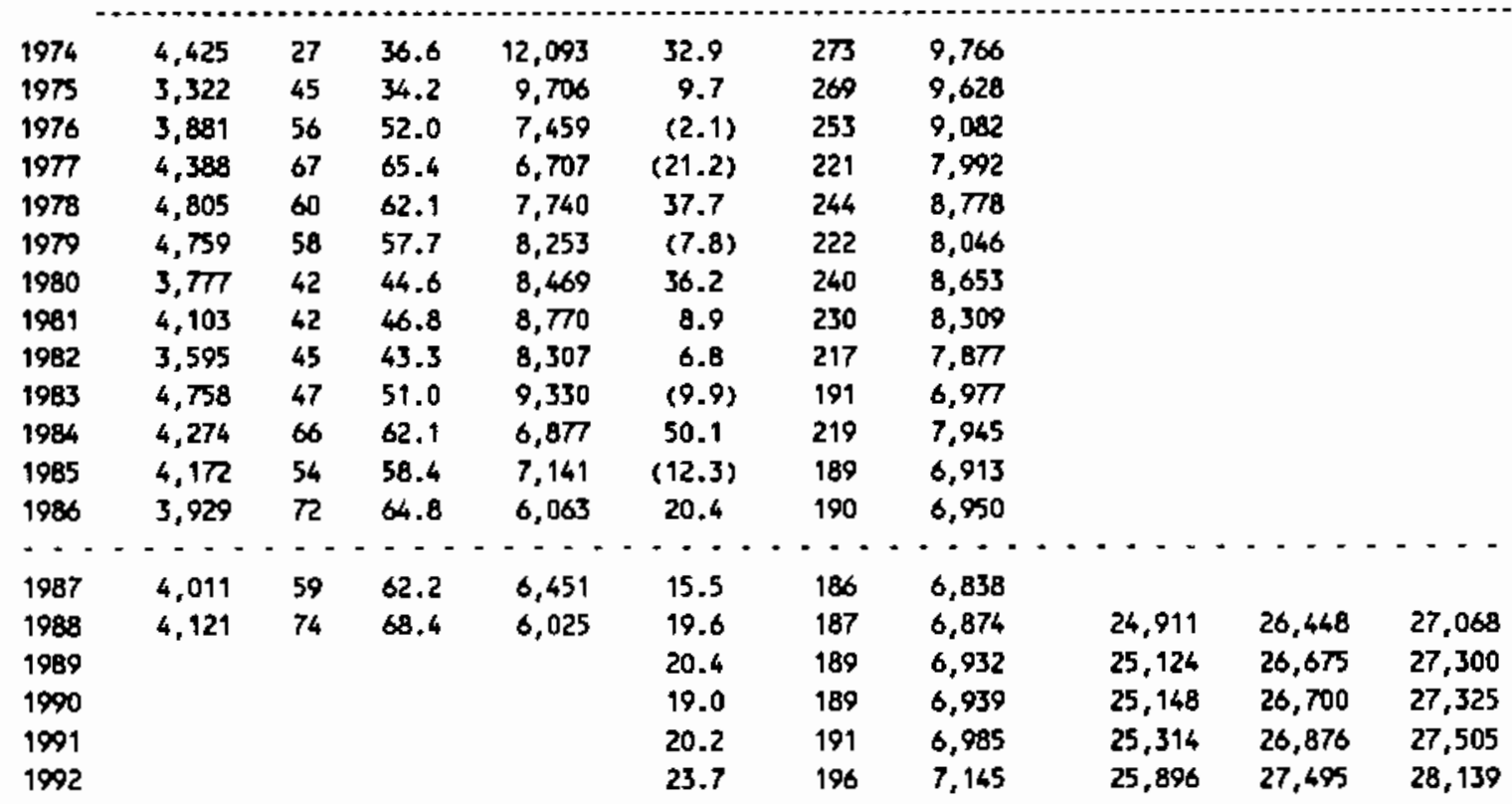

Capacity-Capital Stack Regression: Code 0 RSQ 0.360 Coefficients: Const 552.0 Stock 33.7 Ave. Neekly Hours (High) 40.0 Shife factor 3.94 Ave Weekly Hours (LOW) 40.0 Shift Factor 3.94 Note: Output, Investment, and Capital stock extrapolated for 1987 and 1988.

\section{Mobile homes}

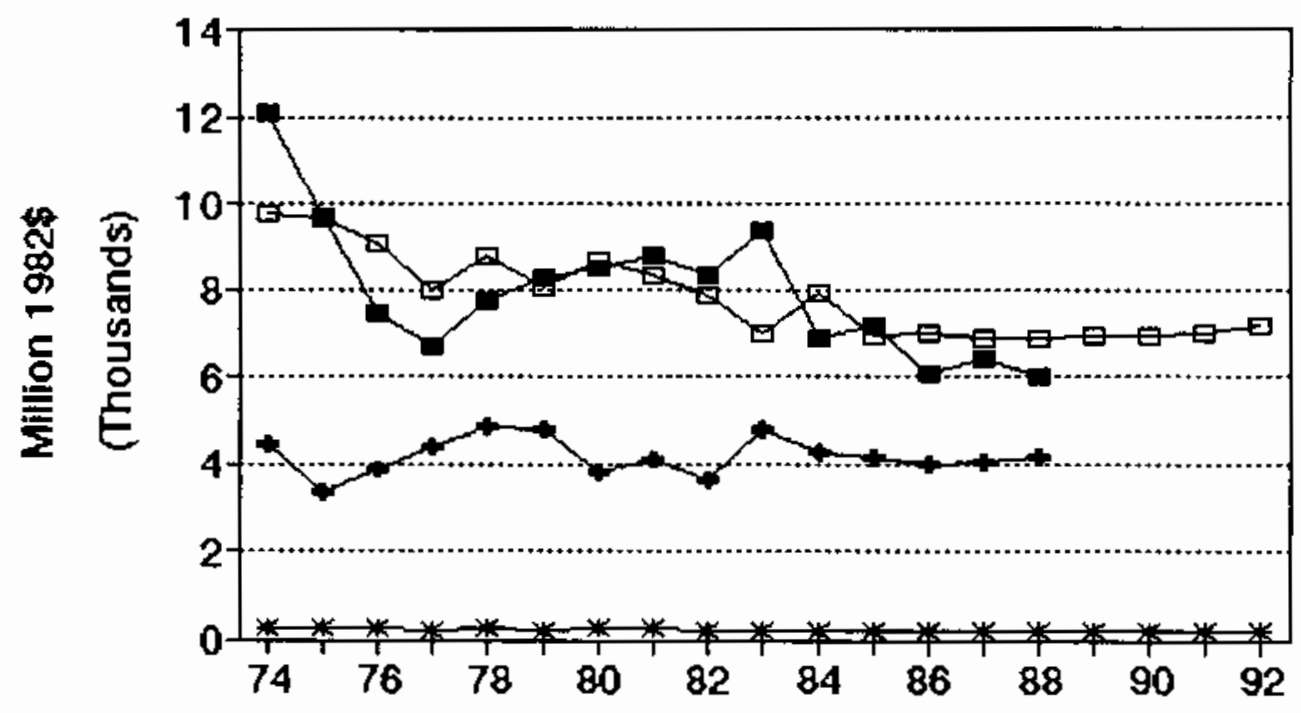

$\rightarrow$ - Outpur $\rightarrow$-mpled Cepecin $\rightarrow$ - Copital Slock $\square$-Pred Capacity




\section{No. 128 SIC 2452 Prefabricated wood buildings}

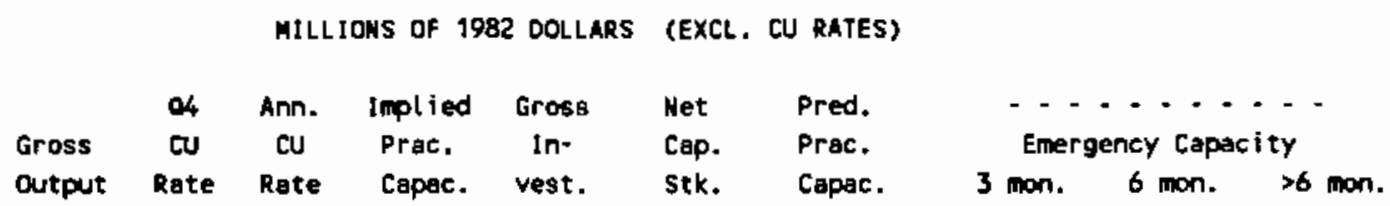

\begin{tabular}{|c|c|c|c|c|c|c|c|c|c|c|}
\hline 1974 & 1,895 & 55 & 61.7 & 3,073 & $(69.1)$ & 176 & 2,996 & & & \\
\hline 1975 & 1,598 & 57 & 56.0 & 2,961 & $(3.5)$ & 160 & 2,795 & & & \\
\hline 1976 & 1,755 & 46 & 50.4 & 3,481 & 14.0 & 160 & 2,790 & & & \\
\hline 1977 & 2,866 & 67 & 60.0 & 4,776 & 98.3 & 237 & 3,734 & & & \\
\hline 1978 & 2,714 & 56 & 59.9 & 4,532 & 50.1 & 265 & 4,082 & & & \\
\hline 1979 & 2,383 & 54 & 54.3 & 4,387 & 28.8 & 271 & 4,150 & & & \\
\hline 1980 & 1,877 & 51 & 50.8 & 3,697 & 9.5 & 250 & 3,998 & & & \\
\hline 1981 & 1,648 & 63 & 60.8 & 2,711 & $(1.1)$ & 235 & 3,713 & & & \\
\hline 1982 & 1,348 & 25 & 38.8 & $3,4 \pi$ & 29.3 & 238 & 3,741 & & & \\
\hline 1983 & 1,427 & $52 *$ & 42.0 & 3,401 & 23.1 & 231 & 3,659 & & & \\
\hline 1984 & 1,551 & 61 & 58.9 & 2,633 & $(34.5)$ & 173 & 2,958 & & & \\
\hline 1985 & 1,523 & 62 & 61.6 & 2,471 & 23.0 & 173 & 2,953 & & & \\
\hline 1986 & 1,717 & 60 & 60.7 & 2,829 & 13.8 & 165 & 2,856 & & & \\
\hline $\begin{array}{l}-\cdot \\
1987\end{array}$ & 1,742 & $\dot{0}$ & 63.1 & 2,763 & 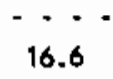 & 159 & 2,788 & $\cdots$ & -- & $\because \cdot$ \\
\hline 1988 & 1,776 & 44 & 52.3 & 3,396 & 17.5 & 156 & 2,742 & 6,981 & 7,366 & 7,521 \\
\hline 1989 & & & & & 17.7 & 152 & 2,704 & 6,884 & 7,263 & 7,416 \\
\hline 1990 & & & & & 17.4 & 149 & 2,667 & 6,790 & 7,164 & 7,315 \\
\hline 1991 & & & & & 17.6 & 147 & 2,638 & 6,717 & 7,087 & 7,236 \\
\hline 1992 & & & & & 18.5 & 146 & 2,623 & 6,679 & 7,067 & 7,196 \\
\hline
\end{tabular}

Capecity-Capital Stock Regression: Code 0 RSQ 0.500

Coefficients: Const 843.5 stock 12.2

Ave. Heekly Hours (High) 57.4 Shift factor 2.74 Ave Heekly Hours (Lou) 50.6 Shife Factor 3.11

* Imputed Mote: Output, Imestment, and Capital stock extrapolated for 1987 and 1988.

\section{Prefabricated wood buildings}

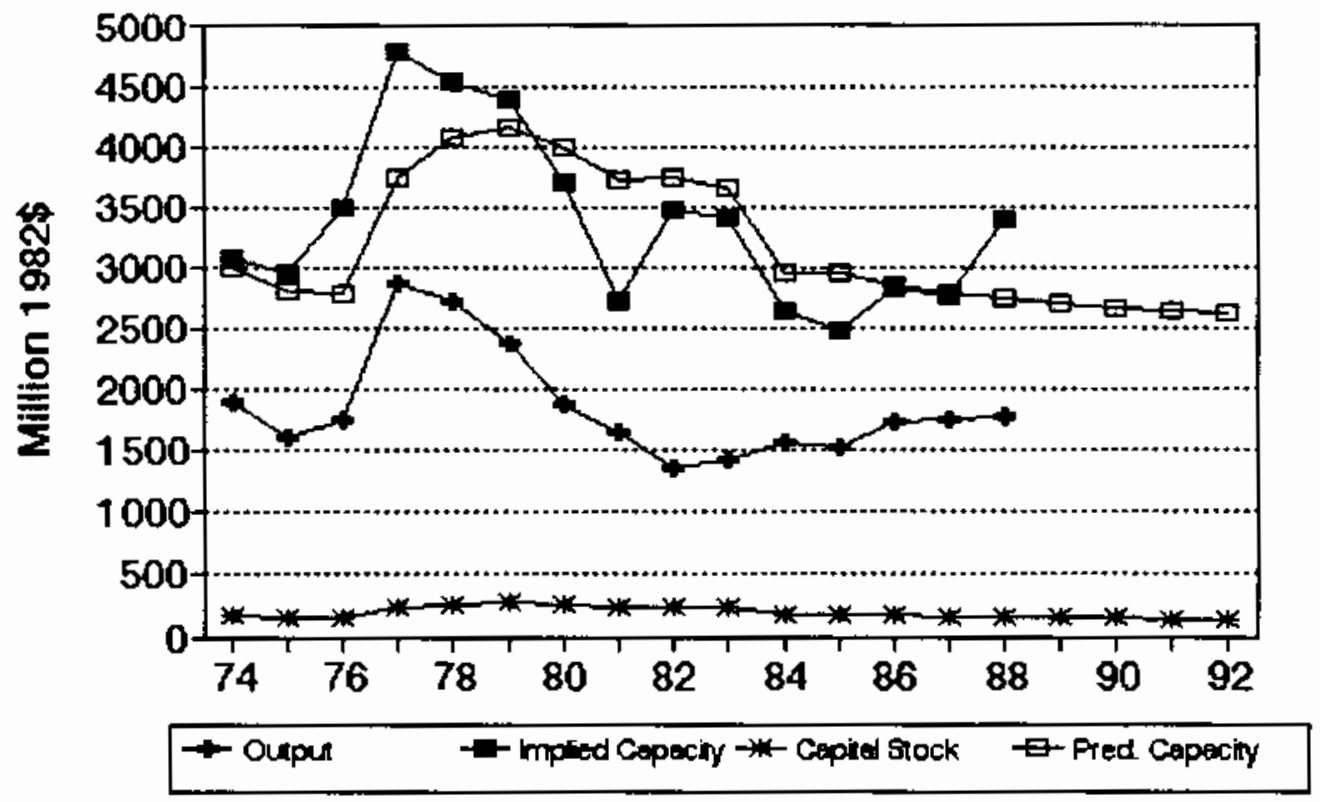




\section{Ho. 129 BIC 2491 wood preserving}

MILLIONS OF 1982 DOLLARS (EXCL. CU RATES)

$\begin{array}{lccccccc} & 04 & \text { Ann. Implied Gross } & \text { Net } & \text { Pred. } & -\ldots & -\ldots \\ \text { Gross } & \text { CU } & \text { CU } & \text { Prac. } & \text { In- } & \text { Cap. } & \text { Prac. } & \text { Emergency Capacity } \\ \text { Output Rate } & \text { Rate } & \text { Capac. } & \text { vest. } & \text { Stk. } & \text { Capac. } & 3 \text { mon. } 6 \text { mon. }\end{array}$

\begin{tabular}{|c|c|c|c|c|c|c|c|c|c|c|}
\hline 1974 & 1,361 & $46 \cdot$ & 52.1 & 2,609 & 68.1 & 349 & 2,523 & & & \\
\hline 1975 & 1,097 & 42 & 41.4 & 2,646 & 56.3 & 373 & 2,526 & & & \\
\hline 1976 & 988 & 33 & 36.7 & $2,6 \% 5$ & 28.6 & 370 & 2,528 & & & \\
\hline 1977 & 1,196 & 43 & 39.9 & 2,995 & 73.7 & 407 & 2,531 & & & \\
\hline 1978 & 1,229 & 52 & 48.4 & 2,536 & 85.8 & 452 & 2,533 & & & \\
\hline 1970 & 1,192 & 80 & 69.0 & 1,728 & 74.7 & 483 & 2,536 & & & \\
\hline 1980 & 1,423 & $6 B$ & 70.3 & 2,023 & 50.5 & 487 & 2,538 & & & \\
\hline 1981 & 1,347 & 59 & 4.6 & 2,077 & 49.0 & 487 & 2,540 & & & \\
\hline 1982 & 1,374 & 31 & $4 t .1$ & 3,343 & $\pi .3$ & 508 & 2,543 & & & \\
\hline 1983 & 1,213 & 57 & 47.9 & 2,531 & 24.0 & $4 \pi 5$ & 2,545 & & & \\
\hline 1984 & 1,492 & 64 & 63.1 & 2,364 & $(16.3)$ & 410 & 2,548 & & & \\
\hline 1985 & 1,565 & 58 & 60.2 & 2,599 & 14.4 & 379 & 2,550 & & & \\
\hline 1986 & 1,614 & 56 & 56.7 & 2,848 & 30.2 & 364 & 2,553 & & & \\
\hline$-\cdots$ & & $\because$ & $\cdots$ & $\cdots$ & $-\cdot-$ & $\cdots$ & --- & --- & --- & -- \\
\hline 1987 & 1,630 & 53 & 53.5 & 3,049 & 47.2 & 367 & 2,555 & & & \\
\hline 1988 & 1,049 & 59 & 56.8 & 2,906 & 61.2 & 384 & 2,557 & 4,657 & 4,880 & 4,969 \\
\hline 1989 & & & & & 63.9 & 401 & 2,560 & 4,602 & 4,884 & 4,974 \\
\hline 1990 & & & & & 59.2 & 411 & 2,562 & 4,666 & 4,889 & 4,979 \\
\hline 1991 & & & & & 63.3 & 425 & 2,565 & 4.670 & 4,893 & 4,983 \\
\hline 1992 & & & & & $\pi .5$ & 449 & 2,567 & 4,675 & 4,898 & 4,988 \\
\hline
\end{tabular}

Capecity-Capital Stock Regression: Code -2 RSQ 0.000 Coefficients: Const 2521.0 Time 2.4

Ave. Capacity/Stock $(74-86): 6.0$ Ave. Weekly Hours (High) 81.1 Shift Factor 1.84 Ave Weekly Hours (Low) 71.4 Shift Factor 2.21 * Imputed Note: Output, Investment, and Capital Stock extrapolated for 1987 and 1988.

\section{Wood preserving}

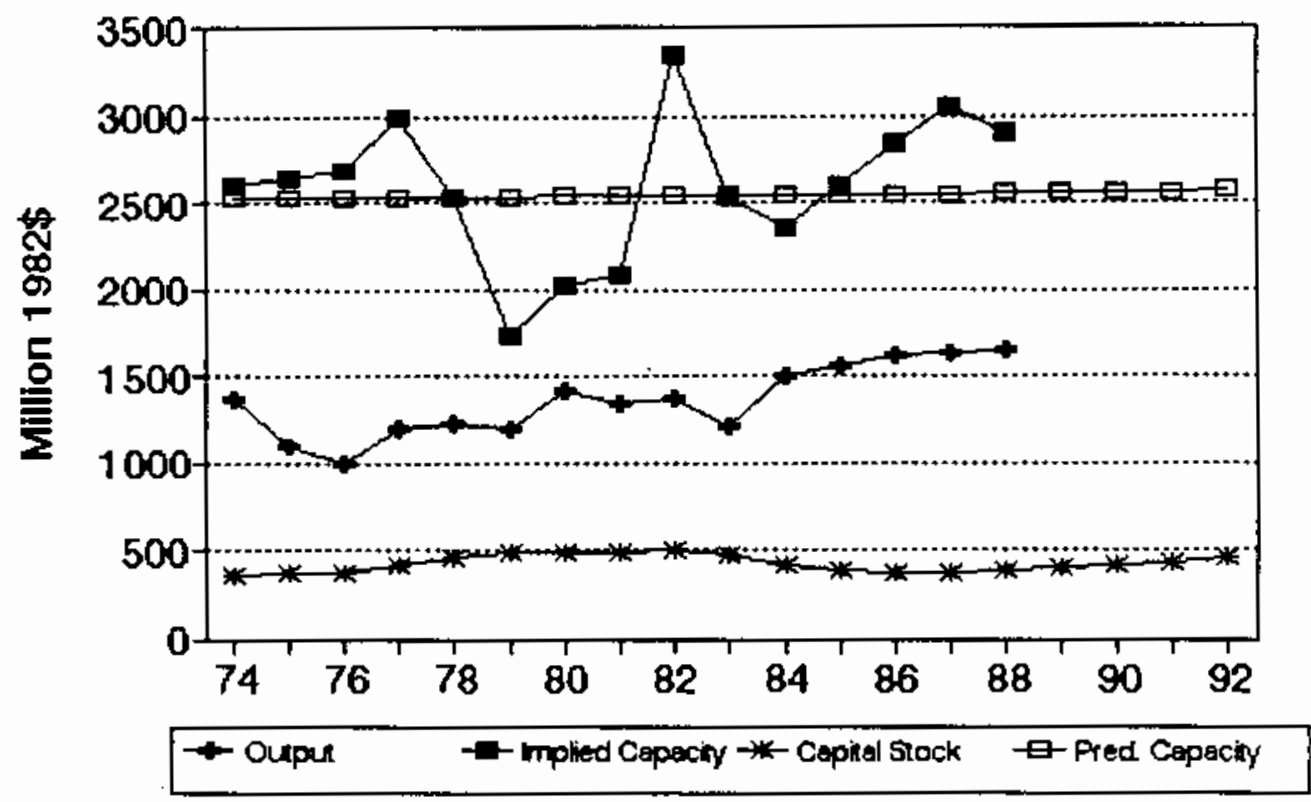




\section{No. 130 SIC 2692 Particleboard}

MILLIOWS OF 1982 DOLLARS (EXCL. CU RATES)

$\begin{array}{lccccccc} & 04 & \text { Ann. Implied Gross } & \text { Net } & \text { Pred. } & \ldots . . . . . . \\ \text { Gross } & \alpha & \text { CU } & \text { Prac. } & \text { In- } & \text { Cap. } & \text { Prac. } & \text { Emergency Capacity } \\ \text { Output Rate } & \text { Rate } & \text { Capac. } & \text { vest. } & \text { Stk. } & \text { Capac. } & 3 \text { mon. } 6 \text { mon. }>6 \text { mon. }\end{array}$

\begin{tabular}{|c|c|c|c|c|c|c|c|c|c|c|}
\hline 1974 & 645 & $37 *$ & 43.7 & 1,476 & 177.8 & 754 & 935 & & & \\
\hline 1973 & 507 & 36 & 34.2 & 1,481 & 32.6 & 738 & 923 & & & \\
\hline 1976 & 562 & 53 & 46.3 & 1,213 & 8.0 & 698 & 893 & & & \\
\hline 1977 & 656 & $\pi *$ & 68.3 & 962 & 79.7 & 723 & 911 & & & \\
\hline 1978 & 688 & 86 & 81.5 & 844 & 42.6 & 706 & 899 & & & \\
\hline $19 \pi 9$ & 686 & 81 & 82.0 & 837 & 80.3 & 718 & 908 & & & \\
\hline 1980 & 570 & 96 & 87.4 & 652 & $(54.3)$ & 603 & 821 & & & \\
\hline 1981 & 513 & $91 *$ & 98.0 & 524 & 83.6 & 616 & 831 & & & \\
\hline 1982 & 553 & 90 & 89.8 & 616 & 208.0 & 745 & 928 & & & \\
\hline 1983 & 652 & 90 & 94.2 & 693 & 44.1 & 708 & 900 & & & \\
\hline 1984 & 757 & 90 & 93.4 & 811 & 272.3 & 883 & 1,033 & & & \\
\hline 1985 & 898 & 91 & 90.6 & 990 & 172.2 & 955 & 1,087 & & & \\
\hline 1986 & 1,066 & 95 & 93.3 & 1,143 & 213.3 & 1,066 & 1,171 & & & \\
\hline$-\cdots$ & $-\cdot-$ & $\cdots$ & $\cdots$ & $-\cdots$ & $\cdots$ & $\cdots$ & $\cdots$ & $\cdots \cdot$ & -- & $-\cdot$ \\
\hline 1987 & 1,089 & 96 & 94.2 & 1,157 & 190.0 & 1,148 & 1,234 & & & \\
\hline 1988 & 1,119 & 92 & 93.6 & 1,196 & 202.3 & 1,235 & 1,299 & 1,283 & 1,295 & 1,299 \\
\hline 1989 & & & & & 214.5 & 1,325 & 1,368 & 1,350 & 1,363 & 1,368 \\
\hline 1990 & & & & & 226.8 & 1,418 & 1,438 & 1,420 & 1,433 & 1,438 \\
\hline 1991 & & & & & 239.0 & 1,514 & 1,511 & 1,492 & 1,506 & 1,511 \\
\hline 1992 & & & & & 251.3 & 1,613 & 1,586 & 1,565 & 1,580 & 1,586 \\
\hline
\end{tabular}

Capecity-Capital Stock Regression: Code O RSQ 0.100

Coefficients: Const 364.2 stock 0.8 Ave. Copocity/Stock (74-86): 1.2 Ave. Heekly Hours (High) 168.0 Shift Factor 1.00 Ave Meekly Hours (LOW) 168.0 Shift factor 1.00 - Imputed Mote: Output, Investment, and Capital Stock extrapolated for 1987 and 1988.

\section{Particleboard}
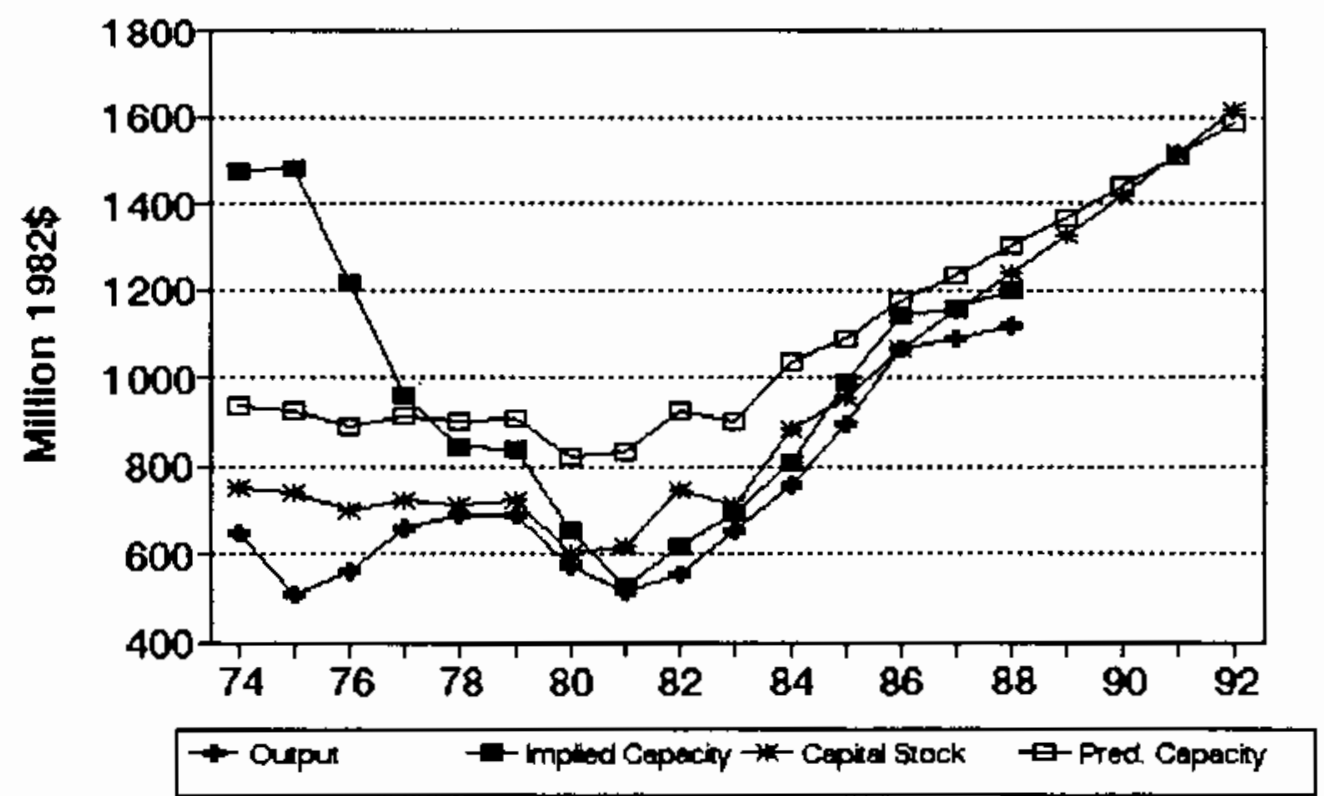
No. 131 sIC 2499 Wood products, n.e.c.

MILLIONS OF 1982 DOLLARS (EXCL. CU RATES)

$\begin{array}{lccccccc} & \text { Q4 } & \text { Ann. } & \text { Implied Gross } & \text { Net } & \text { Pred. } & \ldots \ldots \ldots \\ \text { Gross } & \text { dU } & \text { CU } & \text { Prac. } & \text { In- } & \text { Cap. } & \text { Prac. } & \text { Emergency Capacity } \\ \text { Output } & \text { Rate } & \text { Rate } & \text { Capac. } & \text { vest. } & \text { Stk. } & \text { Capac. } & 3 \text { mon. } 6 \text { mon. }>6 \text { mon. }\end{array}$

\begin{tabular}{|c|c|c|c|c|c|c|c|c|c|c|}
\hline 1974 & 3,295 & 69 & 69.1 & 4,772 & 44.4 & 1,312 & 4,571 & & & \\
\hline 1973 & 3,366 & 77 & 74.0 & 4,520 & 272.4 & 1.457 & 4.657 & & & \\
\hline 1976 & 3,034 & 82 & 80.1 & 4,784 & 146.4 & 1,467 & 4,742 & & & \\
\hline 1977 & 3,877 & 92 & 88.3 & 4,392 & 218.7 & 1,548 & 4,828 & & & \\
\hline 1978 & 3,828 & 69 & 77.6 & 4,931 & 133.3 & 1,540 & 4,913 & & & \\
\hline 1970 & 3,946 & 75 & 72.8 & 5,425 & 130.1 & 1,530 & 4,999 & & & \\
\hline 1980 & 3,545 & 67 & 70.0 & 5,065 & 175.5 & 1,564 & 5,085 & & & \\
\hline 1981 & 3,540 & 63 & 64.5 & 5,487 & 158.3 & 1,573 & 5,170 & & & \\
\hline 1982 & 3,363 & 75 & 70.5 & 4,770 & 75.3 & 1,498 & 5,256 & & & \\
\hline 1983 & 3,748 & 70 & 71.9 & 5,213 & 6.6 & 1,355 & 5,341 & & & \\
\hline 1984 & 4,041 & $\pi$ & 71.3 & 5,670 & 202.1 & 1,392 & 5,427 & & & \\
\hline 1985 & 3,752 & $69 *$ & 70.0 & 5,357 & 107.2 & 1,338 & 5,512 & & & \\
\hline 1986 & 3,796 & 65 & 66.5 & 5,712 & 104.6 & 1,285 & 5,598 & & & \\
\hline-- & $-\cdot-$ & $-\cdots$ & $\because \cdot$ & $\because \cdot \cdot$ & $\cdots$ & $\because-$ & $\because \cdots$ & $\cdot \cdot \cdot$ & $\cdots$ & $\cdots$ \\
\hline 1987 & 3,821 & 62 & 63.1 & 6,053 & 138.4 & 1,267 & 5,684 & & & \\
\hline 1988 & 3,854 & $\pi$ & 68.3 & 5.646 & 158.6 & 1,271 & 5,769 & 8,537 & 8,883 & 9,023 \\
\hline 1989 & & & & & 162.6 & 1,279 & 5,855 & 8,664 & 9,015 & 9,157 \\
\hline 1990 & & & & & 155.7 & 1,270 & 5,940 & 8,790 & 9,147 & 9,291 \\
\hline 1991 & & & & & 161.6 & 1,285 & 6,026 & 8,917 & 9.270 & 9,424 \\
\hline 1992 & & & & & 179.3 & 1,308 & 6,111 & 9,044 & 9,410 & 9,558 \\
\hline
\end{tabular}

Capacity-Capital Stock Regression: Code -2 RSO 0.600 Coefficients: Const 4485.5 Time 85.6

Ave. Capacity/Stock $(74-86): 3.5$ Ave. Weekly Hours (High) 100.7 Shift Factor 1.56 Ave Heekly Hours (Low) 95.0 Shift Factor 1.66 * Imputed Note: Output, Investment, and Capital stock extrapolated for 1987 and 1988.

2499 Wood products, n.e.c.

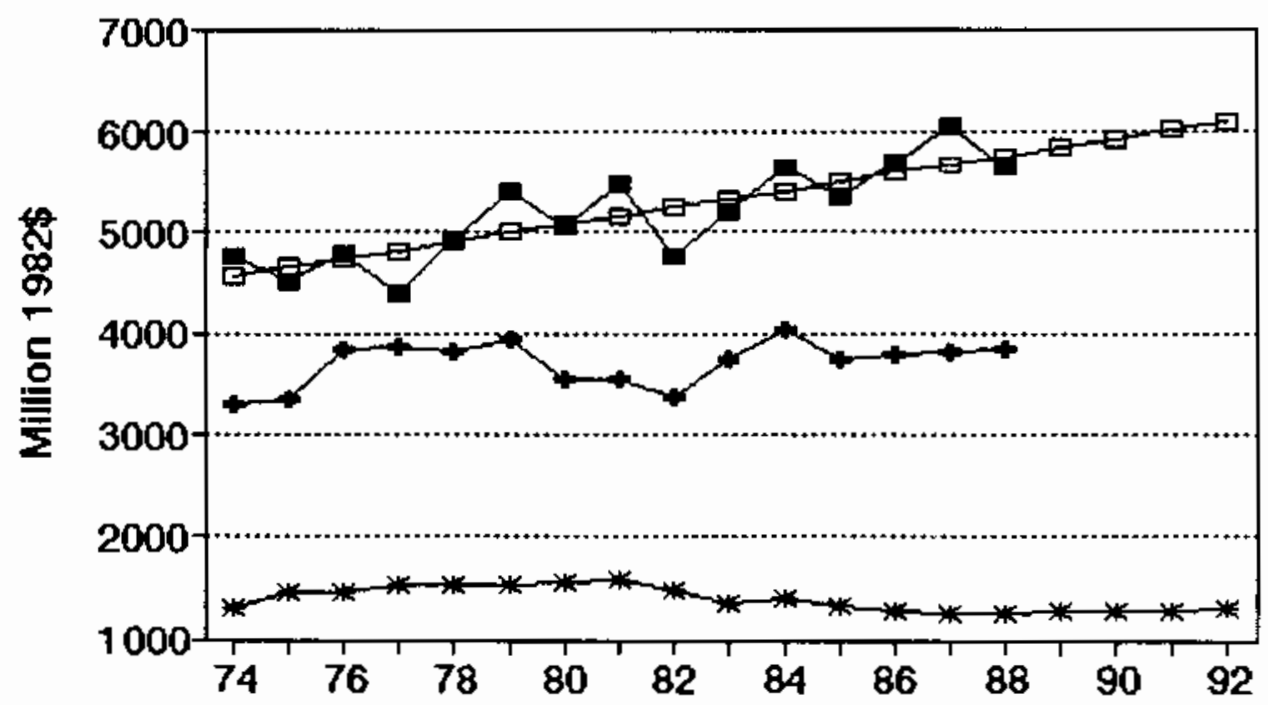

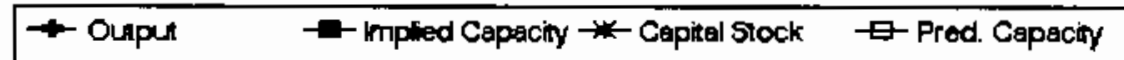


No. 132 sIC 2511 Wood household furniture

MILLLIONS OF 1982 DOLLARS (EXCL, CU RATES)

$\begin{array}{lccccccc} & Q 4 & \text { Ann. Implied } & \text { Gross } & \text { Net } & \text { Pred. } & \ldots \\ \text { Gross } & \text { CJ } & \text { CU } & \text { Prac. } & \text { In- } & \text { Cap. } & \text { Prac. } & \text { Emergency Capacity } \\ \text { Dutput Rate } & \text { Rate } & \text { Capac. } & \text { vest. } & \text { Stk. } & \text { Capac. } & 3 \text { mon. } 6 \text { mon. }>6 \text { mon. }\end{array}$

\begin{tabular}{|c|c|c|c|c|c|c|c|c|c|c|}
\hline 1974 & 5,967 & 7 & 84.1 & 7,093 & 176.2 & 1,229 & 7,621 & & & \\
\hline 1975 & 5,030 & 70 & 70.3 & 7,155 & 126.6 & 1,265 & 7,738 & & & \\
\hline 1976 & 5,950 & 73 & 71.9 & 8,274 & 73.3 & 1,244 & 7,856 & & & \\
\hline 1977 & 6,212 & 79 & 77.8 & 7,985 & 244.9 & 1,380 & 7,973 & & & \\
\hline 1978 & 6,762 & 78 & 78.2 & 8,653 & 22.9 & 1,299 & 8,090 & & & \\
\hline 1979 & 6,245 & $n$ & 76.9 & 8,121 & 103.7 & 1,294 & 8,208 & & & \\
\hline 1980 & 5,637 & 66 & 68.6 & 8,213 & 109.8 & 1,291 & 8,325 & & & \\
\hline 1981 & 5,646 & 63 & 65.9 & 8,568 & 125.2 & 1,297 & 8,442 & & & \\
\hline 1982 & 5,093 & 52 & 55.9 & 9,116 & 90.7 & 1,265 & 8,560 & & & \\
\hline 1983 & 5,505 & 61 & 58.7 & 9,383 & 108.3 & 1,245 & 8,677 & & & \\
\hline 1984 & 6,105 & 67 & 66.1 & 9,239 & 107.0 & 1,224 & 8,795 & & & \\
\hline 1985 & 5.925 & 76 & 71.6 & 8,299 & 142.8 & 1,236 & 8,912 & & & \\
\hline 1986 & 6,310 & 80 & 77.6 & 8,129 & 118.5 & 1,225 & 9,029 & & & \\
\hline$\cdots$ & $\cdots$ & -- & -- & $\cdots$ & $\cdots-$ & $\therefore-$ & $\therefore$ & $-\cdots$ & --- & $\cdot \cdot$ \\
\hline 1987 & 6,362 & 82 & 80.5 & 7,899 & 152.0 & 1,248 & 9,147 & & & \\
\hline 1988 & 6,429 & 63 & 70.2 & 9,161 & 169.9 & 1,286 & 9,264 & 19.069 & 20,004 & 20,381 \\
\hline 1989 & & & & & 173.6 & 1,324 & 9,381 & 19,311 & 20,258 & 20,639 \\
\hline 1990 & & & & & 166.3 & 1,351 & 9,499 & 19,553 & $20,51 t$ & 20,897 \\
\hline 1991 & & & & & 177.2 & 1,386 & 9.616 & 19,794 & 20,764 & 21,155 \\
\hline 1992 & & & & & 162.6 & 1.402 & 9,734 & 20,036 & 21,018 & 21,414 \\
\hline
\end{tabular}

Capecity-Capital stock Regression: Code -2 RSO 0.430

Coefficients: Const 7303.5 Time 117.4

Ave. Capacity/Stock (74-86): 6.6 Ave. Heekly Hours (High) 71.6 shift factor 2.20 Ave Heekly Hours (LOW) 67.8 Shift Factor 2.32 Note: Dutput, Investment, and Capital Stock extrapolated for 1987 and 1988.

\section{Wood household fumiture}

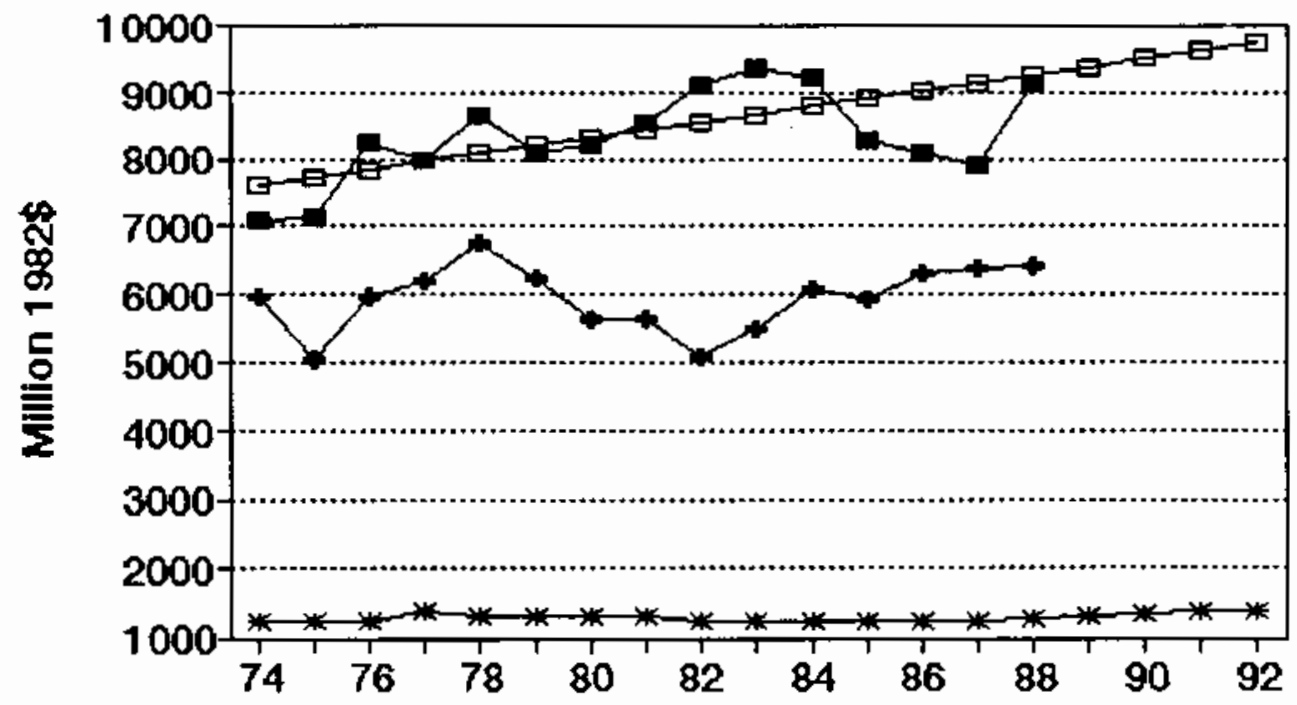

- Oupu $\quad \rightarrow$ - mplied Capecin $\rightarrow$ - Ceppial Stock $\quad \square$ Pred. Cepaciny


No. 133 BIC 2512 Upholstered household furniture

HILLIOWS OF 1982 DOLLARS (EXCL. CU RATES)

$\begin{array}{lccccccc} & \text { Q4 } & \text { Ann. Implied Gross } & \text { Net } & \text { Pred. } & \ldots \ldots \\ \text { Gross } & \text { CU } & \text { CU } & \text { Prac. } & \text { In } & \text { Cap. } & \text { Prac. } & \text { Emergency Capacity } \\ \text { Output Rate } & \text { Rate } & \text { Capac. } & \text { vest. } & \text { Stk. } & \text { Capac. } & 3 \text { mon. } 6 \text { mon. }>6 \text { mon. }\end{array}$

\begin{tabular}{|c|c|c|c|c|c|c|c|c|c|c|}
\hline 1974 & 3,396 & 65 & 65.0 & 5,224 & 71.2 & 610 & 4,739 & & & \\
\hline 1975 & 3,183 & 63 & 63.8 & 4,993 & 64.3 & 630 & 4,831 & & & \\
\hline 1976 & 3,254 & 52 & 56.1 & 5,700 & 111.5 & 695 & 5,128 & & & \\
\hline 1977 & 3,338 & 60 & 57.0 & 5,857 & 56.8 & 701 & 5,157 & & & \\
\hline 1978 & 3,422 & 72 & 67.5 & 5,069 & 63.4 & 714 & 5,215 & & & \\
\hline 1979 & 3,364 & 78 & 75.8 & 4,440 & $(9.0)$ & 660 & 4,966 & & & \\
\hline 1980 & 3,356 & 84 & 81.8 & 4,106 & 57.3 & 669 & 5,009 & & & \\
\hline 1981 & 3,446 & 85 & 84.6 & 4.072 & 28.9 & 649 & 4,920 & & & \\
\hline 1982 & 3,369 & 66 & $\pi .1$ & 4,607 & 74.6 & 672 & 5,021 & & & \\
\hline 1983 & 3,285 & 76 & 72.3 & 4,566 & 35.6 & 655 & 4,945 & & & \\
\hline 1984 & 3,801 & $\infty$ & 69.8 & 5,449 & 49.8 & 652 & 4,931 & & & \\
\hline 1985 & 3,699 & $\infty 6$ & 66.0 & 5,604 & 47.4 & 646 & 4,906 & & & \\
\hline 1986 & 3,935 & 41 & 50.4 & 7,811 & 65.5 & 658 & 7,811 & & & \\
\hline$\because-$ & $-\cdot-$ & -- & -- & $\cdots \cdot$ & $-\cdot-$ & $-\cdots$ & $\because \cdots$ & ---- & --- & $=--$ \\
\hline 1987 & 4,109 & 53 & 48.5 & 8,473 & 51.5 & 655 & 7,800 & & & \\
\hline 1988 & 4.347 & 62 & 58.6 & 7,414 & 58.8 & 660 & 7,823 & 10,556 & 15,119 & 15,184 \\
\hline 1989 & & & & & 70.8 & 677 & 7,899 & 10,659 & 15,266 & 15,332 \\
\hline 1990 & & & & & 63.5 & 685 & 7,935 & 10,708 & 15,336 & 15,402 \\
\hline 1991 & & & & & 67.7 & 696 & 7,988 & 10,779 & 15,438 & 15,504 \\
\hline 1992 & & & & & 62.5 & 702 & 8,012 & 10,812 & 15,485 & 15,551 \\
\hline
\end{tabular}

Capacity-Capital Stock Regression: Code -1 RSO 0.650

Coefficients: Const 1948.7 stock 4.6 D(86-86) $2853.4 \quad$ Ave. Capacity/Stock $(74-86): 7.8$ Ave. Hekly Hours (High) 81.1 Shift Factor 1.94 Ave Heekly Hours (LOW) 72.7 Shift factor 2.17 Hote: Output, Investment, and Capital stock extrapolated for 1987 and 1988.

\section{Upholstered household furniture}

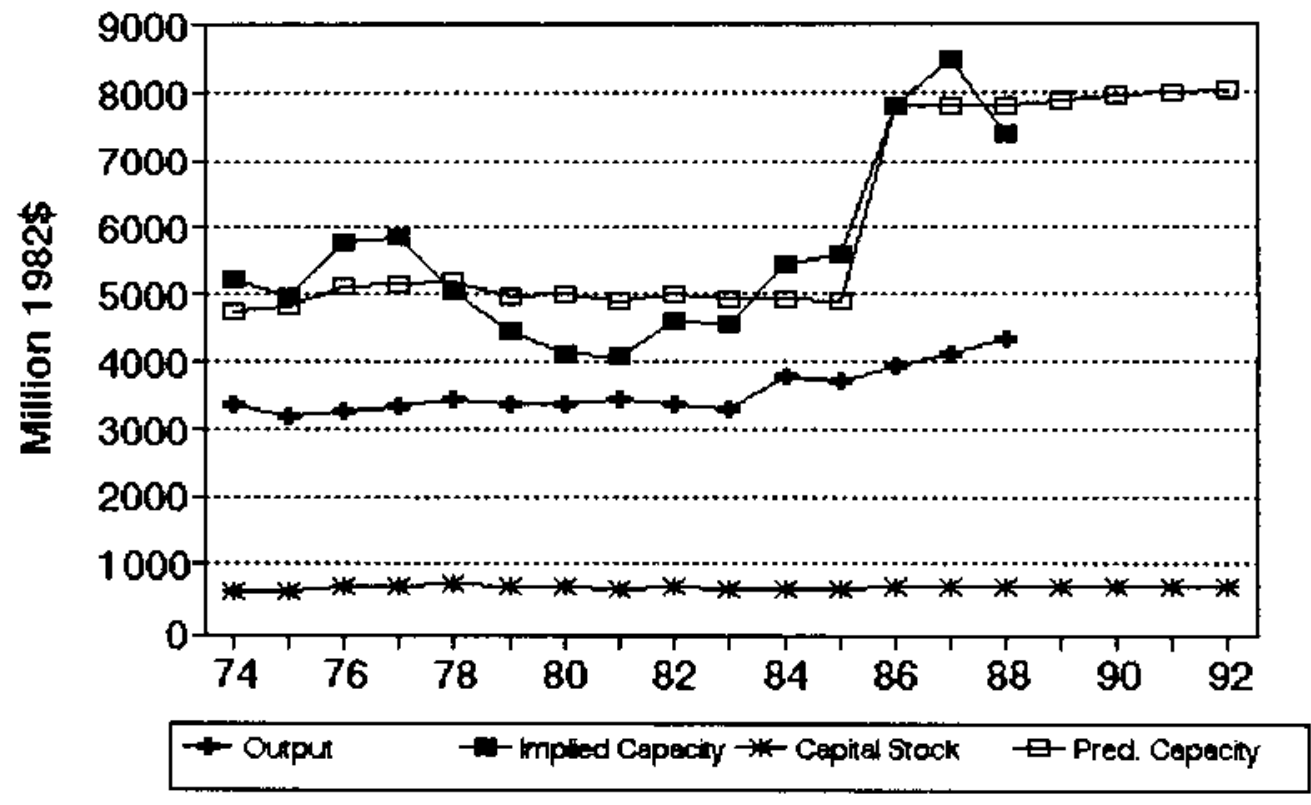


No. 134 BIC 2514 Metal hougehold furniture

MILLIONS OF 1982 DOLLARS (EXCL. CU RATES)

$\begin{array}{lccccccc} & \text { Q4 } & \text { Am. } & \text { Implied } & \text { Gross } & \text { Net } & \text { Pred. } & \ldots \\ \text { Gross } & \text { CU } & \text { CU } & \text { Prac. } & \text { In- } & \text { Cap. } & \text { Prac. } & \text { Emergency Capacity } \\ \text { Output Rate } & \text { Rate } & \text { Capac. } & \text { vest. } & \text { Stk. } & \text { Capac. } & 3 \text { mon. } 6 \text { mon. }>6 \text { mon. }\end{array}$

\begin{tabular}{|c|c|c|c|c|c|c|c|c|c|c|}
\hline 1974 & 1,673 & 46 & 47.9 & 3,494 & 9.0 & 256 & 2,575 & & & \\
\hline 1975 & 1,396 & 74 & 62.7 & 2,225 & 7.2 & 244 & 2,569 & & & \\
\hline 1976 & 1,572 & 60 & 65.4 & 2,405 & 17.1 & 241 & 2,562 & & & \\
\hline 1977 & 1,767 & 78 & 71.7 & 2,466 & 33.9 & 254 & 2,556 & & & \\
\hline 1978 & 1,770 & 70 & 72.9 & 2,428 & 31.1 & 262 & 2,549 & & & \\
\hline 1979 & 1,673 & 72 & 70.8 & 2,364 & 38.3 & 276 & 2,543 & & & \\
\hline 1980 & 1,546 & 65 & 66.8 & 2,314 & 20.7 & 272 & 2,537 & & & \\
\hline 1981 & 1,575 & 64 & 65.2 & 2,417 & 19.3 & 267 & 2,530 & & & \\
\hline 1982 & 1,602 & 66 & 65.2 & 2,459 & 58.2 & 296 & 2,524 & & & \\
\hline 1883 & 1.694 & 77 & 73.4 & 2,307 & $(9.5)$ & 260 & 2,517 & & & \\
\hline 1984 & 1,804 & $70 *$ & 73.2 & 2,466 & $(9.3)$ & 228 & 2,511 & & & \\
\hline 1985 & 1,815 & 64 & 6.1 & 2,745 & 33.6 & 238 & 2,505 & & & \\
\hline 1986 & 1,897 & $67 *$ & 65.7 & 2,886 & 20.7 & 235 & 2,498 & & & \\
\hline$\because-$ & $--\cdot$ & $\cdots$ & $-\cdots$ & $\cdots$ & $=--$ & $\cdots$ & $\cdots-$ & $\cdots$ & $-\cdots$ & -- \\
\hline 1987 & 1,913 & $\infty$ & 67.3 & 2,843 & 26.4 & 238 & 2,492 & & & \\
\hline 1988 & 1,933 & 55 & 59.9 & 3,228 & 28.9 & 243 & 2,485 & 4,262 & 4,434 & 4,504 \\
\hline 1989 & & & & & 29.4 & 249 & 2,479 & 4,251 & 4,423 & 4,492 \\
\hline 1990 & & & & & 28.3 & 252 & 2,473 & 4,240 & 4,411 & 4,480 \\
\hline 1991 & & & & & 29.9 & 257 & 2,466 & 4,229 & 4,400 & 4,469 \\
\hline 1992 & & & & & 27.8 & 259 & 2,460 & 4,218 & 4,388 & 4,457 \\
\hline
\end{tabular}

Capacity-Capital Stock Regression: Code -2 RSO 0.000 Coefficients: Const 2581.3 Time -6.4 Ave. Capacity/Stock $(74-86): 9.9$ Ave. Weekly Hours (High) 86.9 Shift factor 1.81 Ave Weekly Hours (LOW) 83.2 Shift Factor 1.89 - Imputed Note: Output, Investment, and Capital Stock extrapolated for 1987 and 1988.

\section{Metal household fumiture}

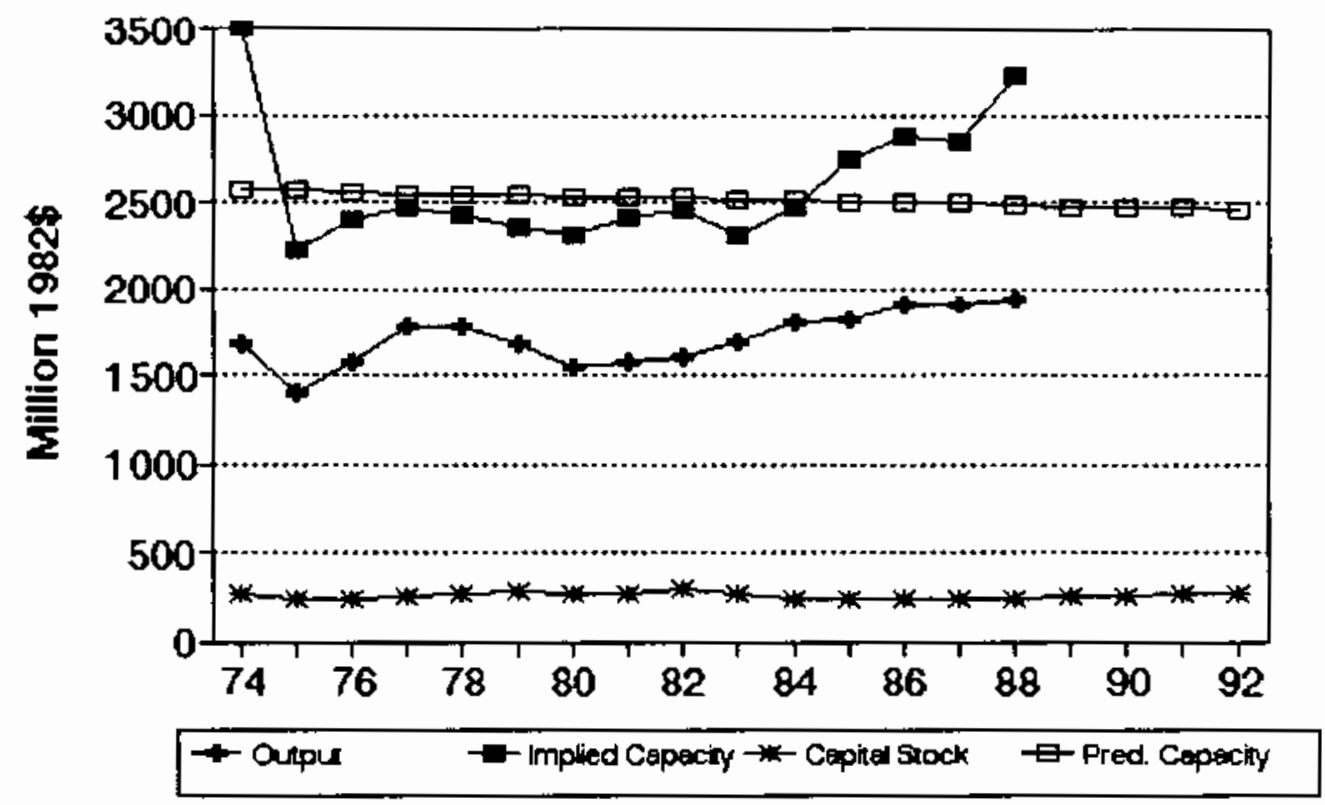




\section{ro. 135 sIC 2515 Mattresses and beadsprings}

MILLIONS OF 1982 DOLLARS (EXCL. CU RATES)

$\begin{array}{lccccccc} & 04 & \text { Amn. Implied } & \text { Gross } & \text { Net } & \text { Pred. } & \ldots & \ldots \ldots \ldots \\ \text { Gross } & \text { CU } & \text { CU } & \text { Prac. } & \text { In. } & \text { Cap. } & \text { Prac. } & \text { Emergency Capacity } \\ \text { Output } & \text { Rete } & \text { Rate } & \text { Capac. } & \text { vest. } & \text { Stk. } & \text { Capac. } & 3 \text { mon. } 6 \text { mon. }>6 \text { mon. }\end{array}$

\begin{tabular}{|c|c|c|c|c|c|c|c|c|c|c|}
\hline 1974 & 1,893 & 88 & 88.8 & 2,133 & $(14.4)$ & 234 & 2,468 & & & \\
\hline 1975 & 1,730 & 64 & 72.8 & 2,378 & 16.5 & 233 & 2,535 & & & \\
\hline 1976 & 1,857 & 74 & 70.2 & 2,644 & 14.2 & 227 & 2,602 & & & \\
\hline 1977 & 2,002 & 75 & 74.7 & 2,679 & 17.5 & 225 & 2,668 & & & \\
\hline 1978 & 1,982 & 78 & 76.9 & 2,579 & 8.0 & 213 & 2,735 & & & \\
\hline 1979 & 2,211 & 85 & 82.3 & 2,686 & 39.2 & 229 & 2,802 & & & \\
\hline 1980 & 2,258 & 63 & 71.1 & 3,176 & 9.2 & 215 & 2,869 & & & \\
\hline 1981 & 2,329 & 65 & 64.4 & 3,616 & 19.9 & 212 & 2,936 & & & \\
\hline 1982 & 1,921 & 57 & 60.0 & 3,203 & 21.8 & 210 & 3,002 & & & \\
\hline 1983 & 2,051 & $58 *$ & 57.4 & 3,573 & 14.6 & 201 & 3,069 & & & \\
\hline 1984 & 1,877 & 58 & 57.9 & 3,240 & $(10.3)$ & 171 & 3,136 & & & \\
\hline 1985 & 1,806 & 71 & 66.1 & 2,730 & 19.0 & 171 & 3.203 & & & \\
\hline 1986 & 1,920 & 73 & 7.2 & 2,657 & 13.2 & 164 & 3,270 & & & \\
\hline$-\cdots$ & $=-\cdot$ & $\cdots$ & -- & $\because-$ & - . . & $\cdots$ & $-\cdots$ & $\cdots-$ & -- & $=\cdot$ \\
\hline 1987 & 1,932 & 55 & 61.7 & 3,132 & 14.8 & 160 & 3,336 & & & \\
\hline 1988 & 1,948 & 67 & 62.5 & 3,117 & 15.0 & 156 & 3,403 & 4,524 & 4,715 & 4,792 \\
\hline 1989 & & & & & 15.2 & 153 & 3,470 & 4,613 & 4,807 & 4,886 \\
\hline 1990 & & & & & 15.4 & 151 & 3,537 & 4,702 & 4,900 & 4,980 \\
\hline 1991 & & & & & 15.6 & 149 & 3,603 & 4,791 & 4,992 & 5,074 \\
\hline 1992 & & & & & 15.8 & 147 & 3,670 & 4.879 & 5,085 & 5,168 \\
\hline
\end{tabular}

Capacity-Capital Stock Regression: Cade -2 RSQ 0.330 Coefficients: Const 2401.3 Time 66.8 Ave. Capacity/stock $(74-86): 13.8$ Ave. Weekly Hours (High) 111.9 Shift factor 1.41 Ave Ueekly Hours (Low) 80.8 shift Factor 1.95 * Imputed Note: Output, Investment, and Capital stock extrapolated for 1987 and 1988.

\section{Mattresses and beadsprings}

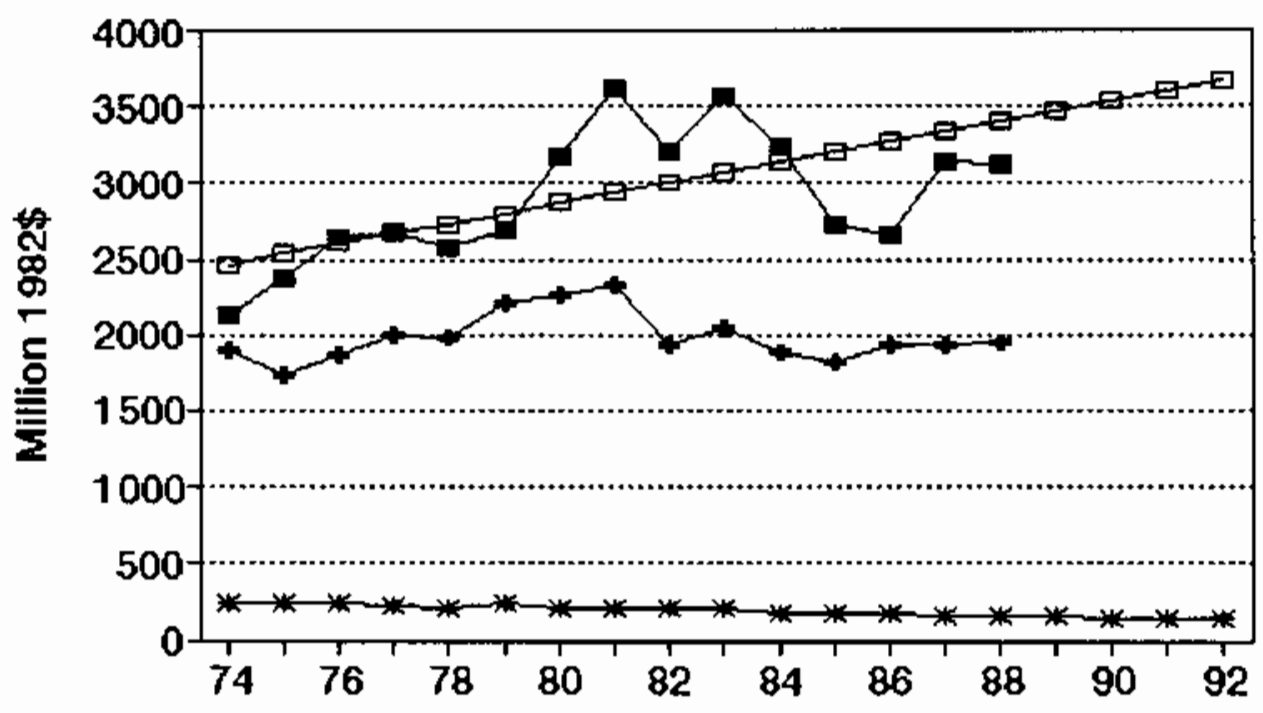

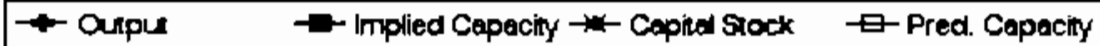




\section{No. 136 sIC 2517 wood IV and radio cabinets}

MILLIONS OF 1982 DOLLARS (EXCL. CU RATES)

$\begin{array}{lccccccc} & \alpha & \text { Ann. Implied Gross } & \text { Net } & \text { Pred. } & \ldots \ldots \ldots \\ \text { Gross } & \text { CU } & \text { CU } & \text { Prac. } & \text { In- } & \text { Cap. } & \text { Prac. } & \text { Emergency Capacity } \\ \text { Output } & \text { Rate } & \text { Rate } & \text { Capac. } & \text { vest. } & \text { Stk. } & \text { Capac. } & 3 \text { mon. } 6 \text { mon. }>6 \text { mon. }\end{array}$

\begin{tabular}{|c|c|c|c|c|c|c|c|c|c|c|}
\hline 1974 & 608 & 61 & 61.0 & $\infty 97$ & 30.0 & 119 & 1,034 & & & \\
\hline 1975 & 374 & 64 & 62.9 & 595 & $(25.3)$ & 87 & 694 & & & \\
\hline 1976 & 456 & 59 & 60.9 & 749 & 5.4 & 85 & 676 & & & \\
\hline 1977 & 449 & 64 & 62.1 & 723 & 9.7 & 87 & 695 & & & \\
\hline 1978 & 484 & 61 & 62.1 & 779 & 3.2 & 82 & 645 & & & \\
\hline 1979 & 318 & $65 *$ & 63.2 & 503 & $(4.9)$ & 71 & 520 & & & \\
\hline 1980 & 316 & $\omega=$ & 65.7 & 678 & 6.1 & 69 & 506 & & & \\
\hline 1981 & 307 & $70 *$ & 68.4 & 648 & 4.6 & 67 & 476 & & & \\
\hline 1982 & 307 & $\pi$ & 71.0 & 433 & 6.8 & $\infty 6$ & 466 & & & \\
\hline 1983 & 301 & $76 *$ & 74.4 & 405 & 4.2 & 62 & 431 & & & \\
\hline 1984 & 368 & 79 & 7.9 & 473 & 9.3 & 64 & 449 & & & \\
\hline 1985 & 325 & 61 & 67.8 & 480 & 11.4 & 68 & 485 & & & \\
\hline 1986 & 340 & $\pi$ & 71.0 & 479 & 5.6 & $\infty$ & 465 & & & \\
\hline$\cdots-$ & $-\cdot-\cdot$ & $\cdots$ & $-\cdot$ & $-\cdot-$ & $\cdots$ & -- & $-\cdots$ & $\cdots \cdot \cdot$ & --- & ... \\
\hline 1987 & 329 & 56 & 63.9 & 515 & 15.3 & 73 & 547 & & & \\
\hline 1988 & 316 & 56 & 55.7 & 567 & 21.5 & 86 & 685 & 989 & 1,038 & 1,058 \\
\hline 1989 & & & & & 22.7 & $\boldsymbol{\phi}$ & 821 & 1,184 & 1,243 & 1,267 \\
\hline 1990 & & & & & 20.2 & 108 & 914 & 1,317 & 1,384 & 1,410 \\
\hline 191 & & & & & 24.0 & 119 & 1,036 & 1,493 & 1,569 & $1,5 \%$ \\
\hline 1992 & & & & & 19.0 & 124 & 1,090 & 1,572 & 1,651 & 1,683 \\
\hline
\end{tabular}

Capecity-Capital Stock Regression: Code 0 RSO 0.890

Coefficients: Const -235.4 stock 10.7

Ave. Capacity/Stock $(74-86): 7.6$

Ave. Weekly Hours (High) 102.0 Shift Factor 1.54 Ave Weekly Hours (Low) 100.0 Shift Factor 1.58

- Imputed Note; Output, Imestment, and Capital stock extrapolated for 1987 and 1988.

\section{Wood TV and radio cabinets}

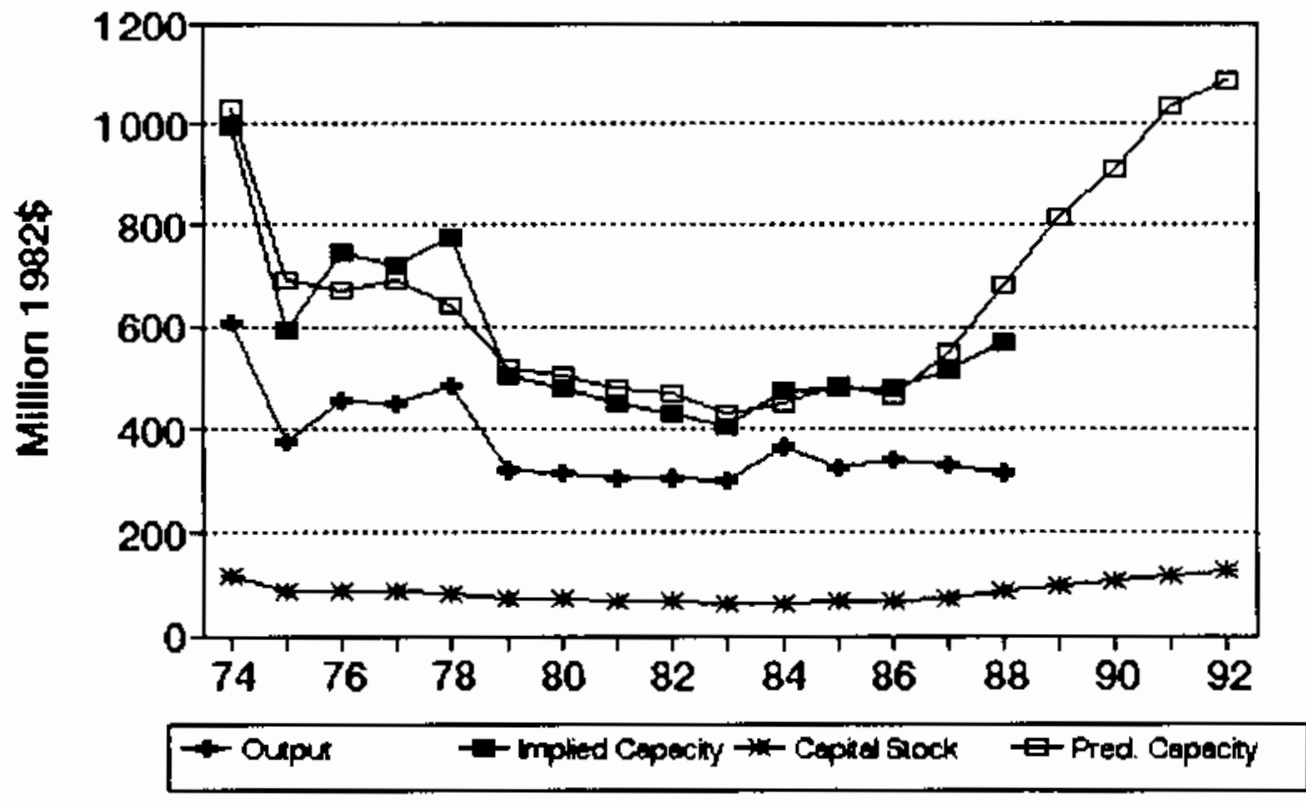


ko. 137 sIC 2519 Household furmiture, n.e.c.

MILLIONS OF 1992 DOLLARS (EXCL. CU RATES)

$\begin{array}{lccccccc} & \text { Q4 } & \text { Ann. Implied } & \text { Gross } & \text { Het } & \text { Pred. } & \ldots & \ldots \\ \text { Gross } & \text { CU } & \text { CU } & \text { Prac. } & \text { In- } & \text { Cap. } & \text { Prac. } & \text { Emergency Capacity } \\ \text { Output } & \text { Rate } & \text { Rate } & \text { Capac. } & \text { vest. } & \text { Stk. } & \text { Capac. } & 3 \text { mon. } 6 \text { mon. }>6 \text { mon. }\end{array}$

\begin{tabular}{|c|c|c|c|c|c|c|c|c|c|c|}
\hline 1974 & 301 & 55 & 55.0 & 548 & 22.1 & 95 & 554 & & & \\
\hline 1975 & 312 & $\pi$ & 67.5 & 463 & 9.9 & 97 & 563 & & & \\
\hline 1976 & 317 & $68 *$ & 70.7 & 448 & 10.8 & 101 & 573 & & & \\
\hline 1977 & 433 & 6 & 66.8 & 648 & 49.6 & 138 & 700 & & & \\
\hline 1978 & 444 & 63 & 4.1 & 692 & 9.0 & 136 & 693 & & & \\
\hline 1979 & 426 & $65 *$ & 64.2 & 663 & $(6.5)$ & 120 & 640 & & & \\
\hline 1980 & 466 & $68 \div$ & 66.6 & 699 & 18.5 & 129 & 668 & & & \\
\hline 1981 & 493 & $79 *$ & 69.5 & 709 & 14.7 & 133 & 682 & & & \\
\hline 1982 & 382 & $72 \star$ & 71.5 & 535 & $(9.8)$ & 114 & 620 & & & \\
\hline 1983 & 465 & $\pi$ & 72.6 & 640 & $(1.6)$ & 105 & 587 & & & \\
\hline 1984 & 392 & 68 & 69.9 & 561 & $(8.1)$ & 89 & 536 & & & \\
\hline 1985 & 410 & 63 & 64.9 & 632 & 19.6 & 101 & 574 & & & \\
\hline 1986 & 402 & 49 & 54.3 & 742 & 12.9 & 105 & 588 & & & \\
\hline$\because-$ & $-\cdots$ & $\cdots$ & $\cdots$ & $\cdots$ & $-\cdots$ & $-\cdots$ & $\cdots$ & $\cdots \cdot \cdot-\cdot$ & $-\cdot--$ & $--\cdot-$ \\
\hline 1987 & 422 & 52 & 50.9 & 830 & 21.6 & 118 & 632 & & & \\
\hline 1988 & 449 & 85 & 72.6 & 618 & 28.5 & 137 & 695 & 881 & 913 & 926 \\
\hline 1989 & & & & & 29.8 & 155 & 757 & 960 & 995 & 1,009 \\
\hline 1990 & & & & & 27.1 & 170 & 805 & 1,021 & 1,058 & 1,073 \\
\hline 1991 & & & & & 31.2 & 187 & 863 & 1,085 & 1,134 & 1,150 \\
\hline 1992 & & & & & 25.7 & 197 & 897 & 1,138 & 1,180 & 1,196 \\
\hline
\end{tabular}

Capacity-Capital Stock Regression: Code 1 RSO 0.360

Coefficients: Const 236.5 stock 3.4

Ave. Capacity/Stock (74-86); 5.5

Ave. Weekly Hours (High) 118.5 Shift Factor 1.33 Ave Weekly Hours (Low) 112.0 Shift Factor 1.41

* Imputed Note: Dutput, Investment, and Capital Stock extrapolated for 1987 and 1988.

2519 Household furniture, n.e.c.

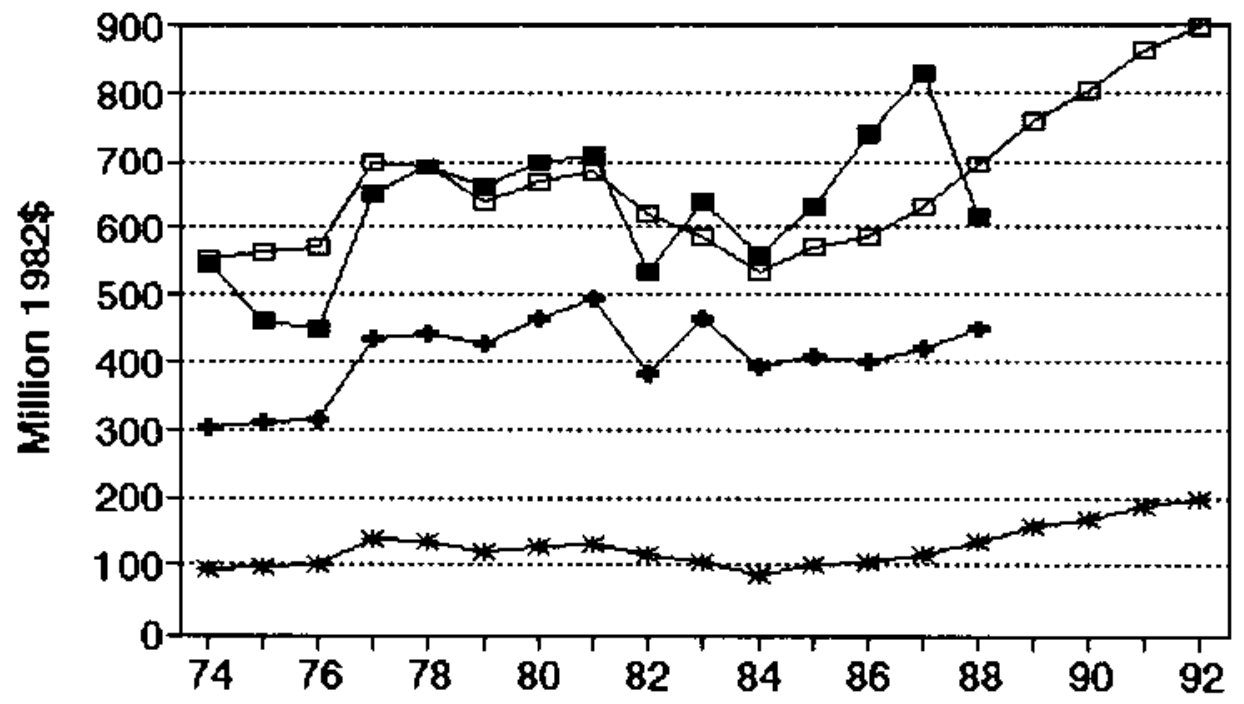

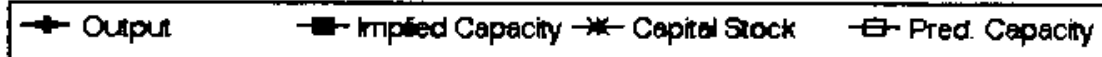




\section{No. 138 sIC 2521 Wood office furniture}

MILLIONS OF 1982 dOLLARS (EXCL. CU RATES)

\begin{tabular}{|c|c|c|c|c|c|c|c|}
\hline & $\begin{array}{l}\mathrm{Q} \\
\mathrm{CU}\end{array}$ & $\begin{array}{l}\text { Ann. } \\
\text { CU }\end{array}$ & $\begin{array}{l}\text { Implied } \\
\text { Prec. }\end{array}$ & $\begin{array}{l}\text { Gross } \\
\text { In- }\end{array}$ & $\begin{array}{l}\text { Net } \\
\text { Cep. }\end{array}$ & $\begin{array}{l}\text { Pred. } \\
\text { Prac. }\end{array}$ & $\begin{array}{l}\text { Emergency Capacity } \\
\text { En }\end{array}$ \\
\hline & Rate & Rate & Capac & est. & stk. & & \\
\hline
\end{tabular}

\begin{tabular}{|c|c|c|c|c|c|c|c|c|c|c|}
\hline 1974 & 626 & $\pi$ & 81.3 & 770 & 4.8 & 90 & 833 & & & \\
\hline 1975 & 499 & 79 & 76.8 & 649 & 6.0 & 91 & 834 & & & \\
\hline 1976 & 584 & 67 & 71.6 & 816 & 5.6 & 90 & 829 & & & \\
\hline 1977 & 948 & 92 & 83.2 & 1,139 & 43.9 & 126 & 1,167 & & & \\
\hline 1978 & 1,007 & 89 & 90.0 & 1,120 & 37.7 & 156 & 1,440 & & & \\
\hline 1979 & 1,307 & 89 & 88.7 & 1,474 & 16.0 & 163 & 1,511 & & & \\
\hline 1980 & 1,202 & 71 & 76.7 & 1,567 & $(4.4)$ & 152 & 1,402 & & & \\
\hline 1981 & 1,113 & 72 & 72.8 & 1,529 & $(14.8)$ & 130 & 1,204 & & & \\
\hline 1982 & 1,093 & 71 & 71.2 & 1,534 & 29.4 & 151 & 1,398 & & & \\
\hline 1983 & 1,130 & 64 & 67.6 & 1,672 & 22.9 & 164 & 1,518 & & & \\
\hline 1984 & 1,390 & 78 & 73.6 & $1,8 B 8$ & 41.9 & 194 & 1,792 & & & \\
\hline 1985 & 1,340 & 68 & 71.7 & 1,868 & 23.8 & 206 & 1,888 & & & \\
\hline 1986 & 1,317 & 79 & 74.8 & 1,762 & 23.2 & 213 & 1,971 & & & \\
\hline$=-$ & $\cdots$ & $=$ & $\cdots$ & $-\cdots$ & $\cdots$ & $\because \cdot$ & $-\cdots$ & $\cdots$ & -- & $--\cdot$ \\
\hline 1987 & 1,391 & 87 & 83.6 & 1,665 & 33.0 & 232 & 2,147 & & & \\
\hline 1988 & 1,492 & 78 & 81.4 & 1,832 & 41.8 & 258 & 2,392 & 4,883 & 5,134 & 5,235 \\
\hline 1989 & & & & & 43.6 & 285 & 2,638 & 5,384 & 5,661 & $5,7 \pi 2$ \\
\hline 1990 & & & & & 40.0 & 306 & 2,834 & 5,785 & 6,082 & 6,201 \\
\hline 1991 & & & & & 45.6 & 331 & 3,067 & 6,260 & 6,581 & 6,711 \\
\hline 1992 & & & & & 38.2 & 347 & 3,218 & 6,568 & 6,906 & 7,041 \\
\hline
\end{tabular}

Capacity-Capital Stock Regression: Code 0 RSO 0.830

Coefficients: Const -7.6 Stock 9.3

Ave. Capacity/Stock (74-86): 9.2

Ave. Weekly Hours (High) 72.0 Shift Factor 2.19 Ave Weekly Hours (Lou) 62.0 Shift Factor 2.54 Note: Output, Imvestment, and Capital Stock extrapolated for 1987 and 1988.

\section{Wood office furniture}

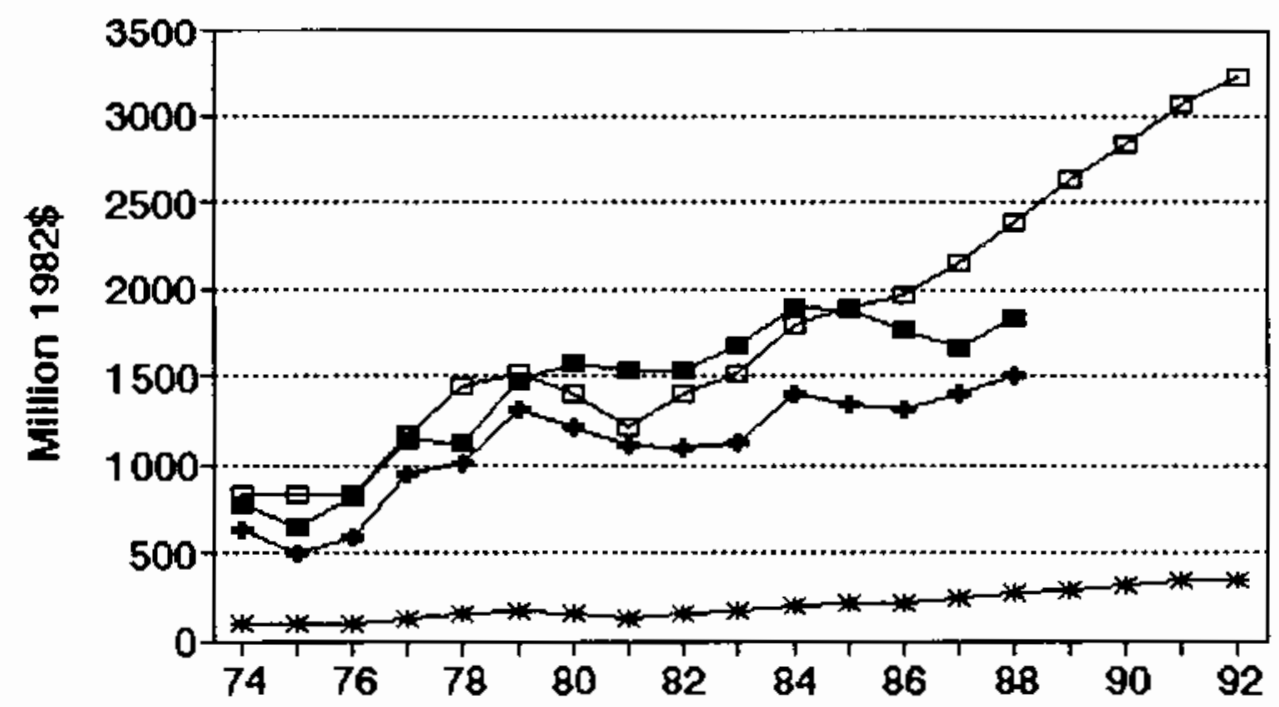

$$
\text { +- Ourpu } \rightarrow \text { Impied Capecity } \rightarrow \text { Capial Stock } \quad \text { E-Pred. Capecity }
$$




\section{No. 139 sIC 2522 Metal office furniture}

MILLIONS OF 1982 DOLLARS (EXCL. CU RATES)

$\begin{array}{lccccccc} & \text { QU } & \text { Ann. } & \text { Implied Gross } & \text { Het } & \text { Pred. } & \ldots \ldots . \ldots \\ \text { Gross } & \text { CU } & \text { CU } & \text { Prac. } & \text { In- } & \text { Cap. } & \text { Prac. } & \text { Emergency Capacity } \\ \text { Output } & \text { Rate } & \text { Rate } & \text { Capac. } & \text { vest. } & \text { Stk. } & \text { Capac. } & 3 \text { mon. } 6 \text { mon. }>6 \text { mon. }\end{array}$

\begin{tabular}{|c|c|c|c|c|c|c|c|c|c|c|}
\hline 1974 & 1,916 & 83 & 83.0 & 2,309 & 20.3 & 327 & 2,385 & & & \\
\hline 1975 & 1,590 & 72 & 76.1 & 2,089 & 25.3 & 327 & 2,381 & & & \\
\hline 1976 & 1,711 & 70 & 70.8 & 2,418 & 25.6 & 326 & 2,376 & & & \\
\hline 1977 & 2,035 & 68 & 68.8 & 2,960 & 79.1 & 374 & 2,732 & & & \\
\hline 1978 & 2,194 & 77 & 73.6 & 2,980 & 48.0 & 389 & 2,844 & & & \\
\hline 1979 & 2,229 & $\pi$ & 75.8 & 2,942 & 43.3 & 400 & 2,926 & & & \\
\hline 1980 & 2,617 & $\pi$ & 76.3 & 3,432 & 112.4 & 474 & 3,482 & & & \\
\hline 1981 & 2,770 & 72 & 73.9 & 3,749 & 71.8 & 506 & 3,717 & & & \\
\hline 1982 & 3,084 & 63 & 66.4 & 4,640 & 138.8 & 597 & 4,394 & & & \\
\hline 1983 & 3,275 & 84 & 76.1 & 4.302 & 91.2 & 640 & 4,713 & & & \\
\hline 1984 & 3,819 & 72 & 76.5 & 4,993 & 95.4 & 684 & 5,043 & & & \\
\hline 1985 & 4,138 & 67 & 68.9 & 6,007 & 139.8 & 769 & 5,679 & & & \\
\hline 1986 & 4,190 & 66 & 66.4 & 6,312 & 165.2 & 875 & 6,468 & & & \\
\hline$-\cdot$ & $\cdots-$ & $\because$ & $\cdots$ & - - - & $--\overline{-}$ & $\cdots$ & $-\cdots$ & $-\cdots$ & $-\cdots$ & $-\cdots$ \\
\hline 1987 & 4,381 & 69 & 67.9 & 6,455 & 80.9 & 895 & 6,619 & & & \\
\hline 1988 & 4,642 & 71 & 70.3 & 6,608 & 81.9 & 915 & 6,767 & 8,982 & 9,303 & 9,433 \\
\hline 1969 & & & & & 82.1 & 934 & 6,906 & 9,167 & 9.495 & 9,627 \\
\hline 1990 & & & & & 81.7 & 951 & 7.032 & 9,335 & 9,668 & 9,803 \\
\hline 1991 & & & & & 82.3 & 967 & 7,154 & 9,497 & 9,836 & 9,973 \\
\hline 1992 & & & & & 81.5 & 982 & 7,262 & 9,640 & 9,984 & 10,123 \\
\hline
\end{tabular}

Capacity-Capital Stock Regression: Code 0 RSo 0.980

Coefficients: Const $\mathbf{5 3 . 0}$ stock 7.5

Ave. Capacity/Stock $(74-86): 7.3$ Ave. Week!y Hours (High) 113.1 Shift Factor 1.39 Ave Heekly Hours (LoW) 108.9 Shift Factor 1.45 Note: Output, Investment, and Capital Stock extrapolated for 1987 and 1988.

\section{Metal office furniture}

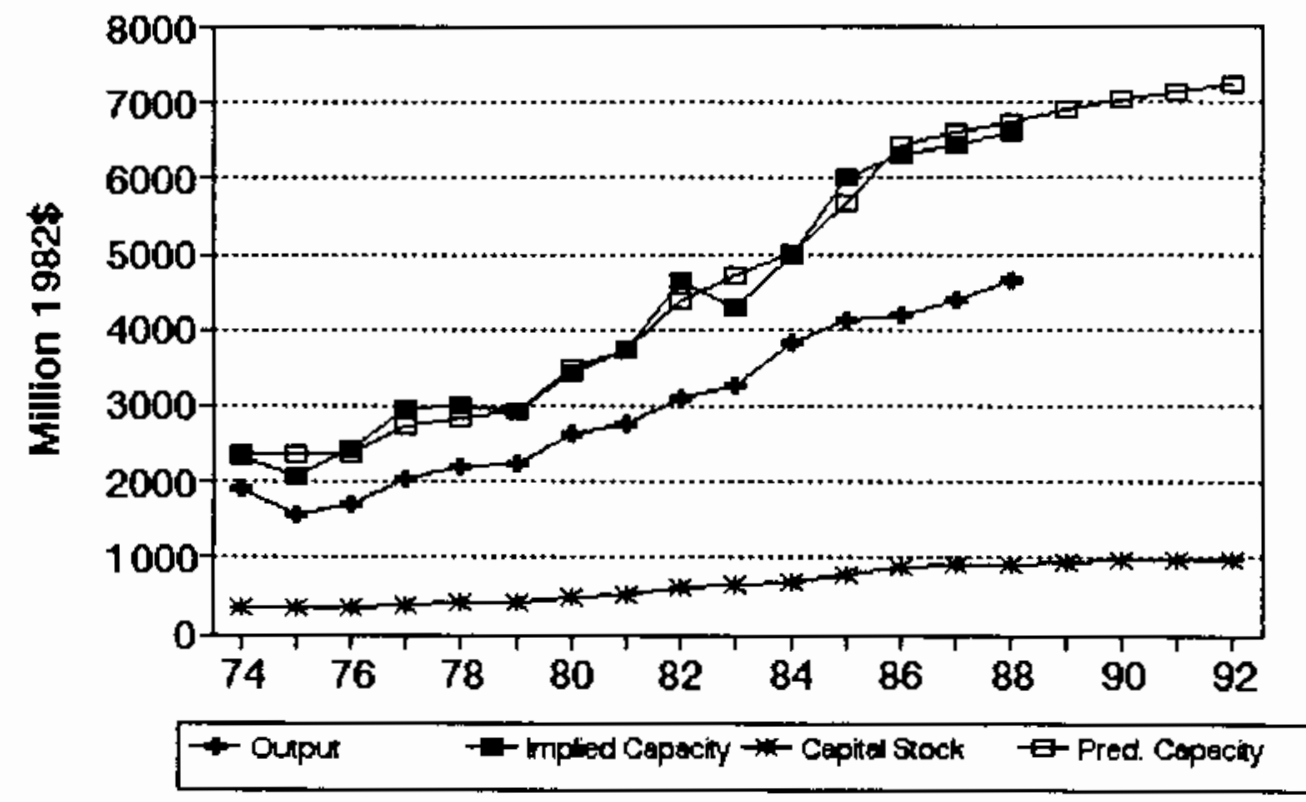


No. 140 sIC 2531 Public building and related furntture

MILLIONS OF 1982 DOLLARS (EXCL. CU RATES)

$\begin{array}{lccccccc} & \text { Q4 } & \text { Ann. Implied Gross } & \text { Net } & \text { Pred. } & \ldots . . . \\ \text { Gross } & \text { CU } & \text { CU } & \text { Prac. } & \text { In- } & \text { Cap. } & \text { Prac. } & \text { Emergency Capacity } \\ \text { Output } & \text { Rate } & \text { Rate } & \text { Capac. } & \text { vest. } & \text { Stk. } & \text { Capac. } & 3 \text { mon. } 6 \text { mon. }>6 \text { mon. }\end{array}$

\begin{tabular}{|c|c|c|c|c|c|c|c|c|c|c|}
\hline 1974 & 1,157 & 68 & 72.6 & 1,593 & 16.6 & 196 & 1,682 & & & \\
\hline 1975 & 1,063 & 71 & 68.3 & 1,556 & 5.9 & 186 & 1,634 & & & \\
\hline 1976 & 1,187 & 69 & 69.8 & 1,700 & 27.6 & 197 & 1,687 & & & \\
\hline 1977 & 1,178 & 67 & 68.5 & 1,721 & 15.7 & 196 & 1,682 & & & \\
\hline 1978 & 1,180 & 79 & 74.4 & 1,587 & 34.1 & 212 & 1,762 & & & \\
\hline 1979 & 1,298 & $84 *$ & 81.9 & 1,585 & $(3.4)$ & 192 & 1,664 & & & \\
\hline 1980 & 1,307 & 7 & 76.0 & 1,719 & 34.7 & 208 & 1,746 & & & \\
\hline 1981 & 1,225 & $76 *$ & 76.5 & 1,602 & 9.8 & 200 & 1,704 & & & \\
\hline 1982 & 1,097 & 67. & 70.3 & 1,562 & (6.6) & 177 & 1,588 & & & \\
\hline 1983 & 1,160 & 88 & 81.1 & 1,430 & 9.0 & 170 & 1,552 & & & \\
\hline 1984 & 1,222 & 63 & 73.7 & 1,658 & 30.1 & 182 & 1,614 & & & \\
\hline 1985 & 1,264 & 69 & 66.8 & 1,893 & 26.2 & 190 & 1,655 & & & \\
\hline 1986 & 1,432 & 70 & 69.6 & 2,059 & 25.3 & 198 & 1.692 & & & \\
\hline$\cdots$ & --- & -- & $-\cdot$ & $\cdots$ & $\cdots$ & $\cdots$ & $-\cdots$ & -- & $\cdots$ & $\cdots$ \\
\hline 1987 & 1,443 & 92 & 83.2 & 1,734 & 29.9 & 209 & 1,730 & & & \\
\hline 1988 & 1,458 & 9 & 96.4 & 1,512 & 37.4 & 227 & 1,840 & 3,140 & 3,264 & 3,314 \\
\hline 1989 & & & & & 38.9 & 245 & 1,930 & 3,292 & 3,422 & 3,475 \\
\hline 1990 & & & & & 35.8 & 258 & 1,995 & 3,404 & 3,539 & 3,593 \\
\hline 1991 & & & & & 40.4 & 273 & 2,078 & 3,545 & 3,685 & 3,742 \\
\hline 1992 & & & & & 34.3 & 284 & 2,122 & 3,620 & 3,764 & 3,822 \\
\hline
\end{tabular}

Capecity-Capital Stock Regression: Code 1 RSO 0.130

Coefficients: Const 704.8 stock $5.0 \quad$ Ave. Capacity/Stock (74-86): 8.7 Ave. Weekly Hours (High) 87.4 Shift Factor 1.80 Ave Weekly Hours (LOW) 85.2 Shift factor 1.85 * Imputed Note: Output, Imvestment, and Capital stock extrapolated for 1987 and 1988.

2531 Public building and related furnit

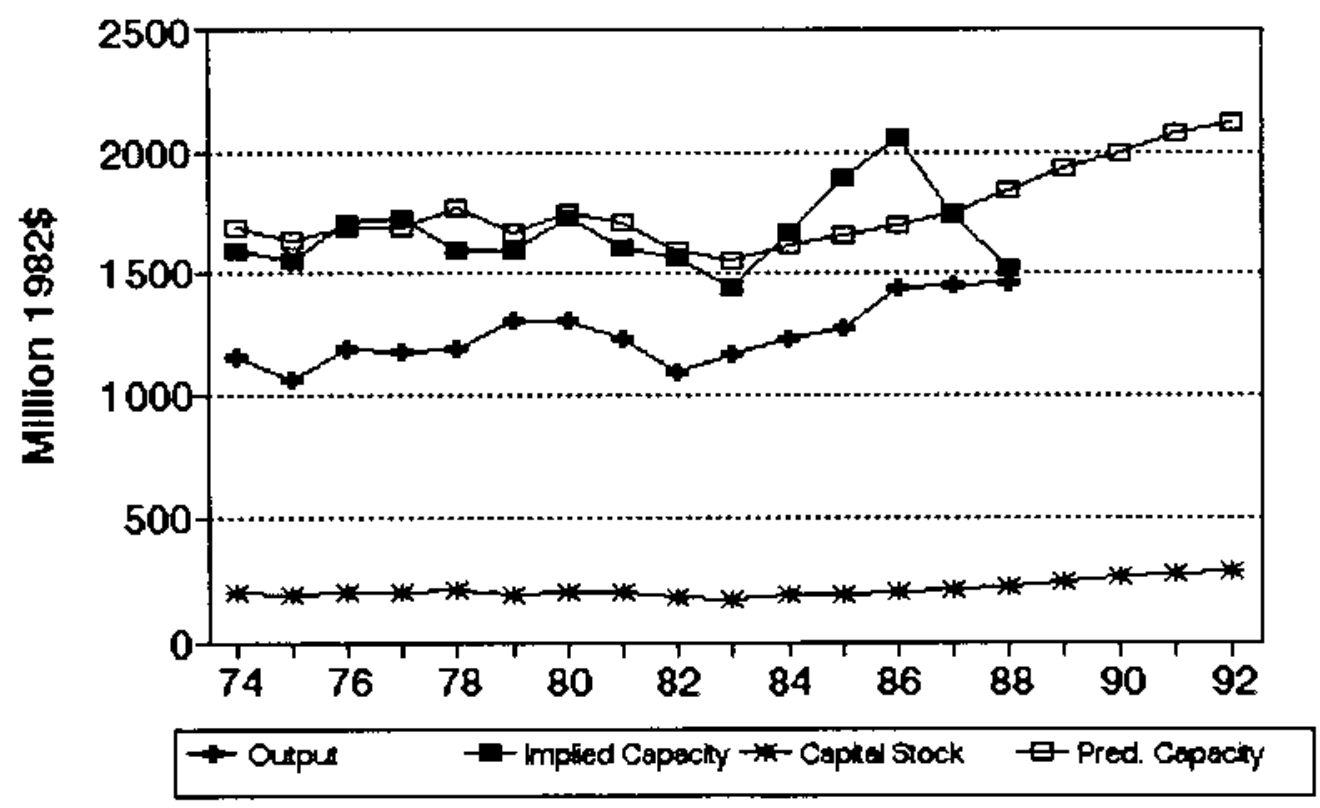




\section{No. 141 sIC 2541 Wood partitions and fixtures}

MILLIONS OF 1982 DOLLARS (EXCL. CU RATES)

$\begin{array}{lcccclcc} & \text { Q4 } & \text { Ann. Implied Gross } & \text { Net } & \text { Pred. } & -\ldots & \ldots . . . \\ \text { Gross } & \text { CU } & \text { CU } & \text { Prac. } & \text { In- } & \text { Cap. } & \text { Prac. } & \text { Emergency Capacity } \\ \text { Output } & \text { Rate } & \text { Rate } & \text { Capac. } & \text { vest. } & \text { Stk. } & \text { Capac. } & 3 \text { mon. } 6 \text { mon. }>6 \text { mon. }\end{array}$

\begin{tabular}{|c|c|c|c|c|c|c|c|c|c|c|}
\hline 1974 & 1,669 & 64 & 64.0 & 2,608 & $(10.0)$ & 175 & 2,193 & & & \\
\hline 1975 & 1,476 & 51 & 55.9 & 2,642 & 13.2 & 174 & 2,191 & & & \\
\hline 1976 & 1,615 & 76 & 66.6 & 2,424 & 26.2 & 186 & 2,303 & & & \\
\hline 1977 & 1,704 & 72 & 73.5 & 2,319 & 13.0 & 184 & 2,286 & & & \\
\hline 1978 & 1,861 & 76 & 74.5 & 2,498 & 27.9 & 196 & 2,403 & & & \\
\hline 1979 & 1,622 & 97 & 89.1 & 1,820 & 23.1 & 202 & 2,456 & & & \\
\hline 1980 & 1,666 & 73 & 82.0 & 2,032 & $(3.4)$ & 182 & 2,264 & & & \\
\hline 1981 & 1,722 & 71 & 71.8 & 2,400 & 34.5 & 197 & 2,410 & & & \\
\hline 1982 & 1,765 & 81 & 77.3 & 2,285 & 36.9 & 212 & 2,557 & & & \\
\hline 1983 & 1,783 & 85 & 83.5 & 2,135 & 9.8 & 201 & 2,447 & & & \\
\hline 1984 & 2,119 & $70 *$ & 75.3 & 2,814 & 68.5 & 245 & 2,869 & & & \\
\hline 1985 & 2,029 & 54 & 59.8 & 3,392 & 44.5 & 264 & 3,055 & & & \\
\hline 1986 & 1,984 & 61 & 58.4 & 3,398 & 54.4 & 293 & 3,333 & & & \\
\hline - - - & $\cdots \cdot$ & $\cdots$ & --- & - - - - & $\cdots \cdot$ & $\cdots \cdot$ & $\cdots$ & $-\cdots-\cdot-$ & ---- & $\cdots$ \\
\hline 1987 & 2,009 & 58 & 59.1 & 3,398 & 28.6 & 295 & 3,350 & & & \\
\hline 1988 & 2,042 & 62 & 60.5 & 3,375 & 30.6 & 298 & 3,385 & 4,654 & 4,847 & 4,925 \\
\hline 1989 & & & & & 31.0 & 302 & 3,421 & 4,704 & 4,899 & 4,978 \\
\hline 1990 & & & & & 30.2 & 305 & 3,446 & 4,738 & 4,934 & 5,014 \\
\hline 1991 & & & & & 31.4 & 308 & 3,480 & 4,785 & 4,984 & 5,064 \\
\hline 1992 & & & & & 29.8 & 310 & 3,496 & 4,806 & 5,006 & 5,086 \\
\hline
\end{tabular}

Capacity-Capital Stock Regression: Code 0 RSO 0.560

Coefficients: Const 509.9 stock 9.6

Ave. Capacity/Stock $(74-86): 12.1$ Ave, Ueekly Hours (High) 108.3 Shift Factor 1.46 Ave Heekly Hours (Low) 104.0 shift Factor 1.51 * Imputed Note: Output, Investment, and Capital stock extrapolated for 1987 and 1988.

\section{Wood partitions and fixtures}

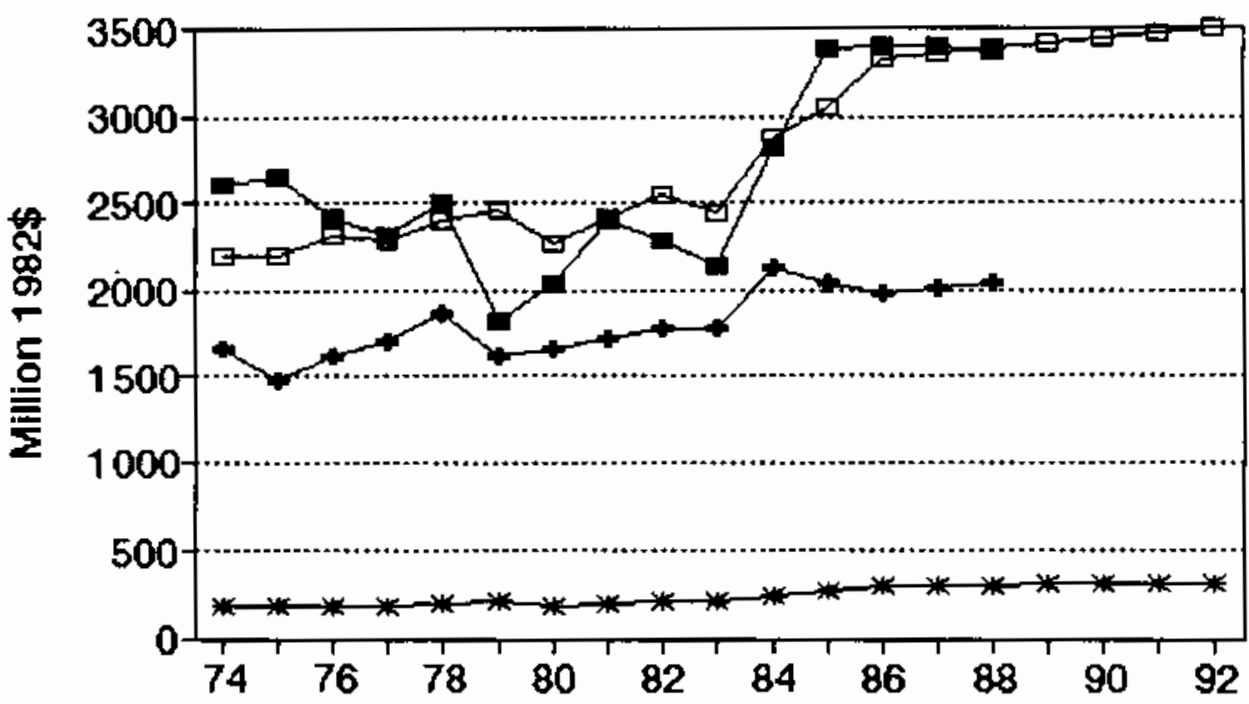

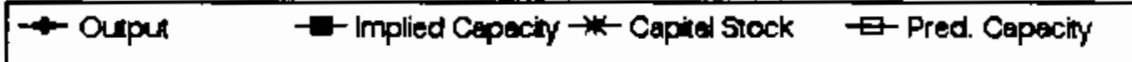




\section{yo. 142 gIC 2542 Metal partitions and fixtures}

MILLIONS OF 1982 DOLLARS (EXCL. CU RATES)

$\begin{array}{lccccccc} & 04 & \text { Ann. Implied } & \text { Gross } & \text { Het } & \text { Pred. } & \ldots & \ldots \\ \text { Gross } & \text { CU } & \text { CU } & \text { Prac. } & \text { In- } & \text { Cap. } & \text { Prac. } & \text { Emergency Capacity } \\ \text { Output } & \text { Rate } & \text { Rate } & \text { Capac. } & \text { vest. } & \text { Stk. } & \text { Capac. } & 3 \text { mon. } 6 \text { mon. }>6 \text { mon. }\end{array}$

\begin{tabular}{|c|c|c|c|c|c|c|c|c|c|c|}
\hline 1974 & 1,765 & 63 & 60.0 & 2,595 & 9.0 & 305 & 2,610 & & & \\
\hline 1975 & 1,396 & 57 & 57.6 & 2,422 & 39.8 & 310 & 2,754 & & & \\
\hline 1976 & 1,509 & 55 & 55.8 & 2,704 & 21.9 & 315 & 2,711 & & & \\
\hline 1977 & 1,942 & 61 & 59.4 & 3,268 & 100.2 & 384 & 3,385 & & & \\
\hline 1978 & 1,951 & $\infty$ & 64.0 & 3,050 & 29.2 & 382 & 3,371 & & & \\
\hline 1979 & 2,062 & 50 & 55.7 & 3.704 & 22.6 & 374 & 3,287 & & & \\
\hline 1980 & 2,016 & 52 & 50.4 & 4,002 & 38.6 & 380 & 3,353 & & & \\
\hline 1981 & 1,912 & 55 & 55.2 & 3,465 & 44.6 & 391 & 3,461 & & & \\
\hline 1982 & 1,074 & 50 & 51.7 & 3,818 & 63.0 & 418 & 3,718 & & & \\
\hline 1983 & 1,933 & 55 & 54.0 & 3,579 & 53.2 & 431 & 3,846 & & & \\
\hline 1984 & 2,248 & $58 *$ & 57.6 & 3,903 & 29.5 & 421 & 3,750 & & & \\
\hline 1985 & 2,220 & 60 & 59.1 & 3,738 & 43.7 & 426 & 3,802 & & & \\
\hline 1986 & 2,260 & 62 & 61.2 & 3,694 & 50.8 & 438 & 3,914 & & & \\
\hline $\begin{array}{l}\cdots \\
1987\end{array}$ & 2,315 & 68 & $\begin{array}{l}--1 \\
65.3\end{array}$ & 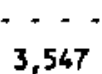 & $\begin{array}{r}--. \\
54.2\end{array}$ & 452 & $\cdots$ & $\cdots$ & $\cdots$ & $\cdots$ \\
\hline 1988 & 2,388 & 68 & 68.0 & 3,511 & 56.0 & 468 & 4,209 & 5,914 & 6,175 & 6,280 \\
\hline 1969 & & & & & 57.9 & 483 & 4,364 & 6,132 & 6,402 & 6,511 \\
\hline 1990 & & & & & 59.8 & 500 & 4,524 & 6,356 & 6,637 & 6,730 \\
\hline 1991 & & & & & 61.7 & 516 & 4,688 & 6,587 & 6.877 & 6,994 \\
\hline 1992 & & & & & 63.6 & 533 & 4,855 & 6.822 & 7.123 & 7,244 \\
\hline
\end{tabular}

Capacity-Capital Stock Regression: Code 0 RSO 0.710

Coefficients: Const -382.6 Stock 9.8

Ave. Capacity/stock $(74-86): 8.8$ Ave. Heekly Hours (High) 105.6 Shift Factor 1.49 Ave Ueekly Hours (Low) 99.8 Shift Factor 1.58 * Imputed Note: Output, Investment, and Capital Stock extrapolated for 1987 and 1988.

\section{Metal partitions and fixtures}

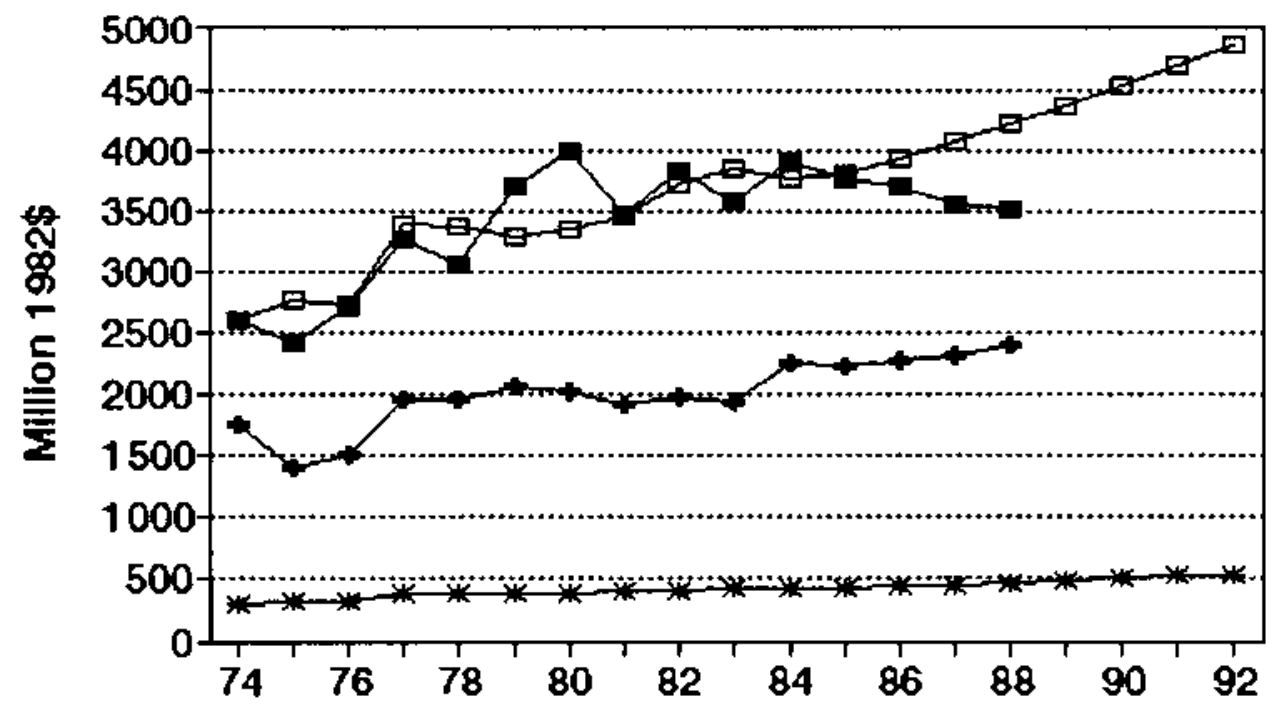

\begin{tabular}{|c|c|c|}
\hline$\rightarrow$ - arpur & $\rightarrow-$ mpled Copecity tim- Cepind Sock & E-Pred. Capaciny \\
\hline
\end{tabular}




\section{No. 143 sIC 2591 Drapery hardware and blinds and shades}

MILLIONS OF 1982 DOLLARS (EXCL. CU RATES)

\begin{tabular}{|c|c|c|c|c|c|c|c|}
\hline & $\begin{array}{l}04 \\
\text { CU }\end{array}$ & Ann. & $\begin{array}{l}\text { Impl ied } \\
\text { Prac. }\end{array}$ & $\begin{array}{l}\text { Gross } \\
\text { In- }\end{array}$ & $\begin{array}{l}\text { Net } \\
\text { Cap. }\end{array}$ & $\begin{array}{l}\text { Pred. } \\
\text { Prac. }\end{array}$ & 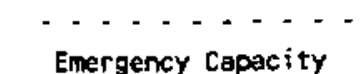 \\
\hline tout & Rate & Rate & Capac & vest. & stk. & Capac. & \\
\hline
\end{tabular}

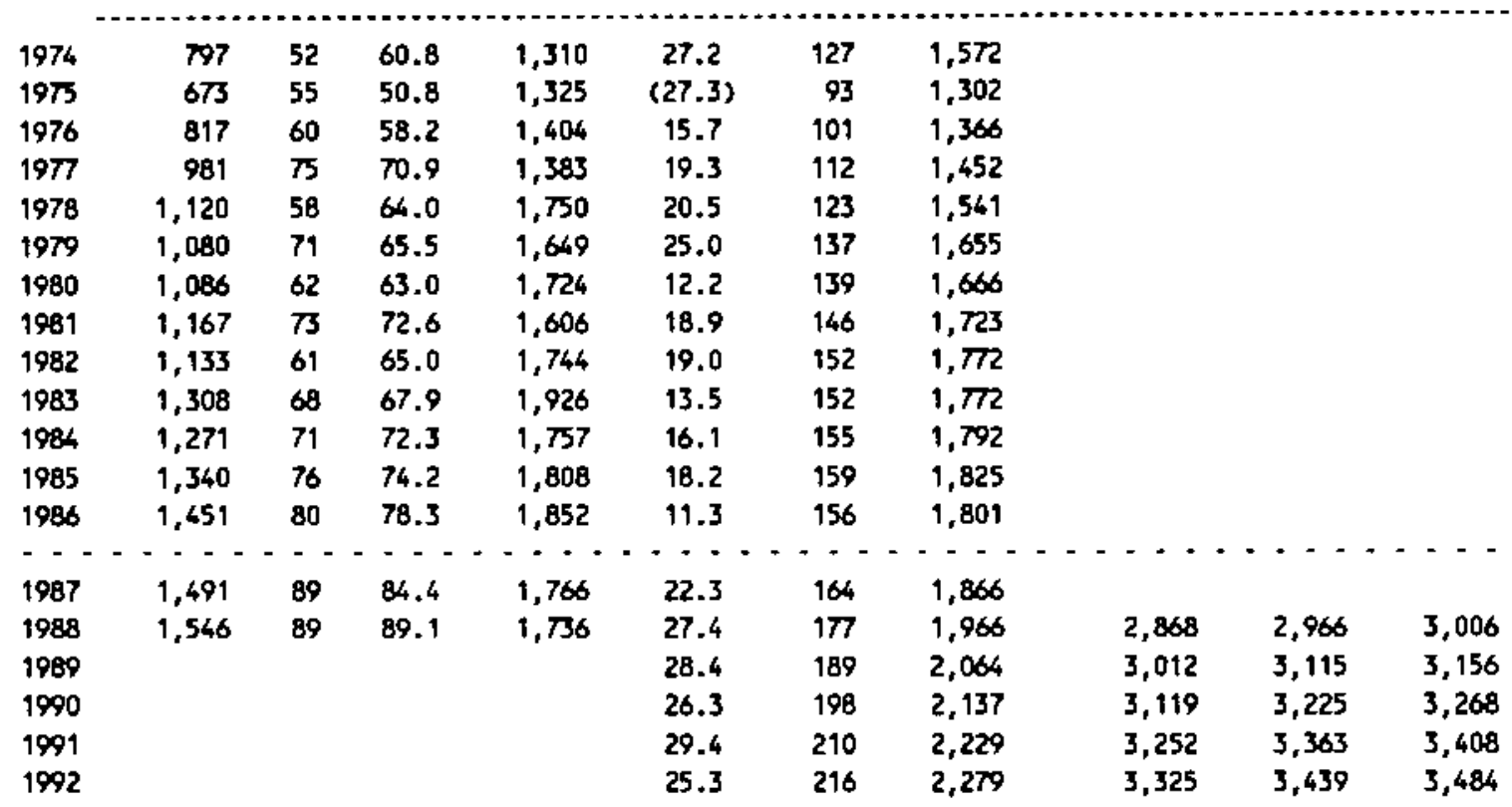

Capacity-Capital Stock Regression: Code 0 RSO 0.690

Coefficients: Const 568.0 stock 7.9

Ave. Capecity/stock (74-86): 12.1

Ave. Weekly Hours (High) 103.0 Shift Factor 1.53 Ave Weekly Hours (Low) 87.2 Shift Factor 1.81

Note: Output, Investment, and Capital Stock extrapolated for 1987 and 1988.

\section{Drapery hardware and blinds and sh}

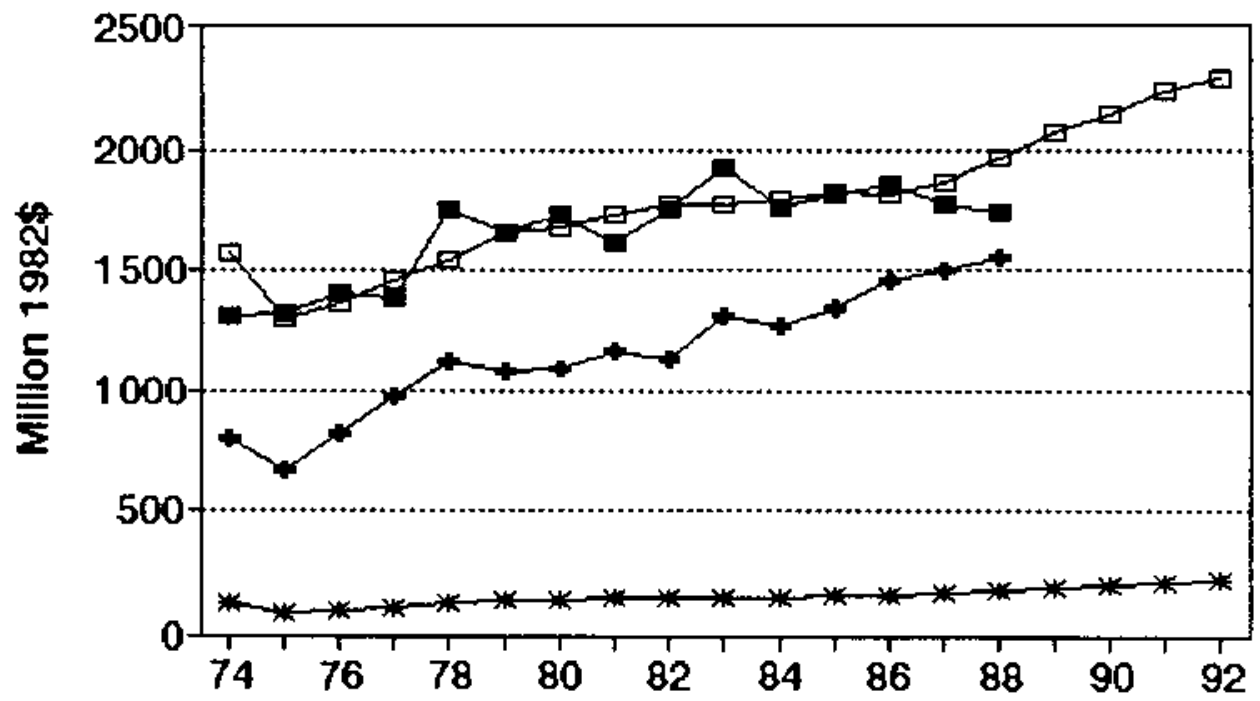

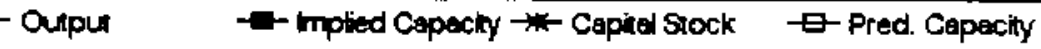


No. 144 sIC 2599 Furniture and fixtures, n.e.c.

MILLIONS OF 1982 DOLLARS (EXCL. CU RATES)

$\begin{array}{lccccccc} & \text { Q4 Amn. Implied Gross } & \text { Het } & \text { Pred. } & \ldots \ldots \ldots \\ \text { Gross } & \text { a } & \text { CU } & \text { Prac. } & \text { In- } & \text { Cap. } & \text { Prac. } & \text { Emergency Capacity } \\ \text { Output } & \text { Rate } & \text { Rate } & \text { Capac. } & \text { vest. } & \text { Stk. } & \text { Capac. } & 3 \text { mon. } 6 \text { mon. }>6 \text { mon. }\end{array}$

\begin{tabular}{|c|c|c|c|c|c|c|c|c|c|c|}
\hline 1974 & $B 50$ & 63 & 68.4 & 1,242 & 19.6 & 114 & 1,558 & & & \\
\hline 1975 & 828 & 57 & 57.5 & 1,441 & 9.8 & 115 & 1,566 & & & \\
\hline 1976 & 806 & 63 & 60.7 & 1,327 & $(0.9)$ & 106 & 1,492 & & & \\
\hline 1977 & 1,071 & 47 & 53.8 & 1,990 & 36.0 & $\uparrow 32$ & 1,711 & & & \\
\hline 1978 & 1,190 & 59 & 54.4 & 2,190 & 21.8 & 143 & 1,804 & & & \\
\hline 1979 & 1,085 & 63 & 61.2 & 1,775 & (12.2) & 123 & 1,632 & & & \\
\hline 1980 & 1,154 & 58 & 58.7 & 1,965 & 47.2 & 158 & 1,927 & & & \\
\hline 1981 & 1,032 & 64 & 63.4 & $1,62 B$ & 6.6 & 154 & 1,891 & & & \\
\hline 1982 & 1,297 & 53 & 56.9 & 2,280 & 49.8 & 189 & 2,189 & & & \\
\hline 1983 & 1,422 & 57 & 56.5 & 2,516 & 19.8 & 194 & 2,225 & & & \\
\hline 1984 & 1,477 & 68 & 65.1 & 2,270 & 29.5 & 208 & 2,343 & & & \\
\hline 1985 & 1,547 & 60 & 63.0 & 2,456 & 31.6 & 223 & 2,470 & & & \\
\hline 1986 & 1,487 & 69 & 65.5 & 2,270 & 25.9 & 231 & 2,540 & & & \\
\hline$-\cdot$ & $\cdots \cdot$ & $=$ & $\because \cdot$ & $\cdots-$ & $-\cdots$ & -- & $-\cdots$ & $-\cdots$ & $\cdots$ & $\cdots$ \\
\hline 1987 & 1,551 & 70 & 69.1 & 2,246 & 25.1 & 230 & 2,606 & & & \\
\hline 1988 & 1,638 & 88 & 81.3 & 2,015 & 27.1 & 248 & 2,693 & 4,532 & 4.746 & 4.832 \\
\hline 1989 & & & & & 27.5 & 257 & 2,758 & 4,659 & 4,879 & 4,968 \\
\hline 1990 & & & & & 26.7 & 265 & 2,821 & 4.765 & 4,990 & 5,080 \\
\hline 1991 & & & & & 28.0 & 273 & 2,889 & 4,880 & 5,111 & 5,204 \\
\hline 1992 & & & & & 26.3 & 279 & 2,939 & 4,963 & 5,198 & 5,292 \\
\hline
\end{tabular}

Capacity-Capital stock Regression: Code 0 RSO 0.720

Coefficients: Const 599.7 Stock 8.4

Ave. Capacity/Stock (74-86): 12.1 Ave. Weekly Hours (High) 87.5 Shift factor 1.80 Ave Weekly Hours (Low) 73.6 Shift Factor 2.14 Note: Output, Imvestment, and Capital Stock extrapoleted for 1987 and 1988.

\section{Fumiture and fixtures, n.e.c.}

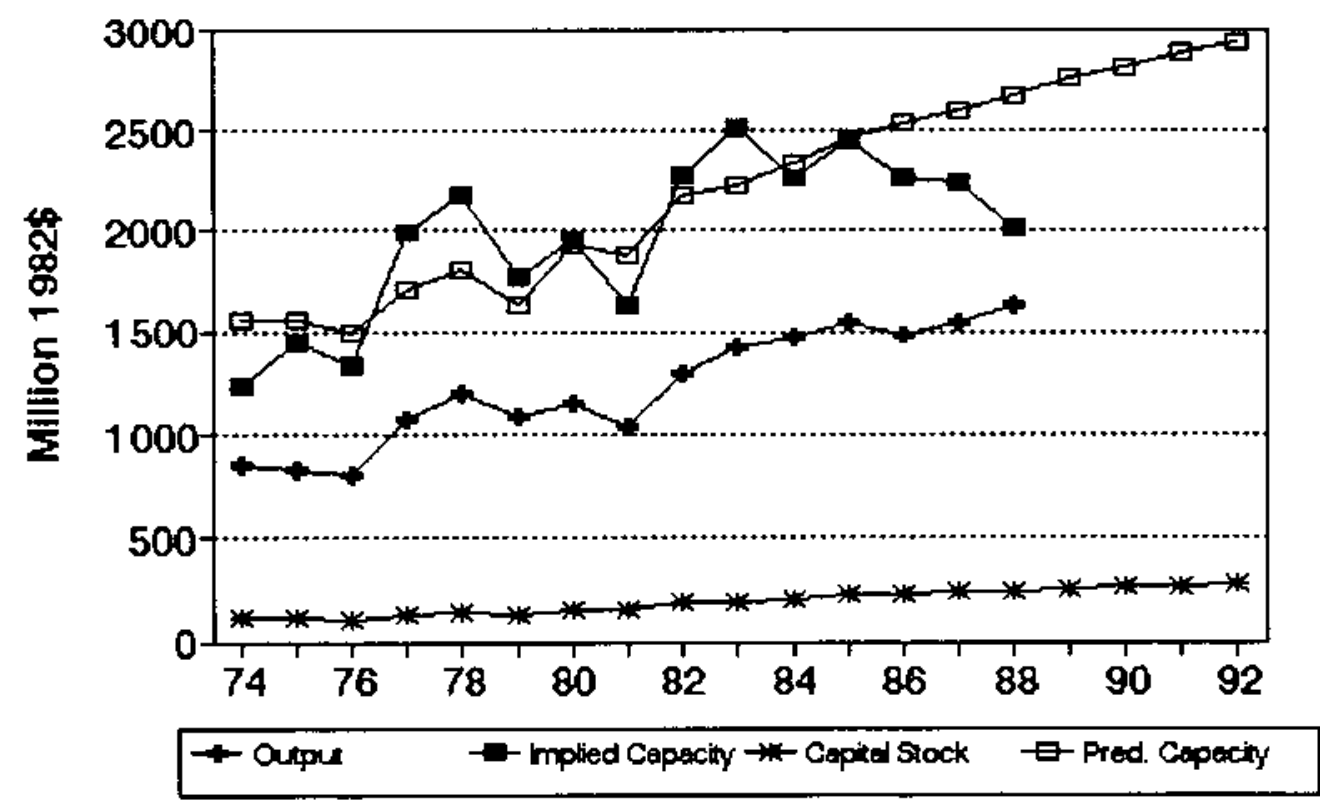




\section{No. 145 sIC 2611 Pulp aills}

MILLLIONS OF 1982 DOLLARS (EXCL. CU RATES)

$\begin{array}{lccccccc} & \text { O4 } & \text { Ann. } & \text { Implied Gross } & \text { Net } & \text { Pred. } & \ldots \ldots \ldots \\ \text { Gross } & \text { CU } & \text { CU } & \text { Prac. } & \text { In- } & \text { Cap. } & \text { Prac. } & \text { Emergency Capacity } \\ \text { Output Rate } & \text { Rate } & \text { Capac. } & \text { vest. } & \text { Stk. } & \text { Capac. } & 3 \text { mon. } 6 \text { mon. }>6 \text { mon. }\end{array}$

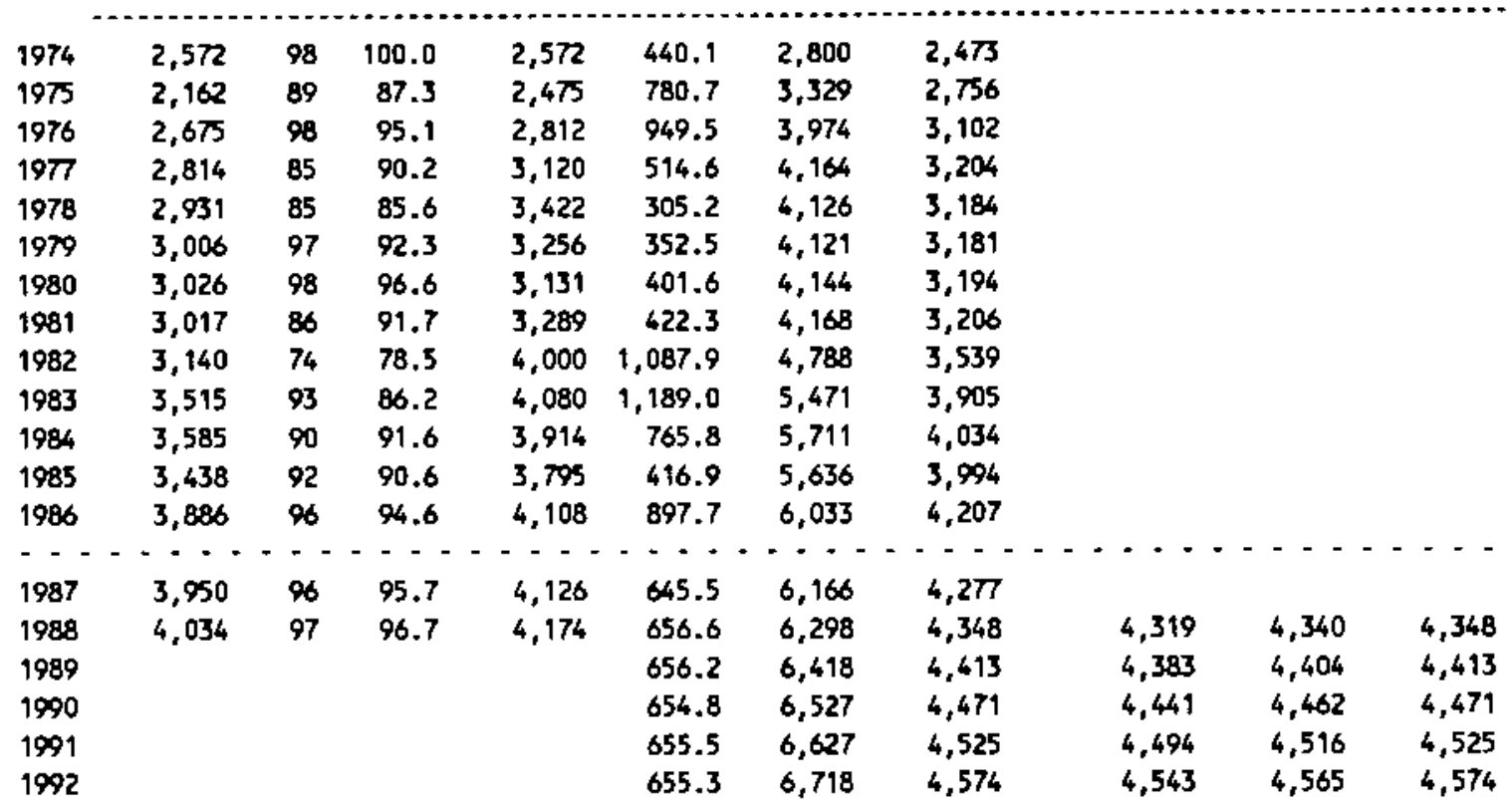

Capecity-Capital stock Regression: Code 0 RSO 0.850 Coefficients: Const 971.3 Stock 0.5

Ave. Capacity/stock $(74-86): 0.8$ Ave. Weekly Hours (High) 168.0 Shift Factor 1.00 Ave Heekly Hours (Low) 167.6 Shift Factor 1.00 Hote: Output, Investment, and Capital Stock extrapolated for 1987 and 1988.

\section{Pulp mills}

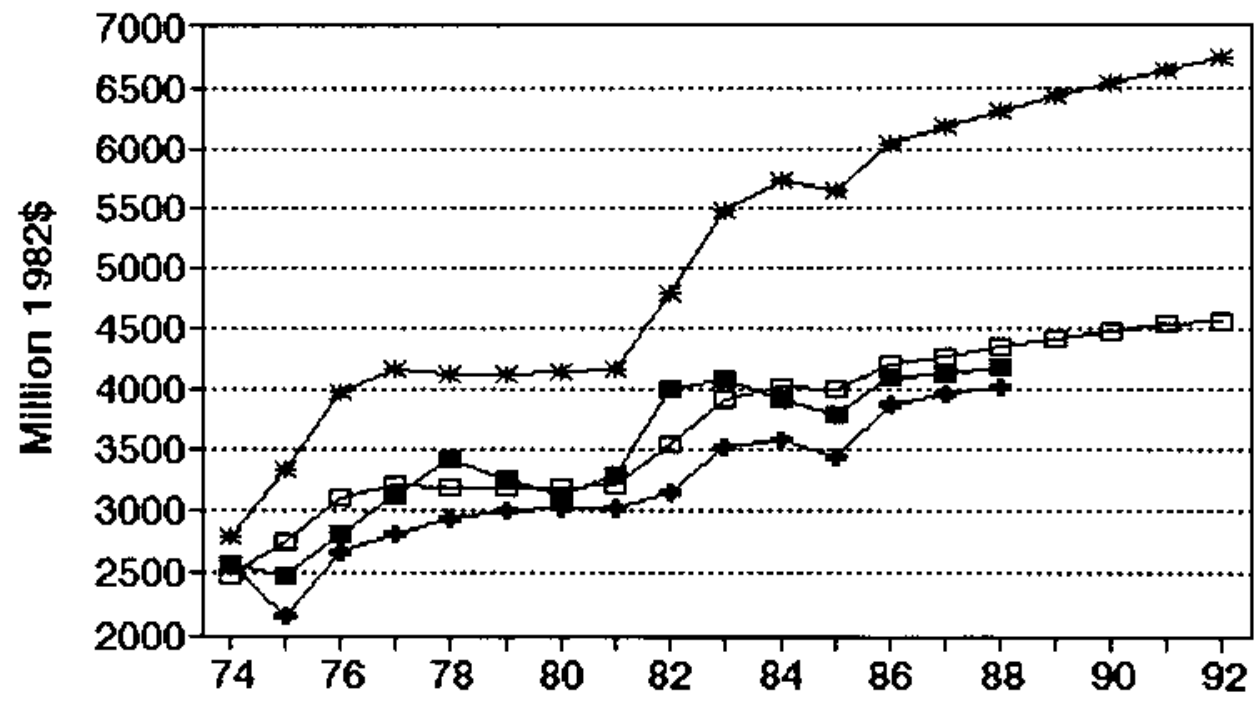

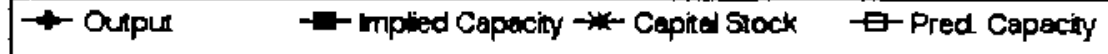




\section{NO. 146 sIC 2621 Paper alls, except building paper}

MILLIONS Of 1982 DOLLARS (EXCL. CU RATES)

$\begin{array}{lccccccc} & 04 & \text { Ann. Implied } & \text { Gross } & \text { Net } & \text { Pred. } & \ldots & -\ldots \\ \text { Gross } & \text { CU } & \text { CU } & \text { Prac. } & \text { In- } & \text { Cap. } & \text { Prac. } & \text { Emergency Capecity } \\ \text { Output Rate } & \text { Rate } & \text { Capac. } & \text { vest. } & \text { Stk. } & \text { Capac. } & 3 \text { mon. } 6 \text { mon. }>6 \text { mon. }\end{array}$

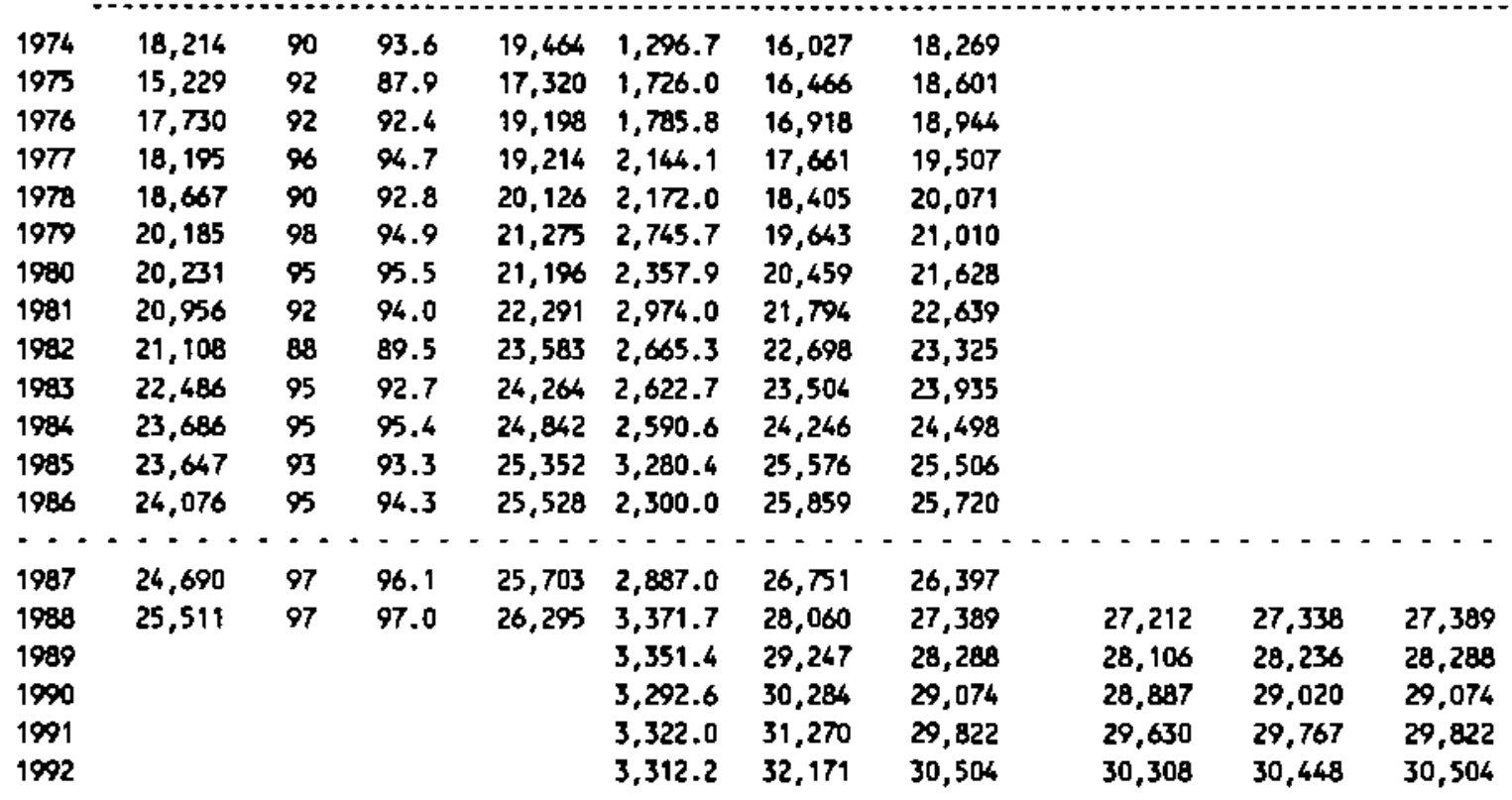

Capacity-Capital Stock Regression: Code 0 RSQ 0.950

Coefficients: Const 6121.6 stock 0.8

Ave. Capacity/Stock (74-86): 1.1 Ave. Weekly Hours (High) 165.6 Shift Factor 1.00 Ave Weekly Hours (Low) 165.6 Shift Factor 1.00 Wote: Output, Imvestment, and Capital stock extrapolated for 1987 and 1988.

\section{Paper mills, except building paper}

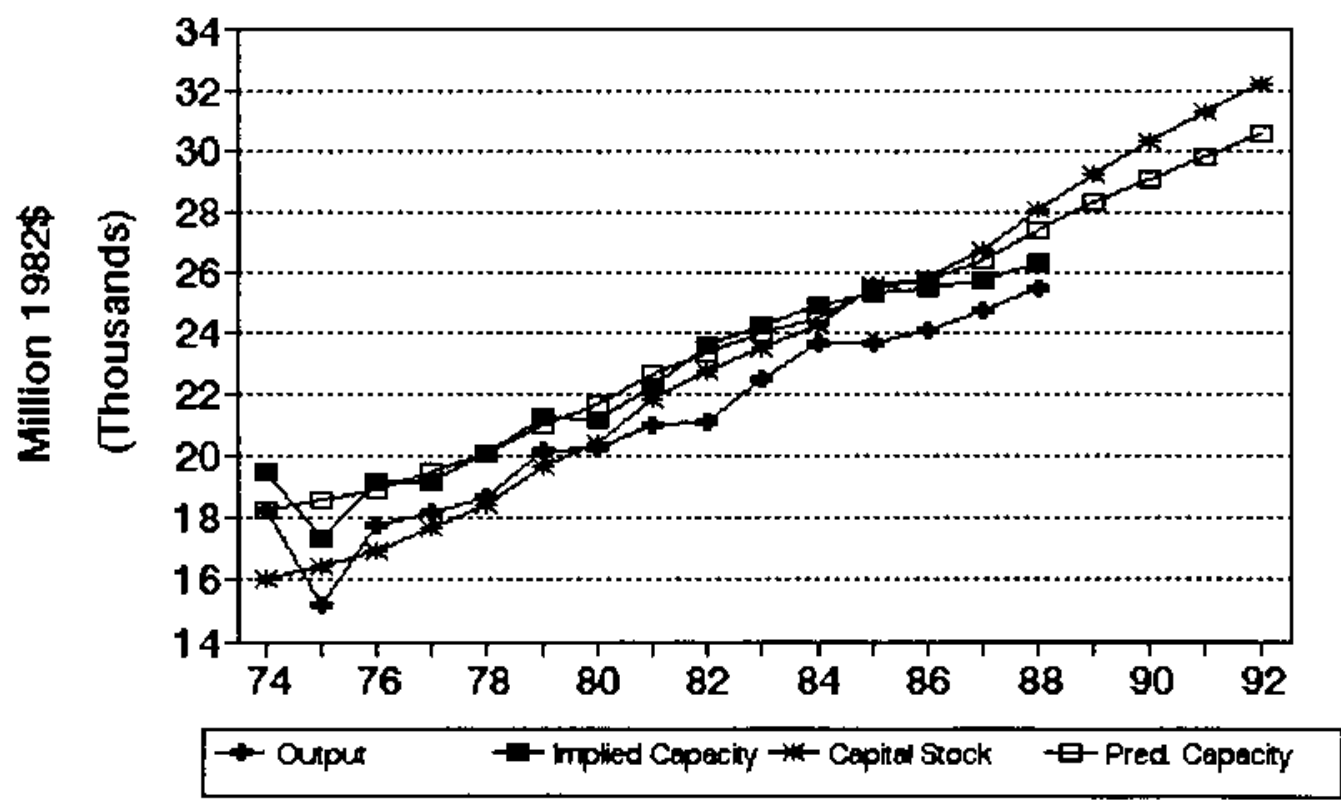


No. 147 SIC 2631 Paperboard mills

MILLIONS OF 1982 DOLLARS (EXCL. CU RATES)

$\begin{array}{lccccccc} & \text { Q4 } & \text { Am. } & \text { Implied } & \text { Gross } & \text { Net } & \text { Pred. } & \ldots \\ \text { Gross } & \text { CU } & \text { CU } & \text { Prac. } & \text { In- } & \text { Cap. } & \text { Prac. } & \text { Emergency Capacity } \\ \text { Output Rate } & \text { Rate } & \text { Capac. } & \text { vest. } & \text { Stk. } & \text { Capac. } & 3 \text { mon. } 6 \text { mon. }>6 \text { mon. }\end{array}$

\begin{tabular}{|c|c|c|c|c|c|c|c|c|c|c|}
\hline 1974 & 11,217 & 84 & 90.4 & 12,410 & $1,863,8$ & 11,542 & 11,624 & & & \\
\hline 1975 & 9.183 & 88 & 80.5 & 11,407 & 913.0 & 11,693 & 11,624 & & & \\
\hline 1976 & 9,860 & $8 B$ & 88.6 & 11,123 & $1,018.4$ & 11,939 & 11,624 & & & \\
\hline 1977 & 10,298 & 91 & 90.2 & 11,414 & 1.546 .9 & $12, \infty 67$ & 11,624 & & & \\
\hline 1978 & 10,847 & 93 & 93.1 & 11,646 & $1,335.9$ & 13,148 & 11,624 & & & \\
\hline 1979 & 10,658 & 97 & 95.3 & 11,187 & $1,086.9$ & 13,378 & 11,624 & & & \\
\hline 1980 & 10,729 & 92 & 92.6 & 11,582 & $1,718.0$ & 14,197 & 11,624 & & & \\
\hline 1981 & 10,791 & 85 & 89.2 & 12,098 & 330.5 & 13,609 & 11,624 & & & \\
\hline 1982 & 9,575 & 81 & 82.5 & 11,605 & 672.4 & 13,373 & 11,624 & & & \\
\hline 1983 & 10,238 & $B B$ & 85.9 & 11,915 & 660.9 & 13,062 & 11,624 & & & \\
\hline 1984 & 10,801 & 89 & 89.2 & 12,106 & 696.5 & 12,744 & 11,624 & & & \\
\hline 1985 & 9,767 & 90 & 88.8 & 11,000 & 814.5 & 12,526 & 11,624 & & & \\
\hline 1986 & 10,411 & 96 & 93.8 & 11,094 & 863.6 & 12,327 & 11,094 & & & \\
\hline$\cdots$ & $-\cdot-$ & -- & -- & $-\cdot-$ & $-\cdots$ & $\cdots \cdot$ & $\cdots$ & --- & $\cdots$ & $\cdots$ \\
\hline 1987 & 10,491 & 95 & 95.0 & 11,039 & 665.6 & 11,951 & 11,094 & & & \\
\hline 1988 & 10,596 & 96 & 95.7 & 11,077 & 615.8 & 11,559 & 11,094 & 11,047 & 11,081 & 11,094 \\
\hline 1989 & & & & & 568.0 & 11,153 & 11,094 & 11,047 & 11,081 & 11,094 \\
\hline 1990 & & & & & 520.2 & 10,733 & 11,094 & 11,047 & 11,081 & 11,094 \\
\hline 1991 & & & & & 472.5 & 10,300 & 11,094 & 11,047 & 11,081 & 11,094 \\
\hline 1992 & & & & & 424.7 & 9,856 & 11,004 & 11,047 & 11,081 & 11,094 \\
\hline
\end{tabular}

Capecity-Capital Stock Regression: Code - 1 RSQ 0.110

Coefficients: Const 11624.6 Stock 0.0 D(86-86) -530.3 Ave. Capacity/Stock (74-86): 0.9 Ave. Weekly Hours (High) 165.6 Shift Factor $\{.00$ Ave Ueekly Hours (Low) 165.6 Shift factor 1.00 Note: Output, Investment, and Capital Stock extrepolated for 1987 and 1988.

\section{Paperboard mills}

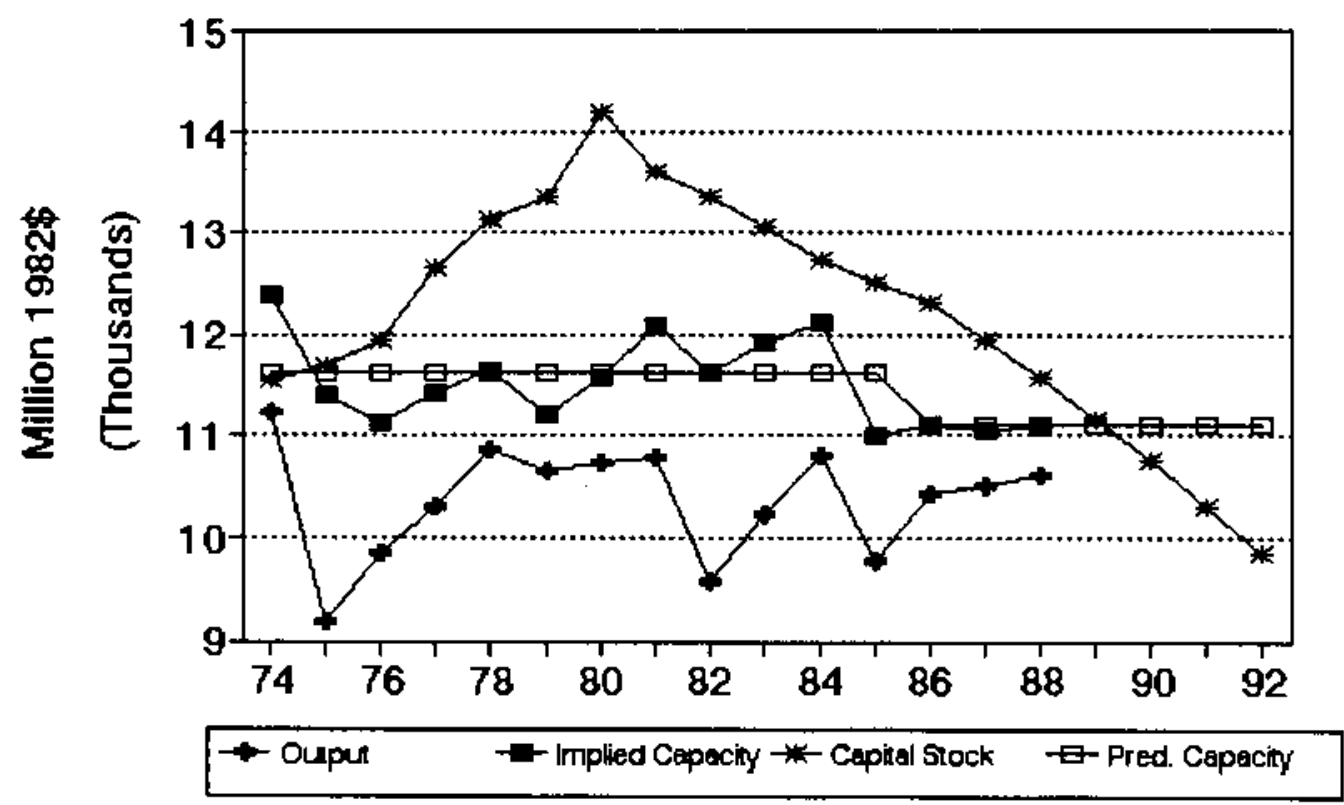




\section{No. 148 sIC 2641 Paper coating and glazing}

MILLIONS OF 1982 DOLLARS (EXCL. CU RATES)

$\begin{array}{lccccccc} & \text { Q4 } & \text { Ann. Implied Gross } & \text { Net } & \text { Pred. } & -\ldots \ldots \ldots \\ \text { Gross } & \text { CU } & \text { CU } & \text { Prac. } & \text { In- } & \text { Cap. } & \text { Prac. } & \text { Emergency Capacity } \\ \text { Output Rate } & \text { Rate } & \text { Capac. } & \text { vest. } & \text { Stk. } & \text { Capac. } & 3 \text { mon. } 6 \text { mon. }>6 \text { mon. }\end{array}$

\begin{tabular}{|c|c|c|c|c|c|c|c|c|c|c|}
\hline 1974 & 4,946 & 83 & 87.3 & 5,664 & 138.2 & 1,354 & 6,031 & & & \\
\hline 1975 & 4,190 & 70 & 71.2 & 5,886 & 177.3 & 1,427 & 6,322 & & & \\
\hline 1976 & 4.785 & 73 & 72.2 & 0,625 & 22.5 & 1,349 & 6,010 & & & \\
\hline 1977 & 5,052 & 74 & 73.8 & 6,843 & 241.5 & 1,473 & 6,506 & & & \\
\hline 1978 & 5,263 & 82 & 79.5 & 6,595 & 102.6 & 1,454 & 6,432 & & & \\
\hline 1979 & 5,362 & 73 & 76.3 & 7,032 & 336.9 & 1,651 & 7,217 & & & \\
\hline 1980 & 5,127 & $\pi$ & 74.8 & 6,852 & 243.2 & 1,746 & 7,596 & & & \\
\hline 1981 & 5,272 & 75 & 76.7 & 6.875 & 145.9 & 1,746 & 7,595 & & & \\
\hline 1982 & 5,473 & 74 & 74.4 & 7,358 & 197.0 & 1,793 & 7,784 & & & \\
\hline 1983 & 5,768 & 85 & 81.2 & 7,106 & 82.8 & 1,730 & 7,532 & & & \\
\hline 1984 & 6,041 & 78 & 81.0 & 7,458 & $(27.4)$ & 1,572 & 6,901 & & & \\
\hline 1985 & 6,553 & $\pi$ & 76.9 & 8,525 & 441.9 & 1,863 & 8,063 & & & \\
\hline 1986 & 7,181 & 78 & 77.7 & 9,242 & 148.3 & 1,866 & 8,073 & & & \\
\hline$\because \cdots$ & $\begin{array}{c}--- \\
7332\end{array}$ & $\begin{array}{l}-- \\
82\end{array}$ & $\begin{array}{l}-\cdots \\
80.3\end{array}$ & $\because \cdots$ & $\because 250$. & $7 .-7$ & $\begin{array}{r}--. \\
8.483\end{array}$ & $\cdot \cdot \cdot$ & -- & $-\cdots$ \\
\hline 1988 & 7,534 & 82 & 82.0 & 9,185 & 316.5 & 2,129 & 9,121 & $10,70 B$ & 10,942 & 11,037 \\
\hline 1989 & & & & & 313.8 & 2,273 & 9,698 & 11,385 & 11,635 & 11,735 \\
\hline 1990 & & & & & 305.8 & 2,398 & 10,198 & 11,971 & 12,234 & 12,339 \\
\hline 1991 & & & & & 309.8 & 2,518 & 10,673 & 12,529 & 12,804 & 12,915 \\
\hline 1992 & & & & & 308.5 & 2,626 & 11,106 & 13,037 & 13,323 & 13,438 \\
\hline
\end{tabular}

Capecity-Capital stock Regression: Code i RSO 0.620

Coefficients: Const 629.9 stock 4.0

Ave. Capecity/Stock $(74-86): 4.4$ Ave. Heekly Hours (High) 131.0 Shift Factor 1.21 Ave Ueekly Hours (Low) 128.8 Shift Factor 1.23 Note: Output, Investment, and Capital Stock extrapolated for 1987 and 1988.

\section{Paper coating and glazing}

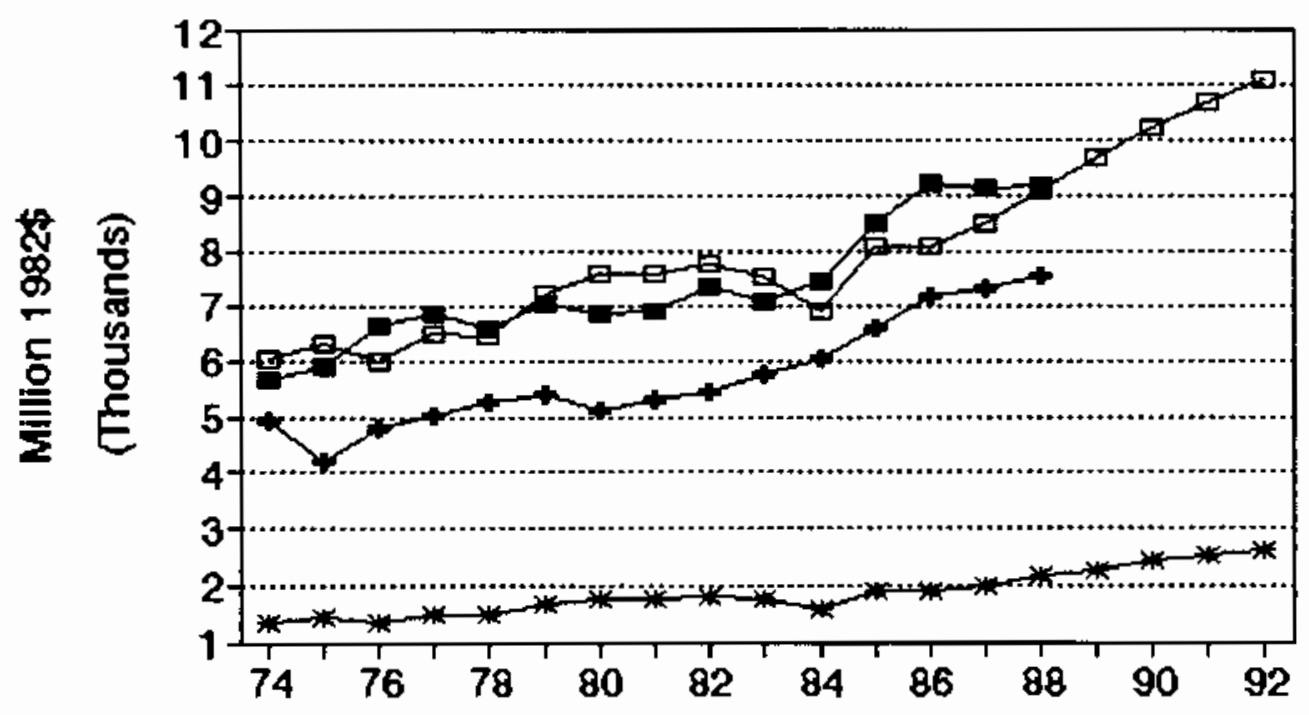

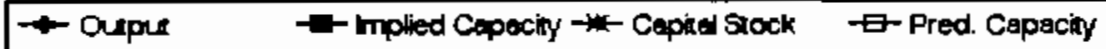




\section{No. 149 SIC 2642 Envelopes}

MILLIOWS OF 1982 DOLLARS (EXCL. CU RATES)

$\begin{array}{lccccccc} & \text { Q4 } & \text { Ann. } & \text { Implied } & \text { Gross } & \text { Net } & \text { Pred. } & \ldots \ldots \ldots \\ \text { Gross } & \text { CU } & \text { CU } & \text { Prac. } & \text { In- } & \text { Cap. } & \text { Prac. } & \text { Emergency Capacity } \\ \text { Output } & \text { Rate } & \text { Rate } & \text { Capac. } & \text { vest. } & \text { Stk. } & \text { Capac. } & 3 \text { mon. } 6 \text { mon. }>6 \text { mon. }\end{array}$

\begin{tabular}{|c|c|c|c|c|c|c|c|c|c|c|}
\hline 1974 & 1,441 & 82 & 87.5 & 1,647 & 66.7 & 438 & 1,728 & & & \\
\hline 1975 & 1,244 & 67 & 68.0 & 1,829 & 50.8 & 447 & 1,787 & & & \\
\hline 1976 & 1,311 & 77 & 73.7 & 1,778 & 27.6 & 436 & 1,711 & & & \\
\hline 1977 & 1,474 & $\pi$ & 76.0 & 1,939 & 59.5 & 454 & 1,830 & & & \\
\hline 1978 & 1,608 & $\pi$ & 78.1 & 2,057 & 41.1 & 456 & 1,839 & & & \\
\hline 1979 & 1,643 & 98 & 90.7 & 1,812 & 41.9 & 459 & 1,860 & & & \\
\hline 1980 & 1,655 & 95 & 95.0 & 1,742 & 71.2 & 490 & 2,060 & & & \\
\hline 1981 & 1,678 & 90 & 93.3 & 1,798 & 28.2 & 479 & 1,989 & & & \\
\hline 1982 & 1,789 & 91 & 90.6 & 1,974 & 64.3 & 501 & 2,133 & & & \\
\hline 1983 & 1,881 & 90 & 91.0 & 2,067 & 33.0 & 492 & 2,073 & & & \\
\hline 1984 & 2,019 & 95 & 93.7 & 2,155 & 50.3 & 500 & 2,125 & & & \\
\hline 1985 & 2,026 & 84 & 87.4 & 2,319 & 60.3 & 519 & 2,244 & & & \\
\hline 1986 & 2,173 & 87 & 86.0 & 2,528 & 44,9 & 522 & 2,266 & & & \\
\hline$\cdots$ & $\ldots$ & $\cdot$ & $\cdots$ & $\because \cdots$ & $\cdots$ & $\cdots$ & $-\cdots$ & $-\cdot-$ & $\cdots$ & $\cdot \cdot$ \\
\hline 1987 & 2,231 & 90 & 88.6 & 2,518 & 57.0 & 538 & 2,365 & & & \\
\hline 1988 & 2,307 & 87 & 88.2 & 2,618 & 64.7 & 559 & 2,504 & 3,507 & 3,586 & 3,631 \\
\hline 1989 & & & & & 64.4 & 579 & 2,631 & 3,686 & 3,77 & 3,815 \\
\hline 1990 & & & & & 63.5 & 596 & 2,742 & 3,839 & 3,936 & 3,975 \\
\hline 1991 & & & & & 63.9 & 613 & 2,846 & 3,986 & 4,086 & 4,127 \\
\hline 1992 & & & & & 63.8 & 627 & 2,942 & 4,119 & 4,223 & 4,265 \\
\hline
\end{tabular}

Capacity-Capital Stock Regression: Code 0 RSO 0.580

Coefficients: Const - 1083.8 Stock $6.4 \quad$ Ave. Capacity/Stock (74-86): 4.1 Ave. Weekly Hours (High) 108.6 Shift factor 1.45 Ave Weekly Hours (Low) 104.8 Shift factor 1.50 Note: Output, Investment, and Capital Stock extrapolated for 1987 and 1988.

\section{Envelopes}

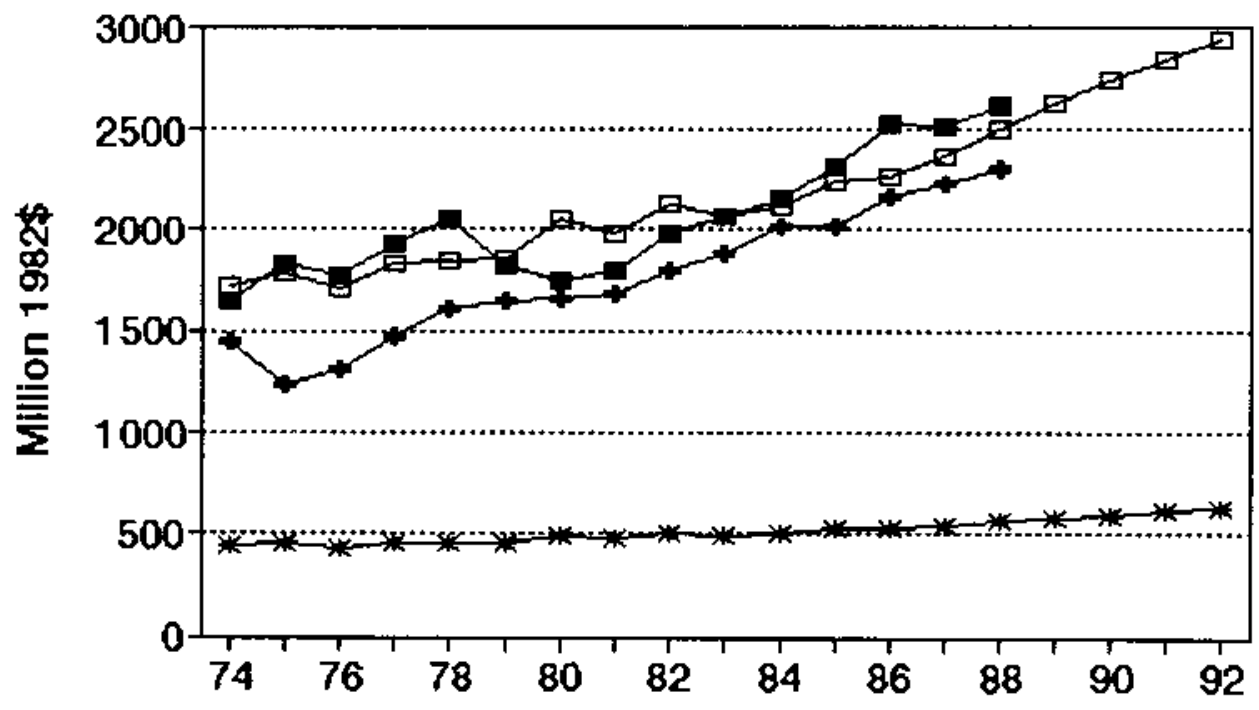




\section{Mo, 150 sIC 2643 Bags, except textile bags}

MILLIONS OF 1982 DOLLARS (EXCL. CU RATES)

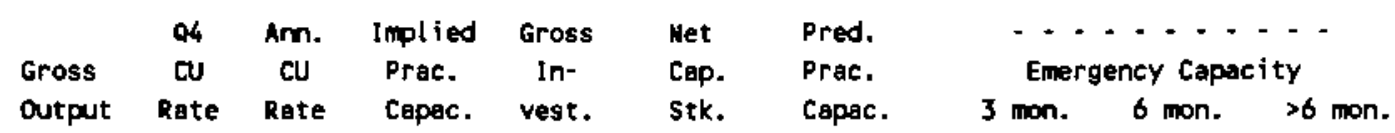

\begin{tabular}{|c|c|c|c|c|c|c|c|c|c|c|}
\hline 1974 & 5,715 & 81 & 82.1 & 6,963 & 125.2 & 1,303 & 6,252 & & & \\
\hline 1975 & 5,266 & 79 & 78.8 & 6,686 & 173.2 & 1,381 & 6,623 & & & \\
\hline 1976 & 5,480 & 84 & 82.2 & 6,664 & 184.1 & 1,458 & 6,907 & & & \\
\hline 1977 & 5,208 & 84 & 84.1 & 6,195 & 65.3 & 1,418 & 6,804 & & & \\
\hline 1978 & 5,386 & 82 & 82.9 & 6,497 & 143.7 & 1,453 & 6,973 & & & \\
\hline 1979 & 5,438 & $\pi$ & 77.6 & 7,008 & 114.1 & 1,456 & 6,986 & & & \\
\hline 1980 & 5,505 & 83 & 79.8 & 6,898 & 175.8 & 1,513 & 7,259 & & & \\
\hline 1981 & 5,455 & 71 & 73.7 & 7,203 & 182.2 & 1,567 & 7,520 & & & \\
\hline 1982 & 5,035 & 69 & 69.8 & 7,218 & 100.6 & 1,537 & 7,373 & & & \\
\hline 1983 & 5,523 & 78 & 74.7 & 7,394 & 104.9 & 1,509 & 7,241 & & & \\
\hline 1984 & 5,439 & 68 & 71.8 & 7,571 & 152.2 & 1,525 & 7,321 & & & \\
\hline 1985 & 5,554 & 68 & 67.9 & 8,182 & 180.8 & 1,568 & 7,524 & & & \\
\hline 1986 & 5,963 & 7 & 73.6 & 8,098 & 174.6 & 1,605 & 7,702 & & & \\
\hline$\because$ & $\cdots \cdot$ & $=-$ & -- & --- & -- & $-\cdots$ & --- & $\cdots$ & $\cdots$ & $\cdots$ \\
\hline 1987 & 6,010 & 81 & 79.4 & 7,565 & 176.7 & 1,642 & 7,880 & & & \\
\hline 1988 & 6,072 & 84 & 82.9 & 7,326 & 208.4 & 1,707 & 8,195 & 8,307 & 8,473 & 8,539 \\
\hline 1989 & & & & & 207.1 & 1,765 & 8,476 & 8,593 & 8,764 & 8,832 \\
\hline 1990 & & & & & 203.2 & 1,815 & 8,715 & 8,834 & 9,010 & 9,081 \\
\hline 1091 & & & & & 205.1 & 1,862 & 8,942 & 9,064 & 9,244 & 9,317 \\
\hline 1992 & & & & & 204.5 & 1,904 & 9,145 & 9,271 & 9,455 & 9,530 \\
\hline
\end{tabular}

Capacity-Capital stock Regression: Code 1 RSO 0.480

Coefficients: Const -24.5 stock 4.8 .

Ave. Capacity/Stock $(74-86): 4.8$ Ave. Weekly Hours (High) 153.3 Shift Factor 1.04 Ave Weekly Hours (Low) 146.9 Shift Factor 1.09 Note: Output, Investment, and Capital stock extrapolated for 1987 and 1988.

\section{Bags, except textile bags}

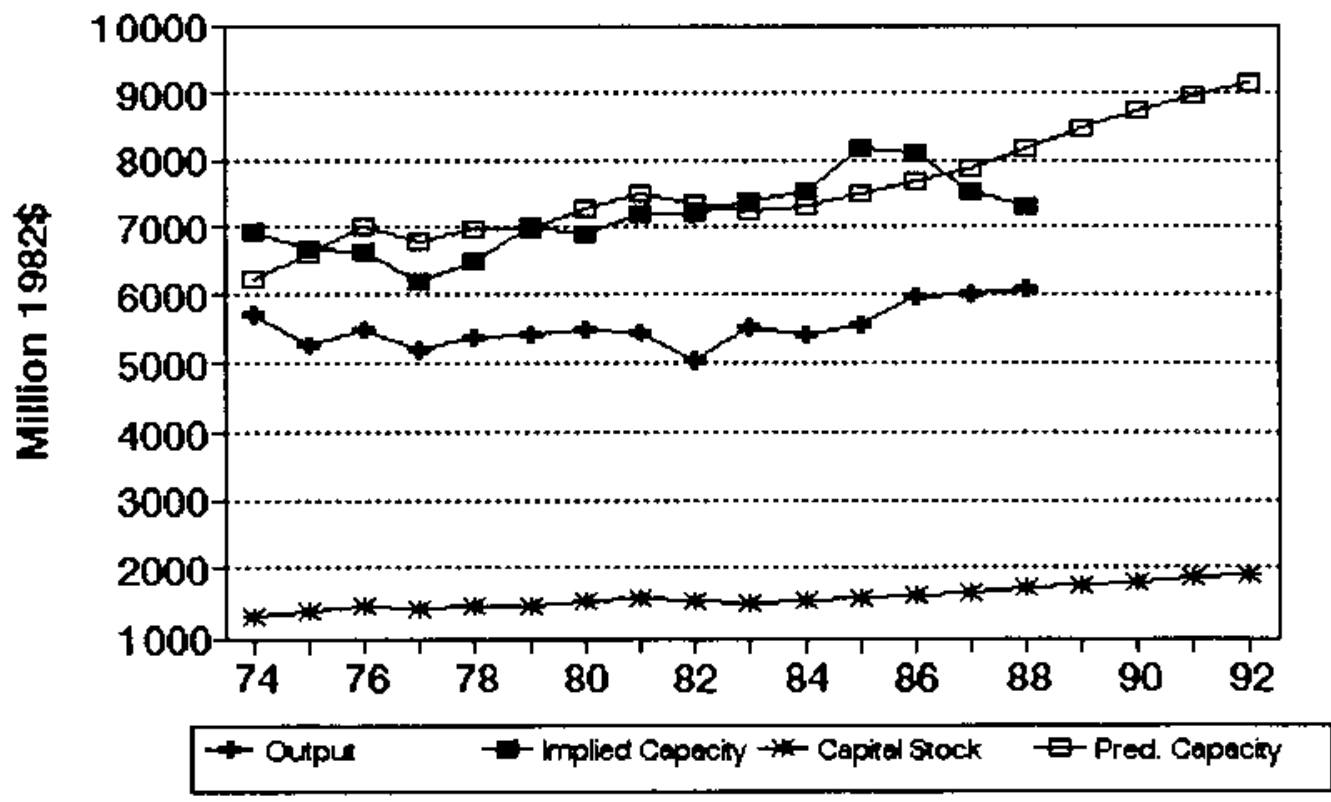




\section{No. 151 SIC 2645 Die-cut paper and board}

MILLIONS OF 1982 DOLLARS (EXCL. CU RATES)

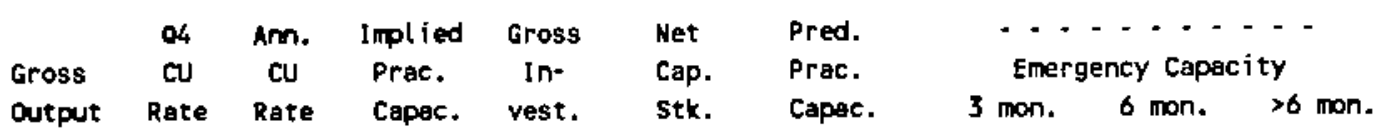

\begin{tabular}{|c|c|c|c|c|c|c|c|c|c|c|}
\hline 1974 & 1,578 & 64 & 69.8 & 2,263 & 18.4 & 277 & 2,406 & & & \\
\hline 1975 & 1,400 & 67 & 60.5 & 2,314 & 32.3 & 287 & 2,442 & & & \\
\hline 1976 & 1,537 & 71 & 70.1 & 2,193 & 15.9 & 281 & 2,422 & & & \\
\hline 1977 & 1,552 & 71 & 71.3 & 2,175 & $(4.0)$ & 257 & 2,335 & & & \\
\hline 1978 & 1,659 & 65 & 68.1 & 2,437 & 28.1 & 264 & 2,359 & & & \\
\hline 1979 & 1,885 & 62 & 63.0 & 2,994 & 28.8 & 271 & 2,384 & & & \\
\hline 1980 & 1,665 & 66 & 63.6 & 2,620 & 44.0 & 291 & 2,456 & & & \\
\hline 1981 & 1,770 & 72 & 71.3 & 2,483 & 34.0 & 299 & 2,483 & & & \\
\hline 1982 & 1,681 & 71 & 71.4 & 2,355 & 22.8 & 295 & 2,470 & & & \\
\hline 1983 & 1,877 & 74 & 73.5 & 2,554 & 28.3 & 296 & 2,473 & & & \\
\hline 1984 & 1,800 & 86 & 82.2 & 2,191 & $(11.6)$ & 261 & 2,348 & & & \\
\hline 1985 & 1,594 & 81 & 82.0 & 1,945 & 50.3 & 285 & 2,012 & & & \\
\hline 1986 & 1,644 & 79 & 79.9 & 2,058 & 20.3 & 279 & 1,991 & & & \\
\hline$\cdots$ & --- & -- & $\because \cdot$ & $\cdots \cdot$ & $-\cdot-$ & -- & $\cdots$ & 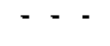 & $\cdots-$ & $-\cdots$ \\
\hline 1987 & 1,656 & 83 & 81.2 & 2,040 & 32.8 & 286 & 2,015 & & & \\
\hline 1988 & 1,671 & 73 & 76.8 & 2,177 & 41.5 & 300 & 2,068 & 3,161 & 3,271 & 3,315 \\
\hline 1989 & & & & & 41.1 & 313 & 2,115 & 3,232 & 3,345 & 3,390 \\
\hline 1990 & & & & & 40.1 & 324 & 2,153 & 3,291 & 3,405 & 3,452 \\
\hline 1991 & & & & & 40.6 & 335 & 2,190 & 3,347 & 3,464 & 3,511 \\
\hline 1992 & & & & & 40.4 & 344 & 2,223 & 3,397 & 3,515 & 3,563 \\
\hline
\end{tabular}

Capecity-Cepital Stock Regression: Code -1 RSa 0.360

Coefficients: Const 1418.0 stock $3.6 \quad D(85-86)-421.5$

Ave. Capacity/stock (74-86): 8.4 Ave. Heekly Hours (High) 98.2 Shift Factor 1.60 Ave Heekly Hours (Low) 95.4 Shift Factor 1.65 Note: Output, Investment, and Capital Stock extrapolated for 1987 and 1988.

\section{Die-cut paper and board}

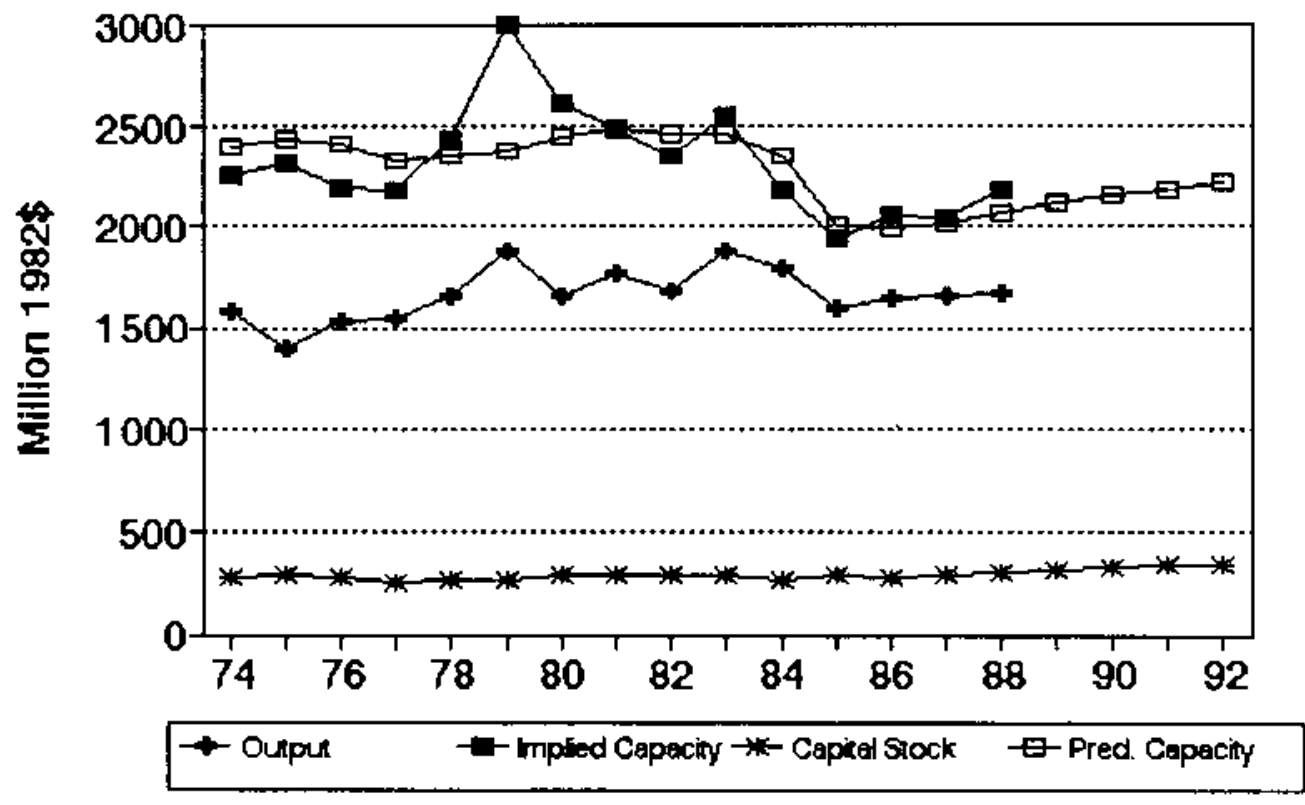


No. 152 BIC 2646 Pressed and solded pulp goods

MILLIONS OF 1982 DOLLARS (EXCL. CU RATES)

\begin{tabular}{|c|c|c|c|c|c|c|c|}
\hline & $\begin{array}{l}\text { B4 } \\
\mathrm{CU}\end{array}$ & $\begin{array}{l}\text { Ann. } \\
\text { CU }\end{array}$ & $\begin{array}{l}\text { Implied } \\
\text { Prac. }\end{array}$ & $\begin{array}{l}\text { Gross } \\
\text { In- }\end{array}$ & $\begin{array}{l}\text { Net } \\
\text { Cap. }\end{array}$ & $\begin{array}{l}\text { Pred. } \\
\text { Prac. }\end{array}$ & $\begin{array}{c}- \\
\text { Emergency Capacity }\end{array}$ \\
\hline utput & Rate & Rate & Capac. & vest. & stk. & Capac. & \\
\hline
\end{tabular}

\begin{tabular}{|c|c|c|c|c|c|c|c|c|c|c|}
\hline 1974 & 364 & 94 & 97.9 & 372 & 20.8 & 318 & 413 & & & \\
\hline $19 \pi$ & 345 & 86 & 85.6 & 403 & 12.9 & 306 & 407 & & & \\
\hline 1976 & 334 & 72 & 77.8 & 429 & 15.8 & 292 & 402 & & & \\
\hline 1977 & 366 & $84 *$ & 79.8 & 458 & 16.2 & 270 & 397 & & & \\
\hline 1078 & 327 & $82 *$ & 83.2 & 393 & 18.1 & 269 & 393 & & & \\
\hline 1979 & 267 & $71 *$ & 75.0 & 356 & 52.2 & 287 & 400 & & & \\
\hline 1980 & 249 & $62 \star$ & 65.0 & 383 & 13.6 & 267 & 392 & & & \\
\hline 1981 & 241 & 64 & 63.9 & 377 & 6.3 & 240 & 381 & & & \\
\hline 1982 & 243 & $54 *$ & 57.7 & 420 & 28.9 & 234 & 370 & & & \\
\hline 1983 & 209 & 59 & 57.3 & 365 & $(42.3)$ & 168 & 351 & & & \\
\hline 1984 & 227 & 79 & 71.8 & 316 & (1.6) & 145 & 342 & & & \\
\hline 1985 & 234 & 77 & 77.3 & 302 & 16.9 & 141 & 340 & & & \\
\hline 1986 & 255 & 69 & $R .1$ & 354 & $(8.1)$ & 116 & 330 & & & \\
\hline$\because 987$ & 248 & -- & 78.8 & $\because 315$ & 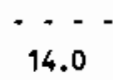 & 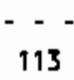 & 329 & - & -- & \\
\hline 1988 & 239 & 88 & 86.9 & 276 & 17.1 & 113 & 329 & 321 & 327 & 329 \\
\hline 1989 & & & & & 17.0 & 114 & 329 & 321 & 327 & 329 \\
\hline 1990 & & & & & 16.6 & 114 & 329 & 321 & 327 & 329 \\
\hline 1991 & & & & & 16.8 & 114 & 328 & 321 & 327 & 329 \\
\hline 1992 & & & & & 16.7 & 114 & 329 & 321 & 327 & 329 \\
\hline
\end{tabular}

Capacity-Capital stock Regression: Code 1 RSO 0.430

Coefficients: Const 282.5 Stock 0.4

Ave. Capacity/Stock $(74-86): 1.6$ Ave. Weekly Hours (High) 168.0 Shift factor 1.00 Ave Weekly Hours (LOH) 168.0 Shift Factor 1.00 * Imputed Note: Output, Imvestment, and Capital Stock extrapolated for 1987 and 1988.

\section{Pressed and molded pulp goods}

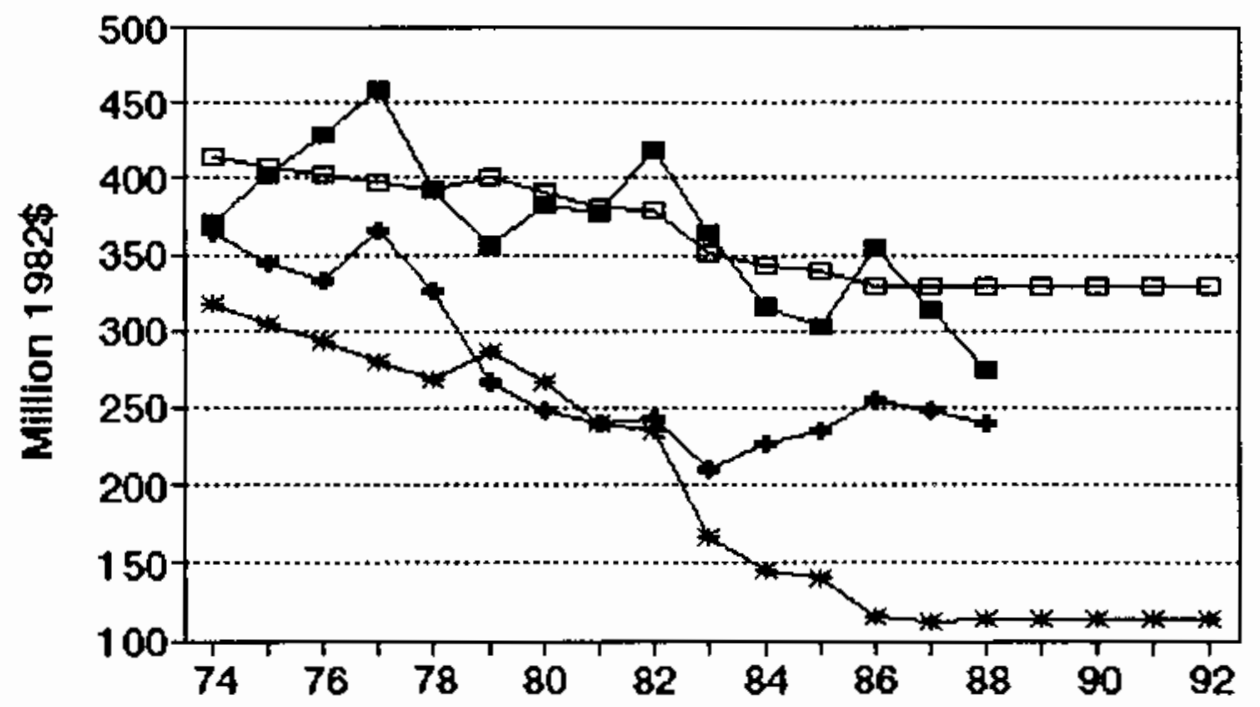

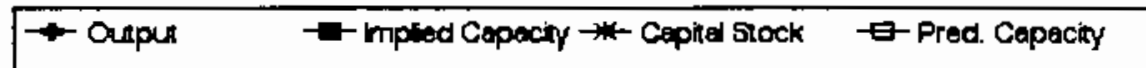


No. 153 SIC 2647 Sanitary paper producte

MILLIONS OF 1982 DOLLARS (EXCL. CU RATES)

$\begin{array}{lccccccc} & \text { Q4 } & \text { Am. Implied } & \text { Gross } & \text { Net } & \text { Pred. } & \ldots & \ldots \ldots \ldots \\ \text { Gross } & \text { CU } & \text { CU } & \text { Prac. } & \text { In- } & \text { Cap. } & \text { Prac. } & \text { Emergency Capacity } \\ \text { Output } & \text { Rate } & \text { Rate } & \text { Capac. } & \text { vest. } & \text { Stk. } & \text { Capac. } & 3 \text { mon. } 6 \text { mon. }>6 \text { mon. }\end{array}$

\begin{tabular}{|c|c|c|c|c|c|c|c|c|c|c|}
\hline 1974 & 7,478 & 94 & 94.0 & 7,956 & 101.6 & 1,407 & 7,236 & & & \\
\hline 1975 & 7,036 & 93 & 93.4 & 7,536 & 134.4 & 1,470 & 7,640 & & & \\
\hline 1976 & 7,223 & 87 & 89.3 & 8,092 & 127.8 & 1,521 & 8,045 & & & \\
\hline 1977 & 7,410 & 94 & 91.4 & 8,110 & 255.7 & 1,690 & 8,450 & & & \\
\hline 1978 & 7,819 & 94 & 94.0 & 8,318 & 209.1 & 1.806 & 8,854 & & & \\
\hline 1979 & 8,353 & 89 & 90.9 & 9,192 & 412.1 & 2,105 & 9,259 & & & \\
\hline 1980 & 8,309 & 85 & 86.5 & 9,606 & 219.3 & 2,201 & 9,664 & & & \\
\hline 1981 & 8,443 & 87 & 86.3 & 9,788 & 138.6 & 2,210 & 10,068 & & & \\
\hline 1982 & 9,049 & 82 & 83.9 & 10,789 & 191.4 & 2,260 & 10,473 & & & \\
\hline 1983 & 9,107 & 86 & 86.5 & 10,778 & 281.4 & 2,391 & 10,878 & & & \\
\hline 1984 & 10,013 & 88 & 87.3 & 11,477 & 160.8 & 2,395 & 11,282 & & & \\
\hline 1985 & 10,126 & 83 & 84.9 & 11,931 & 278.3 & 2,515 & 11,687 & & & \\
\hline 1986 & 10,382 & 88 & 86.1 & 12,055 & 363.3 & 2,707 & 12,092 & & & \\
\hline$\because-$ & $-\cdot-$ & $\cdots$ & $\because-$ & --- & $\cdots$ & $\cdots-$ & $\cdots$ & --- & $-\cdot \cdot$ & ---- \\
\hline 1987 & 10,697 & 86 & 86.8 & 12,331 & 275.0 & 2,811 & 12,496 & & & \\
\hline 1988 & 11,121 & 87 & 86.6 & 12,838 & 318.5 & 2,952 & 12,901 & 13,458 & 13,631 & 13,701 \\
\hline 1989 & & & & & 316.6 & 3,082 & 13,306 & $13,8 B 0$ & 14,059 & 14,131 \\
\hline 1990 & & & & & 311.4 & 3,199 & 13,710 & 14,303 & 14,486 & 14,560 \\
\hline 1991 & & & & & 314.0 & 3,311 & 14,115 & 14,725 & 14,914 & 14.990 \\
\hline 1992 & & & & & 313.1 & 3,415 & 14,520 & 15,147 & 15,341 & 15,420 \\
\hline
\end{tabular}

Capacity-Capital stock Regression: Code -2 RSO 0.960

Coefficients: Const 6830.9 Time 404.7

Ave. Capacity/Stock (74-86): 4.7 Ave. Weekly Hours (High) 150.3 Shift Factor 1.06 Ave Weekly Hours (Low) 146.3 Shift Factor 1.09 Note: Output, Investment, and Capital Stock extrapolated for 1987 and 1988.

\section{Sanitary paper products}

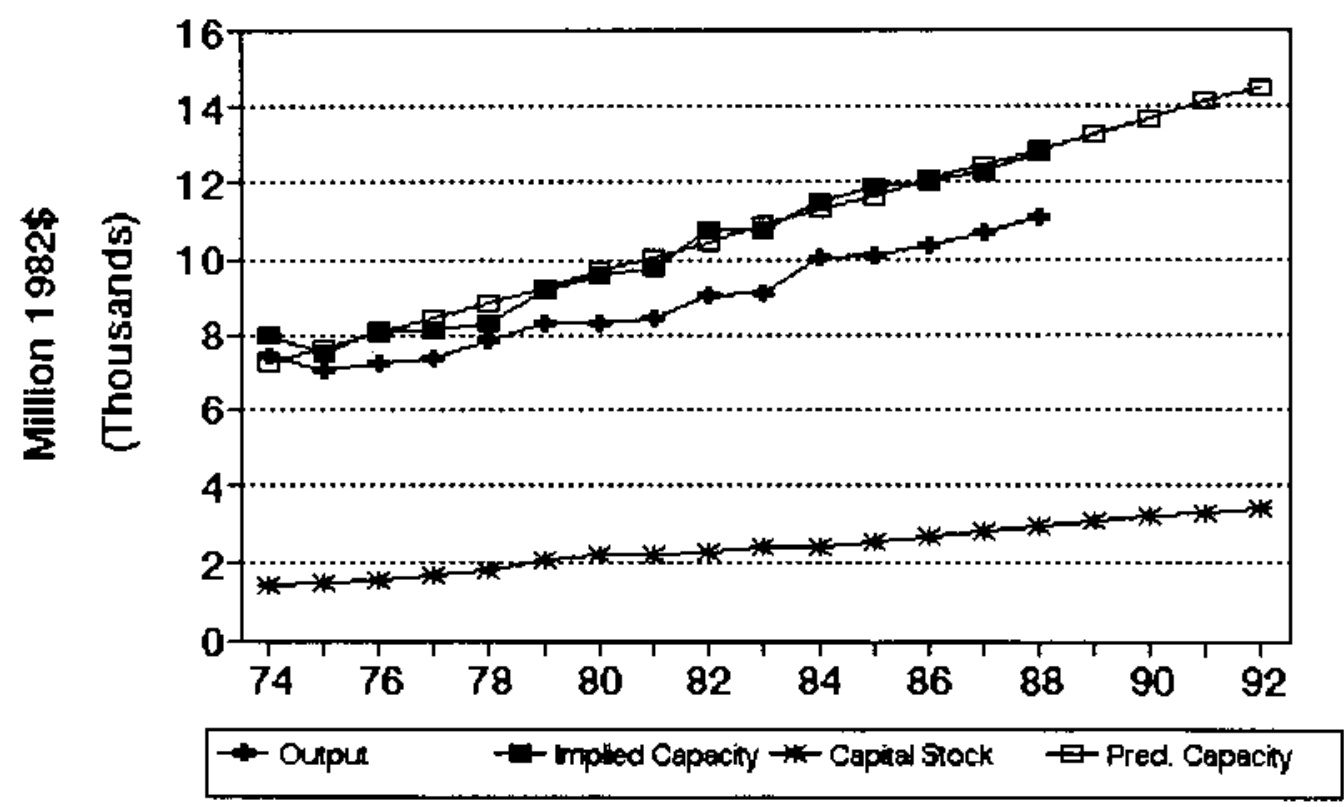




\section{No. 154 SIC 2648 stationery products}

MILLIONS OF 1982 DOLLARS (EXCL. CU RATES)

\begin{tabular}{|c|c|c|c|c|c|c|c|}
\hline & $\begin{array}{l}Q 4 \\
\mathrm{CU}\end{array}$ & Ann. & $\begin{array}{l}\text { Implied } \\
\text { Prac. }\end{array}$ & $\begin{array}{l}\text { Gross } \\
\text { In- }\end{array}$ & $\begin{array}{l}\text { Het } \\
\text { Cap. }\end{array}$ & $\begin{array}{l}\text { Pred. } \\
\text { Prac. }\end{array}$ & Emergency Capeci ty \\
\hline & Rate & Rate & Capac & vest. & & Cange. & \\
\hline
\end{tabular}

\begin{tabular}{|c|c|c|c|c|c|c|c|c|c|c|}
\hline 1974 & 1,071 & $43 \div$ & 53.1 & 2,016 & 0.7 & 201 & 2,080 & & & \\
\hline 1975 & 942 & 49 & 36.4 & 2,586 & 5.5 & 192 & 2,030 & & & \\
\hline 1976 & 1,063 & 59 & 56.4 & $1,8 \mathrm{A3}$ & 21.1 & 197 & 1,981 & & & \\
\hline 1977 & 1,014 & 55 & 57.2 & $1, \pi 7$ & 11.1 & 192 & 1,931 & & & \\
\hline 1978 & 1,086 & 61 & 60.4 & $1,7 \%$ & 28.9 & 202 & 1,882 & & & \\
\hline 1979 & 971 & 59 & 59.4 & 1,636 & 12.9 & 196 & 1,832 & & & \\
\hline 1980 & 949 & 73 & 65.5 & 1,448 & 10.5 & 188 & 1,783 & & & \\
\hline 1981 & 927 & 52 & 63.0 & 1,471 & 9.8 & 179 & 1,733 & & & \\
\hline 1982 & 1,061 & 48 & 49.5 & 2,143 & 42.2 & 199 & 1,684 & & & \\
\hline 1983 & 1,150 & 68 & 61.4 & 1,873 & 30.9 & 206 & 1.634 & & & \\
\hline 1984 & 1,114 & 73 & 72.4 & 1,540 & 22.6 & 207 & 1,584 & & & \\
\hline 1985 & 1,049 & 73 & 71.0 & 1,477 & 31.7 & 218 & 1,535 & & & \\
\hline 1986 & 1,131 & 74 & 73.9 & 1,529 & 29.7 & 227 & 1,485 & & & \\
\hline$\cdots$ & $\cdots$ & $\cdots$ & $\cdots$ & $\cdots$ & $\cdots$ & $\cdots$ & $\cdots$ & $\cdots$ & $\because \cdot$ & 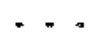 \\
\hline 1987 & 1,135 & 69 & 70.2 & 1,616 & 28.6 & 234 & 1,436 & & & \\
\hline 1988 & 1,141 & 61 & 64.0 & 1,783 & 37.0 & 249 & 1,386 & 1,823 & 1,874 & 1,894 \\
\hline 1989 & & & & & 36.6 & 262 & 1,337 & 1,758 & 1,807 & 1,826 \\
\hline 1990 & & & & & 35.6 & 272 & 1,287 & 1,693 & 1,740 & $1, \pi 59$ \\
\hline 1991 & & & & & 36.1 & 283 & 1,238 & 1,628 & 1,673 & 1,691 \\
\hline 1992 & & & & & 35.9 & 292 & 1,188 & 1,563 & 1,606 & 1,623 \\
\hline
\end{tabular}

Capacity-Capital stock Regression: Code -2 RSQ 0.340

Coefficients: Const 2129.1 Time -49.5

Ave. Capacity/Stock $(74-86): 8.9$ Ave. Weekly Hours (High) 115.5 Shift Factor 1.37 Ave Heekly Hours (Low) 112.5 Shift factor 1.40 * Imputed Note: Output, Investment, and Capital Stock extrapolated for 1987 and 1988.

\section{Stationery products}

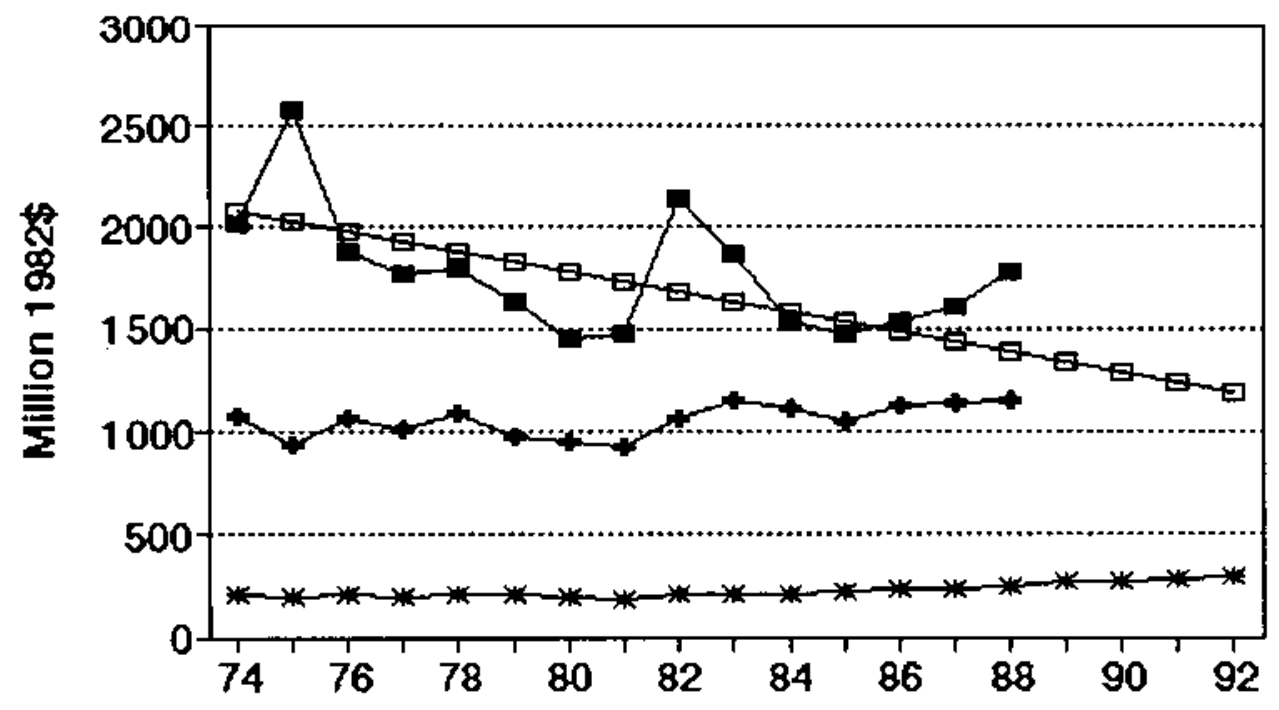

\begin{tabular}{|c|c|c|}
\hline - Oupul & -6- molied Cepeciny -ix- Capided Stock & E-Pred Copecity \\
\hline
\end{tabular}


No. 155 SIC 2649 Converted paper products, n.e.c.

MILLIONS OF 1982 DOLLARS (EXCL. CU RATES)

$\begin{array}{lccccccc} & \text { Q4 } & \text { Am. Implied Gross } & \text { Wet } & \text { Pred. } & \ldots & \ldots \\ \text { Gross } & \text { CU } & \text { CU } & \text { Prac. } & \text { In- } & \text { Cap. } & \text { Prac. } & \text { Emergency Capacity } \\ \text { Output } & \text { Rate } & \text { Rate } & \text { Capac. } & \text { vest. } & \text { Stk. } & \text { Capac. } & 3 \text { mon. } 6 \text { mon. }\end{array}$

\begin{tabular}{|c|c|c|c|c|c|c|c|c|c|c|}
\hline 1974 & 1,837 & 79 & 79.0 & 2,325 & 50.7 & 351 & 2,416 & & & \\
\hline 1975 & 1,700 & 65 & 70.3 & 2,419 & 187.9 & 504 & 2,677 & & & \\
\hline 1976 & 1,952 & 73 & 70.0 & 2,789 & 7.6 & 476 & 2,937 & & & \\
\hline 1977 & 2,596 & 84 & 79.9 & 3,250 & 140.8 & 574 & 3,198 & & & \\
\hline 1978 & 2,753 & 77 & 79.6 & 3,457 & 146.7 & 674 & 3,458 & & & \\
\hline 1979 & 2,484 & 61 & 67.0 & 3,707 & 9.3 & 640 & 3,719 & & & \\
\hline 1980 & 2,584 & 60 & 60.4 & 4,281 & 32.2 & 630 & 3,979 & & & \\
\hline 1981 & 2,684 & 50 & 53.8 & 4,994 & 47.7 & 634 & 4,240 & & & \\
\hline 1982 & 2,377 & 55 & 53.1 & 4,474 & 12.8 & 604 & 4,500 & & & \\
\hline 1983 & 2,605 & 56 & 55.6 & 4,683 & 27.8 & 587 & 4.761 & & & \\
\hline 1984 & 2,799 & 56 & 56.0 & 4,998 & 7.2 & 611 & 5,021 & & & \\
\hline 1985 & 2,855 & $56 *$ & 56.0 & 5,100 & 17.5 & 581 & 5,282 & & & \\
\hline 1986 & 2,941 & $56 \star$ & 56.0 & 5.251 & 48.6 & 581 & 5,542 & & & \\
\hline$\because \cdot$ & $-\cdot-\cdot$ & & $\because \cdot$ & $-\cdot-$ & $\cdots$ & $\therefore$ & $\because \cdots$ & -- & $-\cdots$ & $\cdots$ \\
\hline 1987 & 3,053 & $56 *$ & 56.0 & 5,448 & 29.8 & 563 & 5,803 & & & \\
\hline 1988 & 3,204 & $56 *$ & 56.0 & 5,720 & 26.0 & 543 & 6,063 & 5,795 & 5,986 & 6,063 \\
\hline 1989 & & & & & 22.2 & 521 & 6,324 & 6,043 & 6,243 & 6,324 \\
\hline 1990 & & & & & 18.4 & 496 & 6,584 & 6,292 & 6,500 & 6,584 \\
\hline 1991 & & & & & 14.6 & 470 & 6,845 & 6,541 & 6,758 & 6,845 \\
\hline 1992 & & & & & 10.8 & 442 & 7,105 & 6,790 & 7,015 & 7,105 \\
\hline
\end{tabular}

Capacity-Capital Stock Regression: Code -2 RSO 0.930

Coefficients: Const 2155.4 Time 260.5

Ave. Capacity/Stock $(74-86): 6.9$ Ave. Heekly Hours (High) 1983.0 Shift Factor 1.00 Ave Weekly Hours (Low) 1983.0 Shift factor 1.0 * Imputed Note: Output, Investment, and Capital Stock extrapolated for 1987 and 1988.

\section{Converted paper products, n.e.c.}

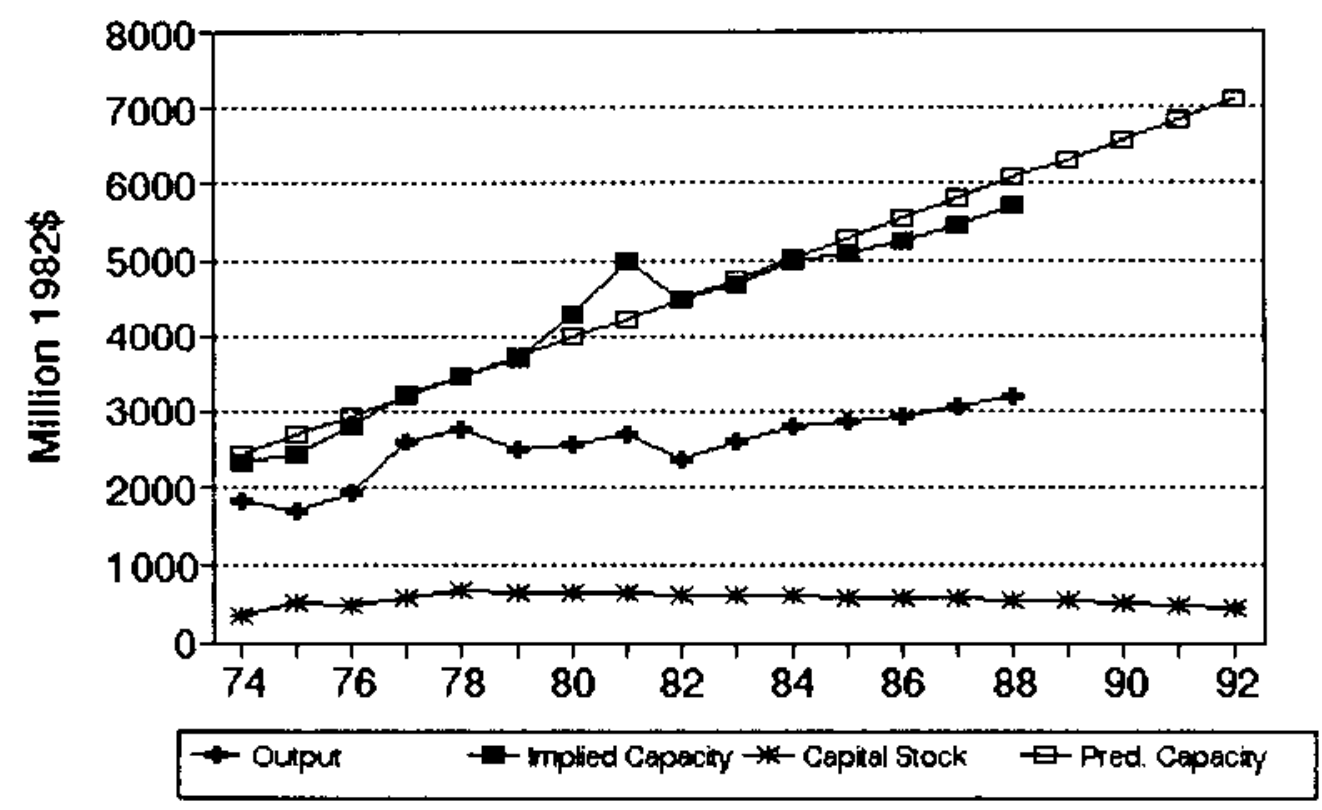




\section{No. 156 sIC 2651 Folding paperboard boxes}

MILLIONS OF 1982 DOLLARS (EXCL. CU RATES)

$\begin{array}{lccccccc} & \text { Q4 } & \text { Ann. } & \text { Implied Gross } & \text { Net } & \text { Pred. } & \ldots & \ldots \\ \text { Gross } & \text { CU } & \text { CU } & \text { Prac. } & \text { In- } & \text { Cap. } & \text { Prac. } & \text { Emergency Capacity } \\ \text { Output } & \text { Rate } & \text { Rete } & \text { Capac. } & \text { vest. } & \text { Stk. } & \text { Capac. } & 3 \text { mon. } 6 \text { mon. }>0 \text { mon. }\end{array}$

\begin{tabular}{|c|c|c|c|c|c|c|c|c|c|c|}
\hline 1974 & 3,620 & 66 & 68.4 & 5,290 & 92.7 & 1,070 & 5,103 & & & \\
\hline 1975 & 3,546 & 74 & 68.6 & 5,167 & 100.1 & 1,081 & 5,125 & & & \\
\hline 1976 & 4,118 & $\pi$ & $\pi .6$ & 5,592 & 268.2 & 1,230 & 6,252 & & & \\
\hline 1977 & 4,704 & $\pi$ & 73.1 & 6,431 & 34.8 & 1,152 & 5,658 & & & \\
\hline 1978 & 5,034 & $\pi$ & 73.4 & 6,862 & 149.3 & 1,182 & 5,893 & & & \\
\hline 1979 & 4,412 & 71 & 71.7 & 6,156 & 88.1 & 1,149 & 5,640 & & & \\
\hline 1980 & 4,328 & 77 & 74.3 & 5,826 & 103.3 & 1,131 & 5,499 & & & \\
\hline 1981 & 3,926 & 82 & 80.9 & 4,856 & 138.9 & 1,141 & 5,581 & & & \\
\hline 1982 & 3,891 & $\pi$ & 78.9 & 4,933 & 119.7 & 1,130 & 5,493 & & & \\
\hline 1983 & 3,902 & $\pi$ & 77.3 & 5,050 & 97.1 & 1,097 & 5,240 & & & \\
\hline 1984 & 4,128 & 78 & 77.9 & 5,300 & 160.8 & 1,126 & 5,466 & & & \\
\hline 1985 & 4,007 & 80 & 78.9 & 5,080 & 127.0 & 1,128 & 5,478 & & & \\
\hline 1986 & 4.191 & 77 & 78.2 & 5,360 & 122.9 & 1,128 & $5,4 \pi$ & & & \\
\hline$\cdots$ & $-\cdots$ & - & -- & $\cdots$ & $\because$ & $\because-$ & $-\cdots$ & -- & $\therefore \cdot$ & -- \\
\hline 1987 & 4,227 & 79 & 78.1 & 5,412 & 139.8 & 1,143 & 5,595 & & & \\
\hline 1988 & 4,274 & 81 & 80.3 & 5,325 & 157.4 & 1.175 & 5,835 & 6,893 & 7,087 & 7,165 \\
\hline 1989 & & & & & 156.7 & 1,202 & 6,042 & 7,138 & 7,339 & 7,420 \\
\hline 1990 & & & & & 154.6 & 1,224 & 6,210 & 7,337 & 7,543 & 7,626 \\
\hline 1991 & & & & & 155.6 & 1,245 & 6,368 & 7,524 & 7,735 & 7,020 \\
\hline 1992 & & & & & 155.3 & 1,263 & 6,506 & 7,686 & 7,902 & 7,989 \\
\hline
\end{tabular}

Capecity-Capital Stock Regression: Code 0 RSO 0.250

Coefficients: Const -3099.7 stock 7.6

Ave. Capacity/Stock $(74-86): 4.9$ Ave. Weekly Hours (High) 129.0 Shift Factor 1.23 Ave Ueekly Hours (Low) 119.0 Shift Factor 1.33 Note: Output, Investment, and Capital stock extrapolated for 1987 and 1988.

\section{Folding paperboard boxes}

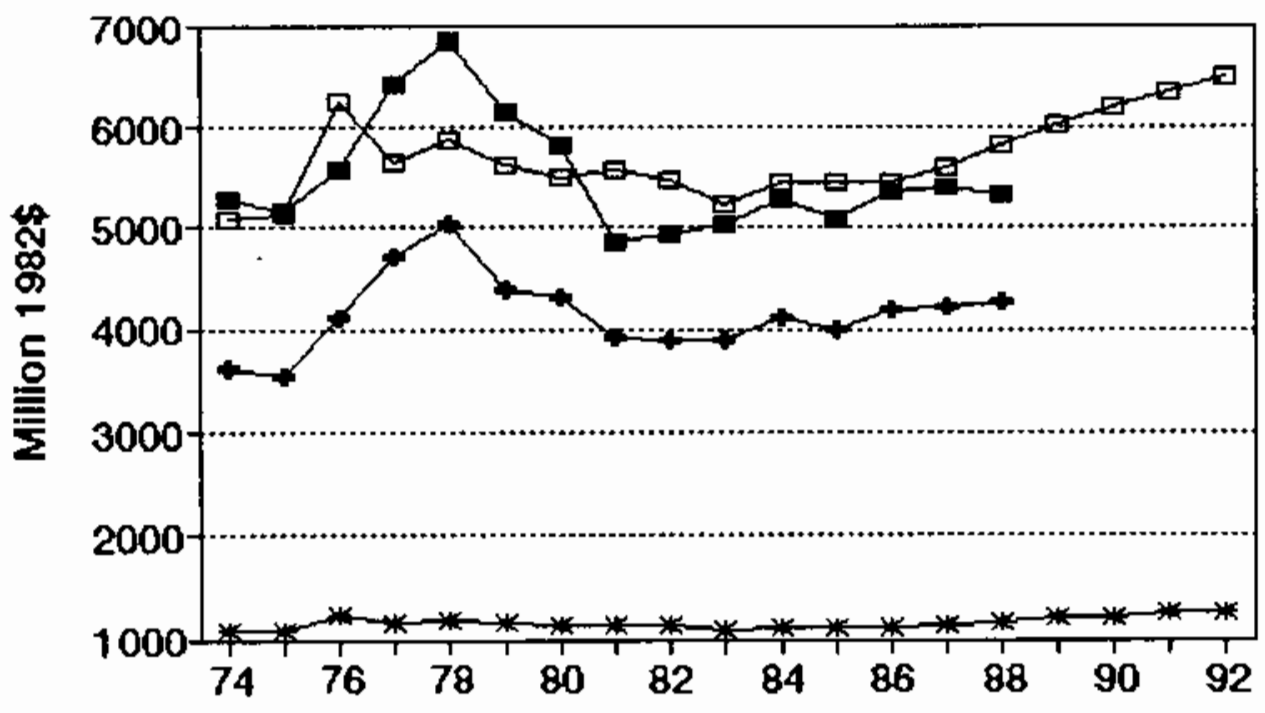

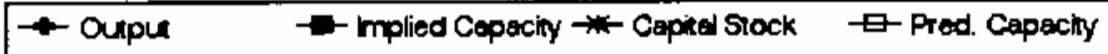




\section{Ko. 157 SIC 2652 set-up paperboard boxes}

MILLIONS OF 1982 DOLLARS (EXCL. CU RATES)

$\begin{array}{lccccccc} & \text { Q4 } & \text { Arn. Implied Gross } & \text { Net } & \text { Pred. } & \ldots \ldots \\ \text { Gross } & \text { CU } & \text { CU } & \text { Prac. } & \text { In- } & \text { Cap. } & \text { Prac. } & \text { Emergency Capacity } \\ \text { Output } & \text { Rate } & \text { Rate } & \text { Capac. } & \text { vest. } & \text { StK. } & \text { Capac. } & 3 \text { mon. } 6 \mathrm{mon} . \quad 76 \mathrm{mon} .\end{array}$

\begin{tabular}{|c|c|c|c|c|c|c|c|c|c|c|}
\hline 1974 & $\pi 7$ & 73 & 81.6 & 928 & 4.4 & 181 & 816 & & & \\
\hline 1975 & 626 & 68 & 62.3 & 1,005 & 22.2 & 185 & 835 & & & \\
\hline 1976 & 693 & $\pi$ & 74.4 & 932 & 4.7 & 171 & 762 & & & \\
\hline $19 \pi$ & 580 & 84 & 81.9 & 709 & 19.7 & 175 & 786 & & & \\
\hline 1978 & 598 & 90 & 89.0 & 672 & 19.5 & 178 & 801 & & & \\
\hline 1979 & 560 & 71 & 7.9 & 719 & 7.3 & 170 & 758 & & & \\
\hline 1980 & 512 & 73 & 70.9 & 722 & 14.4 & 168 & 749 & & & \\
\hline 1981 & 513 & $\pi$ & 76.4 & 672 & 10.3 & 162 & 718 & & & \\
\hline 1982 & 427 & 74 & 74.6 & 574 & $(25.8)$ & 125 & 528 & & & \\
\hline 1983 & 560 & 76 & 76.2 & 735 & 24.0 & 136 & 583 & & & \\
\hline 1984 & 433 & 79 & 78.7 & 551 & 44.7 & 163 & 724 & & & \\
\hline 1985 & 440 & 82 & 79.8 & 552 & 3.4 & 151 & 661 & & & \\
\hline 1986 & 497 & 88 & 85.9 & 579 & 8.8 & 144 & 627 & & & \\
\hline 1987 & $\begin{array}{c}\cdots 83 \\
4 . \cdots\end{array}$ & 89 & 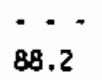 & $-7 \cdot-\cdot$ & 14.6 & $\because-\overline{1}$ & 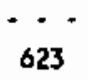 & $\cdots-$ & $\cdots$ & $-\cdots$ \\
\hline 1988 & 465 & 91 & 90.3 & 515 & 17.4 & 146 & 635 & 1,644 & 1,732 & 1,767 \\
\hline 1989 & & & & & 17.3 & 148 & 644 & 1,669 & 1,758 & 1,793 \\
\hline 1990 & & & & & 16.9 & 149 & 651 & 1,687 & 1,776 & 1,812 \\
\hline 1991 & & & & & 17.1 & 150 & 658 & 1,705 & 1,795 & 1,831 \\
\hline 1992 & & & & & 17.1 & 152 & 664 & 1,720 & 1,811 & 1,848 \\
\hline
\end{tabular}

Capacity-Capital Stock Regression: Code 1 RSO 0.390

Coefficients: Const -113.9 stock 5.1

Ave. Capecity/Stock $(74-86): 4.4$ Ave. Heekly Hours (High) 56.6 Shift Factor 2.78 Ave Weekly Hours (Low) 46.0 Shift Factor 3.43 Note: Qutput, Investment, and Capital stock extrapolated for 1987 and 1988.

\section{Set-up paperboard boxes}

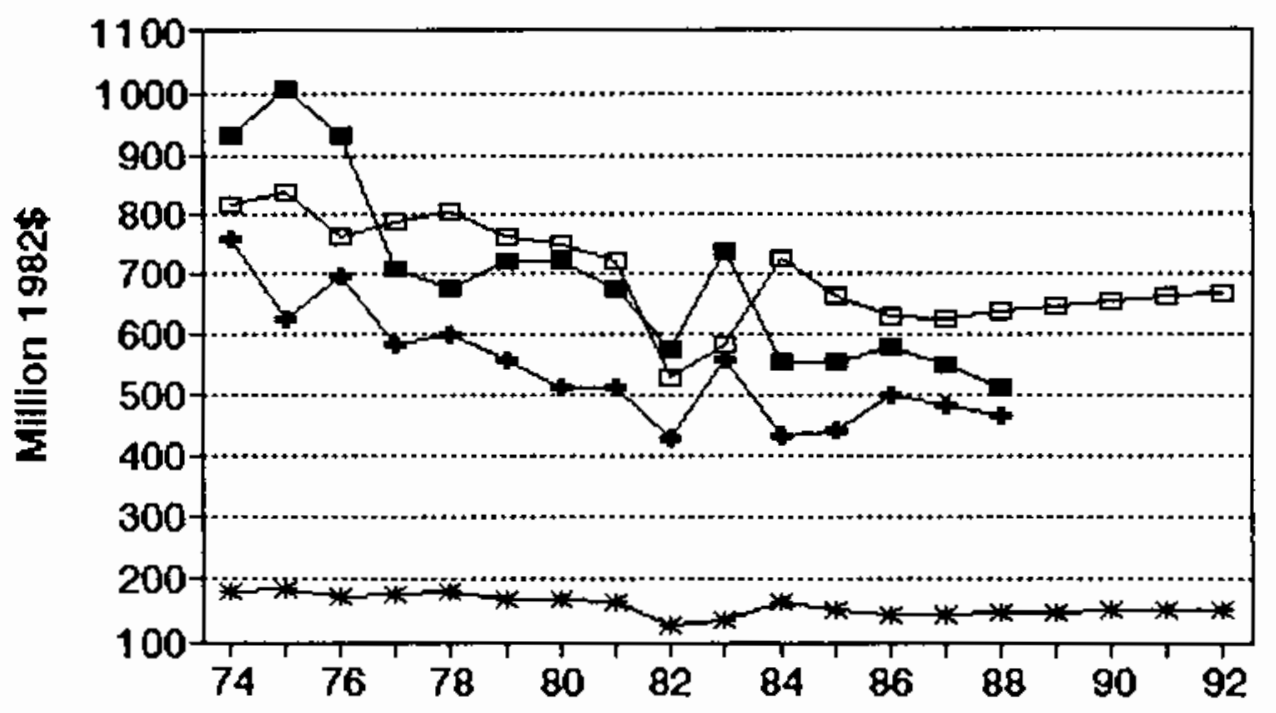

$\rightarrow$ - Outpu $\rightarrow$ - mplied Cepecin $\rightarrow$ - Capted Siock $\quad$-Pred. Cepecity


No. 158 sIC 2653 Corrugated and solid fiber boxes

$\begin{array}{lccccccc} & \text { a4 } & \text { Ann. Implied } & \text { Gross } & \text { Net } & \text { Pred. } & \ldots . \\ \text { Gross } & \text { cu } & \text { CU } & \text { Prac. } & \text { In- } & \text { Cap. } & \text { Prac. } & \text { Emergency Capacity } \\ \text { Output } & \text { Rate } & \text { Rate } & \text { Capac. } & \text { vest. } & \text { Stk. } & \text { Capac. } & 3 \text { mon. } 6 \text { mon. }>6 \text { mon. }\end{array}$

\begin{tabular}{|c|c|c|c|c|c|c|c|c|c|c|}
\hline 1974 & 9,840 & 67 & 67.3 & 16,615 & 92.3 & 2,833 & 13,896 & & & \\
\hline 1975 & 8,797 & 73 & 70.4 & 12,490 & 564.9 & 3,150 & 14,520 & & & \\
\hline 1976 & 9,657 & 69 & 70.5 & 13,691 & 271.5 & 3,160 & 14,540 & & & \\
\hline 1977 & 10,612 & 73 & 71.5 & 16,839 & 312.2 & 3,201 & 14,622 & & & \\
\hline 1978 & 11,170 & 78 & 76.2 & 16,664 & 392.5 & 3,305 & 14,825 & & & \\
\hline 1979 & 10,899 & 70 & 73.0 & 14,933 & 364.7 & 3,364 & 14,943 & & & \\
\hline 1980 & 10,430 & 72 & 71.2 & 14,652 & 296.0 & 3,362 & 14,939 & & & \\
\hline 1981 & 10,862 & 69 & 70.2 & 15,472 & 228.8 & 3,282 & 14,781 & & & \\
\hline 1982 & 10,578 & 70 & 69.6 & 15,192 & 361.3 & 3,313 & 14,843 & & & \\
\hline 1983 & 11,660 & 74 & 72.5 & 16,077 & 276.2 & 3,250 & 14,717 & & & \\
\hline 1984 & 12,407 & 73 & 73.4 & 16,901 & 433.1 & 3,336 & 17,391 & & & \\
\hline 1985 & 11,943 & 67 & 69.2 & 17,257 & 359.2 & 3,350 & 17,418 & & & \\
\hline 1986 & 12,605 & 71 & 69.5 & 18,135 & 376.8 & 3,384 & 17,684 & & & \\
\hline$\because-$ & $-\cdots$ & $\cdots$ & $=-$ & $-\cdot-$ & $\cdots$ & $\cdots-$ & $-\cdots$ & $=-\cdot$ & $--\cdot$ & $\cdot \cdot \cdot$ \\
\hline 1987 & 12,832 & 65 & 67.2 & 19,085 & 389.3 & 3,427 & 17,569 & & & \\
\hline 1988 & 13,132 & 74 & 70.6 & 18,594 & 444.2 & 3,520 & 17,754 & 23,094 & 23,831 & 24,128 \\
\hline 1989 & & & & & 441.9 & 3,602 & 17,915 & 23,304 & 24,047 & 24,347 \\
\hline 1990 & & & & & 435.2 & 3,669 & 18,047 & 23,475 & 24,224 & 24,526 \\
\hline 1991 & & & & & 438.5 & 3,732 & 18,172 & 23,637 & 26,391 & 24,695 \\
\hline 1992 & & & & & 437.4 & 3,788 & 18,281 & 23,780 & 24,539 & 24,844 \\
\hline
\end{tabular}

Capacity-Capital Stock Regression: Code - 1 RSO 0.680

Coefficients: Const 8310.7 stock $2.0 \quad 0(84-86) 2503.2$

Ave. Capacity/Stock $(74-86): 4.7$ Ave. Weekly Hours (High) 116.1 Shift Factor 1.36 Ave Weekly Hours (Low) 114.2 Shift Factor 1.38 Note: Output, Imvestment, and Capital stock extrapolated for 1987 and 1988.

2653 Corrugated and solid fiber boxes

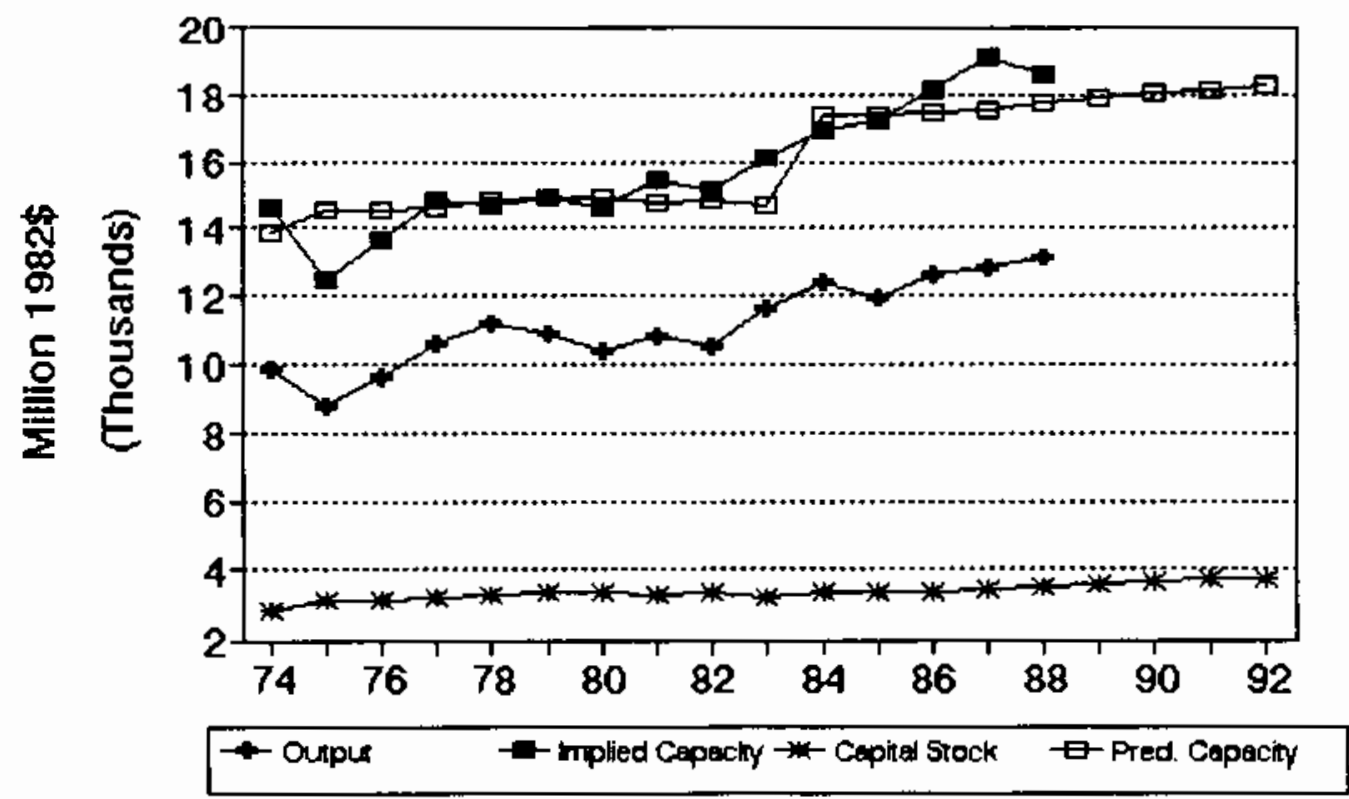


No. 159 sIC 2654 sanitary food containers

MILLIONS OF 1982 DOLLARS (EXCL. CU RATES)

\begin{tabular}{|c|c|c|c|c|c|c|c|}
\hline & $\alpha$ & Ann. & $\begin{array}{l}\text { Implied } \\
\text { Prac. }\end{array}$ & $\begin{array}{c}\text { Gross } \\
\text { In- }\end{array}$ & $\begin{array}{l}\text { Net } \\
\text { Cap. }\end{array}$ & $\begin{array}{l}\text { Pred. } \\
\text { Prac. }\end{array}$ & Energency Capacity \\
\hline I & Rate & Rate & Capec. & vest. & stk. & Capac. & 6 mon. \\
\hline
\end{tabular}

\begin{tabular}{|c|c|c|c|c|c|c|c|c|c|c|}
\hline 1974 & 3,341 & 66 & 66.0 & 5,062 & $(11.9)$ & 1,100 & 4,853 & & & \\
\hline 1975 & 3,130 & 70 & 68.5 & 4,570 & 41.2 & 1,037 & 4,652 & & & \\
\hline 1976 & 3,346 & 71 & 70.6 & 4,738 & 111.1 & 1,040 & 4,660 & & & \\
\hline 1977 & 3,263 & 58 & 62.9 & 5,190 & 43.3 & $9 \pi$ & 4,453 & & & \\
\hline 1978 & 3,165 & 72 & 66.8 & 4,741 & 109.7 & 975 & 4,454 & & & \\
\hline 1979 & 3,179 & 82 & 78.3 & 4,062 & 110.0 & 973 & 4,447 & & & \\
\hline 1980 & 2,872 & 81 & 81.4 & 3,529 & 27.6 & 895 & 4,196 & & & \\
\hline 1981 & 2,862 & 79 & 79.8 & $3,58 \mathrm{~B}$ & $\pi .8$ & 866 & 4,104 & & & \\
\hline 1982 & 2,856 & 82 & 80.9 & 3,531 & 44.0 & 810 & 3,926 & & & \\
\hline 1983 & 2,901 & 73 & 76.4 & 3,798 & 24.2 & 743 & 3,711 & & & \\
\hline 1984 & 2,732 & 74 & 73.6 & 3,710 & 46.3 & 702 & 3,580 & & & \\
\hline 1985 & 2,800 & 69 & 70.9 & 3,051 & 69.7 & 691 & 3,545 & & & \\
\hline 1986 & 2,592 & $\pi$ & 70.9 & 3,657 & 79.1 & 692 & 3,548 & & & \\
\hline$\because-$ & & -1 & $-\cdots$ & $\because \cdot$ & $\because-$ & $\cdots$ & $\because-\cdot$ & $\cdots-$ & $\cdot$ & $\cdots$ \\
\hline 1987 & 2,571 & 70 & 70.8 & 3,633 & 65.3 & 678 & 3,503 & & & \\
\hline 1988 & 2,543 & 70 & 70.0 & 3,633 & 68.3 & 669 & 3,473 & 3,999 & 4,107 & 4,150 \\
\hline 1989 & & & & & 68.2 & 660 & 3,446 & 3,968 & 4,074 & 4,118 \\
\hline 1990 & & & & & 67.8 & 652 & 3,420 & 3,939 & 4,045 & 4,087 \\
\hline 1991 & & & & & $\$ 8.0$ & 646 & 3,399 & 3,913 & 4,019 & 4,061 \\
\hline 1992 & & & & & 68.0 & 640 & 3,379 & 3,891 & 3,996 & 4.038 \\
\hline
\end{tabular}

Capacity-Capital stock Regression: Code O RSO 0.580

Coefficients: Const 1332.8 stock 3.2

Ave. Capacity/Stock $(74-86): 4.7$ Ave. Heekly Hours (High) 132.7 Shift factor 1.20 Ave Neekly Hours (LOW) 130.4 Shift Factor 1.22 Note: Output, Imvestment, and Capital stock extrapolated for 1987 and 1988.

\section{Sanitary food containers}

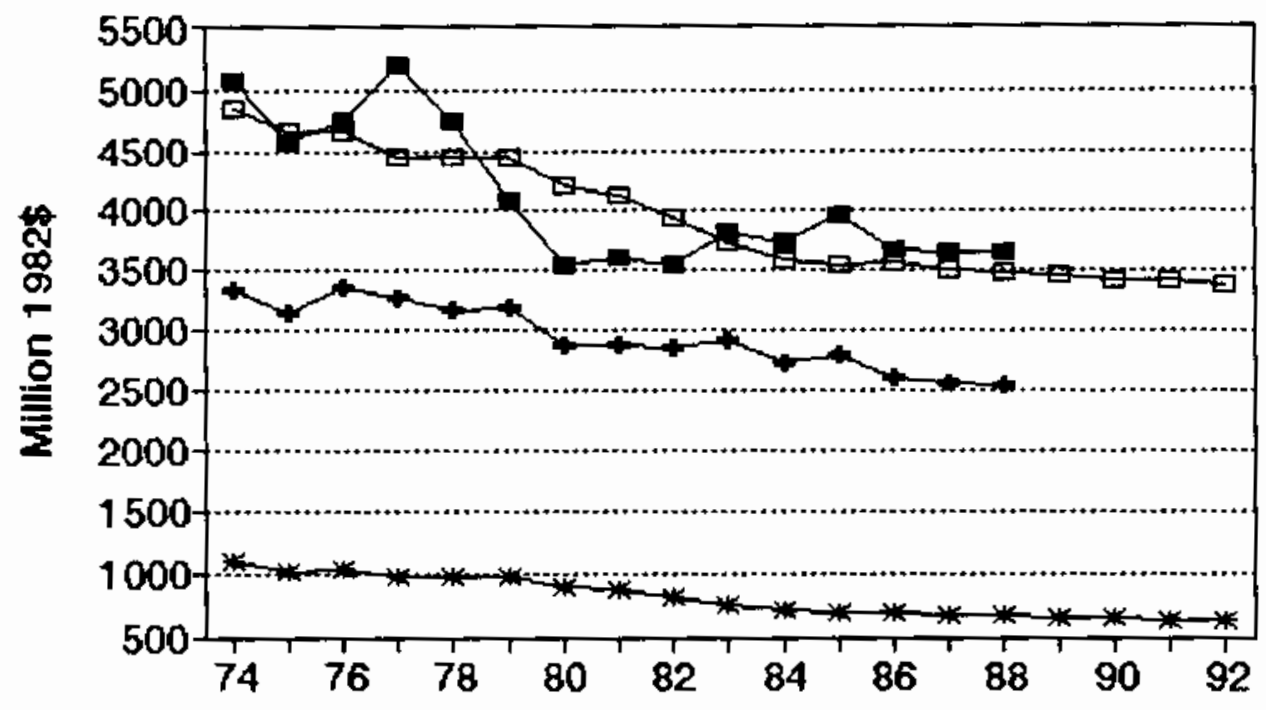

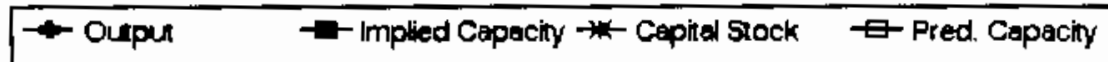


No. 160 sIC 2655 Fiber cans, druns, and similar products MILLIONS OF 1982 DOLLARS (EXCL. CU RATES)

$\begin{array}{lccccccc} & 04 & \text { Ann. Implied } & \text { Gross } & \text { Het } & \text { Pred. } & -\ldots \ldots \ldots \\ \text { Gross } & \text { CU } & \text { CU } & \text { Prac. } & \text { In. } & \text { Cap. } & \text { Prac. } & \text { Emergency Capecity } \\ \text { Output } & \text { Rate } & \text { Rate } & \text { Capac. } & \text { vest. } & \text { Stk. } & \text { Capac. } & 3 \text { mon. } 6 \text { mon. }\end{array}$

\begin{tabular}{|c|c|c|c|c|c|c|c|c|c|c|}
\hline 1974 & 1,744 & 81 & 81.0 & 2,153 & 62.5 & 419 & 2,039 & & & \\
\hline 1975 & 1,494 & 92 & 87.9 & 1,700 & 29.2 & 414 & 2,051 & & & \\
\hline 1976 & 1,563 & 65 & 75.1 & 2,081 & 10.6 & 390 & 2,107 & & & \\
\hline 1977 & 1,570 & 48 & 54.4 & 2,887 & 32.0 & 386 & 2,116 & & & \\
\hline 1978 & 1,617 & 86 & 71.8 & 2,254 & 29.3 & 377 & 2,137 & & & \\
\hline 1979 & 1,707 & 87 & 86.6 & 1,970 & 55.5 & 390 & 2,106 & & & \\
\hline 1980 & 1,609 & $\pi$ & BO.8 & 1,993 & 50.8 & 396 & 2,092 & & & \\
\hline 1981 & 1,643 & 71 & 73.3 & 2,243 & 24.0 & 376 & 2,139 & & & \\
\hline 1982 & 1,474 & 81 & 77.3 & 1,908 & 35.0 & 368 & 2,158 & & & \\
\hline 1983 & 1,545 & 81 & 81.0 & 1,907 & 47.1 & 371 & 2,153 & & & \\
\hline 1984 & 1,550 & 48 & 60.4 & 2,567 & 2.3 & 333 & 2,667 & & & \\
\hline 1985 & 1,494 & 56 & 53.0 & 2,818 & 70.3 & 363 & 2,597 & & & \\
\hline 1986 & 1,384 & 57 & 56.6 & 2,444 & 52.6 & 376 & 2,566 & & & \\
\hline$\cdots-$ & $-\cdot-$ & -- & -- & $\therefore-$ & $\cdots$ & $\cdots$ & $\because \cdots$ & -- & -- & $-\cdot$ \\
\hline 1987 & 1,385 & 68 & 63.9 & 2,168 & 47.9 & 383 & 2,550 & & & \\
\hline 1988 & 1,386 & 65 & 66.1 & 2,096 & 56.0 & 397 & 2,515 & 3,993 & 4,186 & 4,264 \\
\hline 1989 & & & & & 55.7 & 410 & 2,486 & 3,946 & 4,137 & 4,214 \\
\hline 1990 & & & & & 54.7 & 420 & 2,462 & 3,908 & 4,097 & 4,173 \\
\hline 1991 & & & & & 55.2 & 429 & 2,439 & 3,873 & 4,060 & 4,135 \\
\hline 1992 & & & & & 55.0 & 438 & 2,420 & 3,841 & 4,027 & 4,102 \\
\hline
\end{tabular}

Capacity-Capital Stock Regression: Code - 1 RSO 0.380

Coefficients: Const 3030.2 stock $-2.4 \quad 0(84-86) \quad 426.2$

Ave. Capecity/Stock $(74-86): 5.8$ Ave. Weekly Hours (High) 92.9 Shift Factor 1.70 Ave Weekly Hours (Low) 85.6 Shift Factor 1.84 Note: Output, Investment, and Capital Stock extrapolated for 1987 and 1988.

\section{Fiber cans, drums, and similar pro}

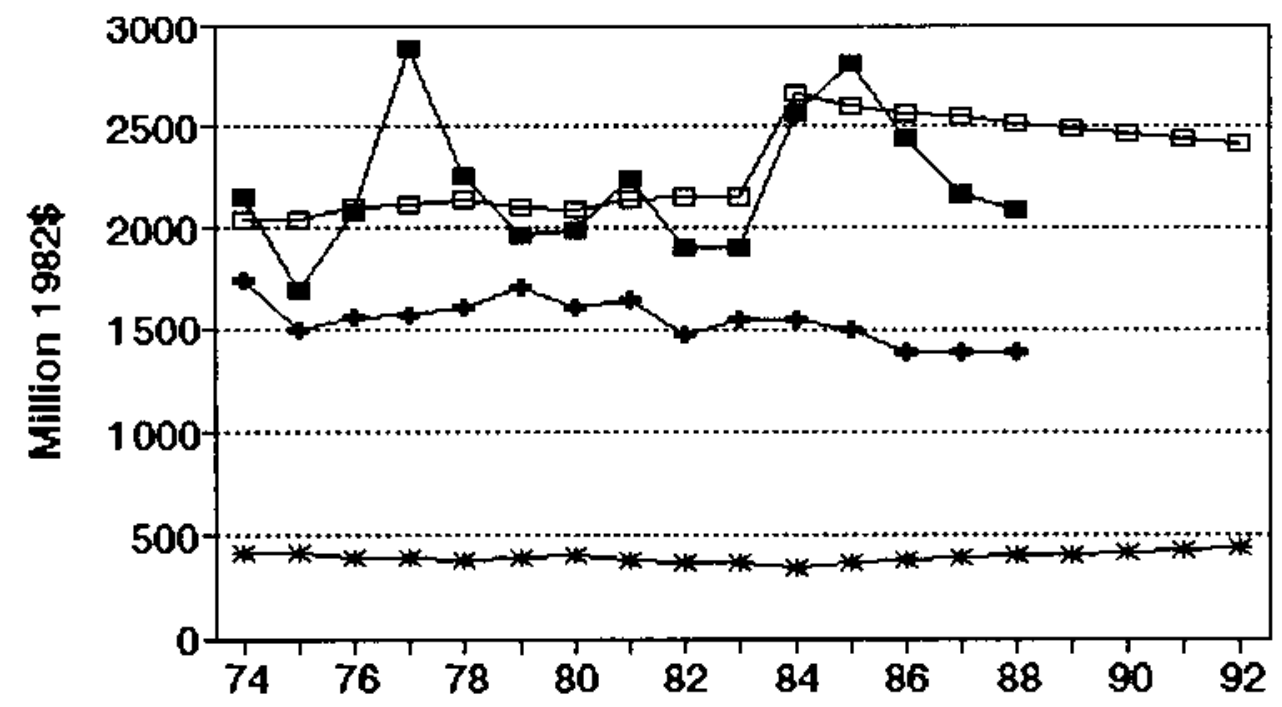

$\rightarrow$ - Oupur $\rightarrow$ mplied Cepecty $\rightarrow-$ Cepital Stock $\square$ Pred. Capacity 
No. 161 sIC 2661 Building paper and board mills

MILLIONS OF 1982 DOLLARS (EXCL. CU RATES)

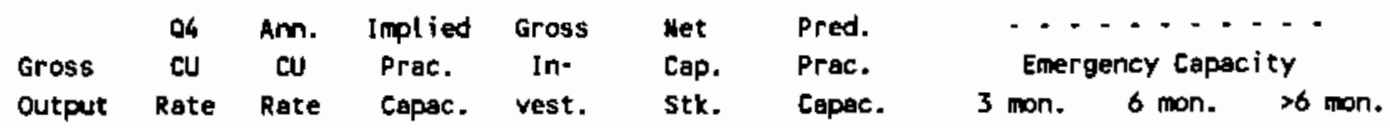

\begin{tabular}{|c|c|c|c|c|c|c|c|c|c|c|}
\hline 1974 & 1,054 & 82 & 88.0 & 1,198 & 110.5 & 668 & 1,170 & & & \\
\hline 1975 & 885 & 83 & 77.1 & 1,148 & 14.1 & 625 & 1,082 & & & \\
\hline 1976 & 850 & 90 & 88.0 & 966 & 6.3 & 579 & 987 & & & \\
\hline 1977 & 687 & 97 & 94.7 & 725 & $(34.3)$ & 500 & 825 & & & \\
\hline 1978 & 664 & 96 & 97.3 & 683 & 43.8 & 498 & $B 22$ & & & \\
\hline 1979 & 714 & 86 & 89.6 & 797 & 68.3 & 520 & Q6B & & & \\
\hline 1980 & 620 & 70 & 75.0 & 827 & 25.8 & 502 & 830 & & & \\
\hline 1981 & 567 & 58 & 63.5 & 893 & 10.8 & 470 & 765 & & & \\
\hline 1982 & 355 & 46 & 50.5 & 704 & $(15.7)$ & 416 & 654 & & & \\
\hline 1983 & 403 & 63 & 56.8 & 709 & $(4.2)$ & 37 & 573 & & & \\
\hline 1984 & 436 & 85 & 77.3 & 564 & 13.0 & 355 & 530 & & & \\
\hline 1985 & 399 & 76 & 78.7 & 507 & 27.2 & 347 & 513 & & & \\
\hline 1986 & 277 & 80 & 78.6 & 352 & 5.9 & 319 & 455 & & & \\
\hline$\cdots$ & $-\cdots$ & - & $-\cdots$ & $-\cdots$ & $-\cdots$ & $\because$ & $\cdots$ & $\cdots$ & $\cdot$ & - - \\
\hline 1987 & 263 & 79 & 79.1 & 332 & 6.0 & 292 & 399 & & & \\
\hline 1988 & 245 & 81 & 80.3 & 305 & 6.0 & 267 & 349 & 341 & 349 & 351 \\
\hline 1989 & & & & & 6.0 & 245 & 304 & 298 & 304 & 306 \\
\hline 1990 & & & & & 6.0 & 226 & 264 & 258 & 264 & 266 \\
\hline 1991 & & & & & 6.0 & 208 & 228 & 223 & 228 & 230 \\
\hline 1992 & & & & & 6.0 & 192 & 196 & 191 & 195 & 197 \\
\hline
\end{tabular}

Capacity-Capital stock Regression: Code 0 Rso 0.870

Coefficients: Const -198.5 stock 2.1

Ave. Capacity/Stock $(74-86): 1.6$ Ave. Weekly Hours (High) 166.8 Shift Factor 1.01 Ave Weekly Hours (Low) 165.6 Shift factor 1.01 Mote: Output, Investment, and Capital stock extrapolated for 1987 and 1988.

\section{Building paper and board mills}

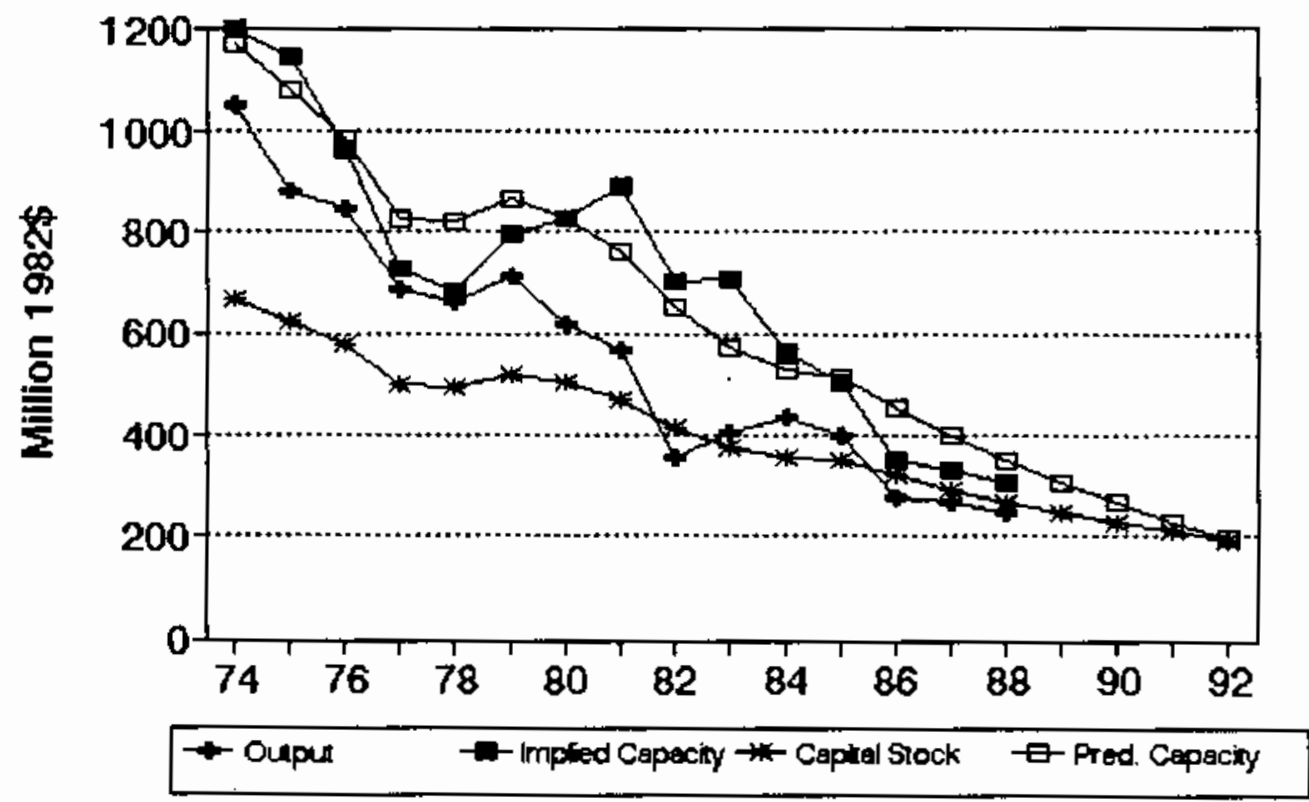




\section{NO. 162 SIC 2711 Hewspapers}

MILLIONS OF 1982 DOLLARS (EXCL. CU RATES)

$\begin{array}{lccccccc} & \text { Q4 } & \text { Ann. Implied Gross } & \text { Het } & \text { Pred. } & \ldots . \ldots \ldots \\ \text { Gross } & \text { CU } & \text { CU } & \text { Prac. } & \text { In- } & \text { Cap. } & \text { Prac. } & \text { Emergency Capacity } \\ \text { Output Rate } & \text { Rate } & \text { Capac. } & \text { vest. } & \text { Stk. } & \text { Capoc. } & 3 \text { mon. } 6 \text { mon. }>6 \text { mon. }\end{array}$

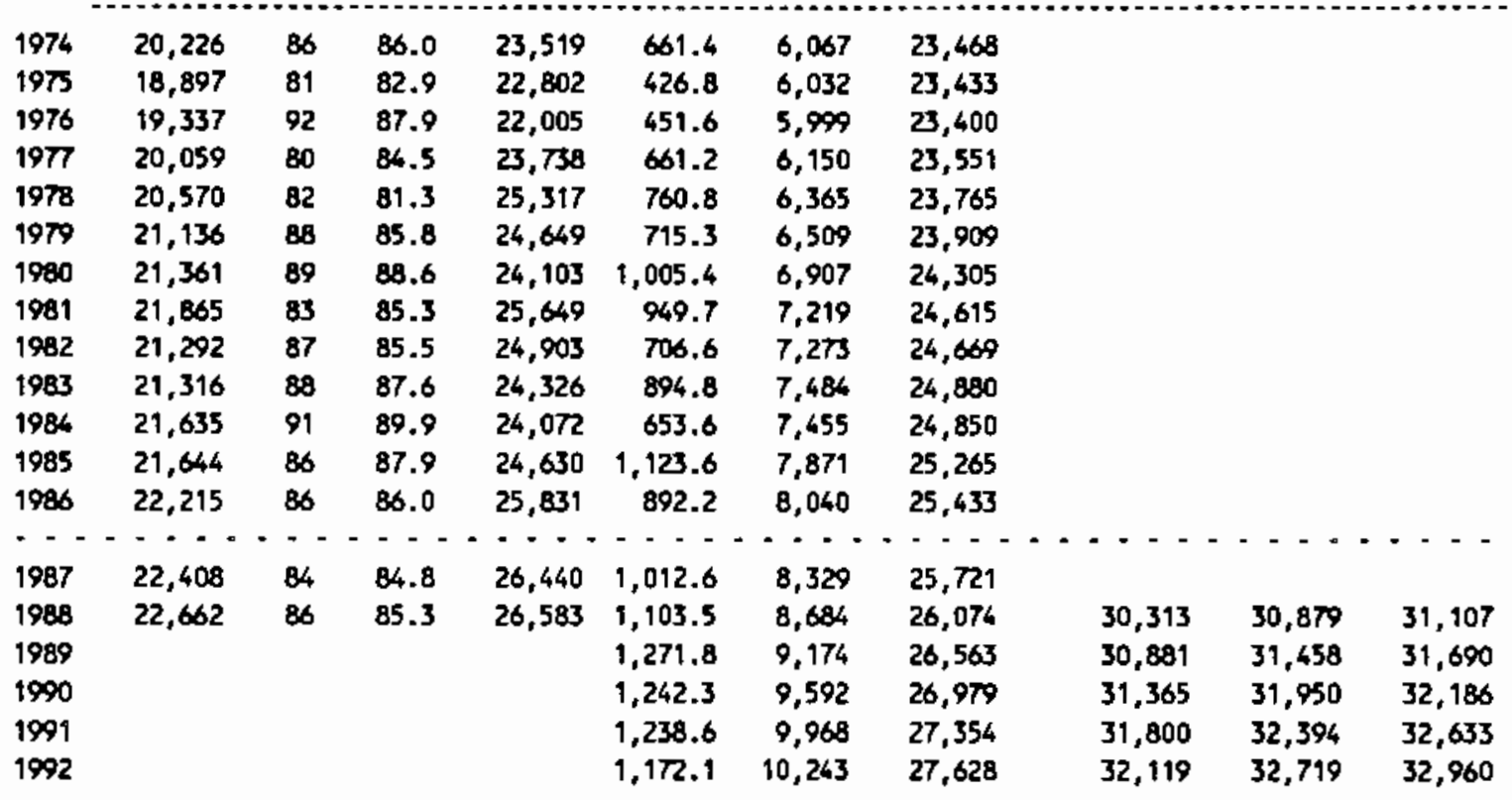

Capacity-Capital Stock Regression: Code 0 RSQ 0.440 Coefficients: Const 17424.3 Stock 1.0

Ave. Capacity/Stock $(74-86): 3.5$ Ave. Weekly Hours (High) 132.9 Shift Factor 1.19 Ave Weekly Hours (Low) 125.1 Shift Factor 1.27 Note: Output, Imestment, and Capitat Stock extrapolated for 1987 and 1988.

\section{Newspapers}

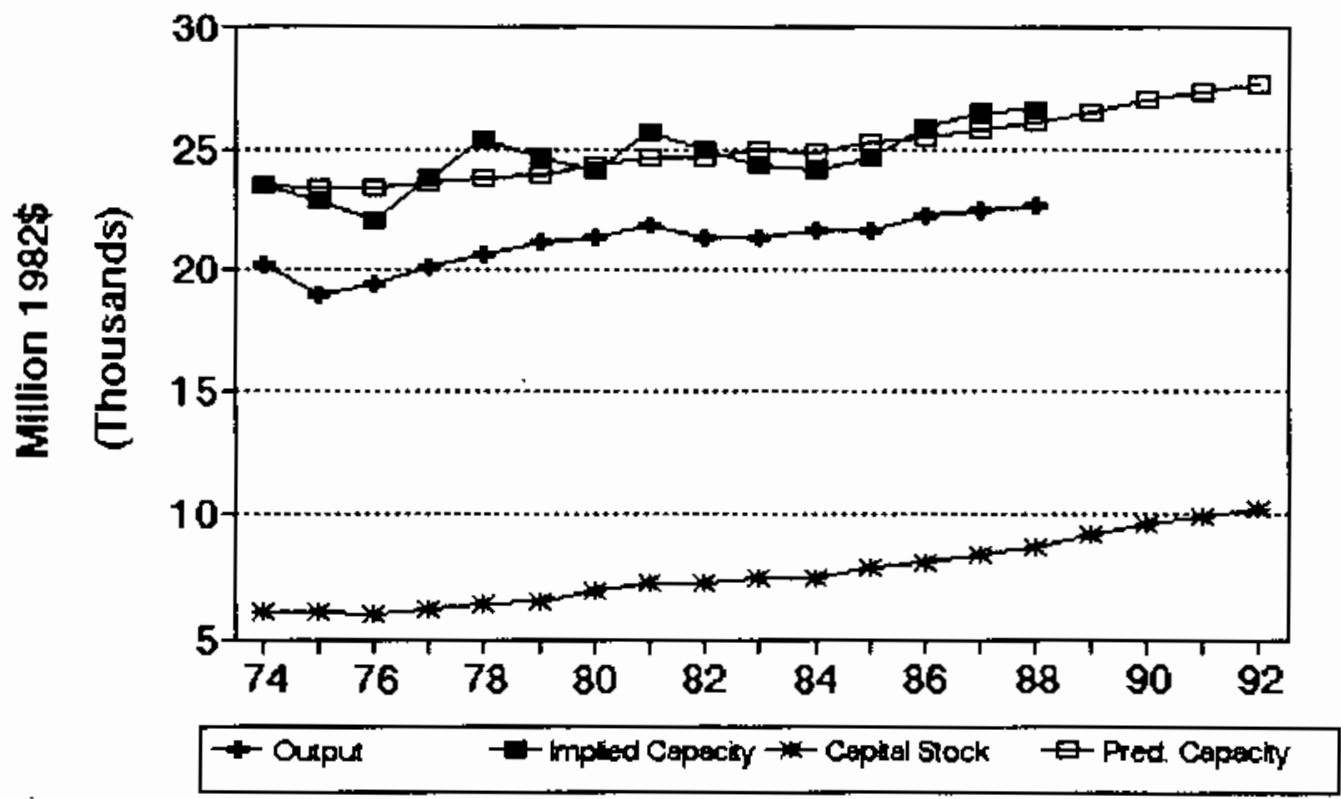




\section{No, 163 SIC 2721 Periodicals}

MILLIONS OF 1982 DOLLARS (EXCL. CU RATES)

$\begin{array}{lccccccc} & \text { Q4 } & \text { Ann. Implied Gross } & \text { Net } & \text { Pred. } & -\ldots . \ldots . \ldots \\ \text { Gross } & \text { CU } & \text { CU } & \text { Prac. } & \text { In- } & \text { Cap. } & \text { Prac. } & \text { Emergency Capacity } \\ \text { Output Rate } & \text { Rate } & \text { Capac. } & \text { vest. } & \text { Stk. } & \text { Capac. } & 3 \text { mon. } 6 \text { mon. }>6 \text { mon. }\end{array}$

\begin{tabular}{|c|c|c|c|c|c|c|c|c|c|c|}
\hline 1974 & 7,396 & B4 & 84.0 & 8,805 & 17.3 & 709 & 10,979 & & & \\
\hline 1975 & 7,161 & 76 & 79.0 & 9,065 & 68.4 & 714 & 11,015 & & & \\
\hline 1976 & 7,655 & 68 & 71.0 & 10,782 & $\$ 1.3$ & 702 & 10,937 & & & \\
\hline 1977 & 8,835 & 66 & 66.8 & 13,235 & 4.4 & 647 & 10,607 & & & \\
\hline 1978 & 9,833 & 89 & 80.4 & 12,233 & 86.8 & 670 & 10,746 & & & \\
\hline 1979 & 10,454 & 100 & 95.9 & 10,903 & 70.8 & 676 & 10,779 & & & \\
\hline 1980 & 10,457 & 100 & 100.0 & 10,457 & 100.9 & 707 & 10,968 & & & \\
\hline 1981 & 11,006 & 100 & 100.0 & 11,006 & 153.0 & 780 & 11,417 & & & \\
\hline 1982 & 11,555 & 100 & 100.0 & 11,555 & 207.8 & 897 & 12,131 & & & \\
\hline 1983 & 11,510 & 89 & 93.1 & 12,360 & 82.6 & 894 & 12,108 & & & \\
\hline 1984 & 12,028 & 89 & 89.0 & 13,514 & 142.6 & 949 & 12,446 & & & \\
\hline 1985 & 12,252 & 89 & 89.0 & 13,766 & 228.1 & 1,082 & 13,260 & & & \\
\hline 1986 & 11,830 & 89 & 89.0 & 13,292 & 150.2 & 1,135 & 13,582 & & & \\
\hline$-\cdots$ & $--\cdot$ & $\cdots$ & $--\cdot$ & $\cdots \cdot$ & $-\cdots$ & $-\cdots$ & $\div--$ & 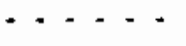 & $-\quad--$ & $\cdots-$ \\
\hline 1987 & 12,062 & 89 & 89.0 & 13,553 & 198.9 & 1,236 & 14,195 & & & \\
\hline 1988 & 12,370 & 89 & 89.0 & 13,899 & 231.5 & 1,360 & 14,954 & 14,712 & 14,885 & 14,954 \\
\hline 1989 & & & & & 291.7 & 1,534 & 16,015 & 15,755 & 15,940 & 16,015 \\
\hline 1990 & & & & & 281.1 & 1,682 & 16,920 & 16,645 & 16,841 & 16,920 \\
\hline 1991 & & & & & 279.8 & 1,816 & 17,737 & 17,469 & 17,655 & 17,737 \\
\hline 1992 & & & & & 256.0 & 1,915 & 18,339 & 18,041 & 18,254 & 18,339 \\
\hline
\end{tabular}

Capacity-Capital Stock Regression: Cade 0 RSO 0.370

Coefficients: Const 6656.1 stock 6.1

Ave. Capacity/stock (74-86): 14.3 Ave. Weekly Hours (High) 1983.0 Shift Factor 1.00 Ave Heexly Hours (LOW) 1983.0 "Shift Factor 1.0 Note: Output, Imvestment, and Capital Stock extrapolated for 1987 and 1988.

\section{Periodicals}

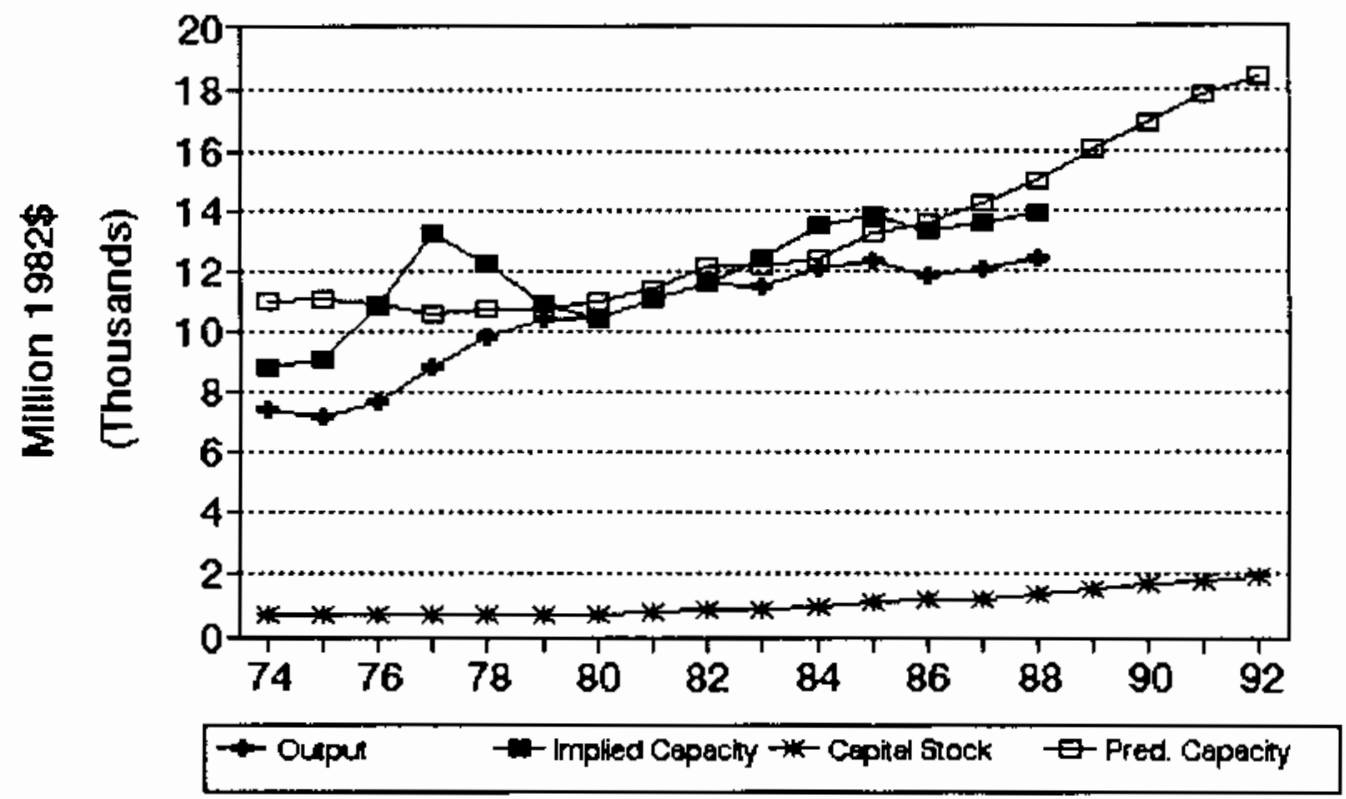




\section{NO. 164 SIC 2731 Book publishing}

MILLIONS OF 1982 DOLLARS (EXCL. CU RATES)

\begin{tabular}{|c|c|c|c|c|c|c|c|}
\hline & $\begin{array}{l}\mathrm{OA} \\
\mathrm{CU}\end{array}$ & $\begin{array}{c}\text { Ann. } \\
\text { CU }\end{array}$ & $\begin{array}{l}\text { Implied } \\
\text { Prac. }\end{array}$ & $\begin{array}{l}\text { Grose } \\
\text { In- }\end{array}$ & $\begin{array}{l}\text { Net } \\
\text { Cap. }\end{array}$ & $\begin{array}{l}\text { Pred. } \\
\text { Prac. }\end{array}$ & $\cdots \cdots \cdots$ \\
\hline & Rate & Rate & & yest & & & \\
\hline
\end{tabular}

\begin{tabular}{|c|c|c|c|c|c|c|c|c|c|c|}
\hline 1974 & 6,526 & 91 & 91.0 & 7,172 & $(47.4)$ & 690 & 6,734 & & & \\
\hline 1973 & 6,181 & 94 & 92.9 & 6,656 & 113.6 & 749 & 7,461 & & & \\
\hline 1976 & 6,359 & 94 & 94.0 & 6,765 & 76.2 & 767 & 7,674 & & & \\
\hline 1977 & 7,222 & 97 & 95.9 & 7,532 & 101.0 & 803 & 8,091 & & & \\
\hline 1978 & 7,465 & 89 & 92.0 & 8,115 & 74.8 & 809 & 8,159 & & & \\
\hline 1979 & 7,151 & 81 & 84.0 & 8,513 & 52.3 & 792 & 7,959 & & & \\
\hline 1980 & 6,969 & 79 & 79.8 & 8,738 & 93.8 & 813 & 8,212 & & & \\
\hline 1981 & 7,285 & 75 & 76.5 & 9,523 & 102.6 & 859 & 8,522 & & & \\
\hline 1982 & 7,820 & 88 & 83.1 & 9,407 & 120.9 & 879 & 8,994 & & & \\
\hline $19 a 3$ & 8,037 & 86 & 86.8 & 9,265 & 141.5 & 933 & 9,625 & & & \\
\hline 1984 & 8,586 & 87 & 86.6 & 9,912 & 132.9 & 975 & 10,126 & & & \\
\hline 1985 & 8,872 & $B B$ & 87.6 & 10,124 & 65.7 & 955 & 9,892 & & & \\
\hline 1986 & 8,870 & 89 & 88.6 & 10,009 & 119.1 & 987 & 10,262 & & & \\
\hline $\begin{array}{l}-\cdots \\
1987\end{array}$ & $\begin{array}{c}- \\
8,971\end{array}$ & 90 & $\begin{array}{l}--. \\
89.6\end{array}$ & $\begin{array}{l}\cdots \\
10,009\end{array}$ & $\begin{array}{l}-\cdots \\
120.5\end{array}$ & 1,019 & $\cdots$ & $-\cdot \cdot$ & $-\cdot \cdot$ & $-\cdots$ \\
\hline 1988 & 9,103 & 90 & 90.0 & 10,114 & 133.0 & 1,061 & 11,143 & 10,951 & $11,0 \mathrm{BB}$ & 11,143 \\
\hline 1989 & & & & & 156.0 & 1,123 & 11,866 & 11,662 & 11,807 & 11.866 \\
\hline 1990 & & & & & 151.9 & 1,175 & 12,477 & 12,262 & 12,416 & 12,477 \\
\hline 1991 & & & & & 151.4 & 1,221 & 13,028 & 12,804 & 12,964 & 13,028 \\
\hline 1992 & & & & & 142.3 & 1,255 & 13,422 & 13,191 & 13,356 & 13,422 \\
\hline
\end{tabular}

Capacity-Cepital Stock Regression: Code 0 RSO 0.780

Coefficients: Const -1381.7 stock 11.8

Ave. Capacity/Stock (74-86): 10.2 Ave. Weekly Hours (High) 1983.0 Shift Factor 1.00 Ave Weekty Hoirs (Low) 1983.0 Shift Factor 1.0 Note: Output, Investment, and Capital stock extrapolated for 1987 and 1988.

\section{Book publishing}

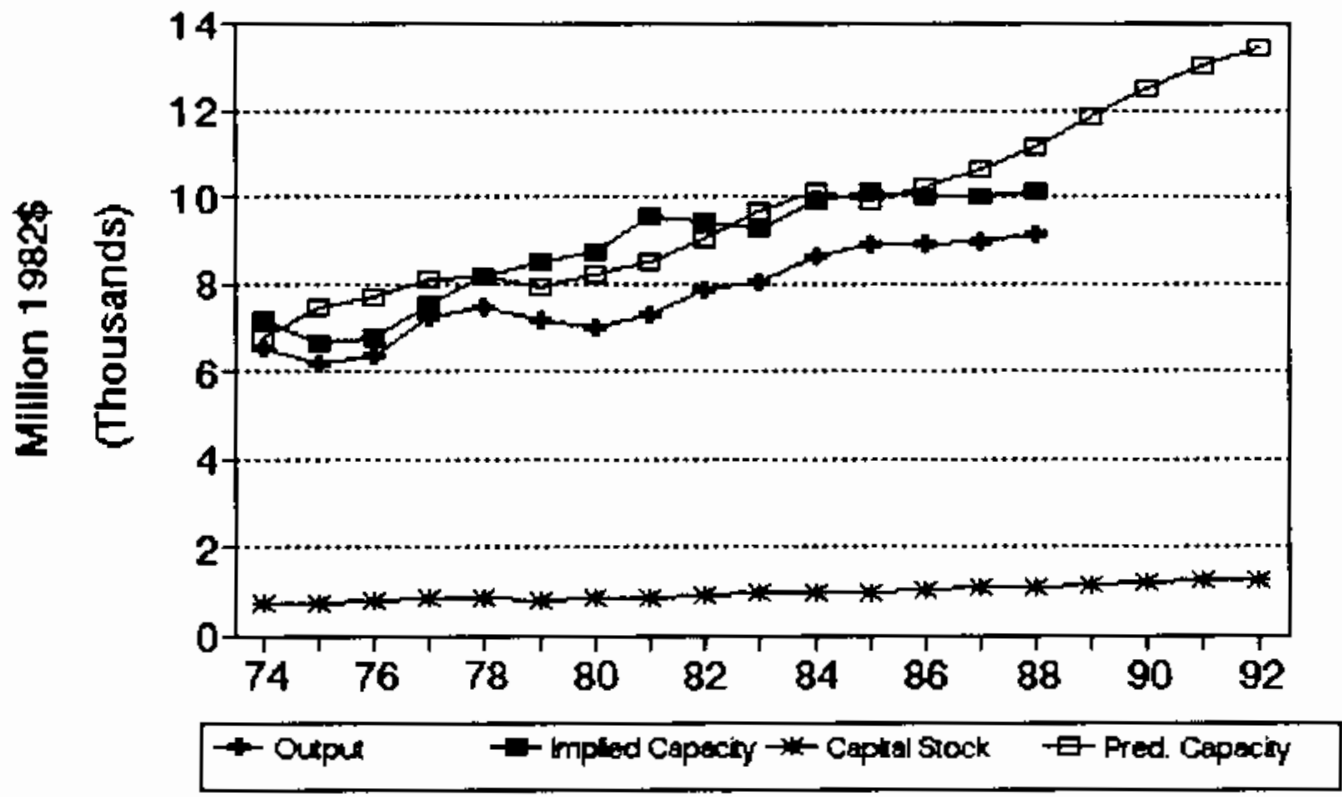




\section{Ko. 165 sIC 2732 Book printing}

MILLIONS OF 1982 DOLLARS (EXCL. CU RATES)

$\begin{array}{lccccccc} & \text { Q4 } & \text { Ann. Implied Gross } & \text { Net } & \text { Pred. } & \ldots \ldots \ldots \\ \text { Gross } & \text { CU } & \text { CU } & \text { Prac. } & \text { In- } & \text { Cap. } & \text { Prac. } & \text { Emergency Capacity } \\ \text { Output Rate } & \text { Rate } & \text { Capac. } & \text { vest. } & \text { Stk. } & \text { Capac. } & 3 \text { mon. } 6 \text { mon. }\end{array}$

\begin{tabular}{|c|c|c|c|c|c|c|c|c|c|c|}
\hline 1974 & 2,061 & 70 & 71.3 & 2,891 & 8.6 & 892 & 3,067 & & & \\
\hline 1975 & 2,164 & $69 *$ & 67.4 & 3,208 & 119.6 & 935 & 3,176 & & & \\
\hline 1976 & 2,174 & 71 & 70.4 & 3,086 & 31.8 & 889 & 3,060 & & & \\
\hline 1977 & 2,639 & $n$ & $\pi .2$ & 3,508 & 153.8 & 953 & 3,220 & & & \\
\hline 1978 & 2,752 & 77 & $n .2$ & 3,565 & 189.5 & 1,042 & 3,445 & & & \\
\hline 1979 & 2,582 & 81 & 79.5 & 3,247 & 7.8 & 1,014 & 3,373 & & & \\
\hline 1980 & 2,608 & 83 & 82.2 & 3,174 & 140.8 & 1,047 & 3,457 & & & \\
\hline 1981 & 2,686 & 81 & 81.9 & 3,282 & 169.1 & 1,097 & 3,584 & & & \\
\hline 1982 & 2,389 & 74 & 76.2 & 3,134 & $(43.2)$ & 951 & 3,217 & & & \\
\hline 1983 & 2,515 & 83 & 79.4 & 3,168 & 76.5 & 925 & 3,151 & & & \\
\hline 1984 & 2,647 & $78 *$ & 80.5 & $3,28 B$ & 150.4 & 968 & 3,258 & & & \\
\hline 1985 & 2,691 & $\pi$ & 75.5 & 3,566 & 180.6 & 1,060 & 3,439 & & & \\
\hline 1986 & 2,733 & 67 & 69.9 & 3,912 & 163.8 & 1,097 & 3,582 & & & \\
\hline : & 2,753 & 69 & 68.4 & 4,026 & 161.8 & 1,148 & 3,710 & $\cdots$ & $\cdots \cdot$ & $-\cdots$ \\
\hline 1988 & 2,779 & 84 & 78.4 & 3,545 & 179.6 & 1,212 & 3,871 & 4,719 & 4,862 & 4,920 \\
\hline 1989 & & & & & 212.8 & 1,302 & 4,098 & 4,996 & 5,148 & 5,209 \\
\hline 1990 & & & & & 206.9 & 1,378 & 4,288 & 5,227 & 5,386 & 5,650 \\
\hline 1991 & & & & & 206.2 & 1,445 & 4,457 & 5,433 & 5,598 & 5,665 \\
\hline 1992 & & & & & 193.1 & 1,492 & 4,576 & 5,578 & 5,748 & 5,816 \\
\hline
\end{tabular}

Cepecity-Capital Stock Regression: Code 0 RSQ 0.470 Coefficients: Const 825.7 stock 2.5 Ave. Weekly Hours (High) 124.5 Shift Factor 1.27 Ave Weekly Hours (Low) 120.5 Shift Factor 1.31 * Imputed Note: Output, Investment, and Capital Stock extrapolated for 1987 and 1988.

\section{Book printing}

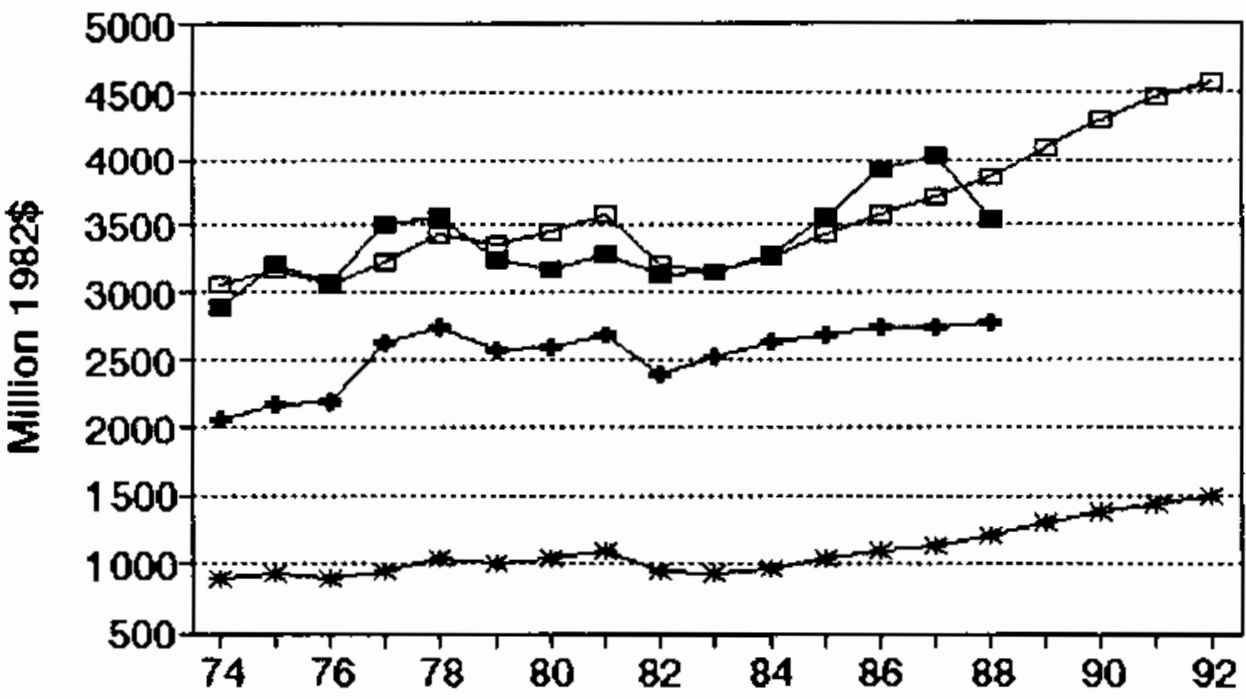

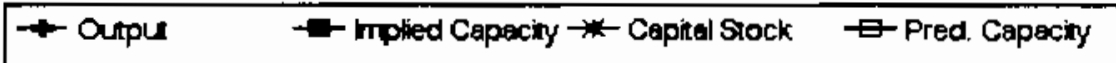


NO. 166 sIc 2741 Misc. publiahing

MILLIONS OF 1982 DOLLARS (EXCL, CU RATES)

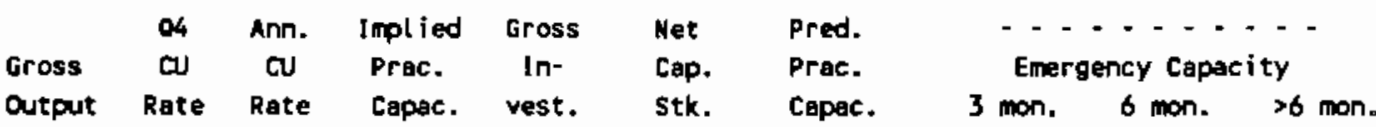

\begin{tabular}{|c|c|c|c|c|c|c|c|c|c|c|}
\hline 1974 & 2,060 & 41 & 43.3 & 4,753 & (3.4) & 317 & 4,151 & & & \\
\hline 1975 & 1,984 & 58 & 47.1 & 4,215 & 44.6 & 339 & 4,072 & & & \\
\hline 1976 & 2,111 & 56 & 57.1 & 3,695 & 52.5 & 366 & 3,969 & & & \\
\hline 1977 & 2,756 & 69 & 65.1 & 4,232 & 74.8 & 413 & 3,791 & & & \\
\hline 1978 & 2,617 & 74 & 72.6 & 3,605 & 37.2 & 423 & 3,755 & & & \\
\hline 1979 & 2,732 & 89 & 83.6 & 3,268 & 35.7 & 429 & 3,733 & & & \\
\hline 1980 & 2,870 & 87 & 87.5 & 3,279 & 33.0 & 431 & 3,726 & & & \\
\hline 1981 & 2,786 & 80 & 82.9 & 3,362 & 29.2 & 427 & 3,738 & & & \\
\hline 1982 & 2.866 & 87 & 83.5 & 3,431 & 96.1 & 483 & 3,528 & & & \\
\hline 1983 & 3,051 & 79 & 82.1 & 3.717 & 54.9 & 496 & 3,481 & & & \\
\hline 1984 & 3,042 & 9 & 93.6 & 3,251 & $(13.9)$ & 444 & 3,677 & & & \\
\hline 1985 & 4,040 & 96 & 95.0 & 4,251 & 108.4 & 508 & 3,435 & & & \\
\hline 1986 & 4,203 & $81 *$ & 86.3 & 4,870 & 69.8 & 533 & 4,870 & & & \\
\hline-- & $\cdots-\cdot$ & $=-$ & $\because \cdot$ & $--\cdot$ & -- & $\because-$ & $\cdots \cdot$ & $-\cdots$ & -- & $\cdot-$ \\
\hline 1987 & 4,267 & $\pi$ & 79.0 & 5,401 & 68.0 & 555 & 4,786 & & & \\
\hline 1988 & 4,351 & 92 & 86.5 & 5,033 & 75.3 & 583 & 4,682 & 7,391 & 7,616 & 7,707 \\
\hline 1989 & & & & & 88.9 & 622 & 4,536 & 7,160 & 7,378 & 7,468 \\
\hline 1990 & & & & & 86.5 & 655 & 4,411 & 6,963 & 7.175 & 7,260 \\
\hline 1991 & & & & & 86.2 & 685 & 4,298 & 6,785 & $6, \infty 91$ & 7,074 \\
\hline 1992 & & & & & 80.8 & 707 & 4,215 & 6,653 & 6,856 & 6,937 \\
\hline
\end{tabular}

Capacity-Capital Stock Regression: Code - 1 RSO 0.440

Coefficients: Const 5342.7 Stock -3.8 D(86-86) $1527.6 \quad$ Ave. Capecity/Stock (74-86): 8.9 Ave. Weekly hours (High) 95.7 Shift Fector 1.65 Ave Veekly Hours (LOW) 88.2 Shift factor 1.78 * Imputed Mote: Output, Investment, end Cepital Stock extrapolated for 1987 and 1988.

\section{Misc. publishing}

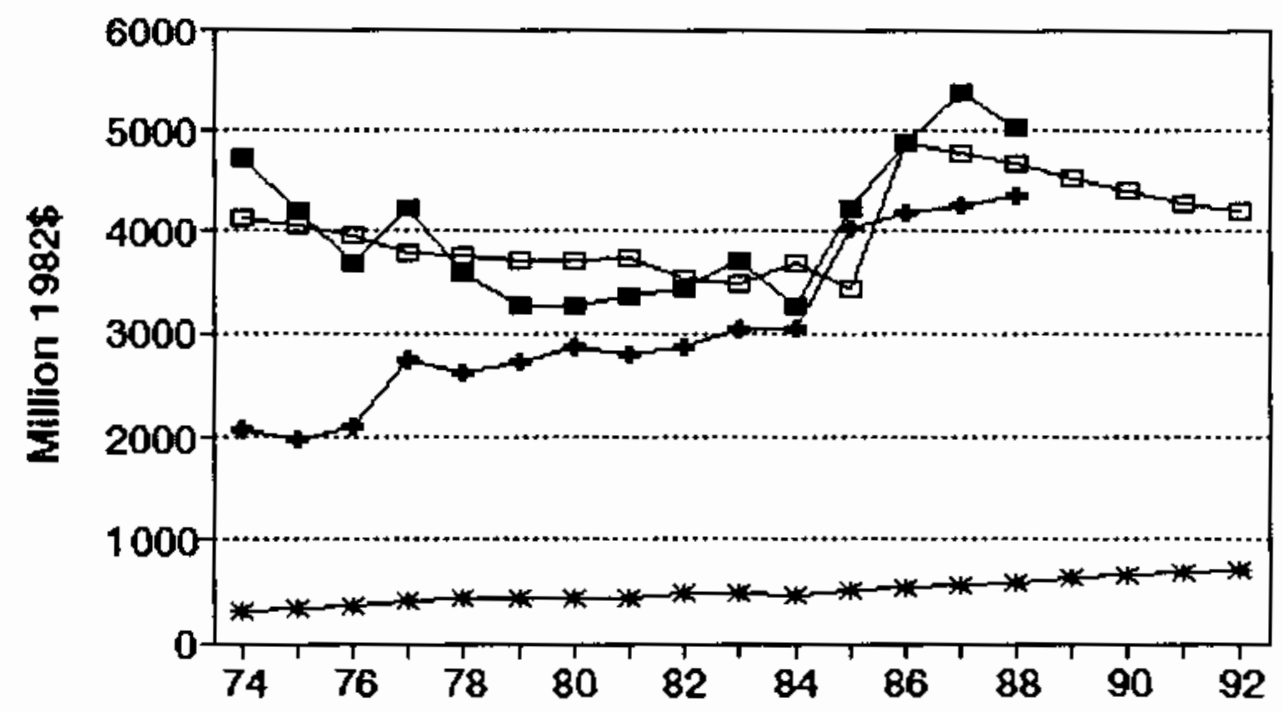

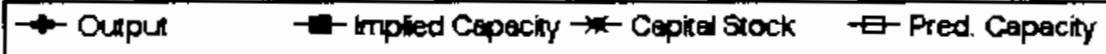




\section{No. 167 sIC 2751 Commercial printing, letterpress}

MILLIONS OF 1982 DOLLARS (EXCL. CU RATES)

$\begin{array}{lccccccc} & \text { Q4 } & \text { Amn. Implied Gross } & \text { Het } & \text { Pred. } & \ldots \ldots \ldots & \ldots \\ \text { Gross } & \text { CU } & \text { CU } & \text { Prac. } & \text { In- } & \text { Cap. } & \text { Prac. } & \text { Emergency Capacity } \\ \text { Output } & \text { Rate } & \text { Rate } & \text { Capac. } & \text { vest. } & \text { Stk. } & \text { Capac. } & 3 \text { mon. } 6 \text { mon. }>6 \text { mon. }\end{array}$

\begin{tabular}{|c|c|c|c|c|c|c|c|c|c|c|}
\hline 1974 & 6,815 & 69 & 72.5 & 9,406 & $(25.8)$ & 2,574 & 9,496 & & & \\
\hline 1975 & 6,910 & 66 & 61.6 & 11,226 & $\pi .4$ & 2,432 & 9,409 & & & \\
\hline 1976 & 7.023 & $\infty 6$ & 66.3 & 10,590 & 115.3 & 2,334 & 9,349 & & & \\
\hline 1977 & 6,238 & 94 & 84.4 & 7,388 & 62.4 & 2,195 & 9,264 & & & \\
\hline 1978 & 6,495 & $\pi$ & 82.7 & 7,858 & 260.0 & 2,261 & 9,292 & & & \\
\hline 1979 & 6,206 & 71 & 72.5 & 8,559 & 96.2 & 2,131 & 9,225 & & & \\
\hline 1980 & 6,208 & 72 & 71.5 & 8,686 & 191.3 & 2,110 & 9,212 & & & \\
\hline 1981 & 6,246 & 61 & 65.3 & 9,572 & 294.9 & 2,176 & 9,253 & & & \\
\hline 1982 & 5,525 & 60 & 59.7 & 9.253 & $(199.7)$ & 1,792 & 9,018 & & & \\
\hline 1983 & 5,677 & 62 & 61.1 & 9.296 & $\$ 5.1$ & 1,701 & 8,962 & & & \\
\hline 1984 & 6,410 & 77 & 72.8 & 8,802 & 185.4 & 1,698 & 8,961 & & & \\
\hline 1985 & 6,857 & 76 & 74.9 & 9,154 & 244.0 & 1,748 & 8,991 & & & \\
\hline 1986 & 7,015 & 72 & 73.1 & 9,591 & 114.5 & 1,679 & 8,949 & & & \\
\hline $\begin{array}{l}--- \\
1987\end{array}$ & 6.991 & 84 & $\begin{array}{l}\cdots \\
79.9\end{array}$ & $\begin{array}{l}--\cdot- \\
8,754\end{array}$ & $\begin{array}{l}\cdots \\
178.1\end{array}$ & 1,673 & 8,945 & 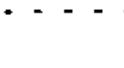 & $-\cdots \cdot-$ & $-\cdots$ \\
\hline 1988 & 6,960 & 71 & 75.9 & 9,174 & 194.8 & 1,683 & 8,951 & 11,173 & 11,484 & 11,610 \\
\hline 1989 & & & & & 225.6 & 1,723 & 8,976 & 11,203 & 11,516 & 11,642 \\
\hline 1990 & & & & & 220.2 & $1, \sqrt{54}$ & 8,995 & 11.227 & 11,540 & 11,666 \\
\hline 1991 & & & & & 219.5 & 1,780 & 9,011 & 11,247 & 11,560 & 11,687 \\
\hline 1992 & & & & & 207.4 & 1,791 & 9,018 & 11,255 & 11,569 & 11,696 \\
\hline
\end{tabular}

Capacity-Capital Stock Regression: Code 0 RSO 0.030 Coefficients: Const 7923.1 stock 0.6

Ave. Capacity/Stock $(74-86): 4.5$ Ave. Yeekly Hours (High) 121.9 Shift Factor 1.30 Ave Weekly Hours (Low) 113.1 Shift Factor 1.39 Mote: Qutput, Investment, and Capital Stock extrapolated for 1987 and 1988.

\section{Commercial printing, letterpress}

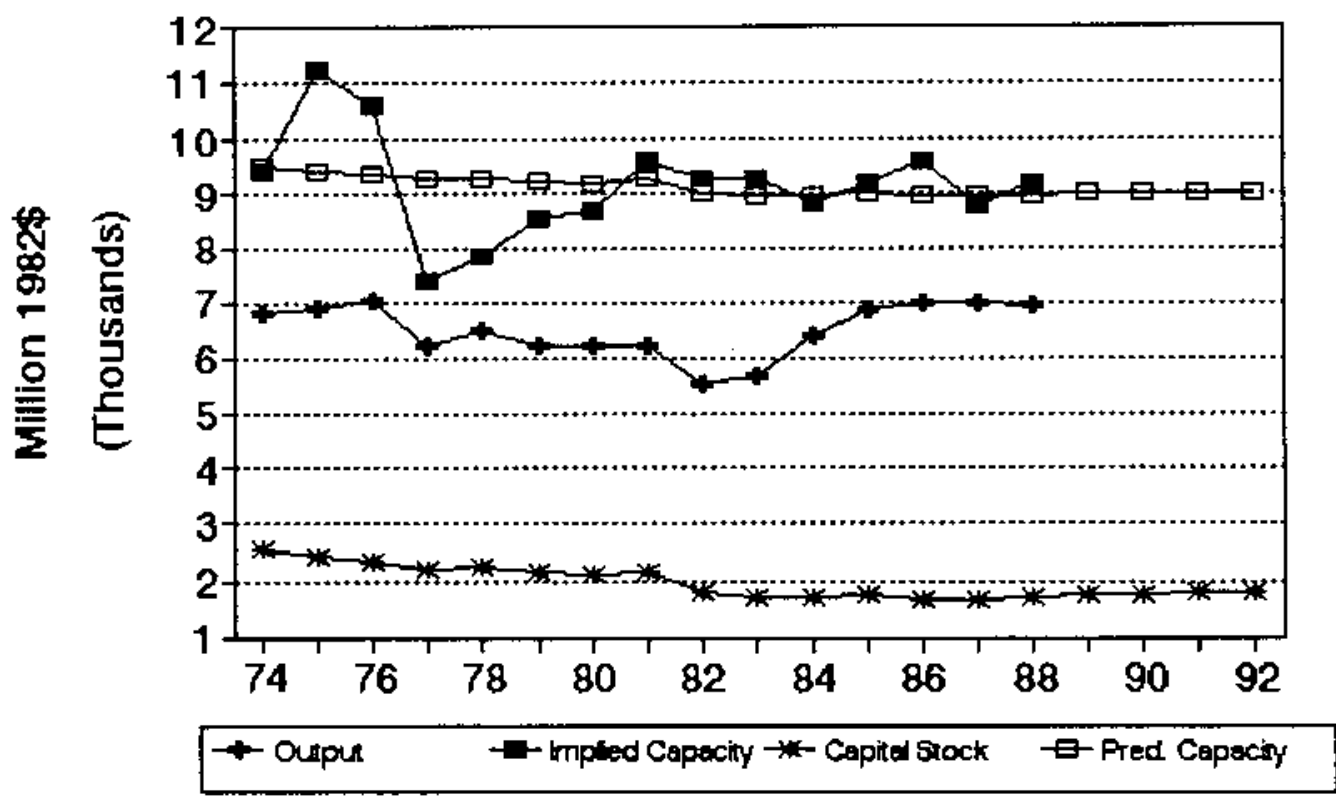




\section{No. 168 SIC 2752 Commexcial printing, lithographic}

MILLIONS OF 1982 DOLLARS (EXCL. CU RATES)

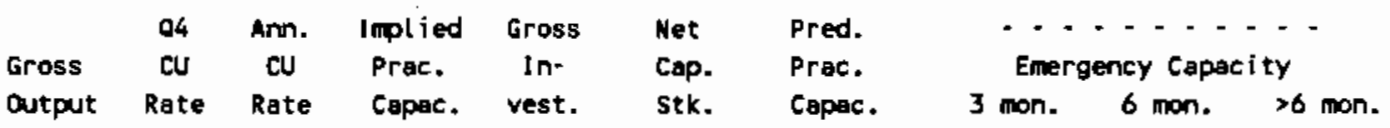

\begin{tabular}{|c|c|c|c|c|c|c|c|c|c|c|}
\hline 1974 & 11,581 & 79 & 79.8 & 14,519 & 147.6 & 4,208 & 14,955 & & & \\
\hline 1975 & 11,149 & 76 & 75.9 & 14,691 & 447.0 & 4,345 & 15,560 & & & \\
\hline 1976 & 12,024 & 78 & 77.3 & 15,559 & 294.3 & 4,308 & 15,395 & & & \\
\hline 1977 & 13,791 & 76 & 77.0 & 17,901 & $1,039.3$ & 4,944 & 18,205 & & & \\
\hline 1978 & 14,411 & 80 & 78.6 & 18,332 & 548.2 & 5,069 & 18,757 & & & \\
\hline 1979 & 15,377 & 79 & 79.4 & 19,372 & 480.2 & 5,127 & 19,013 & & & \\
\hline 1980 & 15,270 & 79 & 79.0 & 19,338 & 568.3 & 5,258 & 19,588 & & & \\
\hline 1981 & $15,72 B$ & 78 & 78.4 & 20,054 & 662.1 & 5,475 & 20,546 & & & \\
\hline 1982 & 19,577 & 67 & 70.9 & 27,608 & $1,824.8$ & 6,747 & 26,167 & & & \\
\hline $19 a 3$ & 20,648 & 69 & 68.2 & 30,290 & 677.5 & 6,873 & 26,721 & & & \\
\hline 1984 & 22,488 & 76 & 73.7 & 30,506 & $1,113.5$ & 7,416 & 29,120 & & & \\
\hline 1985 & 22,794 & 9 & 77.6 & 29,393 & 957.8 & 7,796 & 30,797 & & & \\
\hline 1986 & 23,671 & $\pi$ & 77.7 & 30,477 & $1,165,1$ & 8,344 & 33,217 & & & \\
\hline-- & $\cdots \cdot$ & - & $-\cdot$ & & & $\cdots-$ & $-\cdots$ & $=\cdot-$ & - & $--\cdot$ \\
\hline 1987 & 24,168 & 77 & $\pi .1$ & 31,356 & $1,200.0$ & 8,926 & 35,787 & & & \\
\hline 1988 & 24,830 & 83 & 80.8 & 30,746 & $1,200.0$ & 9,465 & 38,167 & 46,112 & 47,686 & 48,320 \\
\hline 1989 & & & & & $1,200.0$ & 9,964 & 40,371 & 48,774 & 50,439 & 51,110 \\
\hline 1990 & & & & & $1,200.0$ & 10,426 & 42,412 & 51,240 & 52,989 & 53,693 \\
\hline 1991 & & & & & $1,200.0$ & 10,854 & 44,301 & 53,523 & 55,349 & 56,086 \\
\hline 1992 & & & & & $1,200.0$ & 11,250 & 46,051 & 55,636 & 57,535 & 58,301 \\
\hline
\end{tabular}

Capacity-Capital Stock Regression: Code O RSO 0.940

Coefficients: Const $\mathbf{- 3 6 2 9 . 7}$ Stock 4.4

Ave. Capecity/Stock $(74-86): 3.8$ Ave. Weekly Hours (High) 125.1 Shift Factor 1.27 Ave Weekly Hours (Low) 118.8 Shift Factor 1.33 Note: Dutput, Investment, and Capital Stock extrapolated for 1987 and 1988.

\section{Commercial printing, lithographic}

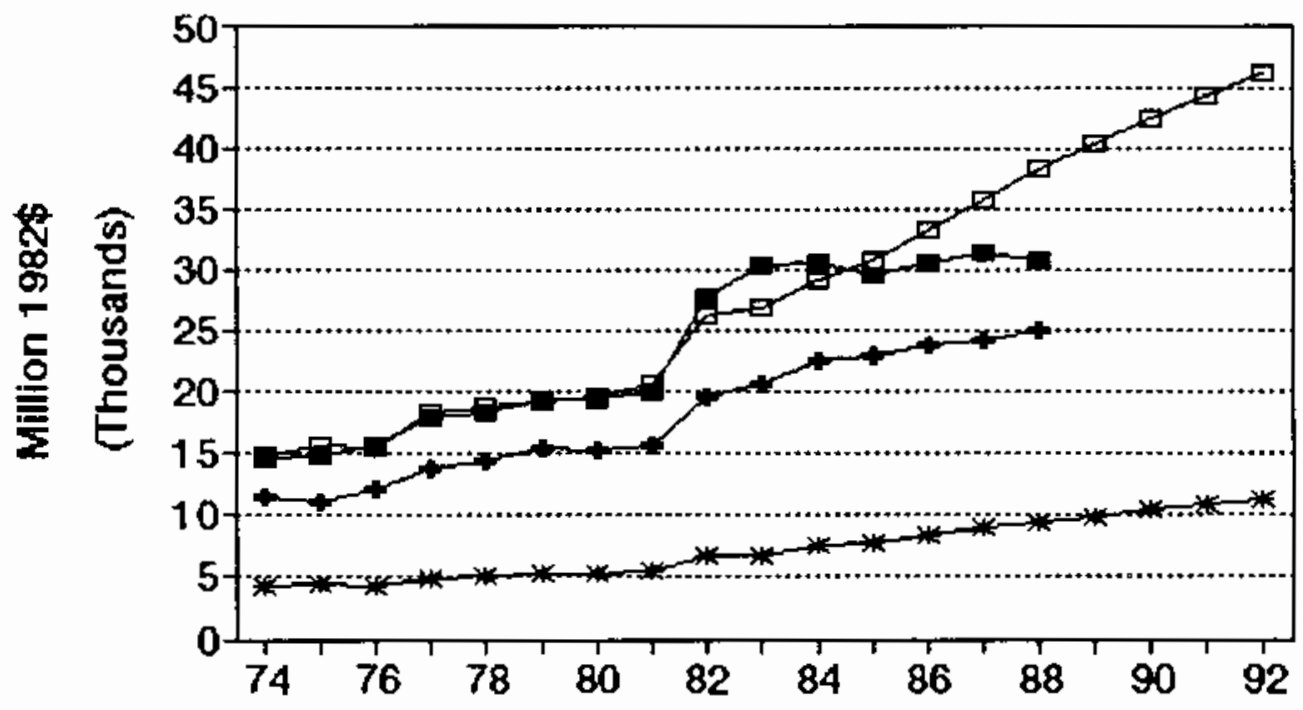

+-Ouput - Implied Capacity - Cepital Stock \&-Pred. Cepecity 


\section{No. 169 SIC 2753 Engraving and plate printing}

MILLIONS OF 1982 DOLLARS (EXCL. CU RATES)

$\begin{array}{lccccccc} & 04 & \text { Ann. } & \text { Implied } & \text { Gross } & \text { Het } & \text { Pred. } & \ldots \ldots \ldots \\ \text { Gross } & \text { CU } & \text { CU } & \text { Prac. } & \text { In- } & \text { Cap. } & \text { Prac. } & \text { Emergency Capacity } \\ \text { Output } & \text { Rate } & \text { Rate } & \text { Capac. } & \text { vest. } & \text { Stk. } & \text { Capac. } & 3 \text { mon. } 6 \text { mon. }>6 \text { mon. }\end{array}$

\begin{tabular}{|c|c|c|c|c|c|c|c|c|c|c|}
\hline 1974 & 452 & 83 & 83.0 & 545 & 6.4 & 128 & 543 & & & \\
\hline 1975 & 418 & 83 & 83.0 & 503 & 9.2 & 127 & 537 & & & \\
\hline 1976 & 453 & 83 & 83.0 & 546 & 13.1 & 128 & 542 & & & \\
\hline 1977 & 462 & 68 & 73.6 & 627 & 30.8 & 146 & 603 & & & \\
\hline 1978 & 483 & 68 & 68.0 & 711 & 27.2 & 160 & 649 & & & \\
\hline 1979 & 413 & 72 & 70.5 & 586 & $(21.0)$ & 128 & 543 & & & \\
\hline 1980 & 380 & 76 & 74.5 & 510 & 10.9 & 128 & 541 & & & \\
\hline 1981 & 373 & 80 & 78.5 & $4 \pi 5$ & 12.3 & 128 & 541 & & & \\
\hline 1982 & 597 & 84 & 82.5 & 724 & 93.2 & 199 & $\pi$ & & & \\
\hline 1983 & 579 & 88 & 86.5 & 669 & $(10.9)$ & 167 & 672 & & & \\
\hline 1984 & 564 & 91 & 89.9 & 627 & 16.3 & 163 & 658 & & & \\
\hline 1985 & 583 & 94 & 92.9 & 628 & 11.9 & 157 & 637 & & & \\
\hline 1986 & 613 & 78 & 84.0 & 730 & 18.1 & 157 & 639 & & & \\
\hline$\cdots$ & $-\cdot-\cdot$ & -- & $\because-$ & $\cdot-$ & - - - - & $\cdots$ & $\cdots$ & 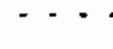 & $-\cdot \cdot$ & $\cdots$ \\
\hline 1987 & 618 & 86 & 83.0 & 744 & 21.0 & 160 & 649 & & & \\
\hline 1989 & 624 & 78 & 81.0 & 770 & 22.5 & 165 & 664 & 1,125 & 1,162 & 1,177 \\
\hline 1989 & & & & & 25.2 & 171 & 686 & 1,162 & 1,200 & 1,216 \\
\hline 1990 & & & & & 24.8 & 176 & 703 & 1,192 & 1,231 & 1,247 \\
\hline 1991 & & & & & 24.7 & 181 & 719 & 1,218 & 1,258 & 1,275 \\
\hline 1992 & & & & & 23.6 & 184 & 729 & 1.235 & 1,276 & 1,293 \\
\hline
\end{tabular}

Capecity-Capital Stock Regression: Code 0 RSO 0.730 Coefficients: Const 116.2 stock 3.3

Ave. Capacity/\$tock $(74-86): 4.1$ Ave. Heekly Hours (High) 88.8 Shift Factor 1.77 Ave Weekly Hours (Lon) 86.5 Shift Factor 1.82 Note: Output, Investment, and Capital stock extrapolated for 1987 and 1988.

\section{Engraving and plate printing}

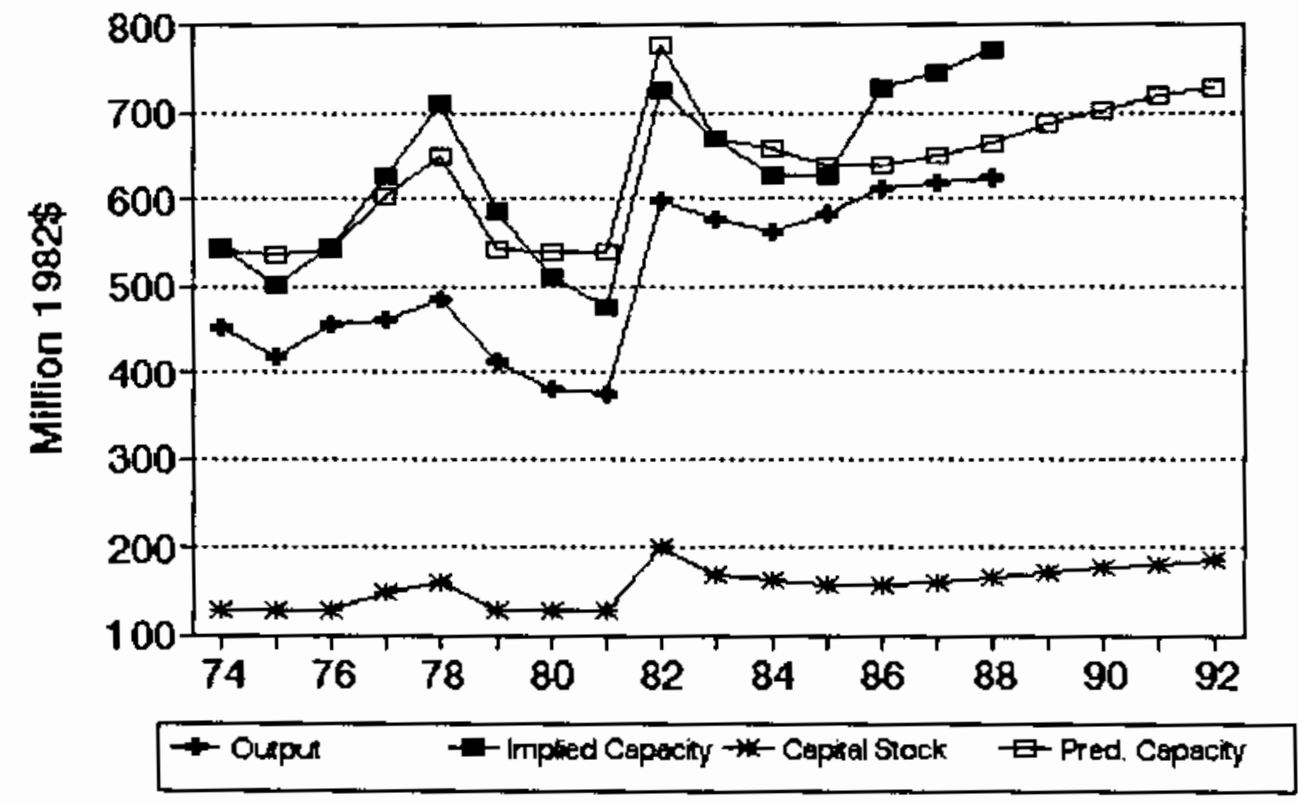




\section{No. 170 src 2754 conmercial printing, gravure}

MILLIONS OF 1982 doLLARS (EXCL. CU RATES)

$\begin{array}{lccccccc} & \text { Q4 } & \text { Am. } & \text { Implied } & \text { Gross } & \text { Net } & \text { Pred. } & \ldots \\ \text { Gross } & \text { CU } & \text { CU } & \text { Prac. } & \text { In- } & \text { Cap. } & \text { Prac. } & \text { Emergency Capacity } \\ \text { Output } & \text { Rate } & \text { Rate } & \text { Capac. } & \text { vest. } & \text { Stk. } & \text { Capac. } & 3 \text { mon. } 6 \text { mon. }>6 \text { mon. }\end{array}$

\begin{tabular}{|c|c|c|c|c|c|c|c|c|c|c|}
\hline 1974 & 1,633 & 69 & 70.4 & 2,320 & 26.9 & 798 & 1,935 & & & \\
\hline 1975 & 1,432 & 81 & 74.0 & 1,935 & 80.2 & 805 & 1,950 & & & \\
\hline 1976 & 1,542 & 84 & 83.0 & 1,859 & 34.7 & 769 & 1,874 & & & \\
\hline 1977 & 1,550 & 81 & 82.8 & 1,873 & 125.5 & 822 & 1,986 & & & \\
\hline 1978 & 1,738 & 82 & 81.9 & 2,123 & 137.8 & 891 & 2,127 & & & \\
\hline 1979 & 1,745 & 79 & 80.1 & 2,178 & 182.1 & 998 & 2,349 & & & \\
\hline 1980 & 1,784 & 88 & 84.6 & 2,110 & 73.6 & $\$ 1$ & 2,336 & & & \\
\hline 1981 & 2,101 & 84 & 85.6 & 2,454 & 207.5 & 1,106 & 2,574 & & & \\
\hline 1982 & 2,198 & $n$ & 76.0 & 2,891 & 216.9 & 1,213 & 2,796 & & & \\
\hline 1983 & 2,493 & 80 & 76.8 & 3,247 & 372.5 & 1,456 & 3,300 & & & \\
\hline 1984 & 2,688 & $\pi$ & 78.9 & 3,407 & 34.4 & 1,370 & 3,120 & & & \\
\hline 1985 & 2,809 & 84 & 80.7 & 3,593 & 288.4 & 1,523 & 3,439 & & & \\
\hline 1986 & 2,883 & B6 & 85.1 & 3,389 & 212.1 & 1,597 & 3,593 & & & \\
\hline$\therefore$ & & $\because$ & $-\cdot$ &..- &.- & $\therefore-$ & $\cdots$ & $\cdot-$ & $-\cdot$ & $\cdots$ \\
\hline 1987 & 2,929 & 85 & 85.6 & 3,424 & 241.6 & 1,699 & 3,804 & & & \\
\hline 1988 & $2, \infty 91$ & 83 & 83.8 & 3,571 & 272.6 & 1,823 & 4,061 & 4,133 & 4,218 & 4,252 \\
\hline 1989 & & & & & 329.9 & 1,993 & 4,414 & 4,493 & 4,585 & 4,622 \\
\hline 1990 & & & & & 319.8 & 2,139 & 4,716 & 4,800 & 4,898 & 4,938 \\
\hline 1991 & & & & & 318.6 & 2,270 & 4,989 & 5,078 & 5,181 & 5,223 \\
\hline 1992 & & & & & 295.9 & 2,368 & 5,191 & 5,283 & 5,391 & 5,435 \\
\hline
\end{tabular}

Capacity-Capital stock Regression: Code 0 RSO 0.920 Coefficients: Const 279.7 stock 2.1 Ave. Weekly Hours (High) 152.4 Shift Factor 1.05 Ave Weekly Hours (Low) 147.6 Shift factor 1.08 Note: Output, Investment, and Capital Stock extrapolated for 1987 and 1988.

\section{Commercial printing, gravure}

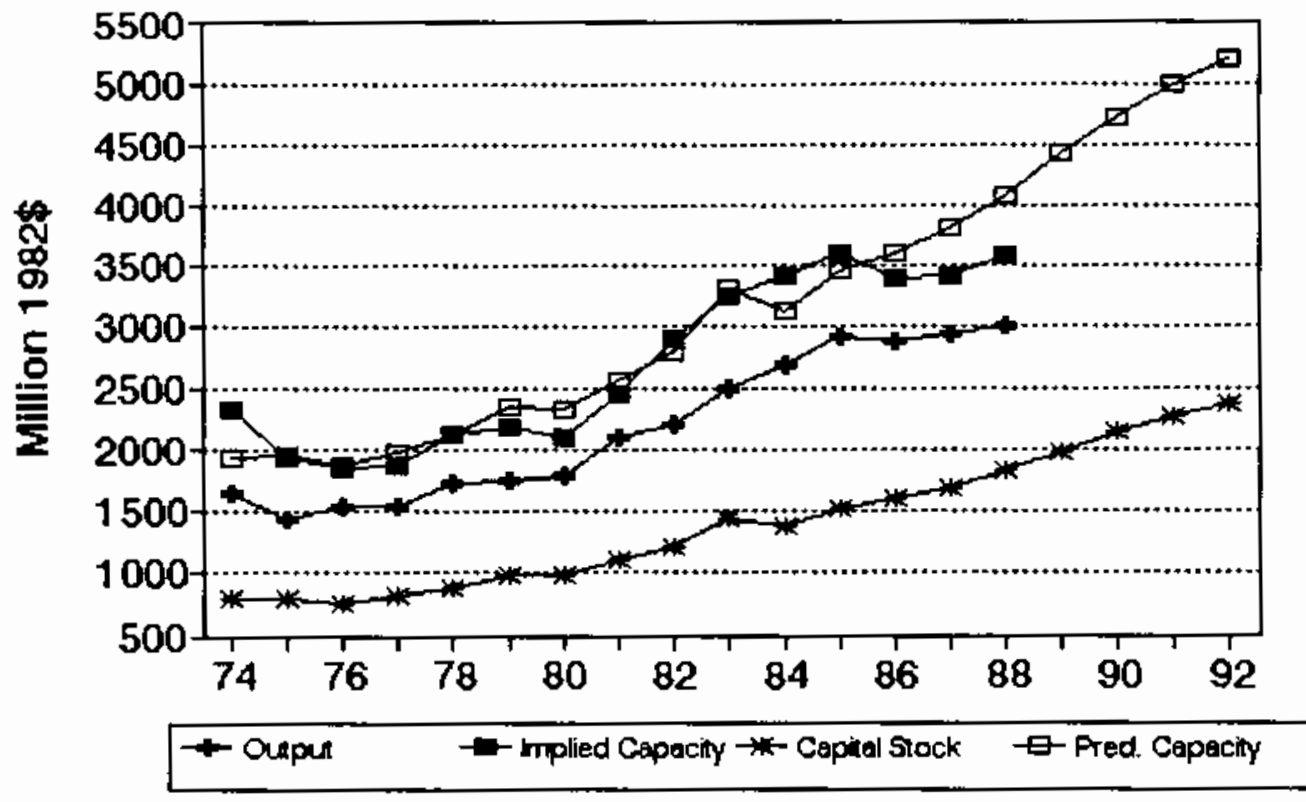




\section{Ho, 171 sIC 2761 Manifold business forms}

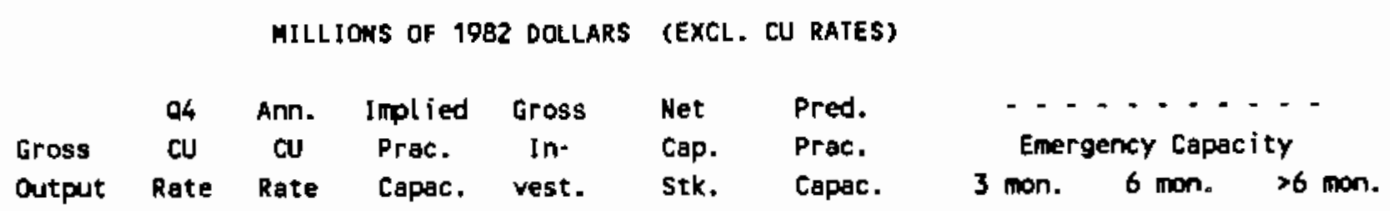

\begin{tabular}{|c|c|c|c|c|c|c|c|c|c|c|}
\hline 1974 & 4,438 & 73 & 74.3 & 5,972 & 144.2 & 1,167 & 4,920 & & & \\
\hline 1975 & 3,643 & $\pi$ & 70.2 & 5,187 & 129.9 & 1,208 & 5,249 & & & \\
\hline 1976 & 3,713 & 80 & 7.0 & 4,821 & 82.8 & 1,195 & 5,147 & & & \\
\hline 1977 & 4,376 & 73 & 76.2 & 5,745 & 230.2 & 1,314 & 6,112 & & & \\
\hline 1978 & 4,641 & 88 & 02.6 & 5,621 & 149.0 & 1,346 & 6,372 & & & \\
\hline $19 \pi 9$ & 4,980 & 83 & 86.9 & 5,868 & 74.2 & 1,304 & 6,031 & & & \\
\hline 1980 & 4,842 & 79 & 80.4 & 6,020 & 147.8 & 1,333 & 6,263 & & & \\
\hline 1981 & 4,933 & 77 & 77.8 & 6,338 & 192.2 & 1,394 & 6,761 & & & \\
\hline 1982 & 5,126 & $\pi$ & 77.9 & 6,578 & 163.8 & 1,419 & 6,958 & & & \\
\hline 1983 & 5,249 & 85 & 82.6 & 6,359 & 59.5 & 1,340 & 6,316 & & & \\
\hline 1986 & 5,842 & 77 & 80.7 & 7,239 & 207.2 & 1,402 & 6,822 & & & \\
\hline 1985 & 6,061 & $\pi$ & 77.7 & 7,804 & 177.5 & 1,435 & 7,091 & & & \\
\hline 1986 & 6,282 & 78 & 78.2 & 8,031 & 200.8 & 1,491 & 7,542 & & & \\
\hline $\begin{array}{l}--\cdot \\
1987\end{array}$ & 6,374 & $\dot{80}$ & $\begin{array}{l}\cdots \\
79.4\end{array}$ & 8,029 & - - & $\cdots$ & $7, \operatorname{283}$ & $=--$ & $\cdots-$ & $\cdots$ \\
\hline 1988 & 6,496 & 74 & 76.3 & 8,520 & 203.7 & 1,585 & 8,304 & 10,143 & 10,413 & 10,521 \\
\hline 1989 & & & & & 229.7 & 1,658 & 8,895 & 10,865 & 11,153 & 11,270 \\
\hline 1990 & & & & & 225.2 & 1,719 & 9,390 & 11,470 & $11, \pi$ & 11,897 \\
\hline 1991 & & & & & 224.6 & 1,773 & 9,832 & 12,009 & 12,328 & 12,457 \\
\hline 1992 & & & & & 214.3 & 1,812 & 10,146 & 12,393 & 12,723 & 12,855 \\
\hline
\end{tabular}

Capacity-Capital Stock Regression: code 1 RSO 0.700 Coefficients: Const -4541.8 stock 8.1 Ave. Weekly Hours (High) 124.9 Shift Factor 1.27 Ave Weekly Hours (LOU) 122.2 Shift Factor 1.29 Note: Output, Investment, and Capital stock extrapoleted for 1987 and 1988. 2761 Manifold business forms

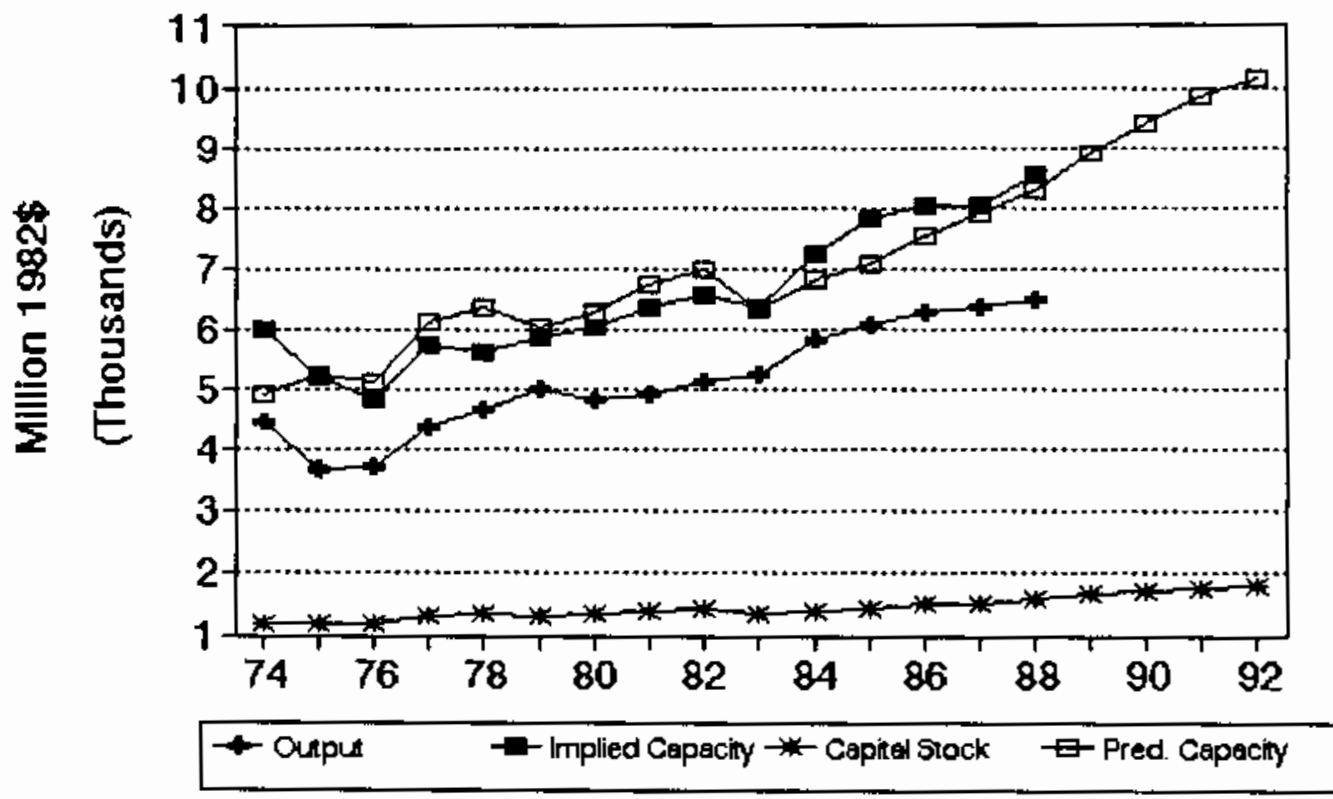




\section{No. 172 8IC 2771 Greeting card publishing}

MILLIONS OF 1982 DOLLARS (EXCL. CU RATES)

$\begin{array}{lccccccc} & \text { Q4 } & \text { Ann. } & \text { Implied Gross } & \text { Het } & \text { Pred. } & -\ldots \\ \text { Gross } & \text { cu } & \text { CU } & \text { Prac. } & \text { In- } & \text { Cap. } & \text { Prac. } & \text { Emergency Capecity } \\ \text { Output } & \text { Rate } & \text { Rate } & \text { Capec. } & \text { vest. } & \text { Stk. } & \text { Capec. } & 3 \text { mon. } 6 \text { mon. }>6 \text { mon. }\end{array}$

\begin{tabular}{|c|c|c|c|c|c|c|c|c|c|c|}
\hline 1974 & 1,486 & 72 & 73.0 & 2,036 & $(32.4)$ & 348 & 2,672 & & & \\
\hline 1975 & 1,322 & 72 & 70.4 & 1,878 & $(25.4)$ & 301 & 2,218 & & & \\
\hline 1976 & 1,355 & 76 & 74.5 & 1,818 & $(20.3)$ & 261 & 1,835 & & & \\
\hline 1977 & 1,455 & 76 & 76.4 & 1,905 & 28.4 & 266 & 1,890 & & & \\
\hline 1978 & 1,597 & 81 & 79.3 & 2,014 & 34.2 & 275 & 1,975 & & & \\
\hline 1979 & 1,700 & 88 & 85.4 & 1,991 & 22.2 & 273 & 1,957 & & & \\
\hline 1980 & 1,782 & 84 & 85.4 & 2,085 & 59.7 & 306 & 2,266 & & & \\
\hline 1981 & 1,977 & 82 & 82.8 & 2,387 & 48.7 & 326 & 2,460 & & & \\
\hline 1992 & 1,891 & 84 & 83.0 & 2,279 & $(6.9)$ & 293 & 2,145 & & & \\
\hline 1983 & 2,265 & 82 & 82.7 & 2,740 & 42.2 & 307 & 2,278 & & & \\
\hline 1984 & 2,275 & 81 & 81.9 & $2, T 77$ & 87.1 & 362 & 2,805 & & & \\
\hline 1985 & 2,555 & $84 *$ & 82.7 & 3,092 & 36.3 & 364 & 2,825 & & & \\
\hline 1986 & 2,516 & 81 & 82.2 & 3,063 & 25.2 & 355 & 2,737 & & & \\
\hline$-\cdot$ & $\cdot-\cdot$ & $\cdot \cdot$ & $\cdot-\cdot$ & $\cdots$ & $\cdots$ & $\because-$ & $\cdots$ & $-\cdots$ & $\cdot-$ & $-\cdots$ \\
\hline 1987 & 2,554 & 81 & 81.1 & 3,149 & 58.7 & 380 & 2,975 & & & \\
\hline 1988 & 2,604 & $84 *$ & 83.2 & 3.131 & 70.0 & 413 & 3,298 & 4,228 & 4,348 & 4,396 \\
\hline 1989 & & & & & 94.0 & 465 & 3,792 & 4,860 & 4,909 & 5,054 \\
\hline 1990 & & & & & 87.3 & 508 & 4,203 & 5,387 & 5,541 & 5,602 \\
\hline 1991 & & & & & 86.9 & 546 & 4,570 & 5,859 & 6,025 & 6,092 \\
\hline 1992 & & & & & 78.6 & 573 & 4,823 & 6,183 & 6,359 & 6,429 \\
\hline
\end{tabular}

Capecity-Capital stock Regression: Code 1 RSQ 0.610

Coefficients: Const -658.9 Stock 9.6

Ave. Capacity/Stock $(74-86): 7.4$

Ave. Heekly Hours (High) 120.0 Shift Factor 1.33 Ave Heekly Hours (LoW) 120.0 Shift Factor 1.33

* Imputed Note: Output, Investment, and Capital Stock extrapolated for 1987 and 1988.

\section{Greeting card publishing}
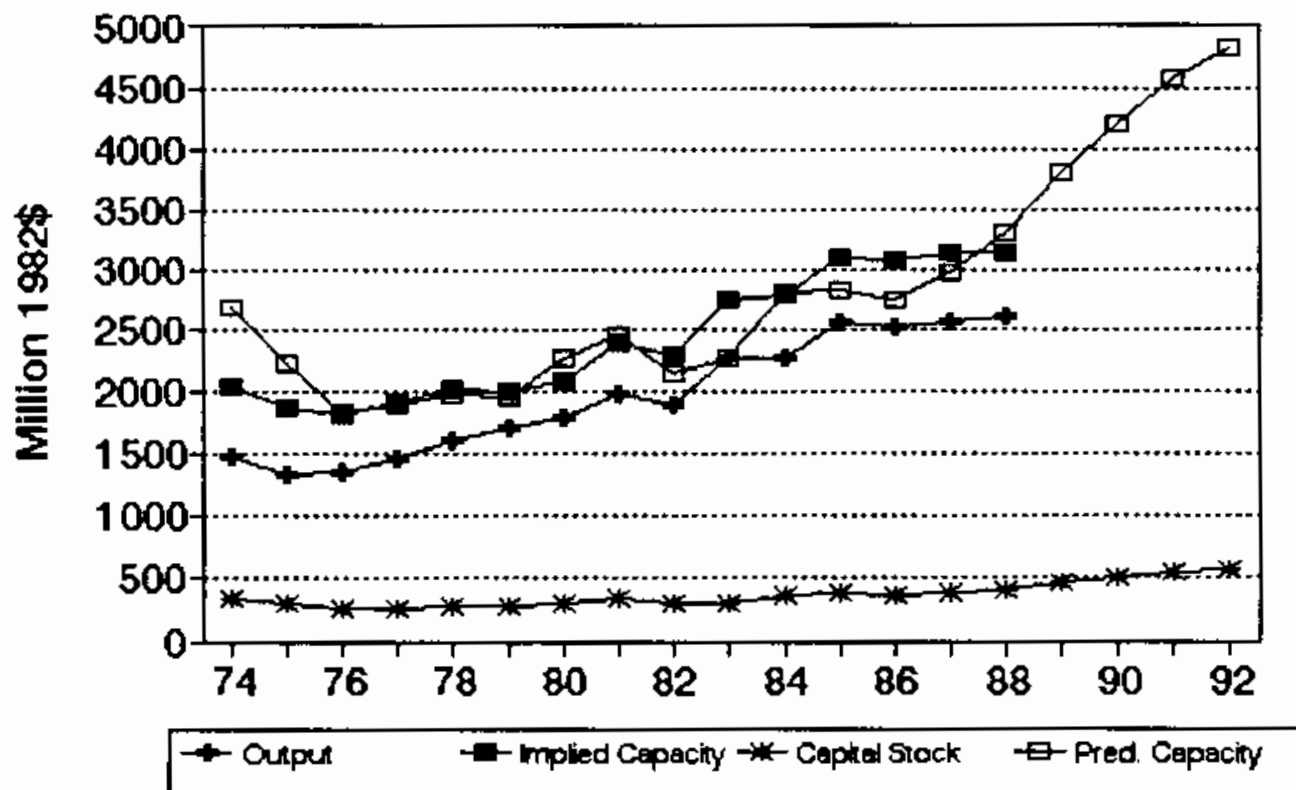
No. 173 sIC 2782 Blankbooks and looseleaf binders

\begin{tabular}{|c|c|c|c|c|c|c|c|}
\hline & & MILL & DNS OF 19 & 2 DOLLARS & GEXCL. & U RATES) & \\
\hline ross & $\begin{array}{l}\text { Q4 } \\
\mathrm{CU}\end{array}$ & $\begin{array}{c}\text { Ann. } \\
\text { CU }\end{array}$ & $\begin{array}{l}\text { Implied } \\
\text { Prac. }\end{array}$ & $\begin{array}{l}\text { Gross } \\
\text { In* }\end{array}$ & $\begin{array}{l}\text { Het } \\
\text { Cap. }\end{array}$ & $\begin{array}{l}\text { Pred. } \\
\text { Prac. }\end{array}$ & $\begin{array}{l}- \\
\text { Energency Capaci ty }\end{array}$ \\
\hline utput & Rate & Rate & Capac. & vest. & sek. & Capec. & 3 mon. $\quad 6$ mon. $>6$ mon. \\
\hline
\end{tabular}

\begin{tabular}{|c|c|c|c|c|c|c|c|c|c|c|}
\hline 1974 & 1,338 & 78 & 79.4 & 1,685 & 20.7 & 300 & 1,394 & & & \\
\hline 1975 & 1,292 & $\pi$ & 73.8 & $1, \pi 50$ & 40.6 & 315 & 1,582 & & & \\
\hline 1976 & 1,361 & 82 & 79.4 & 1,714 & 25.8 & 314 & 1.574 & & & \\
\hline 1977 & 1,672 & 85 & 84.4 & 1,981 & 103.8 & 385 & 2,443 & & & \\
\hline 1978 & 1,798 & 88 & 87.1 & 2,065 & 42.6 & 394 & 2,555 & & & \\
\hline 1979 & 1,846 & 74 & 79.2 & 2,330 & 42.3 & 403 & 2,663 & & & \\
\hline 1980 & 1,950 & 71 & 72.1 & 2,706 & 70.7 & 438 & 3,093 & & & \\
\hline 1981 & 1,917 & 71 & 71.1 & 2,696 & 16.7 & 420 & 2,865 & & & \\
\hline 1982 & 2,066 & 62 & 65.0 & 3,177 & 46.0 & 429 & 2,973 & & & \\
\hline 1983 & 2,298 & 67 & 65.0 & 3,537 & 50.2 & 440 & 3,109 & & & \\
\hline 1984 & 2,106 & 63 & 65.1 & 3,236 & 43.0 & 444 & 3,158 & & & \\
\hline 1985 & 2,250 & 58 & 59.4 & 3,789 & 66.6 & 469 & 3,465 & & & \\
\hline 1986 & 2,310 & 62 & 60.4 & 3,825 & 55.3 & 481 & 3,621 & & & \\
\hline$\cdots$ & & $\cdot-$ & $-\cdot$ & - - - - & $\because$ & $\cdots$ & $\cdots$ & $-\cdot$ & $\cdots-$ & $-\cdots$ \\
\hline 1987 & 2,348 & 60 & 60.9 & 3,858 & 59.2 & 498 & 3,826 & & & \\
\hline 1988 & 2,399 & 67 & 64.4 & 3,726 & 63.5 & 517 & 4,062 & 7.166 & 7,518 & 7,661 \\
\hline 1989 & & & & & 71.6 & 543 & 4,377 & 7,723 & 8,102 & 8,256 \\
\hline 1990 & & & & & 70.1 & 565 & 4,648 & 8,200 & 8,603 & 8,765 \\
\hline 1991 & & & & & 70.0 & 585 & 4,892 & 8,630 & 9.055 & 9,226 \\
\hline 1992 & & & & & 66.8 & 600 & 5,076 & 8,955 & 9,395 & 9,573 \\
\hline
\end{tabular}

Capacity-Capital stock Regression: Code 0 RSO 0.830

Coefficients: Const -2276.4 stock 12.3

Ave. Capacity/Stock $(74-86): 6.6$ Ave. Weekly Hours (High) 83.5 Shift Factor 1.89 Ave Weekly Hours (Low) 80.3 Shift Factor 1.96 Note: Output, Investment, and Capital Stock extrapolated for 1987 and 1988.

\section{Blankbooks and looseleaf binders}

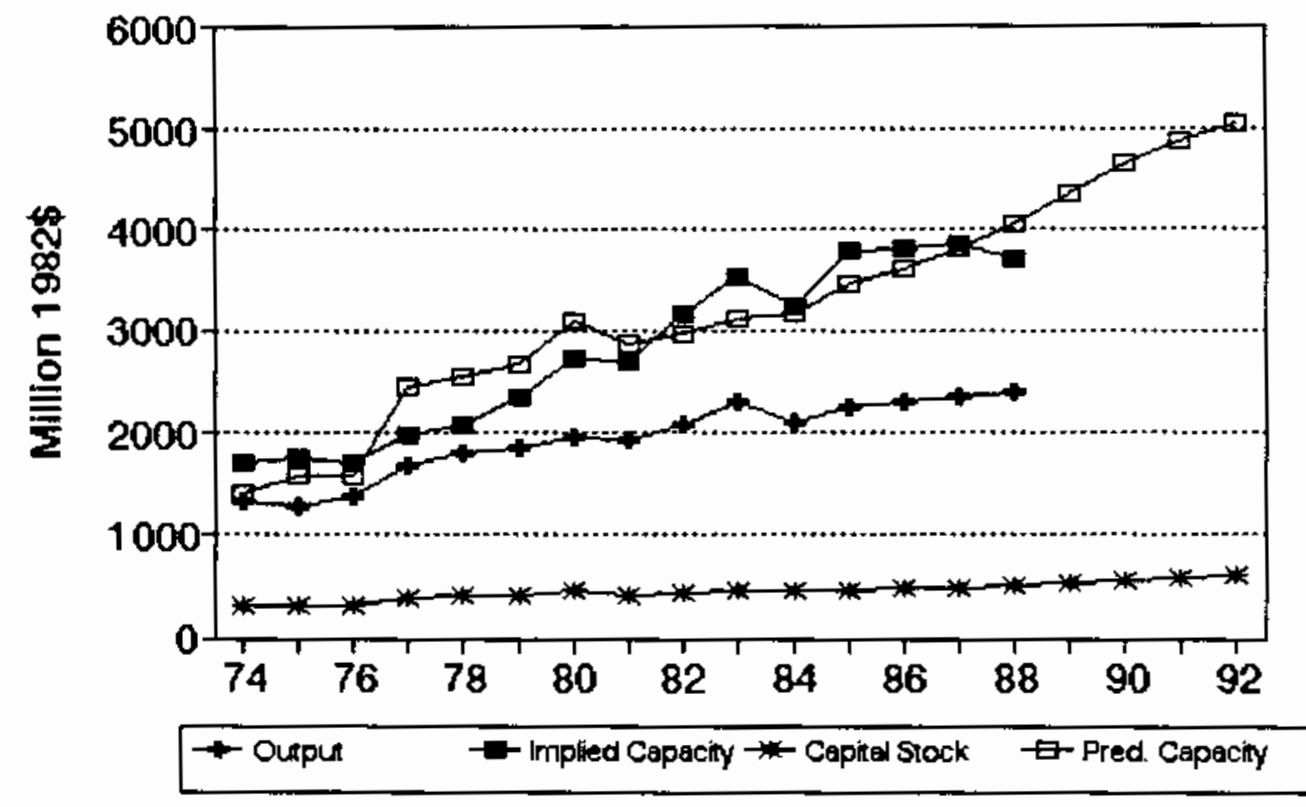




\section{No. 174 SIC 2789 Bookbinding and related work}

MILLIONS OF 1982 DOLLARS (EXCL. CU RATES)

$\begin{array}{lccccccc} & 04 & \text { Ann. Implied Gross } & \text { Het } & \text { Pred. } & \ldots \ldots \ldots \ldots \\ \text { Gross } & \text { CU } & \text { CU } & \text { Prac. } & \text { In- } & \text { Cap. } & \text { Prac. } & \text { Emergency Capacity } \\ \text { Output } & \text { Rate } & \text { Rate } & \text { Capac. } & \text { vest. } & \text { Stk. } & \text { Capac. } & 3 \text { mon. } 6 \text { mon. }>6 \text { mon. }\end{array}$

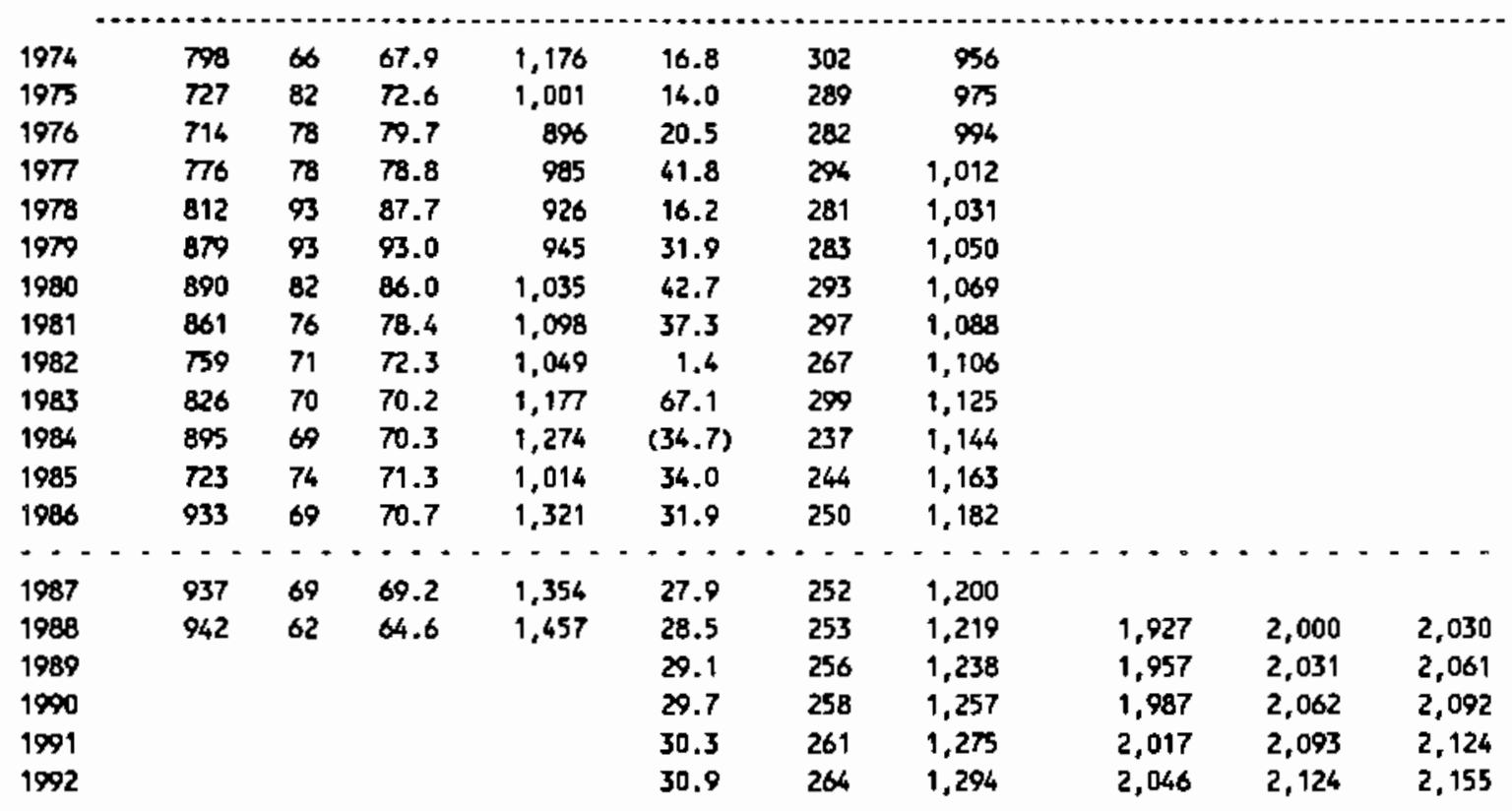

Capecity-Capital Stock Regression: Code -2 RSQ 0.300

Coefficients: Const 937.3 Time 18.8 Ave. Capacity/Stock (74-86): 3.8 Ave. Weekly Hours (High) 94.6 Shift Factor 1.66 Ave Veekly Hours (Low) 93.8 Shift Factor 1.68 Note: Output, Investment, and Capital Stock extrapolated for 1987 and 1988.

\section{Bookbinding and related work}

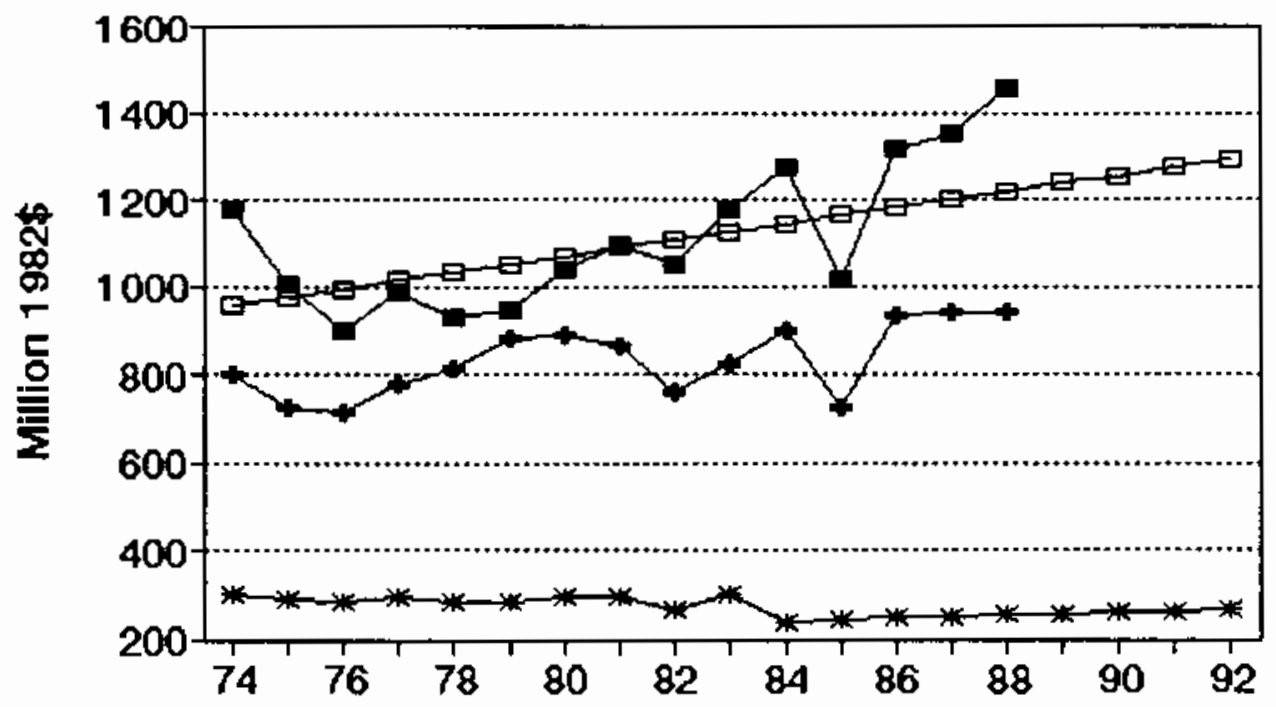

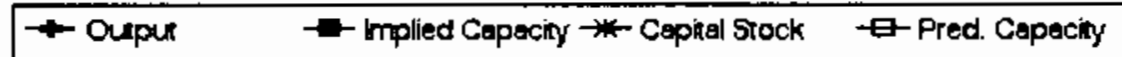




\section{No. 175 sIC 2791 TYpesetting}

MILLIONS OF 1982 DOLLARS (EXCL. CU RATES)

$\begin{array}{lccccccc} & \text { Q4 } & \text { Ann. Implied } & \text { Gross } & \text { Met } & \text { Pred. } & \ldots \ldots \ldots & \ldots \\ \text { Gross } & \text { CU } & \text { CU } & \text { Prac. } & \text { In- } & \text { Cap. } & \text { Prac. } & \text { Emergency Capacity } \\ \text { Output } & \text { Rate } & \text { Rete } & \text { Capac. } & \text { vest. } & \text { Stk. } & \text { Capac. } & 3 \text { mon. } 6 \text { mon. }>6 \text { mon. }\end{array}$

\begin{tabular}{|c|c|c|c|c|c|c|c|c|c|c|}
\hline 1974 & 796 & 69 & 69.0 & 1,153 & 74.6 & 377 & 1,272 & & & \\
\hline 1975 & 726 & 83 & 77.8 & 934 & $(6.9)$ & 344 & 1,108 & & & \\
\hline 1976 & 767 & 69 & 74.3 & 1,033 & 18.9 & 338 & 1,077 & & & \\
\hline 1977 & 1,021 & 49 & 56.5 & 1,807 & 91.8 & 399 & 1,379 & & & \\
\hline 1978 & 1,027 & 86 & 59.6 & 1,723 & 75.7 & 440 & 1,584 & & & \\
\hline 1979 & 1,099 & 87 & 79.1 & 1,389 & $(20.5)$ & 391 & 1,338 & & & \\
\hline 1980 & 1,191 & 77 & 80.8 & 1,474 & 53.1 & 413 & 1,449 & & & \\
\hline 1981 & 1,132 & 83 & 80.8 & 1,402 & 20.6 & 403 & 1,400 & & & \\
\hline 1982 & 1,273 & 78 & 79.9 & 1,594 & 86.5 & 453 & 1,648 & & & \\
\hline 1983 & 1,353 & $84 *$ & 81.9 & 1,653 & 28.1 & 445 & 1,609 & & & \\
\hline 1984 & 1,360 & 88 & 86.6 & 1,571 & 57.9 & 464 & 1,703 & & & \\
\hline 1985 & 1,368 & $\infty 6$ & 86.8 & 1,577 & 34.5 & 460 & 1,683 & & & \\
\hline 1986 & 1,451 & $87 \star$ & 86.7 & 1,674 & 49.7 & 470 & 1,734 & & & \\
\hline$\cdots$ & & -- & $\cdots$ & $\cdots-$ & $\cdots$ & $\cdots$ & $\cdots$ & -- & $-\cdots$ & $\cdots$ \\
\hline 1987 & 1,472 & 73 & 78.3 & 1,880 & 50.6 & 482 & 1,700 & & & \\
\hline 1988 & 1,500 & 76 * & 75.0 & 2,000 & 53.5 & 495 & 1,855 & 2,099 & 2,143 & 2,161 \\
\hline 1989 & & & & & 58.7 & 512 & 1,940 & 2,196 & 2,242 & 2,261 \\
\hline 1990 & & & & & 57.8 & 527 & 2,014 & 2,279 & 2,327 & 2,347 \\
\hline 1991 & & & & & 57.7 & 540 & 2,081 & 2,355 & 2,405 & 2,425 \\
\hline 1992 & & & & & 55.6 & 551 & 2,133 & 2,413 & 2,464 & 2,484 \\
\hline
\end{tabular}

Capacity-Capital Stock Regression: Code 0 RSO 0.670

Coefficients: Const -603.3 stock 5.0

Ave. Capacity/Stock $(74-86): 3.5$ Ave. Weekly Hours (High) 136.3 Shift Factor 1.16 Ave Weekly Hours (Low) 87.7 Shift Factor 1.79 * Imputed Note: Output, Investment, and Capital Stock extrapolated for 1987 and 1988.

\section{Typesetting}

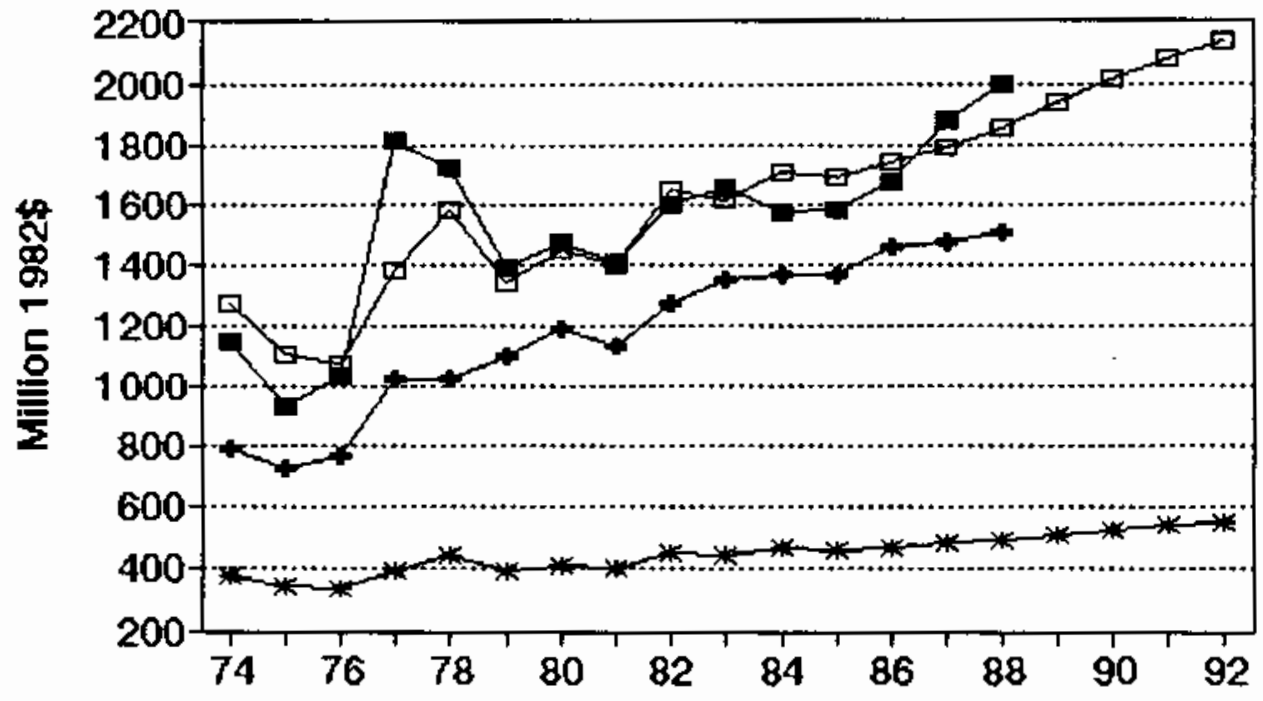

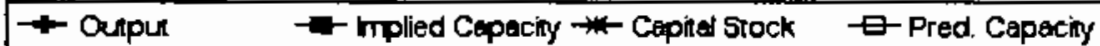


No. 176 SIC 2793 Photoongraving, electrotyping and stereotyping MILLIONS OF 1982 DOLLARS (EXCL. CU RATES)

\begin{tabular}{|c|c|c|c|c|c|c|c|}
\hline & $\begin{array}{l}04 \\
\text { CU }\end{array}$ & $\begin{array}{l}\text { Ann. } \\
\text { cu }\end{array}$ & $\begin{array}{l}\text { Implied } \\
\text { Prac. }\end{array}$ & $\begin{array}{l}\text { Gross } \\
\text { In- }\end{array}$ & $\begin{array}{l}\text { Net } \\
\text { Cop. }\end{array}$ & $\begin{array}{l}\text { Pred. } \\
\text { Prac. }\end{array}$ & $\begin{array}{c}- \\
\text { Emergency Capacity }\end{array}$ \\
\hline utput & Rate & Rate & Capac. & vest. & stk. & Capac. & \\
\hline
\end{tabular}

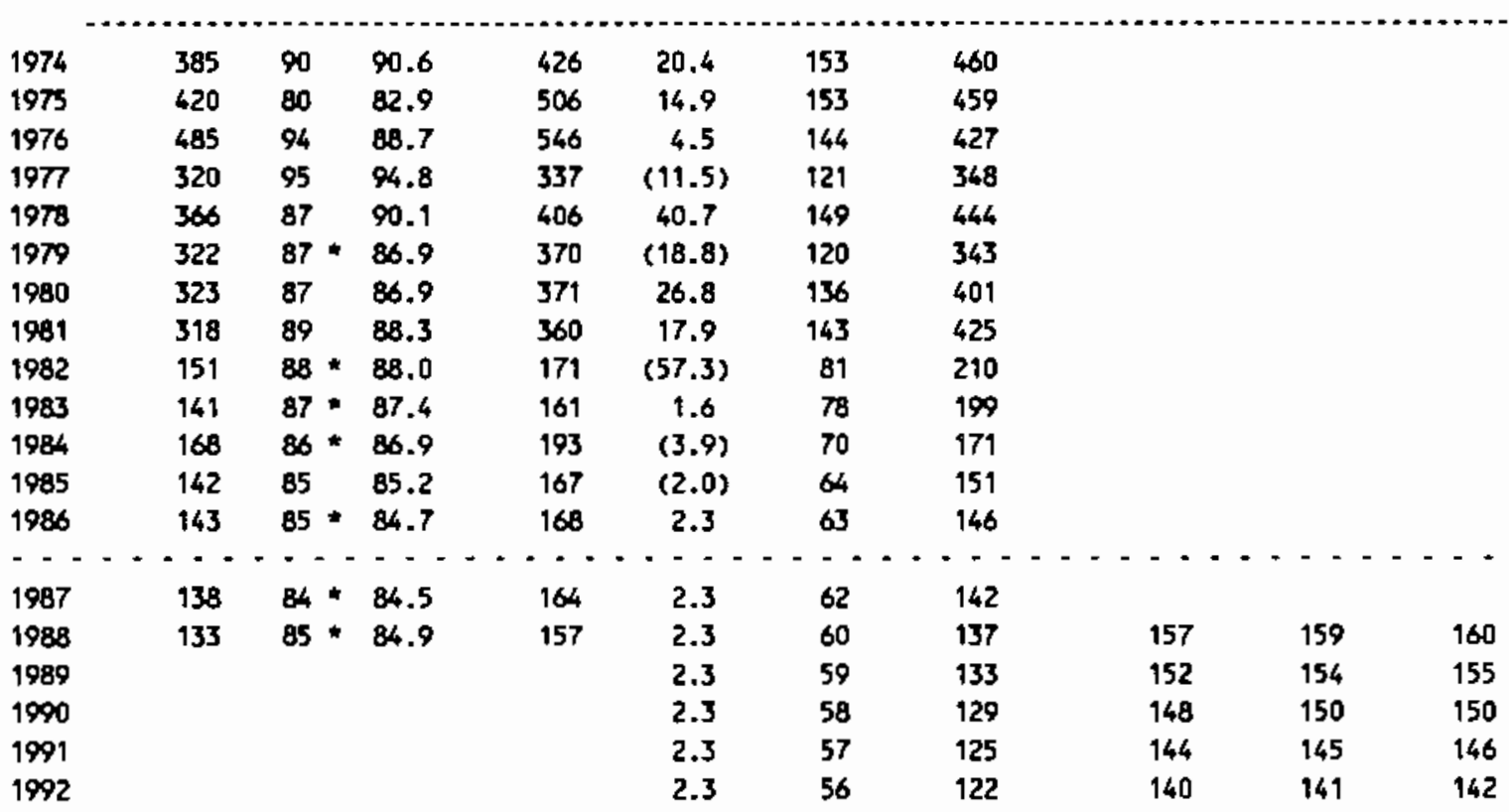

Capacity-Capital Stock Regression: Code 0 RSO 0.870 Coefficients: Const $\cdot 72.4$ stock 3.5

Ave. Capacity/Stock $(74-86): 2.8$ Ave. Weekly Hours (High) 136.3 Shift Factor 1.16 Ave Heekly Hours (Low) 87.7 Shift Factor 1.79 * Imputed Wote: Output, Investment, and Capital Stock extrapolated for 1987 and 1988.

\section{Photoengraving, electrotyping and}

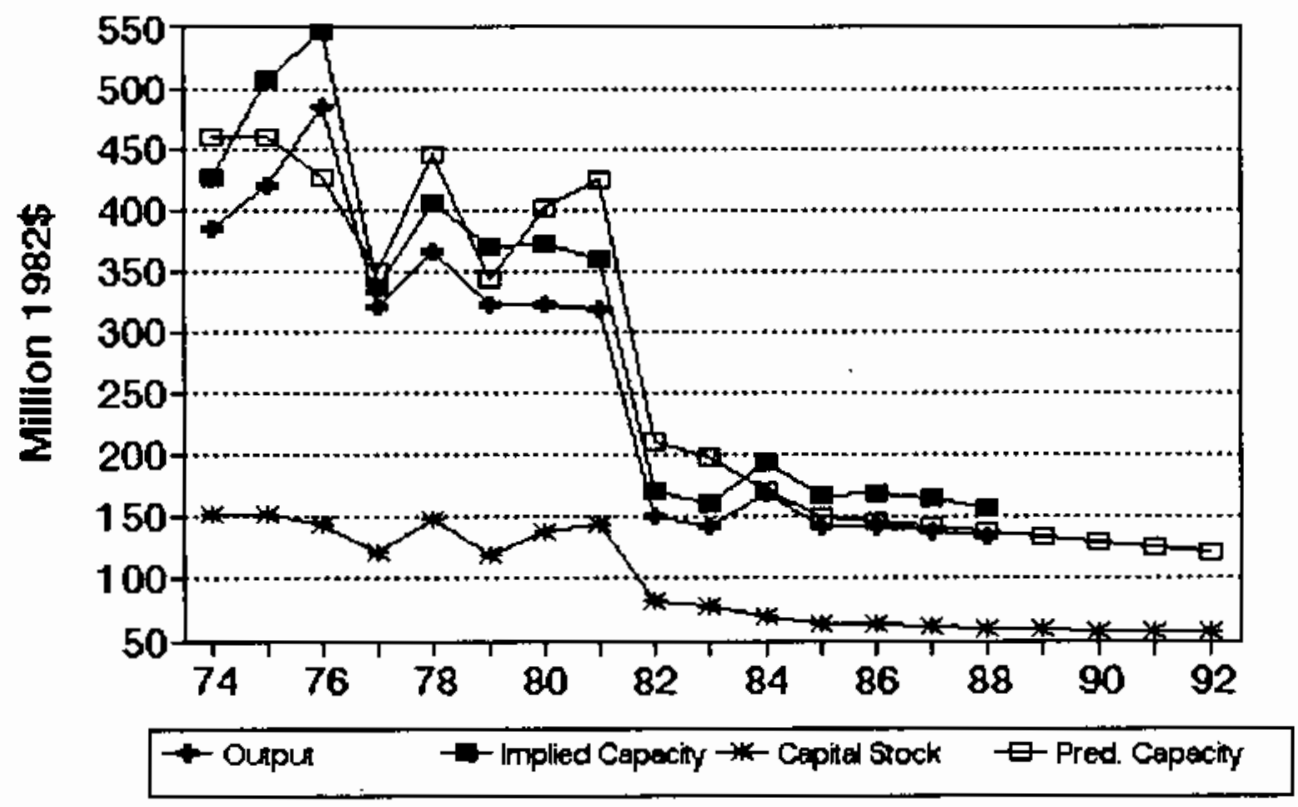


No. 177 sIC 2795 dithographic platemaking services

MILLIONS OF 1982 DOLLARS (EXCL. CU RATES)

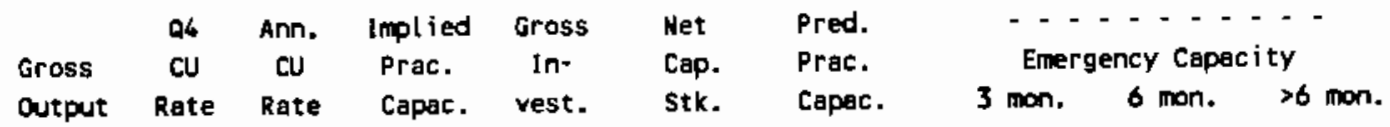

\begin{tabular}{|c|c|c|c|c|c|c|c|c|c|c|}
\hline 1974 & 574 & 62 & $\$ 4.0$ & 898 & 63.4 & 187 & 917 & & & \\
\hline 1975 & 538 & 50 & 51.5 & 1,044 & 34.0 & 204 & 966 & & & \\
\hline 1976 & 645 & 61 & 56.9 & 1,136 & 36.2 & 223 & 1,021 & & & \\
\hline 1977 & 921 & 70 & 67.3 & 1,369 & 135.0 & 334 & 1,337 & & & \\
\hline 1978 & 1,035 & 83 & 78.4 & 1,319 & 18.8 & 329 & 1,323 & & & \\
\hline 1979 & 1,068 & 82 & 82.4 & 1,296 & 22.2 & 329 & 1,324 & & & \\
\hline 1980 & 1,187 & 83 & 82.5 & 1,438 & 53.7 & 358 & 1,406 & & & \\
\hline 1981 & 1,233 & 95 & 90.8 & 1,358 & 39.7 & 371 & 1,443 & & & \\
\hline 1982 & 1,183 & 87 & 89.2 & 1,326 & 44.8 & 387 & 1,488 & & & \\
\hline 1983 & 1,271 & 85 & 85.6 & 1,485 & 39.4 & 396 & 1,514 & & & \\
\hline 1984 & 1,328 & 78 & 81.8 & 1,623 & 123.3 & 481 & $1, \pi 57$ & & & \\
\hline 1985 & 1,463 & 71 & 72.6 & 2,014 & 119.0 & 557 & 1.973 & & & \\
\hline 1986 & 1,561 & 69 & 69.5 & 2,245 & 83.5 & 594 & 2,080 & & & \\
\hline$-\cdot$ & $\cdots \cdot$ & - & $\cdots$ & - - & $\because$ & -- & - - - - & $\cdot-$ & -- & $-\cdots$ \\
\hline 1987 & 1,613 & 67 & 68.0 & 2,373 & 101.2 & 650 & 2,238 & & & \\
\hline 1988 & 1,682 & 72 & 70.2 & 2,398 & 115.2 & 715 & 2,423 & 2,716 & 2,792 & 2,823 \\
\hline 1989 & & & & & 141.3 & 801 & 2,668 & 2,991 & 3,075 & 3,109 \\
\hline 1990 & & & & & 136.7 & 876 & 2,882 & 3,230 & 3,321 & 3,357 \\
\hline 1991 & & & & & 136.2 & 944 & 3,077 & 3,449 & 3,546 & 3,585 \\
\hline 1992 & & & & & 125.9 & 997 & 3,228 & 3,618 & 3,720 & 3,761 \\
\hline
\end{tabular}

Capacity-Capital Stock Regression: Code 0 RSO 0.940 Coefficients: Const 385.2 Stock 2.9

Ave. Capacity/Stock $(74-86): 3.9$ Ave. Weekly Hours (High) 136.3 Shift Factor 1.16 Ave Weekly Hours (Low) 87.7 Shift Factor 1.79 Note: Output, Investment, and Capital Stock extrapolated for 1987 and 1988.

\section{Lithographic platemaking services}

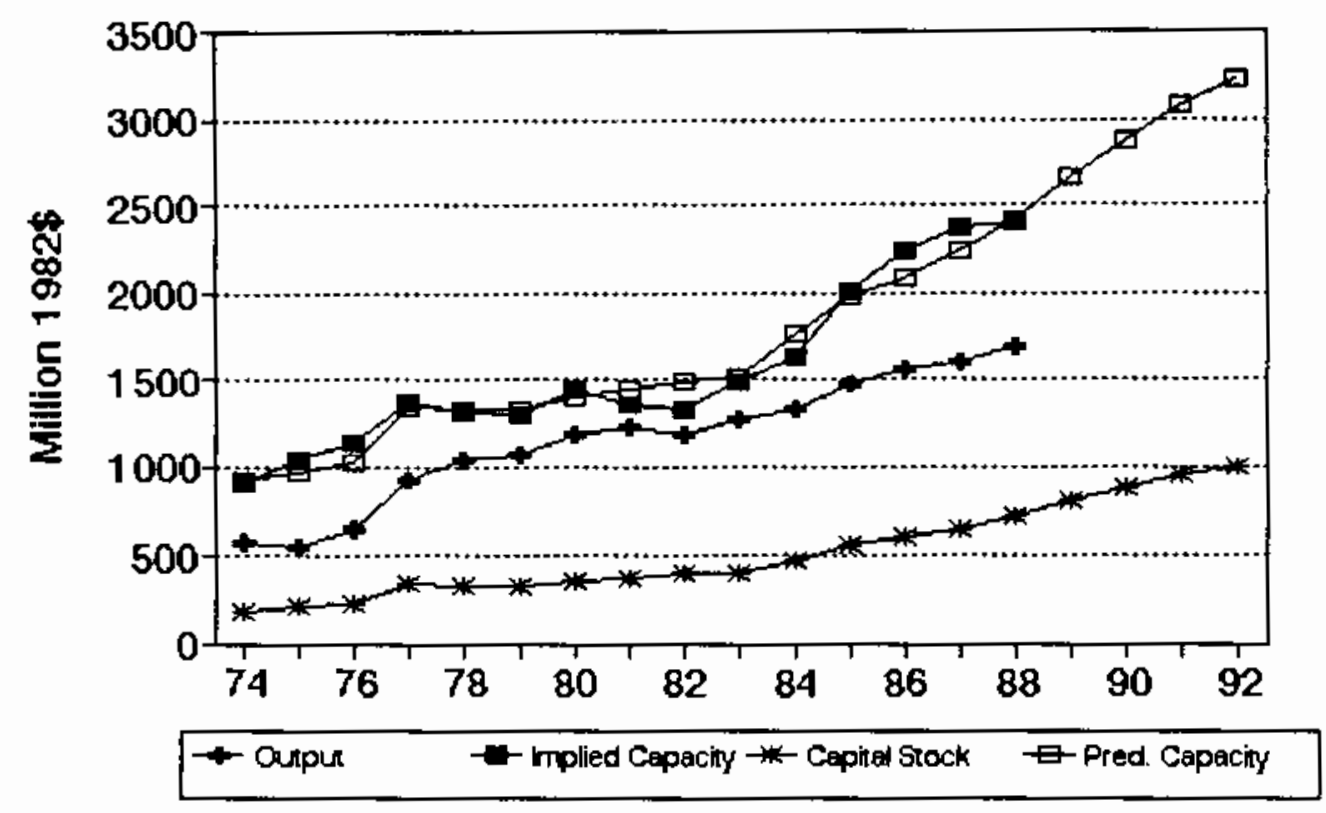




\section{No. 178 sIc 2812 Alkalies and chlorine}

MILLIONS OF 1982 DOLLARS (EXCL. CU RATES)

$\begin{array}{lccccccc} & \text { Q4 } & \text { Ann. Implied Gross } & \text { Net } & \text { Pred. } & -\ldots \ldots \ldots \\ \text { Gross } & \text { CU } & \text { CU } & \text { Prac. } & \text { In. } & \text { Cap. } & \text { Prac. } & \text { Emergency Capacity } \\ \text { Output Rate } & \text { Rate } & \text { Capac. } & \text { vest. } & \text { Stk. } & \text { Capac. } & 3 \text { mon. } 6 \text { mon. }>6 \text { mon. }\end{array}$

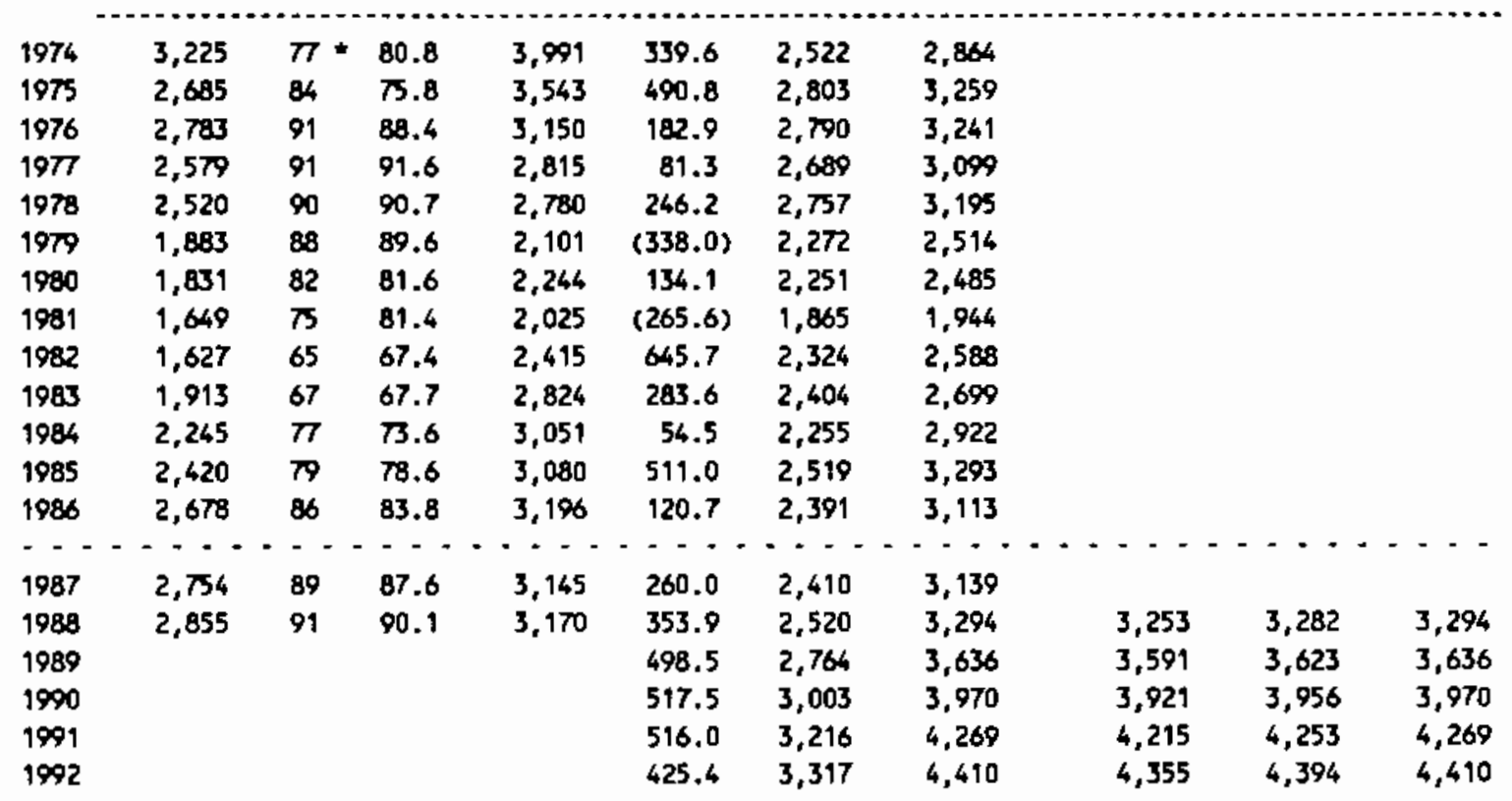

Cepacity-Capital stock Regression: Code 1 RSO 0.500

Coefficients: Const -668.8 stock $1.4 \mathrm{D}(84-86) 431.6$ Ave. Capacity/Stock (74-86): 1.2 Ave. Weekly Hours (High) 168.0 Shift Factor 1.00 Ave Weekly Hours (LOW) 168.0 Shift Factor 1.00 - Imputed Note: Output, Investment, and Capital stock extrapolated for 1987 and 1988.

\section{Alkalies and chlorine}

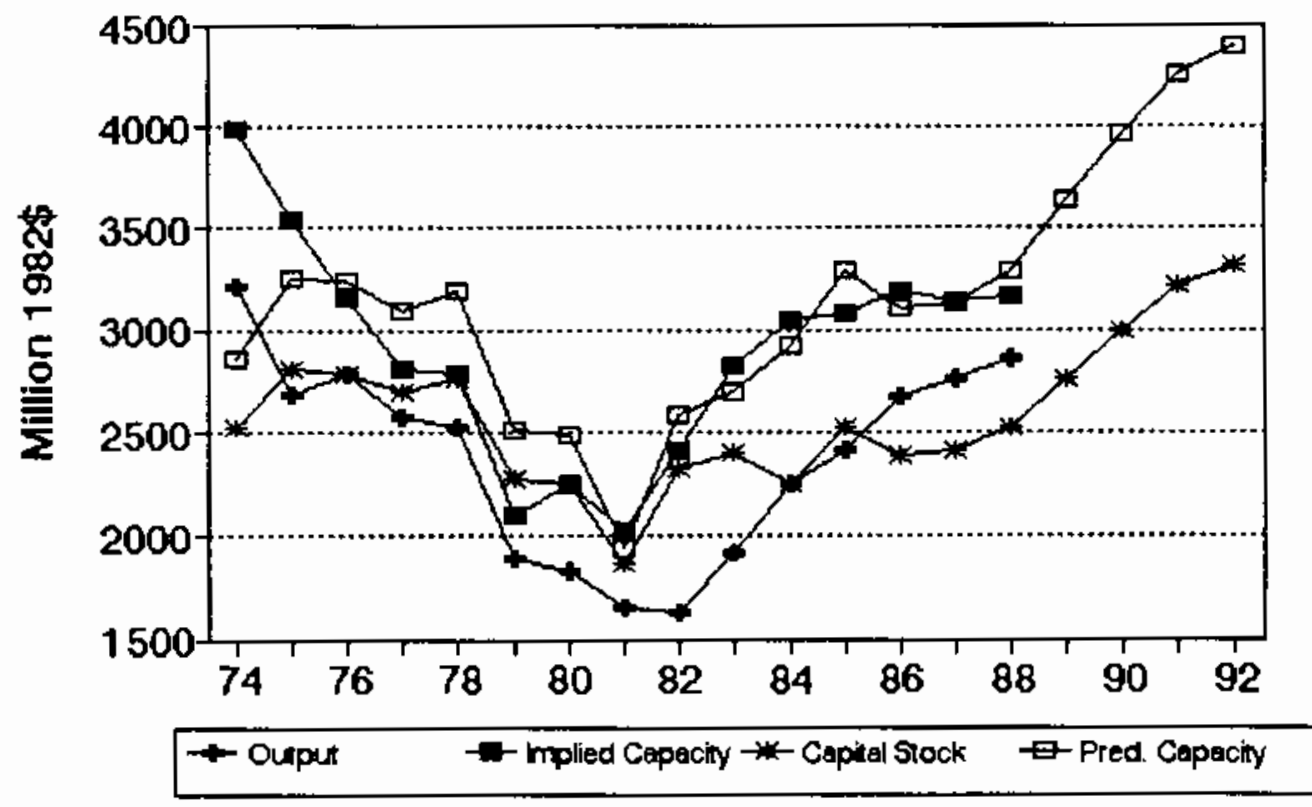


พ0. 179 sIC 2813 Industrial gases

MILLIDNS DF 1982 DOLLARS (EXCL. CU RATES)

$\begin{array}{lccccccc} & \text { Q4 } & \text { Ann. } & \text { Implied Gross } & \text { Net } & \text { Pred. } & -\ldots \\ \text { Gross } & \text { CU } & \text { CU } & \text { Prac. } & \text { In- } & \text { Cap. } & \text { Prac. } & \text { Emergency Capacity } \\ \text { Output } & \text { Rate } & \text { Rate } & \text { Capac. } & \text { vest. } & \text { Stk. } & \text { Copac. } & 3 \text { mon. } 6 \text { mon. }>6 \text { mon. }\end{array}$

\begin{tabular}{|c|c|c|c|c|c|c|c|c|c|c|}
\hline 1974 & 1,353 & 50 & 50.1 & 2,700 & (36.3) & 1,827 & 2,367 & & & \\
\hline 1975 & 1,272 & 52 & 51.1 & 2,487 & 436.6 & 2,073 & 2,427 & & & \\
\hline 1976 & 1,455 & 67 & 61.4 & 2,371 & 252.2 & 2,126 & 2,440 & & & \\
\hline 1977 & 1,686 & 62 & 63.9 & 2,638 & 588.1 & 2,494 & 2,530 & & & \\
\hline 1978 & 1,717 & 74 & 69.5 & 2,470 & 549.2 & 2,792 & 2,603 & & & \\
\hline 1979 & 1,862 & 82 & 79.0 & 2,356 & 31.4 & 2,593 & 2,554 & & & \\
\hline 1980 & 1,756 & 83 & 82.5 & 2,125 & 261.2 & 2,625 & 2,562 & & & \\
\hline 1981 & 1,964 & 80 & 81.3 & 2,417 & 277.3 & 2,666 & 2,572 & & & \\
\hline 1982 & 2,030 & 71 & 74.3 & 2,731 & 677.9 & 3,058 & 2,668 & & & \\
\hline 1983 & 2,093 & 77 & 74.8 & 2,798 & 268.9 & 3,028 & 2,600 & & & \\
\hline 1984 & 2,284 & 84 & 81.4 & 2,807 & 836.1 & 3,522 & 2,781 & & & \\
\hline 1985 & 2,321 & 78 & 80.3 & 2,892 & 269.8 & 3,452 & 2,764 & & & \\
\hline 1986 & 2,195 & 76 & 76.8 & 2,860 & 191.2 & 3,301 & 2,727 & & & \\
\hline$\ddot{1987}$ & 2,240 & 76 & $\begin{array}{l}--- \\
76.0\end{array}$ & $\cdots$ & $\begin{array}{l}\cdots \\
416.0\end{array}$ & 3,384 & 2,747 & -- & -- & $-\cdots$ \\
\hline 1988 & 2,300 & 75 & 75.4 & 3,052 & 544.0 & 3,586 & 2,797 & 2,765 & 2,800 & 2,814 \\
\hline 1989 & & & & & 741.0 & 3,965 & 2,889 & 2,857 & 2,892 & 2,907 \\
\hline 1990 & & & & & 767.0 & 4,331 & 2,979 & 2,965 & 2,982 & 2,997 \\
\hline 1991 & & & & & 764.8 & 4,658 & 3,059 & 3,025 & 3,062 & 3,077 \\
\hline 1992 & & & & & 641.4 & 4,829 & 3,101 & 3,066 & 3,104 & 3,119 \\
\hline
\end{tabular}

Capacity-Capital Stack Regression: Code O RSQ 0.310

Coefficients: Const 1919.9 stock 0.3

Ave. Capacity/Stock (74-86): 0.9 Ave. Heekly Hours (High) 166.9 Shift factor 1.01 Ave Heekly Hours (Low) 164.6 Shift Factor 1.02 Note: Output, Investment, and Capital Stock extrapolated for 1987 and 1988.

\section{Industrial gases}

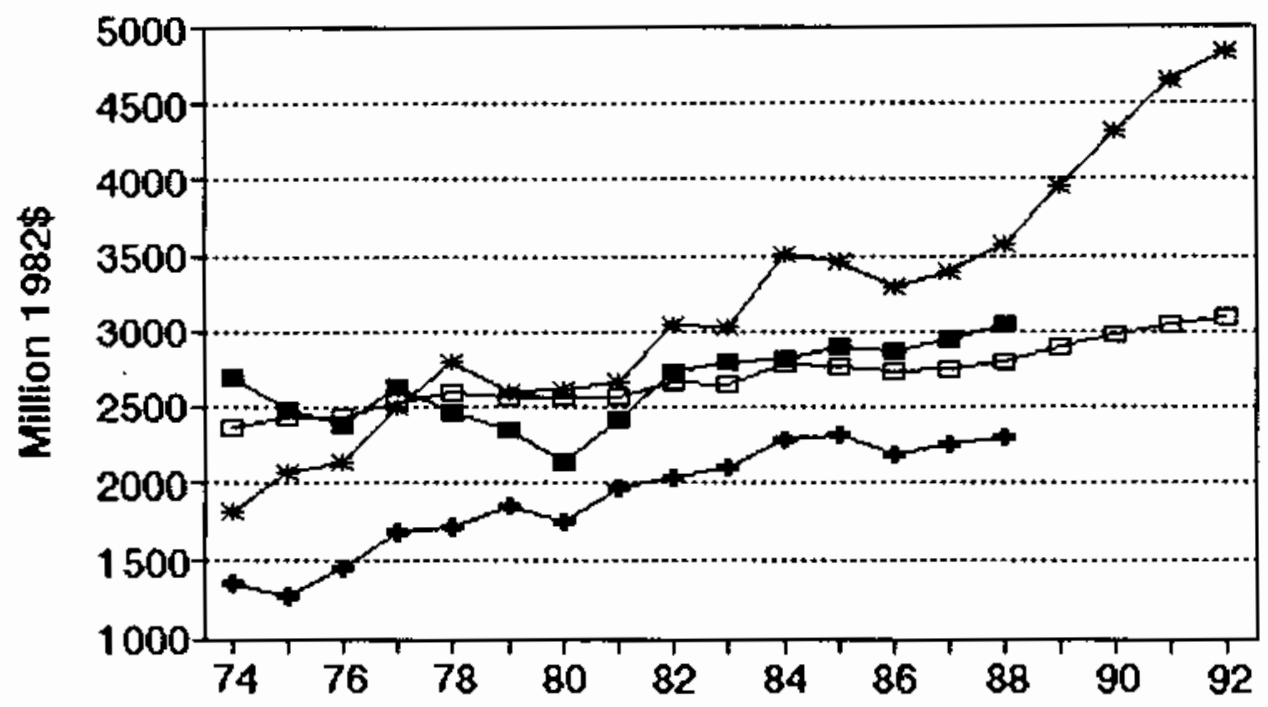

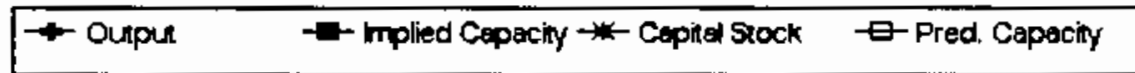




\section{No. 180 SIC 2816 Inorganic chemicals}

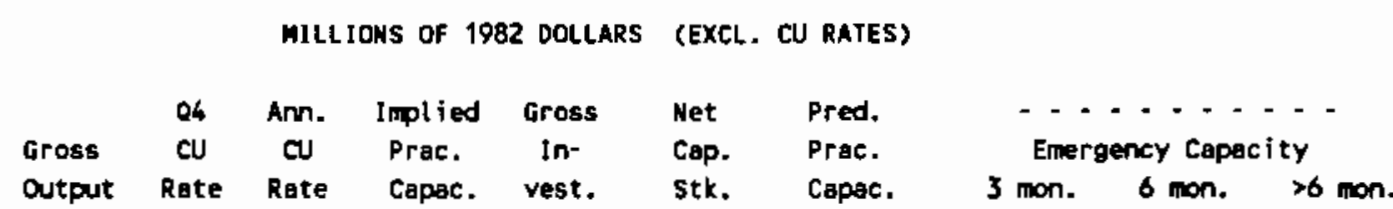

\begin{tabular}{|c|c|c|c|c|c|c|c|c|c|c|}
\hline 1974 & 3,006 & 49 & 50.7 & 5,929 & 254.4 & 1,370 & 3,018 & & & \\
\hline 1975 & 2,151 & 64 & 56.1 & 3,833 & 125.2 & 1,408 & 3,184 & & & \\
\hline 1976 & 2,130 & 73 & 69.6 & 3,061 & 91.3 & 1,410 & 3,194 & & & \\
\hline 1977 & 1,883 & 75 & 74.6 & 2,526 & 105.7 & 1,429 & 3,275 & & & \\
\hline 1978 & 2,008 & 79 & 77.6 & 2,586 & 74.4 & 1,410 & 3,191 & & & \\
\hline 1979 & 1,883 & 81 & 80.7 & 2,333 & $(114.8)$ & 1,214 & 2,336 & & & \\
\hline 1980 & 1,867 & 75 & 75.8 & 2,465 & 118.2 & 1,243 & 2,461 & & & \\
\hline 1981 & 1,857 & 76 & 77.8 & 2,386 & 234.3 & 1,367 & 3,004 & & & \\
\hline 1982 & 1,660 & 68 & 70.2 & 2,366 & 102.6 & 1,357 & 2,960 & & & \\
\hline 1983 & 1,783 & 78 & 75.1 & 2,375 & 116.9 & 1,352 & 2,940 & & & \\
\hline 1984 & 1,787 & Bo & 79.5 & 2,248 & 20.2 & 1,255 & 2,513 & & & \\
\hline 1985 & 1,952 & B2 & B1. 5 & 2,397 & 132.3 & 1,263 & 2,549 & & & \\
\hline 1986 & 1,974 & 79 & 80.4 & 2,455 & 76.1 & 1,214 & 2,336 & & & \\
\hline $\begin{array}{l}-\cdots \\
1987\end{array}$ & 2, & $\begin{array}{l}-- \\
88\end{array}$ & $\begin{array}{l}--. \\
84.4\end{array}$ & $\begin{array}{c}\cdots, 397 \\
2\end{array}$ & $\begin{array}{l}\cdots \\
100.0\end{array}$ & $\begin{array}{l}--\cdot- \\
1.192\end{array}$ & 2,239 & $\cdots$ & -- & $\because \cdot$ \\
\hline 1988 & 2,089 & 85 & 86.0 & 2,429 & 100.0 & 1,172 & 2,152 & 2,228 & 2,239 & 2,243 \\
\hline 1989 & & & & & 100.0 & 1,154 & 2,074 & 2,147 & 2,157 & 2,161 \\
\hline 1900 & & & & & 100.0 & 1,138 & 2,004 & 2,075 & 2,084 & $2,08 B$ \\
\hline 1991 & & & & & 100.0 & 1,124 & 1,941 & 2,009 & 2,018 & 2,022 \\
\hline 1992 & & & & & 100.0 & 1,111 & 1,884 & 1,950 & 1,959 & 1.963 \\
\hline
\end{tabular}

Capacity-Capital Stock Regression: Code 0 RSO 0.120

Coefficients: Const -2971.2 stock 4.4

Ave. Capecity/Stock $(74-86): 2.1$ Ave. Heekly Hours (High) 153.2 Shift Factor 1.04 Ave Heekly Hours (Low) 147.2 Shift Factor 1.08 Note: Output, Investment, and Capital stock extrapolated for 1987 and 1988.

\section{Inorganic chemicals}

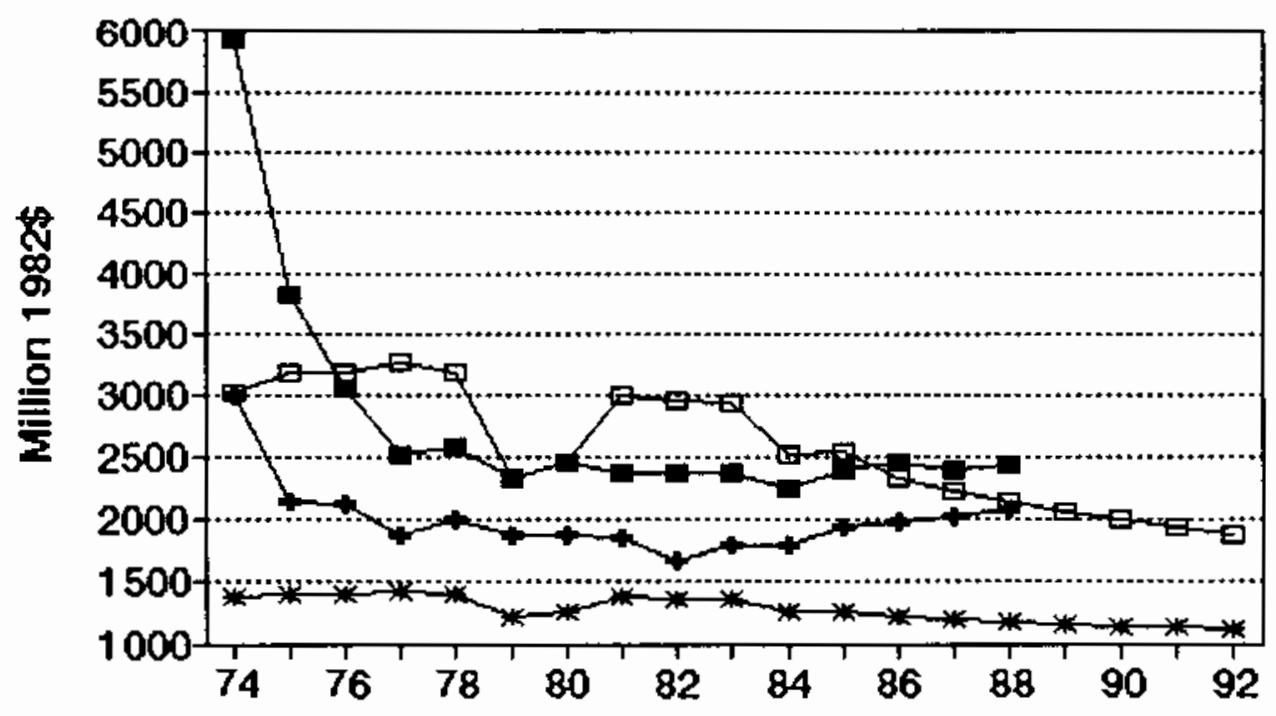

\begin{tabular}{|c|c|c|}
\hline+ Ouput & -6- hrolied Capacty $\rightarrow$ - Copita Srock & E-Pred Copacity \\
\hline
\end{tabular}


No. 181 SIC 2819 Industrial inorganic chemicals, n.e.c MILLIONS OF 1982 DOLLARS (EXCL. CU RATES)

$\begin{array}{lccccccc} & \text { Q4 } & \text { Ann. } & \text { inplied Gross } & \text { Net } & \text { Pred. } & \ldots & \ldots . \\ \text { Gross } & \text { CU } & \text { CU } & \text { Prac. } & \text { In- } & \text { Cap. } & \text { Prac. } & \text { Emergency Capacity } \\ \text { Output } & \text { Rate } & \text { Rate } & \text { Capac. } & \text { vest. } & \text { Stk. } & \text { Capac. } & 3 \text { mon. } 6 \text { mon. }>6 \text { mon. }\end{array}$

\begin{tabular}{|c|c|c|c|c|c|c|c|c|c|c|}
\hline 1974 & 14,120 & 63 & 63.6 & 22,205 & 810.4 & 4,696 & 18,955 & & & \\
\hline 1975 & 12,052 & 73 & 68.5 & 17,594 & 554.1 & 4,834 & 19,241 & & & \\
\hline 1976 & 13,916 & 79 & 76.7 & 18,133 & 642.6 & 5,050 & 19.686 & & & \\
\hline 1977 & 15,730 & 76 & 77.2 & 20,373 & 984.0 & 5,561 & 20,738 & & & \\
\hline 1978 & 16,831 & $\pi$ & 75.4 & 22,319 & 890.0 & 5,961 & 21,564 & & & \\
\hline 1979 & 16,377 & 85 & 81.4 & 20,123 & 97.5 & 5,614 & 20,848 & & & \\
\hline 1980 & 15,610 & 76 & 79.0 & $19, \pi / 2$ & 598.1 & 5,758 & 21,145 & & & \\
\hline 1981 & 13,984 & 63 & 88.4 & 20,449 & 547.1 & 5,843 & 21,320 & & & \\
\hline 1982 & 12,274 & 60 & 61.0 & 20,138 & 210.5 & 5,593 & 20,806 & & & \\
\hline 1983 & 12,655 & 66 & 63.9 & 19.794 & 485.4 & 5,595 & 20,808 & & & \\
\hline 1984 & 14,157 & 56 & 59.8 & 23,670 & 720.4 & 5,792 & 21,216 & & & \\
\hline 1985 & 13,577 & 61 & 59.2 & 22,949 & 652.3 & 5,897 & 21,432 & & & \\
\hline 1986 & 12,949 & 60 & 60.4 & 21,429 & 438.3 & 5,780 & 21,191 & & & \\
\hline - - - & $\cdots \cdot$ & $\because$ & $\because \cdot$ & --- & $-\cdot-$ & $-\cdots$ & --- & --- & --- & $\cdots-\cdot$ \\
\hline 1987 & 13,279 & 66 & 63.7 & 20,847 & 678.3 & 5,912 & 21,464 & & & \\
\hline 1988 & 13,720 & 63 & 64.1 & 21,403 & 835.7 & 6,189 & 22,035 & 21,892 & 22,497 & 22,740 \\
\hline 1989 & & & & & $1,077.8$ & 6,682 & 23,052 & 22,902 & 23,535 & 23,789 \\
\hline 1990 & & & & & $1,109.7$ & 7,161 & 24,038 & 23,823 & 24,542 & 26,808 \\
\hline 1991 & & & & & $1,107.0$ & 7,591 & 26,926 & 24,765 & 25,448 & 25,724 \\
\hline 1992 & & & & & 955.4 & 7,830 & 25,417 & 25,253 & 25,950 & 26,231 \\
\hline
\end{tabular}

Capacity-Capital Stock Regression: Cade O RSQ 0.230

Coefficients: Const 9271.8 stock 2.1

Ave. Capecity/Stock $(74-86): 3.7$ Ave. Weekly Hours (High) 154.8 Shift Factor 1.03 Ave Veekly Hours (Low) 146.7 Shift Factor 1.09 Note: Output, Investment, and Capital stock extrapolated for 1987 and 1988.

\section{Industrial inorganic chemicals, $n$.}

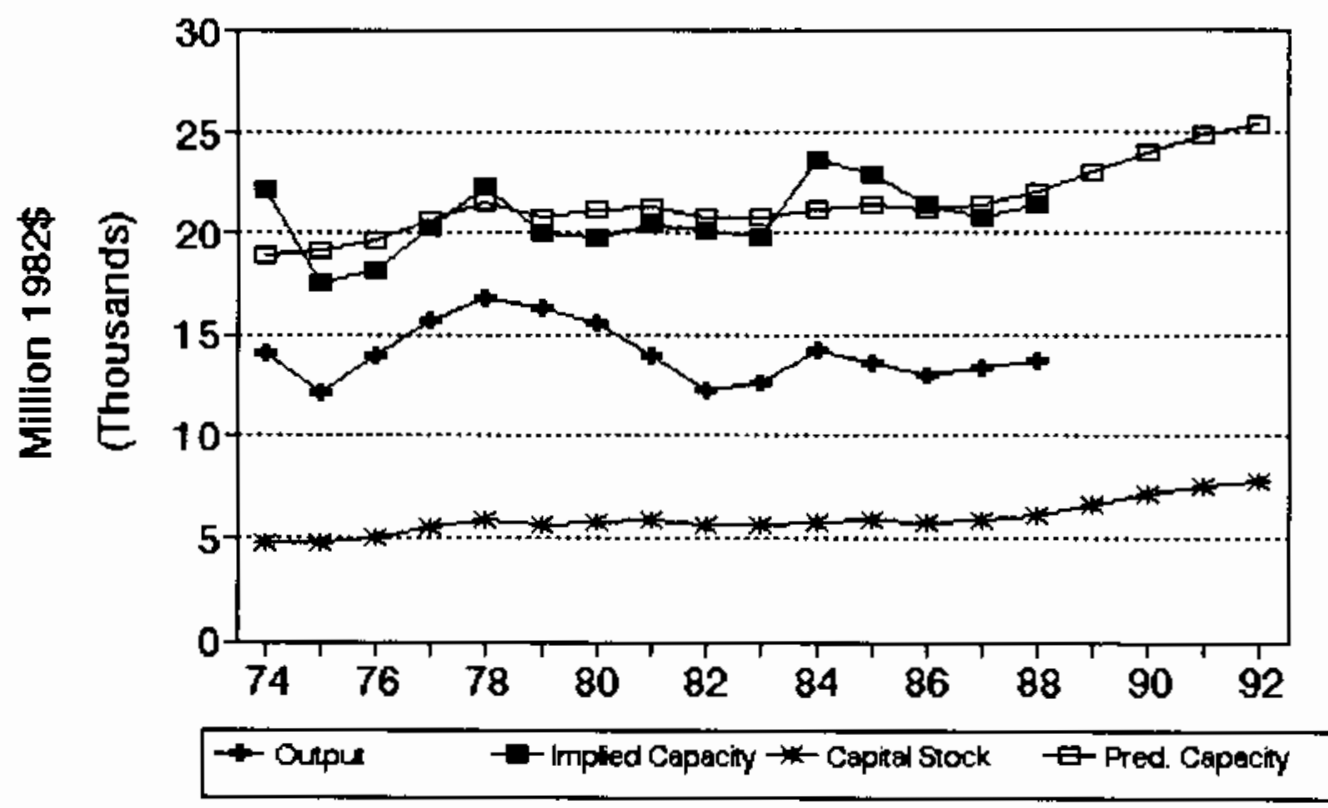




\section{No. 182 SIC 2821 plastics materials and resins}

MILLIONS OF 1982 DOLLARS (EXCL. CU RATES)

$\begin{array}{lccccccc} & \text { Q4 Ann. Implied Gross } & \text { Net } & \text { Pred. } & \ldots & -\ldots \\ \text { Gross } & \text { CU } & \text { CU } & \text { Prac. } & \text { In- } & \text { Cap. } & \text { Prac. } & \text { Emergency Capacity } \\ \text { Output } & \text { Rate } & \text { Rate } & \text { Capac. } & \text { vest. } & \text { Stk. } & \text { Capac. } & 3 \text { mon. } 6 \text { mon. }>6 \text { mon. }\end{array}$

\begin{tabular}{|c|c|c|c|c|c|c|c|c|c|c|}
\hline 1974 & 15,377 & 74 & 78.6 & 19,563 & $1,502.5$ & 8,384 & 17,518 & & & \\
\hline 1975 & 11,091 & 76 & 69.5 & 15,968 & 799.6 & 8,638 & 17,982 & & & \\
\hline 1976 & 13,733 & 77 & 76.7 & 17,917 & 994.6 & 9,083 & 18,795 & & & \\
\hline 1977 & 15,721 & 79 & 78.8 & 19,939 & $1,357.3$ & 9,857 & 20,209 & & & \\
\hline 1978 & 16,970 & 78 & 78.7 & $21,57 \mathrm{~s}$ & $1,478.4$ & 10,706 & 21,739 & & & \\
\hline 1979 & 17,272 & 80 & 80.1 & 21,564 & $1,552.1$ & 11,570 & 23,337 & & & \\
\hline 1980 & 15,315 & 73 & 72.9 & 20,998 & $1,047.4$ & 11,894 & 23,929 & & & \\
\hline 1981 & 16,469 & 65 & 71.8 & 22,947 & 745.8 & 11,870 & 23,885 & & & \\
\hline 1982 & 15,829 & 55 & 57.4 & 27,581 & 446.8 & 11,507 & 23,222 & & & \\
\hline 1993 & 18,625 & 78 & 70.7 & 26,358 & $1,202.4$ & 11,806 & 23,768 & & & \\
\hline 1984 & 19,283 & $\pi$ & 76.6 & 25,171 & $1,021.1$ & 11,863 & 23,873 & & & \\
\hline 1985 & 19,102 & 78 & 7.2 & 24,734 & $1,960.5$ & 12,746 & 25,486 & & & \\
\hline 1986 & 20,585 & 82 & 81.0 & 25,425 & $1,391.2$ & 13,014 & 25,976 & & & \\
\hline$\cdots$ & $\cdots$ & -- & -- & $\cdots$ & $\cdots \cdot$ & $\because \cdot \cdot$ & $\cdots \cdot$ & $=\cdot$ & $\cdot \cdot \cdot$ & $\ldots+\cdot$ \\
\hline 1987 & 20,695 & 84 & 82.9 & 24,953 & $1,332.0$ & 13,235 & 26,378 & & & \\
\hline 1988 & 20,838 & 86 & 85.0 & 24,503 & $1,355.8$ & 13,460 & 26,790 & 26,400 & 26,811 & 26,977 \\
\hline 1989 & & & & & $1,379.6$ & 13,690 & 27,209 & 26,814 & 27,231 & 27,400 \\
\hline 1990 & & & & & $1,403.3$ & 13,924 & 27,637 & 27,235 & 27,659 & 27,830 \\
\hline 1991 & & & & & $1,427.1$ & 14,162 & 28,071 & 27,663 & 28,094 & 28,267 \\
\hline 1992 & & & & & $1,450.8$ & 14,403 & 28,511 & 28,097 & 28,534 & 28,711 \\
\hline
\end{tabular}

Capacity-Capital Stock Regression: Code 0 RSO 0.650

Coefficients: Const 2205.7 Stock 1.8

Ave. Capacity/Stock $(74-86): 2.0$ Ave. Weekly Hours (High) 166.8 Shift Factor 1.01 Ave Heekly Hours (LOH) 164.4 Shift Factor 1.02 Note: Output, Investment, and Capital Stock extrapolated for 1987 and 1988.

\section{Plastics materials and resins}

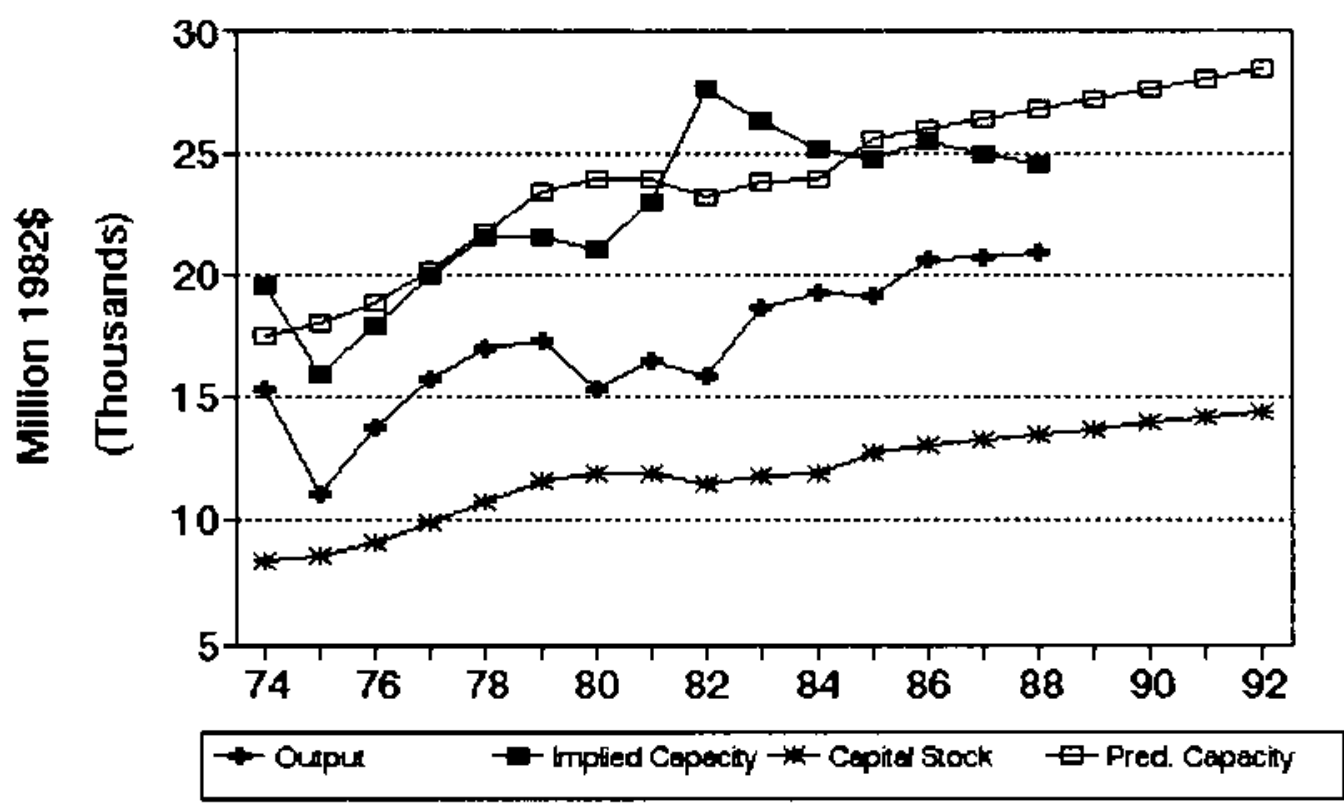


yo. 183 sIC 2822 synthetic rubber

MILLIONS OF 1982 DOLLARS (EXCL. CU RATES)

$\begin{array}{lccccccc} & 04 & \text { Ann. } & \text { Implied Gross } & \text { Met } & \text { Pred. } & \ldots & \ldots \\ \text { Gross } & \text { CU } & \text { CU } & \text { Prac. } & \text { In- } & \text { Cap, } & \text { Prac. } & \text { Emergency Capacity } \\ \text { Output } & \text { Rate } & \text { Rate } & \text { Capac, } & \text { vest. } & \text { Stk. } & \text { Capac. } & 3 \text { mon. } 6 \text { mon. }\end{array}$

\begin{tabular}{|c|c|c|c|c|c|c|c|c|c|c|}
\hline 1974 & 3,439 & 74 & 77.8 & 4,420 & 164.7 & 1,709 & 4,553 & & & \\
\hline 1975 & 2,921 & 86 & 76.6 & 3,815 & $(270.2)$ & 1,344 & 3.874 & & & \\
\hline 1976 & 3,285 & 88 & 87.3 & 3.764 & 87.1 & 1,330 & 3,848 & & & \\
\hline 1977 & 3,337 & 74 & 79.8 & 4,183 & 101.8 & 1,321 & 3,835 & & & \\
\hline 1978 & 3,292 & 93 & 86.0 & 3,828 & 78.4 & 1,286 & 3,767 & & & \\
\hline 1979 & 3,715 & 88 & 90.7 & 4,098 & 282.5 & 1,425 & 4,026 & & & \\
\hline 1980 & 3,080 & 80 & 80.6 & 3,823 & 116.3 & 1,397 & 3,974 & & & \\
\hline 1981 & 3,072 & 70 & 77.1 & 3,987 & 208.3 & 1,454 & 4,080 & & & \\
\hline 1982 & 3,269 & 60 & 62.6 & 5,224 & 297.4 & 1,671 & 4,483 & & & \\
\hline 1983 & 3,235 & 84 & 76.1 & 4,250 & 148.7 & 1,718 & 4,571 & & & \\
\hline 1984 & 3,536 & 79 & 81.3 & 4,349 & 29.4 & 1,647 & 4,438 & & & \\
\hline 1985 & 2,885 & 69 & 73.1 & 3,848 & $(101.8)$ & 1,454 & 4,079 & & & \\
\hline 1986 & 2,819 & 80 & 76.2 & 3,831 & 49.2 & 1,409 & 3,995 & & & \\
\hline$\cdots$ & $\cdots$ & $\cdot \cdot$ & $* \cdot$ & $\cdots$ & 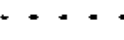 & $\cdots \cdots$ & $\cdots-$ & --- & --- & $-\cdot$ \\
\hline 1987 & 2,962 & 84 & 82.2 & 3,603 & 101.5 & 1,419 & 4,015 & & & \\
\hline 1988 & 3,019 & 89 & 86.9 & 3,474 & 102.6 & 1,430 & 4,035 & 3,939 & 4,008 & 4,035 \\
\hline 1989 & & & & & 103.6 & 1,441 & 4,056 & 3,960 & 4,028 & 4,056 \\
\hline 1990 & & & & & 104.6 & 1,453 & 4,077 & 3,980 & 4,049 & 4,077 \\
\hline 1991 & & & & & 105.7 & 1.465 & 4,099 & 4,002 & 4,071 & 4,099 \\
\hline 1992 & & & & & 106.7 & 1,477 & 4,121 & 4,024 & 4,093 & 4,121 \\
\hline
\end{tabular}

Capacity-Capital Stock Regression: Code 0 RSO 0.530

Coefficients: Const 1374.2 stock 1.9

Ave. Capacity/Stock (74-86): 2.8 Ave. Weekty Hours (High) 168.0 Shift Factor 1.00 Ave Weekly Hours (LOW) 168.0 Shift Factor 1.00 Note: Output, Investment, and Capital stock extrapolated for 1987 and 1988.

\section{Synthetic rubber}

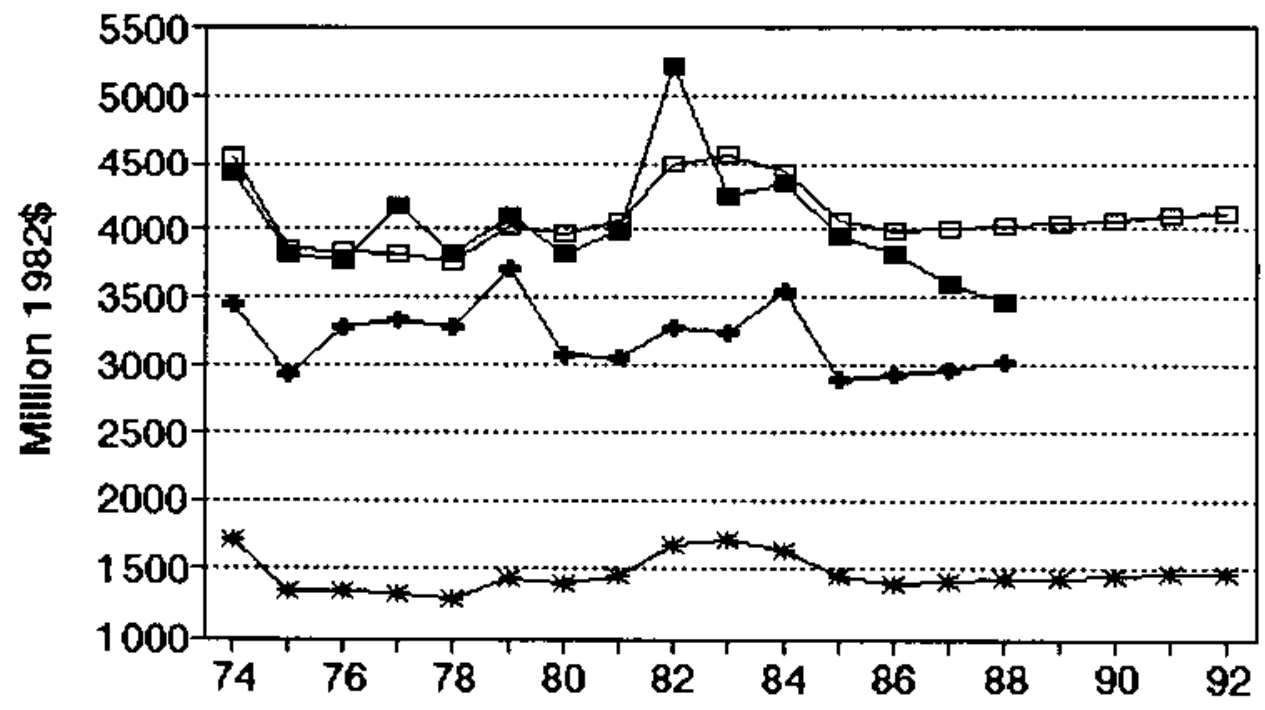

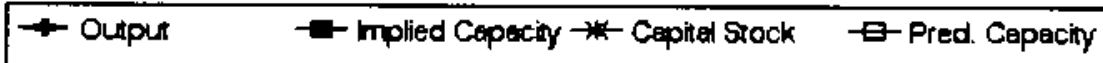


No. 184 GIC 2823 Cellulosic mannade fibera

MILLIONS OF 1982 DOLLARS (EXCL. CU RATES)

$\begin{array}{lccccccc} & \alpha & \text { Ann. Implied Gross } & \text { Met } & \text { Pred. } & \ldots \\ \text { Gross } & \alpha & \alpha & \text { Prac. } & \text { In- } & \text { Cap. } & \text { Prac. } & \text { Emergency Capacity } \\ \text { Output Rate } & \text { Rate } & \text { Capac. } & \text { vest. } & \text { Stk. } & \text { Capac. } & 3 \text { mon. } 6 \text { mon. }>6 \text { mon. }\end{array}$

\begin{tabular}{|c|c|c|c|c|c|c|c|c|c|c|}
\hline 1974 & 1,681 & 71 & 75.6 & 2,223 & 355.1 & 1,354 & 2,118 & & & \\
\hline 1975 & 1,273 & 71 & 65.2 & 1,952 & 266.7 & 1,473 & 2,286 & & & \\
\hline 1976 & 1,421 & 74 & $R .9$ & 1,949 & $(74.4)$ & 1,266 & $1, \infty 3$ & & & \\
\hline 1977 & 1,487 & 85 & 81.5 & 1,824 & 16.2 & 1,150 & 1,830 & & & \\
\hline 1978 & 1,542 & 90 & 88.6 & 1,744 & 43.0 & 1,064 & 1,709 & & & \\
\hline 1979 & 1,629 & 93 & 92.9 & 1,753 & 110.3 & 1,045 & 1,682 & & & \\
\hline 1980 & 1,536 & 93 & 89.7 & 1,713 & 91.3 & 1,009 & 1,632 & & & \\
\hline 1981 & 1,471 & 76 & 87.2 & 1,688 & 159.8 & 1,038 & 1,672 & & & \\
\hline 1982 & 1,278 & 57 & 62.4 & 2,048 & 139.6 & 1,063 & 1,670 & & & \\
\hline 1983 & 1,494 & 86 & 76.5 & 1,953 & 60.2 & 972 & 1,570 & & & \\
\hline 1984 & 1,034 & 82 & 84.1 & 1,230 & 1.1 & 858 & 1,418 & & & \\
\hline 1985 & 915 & 92 & 88.7 & 1,031 & 73.3 & 819 & 1,363 & & & \\
\hline 1986 & 1,063 & 93 & 93.2 & 1,161 & 52.7 & 766 & 1,288 & & & \\
\hline$\therefore$ & $\because \cdots$ & - - & -- & $-\cdots$ & $\cdots$ & $\cdots$ & $\because-$ & -- & $\because \cdot$ & -- \\
\hline 1987 & 1,067 & 89 & 90.2 & 1,183 & 133.3 & 704 & 1,328 & & & \\
\hline 1988 & 1,072 & 96 & 93.1 & 1,151 & 187.3 & 872 & 1,438 & 1,403 & 1,428 & 1,438 \\
\hline 1989 & & & & & 270.3 & 1,022 & 1,650 & 1,610 & 1,638 & 1,650 \\
\hline 1990 & & & & & 281.3 & 1,163 & 1,848 & 1,804 & 1,835 & 1,848 \\
\hline 1991 & & & & & 280.6 & 1,283 & 2,017 & 1,969 & 2,004 & 2,017 \\
\hline 1992 & & & & & 228.3 & 1,335 & 2,090 & 2,040 & 2,076 & 2,090 \\
\hline
\end{tabular}

Capocity-Capital Stock Regression: Code 1 RSO 0.630

Coefficients: Const 208.2 stock 1.4

Ave. Capacity/Stock $(74-86): 1.6$ Ave. Weekly Hours (High) 168.0 Shift Factor 1.00 Ave Weekly Hours (Low) 168.0 Shift Factor 1.00 Note: Output, Imvestment, and Capital stock extrapolated for 1987 and 1988.

\section{Cellulosic manmade fibers}

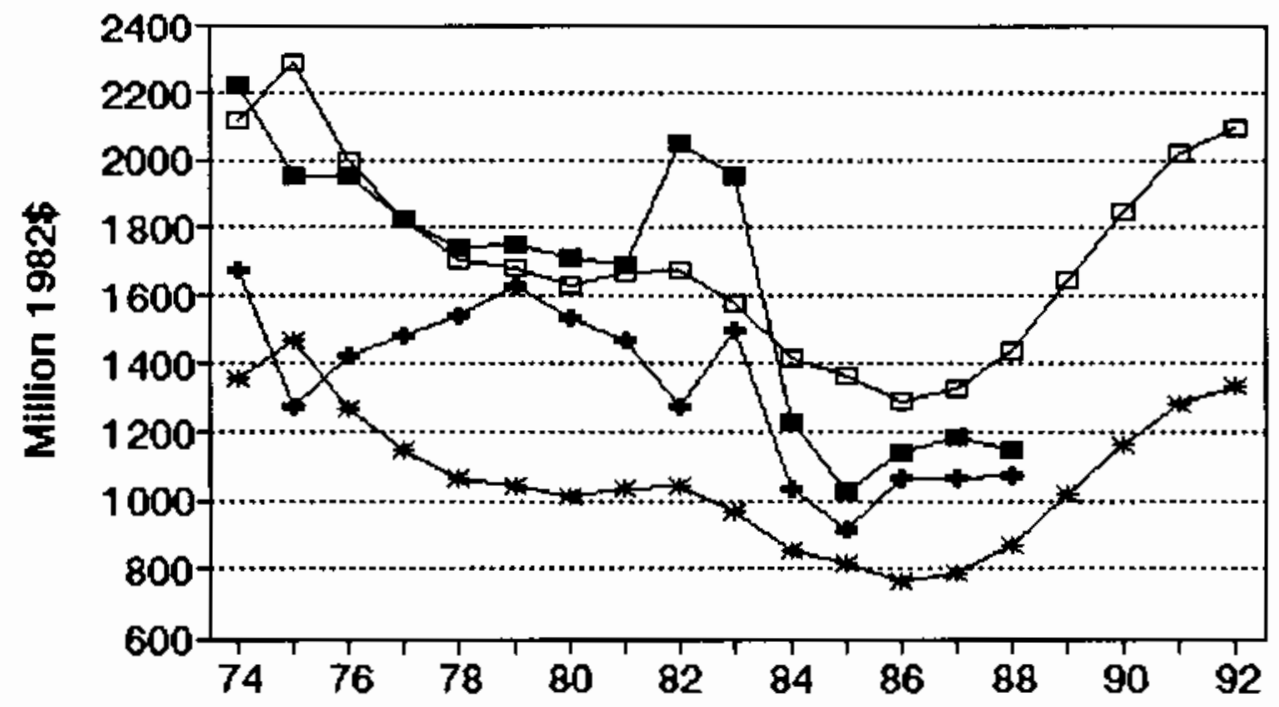

$\rightarrow$ - Oupue $\rightarrow$ - Implied Cepaciny $\rightarrow *$-Cepital Stock $\quad \boxminus$-Pred. Cepaciny




\section{No. 185 sIc 2824 Organic fibers, noncellulosic}

MILLIONS OF 1982 DOLLARS (EXCL. CU RATES)

$\begin{array}{lccccccc} & 04 & \text { Ann. } & \text { Implied } & \text { Gross } & \text { Net } & \text { Pred. } & -\ldots \ldots \\ \text { Gross } & \text { QU } & \text { CU } & \text { Prac. } & \text { In* } & \text { Cap. } & \text { Prac. } & \text { Emergency Capacity } \\ \text { Output } & \text { Rate } & \text { Rate } & \text { Capec. } & \text { vest. } & \text { Stk. } & \text { Capec. } & 3 \text { mon. } 6 \text { mon. }\end{array}$

\begin{tabular}{|c|c|c|c|c|c|c|c|c|c|c|}
\hline 1974 & 7,703 & 71 & 73.8 & 10,435 & 723.0 & 9,536 & 10,669 & & & \\
\hline 1975 & 7,540 & 89 & 78.5 & 9,602 & $1,111.3$ & 10,056 & 10,830 & & & \\
\hline 1976 & 8,512 & 77 & 81.6 & 10,434 & 788.7 & 10,196 & 10,990 & & & \\
\hline 1977 & 9,723 & 83 & 81.1 & 11,985 & 213.2 & 9,735 & 11,150 & & & \\
\hline 1978 & 10,418 & 89 & 86.9 & 11,980 & 878.8 & 9,803 & 11,310 & & & \\
\hline 1979 & 11,276 & 90 & 90.2 & 12,497 & 641.9 & 9,743 & 11,470 & & & \\
\hline 1980 & 10,333 & 88 & 86.8 & 11,903 & 0.8 & 8,989 & 11,631 & & & \\
\hline 1981 & 9,902 & 77 & B3.9 & 11,808 & 338.4 & 8,555 & 11,791 & & & \\
\hline 1982 & 8,565 & 70 & 71.7 & 11,953 & 698.3 & 8,425 & 11,951 & & & \\
\hline 1983 & 9,873 & 85 & 80.4 & 12,284 & 310.4 & 7,891 & 12,111 & & & \\
\hline 1984 & 10,289 & 82 & 83.4 & 12,331 & 607.9 & 7,625 & 12,271 & & & \\
\hline 1985 & 9,798 & 83 & 82.9 & 11,823 & 533.2 & 7,279 & 12,432 & & & \\
\hline 1986 & 10,582 & 89 & 87.1 & 12,156 & 502.5 & 6,928 & 12,592 & & & \\
\hline$\cdots$ & $-\cdots$ & $\because$ & $\cdots$ & $\cdots$ & $\cdots$ & $-\cdots$ & $--\cdot$ & $--\cdot$ & $-=-$ & $\cdots$ \\
\hline 1987 & 10,813 & 93 & 91.3 & 11,850 & 295.8 & 6,379 & 12,752 & & & \\
\hline 1988 & 11,120 & 93 & 92.9 & 11,976 & 253.6 & 5,854 & 12,912 & 12,744 & 12,864 & 12,912 \\
\hline 1989 & & & & & 211.4 & 5,352 & 13,072 & 12,902 & 13,023 & 33,072 \\
\hline 1990 & & & & & 169.1 & 4,868 & 13,233 & 13,060 & 13,183 & 13,233 \\
\hline 1991 & & & & & 126.9 & 4,401 & 13,393 & 13,218 & 13,343 & 13,393 \\
\hline 1992 & & & & & 84.7 & 3,949 & 13,553 & 13,376 & 13,502 & 13,553 \\
\hline
\end{tabular}

Capacity-Capital Stock Regression: Code -2 RSO 0.500 Coefficients: Const 10509.1 Time 160.2

Ave. Capacity/Stock $(74-86): 1.3$ Ave. Weekly Hours (High) 168.0 Shift Factor 1.00 Ave Weekly Hours (Low) 164.7 Shift Factor 1.02 Note: Output, Investment, and Capital stock extrapolated for 1987 and 1988.

\section{Organic fibers, noncellulosic}

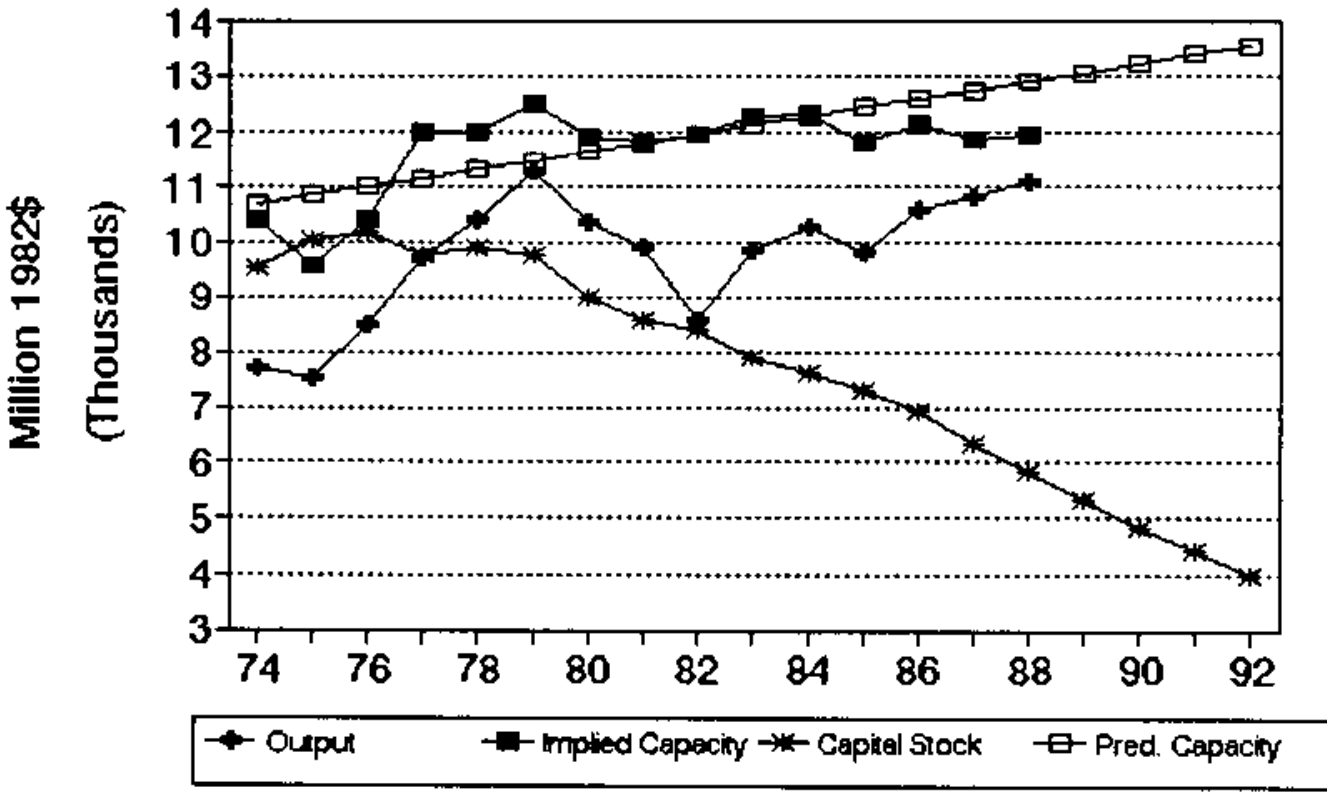


No. 186 BIC 2831 Biological products

MILLIONS OF 1982 DOLLARS (EXCL. CU RATES)

$\begin{array}{lccccccc} & 04 & \text { Ann. Implied Gross } & \text { Net } & \text { Pred. } & \ldots . \ldots . . . \\ \text { Gross } & \text { CU } & \text { CU } & \text { Prac. } & \text { In+ } & \text { Cap. } & \text { Prac. } & \text { Emergency Capacity } \\ \text { Output } & \text { Rate } & \text { Rate } & \text { Capac. } & \text { vest. } & \text { StK. } & \text { Capac. } & 3 \text { mon. } 6 \text { mon. }>6 \text { mon. }\end{array}$

\begin{tabular}{|c|c|c|c|c|c|c|c|c|c|c|}
\hline 1974 & 908 & 84 & 87.3 & 1,041 & 225.9 & 379 & 1,965 & & & \\
\hline 1975 & 992 & 67 & 69.6 & 1,426 & $(46.5)$ & 313 & 1,373 & & & \\
\hline 1976 & 984 & 83 & 76.9 & 1,279 & (21.5) & 275 & 1,032 & & & \\
\hline 1977 & 1,237 & 91 & 88.4 & 1,399 & 60.0 & 316 & 1,395 & & & \\
\hline 1978 & 1,319 & 80 & 84.4 & 1,564 & 40.4 & 336 & 1,574 & & & \\
\hline 1979 & 1,436 & 87 & 84.9 & 1,690 & 34.9 & 349 & 1,697 & & & \\
\hline 1980 & 1,581 & $\pi$ & 79.0 & 2,002 & 54.2 & 380 & 1,972 & & & \\
\hline 1981 & 1,622 & 65 & 71.8 & 2,260 & 48.3 & 403 & 2,178 & & & \\
\hline 1982 & 2,430 & 65 & 64.2 & 3,784 & 141.5 & 511 & 3,143 & & & \\
\hline 1983 & 2,764 & 62 & 64.1 & 4,312 & 147.0 & 614 & 4,071 & & & \\
\hline 1984 & 2,626 & 56 & 58.5 & 4,489 & 34.8 & 606 & 3,998 & & & \\
\hline 1985 & 2,299 & 63 & 60.5 & 3,797 & 41.4 & 604 & 3,983 & & & \\
\hline 1986 & 2,562 & 70 & 67.6 & 3,791 & 99.3 & 656 & 4,453 & & & \\
\hline-- & $-\cdots$ & -- & $-\cdot$ & $\cdots$ & $\cdots$ & $-\cdots$ & $\cdots$ & $-\cdot-$ & $-\cdot-$ & $-\cdot-$ \\
\hline 1987 & 2,689 & 64 & 66.1 & 4,066 & 66.3 & 676 & 4,627 & & & \\
\hline 1988 & 2,861 & 78 & 72.6 & 3,944 & 70.9 & 698 & 4,830 & 10,133 & 10,671 & 10,887 \\
\hline 1989 & & & & & 78.1 & 727 & 5,083 & 10,663 & 11,229 & 11,457 \\
\hline 1990 & & & & & 79.0 & 754 & 5,326 & 11,173 & 11,766 & 12,005 \\
\hline 1901 & & & & & 78.9 & 779 & 5,551 & 11,645 & 12,263 & 12,512 \\
\hline 1992 & & & & & 74.5 & 797 & 5,720 & 11,999 & 12,636 & 12,892 \\
\hline
\end{tabular}

Capacity-Capital stock Regression: Code 0 RSQ 0.890 Coefficients: Const -1440.9 stock 9.0

Ave, Capacity/stock $(74-86): 5.7$ Ave. Heekly Hours (High) 69.9 Shift Factor 2.25 Ave Heekly Hours (Low) 59.8 Shift Factor 2.63 Note: Output, Investment, and Capital Stock extrapolated for 1987 and 1988.

\section{Biological products}

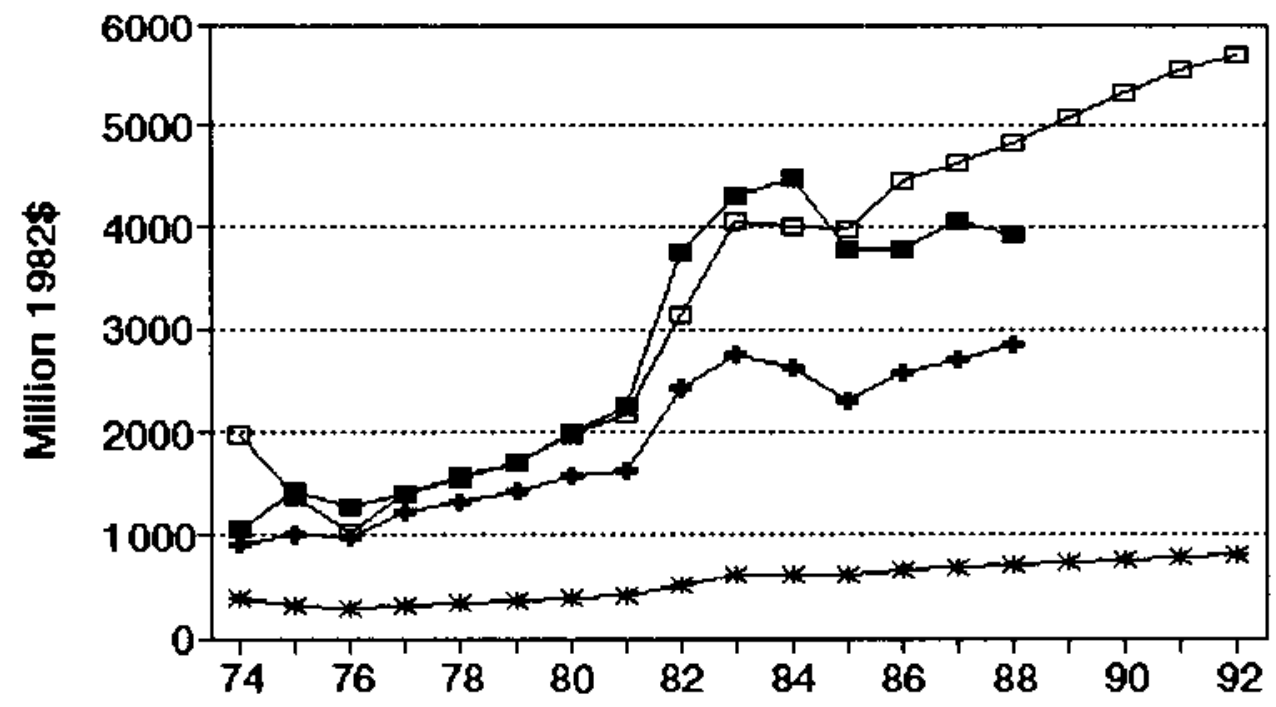

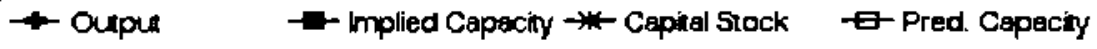




\section{No, 187 SIC 2833 Medicinals and botanicals}

MILLIONS OF 1982 DOLLARS (EXCL. CU RATES)

$\begin{array}{lccccccc} & 04 & \text { Ann. } & \text { Implied } & \text { Gross } & \text { Net } & \text { Pred. } & \ldots \\ \text { Gross } & \text { CU } & \text { dU } & \text { Prac. } & \text { In- } & \text { Cap. } & \text { Prac. } & \text { Emergency Capacity } \\ \text { Qutput Rate } & \text { Rate } & \text { Capac. } & \text { vest. } & \text { Stk. } & \text { Capac. } & 3 \text { mon. } 6 \text { mon. }>6 \text { mon. }\end{array}$

\begin{tabular}{|c|c|c|c|c|c|c|c|c|c|c|}
\hline 1974 & 1,975 & 80 & 80.1 & 2,467 & 220.5 & 1,073 & 2,080 & & & \\
\hline 1975 & 1,714 & 69 & 73.1 & 2,346 & 251.2 & 1,240 & 2,514 & & & \\
\hline 1976 & 2,076 & 87 & 80.3 & 2,587 & 173.6 & 1,327 & 2,740 & & & \\
\hline 1977 & 2,469 & 88 & 87.6 & 2,817 & 236.0 & 1,467 & 3,101 & & & \\
\hline 1978 & 2,502 & 80 & 83.0 & 3,014 & 166.9 & 1,532 & 3,270 & & & \\
\hline 1979 & 2,776 & 68 & $\pi 2.5$ & 3,829 & 211.4 & 1,630 & 3,525 & & & \\
\hline 1980 & 3,128 & 84 & 78.0 & 4,011 & 360.3 & 1,857 & 4,114 & & & \\
\hline 1981 & 3,372 & 60 & 69.0 & 4.884 & 370.1 & 2,077 & 4,685 & & & \\
\hline 1982 & 3,418 & 70 & 66.2 & 5,160 & 231.4 & 2,158 & 4,897 & & & \\
\hline 1983 & 3,369 & $\pi$ & 74.4 & 4,529 & 170.9 & 2,171 & 4,930 & & & \\
\hline 1984 & 3,612 & 74 & 75.1 & 4,808 & 204.4 & 2,212 & 5,035 & & & \\
\hline 1985 & 3,529 & $\pi$ & 75.9 & 4,651 & 3.8 & 2,053 & 4,622 & & & \\
\hline 1986 & 3,549 & 68 & 71.4 & 4,972 & 148.9 & 2,029 & 4,560 & & & \\
\hline $\begin{array}{l}-1987 \\
1987\end{array}$ & $\begin{array}{l}-- \\
3.694\end{array}$ & 79 & 74.9 & 4.934 & $\because \cdots$ & $\because 2$. & 4.756 & $\cdots$ & $-\cdots$ & 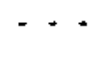 \\
\hline 1988 & 3,891 & 61 & 67.8 & 5,743 & 295.0 & 2,227 & 5,075 & 4,963 & 5,043 & 5,075 \\
\hline 1989 & & & & & 378.4 & 2,422 & 5,581 & 5,459 & 5,546 & 5,581 \\
\hline 1990 & & & & & 389.3 & 2,612 & 6,074 & 5,941 & 6,036 & 6,074 \\
\hline 1991 & & & & & 388.4 & 2,786 & 0,524 & 6,381 & 0,483 & 6,524 \\
\hline 1992 & & & & & 336.7 & 2,893 & 6,803 & 6,654 & 6,760 & 6,803 \\
\hline
\end{tabular}

Capecity-Capital Stock Regression: Code 0 RSO 0.930

Coefficients: Const -703.7 stock 2.6

Ave. Capacity/stock $(74-86): 2.2$ Ave. Weekly Hours (High) 162.1 Shift Factor 1.00 Ave Yeekly Hours (Low) 156.0 Shift factor 1.04 Note: Output, Investment, and Capital Stock extrapolated for 1987 and 1988.

2833 Medicinals and botanicals

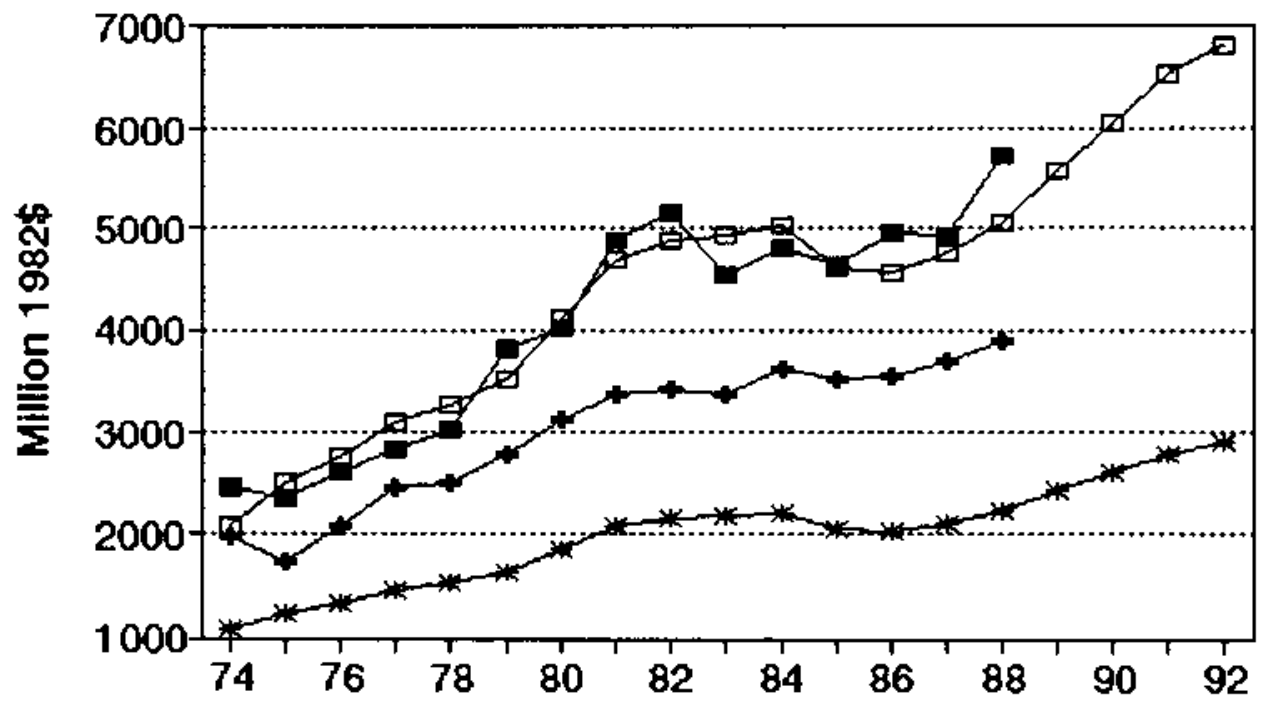

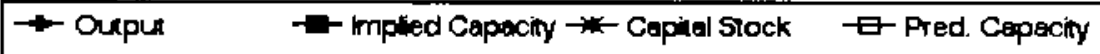


HO. 188 BIC 2834 Pharaceutical preparations

MILLIONS OF 1982 DOLLARS (EXCL. CU RATES)

$\begin{array}{lccccccc} & \alpha 4 & \text { Ann. Implied Gross } & \text { Het } & \text { Pred. } & \ldots \ldots \\ \text { Gross } & \text { a } & \text { Cu } & \text { Prac. } & \text { In- } & \text { Cap. } & \text { Prac. } & \text { Emergency Capacity } \\ \text { Output } & \text { Rate } & \text { Rate } & \text { Capac. } & \text { vest. } & \text { Stk. } & \text { Capac. } & 3 \text { mon. } 6 \text { mon. }>6 \text { mon. }\end{array}$

\begin{tabular}{|c|c|c|c|c|c|c|c|c|c|c|}
\hline 1974 & 15,586 & 76 & 76.0 & 20,507 & 300.2 & 2,862 & 21,098 & & & \\
\hline 1975 & 15,923 & 74 & 74.8 & 21,301 & 344.0 & 2,976 & 21,746 & & & \\
\hline 1976 & 17,438 & $\pi 2$ & 72.8 & 23,970 & 421.4 & 3,145 & 22,712 & & & \\
\hline 1977 & 17,598 & 68 & 69.5 & 25,321 & 461.3 & 3,340 & 23,830 & & & \\
\hline 1978 & 18,472 & 69 & 68.6 & 26,917 & 845.9 & 3,881 & 26,921 & & & \\
\hline 1979 & 18,355 & 72 & 70.9 & 25,898 & 169.7 & 3,762 & 26,245 & & & \\
\hline 1980 & 19,033 & 73 & 72.6 & 26,208 & 553.1 & 4,010 & 27,664 & & & \\
\hline 1981 & 19,157 & 67 & 69.3 & 27,663 & 464.5 & 4,158 & 28,509 & & & \\
\hline 1982 & 18,957 & 67 & 67.0 & 28,295 & 467.9 & 4,298 & 29,306 & & & \\
\hline 1983 & 19,681 & 59 & 62.0 & 31,744 & 582.9 & 4,533 & 30,652 & & & \\
\hline 1984 & 19,678 & 58 & 58.4 & 33,710 & 748.1 & 4,507 & 32,793 & & & \\
\hline 1985 & 20,021 & 56 & 56.8 & 35,279 & 830.0 & 5,334 & 35,231 & & & \\
\hline 1986 & 22,273 & 63 & 60.4 & 36,891 & 742.8 & 5,643 & 37,001 & & & \\
\hline-- & --- & -- & - - - & $-\cdots$ & -- & --- & $-\cdots$ & --- & ---- & $-\cdot-$ \\
\hline 1987 & 22,614 & 62 & 62.4 & 36,255 & 778.7 & 5,992 & 38,993 & & & \\
\hline 1988 & 23,064 & 66 & 64.5 & 35,759 & 814.0 & 6,349 & 41,036 & 54,404 & 56,722 & 57,656 \\
\hline 1989 & & & & & 849.2 & 6,714 & 43,125 & 57,173 & 59,608 & 60,590 \\
\hline 1990 & & & & & 884.5 & 7,087 & 45,255 & 59,998 & 62,554 & 63,584 \\
\hline 1991 & & & & & 919.7 & 7,466 & 47,425 & 62,875 & 65,553 & 66,633 \\
\hline 1992 & & & & & 955.0 & 7.852 & 49,632 & 65,800 & 68,603 & 69,733 \\
\hline
\end{tabular}

Capacity-Capital stock Regression: Code 0 RSO 0.970 Coefficients: Const 4729.1 stock 5.7

Ave. Capacity/Stock $(74-86): 6.9$ Ave. Weekly Hours (High) 112.2 Shift Factor 1.40 Ave Heekly Hours (LOW) 109.0 Shift Factor 1.45 Note: Output, Investment, and Capital Stock extrapolated for 1987 and 1988.

\section{Pharmaceutical preparations}

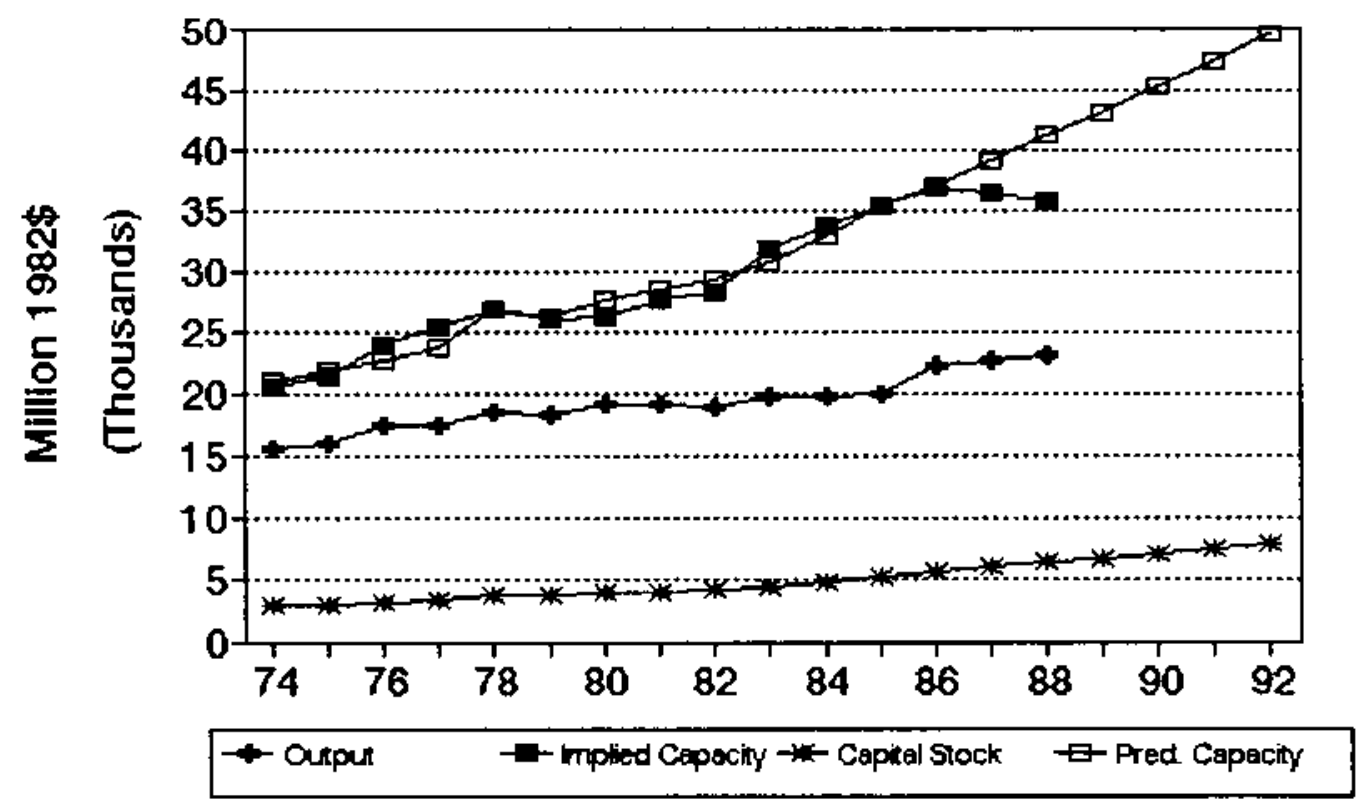


No. 189 sIC 2841 soap and other detergents

MILLIONS OF 1982 DOLLARS (EXCL. CU RATES)

$\begin{array}{lccccccc} & 04 & \text { Ann. } & \text { Implied Gross } & \text { Het } & \text { Pred. } & -\ldots . \ldots \\ \text { Gross } & \text { CU } & \text { CU } & \text { Prac. } & \text { In- } & \text { Cap. } & \text { Prac. } & \text { Emergency Capacity } \\ \text { Output Rate } & \text { Rate } & \text { Capac. } & \text { vest. } & \text { Stk. } & \text { Capac. } & 3 \text { mon. } 6 \text { mon. } & \end{array}$

\begin{tabular}{|c|c|c|c|c|c|c|c|c|c|c|}
\hline 1974 & 8,643 & 62 & 62.0 & 13,941 & 176.3 & 1,553 & 13,411 & & & \\
\hline 1975 & 7,809 & 62 & 62.0 & 12,595 & 192.4 & 1,625 & 13,499 & & & \\
\hline 1976 & 8,316 & 54 & 57.0 & 14,590 & 151.7 & 1,653 & 13,532 & & & \\
\hline 1977 & 8,552 & 58 & 56.5 & 15,136 & 250.6 & 1,768 & 13,672 & & & \\
\hline 1978 & 9,208 & 66 & 63.0 & 14,615 & 221.7 & 1,845 & 13,764 & & & \\
\hline 1979 & 9,095 & $\pi$ & 71.6 & 12,698 & 203.9 & 1,896 & 13,826 & & & \\
\hline 1980 & 9,657 & 78 & 76.9 & 12,561 & 203.5 & 1,942 & 13,881 & & & \\
\hline 1981 & 9,614 & 70 & 73.0 & 13,170 & 159.4 & 1,938 & 13,877 & & & \\
\hline 1982 & 9.170 & 68 & 68.8 & 13,351 & 172.6 & 1,947 & 13,888 & & & \\
\hline 1983 & 8,741 & $64 *$ & 65.4 & 13,361 & 255.6 & 2,030 & 13,988 & & & \\
\hline 1984 & 9,091 & 66 & 65.2 & 13,942 & 304.0 & 2,153 & 14,136 & & & \\
\hline 1985 & 9,321 & 60 & 62.3 & 14,973 & 332.8 & 2,293 & 14,306 & & & \\
\hline 1986 & 9,633 & 65 & 63.1 & 15,260 & 290.4 & 2,383 & 14,414 & & & \\
\hline - - - & $\cdots$ & $\because$ & $-\cdots$ & $\cdots$ & $\because-$ & $\cdots$ & - - - & $-\cdots$ & ---- & $\cdots-$ \\
\hline 1987 & 9,689 & 65 & 65.0 & 14,906 & 306.6 & 2,489 & 14,542 & & & \\
\hline 1988 & 9.763 & 70 & 68.1 & 14,331 & 319.0 & 2,599 & $14,6 \pi 5$ & 17,657 & 18,240 & 18,476 \\
\hline 1989 & & & & & 331.3 & 2,711 & 14,811 & 17,821 & 18,410 & 18,647 \\
\hline 1990 & & & & & 343.7 & 2,827 & 14,953 & 17,989 & 18,584 & 18,823 \\
\hline 1991 & & & & & 356.0 & 2,945 & 15,094 & 18,161 & 18,761 & 19,003 \\
\hline 1992 & & & & & 368.4 & 3,066 & 15,240 & 18,336 & 18,942 & 19,187 \\
\hline
\end{tabular}

Capacity-Capital Stock Regression: Code 0 RSO 0.090

Coefficients: Const 11534.9 stock 1.2

Ave. Capacity/stock $(74-86): 7.2$ Ave. Weekly Hours (High) 125.7 Shift factor 1.26 Ave Heekly Hours (Low) 121.2 Shift Factor 1.30 - Imputed Note: Output, Investment, and Capital Stock extrapolated for 1987 and 1988.

\section{Soap and other detergents}

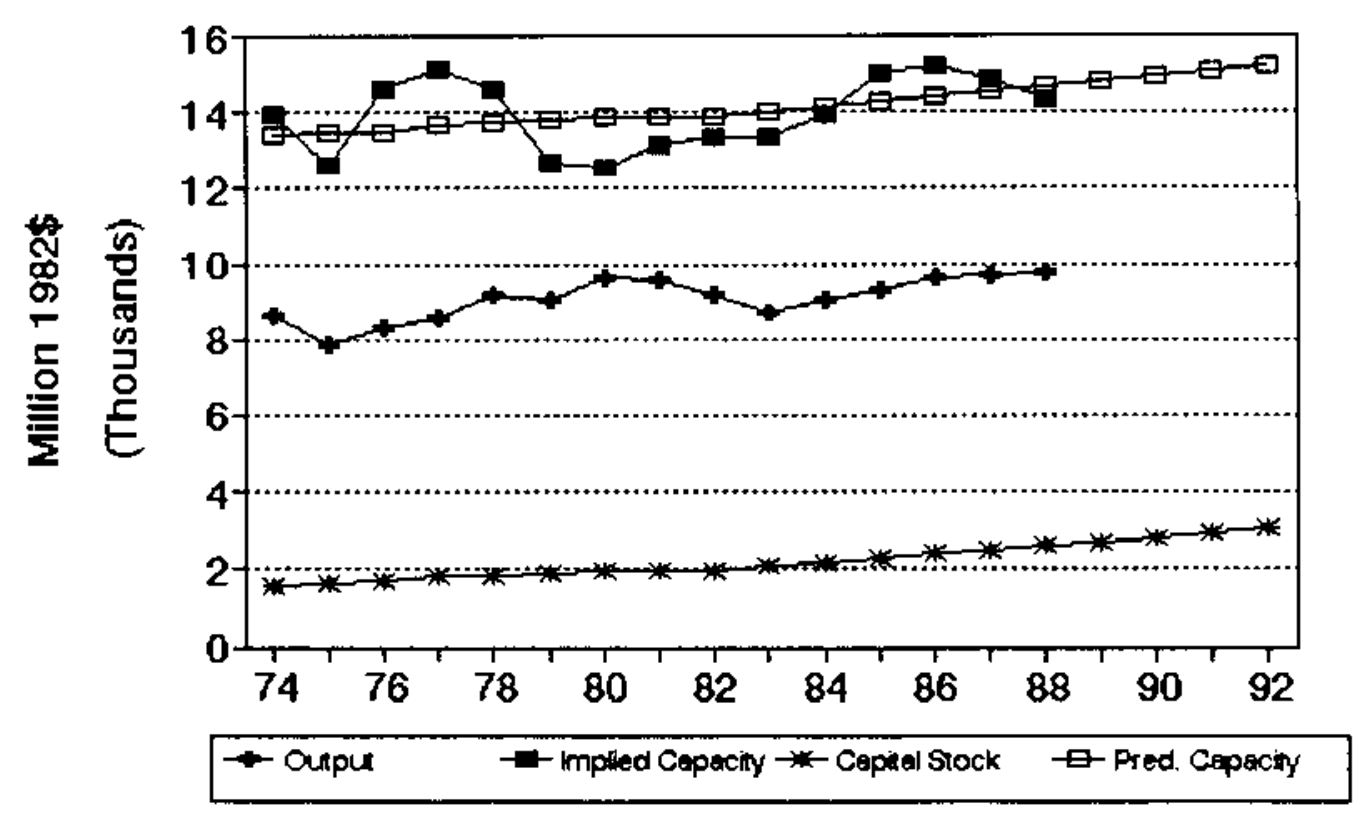


No. 190 sIC 2842 Polishes and anitation goods

MILLIONS OF 1982 DOLLARS (EXCL. CU RATES)

$\begin{array}{lccccccc} & \text { Q4 } & \text { Ann. Implied Gross } & \text { Net } & \text { Pred. } & \ldots & \ldots \\ \text { Gross } & \text { QU } & \text { CU } & \text { Prac. } & \text { In- } & \text { Cap. } & \text { Prac. } & \text { Emergency Capacity } \\ \text { Output } & \text { Rate } & \text { Rate } & \text { Capac. } & \text { vest. } & \text { Stk. } & \text { Capac. } & 3 \text { mon. } 6 \text { mon. }\end{array}$

\begin{tabular}{|c|c|c|c|c|c|c|c|c|c|c|}
\hline 1974 & 4,517 & 55 & 55.0 & 8,212 & 38.5 & 438 & 7,971 & & & \\
\hline 1975 & 4,418 & 64 & 60.6 & 7,288 & 42.5 & 442 & 7,972 & & & \\
\hline 1976 & 4,628 & 58 & 60.3 & 7,682 & 46.1 & 446 & 7,972 & & & \\
\hline 1977 & 4,832 & 55 & 56.1 & 8,609 & 72.0 & 472 & 7,975 & & & \\
\hline 1978 & 5,165 & 55 & 55.0 & 9,390 & 75.4 & 496 & 7,978 & & & \\
\hline 1979 & 5,023 & 62 & 59.4 & 8,459 & 42.8 & 489 & 7,977 & & & \\
\hline 1980 & 4,793 & 66 & 64.5 & 7.431 & 100.9 & 532 & 7,982 & & & \\
\hline 1981 & 5,009 & 77 & 72.9 & 6,874 & 75.2 & 548 & 7,984 & & & \\
\hline 1982 & 4,644 & 67 & 70.8 & 6,563 & 11.3 & 504 & 7.979 & & & \\
\hline 1983 & 4,554 & 55 & 59.5 & 7,653 & 6.5 & 459 & 7,974 & & & \\
\hline 1984 & 4,649 & 41 & 46.3 & 10,052 & 114.5 & 516 & 7,981 & & & \\
\hline 1985 & 4,210 & 58 & 51.6 & 8,154 & $(7.7)$ & 456 & 7,973 & & & \\
\hline 1986 & 4,568 & 65 & 62.4 & 7,324 & 54.1 & 459 & 7,974 & & & \\
\hline $\begin{array}{l}\cdots \\
1987\end{array}$ & $\cdots$ & 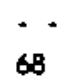 & 66.9 & 6,840 & 54.9 & $\because 462$ & $\cdots$ & $\cdots \cdot$ & --- & --- \\
\hline 1988 & 4,581 & $68 *$ & 68.3 & 6,712 & 64.7 & 474 & 7,976 & 10,084 & 10,491 & 10,655 \\
\hline 1989 & & & & & 79.9 & 500 & 7,979 & 10,088 & 10,495 & 10,659 \\
\hline 1990 & & & & & 81.9 & 526 & 7,982 & 10,092 & 10,499 & 10,664 \\
\hline 1991 & & & & & 81.8 & 548 & 7,984 & 10,096 & 10,503 & 10,667 \\
\hline 1992 & & & & & $\pi .3$ & 558 & 7,986 & 10,097 & 10,505 & 10,669 \\
\hline
\end{tabular}

Capacity-Capital stock Regression: Code 1 RSQ 0.000 Coefficients: Const 7918.8 stock 0.1

Ave. Cepacity/Stock ( $74-86): 16.6$ Ave. Weekly Hours (High) 118.2 Shift Factor 1.34 Ave Weekly Hours (Low) 93.6 Shift Factor 1.68 - Inputed Note: Output, Investment, and Capital stock extrapolated for 1987 and 1988.

\section{Polishes and sanitation goods}

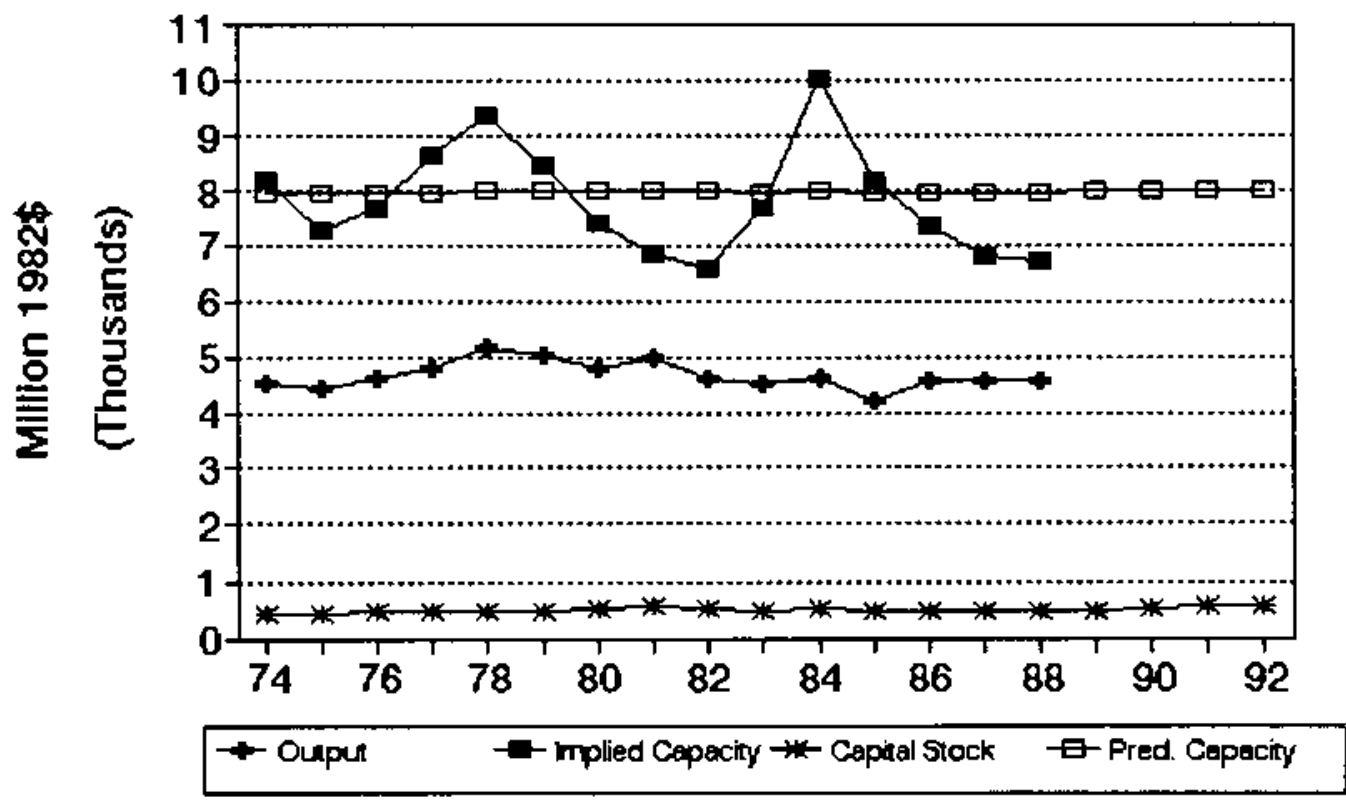


No. 191 SIC 2843 Surface active agents

MILLIONS OF 1982 DOLLARS (EXCL. CU RATES)

$\begin{array}{lccccccc} & \text { Q4 } & \text { Ann. } & \text { Implied Gross } & \text { Net } & \text { Pred. } & \ldots \ldots \\ \text { Gross } & \text { CU } & \text { CU } & \text { Prac. } & \text { In- } & \text { Cap. } & \text { Prac. } & \text { Emergency Capacity } \\ \text { Output } & \text { Rate } & \text { Rate } & \text { Capac. } & \text { vest. } & \text { Stk. } & \text { Capac. } & 3 \text { mon. } 6 \text { mon. }>6 \text { mon. }\end{array}$

\begin{tabular}{|c|c|c|c|c|c|c|c|c|c|c|}
\hline 1974 & 1,666 & 58 & 58.7 & 2,837 & 102.3 & 384 & 2,451 & & & \\
\hline 1975 & 1,169 & 53 & 54.0 & 2,163 & 16.5 & 377 & 2,400 & & & \\
\hline 1976 & 1,390 & 69 & 63.0 & 2,208 & 44.8 & 395 & 2,523 & & & \\
\hline 1977 & 1,458 & 47 & 55.3 & 2,634 & 45.5 & 416 & 2,644 & & & \\
\hline 1978 & 1,770 & 71 & 62.0 & 2,854 & 51.9 & 437 & 2,794 & & & \\
\hline 1979 & 1,745 & 52 & 59.2 & 2,945 & 38.0 & 445 & 2,849 & & & \\
\hline 1980 & 1,976 & 57 & 54.8 & 3,608 & 72.9 & 485 & 3,107 & & & \\
\hline 1981 & 2,197 & 73 & 67.7 & 3,247 & 114.7 & 557 & 3,577 & & & \\
\hline 1982 & 2,131 & 59 & 64.0 & 3,332 & 99.0 & 611 & 3,930 & & & \\
\hline 1983 & 2,243 & 57 & 58.0 & 3,867 & 112.9 & 673 & 4,331 & & & \\
\hline 1984 & 2,485 & 60 & 58.9 & 4,216 & 19.8 & 641 & 4,125 & & & \\
\hline 1985 & 2,441 & 50 & 53.8 & 4,537 & 90.8 & 673 & 4,349 & & & \\
\hline 1988 & 2,656 & 54 & 52.6 & 5,053 & 71.2 & 687 & 4,422 & & & \\
\hline i & $\begin{array}{c}2,727 \\
2,-\end{array}$ & $\begin{array}{l}- \\
57\end{array}$ & $\begin{array}{l}\cdots \\
55.8\end{array}$ & 4.886 & $\begin{array}{r}--\cdot \\
73.5\end{array}$ & 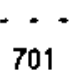 & $\begin{array}{l}\cdots \\
4,519\end{array}$ & $\cdots$ & -- & $-\cdots$ \\
\hline 1988 & 2,823 & 70 & 65.1 & 4,339 & 87.5 & 729 & 4.699 & 4,769 & 4,913 & 4,971 \\
\hline 1989 & & & & & 109.1 & 776 & 5,004 & 5,079 & 5,232 & 5,294 \\
\hline 1990 & & & & & 112.0 & 822 & 5,302 & 5,381 & 5,544 & 5,609 \\
\hline 1991 & & & & & 111.7 & 863 & 5,572 & 5,656 & 5,827 & 5,896 \\
\hline 1992 & & & & & 98.2 & 888 & 5,732 & 5,818 & 5,994 & 6,064 \\
\hline
\end{tabular}

Capacity-Capital stock Regression: Code 0 RSO 0.820

Coefficients: Const -55.3 stock 6.5 Ave. Capacity/Stock $(74-86): 6.4$

Ave. Heekly Hours (High) 150.7 Shift Factor 1.06 Ave Heekly Hours (Low) 143.5 Shift Factor 1.11 Note: Output, lnvestment, and Capital Stock extrapolated for 1987 and 1988.

\section{Surface active agents}

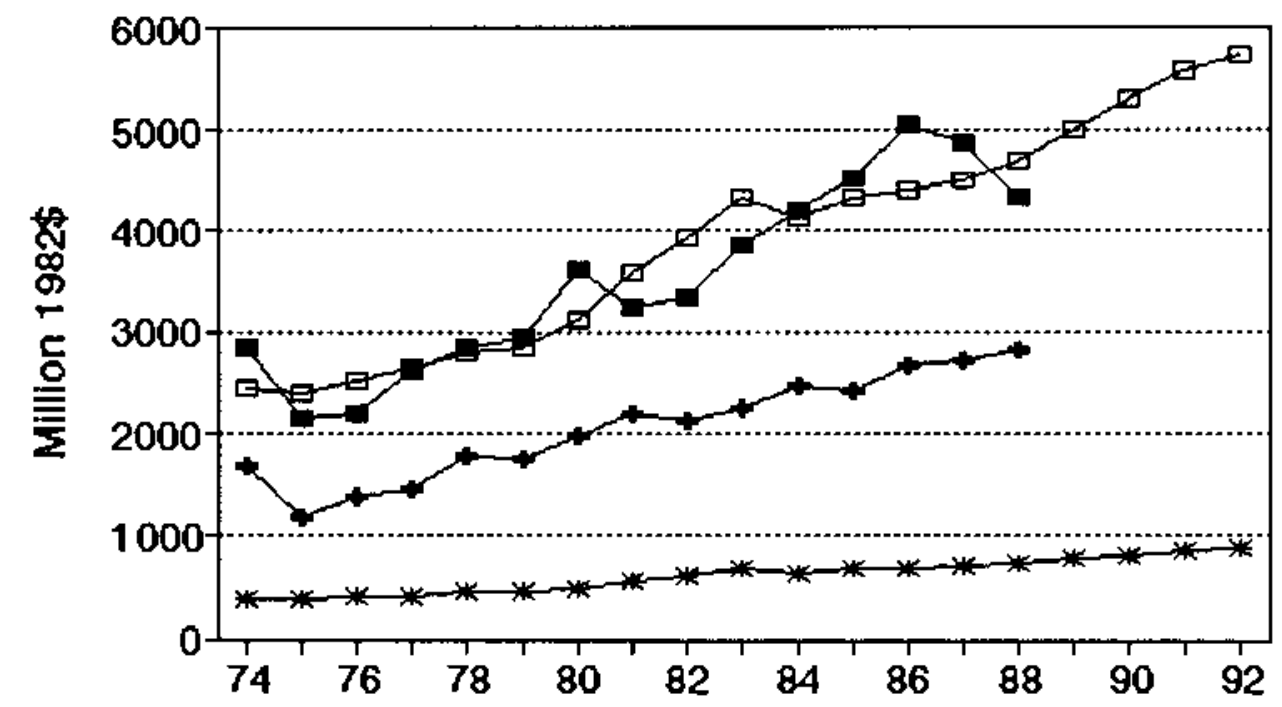

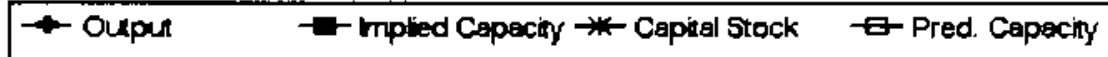




\title{
No. 192 sIC 2844 Toilet preparations
}

\author{
MILLIONS OF 1982 DOLLARS (EXCL. CU RATES)
}

$\begin{array}{lccccccc} & \text { Q4 Am. Implied Gross } & \text { Net } & \text { Pred. } & -\ldots & -\ldots \\ \text { Gross } & \text { CU } & \text { CU } & \text { Prac. } & \text { In- } & \text { Cap. } & \text { Prac. } & \text { Emergency Capacity } \\ \text { Output Rate } & \text { Rate } & \text { Capac. } & \text { vest. } & \text { Stk. } & \text { Capac. } & 3 \text { mon. } 6 \text { mon. }>6 \text { mon. }\end{array}$

\begin{tabular}{|c|c|c|c|c|c|c|c|c|c|c|}
\hline 1974 & 9,162 & 43 & 43.0 & 21,306 & 100.2 & 818 & 21,306 & & & \\
\hline 1975 & 9,093 & 73 & 61.8 & 14,726 & 113.3 & 868 & 15,502 & & & \\
\hline 1976 & 9.972 & 63 & 66.8 & 14,940 & 67.9 & 870 & 15,503 & & & \\
\hline 1977 & 10,488 & 67 & 65.5 & 16,012 & 131.7 & 931 & 15,517 & & & \\
\hline 1978 & 10,988 & 59 & 62.0 & 17,723 & 140.9 & 997 & 15,533 & & & \\
\hline 1979 & 10,482 & 67 & 64.0 & 16,378 & 179.9 & 1,093 & 15,556 & & & \\
\hline 1980 & 9,344 & 64 & 65.1 & 14,348 & 121.1 & 1,129 & 15,564 & & & \\
\hline 1981 & 9,421 & 67 & 65.9 & 14,302 & 60.7 & 1,105 & 15,558 & & & \\
\hline 1982 & 10,200 & 62 & 63.9 & 15,983 & 221.1 & 1,231 & 15,588 & & & \\
\hline 1983 & 9,532 & 62 & 62.0 & 15,375 & 202.4 & 1,328 & 15,611 & & & \\
\hline 1984 & 9,899 & 63 & 62.6 & 15,806 & 86.8 & 1,311 & 15,607 & & & \\
\hline 1985 & 10,221 & 70 & 87.4 & 15,171 & 227.5 & 1,429 & 15,635 & & & \\
\hline 1986 & 10,540 & 63 & 85.6 & 16,061 & 181.2 & 1,493 & 15,650 & & & \\
\hline$\cdots$ & $\cdots \cdot$ & $\cdot$ & $\cdots$ & $\cdots$ & $\cdots$ & $\cdots$ &.-- & --- & ---- & $-\cdot \cdot$ \\
\hline 1987 & 10,604 & 69 & 66.8 & 15,885 & 197.0 & 1,574 & 15,669 & & & \\
\hline 1988 & 10,687 & 68 & 68.4 & 15,630 & 205.1 & 1,657 & 15,689 & 24,188 & 25,187 & 25,589 \\
\hline 1989 & & & & & 213.3 & 1,742 & 15,709 & 24,219 & 25,219 & 25,622 \\
\hline 1990 & & & & & 221.4 & 1,828 & 15,730 & 24,251 & 25,252 & 25,655 \\
\hline 1991 & & & & & 229.5 & 1,916 & 15,750 & 24,283 & 25,285 & 25,689 \\
\hline 1992 & & & & & 237.7 & 2,005 & 15,771 & 24,315 & 25,319 & 25,723 \\
\hline
\end{tabular}

Capacity-Capital Stock Regression: Code -1 RSO 0.750

Coefficients: Const 21112.0 Stock 0.2 D(75-86) -5815.5 Ave. Capecity/stock $(74-86): 14.3$ Ave. Weekly Hours (High) 96.5 shift Factor 1.63 Ave Ueekly Hours (Low) 92.9 Shift Factor 1.70 Note: Output, Imvestment, and Capital Stock extrapolated for 1987 and 1988.

\section{Toilet preparations}

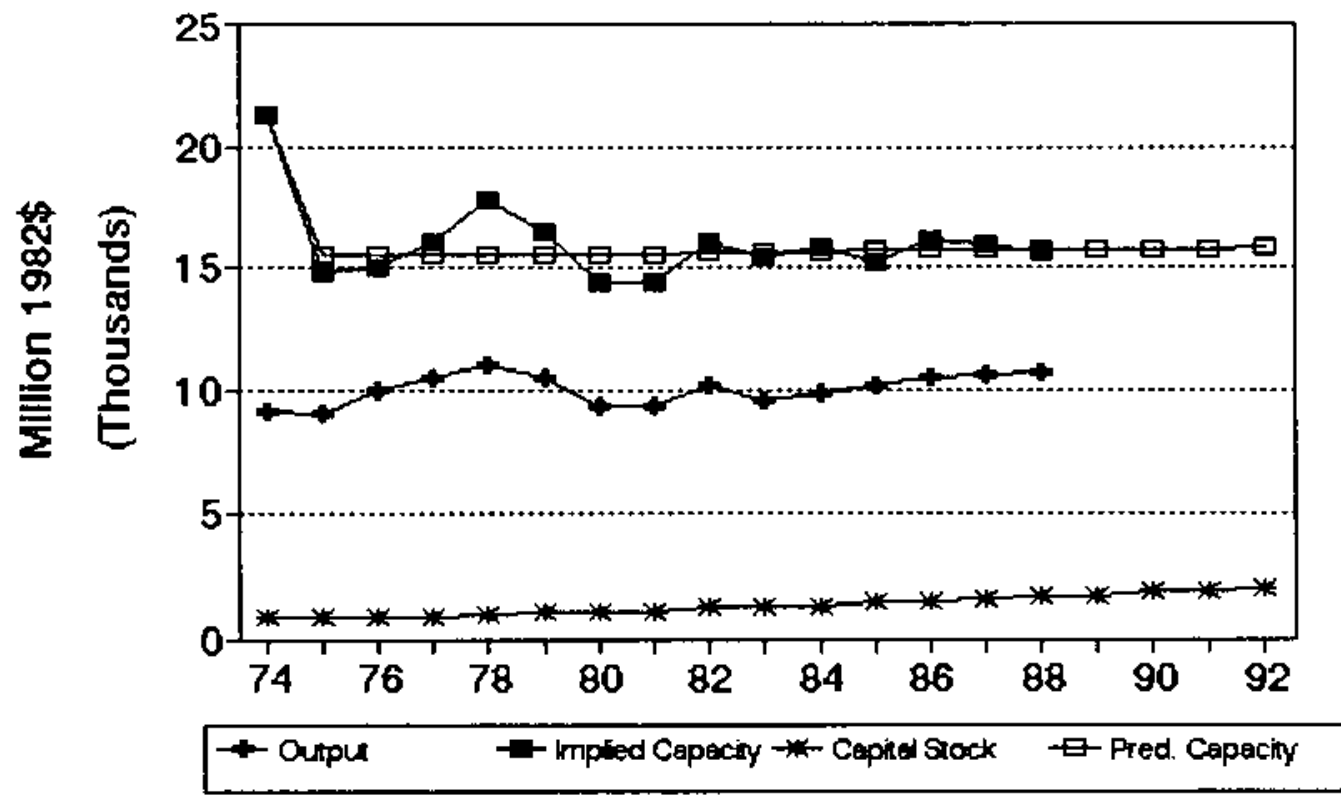




\section{No. 193 SIC 2851 Paints and allied products}

MILLIONS OF 1982 dollars (EXCL. CU RATES)

$\begin{array}{lccccccc} & \text { Q4 } & \text { Ann. Implied Gross } & \text { Net } & \text { Pred. } & \ldots & \ldots . . . \\ \text { Gross } & \text { CU } & \text { CU } & \text { Prac. } & \text { In- } & \text { Cap. } & \text { Prac. } & \text { Emergency Capacity } \\ \text { Output } & \text { Rate } & \text { Rate } & \text { Capac. } & \text { vest. } & \text { Stk. } & \text { Capac. } & 3 \text { mon. } 6 \text { mon. }>6 \text { mon. }\end{array}$

\begin{tabular}{|c|c|c|c|c|c|c|c|c|c|c|}
\hline 1974 & 9,037 & 76 & 76.0 & 11,891 & 112.6 & 1,209 & 11,235 & & & \\
\hline 1973 & 7,963 & 71 & 72.9 & 10,927 & 149.5 & 1,251 & 12,046 & & & \\
\hline 1976 & 9,013 & 70 & 70.4 & 12,806 & 126.6 & 1,263 & 12,290 & & & \\
\hline 1977 & 9,594 & 72 & 71.3 & 13,465 & 157.7 & 1,304 & 13,099 & & & \\
\hline 1978 & 9,768 & 71 & 71.4 & 13,685 & 154.8 & 1,336 & 13,731 & & & \\
\hline 1979 & 10,134 & 71 & 71.0 & 14,274 & 193.0 & 1,397 & 14,930 & & & \\
\hline 1980 & 9,241 & 66 & 67.9 & 13,615 & 183.3 & 1,463 & 15,830 & & & \\
\hline 1981 & 9,656 & 67 & 66.6 & 14,494 & 216.4 & 1,513 & 17,214 & & & \\
\hline 1982 & 9,216 & 60 & 62.6 & 14,717 & 93.6 & 1,460 & 16,174 & & & \\
\hline 1983 & 10,197 & 60 & 60.0 & 16,995 & 149.2 & 1,457 & 16,111 & & & \\
\hline 1984 & 10,481 & 56 & 57.5 & 18,227 & 149.9 & 1,454 & 16,044 & & & \\
\hline 1985 & 10,838 & 54 & 54.8 & 19,796 & 206.6 & 1,503 & 17,007 & & & \\
\hline 1986 & 10,789 & 63 & 59.6 & 18,094 & 173.7 & 1,516 & 17,275 & & & \\
\hline$\therefore$ & - - - - & -- & 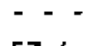 & $\because \cdot \cdot$ & $-\overline{-}$ & $\cdots$ & "- & $\cdots$ & --- & $-\cdot \cdot \cdot$ \\
\hline 1987 & 40,973 & 54 & 57.4 & 19,124 & 162.3 & 1,519 & 17,327 & & & \\
\hline 1988 & 11,216 & 59 & 57.1 & 19.634 & 182.9 & 1,542 & $17, \pi 79$ & 25,683 & 26,753 & 27,184 \\
\hline 1989 & & & & & 214.6 & 1,594 & 18,809 & 27,171 & 28,303 & 28,759 \\
\hline 1990 & & & & & 218.8 & 1,645 & 19,813 & 28,621 & 29,813 & 30,294 \\
\hline 1991 & & & & & 218.5 & 1,690 & 20,704 & 29,908 & 31,154 & 31,656 \\
\hline 1992 & & & & & 198.6 & 1,711 & 21,110 & 30,495 & 31,765 & 32,277 \\
\hline
\end{tabular}

Capacity-Capital Stock Regression: Code 1 RSQ 0.630

Coefficients: Const -12572.5 Stock 19.7

Ave. Capacity/Stock $(74-86): 10.7$ Ave. Weekly Hours (High) 103.0 Shift Factor 1.53 Ave Heekly Hours (Low) 100.0 Shift Factor 1.58 Note: Output, Investment, and Capital stock extrapolated for 1987 and 1988.

\section{Paints and allied products}

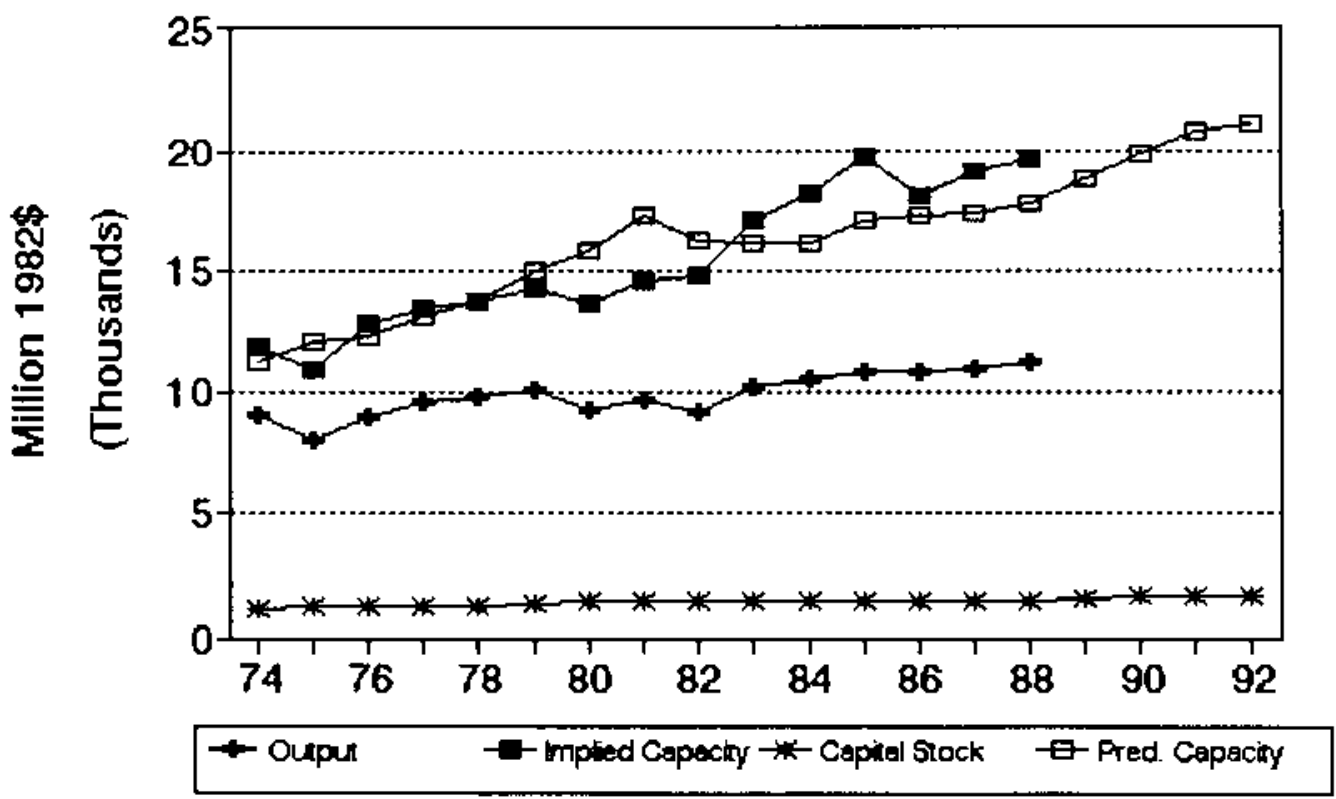


No. 194 SIC 2861 Gu and wood chemicals

MILLIONS OF 1982 DOLLARS (EXCL. CU RATES)

$\begin{array}{lccccccc} & 04 & \text { Amn. Implied Gross } & \text { Net } & \text { Pred. } & \ldots \\ \text { Gross } & \text { CU } & \text { CU } & \text { Prac. } & \text { In- } & \text { Cap. } & \text { Prac. } & \text { Emergency Capacity } \\ \text { Output } & \text { Rate } & \text { Rate } & \text { Capac. } & \text { vest. } & 5 t k . & \text { Capac. } & 3 \text { mon. } 6 \text { mon. }>6 \text { mon. }\end{array}$

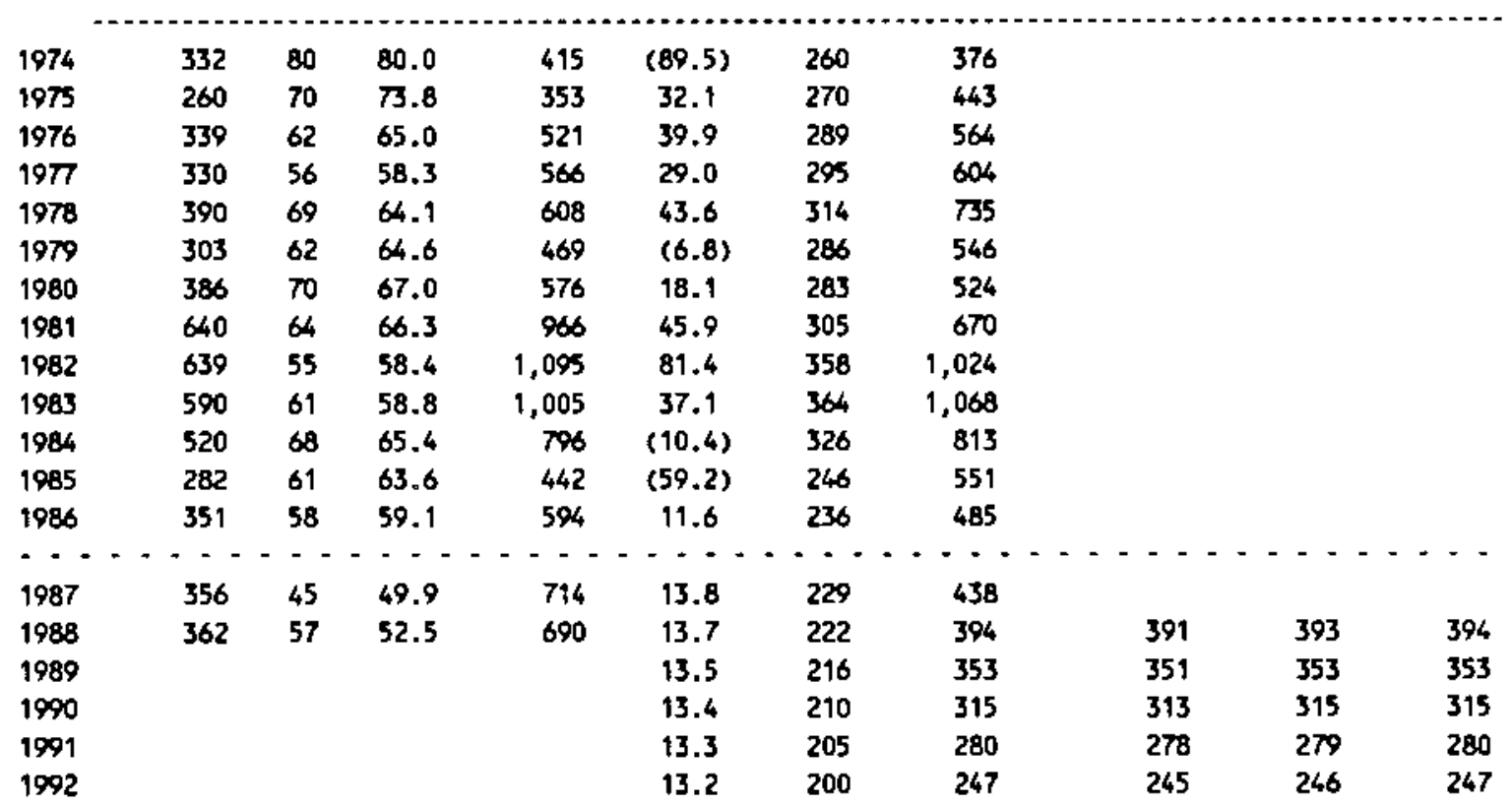

Capacity-Capital Stock Regression: Code -1 RSO 0.770

Coefficients: Const -1355.5 stock 6.7 D(85-86) 272.2 Ave. Capecity/Stock (74-86): 2.2 Ave. Weekly Hours (High) 168.0 Shift factor 1.00 Ave Veekly Hours (Low) 167.6 Shift Factor 1.00 Note: Dutput, Imvestment, and Capital stock extrapolated for 1987 and 1988.

\section{Gum and wood chemicals}

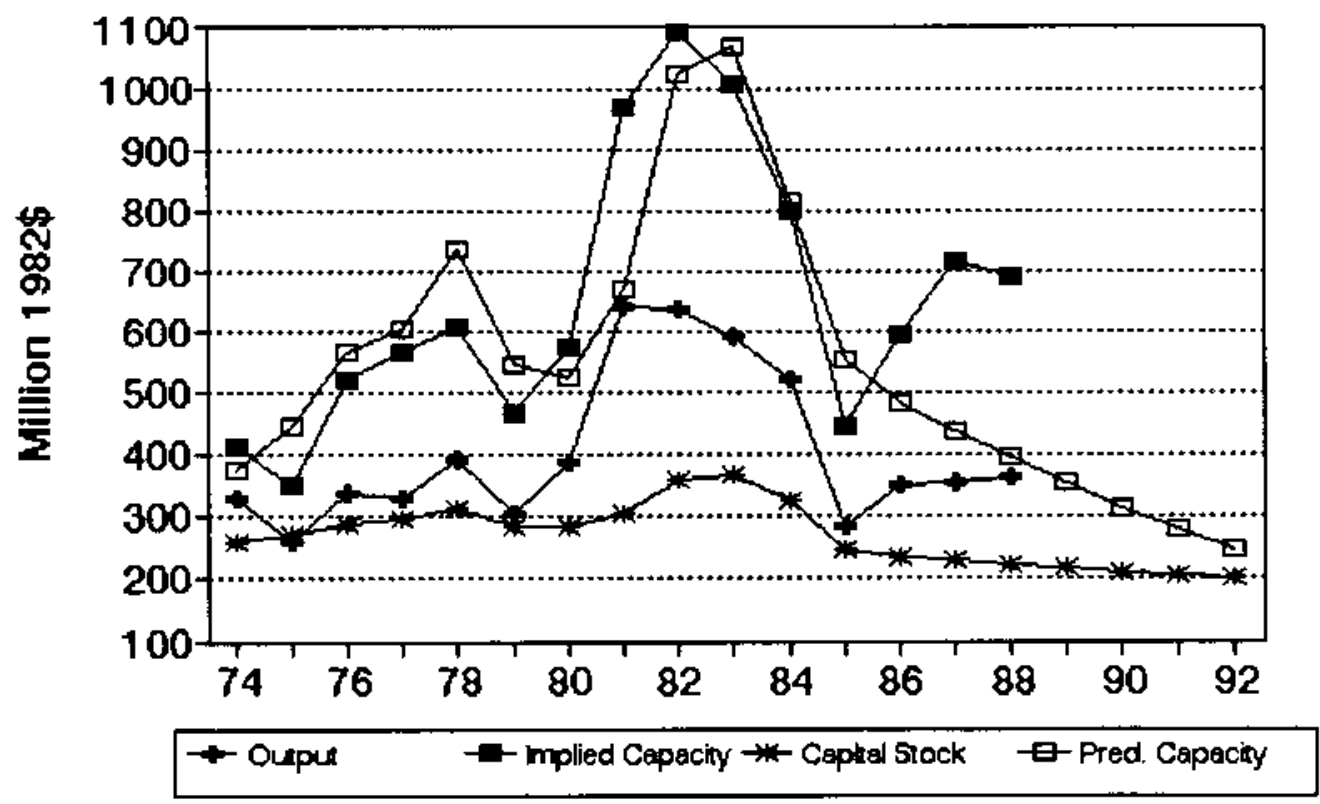




\section{No. 195 SIC 2865 Cyclic crudes and intermediates}

HILLIONS OF 1982 DDLLARS (EXCL. CU RATES)

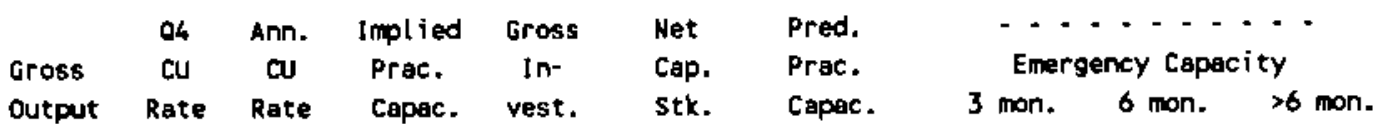

\begin{tabular}{|c|c|c|c|c|c|c|c|c|c|c|}
\hline 1974 & 7,273 & 69 & 73.9 & 9,850 & 621.8 & 4,415 & 8,613 & & & \\
\hline 1973 & 6,468 & 81 & 70.1 & 9,228 & $1,120.4$ & 5,204 & 10,056 & & & \\
\hline 1976 & 7,637 & 78 & 79.2 & 9,642 & 519.4 & 5,387 & 10,389 & & & \\
\hline 1977 & 9,017 & 73 & 75.5 & 11,937 & $1,136.6$ & 6,129 & 11,745 & & & \\
\hline 1978 & 9,190 & 80 & 77.6 & 11,839 & 382.4 & 6,113 & 11,717 & & & \\
\hline 1979 & 7,727 & 73 & 76.5 & 10,100 & $(257.0)$ & 5,489 & 10,576 & & & \\
\hline 1980 & 7,656 & 69 & 67.7 & 11,311 & $1,049.0$ & 6,096 & 11,685 & & & \\
\hline 1981 & 7,566 & 61 & 68.1 & 11,112 & 265.5 & 5,914 & 11,352 & & & \\
\hline 1982 & 7,209 & 50 & 52.6 & 13,694 & 538.7 & 5,966 & 11,449 & & & \\
\hline 1983 & 7,337 & 73 & 65.8 & 11,160 & 391.4 & 5,862 & 11,258 & & & \\
\hline 1984 & 7,598 & 74 & 74.1 & 10,250 & $(32.4)$ & 5,342 & 10,307 & & & \\
\hline 1985 & 7,885 & 68 & 70.6 & 11,162 & 893.5 & 5,680 & 10,926 & & & \\
\hline 1986 & 6,987 & 79 & $\pi .3$ & 9,277 & 321.7 & 5,440 & 10,487 & & & \\
\hline$-\cdot$ & $\cdots$ & $\cdots$ & -- & --- & $\cdots$ & $\cdots \cdot$ & $-\cdots$ & $-\cdots \cdots$ & --- & --- \\
\hline 1987 & 6,992 & 83 & 81.1 & 8,618 & 500.0 & 5,394 & 10,402 & & & \\
\hline 1988 & 7,000 & 85 & 84.0 & 8,330 & 500.0 & 5,352 & 10,326 & 10,126 & 10,357 & 10,450 \\
\hline 1989 & & & & & 500.0 & 5,314 & 10,257 & 10,058 & 10,288 & 10,380 \\
\hline 1990 & & & & & 500.0 & 5,281 & 10,195 & 9.997 & 10,226 & 10,318 \\
\hline 1991 & & & & & 500.0 & 5,250 & 10,140 & 9,943 & 10,170 & 10,261 \\
\hline 1992 & & & & & 500.0 & 5,223 & 10,090 & 9,894 & 10,120 & 10,211 \\
\hline
\end{tabular}

Capacity-Capital Stock Regression: Code 0 RSO 0.490

Coefficients: Const 544.9 stock 1.8 Ave. Capacity/Stock $(74-86): 1.9$ Ave. Weekly Hours (High) 163.8 Shift Factor 1.01 Ave Weekly Hours (LOU) 161.2 Shift Factor 1.03 Note: Output, Investment, and Capital Stock extrapolated for 1987 and 1988.

\section{Cyclic crudes and intermediates}

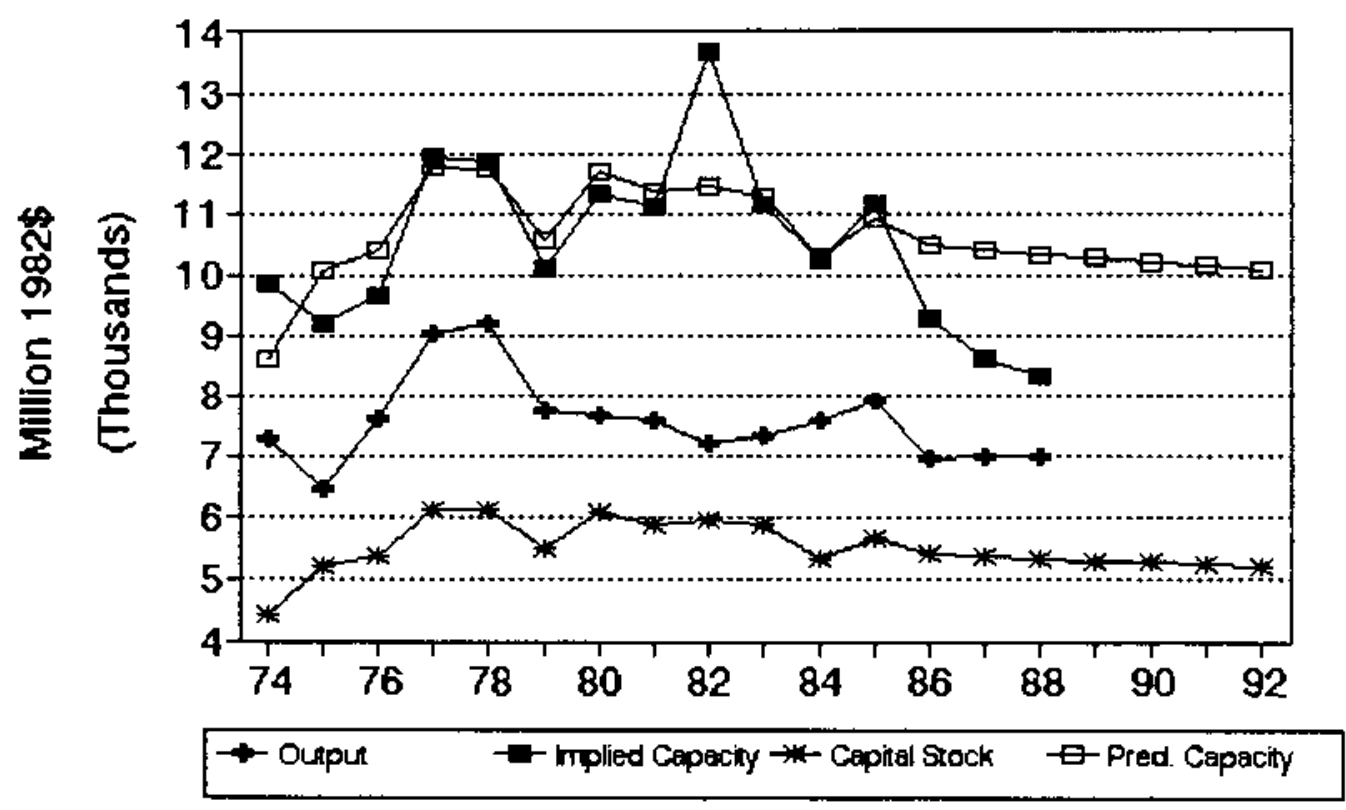


No. 196 SIC 2869 Industrial organic chenicale, n.e.c.

MILLIONS OF 1982 DOLLARS (EXCL. CU RATES)

$\begin{array}{lccccccc} & \alpha 4 & \text { Ann. Implied Gross } & \text { Het } & \text { Pred. } & \ldots \\ \text { Gross } & \text { a } & \text { CU } & \text { Prac. } & \text { In- } & \text { Cap. } & \text { Prac. } & \text { Emergency Capacity } \\ \text { Output Rate } & \text { Rate } & \text { Capac. } & \text { vest. } & \text { Stk. } & \text { Capac. } & 3 \text { mon. } 6 \text { mon. }>6 \text { mon, }\end{array}$

\begin{tabular}{|c|c|c|c|c|c|c|c|c|c|c|}
\hline 1974 & 34,249 & 88 & 93.5 & 36,649 & $1,724.9$ & 19,705 & 33,850 & & & \\
\hline 1975 & 27.233 & 81 & 77.0 & 35,385 & $3,345.9$ & 21,583 & 36,816 & & & \\
\hline 1976 & 32,259 & 80 & 80.4 & 40,113 & $4,073.2$ & 24,064 & 40,734 & & & \\
\hline 1977 & 37,561 & 78 & 79.4 & 47,333 & 5.634 .5 & 27,903 & 46,798 & & & \\
\hline 1978 & 37,608 & 78 & 78.3 & 48,050 & $2,600.9$ & 28,718 & 48,086 & & & \\
\hline 1979 & 40,104 & 82 & 81.4 & 49,291 & $2,453.2$ & 29,233 & 48,900 & & & \\
\hline 1980 & 35,606 & 75 & 74.9 & 47,554 & $2,467.2$ & 29,673 & 49,598 & & & \\
\hline 1981 & 36,840 & 69 & 75.2 & $48, \infty 91$ & $1,897,0$ & 29,529 & 49,366 & & & \\
\hline 1982 & 30,402 & 57 & 60.1 & 50,626 & $1,826.7$ & 29,248 & 48,923 & & & \\
\hline 1983 & 33,359 & 66 & 6.1 & 52,067 & $3,180.8$ & 30,065 & 50,213 & & & \\
\hline 1984 & 34,690 & 71 & 69.5 & 49,912 & $1,233.9$ & 28,868 & 48,323 & & & \\
\hline 1985 & 31,666 & 73 & 72.6 & 43,626 & 441.3 & 26,901 & 45,215 & & & \\
\hline 1986 & 30,907 & 77 & 75.9 & 40,705 & $1,369.4$ & 25,801 & 43,478 & & & \\
\hline-- & $\cdots$ & $\cdots$ & -- & $-\cdots$ & $\therefore \cdot$ & $-\cdots$ & $\cdots$ & $\cdot---\cdot$ & $--\cdot$ & 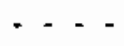 \\
\hline 1987 & 31,097 & 81 & 79.2 & 39,275 & $2,837.2$ & 26,248 & 44,185 & & & \\
\hline 1988 & 31,344 & 87 & 84.5 & 37,089 & $3,416.6$ & 27,234 & 45,741 & 44,471 & 45,442 & 45,833 \\
\hline 1989 & & & & & $4,307.9$ & 29,019 & 48,562 & 47,213 & 48,243 & 48,659 \\
\hline 1990 & & & & & $4,425.5$ & 30,757 & 51,306 & 49,881 & 50,970 & 51,409 \\
\hline 1991 & & & & & $4,415.7$ & 32,324 & 53,781 & 52,288 & 53,429 & 53,889 \\
\hline 1992 & & & & & $3,857.3$ & 33,187 & 55,145 & 53,613 & 54,784 & 55,255 \\
\hline
\end{tabular}

Capacity-Capital Stock Regression: Code 0 RSO 0.910 Coefficients: Const 2724.3 stock 1.6

Ave. Capacity/Stock $(74-86): 1.7$ Ave. Weekly Hours (High) 164.6 Shift Factor 1.00 Ave Heekly Hours (Low) 161.8 Shift Factor 1.02 Hote: Output, Investment, and Capital Stock extrapolated for 1987 and 1988.

2869 Industrial organic chemicals, n.e.

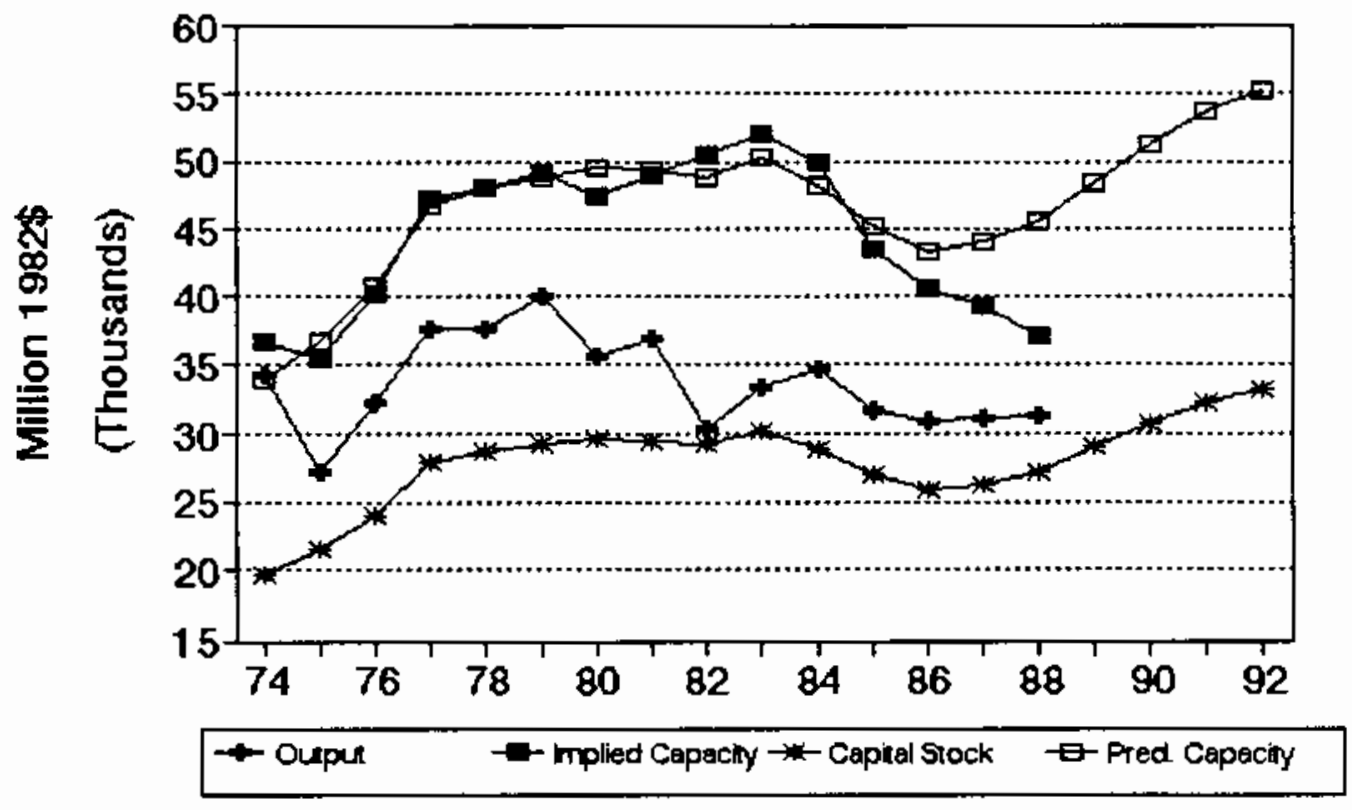




\section{No. 197 sIC 2873 Nitrogenous fertilizers}

MILLIONS OF 1982 DOLLARS (EXCL. CU RATES)

$\begin{array}{lccccccc} & \text { Q4 } & \text { Am. Implied Gross } & \text { Net } & \text { Pred. } & \ldots \ldots \ldots \\ \text { Gross } & \text { CU } & \text { CU } & \text { Prac. } & \text { In- } & \text { Cap. } & \text { Prac. } & \text { Emergency Capacity } \\ \text { Output } & \text { Rate } & \text { Rate } & \text { Capac. } & \text { vest. } & \text { Stk. } & \text { Capac. } & 3 \text { mon. } 6 \text { mon. }>6 \text { mon. }\end{array}$

\begin{tabular}{|c|c|c|c|c|c|c|c|c|c|c|}
\hline 1974 & 2,952 & 71 & 72.1 & 4,093 & 289.8 & 2,720 & 3,839 & & & \\
\hline 1975 & 2,999 & 81 & 75.8 & 3,954 & 798.0 & 3,369 & 3,888 & & & \\
\hline 1976 & 3,254 & 82 & 81.6 & 3,987 & 800.7 & 4,032 & 3,938 & & & \\
\hline 1977 & 3,503 & 92 & 88.4 & 3,962 & $1,279.4$ & 5,159 & 3,987 & & & \\
\hline 1978 & 3,581 & 97 & 95.2 & 3,761 & $(19.9)$ & 4,966 & 4,037 & & & \\
\hline 1979 & 3,421 & 89 & 92.2 & 3,709 & $(236.6)$ & 4,554 & 4,086 & & & \\
\hline 1980 & 3,819 & 88 & 87.6 & 4,359 & 55.9 & 4,414 & 4.136 & & & \\
\hline 1981 & 3,696 & 86 & 87.9 & 4,206 & 96.8 & 4,293 & 4,186 & & & \\
\hline 1982 & 3,425 & 75 & 78.7 & 4,351 & 124.4 & 4.170 & 4,235 & & & \\
\hline 1983 & 3,082 & 84 & 81.0 & 3,804 & $(69.7)$ & 3,835 & 4,285 & & & \\
\hline 1984 & 3,678 & 84 & 84.1 & 4,372 & 346.9 & 3,863 & 4,334 & & & \\
\hline 1985 & 3,621 & 74 & 77.9 & 4,652 & 18.7 & 3,552 & 4,384 & & & \\
\hline 1986 & 2,610 & 67 & 57.3 & 4,558 & 85.8 & 3,295 & 4,434 & & & \\
\hline-- & $\cdots$ & - & $\cdots$ & $\cdots$ & $-\cdot-$ & $-\cdots$ & $\cdots$ & $-\cdots$ & -- & $-\cdots$ \\
\hline 1987 & 2,675 & 87 & 71.8 & 3,725 & $(276.5)$ & 2,695 & 4,483 & & & \\
\hline 1988 & 2,761 & 89 & 88.2 & 3,132 & (87.3) & 2,342 & 4,533 & 4,366 & 4,485 & 4,533 \\
\hline 1989 & & & & & 260.1 & 2,372 & 4,582 & 4,414 & 4,534 & 4,582 \\
\hline 1990 & & & & & 736.4 & 2,895 & 4,632 & 4,462 & 4,583 & 4,632 \\
\hline 1991 & & & & & 768.8 & 3,379 & 4,682 & 4,510 & 4,632 & 4,682 \\
\hline 1992 & & & & & 657.1 & 3,703 & 4,731 & 4,557 & 4,681 & 4,731 \\
\hline
\end{tabular}

Capacity-Capital Stock Regression: Code -2 RSO 0.400

Coefficients: Const 3788.9 Time 49.6

Ave, Capacity/Stack $(74-86): 1.0$ Ave. Weekly Hours (High) 168.0 Shift Factor 1.00 Ave reekly Hours (Low) 168.0 Shift Factor 1.00 Note: Output, Investment, and Capital stock extrapolated for 1987 and 1988.

\section{Nitrogenous fertilizers}

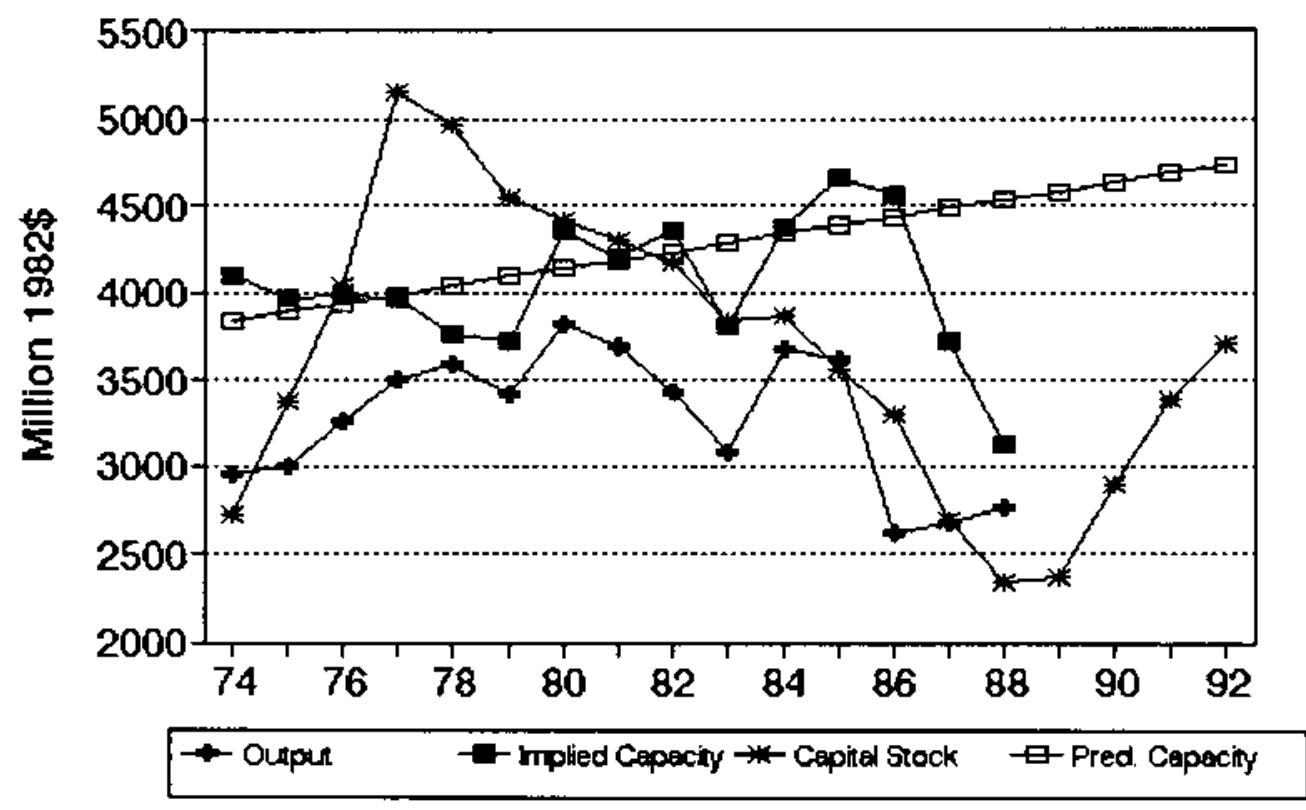




\title{
No. 198 sIC 2874 Phosphatic fertilizers
}

\author{
MILLIONS OF 1982 DOLLARS (EXCL. CU RATES)
}

$\begin{array}{lccccccc} & a 4 & \text { Ann. Implied Gross } & \text { Wet } & \text { Pred. } & -\ldots \ldots \\ \text { Gross } & \text { Cu } & \text { CU } & \text { Prac. } & \text { In- } & \text { Cap. } & \text { Prac. } & \text { Emergency Capacity } \\ \text { Output } & \text { Rate } & \text { Rate } & \text { Capac. } & \text { vest. } & \text { Stk. } & \text { Capac. } & 3 \text { mon. } 6 \text { mon. }>6 \text { mon. }\end{array}$

\begin{tabular}{|c|c|c|c|c|c|c|c|c|c|c|}
\hline 1974 & 4,512 & 92 & 96.2 & 4,693 & 462.0 & 1,747 & 5,201 & & & \\
\hline 1975 & 3,895 & 76 & 7.1 & 5,051 & 842.4 & 2,431 & 5,578 & & & \\
\hline 1976 & 4,013 & 56 & 63.6 & 6,306 & 192.3 & 2,459 & 5,593 & & & \\
\hline 1977 & 4,571 & 73 & 67.0 & 6,826 & 361.5 & 2,632 & 5,688 & & & \\
\hline 1978 & 4,587 & $\pi$ & $\pi .7$ & 6,062 & 358.1 & 2,793 & $5, \pi 7$ & & & \\
\hline 1979 & 4,627 & 92 & 87.1 & 5,314 & 130.6 & 2,717 & 5,735 & & & \\
\hline 1980 & 4,902 & 89 & 87.9 & 5,579 & 392.9 & 2,896 & 5,834 & & & \\
\hline 1981 & 4,356 & 61 & 74.2 & 5,869 & $\pi 0.1$ & 3,422 & 6,123 & & & \\
\hline 1982 & 3,837 & 59 & 58.9 & 6,517 & 574.1 & 3,721 & 6,288 & & & \\
\hline 1983 & 4,359 & 87 & $\pi .4$ & 5,632 & 186.8 & 3,614 & 6,229 & & & \\
\hline 1984 & 5,130 & 79 & 82.4 & 6,226 & $(20.6)$ & 3,305 & 6,059 & & & \\
\hline 1985 & $4, \pi 55$ & 70 & 73.6 & 6,458 & 455.9 & 3,439 & 6,133 & & & \\
\hline 1986 & 3,962 & 67 & 68.4 & 5,790 & 243.4 & 3,353 & 6,085 & & & \\
\hline$\cdots$ & $\cdots--$ & $-\cdot$ & $\cdots$ & $-\cdots$ & $-\cdots$ & $\cdots$ & $-\cdots$ & $\cdot-$ & -- & $\cdots$ \\
\hline 1987 & 4,024 & 76 & 72.4 & 5,562 & 181.2 & 3,213 & 6,008 & & & \\
\hline 1988 & 4,106 & 83 & 80.2 & 5,121 & 245.6 & 3,150 & 5,974 & 5,798 & 5,923 & 5,974 \\
\hline 1989 & & & & & 363.8 & 3,212 & 6,007 & 5,831 & 5.957 & 6,007 \\
\hline 1990 & & & & & 532.7 & 3,437 & 6,131 & 5,951 & 6,079 & 6,131 \\
\hline 1991 & & & & & 536.9 & 3,644 & 6,245 & 6,062 & 6,193 & 6,245 \\
\hline 1992 & & & & & 498.9 & 3,793 & 6,328 & 6,142 & 6,274 & 6,328 \\
\hline
\end{tabular}

Capacity-Capital Stock Regression: Code 1 RSO 0.260

Coefficients: Const $\mathbf{4 2 5 9 . 3}$ stock 0.6

Ave. Capacity/5tock $(74-86): 2.0$ Ave. Weekly Hours (High) 168.0 Shift Factor 1.00 Ave Weekly Hours (Low) 167.6 Shift Factor 1.00 Note: Output, Imvestment, and Capital stock extrapolated for 1987 and 1988.

\section{Phosphatic fertilizers}

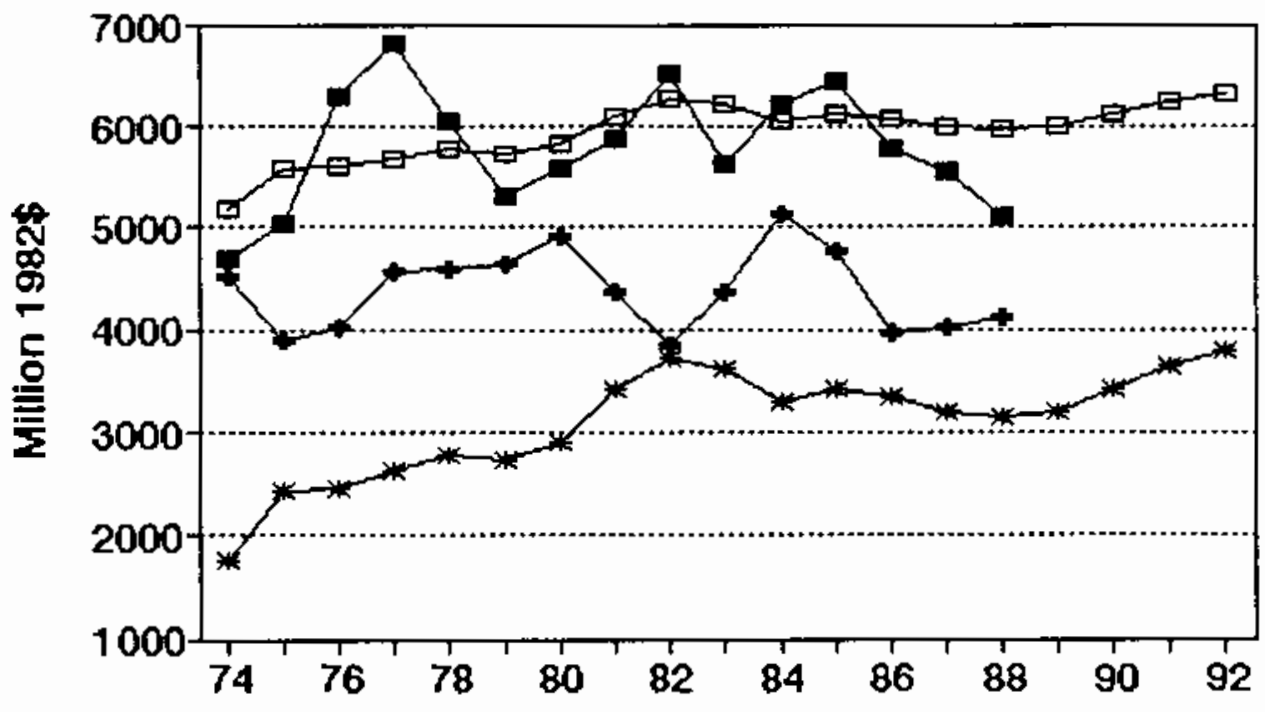

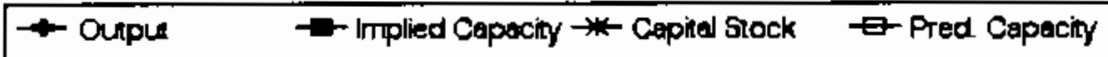


No. 199 sIC 2875 Fertilizers, mixing only

MILLIONS OF 1982 DOLLARS (EXCL. CU RATES)

$\begin{array}{lccccccc} & 04 & \text { Ann. } & \text { Implied Gross } & \text { Net } & \text { Pred. } & -\ldots \ldots \ldots \\ \text { Gross } & \text { CU } & \text { CU } & \text { Prac. } & \text { In- } & \text { Cap. } & \text { Prac. } & \text { Emergency Capacity } \\ \text { Output } & \text { Rate } & \text { Rate } & \text { Capac. } & \text { vest. } & \text { Stk. } & \text { Capac. } & 3 \text { mon. } 6 \text { mon. }>6 \text { mon. }\end{array}$

\begin{tabular}{|c|c|c|c|c|c|c|c|c|c|c|}
\hline 1974 & 2,423 & 40 & 40.0 & 6,057 & $(22.7)$ & 340 & 5,335 & & & \\
\hline 1975 & 2,453 & 58 & 51.3 & 4,787 & 110.9 & 418 & 4,633 & & & \\
\hline 1976 & 2,490 & 66 & 63.0 & 3,953 & $(5.3)$ & 383 & 4,950 & & & \\
\hline 1977 & 2,948 & 60 & 62.3 & 4,736 & 72.0 & 422 & 4,601 & & & \\
\hline 1978 & 2,601 & $\pi$ & 69.4 & 3,749 & 30.7 & 420 & 4,618 & & & \\
\hline 1979 & 2,725 & 61 & 66.3 & 4,113 & 124.9 & 507 & 3,837 & & & \\
\hline 1980 & 2,887 & 70 & 66.6 & 4,333 & 53.7 & 520 & 3,716 & & & \\
\hline 1981 & 2,490 & 48 & 56.3 & 4,427 & 17.7 & 498 & 3,912 & & & \\
\hline 1982 & 1,964 & 52 & 50.5 & 3,889 & $(23.1)$ & 441 & 4,428 & & & \\
\hline 1983 & 2,517 & 67 & 61.4 & 4,100 & $(33.4)$ & 378 & 4,999 & & & \\
\hline 1984 & 2,441 & $55 *$ & 59.6 & 4,100 & (21.8) & 329 & 5,441 & & & \\
\hline 1985 & 2,445 & $38 *$ & 44.2 & 5,527 & 25.5 & 325 & 5,474 & & & \\
\hline 1986 & 2,370 & 26 & 30.4 & 7,827 & 9.8 & 305 & 5,651 & & & \\
\hline$\cdots$ & $\cdots$ & $\cdots$ & $\cdots$ & $\cdots \cdot$ & $\cdots$ & $\cdots$ & $\cdots$ & $-\cdot-\cdot$ & $-\cdots$ & $\cdot \cdot$ \\
\hline 1987 & 2,406 & $33 *$ & 30.1 & 7,993 & $(2.1)$ & 275 & 5,927 & & & \\
\hline 1988 & 2,441 & $35 *$ & 33.8 & 7,225 & 8.3 & 257 & 6,082 & 13,191 & 13,986 & 14,306 \\
\hline 1989 & & & & & 27.5 & 281 & 6,051 & 13,123 & 13,913 & 14,232 \\
\hline 1990 & & & & & 54.9 & 291 & 5,776 & 12,526 & 13,281 & 13,585 \\
\hline 1991 & & & & & 55.6 & 320 & 5,520 & 11,972 & 12,693 & 12,984 \\
\hline 1992 & & & & & 49.4 & 339 & 5,344 & 11,590 & 12,288 & 12,570 \\
\hline
\end{tabular}

Capacity-Capital Stock Regression: Code 0 RSO 0.320

Coefficients: Const 8398.3 Stock -9.0

Ave. Capacity/Stock $(74-86): 11.7$ Ave. Weekly Hours (High) 67.0 Shift Factor 2.35 Ave Heekly Hours (LOW) 67.0 Shift Factor 2.35 * Imputed Note: Output, Investment, and Capital Stock extrapolated for 1987 and 1988.

\section{Fertilizers, mixing only}

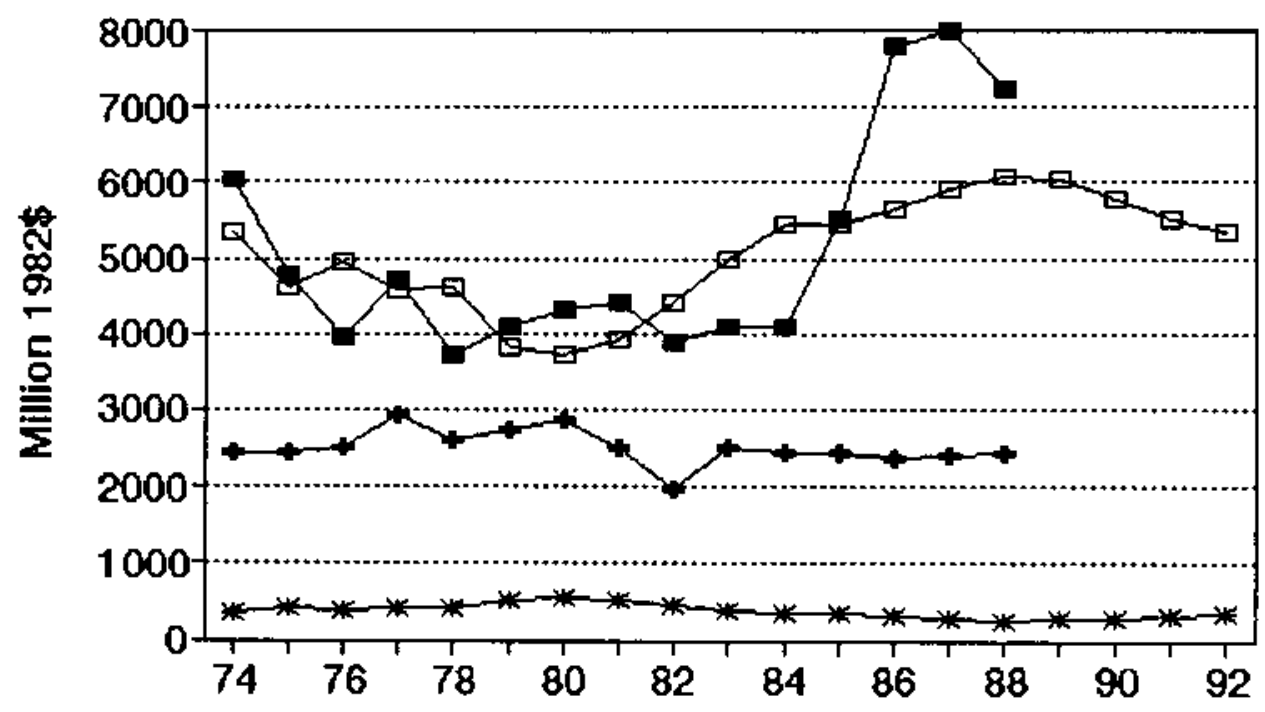

- Outpur - Implied Cepacity * Cepites Stock $\rightarrow$ Pred. Capaciny 
No. 200 sIC 2879 Agricultural chenicals, n.e.c.

MILLIONS OF 1982 DOLLARS (EXCL. CU RATES)

$\begin{array}{lccccccc} & Q 4 & \text { Ann. Implied Gross } & \text { Met } & \text { Pred. } & -\ldots & - \\ \text { Gross } & \text { CU } & \text { CU } & \text { Prac. } & \text { In- } & \text { Cap. } & \text { Prac. } & \text { Emergency Capacity } \\ \text { Output Rate } & \text { Rate } & \text { Capac. } & \text { vest. } & \text { Stk. } & \text { Capac. } & 3 \text { mon. } 6 \text { mon. }>6 \text { mon. }\end{array}$

\begin{tabular}{|c|c|c|c|c|c|c|c|c|c|c|}
\hline 1974 & 6,035 & 71 & 75.7 & 7,976 & 88.4 & 997 & 5,594 & & & \\
\hline 1975 & 4,788 & 81 & 71.2 & 6,727 & 220.6 & 1,165 & 5,963 & & & \\
\hline 1976 & 4,427 & 84 & 82.9 & 5,341 & 405.8 & 1,497 & 6,695 & & & \\
\hline 1977 & 4,396 & 69 & 75.3 & 5,841 & 238.6 & 1,657 & 7,046 & & & \\
\hline 1978 & 4,609 & 87 & 80.4 & 5,733 & 286.7 & 1,855 & 7,484 & & & \\
\hline 1979 & 5,043 & 70 & 77.2 & 6,534 & 271.0 & 2,025 & 7,856 & & & \\
\hline 1980 & 4,768 & 58 & 60.1 & 7,939 & 137.4 & 2,052 & 7,917 & & & \\
\hline 1981 & 6,232 & 58 & 61.5 & 10,141 & 741.8 & 2,637 & 9,205 & & & \\
\hline 1982 & 5,427 & 49 & 51.1 & 10,623 & 287.1 & 2,751 & 9,456 & & & \\
\hline 1983 & 4,958 & 56 & 54.7 & 9,060 & 64.4 & 2,630 & 9,189 & & & \\
\hline 1984 & 5,867 & 61 & 59.5 & 9,866 & 194.9 & 2,627 & 9,182 & & & \\
\hline 1985 & 5,397 & 54 & 56.9 & 9,480 & t88.4 & 2,604 & 9,132 & & & \\
\hline 1986 & 5,554 & 71 & 64.9 & 8,556 & 209.0 & 2,590 & 9,100 & & & \\
\hline$\because 987$ & 5,627 & $\begin{array}{l}-- \\
65\end{array}$ & $\begin{array}{l}-1 . \\
67.1\end{array}$ & 8,391 & $\ddot{123.1}$ & 2,496 & 8,894 & --- & $\cdots \cdot$ & --- \\
\hline 1988 & 5,724 & 76 & 71.6 & 7,994 & 165.7 & 2,453 & 8,799 & 9,252 & 9,513 & 9,618 \\
\hline 1989 & & & & & 243.8 & 2,492 & 8,884 & 9,341 & 9,604 & 9,710 \\
\hline 1990 & & & & & 355.4 & 2,639 & 9,208 & 9,681 & 9,954 & 10,064 \\
\hline 1991 & & & & & 358.2 & 2,776 & 9,510 & 9,999 & 10,281 & 10,395 \\
\hline 1992 & & & & & 333.1 & 2,877 & 9,732 & 10,232 & 10,521 & 10,637 \\
\hline
\end{tabular}

Capacity-Capital Stock Regression: Code 0 RSO 0.550 Coefficients: Const 3399.6 stock 2.2

Ave. Capacity/Stock $(74-86): 3.8$ Ave. Heekly Hours (High) 145.7 Shift Factor 1.09 Ave Neekly Hours (Low 142.8 Shift Factor 1.11 Note: Output, Investment, and Capital stock extrapolated for 1987 and 1988.

\section{Agricultural chemicals, n.e.c.}

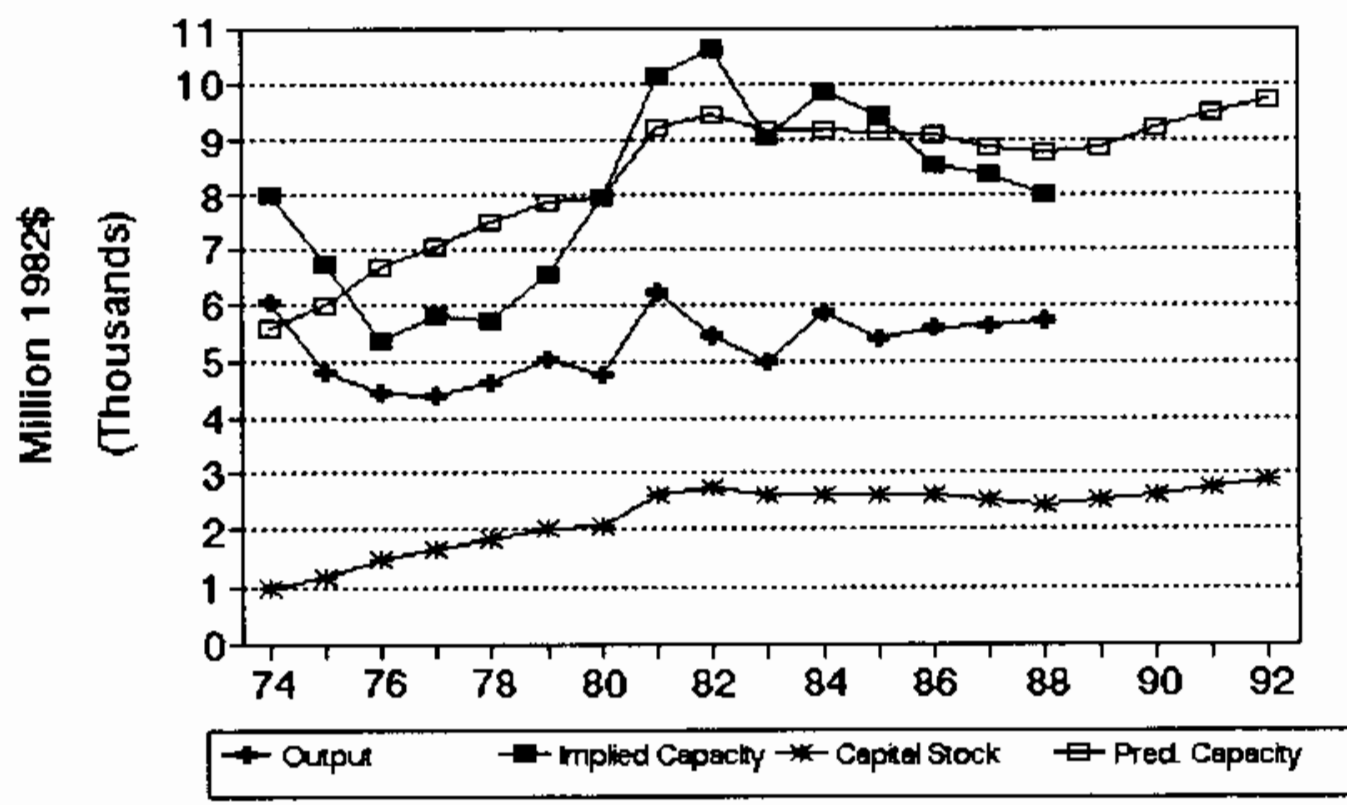




\section{No. 201 SIC 2891 Adhesives and sealants}

MILLIONS OF 1982 DOLLARS (EXCL. CU RATES)

$\begin{array}{lccccccc} & 04 & \text { Ann. } & \text { Implied } & \text { Gross } & \text { Net } & \text { Pred. } & \ldots \ldots \\ \text { Gross } & \text { CU } & \text { CU } & \text { Prac. } & \text { In- } & \text { Cap. } & \text { Prac. } & \text { Emergency Capacity } \\ \text { Qutput Rate } & \text { Rate } & \text { Capac. } & \text { vest. } & \text { Stk. } & \text { Capac. } & 3 \text { mon. } 6 \text { mon. }>6 \text { mon. }\end{array}$

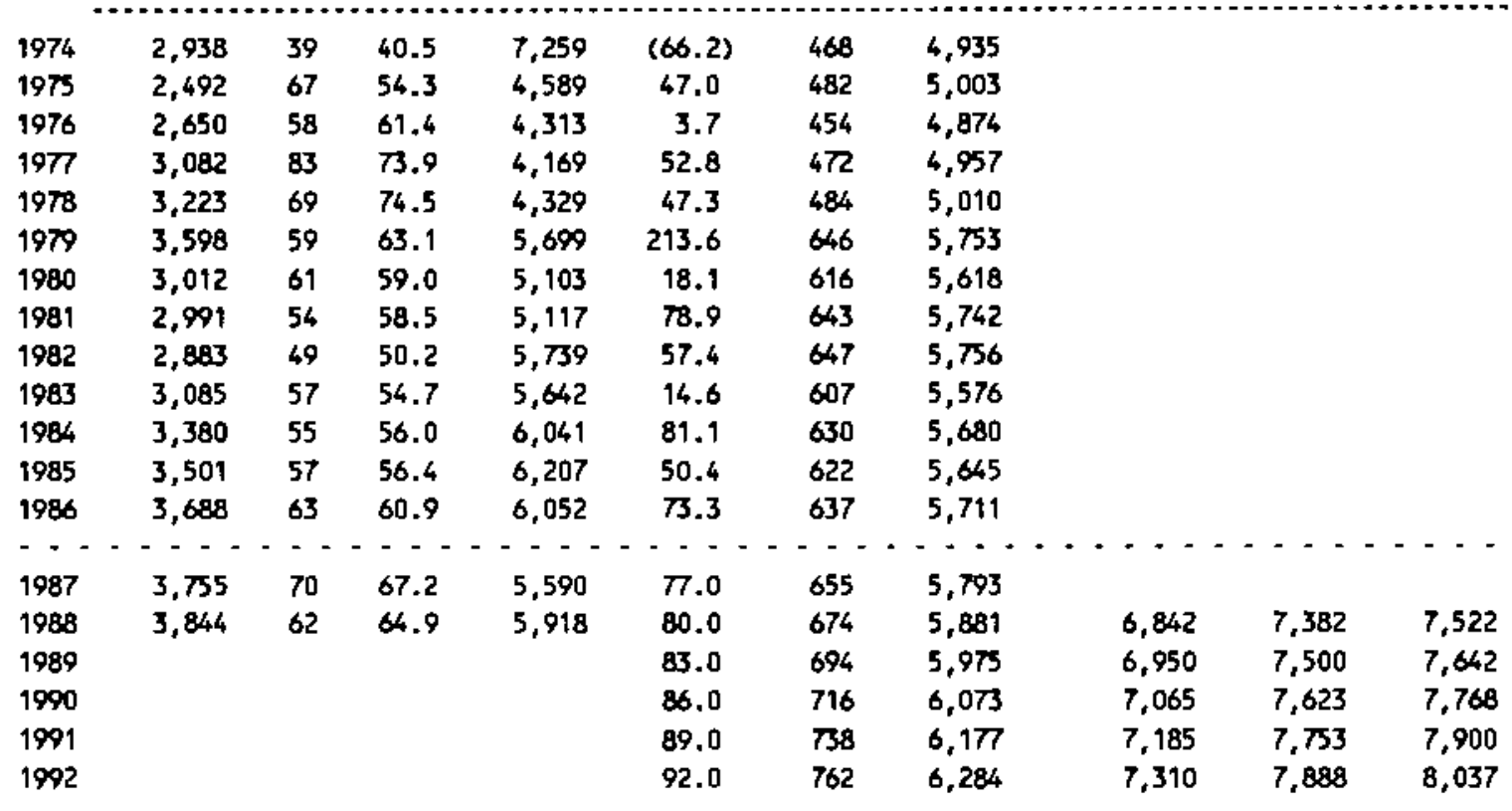

Capacity-Capital Stock Regression: Code 0 RSO 0.170 Coefficients: Const 2789.9 stock 4.6

Ave. Capacity/stock $(74-86): 9.5$ Ave. Weekly Hours (High) 123.7 Shift Factor 1.28 Ave Heekly Hours (Low) 114.3 Shift factor 1.38 Note: Output, Investment, and Capital Stock extrapolated for 1987 and 1988.

\section{Adhesives and sealants}

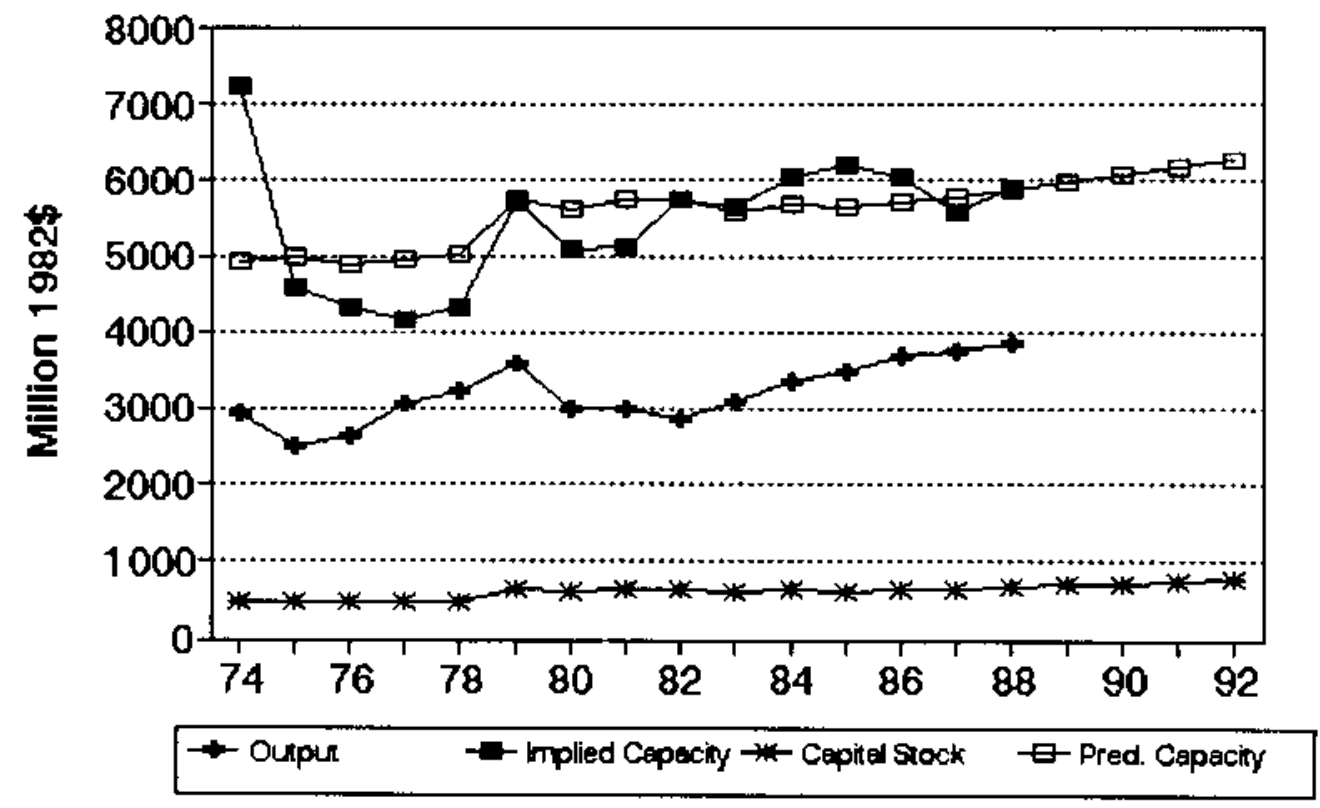


No. 202 SIC 2892 Explosives

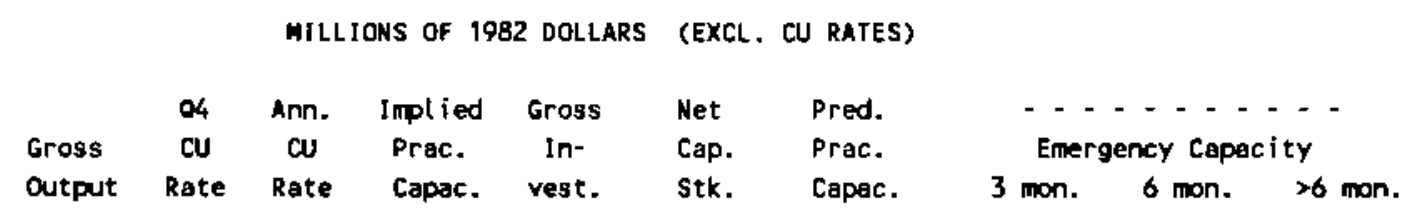

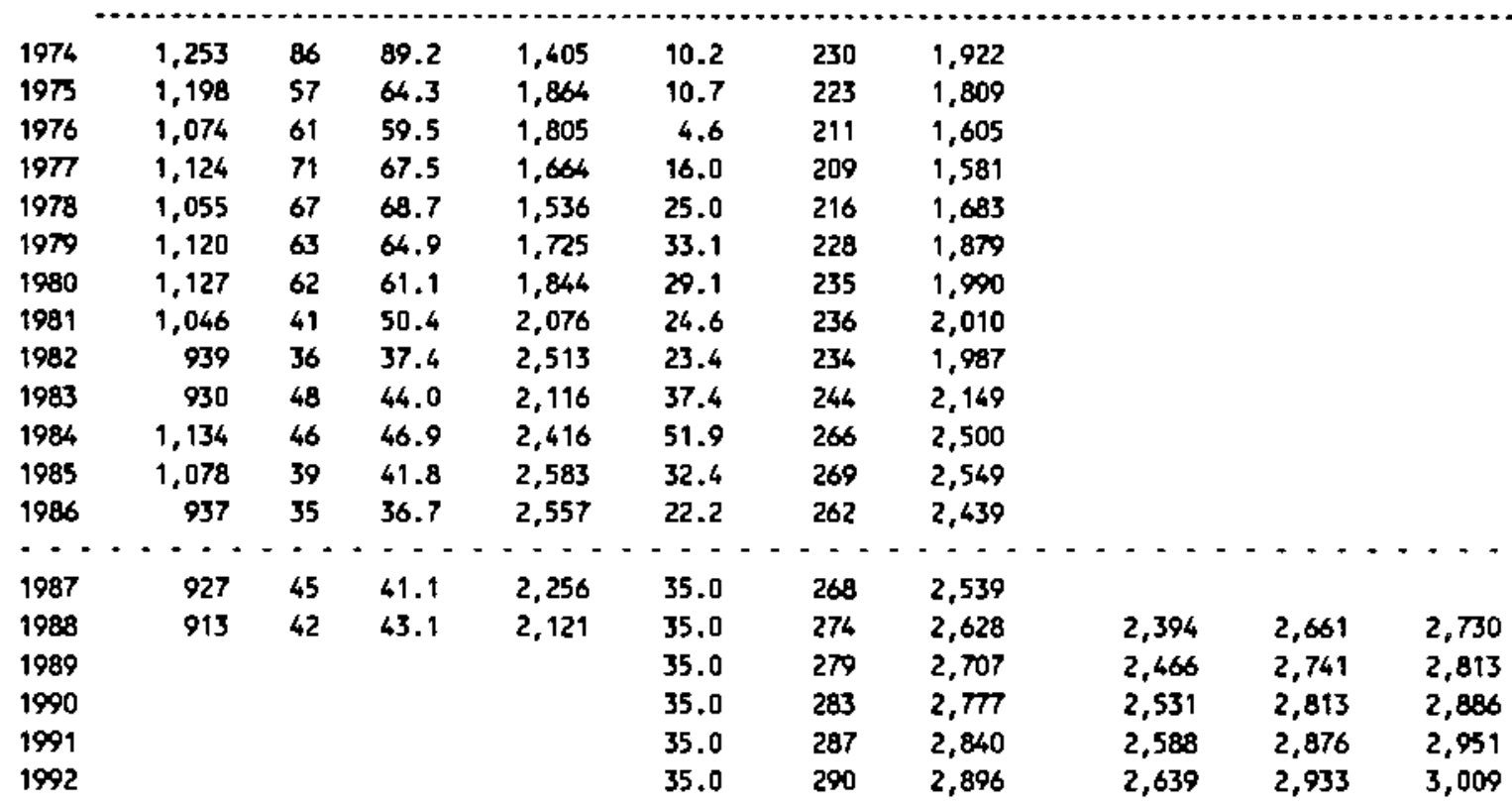

Capecity-Capital Stock Regression: Code 0 RSO 0.650

Coefficients: Const -1806.4 stock 16.2

Ave. Capacity/Stock $(74-86): 8.5$

Ave. Weekly Hours (High) 153.7 Shift Factor 1.04 Ave Heek(y Hours (LOW) 151.3 Shift Factor 1.05 Mote: Output, Investment, and Capital Stock extrapolated for 1987 and 1988.

\section{Explosives}

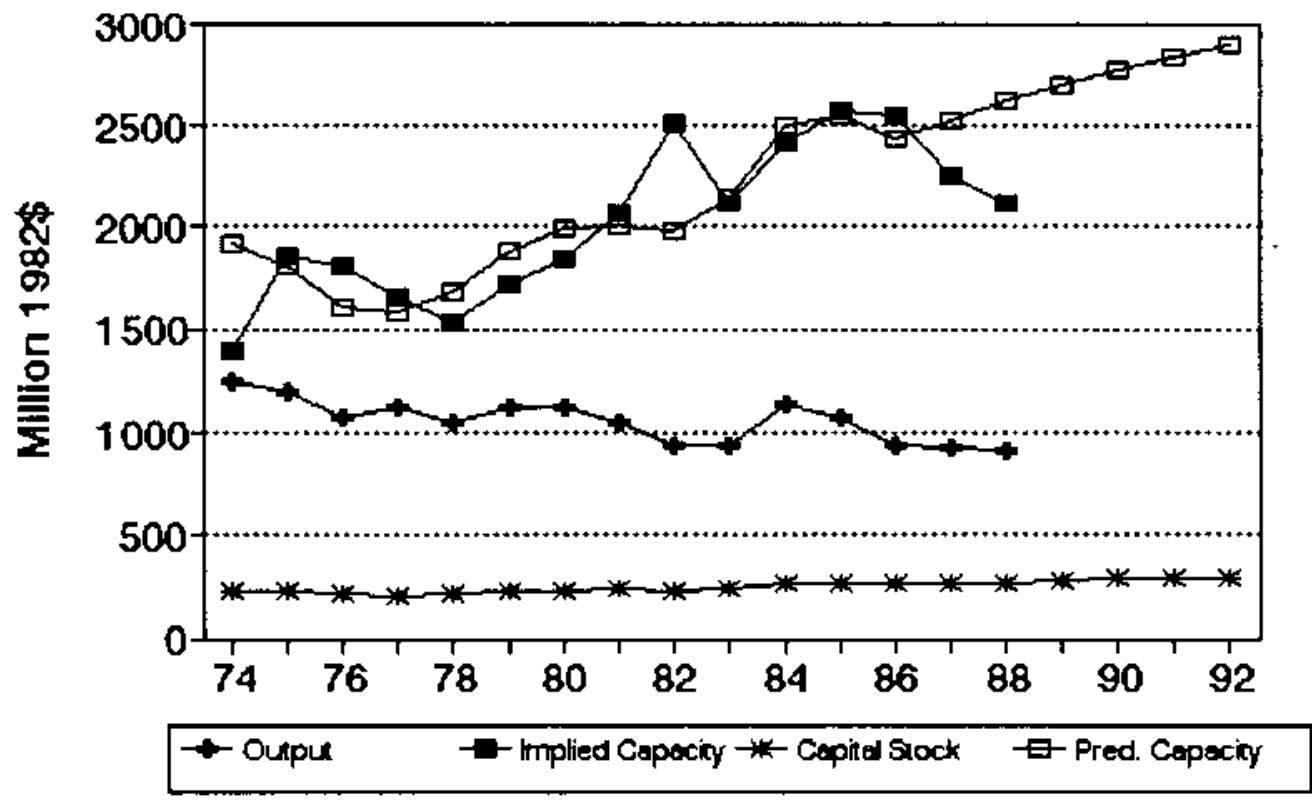




\section{No, 203 sIC 2893 Printing ink}

MILLIONS DF 1982 dOLLARS (EXCL. CU RATES)

$\begin{array}{lccccccc} & \text { Q4 } & \text { Ann. } & \text { Implied Gross } & \text { Net } & \text { Pred. } & \ldots & \ldots \\ \text { Gross } & \text { CU } & \text { CU } & \text { Proc. } & \text { In- } & \text { Cap. } & \text { Prac. } & \text { Emergenty Capacity } \\ \text { Output Rate } & \text { Rate } & \text { Capac. } & \text { vest. } & \text { Stk. } & \text { Capac. } & 3 \text { mon. } 6 \text { mon. }>6 \text { mon. }\end{array}$

\begin{tabular}{|c|c|c|c|c|c|c|c|c|c|c|}
\hline 1974 & 1,319 & 65 & 70.1 & 1,882 & $(20.7)$ & 163 & 1,674 & & & \\
\hline 1975 & 1,220 & 76 & 65.2 & 1.871 & 28.4 & 178 & 2,033 & & & \\
\hline 1976 & 1,214 & 67 & 70.5 & 1,722 & 18.5 & 182 & 2,136 & & & \\
\hline 1977 & 1,503 & 65 & 66.4 & 2,264 & 22.6 & 190 & 2,315 & & & \\
\hline 1978 & 1,708 & 62 & 63.4 & 2,692 & 8.9 & 184 & 2,184 & & & \\
\hline 1979 & 1,639 & 61 & 62.2 & 2,636 & 34.6 & 202 & 2,612 & & & \\
\hline 1980 & 1,492 & 61 & 58.4 & 2,557 & 21.1 & 206 & 2,703 & & & \\
\hline 1981 & 1,464 & 56 & 62.0 & 2,359 & 33.7 & 221 & 3,059 & & & \\
\hline 1982 & 1,592 & 50 & 50.8 & 3,137 & 30.1 & 230 & 3,289 & & & \\
\hline 1983 & 1,659 & 49 & 51.2 & 3,240 & 22.7 & 232 & 3,327 & & & \\
\hline 1984 & 1,838 & 47 & 48.2 & 3,818 & 13.3 & 225 & 3,167 & & & \\
\hline 1985 & 1,836 & 54 & 51.7 & 3,553 & 25.9 & 231 & 3,306 & & & \\
\hline 1986 & 1,963 & 55 & 55.0 & 3,566 & 28.3 & 239 & 3,490 & & & \\
\hline$-"$ & $\cdots-$ & $\therefore$ & $\cdots$ & $-\cdots$ & - - - - & $\cdots$ & $\cdots$ & $\cdots \cdot \cdot$ & 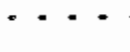 & $\cdots$ \\
\hline 1987 & 1,981 & 86 & 73.7 & 2,690 & 21.0 & 239 & 3,498 & & & \\
\hline 1988 & 2,006 & $\pi$ & 80.3 & 2,500 & 24.1 & 243 & 3,579 & 8,958 & 10,212 & 10,536 \\
\hline 1989 & & & & & 28.9 & 250 & 3,767 & 9,428 & 10,748 & 11,089 \\
\hline 1990 & & & & & 29.5 & 258 & 3,953 & 9,895 & 11,280 & 11,638 \\
\hline 1991 & & & & & 29.4 & 265 & 4,123 & 10,319 & 11,763 & 12,137 \\
\hline 1992 & & & & & 26.5 & 269 & 4,206 & 10,527 & 12,001 & 12,382 \\
\hline
\end{tabular}

Capecity-Capital stock Regression: Code 0 RSQ 0.740 Coefficients: Const -2222.0 stock 23.9

Ave. Capacity/Stock ( $74-86): 13.2$ Ave. Weekly Hours (High) $\mathbf{5 3 . 5}$ Shift Factor 2.94 Ave Heekly Hours (Low) 51.8 Shift Factor 3.04 Note: Output, Investment, and Capital Stock extrapolated for 1987 and 1988.

\section{Printing ink}

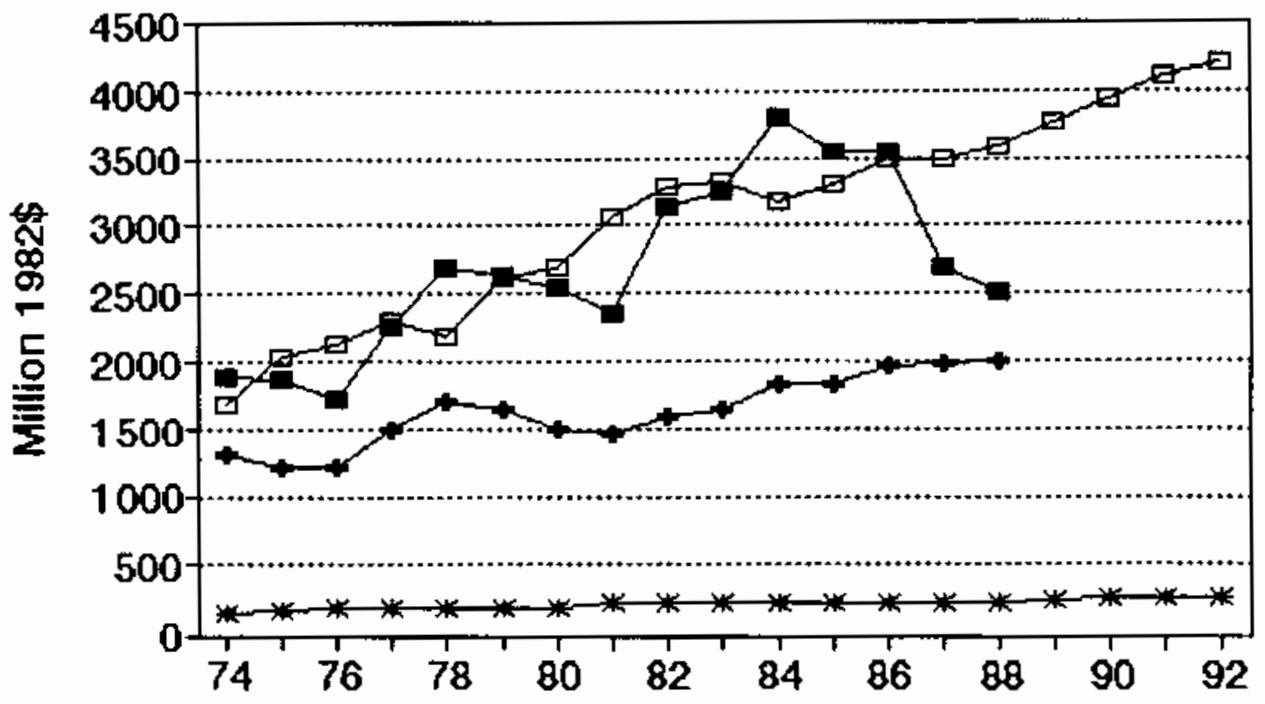

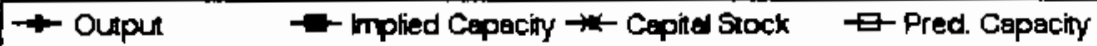




\section{No. 204 sIC 2895 Carbon black}

MILLIONS OF 1982 DOLLARS (EXCL. CU RATES)

$\begin{array}{lccccccc} & Q 4 & \text { Ann. } & \text { Implied } & \text { Gross } & \text { Net } & \text { Pred, } & \ldots \\ \text { Gross } & \text { cu } & \text { cu } & \text { Prac. } & \text { In- } & \text { Cap. } & \text { Prac. } & \text { Emergency Capacity } \\ \text { Output } & \text { Rate } & \text { Rate } & \text { Capac. } & \text { vest. } & \text { Stk. } & \text { Capac. } & 3 \text { mon. } 6 \text { mon. }>6 \text { mon. }\end{array}$

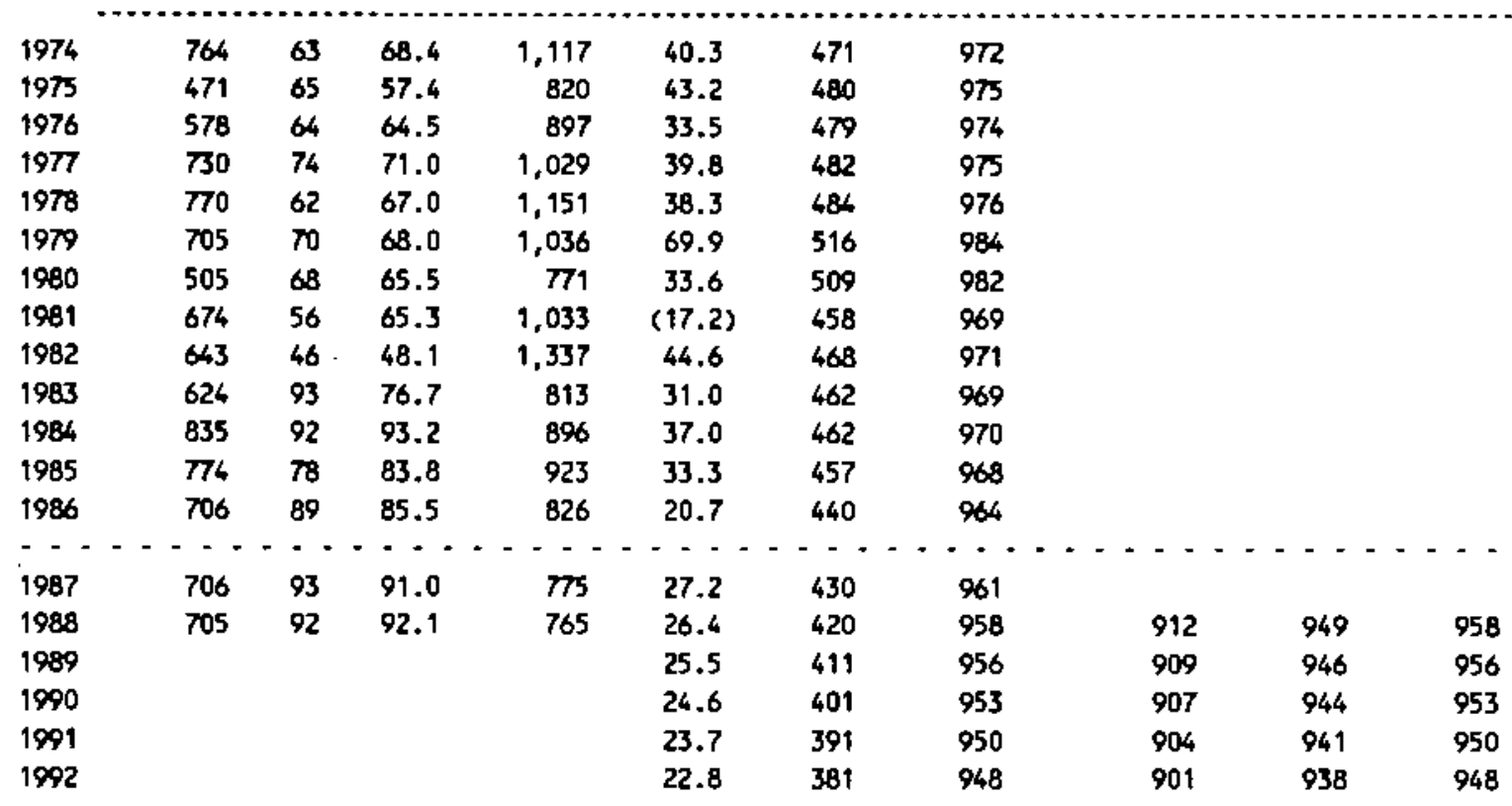

Capacity-Capital Stock Regression: Code 0 RSQ 0.000

Coefficients: Const 845.4 Stock 0.3

Ave. Capacity/Stock $(74-86): 2.1$ Ave. Heekly Hours (High) 168.0 Shift Factor 1.00 Ave Heekly Hours (Low) 168.0 shift Factor 1.00 Note: Output, Investment, and Capital stock extrapolated for 1987 and 1988.

\section{Carbon black}

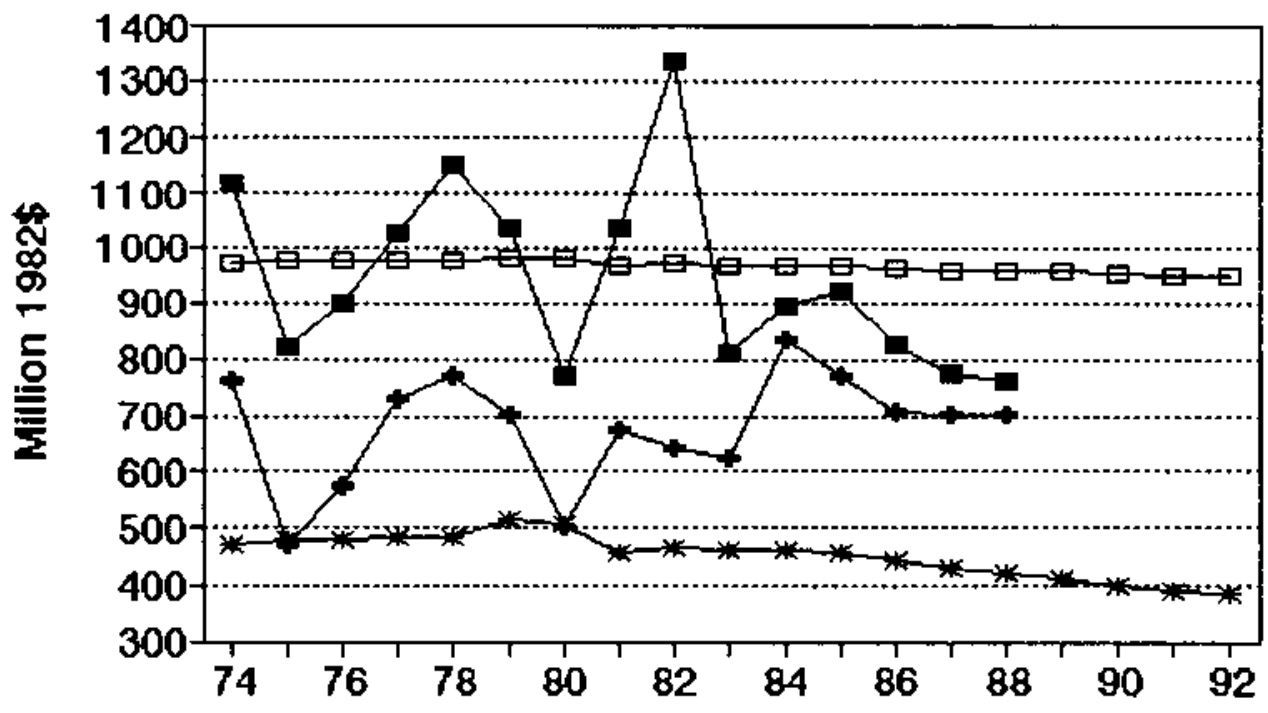

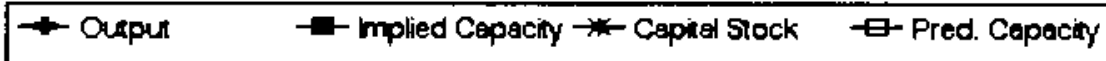


Ho. 205 SIC 2899 Chenical preparation, n.e.c.

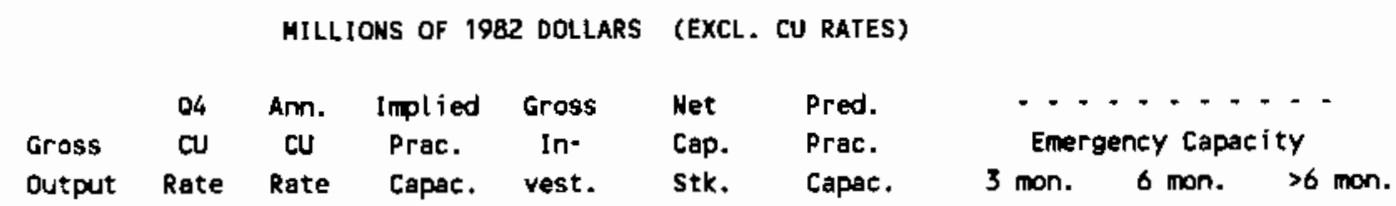

\begin{tabular}{|c|c|c|c|c|c|c|c|c|c|c|}
\hline 1974 & 3,907 & 72 & 74.9 & 5,214 & 91.8 & 1,394 & 3,962 & & & \\
\hline 1975 & 3,936 & 71 & 67.8 & 5,808 & 200.5 & 1,506 & 5,126 & & & \\
\hline 1976 & 5,014 & 81 & 77.2 & 6,494 & 390.3 & 1,798 & 8,176 & & & \\
\hline 1977 & 5,261 & $\pi$ & 78.9 & 6,669 & $(9.0)$ & 1,693 & 7,083 & & & \\
\hline 1978 & 6,234 & 71 & 73.4 & 8,489 & 267.0 & 1,850 & 8,717 & & & \\
\hline 1979 & 7,430 & 80 & 77.2 & 9,628 & 503.2 & 2,214 & 12,514 & & & \\
\hline 1980 & 6,164 & 73 & 73.9 & 8,344 & $(130.2)$ & 1,962 & 9,891 & & & \\
\hline 1981 & 6,248 & 61 & 67.8 & 9,221 & 287.1 & 2,115 & 11,489 & & & \\
\hline 1982 & 6,454 & 45 & 50.2 & 12,861 & 429.4 & 2,380 & 14,255 & & & \\
\hline 1983 & 6,978 & 68 & 60.0 & 11,636 & 125.9 & 2,332 & 13,749 & & & \\
\hline 1984 & 8,246 & 55 & 60.2 & 13,705 & 45.5 & 2,208 & 12,458 & & & \\
\hline 1985 & 7,488 & 37 & 43.9 & 17,054 & 192.7 & 2,223 & 12,608 & & & \\
\hline 1986 & 8,105 & 49 & 44.6 & 18,164 & $24 B .6$ & 2,285 & 13,259 & & & \\
\hline$\because \cdot$ & $\cdots+$ & -- & $-\cdots$ & $-\cdots$ & $\cdots$ & $\cdots \cdot$ & $\cdots \cdot$ & $-\cdots$ & $-\quad--$ & --- \\
\hline 1987 & 8,319 & 39 & 42.7 & 19,477 & 227.8 & 2,328 & 13,708 & & & \\
\hline 1988 & $B, 605$ & 82 & 65.5 & 13,133 & 232.6 & 2,372 & 14,171 & 14,955 & 16,100 & 16,396 \\
\hline 1989 & & & & & 237.4 & 2,418 & 14.647 & 15,457 & 16,641 & 16,947 \\
\hline 1990 & & & & & 242.2 & 2,465 & 15,134 & 15,971 & 17,194 & 17,511 \\
\hline 1991 & & & & & 247.0 & 2,512 & 15,633 & 16,497 & 17,761 & 18,087 \\
\hline 1992 & & & & & 251.8 & 2,561 & 16,141 & 17,034 & 18,338 & 18,675 \\
\hline
\end{tabular}

Capacity-Capital Stock Regression: Code 0 RSO 0.650

Coefficients: Const $-\$ 0590.7$ Stock 10.4

Ave. Capacity/Stock $(74-86): 5.1$ Ave. Weekly Hours (High) 137.3 Shift Factor 1.16 Ave Heekly Hours (LOW) 130.5 Shift Factor 1.22 Note: Output, Investment, and Capital Stock extrapolated for 1987 and 1988.

2899 Chemical preparation, n.e.c.

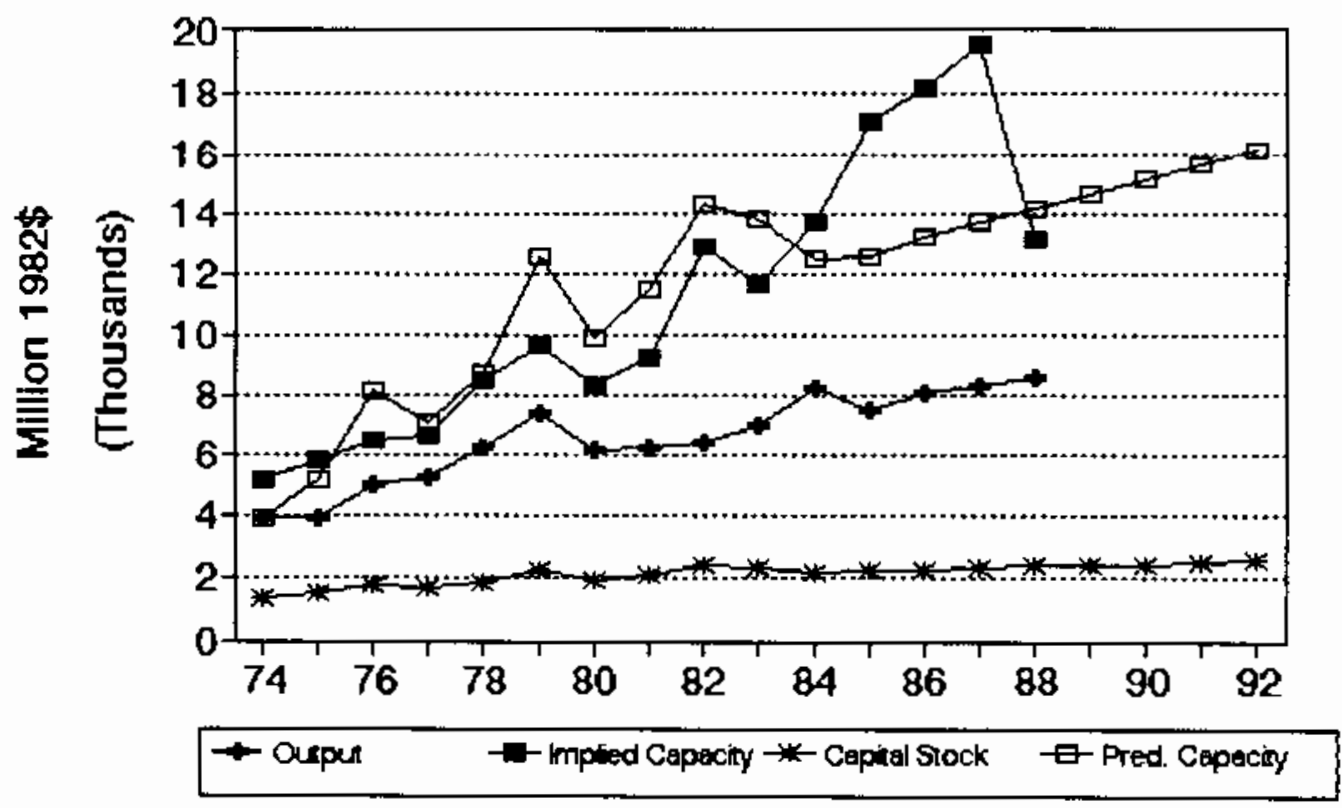




\section{No. 206 sIC 2911 Petroleun refining}

MILLIONS OF 1982 DOLLARS (EXCL. CU RATES)

$\begin{array}{lccccccc} & \text { Q4 } & \text { Ann. Implied Gross } & \text { Net } & \text { Pred. } & \ldots \ldots \\ \text { Gross } & \text { CU } & \text { CU } & \text { Prac. } & \text { In. } & \text { Cap. } & \text { Prac. } & \text { Emergency Capacity } \\ \text { Output Rete } & \text { Rate } & \text { Capac. } & \text { vest. } & \text { Stk. } & \text { Capac. } & 3 \text { mon. } 6 \text { mon. }>6 \text { mon. }\end{array}$

\begin{tabular}{|c|c|c|c|c|c|c|c|c|c|c|}
\hline 1974 & 187,440 & 87 & 87.6 & 213,972 & $3,580.3$ & 22,354 & 227,877 & & & \\
\hline 1975 & 191,346 & 89 & 87.5 & 218,797 & $2,624.6$ & 23,446 & 231,087 & & & \\
\hline 1976 & 211,509 & 90 & 93.4 & 226,530 & $2,724.8$ & 24,578 & 234,415 & & & \\
\hline 1977 & 225,030 & 88 & 93.2 & 241,503 & $2,689.7$ & 25,600 & 237,420 & & & \\
\hline 1978 & 223,720 & 90 & 88.8 & 252,049 & $1,920.0$ & 25,776 & 237,938 & & & \\
\hline 1979 & 240,119 & 87 & 87.9 & 273,282 & $4,636.8$ & 28,490 & 245,917 & & & \\
\hline 1980 & 213,923 & 80 & 82.4 & 259,664 & $3,062.2$ & 29,518 & 248,938 & & & \\
\hline 1981 & 203,631 & 75 & 77.8 & 261,905 & $3,868,5$ & 31,219 & 253,939 & & & \\
\hline 1982 & 202,530 & $\pi$ & 73.5 & 275,743 & $6,314,1$ & 35,066 & 265,251 & & & \\
\hline 1983 & 199,255 & 76 & $\pi .1$ & 265,312 & $4,147.4$ & 36,512 & 269,501 & & & \\
\hline 1984 & 201,708 & 7 & 78.5 & 256,911 & $1,158.7$ & 34,932 & 264,856 & & & \\
\hline 1985 & 201,524 & 82 & 79.7 & 252,956 & $1,936.6$ & 34,035 & 262,218 & & & \\
\hline 1986 & 206,123 & 86 & 85.6 & 240,944 & $2,251.0$ & 33,352 & 260,211 & & & \\
\hline 1987 & 210,777 & $\because 7$ & $=\cdots$ & 244,283 & $\cdots$ & $\begin{array}{l}\cdots \\
32,642\end{array}$ & 258, 123 & $\cdot$. & $=\cdot$ & $\cdots \cdot$ \\
\hline 1988 & 216,977 & 92 & 90.3 & 240,274 & $2,188.4$ & 32,036 & 256,341 & 249,059 & 254,845 & 256,341 \\
\hline 1989 & & & & & $2,434.5$ & 31,728 & 255,435 & 248,179 & 253,945 & 255,435 \\
\hline 1990 & & & & & $2,556.2$ & 31,568 & 254,965 & 247,722 & 253,477 & 254,965 \\
\hline 1991 & & & & & $2, \pi / 5.3$ & 31,640 & 255,179 & 247,929 & 253,690 & 255,179 \\
\hline 1992 & & & & & $2,677.9$ & 31,609 & 255,088 & 247,841 & 253,599 & 255,088 \\
\hline
\end{tabular}

Capacity-Cepital Stock Regression: Code 0 RSO 0.530

Coefficients: Const 162152.6 stock 2.9

Ave. Capacity/Stock $(74-86): 8.4$ Ave. Weekty Hours (High) 168.0 Shift Factor 1.00 Ave Weekly Hours (LOW) 168.0 Shift Factor 1.00 Note: Output, Investment, and Capital Stock extrapolated for 1987 and 1988.

\section{Petroleum refining}

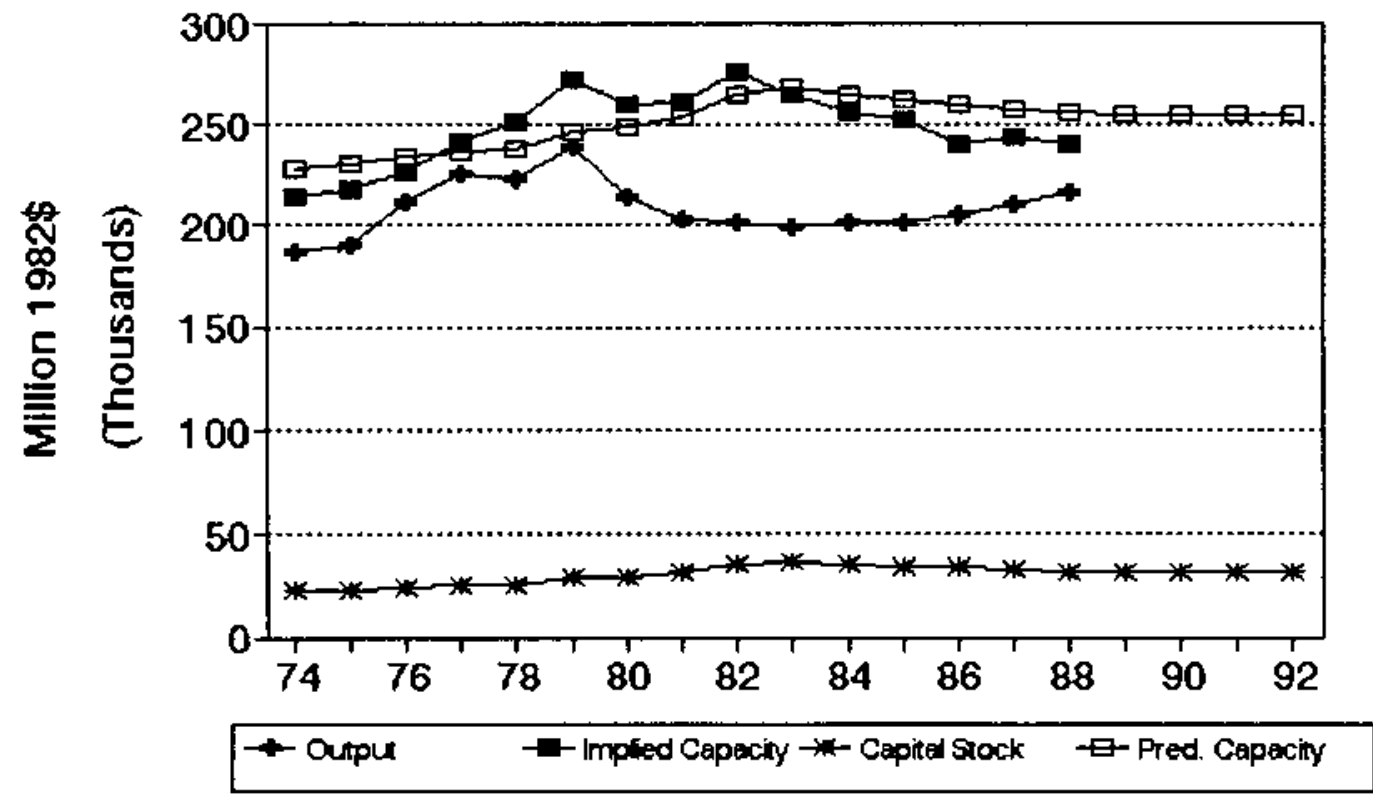




\title{
No. 207 SIC 2951 Paving aixtures and blocks
}

\author{
MILLIONS OF 1982 DOLLARS (EXCL. CU RATES)
}

$\begin{array}{lccccccc} & 04 & \text { Ann. } & \text { Implied Gross } & \text { Net } & \text { Pred. } & \ldots . \ldots . . . \\ \text { Gross } & \text { CU } & \text { CU } & \text { Prac. } & \text { In- } & \text { Cap. } & \text { Prac. } & \text { Emergency Capacity } \\ \text { Output Rate } & \text { Rate } & \text { Capac. } & \text { vest. } & \text { Stk. } & \text { Capac. } & 3 \text { mon. } 6 \text { mon. }>6 \text { mon. }\end{array}$

\begin{tabular}{|c|c|c|c|c|c|c|c|c|c|c|}
\hline 1974 & 2,860 & 53 & 53.0 & 5,391 & 165.7 & 649 & 3,828 & & & \\
\hline 1975 & 2,463 & $59 *$ & 56.4 & 4,364 & 47.9 & 837 & 3,725 & & & \\
\hline 1976 & 2,401 & $63 *$ & 61.5 & 3,906 & 106.3 & 877 & 4,066 & & & \\
\hline 1977 & 2,885 & $72 *$ & 68.7 & 4,199 & 120.9 & 925 & 4,465 & & & \\
\hline 1978 & 3,576 & 75 & 73.7 & 4,850 & 134.7 & 979 & 4,917 & & & \\
\hline 1979 & 2,892 & 74 & 74.4 & 3,889 & 28.0 & 928 & 4,486 & & & \\
\hline 1980 & 2,568 & 78 & 76.5 & 3,358 & 77.8 & 921 & 4,434 & & & \\
\hline 1981 & 2,243 & 73 & 75.0 & 2,903 & 79.8 & 912 & 4,351 & & & \\
\hline 1982 & 3,142 & 49 & 58.0 & 5,420 & 298.8 & 1,092 & 5,868 & & & \\
\hline 1983 & 3.257 & 57 & 54.0 & 6,030 & 77.5 & 1,050 & 5,509 & & & \\
\hline 1984 & 3,372 & 56 & 56.5 & 5,967 & 84.1 & 1,022 & 5,281 & & & \\
\hline 1985 & 3,656 & 59 & 57.8 & 6,321 & 175.2 & 1,088 & 5,830 & & & \\
\hline 1986 & 3,825 & 65 & 62.8 & 6,088 & 132.1 & 1,110 & 6,015 & & & \\
\hline$\because$ & $\cdots \cdot$ & 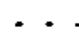 & - - & & $-\cdots$ & -- & $\therefore$ & $\cdots \cdot$ & $\cdot \cdot \cdot$ & $\cdots$ \\
\hline 1987 & 3,855 & 54 & 58.1 & 6,635 & 97.1 & 1,096 & 5,890 & & & \\
\hline 1988 & 3,893 & 63 & 59.6 & 6,529 & 98.0 & 1,085 & 5,802 & 12,702 & 14,290 & 16,701 \\
\hline 1989 & & & & & 103.1 & 1,079 & $5, \pi 57$ & 12,603 & 16,180 & 14,587 \\
\hline 1990 & & & & & 105.6 & 1,077 & 5,737 & 12,561 & 14,132 & 14,538 \\
\hline 1991 & & & & & 110.1 & 1,079 & 5,738 & 12,606 & 14,182 & 14,590 \\
\hline 1992 & & & & & 108.1 & 1,080 & 5,759 & 12,609 & 14,186 & 14,594 \\
\hline
\end{tabular}

Capacity-Capitat Stock Regression: Code 0 RSQ 0.500 Coefficients: Const -3288.0 stock 8.4

Ave. Capacity/Stock $(74-86): 5.0$ Ave. Weekly Hours (High) 62.2 Shift Factor 2.53 Ave Weekly Hours (Low) 54.8 Shift factor 2.88 - Imputed Note: Output, Investment, and Copital Stock extrapolated for 1987 and 1988.

\section{Paving mixtures and blocks}

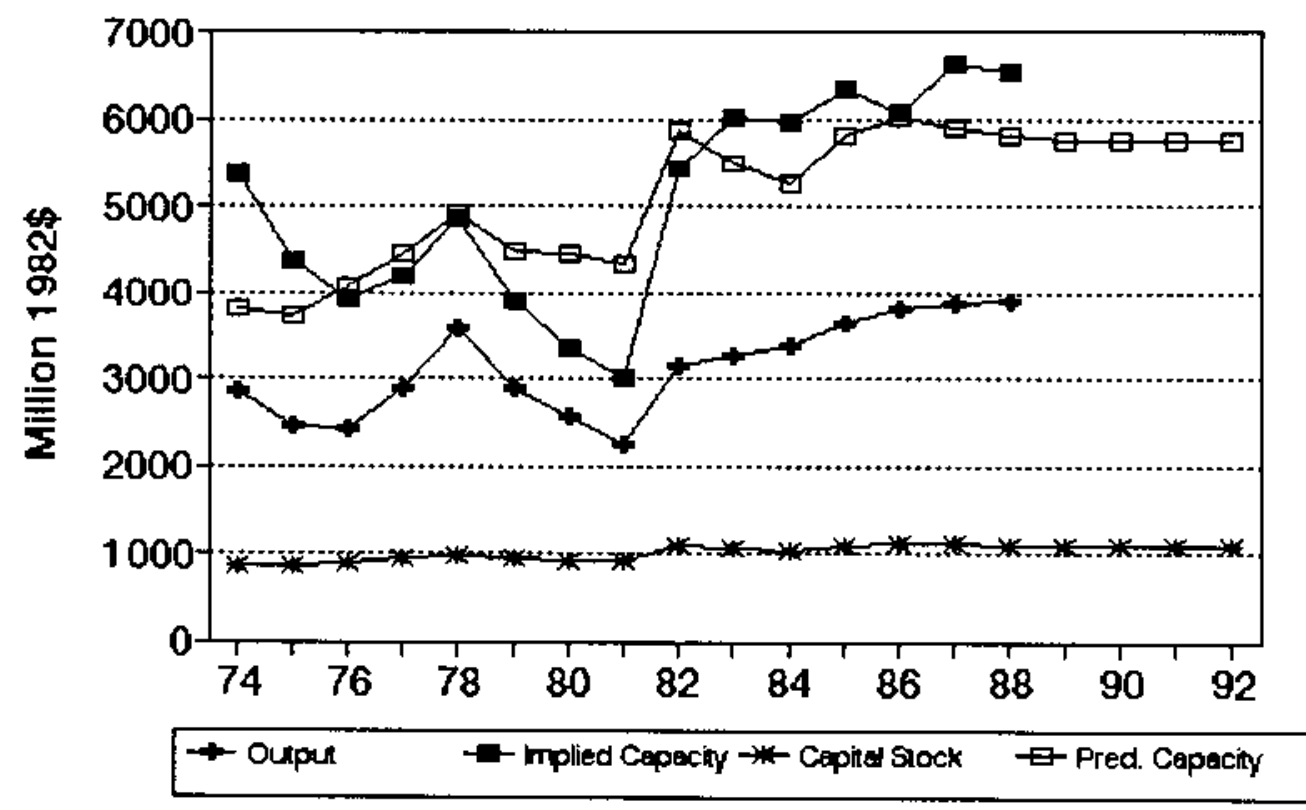




\section{No. 208 SIC 2952 Asphalt felts and coating}

\section{MILLIONS OF 1982 DOLLARS (EXCL. CU RATES)}

$\begin{array}{lccccccc} & \text { Q4 } & \text { Ann. } & \text { Implied Gross } & \text { Met } & \text { Pred. } & -\ldots \ldots \ldots \\ \text { Gross } & \text { CU } & \text { CU } & \text { Prac. } & \text { In- } & \text { Cap. } & \text { Prac. } & \text { Emergency Capacity } \\ \text { Output } & \text { Rate } & \text { Rate } & \text { Capac. } & \text { vest. } & \text { Stk. } & \text { Capac. } & 3 \text { mon. } 6 \text { mon. }>6 \text { mon. }\end{array}$

\begin{tabular}{|c|c|c|c|c|c|c|c|c|c|c|}
\hline 1974 & 3,085 & 66 & 66.7 & 4,626 & 78.9 & 501 & 4,374 & & & \\
\hline 1975 & 2,810 & $6 B$ & 66.4 & 4,234 & 58.7 & 521 & 4,416 & & & \\
\hline 1976 & 3,152 & 59 & 66.3 & 4,756 & 112.2 & 593 & 4,561 & & & \\
\hline 1977 & 3,271 & 85 & 79.9 & 4,093 & 103.4 & 651 & 4.680 & & & \\
\hline 1978 & 3,655 & 70 & 75.0 & 4,871 & 82.2 & 688 & 4,754 & & & \\
\hline 1979 & 3,617 & 82 & 77.4 & 4,673 & 47.3 & 689 & 4,758 & & & \\
\hline 1980 & 2,915 & 63 & 69.6 & 4,186 & 60.1 & 702 & 4,783 & & & \\
\hline 1981 & 2,844 & 55 & 59.1 & 4,815 & 116.6 & 764 & 4,909 & & & \\
\hline 1982 & 2,823 & 64 & 60.2 & 4,688 & 8.1 & 717 & 4.814 & & & \\
\hline 1983 & 3,447 & 56 & 59.3 & 5,811 & 50.6 & 710 & 4,800 & & & \\
\hline 1984 & 3,315 & 61 & 61.4 & 5,402 & 105.5 & 31 & 4,883 & & & \\
\hline 1985 & 3,260 & 74 & 68.5 & 4,761 & 34.0 & 724 & 4,827 & & & \\
\hline 1986 & 3,344 & 72 & 74.2 & 4,508 & 83.1 & 743 & 4,867 & & & \\
\hline$-\cdots$ & $--\cdot-$ & -- & -- & $-\cdots$ & -- & $\cdots$ & $\cdots$ & $\cdots$ & $-\cdots$ & $\cdots$ \\
\hline 1987 & 3,360 & $\pi$ & 73.4 & 4,576 & 62.8 & 742 & 4.866 & & & \\
\hline 1988 & 3,381 & $n$ & 76.5 & 4,420 & 61.7 & 741 & 4,862 & 5,991 & 6,462 & 6,584 \\
\hline 1989 & & & & & 60.6 & 738 & 4,857 & 5,985 & 6,455 & 6,577 \\
\hline 1990 & & & & & 59.5 & 735 & 4.850 & 5,976 & 6,446 & 6,567 \\
\hline 1991 & & & & & 58.4 & 731 & 4,842 & 5,966 & 6,434 & 6,556 \\
\hline 1992 & & & & & 57.3 & 726 & 4,831 & 5,953 & 6,421 & 6,542 \\
\hline
\end{tabular}

Capacity-Capital Stock Regression: Code 0 RSQ 0.130 Coefficients: Const 3356.8 stock 2.0

Ave. Capacity/Stock $(74-86): 7.0$ Ave. Weekly Hours (High) 116.6 Shift Factor 1.35 Ave Weekly Hours (Low) 112.2 Shift Factor 1.40 Mote: Output, Investment, and Capital Stock extrapolated for 1987 and 1988.

\section{Asphalt felts and coating}

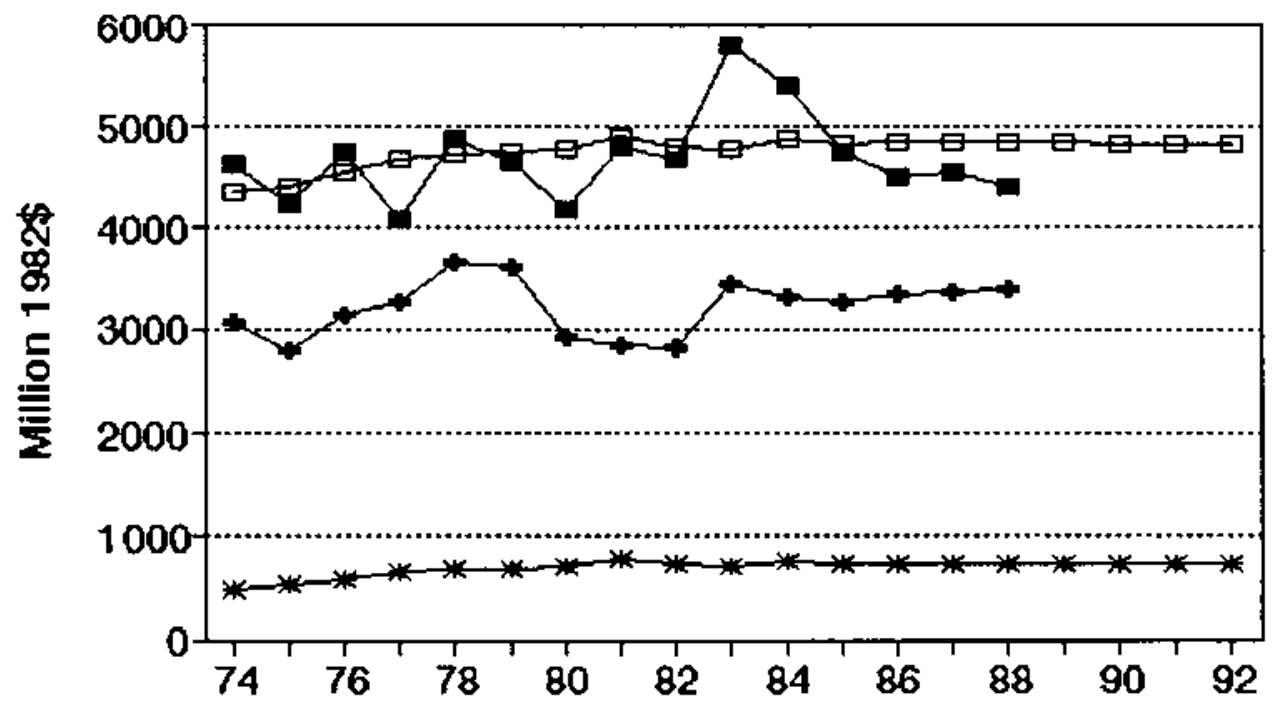

\begin{tabular}{|c|c|}
\hline$\rightarrow$ Output & 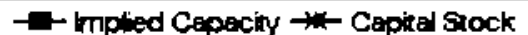 \\
\hline
\end{tabular}


No. 209 SIC 2992 Lubricating oils and greases

MILLIONS OF 1982 DOLLARS (EXCL. CU RATES)

$\begin{array}{lccccccc} & \text { Q4 } & \text { Am. Implied Gross } & \text { Met } & \text { Pred. } & \ldots & \ldots . . . \\ \text { Gross } & \text { CU } & \text { CU } & \text { Prac. } & \text { In- } & \text { Cap. } & \text { Prac. } & \text { Emergency Capacity } \\ \text { Output } & \text { Rate } & \text { Rate } & \text { Capac. } & \text { vest. } & \text { Stk. } & \text { Capac. } & 3 \text { mon. } 6 \text { mon. }>6 \text { mon. }\end{array}$

\begin{tabular}{|c|c|c|c|c|c|c|c|c|c|c|}
\hline 1974 & 2,254 & 79 & 79.2 & 2,845 & 70.5 & 277 & 3,523 & & & \\
\hline 1975 & 2,252 & 77 & 77.5 & 2,906 & 49.5 & 306 & 3,721 & & & \\
\hline 1976 & 2,773 & 66 & 71.1 & 3,899 & 31.9 & 316 & 3,789 & & & \\
\hline 1977 & 3,005 & 66 & 67.2 & 4,475 & 56.5 & 348 & 4,006 & & & \\
\hline 1978 & 3,182 & $73 *$ & 70.5 & 4,517 & 34.9 & 358 & 4,078 & & & \\
\hline 1979 & 3,560 & 80 & 77.5 & 4,596 & (11.5) & 324 & 3,846 & & & \\
\hline 1980 & 3,102 & 76 & 77.4 & 4,008 & 65.1 & 365 & 4,126 & & & \\
\hline 1981 & 3,162 & 67 & 70.6 & 4,476 & 70.1 & 408 & 4,417 & & & \\
\hline 1982 & 3,116 & 62 & 63.8 & 4,886 & 43.0 & 421 & 4,511 & & & \\
\hline 1983 & 2,872 & 76 & 70.8 & 4,056 & 20.6 & 412 & 4,446 & & & \\
\hline 1984 & 3,148 & 53 & 62.2 & 5,057 & 244.4 & 609 & 5,795 & & & \\
\hline 1985 & 3,218 & 54 & 53.5 & 6,014 & 9.5 & $5 \pi$ & 5,558 & & & \\
\hline 1986 & 3,174 & 55 & 54.9 & 5,785 & 65.3 & 596 & 5,703 & & & \\
\hline-- & $\cdots$ & $\cdots$ & $\cdots$ & $\therefore-$ & -- & $\cdots$ & $\cdots$ & $\cdots$ & $\cdots$ & $\cdot \cdot$ \\
\hline 1987 & 3,216 & 56 & 55.5 & 5,790 & 54.1 & 605 & 5,766 & & & \\
\hline 1988 & 3,271 & 77 & 69.2 & 4,730 & 54.2 & 613 & 5,824 & 7,502 & 8,265 & 8,462 \\
\hline 1989 & & & & & 54.3 & 621 & 5,879 & 7,572 & 8,343 & 8,542 \\
\hline 1990 & & & & & 54.4 & 629 & 5,930 & 7,638 & 8,415 & 8,616 \\
\hline 1991 & & & & & 54.5 & 636 & 5,978 & 7,701 & 8,484 & 8,686 \\
\hline 1992 & & & & & 54.5 & 642 & 6,023 & 7,738 & 8,547 & 8,751 \\
\hline
\end{tabular}

Capacity-Capital Stock Regression: Code 0 RSO 0.690 Coefficients: Const 1627.4 stock 6.8

Ave. Capacity/Stock $(74-86): 10.8$ Ave. Heek(y Hours (High) 108.4 Shift factor 1.45 Ave Weekly Hours (LOW) 99.2 Shift factor 1.59 * Imputed Note: Output, Investment, and Capital Stock extrapolated for 1987 and 1988.

\section{Lubricating oils and greases}

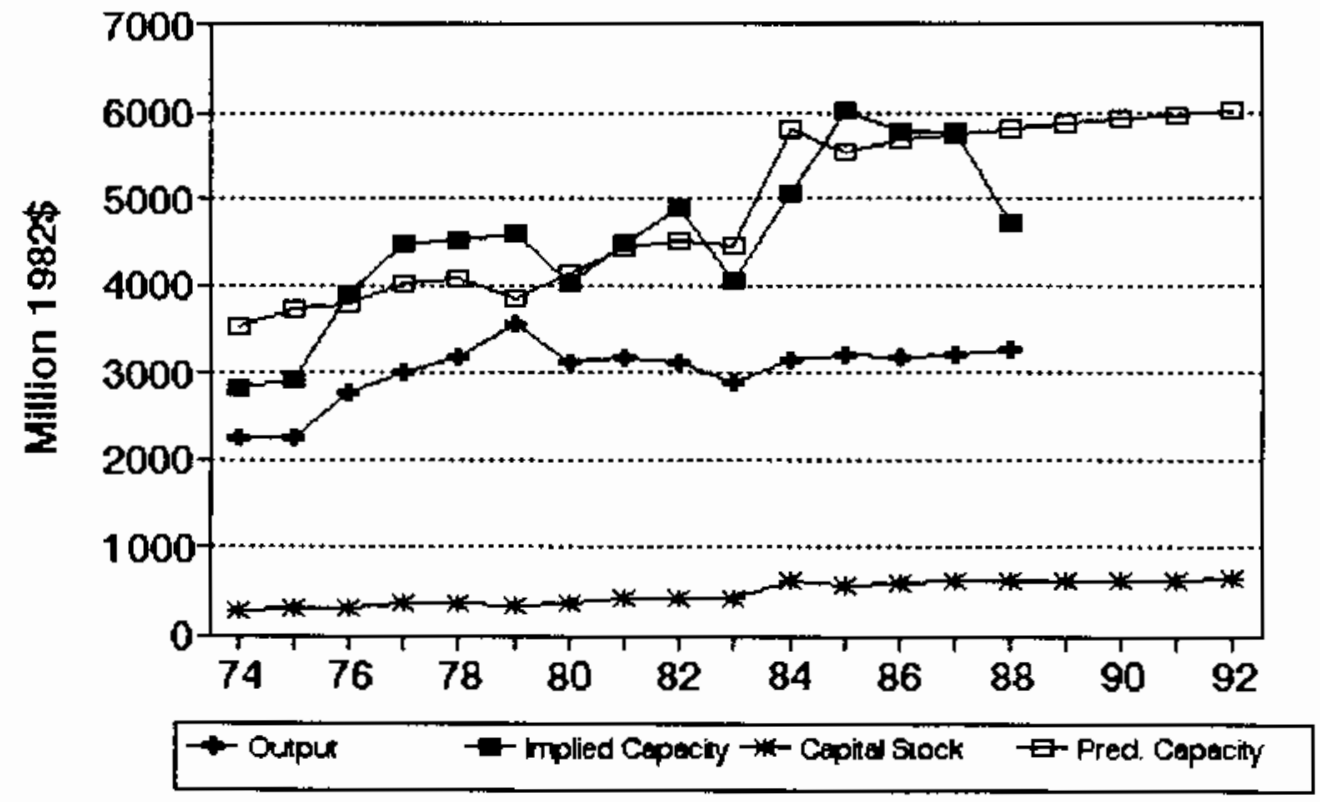


No. 210 gIC 2999 Petrolew and coal products, n.e.c. MILLIONS OF 1982 DOLLARS (EXCL. CU RATES)

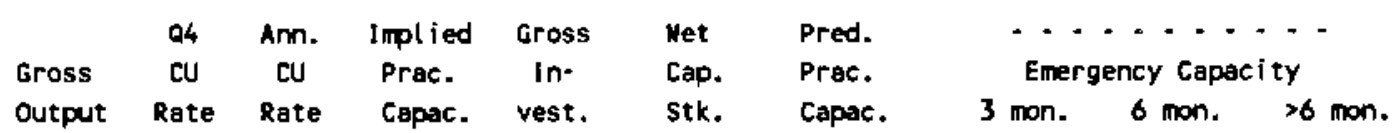

\begin{tabular}{|c|c|c|c|c|c|c|c|c|c|c|}
\hline 1974 & 376 & $93 *$ & 93.5 & 402 & 20.3 & 135 & 394 & & & \\
\hline 1975 & 290 & $90 *$ & 90.3 & 321 & 29.8 & 156 & 442 & & & \\
\hline 1976 & 410 & $76 *$ & 84.8 & 484 & 60.3 & 208 & 558 & & & \\
\hline 1977 & 568 & $76 \star$ & 80.0 & 710 & 42.2 & 238 & 626 & & & \\
\hline 1978 & 533 & $88 \div$ & 82.8 & 644 & 43.3 & 268 & 696 & & & \\
\hline 1979 & 620 & 98 & 94.0 & 660 & 44.7 & 298 & 761 & & & \\
\hline 1980 & 744 & 83 & 88.3 & 843 & 43.7 & 326 & 823 & & & \\
\hline 1981 & 895 & 73 & 7.7 & 1,153 & 27.4 & 334 & 841 & & & \\
\hline 1982 & 758 & $70 \star$ & 70.7 & 1,072 & 91.4 & 401 & 991 & & & \\
\hline 1983 & 702 & 60 & 63.9 & 1,099 & 81.5 & 458 & 1,118 & & & \\
\hline 1984 & 755 & 69 & 67.3 & 1,123 & 123.0 & 545 & 1,314 & & & \\
\hline 1985 & 707 & 64 & 53.2 & 1,329 & 66.3 & 575 & 1,381 & & & \\
\hline 1986 & 795 & 62 & 55.9 & 1,422 & 13.5 & 547 & 1,320 & & & \\
\hline-- & $-\cdots$ & $\cdots$ & $\because \cdot$ & $\cdots \cdot$ & $\cdots$ & $-\cdots$ & --- & $----\cdot$ & --- & $\cdots$ \\
\hline 1987 & 791 & 68 & 65.5 & 1,207 & 34.3 & 544 & 1,312 & & & \\
\hline 1988 & 785 & 83 & 7.5 & 1,013 & 35.0 & 561 & 1,306 & 1,271 & 1,359 & 1,382 \\
\hline 1989 & & & & & 38.9 & 543 & 1,309 & 1.275 & 1,362 & 1,385 \\
\hline 1990 & & & & & 40.8 & 546 & 1,317 & 1,282 & 1.370 & 1,393 \\
\hline 1991 & & & & & 44.4 & 553 & 1,332 & 1,296 & 1,386 & 1,409 \\
\hline 1992 & & & & & 42.8 & 557 & 1,342 & 1,306 & 1,396 & 1,420 \\
\hline
\end{tabular}

Capacity-Capital Stock Regression: Code 0 RSO 0.870

Coefficients: Const 92.9 Stock 2.2 Ave. Capacity/Stock $(74-86): 2.5$ Ave. Weekly Hours (High) 158.4 Shift Factor 1.06 Ave Heekly Hours (Low) 147.6 Shift Factor 1.13 - Imputed Note: Output, Investment, and Capital Stock extrapolated for 1987 and 1988.

2999 Petroleum and coal products, n.e.c

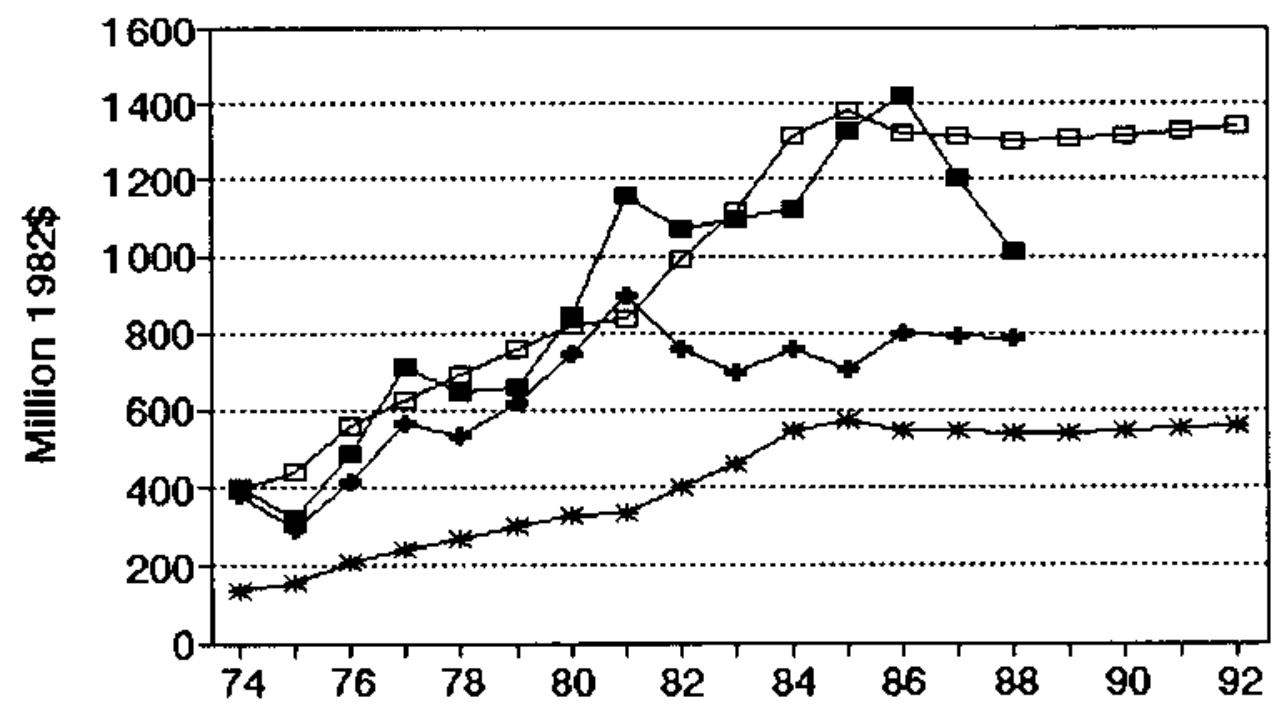

- Outpu $\quad \rightarrow$ impied Cepeciny $\rightarrow$ - Cepite Srock $\quad \square$ Pred. Capacity 


\section{No. 211 sIC 3011 Tires and inner tubes}

MILLIONS OF 1982 DOLLARS (EXCL. CU RATES)

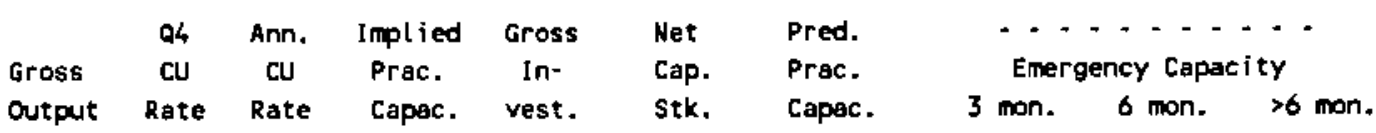

\begin{tabular}{|c|c|c|c|c|c|c|c|c|c|c|}
\hline 1974 & 14,405 & 80 & 87.0 & 16,558 & 976.2 & 6,523 & 15,852 & & & \\
\hline 1975 & 12,422 & 78 & 72.7 & 17,086 & 440.2 & 6,494 & 15,807 & & & \\
\hline 1976 & 11,914 & 87 & 80.2 & 14,851 & 432.3 & 6,438 & 15,718 & & & \\
\hline 1977 & 13,792 & 82 & 85.4 & 16,155 & 568.9 & 6,488 & 15,797 & & & \\
\hline 1978 & 12,664 & 86 & 84.2 & 15,036 & 24.2 & 6,017 & 15,051 & & & \\
\hline 1979 & 11,892 & 80 & 82.8 & 14,362 & 427.0 & 5,932 & 14,916 & & & \\
\hline 1980 & 9,375 & 79 & 76.0 & 12,332 & $(129.5)$ & 5,331 & 13,964 & & & \\
\hline 1981 & 9,903 & 73 & 77.3 & 12,811 & 426.3 & 5,259 & 13,850 & & & \\
\hline 1982 & 9,466 & 70 & 70.7 & 13,394 & 99.4 & 4,870 & 13,233 & & & \\
\hline 1983 & 10,390 & 89 & 81.3 & 12,783 & 228.3 & 4,609 & 12,820 & & & \\
\hline 1984 & 11,345 & 89 & 90.1 & 12,586 & 279.2 & 4,404 & 12,496 & & & \\
\hline 1985 & 11,209 & 80 & 82.8 & 13,530 & 582.6 & 4,493 & 12,636 & & & \\
\hline 1986 & 10,637 & 83 & 81.3 & 13,084 & 350.5 & 4,363 & 12,429 & & & \\
\hline 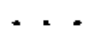 & $\cdots$ & $\because-$ & $\cdots$ & $-\cdots$ & $-\cdots$ & -- & $\cdots$ & $\cdots-$ & $-\cdot--$ & $-\cdots$ \\
\hline 1987 & 10,615 & 92 & 88.4 & 12,010 & 500.0 & 4,382 & 12,460 & & & \\
\hline 1988 & 10,587 & 93 & 92.8 & 11,412 & 500.0 & 4,400 & 12,488 & 12,696 & 13,077 & 13,175 \\
\hline 1989 & & & & & 500.0 & 4,415 & 12,513 & 12,721 & 13,103 & 13,201 \\
\hline 1990 & & & & & 500.0 & 4,429 & 12,535 & 12,744 & 13,126 & 13,224 \\
\hline 1991 & & & & & 500.0 & 4,442 & 12,554 & 12,764 & 13,146 & 13,245 \\
\hline 1992 & & & & & 500.0 & 4,453 & 12,572 & 12,781 & 13,164 & 13,263 \\
\hline
\end{tabular}

Capacity-Capital Stock Regression: Code I RSQ 0.730

Coefficients: Const 5517.2 Stock 1.6

Ave. Capacity/Stock $(74-86): 2.6$ Ave. Weekly Hours (High) 151.2 Shift Factor 1.05 Ave Weekly Hours (Low) 150.0 Shift Factor 1.06 Note: Output, Imestment, and Capital Stock extrapolated for 1987 and 1988.

\section{Tires and inner tubes}

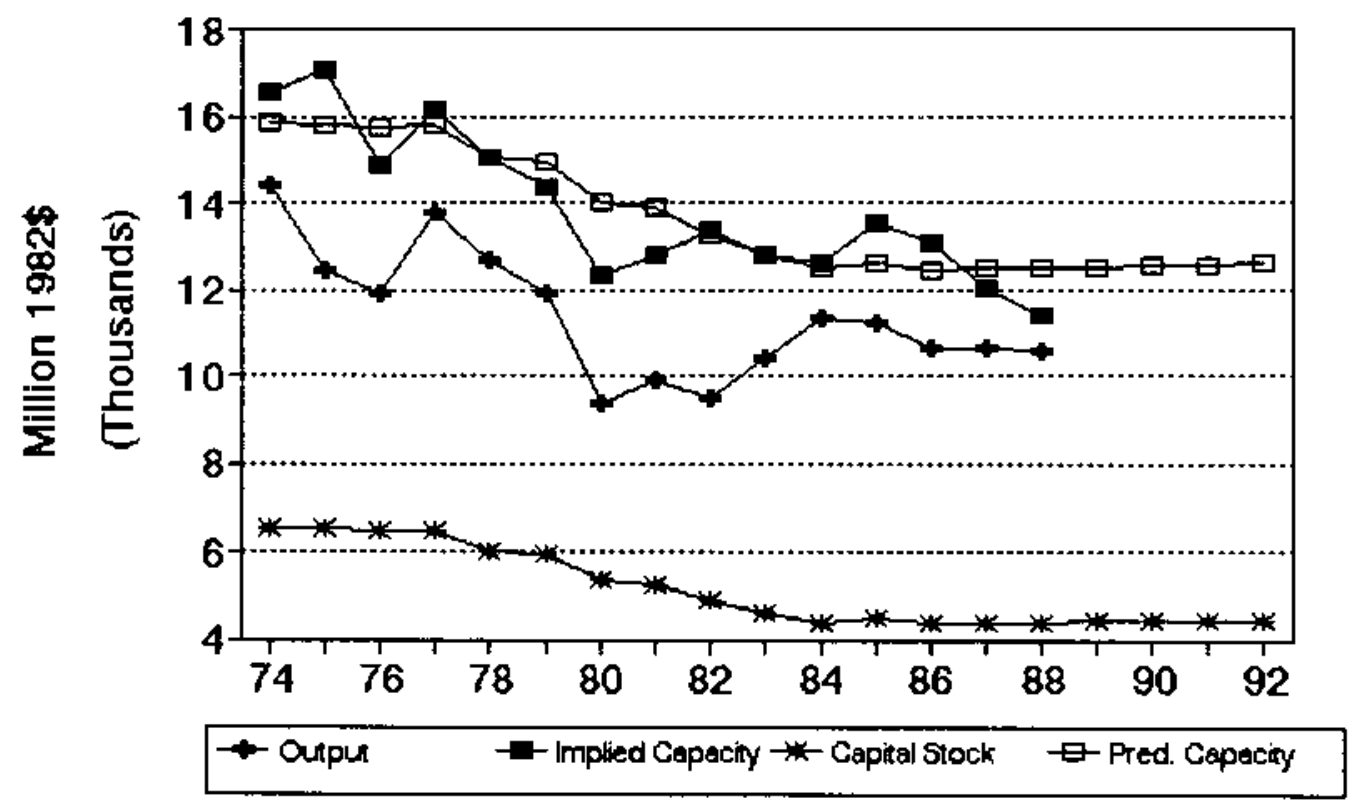




\section{No. 212 sIc 3021 Rubber and plastics footwear}

MILLIONS OF 1982 DOLLARS (EXCL. CU RATES)

$\begin{array}{lccccccc} & Q 4 & \text { Ann. Implied Gross } & \text { Net } & \text { Pred. } & \ldots \ldots \ldots \\ \text { Gross } & \text { CU } & \text { CU } & \text { Prac. } & \text { In- } & \text { Cap. } & \text { Prac. } & \text { Emergency Capacity } \\ \text { Output Rate } & \text { Rate } & \text { Capac. } & \text { vest. } & \text { Stk. } & \text { Capac. } & 3 \text { mon. } 6 \text { mon. }>6 \text { mon. }\end{array}$

\begin{tabular}{|c|c|c|c|c|c|c|c|c|c|c|}
\hline 1974 & 851 & 83 & 90.2 & 943 & $(43.9)$ & 242 & 1,161 & & & \\
\hline 1975 & 774 & 67 & 67.0 & 1,155 & 13.5 & 238 & 1,147 & & & \\
\hline 1976 & 748 & 48 & 53.4 & 1,403 & 18.0 & 237 & 1,144 & & & \\
\hline 1977 & 667 & 71 & 63.4 & 1,053 & 8.0 & 226 & 1,106 & & & \\
\hline 1978 & 553 & 62 & 65.2 & 848 & $(12.0)$ & 197 & 1,011 & & & \\
\hline 1979 & 610 & 61 & 61.8 & 986 & $(18.0)$ & 165 & 906 & & & \\
\hline 1980 & 608 & 68 & 62.7 & 970 & 7.0 & 157 & 881 & & & \\
\hline 1981 & 723 & 74 & 73.7 & 981 & 21.4 & 162 & 897 & & & \\
\hline 1982 & 702 & 62 & 6.0 & 1,004 & 13.5 & 159 & 885 & & & \\
\hline 1983 & 766 & $\pi$ & 70.9 & 1,080 & 17.9 & 158 & 883 & & & \\
\hline 1984 & 650 & $84 \div$ & 82.2 & 791 & 20.8 & 160 & 888 & & & \\
\hline 1985 & 629 & 92 & 88.5 & 710 & 8.0 & 149 & 853 & & & \\
\hline 1986 & 523 & $89+$ & 89.6 & 584 & 3.9 & 135 & 808 & & & \\
\hline$\because-$ & $\therefore$ & -- & $-\cdots$ & $\because-$ & $\cdots$ & $\because-$ & $-\ldots$ & -- & $\cdots-$ & $-\cdot$ \\
\hline 1987 & 507 & 86 & 87.0 & 583 & 18.3 & 137 & 812 & & & \\
\hline 1988 & 487 & 86 & 86.1 & 565 & 20.2 & 139 & 821 & 1,908 & 2,165 & 2,231 \\
\hline 1989 & & & & & 22.1 & 144 & 836 & 1,942 & 2,203 & 2,270 \\
\hline 1990 & & & & & 24.0 & 150 & 855 & 1,986 & 2,253 & 2,322 \\
\hline 1991 & & & & & 25.9 & 157 & 878 & 2,039 & 2,313 & 2,384 \\
\hline 1992 & & & & & 27.8 & 164 & 904 & 2,100 & 2,382 & 2,455 \\
\hline
\end{tabular}

Capacity-Capital stock Regression: Code 1 RSO 0.380

Coefficients: Const 362.1 stock 3.3

Ave. Capacity/Stock $(74-86): 5.3$ Ave. Weekly Hours (High) 58.0 shift Factor 2.72 Ave Weekly Hours (Low) 58.0 Shift Factor 2.72 * Imputed Note: Output, Imvestment, and Capital Stock extrapolated for 1987 and 1988.

3021 Rubber and plastics footwear

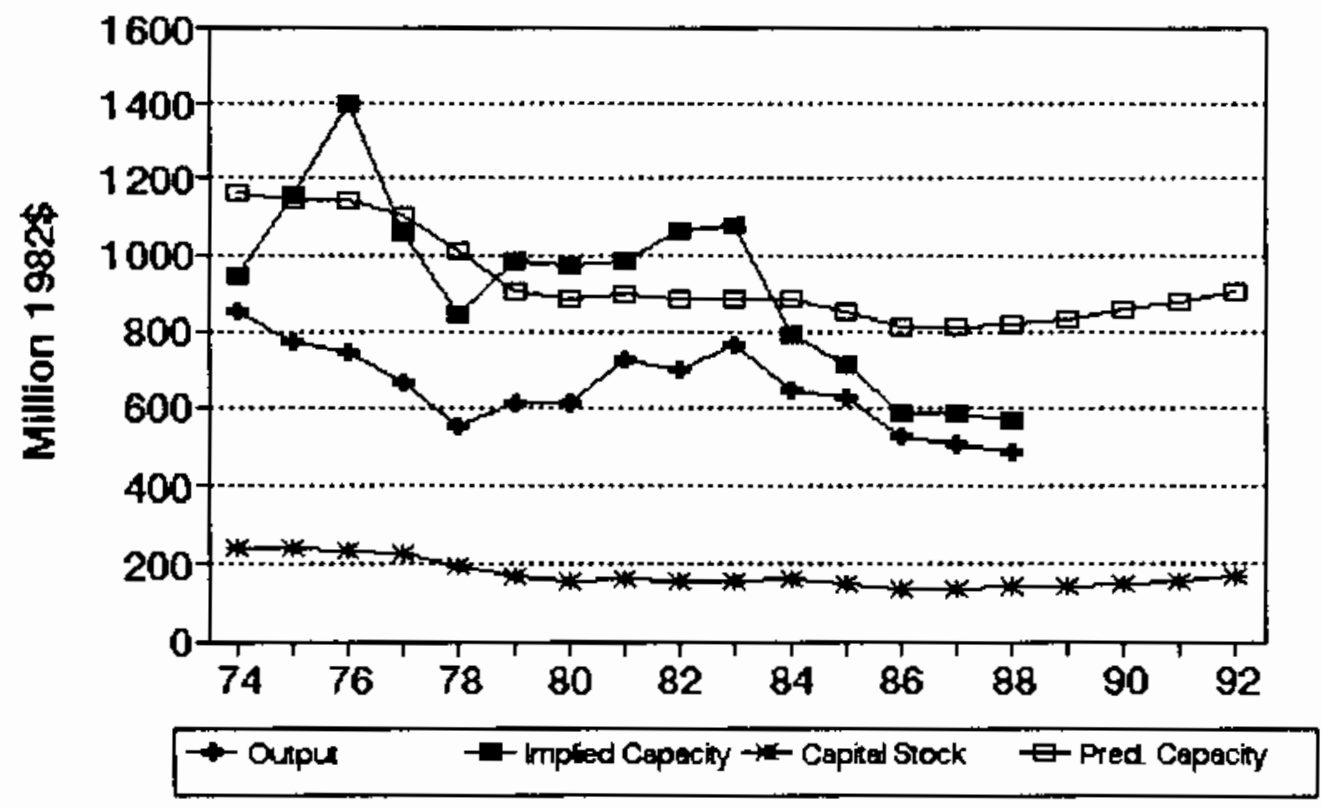


No. 213 sIC 3031 Reclained rubber

MILLIONS OF 1982 DOLLARS (EXCL. CU RATES)

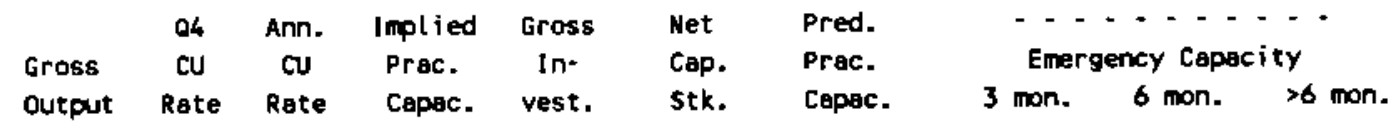

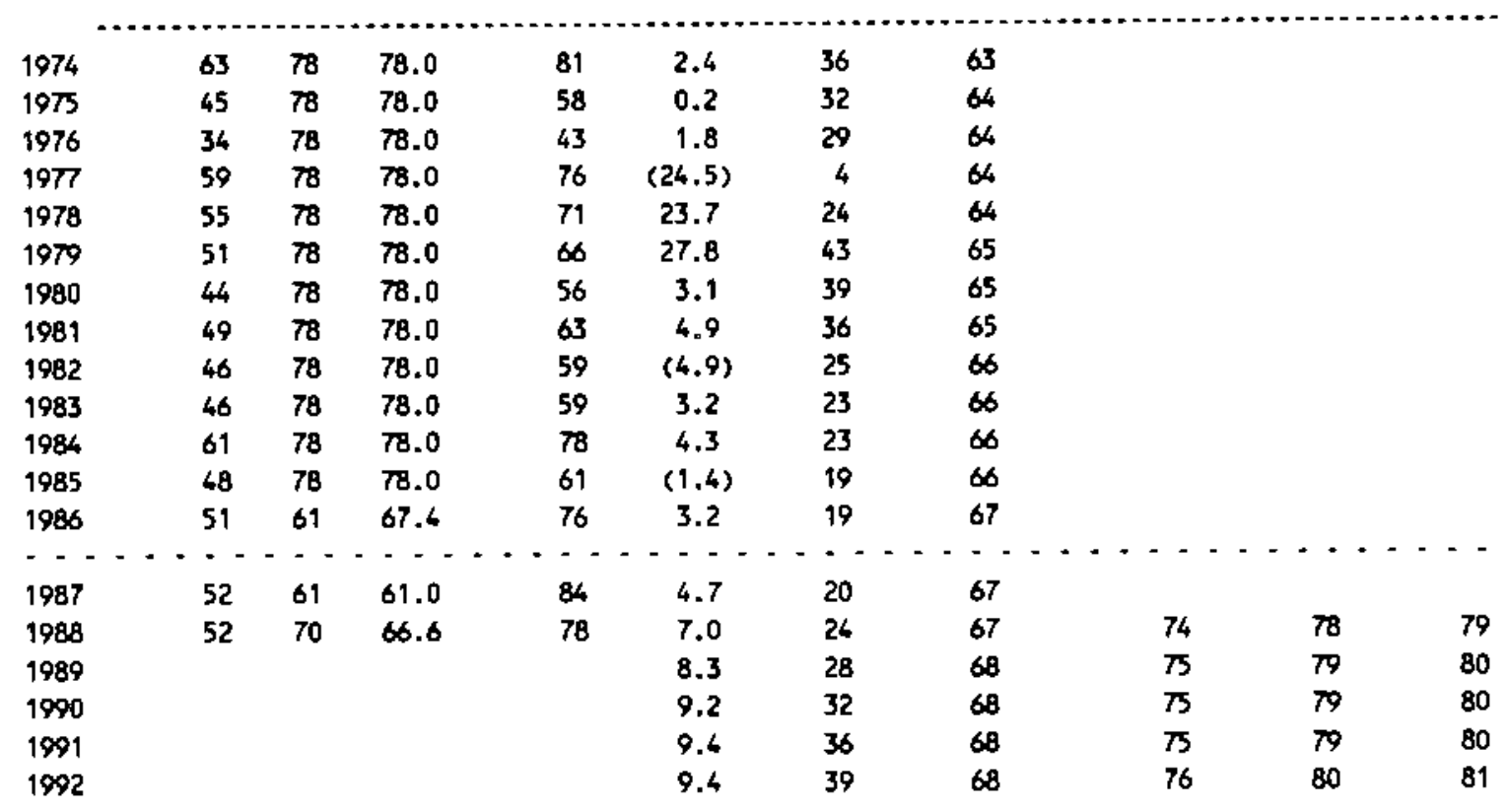

Capacity-Capital Stock Regression: Code -2 RSO 0.0 t0 Coefficients: Const 63.0 Time 0.3

Ave. Capacity/Stock (74-86): 2.4 Ave. Weekly Hours (High) 134.4 Shift factor 1.18 Ave Weekly Hours (Low) 127.2 shift Factor 1.25 Note: Output, Investment, and Capital Stock extrapolated for 1987 and 1988.

3031 Reclaimed rubber

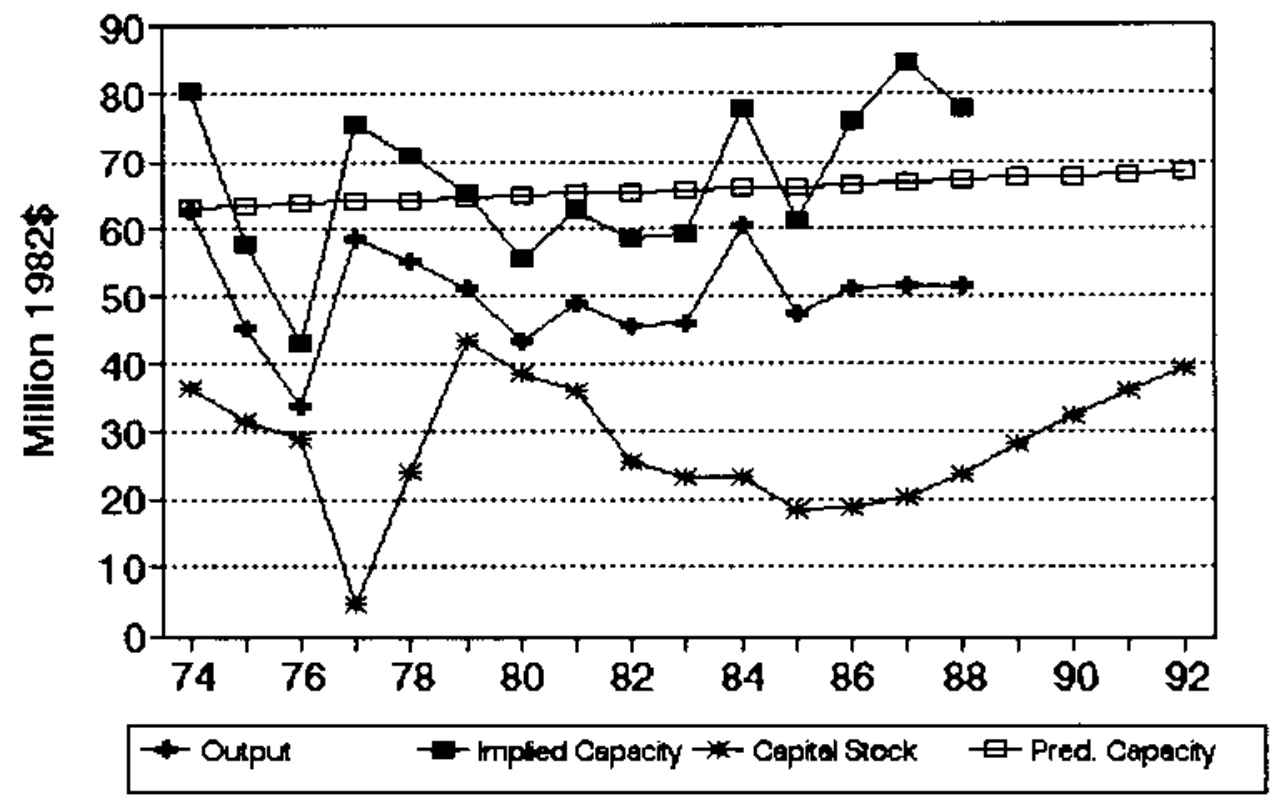


No. 214 sIC 3041 Rubber and plastics hose and belting

MILLIONS OF 1982 DOLLARS (EXCL. CU RATES)

$\begin{array}{lccccccc} & 04 & \text { Ann. } & \text { Implied Gross } & \text { Net } & \text { Pred. } & \ldots \ldots \ldots \\ \text { Gross } & \text { CU } & \text { CU } & \text { Prac. } & \text { In- } & \text { Cap. } & \text { Prac. } & \text { Energency Capacity } \\ \text { Output } & \text { Rate } & \text { Rate } & \text { Capac. } & \text { vest. } & \text { Stk. } & \text { Capac. } & 3 \text { mon. } 6 \text { mon. }>6 \text { mon. }\end{array}$

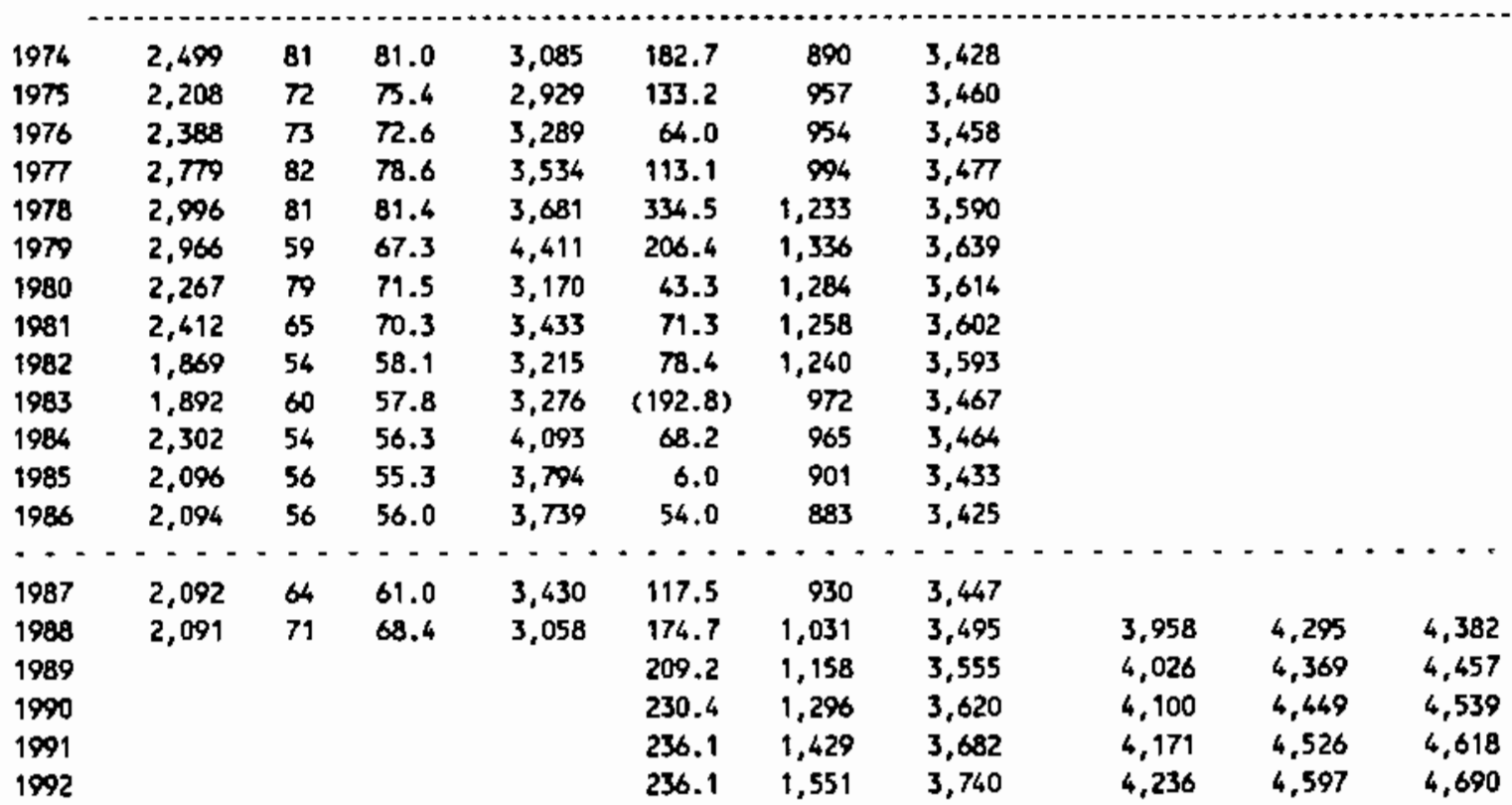

Capacity-Capital Stock Regression; Code 1 RSO 0.040

Coefficients: Const 3008.9 stock 0.5

Ave. Capacity/Stock $(74-86): 3.3$

Ave. Weekly Hours (High) 126.3 Shift Factor 1.25 Ave Weekly Hours (Low) 112.8 Shift Factor 1.40 Mote: Dutput, Investment, and Capital Stock extrapoleted for 1987 and 1988.

\section{Rubber and plastics hose and belti}

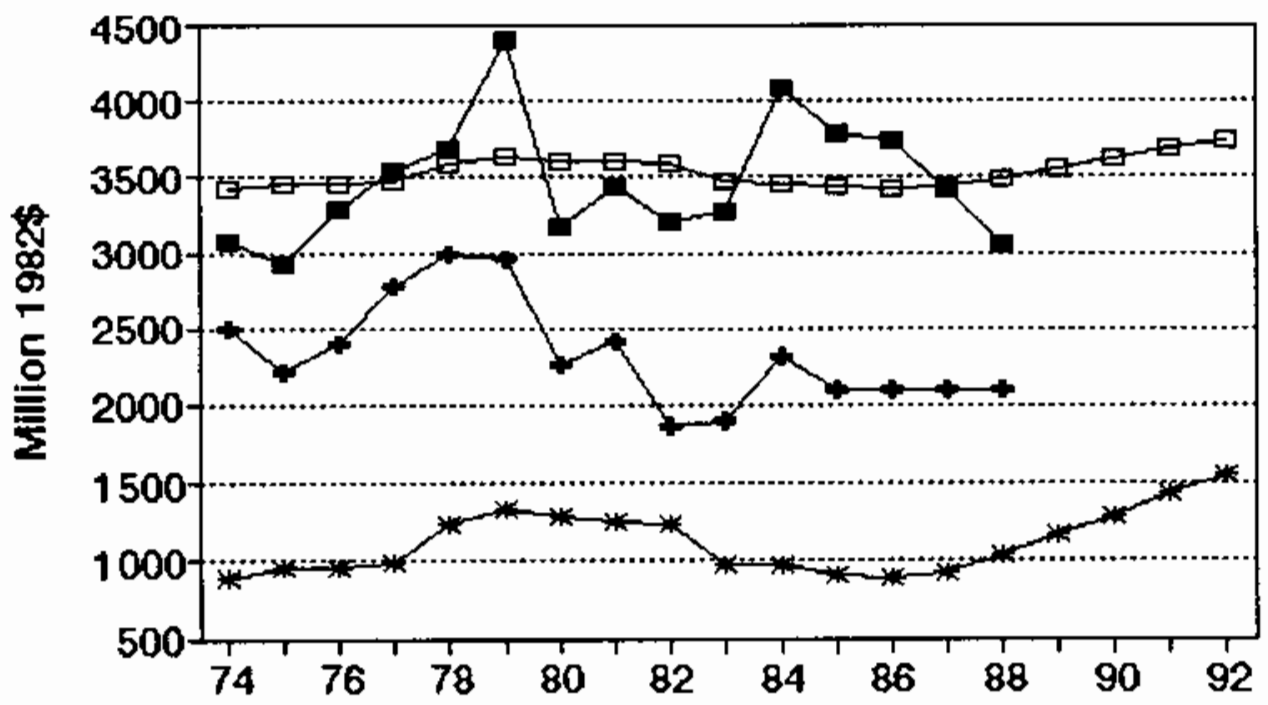

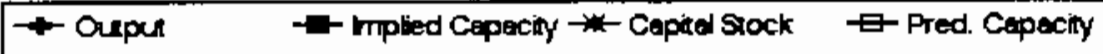


No. 215 sIc 3069 Fabricated rubber products, n.e.c. MILLIONS OF 1982 DOLLARS (EXCL. CU RATES)

$\begin{array}{lccccccc} & 04 & \text { Am. Implied } & \text { Gross } & \text { Net } & \text { Pred. } & \ldots \ldots & \ldots \\ \text { Gross } & \text { CU } & \text { CU } & \text { Prac. } & \text { In- } & \text { Cap. } & \text { Prac. } & \text { Emergency Capacity } \\ \text { Output } & \text { Rate } & \text { Rate } & \text { Capac. } & \text { vest. } & \text { Stk. } & \text { Capac. } & 3 \text { mon. } 6 \text { mon. }>6 \text { mon. }\end{array}$

\begin{tabular}{|c|c|c|c|c|c|c|c|c|c|c|}
\hline 1976 & 6,597 & 73 & 77.5 & 8,509 & 119.5 & 1,852 & 8,674 & & & \\
\hline 1975 & 5,789 & 79 & 72.7 & 7.959 & 102.2 & 1,818 & 8,392 & & & \\
\hline 1976 & 6,374 & 79 & 76.8 & 8,301 & 127.3 & 1,810 & 8,324 & & & \\
\hline 1977 & 7,024 & 79 & 80.0 & 8,780 & 446.1 & 2,089 & 10,636 & & & \\
\hline 1978 & 7,159 & 80 & 79.4 & 9,012 & 206.8 & 2,124 & 10,926 & & & \\
\hline 1979 & 7.077 & 67 & 72.2 & 9,803 & 41.9 & 2,005 & 9,939 & & & \\
\hline 1980 & 6,516 & 63 & 62.5 & 10,425 & 141.5 & 1,981 & 9,739 & & & \\
\hline 1981 & 6,930 & 61 & 62.9 & 11,009 & 242.6 & 2,052 & 10,323 & & & \\
\hline 1982 & 6,463 & 54 & 56.3 & 11,476 & 283.2 & 2,147 & 11,114 & & & \\
\hline 1983 & 6,891 & 69 & 63.0 & 10,942 & 131.0 & 2,089 & 10,634 & & & \\
\hline 1984 & 7,559 & 69 & 69.6 & 10,853 & 124.6 & 2,031 & 10,156 & & & \\
\hline 1985 & 7.817 & 68 & 68.1 & 11,480 & 263.9 & 2,088 & 10,625 & & & \\
\hline 1986 & 7,828 & 67 & 67.0 & 11,670 & 203.7 & 2,103 & 10,749 & & & \\
\hline$-\cdot \cdot$ & - - - & -- & $\because \cdot$ & - - - - & 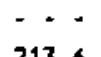 & - - - - & $-\cdots$ & 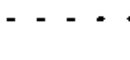 & 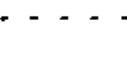 & $\cdots$ \\
\hline 1987 & 7,964 & 73 & 70.6 & 11,278 & 213.6 & 2,128 & 10,956 & & & \\
\hline 1988 & 8,164 & 82 & 78.7 & 10,345 & 249.4 & 2,187 & 11,440 & 13,451 & 14,408 & 14,655 \\
\hline 1989 & & & & & 271.0 & 2,262 & 12,060 & 14,179 & 15,188 & 15,449 \\
\hline 1990 & & & & & 284.4 & 2,343 & 12,734 & 14,971 & 16,037 & 16,312 \\
\hline 1991 & & & & & 287.9 & 2,421 & 13,377 & 15,727 & 16,846 & 17,136 \\
\hline 1992 & & & & & 287.9 & 2,492 & 13,962 & 16,415 & 17,583 & 17,885 \\
\hline
\end{tabular}

Capecity-Capital Stock Regression: Code 1 RSO 0.510

Coefficients: Const -6640.0 stock 8.3

Ave. Capacity/Stock $(74-86): 5.0$ Ave. Weekly Hours (High) 123.4 Shift Factor 1.28 Ave Weekly Hours (Low) 122.9 Shift factor 1.29 Note: output, Investment, and Capital stock extrapolated for 1987 and 1988.

3069 Fabricated rubber products, n.e.c.

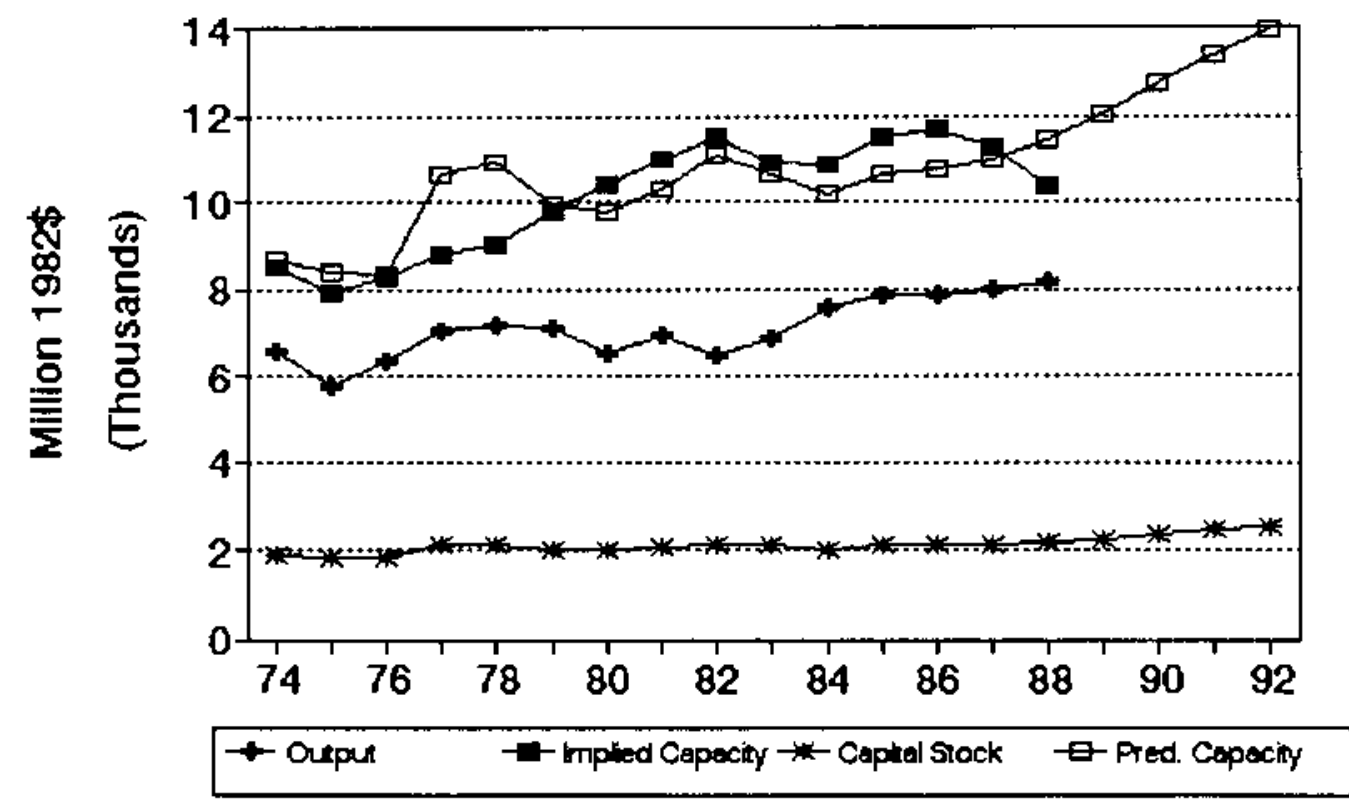




\section{No. 216 sIC 3079 Miscellaneous plastics products}

MILLIONS OF 1982 DOLLARS (EXCL. CU RATES)

$\begin{array}{lccccccc} & \text { Q4 } & \text { Ann. Implied Gross } & \text { Net } & \text { Pred. } & \ldots & \ldots \\ \text { Gross } & \text { CU } & \text { CU } & \text { Prac. } & \text { In- } & \text { Cap. } & \text { Prac. } & \text { Emergency Capacity } \\ \text { Output Rate } & \text { Rate } & \text { Capac. } & \text { vest. } & \text { Stk. } & \text { Capac. } & 3 \text { mon. } 6 \text { mon. }>6 \text { mon. }\end{array}$

\begin{tabular}{|c|c|c|c|c|c|c|c|c|c|c|}
\hline 1974 & 27,102 & 70 & 74.1 & 36,553 & 521.8 & 10,418 & 29,804 & & & \\
\hline 1975 & 22,567 & 70 & 66.4 & 33,981 & $1,089.6$ & 10,866 & 32,401 & & & \\
\hline 1976 & 26,445 & 88 & 78.8 & 33,541 & 995.9 & 11,180 & 34,224 & & & \\
\hline 1977 & 33,302 & 93 & 92.2 & 36,112 & $2,188.8$ & 12,578 & 42,337 & & & \\
\hline 1978 & 35,748 & 79 & 84.1 & 42,506 & $1,601.0$ & 13,333 & 46,716 & & & \\
\hline 1979 & 34,919 & 72 & 75.0 & 46,582 & $1,324.6$ & 13,771 & 49.254 & & & \\
\hline 1980 & 33,251 & 72 & 69.9 & 47,560 & $1,009.1$ & 13,855 & 49.746 & & & \\
\hline 1981 & 35,281 & 63 & 67.6 & 52,194 & $1,327.7$ & 14,201 & 51,752 & & & \\
\hline 1982 & 37,213 & 64 & 63.4 & 58,722 & $2,466.5$ & 15,525 & 59,435 & & & \\
\hline 1983 & 39,529 & 71 & 68.1 & 58,017 & 675.8 & 15,033 & 56,580 & & & \\
\hline 1984 & 45,465 & 73 & 72.9 & 62,351 & $1,791,8$ & 15,613 & 59,942 & & & \\
\hline 1985 & 47,285 & 69 & 70.2 & 67,357 & $2,012.4$ & 16,365 & 64,307 & & & \\
\hline 1986 & 48,953 & 73 & 74.2 & 68,805 & $1,916.6$ & 16,964 & 67,783 & & & \\
\hline$\cdots$ & --- & - & -- & $-\ldots$ & $\because \cdot$ & $\because \cdot$ & $\because \cdot$ & $=\cdot$ & $\cdot \cdot \cdot$ & $\cdot \cdot \cdot$ \\
\hline 1987 & 49,829 & 76 & 74.7 & 66,660 & $1,601.8$ & 17,254 & 69,465 & & & \\
\hline 1988 & 50,991 & 75 & 75.5 & 67,576 & $1,853.9$ & 17,774 & 72,480 & 77,897 & $B 2,750$ & 84,005 \\
\hline 1989 & & & & & $2,006.0$ & 18,405 & 76,145 & 81,835 & $B 6,934$ & 88,252 \\
\hline 1990 & & & & & $2,099.7$ & 19,081 & 80,069 & 86,053 & 91,414 & 92,800 \\
\hline 1991 & & & & & $2,124.7$ & 19,730 & 83,835 & 90,101 & 95,714 & 97,165 \\
\hline 1992 & & & & & $2,124.7$ & 20,329 & 87,310 & 93,836 & 99.682 & 101,193 \\
\hline
\end{tabular}

Capacity-Capital stock Regression: code 0 RSO 0.930

Coefficients: Const -30647.9 stock 5.8

Ave. Copacity/Stock $(74-86): 3.6$ Ave. Heekly Hours (High) 137.0 Shift Factor 1.16 Ave Heekly Hours (Low) 134.4 Shift Factor 1.18 Note: Qutput, Investment, and Capital Stock extrapolated for 1987 and 1988.

\section{Miscellaneous plastics products}

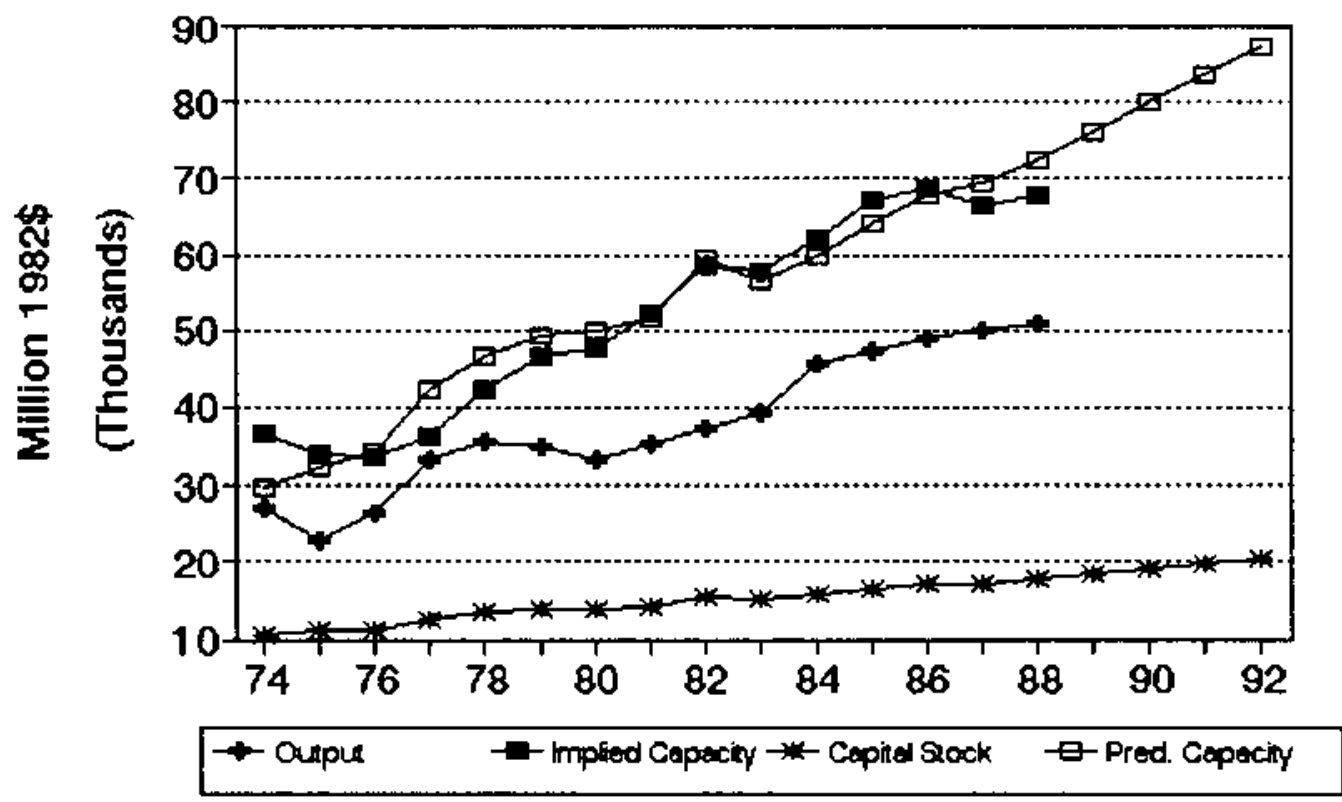




\section{No. 217 sIc 3111 Leather tanning and finishing}

MILLIONS OF 1982 DOLLARS (EXCL. CU RATES)

\begin{tabular}{|c|c|c|c|c|c|c|c|}
\hline Gross & $\begin{array}{l}\mathrm{O4} \\
\mathrm{CU}\end{array}$ & Arn. & $\begin{array}{l}\text { Implied } \\
\text { Prac. }\end{array}$ & $\begin{array}{l}\text { Gross } \\
\text { In- }\end{array}$ & $\begin{array}{l}\text { Net } \\
\text { Cap. }\end{array}$ & $\begin{array}{l}\text { Pred. } \\
\text { Prac. }\end{array}$ & Energency Capacity \\
\hline Output & Rete & Rate & Capec. & vest. & stk. & Capec. & 6 mon. \\
\hline
\end{tabular}

\begin{tabular}{|c|c|c|c|c|c|c|c|c|c|c|}
\hline 1974 & 2,224 & $70 *$ & 69.9 & 3,181 & $(8.4)$ & 255 & 3,370 & & & \\
\hline 1975 & 2,268 & $70 *$ & 69.9 & 3,246 & 39.6 & 270 & 3,259 & & & \\
\hline 1976 & 2,223 & 70. & 70.0 & 3,177 & 24.0 & 270 & 3,149 & & & \\
\hline 1977 & 2,332 & 70 & 70.1 & 3,328 & 33.4 & $2 \pi 7$ & 3,038 & & & \\
\hline 1978 & 2,127 & 70 & 70.0 & 3,038 & 48.3 & 299 & 2,928 & & & \\
\hline 1979 & 1,559 & 68 & 68.8 & 2,268 & 57.5 & 326 & 2,818 & & & \\
\hline 1980 & 1,843 & 64 & 65.5 & 2,814 & 47.4 & 341 & 2,707 & & & \\
\hline 1981 & 1,879 & 69 & 67.1 & 2,800 & 38.1 & 348 & 2,597 & & & \\
\hline 1982 & 1,771 & 69 & 69.0 & 2,567 & 16.5 & 332 & 2,486 & & & \\
\hline 1983 & 1.803 & 69 & 69.0 & 2,613 & $(8.7)$ & 295 & 2,376 & & & \\
\hline 1984 & 1,521 & 69 & 69.0 & 2,204 & $(41.2)$ & 230 & 2,266 & & & \\
\hline 1985 & 1,440 & $69 *$ & 69.0 & 2,087 & 6.4 & 214 & 2,155 & & & \\
\hline 1986 & 1,291 & 69 & 69.0 & 1,872 & 9.1 & 201 & 2,045 & & & \\
\hline$\cdots$ & $-\cdots$ & $-\cdots$ & $-\cdot$ & $\cdots$ & --- & $-\cdots$ & $\cdots$ & $-\quad--$ & $\cdots-$ & -- \\
\hline 1987 & 1,318 & 69 & 69.0 & 1,909 & $(1.0)$ & 178 & 1,935 & & & \\
\hline 1988 & 1,353 & 84 & 78.4 & 1,726 & 2.5 & 161 & 1,824 & 2,317 & 2,491 & 2,536 \\
\hline 1989 & & & & & 14.8 & 158 & 1,714 & 2,177 & 2,340 & 2,382 \\
\hline 1990 & & & & & 20.2 & 161 & 1,603 & 2,036 & 2,189 & 2,229 \\
\hline 1991 & & & & & 25.7 & 169 & 1,493 & 1,896 & 2,038 & 2,075 \\
\hline 1992 & & & & & 14.8 & 165 & 1,383 & 1,756 & 1,888 & 1,922 \\
\hline
\end{tabular}

Capacity-Capital Stock Regression: Code -2 RSQ 0.790

Coefficients: Const 3479.9 Time -110.4

Ave. Capacity/Stock $(74-86): 9.6$ Ave. Heekly Hours (High) 113.4 Shift Factor 1.39 Ave Heekly Hours (Low) 113.4 Shift Factor 1.39 * Imputed Note: Output, Investment, and Capital stock extrapolated for 1987 and 1988.

\section{Leather tanning and finishing}

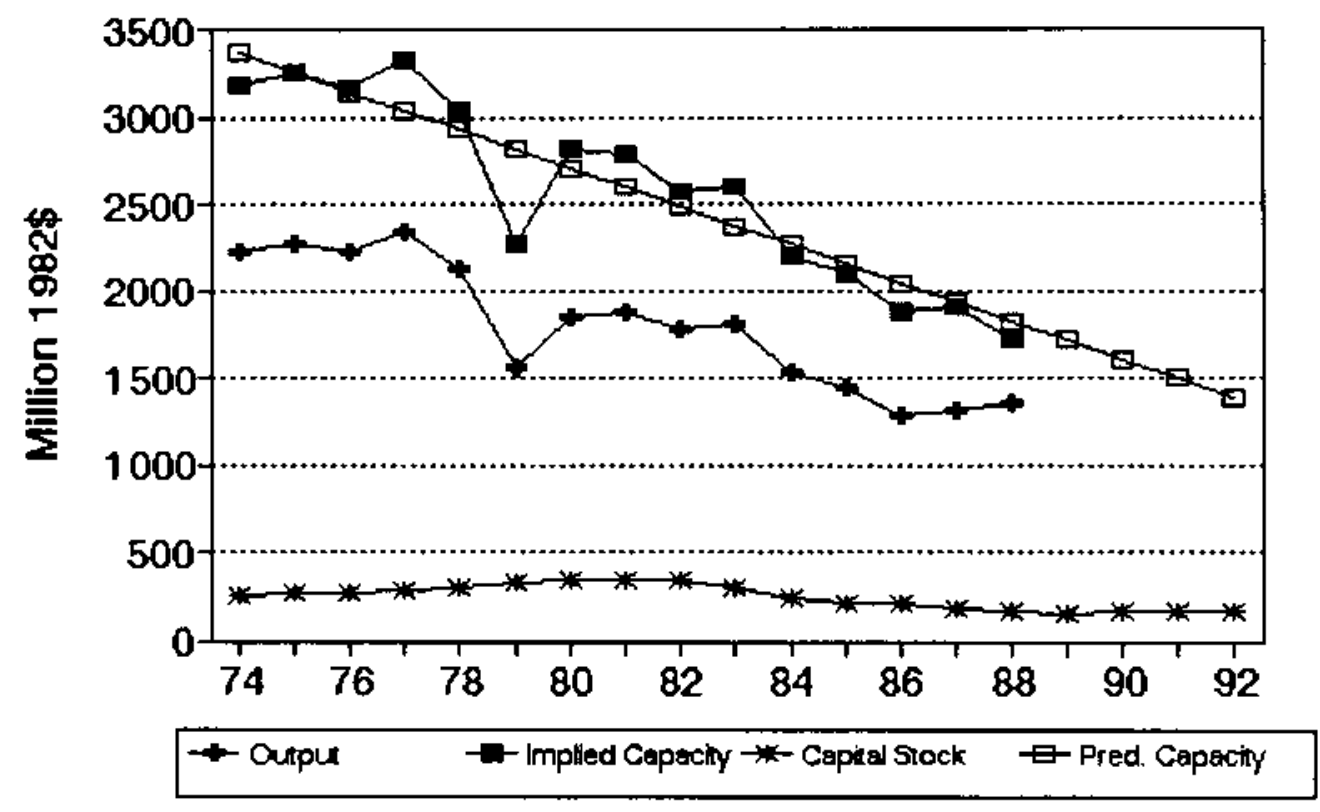


No. 218 sIC 3131 Boot and shoe cut stock and findings

MILLIONS OF 1982 OOLLARS (EXCL. CU RATES)

$\begin{array}{lccccccc} & 04 & \text { Ann. Implied Gross } & \text { Het } & \text { Pred. } & \ldots . . . . . . . . \\ \text { Gross } & \text { CU } & \text { CU } & \text { Prac. } & \text { In- } & \text { Cap. } & \text { Prac. } & \text { Emergency Capacity } \\ \text { Output } & \text { Rate } & \text { Rate } & \text { Capac. } & \text { vest. } & \text { Stk. } & \text { Capac. } & 3 \text { mon. } 6 \text { mon. }>6 \text { mon. }\end{array}$

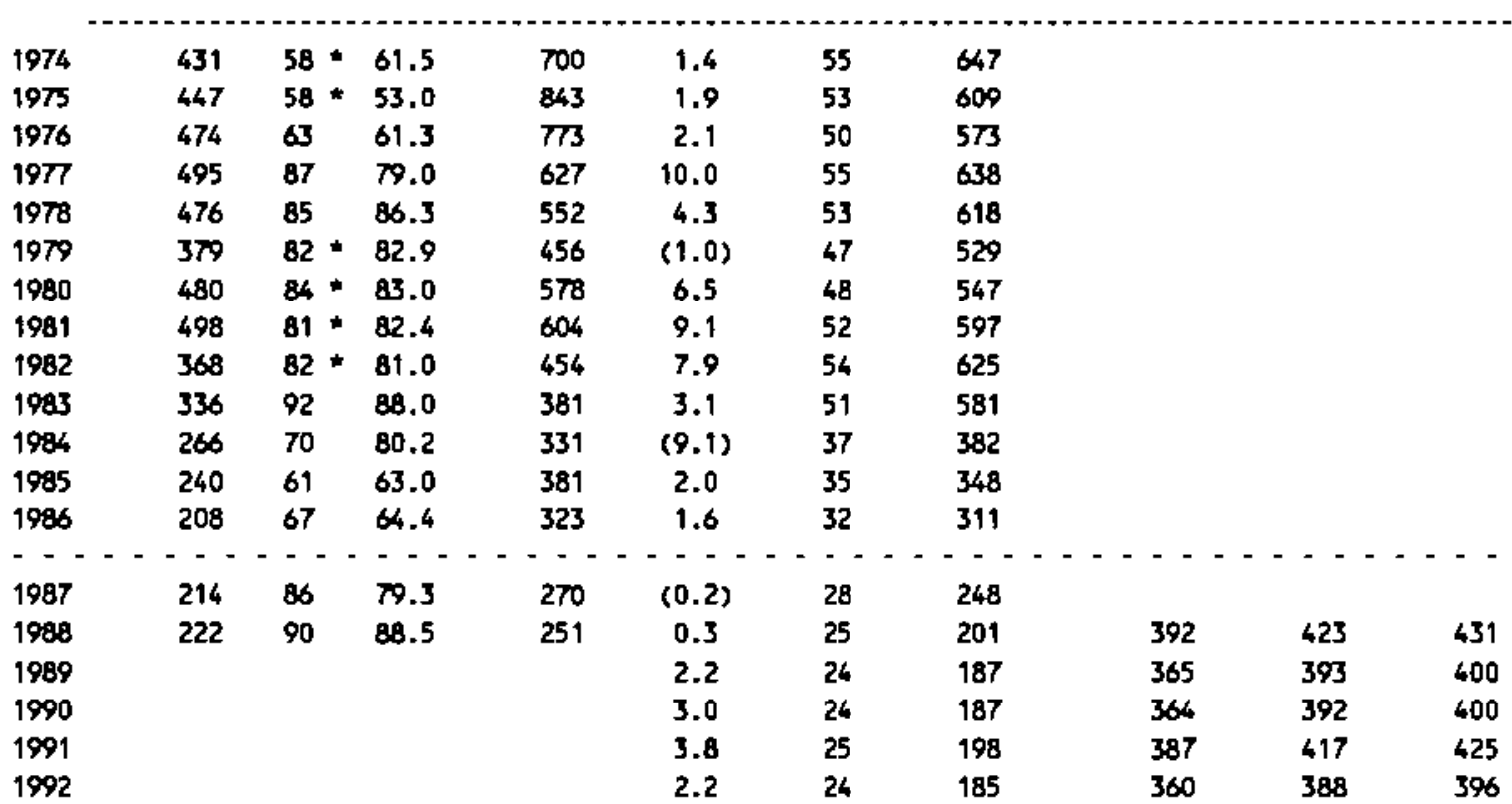

Capacity-Capital Stock Regression: Code i Rso 0.470

Coefficients: Const -166.1 stock 14.7

Ave. Capacity/Stock $(74-86): 11.3$

Ave. Ueekly Hours (High) 73.5 Shift Factor 2.14 Ave Heekly Hours (Low) 57.8 Shift factor 2.73

- Inputed Note: Qutput, Investment, and Capital Stock extrapolated for 1987 and 1988.

\section{Boot and shoe cut stock and findin}

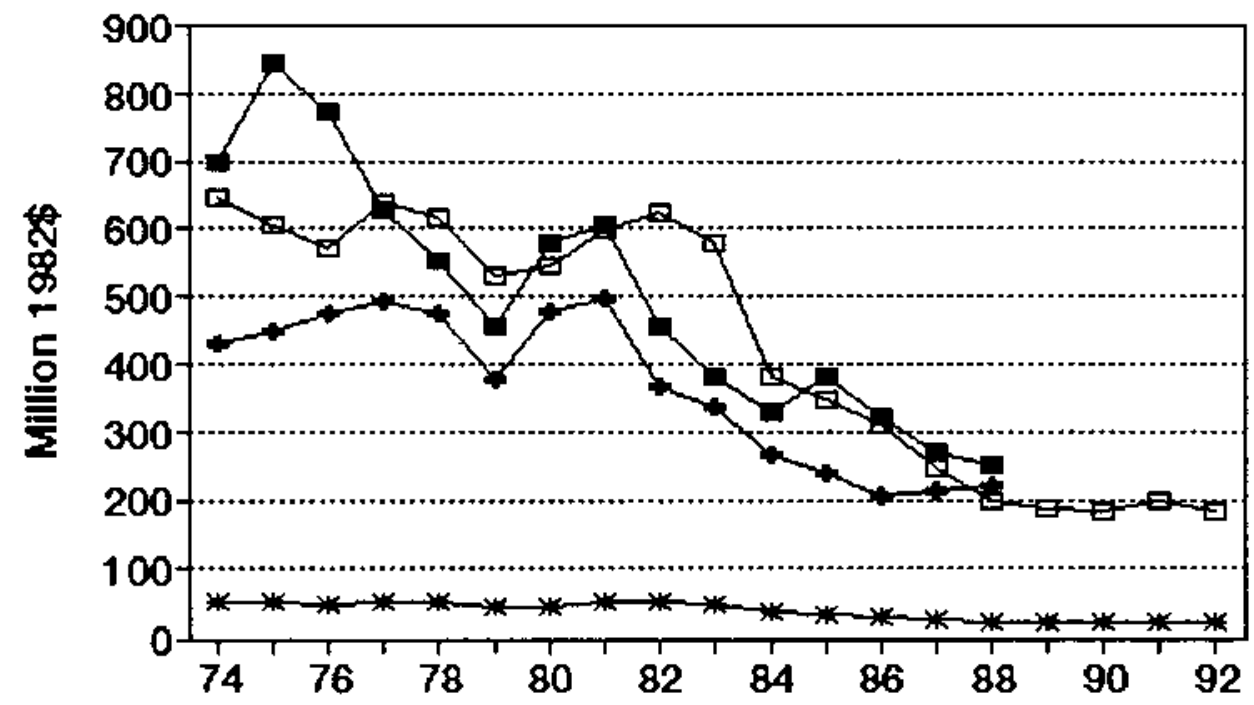

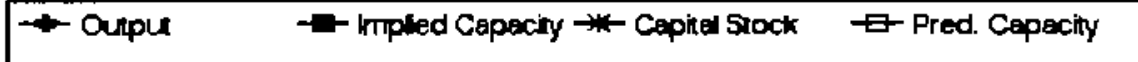




\section{No. 219 SIC 3142 House slippers}

\section{MILLIONS OF 1992 DOLLARS (EXCL. CU RATES)}

$\begin{array}{lccccccc} & \text { Q4 } & \text { Ann. Implied } & \text { Gross } & \text { Net } & \text { Pred. } & \ldots & \ldots \\ \text { Gross } & \text { CU } & \text { CU } & \text { Prac. } & \text { In- } & \text { Cap. } & \text { Prac. } & \text { Emergency Capacity } \\ \text { Output } & \text { Rate } & \text { Rate } & \text { Capac. } & \text { vest. } & \text { Stk. } & \text { Capac. } & 3 \text { mon. } 6 \text { mon. }>6 \text { mon. }\end{array}$

\begin{tabular}{|c|c|c|c|c|c|c|c|c|c|c|}
\hline 1974 & 284 & 73 & 73.0 & 388 & 1.7 & 28 & 381 & & & \\
\hline 1975 & 252 & 80 & 77.4 & 325 & 4.8 & 29 & 381 & & & \\
\hline 1976 & 255 & 70 & 73.8 & 346 & 1.3 & 28 & 381 & & & \\
\hline 1977 & 284 & $\pi$ & 73.1 & 388 & 3.9 & 28 & 381 & & & \\
\hline 1978 & 293 & 74 & 74.4 & 394 & 1.9 & 27 & 381 & & & \\
\hline 1979 & 282 & 76 & 75.3 & 375 & 6.7 & 30 & 355 & & & \\
\hline 1980 & 335 & 77 & 76.6 & 437 & 1.9 & 28 & 351 & & & \\
\hline 1981 & 264 & 74 & $\pi .1$ & 352 & 2.0 & 27 & 346 & & & \\
\hline 1982 & 280 & 72 & 72.8 & 385 & $(0.6)$ & 24 & 342 & & & \\
\hline 1983 & 237 & 71 & 71.4 & 332 & 3.8 & 25 & 338 & & & \\
\hline 1984 & 239 & 71 & 71.0 & 337 & 8.2 & 30 & 333 & & & \\
\hline 1985 & 216 & 73 & 72.3 & 299 & 0.6 & 28 & 329 & & & \\
\hline 1986 & 186 & 68 & 69.9 & 267 & 4.6 & 30 & 325 & & & \\
\hline$=-$ & $\therefore$ & $\because-$ & $\cdots$ & $\therefore$ & -- & -- & $-\cdots$ & -- & $-\quad-$ & • \\
\hline 1987 & 189 & 69 & 68.6 & 275 & 3.7 & 31 & 321 & & & \\
\hline 1988 & 192 & 81 & 76.5 & 250 & 3.7 & 32 & 316 & 832 & 948 & 978 \\
\hline 1989 & & & & & 3.8 & 33 & 312 & 821 & 935 & 964 \\
\hline 1990 & & & & & 3.9 & 33 & 308 & 810 & 922 & 951 \\
\hline 1991 & & & & & 4.0 & 34 & 303 & 798 & 909 & 938 \\
\hline 1992 & & & & & 4.0 & 35 & 299 & 787 & 896 & 924 \\
\hline
\end{tabular}

Capacity-Capital stock Regression: Code -2 RSO 0.240 Coefficients: Const 380.7 Time -4.3 Ave. Yeekly Hours (High) 51.0 Shift factor 3.09 Ave Weekly Hours (Low) 46.7 Shift Factor 3.37 Note: Output, Investment, and Capital Stock extrapolated for 1987 and 1988.

\section{House slippers}

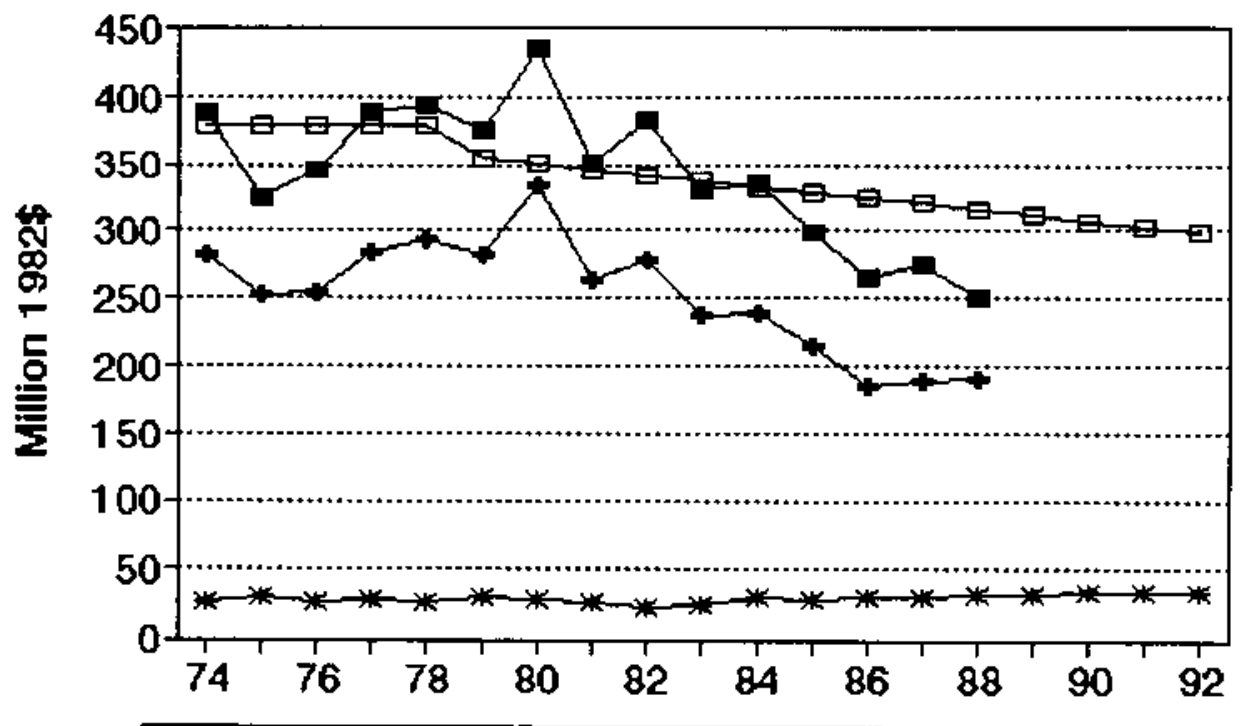




\section{No. 220 sIc 3143 Men's footwear, except athletic}

MILLIONS OF 1982 DOLLARS (EXCL. CU RATES)

$\begin{array}{lccccccc} & \text { Q4 } & \text { Am. Implied Gross } & \text { Net } & \text { Pred. } & \ldots \ldots . . . \\ \text { Gross } & \text { CU } & \text { CU } & \text { Prac. } & \text { In- } & \text { Cap. } & \text { Prac. } & \text { Energency Capacity } \\ \text { Output Rate } & \text { Rate } & \text { Capac. } & \text { vest. } & \text { Stk. } & \text { Capac. } & 3 \text { mon. } 6 \text { mon. } & \end{array}$

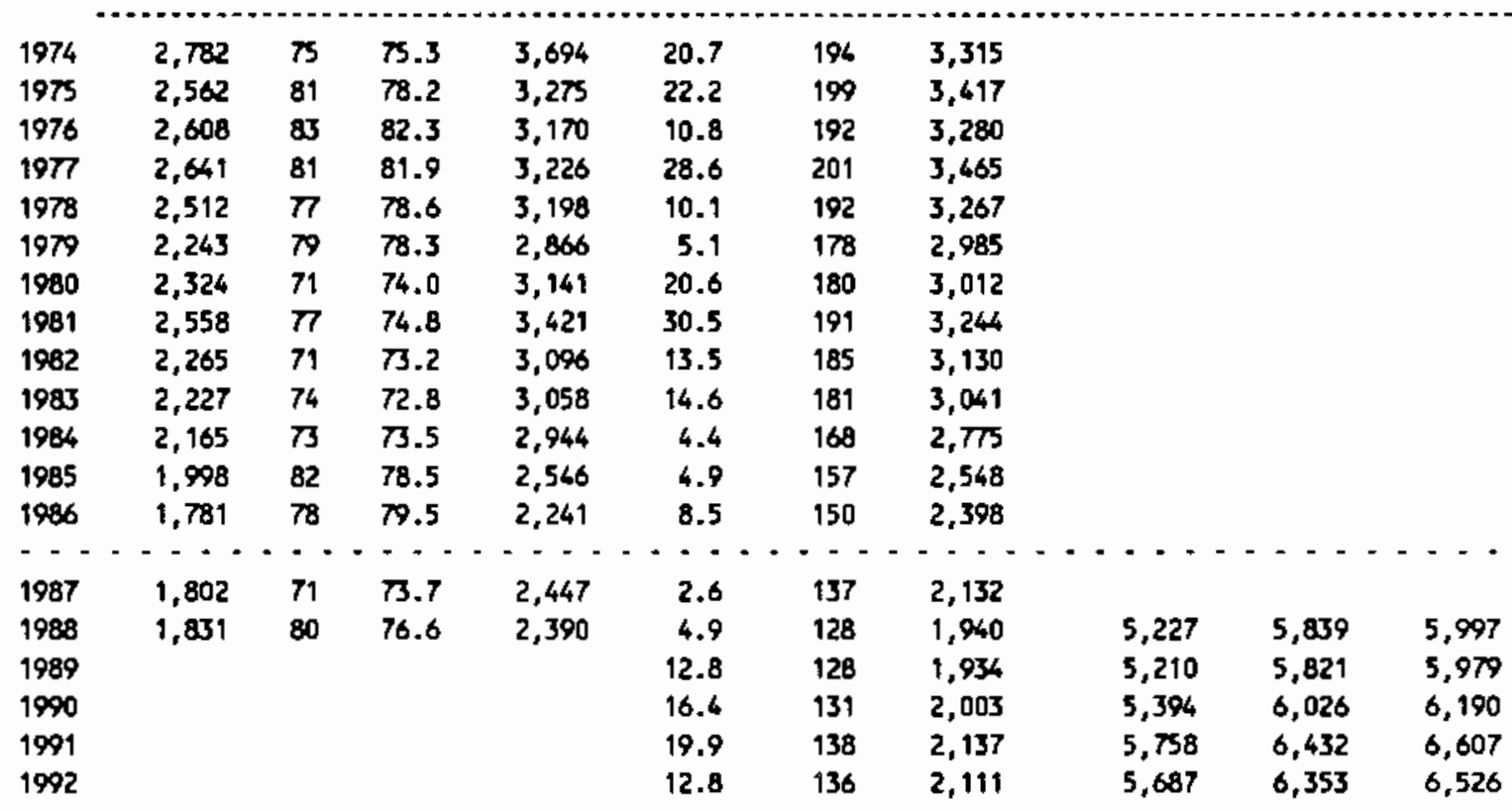

Capacity-Capital Stock Regression: Code 1 RSQ 0.780

Coefficients: Const -721.2 stock 20.8

Ave. Capacity/Stock $(74-86): 16.8$

Ave. Weekly Hours (High) $\mathbf{5 1 . 0}$ Shift factor 3.09 Ave Weekly Hours (Low) 46.7 Shift Factor 3.37 Note: Qutput, Investment, and Capital Stock extrapolated for 1987 and 1988.

\section{Men's footwear, except athletic}

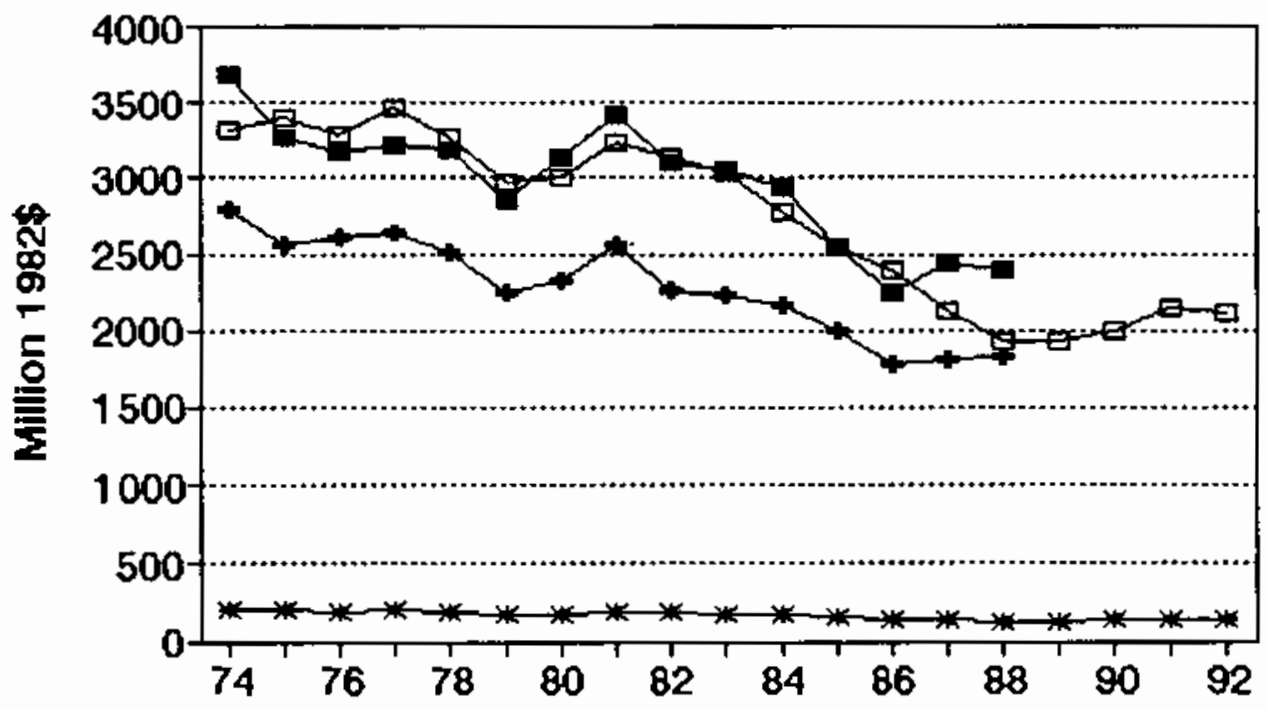

$\rightarrow$ - Oupur $\quad \rightarrow$ - mplied Cepecity $\rightarrow$ - Cepitel Stock $\quad$ E-Pred. Cepocity 


\section{No. 221 SIC 3144 Women' footwear, except athletic}

MILLIOWS OF 1982 DOLLARS (EXCL. CU RATES)

$\begin{array}{lccccccc} & 04 & \text { Arm. Implied Gross } & \text { Net } & \text { Pred. } & -\ldots \ldots \ldots \\ \text { Gross } & \text { CU } & \text { CU } & \text { Prac. } & \text { In. } & \text { Cap. } & \text { Prac. } & \text { Emergency Capacity } \\ \text { Output Rate } & \text { Rate } & \text { Capac. } & \text { vest. } & \text { Stk. } & \text { Capac. } & 3 \text { mon. } 6 \text { mon. }>6 \text { mon. }\end{array}$

\begin{tabular}{|c|c|c|c|c|c|c|c|c|c|c|}
\hline 1974 & 2,173 & 67 & 68.1 & 3,191 & 3.5 & 193 & 3,089 & & & \\
\hline 1975 & 2,039 & 80 & 73.2 & 2,787 & 9.6 & 184 & 2,993 & & & \\
\hline 1976 & 2,071 & 63 & 69.6 & 2,976 & $(7.2)$ & 160 & 2,724 & & & \\
\hline 1977 & 2,039 & 81 & 74.6 & 2,734 & 9.8 & 153 & 2,645 & & & \\
\hline 1978 & 1,971 & 81 & 81.2 & 2,428 & 2.1 & 139 & 2,487 & & & \\
\hline 1979 & 1,816 & 72 & 75.4 & 2,410 & 17.5 & 140 & 2,497 & & & \\
\hline 1980 & 1,861 & 83 & 78.8 & 2,361 & 17.3 & 141 & 2,504 & & & \\
\hline 1981 & 1,997 & 74 & 77.4 & 2,579 & 23.3 & 147 & 2,575 & & & \\
\hline 1982 & 1,913 & 74 & 73.7 & 2,596 & 17.4 & 148 & 2,579 & & & \\
\hline 1983 & 1,906 & 76 & 75.1 & 2,535 & 17.1 & 148 & 2,584 & & & \\
\hline 1984 & 1,741 & $\pi$ & 76.0 & 2,291 & $(9.3)$ & 125 & 2,327 & & & \\
\hline 1985 & 1,598 & 63 & 67.0 & 2,386 & $(0.3)$ & 113 & 2,187 & & & \\
\hline 1986 & 1,393 & 71 & 67.9 & 2,052 & 7.0 & 108 & 2,135 & & & \\
\hline$\cdots-$ & --- & -- & $-\cdot$ & $\cdots$ & $\cdots$ & $\cdots$ & --- & $-\ldots$ & $=$ & -- \\
\hline 1987 & 1,612 & 91 & 83.6 & 1,688 & 3.6 & 100 & 2,044 & & & \\
\hline 1988 & 1,436 & 86 & 87.9 & 1,634 & 4.5 & 96 & 1,973 & 6,598 & 7,529 & 7,770 \\
\hline 1989 & & & & & 7.5 & 92 & 1,944 & 6,502 & 7,419 & 7,656 \\
\hline 1990 & & & & & 8.9 & 91 & 1,934 & 6,467 & 7,380 & 7,616 \\
\hline 1991 & & & & & 10.3 & 91 & 1,940 & 6,488 & 7,403 & 7,640 \\
\hline 1992 & & & & & 7.5 & 89 & 1,915 & 6,403 & 7,307 & 7,540 \\
\hline
\end{tabular}

Capacity-Capital Stock Regression: Code 0 RSO 0.810 Coefficients: Const 906.5 stock 11.3

Ave. Capacity/stock $(74-86): 17.5$ Ave. Weekly Hours (High) 40.0 shift Factor 3.96 Ave Weekly Hours (Low) 40.0 Shift Factor 3.94 Note: Dutput, Investment, and Capital Stock extrapolated for 1987 and 1988.

\section{Women's footwear, except athletic}

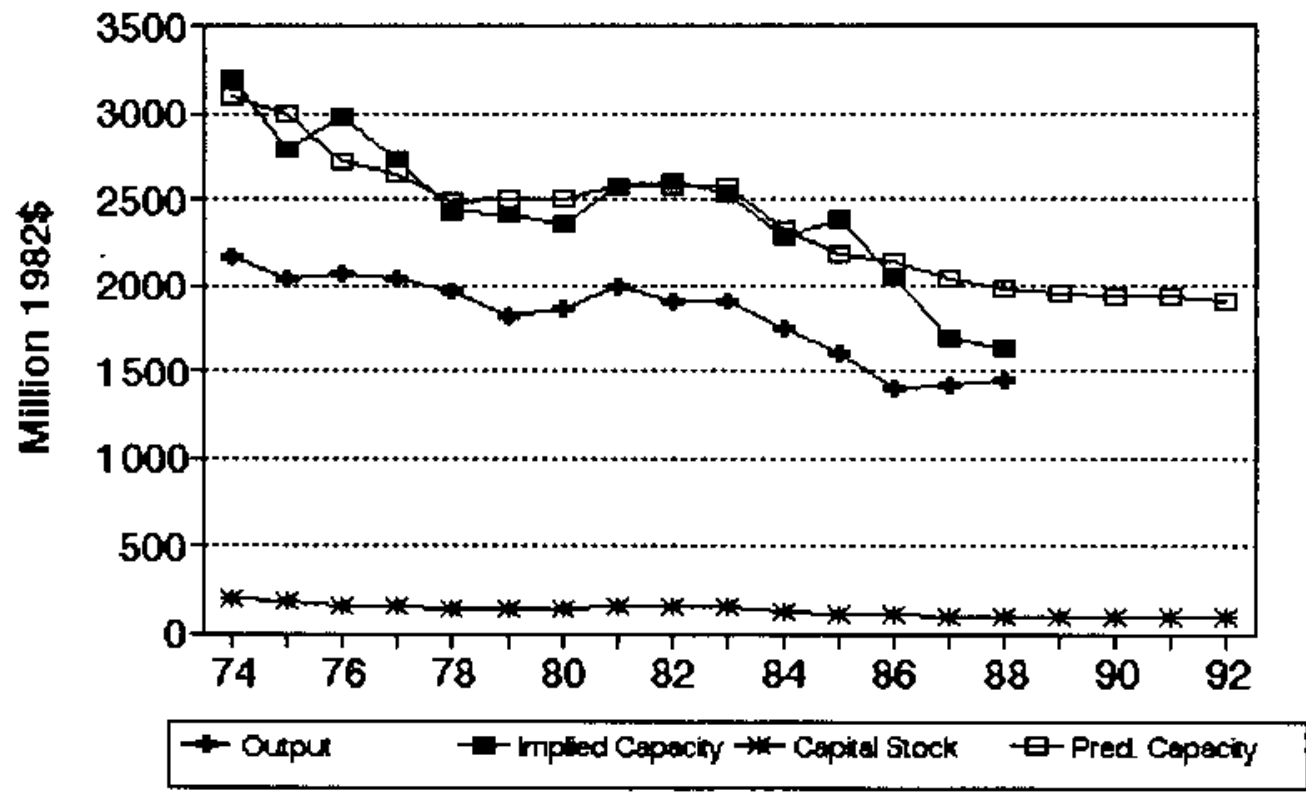


No. 222 SIC 3149 Footwear, except rubber, n.e.c.

MILLIONS OF 1982 DOLLARS (EXCL. CU RATES)

$\begin{array}{lccccccc} & \text { Q4 } & \text { Ann. Implied Gross } & \text { Net } & \text { Pred. } & -\ldots & -\ldots \\ \text { Gross } & \text { CU } & \text { CU } & \text { Prac. } & \text { In- } & \text { Cap. } & \text { Prac. } & \text { Emergency Capacity } \\ \text { Output } & \text { Rate } & \text { Rate } & \text { Capac. } & \text { vest. } & \text { Stk. } & \text { Capac. } & 3 \text { mon. } 6 \text { mon. }>6 \text { mon. }\end{array}$

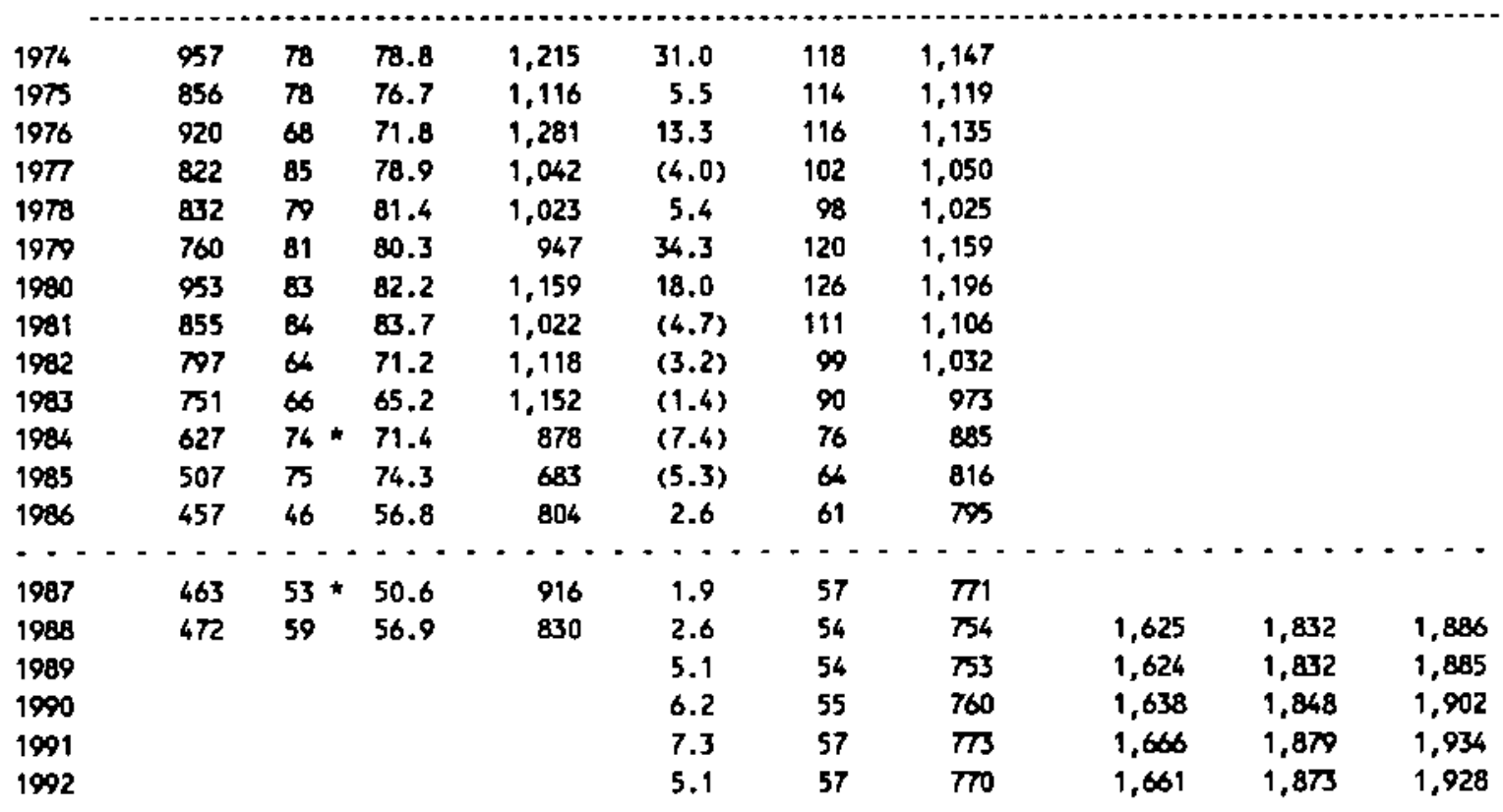

Capecity-Capital Stock Regression: Code 1 RSQ 0.600

Coefficients: Const 420.0 stock 6.2

Ave, Weekly Hours (High) 62.9 Shift Factor 2.50 Ave Veekly Hours (Low) 62.9 Shift Factor 2.50

* Imputed Hote: Output, Imvestment, and Capital stock extropolated for 1987 and 1988.

\section{Footwear, except rubber, n.e.c.}

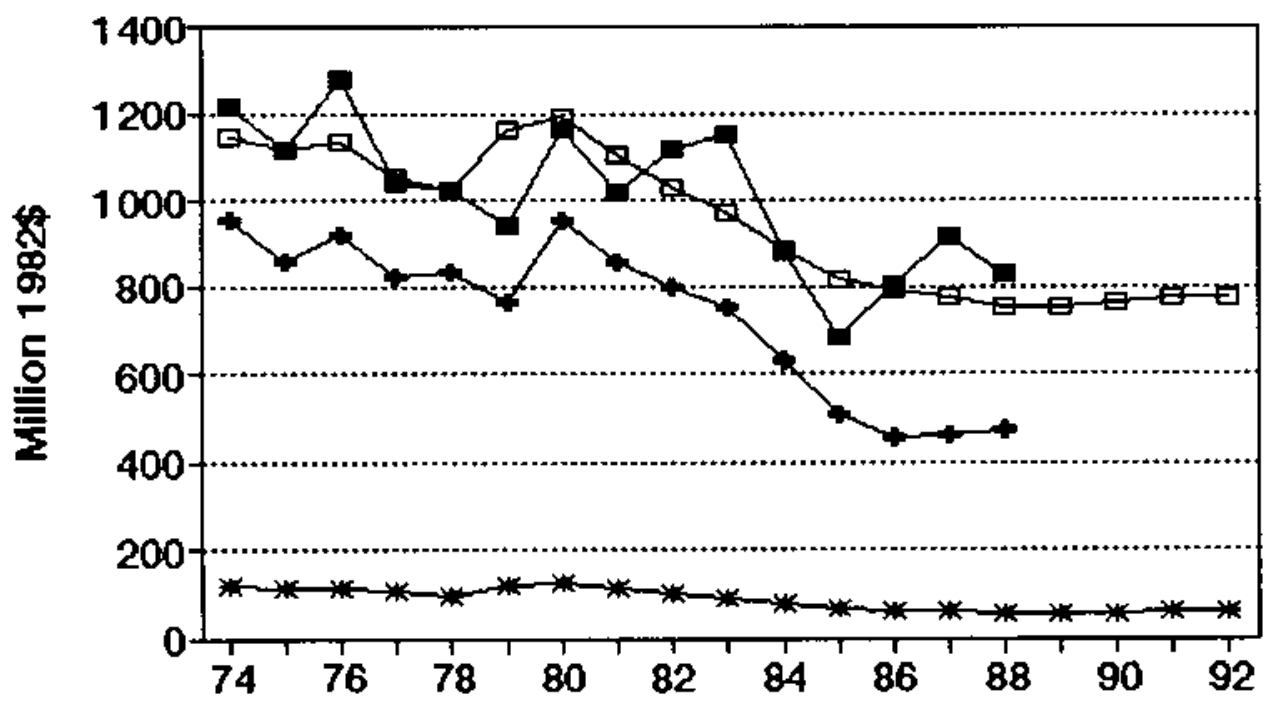

- Oupu t-Implied Cepecty $\rightarrow$ - Capired Srock E-Pred. Cepeciry 


\section{ro. 223 sIC 3151 Leather gloves and mittens}

MILLIONS OF 1982 DOLLARS (EXCL. CU RATES)

$\begin{array}{lccccccc} & \text { Q6 } & \text { Ann. Implied Gross } & \text { Net } & \text { Pred. } & \ldots & \ldots \\ \text { Gross } & \text { CU } & \text { CU } & \text { Prac. } & \text { In- } & \text { Cap. } & \text { Prac. } & \text { Emergency Capacity } \\ \text { Output } & \text { Rate } & \text { Rate } & \text { Capac. } & \text { vest. } & \text { 5tk. } & \text { Capac. } & 3 \text { mon. } 6 \text { mon. }>6 \text { mon. }\end{array}$

\begin{tabular}{|c|c|c|c|c|c|c|c|c|c|c|}
\hline 1974 & 395 & 45 & 46.5 & 848 & 7.5 & 18 & 357 & & & \\
\hline 1975 & 295 & 67 & 55.7 & 529 & 2.3 & 19 & 383 & & & \\
\hline 1976 & 294 & 64 & 65.4 & 450 & 0.5 & 18 & 362 & & & \\
\hline 1977 & 296 & 69 & 67.9 & 436 & 4.4 & 21 & 434 & & & \\
\hline 1978 & 243 & 68 & 68.7 & 354 & 3.3 & 23 & 482 & & & \\
\hline 1979 & 222 & 80 & 75.3 & 294 & $(3.4)$ & 18 & 376 & & & \\
\hline 1980 & 194 & 81 & 80.3 & 242 & 0.6 & 18 & 364 & & & \\
\hline 1981 & 195 & $83 *$ & 82.7 & 235 & 0.5 & 17 & 348 & & & \\
\hline 1982 & 172 & $78 *$ & 79.5 & 217 & 0.7 & 17 & 334 & & & \\
\hline 1983 & 171 & $83 *$ & 81.0 & 211 & 0.2 & 15 & 309 & & & \\
\hline 1984 & 185 & 88 & 87.5 & 212 & $(0.2)$ & 14 & 274 & & & \\
\hline 1985 & 187 & 82 & 83.1 & 225 & 0.0 & 13 & 249 & & & \\
\hline 1986 & 192 & 73 & 76.1 & 252 & 0.6 & 12 & 233 & & & \\
\hline$\cdots$ & $\cdots \cdots$ & - & $\cdots-$ & $-\cdots$ & --- & $\cdots$ & $=-$ & -- & $\cdots$ & $-\cdot$ \\
\hline 1987 & 196 & 78 & 76.4 & 256 & $(0.5)$ & 10 & 193 & & & \\
\hline 1988 & 201 & 83 & 81.2 & 248 & $(0.2)$ & 9 & 165 & 568 & 634 & 651 \\
\hline 1089 & & & & & 0.9 & 9 & 166 & 571 & 637 & 654 \\
\hline 1990 & & & & & 1.4 & 10 & 178 & 613 & 624 & 702 \\
\hline 1991 & & & & & 1.9 & 11 & 201 & 690 & 771 & 792 \\
\hline 1992 & & & & & 0.9 & 11 & 198 & 681 & 760 & 781 \\
\hline
\end{tabular}

Capacity-Capitel Stock Regression: Code 0 RSO 0.140 Coefficients: Const -46.7 stock 23.1

Ave. Capacity/Stock (74-85): 20.3 Ave. Weekly Hours (High) 40.0 shift Factor 3.94 Ave Weekly Hours (Low) 40.0 Shift factor 3.94 * Imputed Note: Output, Investment, and Capital Stock extrapolated for 1987 and 1988.

\section{Leather gloves and mittens}

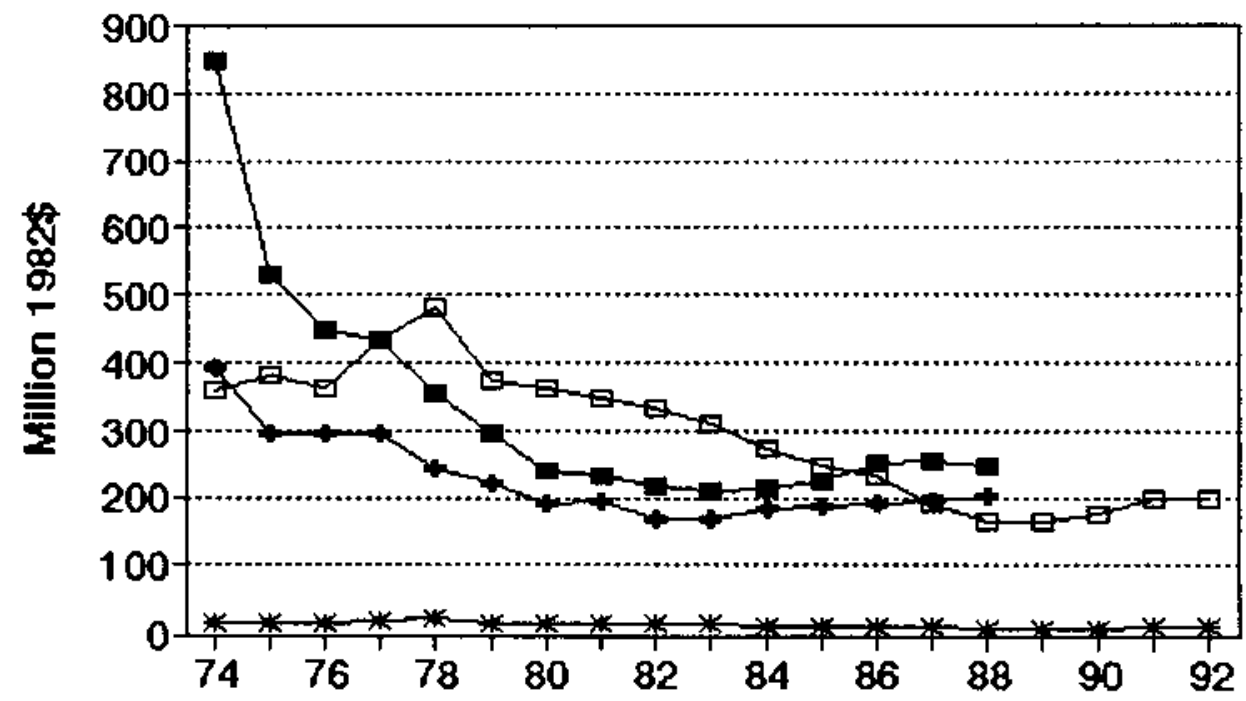

\begin{tabular}{|c|c|c|}
\hline$\rightarrow$ Ourpis & $\rightarrow-$ Impied Cepeciny $\rightarrow$ - Copkal Stock & E-Pred. Cepecity \\
\hline
\end{tabular}


No. 224 SIC 3161 Luggage

MILLIONS OF 1982 DOLLARS (EXCL. CU RATES)

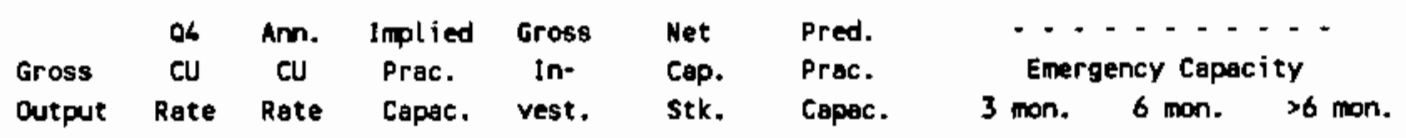

\begin{tabular}{|c|c|c|c|c|c|c|c|c|c|c|}
\hline 1976 & 803 & 87 & 87.0 & 923 & 9.4 & 98 & 1,296 & & & \\
\hline 1973 & 739 & 86 & 86.4 & 855 & 5.3 & 95 & 1,224 & & & \\
\hline 1976 & 849 & $B 7$ & 86.6 & 980 & 5.0 & 92 & 1,137 & & & \\
\hline 1977 & 935 & 73 & 78.3 & 1,194 & 7.3 & 91 & 1,113 & & & \\
\hline 1978 & 956 & 82 & 78.6 & 1,215 & 15.5 & 97 & 1,283 & & & \\
\hline 1979 & 1,095 & $\pi$ & 77.6 & 1,411 & 22.8 & 110 & 1,612 & & & \\
\hline 1980 & 1,153 & 60 & 65.6 & 1,756 & 15.1 & 115 & 1,745 & & & \\
\hline 1981 & 975 & 54 & 56.3 & 1,734 & 2.4 & 108 & 1,575 & & & \\
\hline 1982 & 770 & 59 & 57.1 & 1,347 & (1.1) & $\infty$ & 1,330 & & & \\
\hline 1983 & 771 & 45 & 50.3 & 1,534 & 9.0 & 100 & 1,346 & & & \\
\hline 1984 & 782 & $43 *$ & 43.7 & 1,787 & 11.5 & 102 & 1,415 & & & \\
\hline 1985 & 622 & 51 & 48.0 & 1,297 & $(4.0)$ & 90 & 1,094 & & & \\
\hline 1986 & 599 & 52 & 51.6 & 1,161 & 5.5 & 88 & 1,025 & & & \\
\hline - : & $\begin{array}{c}\cdots 14 \\
614\end{array}$ & $\begin{array}{l}-\cdots \\
51\end{array}$ & $\begin{array}{l}-\cdots \\
51.4\end{array}$ & $\begin{array}{l}-\cdots \\
1,196\end{array}$ & $\begin{array}{l}-. \\
6.0\end{array}$ & - & $\begin{array}{c}\cdots \\
972\end{array}$ & -- & $-\cdots$ & $-\cdots$ \\
\hline 1988 & 635 & 77 * & 67.3 & 942 & 6.3 & 84 & 933 & 1,892 & 2,119 & 2,178 \\
\hline 1989 & & & & & 7.5 & 84 & 928 & 1,882 & 2,107 & 2,166 \\
\hline 1990 & & & & & 8.0 & 84 & 937 & 1,900 & 2,128 & 2,187 \\
\hline 1991 & & & & & 8.5 & 85 & 959 & 1,944 & 2,177 & 2,237 \\
\hline 1992 & & & & & 7.5 & 85 & 951 & 1,929 & 2,160 & 2,220 \\
\hline
\end{tabular}

Capacity-Capital stock Regression: Code 1 RSO 0.480

Coefficients: Const -1302.2 Stock 26.6

Ave. Capacity/Stock $(74-86): 13.4$ Ave. Weekly Hours (High) 67.5 Shift Factor 2.33 Ave Heekty Hours (LOW) 65.5 Shift Factor 2.40 - Imputed Note: Output, Imvestment, and Capital stock extrapolated for 1987 and 1988.

\section{Luggage}

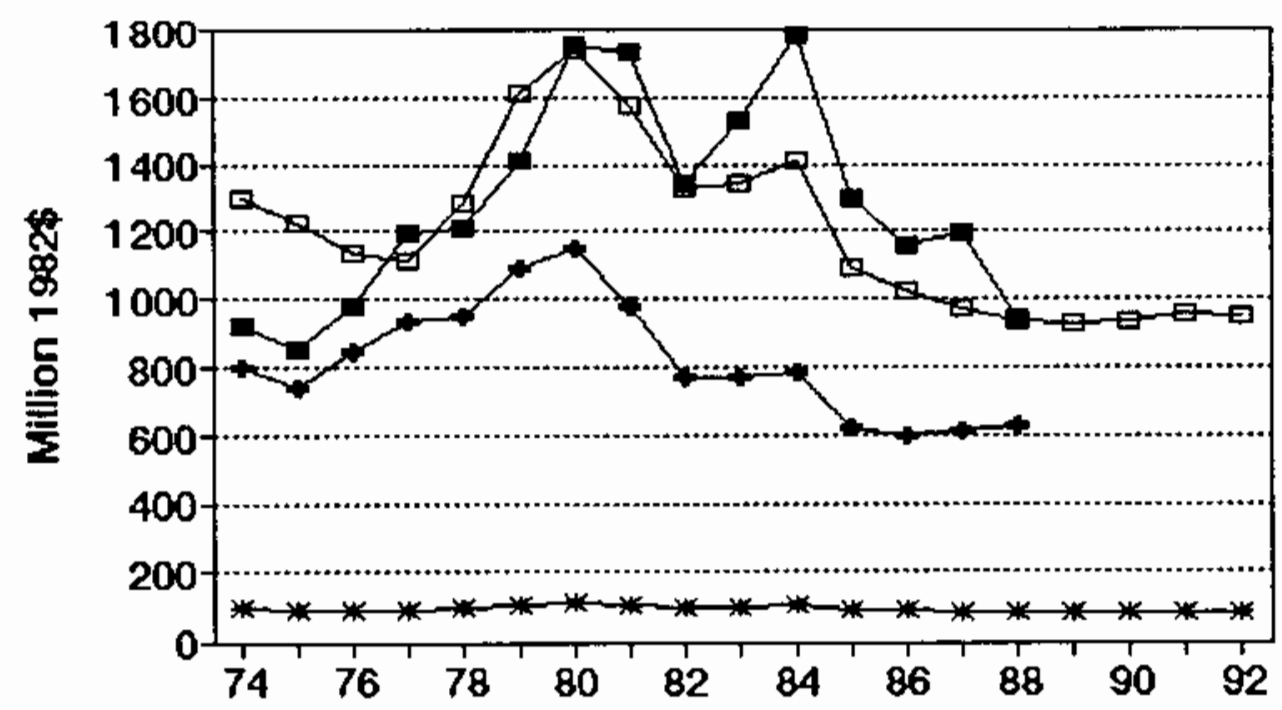

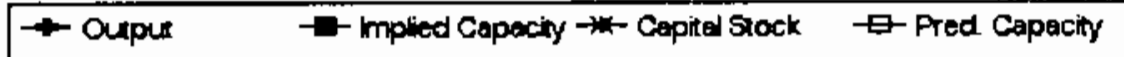




\section{No. 225 src 3171 Women' ' handbags and purses}

MILLIONS OF 1982 DOLLARS (EXCL. CU RATES)

$\begin{array}{lccccccc} & a 4 & \text { Anr. } & \text { Implied Gross } & \text { Met } & \text { Pred. } & \ldots \ldots \ldots \\ \text { Gross } & \text { CU } & \text { CU } & \text { Prac. } & \text { In } & \text { Cap. } & \text { Prac. } & \text { Emergency Capacity } \\ \text { Output } & \text { Rete } & \text { Rate } & \text { Capac. } & \text { vest. } & \text { Stk. } & \text { Capac. } & 3 \text { mon. } 6 \text { mon. }>6 \text { mon. }\end{array}$

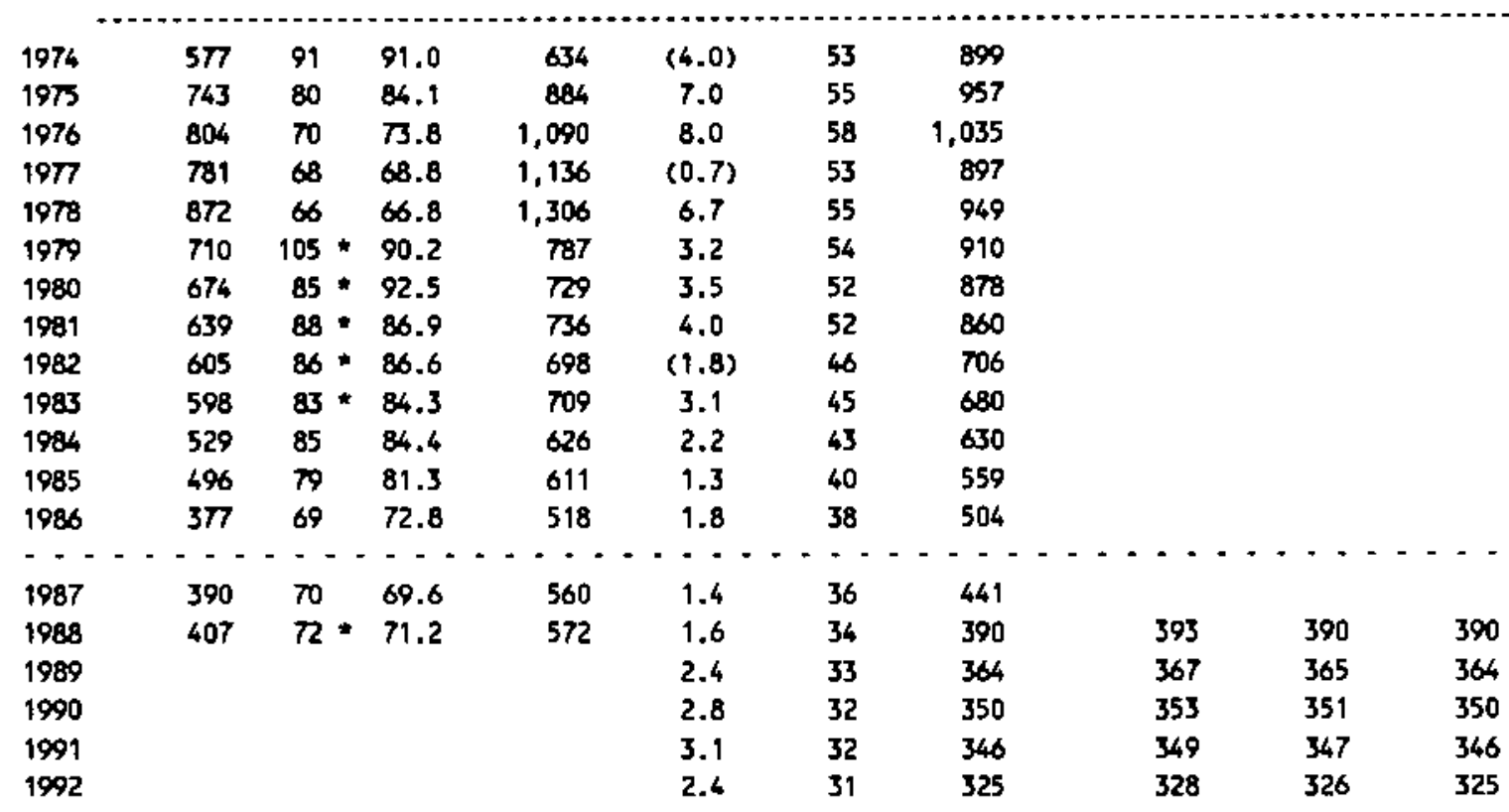

Capacity-Capital stock Regression: Code 1 RSO 0.510

Coefficients: Const -491.8 Stock 26.2

Ave. Capecity/stock $(74-86): 16.2$ Ave. Weekly Hours (High) 1985.0 Shift Factor 1.00 Ave Ueekly Hours (Low) 1985.0 Shift Factor 1.0 - Imputed Note: Output, Investment, and Copital Stock extrapolated for 1987 and 1988.

\section{Women's handbags and purses}

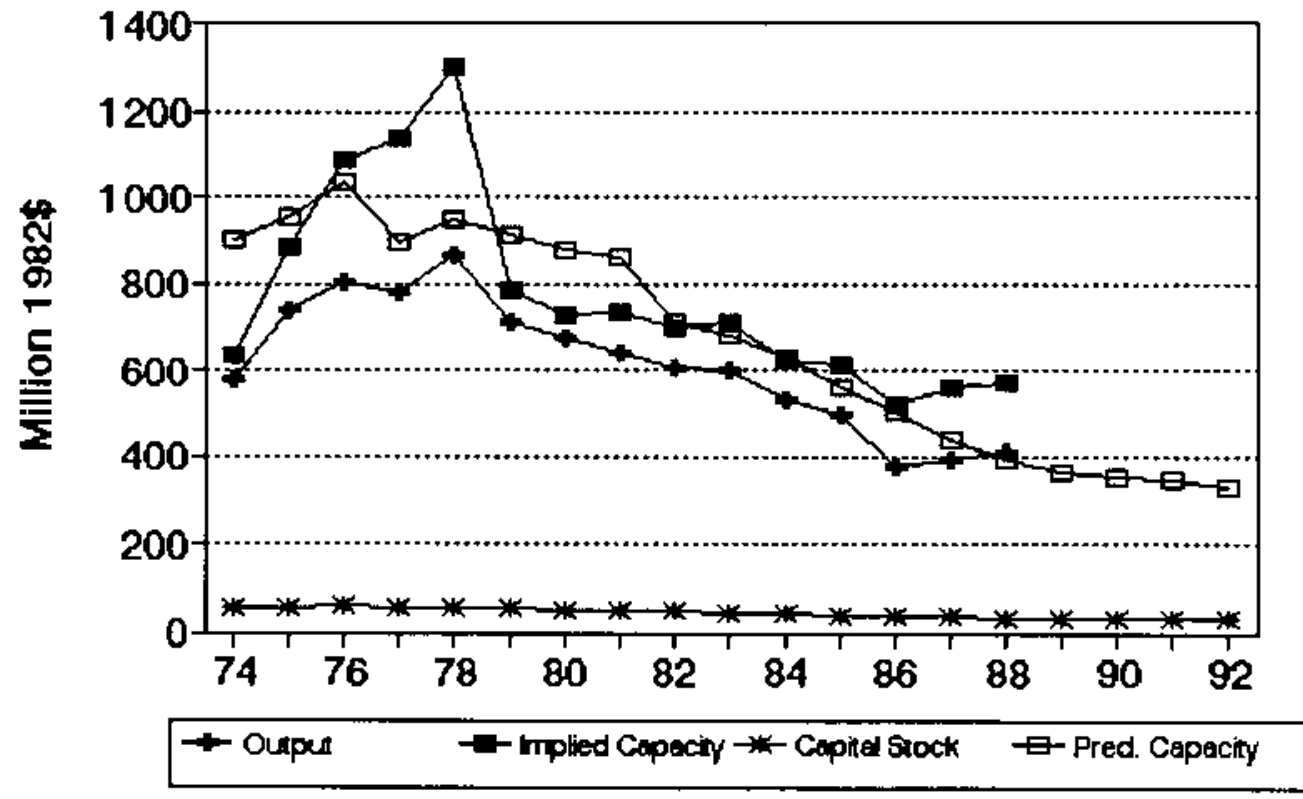




\section{No. 226 sic 3172 Personal leather goods}

MILLIONS OF 1982 DOLLARS (EXCL. CU RATES)

$\begin{array}{lccccccc} & \text { Q4 } & \text { Am. Implied Gross } & \text { Net } & \text { Pred. } & \ldots \ldots \ldots \\ \text { Gross } & \text { CU } & \text { CU } & \text { Prac. } & \text { In- } & \text { Cap. } & \text { Prac. } & \text { Emergency Capacity } \\ \text { Output } & \text { Rate } & \text { Rate } & \text { Capac. } & \text { vest. } & \text { Stk. } & \text { Capac. } & 3 \text { mon. } 6 \text { mon. }>6 \text { mon. }\end{array}$

\begin{tabular}{|c|c|c|c|c|c|c|c|c|c|c|}
\hline 1974 & 471 & 95 & 95.8 & 492 & $(2.2)$ & 42 & 699 & & & \\
\hline 1975 & 489 & 73 & 80.1 & 611 & 3.9 & 41 & 700 & & & \\
\hline 1976 & 504 & 69 & 70.6 & 714 & 5.6 & 42 & 699 & & & \\
\hline 1977 & 546 & 80 & 76.1 & 717 & 4.4 & 42 & 699 & & & \\
\hline 1978 & 584 & 82 & 81.4 & 718 & 11.8 & 49 & 694 & & & \\
\hline 1979 & 520 & 90 & 87.0 & 598 & $(13.3)$ & 32 & 706 & & & \\
\hline 1980 & 447 & 58 & 70.0 & 638 & (1.6) & 28 & 709 & & & \\
\hline 1981 & 442 & $53 *$ & 54.6 & 809 & 5.8 & 31 & 707 & & & \\
\hline 1982 & 428 & 45 & 47.7 & 897 & 5.0 & 33 & 706 & & & \\
\hline 1983 & 436 & $41 *$ & 42.8 & 1,020 & 2.5 & 32 & 707 & & & \\
\hline 1984 & 367 & $39 *$ & 40.2 & 913 & 2.1 & 31 & 707 & & & \\
\hline 1985 & 338 & 72 & 59.5 & 567 & $(0.5)$ & 27 & 710 & & & \\
\hline 1986 & 320 & 68 & 69.4 & 460 & 1.5 & 26 & 711 & & & \\
\hline$-\cdots$ & $-\cdots$ & - - - & -- & $--\cdot$ & --- & - & $-\cdot$ & $-\cdots$ & $\cdots$ & $\cdots$ \\
\hline 1987 & 327 & 70 & 69.3 & 472 & $(0.6)$ & 23 & 713 & & & \\
\hline 1988 & 338 & 57 & 61.9 & 546 & $(0 . t)$ & 20 & 715 & 1,793 & 2,055 & 2,123 \\
\hline 1989 & & & & & 1.5 & 20 & 716 & 1,794 & 2,057 & 2,125 \\
\hline 1990 & & & & & 2.2 & 20 & 716 & 1,794 & 2,057 & 2,125 \\
\hline 1991 & & & & & 2.9 & 21 & 715 & 1,793 & 2,055 & 2,123 \\
\hline 1992 & & & & & 1.5 & 20 & 715 & 1,794 & 2,056 & 2,124 \\
\hline
\end{tabular}

Capacity-Capital Stock Regression: Code 1 RSa 0.000 Coefficients: Const 730.6 stock -0.8

Ave. Capacity/stock $(74-86): 20.1$ Ave. Weekly Hours (High) 53.0 Shift Factor 2.97 Ave Yeekly Hours (Low) 48.5 Shift Factor 3.25 - Imputed Note: Output, Investment, and Capital stock extropolated for 1987 and 1988.

\section{Personal leather goods}

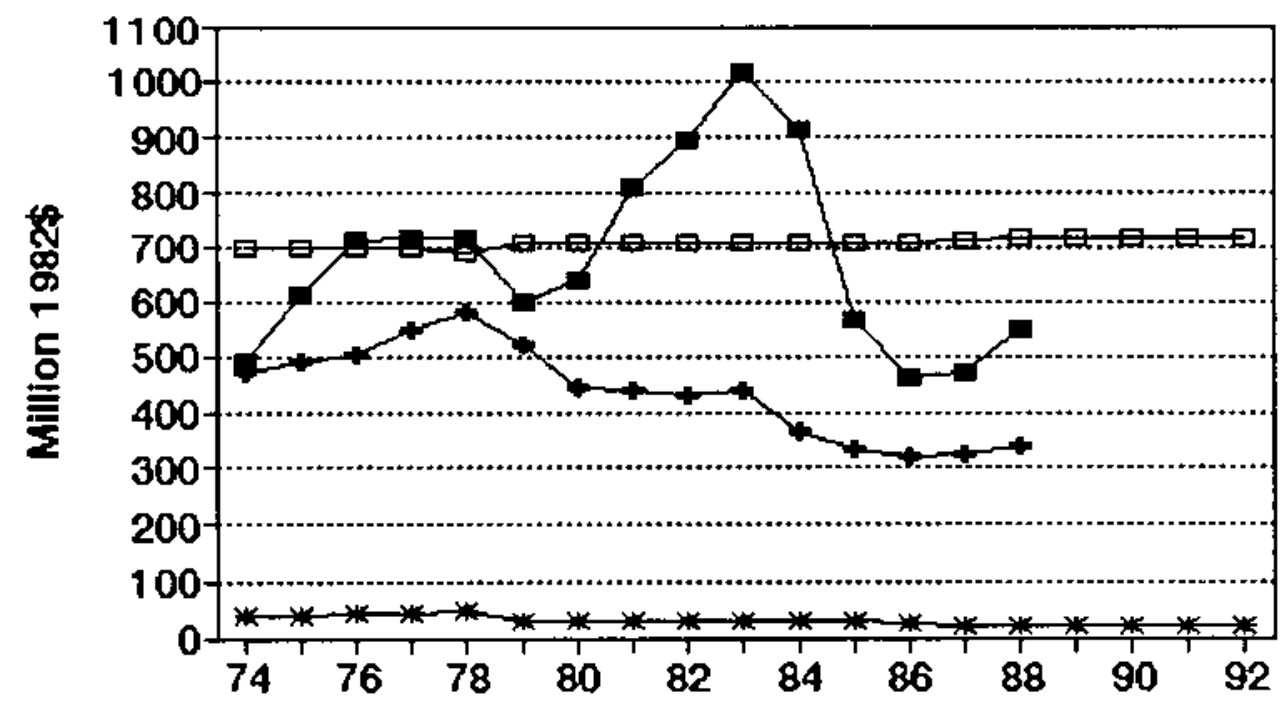

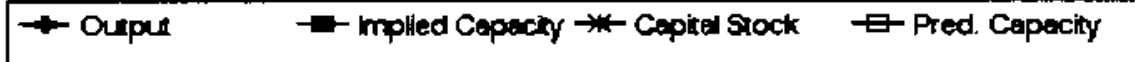


No. 227 sIC 3199 Leather goods, n.e.c.

MILLIOWS OF 1982 DOLLARS (EXCL. CU RATES)

$\begin{array}{lccccccc} & Q 4 & \text { Ann. Implied Gross } & \text { Net } & \text { Pred. } & \ldots \ldots \ldots \\ \text { Gross } & \text { CU } & \text { CU } & \text { Prac. } & \text { In- } & \text { Cap. } & \text { Prac. } & \text { Emergency Capacity } \\ \text { Output Rate } & \text { Rate } & \text { Capac. } & \text { vest. } & \text { Stk. } & \text { Capac. } & 3 \text { mon. } 6 \text { mon. }>6 \text { mon. }\end{array}$

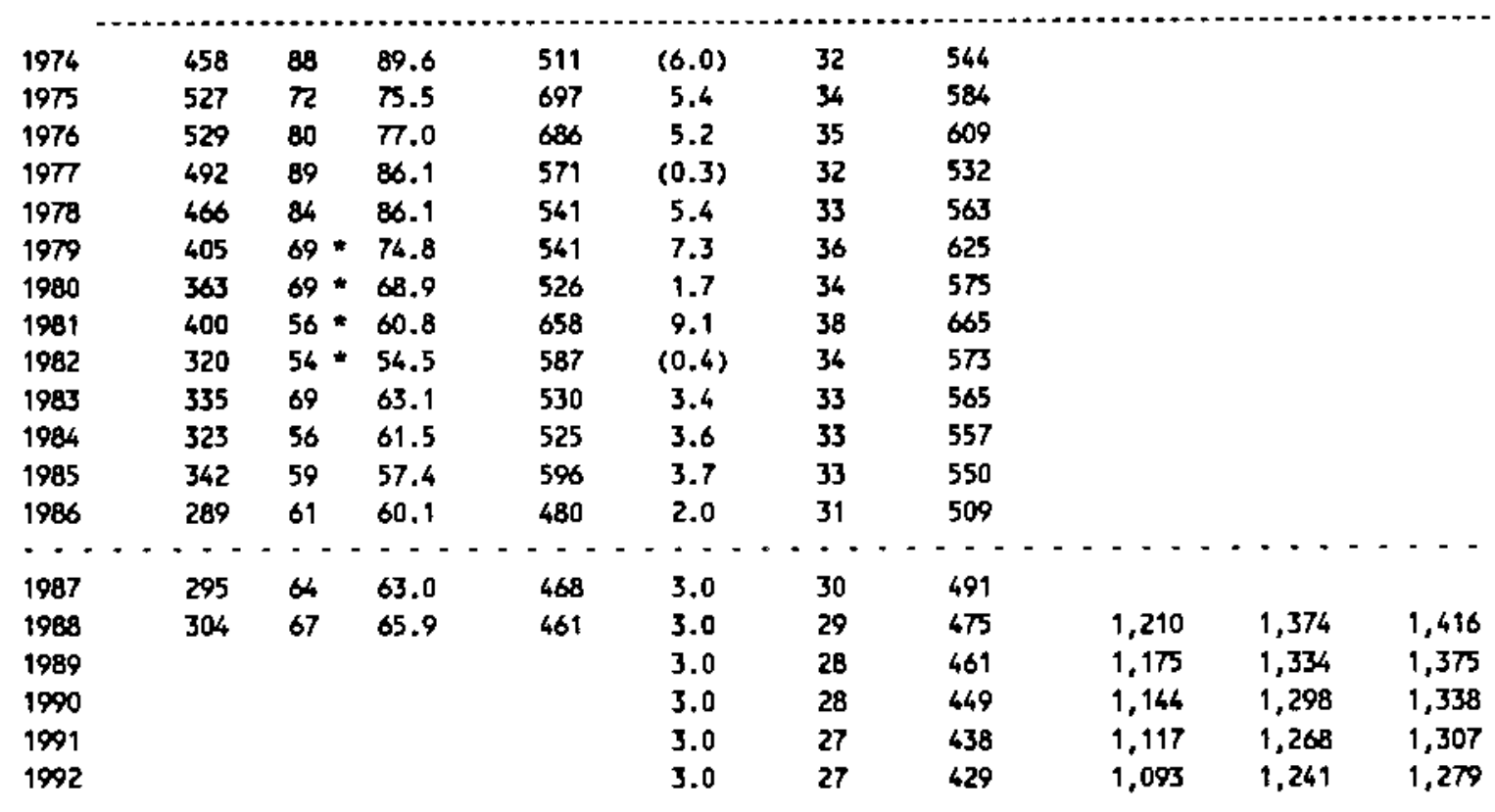

Capacity-Capital Stock Regression: Code 0 RSQ 0.350

Coefficients: Const -126.1 stock 20.8

Ave. Capacity/Stock (74-86): 17.1

Ave. Weekly Hours (High) 52.8 Shift Factor 2.98 Ave Weekly Hours (Low 48.7 Shift Factor 3.23

* Imputed Note: Output, Imvestment, and Capital stock extrapolated for 1987 and 1988.

\section{Leather goods, n.e.c.}

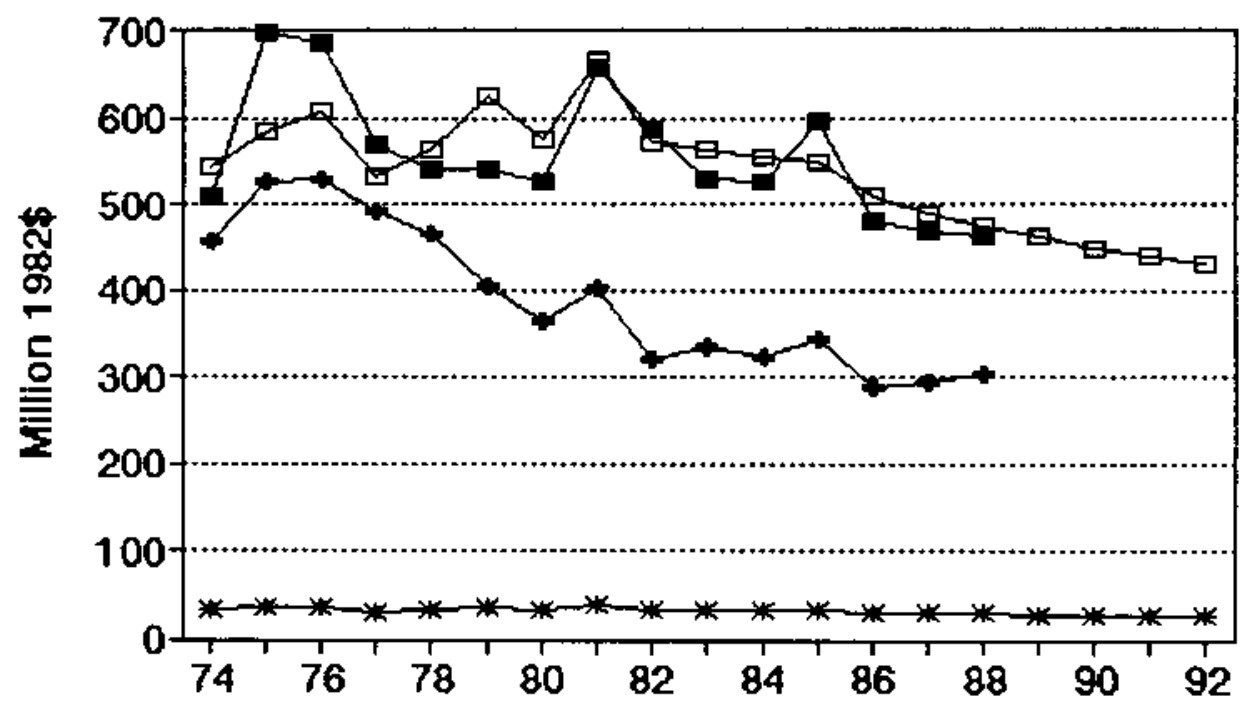

$\rightarrow$ Outpur $\quad-$ Implied Cepecity - Cepked Stock $\quad \square$ - Pred. Cepacty 
No. 228 SIC 3211 Flat glasa

MILLIONS OF 1982 DOLLARS (EXCL. CU RATES)

\begin{tabular}{|c|c|c|c|c|c|c|c|}
\hline & 04 & An. & $\begin{array}{l}\text { Implied } \\
\text { Prac. }\end{array}$ & $\begin{array}{l}\text { Gross } \\
\text { In- }\end{array}$ & $\begin{array}{l}\text { Net } \\
\text { Cap. }\end{array}$ & $\begin{array}{l}\text { Pred. } \\
\text { Prac. }\end{array}$ & Emergency Capocity \\
\hline & Rate & Rate & CaDAC & vest & & Сарас. & \\
\hline
\end{tabular}

\begin{tabular}{|c|c|c|c|c|c|c|c|c|c|c|}
\hline 1974 & 1,600 & 57 & 59.6 & 2,683 & 320.6 & 1.778 & 2,671 & & & \\
\hline 1975 & 1,620 & 71 & 62.1 & 2,600 & 295.8 & 1,919 & 2,651 & & & \\
\hline 1976 & 2,005 & 72 & 72.6 & 2,763 & $(27.4)$ & 1,742 & 2,676 & & & \\
\hline 1977 & 2,203 & 88 & 82.5 & 2,670 & 184.7 & 1,769 & 2,673 & & & \\
\hline 1978 & 2,302 & 86 & 85.9 & 2,679 & 161.7 & 1,768 & 2,673 & & & \\
\hline 1979 & 1,996 & 74 & 79.0 & 2,528 & 97.5 & 1,713 & 2,680 & & & \\
\hline 1980 & 1,752 & 70 & 69.4 & 2,523 & 182.0 & 1,741 & 2,676 & & & \\
\hline 1981 & 1.748 & 54 & 61.3 & 2,856 & 98.9 & 1,683 & 2,685 & & & \\
\hline 1982 & 1,672 & 61 & 58.2 & 2,870 & 96.7 & 1,621 & 2,693 & & & \\
\hline 1983 & 1,890 & B1 & 73.0 & 2,587 & 134.7 & 1,589 & 2,698 & & & \\
\hline 1984 & 2,000 & 81 & 82.7 & 2,429 & 45.7 & 1,472 & 2,714 & & & \\
\hline 1985 & 2,197 & $\pi$ & 7.3 & 2,844 & 265.0 & 1,573 & 2,700 & & & \\
\hline 1986 & 2,316 & 85 & 81.0 & 2,861 & 89.4 & $1,50 t$ & 2,710 & & & \\
\hline 1987 & 2,379 & $-\cdot$ & 86.3 & 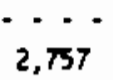 & $\begin{array}{l}\cdots \\
125.8\end{array}$ & 1,468 & 2,715 & & $\cdots$ & -- \\
\hline 1988 & 2,462 & 79 & 82.4 & 2,987 & 150.5 & 1,463 & 2,716 & 2,715 & 2,761 & 2,773 \\
\hline 1989 & & & & & 157.1 & 1,466 & 2,715 & 2,715 & 2,760 & 2,772 \\
\hline 1990 & & & & & 161.0 & 1,471 & 2,714 & 2,716 & 2,760 & 2,771 \\
\hline 1991 & & & & & 197.5 & 1,513 & 2,708 & 2,708 & 2,754 & 2,765 \\
\hline 1992 & & & & & 220.6 & 1,574 & 2,700 & 2,700 & 2,745 & $2, \pi 57$ \\
\hline
\end{tabular}

Capacity-Capital Stock Regression: Code 2 RSO 0.010

Coefficients: Const 2921.6 stock -0.1

Ave. Capecity/Stock $(74-86): 1.6$ Ave. Weekly Hours (High) 164.4 Shift factor 1.02 Ave Ueekly Hours (Low) 158.6 Shift factor 1.06 Note: Output, Investment, and Capital Stock extrapolated for 1987 and 1988.

\section{Flat glass}

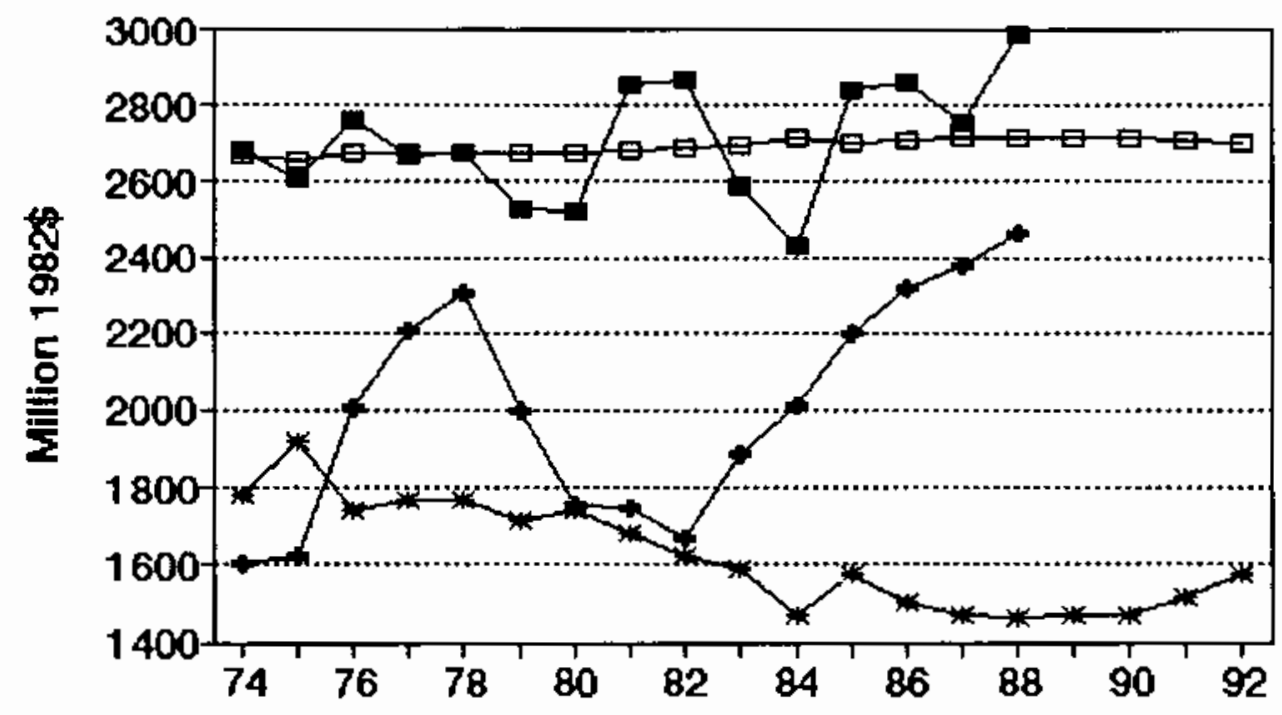

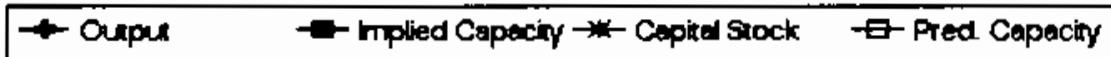




\section{No. 229 SIC 3221 Glass containers}

\begin{tabular}{|c|c|c|c|c|c|c|c|c|c|}
\hline \multirow{3}{*}{$\begin{array}{l}\text { Gross } \\
\text { Output }\end{array}$} & \multirow{3}{*}{$\begin{array}{l}\text { Q6 } \\
\text { CU } \\
\text { Rate }\end{array}$} & \multicolumn{3}{|c|}{ MILLIONS OF 1982 DOLLARS } & \multicolumn{2}{|c|}{ (EXCL. CU RATES) } & \multirow{2}{*}{\multicolumn{3}{|c|}{$\begin{array}{c}-\cdots \\
\text { Emergency Capacity }\end{array}$}} \\
\hline & & $\begin{array}{l}\text { Ann. } \\
\text { Cu }\end{array}$ & $\begin{array}{l}\text { Inplied } \\
\text { Prac. }\end{array}$ & $\begin{array}{l}\text { Gross } \\
\text { In- }\end{array}$ & $\begin{array}{l}\text { Net } \\
\text { Cap. }\end{array}$ & $\begin{array}{l}\text { Pred. } \\
\text { Prac. }\end{array}$ & & & \\
\hline & & Rate & Capac. & vest. & stk. & Capec. & 3 mon. & 6 mon. & $>6$ mon. \\
\hline 5,631 & 81 & 83.1 & $6, \pi / 5$ & 168.7 & 2,131 & 6,971 & & & \\
\hline 6,053 & 86 & 81.9 & 7,391 & 309.9 & 2,273 & 6,881 & & & \\
\hline 6,054 & 85 & 85.7 & 7,068 & 318.7 & 2,410 & 6,795 & & & \\
\hline 6,011 & 89 & 87.2 & 6,897 & 268.8 & 2,486 & 6,747 & & & \\
\hline 5,998 & 86 & 87.3 & 6,870 & 296.2 & 2,57 & 6,689 & & & \\
\hline 5,729 & 85 & 85.5 & 6,703 & 283.0 & 2,645 & 6,646 & & & \\
\hline 5,467 & 86 & 84.7 & 6,457 & 238.5 & 2,662 & 6,636 & & & \\
\hline 5,301 & 81 & 84.1 & 6,308 & 207.7 & 2,643 & 6,648 & & & \\
\hline 5,349 & $\pi$ & 7.2 & 6,929 & 126.9 & 2,547 & 6,708 & & & \\
\hline 4,927 & 83 & 80.6 & 6,116 & 86.0 & 2,411 & 6,794 & & & \\
\hline 4,323 & 83 & 84.3 & 5,126 & (153.8) & 2,061 & 4,793 & & & \\
\hline 4,276 & 91 & 87.7 & 4,877 & 76.8 & 1,946 & 4,866 & & & \\
\hline 4,150 & 91 & 91.2 & 4,550 & 146.5 & 1,890 & 4,895 & & & \\
\hline 4,177 & 86 & $\dot{\theta 8}$ & 4,737 & $\begin{array}{l}-\cdot--- \\
152.7\end{array}$ & 1,861 & 4,919 & $---\cdot$ & $\cdots \cdot-$ & $-\cdots$ \\
\hline 4,214 & 94 & 90.9 & 4,634 & 178.6 & 1,853 & 4,924 & 4.793 & 4,897 & 4,924 \\
\hline & & & & 185.6 & 1,853 & 4,924 & 4,793 & 4,897 & 4,924 \\
\hline & & & & 189.6 & 1,857 & 4,922 & 4,791 & 4,895 & 4,922 \\
\hline & & & & 228.0 & 1,899 & 4,896 & 4.765 & 4,869 & 4,896 \\
\hline & & & & 252.2 & 1,961 & 4,856 & 4,727 & 4,830 & 4,856 \\
\hline
\end{tabular}

Capecity-Capital Stock Regression: Code -1 RSO 0.870

Coefficients: Const 8316.3 Stock -0.6 D(84-86) $-2222.1 \quad$ Ave. Capacity/Stock (74-86): 2.7 Ave. Weekly Hours (High) 168.0 Shift Factor 1.00 Ave Weekly Hours (Low) 168.0 Shift Factor 1.00 Hote: Output, Investment, and Capital Stock extrapolated for 1987 and 1988.

\section{Glass containers}

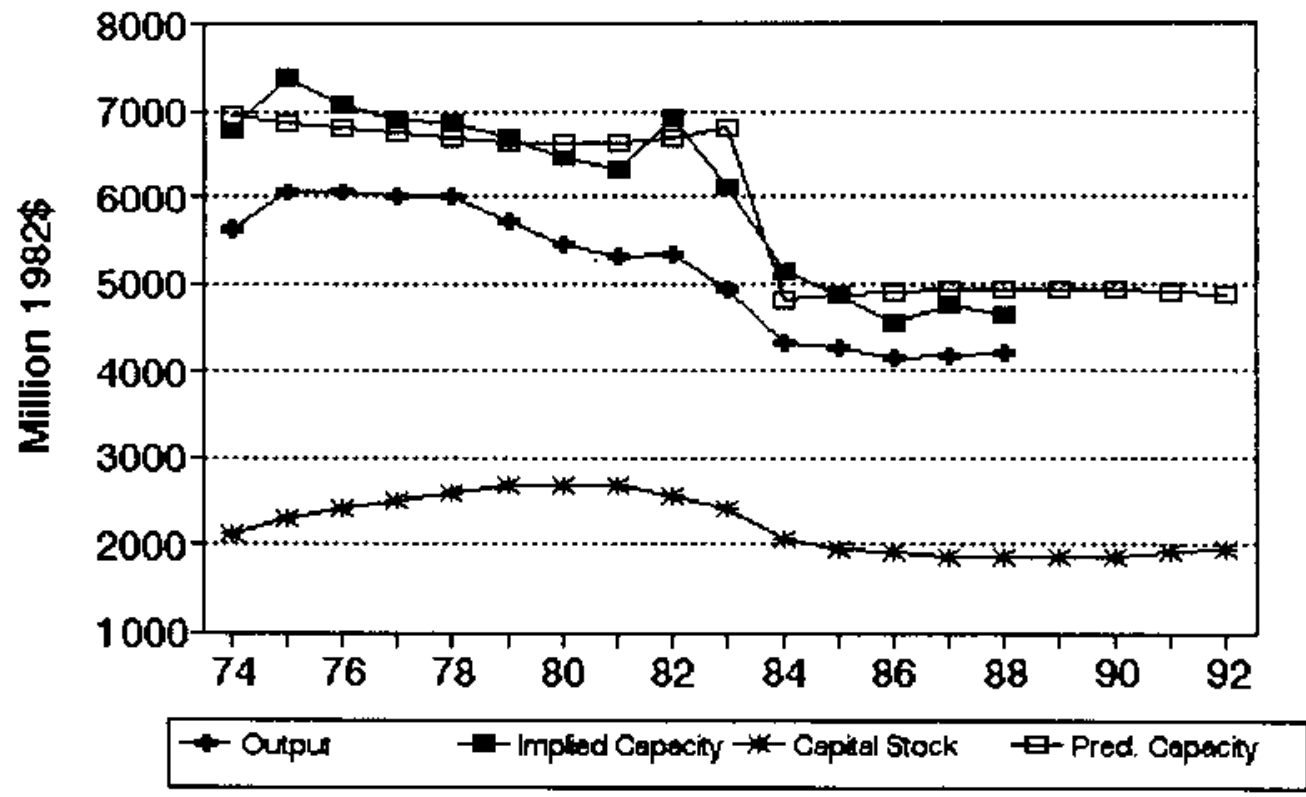


No. 230 sIC 3229 Pressed and blown glass, n.e.c.

MILLIONS OF 1982 DOLLARS (EXCL, CU RATES)

$\begin{array}{lccccccc} & \alpha 4 & \text { Am. } & \text { Implied } & \text { Gross } & \text { Net } & \text { Pred. } & -\ldots \\ \text { Gross } & \text { CU } & \text { CU } & \text { Prac. } & \text { In- } & \text { Cap. } & \text { Prac. } & \text { Emergency Capacity } \\ \text { Output Rate } & \text { Rate } & \text { Capac. } & \text { vest. } & \text { Stk. } & \text { Capac. } & 3 \text { mon. } 6 \text { mon. }>0 \text { mon. }\end{array}$

\begin{tabular}{|c|c|c|c|c|c|c|c|c|c|c|}
\hline 1974 & 3,488 & 87 & 90.1 & 3,871 & 224.5 & 1,621 & 3,942 & & & \\
\hline 1975 & 2,929 & 77 & 77.6 & 3,774 & 148.0 & 1,643 & 3,965 & & & \\
\hline 1976 & 3,267 & 73 & 74.9 & 4,363 & 196.7 & 1,704 & 4,026 & & & \\
\hline 1977 & 3,423 & 80 & 7.0 & 4,449 & 218.4 & 1,776 & 4,099 & & & \\
\hline 1978 & 3,573 & 79 & 79.6 & 4,489 & 197.8 & 1,824 & 4,148 & & & \\
\hline 1979 & 3,602 & 84 & 82.3 & 4,379 & 391.2 & 2,046 & 4,374 & & & \\
\hline 1980 & 3,533 & 74 & 76.5 & 4,621 & 205.1 & 2,077 & 4,403 & & & \\
\hline 1981 & 3,603 & 70 & 72.9 & 4,940 & 184.0 & 2,079 & 4,405 & & & \\
\hline 1982 & 2,707 & 64 & 66.2 & 4,090 & 99.7 & 2,002 & 4,327 & & & \\
\hline 1983 & 2,940 & $\pi$ & 71.5 & 4.110 & 96.6 & 1,922 & 4,247 & & & \\
\hline 1984 & 3,060 & 81 & 80.4 & 3,806 & 179.3 & 1,921 & 4,245 & & & \\
\hline 1985 & 2,943 & 78 & 78.7 & 3,740 & 200.2 & 1,939 & 4,263 & & & \\
\hline 1986 & 2,926 & 67 & 71.4 & 4,100 & 210.3 & 1,964 & 4,288 & & & \\
\hline $\begin{array}{l}-\cdot \cdot \\
1987\end{array}$ & 2,951 & " & $\begin{array}{l}--. \\
74.5\end{array}$ & 3,963 & $\begin{array}{l}\because 72.4 \\
178\end{array}$ & 1,951 & $\begin{array}{l}\cdots \\
4,273\end{array}$ & $\cdots$ & -- & $=-$ \\
\hline 1988 & 2,983 & 88 & 84.5 & 3,529 & 189.6 & 1,957 & 4,281 & 4,065 & 4,261 & 4,311 \\
\hline 1989 & & & & & 194.2 & 1,967 & 4,291 & 4,075 & 4,271 & 4,321 \\
\hline 1990 & & & & & 196.9 & 1,978 & 4,303 & 4,086 & 4,282 & 4,333 \\
\hline 1991 & & & & & 222.4 & 2,014 & 4,339 & 4,120 & 4,318 & 4,370 \\
\hline 1992 & & & & & 238.5 & 2,063 & 4,368 & 4,167 & 4,367 & 4,419 \\
\hline
\end{tabular}

Capacity-Capital Stock Regression: Code 1 RSO 0.190

Coefficients: Const 2305.6 stock 1.0

Ave. Capocity/Stock (76-86): 2.2 Ave. Weekly Hours (High) 166.8 Shift Factor t.01 Ave Heekly Hours (Low) 157.2 Shift Factor 1.07 Note: Output, Investment, and Capital Stock extrapolated for 1987 and 1988.

\section{Pressed and blown glass, n.e.c.}
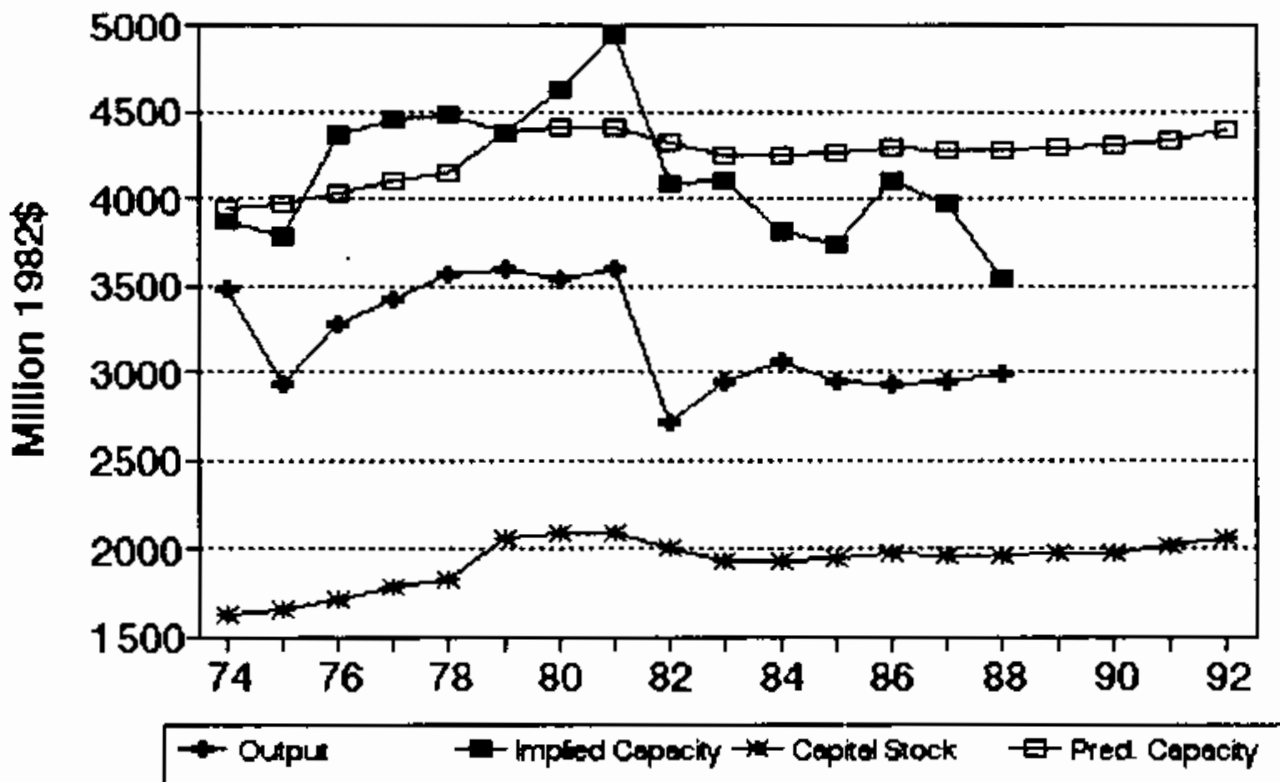


\section{No. 231 SIC 3232 Products of purchased glass}

MILLIONS DF 1982 DOLLARS (EXCL. CU RATES)

$\begin{array}{lccccccc} & Q 4 & \text { Ann. } & \text { Implied Gross } & \text { Net } & \text { Pred. } & \ldots \ldots \ldots \\ \text { Gross } & \text { CU } & \text { CU } & \text { Prac. } & \text { In- } & \text { Cap. } & \text { Prac. } & \text { Energency Capacity } \\ \text { Dutput } & \text { Rate } & \text { Rate } & \text { Capac. } & \text { vest. } & \text { Stk. } & \text { Capac. } & 3 \text { mon. } 6 \text { mon. }>6 \text { mon. }\end{array}$

\begin{tabular}{|c|c|c|c|c|c|c|c|c|c|c|}
\hline 1974 & 2,763 & 75 & 80.1 & 3,447 & 72.0 & 694 & 3,572 & & & \\
\hline 1975 & 2,167 & 73 & 68.4 & 3,169 & 22.2 & 663 & 3,361 & & & \\
\hline 1976 & 2,607 & 88 & 82.9 & 3,146 & 41.2 & 650 & 3,276 & & & \\
\hline 1977 & 2,654 & 85 & 85.3 & 3,111 & 76.5 & 670 & 3,412 & & & \\
\hline 1978 & 3,239 & 83 & 84.2 & 3,848 & 140.9 & 749 & 3,943 & & & \\
\hline 1979 & 3,100 & 63 & 70.6 & 4,392 & 96.4 & 78 & 4,149 & & & \\
\hline 1980 & 2,898 & 68 & 64.4 & 4,500 & 74.8 & 787 & 4,205 & & & \\
\hline 1981 & 2,977 & 65 & 68.9 & 4,320 & 69.2 & 788 & 4,212 & & & \\
\hline 1982 & 2,990 & 54 & 58.0 & 5,159 & 89.7 & 808 & 4,346 & & & \\
\hline 1983 & 3,138 & 70 & 65.2 & 4,810 & 89.9 & 822 & 4,440 & & & \\
\hline 1984 & 3,482 & 81 & 79.9 & 4,360 & 62.1 & 809 & 4,354 & & & \\
\hline 1985 & 3,565 & 88 & 84.5 & 4,217 & 100.9 & 835 & 4,528 & & & \\
\hline 1986 & 3,792 & 79 & 82.9 & 4,576 & 185.9 & 943 & 5,256 & & & \\
\hline $\begin{array}{l}--- \\
1987\end{array}$ & 3,863 & $-\overline{1}$ & 83.3 & $\because, 639$ & $\begin{array}{l}--- \\
131.0\end{array}$ & $\begin{array}{c}--- \\
992\end{array}$ & . & --- & $--=$ & $\cdots$ \\
\hline 1988 & 3,956 & 91 & 89.0 & 4,446 & 137.6 & 1,043 & 5,939 & 6,347 & 6,778 & 6,890 \\
\hline 1989 & & & & & 144.2 & 1,097 & 6,303 & 6,736 & 7,193 & 7,312 \\
\hline 1990 & & & & & 150.8 & 1,153 & 6,690 & 7,139 & 7,623 & 7,749 \\
\hline 1991 & & & & & 157.3 & 1,210 & 7,069 & 7,554 & 8,067 & 8,200 \\
\hline 1992 & & & & & 163.9 & 1,269 & 7,469 & 7,981 & 8,526 & 8,664 \\
\hline
\end{tabular}

Capacity-Capital Stock Regression: Code 1 RSO 0.690

Coefficients: Const -1127.7 Stock 6.8

Ave. Capocity/Stock $(74-86): 5.3$ Ave. Weekly Hours (High) 136.9 Shift Factor 1.16 Ave Weekly Hours (Low) 129.9 shift factor 1.22 Note: Output, Investment, and Capital Stack extrapolated for 1987 and 1988.

3231 Products of purchased glass

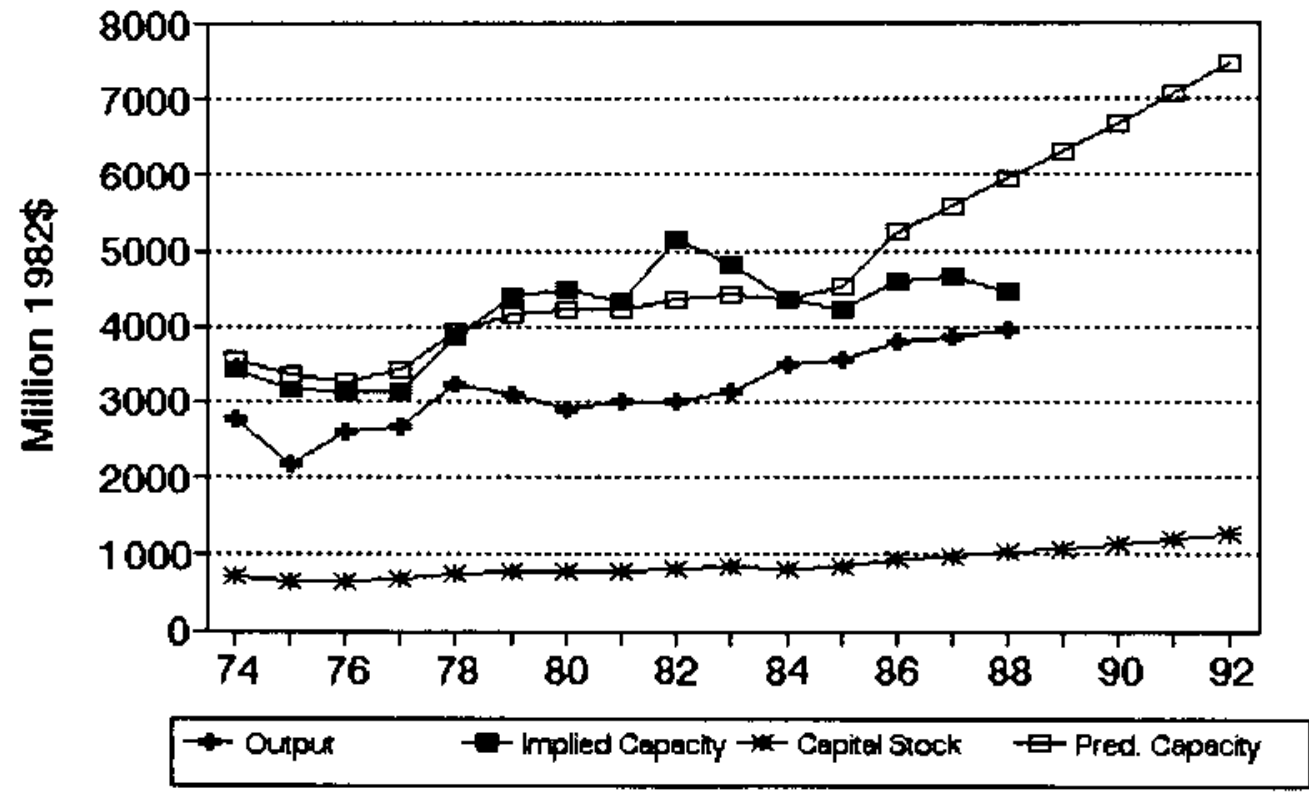


No. 232 gIc 3241 Cement, hydraulic

MILLIONS OF 1982 DOLLARS (EXCL. CU RATES)

$\begin{array}{lccccccc} & 04 & \text { Ann. Implied Gross } & \text { Net } & \text { Pred. } & -\ldots \ldots \ldots \\ \text { Gross } & \text { CU } & \text { CU } & \text { Prac. } & \text { In- } & \text { Cap. } & \text { Prac. } & \text { Emergency Capacity } \\ \text { Output } & \text { Rate } & \text { Rate } & \text { Capac. vest. } & \text { Stk. } & \text { Capac. } & 3 \text { mon. } 6 \text { mon. }>6 \text { mon. }\end{array}$

\begin{tabular}{|c|c|c|c|c|c|c|c|c|c|c|}
\hline 1974 & 4,895 & 81 & 86.6 & 5,650 & 779.4 & 5,723 & 5,484 & & & \\
\hline 1975 & 4,121 & 64 & 64.8 & 6,357 & 832.7 & 6,093 & 5,936 & & & \\
\hline 1976 & 4,163 & 63 & 64.0 & 6,506 & 395.2 & 6,017 & 5,844 & & & \\
\hline 197 & 4,439 & 84 & 75.3 & 5,897 & 411.8 & 5,967 & 5,783 & & & \\
\hline 1978 & 4,808 & 88 & 88.9 & 5,532 & 578.8 & 6,070 & 5,908 & & & \\
\hline 1979 & 4,870 & 89 & 88.9 & 5,480 & 136.5 & 5,735 & 5,499 & & & \\
\hline 1980 & 4,335 & 75 & 7.7 & 5,578 & 612.8 & 5,854 & 5,644 & & & \\
\hline 1981 & 3,878 & 72 & 76.3 & 5,088 & 303.8 & 5,662 & 5,409 & & & \\
\hline 1982 & 3,593 & 64 & 66.9 & 5,373 & 683.8 & 5,810 & 5,591 & & & \\
\hline 1983 & 3,651 & 73 & 71.4 & 5,117 & 246.0 & 5,501 & 5,213 & & & \\
\hline 1984 & 3,993 & 88 & 85.6 & 4,667 & 707.8 & 5,617 & 5,354 & & & \\
\hline 1985 & 3,991 & 78 & 80.9 & 4,933 & 65.5 & 5,112 & 4,738 & & & \\
\hline 1986 & 4,002 & 93 & 87.8 & 4,556 & 225.3 & 4,777 & 4,329 & & & \\
\hline-- & $--\ldots$ & $\cdots$ & $\cdots$ & $-\cdots$ & $-\cdots$ & $\therefore$ & $\therefore-$ & $-\cdots$ & - - - & -- \\
\hline 1987 & 4,074 & 96 & 94.8 & 4,300 & 429.3 & 4,660 & 4,186 & & & \\
\hline 1988 & 4.170 & 94 & 94.6 & 4,407 & 458.8 & 4,588 & 4,095 & 4,109 & 4,098 & 4,095 \\
\hline 1989 & & & & & 466.8 & 4,529 & 4,024 & 4,038 & 4,027 & 4,024 \\
\hline 1990 & & & & & 471.4 & 4,482 & 3,968 & 3,981 & 3,970 & 3,968 \\
\hline 1991 & & & & & 515.1 & 4,485 & 3,971 & 3,984 & 3,973 & 3,971 \\
\hline 1992 & & & & & 542.7 & 4,514 & 4,007 & 4,021 & 4,010 & 4,007 \\
\hline
\end{tabular}

Capacity-Capital Stock Regression: Code 1 RSO 0.630

Coefficients: Const -1509.7 Stock 1.2

Ave. Capacity/Stock $(74-86): 1.0$ Ave. Heekly Hours (High) 167.3 Shift Factor 1.00 Ave Weekly Hours (Low) 167.3 Shift Factor 1.00 Note: Output, Imvestment, and Capital Stock extropolated for 1987 and 1988.

\section{Cement, hydraulic}

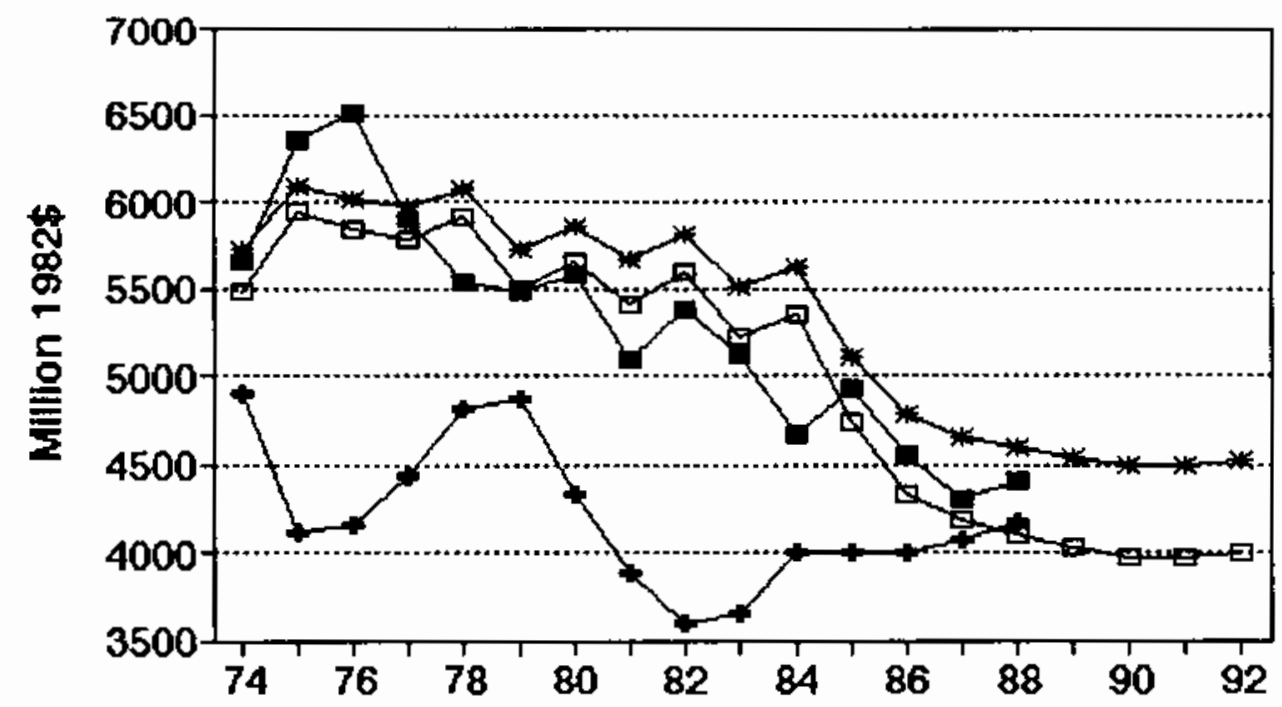

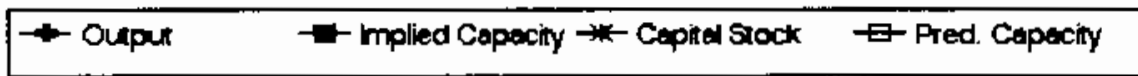




\section{No. 233 BIC 3251 Brick and atructural clay tile}

MILLIONS OF 1982 DOLLARS (EXCL. CU RATES)

$\begin{array}{lccccccc} & \text { Q4 } & \text { Ann. } & \text { Implied } & \text { Gross } & \text { Het } & \text { Pred. } & -\ldots \ldots \ldots \\ \text { Gross } & \text { CU } & \text { CU } & \text { Prac. } & \text { In- } & \text { Cap. } & \text { Prac. } & \text { Emergency Capacity } \\ \text { Output } & \text { Rate } & \text { Rate } & \text { Capac. } & \text { vest. } & \text { Stk. } & \text { Capac. } & 3 \text { mon. } 6 \text { mon. }>6 \text { mon. }\end{array}$

\begin{tabular}{|c|c|c|c|c|c|c|c|c|c|c|}
\hline 1974 & 1,228 & 87 & 92.1 & 1,333 & 145.9 & 748 & 1,215 & & & \\
\hline 1975 & 990 & $\pi$ & 74.4 & 1,332 & 42.2 & 725 & 1,244 & & & \\
\hline 1976 & 1,114 & 91 & 85.4 & 1,304 & 14.2 & 676 & 1,305 & & & \\
\hline 1977 & 1,137 & 91 & 90.2 & 1,260 & 77.1 & 689 & 1,289 & & & \\
\hline 1978 & 1,195 & 96 & 94.5 & 1,265 & 68.3 & 692 & 1,285 & & & \\
\hline 1979 & 1,105 & 98 & 97.5 & 1,133 & 160.9 & $7 B 0$ & 1,176 & & & \\
\hline 1980 & $\$ 51$ & 79 & 83.7 & 1,136 & 87.5 & 793 & 1,160 & & & \\
\hline 1981 & 804 & 67 & 73.9 & 1,088 & 37.3 & 757 & 1,204 & & & \\
\hline 1982 & 643 & 45 & 53.0 & 1,215 & 71.8 & 748 & 1,215 & & & \\
\hline 1983 & 778 & 76 & 64.9 & 1,199 & 39.2 & 708 & 1,265 & & & \\
\hline 1984 & 913 & 62 & 70.0 & 1,305 & 132.1 & 731 & 1,212 & & & \\
\hline 1985 & 1,072 & 54 & 56.5 & 1,897 & 110.1 & 770 & 1,907 & & & \\
\hline 1986 & 1,114 & 58 & 56.8 & 1,962 & 52.7 & 733 & 1,952 & & & \\
\hline - - - & & -- & -- & & -- & $-\cdots$ & $\cdots-$ & $\cdots$ & -- & $\cdots \cdot$ \\
\hline 1987 & 1,132 & $59 *$ & 58.7 & 1,927 & 66.9 & 712 & 1,978 & & & \\
\hline 1988 & 1,155 & $63 *$ & 61.5 & 1,878 & 75.7 & 703 & 1,990 & 2,324 & 2,535 & 2,590 \\
\hline 1989 & & & & & 78.0 & 696 & 1,998 & 2,333 & 2,545 & 2,600 \\
\hline 1990 & & & & & 79.4 & 692 & 2,003 & 2,339 & 2,551 & 2,606 \\
\hline 1991 & & & & & 92.4 & 702 & 1,992 & 2,326 & 2,537 & 2,591 \\
\hline 1992 & & & & & 100.6 & 718 & 1,971 & 2,302 & 2,511 & 2,564 \\
\hline
\end{tabular}

Capacity-Capital Stock Regression: Code -1 RSO 0.940

Coefficients: Const 2144.3 stock -1.2 O(B5-86) 718.7 Ave. Capacity/Stock $(74-86): 1.8$ Ave. Ueekly Hours (High) 121.6 Shift Factor 1.30 Ave Weekly Hours (Low) 121.6 Shift Factor 1.30 * Imputed Note: Output, Investment, and Capital Stock extrapolated for 1987 and 1988.

\section{Brick and structural clay tile}

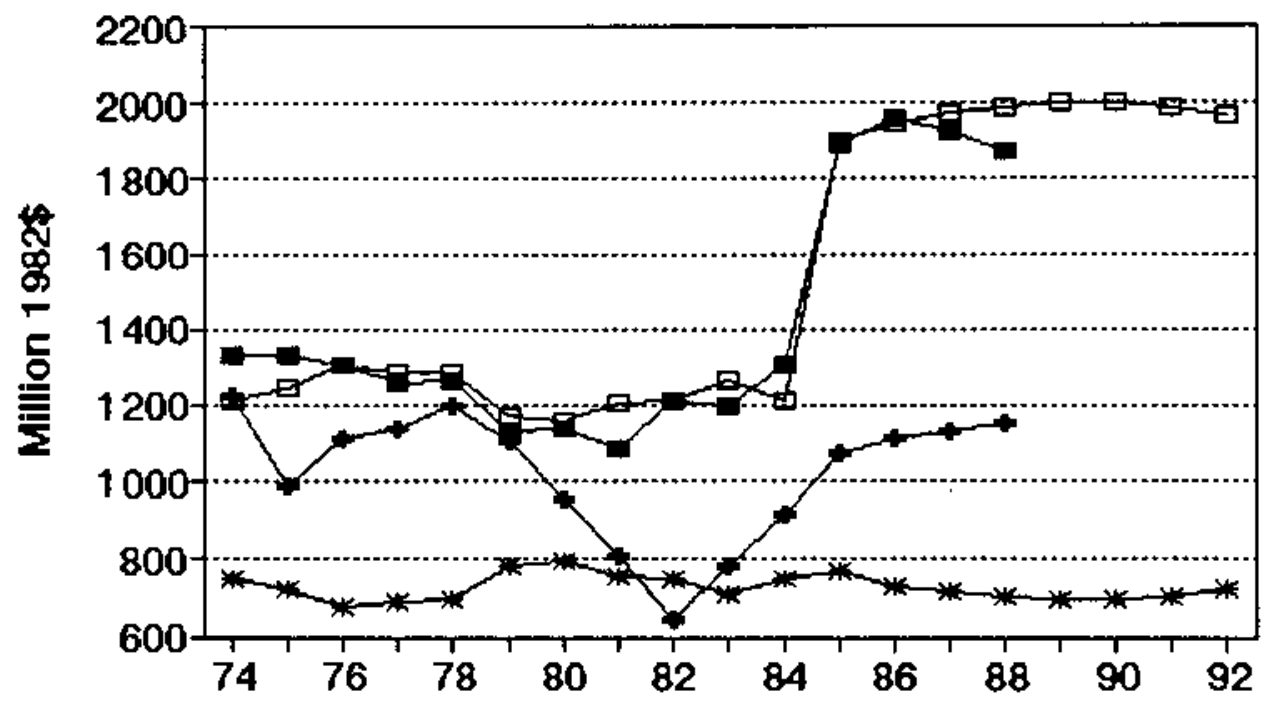

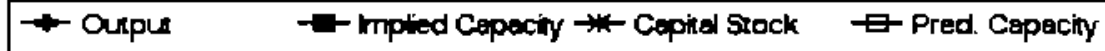




\section{No. 234 gIC 3253 Ceramic wall and floor tile}

MILLIONS OF 1982 DOLLARS (EXCL. CU RATES)

$\begin{array}{lccccccc} & \text { Q4 } & \text { Ann. Implied Gross } & \text { Net } & \text { Pred. } & \ldots \ldots \ldots \\ \text { Gross } & \text { CU } & \text { CU } & \text { Prac. } & \text { In- } & \text { Cap. } & \text { Prac. } & \text { Emergency Capacity } \\ \text { Output } & \text { Rate } & \text { Rate } & \text { Capac. } & \text { vest. } & \text { Stk. } & \text { Capac. } & 3 \text { mon. } 6 \text { mon. }>6 \text { mon. }\end{array}$

\begin{tabular}{|c|c|c|c|c|c|c|c|c|c|c|}
\hline 1974 & 326 & 87 & 94.0 & 347 & 37.8 & 202 & 514 & & & \\
\hline 1975 & 299 & 75 & 72.4 & 413 & 14.6 & 196 & 503 & & & \\
\hline 1976 & 318 & 91 & 85.6 & 372 & 19.9 & 195 & 503 & & & \\
\hline 1977 & 321 & 91 & 90.0 & 357 & (3.3) & 174 & 466 & & & \\
\hline 1978 & 372 & 96 & 94.6 & 393 & 24.9 & 181 & 47 & & & \\
\hline 1979 & 413 & $\pi$ & 81.7 & 505 & 32.3 & 193 & 499 & & & \\
\hline 1980 & 441 & 69 & 68.2 & 646 & 21.0 & 195 & 502 & & & \\
\hline 1981 & 470 & 63 & 68.5 & 686 & 35.7 & 208 & 525 & & & \\
\hline 1982 & 410 & 52 & 55.9 & 733 & 22.8 & 207 & 523 & & & \\
\hline 1983 & 458 & 81 & 71.4 & 642 & 14.6 & 198 & 507 & & & \\
\hline 1994 & 533 & 80 & 84.4 & 632 & 118.9 & 292 & 671 & & & \\
\hline 1985 & 504 & 80 & 79.1 & 637 & 29.1 & 294 & 675 & & & \\
\hline 1986 & 593 & 82 & 81.8 & 725 & 54.4 & 321 & 722 & & & \\
\hline $\begin{array}{l}--- \\
1987\end{array}$ & $\cdots$ & $\begin{array}{l}-- \\
84\end{array}$ & $\begin{array}{l}-\cdots \\
\text { as.1 }\end{array}$ & $=$ & $\begin{array}{c}* \\
55.3\end{array}$ & $\begin{array}{l}--\cdot \\
347\end{array}$ & $\begin{array}{c}\cdots \\
769\end{array}$ & $\cdots$ & $\cdots$ & $\cdots$ \\
\hline 1988 & 633 & 90 & 87.6 & 723 & 58.5 & 373 & 817 & 981 & 1,044 & 1,060 \\
\hline 1989 & & & & & 61.7 & 403 & 867 & 1,040 & 1,107 & 1,124 \\
\hline 1990 & & & & & 64.9 & 432 & 917 & 1,101 & 1,172 & 1,190 \\
\hline 1991 & & & & & 68.1 & 462 & 969 & 1,163 & 1,238 & 1,257 \\
\hline 1992 & & & & & 71.3 & 492 & 1,022 & 1,227 & 1,305 & 1,326 \\
\hline
\end{tabular}

Capacity-Capital Stock Regression: Code O R50 0.320

Coefficients: Const 161.5 stock 1.8

Ave. Capecity/Stock $(74-86): 2.5$

Ave. Weekly Hours (High) 121.9 Shift Factor 1.30 Ave Weekly Hours (Low) 115.8 Shift Factor 1.36 Note: Output, Investment, and Capital Stock extrapolated for 1987 and 1988.

\section{Ceramic wall and floor tile}

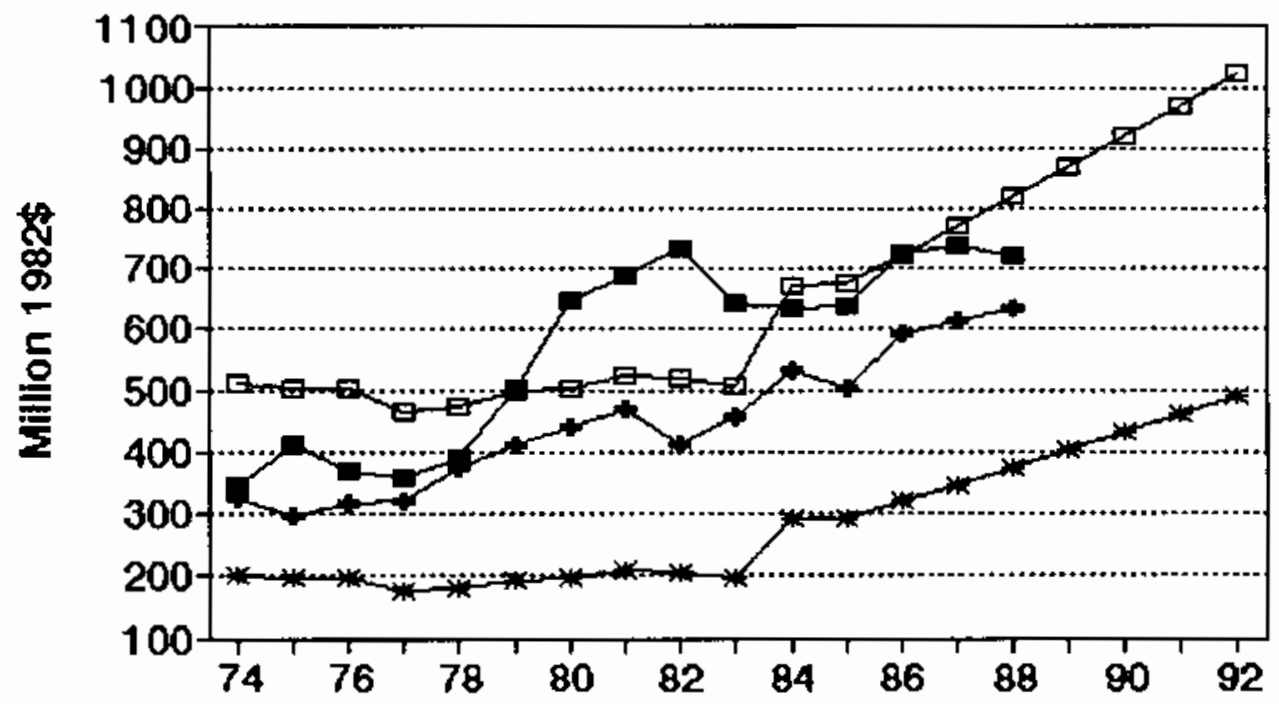

\begin{tabular}{|c|c|c|}
\hline - Ourour & Copociny $-M-C$ & \\
\hline
\end{tabular}


No. 235 sIc 3255 clay refractories

MILLIONS OF 1982 DOLLARS (EXCL. CU RATES)

$\begin{array}{lccccccc} & 04 & \text { Ann. Implied Gross } & \text { Net } & \text { Pred. } & \ldots \ldots \\ \text { Gross } & \text { CU } & \text { CU } & \text { Prac. } & \text { In- } & \text { Cap. } & \text { Prac. } & \text { Emergency Capacity } \\ \text { Qutput } & \text { Rate } & \text { Rate } & \text { Capac. } & \text { vest. } & \text { Stk. } & \text { Capac. } & 3 \text { mon. } 6 \text { mon. }\end{array}$

\begin{tabular}{|c|c|c|c|c|c|c|c|c|c|c|}
\hline 1976 & 1,212 & 96 & 96.0 & 1,262 & 28.7 & 382 & 1,949 & & & \\
\hline 1975 & 1,055 & 37 & 59.1 & 1,784 & 99.4 & 435 & 1,969 & & & \\
\hline 1976 & 987 & 35 & 35.8 & 2,761 & 15.1 & 405 & 1,958 & & & \\
\hline 1977 & 1,054 & 30 & 31.9 & 3,308 & 26.1 & 389 & 1,952 & & & \\
\hline 1978 & 1,158 & 36 & 33.8 & 3,431 & 43.0 & 390 & 1,952 & & & \\
\hline 1979 & 1,133 & 86 & 67.3 & 1,685 & 70.1 & 415 & 1,962 & & & \\
\hline 1980 & 991 & 69 & $\pi .4$ & 1,315 & 26.4 & 398 & 1,955 & & & \\
\hline 1981 & 974 & 63 & 65.3 & 1,493 & 55.7 & 408 & 1,959 & & & \\
\hline 1982 & 694 & 41 & 69.3 & 1,410 & 45.3 & 405 & 1,958 & & & \\
\hline 1983 & 749 & 62 & 54.1 & 1,383 & 11.7 & 370 & 1,945 & & & \\
\hline 1984 & 826 & 41 & 48.9 & 1,691 & 17.6 & 343 & 1,935 & & & \\
\hline 1985 & 721 & 47 & 44.8 & 1,610 & $(17.6)$ & 287 & 1,914 & & & \\
\hline 1986 & 753 & 27 & 34.5 & 2,182 & 18.0 & 268 & 1,907 & & & \\
\hline $\begin{array}{l}--- \\
1987\end{array}$ & $\cdots \frac{\cdots}{734}$ & 38 & $\begin{array}{l}-- \\
33.9\end{array}$ & $\begin{array}{l}\cdots, 224 \\
2,24\end{array}$ & 29.7 & 261 & 1,904 & $\cdots$ & $-\cdot$ & - - - \\
\hline 1988 & 755 & 34 & 35.5 & 2,125 & 31.8 & 257 & 1,903 & 2,388 & 2,683 & 2,759 \\
\hline 1989 & & & & & 32.3 & 255 & 1,902 & 2,387 & 2,681 & 2,757 \\
\hline 1990 & & & & & 32.7 & 253 & 1,901 & 2,386 & 2,680 & 2,756 \\
\hline 1991 & & & & & 35.7 & 254 & 1,901 & 2,387 & 2,681 & 2,757 \\
\hline 1992 & & & & & 37.7 & 257 & 1,903 & 2,388 & 2,683 & 2,759 \\
\hline
\end{tabular}

Capacity-Capital stock Regression: Code 1 RSa 0.000

Coefficients: Const 1806.1 Stock 0.4

Ave, Capacity/Stock $(74-86): 5.2$

Ave. Weekly Hours (High) 108.6 Shift factor 1.45 Ave Weekly Hours (LOW) 95.8 Shift Factor 1.64 Note: Output, Investment, and Capital Stock extrapolated for 1987 and 1988.

\section{Clay refractories}

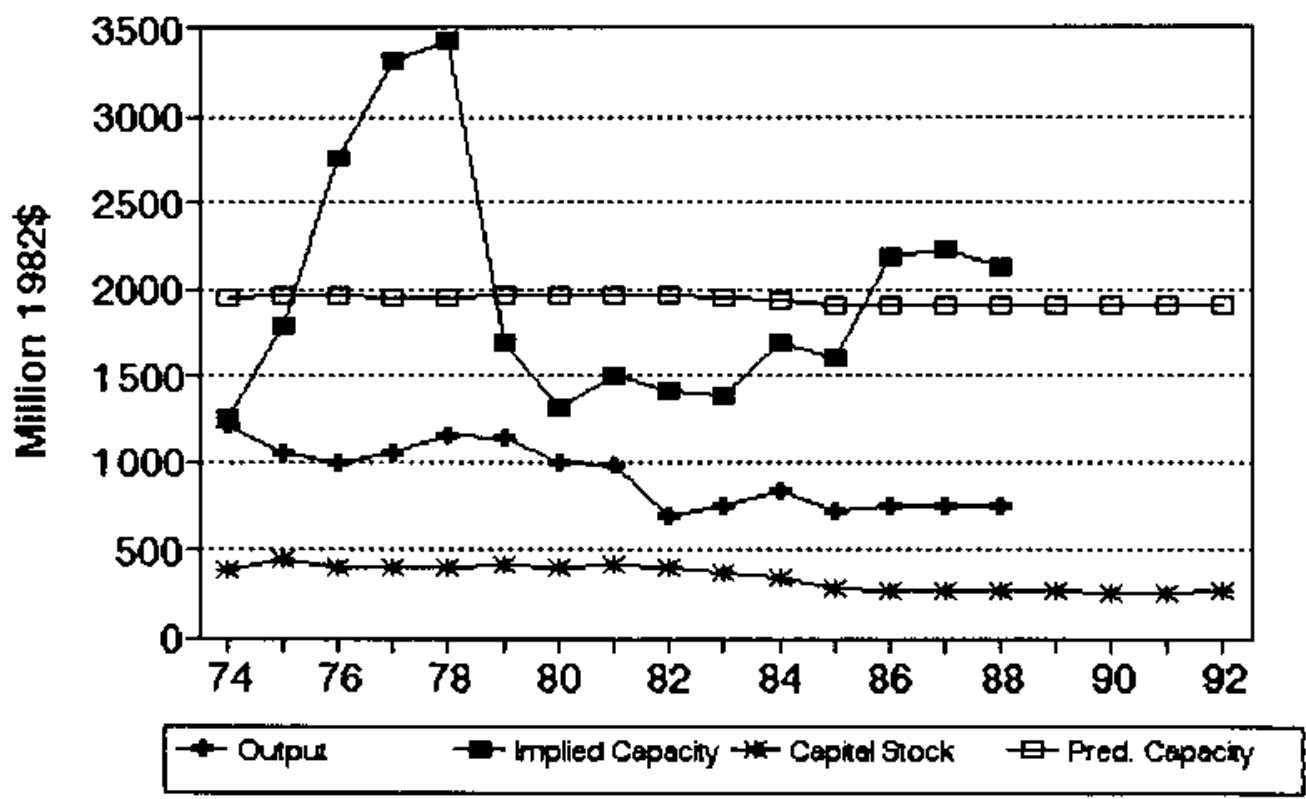


No. 236 SIC 3259 structural clay products, n.e.c.

MILLIONS OF 1982 DOLLARS (EXCL. CU RATES)

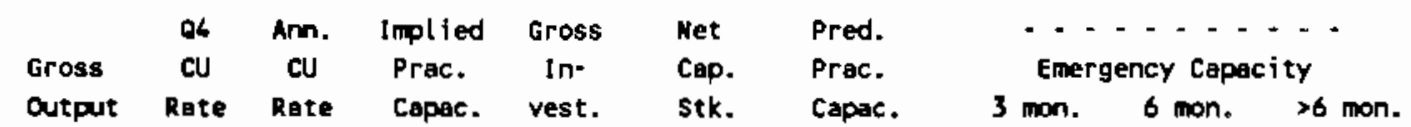

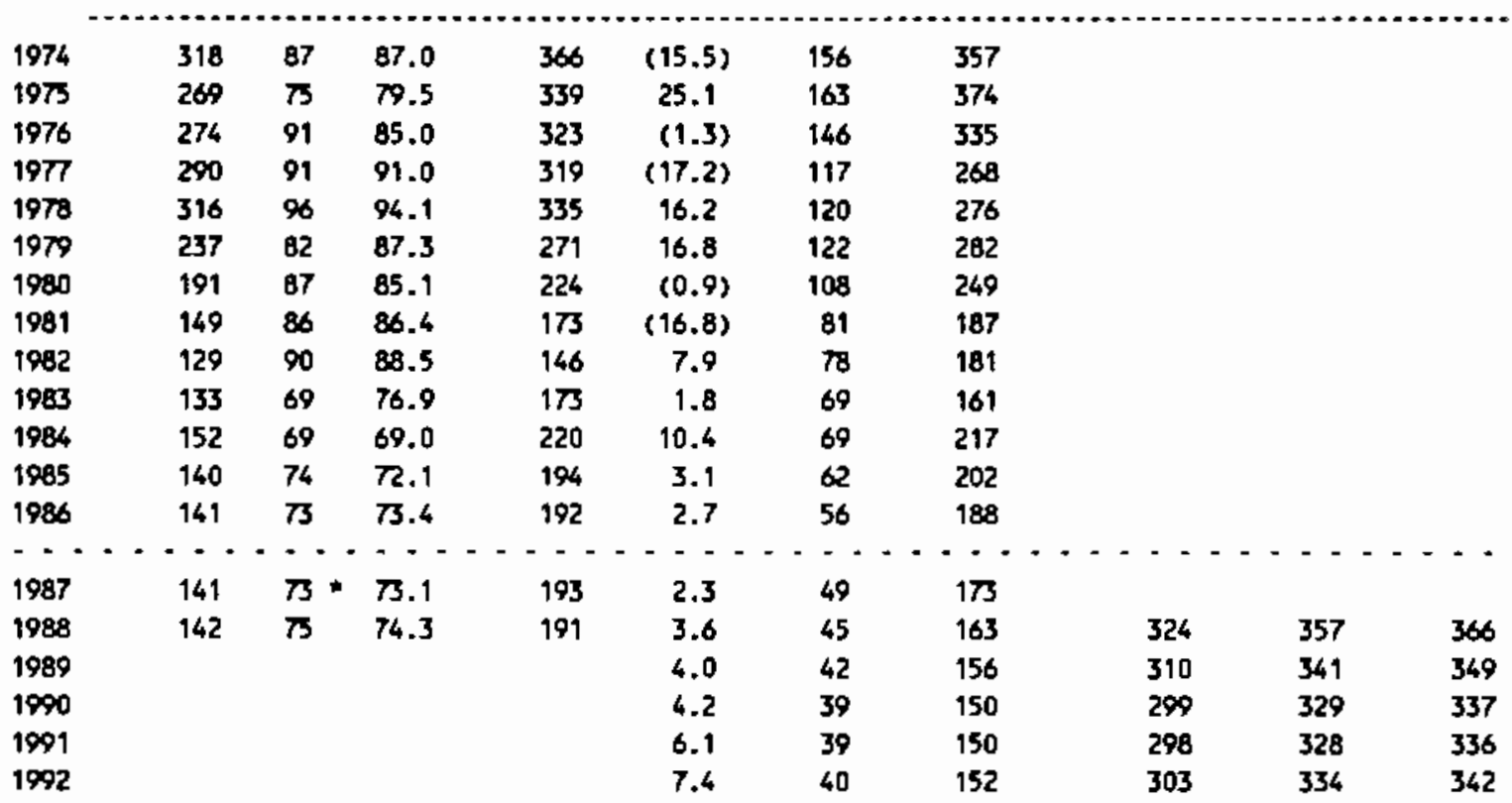

Capacity-Capital Stock Regression: Code -1 RSQ 0.860

Coefficients: Const 4.2 stock 2.3 D(84-86) 57.6

Ave. Capacity/Stock $(74-86): 2.4$ Ave. Ueekly Hours (High) 70.2 Shift Factor 2.24 Ave Weekly Hours (LOH) 47.0 Shift factor 3.35 - Inputed Note: Output, Investment, and Capital Stock extrapolated for 1987 and 1988.

3259 Structural clay products, n.e.c.

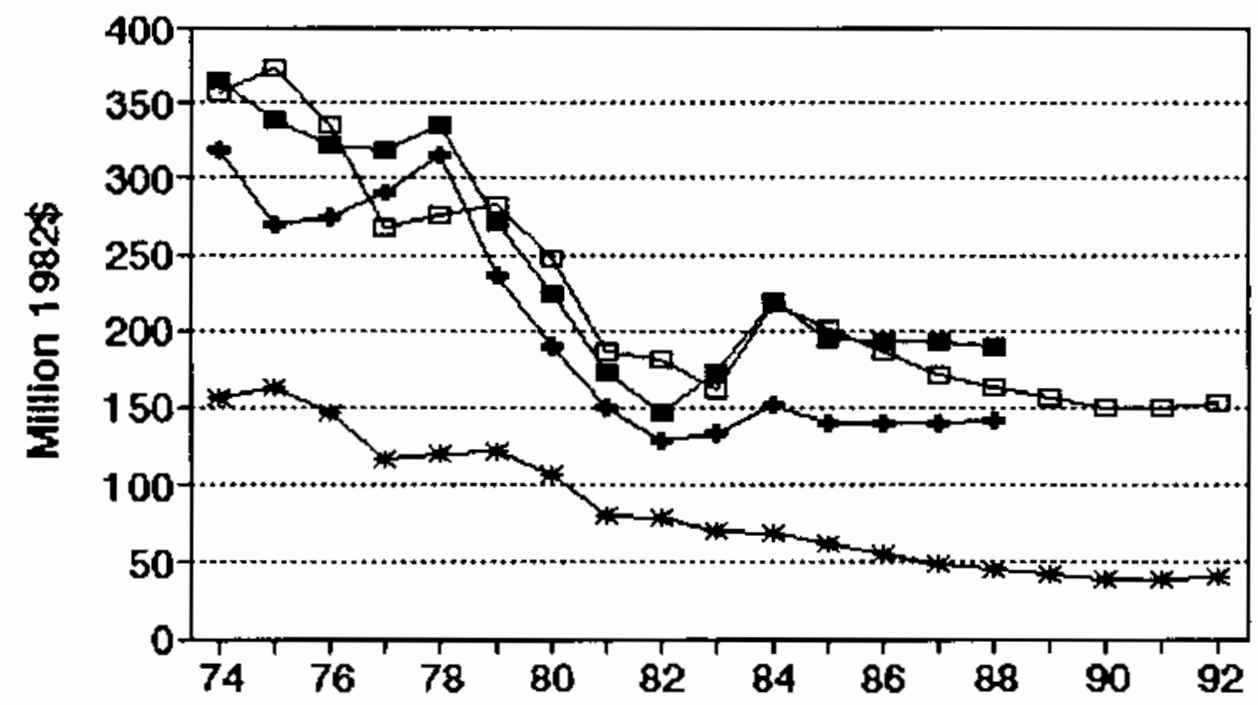

$\rightarrow$ Oupu $\rightarrow$ - mpled Cepaciny $\rightarrow$ - Capiel Slock $\quad$ E Pred. Ceapeciny 


\section{No. 237 sIC 3261 Vitreous plumbing fixtures}

\section{MILLIONS OF 1982 DOLLARS (EXCL. CU RATES)}

$\begin{array}{lccccccc} & 04 & \text { Ann. Implied Gross } & \text { Net } & \text { Pred. } & -\ldots \ldots \ldots \\ \text { Gross } & \text { CU } & \text { CU } & \text { Prac. } & \text { In- } & \text { Cap. } & \text { Prac. } & \text { Emergency Capacity } \\ \text { Output } & \text { Rate } & \text { Rate } & \text { Capac. } & \text { vest. } & \text { Stk. } & \text { Capac. } & 3 \text { mon. } 6 \text { mon. }>6 \text { mon. }\end{array}$

\begin{tabular}{|c|c|c|c|c|c|c|c|c|c|c|}
\hline 1974 & 696 & 87 & 93.9 & 761 & 9.7 & 161 & 798 & & & \\
\hline 1975 & 539 & 86 & 79.1 & 681 & 12.1 & 161 & 801 & & & \\
\hline 1976 & 581 & 76 & 80.9 & 718 & 12.2 & 160 & 807 & & & \\
\hline 1977 & 618 & 83 & 79.5 & 77 & 9.9 & 156 & 829 & & & \\
\hline 1978 & 652 & 90 & 87.8 & 743 & 18.0 & 150 & 810 & & & \\
\hline 1979 & 667 & $\pi$ & 78.9 & 845 & 15.8 & 160 & 807 & & & \\
\hline 1980 & 599 & 54 & 58.4 & 1,025 & 10.1 & 154 & 838 & & & \\
\hline 1981 & 563 & $54 *$ & 57.0 & 988 & 14.5 & 153 & 845 & & & \\
\hline 1982 & 486 & $54 \div$ & 54.2 & 897 & 18.6 & 157 & 825 & & & \\
\hline 1983 & 550 & 81 & 72.3 & 761 & 44.4 & 182 & 680 & & & \\
\hline 1984 & 592 & 89 & 90.0 & 658 & 18.6 & 181 & 685 & & & \\
\hline 1985 & 617 & 84 & 84.9 & 726 & $(13.0)$ & 153 & 849 & & & \\
\hline 1986 & 670 & 86 & 85.8 & 781 & 30.0 & 166 & 770 & & & \\
\hline$\because-$ & $--\cdot-$ & $\cdots$ & $\cdots$ & $\cdots$ & $\cdots$ & $-\cdots$ & $\cdots$ & $\cdots$ & $\cdots-$ & $-\cdot-$ \\
\hline 1987 & 671 & 78 & 80.9 & 830 & 18.4 & 168 & 760 & & & \\
\hline 1988 & 672 & 82 & 80.4 & 836 & 18.7 & 170 & 749 & 900 & 941 & 952 \\
\hline 1989 & & & & & 19.0 & 172 & 738 & 886 & 927 & 937 \\
\hline 1990 & & & & & 19.4 & 174 & 725 & 872 & 912 & 922 \\
\hline 1991 & & & & & 19.7 & 176 & 713 & 857 & 896 & 906 \\
\hline 1992 & & & & & 20.0 & 179 & 700 & 841 & 879 & 899 \\
\hline
\end{tabular}

Capecity-Capital stock Regression: Code 1 RSQ 0.230

Coefficients: Const 1717.3 Stock $\mathbf{- 5 . 7}$

Ave. Capacity/5tock $(74-86): 4.9$ Ave. Weekly Hours (High) 124.4 Shift Factor 1.27 Ave Heekly Hours (Low) 120.0 Shift Factor 1.32 - Imputed Note: Output, Imvestment, and Capital Stock extrapolated for 1987 and 1988.

\section{Vitreous plumbing fixtures}
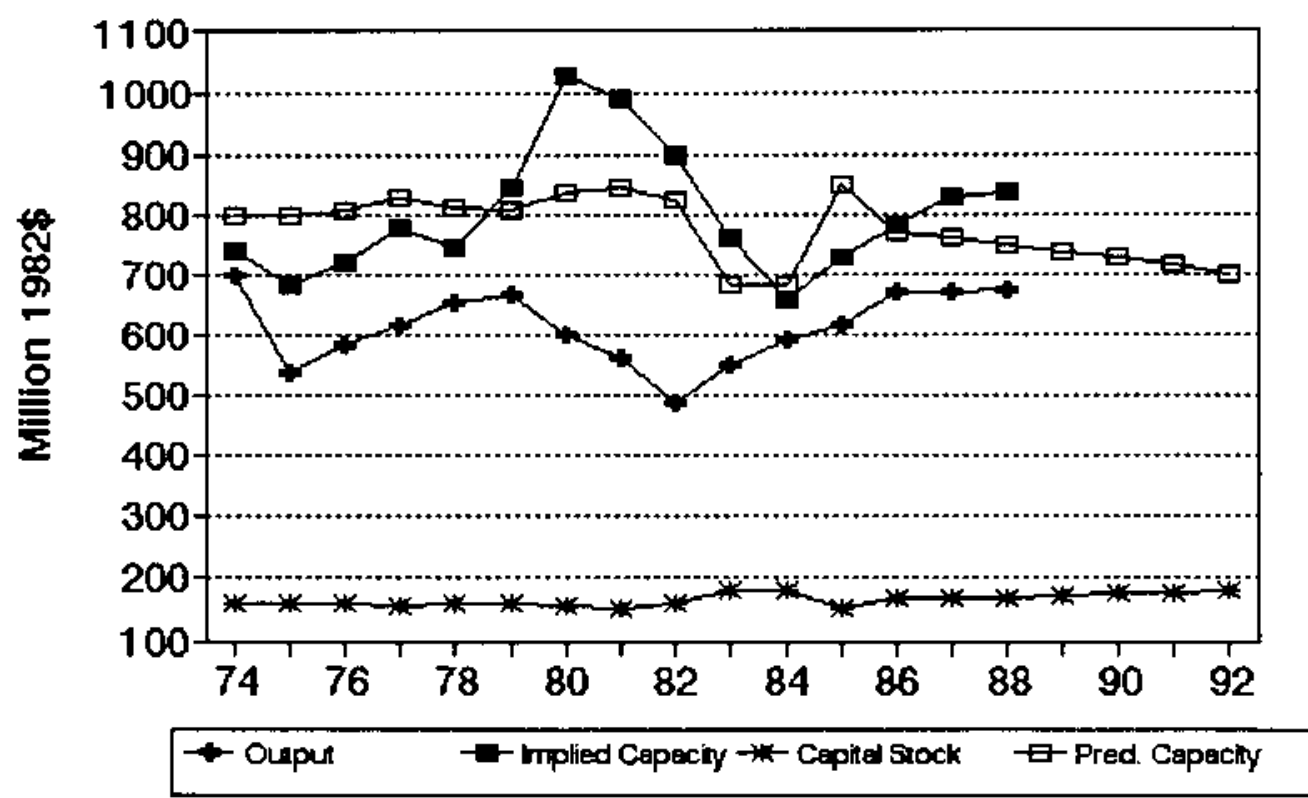


\section{No. 238 sIC 3262 vitreous china food utensils}

MILLIONS OF 1982 DOLLARS (EXCL. CU RATES)

$\begin{array}{lcccclcc} & \text { Q4 Amn. Implied Gross } & \text { Het } & \text { Pred. } & \ldots \ldots \\ \text { Gross } & \text { CU } & \text { CU } & \text { Prac. } & \text { In- } & \text { Cap. } & \text { Prac. } & \text { Emergency Capacity } \\ \text { Output Rate } & \text { Rate } & \text { Capac. } & \text { vest. } & \text { 5tk. } & \text { Capac. } & 3 \text { mon. } 6 \text { mon. }>6 \text { mon. }\end{array}$

\begin{tabular}{|c|c|c|c|c|c|c|c|c|c|c|}
\hline 1974 & 219 & 89 & 90.1 & 243 & 13.3 & 53 & 285 & & & \\
\hline 1975 & 195 & 65 & 73.0 & 267 & 6.9 & 55 & 288 & & & \\
\hline 1976 & 236 & 73 & 70.1 & 337 & 19.6 & 69 & 304 & & & \\
\hline 1977 & 265 & $\pi *$ & 73.8 & 350 & 1.7 & 65 & 300 & & & \\
\hline 1978 & 269 & $76 *$ & 75.5 & 345 & 13.1 & $\pi$ & 308 & & & \\
\hline 1979 & 261 & 77 & 76.7 & 340 & 23.6 & 88 & 326 & & & \\
\hline 1980 & 294 & 90 & 84.7 & 347 & 11.6 & 91 & 331 & & & \\
\hline 1981 & 280 & 73 & 79.9 & 351 & 9.8 & 93 & 333 & & & \\
\hline 1982 & 244 & 74 & 73.6 & 332 & 6.8 & 91 & 330 & & & \\
\hline 1983 & 266 & 91 & 84.8 & 314 & 5.9 & 88 & 327 & & & \\
\hline 1984 & 265 & 77 & 82.9 & 296 & 7.2 & 87 & 325 & & & \\
\hline 1985 & 243 & 80 & 78.7 & 308 & 8.9 & 86 & 325 & & & \\
\hline 1986 & 220 & 84 & 82.6 & 267 & 8.2 & 85 & 323 & & & \\
\hline - & $\begin{array}{c}-\cdots \\
222\end{array}$ & 87 & 85.9 & - - & . & 85 & 323 & - & $\cdots$ & -- \\
\hline 1988 & 225 & 81 & 83.2 & 270 & 9.9 & 86 & 326 & 508 & 559 & 572 \\
\hline 1989 & & & & & 10.1 & 86 & 325 & 509 & 561 & 574 \\
\hline 1990 & & & & & 10.2 & 87 & 326 & 511 & 562 & 576 \\
\hline 1991 & & & & & 11.3 & 89 & 328 & 514 & 566 & 580 \\
\hline 1992 & & & & & 12.0 & 91 & 331 & 519 & 571 & 584 \\
\hline
\end{tabular}

Cepacity-Capital 5tock Regression: Code 0 RSQ 0.200

Coefficients: Const 223.4 stock 1.2

Ave. Capecity/stock $(74-86): 4.0$ Ave. Weekly Hours (High) 89.1 Shift Factor 1.77 Ave Weekly Hours (Low) 85.3 shift Factor 1.85 - Imputed Note: Output, Investment, and Capital Stock extrapolated for 1987 and 1988.

\section{Vitreous china food utensils}

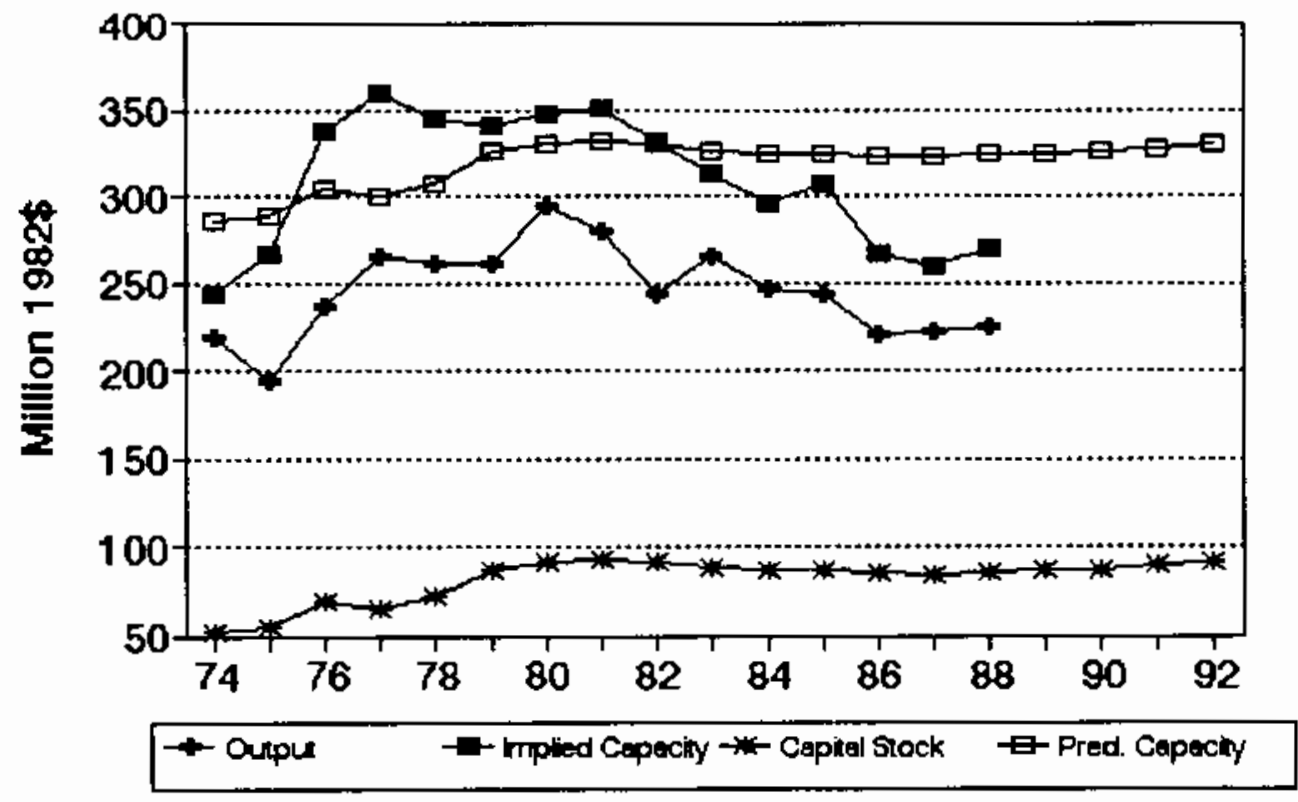




\section{No. 239 sIC 3263 Pine earthenware food utensils}

MILLIONS OF 1982 DOLLARS (EXCL. CU RATES)

$\begin{array}{lccccccc} & \text { Q4 } & \text { Ann. Implied Gross } & \text { Net } & \text { Pred. } & \ldots \\ \text { Gross } & \text { CU } & \text { CU } & \text { Prac. } & \text { In* } & \text { Cap. } & \text { Prac. } & \text { Emergency Capacity } \\ \text { Output Rate } & \text { Rate } & \text { Capac. } & \text { vest. } & \text { Stk. } & \text { Capac. } & 3 \text { mon. } 6 \text { mon. }>6 \text { mon. }\end{array}$

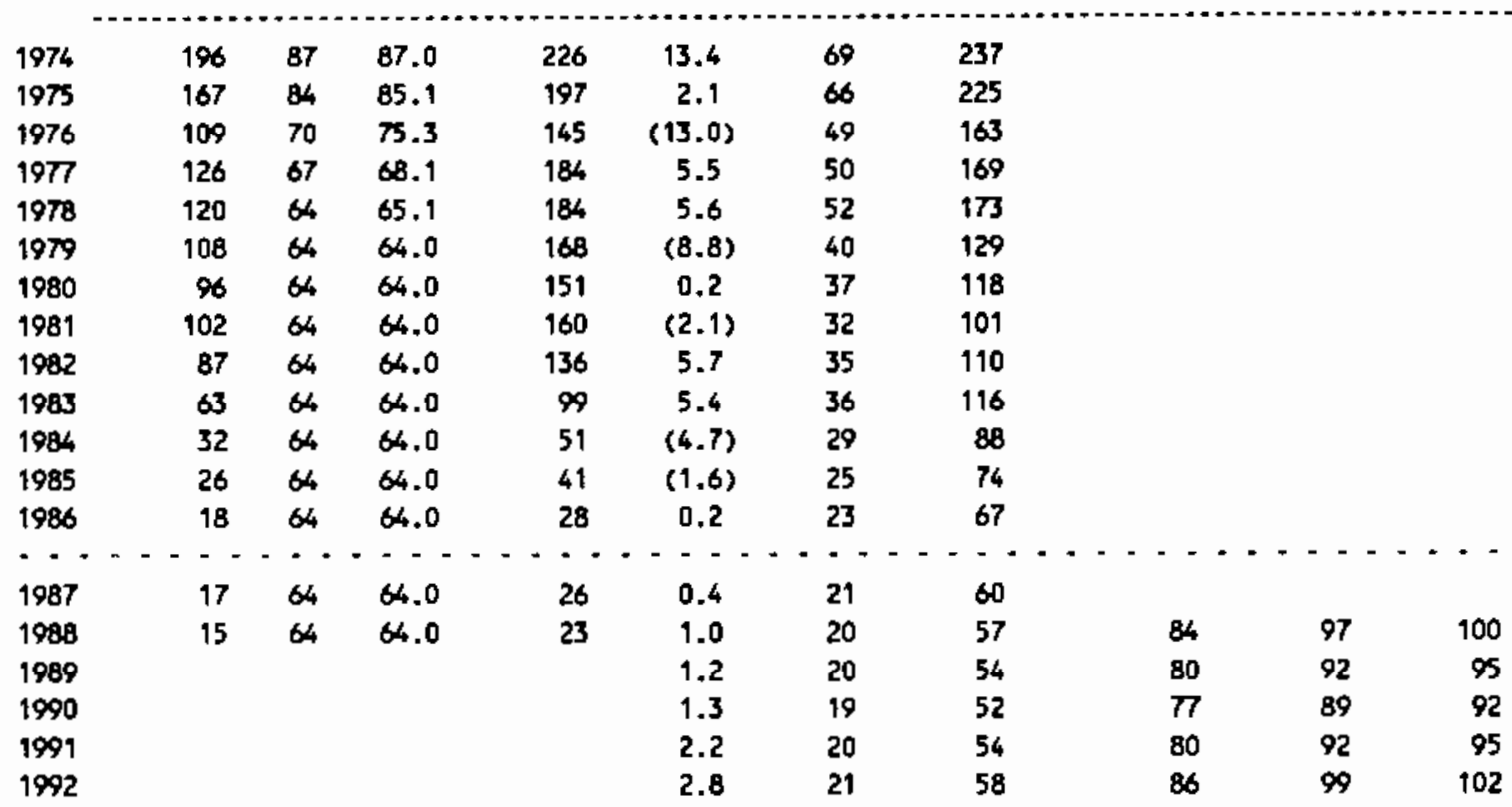

Capecity-Capital Stock Regression: Code 1 RSO 0.740

Coefficients: Const -19.1 stock 3.7

Ave. Capacity/Stock $(74-86): 3.3$ Ave. Weekly Hours (High) 89.1 Shift Factor 1.77 Ave Heekly Hours (LOW) 85.3 Shift Factor 1.85 Note: Output, Investment, and Capital Stock extrapolated for 1987 and 1988.

\section{Fine earthenware food utensils}

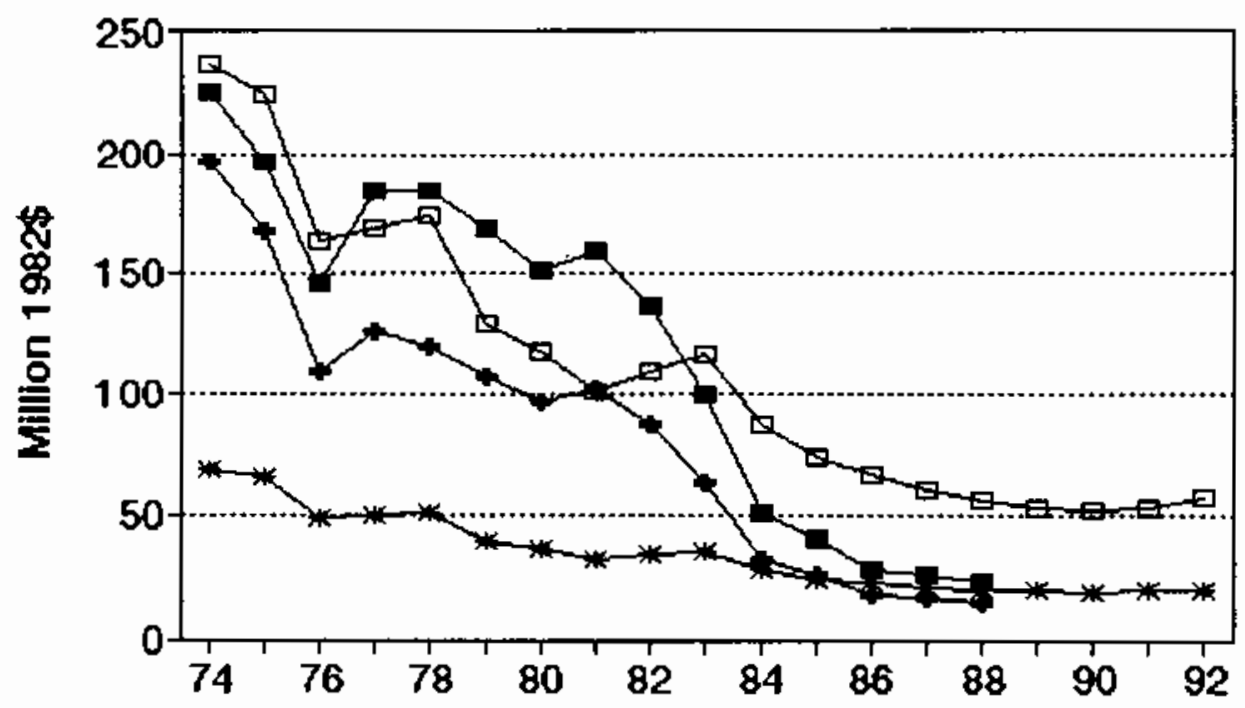

- Ouput t- mplied Cepaciny $\rightarrow$ - Cepite Stock t-Pred. Capacity 


\section{No. 240 SIC 3269 Porcelain electrical suppplie日}

MILLIONS OF 1982 DOLLARS (EXCL. CU RATES)

\begin{tabular}{|c|c|c|c|c|c|c|c|}
\hline & $\begin{array}{l}Q 4 \\
\alpha\end{array}$ & Am. & $\begin{array}{l}\text { Implied } \\
\text { Prac. }\end{array}$ & $\begin{array}{l}\text { Gross } \\
\text { In- }\end{array}$ & $\begin{array}{l}\text { Net } \\
\text { Cap. }\end{array}$ & $\begin{array}{l}\text { Pred. } \\
\text { Prec. }\end{array}$ & $\begin{array}{l}* \\
\text { Energency Capocity }\end{array}$ \\
\hline tput & Rate & Rate & Capec. & & Stk. & apec. & \\
\hline
\end{tabular}

\begin{tabular}{|c|c|c|c|c|c|c|c|c|c|c|}
\hline 1974 & 918 & $56 *$ & 58.1 & 1,579 & 33.7 & 295 & 1,318 & & & \\
\hline 1975 & 692 & 50 & 49.8 & 1,390 & 23.1 & 291 & 1,262 & & & \\
\hline 1976 & 638 & 67 & 60.8 & 1,049 & 9.5 & 273 & 1,016 & & & \\
\hline 1977 & 621 & 61 & 62.9 & 987 & 20.6 & 268 & 936 & & & \\
\hline 1978 & 628 & 66 & 64.3 & 97 & 6.5 & 249 & 670 & & & \\
\hline 1979 & 743 & 84 & 7.4 & 921 & 36.4 & 258 & 805 & & & \\
\hline 1980 & 738 & 72 & $\pi .0$ & 984 & 49.2 & 279 & 1,099 & & & \\
\hline 1981 & 622 & 66 & 69.9 & 890 & 22.8 & 274 & 1,020 & & & \\
\hline 1982 & 538 & 57 & 60.3 & 893 & 29.6 & 274 & 1,027 & & & \\
\hline 1983 & 534 & 65 & 62.7 & 851 & 33.6 & 279 & 1,089 & & & \\
\hline 1984 & 576 & 66 & 67.3 & 855 & 18.1 & 267 & 929 & & & \\
\hline 1985 & 517 & 66 & 65.6 & 788 & 21.7 & 262 & 852 & & & \\
\hline 1986 & 414 & 68 & 67.5 & 613 & 20.4 & 255 & 732 & & & \\
\hline$\because 1987$ & $\begin{array}{c}-- \\
408\end{array}$ & -- & -7 & ‥ & $\because \overline{9}$ & $\begin{array}{l}-- \\
252\end{array}$ & - - - & $\cdots$ & $=-$ & -- \\
\hline 1988 & 401 & 78 & 76.4 & 524 & 24.7 & 250 & 681 & 946 & 1,044 & 1,070 \\
\hline 1989 & & & & & 26.9 & 248 & 659 & 915 & 1,010 & 1,035 \\
\hline 1990 & & & & & 25.0 & 247 & 640 & 889 & 982 & 1,006 \\
\hline 1991 & & & & & 26.0 & 267 & 639 & 888 & 980 & 1,004 \\
\hline 1992 & & & & & 26.7 & 247 & 647 & 899 & 993 & 1,017 \\
\hline
\end{tabular}

Capecity-Capital Stock Regression: Code i RSO 0.560

Coefficients: Const -2825.5 stock 14.1

Ave. Capacity/stock $(74-86): 3.6$ Ave. Weekly Hours (High) 100.3 Shift Factor 1.57 Ave Yeekly Hours (LOW) 91.1 shift Factor 1.73 - Imputed Note: Output, Investment, and Capital Stock extrapolated for 1987 and 1988.

\section{Porcelain electrical suppplies}

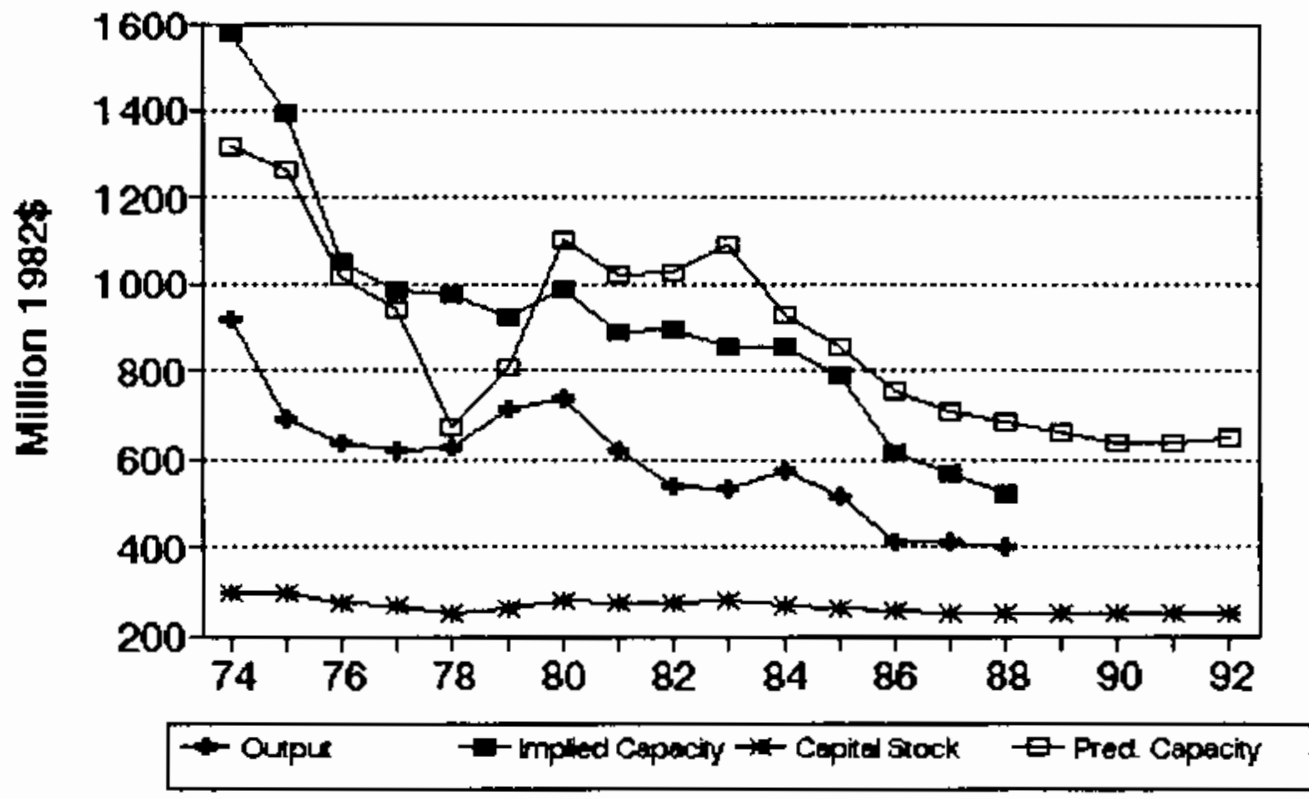




\section{No. 241 SIC 3269 Pottary products, n.e.c.}

MILLIONS OF 1982 DOLLARS (EXCL. CU RATES)

$\begin{array}{lccccccc} & 04 & \text { Amn. Implied Gross } & \text { Net } & \text { Pred. } & -\ldots \ldots \\ \text { Gross } & \text { CU } & \text { CU } & \text { Prac. } & \text { In- } & \text { Cap. } & \text { Prac. } & \text { Emergency Capacity } \\ \text { Output Rate } & \text { Rate } & \text { Capac. } & \text { vest. } & \text { Stk. } & \text { Capac. } & 3 \text { mon. } 6 \text { mon. }>6 \text { mon. }\end{array}$

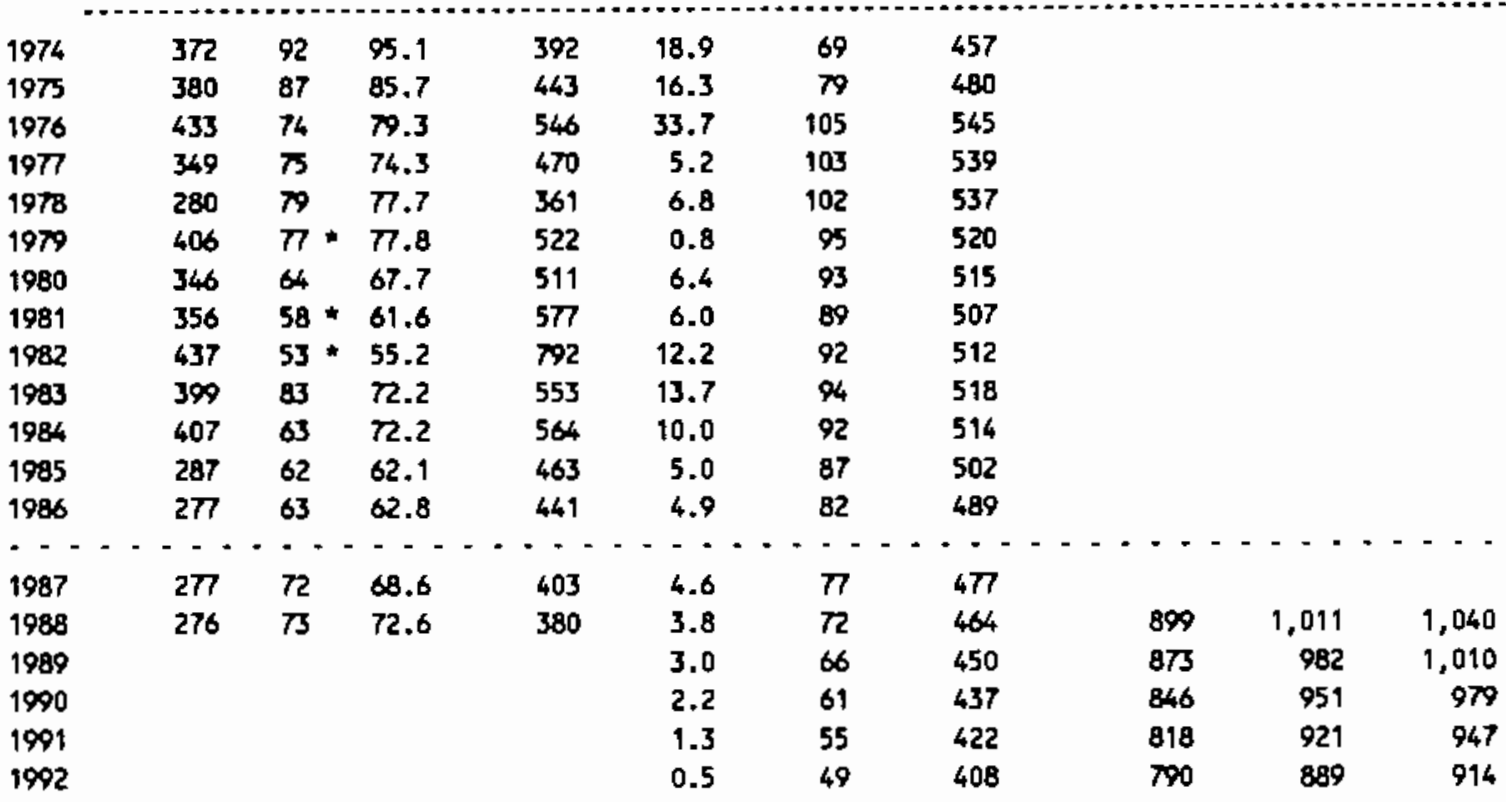

Capacity-Capital stock Regression: Code 1 RSO 0.050

Coefficients: Const- 288.4 stock $\mathbf{2 . 4}$

Ave. Capacity/Stock $(74-86): 5.6$ Ave. Weekly Hours (High) 70.2 Shift Factor 2.24 Ave Weekly Hours (LOW) 65.0 Shift Factor 2.42 - Imputed Note: Output, Investment, and Capitat Stock extrepolated for 1987 and 1988.

3269 Pottery products, n.e.c.

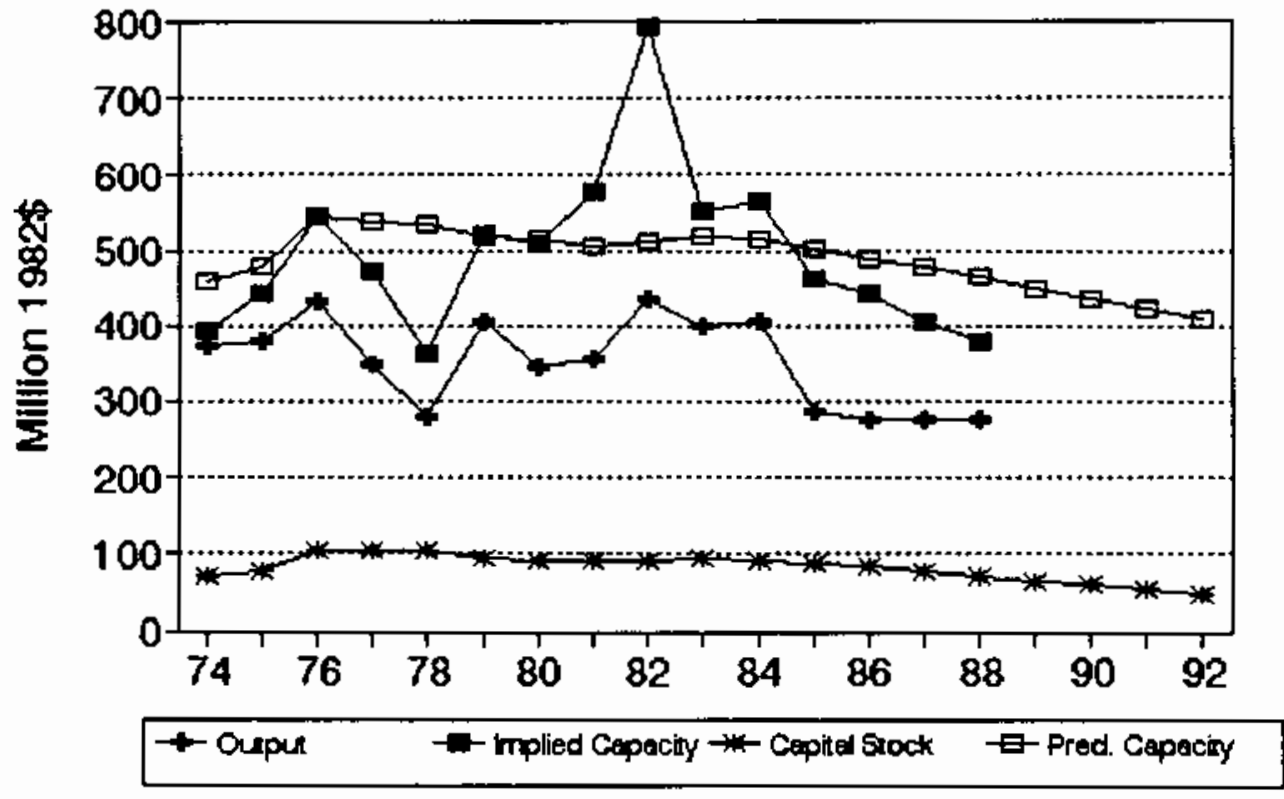


No. 242 SIC 3271 concrete block and brick

MILLIONS OF 1982 DOLLARS (EXCL. CU RATES)

$\begin{array}{lccccccc} & 04 & \text { Ann. } & \text { Implied Gross } & \text { Net } & \text { Pred. } & \ldots \ldots \ldots \\ \text { Gross } & \text { CU } & \text { CU } & \text { Prac. } & \text { In } & \text { Cap. } & \text { Prac. } & \text { Emergency Capacity } \\ \text { Output } & \text { Rate } & \text { Rate } & \text { Capac. } & \text { vest. } & \text { Stk. } & \text { Capac. } & 3 \text { mon. } 6 \text { mon. }>6 \text { mon. }\end{array}$

\begin{tabular}{|c|c|c|c|c|c|c|c|c|c|c|}
\hline 1974 & 1,845 & 49 & 51.2 & 3,604 & 26.4 & 787 & 2,459 & & & \\
\hline 1975 & 1,691 & 46 & 44.9 & 3,766 & 176.0 & 878 & 2,578 & & & \\
\hline 1976 & 1,720 & 74 & 63.5 & 2,707 & 11.6 & 813 & 2,493 & & & \\
\hline 1977 & 1,717 & $\pi$ & 74.1 & 2,316 & 54.8 & 790 & 2,464 & & & \\
\hline 1978 & 1,915 & 71 & 72.8 & 2,632 & 112.6 & 820 & 2,503 & & & \\
\hline 1979 & 1,805 & 70 & 70.5 & 2,561 & 135.7 & 875 & 2,574 & & & \\
\hline 1980 & 1,636 & 71 & 69.3 & 2,360 & 129.4 & 921 & 2,634 & & & \\
\hline 1981 & 1,423 & 65 & 68.9 & 2,064 & 56.4 & 892 & 2,596 & & & \\
\hline 1982 & 1,300 & 65 & 65.0 & 2,000 & 102.4 & 897 & 2,603 & & & \\
\hline 1983 & 1,569 & 73 & 70.9 & 2,211 & 68.0 & 869 & 2,566 & & & \\
\hline 1984 & 1,529 & 85 & 82.5 & 1,853 & $(84.4)$ & 702 & 2,350 & & & \\
\hline 1985 & 1,511 & 69 & 74.5 & 2,029 & 46.7 & 667 & 2,304 & & & \\
\hline 1986 & 1,717 & 78 & 74.9 & 2,293 & 54.1 & 641 & 2,271 & & & \\
\hline 1987 & 1,715 & $\begin{array}{l}-- \\
76\end{array}$ & $\begin{array}{l}--1 \\
76.7\end{array}$ & 2,237 & $\because-$ & 619 & 2,241 & $=\cdot$ & -- & -- \\
\hline 1988 & 1,713 & $\pi$ & 76.6 & 2,238 & 67.8 & 610 & 2,230 & 3,441 & $3, \sqrt{53}$ & 3,834 \\
\hline 1989 & & & & & 70.9 & 606 & 2,224 & 3,432 & 3,743 & 3,824 \\
\hline 1990 & & & & & 72.6 & 603 & 2,222 & 3,428 & 3,739 & 3,819 \\
\hline 1991 & & & & & 89.5 & 618 & 2,241 & 3,458 & 3,772 & 3,852 \\
\hline 1992 & & & & & 100.2 & 642 & 2,272 & 3,506 & 3,824 & 3,906 \\
\hline
\end{tabular}

Capacity-Capital Stock Regression: Code 0 RSO 0.040 Coefficients: Const 1438.3 Stock 1.3

Ave. Capacity/stock $(74-86): 3.1$ Ave. Weekly Hours (High) 91.6 Shift Factor 1.72 Ave Heekly Hours (Low) 84.0 Shift Factor 1.88 Note: Output, Investment, and Capital Stock extrapolated for 1987 and 1988.

\section{Concrete block and brick}

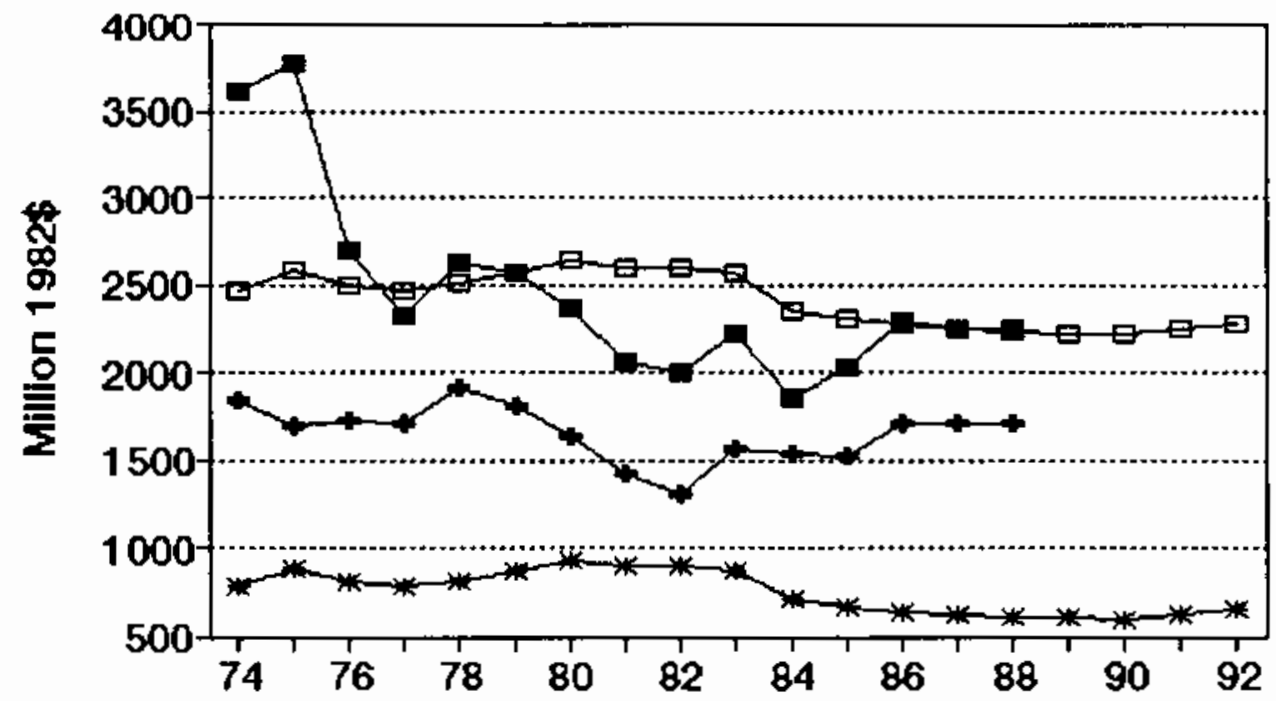

$$
\rightarrow \text { - Oupur } \quad \rightarrow \text {-mplied Cepecity } \rightarrow \text { - Cepitel Stock } \quad \square \text {-Pred Capacity }
$$


No. 243 sIC 3272 Concrete products, n.e.c.

MILLIONS OF 1982 DOLLARS (EXCL. CU RATES)

$\begin{array}{lccccccc} & \text { O4 } & \text { Ann. } & \text { Implied } & \text { Gross } & \text { Net } & \text { Pred. } & \ldots \\ \text { Gross } & \text { CU } & \text { CU } & \text { Prac. } & \text { In- } & \text { Cap. } & \text { Prac. } & \text { Emergency Capacity } \\ \text { Output } & \text { Rate } & \text { Rate } & \text { Capac. } & \text { vest. } & \text { Stk. } & \text { Capac. } & 3 \text { mon. } 6 \text { mon. }>6 \text { mon. }\end{array}$

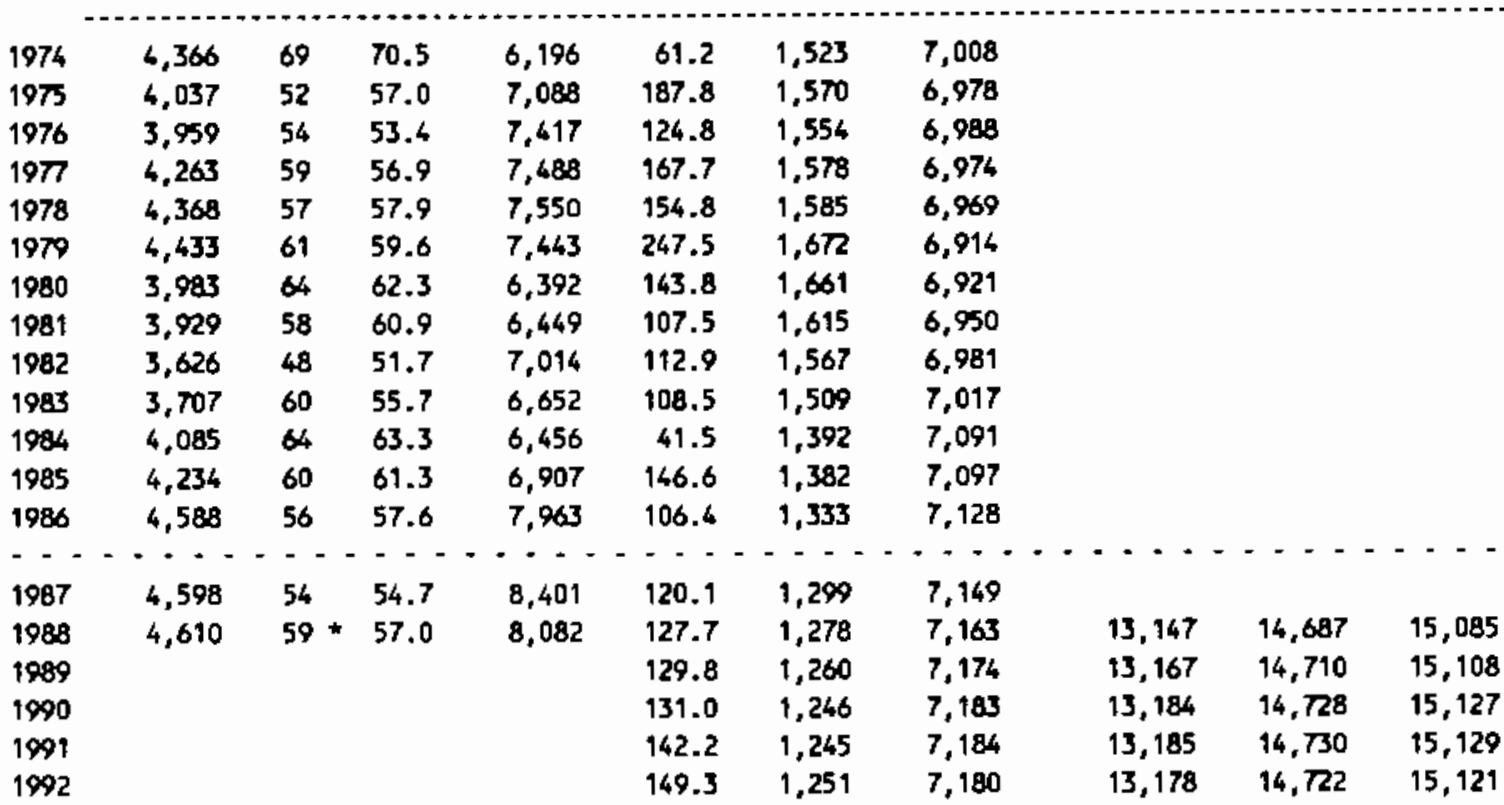

Capacity-Capital Stock Regression: Code 2 RSO 0.010

Coefficients: Const 7969.3 stock -0.6

Ave. Capacity/Stock $(74-86): 4.6$ Ave. Weekly Hours (High) 74.8 Shift Factor 2.11 Ave Ueekly Hours (Low) 70.7 Shift Factor 2.23 * Imputed Note: Output, Investment, and Capital Stock extrapolated for 1987 and 1988.

3272 Concrete products, n.e.c.

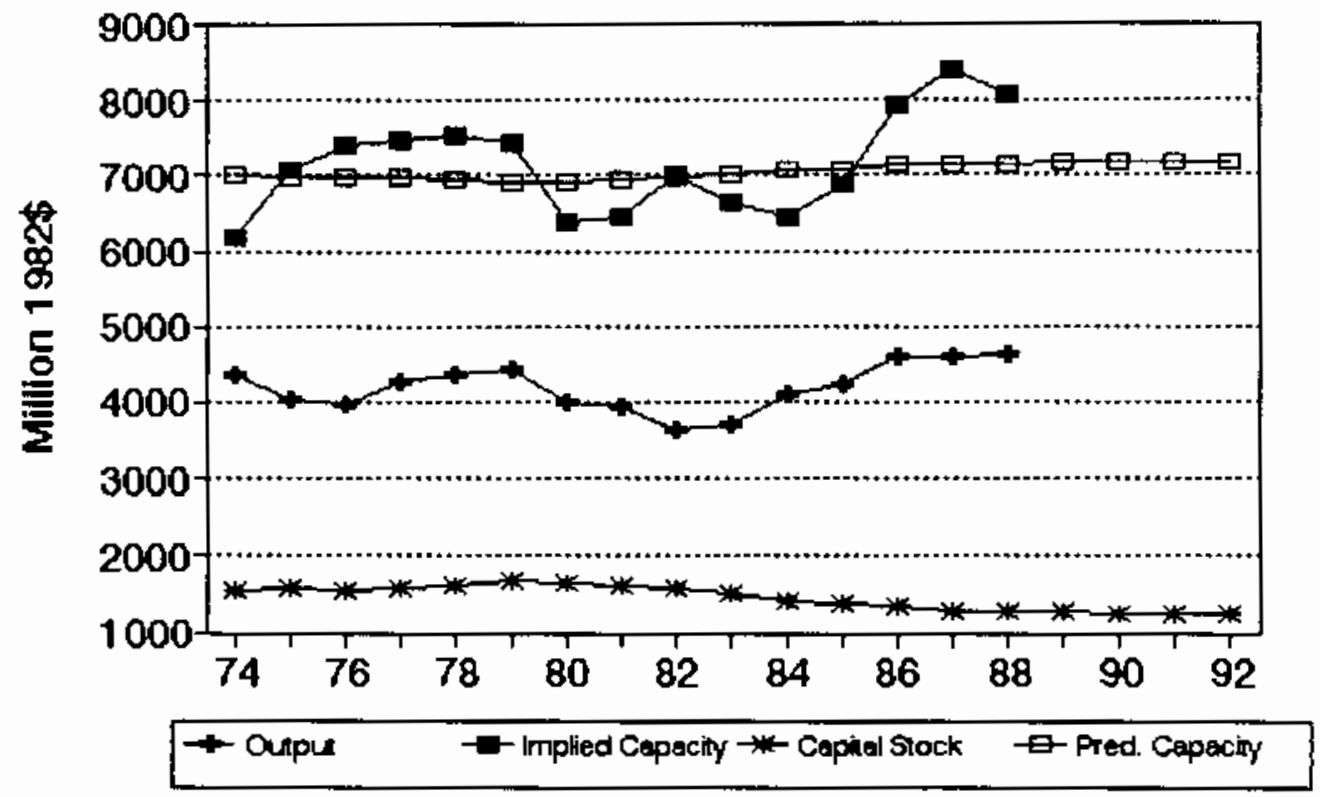




\section{No. 244 BIC 3273 Ready-mixed concrete}

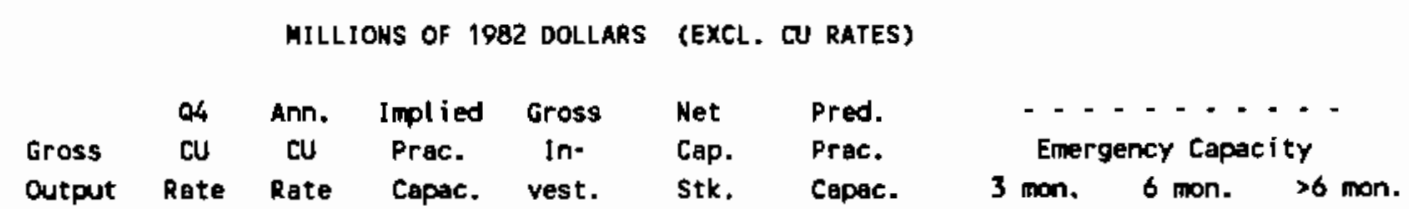

\begin{tabular}{|c|c|c|c|c|c|c|c|c|c|c|}
\hline 1974 & 9,754 & 72 & 74.6 & $\uparrow 3,07 \uparrow$ & 232.8 & 3,706 & 13,002 & & & \\
\hline 1975 & 8,847 & 67 & 6.2 & 13,363 & 539.8 & 3,919 & 13,563 & & & \\
\hline 1976 & 8,818 & 73 & 71.0 & 12,418 & 133.8 & 3,741 & 13,093 & & & \\
\hline 1977 & 10,063 & $\pi$ & 73.9 & 13,627 & 741.2 & 4,129 & 14,116 & & & \\
\hline 1978 & 10,847 & 72 & 73.3 & 14,791 & 455.8 & 4,235 & 14,397 & & & \\
\hline 1979 & 10,985 & 71 & 71.5 & 15,370 & 429.2 & 4,303 & 14,574 & & & \\
\hline 1980 & 9,593 & 62 & 64.3 & 14,928 & 487.1 & 4,401 & 14,834 & & & \\
\hline 1981 & 9,126 & 56 & 59.4 & 15,361 & 488.0 & 4,467 & 15,006 & & & \\
\hline 1982 & 8,170 & 57 & 56.6 & 14,434 & 369.2 & 4,381 & 14.779 & & & \\
\hline 1983 & 8,917 & 54 & 55.9 & 15,961 & 236.0 & 4,151 & 16,782 & & & \\
\hline 1984 & 9,283 & 62 & 60.2 & 15,417 & $(3.3)$ & 3,720 & 15,649 & & & \\
\hline 1985 & 9,429 & 62 & 61.7 & 15,291 & 409.7 & 3,699 & 15,593 & & & \\
\hline 1986 & 9,852 & 57 & 59.1 & 16,677 & 320.0 & 3,597 & 15,323 & & & \\
\hline$-\cdots$ & $\cdots \cdot-$ & - & $\cdots$ & $-\cdots$ & $\cdots \cdot$ & $-\cdots$ & $\cdots$ & $\cdots---$ & $-\cdot \cdot$ & $--\cdot$ \\
\hline 1987 & 9,935 & 56 & 56.3 & 17,636 & 326.8 & 3,503 & 15,076 & & & \\
\hline 1988 & 10,045 & 70 & 64.7 & 15,534 & 343.3 & 3,437 & 14,901 & 29,778 & 33,337 & 34,257 \\
\hline 1989 & & & & & 347.8 & 3,382 & 14,758 & 29,493 & 33,018 & 33,929 \\
\hline 1990 & & & & & 350.4 & 3,337 & 14,639 & 29,255 & 32,751 & 33,655 \\
\hline 1991 & & & & & 374.9 & 3,322 & 14,599 & 29,174 & 32,661 & 33,562 \\
\hline 1992 & & & & & 390.4 & 3,324 & 14,604 & 29,184 & 32,672 & 33,574 \\
\hline
\end{tabular}

Capecity-Capital Stock Regression: Code - 1 RSO 0.760 Coefficients: Const 3240.2 stock $2.6 \quad 0(83-86) 2608.6$ Ave. Capacity/stock $(74-86): 3.6$ Ave. Weekly Hours (High) 68.5 Shift factor 2.30 Ave Weekly Hours (Low) 54.3 Shift factor 2.90 Mote: Output, Investment, and Capital Stock extrapolated for 1987 and 1988.

\section{Ready-mixed concrete}

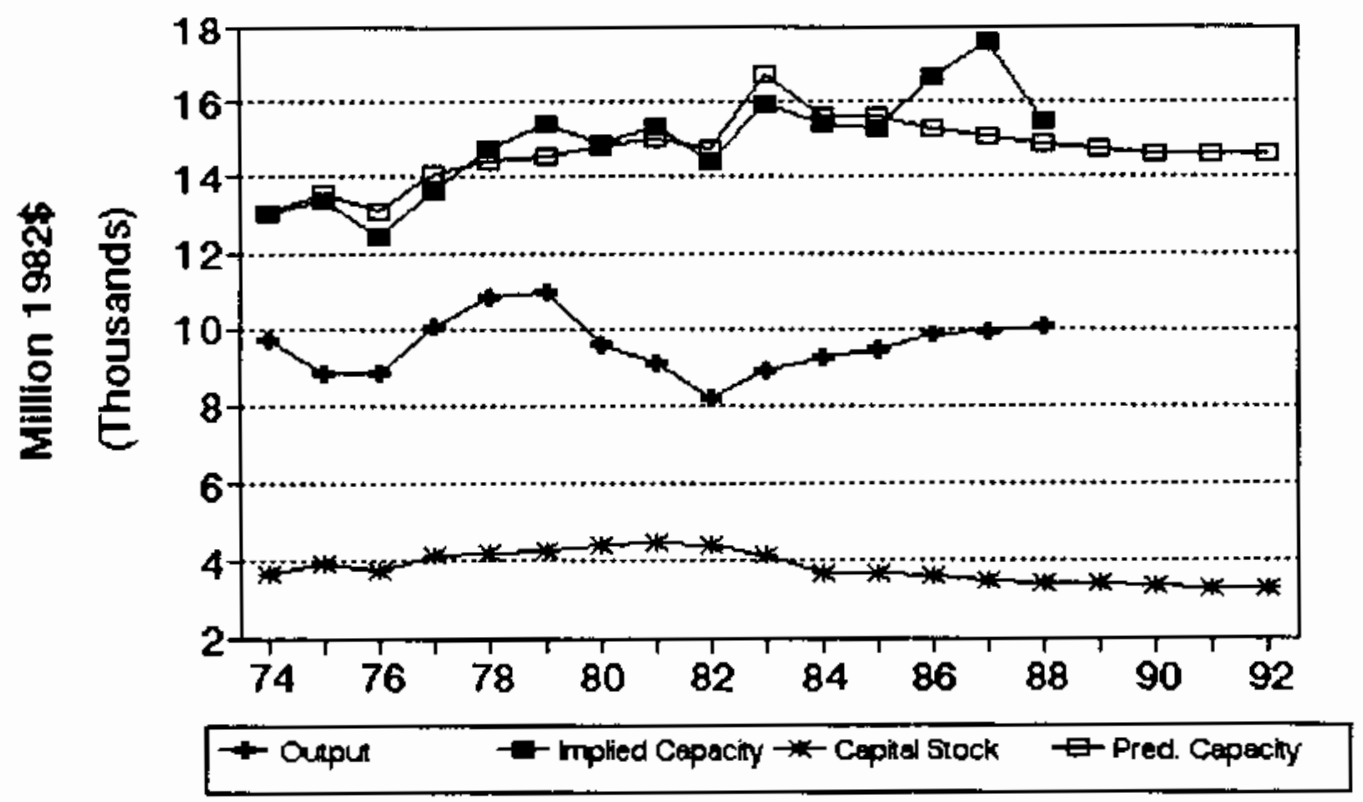


No. 245 SIC 3274 Lime

MILLIONS OF 1982 DOLLARS (EXCL. CI RATES)

$\begin{array}{lccccccc} & \text { Q4 } & \text { Ann. Implied Gross } & \text { Net } & \text { Pred. } & -\ldots-\ldots & -\ldots \\ \text { Gross } & \text { CU } & \text { CU } & \text { Prac. } & \text { In- } & \text { Cap. } & \text { Prac. } & \text { Emergency Capacity } \\ \text { Output Rate } & \text { Rate } & \text { Capac. } & \text { vest. } & \text { Stk. } & \text { Capac. } & 3 \text { mon. } 6 \text { mon. }>6 \text { mon. }\end{array}$

\begin{tabular}{|c|c|c|c|c|c|c|c|c|c|c|}
\hline 1974 & 806 & $79 *$ & 84.8 & 950 & 31.2 & 599 & 880 & & & \\
\hline 1973 & 79 & 76 & 71.2 & 1,123 & 172.3 & 710 & 1,073 & & & \\
\hline 1976 & 761 & $7 *$ & 77.6 & 982 & $(0.3)$ & 649 & 968 & & & \\
\hline 1977 & 736 & $86 \div$ & 81.8 & 926 & 24.2 & 616 & 908 & & & \\
\hline 1978 & aso & 86 & 86.6 & 961 & 118.2 & 671 & 1,005 & & & \\
\hline 1979 & 796 & 81 & B3. 1 & 958 & 72.2 & 676 & 1,011 & & & \\
\hline 1980 & 705 & 58 & 64.2 & 1,099 & 107.8 & 710 & 1,073 & & & \\
\hline 1981 & 712 & 57 & 60.0 & 1,187 & 111.3 & 742 & 1,130 & & & \\
\hline 1982 & 548 & 59 * & 58.0 & 944 & 10.4 & 676 & 1,015 & & & \\
\hline 1983 & 560 & 57 & 59.5 & 940 & 13.8 & 613 & 906 & & & \\
\hline 1984 & $\infty 60$ & 72 & 69.0 & 956 & 92.2 & 629 & 933 & & & \\
\hline 1985 & 688 & 76 & 73.7 & 933 & 116.1 & 663 & 992 & & & \\
\hline 1986 & $6 \pi$ & 76 & 76.5 & 882 & 53.1 & 635 & 943 & & & \\
\hline- - & $\cdots \cdot$ & $\because$ & -- & $-\cdots$ & $\cdots \cdot$ & $\because$ & $\cdots$ & $-=$ & $-\cdot-$ & $\cdots$ \\
\hline 1987 & 675 & 78 & 77.1 & 875 & 56.5 & 611 & 902 & & & \\
\hline 1988 & 675 & 76 & 76.7 & 881 & 67.2 & 601 & 885 & 962 & 1,013 & 1,027 \\
\hline 1989 & & & & & 70.1 & 596 & 875 & $\$ 51$ & 1,002 & 1,015 \\
\hline 1990 & & & & & 71.7 & 592 & 889 & 946 & 995 & 1,008 \\
\hline 1991 & & & & & 87.5 & 605 & 891 & 968 & 1,020 & 1,034 \\
\hline 1992 & & & & & 97.5 & 626 & 928 & 1,008 & 1,063 & \\
\hline
\end{tabular}

Capacity-Capital Stock Regression: Code 1 RSO 0.690

Coefficients: Const -160.5 stock 1.7

Ave. Capacity/stock $(74-86): 1.5$ Ave. Wekly Hours (High) 136.9 Shift Factor 1.16 Ave Weekly Hours (LoW) 136.6 Shift Factor 1.16 * Inputed Note: Output, Investment, and Capital Stock extrapolated for 1987 and 1988.

\section{Lime}
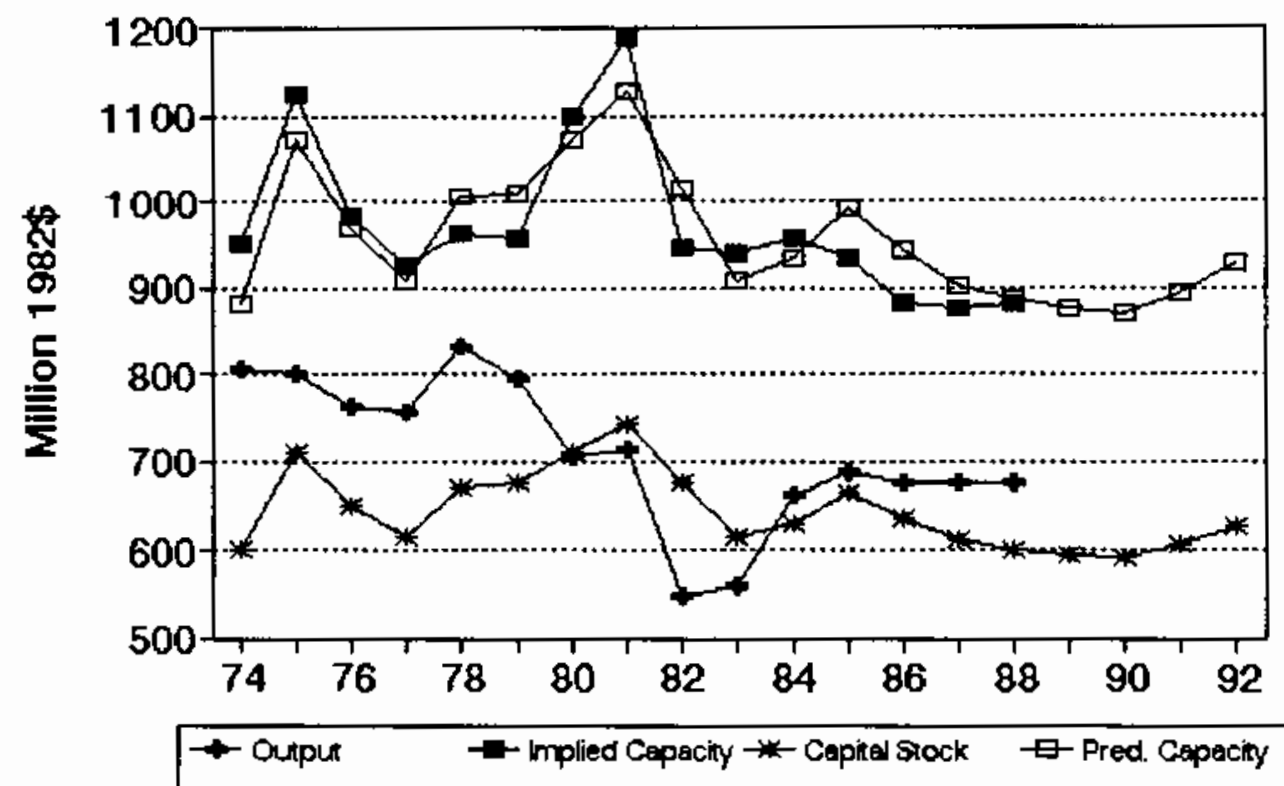
No. 246 SIC 3275 Oypsun products

MILLIONS OF 1982 DOLLARS (EXCL. CU RATES)

$\begin{array}{lccccccc} & \text { Q4 } & \text { Am. Implied Gross } & \text { Net } & \text { Pred. } & \ldots \ldots \\ \text { Gross } & \text { CU } & \text { CU } & \text { Prac. } & \text { In- } & \text { Cap. } & \text { Prac. } & \text { Emergency Capacity } \\ \text { Output } & \text { Rate } & \text { Rate } & \text { Capac. } & \text { vest. } & \text { Stk. } & \text { Capac. } & 3 \text { mon. } 6 \text { mon. }\end{array}$

\begin{tabular}{|c|c|c|c|c|c|c|c|c|c|c|}
\hline 1974 & 1,320 & 77 & 78.8 & 1,676 & 126.6 & $\pi 7$ & 1,593 & & & \\
\hline 1975 & 1,057 & 71 & 71.5 & 1,479 & 11.9 & 695 & 1,574 & & & \\
\hline 1976 & 1,226 & 76 & 74.3 & 1.650 & 36.6 & $\$ 1$ & 1,564 & & & \\
\hline 1977 & 1,375 & 96 & 88.2 & 1,559 & 57.8 & 651 & 1,561 & & & \\
\hline 1978 & 1,545 & 99 & 98.0 & 1,576 & 59.0 & 644 & 1,559 & & & \\
\hline 1979 & 1,572 & 94 & 96.0 & 1,639 & 113.1 & 690 & 1,573 & & & \\
\hline 1980 & 1,331 & 86 & 88.1 & 1,512 & 112.4 & 739 & 1,588 & & & \\
\hline 1981 & 4,313 & 81 & 83.9 & 1,565 & 93.6 & 769 & 1,597 & & & \\
\hline 1982 & $t, 301$ & 92 & 87.9 & 1,480 & 9.1 & 719 & 1,582 & & & \\
\hline 1983 & 1,535 & 94 & 93.9 & 1,635 & 59.6 & 713 & 1,580 & & & \\
\hline 1984 & 1,631 & 87 & 90.9 & 1,793 & 68.7 & 712 & 1,926 & & & \\
\hline 1985 & 1,892 & 94 & 91.1 & 2,078 & 119.0 & 759 & 1,941 & & & \\
\hline 1986 & 1,819 & 92 & 92.9 & 1,957 & 137.4 & 822 & 1,960 & & & \\
\hline$-\cdots$ & $\therefore--$ & $\cdots$ & - - & $-\cdots$ & $\ldots$ & $\cdots$ & $\therefore-$ & $\cdots$ & $-\cdots$ & $=-$ \\
\hline 1987 & 1,854 & 94 & 93.2 & 1,989 & 68.9 & 814 & 1,958 & & & \\
\hline 1988 & 1,900 & 82 & 86.5 & 2,197 & 76.3 & 815 & 1,958 & 2,035 & 2,059 & 2,066 \\
\hline 1989 & & & & & 78.3 & 817 & 1,959 & 2,036 & 2,060 & 2,068 \\
\hline 1990 & & & & & 79.5 & 820 & 1,960 & 2,037 & 2,061 & 2,068 \\
\hline 1991 & & & & & 90.4 & 834 & 1,964 & 2,061 & 2,066 & 2,072 \\
\hline 1992 & & & & & 97.3 & 856 & 1,970 & 2,048 & 2,072 & 2,078 \\
\hline
\end{tabular}

Capacity-Copital Stock Regression: Code -1 RSO 0.790

Coefficients: Const 1359.8 stock $0.3 \quad D(84-86) 346.8$

Ave. Capacity/Stock $(74-86): 2.3$ Ave. Weekly Hours (High) 151.2 Shift Factor 1.05 Ave Weekly Hours (Low) 148.8 Shift factor 1.07 Note: Output, Investment, and Capital Stock extrapolated for 1987 end 1988.

\section{Gypsum products}

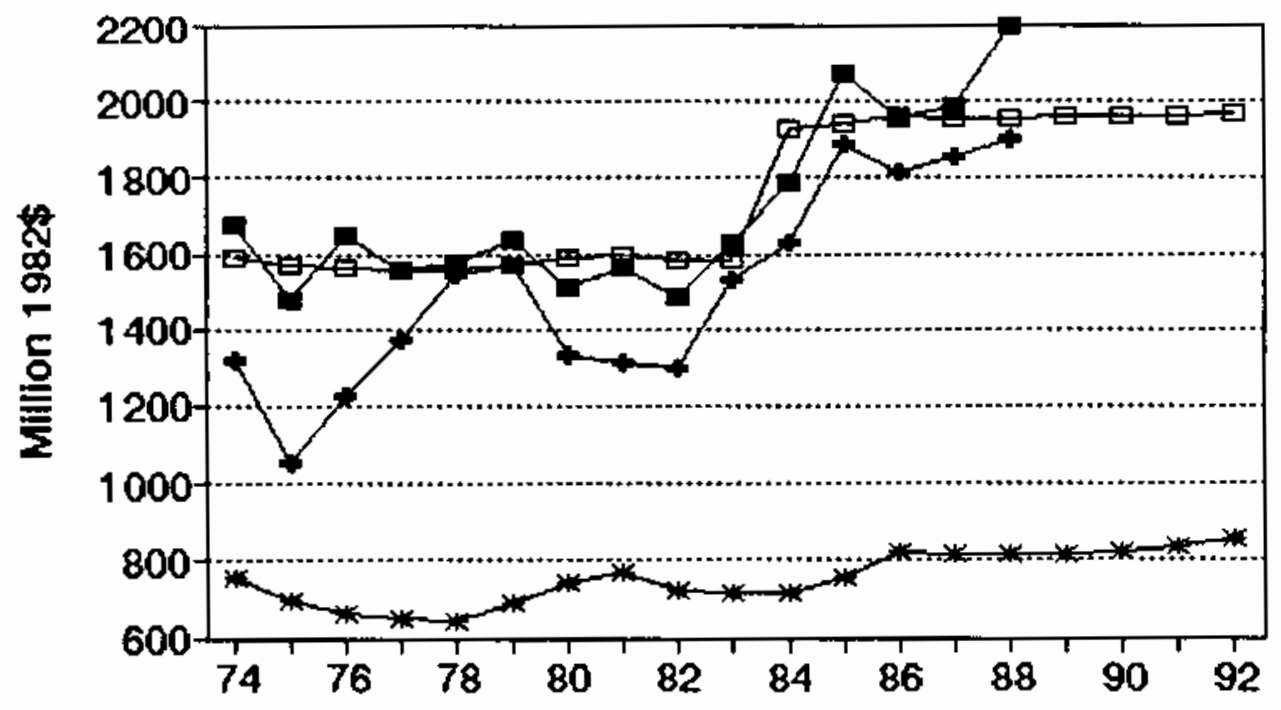

\begin{tabular}{|c|c|c|}
\hline - Oupu & -E knoled Cepaciny $\rightarrow$ - Caphal Stock & Ð-Pred Capaciky \\
\hline
\end{tabular}


No. 247 SIC 3281 Cut stone and stone products

MILLIONS OF 1982 DOLLARS (EXCL. CU RATES)

$\begin{array}{lcccclcc} & \text { Q4 } & \text { Ann. Implied Gross } & \text { Net } & \text { Pred. } & -\ldots \ldots \\ \text { Gross } & \text { CU } & \text { CU } & \text { Prac. } & \text { In- } & \text { Cap. } & \text { Prac. } & \text { Emergency Capacity } \\ \text { Output } & \text { Rate } & \text { Rate } & \text { Capac. } & \text { vest. } & \text { Stk. } & \text { Capac. } & 3 \text { mon. } 6 \text { mon. }>6 \text { mon. }\end{array}$

\begin{tabular}{|c|c|c|c|c|c|c|c|c|c|c|}
\hline 1974 & 573 & 52 & 54.8 & 1,046 & $(0.5)$ & 214 & 788 & & & \\
\hline 1975 & 644 & 100 & 78.3 & $B 22$ & 39.7 & 232 & 786 & & & \\
\hline 1976 & 692 & 98 & 99.5 & 696 & 24.3 & 236 & 785 & & & \\
\hline 1977 & 581 & 82 & 87.4 & 665 & 3.4 & 220 & 783 & & & \\
\hline 1978 & 606 & 98 & 92.3 & 754 & 47.8 & 247 & 781 & & & \\
\hline 1979 & 555 & 90 & 93.2 & 596 & (17.1) & 212 & 779 & & & \\
\hline 1980 & 544 & 80 & 81.7 & 666 & 8.2 & 202 & $\pi 7$ & & & \\
\hline 1981 & 533 & 70 & 76.0 & 702 & 14.9 & 198 & $\pi / 3$ & & & \\
\hline 1982 & 562 & 60 & 63.6 & 893 & 25.6 & 205 & 773 & & & \\
\hline 1983 & 558 & 65 & 64.3 & 869 & 6.6 & 192 & 71 & & & \\
\hline 1984 & 599 & 70 & 70.3 & 852 & 42.8 & 213 & 769 & & & \\
\hline 1985 & 600 & 74 & 71.9 & 833 & 15.0 & 208 & 767 & & & \\
\hline 1986 & 594 & 89 & 83.7 & 710 & 31.3 & 219 & 765 & & & \\
\hline 1987 & $\begin{array}{c}-\cdots \\
593\end{array}$ & $\begin{array}{l}--- \\
86\end{array}$ & $\begin{array}{l}\cdots 7.0 \\
87.0\end{array}$ & $\begin{array}{c}-\cdots \\
681\end{array}$ & 24.9 & $\begin{array}{c}\cdots \\
224\end{array}$ & $\begin{array}{c}\cdots \\
763\end{array}$ & $-\cdot-\cdot-$ & $\cdots \cdot$ & $-\cdot-$ \\
\hline 1988 & 591 & 80 & 82.2 & 720 & 25.8 & 229 & 761 & 1,485 & 1,646 & 1,688 \\
\hline 1989 & & & & & 26.8 & 234 & 759 & 1,481 & 1,642 & 1,683 \\
\hline 1990 & & & & & 27.7 & 240 & 57 & 1,477 & 1,637 & 1,679 \\
\hline 1991 & & & & & 28.7 & 246 & 755 & 1,473 & 1,633 & 1,674 \\
\hline 1992 & & & & & 29.6 & 253 & 753 & 1,469 & 1,629 & 1,670 \\
\hline
\end{tabular}

Capacity-Capital Stock Regression: Code -2 RSO 0.000

Coefficients: Const 790.4 Time -2.0

Ave. Capacity/Stock $(74-86): 3.6$ Ave. Weekty Hours (High) 71.0 Shift factor 2.22 Ave Weekly Hours (Low) 70.2 Shift Factor 2.24 Note: Output, Investment, and Capital Stock extrapolated for 1987 and 1988.

\section{Cut stone and stone products}

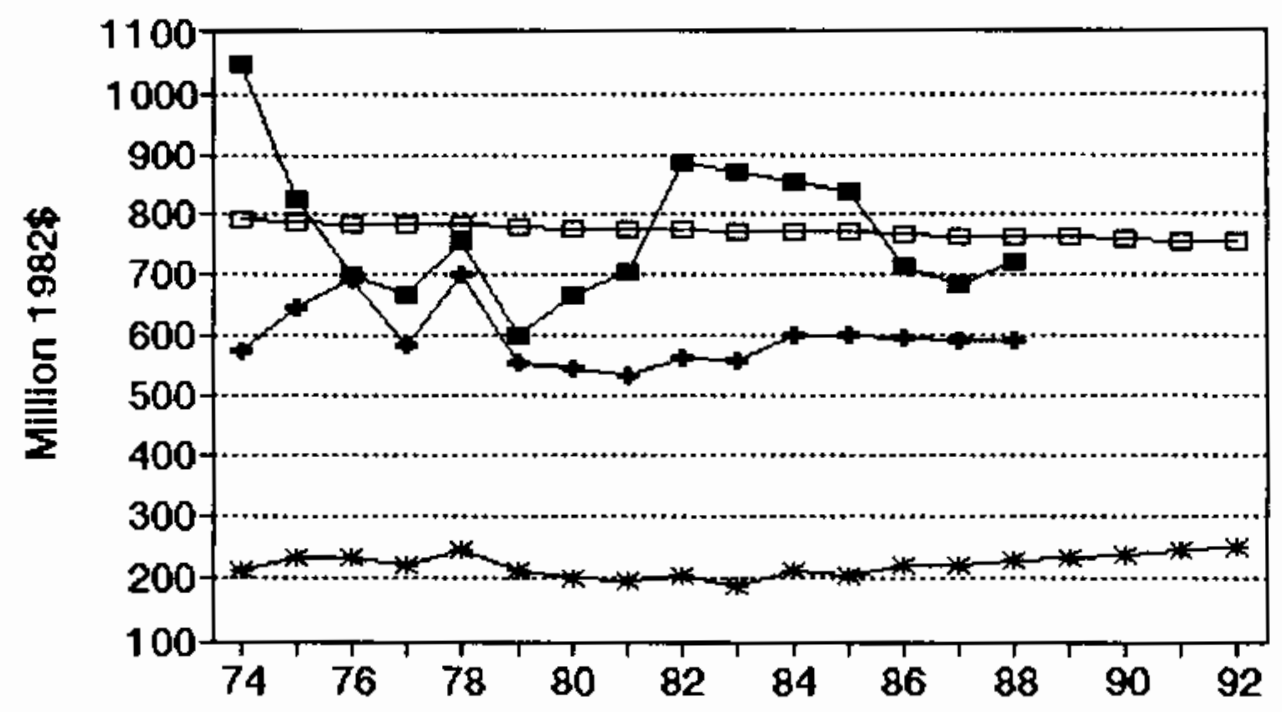

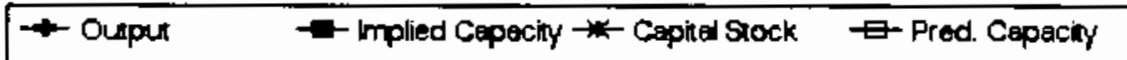




\section{No. 248 SIC 3291 Abrasive products}

MILLIONS OF 1982 DOLLARS (EXCL. CU RATES)

$\begin{array}{lccccccc} & \text { Q4 } & \text { Am. Implied } & \text { Gross } & \text { Met } & \text { Pred. } & \ldots \ldots \ldots \\ \text { Gross } & \text { CU } & \text { CU } & \text { Prac. } & \text { In- } & \text { Cap. } & \text { Prac. } & \text { Emergency Capacity } \\ \text { Output } & \text { Rate } & \text { Rate } & \text { Capac. } & \text { vest. } & \text { 5tk. } & \text { Capac. } & 3 \text { mon. } 6 \text { mon. }>6 \text { mon. }\end{array}$

\begin{tabular}{|c|c|c|c|c|c|c|c|c|c|c|}
\hline 1974 & 2,542 & 83 & 87.1 & 2,918 & 30.0 & 601 & 3,186 & & & \\
\hline 1975 & 2,219 & 62 & 65.9 & 3,367 & 91.1 & 629 & 3,425 & & & \\
\hline 1976 & 2,436 & 78 & 72.3 & 3,372 & 49.6 & 618 & 3,332 & & & \\
\hline 1977 & 3,091 & $\pi$ & 75.6 & 4,089 & 170.1 & 717 & 4,179 & & & \\
\hline 1978 & 3,186 & 75 & 75.3 & 4,233 & 81.5 & 727 & 4,266 & & & \\
\hline 1979 & 3,522 & 83 & 80.2 & 4,392 & 112.1 & 767 & 4,611 & & & \\
\hline 1980 & 3,239 & $\pi$ & 76.3 & 4,247 & 123.4 & 817 & 5,043 & & & \\
\hline 1981 & 3,354 & 67 & 72.0 & 4,662 & 78.1 & 818 & 5,051 & & & \\
\hline 1982 & 2,783 & 53 & 58.1 & 4,790 & 38.8 & 783 & 4,746 & & & \\
\hline 1983 & 3,083 & 64 & 60.7 & 5,080 & 46.5 & 754 & 4,502 & & & \\
\hline 1984 & 3,715 & 68 & 68.5 & 5,426 & 102.7 & 782 & 4,736 & & & \\
\hline 1985 & 3,445 & 66 & 66.3 & 5,199 & 69.6 & 776 & 4.686 & & & \\
\hline 1986 & 3,211 & 69 & 68.2 & 4.711 & 81.6 & 780 & 4,722 & & & \\
\hline$-\cdots$ & $\cdots \cdot$ & -- & $\cdots$ & 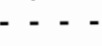 & $\cdots$ & $-\cdot$ & $\cdots$ & -- & -- & - - \\
\hline 1987 & 3,281 & 76 & 73.3 & 4,475 & 74.6 & 778 & 4,705 & & & \\
\hline 1988 & 3,374 & 76 & 75.9 & 4,445 & 78.5 & 780 & 4,722 & 7,317 & 8,028 & 8,212 \\
\hline 1989 & & & & & 79.5 & 783 & 4,747 & 7,356 & 8,071 & 8,256 \\
\hline 1990 & & & & & 80.1 & $78 S$ & $4, \pi 7$ & 7,399 & 8,118 & 8,304 \\
\hline 1991 & & & & & 85.9 & 795 & 4,850 & 7,515 & 8,245 & 8,434 \\
\hline 1992 & & & & & 89.5 & 806 & 4,949 & 7,668 & 8,413 & 8,606 \\
\hline
\end{tabular}

Capacity-Capital Stock Regression: Code 0 RSO 0.710

Coefficients: Const -1992.1 Stock 8.6

Ave. Copacity/Stock $(74-86): 5.9$

Ave. Weekly Hours (High) 90.6 Shift Factor 1.74 Ave Weekly Hours (Low) 81.3 Shift factor 1.94 Note: Output, Investment, and Capital Stock extrapolated for 1987 and 1988.

\section{Abrasive products}

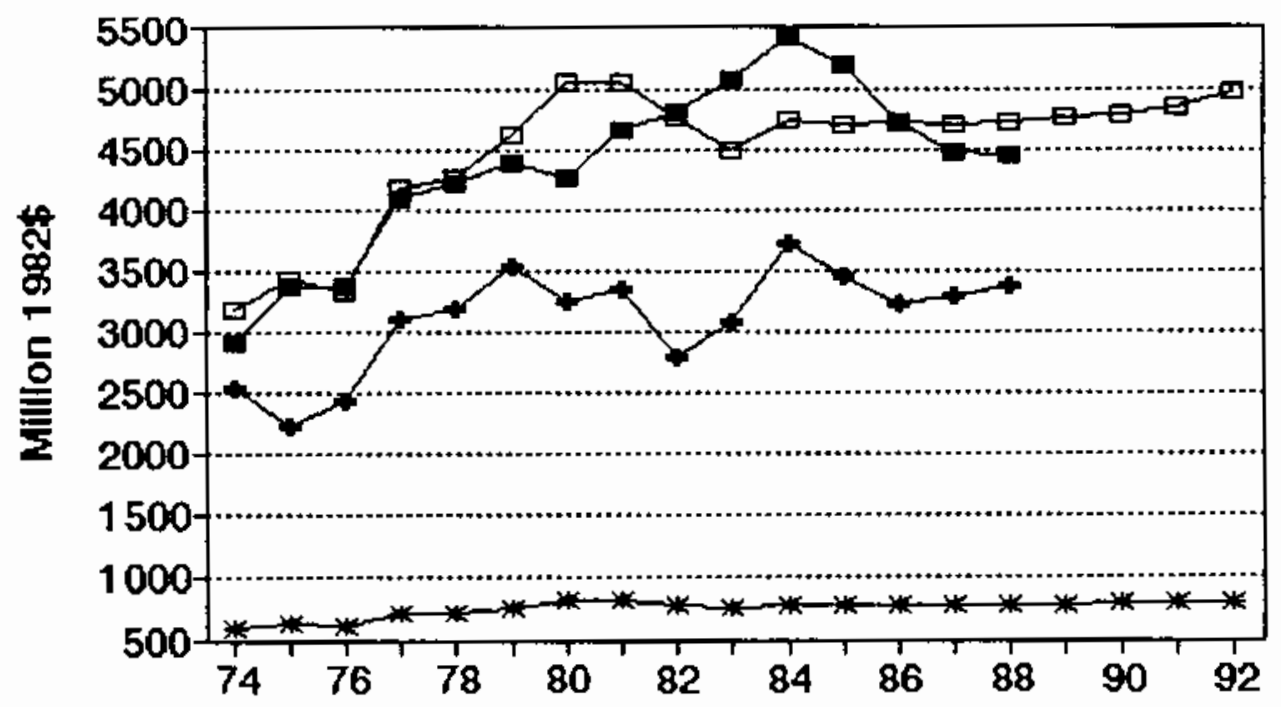

Oupu

t- Impled Cepeclyy $\rightarrow$ - Copied Siock

๑ Pred. Cepechy 
No. 249 aIc 3292 Aabestos products

MILLIONS OF 1982 DOLLARS (EXCL. CU RATES)

$\begin{array}{lcccclcc} & \text { Q4 Am. Implied Gross } & \text { Net } & \text { Pred. } & -\ldots \ldots \\ \text { Gross } & \text { a } & \text { CU } & \text { Prac. } & \text { In- } & \text { Cap. } & \text { Prac. } & \text { Emergency Capacity } \\ \text { Output } & \text { Rate } & \text { Rate } & \text { Capac. } & \text { vest. } & \text { Stk. } & \text { Capac. } & 3 \text { mon. } 6 \text { mon. }>6 \text { mon. }\end{array}$

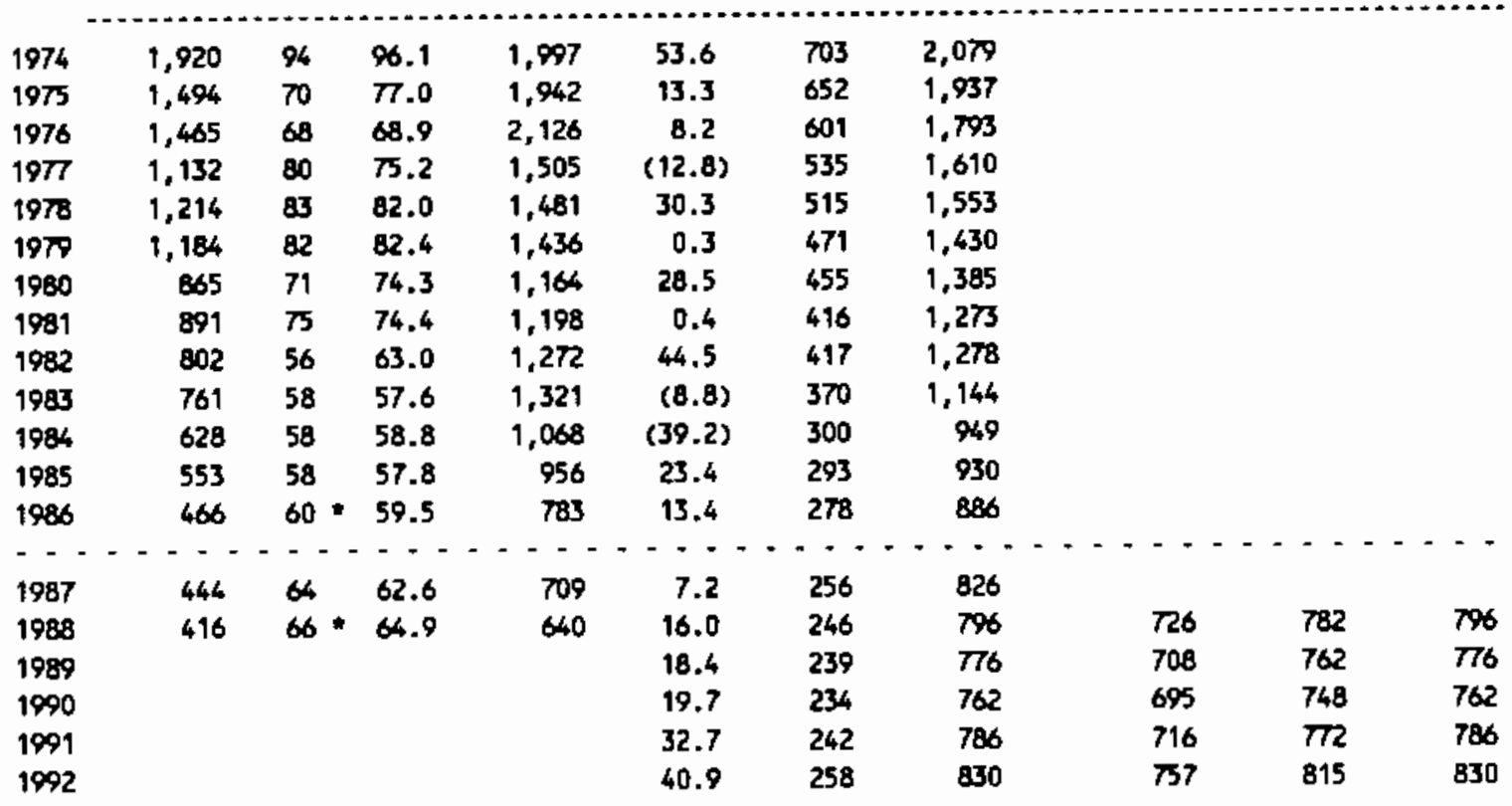

Capacity-Capital stock Regression: Code 0 RSO 0.880 Coefficients: Const 106.0 stock 2.8

Ave. Capacity/stock $(74-86): 3.0$ Ave. Weekly Hours (High) 1983.0 shift Factor 1.00 Ave Weekly Hours (Lon) 1983.0 Shift factor 1.0 * Imputed Mote: Output, Investment, and Capital stock extrapolated for 1987 and 1988.

3292 Asbestos products

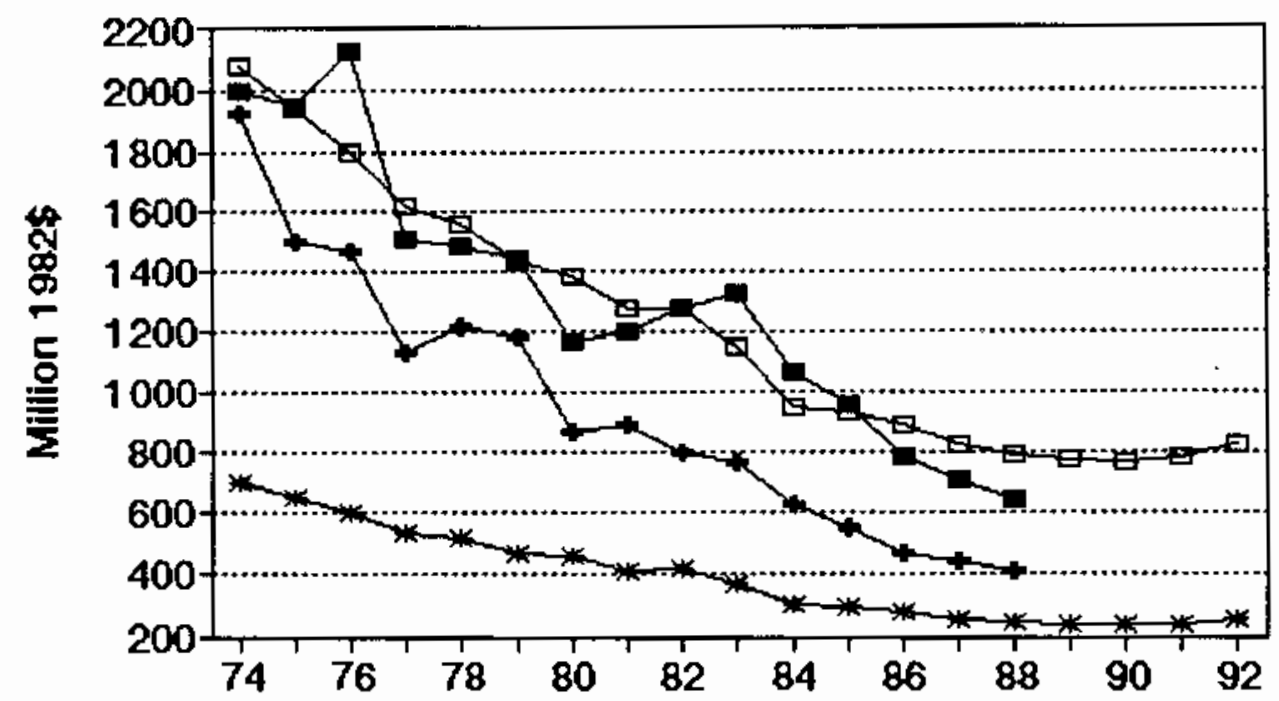

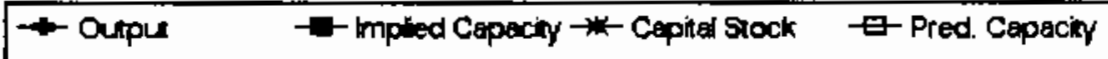




\section{No. 250 BIC 3293 Gaskets, packing and sealing devices}

MILLIONS OF 1982 DOLLARS (EXCL. CU RATES)

$\begin{array}{lccccccc} & \text { Q4 } & \text { Amn. Implied Gross } & \text { Het } & \text { Pred. } & \ldots \ldots & \ldots \\ \text { Grogs } & \text { CU } & \text { CU } & \text { Prac. } & \text { In- } & \text { Cap. } & \text { Prac. } & \text { Emergency Capacity } \\ \text { Output } & \text { Rate } & \text { Rate } & \text { Capac. } & \text { vest. } & \text { Stk. } & \text { Capac. } & 3 \text { mon. } 6 \text { mon. }>6 \text { mon. }\end{array}$

\begin{tabular}{|c|c|c|c|c|c|c|c|c|c|c|}
\hline 1974 & 2,010 & 85 & 92.3 & 2,177 & 59.4 & 401 & 2,259 & & & \\
\hline 1975 & 1,741 & 79 & 73.7 & 2,362 & 6.4 & 427 & 2,467 & & & \\
\hline 1976 & 2,046 & 79 & 80.0 & 2,558 & 43.7 & 432 & 2,503 & & & \\
\hline 1977 & 2,287 & 81 & 79.3 & 2,885 & 97.4 & 486 & 2,927 & & & \\
\hline 1978 & 2,415 & 79 & 80.3 & 3,009 & 74.8 & 514 & 3,153 & & & \\
\hline 1979 & 2,490 & 62 & 68.5 & 3,636 & 62.9 & 529 & 3,265 & & & \\
\hline 1980 & 1,978 & 56 & 56.1 & 3,523 & 43.3 & 523 & 3,225 & & & \\
\hline 1961 & 1,920 & 51 & 55.8 & 3,444 & 50.8 & 524 & 3,230 & & & \\
\hline 1982 & 1,650 & 38 & 42.6 & 3,870 & 149.9 & 610 & 3,909 & & & \\
\hline 1983 & 1,815 & 54 & 69.2 & 3,693 & 30.6 & 577 & 3,643 & & & \\
\hline 1984 & 2,044 & 63 & 62.6 & 3,268 & 10.5 & 528 & 3,263 & & & \\
\hline 1985 & 2,133 & $\infty$ & 64.1 & 3,328 & 87.9 & 557 & 3,489 & & & \\
\hline 1986 & 2,098 & 69 & 68.6 & 3,066 & 59.5 & 557 & 3,486 & & & \\
\hline $\begin{array}{l}\cdots \\
1987\end{array}$ & 2,097 & 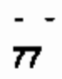 & 73. & 2,838 & 72.2 & $\begin{array}{l}--\cdot \\
569\end{array}$ & 3,586 & $\cdots$ & -- & $\cdots$ \\
\hline 1988 & 2,098 & $\pi$ & 73.8 & 2,843 & 74.0 & 582 & 3,689 & 3,981 & 4,320 & 4,408 \\
\hline 1989 & & & & & 75.8 & 596 & 3,795 & 4,095 & 4,464 & 4,535 \\
\hline 1990 & & & & & $n .5$ & 609 & 3,903 & 4,212 & 4,571 & 4,664 \\
\hline 1991 & & & & & 79.3 & 624 & 4,014 & 4,332 & 4,701 & 4,797 \\
\hline 1992 & & & & & 81.1 & 638 & 4,127 & 4,453 & 4,833 & 4,931 \\
\hline
\end{tabular}

Capacity-Capital Stock Regression: Code 0 RSO 0.840 Coefficients: Const -900.8 stock 7.9 Ave. Weekly Hours (High) 132.7 Shift Factor 1.20 Ave Heekly Hours (Low) 126.2 Shift Factor 1.25 Note: Output, Imvestment, and Capital Stock extrapolated for 1987 and 1988.

\section{Gaskets, packing and sealing devic}

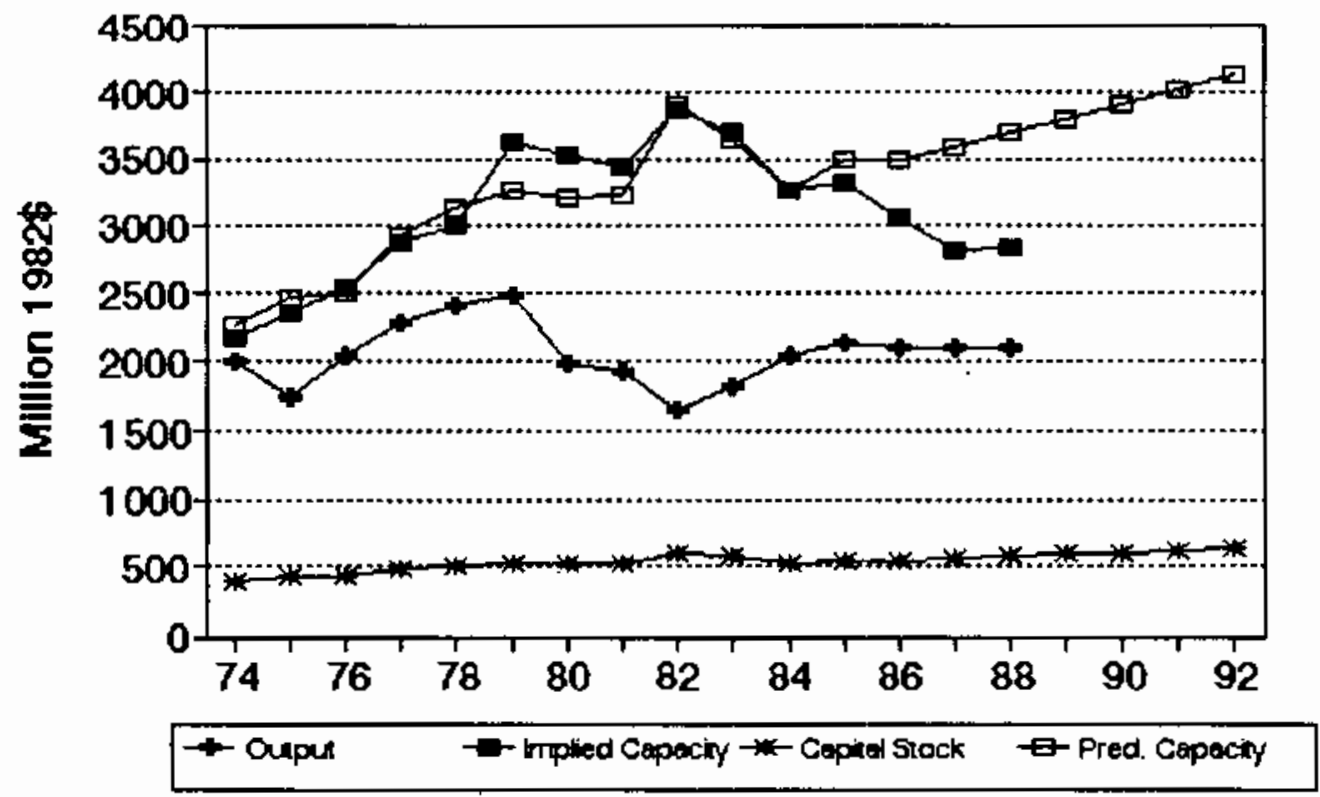




\section{No. 251 BIC 3295 Minerals, ground or treated}

MILLIONS OF 1982 DOLLARS (EXCL. CU RATES)

$\begin{array}{lccccccc} & \text { Q4 } & \text { Ann. Implied } & \text { Gross } & \text { Net } & \text { Pred. } & \ldots \\ \text { Gross } & \text { CU } & \text { CU } & \text { Prac. } & \text { In- } & \text { Cap. } & \text { Prac. } & \text { Emergency Capacity } \\ \text { Output Rate } & \text { Rate } & \text { Capac. } & \text { vest. } & \text { Stk. } & \text { Capac. } & 3 \text { mon. } 6 \text { mon. }>6 \text { mon. }\end{array}$

\begin{tabular}{|c|c|c|c|c|c|c|c|c|c|c|}
\hline 1974 & 1,442 & 83 & 83.5 & 1,727 & 117.6 & 693 & 1,534 & & & \\
\hline 1975 & 1,423 & 83 & 82.5 & 1,725 & 202.9 & 828 & 1,978 & & & \\
\hline 1976 & 1,781 & 90 & 87.4 & 2,037 & 130.1 & 887 & 2,172 & & & \\
\hline 1977 & 1,795 & 58 & 70.0 & 2,566 & 3.8 & 823 & 1,961 & & & \\
\hline 1978 & 2,059 & 61 & 59.9 & 3,437 & 168.1 & 913 & 2,259 & & & \\
\hline 1979 & 2,023 & 89 & 78.5 & 2,576 & 68.1 & 903 & 2,225 & & & \\
\hline 1980 & 1,457 & 84 & 85.6 & 1,702 & 65.8 & 892 & 2,188 & & & \\
\hline 1981 & 1,315 & 62 & 70.5 & 1,867 & 45.4 & 861 & 2,087 & & & \\
\hline 1982 & 1,306 & 71 & 67.6 & 1,931 & 80.1 & 859 & 2,081 & & & \\
\hline 1983 & 1,388 & 80 & 76.7 & 1,808 & 182.6 & 940 & 2,346 & & & \\
\hline 1984 & 1,200 & 64 & 70.3 & 1,707 & 7.0 & 849 & 2,049 & & & \\
\hline 1985 & 1,066 & 69 & 67.1 & 1,590 & $(20.3)$ & 743 & 1,700 & & & \\
\hline 1986 & 1,116 & 79 & $\pi .3$ & 1,482 & 47.5 & 706 & 1,576 & & & \\
\hline & $\cdots$ & $\cdots$ & -- & & $\cdots \cdot$ & -- & $\cdots$ & $=-$ & $-\cdots$ & $\cdots \cdot$ \\
\hline 1987 & 1,114 & 82 & 80.9 & 1,378 & 50.0 & 673 & 1,467 & & & \\
\hline 1988 & 1,112 & 90 & 87.0 & 1,279 & 50.0 & 644 & 1,371 & 1,544 & 1,622 & 1,642 \\
\hline 1989 & & & & & 50.0 & 618 & 1,286 & 1,449 & 1,522 & 1,541 \\
\hline 1990 & & & & & 50.0 & 595 & 1,211 & 1,364 & 1,433 & 1,451 \\
\hline 1991 & & & & & 50.0 & 573 & 1,145 & 1,290 & 1,355 & 1,372 \\
\hline 1992 & & & & & 50.0 & 557 & 1,087 & 1,224 & 1,286 & 1,302 \\
\hline
\end{tabular}

Capacity-Capital Stock Regression: Code 0 RSQ 0.230

Coefficients: Const -748.7 Stock 3.3

Ave. Capacity/Stock $(74-86): 2.4$ Ave. Weekly Hours (High) 132.4 Shift Factor 1.20 Ave Weekly Hours (Low) 122.8 Shift Factor 1.29 Note: Output, Investment, and Cepital Stock extrapolated for 1987 and 1988.

\section{Minerals, ground or treated}

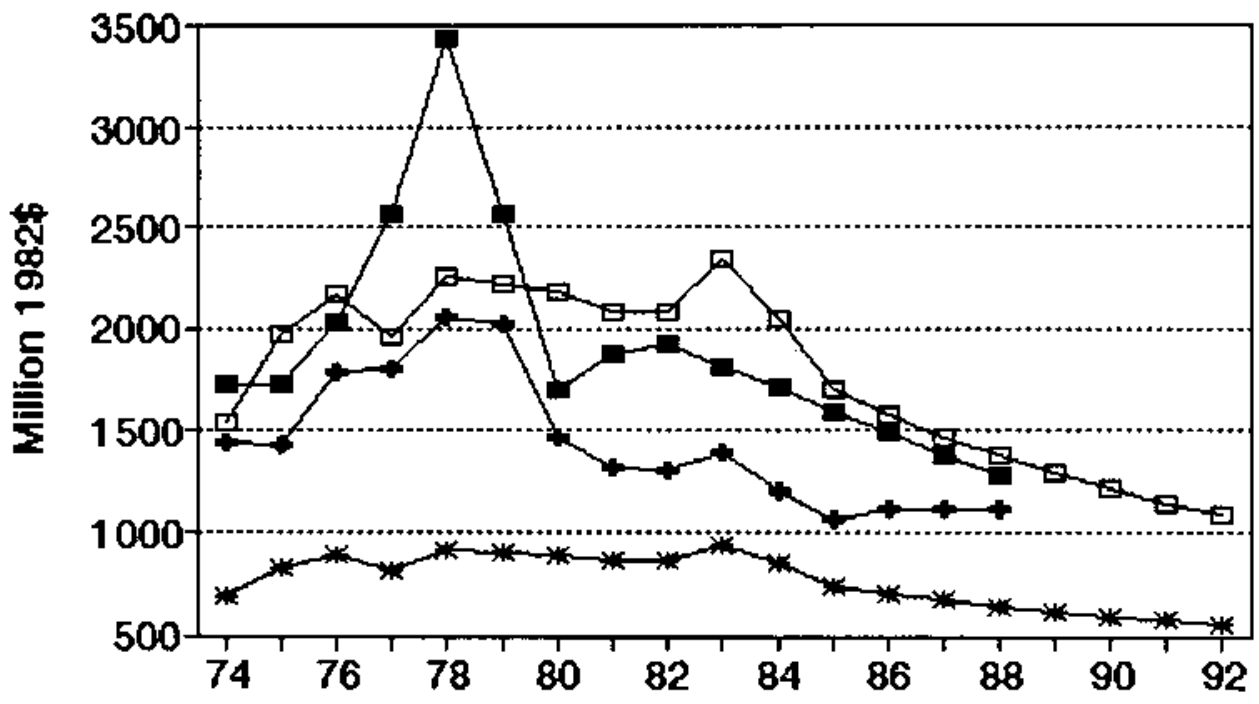

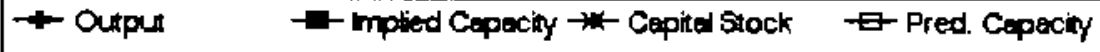


Ho, 252 sIC 3296 Mineral wool

MILLIONS OF 1982 doLlaRS (EXCL. CU RATES)

$\begin{array}{lccccccc} & 04 & \text { Ann. Implied Gross } & \text { Net } & \text { Pred. } & -\ldots . \ldots \\ \text { Gross } & \text { CU } & \text { CU } & \text { Prac. } & \text { In- } & \text { Cap. } & \text { Prac. } & \text { Emergency Capacity } \\ \text { Output } & \text { Rate } & \text { Rate } & \text { Capac. } & \text { vest. } & \text { Stk. } & \text { Capac. } & 3 \text { mon. } 6 \text { mon. }>6 \text { mon. }\end{array}$

\begin{tabular}{|c|c|c|c|c|c|c|c|c|c|c|}
\hline 1974 & 2,374 & 80 & 85.7 & 2,770 & 156.2 & 942 & 2,652 & & & \\
\hline 1975 & 2,034 & 81 & 74.6 & 2,726 & 155.6 & 1,023 & 2,866 & & & \\
\hline 1976 & 2,293 & 89 & 86.7 & 2,644 & $7 B .8$ & 1,020 & 2,860 & & & \\
\hline 1977 & 2,657 & 92 & 90.0 & 2,954 & 114.2 & 1,051 & 2,941 & & & \\
\hline 1978 & 2,865 & 92 & 92.5 & 3,098 & 234.9 & 1,199 & 3,332 & & & \\
\hline 1979 & 3,002 & 91 & 91.6 & 3,277 & 188.3 & 1,285 & 3,559 & & & \\
\hline 1980 & 2,728 & 5 & 78.3 & 3,483 & 131.9 & 1,306 & 3,615 & & & \\
\hline 1981 & 2,589 & 60 & 68.3 & 3,791 & 95.4 & 1,281 & 3,551 & & & \\
\hline 1982 & 2,277 & 64 & 62.5 & 3,641 & 71.7 & 1,225 & 3,402 & & & \\
\hline 1983 & 2,467 & 76 & 73.2 & 3,369 & 80.2 & 1,168 & 3,251 & & & \\
\hline 1984 & 2,923 & 85 & 85.0 & 3,438 & 104.9 & 1,135 & 3,164 & & & \\
\hline 1985 & 3,131 & 84 & 83.5 & 3.749 & 167.6 & 1,164 & 3,035 & & & \\
\hline 1986 & 3,354 & 83 & 83.9 & 3,999 & 166.1 & 1,194 & 3,913 & & & \\
\hline$\cdots$ & --- & $\because$ & $\cdot-$ & $-\cdots$ & $\cdots$ & $\cdots \cdot$ & $\cdots$ & $-\cdots$ & $\cdots$ & -- \\
\hline 1987 & 3,428 & 86 & 84.8 & 4,044 & 115.1 & 1,170 & 3,850 & & & \\
\hline 1988 & 3,526 & 88 & 87.1 & 4,048 & 128.6 & 1,162 & 3,829 & 3,779 & 3,825 & 3,837 \\
\hline 1989 & & & & & 132.2 & 1,159 & 3,821 & 3,771 & 3,817 & 3,828 \\
\hline 1990 & & & & & 134.4 & 1,158 & 3,819 & 3,769 & 3,815 & 3,826 \\
\hline 1991 & & & & & 154.3 & 1,178 & 3,870 & 3,820 & 3,866 & 3,878 \\
\hline 1992 & & & & & 167.0 & 1,207 & 3,949 & 3,897 & 3,944 & 3,957 \\
\hline
\end{tabular}

Capacity-Capital Stock Regression: Code -1 RSO 0.810

Coefficients: Const 161.2 stock $2.6 \mathrm{O}(85-86) 593.6 \quad$ Ave. Capacity/Stock $(74-86): 2.9$ Ave. Weekly Hours (High) 164.6 Shift Factor 1.00 Ave Weekly Hours (Low) 156.7 Shift Factor 1.05 Note: Output, Investment, and Cepital 5tock extrapolated for 1987 and 1988.

\section{Mineral wool}

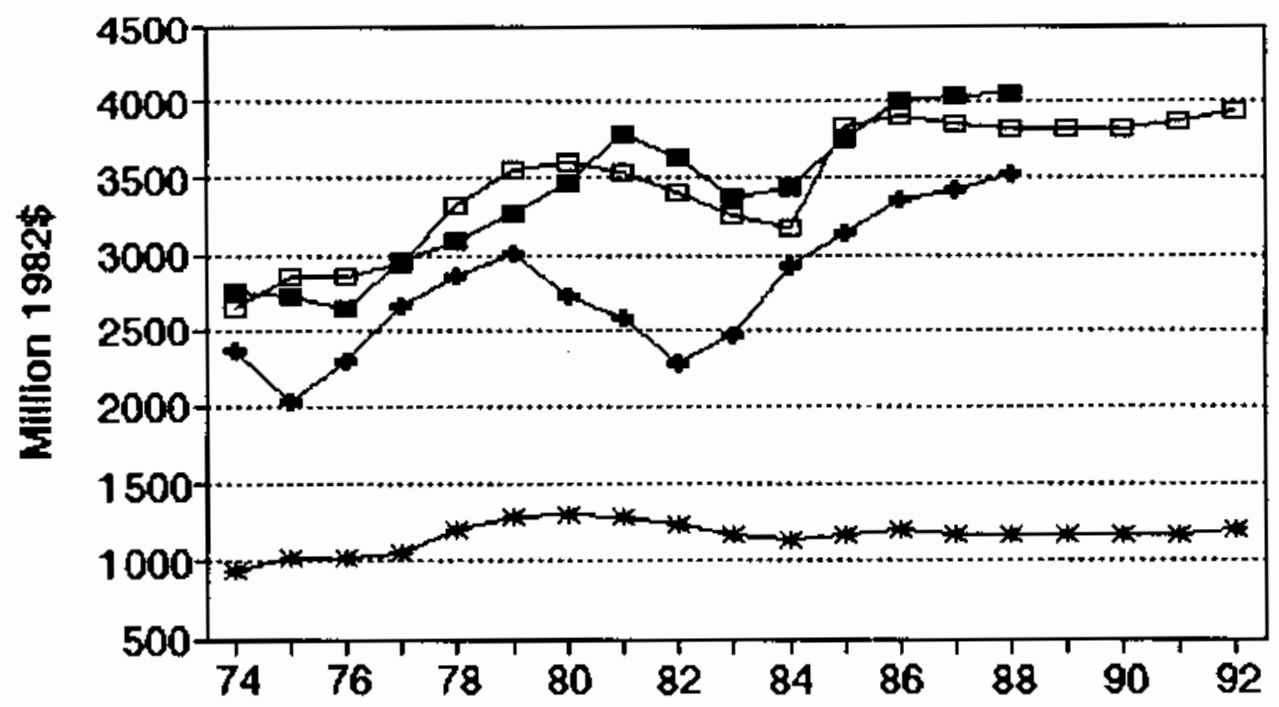

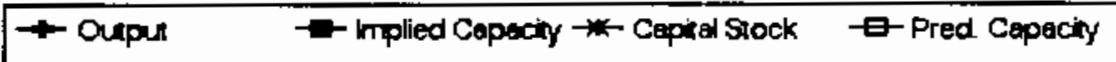


yo. 253 sIC 3297 Nonclay refractories

MILLIONS OF 1982 DOLLARS (EXCL. CU RATES)

$\begin{array}{lccccccc} & \text { Q4 Ann. Implied } & \text { Gross } & \text { Het } & \text { Pred. } & \ldots \ldots \ldots \\ \text { Gross } & \text { CU } & \text { CU } & \text { Prac. } & \text { In- } & \text { Cap. } & \text { Prac. } & \text { Emergency Capacity } \\ \text { Output } & \text { Rate } & \text { Rate } & \text { Capac. } & \text { vest. } & \text { Stk. } & \text { Capac. } & 3 \text { mon. } 6 \text { mon. }>6 \text { mon. }\end{array}$

\begin{tabular}{|c|c|c|c|c|c|c|c|c|c|c|}
\hline 1976 & 1,500 & 58 & 64.4 & 2,330 & 93.1 & 497 & 1,846 & & & \\
\hline 1975 & 1,085 & 53 & 48.2 & 2,250 & 36.6 & 487 & 1,814 & & & \\
\hline 1976 & 1,149 & 56 & 55.7 & 2,062 & 42.0 & 482 & 1,797 & & & \\
\hline 1977 & 1,111 & 78 & 68.6 & 1,621 & 27.6 & 466 & 1,746 & & & \\
\hline 1978 & 1,322 & 85 & 82.9 & 1,594 & 54.3 & 477 & 1,779 & & & \\
\hline 1979 & 1,301 & 74 & 78.4 & 1,660 & 36.5 & 467 & 1,746 & & & \\
\hline 1980 & 1,220 & 61 & 62.7 & 1,945 & 119.3 & 532 & 1,963 & & & \\
\hline 1981 & 1,118 & 58 & 63.7 & 1,756 & 53.0 & 530 & 1,958 & & & \\
\hline 1982 & 666 & 32 & 41.1 & 1,619 & 62.7 & 536 & 1.977 & & & \\
\hline 1983 & 578 & 53 & 46.5 & 1,245 & $(109.2)$ & 384 & 1,468 & & & \\
\hline 1984 & 628 & 66 & 64.9 & 969 & $(87.7)$ & 267 & 1,077 & & & \\
\hline 1985 & 682 & 66 & 65.0 & 1,049 & 19.9 & 258 & 1,047 & & & \\
\hline 1986 & 701 & 62 & 63.9 & 1,097 & 8.7 & 239 & 981 & & & \\
\hline $\begin{array}{l}-7- \\
1987\end{array}$ & $\begin{array}{c}--- \\
686\end{array}$ & $\begin{array}{l}-\cdots \\
60\end{array}$ & 60.5 & $\because \ldots$ & $=$ & $\begin{array}{l}-\cdots \\
213\end{array}$ & $\begin{array}{c}\cdots \\
896\end{array}$ & $-\cdots$ & $-\cdots$ & -- \\
\hline 1988 & 667 & $65 *$ & 63.2 & 1,055 & 23.0 & 212 & 890 & 892 & 932 & 943 \\
\hline 1989 & & & & & 28.6 & 216 & 904 & 906 & 947 & 958 \\
\hline 1990 & & & & & 31.8 & 223 & 927 & 929 & 971 & 982 \\
\hline 1991 & & & & & 62.4 & 259 & 1,050 & 1,052 & 1,100 & 1,112 \\
\hline 1992 & & & & & 81.7 & 311 & 1,224 & 1,226 & 1,282 & 1,296 \\
\hline
\end{tabular}

Capacity-Capital Stock Regression: Code 0 RSO 0.670 Coefficients: Const 181.1 stock 3.4

Ave. Capacity/Stock $(74-86): 3.8$ Ave. Weekly Hours (High) 150.6 Shift Factor 1.06 Ave Weekly Hours (LOW) 150.6 Shift Factor 1.06 * Imputed Note: Output, Investment, and Capital stock extrapolated for 1987 and 1988.

\section{Nonclay refractories}

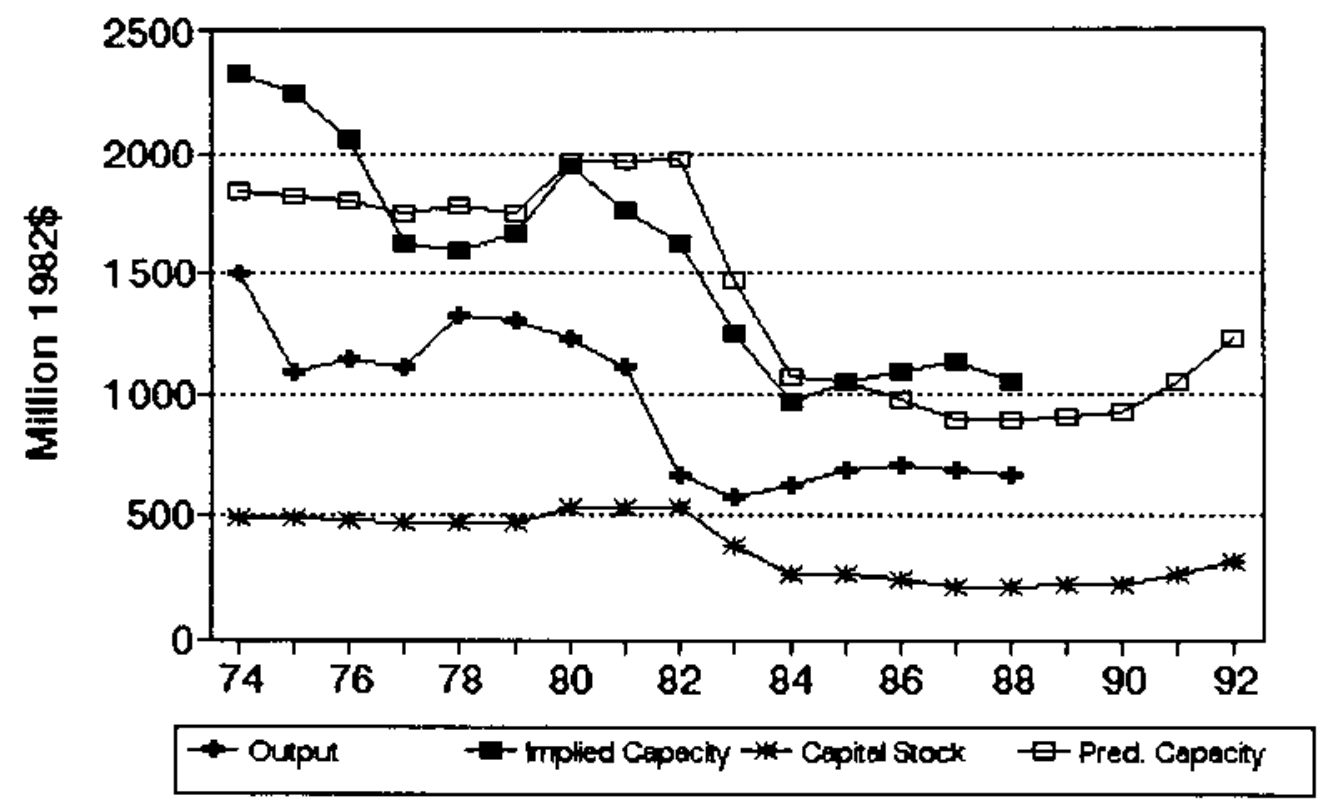


No. 254 sIC 3299 Nonmetallic nineral products, n.e.c.

MILLIOWS OF 1982 DOLLARS (EXCL. CU RATES)

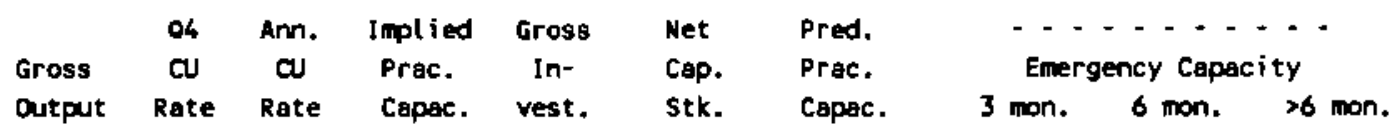

\begin{tabular}{|c|c|c|c|c|c|c|c|c|c|c|}
\hline 1974 & 497 & 81 & 88.5 & 562 & 69.2 & 196 & 648 & & & \\
\hline 1975 & 460 & 86 & 76.0 & 606 & $(37.4)$ & 150 & 562 & & & \\
\hline 1976 & 565 & 73 & 79.3 & 713 & 92.3 & 230 & 712 & & & \\
\hline 1977 & 628 & 73 & 72.1 & 871 & 52.4 & 267 & 781 & & & \\
\hline 1978 & 620 & 94 & 86.5 & 717 & 32.6 & 284 & 814 & & & \\
\hline 1979 & 653 & $B 2$ & 86.7 & 753 & 29.6 & 296 & 837 & & & \\
\hline 1980 & 619 & 59 & 64.6 & 959 & 62.7 & 342 & 923 & & & \\
\hline 1981 & 492 & 53 & 58.6 & 841 & $(17.8)$ & 306 & 855 & & & \\
\hline 1982 & 440 & 37 & 42.7 & 1,031 & (19.5) & 270 & 787 & & & \\
\hline 1983 & 388 & 74 & 60.8 & 638 & 16.9 & 268 & 784 & & & \\
\hline 1986 & 556 & 69 & 75.2 & 740 & 19.1 & 266 & 780 & & & \\
\hline 1985 & 566 & 70 & 68.7 & 824 & 42.3 & 284 & 814 & & & \\
\hline 1986 & 576 & 67 & 68.7 & 839 & 16.9 & 275 & 797 & & & \\
\hline - & $\because 585$ & $\ddot{68}$ & . & Fs6 & $\begin{array}{r}\cdots \\
20.9\end{array}$ & $\because 271$ & $\cdots$ & $\cdots$ & -- & -- \\
\hline 1988 & $5 \%$ & $\pi$ & 73.4 & 811 & 25.8 & 273 & 793 & 931 & 992 & 1,008 \\
\hline 1989 & & & & & 27.1 & 275 & 798 & 937 & 998 & 1,014 \\
\hline 1990 & & & & & 27.8 & 279 & 806 & 944 & 1,005 & 1,021 \\
\hline 1991 & & & & & 35.0 & 289 & 822 & 966 & 1,029 & 1,045 \\
\hline 1992 & & & & & 39.5 & 302 & 848 & 996 & 1,061 & 1,078 \\
\hline
\end{tabular}

Capacity-Capital stock Regression: Code 0 RSO 0.460

Coefficients: Const 279.2 stock 1.9

Ave. Capacity/stock $(74-86): 2.9$

Ave. Weekly Hours (High) 126.5 Shift factor 1.27 Ave Weekly Hours (LOW) 118.4 Shift factor 1.33 Note: Output, Investment, and Capital stock extrapolated for 1987 and 1988.

3299 Nonmetallic mineral products, n.e.

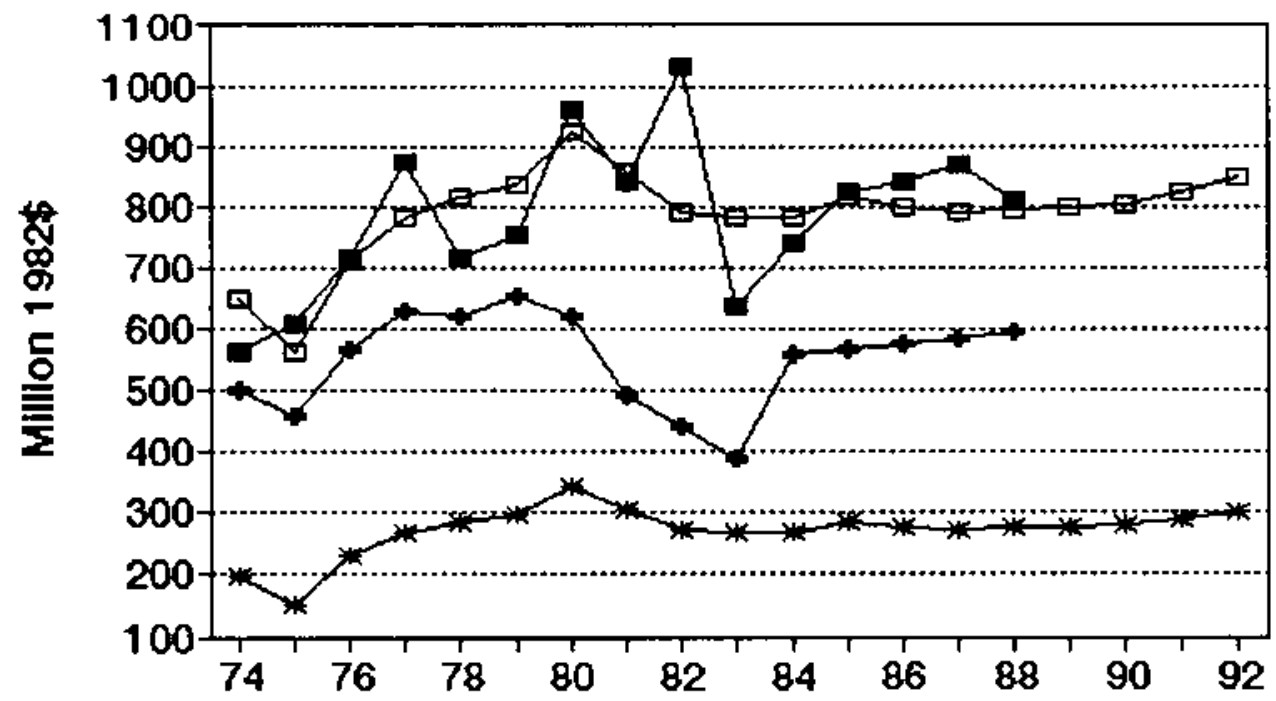

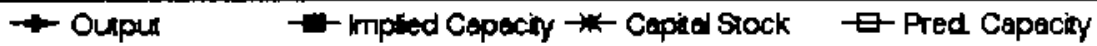


No. 255 sIC 3312 Blast furnaces and steel wills

MILLIONS OF 1982 DOLLARS (EXCL. CU RATES)

$\begin{array}{lccccccc} & 04 & \text { Ann. } & \text { Implied Gross } & \text { Net } & \text { Pred. } & \ldots \ldots \ldots \\ \text { Gross } & \text { CU } & \text { aJ } & \text { Prac. } & \text { In- } & \text { Cap. } & \text { Prac. } & \text { Emergency Capacity } \\ \text { Output } & \text { Rate } & \text { Rate } & \text { Capac. } & \text { vest. } & \text { Stk. } & \text { Capac. } & 3 \text { mon. } 6 \text { mon. }>6 \text { mon. }\end{array}$

\begin{tabular}{|c|c|c|c|c|c|c|c|c|c|c|}
\hline 1974 & 84,567 & 89 & 92.6 & 91,281 & $4,487.5$ & 49,745 & 89,636 & & & \\
\hline 1975 & 63,256 & 64 & 67.4 & 93,820 & $4,507.7$ & 49,828 & 89,830 & & & \\
\hline 1976 & 65,950 & 68 & 70.9 & 92,981 & $4,978.5$ & 50,261 & 90,841 & & & \\
\hline 1977 & 62,237 & 77 & 73.8 & 84,331 & $2,997,4$ & 48,780 & 87,381 & & & \\
\hline 1978 & 67,062 & 90 & 82.1 & 81,667 & 3.642 .3 & 47,945 & 85,432 & & & \\
\hline 1979 & 68,049 & 79 & 82.6 & 82,400 & $3,202.7$ & 46,719 & 82,568 & & & \\
\hline 1980 & 57,324 & 76 & 69.4 & 82,593 & $3,486.1$ & 45,821 & 80,471 & & & \\
\hline 1981 & 59,849 & 66 & 73.7 & 81,227 & $3,912.6$ & 45,338 & 79,344 & & & \\
\hline 1982 & 36,250 & 44 & 50.2 & 72,253 & $2,247.2$ & 43,275 & 74,527 & & & \\
\hline 1983 & 35,383 & 66 & 58.4 & 60,622 & $1,146.6$ & 40,317 & 67,619 & & & \\
\hline 1984 & 38,152 & 59 & 63.3 & 60,234 & 585.5 & 37,032 & 59,948 & & & \\
\hline 1985 & 35,559 & 67 & 63.9 & 55,671 & $2,105.8$ & 35,382 & 56,093 & & & \\
\hline 1986 & 34,047 & 62 & 62.3 & 54,665 & $1,082.5$ & 32,796 & 50,055 & & & \\
\hline$\cdots$ & $\ldots$ & - & $-\cdots$ & $-\cdots$ & $\ldots$ & $\cdots$ & $\cdots$ & $\cdot \cdot \cdot$ & --- & --- \\
\hline 1987 & 34,784 & 79 & 70.2 & 49,560 & $1,695.8$ & 30,917 & 45,667 & & & \\
\hline 1988 & 35,766 & 82 & 78.9 & 45,322 & $2,510.3$ & 30,057 & 43,659 & 42,483 & 43,695 & 44,008 \\
\hline 1989 & & & & & $2,707.7$ & 29,488 & $42,33 t$ & 41,191 & 42,366 & 42,670 \\
\hline 1990 & & & & & $3,614.6$ & 29,889 & 43,266 & 42,101 & 43,301 & 43,612 \\
\hline 1991 & & & & & $3,312.3$ & 29,943 & 43,392 & 42,224 & 43,428 & 43,739 \\
\hline 1992 & & & & & $2,802.2$ & 29,481 & 42,314 & 41,175 & 42,349 & 42,653 \\
\hline
\end{tabular}

Cepacity-Capital stock Regression: Code 0 RSO 0.950

Coefficients: Const -26532.0 stock 2.3

Ave. Capacity/Stock (74-86): 1.7 Ave. Heekty Hours (High) 161.8 Shift Factor 1.01 Ave Heekly Hours (Low) 159.0 Shift factor 1.02 Note: Output, Investment, and Capital Stock extrapolated for 1987 and 1988.

\section{Blast furnaces and steel mills}

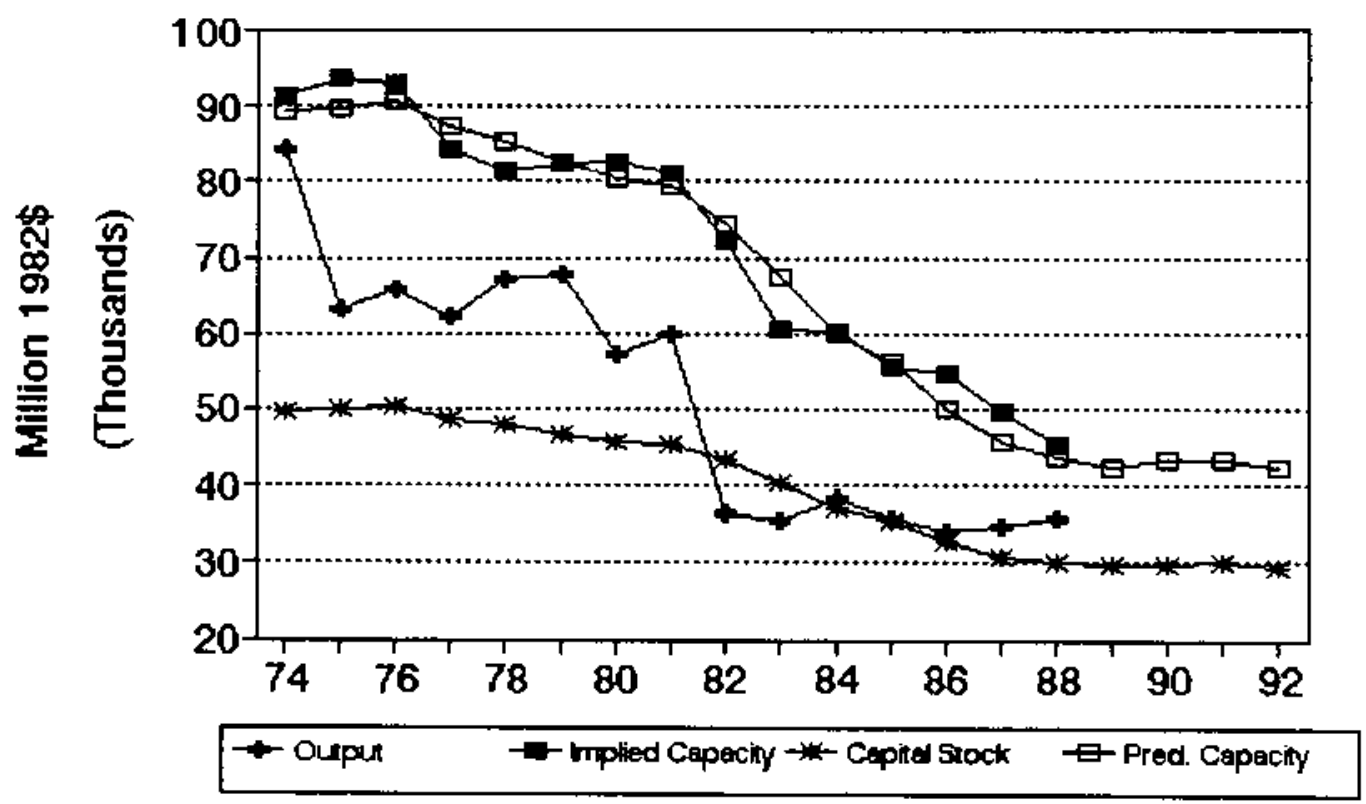


No. 256 grc 3313 Electrometallurgical products

MILLIONS OF 1982 DOLLARS (EXCL. OU RATES)

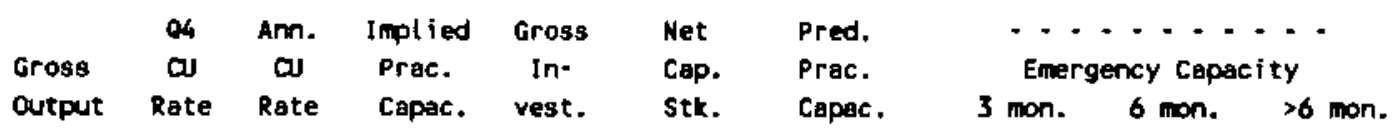

\begin{tabular}{|c|c|c|c|c|c|c|c|c|c|c|}
\hline 1974 & 1,620 & 97 & 100.0 & 1,620 & 98.6 & 1,021 & 1,892 & & & \\
\hline 1975 & 1,149 & 42 & 52.9 & 2,171 & 154.7 & 1,097 & 1,931 & & & \\
\hline 1976 & 1,087 & 60 & 58.7 & 1,852 & 98.3 & 1,114 & 1,940 & & & \\
\hline 1977 & 1,218 & 60 & 60.4 & 2,018 & 171.0 & 1,195 & 1,982 & & & \\
\hline 1978 & 1,281 & 76 & 66.3 & 1.932 & 55.7 & 1,161 & 1,965 & & & \\
\hline 1979 & 1,441 & 88 & 83.4 & 1,727 & (38.6) & 1,036 & 1,900 & & & \\
\hline 1980 & 1,258 & 70 & 64.5 & 1,951 & 59.8 & 1,005 & 1,884 & & & \\
\hline 1981 & 1,259 & 50 & 62.8 & 2,003 & $(106.6)$ & 834 & 1,795 & & & \\
\hline 1992 & 658 & 27 & 34.5 & 1,906 & $(102.2)$ & 678 & 1,715 & & & \\
\hline 1983 & 650 & 37 & 37.7 & 1,726 & 8.7 & 633 & 1,691 & & & \\
\hline 1984 & 898 & 63 & 55.5 & 1,619 & $(7.3)$ & 575 & 1,661 & & & \\
\hline 1985 & 236 & 40 & 48.9 & 1,711 & $(5.5)$ & 523 & 1,634 & & & \\
\hline 1986 & 729 & 64 & 53.1 & 1,373 & 17.1 & 495 & 1,620 & & & \\
\hline 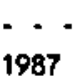 & TOB & 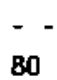 & $\begin{array}{l}-\cdots \\
70.7\end{array}$ & $\because \cdots$ & $(7.7)$ & 442 & - - - & $\cdots$ & $\cdots$ & -- \\
\hline 1988 & 682 & 82 & 78.3 & 871 & 20.2 & 422 & 1,582 & 1,416 & 1,548 & 1,582 \\
\hline 1989 & & & & & 26.9 & 411 & 1,576 & 1,410 & 1,542 & 1,576 \\
\hline 1990 & & & & & 57.9 & 432 & 1,587 & 1,420 & 1,553 & 1,587 \\
\hline 1991 & & & & & 47.5 & 440 & 1,591 & 1,424 & 1,557 & 1,591 \\
\hline 1992 & & & & & 30.1 & 430 & 1,586 & 1,419 & 1,552 & 1,586 \\
\hline
\end{tabular}

Capacity-Copital stock Regression: Code 0 RSO 0.400

Coefficients: Const 1363.4 stock 0.5

Ave. Capacity/Stock $(74-86): 2.1$

Ave. Weekly Hours (High) 165.6 Shift Factor 1.00 Ave Weekly Hours (Low) 165.6 Shift Factor 1.00 Note: Output, Imvestment, and Capital Stock extrapolated for 1987 and 1988.

\section{Electrometallurgical products}

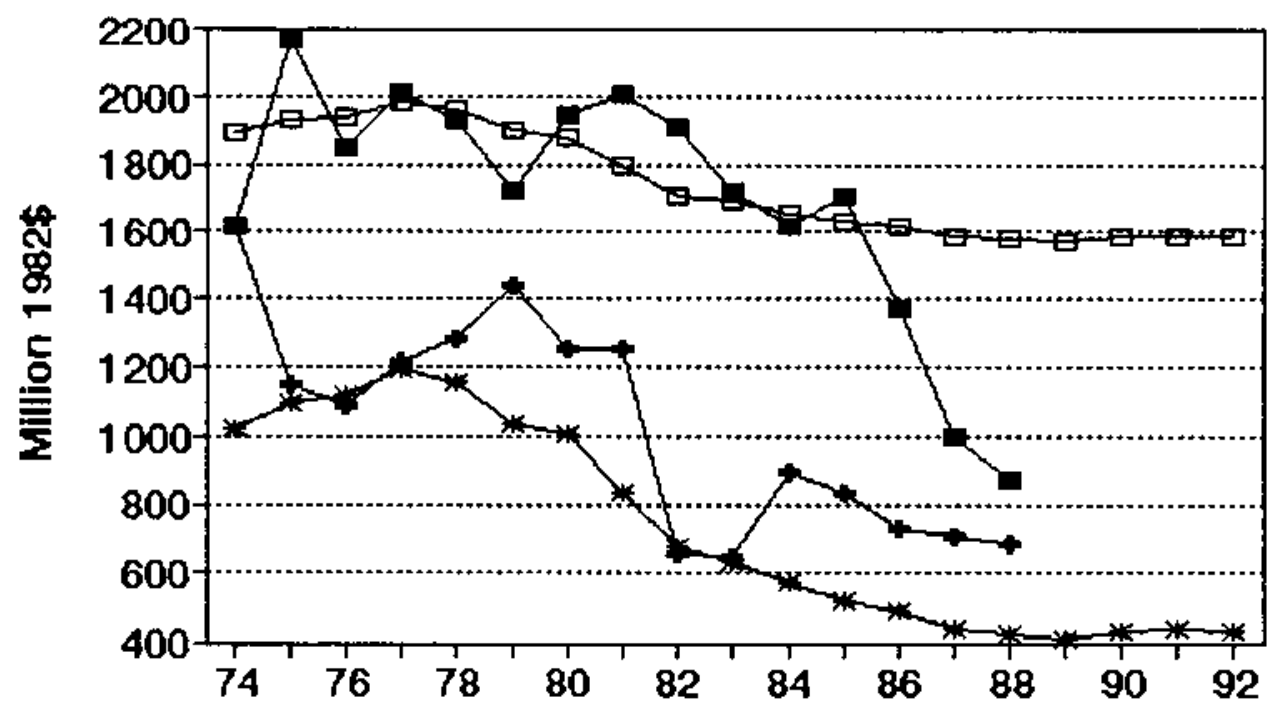

$\rightarrow$ - Oupu $\quad \rightarrow-$ mplied Cepacity $\rightarrow$ - Caphal Stock $\quad$ - Pred. Cepecity 


\section{No. 257 sIC 3315 steel wire and related products}

MILLIONS OF 1982 DOLLARS (EXCL. CU RATES)

$\begin{array}{lccccccc} & 04 & \text { Arn. } & \text { Implied Gross } & \text { Net } & \text { Pred. } & \ldots \ldots . . . \\ \text { Gross } & \text { CU } & \text { CU } & \text { Prac. } & \text { In- } & \text { Cap. } & \text { Prac. } & \text { Emergency Capacity } \\ \text { Output Rate } & \text { Rate } & \text { Capac. } & \text { vest. } & \text { Stk. } & \text { Capac. } & 3 \text { mon. } 6 \text { mon. }>6 \text { mon. }\end{array}$

\begin{tabular}{|c|c|c|c|c|c|c|c|c|c|c|}
\hline 1974 & $3,4 \pi$ & 78 & 80.5 & 4,311 & 26.0 & 826 & 4,392 & & & \\
\hline 1975 & 2,765 & 62 & 63.9 & 4,331 & 143.9 & 904 & 4,540 & & & \\
\hline 1976 & 3,069 & 66 & 67.8 & 4,529 & 94.5 & 929 & 4,586 & & & \\
\hline 1977 & 3,194 & $\pi$ & 73.0 & 4,376 & 106.2 & 962 & 4,649 & & & \\
\hline 1978 & 3,354 & 79 & 76.2 & 4,404 & 116.0 & 1,001 & $4, \pi 4$ & & & \\
\hline 1979 & 3,504 & 74 & 75.5 & 4,639 & 115.5 & 1,036 & 4,790 & & & \\
\hline 1980 & 3,251 & 65 & 63.0 & 5,161 & 90.3 & 1,042 & 4,803 & & & \\
\hline 1981 & 3,233 & 67 & 69.2 & $4,6 \pi$ & 56.1 & 1,014 & 4.749 & & & \\
\hline 1982 & 2,381 & 44 & 50.7 & 4,693 & 5.3 & 939 & 4,605 & & & \\
\hline 1983 & 2,568 & 50 & 48.5 & 5,298 & 67.6 & 924 & $4,5 \pi$ & & & \\
\hline 1984 & 2,708 & 80 & 70.0 & 3,860 & 62.7 & 905 & 3,770 & & & \\
\hline 1985 & 2,572 & $\infty 6$ & 71.3 & 3,608 & 45.1 & 870 & 3,703 & & & \\
\hline 1986 & 2,705 & 81 & 74.1 & 3,651 & 55.0 & 844 & 3,655 & & & \\
\hline$-\cdot-$ & $\cdots-\cdot$ & $\because$ & $\cdots$ & $-\cdot-$ & $\cdots$ & $\cdots \cdot$ & $\because \cdots$ & -- & -- & $-\cdots$ \\
\hline 1987 & 2,742 & 80 & 78.7 & 3,486 & 56.5 & 821 & 3,611 & & & \\
\hline 1988 & 2,791 & 86 & 82.2 & 3,398 & 69.8 & 814 & 3,598 & 4,070 & 4,301 & 4,360 \\
\hline 1989 & & & & & 73.0 & 811 & 3,591 & 4,063 & 4,293 & 4,352 \\
\hline 1990 & & & & & 87.8 & 822 & 3,613 & 4,088 & 4,319 & 4,379 \\
\hline 1991 & & & & & 82.9 & 828 & 3,624 & 4,100 & 4,332 & 4,392 \\
\hline 1992 & & & & & 74.5 & 825 & 3,618 & 4,093 & 4,325 & 4,385 \\
\hline
\end{tabular}

Capacity-Capital Stock Regression: Code -1 RSO 0.700

Coefficients: Const 2819.0 stock $1.90(84-86)-770.6$

Ave. Capacity/Stock (74-86): 4.7 Ave. Heekly Hours (High) 130.8 Shift factor 1.21 Ave Heekly Hours (Low) 123.4 Shift Factor 1.28 Note: Output, Investment, and Capital stock extrapolated for 1987 and 1988.

\section{Steel wire and related products}

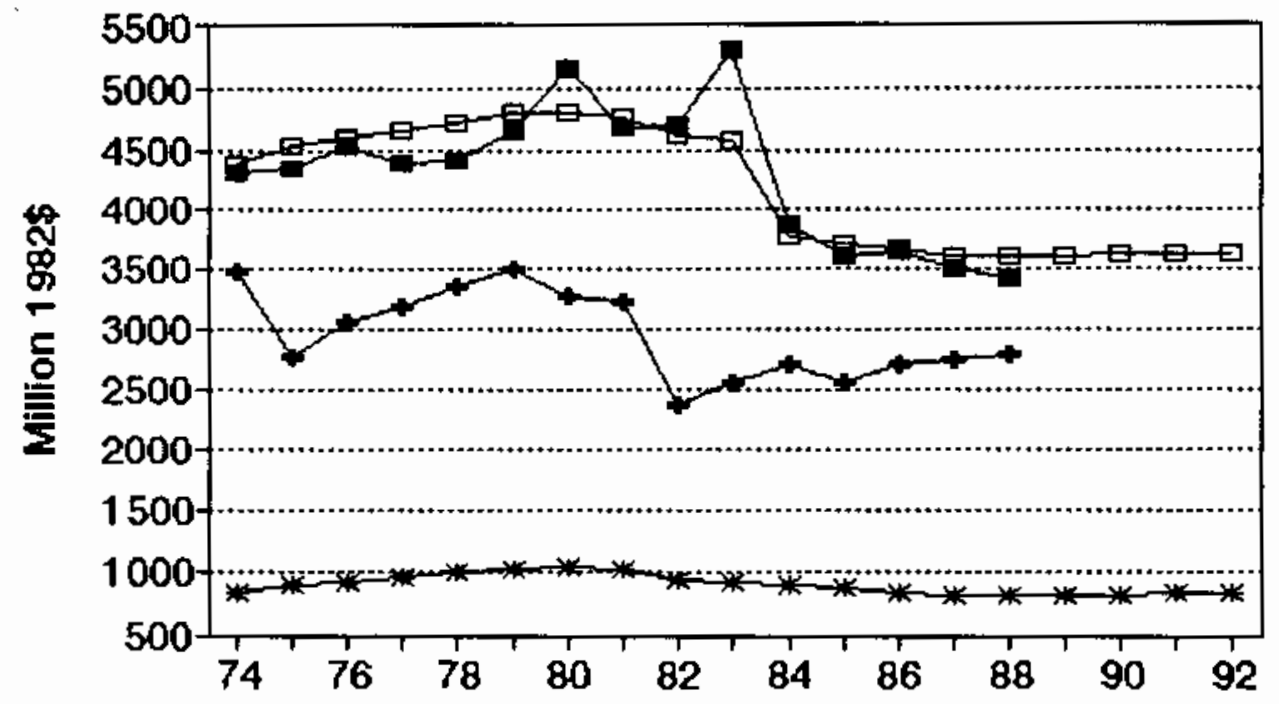

Ouput

$\rightarrow$ - Mplied Capeciny $\rightarrow$ - Ceppital Stock

Æ-Pred. Capecity 


\section{No. 258 stc 3316 cold finishing of steel shapes}

MILLIONS OF 1982 DOLLARS (EXCL. CU RATES)

$\begin{array}{lccccccc} & 04 & \text { Amm. } & \text { Implied Gross } & \text { Net } & \text { Pred, } & \ldots \ldots \ldots \\ \text { Gross } & \text { CU } & \text { CU } & \text { Prac. } & \text { In- } & \text { Cap. } & \text { Prac. } & \text { Emergency Capacity } \\ \text { Output } & \text { Rate } & \text { Rate } & \text { Capac. } & \text { vest. } & \text { Stk. } & \text { Capac. } & 3 \text { mon. } 6 \text { mon. }>6 \text { mon. }\end{array}$

\begin{tabular}{|c|c|c|c|c|c|c|c|c|c|c|}
\hline 1974 & 5,37 & 89 & 92.9 & $5,78 B$ & 102.5 & 1,192 & 5,743 & & & \\
\hline 1975 & 2,961 & 60 & 64.5 & 4,588 & (128.5) & 994 & 5,508 & & & \\
\hline 1976 & 3,716 & 79 & 76.7 & 4,846 & 26.7 & 949 & 5,455 & & & \\
\hline 1977 & 4,026 & 80 & 79.9 & 5,038 & 249.1 & 1,105 & 5,640 & & & \\
\hline 1978 & 4,292 & 82 & 78.4 & 5,476 & 89.5 & 1,097 & 5,630 & & & \\
\hline 1979 & 4,394 & 73 & 75.9 & 5,791 & 90.8 & 1,086 & 5,617 & & & \\
\hline 1980 & 3,474 & 67 & 61.8 & 5,622 & 79.9 & 1,063 & 5,590 & & & \\
\hline 1981 & 3,554 & 53 & 61.7 & 5,359 & 97.4 & 1,055 & 5,581 & & & \\
\hline 1982 & 3,166 & 38 & 42.1 & 7,515 & 25.6 & 978 & 5,490 & & & \\
\hline 1983 & 3,146 & 64 & 54.6 & 5,760 & 59.4 & 936 & 5,439 & & & \\
\hline 1984 & 3,650 & 66 & 67.2 & 5,436 & 69.5 & 905 & 5,403 & & & \\
\hline 1985 & 3,168 & 59 & 61.6 & 5,141 & 72.0 & 880 & 5,373 & & & \\
\hline 1986 & 3,301 & 72 & 65.5 & 5,040 & 56.1 & 842 & 5,328 & & & \\
\hline 1987 & 3,370 & $\ddot{81}$ & $\overline{3.2}$ & 4,493 & 57.6 & 805 & F, 285 & --- & -- & $\cdots$ \\
\hline 1988 & 3,483 & 87 & 82.6 & 4,218 & 63.6 & 779 & 5,254 & 5,098 & 5,389 & 5,464 \\
\hline 1989 & & & & & 65.1 & 37 & 5,228 & 5,073 & 5,362 & 5,437 \\
\hline 1990 & & & & & 71.8 & 745 & 5,213 & 5,058 & 5,347 & 5,422 \\
\hline 1991 & & & & & 69.6 & 731 & 5,197 & 5,043 & 5,331 & 5,405 \\
\hline 1992 & & & & & 65.8 & 715 & 5,179 & 5,024 & 5,311 & 5,386 \\
\hline
\end{tabular}

Capecity-Cepital Stock Regression: Code 0 RSO 0.030

Coefficients: Const 4331.0 stock 1.2

Ave. Capecity/Stock $(74-86): 5.5$ Ave. Weekly Hours (High) 153.6 Shift Factor 1.04 Ave Weekly Hours (Low) 148.8 Shift Factor 1.07 Note: Output, Investment, and Capital Stock extrapolated for 1987 and 1988.

\section{Cold finishing of steel shapes}
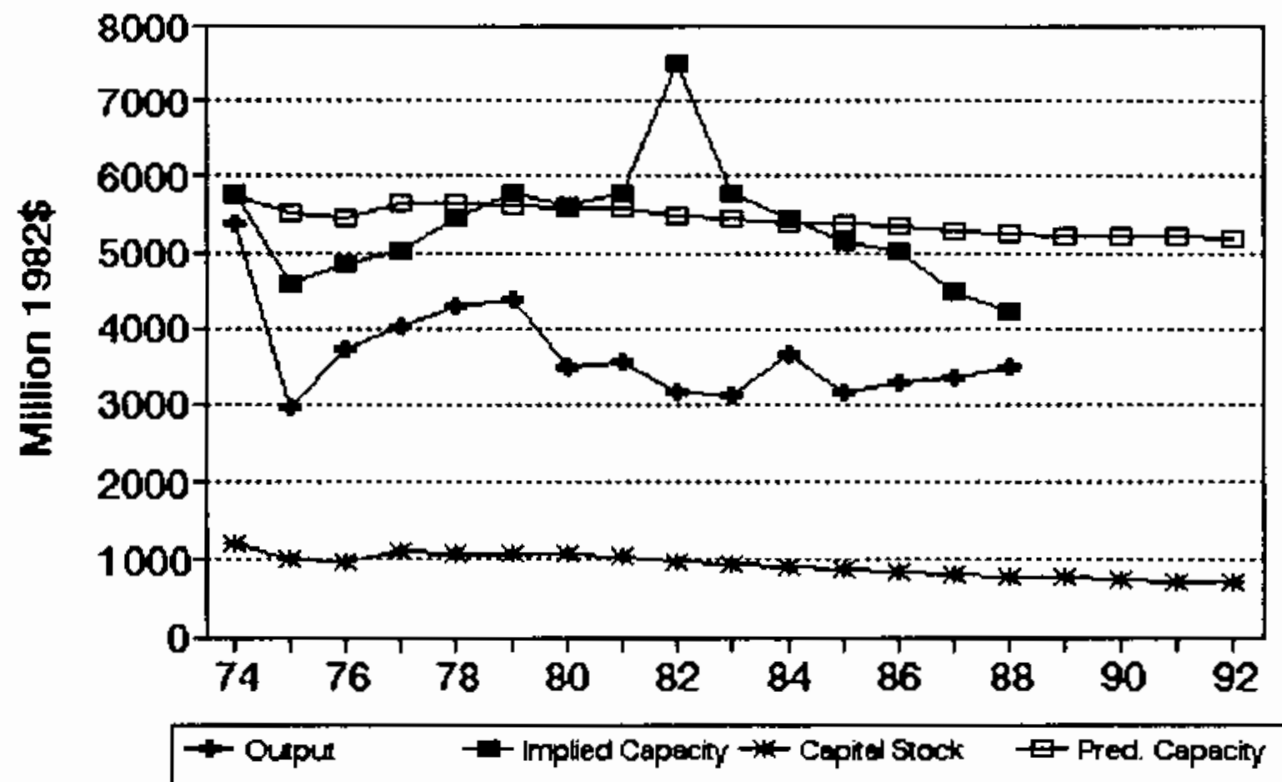


\section{No. 259 sIC 3317 steel pipe and tubes}

MILLIONS OF 1982 dollars (EXCL. CU RATES)

$\begin{array}{lccccccc} & 04 & \text { Ann. } & \text { Implied Gross } & \text { Net } & \text { Pred. } & \ldots \\ \text { Gross } & \text { CU } & \text { CU } & \text { Prac. } & \text { In- } & \text { Cap. } & \text { Prac. } & \text { Emergency Capacity } \\ \text { Output } & \text { Rate } & \text { Rate } & \text { Capac. } & \text { vest. } & \text { Stk. } & \text { Capac. } & 3 \text { mon. } 6 \text { mon. }>6 \text { mon. }\end{array}$

\begin{tabular}{|c|c|c|c|c|c|c|c|c|c|c|}
\hline 1974 & 5,037 & 85 & 88.6 & 5,684 & 188.2 & 918 & 5,441 & & & \\
\hline 1975 & 4,042 & 72 & 70.9 & 5,698 & 57.1 & 895 & 5,304 & & & \\
\hline 1976 & 3,149 & 63 & 71.3 & 4,416 & $(9.4)$ & 814 & 4,817 & & & \\
\hline 1977 & 4,357 & 81 & 74.4 & 5,860 & 385.2 & 1,106 & 6,572 & & & \\
\hline 1978 & 4,705 & 75 & 74.7 & 6,296 & 100.4 & 1,111 & 6,601 & & & \\
\hline 1979 & 5,038 & 75 & 74.7 & 6,746 & 156.7 & 1,170 & 6,954 & & & \\
\hline 1980 & 4,848 & 70 & 64.4 & 7,531 & 161.5 & 1,231 & 7,320 & & & \\
\hline 1981 & 5,386 & 75 & 7.7 & 6,932 & 118.5 & 1,249 & 7,428 & & & \\
\hline 1982 & 3,869 & 33 & 45.2 & 8,561 & 195.8 & 1,336 & 7,954 & & & \\
\hline 1983 & 3,223 & 48 & 43.0 & 7,491 & 64.8 & 1,298 & 7,725 & & & \\
\hline 1984 & 3,626 & 57 & 55.1 & 6,584 & 95.5 & 1,286 & 7.650 & & & \\
\hline 1985 & 3,449 & 37 & 44.6 & 7,729 & 38.7 & 1,216 & 7,235 & & & \\
\hline 1986 & 3,383 & 43 & 39.8 & 8,507 & 76.0 & 1,183 & 7,035 & & & \\
\hline$\because-$ & & $-\cdot$ & $-\cdot$ & $\cdots \cdot$ & $\cdots-$ & $-\cdots$ & $\cdots$ & ---- & $-\cdot \cdot$ & --- \\
\hline 1987 & 3,447 & 60 & 51.6 & 6,679 & 90.9 & 1,167 & 6,937 & & & \\
\hline 1988 & 3,531 & 61 & 59.1 & 5,974 & 109.8 & 1,171 & 6,962 & 9,772 & 10,898 & 11,189 \\
\hline 1989 & & & & & 114.3 & 1,179 & 7,013 & 9,842 & 10,976 & 11,269 \\
\hline 1990 & & & & & 135.4 & 1,208 & 7,185 & 10,084 & 11,245 & 11,546 \\
\hline 1991 & & & & & 128.4 & 1,227 & 7,299 & 10,244 & 11,424 & 11,729 \\
\hline 1992 & & & & & 116.5 & 1,233 & 7,332 & 10,290 & 11,476 & 11,782 \\
\hline
\end{tabular}

Capacity-Capital Stock Regression: Code 0 RSO 0.690 Coefficients: Const -74.3 stock 6.0

Ave. Capacity/Stock $(74-86): 5.9$ Ave. Ueekly Hours (High) 98.0 Shift factor 1.61 Ave Ueekly Hours (Low) 92.4 Shift Factor 1.71 Note: Output, Investment, and Capital Stock extrapolated for 1987 and 1988.

\section{Steel pipe and tubes}

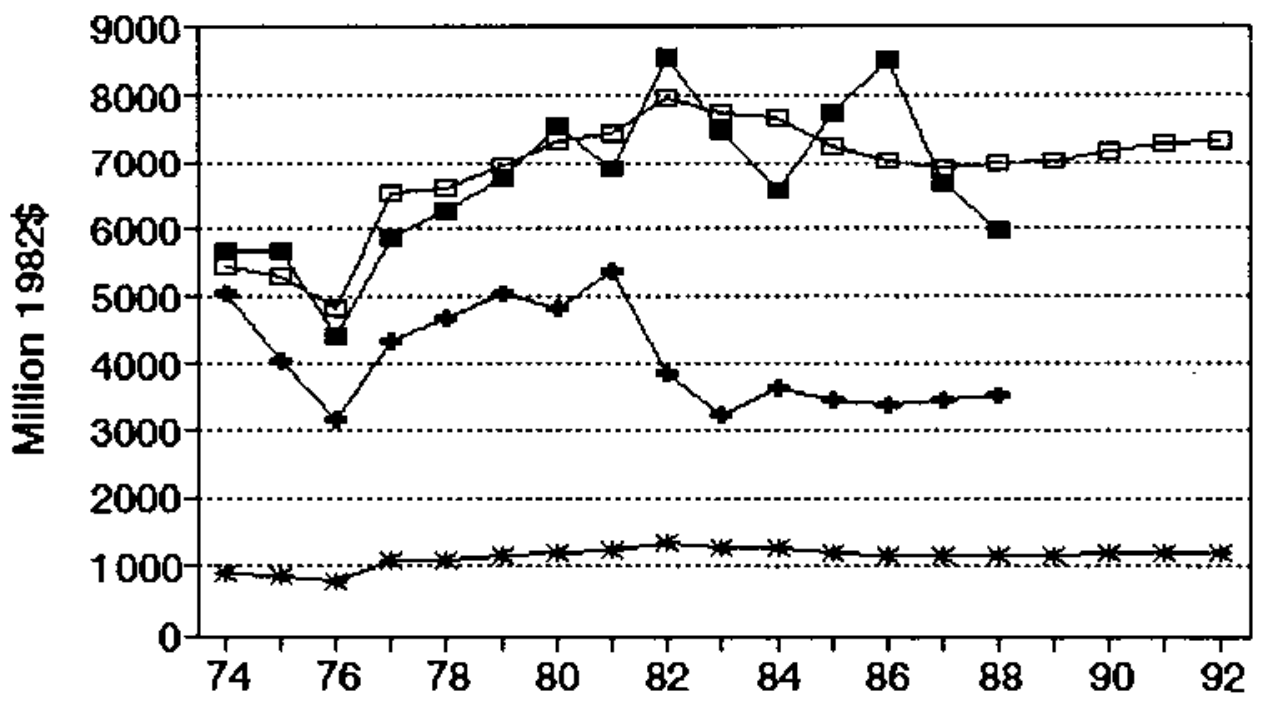

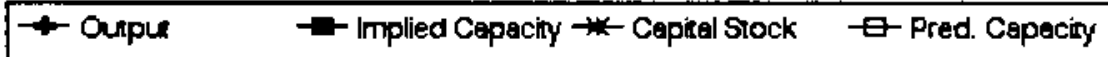


No. 260 sIC 3321 Gray iron foundries

MILLIONS OF 1982 DOLLARS (EXCL. CU RATES)

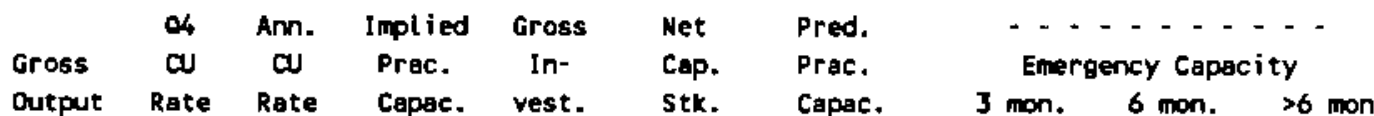

\begin{tabular}{|c|c|c|c|c|c|c|c|c|c|c|}
\hline 1974 & 11,445 & 86 & 89.4 & 12,799 & 706.6 & 4,810 & 11,298 & & & \\
\hline 1975 & 9,629 & 71 & 71.0 & 13,561 & 626.3 & 5,124 & 12,221 & & & \\
\hline 1976 & 9,671 & $\pi$ & 78.2 & 12,362 & 300.8 & 5,102 & 12,155 & & & \\
\hline 197 & 10,392 & 81 & 79.0 & 13,160 & 753.1 & 5,496 & 13,316 & & & \\
\hline 1978 & 11,393 & 82 & 79.0 & 14,422 & 979.7 & 6,074 & 15,011 & & & \\
\hline 1979 & 10,871 & 65 & 70.8 & 15,357 & 761.3 & 6,397 & 15,961 & & & \\
\hline 1980 & 8,514 & 57 & 54.2 & 15.724 & 442.1 & 6,391 & 15,942 & & & \\
\hline 1981 & 8,880 & 46 & 52.8 & 16,822 & 650.3 & 6,569 & 16,465 & & & \\
\hline 1982 & 6,131 & 40 & 41.4 & 14,815 & $(664.7)$ & 5,496 & 13,314 & & & \\
\hline 1983 & 6,311 & 59 & 52.4 & 12,045 & 175.7 & 5,245 & 12,577 & & & \\
\hline 1984 & 7,472 & 73 & 69.4 & 10.765 & 178.0 & 4,988 & 11,819 & & & \\
\hline 1985 & 6,604 & 66 & 68.6 & 9.625 & 68.4 & 4,628 & 10,762 & & & \\
\hline 1986 & 6,253 & 67 & 65.1 & 9,608 & 249.8 & 4,446 & 10,228 & & & \\
\hline$\because$ & $\cdots \cdot$ & - & -- & $\cdots$ & $\cdots \cdot$ & $\cdots$ & --- & --- & --- & $\cdots$ \\
\hline 1987 & 6,353 & 77 & 71.1 & 8,938 & 109.3 & 4,134 & 9,310 & & & \\
\hline 1988 & 6,485 & 87 & 81.3 & 7,975 & 284.2 & 4,026 & 8,994 & 13,316 & 14,549 & 14,867 \\
\hline 1989 & & & & & 326.6 & 3,971 & 8,832 & 13.077 & 14,287 & 14,600 \\
\hline 1990 & & & & & 521.3 & 4,116 & 9,258 & 13,708 & 14,976 & 15,304 \\
\hline 1991 & & & & & 456.4 & 4,183 & 9,453 & 13,996 & 15,291 & 15,626 \\
\hline 1992 & & & & & 346.9 & 4,133 & 9.307 & 13,780 & 15,055 & 15,385 \\
\hline
\end{tabular}

Capacity-Capital Stock Regression: Code 1 R50 0.830

Coefficients: Const -2839.4 5tock 2.8

Ave. Capacity/Stock $(74-86): 2.4$ Ave. Weekly Hours (High) 95.3 Shift factor 1.65 Ave Heekly Hours (LOH) 93.1 Shift Factor 1.69 Note: Output, Investment, and Capital stock extrapolated for 1987 and 1988.

\section{Gray iron foundries}

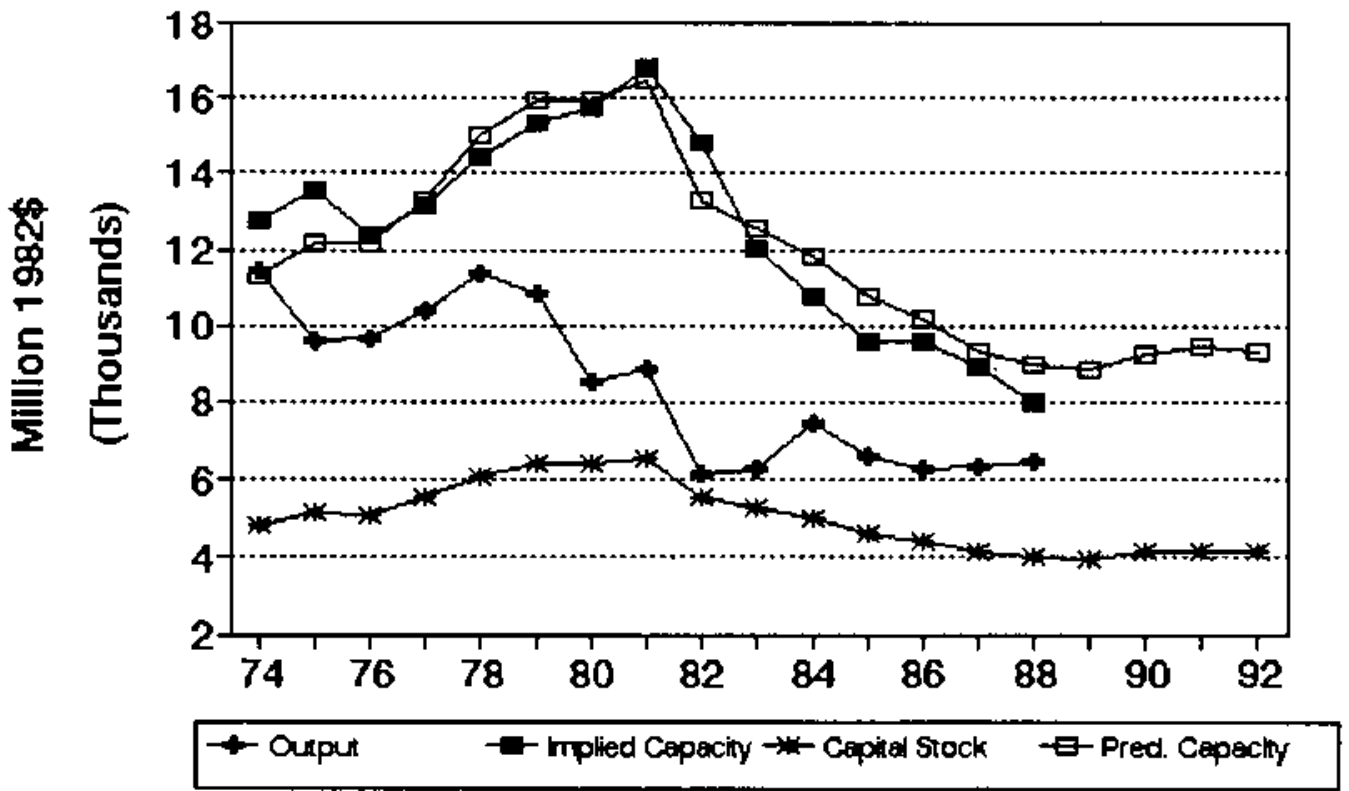




\section{No. 261 sIc 3322 Malleable iron foundries}

MILLIONS OF 1982 DOLLARS (EXCL. CU RATES)

$\begin{array}{lcccclll} & 04 & \text { Ann. Implied Gross } & \text { Net } & \text { Pred. } & \ldots & -\ldots \\ \text { Gross } & \text { CU } & \text { CU } & \text { Prac. } & \text { In- } & \text { Cap. } & \text { Prac. } & \text { Emergency Capacity } \\ \text { Output } & \text { Rate } & \text { Rate } & \text { Capac. } & \text { vest. } & \text { StK. } & \text { Capac. } & 3 \text { mon. } 6 \text { mon. }>6 \text { mon. }\end{array}$

\begin{tabular}{|c|c|c|c|c|c|c|c|c|c|c|}
\hline 1974 & 1,219 & 69 & $\pi .1$ & 1,691 & 39.4 & 541 & 1,440 & & & \\
\hline 1975 & 955 & 65 & 61.4 & 1,556 & 41.5 & 542 & 1,443 & & & \\
\hline 1976 & 1,014 & 71 & 73.8 & 1,374 & 47.3 & 546 & 1,454 & & & \\
\hline 1977 & 1,016 & 61 & 65.1 & 1,561 & 9.5 & 511 & 1,349 & & & \\
\hline 1978 & 1,003 & 9 & 78.7 & 1,274 & 6.1 & 476 & 1,243 & & & \\
\hline 1979 & 859 & 73 & 80.5 & 1,068 & 74.9 & 504 & 1,328 & & & \\
\hline 1980 & 557 & 58 & 56.5 & 984 & 19.9 & 476 & 1,243 & & & \\
\hline 1981 & 492 & $52 *$ & 57.8 & $85 t$ & $(54.9)$ & 383 & 960 & & & \\
\hline 1982 & 314 & 40 & 43.3 & 726 & $(12.8)$ & 336 & 815 & & & \\
\hline 1983 & 318 & 58 & 52.3 & 607 & $(5.7)$ & 297 & 697 & & & \\
\hline 1984 & 417 & 55 & 57.9 & 720 & 19.9 & 284 & 658 & & & \\
\hline 1985 & 410 & 53 & 53.7 & 763 & 25.4 & 276 & 633 & & & \\
\hline 1986 & 390 & 67 & 60.2 & 648 & 8.8 & 253 & 563 & & & \\
\hline$\cdots-$ & --- & -- & -- & $\cdots$ & $\because \cdot$ & $-\cdots$ & --- & -- & $--\cdots$ & $-\cdots$ \\
\hline 1987 & 405 & 76 & 70.3 & $5 \pi$ & 8.5 & 230 & 493 & & & \\
\hline 1988 & 424 & 86 & 80.1 & 529 & 14.0 & 215 & 448 & 763 & 824 & 840 \\
\hline 1989 & & & & & 15.3 & 204 & 414 & 704 & 760 & 775 \\
\hline 1990 & & & & & 21.5 & 200 & 402 & 683 & 738 & 752 \\
\hline 1991 & & & & & 19.4 & 195 & 385 & 655 & 708 & 721 \\
\hline 1992 & & & & & 16.0 & 186 & 360 & 613 & 662 & 675 \\
\hline
\end{tabular}

Capacity-Capital Stock Regression: Code 1 RSa 0.820

Coefficients: Const -207.4 stock 3.0

Ave. Cepacity/Stock (74-86): 2.5

Ave. Weekly Hours (High) 84.1 Shift factor 1.87 Ave Heekly Hours (Low) 72.8 Shift Factor 2.16

* Impured Note: Output, Investment, and Capital Stock extrapolated for 1987 and 1988.

\section{Malleable iron foundries}

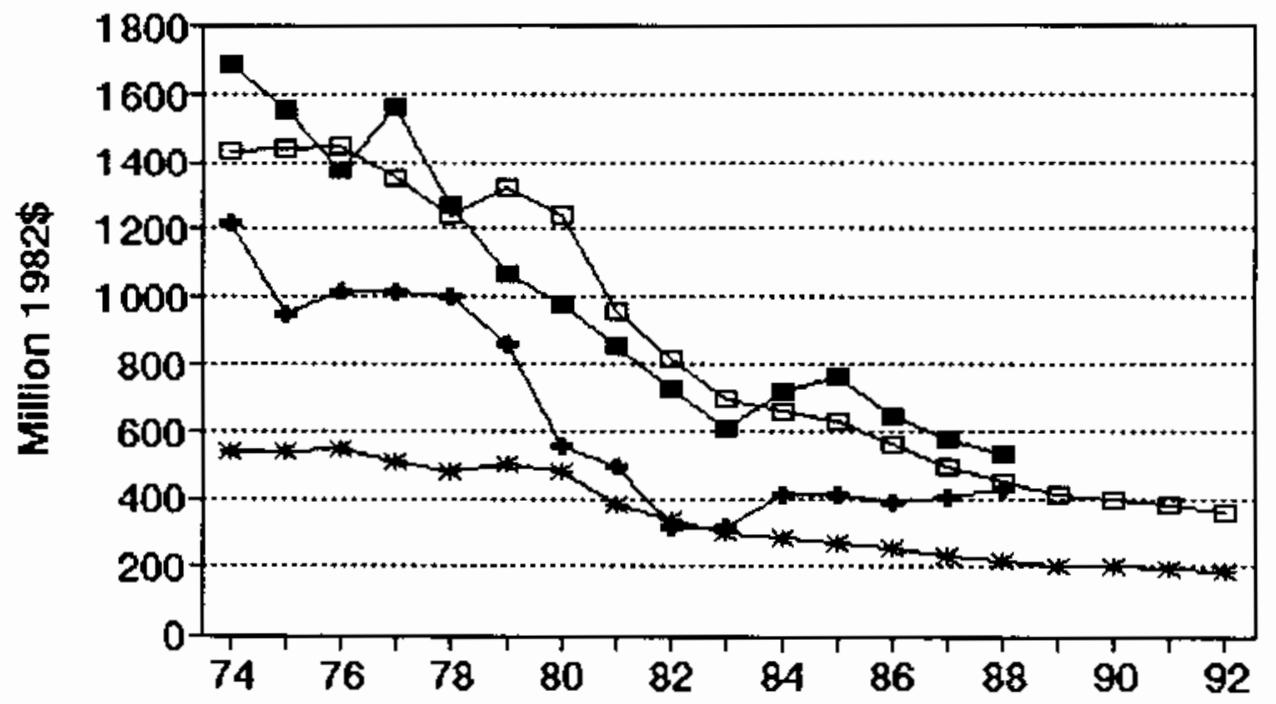

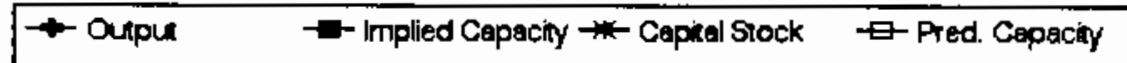




\section{No. 262 sIC 3324 steel 1nvestment foundries}

MILLIONS OF 1982 dOLLARS (EXCL. CU RATES)

$\begin{array}{lccccccc} & \text { Q4 } & \text { Ann. Implied Gross } & \text { Net } & \text { Pred. } & \ldots \ldots . . . \\ \text { Gross } & \text { CU } & \text { CU } & \text { Prac. } & \text { In- } & \text { Cap. } & \text { Prac. } & \text { Emergency Capacity } \\ \text { Output Rate } & \text { Rate } & \text { Capac. } & \text { vest. } & \text { Stk. } & \text { Capac. } & 3 \text { mon. } 6 \text { mon. }>6 \text { mon. }\end{array}$

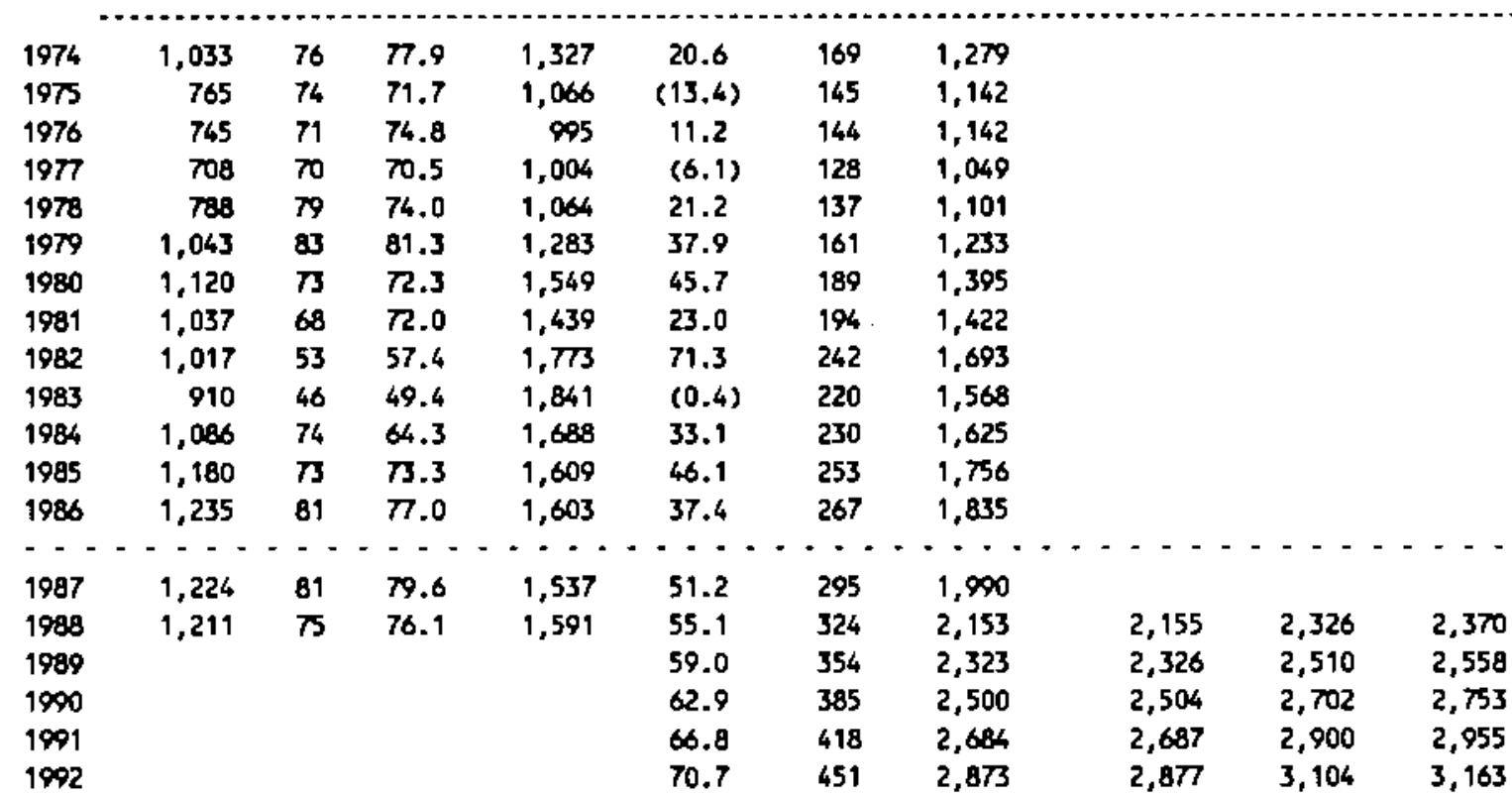

Capacity-Capital Stock Regression: Code 0 RSO 0.800

Coefficients: Const 326.7 Stock 5.6

Ave. Capacity/Stock $(74-86): 7.4$ Ave. Weekly Hours (High) 144.6 Shift Factor 1.10 Ave Heekly Hours (Low) 138.4 Shift Factor 1.15 Note: Output, Investment, and Capital stock extrapolated for 1987 and 1988.

\section{Steel investment foundries}

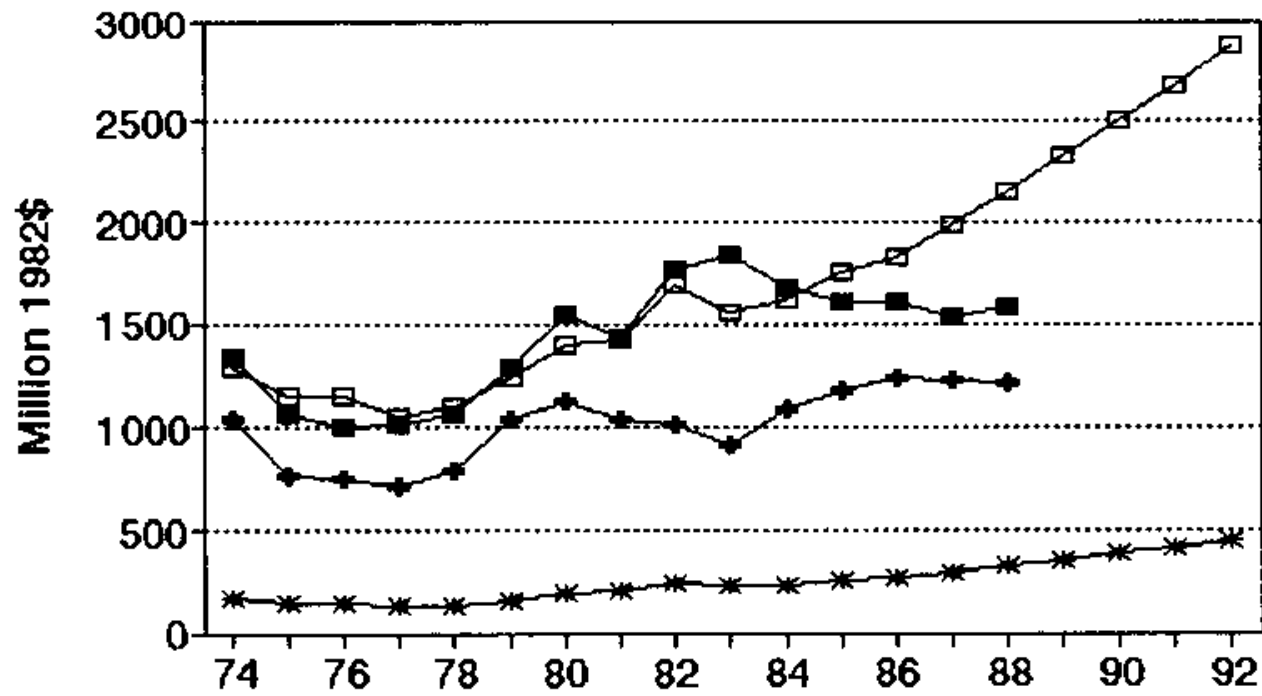

- Oupur $\quad \rightarrow$-mplied Cepacity $\rightarrow$ - Cepital Skock $\square$-Pred. Cepaciny 


\section{No. 263 sIC 3325 steel foundries, n.e.c.}

MILLIONS OF 1982 DOLLARS (EXCL. CU RATES)

$\begin{array}{lccccccc} & 04 & \text { Ann. } & \text { Implied } & \text { Gross } & \text { Net } & \text { Pred. } & -\ldots \ldots \ldots \\ \text { Gross } & \text { CU } & \text { CU } & \text { Prac. } & \text { In- } & \text { Cap. } & \text { Prac. } & \text { Emergency Capacity } \\ \text { Output } & \text { Rate } & \text { Rate } & \text { Capac. } & \text { vest. } & \text { Stk. } & \text { Capac. } & 3 \text { mon. } 6 \text { mon. }>6 \text { mon. }\end{array}$

\begin{tabular}{|c|c|c|c|c|c|c|c|c|c|c|}
\hline 1974 & 3,903 & 96 & 100.0 & 3,903 & 106.3 & 1,080 & 4,252 & & & \\
\hline 1975 & 4,066 & 81 & 77.7 & 5,234 & 269.6 & 1,254 & 4,721 & & & \\
\hline 1976 & 3,786 & 78 & 87.3 & 4,336 & 342.2 & 1,500 & 5,384 & & & \\
\hline 1977 & 3,847 & 74 & 76.0 & 5,006 & 1.8 & 1,410 & 5,142 & & & \\
\hline 1978 & 3,890 & 85 & 77.1 & 5,046 & 110.6 & 1,427 & 5,187 & & & \\
\hline 1979 & 4,282 & 85 & 84.6 & 5,061 & 190.4 & 1,518 & 5,433 & & & \\
\hline 1980 & 4,013 & 64 & 61.5 & 6,524 & 214.8 & 1,624 & 5,720 & & & \\
\hline 1981 & 3,241 & 58 & 65.5 & 4,949 & 80.9 & 1,594 & 5,638 & & & \\
\hline 1982 & 2,082 & 22 & 32.0 & 6,506 & $(48.5)$ & 1,440 & 5,224 & & & \\
\hline 1983 & 1,486 & $18 *$ & 22.6 & 6,566 & 1.5 & 1,335 & 4,939 & & & \\
\hline 1984 & $1, \pi 57$ & 71 & 52.7 & 3,333 & 95.3 & 1,315 & 4,885 & & & \\
\hline 1985 & 1,542 & 66 & 67.9 & 2,272 & $(57.3)$ & 1,150 & 2,374 & & & \\
\hline 1986 & 1,373 & 61 & 60.5 & 2,270 & 32.6 & 1,073 & 2,168 & & & \\
\hline 1987 & 1,407 & $\begin{array}{l}-- \\
68\end{array}$ & 63.0 & 2,235 & 19.4 & $\begin{array}{c}-788 \\
988\end{array}$ & 1,938 & $\cdots$ & $-\cdots$ & $\cdots$ \\
\hline 1988 & 1,453 & $\pi$ & 70.0 & 2,076 & 65.6 & 957 & 1,855 & 2,453 & 2,634 & 2,680 \\
\hline 1998 & & & & & 76.9 & 941 & 1,810 & 2,394 & 2,570 & 2,616 \\
\hline 1990 & & & & & 128.4 & 977 & 1,909 & 2,525 & 2,710 & 2,758 \\
\hline 1991 & & & & & 111.2 & 993 & 1,952 & 2,581 & 2,771 & 2,820 \\
\hline 1992 & & & & & 82.2 & 979 & 1,912 & 2,529 & 2,715 & 2,763 \\
\hline
\end{tabular}

Capacity-Capital Stock Regression: Code - 1 RSQ 0.620

Coefficients: Const 1340.9 stock 2.7 D $(85-86)-2066.8$ Ave. Capacity/Stock $(74-86): 3.4$ Ave. Heekly Hours (High) 109.0 Shift factor 1.45 Ave Weekly Hours (Low) 108.8 Shift Factor 1.45 * Imputed Note: Output, Investment, and Capital stock extrapolated for 1987 and 1988.

3325 Steel foundries, n.e.c.

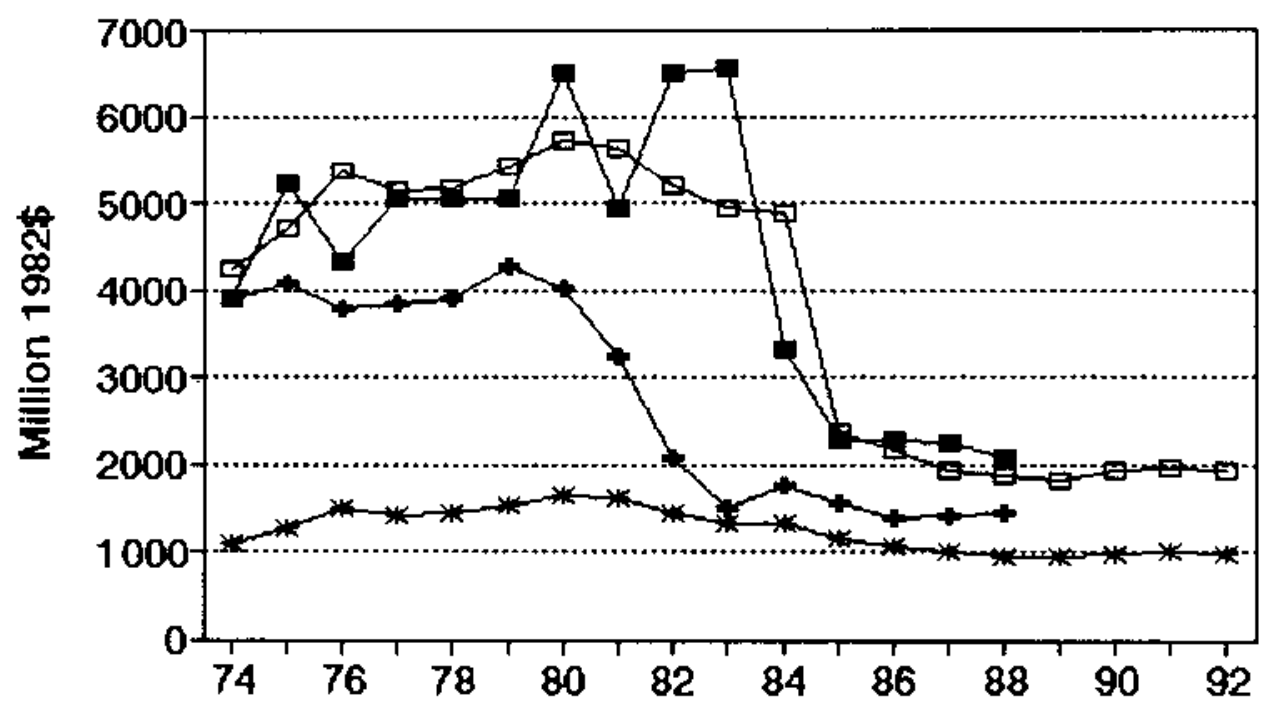

- Oupur $\quad \rightarrow$ - mplied Cepacity $\rightarrow$ - Cepited Stock $\square$-Pred. Capacity 


\section{No. 264 sIC 3331 Primary copper}

MILLIONS OF 1982 DOLLARS (EXCL. CU RATES)

$\begin{array}{lccccccc} & \text { O4 } & \text { Am. Implied } & \text { Gross } & \text { Net } & \text { Pred. } & \ldots \ldots \ldots \\ \text { Gross } & \text { CU } & \text { CU } & \text { Prac. } & \text { In- } & \text { Cap. } & \text { Prac. } & \text { Emergency Capacity } \\ \text { Output } & \text { Rate } & \text { Rate } & \text { Capac. } & \text { vest. } & \text { Stk. } & \text { Capac. } & 3 \text { mon. } 6 \text { mon. }>6 \text { mon. }\end{array}$

\begin{tabular}{|c|c|c|c|c|c|c|c|c|c|c|}
\hline 1974 & 4,160 & 82 & 83.4 & 4,990 & 143.0 & 1,488 & 4,337 & & & \\
\hline $19 \pi 5$ & 3,282 & 63 & 67.9 & 4,835 & 311.0 & 1,722 & 4,827 & & & \\
\hline 1976 & 3,576 & 65 & 65.8 & 5,433 & 26.4 & 1,663 & 4,703 & & & \\
\hline 1977 & 4,467 & 79 & 73.8 & 6,053 & 906.0 & 2,437 & 6,324 & & & \\
\hline 1978 & 4,469 & 70 & 72.4 & 6,173 & 254.0 & 2,534 & 6,528 & & & \\
\hline 1979 & 4,649 & 81 & 76.8 & 6,054 & 189.1 & 2,545 & 6,550 & & & \\
\hline 1980 & 3,731 & 47 & 57.3 & 6,509 & $(56.0)$ & 2,306 & 6,050 & & & \\
\hline 1981 & 4,688 & 67 & 60.9 & 7,703 & 73.4 & 2,183 & 5,792 & & & \\
\hline 1982 & 2,858 & 67 & 66.4 & 4,307 & $(123.4)$ & 1,876 & 5,150 & & & \\
\hline 1983 & 3,241 & 69 & 68.6 & 4,726 & 202.6 & 1,900 & 5,200 & & & \\
\hline 1984 & 2,872 & $\pi$ & 73.4 & 3,912 & 13.5 & 1,746 & 4.877 & & & \\
\hline 1985 & 2,293 & 63 & 67.5 & 3,396 & $(408.9)$ & 1,223 & $3,7 \mathrm{B2}$ & & & \\
\hline 1986 & 2,175 & 60 & 60.6 & 3,590 & 7.9 & 1,117 & 3,561 & & & \\
\hline$\cdots$ & $-\cdot-$ & -- & -- & $\cdots$ & $\cdots$ & $\therefore-$ & $\therefore-$ & -- & -- & $\cdots$ \\
\hline 1987 & 2,213 & $70 *$ & 65.1 & 3,400 & 114.2 & 1,123 & 3,572 & & & \\
\hline 1988 & 2,262 & 94 & 84.0 & 2,692 & 121.4 & 1,135 & 3,597 & 3,350 & 3,547 & 3,597 \\
\hline 1989 & & & & & 126.5 & 1,151 & 3,631 & 3,382 & 3,580 & 3,631 \\
\hline 1990 & & & & & 128.1 & 1,167 & 3,664 & 3,413 & 3,613 & 3,664 \\
\hline 1991 & & & & & 128.3 & 1,182 & 3,695 & 3,441 & 3,643 & 3,695 \\
\hline 1992 & & & & & 126.5 & 1,193 & 3,719 & 3,464 & 3,667 & 3,719 \\
\hline
\end{tabular}

Capacity-Capital Stock Regression: Code 0 RSO 0.620

Coefficients: Const 1220.0 stock 2.1

Ave. Capacity/stock $(74-86): 2.7$ Ave. Heekly Hours (High) 168.0 Shift factor 1.00 Ave Ueekly Hours (LOW) 168.0 Shift Factor 1.00 - Imputed Note: Output, Imestment, and Capital stock extrapolated for 1987 and 1988.

3331 Primary copper

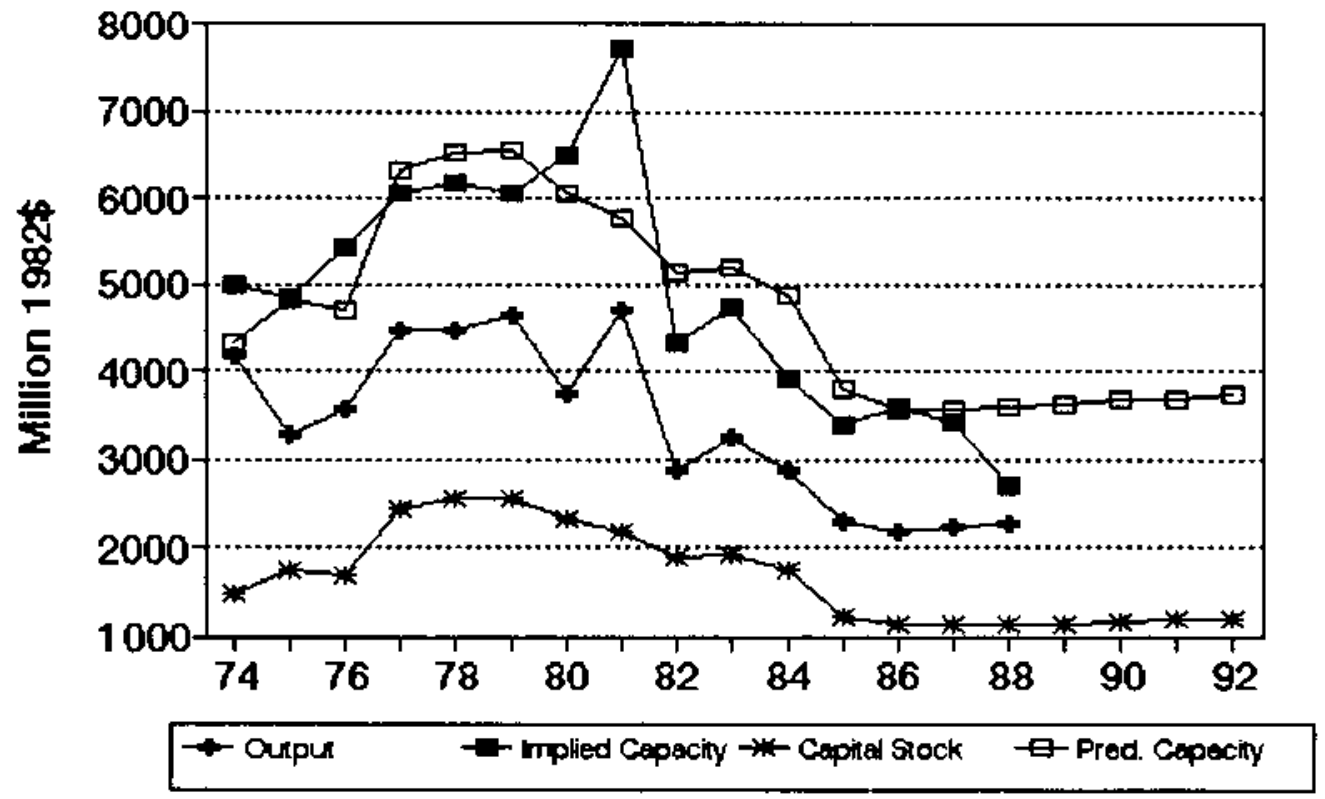


No. 265 src 3333 Primary zinc

MILLIONS OF 1982 DOLLARS (EXCL. CU RATES)

$\begin{array}{lccccccc} & \text { Q4 } & \text { Ann. Inplied Gross } & \text { Net } & \text { Pred. } & \ldots \\ \text { Gross } & \text { CU } & \text { CU } & \text { Prac. } & \text { In* } & \text { Cap. } & \text { Prac. } & \text { Emergency Capacity } \\ \text { Output Rate } & \text { Rate } & \text { Capac. } & \text { vest. } & \text { Stk. } & \text { Capac. } & 3 \text { mon. } 6 \text { mon. }>6 \text { mon. }\end{array}$

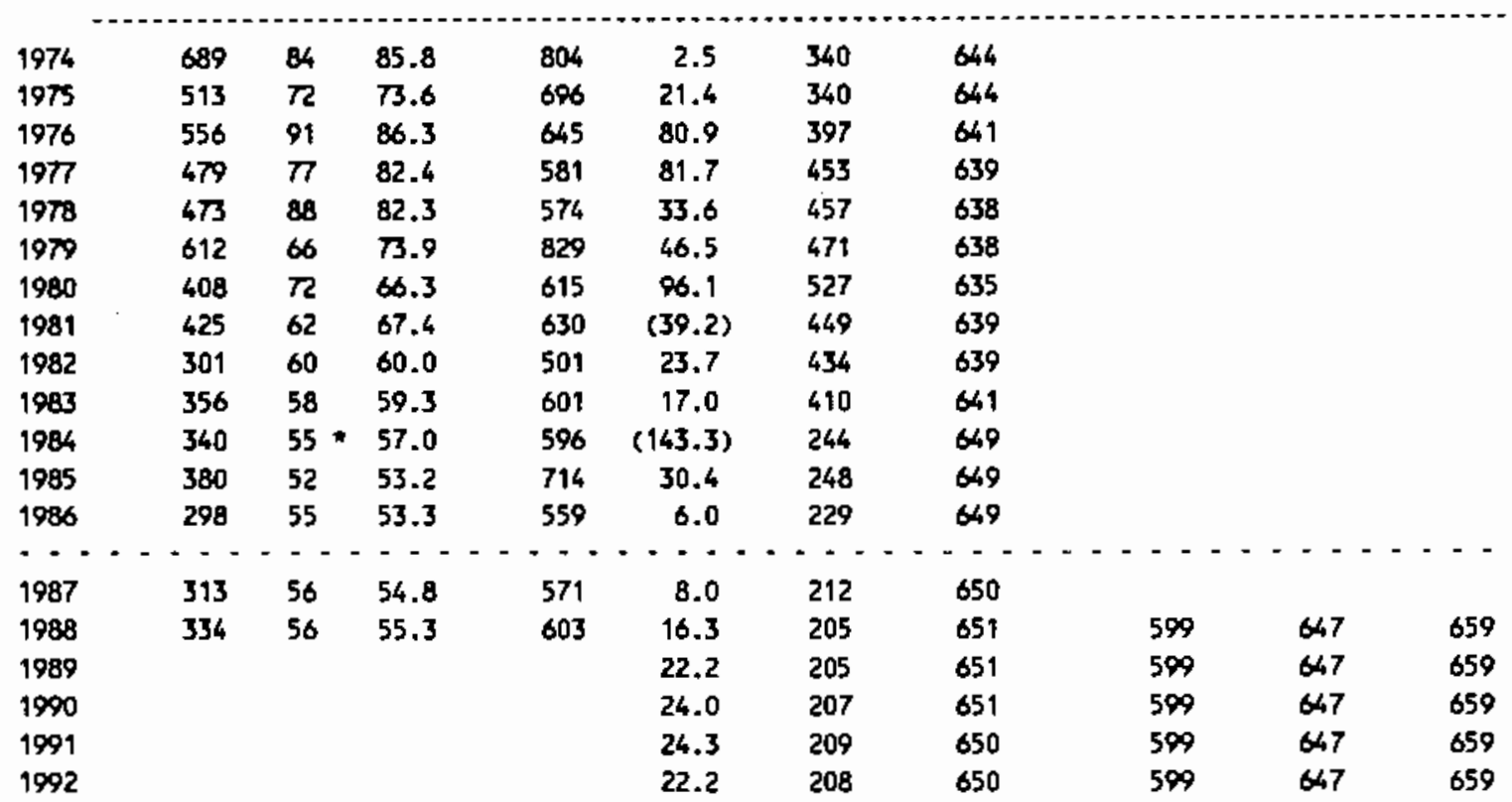

Capacity-Capital stock Regression: Code 2 RSO 0.000 Coefficients: Const 660.5 stock -0.1

Ave. Capecity/stock (74-86): 1.7 Ave. Weekly Hours (High) 157.8 Shift Factor 1.01 Ave Heekly Hours (Low) 146.5 Shift Factor 1.09 * Imputed Note: Output, Investment, and Capital stock extrapolated for 1987 and 1988.

З३З3 Primary zinc

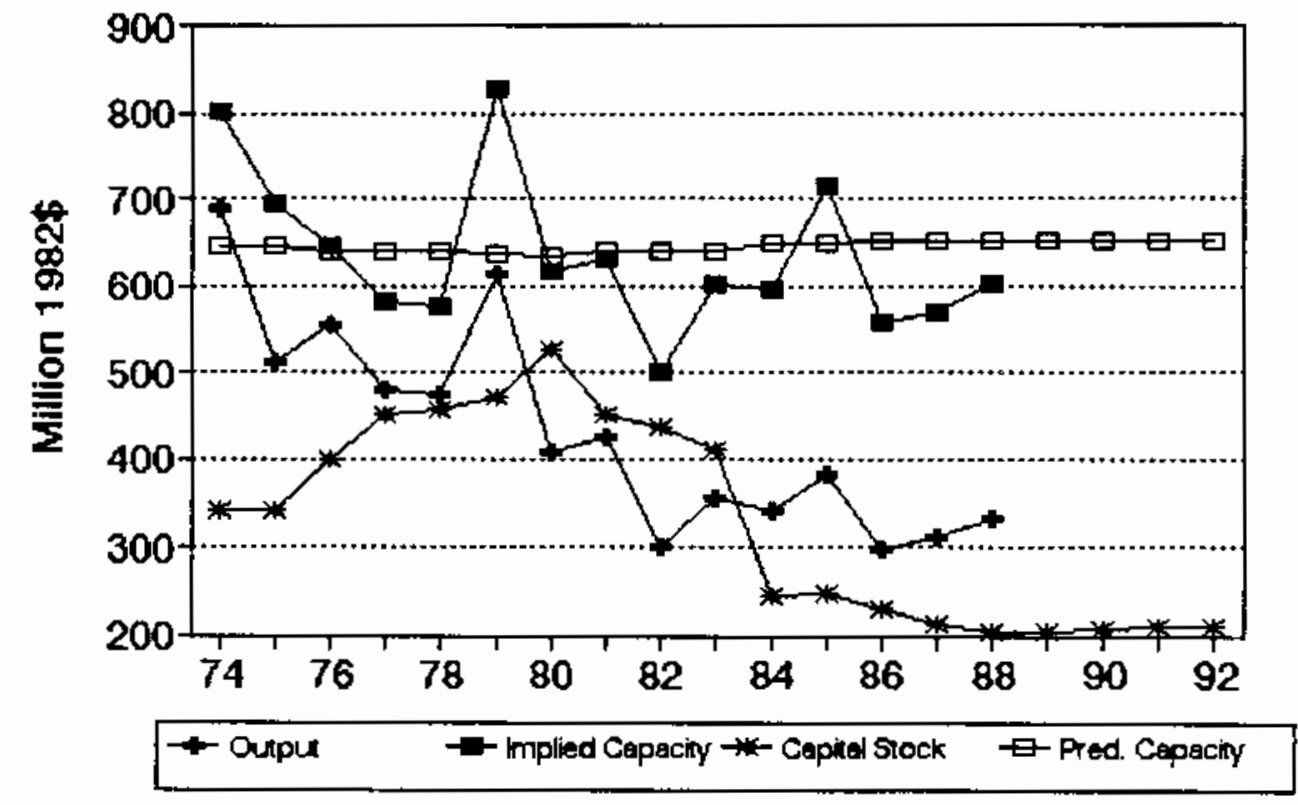


NO. 266 SIC 3334 Primary aluminum

MILLIONS OF 1992 DOLLARS (EXCL. CU RATES)

$\begin{array}{lccccccc} & \text { Q4 } & \text { Ann. } & \text { Implied } & \text { Gross } & \text { Net } & \text { Pred. } & -\ldots \ldots \ldots \\ \text { Gross } & \text { CU } & \text { CU } & \text { Prac. } & \text { In- } & \text { Cap. } & \text { Prac. } & \text { Emergency Capacity } \\ \text { Output } & \text { Rate } & \text { Rate } & \text { Capac. } & \text { vest. } & \text { Stk. } & \text { Capac. } & 3 \text { mon. } 6 \text { mon. }>6 \text { mon. }\end{array}$

\begin{tabular}{|c|c|c|c|c|c|c|c|c|c|c|}
\hline 1974 & 6,140 & 98 & 100.0 & 6,140 & 78.3 & 3,689 & 6,790 & & & \\
\hline 1975 & 5,954 & 59 & 69.6 & 8,559 & 732.6 & 4,116 & 7,749 & & & \\
\hline 1976 & 6,636 & 81 & 75.3 & 8,811 & 310.6 & 4,126 & 7,770 & & & \\
\hline 1977 & 7,049 & 78 & 79.3 & 8,890 & 701.6 & 4,491 & 8,589 & & & \\
\hline 1978 & 7,238 & 79 & 76.9 & 9,407 & $(62.0)$ & 4,120 & 7,756 & & & \\
\hline 1979 & 7,260 & 96 & 89.6 & 8,107 & 472.1 & 4,268 & 8,089 & & & \\
\hline 1980 & 7,324 & 96 & 89.0 & 8,230 & 399.2 & 4,326 & 8,220 & & & \\
\hline 1981 & 6,547 & 80 & 88.0 & 7,442 & 507.6 & 4,499 & 8,608 & & & \\
\hline 1982 & 5,132 & 58 & 64.6 & 7,947 & (7.6) & 4,163 & 7,853 & & & \\
\hline 1983 & 5,661 & 83 & 73.6 & 7,663 & 604.5 & 4,421 & 8,433 & & & \\
\hline 1984 & 6,018 & 85 & 85.5 & 7,037 & 130.0 & 4,202 & 7.941 & & & \\
\hline 1985 & 4,323 & 76 & 79.4 & 5,446 & (622.1) & 3,300 & 5,917 & & & \\
\hline 1986 & 4,085 & $\pi$ & 74.3 & 5,497 & 75.5 & 3,095 & 5,457 & & & \\
\hline$\because \cdot$ & $\because \cdot$ & $\cdot$ & $\cdots$ & $\cdots$ & $\because \cdot \cdot$ & $\cdots$ & $\cdots$ & $\cdots$ & $\cdots$ & $\cdots$ \\
\hline 1987 & 4,184 & 92 & 83.8 & 4,994 & 175.2 & 2,999 & 5,242 & & & \\
\hline 1988 & 4,315 & 85 & 86.3 & 5,002 & 253.5 & 2,990 & 5,221 & 5,053 & 5,187 & 5,221 \\
\hline 1989 & & & & & 308.8 & 3,037 & 5,326 & 5,155 & 5,291 & 5,326 \\
\hline 1990 & & & & & 326.0 & 3,097 & 5,461 & 5,285 & 5,425 & 5,461 \\
\hline 1991 & & & & & 328.5 & 3,154 & 5,589 & 5,410 & 5,552 & 5,589 \\
\hline 1992 & & & & & 308.8 & 3,187 & 5,662 & 5,480 & 5,625 & 5,662 \\
\hline
\end{tabular}

Capacity-Capital stock Regression: Code 0 RSO 0.590

Coefficients: Const -1491.9 stock 2.2

Ave. Capacity/Stock $(74-86): 1.9$

Ave. Weekly Hours (High) 165.6 Shift factor 1.00 Ave Neekly Hours (LOW) 165.6 Shift factor 1.00 Note: Output, Investment, and Capital stock extrapolated for 1987 and 1988.

\section{Primary aluminum}

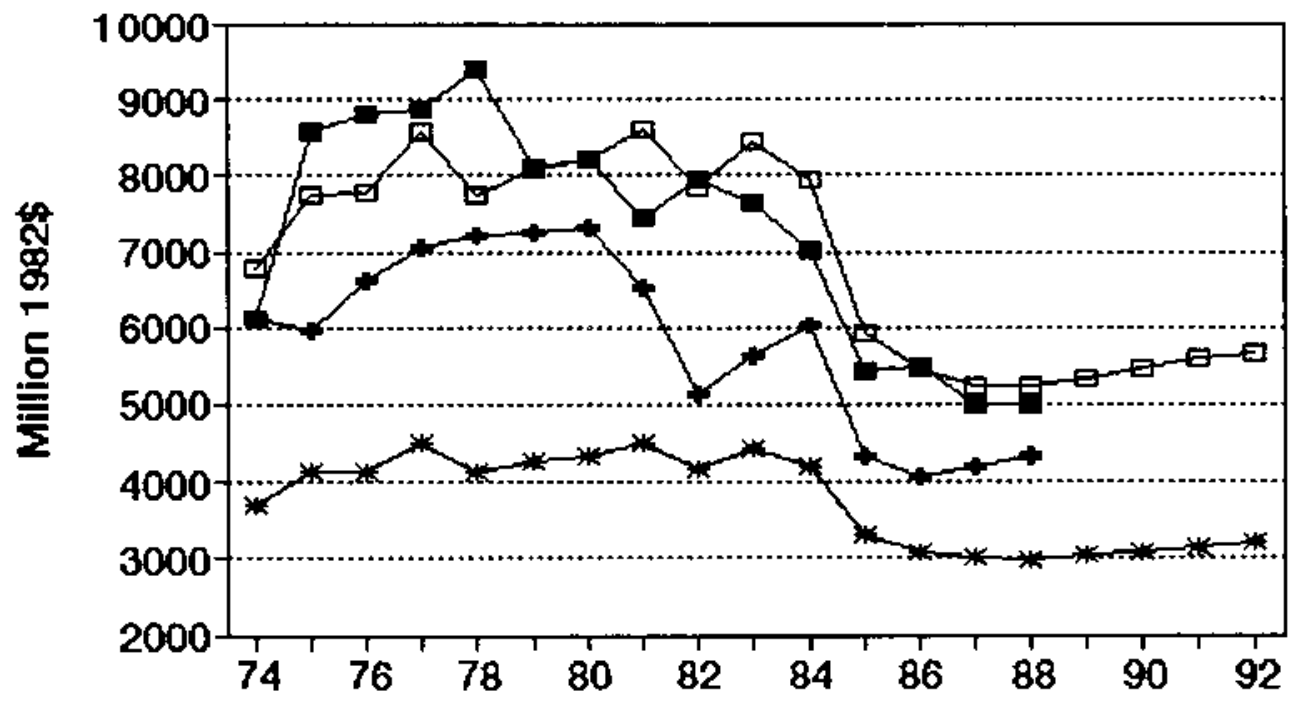

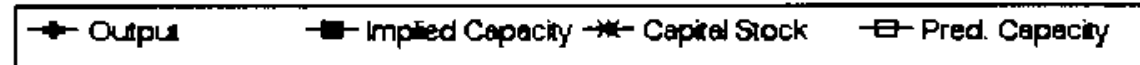


No. 267 SIC 3339 Prinary nonferrous metals, n.e.c.

MILLIONS OF 1982 DOLLARS (EXCL. CU RATES)

$\begin{array}{lccccccc} & 04 & \text { Ann. Implied Gross } & \text { Net } & \text { Pred. } & \ldots . . . . . . . \\ \text { Gross } & \text { CU } & \text { CU } & \text { Prac. } & \text { In- } & \text { Cap. } & \text { Prac. } & \text { Emergency Capacity } \\ \text { Output Rate } & \text { Rate } & \text { Capec. } & \text { vest. } & \text { Stk. } & \text { Capac. } & 3 \text { mon. } 6 \text { mon. }>6 \text { mon. }\end{array}$

\begin{tabular}{|c|c|c|c|c|c|c|c|c|c|c|}
\hline 1974 & 2,905 & 69 & 69.9 & 4.157 & 533.2 & 1,103 & 4,625 & & & \\
\hline 1975 & 3,094 & 48 & 54.4 & 5,685 & 522.6 & 1,555 & 5,592 & & & \\
\hline 1976 & 3,918 & 67 & 60.9 & 6,436 & 287.0 & 1,759 & 6,029 & & & \\
\hline 1977 & 2,630 & 38 & 49.0 & 5,368 & $(589.8)$ & 1,120 & 4,662 & & & \\
\hline 1978 & 2,892 & 61 & 51.7 & 5,597 & 55.1 & 1,120 & 4,663 & & & \\
\hline 1979 & 3,269 & 78 & 71.6 & 4,566 & $(32.5)$ & 1,036 & 4,481 & & & \\
\hline 1980 & 4,467 & 84 & 79.3 & 5,632 & $(84.9)$ & 904 & 4,199 & & & \\
\hline 1981 & 2,648 & 86 & 86.6 & 3,057 & 98.2 & 946 & 4,289 & & & \\
\hline 1982 & 2,654 & 71 & 75.8 & 3,502 & 401.1 & 1,251 & 4,942 & & & \\
\hline 1983 & 2,960 & 65 & 67.6 & 4,380 & 39.2 & 1,187 & 4,805 & & & \\
\hline 1984 & 2,817 & 57 & 60.4 & 4,661 & 59.4 & 1,138 & 4,701 & & & \\
\hline 1985 & 2,732 & 46 & 50.1 & 5,449 & 166.9 & 1,193 & 4,818 & & & \\
\hline 1986 & 2,275 & 62 & 55.7 & 4,087 & 94.9 & 1,172 & 4,773 & & & \\
\hline$=-$ & --- & $\cdot$ & $\cdots-$ & $--\cdot-$ & $-\cdots$ & $\cdots$ & $\cdots$ & --- & -- & $-\cdots$ \\
\hline 1987 & 2,313 & 88 & 77.3 & 2,993 & $(136.8)$ & 922 & 4,238 & & & \\
\hline 1988 & 2,362 & 92 & 89.8 & 2,630 & 67.3 & 900 & 4,191 & 3,853 & 4.122 & 4,191 \\
\hline 1989 & & & & & 211.4 & 1,025 & 4,458 & 4,098 & 4,384 & 4,458 \\
\hline 1990 & & & & & 256.2 & 1,182 & 4,755 & 4,407 & 4,715 & 4,795 \\
\hline 1991 & & & & & 262.6 & $1, \mathbf{3 3 1}$ & 5,112 & 4,700 & 5,028 & 5,112 \\
\hline 1992 & & & & & 211.4 & 1,414 & 5,290 & 4,863 & 5,202 & 5,290 \\
\hline
\end{tabular}

Capecity-Capital Stock Regression: Code 0 RSQ 0.260

Coefficients: Const 2264.6 stock 2.1

Ave. Capacity/Stock $(74-86): 4.0$ Ave. Weekty Hours (High) 168.0 Shift Factor 1.00 Ave Heekly Hours (LOW) 165.4 Shift Factor 1.01 Note: Output, Investment, and Capital Stock extrapolated for 1987 and 1988.

3339 Primary nonferrous metals, n.e.c.
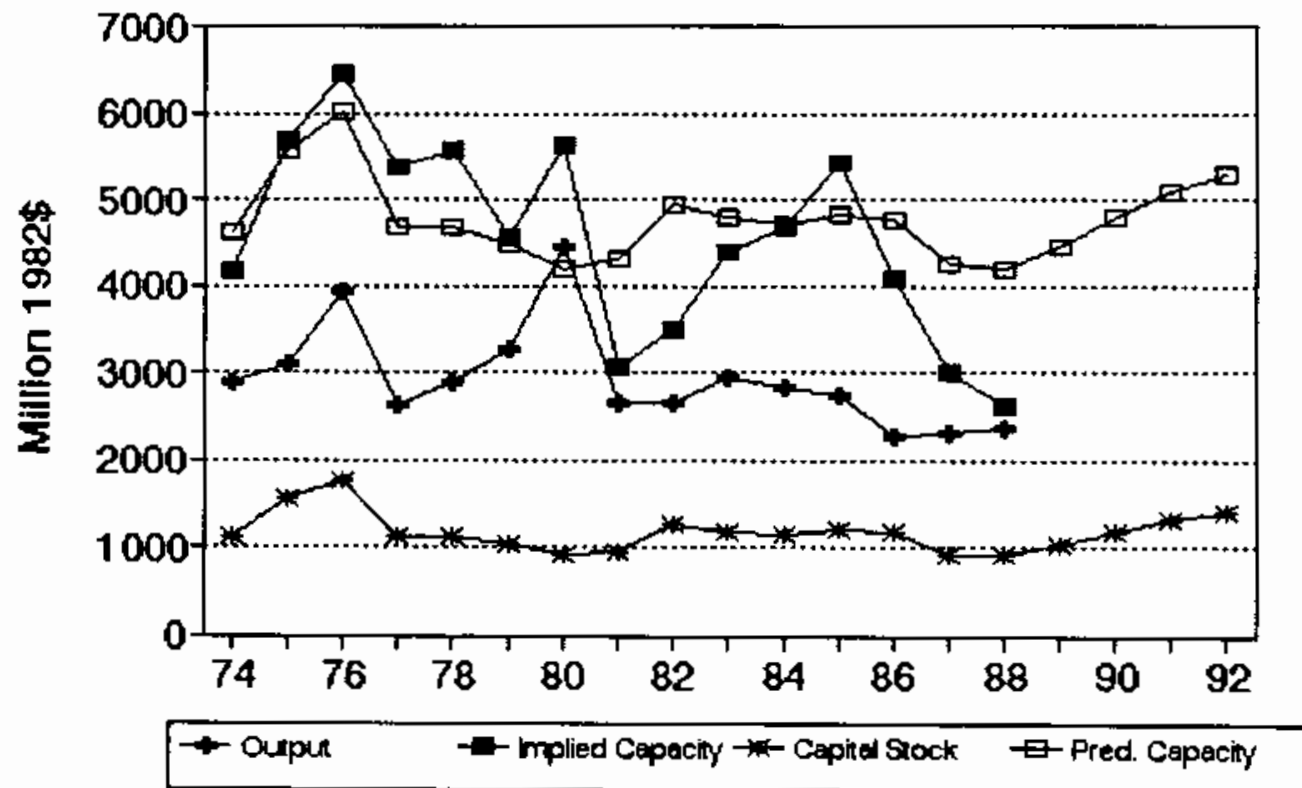


\section{No. 268 SIC 3341 secondary nonferrous eetals}

MILLIONS OF 1982 DOLLARS (EXCL. CU RATES)

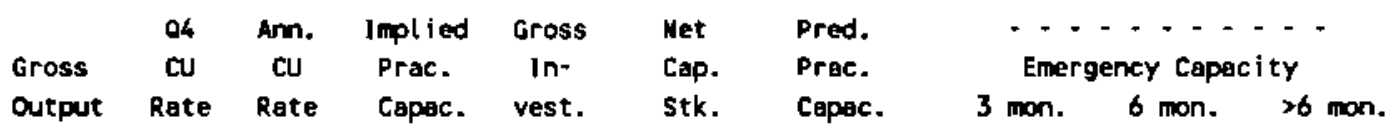

\begin{tabular}{|c|c|c|c|c|c|c|c|c|c|c|}
\hline 1974 & 5,234 & 61 & 62.3 & 8,406 & 225.2 & 748 & 7,381 & & & \\
\hline 1975 & 4,123 & 59 & 57.7 & 7,148 & 1.5 & 703 & 7,545 & & & \\
\hline 1976 & 4,739 & 57 & 59.6 & 7,958 & 114.6 & $m 1$ & 7,297 & & & \\
\hline 197 & 4,494 & 72 & 66.4 & 6,767 & 228.0 & 940 & 6,680 & & & \\
\hline 1978 & 4,422 & 65 & 66.5 & 6,648 & 130.0 & 1,007 & 6,435 & & & \\
\hline 1979 & 4,253 & 83 & 76.2 & 5,580 & 123.5 & 1,061 & 6,236 & & & \\
\hline 1980 & 4,025 & 74 & 73.6 & 5,469 & 20.7 & 1,016 & 6,402 & & & \\
\hline 1981 & 4,049 & 60 & 66.9 & 6,056 & 166.8 & 1,110 & 6,056 & & & \\
\hline 1982 & 4,944 & 50 & 52.9 & 9,355 & 283.4 & 1,302 & 8,387 & & & \\
\hline 1983 & 4,534 & 54 & 52.8 & 8,584 & (8.1) & 1,201 & 8,755 & & & \\
\hline 1984 & 4,906 & $51 *$ & 52.4 & 9,355 & (52.5) & 1,065 & 9.252 & & & \\
\hline 1985 & 4,529 & 47 & 48.3 & 9,377 & $(60.5)$ & 929 & 9,751 & & & \\
\hline 1986 & 4,418 & 48 & 47.1 & 9,377 & 36.5 & 898 & 9,903 & & & \\
\hline-- & --- & $-\cdots$ & $-\cdots$ & $\cdots$ & $\cdots$ & $\cdots \cdot$ & $\cdots$ & $\cdots---$ & --- & $-\cdot-$ \\
\hline 1987 & 4,431 & 44 & 44.9 & 9.868 & 60.5 & 873 & 9,957 & & & \\
\hline 1988 & 4,449 & 60 & 53.4 & 8,336 & 76.1 & 875 & 9,949 & 10,504 & 11,596 & 11,879 \\
\hline 1989 & & & & & 87.2 & 898 & 9,901 & 10,454 & 11,541 & 11,821 \\
\hline 1990 & & & & & 90.6 & 904 & 9,844 & 10,394 & $11,4 \pi 5$ & 11,754 \\
\hline 1991 & & & & & 91.1 & 918 & 9,791 & 10,338 & 11,413 & 11,691 \\
\hline 1992 & & & & & 87.2 & 928 & 9,757 & 10,301 & 11,373 & 11,649 \\
\hline
\end{tabular}

Capacity-Capital Stock Regression: Code -1 RSO 0.840

Coefficients: Const 10121.0 stock -3.7 D(82-86) 3032.8 Ave. Capacity/5tock (74-86): 7.9 Ave. Weekly Hours (High) 132.9 Shift Factor 1.19 Ave Weekly Hours (LOW) 131.4 Shift Factor 1.21 - Imputed Mote: Output, Investment, and Capital Stack extrapolated for 1987 and 1988.

\section{Secondary nonferrous metals}

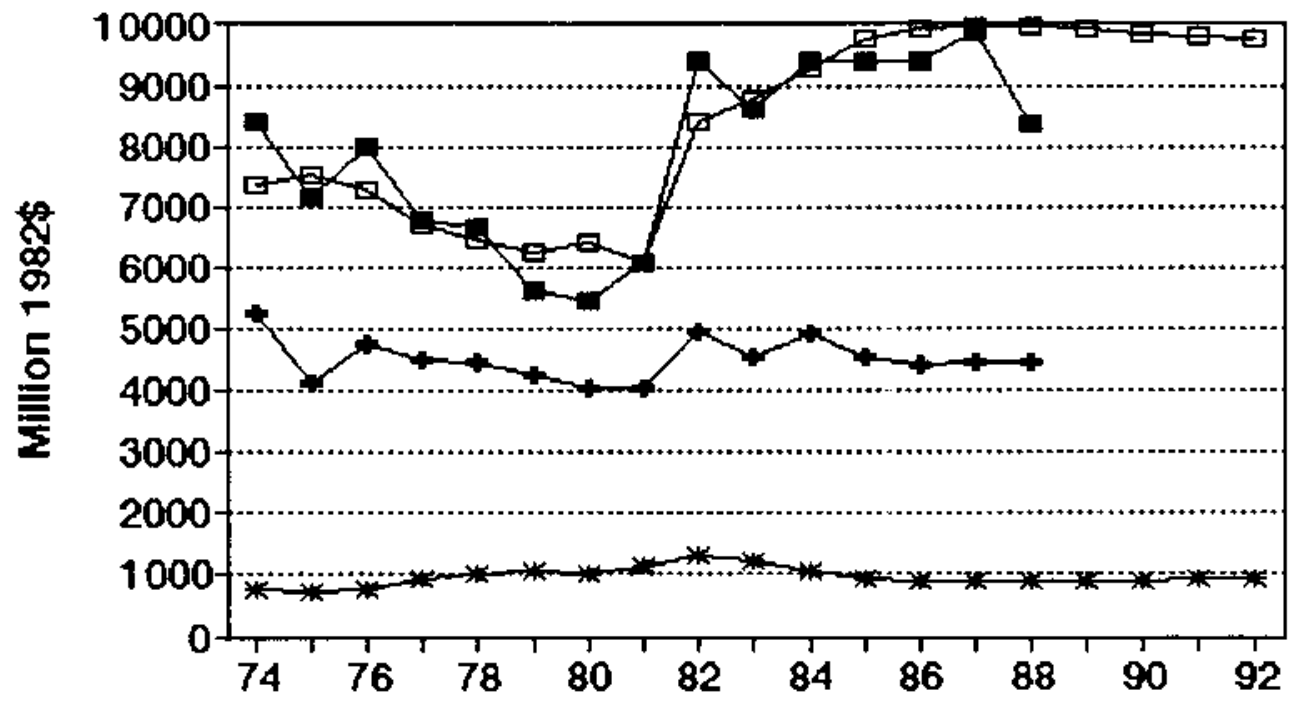

$\rightarrow$ Oupu $\quad-$ - Impled Cepeciny $\rightarrow$ - Capial Stock $\quad$ - Pred. Capeciny




\section{No, 269 sIC 3351 Coppper rolling and drawing}

MILLIONS OF 1982 DOLLARS (EXCL. CU RATES)

\begin{tabular}{|c|c|c|c|c|c|c|c|}
\hline & $\begin{array}{l}04 \\
C U\end{array}$ & $\begin{array}{c}\text { Ann. } \\
\text { CU }\end{array}$ & $\begin{array}{l}\text { Impl ied } \\
\text { Prac. }\end{array}$ & $\begin{array}{c}\text { Gross } \\
\ln ^{+}\end{array}$ & $\begin{array}{l}\text { Net } \\
\text { Cap. }\end{array}$ & $\begin{array}{l}\text { Pred. } \\
\text { Prac. }\end{array}$ & Emergency Capacity \\
\hline & Rate & Rate & Capac. & vest. & stk. & Capa & \\
\hline
\end{tabular}

\begin{tabular}{|c|c|c|c|c|c|c|c|c|c|c|}
\hline 1974 & 5,673 & 68 & 69.8 & 8,125 & 140.2 & 1,829 & 7,398 & & & \\
\hline 1975 & 3,798 & 54 & 56.3 & 6,747 & 163.0 & 1,830 & 7,403 & & & \\
\hline 1976 & 4,649 & 69 & 65.8 & 7,068 & 115.4 & 1,781 & 7,252 & & & \\
\hline 1977 & 4,847 & 69 & 69.1 & 7,010 & $(61.0)$ & 1,573 & 6,610 & & & \\
\hline 1978 & 4,878 & 79 & 73.5 & 6,639 & 116.4 & 1,539 & 6,505 & & & \\
\hline 1979 & 4,992 & 73 & 74.9 & 6,660 & 286.3 & 1,655 & 6,863 & & & \\
\hline 1980 & 4,181 & 69 & 66.0 & 6,332 & 64.2 & 1,556 & 6,557 & & & \\
\hline 1981 & 4,410 & 63 & 67.5 & 6,535 & 118.0 & 1,513 & 6,425 & & & \\
\hline 1982 & 3,278 & 52 & 55.0 & 5,964 & 41.6 & 1,409 & 6,105 & & & \\
\hline 1983 & 3,650 & 65 & 60.4 & 6,039 & 51.7 & 1,325 & 5,845 & & & \\
\hline 1984 & 3,848 & 67 & 67.3 & 5,717 & 81.8 & 1,278 & 5,700 & & & \\
\hline 1985 & 3,451 & 71 & 69.4 & 4,969 & $(45.9)$ & 1,125 & 5,229 & & & \\
\hline 1986 & 3,588 & 69 & 68.7 & 5,220 & 72.7 & 1,094 & 5,134 & & & \\
\hline$\because 987$ & 3,661 & 83 & 76.0 & 4,815 & (7.8) & 982 & 4,790 & $\cdots$ & $\cdots$ & $\cdots$ \\
\hline 1988 & 3,759 & 80 & 79.8 & 4,709 & 65.0 & 954 & 4,703 & 4,670 & 4,835 & 4.877 \\
\hline 1989 & & & & & 116.5 & 980 & 4,783 & 4.730 & 4,917 & 4,960 \\
\hline 1990 & & & & & 132.5 & 1,020 & 4.905 & 4,870 & 5,042 & 5,086 \\
\hline 1991 & & & & & 134.7 & 1,058 & 5,022 & 4,987 & 5,162 & 5,207 \\
\hline 1992 & & & & & 116.5 & 1.074 & 5,071 & 5,036 & 5,213 & 5,259 \\
\hline
\end{tabular}

Capecity-Capital Stock Regression: Code 0 RSO 0.830

Coefficients: Const 1762.6 stock 3.1

Ave. Capacity/stock $(74-86): 4.3$ Ave. Weekly Hours (High) 154.1 Shift Factor 1.04 Ave Heekly Hours (Low) 143.1 Shift factor 1.11 Note: Output, Investment, and Capital Stock extrapolated for 1987 and 1988.

\section{Coppper rolling and drawing}

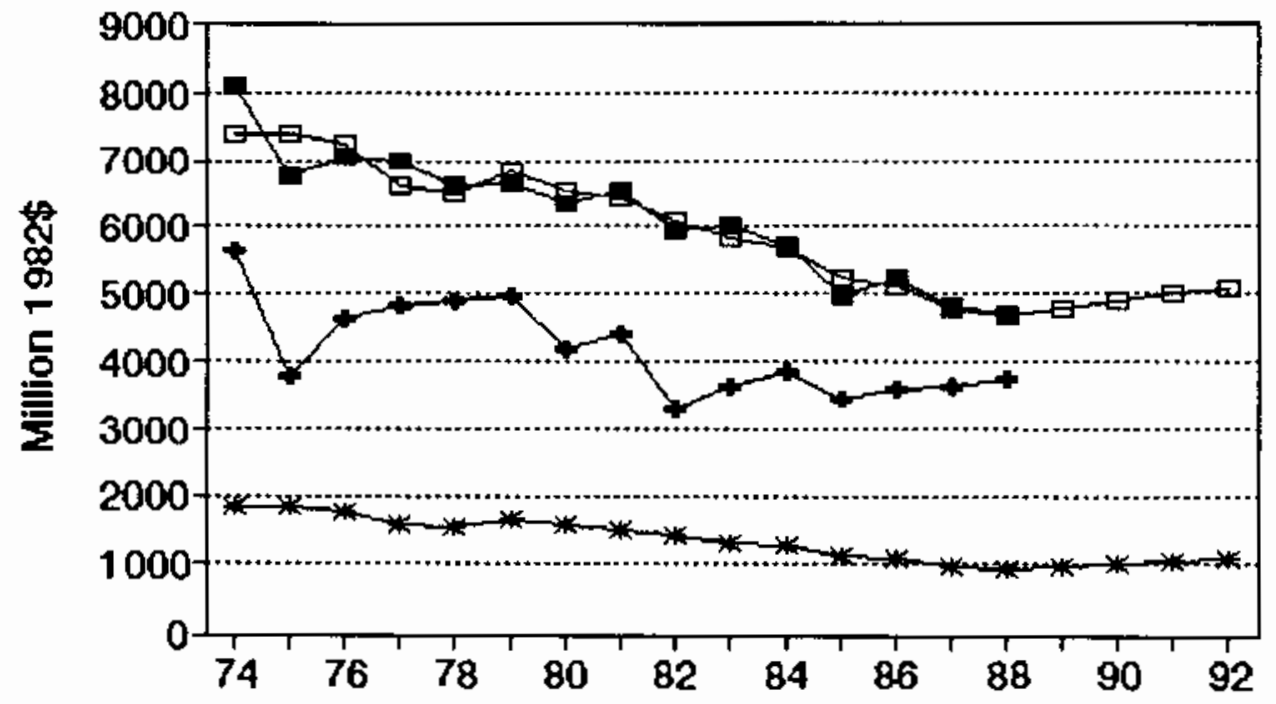

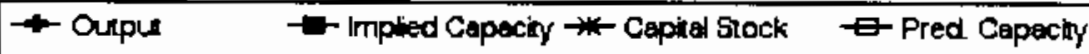


No. 270 sIC 3353 Aluminum sheet plate, and foil

MILLIONS OF 1982 DOLLARS (EXCL. CU RATES)

$\begin{array}{lccccccc} & \alpha 4 & \text { Ann. Implied Gross } & \text { Net } & \text { Pred. } & \ldots & \ldots \\ \text { Gross } & \text { CU } & \text { CU } & \text { Prac. } & \text { In- } & \text { Cap. } & \text { Prac. } & \text { Emergency Capacity } \\ \text { Output } & \text { Rate } & \text { Rate } & \text { Capac. } & \text { vest. } & \text { Stk. } & \text { Capac. } & 3 \text { mon. } 6 \text { mon. }>6 \text { mon. }\end{array}$

\begin{tabular}{|c|c|c|c|c|c|c|c|c|c|c|}
\hline 1974 & 8,919 & 77 & 78.1 & 11,422 & 425.8 & 3,554 & 10,907 & & & \\
\hline 1975 & 7,087 & 71 & 71.5 & 9,916 & 202,3 & 3,472 & 10,969 & & & \\
\hline 1976 & 8,948 & $\pi$ & 75.0 & 11,935 & 219.8 & 3,409 & 11,017 & & & \\
\hline 1977 & 9,157 & 86 & 81.9 & 11,177 & 291.5 & 3,412 & 11,015 & & & \\
\hline 1978 & 9,699 & 93 & 89.3 & 10,866 & 342.7 & 3,464 & 10,975 & & & \\
\hline 1979 & 9,571 & 87 & 89.1 & 10,748 & 449.8 & 3,638 & 10,843 & & & \\
\hline 1980 & 9,497 & 86 & 83.6 & 11,366 & 443.9 & 3,796 & 10,723 & & & \\
\hline 1981 & 9,331 & 72 & 78.5 & 11,883 & 414.0 & 3,914 & 10,633 & & & \\
\hline 1982 & 7,054 & 70 & 70.1 & 10,060 & 184.6 & 3,806 & 10,715 & & & \\
\hline 1983 & 8,220 & 81 & 7.0 & 10,676 & 322.6 & 3,831 & 10,696 & & & \\
\hline 1984 & 7,854 & 79 & 80.4 & 9,771 & 252.2 & 3,791 & 10,727 & & & \\
\hline 1985 & 7,549 & 78 & 78.6 & 9,636 & 382.0 & 3,871 & 10,666 & & & \\
\hline 1986 & 8,593 & 79 & 78.1 & 11,007 & 425.4 & 3,988 & 10,577 & & & \\
\hline 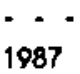 & . & 81 & $\begin{array}{l}--. \\
79.4\end{array}$ & 我 & $\begin{array}{l}\cdots \\
326.1\end{array}$ & 4,003 & $\begin{array}{l}-\cdots \\
10,566\end{array}$ & $=\cdot$ & . . - & $\cdots$ \\
\hline 1988 & 8,945 & 77 & 77.8 & 11,492 & 327.2 & 4,019 & 10,554 & 10,256 & 10,493 & 10,554 \\
\hline 1989 & & & & & 329.4 & 4,036 & 10,541 & 10,243 & 10,479 & 10,541 \\
\hline 1990 & & & & & 330.1 & 4,053 & 10,528 & 10,231 & 10,467 & 10,528 \\
\hline 1991 & & & & & 330.2 & 4,068 & 10,516 & 10,220 & 10,455 & 10,516 \\
\hline 1992 & & & & & 329.4 & 4,082 & 10,506 & 10,210 & 10,445 & 10,506 \\
\hline
\end{tabular}

Capacity-Capital Stock Regression: Code O RSQ 0.040

Coefficients: Const 13606.9 stock $-0.8 \quad$ Ave. Capacity/5tock $(74-86): 2.9$ Ave. Weekly Hours (High) 163.2 Shift Factor 1.00 Ave Weekly Hours (Low) 162.0 Shift Factor 1.01 Note: Output, Investment, and Capital Stock extrapolated for 1987 and 1988.

3353 Aluminum sheet plate, and foil

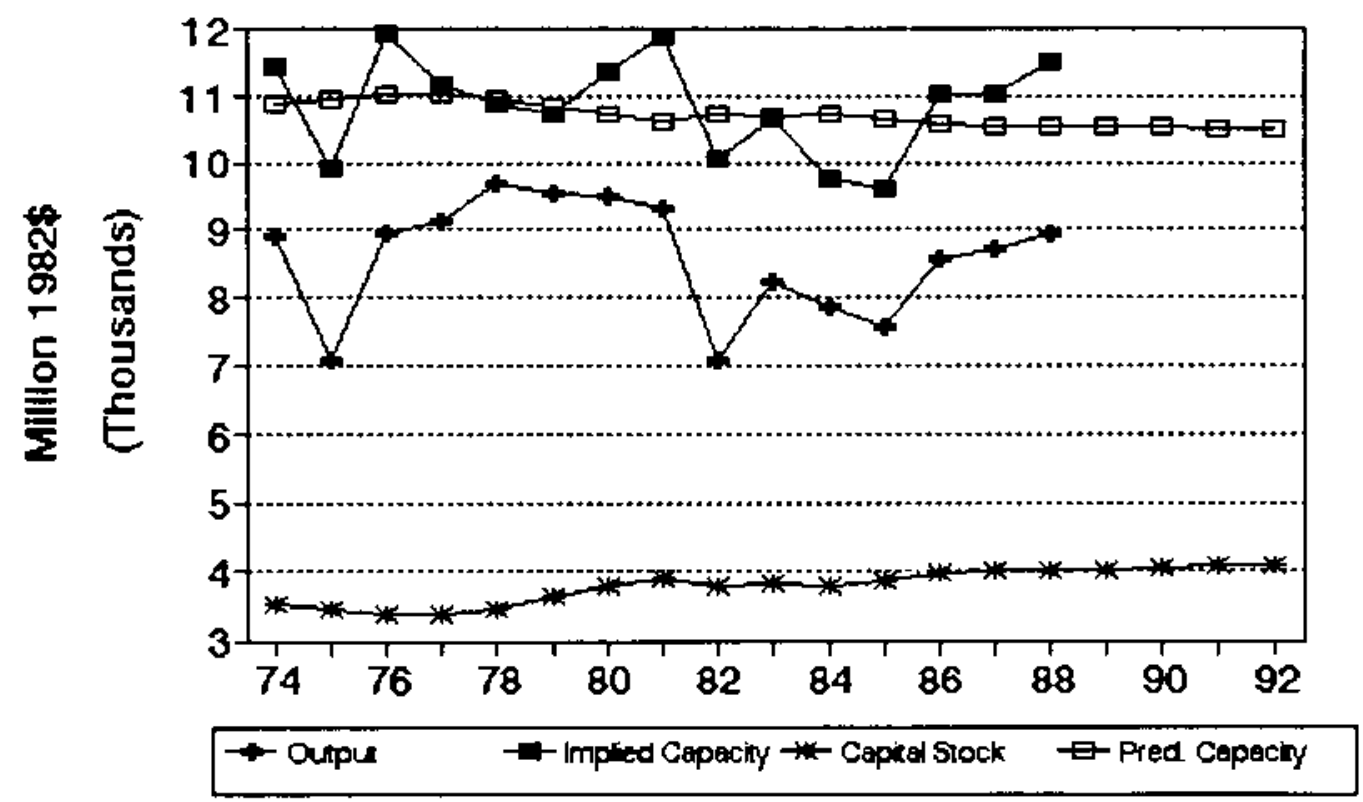




\section{ผo. 271 sIc 3354 Aluminue extruded products}

MILLIONS OF 1982 DOLLARS (EXCL. CU RATES)

$\begin{array}{lccccccc} & Q 4 & \text { Am. Implied Gross } & \text { Net } & \text { Pred. } & \ldots \\ \text { Gross } & \text { CU } & \text { QU } & \text { Prac. } & \text { In- } & \text { Cap. } & \text { Prac. } & \text { Emergency Capacity } \\ \text { Output Rate } & \text { Rate } & \text { Capac. } & \text { vest. } & \text { Stk. } & \text { Capac. } & 3 \text { mon. } 6 \text { mon. }>6 \text { mon. }\end{array}$

\begin{tabular}{|c|c|c|c|c|c|c|c|c|c|c|}
\hline 1974 & 3,287 & 55 & 56.4 & 5.826 & 9.1 & 811 & 4,528 & & & \\
\hline 1973 & 2,168 & 50 & 49.5 & 4,376 & 73.9 & 821 & 4,546 & & & \\
\hline 1976 & 2,677 & 61 & 59.0 & 4,539 & 44.7 & 800 & 4,507 & & & \\
\hline 1977 & 3,002 & 82 & 74.2 & 4,048 & 83.8 & 813 & 4,532 & & & \\
\hline 1978 & 3,214 & 83 & 80.9 & 3,976 & 24.2 & 769 & 4,452 & & & \\
\hline 1979 & 3,348 & 78 & 79.6 & 4,208 & 91.9 & 788 & 4,486 & & & \\
\hline 1980 & 3,041 & 66 & 66.1 & 4,598 & 91.4 & 804 & 4,516 & & & \\
\hline 1981 & 2,904 & 60 & 64.3 & 4,517 & 87.4 & 813 & 4,532 & & & \\
\hline 1982 & 2,689 & 55 & 56.0 & 4,806 & 186.8 & 912 & 4,709 & & & \\
\hline 1983 & 3,018 & 67 & 62.9 & 4,802 & 51.4 & 879 & 4,650 & & & \\
\hline 1984 & 3,333 & 70 & 69.9 & 4,767 & 168.3 & 959 & 4,794 & & & \\
\hline 1985 & 3,313 & 69 & 69.3 & 4,770 & 111.0 & 982 & 4,836 & & & \\
\hline 1986 & 3,352 & 74 & 71.1 & 4,711 & 104.9 & 99 & 4,867 & & & \\
\hline & $\cdots$ & $\because$ & $\because \cdot$ & & $\cdots$ & $\cdots$ & $\cdots$ & $\cdots-$ & -- & -- \\
\hline 1987 & 3,413 & 85 & 79.2 & 4,309 & 138.2 & 1,049 & 4,956 & & & \\
\hline 1988 & 3,495 & 84 & 83.1 & 4,207 & 145.2 & 1,101 & 5,050 & 5,523 & 5,889 & 5,984 \\
\hline 1989 & & & & & 152.2 & 1,155 & 5,148 & 5,631 & 6,004 & 6,100 \\
\hline 1990 & & & & & 159.2 & 1,212 & 5,250 & 5,742 & 6,123 & 6,221 \\
\hline 1991 & & & & & 166.2 & 1,271 & 5,356 & 5,858 & 6,246 & 6,346 \\
\hline 1992 & & & & & 173.2 & 1,331 & 5,465 & 5,977 & 6,373 & 6,476 \\
\hline
\end{tabular}

Capacity-Capital stock Regression: Code 0 RSQ 0.100 Coefficients: Const 3006.1 stock 1.8

Ave. Capacity/Stock $(74-86): 5.4$ Ave. Weekly Hours (High) 133.9 Shift Factor 1.18 Ave Heekly Hours (Low) 132.8 Shift Factor 1.19 Note: Output, Investment, and Capital Stock extrapolated for 1987 and 1988. 3354 Aluminum extruded products

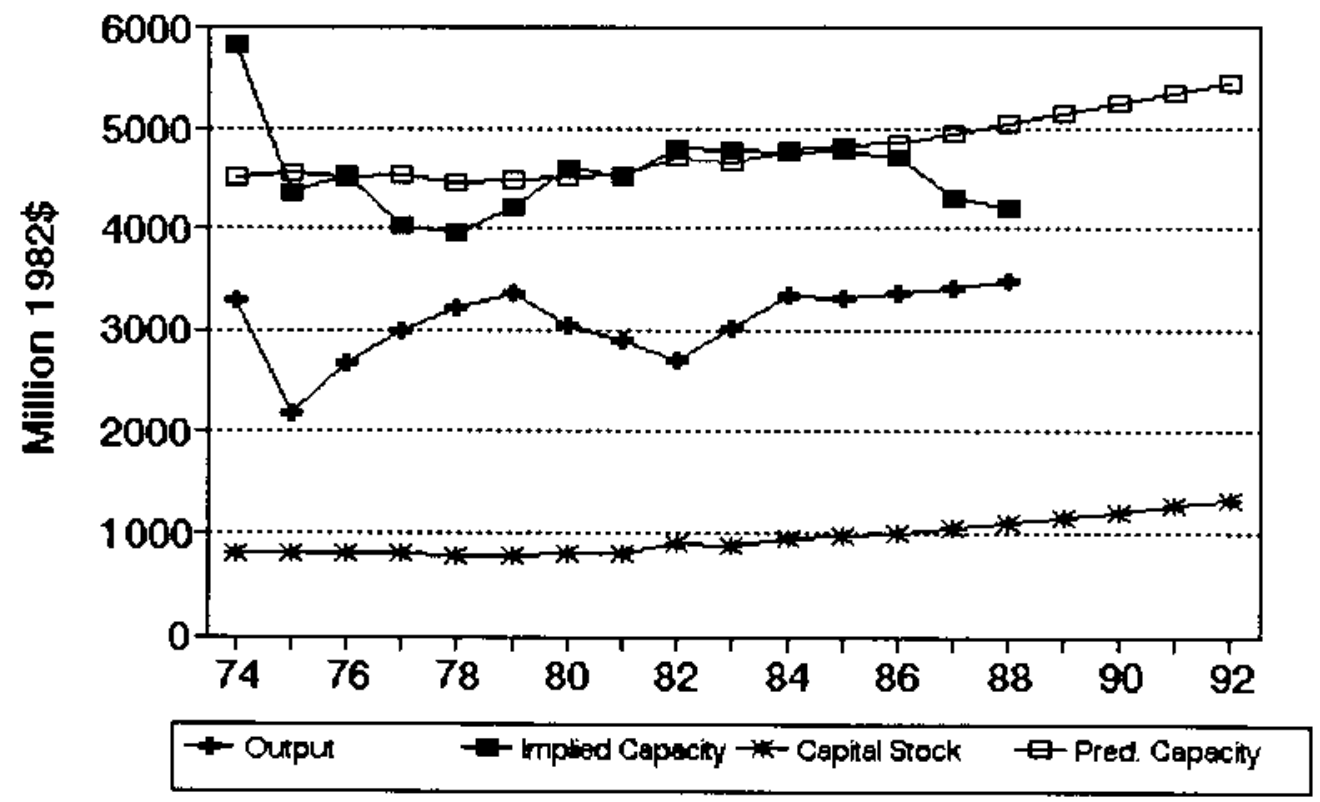




\title{
No. 272 sIC 3355 Aluminum rolling and drawting, nec
}

\begin{abstract}
MILLIONS OF 1982 DOLLARS (EXCL. CU RATES)
\end{abstract}
$\begin{array}{lccccccc} & \text { Q4 Ann. Implied Gross } & \text { Net } & \text { Pred. } & \ldots \ldots \ldots \\ \text { Gross } & \text { CU } & \text { CU } & \text { Prac. } & \text { In- } & \text { Cap. } & \text { Prac. } & \text { Energency Capacity } \\ \text { Output Rate } & \text { Rate } & \text { Capac. } & \text { vest. } & \text { Stk. } & \text { Capac. } & 3 \text { mon. } 6 \text { mon. }>6 \text { mon. }\end{array}$

\begin{tabular}{|c|c|c|c|c|c|c|c|c|c|c|}
\hline 1974 & 1,004 & 57 & 57.5 & 1,745 & 14.3 & 261 & 1,629 & & & \\
\hline 1975 & 745 & 51 & 52.4 & 1,422 & 39.7 & 275 & 1,744 & & & \\
\hline 1976 & 856 & 55 & 54.2 & 1,581 & 40.5 & 289 & 1,850 & & & \\
\hline 1977 & 1,385 & 67 & 62.5 & 2,215 & 45.2 & 304 & 1.972 & & & \\
\hline 1978 & 1,541 & $\pi 5$ & 71.4 & 2,159 & 49.9 & 323 & 2,117 & & & \\
\hline 1970 & 1,558 & 72 & 73.0 & 2,134 & 50.1 & 340 & 2,257 & & & \\
\hline 1980 & 1,622 & 63 & 65.0 & 2,497 & 41.2 & 348 & 2,315 & & & \\
\hline 1981 & 1,366 & 52 & 56.7 & 2,409 & 35.6 & 349 & 2,324 & & & \\
\hline 1982 & 513 & 53 & 52.3 & 980 & $(113.8)$ & 216 & 1,275 & & & \\
\hline 1983 & 842 & 79 & 69.1 & 1,219 & (18.4) & 181 & 1,001 & & & \\
\hline 1984 & 814 & 81 & 80.6 & 1,010 & 24.0 & 187 & 1,047 & & & \\
\hline 1985 & 761 & 63 & 69.8 & 1,091 & 25.7 & 192 & 1,093 & & & \\
\hline 1986 & 609 & 48 & 53.4 & 1,141 & 4.9 & 178 & 979 & & & \\
\hline$\cdots$ & $\therefore \cdot$ & - - & $-\cdots$ & $\cdots$ & $\cdots$ & -- & $-\cdots$ & $\cdots$ & $\because \cdot$ & $-\cdot-$ \\
\hline 1987 & 616 & 55 & 52.0 & 1,184 & $(14.9)$ & 144 & 714 & & & \\
\hline 1988 & 625 & 55 & 54.7 & 1,142 & 12.1 & 141 & 689 & 953 & 1,012 & 1,028 \\
\hline 1989 & & & & & 31.2 & 157 & 817 & 1,130 & 1,200 & 1,218 \\
\hline 1990 & & & & & 37.1 & 178 & 978 & 1,352 & 1,436 & 1,458 \\
\hline 1991 & & & & & 38.0 & 197 & 1,129 & 1,560 & 1,658 & 1,683 \\
\hline 1992 & & & & & 31.2 & 207 & 1,210 & 1.673 & 1,777 & 1,804 \\
\hline
\end{tabular}

Capacity-Capital stock Regression: Code 0 RSO 0.880

Coefficients: Const -419.0 stock 7.9

Ave. Capacity/Stock (74-86): 6.3 Ave. Weekly Hours (High) 105.6 Shift Factor 1.49 Ave Veekly Hours (LOW) 97.0 Shift factor 1.62 Note: Output, Investment, and Capital Stock extrapolated for 1987 and 1988.

\section{Aluminum rolling and drawing, nec}

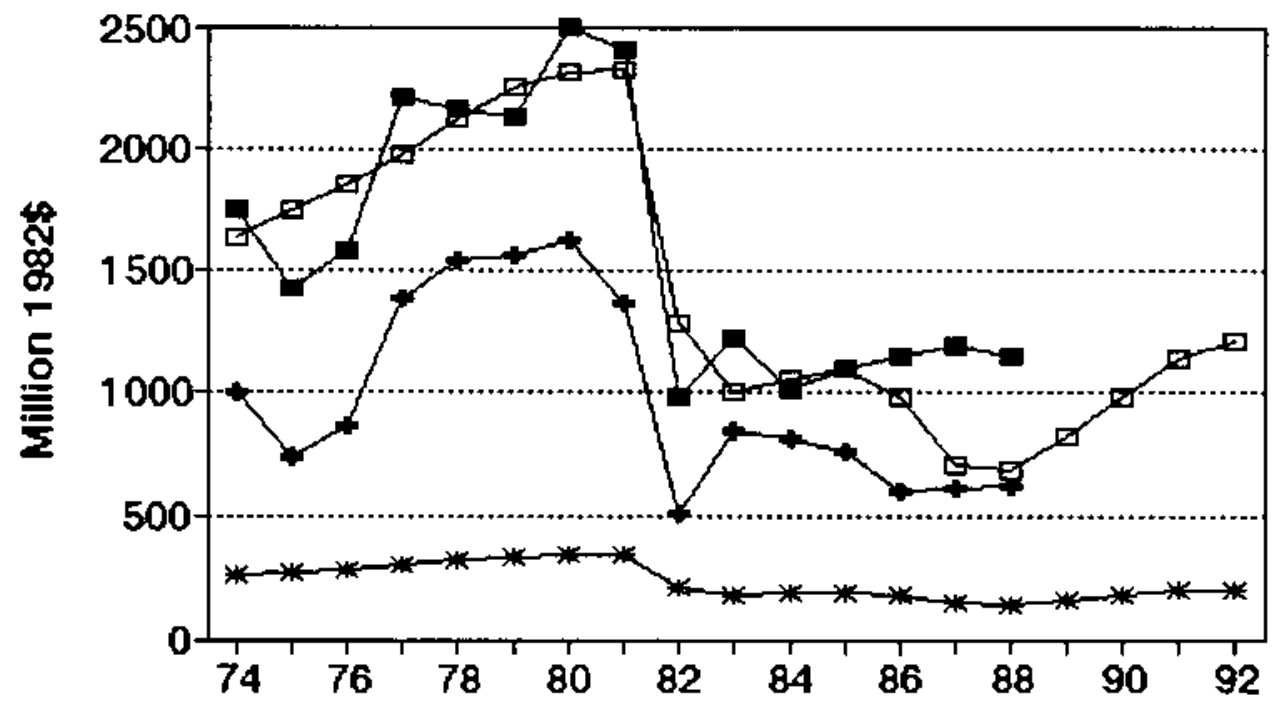

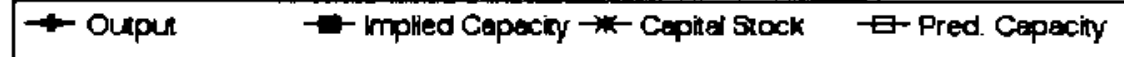


No. 273 sIC 3356 Nonferrous rolling and drawing, nec MILLIONS OF 1982 DOLLARS (EXCL. CU RATES)

$\begin{array}{lccccccc} & \text { Q4 } & \text { Ann. Implied Gross } & \text { Net } & \text { Pred. } & -\ldots \ldots \ldots \\ \text { Gross } & \text { CU } & \text { CU } & \text { Prac. } & \text { In- } & \text { Cap. } & \text { Prac. } & \text { Emergency Capacity } \\ \text { Output Rate } & \text { Rate } & \text { Capac. } & \text { vest. } & \text { Stk. } & \text { Capac. } & 3 \text { mon. } 6 \text { mon. }>6 \text { mon. }\end{array}$

\begin{tabular}{|c|c|c|c|c|c|c|c|c|c|c|}
\hline 1974 & 4,395 & 74 & 74.2 & 5,924 & 109.1 & 1,026 & 6,889 & & & \\
\hline 1975 & 3,521 & 59 & 64.3 & 5,476 & 432.1 & 1,076 & 6,811 & & & \\
\hline 1976 & 3,551 & 49 & 53.0 & 6,706 & 117.4 & 1,105 & 6,733 & & & \\
\hline 1977 & 4,522 & 47 & 47.8 & 9,468 & $(5.6)$ & 1,013 & 6,655 & & & \\
\hline 1978 & 4,328 & 47 & 46.9 & 9,230 & 101.4 & 1,021 & 6.577 & & & \\
\hline 1979 & 3,943 & 74 & 63.9 & 6,172 & 26.6 & 953 & 6,499 & & & \\
\hline 1980 & 3,586 & 70 & 71.1 & 5,046 & 136.1 & 989 & 6,421 & & & \\
\hline 1981 & 3,603 & 65 & 67.1 & 5,371 & 161.5 & 1,049 & 6,343 & & & \\
\hline 1982 & 3,568 & 56 & 59.2 & 6,022 & 178.5 & 1,118 & 6,265 & & & \\
\hline 1983 & 3,200 & 67 & 62.9 & 5,090 & 102.9 & 1,111 & 6.187 & & & \\
\hline 1984 & 3,272 & 55 & 59.6 & 5,492 & 61.3 & 1,066 & 6.109 & & & \\
\hline 1985 & 3,641 & 51 & 52.5 & 6,935 & 127.3 & 1,085 & 6,030 & & & \\
\hline 1986 & 3,495 & 55 & 53.4 & 6,540 & 77.7 & 1,056 & 5,952 & & & \\
\hline-- & $-\cdot-$ & $\because$ & $\because \cdot$ & $-\cdot-\cdot$ & - - - - & $-\cdots$ & $\cdots$ & $\cdots$ & $\cdots$ & $\cdots$ \\
\hline 1987 & 3,501 & 57 & 56.1 & 6,237 & 71.8 & 1,022 & 5,874 & & & \\
\hline 1988 & 3,510 & 58 & 57.5 & 6,100 & 90.8 & 1,010 & 5,796 & 5,794 & 6,206 & 6,312 \\
\hline 1989 & & & & & 104.2 & 1,013 & 5,718 & 5,716 & 6,122 & 6,227 \\
\hline 1990 & & & & & 108.4 & 1,020 & 5,640 & 5,637 & 6,038 & 6,142 \\
\hline 1981 & & & & & 109.0 & 1,027 & 5,562 & 5,559 & 5,955 & 6,057 \\
\hline 1982 & & & & & 104.2 & 1,028 & 5,484 & 5,481 & 5,871 & 5,972 \\
\hline
\end{tabular}

Capacity-Capital Stock Regression: Code -2 RSO 0.050

Coefficients: Const 6967.5 Time -78.1

Ave. Capacity/Stock $(74-86): 6.1$ Ave. Heekly Hours (High) 146.3 Shift Factor 1.09 Ave Leekly Hours (Low) 145.7 Shift Factor 1.09 Note: Dutput, Investment, and Capital Stock extrapolated for 1987 and 1988.

3356 Nonferrous rolling and drawing, ne

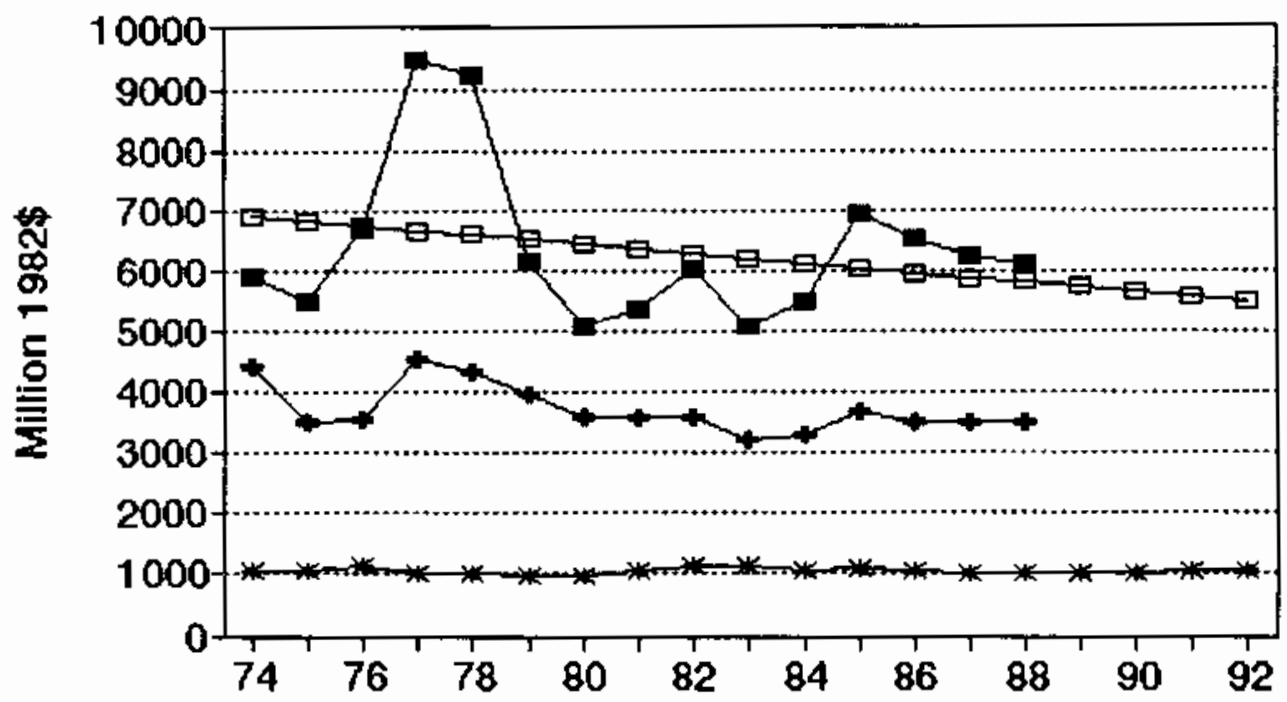

$\rightarrow$ Oupur - Implied Cepecity - Cepitel Stock E-Pred. Capacity 
No. 274 sIC 3357 Nonferrous wire drawing and insulating MILLIONS OF 1982 DOLLARS (EXCL. CU RATES)

\begin{tabular}{|c|c|c|c|c|c|c|c|}
\hline & $\begin{array}{l}04 \\
\mathrm{CU}\end{array}$ & Ann. & $\begin{array}{l}\text { Implied } \\
\text { Prac. }\end{array}$ & $\begin{array}{l}\text { Gross } \\
\text { In- }\end{array}$ & $\begin{array}{l}\text { Net } \\
\text { Cap. }\end{array}$ & $\begin{array}{l}\text { Pred. } \\
\text { Prac. }\end{array}$ & $\begin{array}{l}--------- \\
\text { Energency Capacity }\end{array}$ \\
\hline & Rate & Rate & & & & 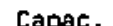 & \\
\hline
\end{tabular}

\begin{tabular}{|c|c|c|c|c|c|c|c|c|c|c|}
\hline 1974 & 8,154 & 69 & 70.2 & 11,611 & 401.0 & 2,981 & 12,192 & & & \\
\hline 1975 & 6,307 & 43 & 50.8 & 12,422 & 122.7 & 2,897 & 12,298 & & & \\
\hline 1976 & 7,375 & 57 & 53.0 & 13,918 & 145.8 & 2,826 & 12,405 & & & \\
\hline 1977 & 8,549 & 73 & 67.0 & 12,754 & 286.6 & 2,875 & 12,511 & & & \\
\hline 1978 & 9,155 & 66 & 67.7 & 13,533 & 33.6 & 2,677 & 12,618 & & & \\
\hline 1979 & 8,846 & 83 & 76.6 & 11,550 & 233.6 & 2,666 & 12,725 & & & \\
\hline 1980 & 8,195 & 70 & 71.7 & 11,424 & 254.4 & 2,666 & 12,831 & & & \\
\hline 1981 & 8,791 & 69 & 70.9 & 12,398 & 190.9 & 2,606 & 12,938 & & & \\
\hline 1982 & 8,185 & 63 & 64.4 & 12,701 & 360.3 & 2,690 & 13,044 & & & \\
\hline 1983 & 8,239 & 63 & 63.4 & $12, \infty 97$ & 199.0 & 2,620 & $\uparrow 3,151$ & & & \\
\hline 1986 & 9,414 & 68 & 66.8 & 14,098 & 119.2 & 2,493 & 13,257 & & & \\
\hline 1985 & 9,150 & 65 & 66.1 & 13,841 & 224.7 & 2,470 & 13,366 & & & \\
\hline 1986 & 8,983 & 68 & 66.3 & 13,556 & 180.2 & 2,407 & 13,471 & & & \\
\hline$\cdots$ & $-\ldots$ & - - & -- & $-\cdot-$ & -- & $\cdots$ & $\cdots$ & $\cdots \cdot \cdot \cdot$ & 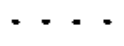 & $=\cdot-$ \\
\hline 1987 & 9,063 & 78 & 73.2 & 12,386 & 213.0 & 2,378 & 13,577 & & & \\
\hline 1988 & 9,168 & 82 & 79.7 & 11,510 & 214.5 & 2,353 & 13,684 & 14,454 & 15,408 & 15,654 \\
\hline 1989 & & & & & 216.1 & 2,332 & 13,790 & 14,567 & 15,528 & 15,776 \\
\hline 1990 & & & & & 217.6 & 2,315 & 13,897 & 14,680 & 15,648 & 15,898 \\
\hline 1991 & & & & & 219.1 & 2,301 & 14,004 & 14,792 & 15,768 & 16,020 \\
\hline 1992 & & & & & 220.6 & 2,290 & 14,110 & 14,905 & 15,888 & 16,142 \\
\hline
\end{tabular}

Capacity-Capital Stock Regression: Code -2 RSQ 0.200

Coefficients: Const 12084.9 Time 106.6

Ave. Capacity/Stock $(74-86): 4.8$

Ave. Weekly Hours (High) 138.9 Shift Factor 1.14 Ave Weekly Hours (Low) 128.5 Shift factor 1.23 Note: Output, Imvestment, and Capital Stock extrapolated for 1987 and 1988.

3357 Nonferrous wire drawing and insula

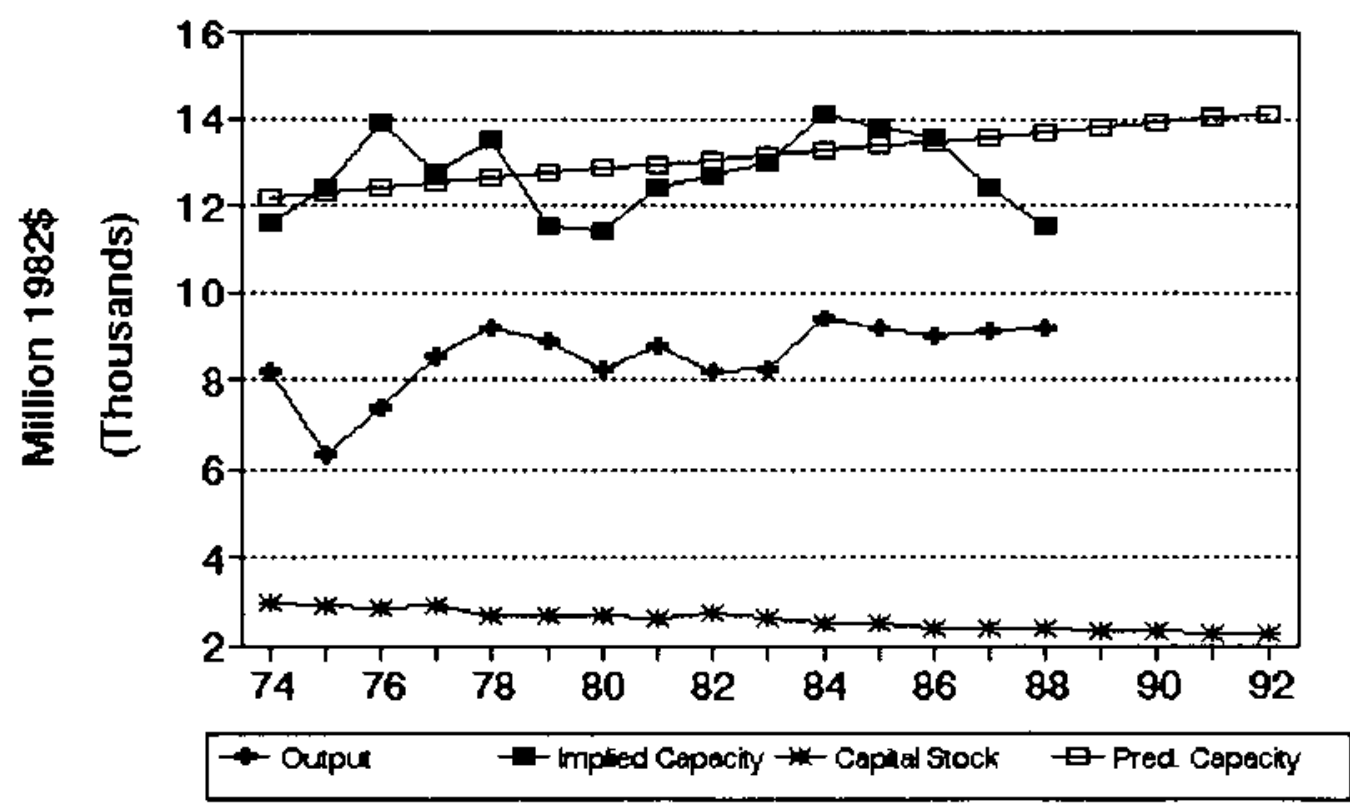


No. 275 sIc 3361 Aluminum foundries

MILLIONS OF 1982 DOLLARS (EXCL. CU RATES)

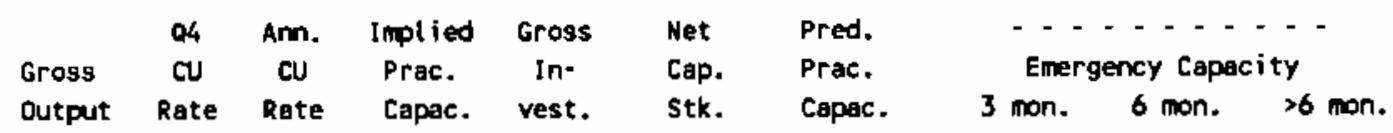

\begin{tabular}{|c|c|c|c|c|c|c|c|c|c|c|}
\hline 1974 & 3,833 & 71 & 73.5 & 5,215 & 149.5 & 989 & 4,541 & & & \\
\hline 1975 & 2,771 & 62 & 61.3 & 4,523 & 223.3 & 1,142 & 5,046 & & & \\
\hline 1976 & 3,312 & 69 & 70.0 & 4,732 & (61.1) & 1,016 & 4,627 & & & \\
\hline 1977 & 3,417 & 81 & 76.6 & 4,459 & 155.1 & 1,099 & 4,904 & & & \\
\hline 1978 & 3,759 & 83 & 79.9 & 4,706 & 213.5 & 1,232 & 5,344 & & & \\
\hline 1979 & 3,988 & 73 & 76.3 & 5,226 & 213.4 & 1,356 & 5,754 & & & \\
\hline 1980 & 3,380 & 61 & 59.9 & 5,644 & 184.7 & 1,442 & 6,037 & & & \\
\hline 1981 & 3,484 & 54 & 59.3 & 5,877 & 135.6 & 1,472 & 6,137 & & & \\
\hline 1982 & 3,023 & 40 & 43.9 & 6,892 & 105.3 & 1,465 & 6,114 & & & \\
\hline 1983 & 3,391 & 58 & 51.5 & 6,581 & $(44.2)$ & 1,315 & 5,619 & & & \\
\hline 1984 & 3,992 & 67 & 65.0 & 6,143 & 141.1 & 1,344 & 5,714 & & & \\
\hline 1985 & 3,853 & 68 & 67.6 & 5,703 & 127.7 & 1,357 & 5,757 & & & \\
\hline 1986 & 3,745 & 69 & $\$ 6.6$ & 5,621 & 109.4 & 1,348 & 5,726 & & & \\
\hline$\cdots$ & --- & $-\cdot$ & $\cdots$ & $\cdots \cdot$ & $=$. & $\cdots$ & $\cdots$ & -- & --- & $-\cdots$ \\
\hline 1987 & 3,786 & 71 & 69.2 & 5,552 & 98.8 & 1,330 & 5,668 & & & \\
\hline 1988 & 3,839 & 73 & 70.8 & 5,426 & 119.5 & 1,335 & 5,683 & 6,967 & 7,532 & 7,678 \\
\hline 1989 & & & & & 134.1 & 1,354 & 5,745 & 7,044 & 7,614 & 7.762 \\
\hline 1990 & & & & & 138.6 & 1,375 & 5,817 & 7,132 & 7,710 & 7,859 \\
\hline 1991 & & & & & 139.3 & 1,396 & 5,885 & 7,215 & 7,800 & 7,951 \\
\hline 1992 & & & & & $134 . t$ & 1,409 & 5,930 & 7,270 & 7,859 & 6,011 \\
\hline
\end{tabular}

Capacity-Capital Stock Regression: Code 0 RSO 0.500 Coefticients: Const 1268.3 stock 3.3

Ave. Capacity/Stock (74-86): 4.3 Ave. Ueekly Hours (High) 116.8 Shift Factor 1.35 Ave Weekly Hours (Low) 112.8 Shift Factor $t .40$ Hote: Output, Investment, and Capital Stock extrapolated for 1987 and 1988.

\section{Aluminum foundries}

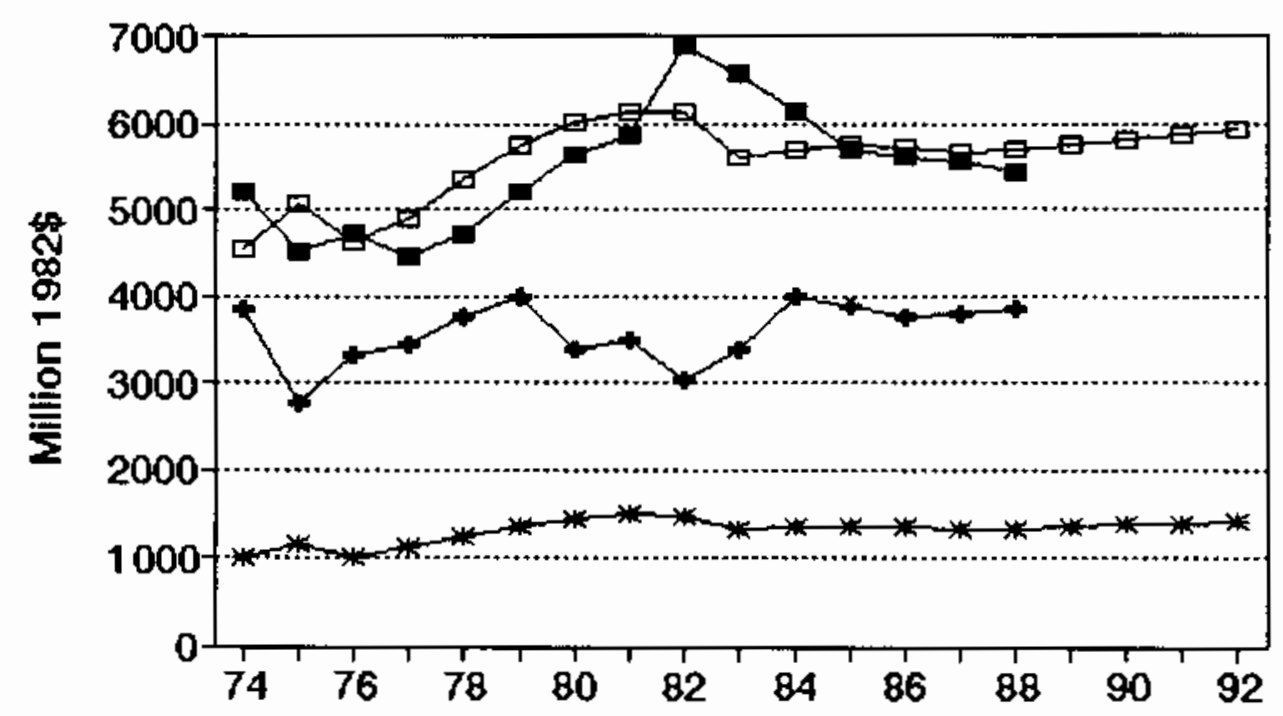

$\rightarrow-$ Oupue $\rightarrow$ Implied Capacity - $\rightarrow$ - Cepiral Stock $\quad \square$-Pred. Ceapaciny 
No. 276 BIC 3362 Brass, bronse, and copper foundries MILLIONS OF 1982 DOLLARS (EXCL. CU RATES)

$\begin{array}{lccccccc} & \text { Q4 } & \text { Am. } & \text { Implied Gross } & \text { Net } & \text { Pred. } & \ldots \ldots \ldots \\ \text { Gross } & \text { CU } & \text { CU } & \text { Prac. } & \text { In- } & \text { Cap. } & \text { Prac. } & \text { Emergency Capacity } \\ \text { Output } & \text { Rate } & \text { Rate } & \text { Capac. } & \text { vest. } & \text { Stk. } & \text { Capac. } & 3 \text { mon. } 6 \text { mon. }>6 \text { mon. }\end{array}$

\begin{tabular}{|c|c|c|c|c|c|c|c|c|c|c|}
\hline 1974 & 977 & 76 & 78.5 & 1,245 & $(8.0)$ & 255 & 1,062 & & & \\
\hline 1975 & 697 & 66 & 65.7 & 1,062 & 39.5 & 275 & 1,084 & & & \\
\hline 1976 & 674 & $\infty$ & 69.4 & 971 & 14.0 & 270 & 1,106 & & & \\
\hline 1977 & 686 & 69 & 68.0 & 1,008 & $(24.8)$ & 229 & 1,127 & & & \\
\hline 1978 & 707 & 72 & 68.9 & 1,025 & 16.1 & 227 & 1,149 & & & \\
\hline 1979 & 763 & 73 & 72.4 & 1,054 & 50.6 & 259 & 1,171 & & & \\
\hline 1980 & 746 & 63 & 61.5 & 1,214 & 30.7 & 269 & 1,193 & & & \\
\hline 1981 & 766 & 58 & 62.5 & 1,225 & $(0.6)$ & 249 & 1,214 & & & \\
\hline 1982 & 699 & 33 & 40.5 & 1,724 & 12.7 & 241 & 1,236 & & & \\
\hline 1983 & 671 & 58 & 48.4 & 1,387 & 34.1 & 252 & 1,258 & & & \\
\hline 1984 & 759 & 64 & 63.0 & 1,206 & $(1.7)$ & 228 & 1,270 & & & \\
\hline 1985 & 731 & 58 & 60.2 & 1,213 & 26.4 & 232 & 1,301 & & & \\
\hline 1986 & 709 & 64 & 60.7 & 1,169 & 10.4 & 220 & 1,323 & & & \\
\hline$\cdots$ & $\cdots \cdot$ & $\cdots$ & $\cdots$ & $-\cdot-$ & -- & -- & $-\cdots$ & $-\cdots$ & $=-$ & $\cdots$ \\
\hline 1987 & 712 & 54 & 56.8 & 1,254 & 2.8 & 201 & 1,344 & & & \\
\hline 1988 & 715 & 67 & 61.0 & 1,173 & 11.2 & 192 & 1,366 & 1,883 & 2,094 & 2,149 \\
\hline 1989 & & & & & 17.1 & 189 & 1,388 & 1,913 & 2,127 & 2,183 \\
\hline 1990 & & & & & 18.9 & 189 & 1,409 & 1,943 & 2,161 & 2,217 \\
\hline 1991 & & & & & 19.1 & 189 & 1,431 & 1,973 & 2,194 & 2,251 \\
\hline 1992 & & & & & 17.1 & 187 & 1,453 & 2,003 & 2,227 & 2,285 \\
\hline
\end{tabular}

Capacity-Capital Stock Regression: Code -2 RSA 0.180 Coefficients: Const 1040.7 Time 21.7

Ave. Capacity/Stock $(74-86): 4.8$ Ave. Weekly Hours (High) 100.1 Shift Factor 1.57 Ave Heekly Hours (LOW) 97.8 Shift Factor 1.61 Note: Output, Imestment, and Capital Stock extrapolated for 1987 and 1988.

\section{Brass, bronze, and copper foundrie}

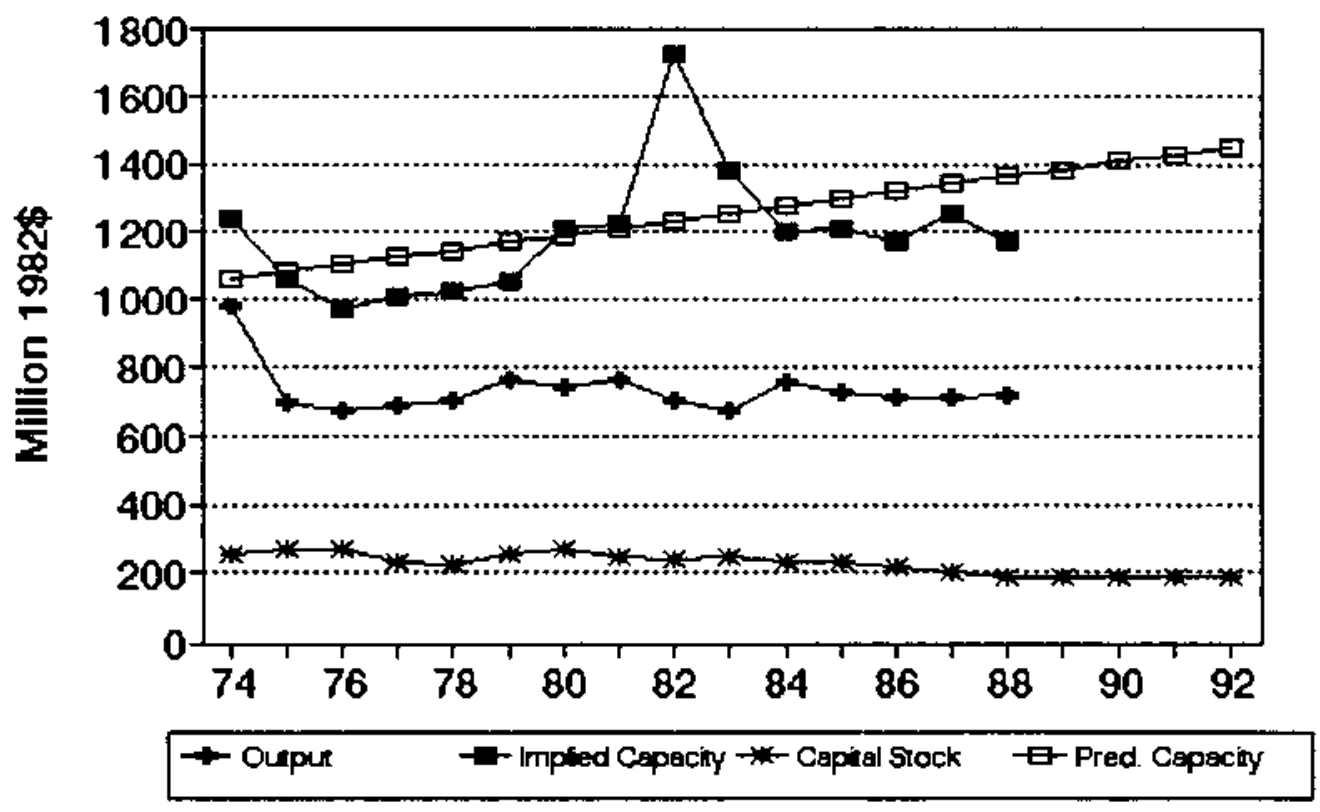


No. 277 sIc 3369 Nonferrous foundries, nec

MILLIONS OF 1982 DOLLARS (EXCL. CU RATES)

$\begin{array}{lccccccc} & \text { Q4 } & \text { Amn. Implied Gross } & \text { Net } & \text { Pred. } & \ldots & \ldots \\ \text { Gross } & \text { CU } & \text { CU } & \text { Prac. } & \text { Ir- } & \text { Cap. } & \text { Prac. } & \text { Emergency Capacity } \\ \text { Output } & \text { Rate } & \text { Rate } & \text { Capac. } & \text { vest. } & \text { Stk. } & \text { Capac. } & 3 \text { mon. } 6 \text { mon. } 76 \text { mon. }\end{array}$

\begin{tabular}{|c|c|c|c|c|c|c|c|c|c|c|}
\hline 1974 & 1,092 & 72 & 73.5 & 1,486 & 12.5 & 319 & 1,917 & & & \\
\hline $19 \pi$ & 965 & 56 & 59.6 & 1,619 & 10.5 & 305 & 1,885 & & & \\
\hline 1976 & 1,076 & 57 & 58.4 & 1,844 & 41.8 & 320 & 1,918 & & & \\
\hline 1977 & 1,048 & 59 & 58.3 & 1,796 & 21.6 & 315 & 1,907 & & & \\
\hline 1978 & 1,162 & 65 & 61.6 & 1,887 & 36.0 & 322 & 1,923 & & & \\
\hline 1979 & 1,202 & 58 & 60.4 & 1,989 & 48.9 & 341 & 1,968 & & & \\
\hline 1980 & 1,051 & 42 & 45.6 & 2,304 & 23.3 & 335 & 1,954 & & & \\
\hline 1981 & 1,049 & 61 & 55.4 & 1,892 & 12.9 & 321 & 1,922 & & & \\
\hline 1982 & 883 & 43 & 48.7 & 1,815 & $(23.0)$ & 275 & 1,819 & & & \\
\hline 1983 & 1,053 & 46 & 45.2 & 2,332 & 43.2 & 294 & 1,851 & & & \\
\hline 1984 & 1,100 & $53 *$ & 51.0 & 2,156 & 54.1 & 320 & 1,921 & & & \\
\hline 1985 & 1,112 & 57 & 55.5 & 2,005 & 30.1 & 323 & 1,926 & & & \\
\hline 1986 & 1,143 & 74 & 67.0 & 1,708 & 21.2 & 316 & 1,911 & & & \\
\hline-- & $-\cdots$ & -- & $-\cdot-$ & $-\cdot-$ & $-\cdot-$ & $\because \cdot$ & $\cdots$ & $-\cdots$ & --- & -- \\
\hline 1987 & 1,169 & 31 & 47.1 & 2,438 & 17.8 & 307 & 1,889 & & & \\
\hline 1988 & 1,156 & 85 & 64.1 & 1,805 & 22.5 & 302 & 1,880 & 3,134 & 3,491 & 3,583 \\
\hline 1989 & & & & & 25.8 & 302 & 1,879 & 3,132 & 3,489 & 3,581 \\
\hline 1990 & & & & & 26.8 & 302 & 1,880 & 3,134 & 3,491 & 3,583 \\
\hline 1991 & & & & & 26.9 & 303 & 1,881 & 3,137 & 3,494 & 3,586 \\
\hline 1992 & & & & & 25.8 & 303 & 1,880 & 3,135 & 3,491 & 3,584 \\
\hline
\end{tabular}

Capacity-Capital Stock Regression: Code 0 RSO 0.020

Coefficients: Const 1193.7 Stock 2.3

Ave. Capacity/stock $(74-86): 6.0$ Ave. Weekly Hours (High) 82.6 Shift Factor 1.91 Ave Weekly Hours (Low) 78.5 Shift factor 2.01

* Imputed Note: Output, Investment, and Capital Stock extrapolated for 1987 and 1988.

\section{Nonferrous foundries, nec}

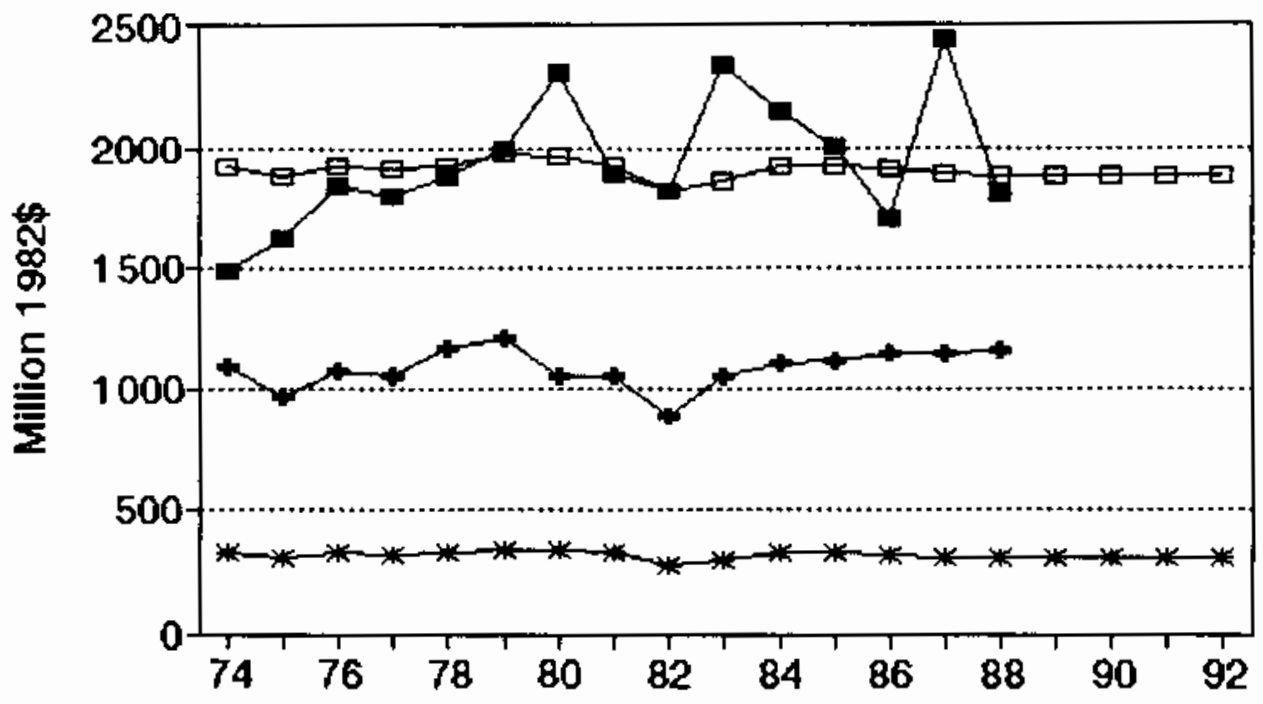

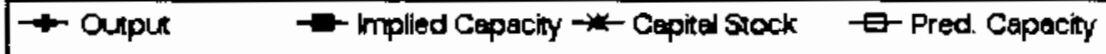




\section{No. 278 sIc 3398 Metal heat treating}

MILLIONS OF 1982 DOLLARS (EXCL. CU RATES)

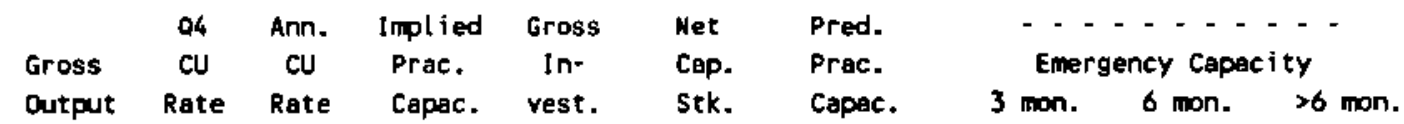

\begin{tabular}{|c|c|c|c|c|c|c|c|c|c|c|}
\hline 1974 & 964 & 84 & 84.9 & 1,136 & $(65.2)$ & 421 & 1,218 & & & \\
\hline 1975 & 919 & 73 & 75.7 & 1,214 & 58.4 & 451 & 1,235 & & & \\
\hline 1976 & 887 & 85 & 81.6 & 1,086 & 14.2 & 435 & 1,226 & & & \\
\hline 1977 & 1,031 & 80 & 81.9 & 1,258 & 83.9 & 485 & 1,254 & & & \\
\hline 1978 & 1,303 & 76 & 76.8 & 1,696 & 101.4 & 544 & 1,288 & & & \\
\hline 1979 & 1,011 & 91 & 85.3 & 1,185 & 86.7 & 583 & 1,310 & & & \\
\hline 1980 & 859 & 85 & 85.2 & 1,009 & 55.5 & 588 & 1,313 & & & \\
\hline 1981 & 1,030 & 83 & 84.8 & 1,215 & 82.1 & 615 & 1,328 & & & \\
\hline 1982 & 1,146 & 76 & 78.0 & 1,468 & (13.7) & 551 & 1,292 & & & \\
\hline 1983 & 1,030 & 66 & 70.1 & 1,471 & 22.8 & 522 & 1,276 & & & \\
\hline 1984 & 1,303 & 83 & 7.0 & 1,692 & 116.5 & 582 & 1,679 & & & \\
\hline 1985 & 1,298 & 82 & 82.4 & 1,576 & 61.4 & 588 & 1,682 & & & \\
\hline 1986 & 1,347 & $n$ & 75.3 & 1,789 & 78.3 & 611 & 1,695 & & & \\
\hline$\because$ & $\because \cdots$ & $\because$ & $-\cdots$ & $-\cdot-$ & $\begin{array}{r}- \\
013\end{array}$ & -- & 1715 & $\cdots$ & $-\cdot$ & -- \\
\hline 1988 & 1,399 & 90 & 86.1 & $\begin{array}{l}1,100 \\
1,625\end{array}$ & $\begin{array}{l}91.3 \\
97.2\end{array}$ & $\begin{array}{l}646 \\
683\end{array}$ & $\begin{array}{l}1,715 \\
1,736\end{array}$ & 1,787 & 1,857 & 1,875 \\
\hline 1989 & & & & & 103.0 & 723 & 1,759 & 1,810 & 1,881 & 1,900 \\
\hline 1990 & & & & & 108.9 & 765 & 1,783 & 1,835 & 1,907 & 1,925 \\
\hline 1991 & & & & & 114.7 & 809 & 1,808 & $1,8<1$ & 1,933 & 1,952 \\
\hline 1992 & & & & & 120.6 & 855 & 1,834 & 1,887 & 1,961 & 1,980 \\
\hline
\end{tabular}

Capacity-Capital Stock Regression: Code -1 RSO 0.500

Coefficients: Const 979.6 Stock 0.6 D(84-86) 369.5 Ave. Capacity/Stock (74-86): 2.6 Ave. Weekly Hours (High) 147.6 Shift Factor 1.08 Ave Weekly Hours (Low) 141.6 Shift factor 1.12 Note: Output, Investment, and Capital Stack extrapolated for 1987 and 1988.

\section{Metal heat treating}

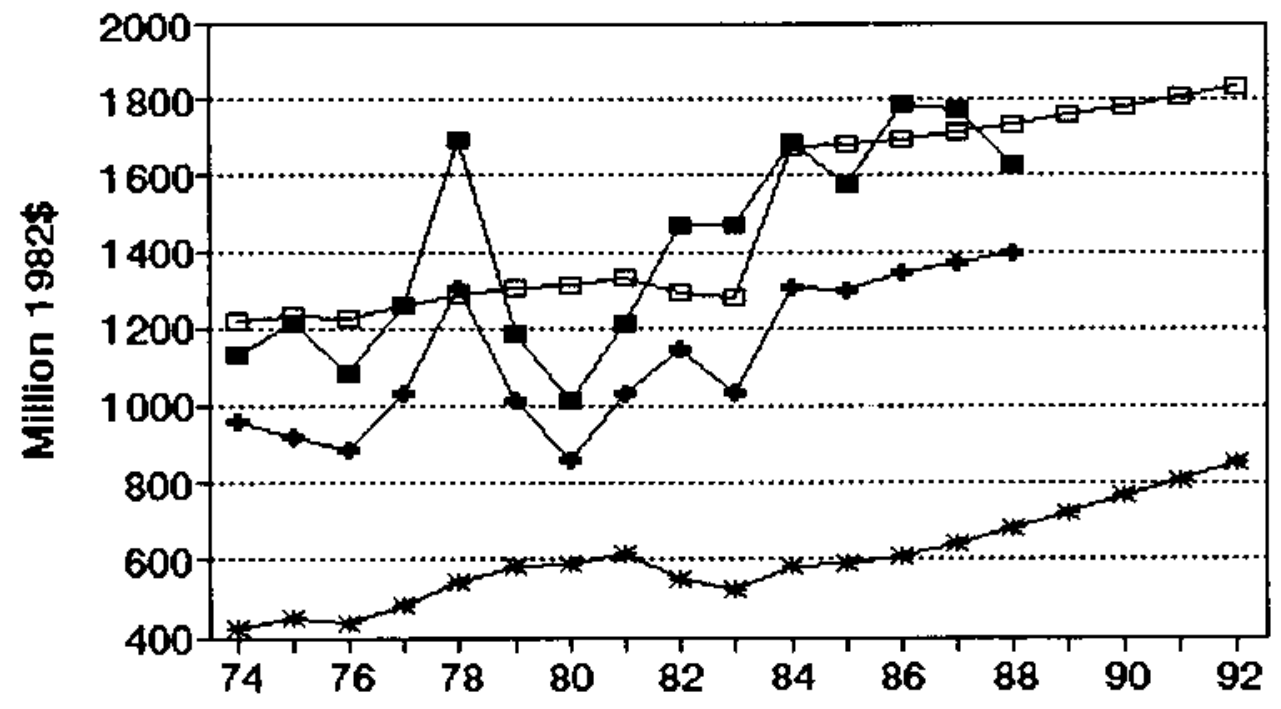

$\rightarrow$ - Output $\quad-$ - Implied Cepacity $*$ - Capial Stock $\quad$ E Pred. Cepacity


No. 279 SIC 3399 Primary metal productB, nec

MILLIONS OF 1982 DOLLARS (EXCL. CU RATES)

$\begin{array}{lccccccc} & \text { Q4 } & \text { Ann. Implied Gross } & \text { Het } & \text { Pred. } & \ldots \ldots \ldots \\ \text { Gross } & \text { CU } & \text { CU } & \text { Prac. } & \text { In- } & \text { Cap. } & \text { Prac. } & \text { Emergency Capacity } \\ \text { Output } & \text { Rate } & \text { Rate } & \text { Capec. } & \text { vest. } & \text { Stk. } & \text { Capac. } & 3 \text { mon. } 6 \text { mon. }>6 \text { mon. }\end{array}$

\begin{tabular}{|c|c|c|c|c|c|c|c|c|c|c|}
\hline 1974 & 984 & 86 & 89.5 & 1,100 & $(12.8)$ & 464 & 1,212 & & & \\
\hline 1975 & 907 & 55 & 61.0 & 1,488 & 4.8 & 445 & 1,338 & & & \\
\hline 1976 & 1,094 & 64 & 64.5 & 1,696 & 46.5 & 464 & 1,464 & & & \\
\hline 1977 & 1,210 & $\pi$ & 69.8 & $1, \pi 34$ & 103.3 & 531 & 1,590 & & & \\
\hline 1978 & 1,295 & 72 & 70.0 & 1,849 & 82.7 & 571 & 1,716 & & & \\
\hline 1979 & 1,569 & 90 & 83.2 & 1,886 & $(36.3)$ & 497 & 1,842 & & & \\
\hline 1980 & 1,383 & 85 & 78.3 & 1,767 & 42.5 & 500 & 1,960 & & & \\
\hline 1981 & 1,354 & 68 & 78.4 & 1,727 & 99.2 & 551 & 2,094 & & & \\
\hline 1982 & 943 & 48 & 53.5 & 1,761 & $(77.5)$ & 437 & 2,220 & & & \\
\hline 1983 & 1,013 & $44 \star$ & 47.5 & 2,133 & 23.0 & 422 & 2,346 & & & \\
\hline 1984 & 1,259 & 57 & 53.4 & 2,361 & 105.5 & 481 & 2,472 & & & \\
\hline 1985 & 1,580 & 49 & 52.0 & 3,038 & 47.0 & 480 & 2,598 & & & \\
\hline 1986 & 1,457 & 49 & 47.8 & 3,046 & 35.7 & 468 & 2,724 & & & \\
\hline-- & $-\cdots$ & $\cdots$ & - . & $\cdots \cdot$ & $--\cdot$ & -- & $\cdots$ & $-\cdots$ & $-\cdot-$ &.$- \cdot$ \\
\hline 1987 & 1,495 & 72 & 61.0 & 2,452 & 44.0 & 465 & 2,850 & & & \\
\hline 1988 & 1,547 & $\pi$ & 70.9 & 2,183 & 45.0 & 462 & 2,976 & 3,173 & 3,469 & 3,545 \\
\hline 1989 & & & & & 46.0 & 461 & 3,102 & 3,310 & 3,616 & 3,695 \\
\hline 1990 & & & & & 46.9 & 462 & 3,228 & 3,444 & 3,763 & 3,845 \\
\hline 1991 & & & & & 47.9 & 463 & 3,354 & 3,579 & 3,910 & 3,995 \\
\hline 1992 & & & & & 48.9 & 465 & 3,481 & 3,713 & 4,057 & 4,145 \\
\hline
\end{tabular}

Capecity-Capital stock Regression: Code -2 RSO 0.770

Coefficients: Const 1085.6 Time 126.0

Ave. Capecity/Stock (74-85): 4.1

Ave. Weekly Hours (High) 133.2 Shift factor 1.19 Ave Weekly Hours (Low) 130.4 Shift Factor 1.22

- Imputed Note: Output, Investment, and Capital stock extrapolated for 1987 and 1988.

3399 Primary metal products, nec

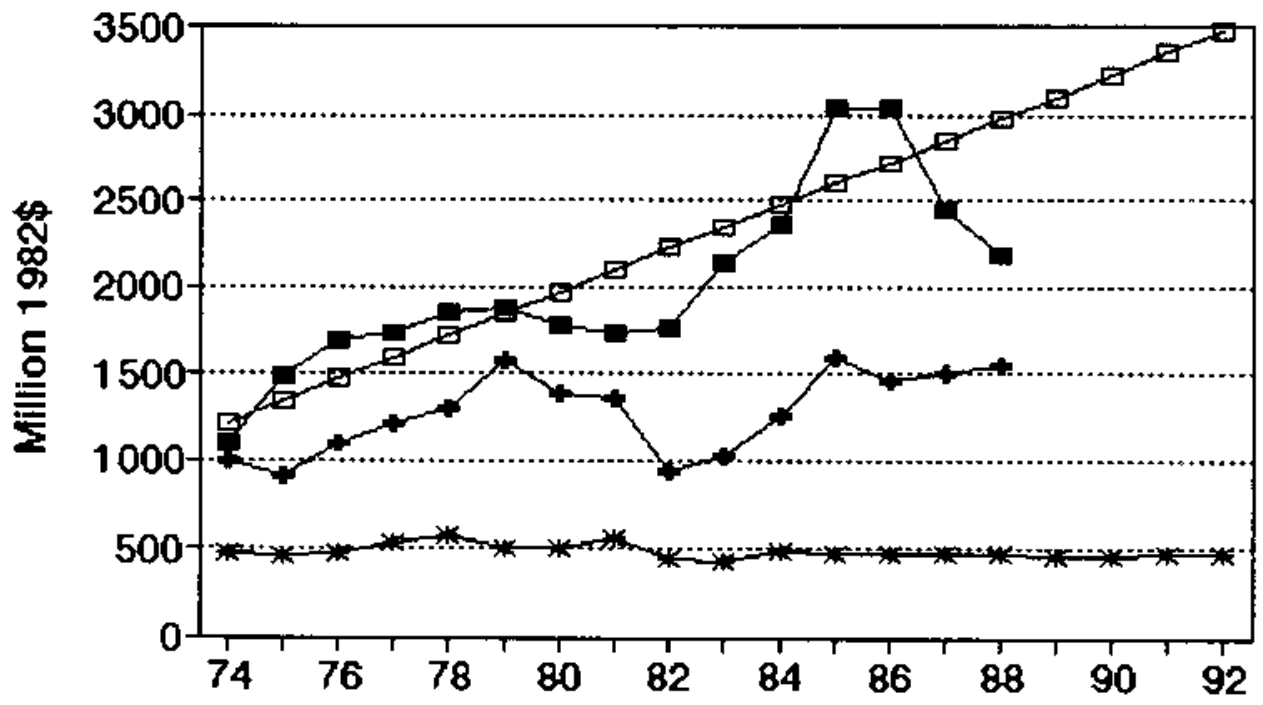

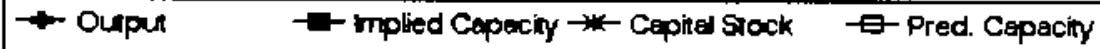


No. 280 BIC 3411 Netal cans

MILLIONS OF 1982 DOLLARS (EXCL. CU RATES)

$\begin{array}{lccccccc} & \text { Q4 } & \text { Am. } & \text { Implied Gross } & \text { Het } & \text { Pred. } & \ldots & \ldots \ldots \ldots \\ \text { Gross } & \text { CU } & \text { CU } & \text { Prac. } & \text { In- } & \text { Cap. } & \text { Prac. } & \text { Emergency Capacity } \\ \text { Output } & \text { Rate } & \text { Rate } & \text { Capac. } & \text { vest. } & \text { Stk. } & \text { Capac, } & 3 \text { mon. } 6 \text { mon. }>6 \text { mon. }\end{array}$

\begin{tabular}{|c|c|c|c|c|c|c|c|c|c|c|}
\hline 1974 & 12,067 & 68 & 68.0 & 17,739 & 265.0 & 3,517 & 18,434 & & & \\
\hline 1975 & 11,376 & 56 & 60.5 & 18,818 & 360.0 & 3,625 & 18,773 & & & \\
\hline 1976 & 11,737 & 67 & 62.9 & 18,667 & 77.5 & 3,443 & 18,201 & & & \\
\hline 1977 & 12,229 & 56 & 60.1 & 20,339 & 323.2 & 3,487 & 18,341 & & & \\
\hline 1978 & 12,002 & 65 & 61.6 & 19,477 & 217.0 & 3,419 & 18,126 & & & \\
\hline 1979 & 11,997 & 69 & 67.5 & 17,771 & 295.3 & 3,410 & 18,098 & & & \\
\hline 1980 & 11,028 & 69 & 69.0 & 15,986 & 242.7 & 3,339 & 17,875 & & & \\
\hline 1981 & 10,803 & 64 & 65.9 & 16,396 & 359.7 & 3,379 & 18,001 & & & \\
\hline 1982 & 11,172 & 62 & 62.7 & 17,807 & 219.5 & 3,275 & 17,673 & & & \\
\hline 1983 & 11,038 & 68 & 65.7 & 16,790 & 147.2 & 3,101 & 17,127 & & & \\
\hline 1984 & 11,107 & $\pi$ & 70.5 & 15,752 & 239.9 & 3,018 & 16,866 & & & \\
\hline 1985 & 10,917 & 58 & 63.3 & 17,259 & 257.1 & 2,956 & 16,674 & & & \\
\hline 1986 & 10,453 & 57 & 57.4 & 18,219 & 362.8 & 3,007 & 16,832 & & & \\
\hline-- & $-\cdots$ & - & - - - & -- & $-\cdot$ & $-\cdots$ & $--\cdot$ & $----\cdot$ & $-\cdots$ & $\cdot-\cdot$ \\
\hline 1987 & 10,544 & 54 & 55.1 & 19,130 & 282.0 & 2,971 & 16,719 & & & \\
\hline 1988 & 10,663 & 59 & 57.1 & 18,665 & 368.0 & 3,024 & 16,887 & 16,768 & 17,869 & 18,154 \\
\hline 1989 & & & & & 381.6 & 3,086 & 17,081 & 16,960 & 18,074 & 18,362 \\
\hline 1990 & & & & & 390.2 & 3,150 & 17,281 & 17,159 & 18,286 & 18,577 \\
\hline 1991 & & & & & 409.2 & 3,226 & 17,519 & 17,396 & 18,538 & 18,833 \\
\hline 1992 & & & & & 398.9 & 3,283 & 17,700 & 17,575 & 18,729 & 19,028 \\
\hline
\end{tabular}

Capacity-Capital Stock Regression: Code 0 RSO 0.250

Coefficients: Const 7394.1 stock 3.1

Ave, Capacity/Stock $(74-86): 5.4$ Ave. Weekly Hours (High) 148.4 Shift Factor 1.08 Ave Weekly Hours (Low) 144.8 Shift Factor 1.10 Note: Output, Investment, and Capital Stock extrapolated for 1987 and 1988.

\section{Metal cans}

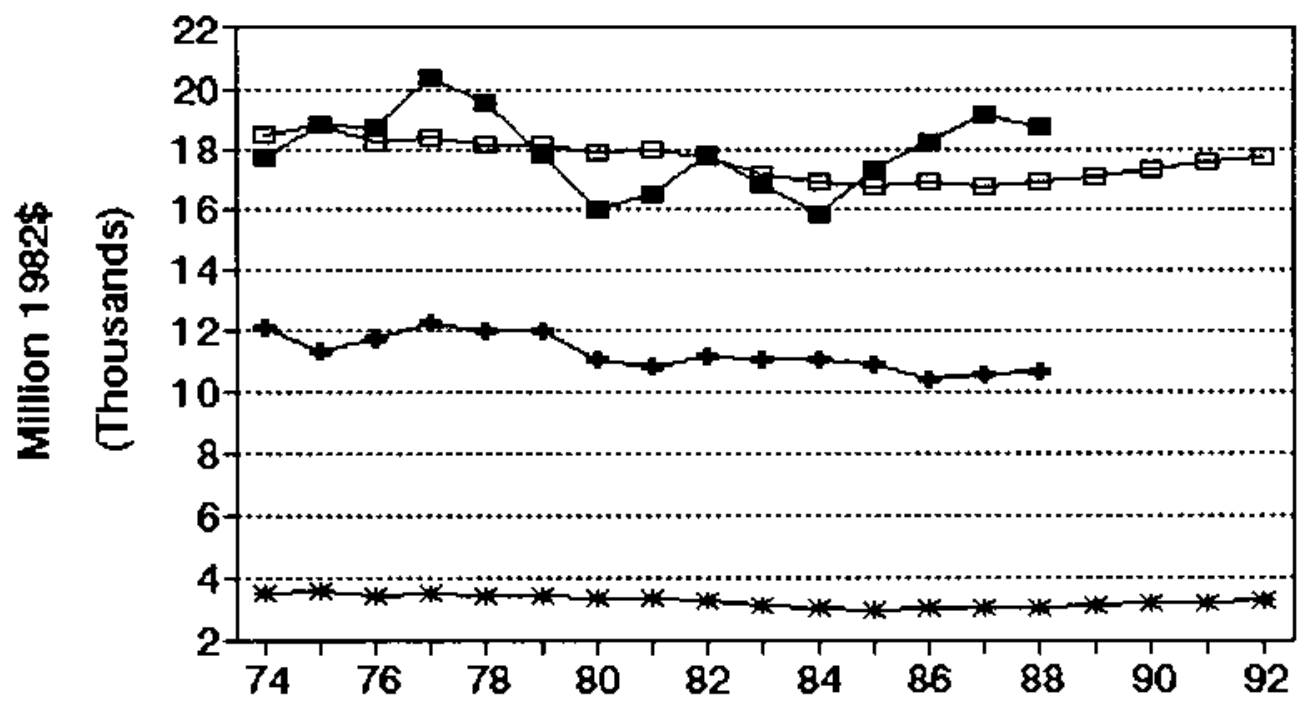

$\rightarrow$ - Ouput $\quad-$ - Implied Capacty tw- Capked Srock $\quad \square$ Pred Capaciny




\section{No. 281 SIC 3412 Metal barrels, drums, and pails}

MILLIONS OF 1982 DOLLARS (EXCL. CU RATES)

$\begin{array}{lccccccc} & \text { Q4 } & \text { Ann. } & \text { Implied } & \text { Gross } & \text { Net } & \text { Pred. } & \ldots \ldots \ldots \ldots \ldots \\ \text { Gross } & \text { CU } & \text { CU } & \text { Prac. } & \text { In- } & \text { Cap. } & \text { Prac. } & \text { Emergency Capacity } \\ \text { Output } & \text { Rate } & \text { Rate } & \text { Capac. } & \text { vest. } & \text { Stk. } & \text { Capac. } & 3 \text { mon. } 6 \text { mon. } 76 \text { mon. }\end{array}$

\begin{tabular}{|c|c|c|c|c|c|c|c|c|c|c|}
\hline 1974 & 1,514 & 50 & 50.5 & 2,997 & 47.8 & 327 & 2,106 & & & \\
\hline 1975 & 1,168 & 64 & 57.9 & 2,018 & 28.5 & 329 & 2,119 & & & \\
\hline 1976 & 1,237 & 53 & 57.3 & 2,159 & 34.7 & 336 & 2,163 & & & \\
\hline 1977 & 1,410 & $\pi$ & 65.4 & 2,155 & 63.6 & 370 & 2,374 & & & \\
\hline 1978 & 1,406 & 49 & 57.9 & 2,428 & 26.0 & 366 & 2,350 & & & \\
\hline 1979 & 1,561 & 60 & 56.0 & 2,787 & 63.8 & 397 & 2,545 & & & \\
\hline 1980 & 1,429 & 50 & 53.4 & $2,6 \pi 7$ & 62.1 & 425 & 2,719 & & & \\
\hline 1981 & 1,316 & 49 & 49.6 & 2,650 & 42.5 & 433 & 2,773 & & & \\
\hline 1982 & 1,049 & 49 & 48.8 & 2,151 & $(40.5)$ & 364 & 2,338 & & & \\
\hline 1983 & 1,177 & 62 & 56.9 & 2,067 & 18.3 & 353 & 2,271 & & & \\
\hline 1984 & 947 & $52 *$ & 55.8 & 1,697 & $(34.7)$ & 294 & 1,901 & & & \\
\hline 1985 & 871 & $59 *$ & 56.0 & 1.555 & (35.1) & 240 & 1,559 & & & \\
\hline 1986 & 836 & $63 *$ & 61.3 & 1,364 & 8.0 & 228 & 1,486 & & & \\
\hline$-\cdots$ & $-\cdot-$ & $\cdots$ & $\cdots$ & $\cdots$ & $-\cdots$ & $-\cdots$ & $--\cdot$ & -- & --- & $-\cdot-$ \\
\hline$\uparrow 987$ & 866 & $65 \star$ & 64.0 & 1,354 & 25.2 & 234 & 1,525 & & & \\
\hline 1988 & 908 & $58 *$ & 60.7 & 1,497 & 37.4 & 252 & 1,637 & 9,502 & 1,609 & 1,637 \\
\hline 1989 & & & & & 39.3 & 270 & 1,752 & 1,607 & 1,722 & 1,752 \\
\hline 1990 & & & & & 40.5 & 288 & 1,864 & 1,710 & 1,833 & 1,864 \\
\hline 1991 & & & & & 43.2 & 307 & 1,984 & 1,821 & 1,950 & 1,984 \\
\hline 1992 & & & & & 41.7 & 324 & 2,085 & 1,913 & 2,050 & 2,085 \\
\hline
\end{tabular}

Capacity-Capital Stock Regression: Code 0 RSQ 0.630 Coefficients: Const 55.2 stock 6.3

Ave. Capacity/Stock $(74-86): 6.4$ Ave. Weekly Hours (High) 1983.0 Shift factor 1.00 Ave Heekly Hours (LOW) 1983.0 Shift Factor 1.0 * Imputed Note: Output, Imvestment, and Capital stock extrapolated for 1987 and 1988.

\section{Metal barrels, drums, and pails}

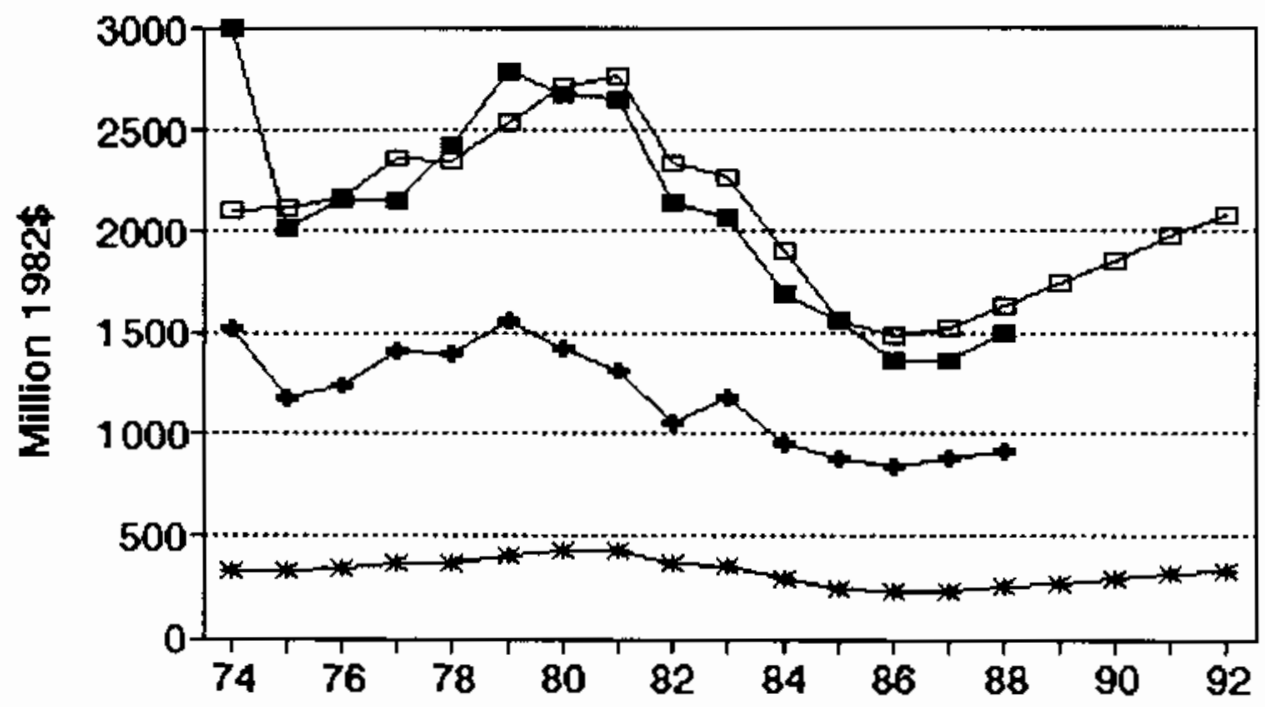

+- Ouput t-mplied Capaciy - Copital Stock E-Pred Capacity 


\section{No. 282 sIC 3421 Cutlery}

MILLIONS OF 1982 DOLLARS (EXCL. CU RATES)

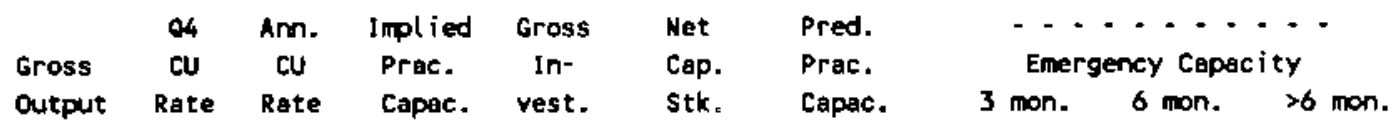

\begin{tabular}{|c|c|c|c|c|c|c|c|c|c|c|}
\hline 1974 & 1,134 & 68 & 69.1 & 1,640 & 38.0 & 265 & 1,479 & & & \\
\hline 1975 & 1,142 & 77 & 71.6 & 1,595 & 15.7 & 261 & 1,479 & & & \\
\hline 1976 & 1,215 & 70 & 72.9 & 1,666 & 21.0 & 261 & 1,479 & & & \\
\hline 1977 & 1,165 & 65 & 66.9 & 1,741 & 21.9 & 262 & 1,479 & & & \\
\hline 1978 & 1,187 & 76 & 71.6 & 1,658 & 32.8 & 272 & 1,479 & & & \\
\hline 1979 & 1,039 & 80 & 78.8 & 1,318 & 43.4 & 290 & 1,479 & & & \\
\hline 1980 & 948 & 85 & 82.3 & 1,151 & $(0.3)$ & 267 & 1,479 & & & \\
\hline 1981 & 932 & 70 & 76.3 & 1,222 & 22.5 & 266 & 1,479 & & & \\
\hline 1982 & 950 & 69 & 68.9 & 1,379 & 71.6 & 308 & 1,479 & & & \\
\hline 1983 & 940 & 66 & 66.9 & 1,406 & 32.9 & 311 & 1,479 & & & \\
\hline 1984 & 962 & $\pi$ & 73.3 & 1,313 & 32.9 & 313 & 1,227 & & & \\
\hline 1985 & 995 & 90 & 85.3 & 1,167 & 29.8 & 313 & 1,204 & & & \\
\hline 1986 & 970 & 82 & 84.8 & 1,144 & 36.3 & 318 & 1,182 & & & \\
\hline $\begin{array}{l}-- \\
1987\end{array}$ & $\begin{array}{c}078 \\
978\end{array}$ & 8 & $\begin{array}{l}\cdots 2.8 \\
82\end{array}$ & $\begin{array}{c}\cdots \\
1,182\end{array}$ & $\begin{array}{l}37.9 \\
37.9\end{array}$ & 326 & - & -- & -- & $\cdots$ \\
\hline 1988 & 988 & 88 & 86.8 & 1,139 & 38.9 & 333 & 1,136 & 2,031 & 2,219 & 2,267 \\
\hline 1989 & & & & & 39.9 & 341 & 1,113 & $1, \infty 90$ & 2,174 & 2,222 \\
\hline 1990 & & & & & 40.9 & 349 & 1,090 & 1,949 & 2,129 & 2,176 \\
\hline 1991 & & & & & 41.9 & 357 & 1,067 & 1,908 & 2,085 & 2,130 \\
\hline 1992 & & & & & 42.9 & 365 & 1,045 & 1,867 & 2,040 & 2,085 \\
\hline
\end{tabular}

Capacity-Capital Stock Regression: Code -2 RSO 0.300

Coefficients: Const 1478.6 Time -22.9

Ave. Heekly Hours (High) 78.9 Shift Factor 2.00 Ave Heekly Hours (Low) 7.5 Shift Factor 2.03 Note: Output, Investment, and Capital stock extrapolsted for 1987 and 1988.

\section{Cutlery}

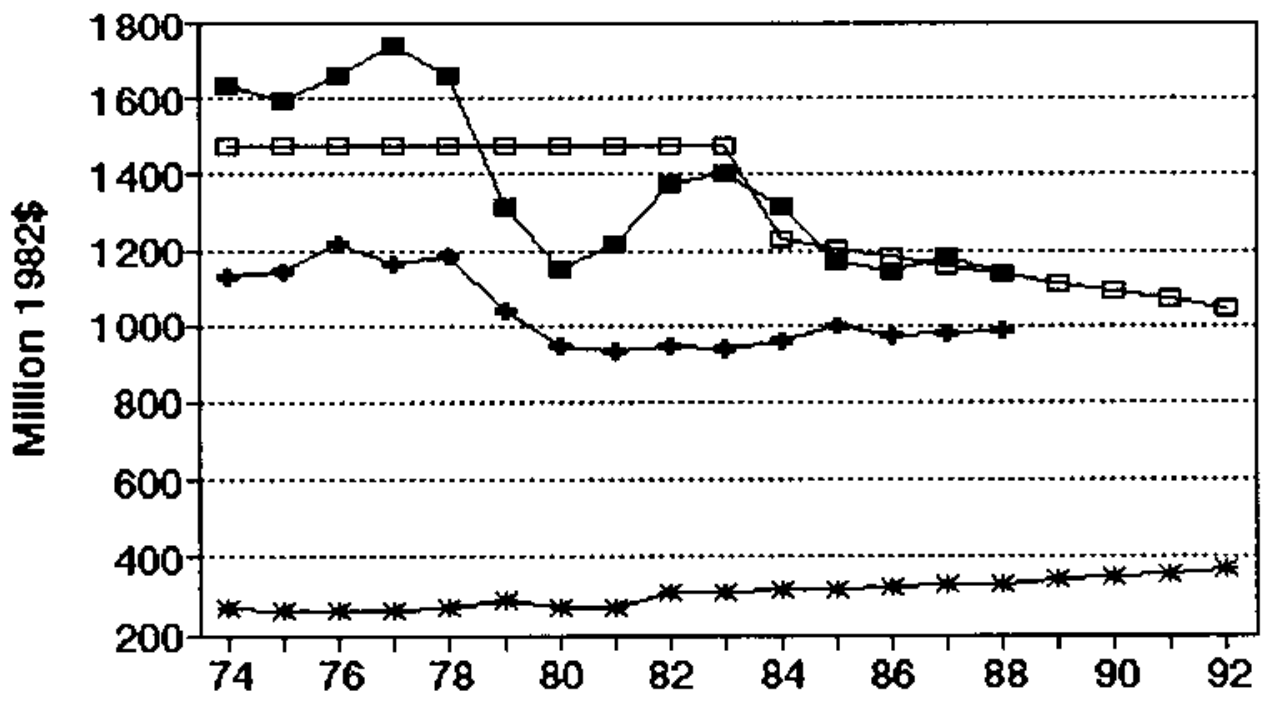

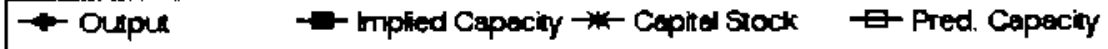


No. 283 sIC 3423 Hand and edge tools, nec

MILLIONS OF 1982 DOLLARS (EXCL. CU RATES)

$\begin{array}{lccccccc} & \text { Q4 } & \text { Ann. } & \text { Inplied Gross } & \text { Net } & \text { Pred. } & \ldots \ldots \ldots \\ \text { Gross } & \text { CU } & \text { CU } & \text { Prac. } & \text { In- } & \text { Cap. } & \text { Prac. } & \text { Emergency Capacity } \\ \text { Output } & \text { Rate } & \text { Rate } & \text { Capac. } & \text { vest. } & \text { 5tk. } & \text { Capac. } & 3 \text { mon. } 6 \text { mon. }>6 \text { mon. }\end{array}$

\begin{tabular}{|c|c|c|c|c|c|c|c|c|c|c|}
\hline 1974 & 3,269 & $\pi$ & 76.7 & 4,262 & 80.8 & 609 & 4,352 & & & \\
\hline 1973 & 2,878 & 69 & 68.4 & 4,208 & 101.5 & 668 & 4,472 & & & \\
\hline 1976 & 3,220 & $n$ & 71.2 & 4,524 & 85.1 & 706 & 4,550 & & & \\
\hline 1977 & 3,834 & 78 & 75.7 & 5,063 & 139.0 & 792 & 4.723 & & & \\
\hline 1978 & 4,009 & 72 & 73.9 & 5,427 & 95.2 & 830 & 4,801 & & & \\
\hline 1979 & 4.153 & 70 & 71.2 & 5.837 & 128.1 & 895 & 4,932 & & & \\
\hline 1980 & 3,567 & 53 & 58.5 & 6,103 & 53.3 & 884 & 4,910 & & & \\
\hline 1981 & 3,520 & 66 & 61.9 & 5,685 & 138.7 & 949 & 5,043 & & & \\
\hline 1982 & 3,012 & 53 & 57.1 & 5,277 & 64.7 & 938 & 5,020 & & & \\
\hline 1983 & 2,776 & 68 & 61.8 & 4,488 & 110.6 & 966 & 5,076 & & & \\
\hline 1984 & 2,911 & 71 & 70.5 & 4,131 & 28.6 & 914 & 4,972 & & & \\
\hline 1985 & 2,883 & 78 & $\pi .5$ & 3,817 & 52.5 & 888 & 4,918 & & & \\
\hline 1986 & 2,916 & 74 & $\pi 3.3$ & 3,874 & 85.3 & 892 & 4,927 & & & \\
\hline$\cdots$ & $\cdots--$ & -- & -- & $\cdots$ & $\cdots$ & $-\cdots$ & --- & $\cdots \cdots$ & $-\quad--$ & $-\cdots$ \\
\hline 1987 & 2,958 & 70 & 70.9 & 4,170 & 74.8 & 887 & 4,917 & & & \\
\hline 1988 & 3,014 & 69 & 69.7 & 4,325 & 73.2 & 881 & 4,904 & 5,601 & 6,068 & 6,189 \\
\hline 1989 & & & & & 71.5 & 873 & 4,889 & 5,584 & 6,040 & 6,170 \\
\hline 1990 & & & & & 69.8 & 865 & 4,871 & 5,564 & 6,028 & 6,148 \\
\hline 1991 & & & & & 68.2 & 855 & 4,852 & 5,543 & 6,004 & 6,124 \\
\hline 1992 & & & & & 66.5 & 845 & 4,832 & 5,519 & 5,979 & 6,098 \\
\hline
\end{tabular}

Capacity-Capital Stock Regression: Code I RSG 0.090

Coefficients: Const 3115.8 stock 2.0

Ave. Capacity/Stock $(74-86): 5.7$ Ave. Weekly Hours (High) 125.4 Shift Factor 1.26 Ave Weekly Hours (LOW) 121.0 Shift Factor 1.31 Note: Output, Investment, and Capital Stock extrapolated for 1987 and 1988.

\section{$3423 \mathrm{Hand}$ and edge tools, nec}

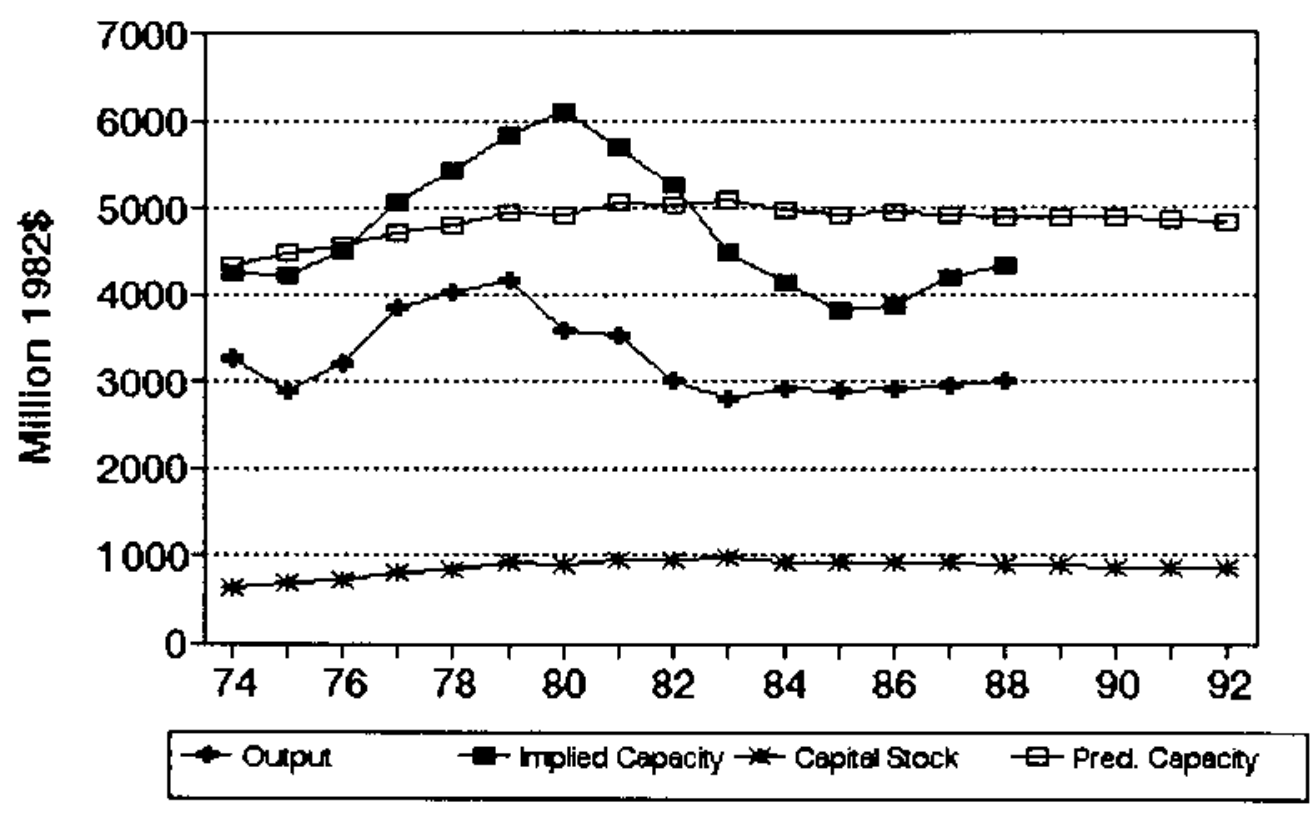




\section{No, 284 BIC 3425 Handsaws and sawblades}

MILLIONS OF 1982 DOLLARS (EXCL. CU RATES)

$\begin{array}{lccccccc} & Q 4 & \text { Ann. Implied } & \text { Gross } & \text { Net } & \text { Pred. } & \ldots \ldots \\ \text { Gross } & \text { CU } & \text { CU } & \text { Prac. } & \text { In- } & \text { Cap. } & \text { Prac. } & \text { Emergency Capacity } \\ \text { Output } & \text { Rate } & \text { Rate } & \text { Capac. } & \text { vest. } & \text { Stk. } & \text { Capac. } & 3 \text { mon. } 6 \text { mon. }>6 \text { mon. }\end{array}$

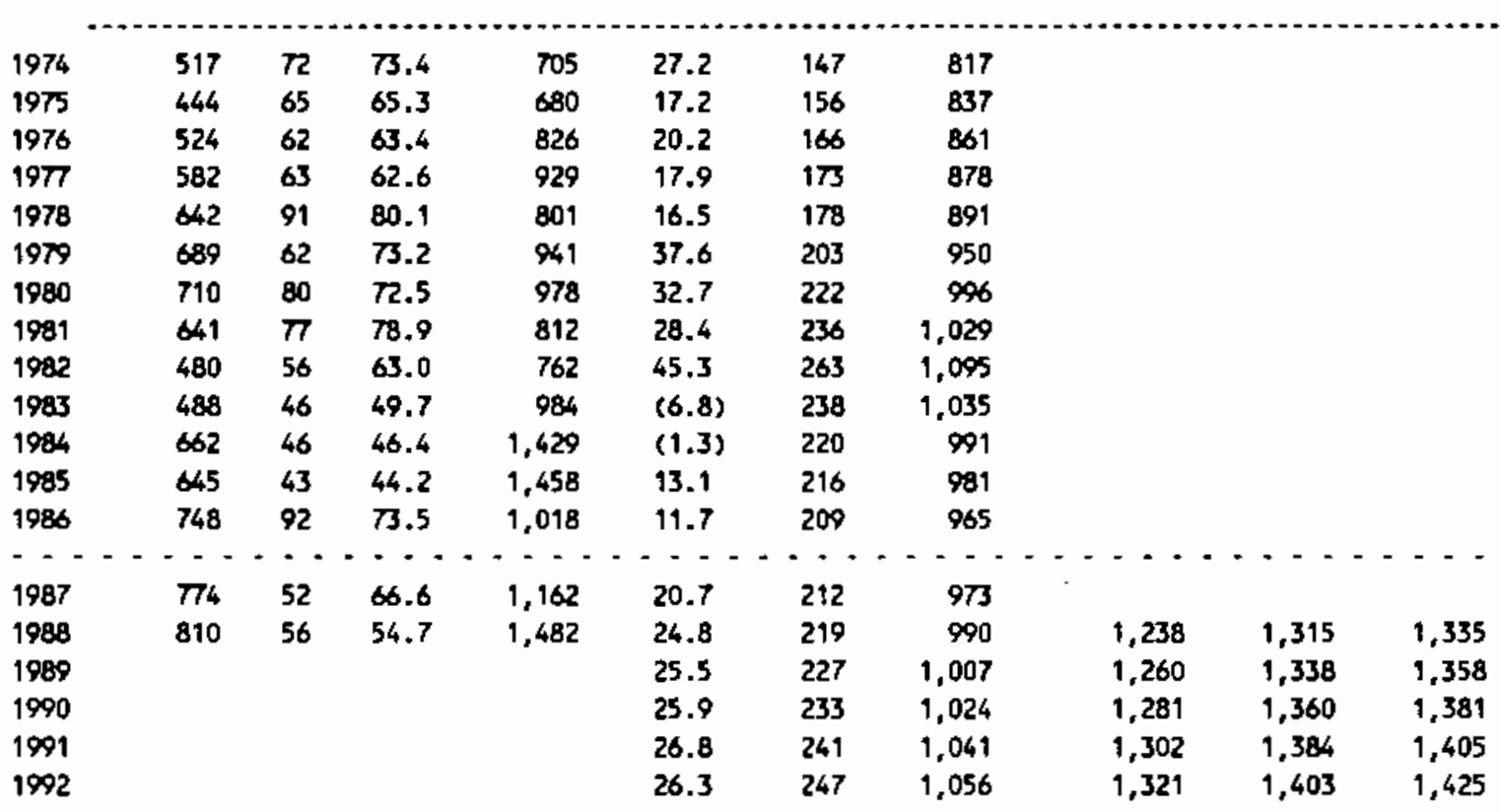

Capacity-Capital Stock Regression: Code 0 RSO 0.120 Coefficients: Const 461.6 stock 2.4

Ave. Capacity/stock $(74-86): 4.7$ Ave. Weekly Hours (High) 117.0 Shift factor 1.35 Ave Veekly Hours (Low) 116.6 Shift Factor 1.35 Note: Output, Imestment, and Capital Stock extrapolated for 1987 and 1988.

\section{Handsaws and sawblades}

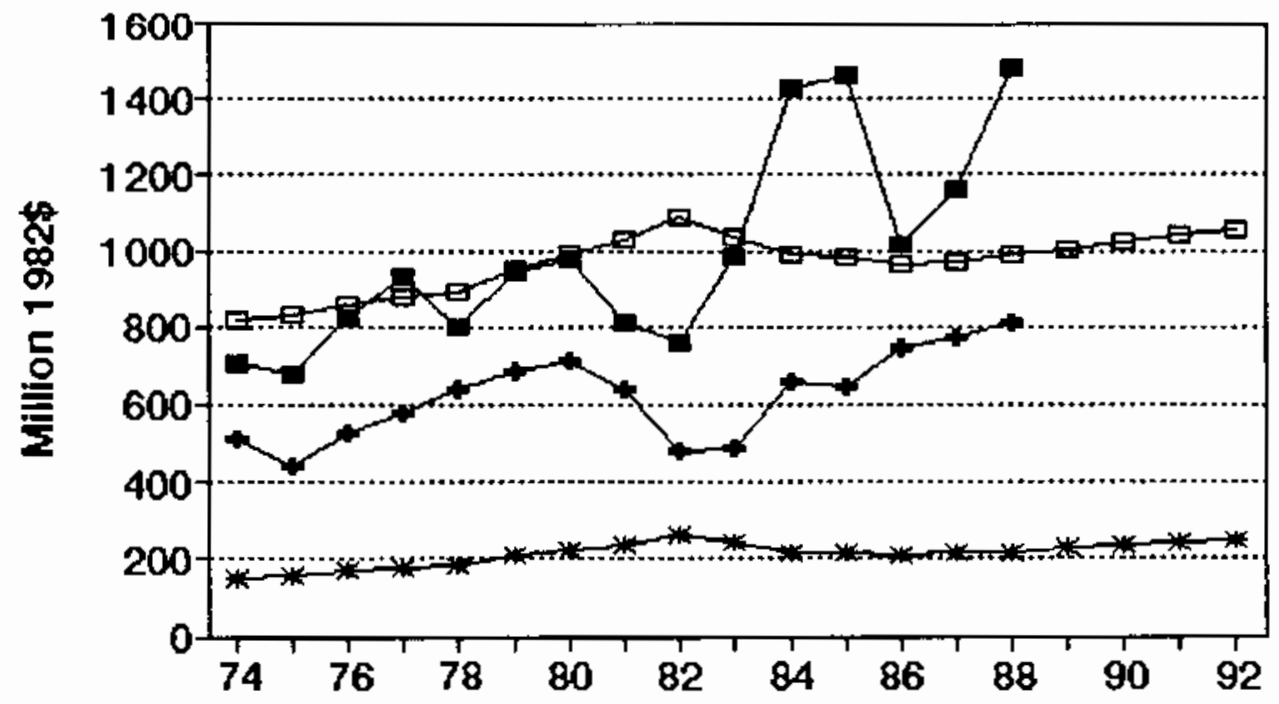

+- Ouput $\rightarrow$ - mplied Capeciny $\rightarrow$ - Cepital Stock $\quad \square$-Pred Capacity


No. 285 SIC 3429 Hardware, n.e.c.

MILLIONS OF 1982 DOLLARS (EXCL. CU RATES)

$\begin{array}{lccccccc} & 04 & \text { Ann. Implied Gross } & \text { Net } & \text { Pred. } & \ldots & \ldots \\ \text { Gross } & \text { CU } & \text { CU } & \text { Prac. } & \text { In- } & \text { Cap. } & \text { Prac. } & \text { Energency Capacity } \\ \text { Output Rate } & \text { Rate } & \text { Capac. } & \text { vest. } & \text { Stk. } & \text { Capac. } & 3 \text { mon. } 6 \text { mon. }>6 \text { mon. }\end{array}$

\begin{tabular}{|c|c|c|c|c|c|c|c|c|c|c|}
\hline 1974 & 6,706 & 68 & 69.6 & 9,633 & 100.2 & 1,801 & 9,690 & & & \\
\hline 1975 & 5,938 & 65 & 63.4 & 9,367 & 225.4 & 1,876 & 9,615 & & & \\
\hline 1976 & 6,977 & $\pi$ & 73.9 & 9,438 & 114.7 & 1,842 & 9,540 & & & \\
\hline 1977 & 7,363 & $\pi$ & 79.0 & 9,294 & 150.9 & 1,845 & 9,465 & & & \\
\hline 1978 & 7,556 & 80 & 79.2 & 9,543 & 196.8 & 1,884 & 9,390 & & & \\
\hline 1979 & 7,541 & 73 & 76.1 & 9,912 & 16.0 & 1,730 & 9,315 & & & \\
\hline 1980 & 6,339 & 68 & 68.9 & 9,204 & 248.2 & 1,842 & 9,240 & & & \\
\hline 1981 & 6,555 & 66 & 67.6 & 9,696 & 36.2 & 1,738 & 9,165 & & & \\
\hline 1982 & 5,743 & 62 & 62.8 & 9,140 & 246.5 & 1,824 & 9,090 & & & \\
\hline 1983 & 6,533 & 85 & 73.6 & 8,639 & 158.2 & 1,816 & 9,015 & & & \\
\hline 1984 & 7,109 & 82 & 84.0 & 8,468 & 170.7 & 1,818 & 8,940 & & & \\
\hline 1985 & 7,234 & 81 & 81.6 & 8,868 & 215.8 & 1,860 & 8,865 & & & \\
\hline 1986 & 7,201 & 81 & 80.8 & 8,916 & 178.8 & 1,864 & 8,790 & & & \\
\hline$\because \cdot$ & & -- & & & -- & $\cdots$ & $\because \cdots$ & $\cdots \cdot$ & $\cdots$ & $=\cdot$ \\
\hline 1987 & 7,308 & 81 & 80.3 & 9,098 & 173.3 & 1,863 & 8,716 & & & \\
\hline 1988 & 7,449 & 84 & 83.2 & 8,950 & 174.6 & 1,863 & 8,639 & 13,158 & 14,187 & 14,454 \\
\hline 1989 & & & & & 175.9 & 1,864 & 8,564 & 13,063 & 14.064 & 14,328 \\
\hline 1990 & & & & & 177.1 & 1,867 & 8,489 & 12,929 & 13,941 & 14,203 \\
\hline 1991 & & & & & 178.4 & 1.871 & 8,416 & 12,815 & 13,818 & 14,077 \\
\hline 1992 & & & & & 179.6 & 1,875 & 8,339 & 12,701 & 13,694 & 13,951 \\
\hline
\end{tabular}

Capacity-Capital Stock Regression: Code -2 RSQ 0.470

Coefficients: Const 9765.3 Time -75.1

Ave. Capacity/Stock $(74-86): 5.1$ Ave. Weekly Hours (High) 94.1 Shift Factor 1.67 Ave Heekly Hours (Low) 93.1 Shift Factor 1.69 Mote: Output, Investment, and Capital Stock extrapolated for 1987 and 1988.

3429 Hardware, n.e.c.
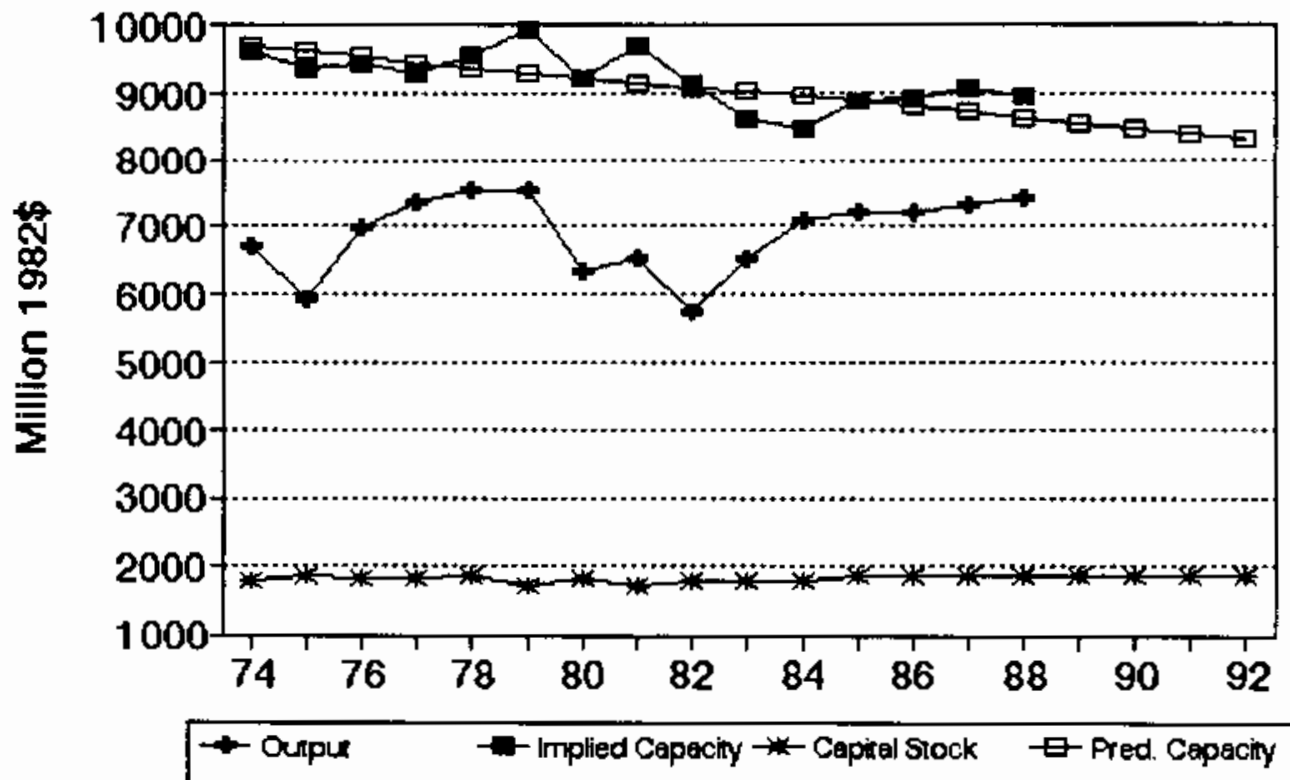


\section{No. 286 SIC 3431 Metal sanitary ware}

MILLIONS OF 1982 DOLLARS (EXCL. CU RATES)

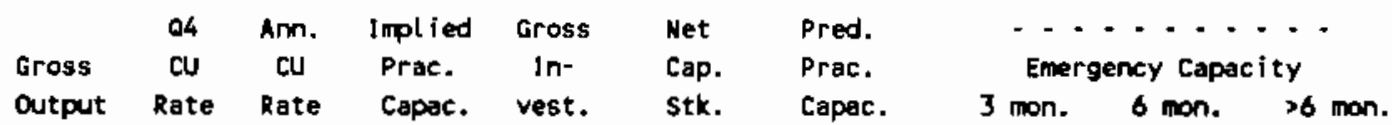

\begin{tabular}{|c|c|c|c|c|c|c|c|c|c|c|}
\hline 1974 & 737 & 52 & 54.1 & 1,361 & 35.9 & 192 & 1,051 & & & \\
\hline 1975 & 616 & 63 & 55.0 & 1,120 & 36.1 & 206 & 1,076 & & & \\
\hline 1976 & 631 & 61 & 62.4 & 1,010 & $(11.3)$ & 177 & 1,024 & & & \\
\hline 1977 & 652 & 75 & 69.6 & 936 & 29.3 & 187 & 1,042 & & & \\
\hline 1978 & 718 & 81 & 78.0 & 920 & 26.6 & 194 & 1,055 & & & \\
\hline 1979 & 671 & 73 & 76.8 & 875 & 8.0 & 184 & 1,038 & & & \\
\hline 1980 & 552 & 69 & 68.8 & 802 & 19.8 & 186 & 1,040 & & & \\
\hline 1981 & 559 & 62 & 66.0 & 846 & 25.0 & 192 & 1,051 & & & \\
\hline 1982 & 652 & 50 & 53.3 & 1,222 & 13.1 & 187 & 1,041 & & & \\
\hline 1983 & 716 & 64 & 57.9 & 1,232 & 16.1 & 183 & 1,036 & & & \\
\hline 1984 & 682 & 59 & 62.1 & 1,099 & 14.3 & 179 & 1,028 & & & \\
\hline 1985 & 728 & 67 & 64.3 & 1,134 & 33.4 & 193 & 1,052 & & & \\
\hline 1986 & 743 & 75 & 71.7 & 1,037 & 23.1 & 196 & 1,059 & & & \\
\hline $\begin{array}{l}\cdots \\
1987\end{array}$ & $\begin{array}{c}\cdots \\
739\end{array}$ & 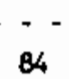 & 79.5 & $\begin{array}{c}-.- \\
930\end{array}$ & $\begin{array}{l}--- \\
18.7\end{array}$ & $\begin{array}{l}--\cdot \\
195\end{array}$ & $\cdots$ & $\cdots$ & -- & $\cdots$ \\
\hline 1988 & 734 & 70 & 76.0 & 966 & 20.6 & 196 & 1,059 & 1,510 & 1,651 & 9,687 \\
\hline 1989 & & & & & 20.9 & 198 & 1,061 & 1,514 & 1,656 & 1,690 \\
\hline 1990 & & & & & 21.1 & 199 & 1,063 & 1,517 & 1,658 & 1,694 \\
\hline 1991 & & & & & 21.5 & 201 & 1,066 & 1,521 & 1,662 & 1,698 \\
\hline 1992 & & & & & 21.3 & 202 & 1,068 & 1,524 & 1,665 & 1,702 \\
\hline
\end{tabular}

Capacity-Capital Stock Regression: Code 0 RSD 0.010 Coefficients: Const 713.5 stock 1.8 Ave, Capacity/Stock (74-86): 5.5 Ave. Weekly Hours (High) 98.9 Shift Factor 1.59 Ave Heekly Hours (Low) 87.0 Shift Factor 1.81 Note: Output, Investment, and Capital stock extrapolsted for 1987 and 1988.

\section{Metal sanitary ware}

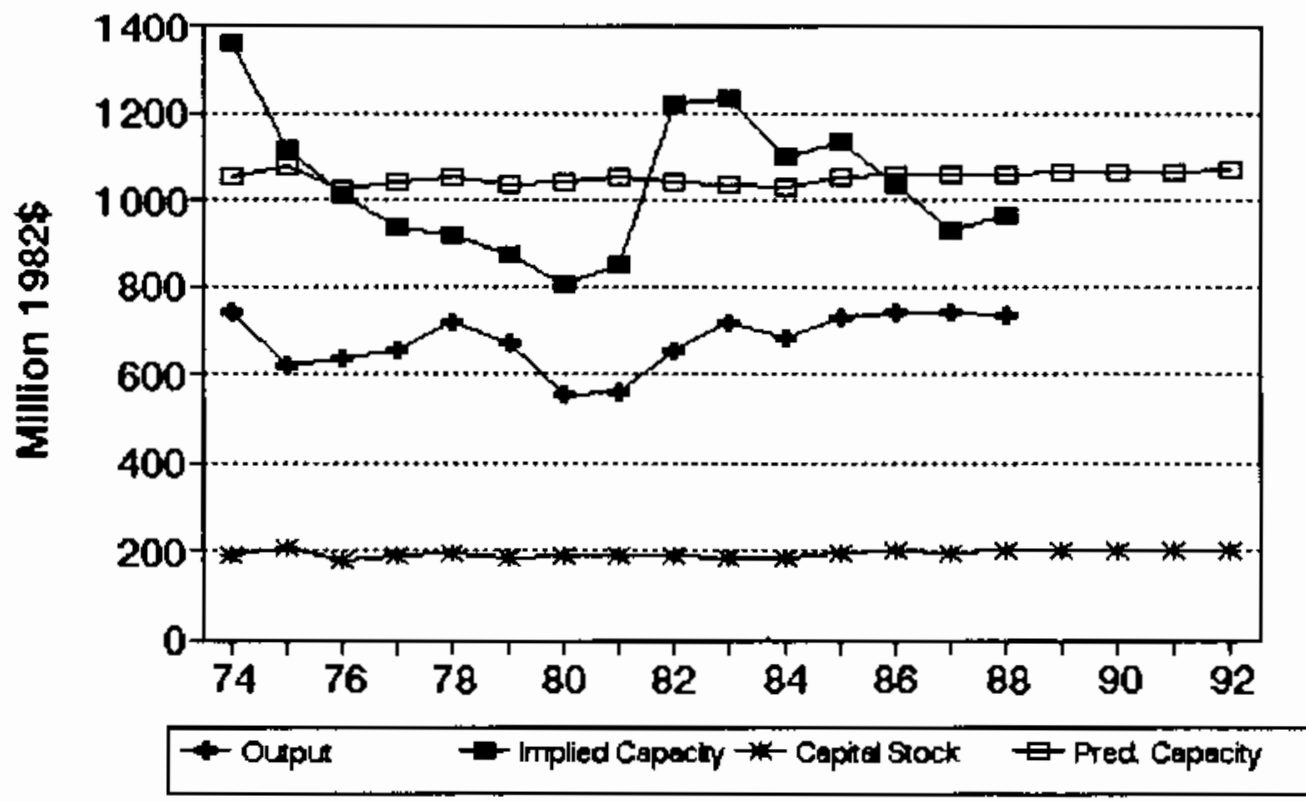




\section{No. 287 SIC 3432 plumbing fittings and brass goods}

MILLIONS OF 1982 DOLLARS (EXCL. CU RATES)

$\begin{array}{lccccccc} & \text { Q4 } & \text { Ann. Implied Gross } & \text { Net } & \text { Pred. } & \ldots \ldots \ldots \\ \text { Gross } & \text { CU } & \text { CU } & \text { Prac. } & \text { In- } & \text { Cap. } & \text { Prac. } & \text { Emergency Capacity } \\ \text { Output } & \text { Rate } & \text { Rate } & \text { Capac. } & \text { vest. } & \text { Stk. } & \text { Capac. } & 3 \text { mon. } 6 \text { mon. }>6 \text { mon. }\end{array}$

\begin{tabular}{|c|c|c|c|c|c|c|c|c|c|c|}
\hline 1974 & 1,380 & 54 & 57.2 & 2,411 & 11.8 & 258 & 2,391 & & & \\
\hline 1975 & 1,398 & 60 & 51.9 & 2,692 & 97.2 & 332 & 2,399 & & & \\
\hline 1976 & 1,678 & 82 & 74.2 & 2,261 & 22.0 & 329 & 2,407 & & & \\
\hline 1977 & 1,772 & 77 & 79.1 & 2,240 & 44.4 & 348 & 2,415 & & & \\
\hline 1978 & 1,751 & 81 & 78.5 & 2,230 & 6.7 & 328 & 2,423 & & & \\
\hline 1979 & 1,227 & 70 & 75.1 & 2,299 & 54.6 & 354 & 2,431 & & & \\
\hline 1980 & 1,505 & 58 & 60.3 & 2,496 & 25.1 & 349 & 2,439 & & & \\
\hline 1981 & 1,475 & 63 & 63.2 & 2,334 & 17.1 & 337 & 2,446 & & & \\
\hline 1982 & 1,284 & 44 & 49.4 & 2,598 & $(5.5)$ & 304 & 2,454 & & & \\
\hline 1983 & 1,529 & 53 & 48.9 & 3,126 & 24.6 & 301 & 2,462 & & & \\
\hline 1984 & 1,704 & 77 & 69.1 & 2,465 & 29.7 & 302 & 2,470 & & & \\
\hline 1985 & 1,711 & 79 & 78.7 & 2,174 & 44.1 & 316 & 2,478 & & & \\
\hline 1986 & 1,745 & 71 & 73.5 & 2,375 & 21.7 & 308 & 2,486 & & & \\
\hline-- & & -- & $-\cdots$ & $-\cdots$ & $\cdots \cdot$ & 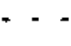 & $-\cdot-$ & $-\cdots$ & $\cdots$ & $-\cdots$ \\
\hline 1987 & 1,778 & $81^{\circ}$ & 75.7 & 2,350 & 29.2 & 307 & 2,494 & & & \\
\hline 1988 & 1,822 & 87 & 85.6 & 2,128 & 29.4 & 307 & 2,502 & 3,614 & 3,937 & 4,021 \\
\hline 1989 & & & & & 29.5 & 307 & 2,510 & 3,625 & 3,950 & 4,033 \\
\hline 1990 & & & & & 29.5 & 307 & 2,518 & 3,637 & 3,962 & 4,046 \\
\hline 1991 & & & & & 29.5 & 307 & 2,526 & 3,648 & 3,975 & 4,059 \\
\hline 1992 & & & & & 29.5 & 307 & 2,534 & 3,660 & 3,987 & 4,072 \\
\hline
\end{tabular}

Capacity-Capital Stock Regression: Code -2 RSD 0.010 Coefficients: Const 2383.0 Time 7.9

Ave. Capacity/Stock $(74-86): 7.6$ Ave. Weekly Hours (High) 98.0 Shift Factor 1.61 Ave Ueekly Hours (LOH) 90.3 Shift Factor 1.75 Note: Output, Investment, and Capital Stock extrapolated for 1987 and 1988.

\section{Plumbing fittings and brass goods}

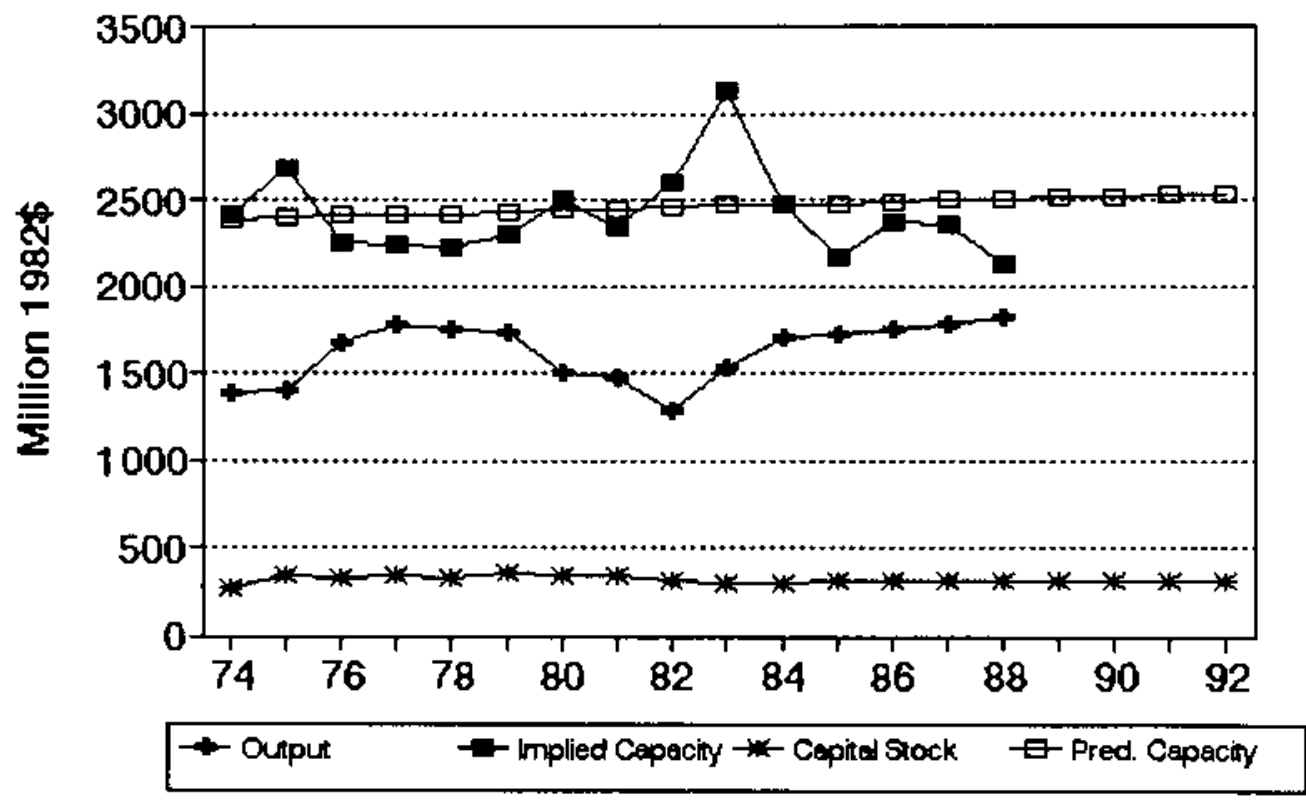




\section{No. 288 sIC 3433 Heating equipent except electric}

MILLIONS OF 1982 DOLLARS (EXCL. CU RATES)

$\begin{array}{lccccccc} & 04 & \text { Anr. Implied } & \text { Gross } & \text { Met } & \text { Pred. } & \ldots & \ldots \\ \text { Gross } & \text { CU } & \text { CU } & \text { Prac. } & \text { In- } & \text { Cap. } & \text { Prac. } & \text { Emergency Capacity } \\ \text { Output Rate } & \text { Rate } & \text { Capac. } & \text { vest. } & \text { Stk. } & \text { Capac. } & 3 \text { mon. } 6 \text { mon. }>6 \text { mon. }\end{array}$

\begin{tabular}{|c|c|c|c|c|c|c|c|c|c|c|}
\hline 1974 & 1,977 & 84 & 86.7 & 2,281 & 57.7 & 408 & 3,194 & & & \\
\hline 1975 & 1,844 & 69 & 70.2 & 2,628 & 26.2 & 397 & 3,218 & & & \\
\hline 1976 & 1,721 & 72 & 71.3 & 2,413 & $(55.4)$ & 312 & 3,399 & & & \\
\hline 1977 & 2,083 & 82 & 78.2 & 2,664 & 80.4 & 357 & 3,302 & & & \\
\hline 1978 & 2,322 & 80 & 80.2 & 2,896 & 53.0 & 373 & 3,268 & & & \\
\hline 1979 & 2,383 & 57 & 66.1 & 3,603 & 0.2 & 341 & 3,336 & & & \\
\hline 1980 & 2,498 & 54 & 54.1 & 4,619 & 59.7 & 366 & 3,284 & & & \\
\hline 1981 & 1,971 & 48 & 51.1 & 3,857 & $(8.7)$ & 327 & 3,367 & & & \\
\hline 1982 & 2,114 & 45 & 45.5 & 4,664 & 99.5 & 388 & 3,237 & & & \\
\hline 1983 & 1.923 & 73 & 61.5 & 3,127 & 6.6 & 357 & 2,861 & & & \\
\hline 1984 & 1,947 & 73 & 74.0 & 2,632 & 55.5 & 374 & 2,825 & & & \\
\hline 1985 & 1,966 & 66 & 68.9 & 2,853 & 39.1 & 375 & 2,823 & & & \\
\hline 1986 & 1,938 & $\pi$ & 71.4 & 2,717 & 39.6 & 377 & 2,820 & & & \\
\hline $\begin{array}{l}\cdots \\
1987\end{array}$ & $\begin{array}{l}\cdots, 962 \\
1,-\end{array}$ & 71 & $\begin{array}{l}\because \cdot \\
71.7\end{array}$ & 2,736 & $\begin{array}{l}--. \\
34.6\end{array}$ & $\cdots$ & 2,827 & $\cdots$ & -- & $\cdots$ \\
\hline 1988 & 1,993 & 72 & 72.1 & 2,766 & 37.0 & 372 & 2,829 & 4,562 & 5,026 & 5,145 \\
\hline 1989 & & & & & 37.4 & 372 & 2,829 & 4,563 & 5,027 & 5,147 \\
\hline 1990 & & & & & 37.6 & 372 & 2,829 & 4,563 & 5,027 & 5,147 \\
\hline 1991 & & & & & 38.2 & 372 & 2,828 & 4,562 & 5,025 & 5,145 \\
\hline 1992 & & & & & 37.9 & 373 & 2,828 & 4,561 & 5,024 & 5,144 \\
\hline
\end{tabular}

Capacity-Capital Stock Regression: Code -1 RSQ 0.080 Coefficients: Const 4063.8 stock -2.1 D(83-86) -441.6 Ave. Capecity/Stock (74-86): 8.6 Ave. Weekly Hours (High) 86.6 Shift Factor 1.82 Ave Hekly Hours (Low) 82.7 Shift Factor 1.90 Wote: Output, Investment, and Capital stock extrapolated for 1987 and 1988.

3433 Heating equipment except electric

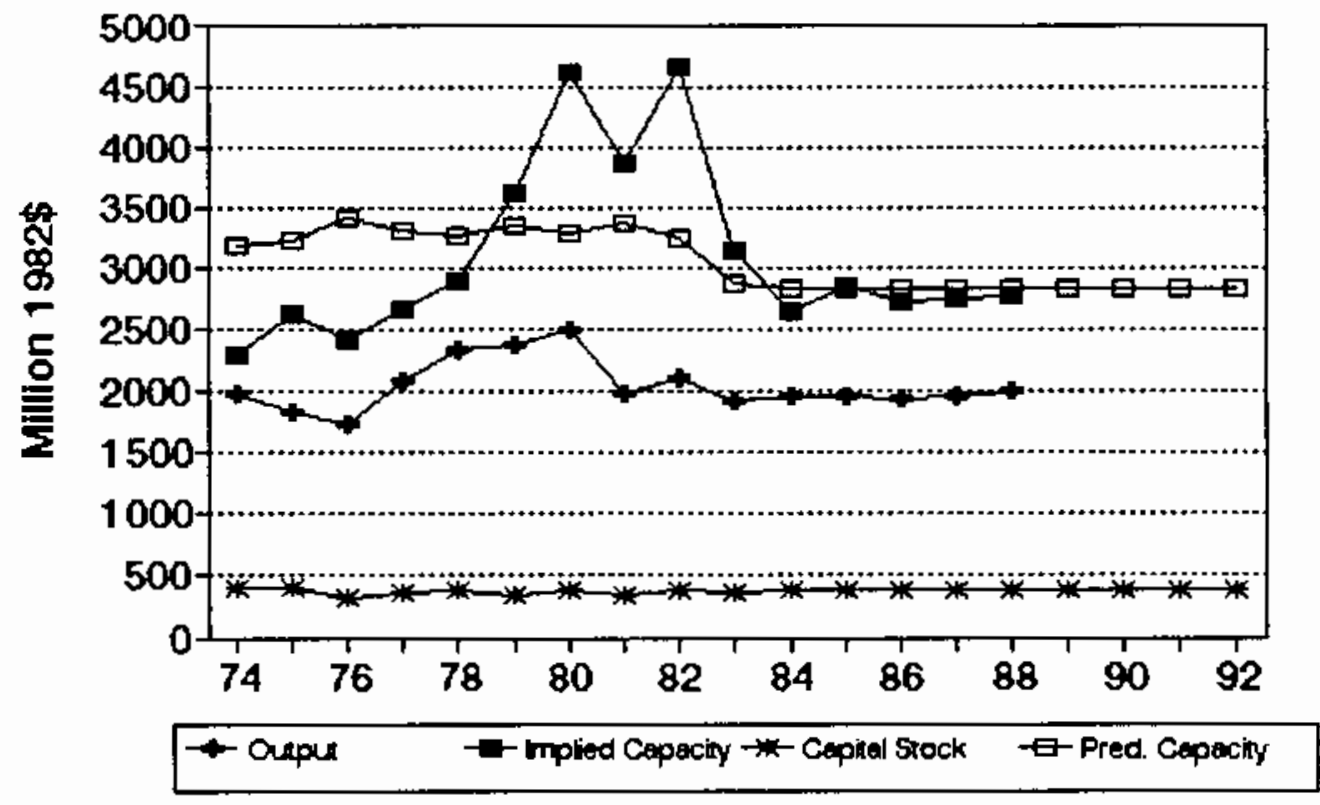




\section{No. 289 SIC 3441 Pabricated structural metal}

MILLIONS OF 1982 DOLLARS (EXCL. CU RATES)

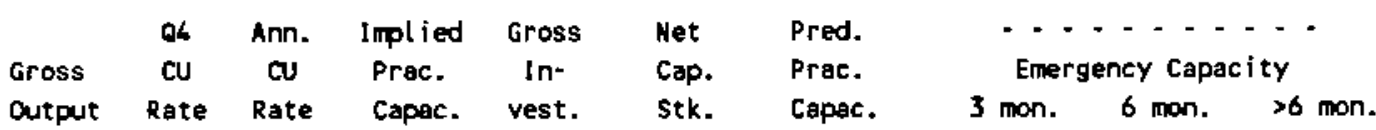

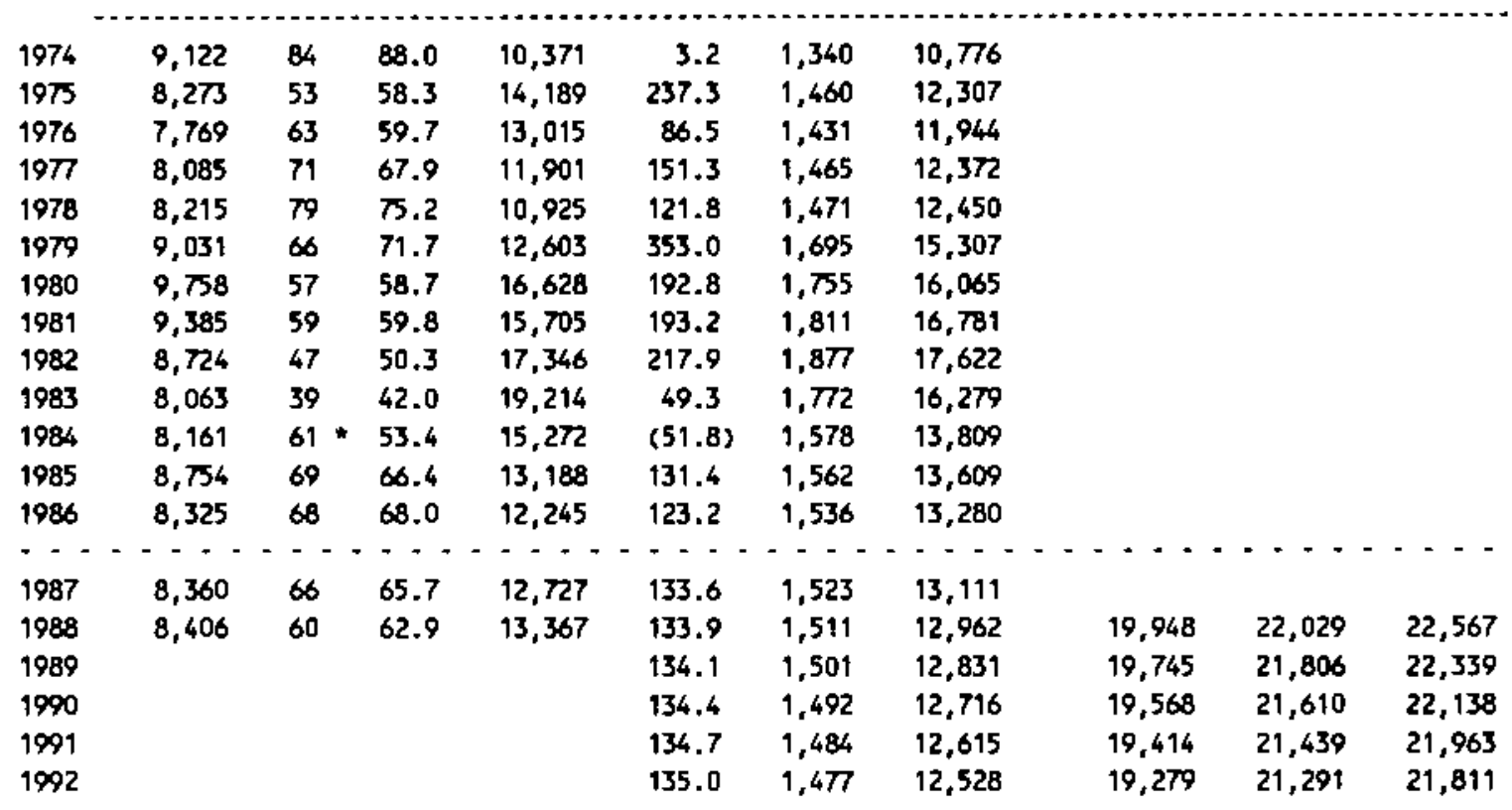

Capacity-Capital Stock Regression: Code 1 RSO 0.660

Coefficients: Const -6290.0 stock 12.7

Ave. Capacity/Stock (74-86): 8.8

Ave. Heekly Hours (High) 90.4 Shift Factor 1.74 Ave Heekly Hours (Low) 87.1 Shift Factor 1.81

* Imputed Note: Output, Investment, and Capital Stock extraoolated for 1987 and 1988.

\section{Fabricated structural metal}

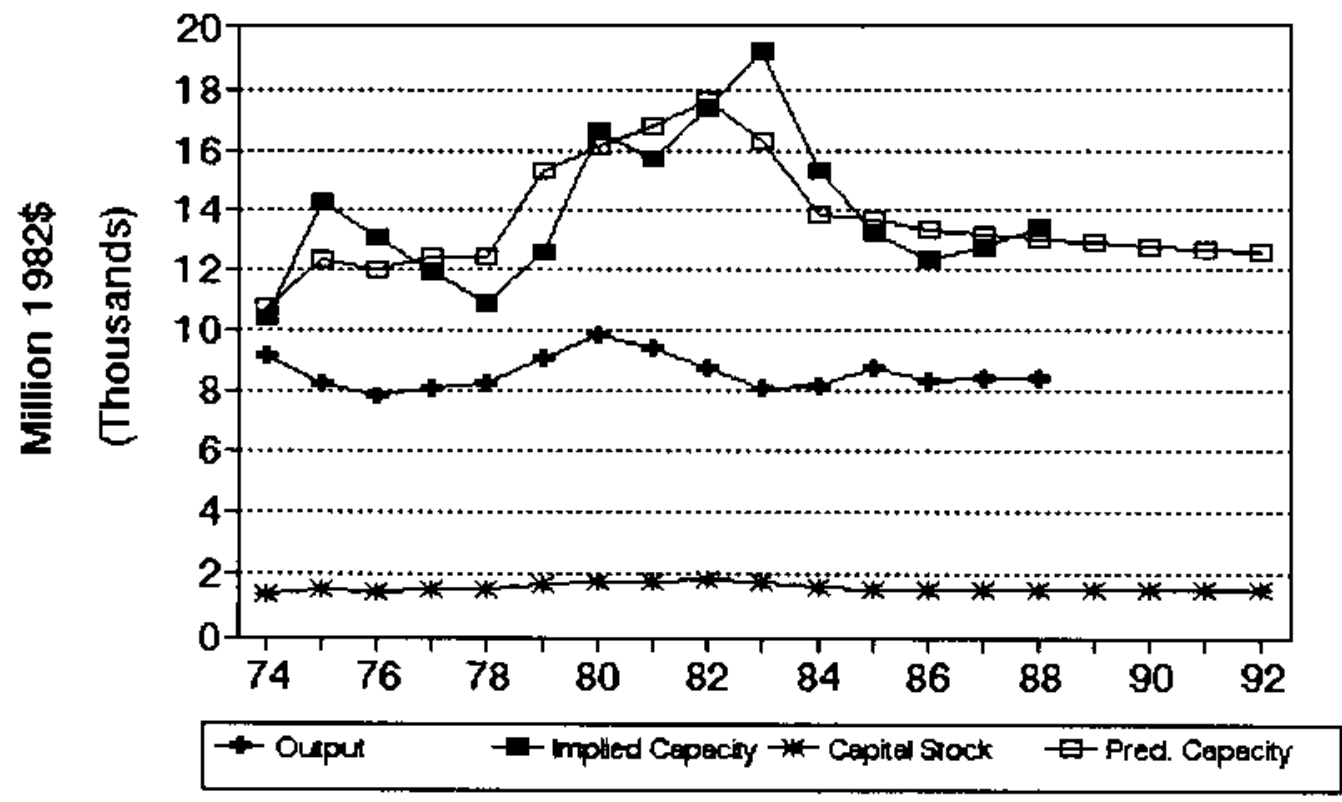


No. 290 BIC 3442 Metal doors, ansh, and trim

MILLIONS OF 1982 DOLLARS (EXCL. CU RATES)

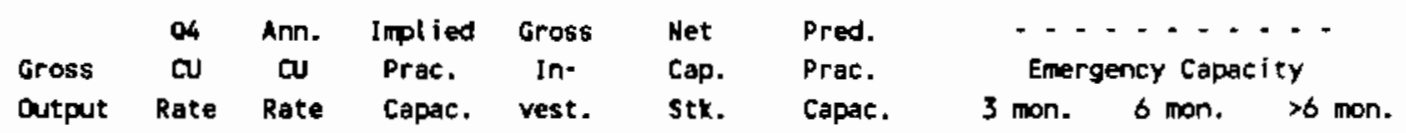

\begin{tabular}{|c|c|c|c|c|c|c|c|c|c|c|}
\hline 1974 & 4,389 & 56 & 57.1 & 7,682 & (26.1) & 564 & 6,984 & & & \\
\hline 1975 & 3,548 & 56 & 54.1 & 6,563 & 5.4 & 522 & 6,737 & & & \\
\hline 1976 & 3,945 & 61 & 59.3 & 6,652 & 71.1 & 544 & 6,865 & & & \\
\hline 1977 & 4,831 & 61 & 61.0 & 7,919 & 107.1 & 595 & 7,173 & & & \\
\hline 1978 & 4,965 & 70 & 66.3 & 7,490 & 66.9 & 605 & 7,232 & & & \\
\hline 1979 & 5,035 & 72 & 71.6 & 7,030 & 94.7 & 639 & 7,436 & & & \\
\hline 1980 & 4,686 & 76 & 73.6 & 6,363 & 95.9 & 672 & 7,630 & & & \\
\hline 1981 & 4,879 & 72 & 74.3 & 6,567 & 82.4 & 689 & 7,729 & & & \\
\hline 1982 & 4,714 & 58 & 62.5 & 7,546 & 135.7 & 749 & 8,089 & & & \\
\hline 1983 & 5,115 & 70 & 65.0 & 7,866 & 38.2 & 715 & 7,884 & & & \\
\hline 1984 & 5,246 & 62 & 65.6 & 7,997 & 52.6 & 697 & 7,780 & & & \\
\hline 1985 & 5,742 & 68 & 65.9 & 8,716 & 112.0 & 734 & $B, 003$ & & & \\
\hline 1986 & 5,946 & 62 & 64.1 & 9.277 & 95.0 & 755 & 8,123 & & & \\
\hline-- & $-\ldots$ & $\because$ & $\cdots$ & $\therefore-$ & $-\cdots$ & $\cdots$ & $\cdots$ & $--\cdot-\cdot$ & ---- & $\cdot \cdot \cdot \cdot$ \\
\hline 1987 & 6,048 & 71 & 67.1 & 9,010 & 117.5 & 796 & 8,372 & & & \\
\hline 1988 & 6,183 & $\pi$ & 72.5 & 8,527 & 124.2 & 841 & 8,636 & 14,531 & 16,036 & 16,426 \\
\hline 1989 & & & & & 131.0 & 887 & 8,914 & 14,998 & 16,552 & 16,954 \\
\hline 1990 & & & & & 137.8 & 936 & 9,204 & 15,486 & 17,091 & 17,506 \\
\hline 1991 & & & & & 144.5 & 986 & 9,505 & 15,993 & 17,650 & 18,079 \\
\hline 1992 & & & & & 151.3 & 1,039 & 9,816 & 16,517 & 18,228 & 18,671 \\
\hline
\end{tabular}

Capacity-Capital Stock Regression: Code 1 RSO 0.290

Coefficients: Const 3623.8 stock 6.0

Ave. Capacity/stock $(74-86): 11.5$

Ave. Weekly Hours (High) 82.8 Shift factor 1.90 Ave Heekly Hours (Low) 73.8 Shift Factor 2.13 Note: Output, Investment, and Capital Stock extrapolated for 1987 and 1988.

\section{Metal doors, sash, and trim}

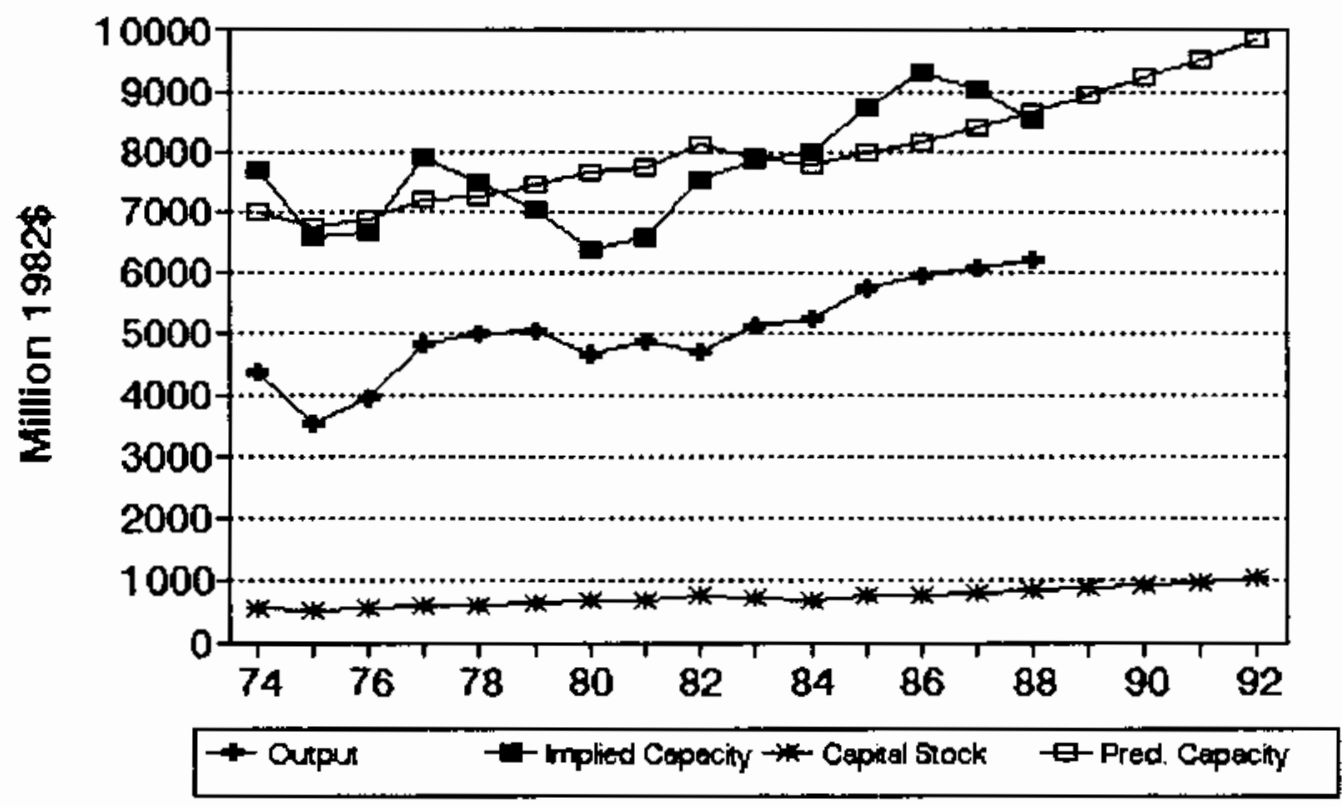




\section{No. 291 SIC 3443 Fabricated plate work (boiler shops)}

MILLIONS OF 1982 DOLLARS (EXCL. CU RATES)

$\begin{array}{lccccccc} & \text { Q6 } & \text { Ann. } & \text { Implied } & \text { Gross } & \text { Net } & \text { Pred. } & \ldots \\ \text { Gross } & \text { CU } & \text { CU } & \text { Prac. } & \text { In- } & \text { Cap. } & \text { Prac. } & \text { Emergency Capacity } \\ \text { Output Rate } & \text { Rate } & \text { Capac. } & \text { vest. } & \text { Stk. } & \text { Capac. } & 3 \text { mon. } 6 \text { mon. }\end{array}$

\begin{tabular}{|c|c|c|c|c|c|c|c|c|c|c|}
\hline 1974 & 9,899 & 65 & 67.8 & 14,600 & 586.9 & 1,828 & 13,022 & & & \\
\hline 1975 & 10,158 & 72 & 64.4 & 15,770 & 305.9 & 1,983 & 13,869 & & & \\
\hline 1976 & 10,606 & 72 & 72.8 & 14,577 & 329.7 & 2,149 & 14,775 & & & \\
\hline 1977 & 11,283 & 67 & 69.0 & 16,354 & 426.4 & 2,385 & 16,068 & & & \\
\hline 1978 & 11,266 & $\pi$ & 71.3 & 15,804 & $3 t 8.4$ & 2,500 & 16,697 & & & \\
\hline 1979 & 11,685 & 70 & 72.6 & 16,093 & 289.1 & 2,576 & 17,109 & & & \\
\hline 1980 & 10,876 & 67 & 66.5 & 16,362 & 145.9 & 2,508 & 16,740 & & & \\
\hline 1981 & 10,076 & 68 & 69.2 & 14,557 & 46.3 & 2,353 & 15,889 & & & \\
\hline 1982 & 8,121 & 41 & 49.3 & 16,465 & B2. 0 & 2,234 & 15,242 & & & \\
\hline 1983 & 6,861 & 37 & 38.3 & 17.894 & 153.2 & 2,177 & 14,928 & & & \\
\hline 1984 & 6,553 & 60 & 51.8 & 12,645 & $(98.5)$ & 1,890 & 13,361 & & & \\
\hline 1985 & 6,455 & 58 & 59.0 & 10,940 & 37.2 & 1,748 & 12,583 & & & \\
\hline 1986 & 5,943 & 58 & 57.7 & 10,299 & 85.1 & 1,655 & 12,076 & & & \\
\hline$-\cdot$ & --- & - & - - - & $-\cdot-$ & $--\cdot$ & $-\cdots$ & $-\cdot-$ & $-=-$ & $--\cdot$ & $-\cdot \cdot$ \\
\hline 1987 & 5,968 & 65 & 61.4 & 9,715 & 50.0 & 1,531 & 11,399 & & & \\
\hline 1988 & 6.001 & 68 & 67.4 & 8,906 & 50.0 & 1,420 & 10,793 & 13,785 & 15,189 & 15,552 \\
\hline 1989 & & & & & 50.0 & 1,321 & 10,250 & 13,093 & 14,426 & 14,771 \\
\hline $19 \% 0$ & & & & & 50.0 & 1,232 & 9,765 & 12,473 & 13,743 & 14,071 \\
\hline 1991 & & & & & 50.0 & 1,153 & 9,331 & 11,918 & 13,132 & 13,446 \\
\hline 1992 & & & & & 50.0 & 1,081 & 8,942 & 11,421 & 12,585 & 12,885 \\
\hline
\end{tabular}

Capacity-Cepital stock Regression: Code 0 RSQ 0.560

Coefficients: Const 3032.6 stack 5.5

Ave. Cepacity/Stock $(74-86): 6.9$ Ave. Weekly Hours (High) 109.3 Shift Factor 1.44 Ave Weekly Hours (Low) 101.6 Shift Factor 1.55 Note: Dutput, Investment, and Capital Stock extrapolated for 1987 and 1988.

\section{Fabricated plate work (boiler shop}

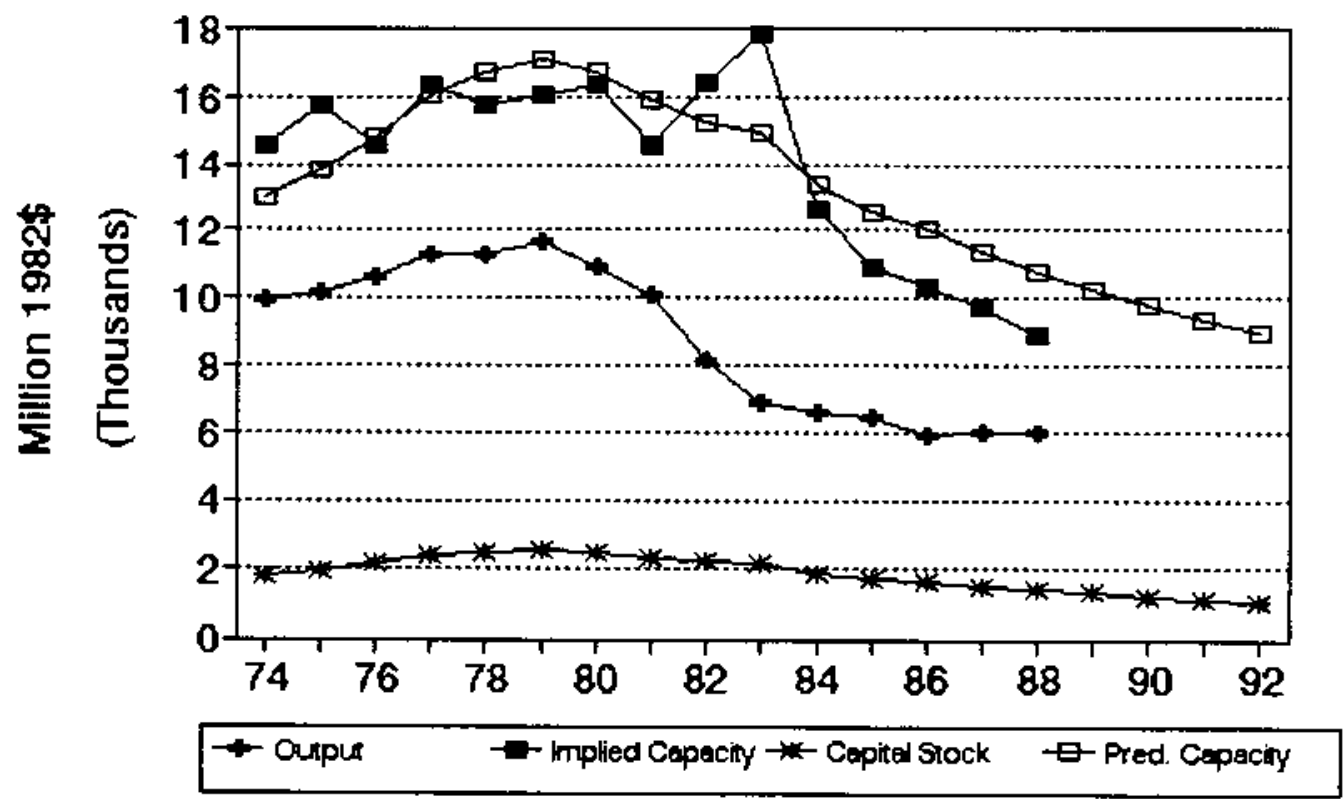




\section{No. 292 SIC 3444 sheet metal work}

MILLIOWS OF 1982 DOLLARS (EXCL. CU RATES)

$\begin{array}{lccccccc} & \text { Q4 } & \text { Ann. } & \text { Implied Gross } & \text { Net } & \text { Pred. } & \ldots \ldots \\ \text { Gross } & \text { CU } & \text { CU } & \text { Prac. } & \text { In- } & \text { Cap. } & \text { Prac. } & \text { Emergency Capacity } \\ \text { Output } & \text { Rate } & \text { Rate } & \text { Capac. } & \text { vest. } & \text { Stk. } & \text { Capac. } & 3 \text { mon. } 6 \text { mon. }>6 \text { mon. }\end{array}$

\begin{tabular}{|c|c|c|c|c|c|c|c|c|c|c|}
\hline 1976 & 6,956 & 80 & 81.7 & 8,517 & 196.3 & 1,039 & 7,260 & & & \\
\hline 1975 & 5,244 & 79 & 76.5 & 6,853 & 77.7 & 1,041 & 7,284 & & & \\
\hline 1976 & 6,259 & 67 & 71.9 & 8,701 & 239.0 & 1,196 & 9,521 & & & \\
\hline 1977 & 6,502 & 81 & 75.7 & 8,596 & 59.8 & 1,168 & 9,115 & & & \\
\hline 1978 & 6,749 & 66 & 71.3 & 9,463 & 142.9 & 1,216 & 9,807 & & & \\
\hline 1979 & 6,824 & 65 & 65.7 & 10,383 & 168.1 & 1,279 & 10,711 & & & \\
\hline 1980 & 6,601 & 64 & 63.6 & 10,382 & 200.2 & 1,361 & 11,895 & & & \\
\hline 1981 & 6,388 & 55 & 59.0 & 10,825 & 112.7 & 1,350 & 11,735 & & & \\
\hline 1982 & 6,896 & 48 & 50.1 & 13,770 & 127.9 & 1,347 & $11,6 \%$ & & & \\
\hline 1983 & 7,252 & 59 & 54.5 & 13,315 & 142.2 & 1,351 & 11,747 & & & \\
\hline 1984 & 7,228 & 62 & 61.4 & 11,781 & 142.1 & 1,352 & 11,770 & & & \\
\hline 1985 & 7,244 & 68 & 65.9 & 10,996 & 115.5 & 1,327 & 11,404 & & & \\
\hline 1986 & 7,363 & 64 & 65.3 & 11,270 & 106.3 & 1,292 & 10,004 & & & \\
\hline$\cdots$ & $-\cdot-\cdot$ & -- & $--\cdot$ & $\cdots$ & $\cdots$ & $\cdots$ & --- & --- & $-\cdot-$ & $\cdots$ \\
\hline 1987 & 7,493 & 59 & 60.4 & 12,398 & 127.5 & 1,281 & 10,737 & & & \\
\hline 1988 & 7,666 & 71 & 66.7 & 11,495 & 126.1 & 1,269 & 10,567 & 18,772 & 20,776 & 21,293 \\
\hline 1989 & & & & & 124.7 & 1,257 & 10,396 & 18,468 & 20,438 & 20,948 \\
\hline 1990 & & & & & 123.3 & 1,245 & 10,223 & 18,160 & 20,098 & 20,599 \\
\hline 1991 & & & & & 121.9 & 1,233 & 10,048 & 17,850 & 19,755 & 20,247 \\
\hline 1992 & & & & & 120.5 & 1,221 & 9,872 & 17,537 & 19,408 & 19,892 \\
\hline
\end{tabular}

Capacity-Capital Stock Regression: Code 0 RSQ 0.720

Coefficients: Const -7703.8 stock 14.4

Ave. Capacity/Stock $(74-86): 8.3$

Ave. Weekly Hours (High) 78.2 Shift Factor 2.02 Ave Weekly Hours (LOw) 67.0 Shift Factor 2.35 Nate: output, Investment, and Capital stock extrapolated for 1987 and 1988.

\section{Sheet metal work}

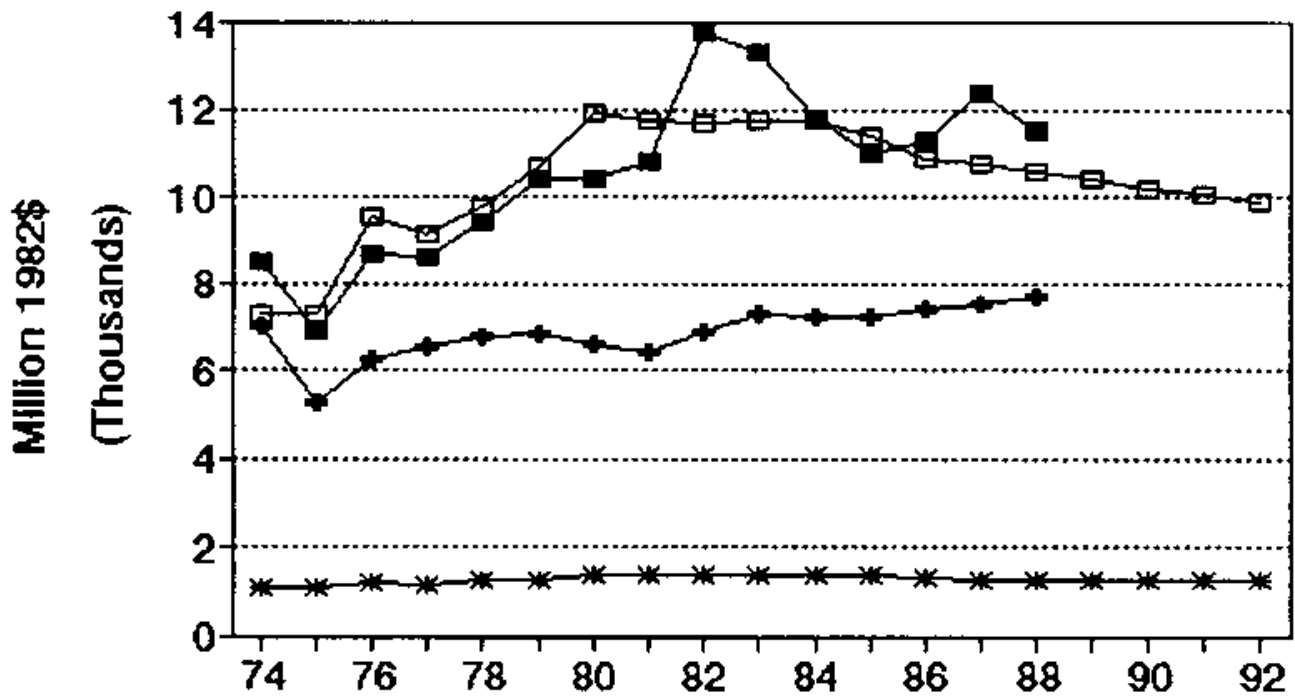

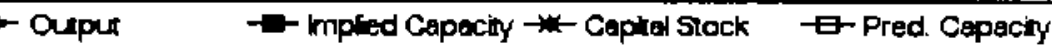




\section{No. 293 SIC 3446 Architectural metal work}

MILLIONS OF 1982 DOLLARS (EXCL. CU RATES)

$\begin{array}{lccccccc} & \text { Q4 } & \text { Ann. } & \text { Implied } & \text { Gross } & \text { Het } & \text { Pred. } & \ldots \ldots \ldots \\ \text { Gross } & \text { CU } & \text { CU } & \text { Prac. } & \text { In- } & \text { Cap. } & \text { Prac. } & \text { Emergency Capacity } \\ \text { Output } & \text { Rate } & \text { Rate } & \text { Capac. } & \text { vest. } & \text { Stk. } & \text { Capac. } & 3 \text { mon. } 6 \text { mon. }>6 \text { mon. }\end{array}$

\begin{tabular}{|c|c|c|c|c|c|c|c|c|c|c|}
\hline 1974 & 1,059 & 76 & 7.1 & 1,374 & $(29.2)$ & 219 & 1,186 & & & \\
\hline 1975 & 978 & 70 & 70.4 & 1,389 & 15.8 & 218 & 1,119 & & & \\
\hline 1976 & 1,174 & 76 & 73.9 & 1,589 & 32.2 & 232 & 1,707 & & & \\
\hline 1977 & 1,240 & 60 & $\$ 6.1$ & 1,877 & 36.2 & 248 & 2,366 & & & \\
\hline 1978 & 1,241 & 74 & 68.5 & 1,812 & 12.6 & 240 & 2,041 & & & \\
\hline 1979 & 1,257 & $\pi$ & 73.0 & 1,721 & 35.5 & 253 & 2,578 & & & \\
\hline 1980 & 1,332 & 68 & 68.9 & 1,934 & 21.6 & 251 & 2,511 & & & \\
\hline 1981 & 1,356 & 64 & $\infty 6.0$ & 2,055 & 33.6 & 260 & 2,866 & & & \\
\hline 1982 & 1,362 & 62 & 62.3 & 2,184 & 7.7 & 243 & 2,178 & & & \\
\hline 1983 & 1,811 & 53 & 56.3 & 3,219 & 29.3 & 246 & 2,316 & & & \\
\hline 1984 & 2,089 & 61 & 58.3 & 3,585 & 34.9 & 255 & 2,657 & & & \\
\hline 1985 & 2,389 & 58 & 59.2 & 4,035 & 44.2 & 272 & 3,370 & & & \\
\hline 1986 & 2,476 & $64 *$ & 61.8 & 4,008 & 39.6 & 284 & 3,887 & & & \\
\hline-- & $--\cdot$ & -- & $-\cdot$ & $\cdots$ & $\cdots-$ & -- & $\cdots$ & $-\cdot \cdot \cdot \cdot$ & $=--$ & $--\cdot$ \\
\hline 1987 & 2,557 & 70 & 67.5 & 3,790 & 25.3 & 282 & 3,784 & & & \\
\hline 1988 & 2,666 & $\pi *$ & $\pi .0$ & 3,701 & 29.2 & 283 & 3,856 & 4,933 & 5,342 & 5,448 \\
\hline 1989 & & & & & 29.9 & 285 & 3,946 & 5,049 & 5,468 & 5,576 \\
\hline 1990 & & & & & 30.3 & 288 & 4,045 & 5,175 & 5,604 & 5,715 \\
\hline 1991 & & & & & $31 . \uparrow$ & 291 & 4,170 & 5,336 & 5,778 & 5,892 \\
\hline 1992 & & & & & 30.7 & 293 & 4,263 & 5,455 & 5,907 & 6,024 \\
\hline
\end{tabular}

Capacity-Cepital stock Regression: Code 0 RSQ 0.630

Coefficients: Const -7950.2 stock 41.7

Ave. Capacity/Stock $(74-86): 9.6$ Ave. Weekly Hours (High) 111.5 Shift Factor 1.41 Ave Heekly Hours (LoH) 111.5 Shift factor 1.41 * Imputed Note: Output, Imvestment, and Capital Stock extrapolated for 1987 and 1988.

\section{Architectural metal work}

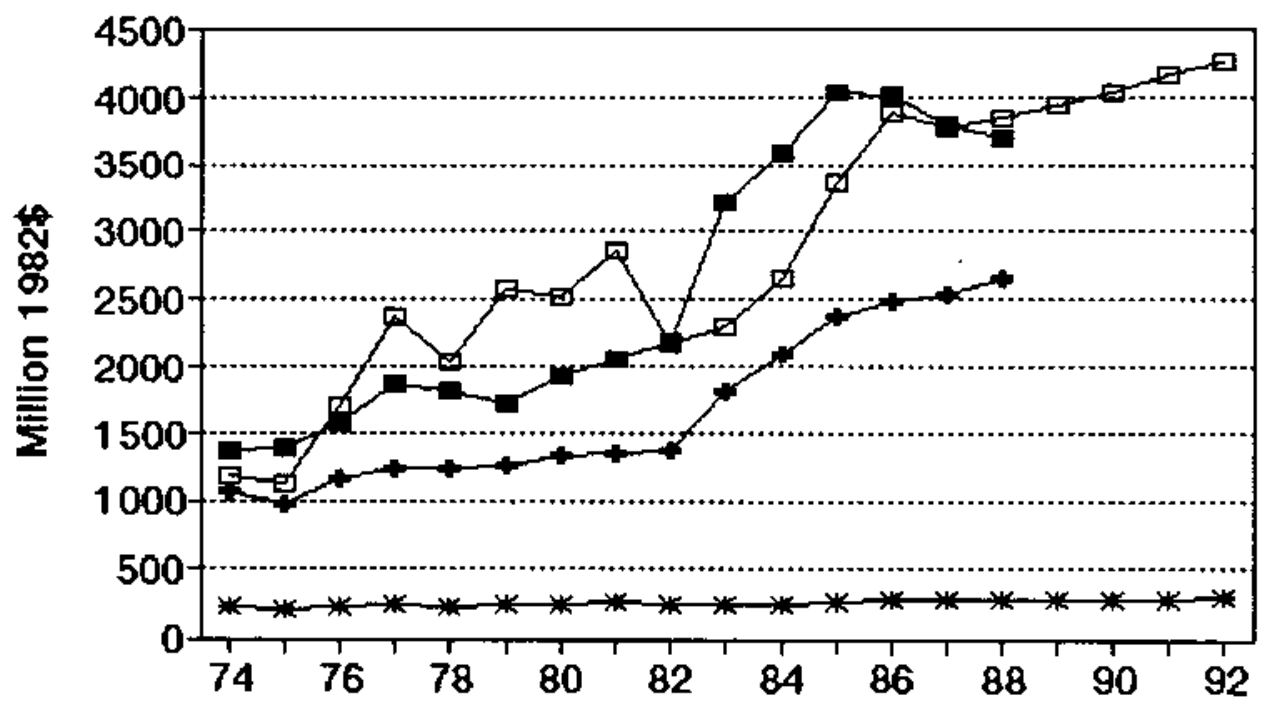

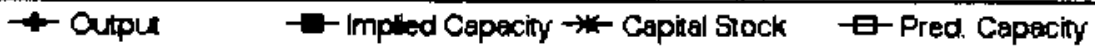




\section{No. 294 SIC 3448 Prefebricated motal buildings}

MILLIONS DF 1982 DOLLARS (EXCL. CU RATES)

$\begin{array}{lcccclcc} & 04 & \text { Am. Implied Gross } & \text { Net } & \text { Pred. } & \ldots \\ \text { Gross } & \text { CU } & \text { CU } & \text { Prac. } & \text { In- } & \text { Cap. } & \text { Prac. } & \text { Emergency Capacity } \\ \text { Output Rate } & \text { Rate } & \text { Capac. } & \text { vest. } & 5 t x . & \text { Capac. } & 3 \text { mon. } 6 \text { mon. }>6 \text { mon. }\end{array}$

\begin{tabular}{|c|c|c|c|c|c|c|c|c|c|c|}
\hline 1974 & 2,226 & $\pi$ & 77.6 & 2,869 & 65.6 & 287 & 2,434 & & & \\
\hline 1975 & 1,426 & 67 & 65.6 & 2,173 & 44.8 & 315 & 2,785 & & & \\
\hline 1976 & 1,669 & 60 & 63.3 & 2,639 & 14.3 & 310 & 2,723 & & & \\
\hline 1977 & 2,647 & 71 & 66.8 & 3,964 & 108.6 & 393 & 3,790 & & & \\
\hline 1978 & 2,822 & 84 & 78.5 & 3,597 & 39.7 & 406 & 3,953 & & & \\
\hline 1979 & 2,701 & 73 & 77.8 & 3,474 & 42.3 & 420 & 4,136 & & & \\
\hline 1980 & 2,455 & 54 & 59.7 & 4,112 & 65.2 & 455 & 4,588 & & & \\
\hline 1981 & 2,521 & 53 & 54.4 & 4,636 & 52.7 & 474 & 4,832 & & & \\
\hline 1982 & 2,320 & 49 & 49.8 & 4,660 & 79.0 & 514 & 5,340 & & & \\
\hline 1983 & 2,350 & 46 & 46.9 & 5,013 & 10.3 & 485 & 4,972 & & & \\
\hline 1984 & 2,422 & 44 & 45.4 & 5,332 & 18.4 & 464 & 4,707 & & & \\
\hline 1985 & 2,524 & 50 & 47.9 & 5,267 & 17.1 & 443 & 4,434 & & & \\
\hline 1986 & 2,738 & 51 & 50.4 & 5,431 & 43.0 & 446 & 4,472 & & & \\
\hline 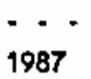 & 2,833 & $\begin{array}{l}-\cdots \\
55 *\end{array}$ & $\begin{array}{l}\cdots \\
53.0\end{array}$ & 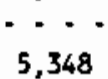 & $\begin{array}{l}\cdots \\
48.2\end{array}$ & $\begin{array}{l}-- \\
455\end{array}$ & 4.586 & $\cdots$ & -- & $\cdots$ \\
\hline 1988 & 2,962 & 54 & 54.8 & 5,402 & 57.7 & 473 & 4.812 & 6,184 & 6,765 & 6,915 \\
\hline 1989 & & & & & 59.2 & 490 & 5,038 & 6,474 & 7.082 & 7,239 \\
\hline 1990 & & & & & 60.2 & 507 & 5,256 & 6,754 & 7,389 & 7,553 \\
\hline 1991 & & & & & 62.3 & 525 & 5,482 & 7,044 & 7,706 & 7,877 \\
\hline 1992 & & & & & 61.1 & 560 & 5,673 & 7,290 & 7,975 & 8,152 \\
\hline
\end{tabular}

Capacity-Capital Stock Regression: Code 1 RSO 0.720 Coefficients: Const -1256.1 stock 12.8 Ave. Weekly Hours (High) 109.6 shift factor 1.44 Ave Weekly Hours (LOW) 99.1 Shift factor 1.59 - Imputed Note: Output, Investment, and Capital 5tock extrapolated for 1987 and 1988.

3448 Prefabricated metal buildings

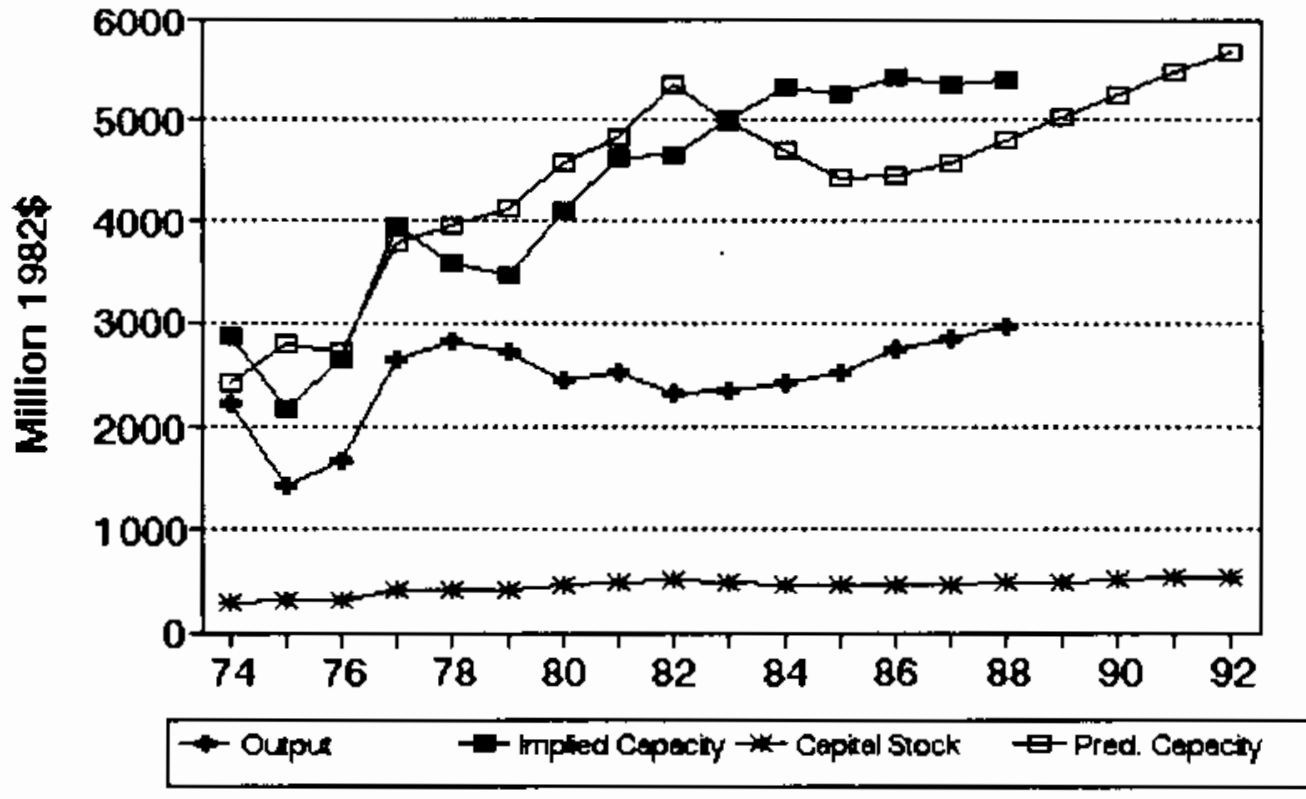




\section{No. 295 sIC 3449 Migcellaneous netal work}

\section{MILLIONS OF 1982 dOLLARS (EXCL. CU RATES)}

$\begin{array}{lccccccc} & \text { Q4 } & \text { Am. Implied } & \text { Gross } & \text { Net } & \text { Pred. } & -\ldots & -\ldots \\ \text { Gross } & \text { CU } & \text { CU } & \text { Prac. } & \text { In- } & \text { Cap. } & \text { Prac. } & \text { Emergency Capacity } \\ \text { Dutput } & \text { Rate } & \text { Rate } & \text { Capac. } & \text { vest. } & \text { Stk. } & \text { Capac. } & 3 \text { mon. } 6 \text { mon. }>6 \text { mon. }\end{array}$

\begin{tabular}{|c|c|c|c|c|c|c|c|c|c|c|}
\hline 1974 & 2,927 & 88 & 88.2 & 3,318 & 16.3 & 290 & 2,701 & & & \\
\hline 1975 & 1,918 & $76 *$ & 80.2 & 2,391 & 46.3 & 312 & 2,913 & & & \\
\hline 1976 & 1,932 & 60 & 71.1 & 2,718 & 41.6 & 325 & 3,050 & & & \\
\hline 1977 & 1,827 & 69 & 68.6 & 2,663 & $(8.7)$ & 290 & 2,704 & & & \\
\hline 1978 & 1,969 & 7 & 74.0 & 2,663 & 38.3 & 301 & 2,808 & & & \\
\hline 1979 & 2,244 & 86 & 82.7 & 2.714 & 29.1 & 301 & 2,805 & & & \\
\hline 1980 & 2,348 & $n$ & 80.3 & 2,925 & 34.2 & 304 & 2,834 & & & \\
\hline 1981 & 2,361 & 92 & 86.5 & 2,730 & 42.3 & 313 & 2,931 & & & \\
\hline 1982 & 2,609 & 69 & $\pi .5$ & 3,367 & 97.5 & 371 & 3,499 & & & \\
\hline 1983 & 2,563 & 75 & 72.7 & 3,525 & 11.2 & 364 & 3,230 & & & \\
\hline 1984 & 2,947 & 87 & 82.6 & 3,570 & 23.4 & 331 & 3,109 & & & \\
\hline 1985 & 2,821 & 76 & 80.2 & 3,520 & 27.3 & 325 & 3,893 & & & \\
\hline 1986 & 2,733 & 54 & 62.2 & 4,392 & 46.6 & 338 & 4,019 & & & \\
\hline $\begin{array}{l}-\cdots \\
1987\end{array}$ & 2,758 & $\begin{array}{l}-- \\
59\end{array}$ & $\because \overline{57.1}$ & $\cdots$ & $\begin{array}{l}--- \\
42.2\end{array}$ & 345 & 4,096 & $-\cdots$ & $\cdots$ & $\cdots \cdot$ \\
\hline 1988 & 2,791 & 72 & 67.1 & 4,156 & 43.3 & 353 & 4,176 & 4,794 & 5,155 & 5,249 \\
\hline 1989 & & & & & 44.5 & 362 & 4,258 & 4,889 & 5,257 & 5,353 \\
\hline 1990 & & & & & 45.6 & 371 & 4,344 & 4,987 & 5,363 & 5,460 \\
\hline 1991 & & & & & 46.7 & 379 & 4,432 & 5,088 & 5,472 & 5,571 \\
\hline 1992 & & & & & 47.9 & 389 & 4,522 & 5,192 & 5,583 & 5,684 \\
\hline
\end{tabular}

Capacity-Capital Stock Regression: Code -1 RSO 0.610

Coefficients: Const -162.4 Stock 9.9 D(85-86) 848.4

Ave. Capacity/Stock $(74-86): 9.8$ Ave. Weekly Hours (High) 126.0 Shift Factor 1.26 Ave Heekly Hours (Low) 114.6 Shift Factor 1.38 * Imputed Hote: Output, Investment, and Capital Stock extrapalated for 1987 and 1988.

\section{Miscellaneous metal work}

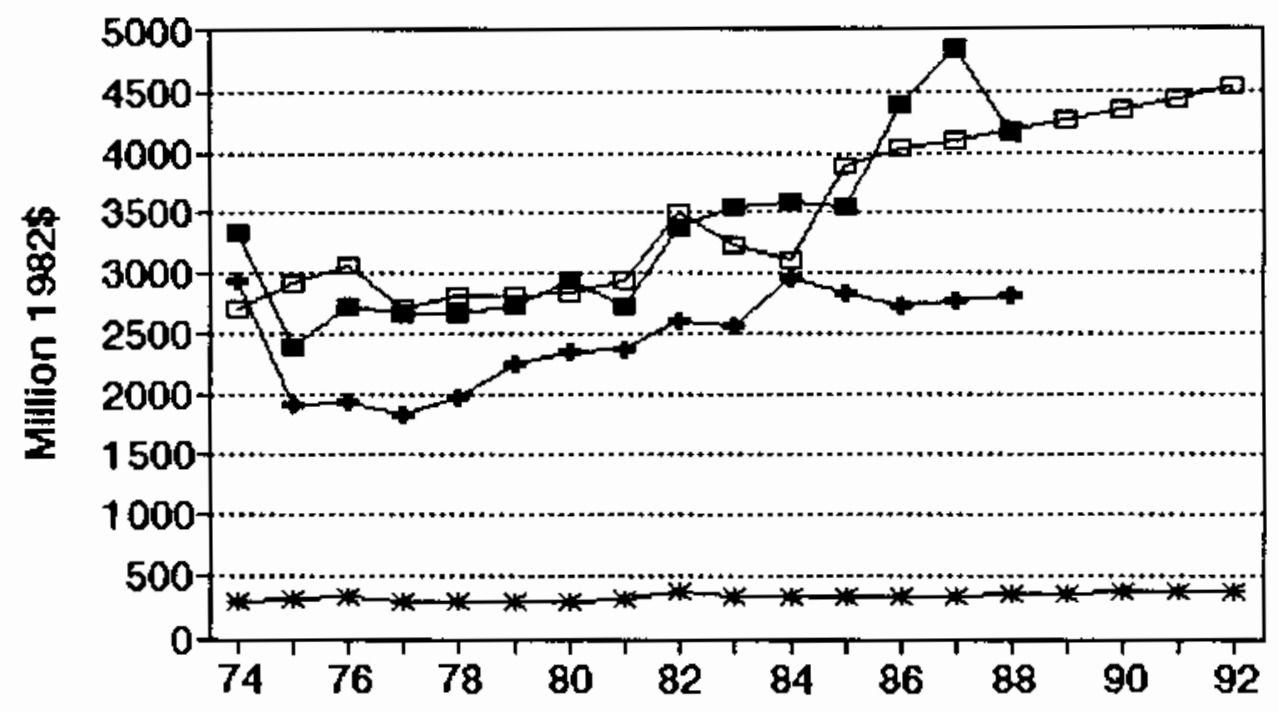

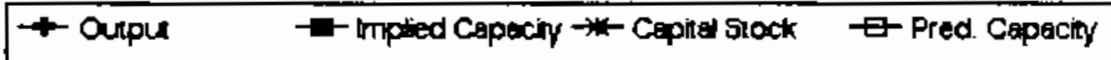




\section{No. 296 SIC 3451 Screw machine products}

MILLIONS OF 1982 DOLLARS (EXCL. CU RATES)

$\begin{array}{lccccccc} & \text { Q4 } & \text { Ann. Implied Gross } & \text { Net } & \text { Pred. } & -\ldots \\ \text { Gross } & \text { CU } & \text { CU } & \text { Prac. } & \text { In- } & \text { Cap. } & \text { Prac. } & \text { Emergency Capacity } \\ \text { Output Rate } & \text { Rate } & \text { Capac. } & \text { vest. } & \text { Stk. } & \text { Capac. } & 3 \text { mon. } 6 \text { mon. }\end{array}$

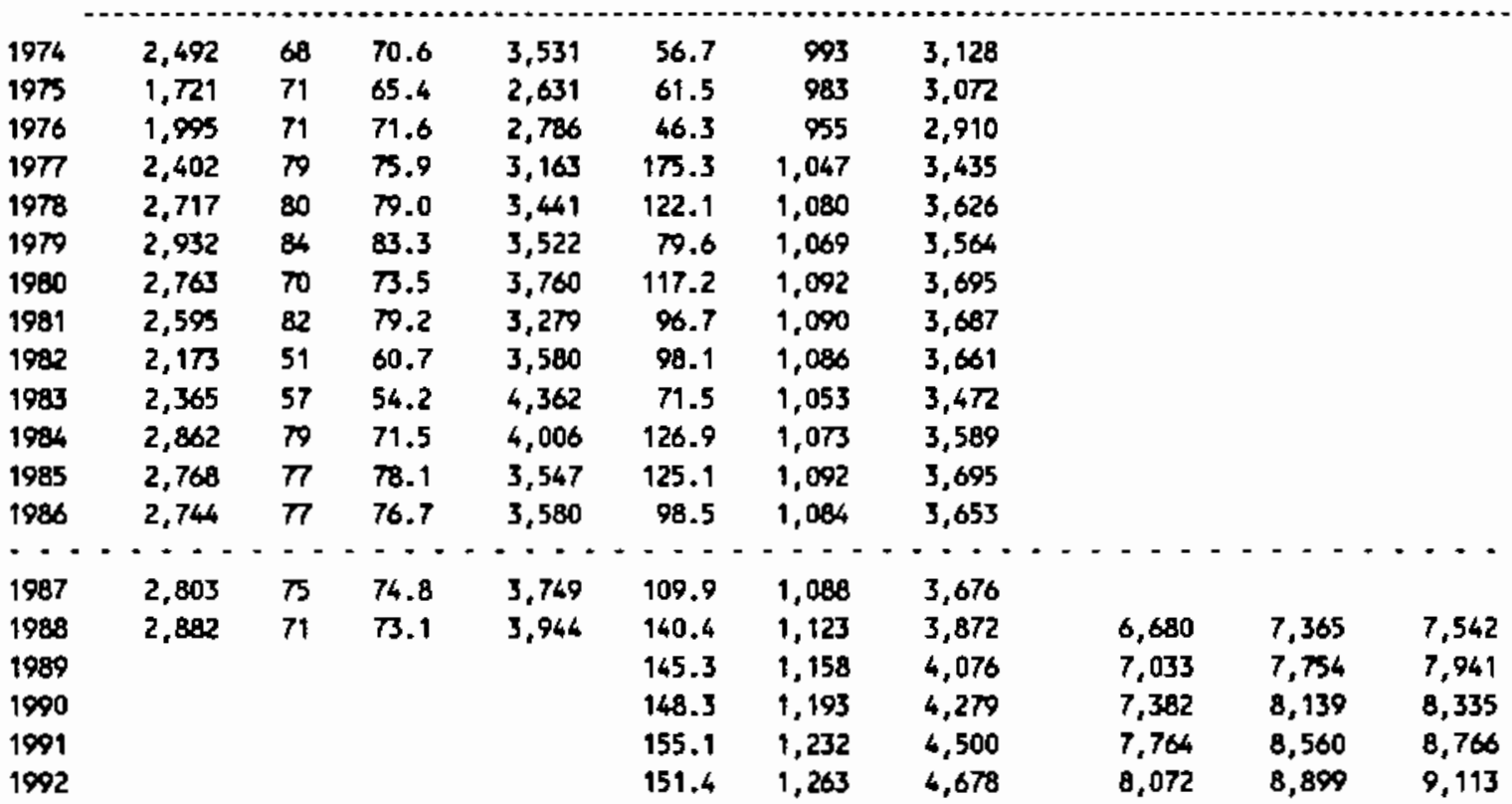

Capacity-Capital Stock Regression: Code 0 RSO 0.340 Coefficients: Const -2575.2 stock 5.7 Ave. Weekly Hours (High) 80.9 Shift Factor 1.95 Ave Heekly Hours (Low) 73.3 Shift Factor 2.15 Note: Output, Investment, and Capital stock extrapolated for 1987 and 1988.

3451 Screw machine products

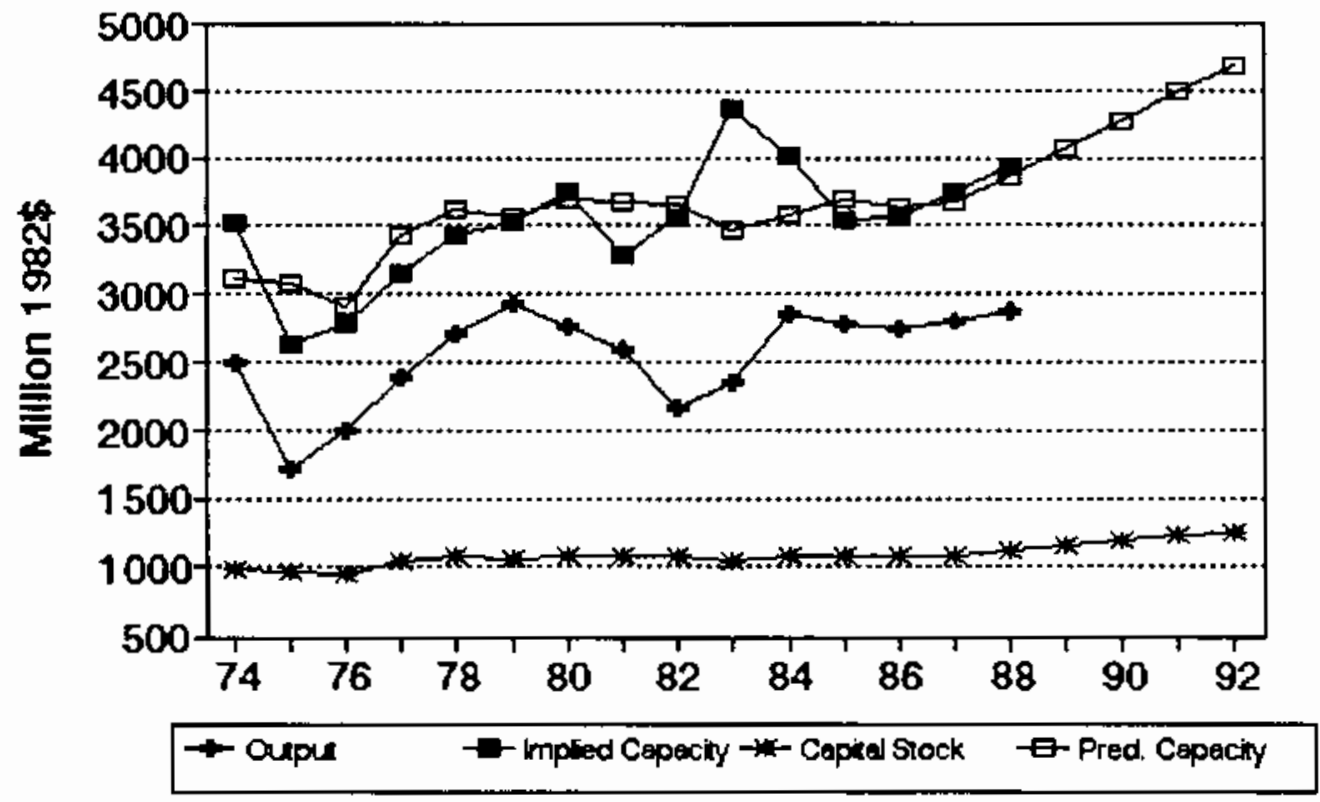


yo. 297 sIC 3452 Bolts, nuts, rivets, and washers

MILLIONS OF 1982 DOLLARS (EXCL. CU RATES)

$\begin{array}{lcccclcc} & \text { Q4 } & \text { Ann. } & \text { Implied Gross } & \text { Net } & \text { Pred. } & -\ldots \ldots \\ \text { Gross } & \text { CU } & \text { CU } & \text { Prac. } & \text { In- } & \text { Cap. } & \text { Prac. } & \text { Emergency Capacity } \\ \text { Output } & \text { Rate } & \text { Rate } & \text { Capac. } & \text { vest, } & \text { Stk. } & \text { Capac. } & 3 \text { mon. } 6 \text { mon. }>6 \text { mon. }\end{array}$

\begin{tabular}{|c|c|c|c|c|c|c|c|c|c|c|}
\hline 1974 & 4,752 & 72 & 74.8 & 6,353 & 262.3 & 1,696 & 7,013 & & & \\
\hline 1975 & 3,499 & 45 & 50.7 & 6,903 & 125.5 & 1,689 & 6,983 & & & \\
\hline 1976 & 4,029 & 58 & 53.3 & 7,554 & 149.6 & 1,700 & 7.028 & & & \\
\hline 1977 & 4,525 & 58 & 58.0 & 7,798 & 286.5 & 1,832 & 7,577 & & & \\
\hline 1978 & 4,925 & 67 & 63.0 & 7,813 & 159.3 & 1,837 & 7,595 & & & \\
\hline 1979 & 5,120 & $\pi$ & 70.8 & 7,233 & 56.8 & 1,745 & 7,213 & & & \\
\hline 1980 & 4,715 & 64 & 65.4 & 7,204 & 195.0 & 1,787 & 7,391 & & & \\
\hline 1981 & 4,605 & 61 & 63.4 & 7,260 & 169.9 & 1,798 & 7,436 & & & \\
\hline 1982 & 3,672 & 50 & 53.1 & 6,922 & 42.9 & 1,687 & 6,976 & & & \\
\hline 1983 & 3,953 & 64 & 58.0 & 6,020 & 103.8 & 1,635 & 6,760 & & & \\
\hline 1984 & 4,478 & $\pi$ & 71.8 & 6,234 & 105.9 & $1,5 B B$ & 6,562 & & & \\
\hline 1985 & 4,625 & 70 & 72.2 & 6,408 & 142.5 & 1,575 & 6,511 & & & \\
\hline 1986 & 4,610 & 64 & 65.9 & 6,992 & 137.4 & 1,560 & 6,448 & & & \\
\hline$\because-$ & $-\cdot-\cdot$ & $\because$ & $\because$ & & -- & $\cdots$ & $-\cdots$ & -- & $-\cdots$ & $\cdots \cdot$ \\
\hline 1987 & 4,692 & 73 & 68.7 & 6,833 & 155.7 & 1,563 & 6,460 & & & \\
\hline 1988 & 4,800 & $\sqrt{3}$ & 74.8 & 6,419 & 198.3 & 1,608 & 6,646 & 8,444 & 9,102 & 9.272 \\
\hline 1989 & & & & & 205.0 & 1,655 & 6,843 & 8,694 & 9,370 & 9,545 \\
\hline 1990 & & & & & 209.3 & 1,702 & 7,037 & 8,941 & 9,637 & 9,817 \\
\hline 1991 & & & & & 218.7 & $1, \pi 34$ & 7.252 & 9,214 & 9,931 & 10,116 \\
\hline 1992 & & & & & 213.5 & 1,796 & 7,424 & 9,432 & 10,167 & 10,357 \\
\hline
\end{tabular}

Capacity-Capital stock Regression: Code 0 RSO 0.590

Coefficients: Const -16.6 stock 4.1

Ave. Capacity/Stock $(74-86): 4.1$

Ave. Weekly Hours (High) 113.0 Shift factor 1.39 Ave Heekly Hours (LOW) 103.6 Shift Factor 1.52 Note: Output, Investment, and Capital Stock extrapolated for 1987 and 1988.

\section{Bolts, nuts, rivets, and washers}

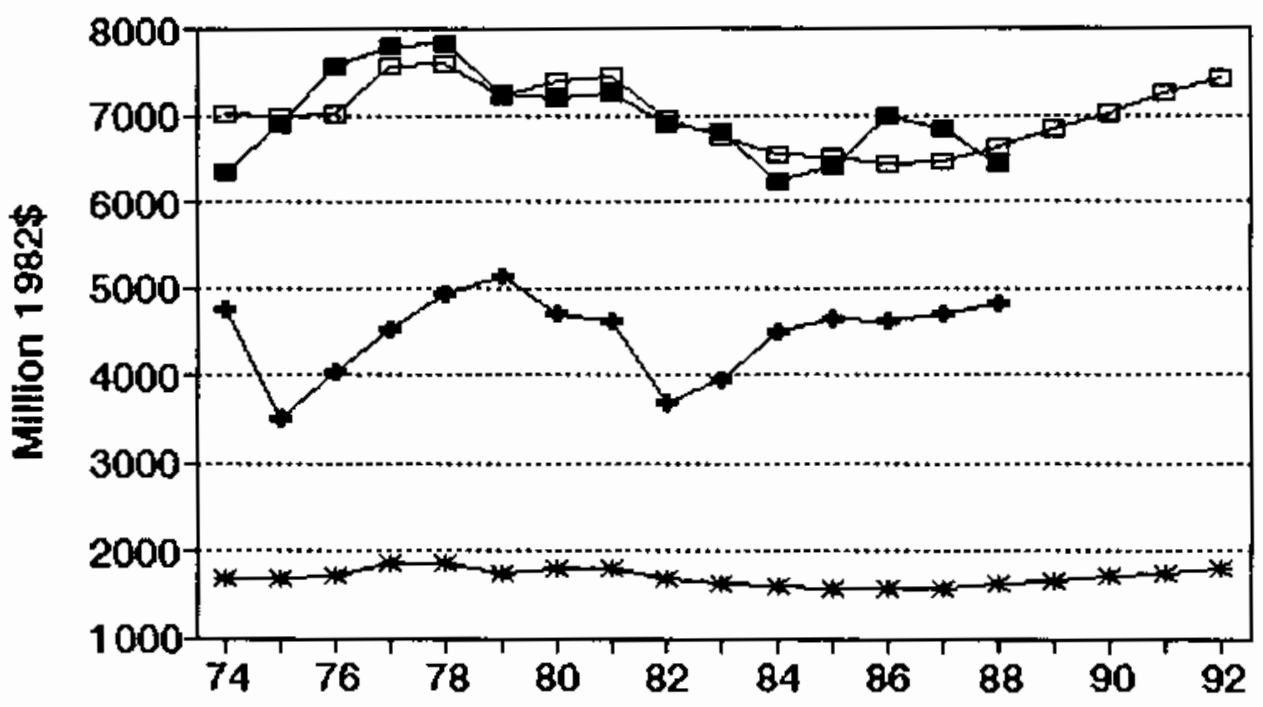

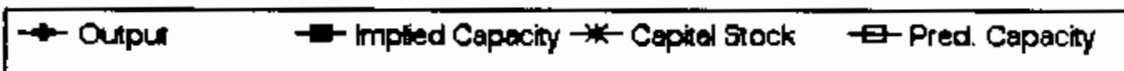


No. 298 sIC 3462 Iron and steel forgings

MILLIONS OF 1982 DOLLARS (EXCL. CU RATES)

$\begin{array}{lccccccc} & \text { Q4 } & \text { Ann. Implied Gross } & \text { Net } & \text { Pred. } & \ldots \ldots \ldots \\ \text { Gross } & \text { CI } & \text { CU } & \text { Prac. } & \text { In- } & \text { Cap. } & \text { Prac. } & \text { Emergency Capecity } \\ \text { Output Rate } & \text { Rate } & \text { Capac. } & \text { vest. } & \text { Stk. } & \text { Capoc. } & 3 \text { mon. } 6 \text { mon. }>6 \text { mon. }\end{array}$

\begin{tabular}{|c|c|c|c|c|c|c|c|c|c|c|}
\hline 1974 & 5,085 & 69 & 73.1 & 6,959 & 152.6 & 1,078 & 6,981 & & & \\
\hline 1975 & 4,392 & 71 & 63.1 & 6,964 & 66.4 & 1,066 & 6,898 & & & \\
\hline 1976 & 4,232 & 67 & 69.8 & 6,064 & 205.6 & 1,187 & 6,815 & & & \\
\hline 1977 & 4,603 & $n$ & 70.1 & 6,563 & 384.6 & 1,469 & 6,732 & & & \\
\hline 1978 & 4,710 & 76 & 73.6 & 6,401 & 9.6 & 1,385 & 0,648 & & & \\
\hline 1979 & 4,590 & 73 & 75.1 & 6,110 & 162.4 & 1,451 & 6,565 & & & \\
\hline 1980 & 3,949 & 54 & 58.9 & 6,710 & 176.2 & 1,526 & 6,482 & & & \\
\hline 1981 & 4,039 & 50 & 53.1 & 7,603 & 179.9 & 1,590 & 6,399 & & & \\
\hline 1982 & 2,925 & 30 & 35.9 & 8,157 & 146.6 & 1,632 & 6,315 & & & \\
\hline 1983 & 2,366 & 62 & 36.7 & 6,443 & 5.3 & 1,524 & 6,232 & & & \\
\hline 1984 & 3,104 & 61 & 54.7 & 5,671 & 495.5 & 1,806 & 6,149 & & & \\
\hline 1985 & 2,817 & 47 & 52.6 & 5,357 & 2.2 & 1,720 & 6,066 & & & \\
\hline 1986 & 2,652 & 53 & 50.4 & 5,262 & 89.0 & 1,655 & 5,982 & & & \\
\hline$\cdots$ & & $\cdots$ & $\cdots$ & & $\cdots$ & $\cdots$ & $\cdots$ & $\cdots \cdots$ & - - - - & $\cdots$ \\
\hline 1987 & 2,641 & 48 & 48.9 & 5,399 & 157.2 & 1,664 & 5,890 & & & \\
\hline 1988 & 2,627 & 65 & 59.0 & 4,653 & 168.0 & 1,682 & 5,816 & 7,236 & 8,067 & 8,282 \\
\hline 1989 & & & & & 169.7 & 1,701 & 5,733 & 7,132 & 7,952 & 8,163 \\
\hline 1990 & & & & & 170.8 & 1,719 & 5,650 & 7,029 & 7,836 & 8,045 \\
\hline 1991 & & & & & 173.2 & 1,738 & 5,566 & 6,925 & 7,721 & 7,926 \\
\hline 1992 & & & & & 171.9 & $1, \pi 34$ & 5,483 & 6,822 & 7,605 & 7,808 \\
\hline
\end{tabular}

Capacity-Capital Stock Regression: Code -2 RSQ 0.150 Coefficients: Const 7064.4 Jime $\mathbf{- 8 3 . 2}$

Ave. Capecity/Stock $(74-86): 4.4$ Ave. Weekly Hours (High) $\$ 10.6$ Shift Factor 1.42 Ave Weekly Hours (LOw) 99.0 Shift Factor 1.59 Note: Output, Investment, and Capital Stock extrapoleted for 1987 and 1988.

\section{Iron and steel forgings}

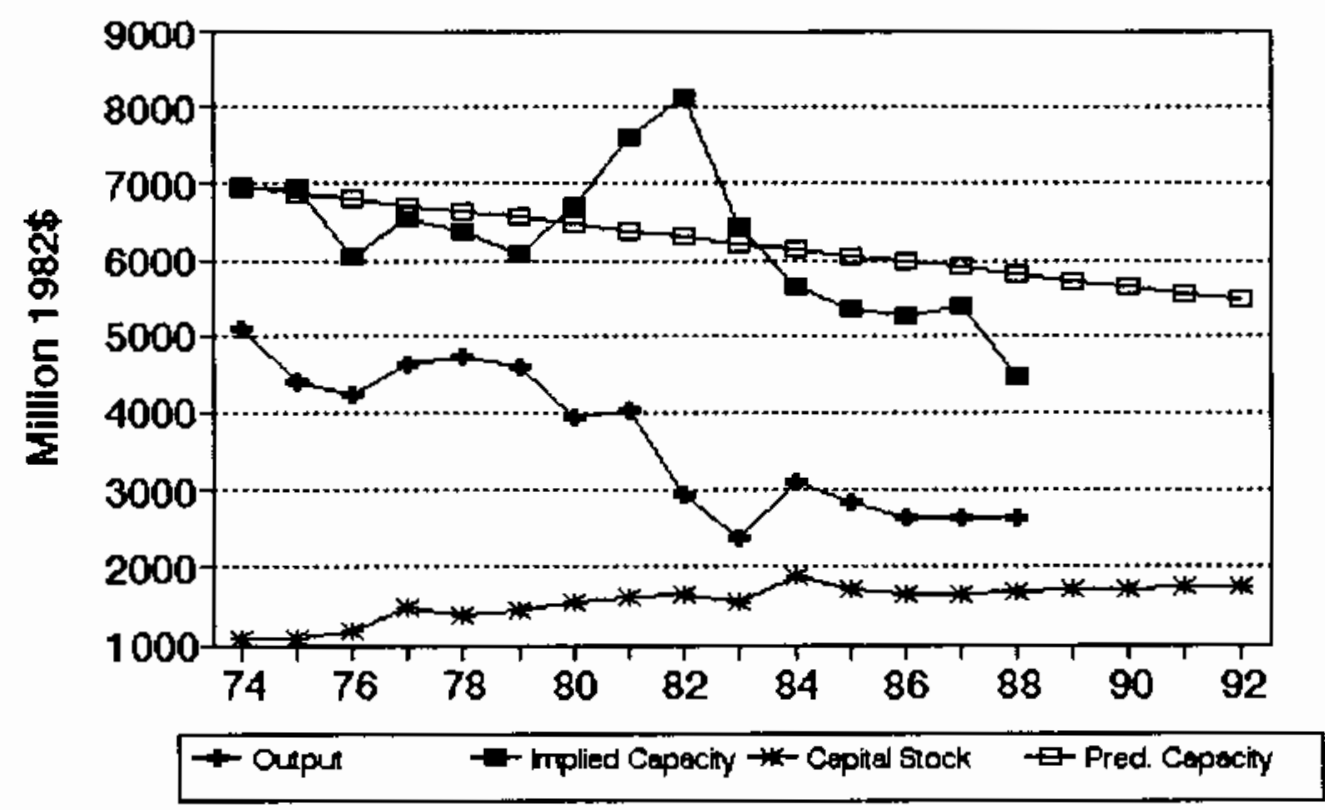




\section{No. 299 sIC 3463 Nonferrous forgings}

MILLIONS OF 1982 DOLLARS (EXCL. CU RATES)

$\begin{array}{lccccccc} & \text { Q4 } & \text { Ann. Implied Gross } & \text { Net } & \text { Pred. } & \ldots \ldots & \ldots \ldots \\ \text { Gross } & \text { CU } & \text { CU } & \text { Prac. } & \text { In- } & \text { Cap. } & \text { Prac. } & \text { Emergency Capacity } \\ \text { Output } & \text { Rate } & \text { Rete } & \text { Capac. } & \text { vest. } & \text { Stk. } & \text { Capac. } & 3 \text { mon. } 6 \text { mon. }>6 \text { mon. }\end{array}$

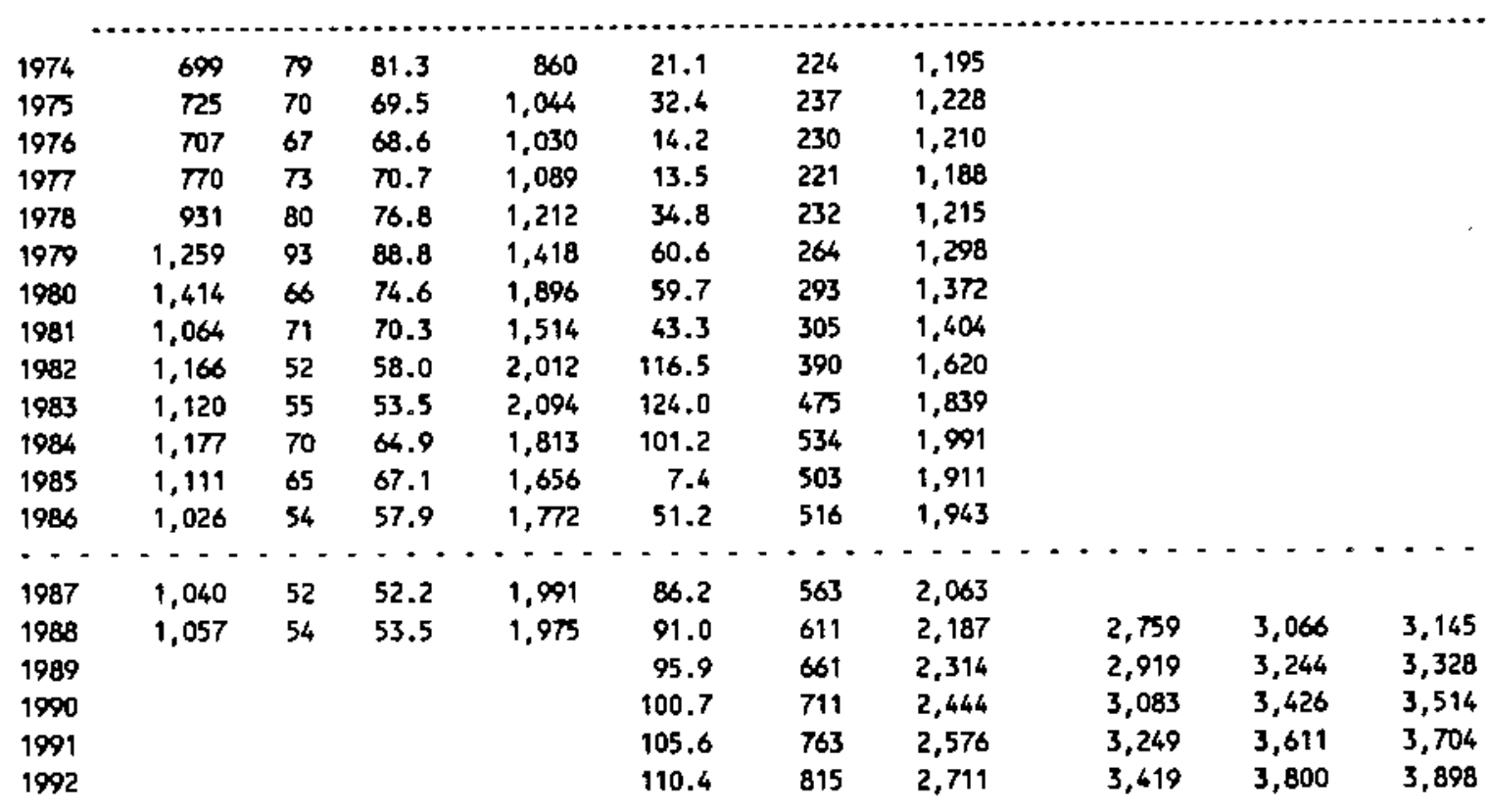

Capacity-Capital Stock Regression: Code 1 RSQ 0.600

Coefficients: Const 620.7 Stock 2.6

Ave. Capacity/Stock $(74-86): 4.4$ Ave. Weekly Hours (High) 109.6 Shift factor 1.44 Ave Weekly Hours (Low) 103.3 Shift Factor 1.52 Note: Output, Investment, and Capital Stock extrapolated for 1987 and 1988.

\section{Nonferrous forgings}

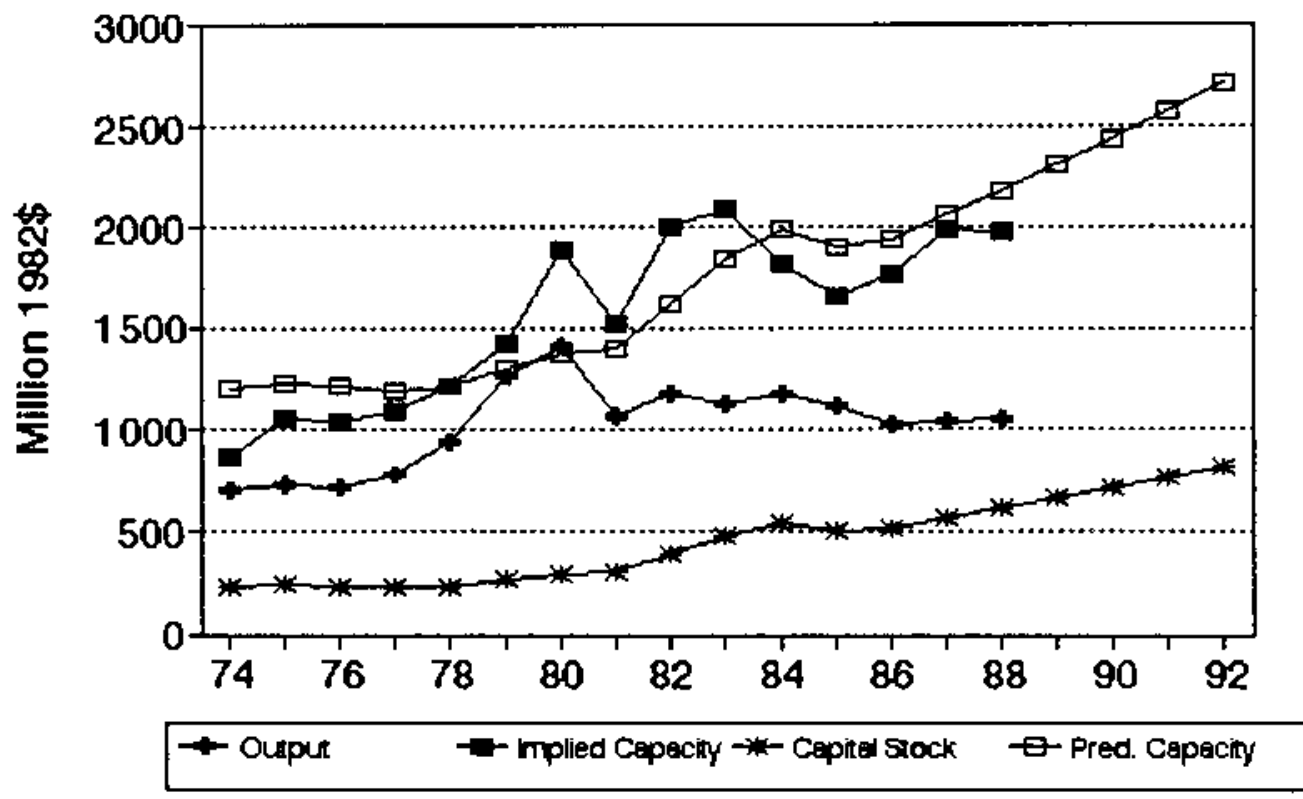




\section{No. 300 SIC 3465 Autonotive stampings}

MILLIONS OF 1982 DOLLARS (EXCL. CU RATES)

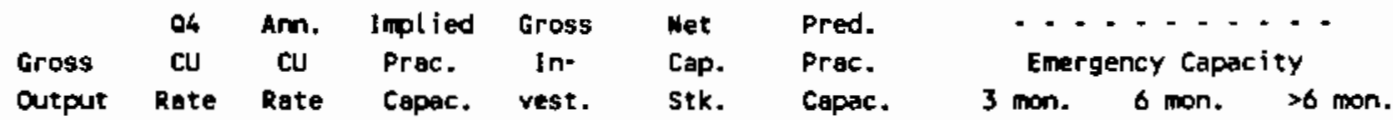

\begin{tabular}{|c|c|c|c|c|c|c|c|c|c|c|}
\hline 1974 & 10,789 & 81 & 83.2 & 12,962 & 928.6 & 5,285 & 14,406 & & & \\
\hline 1975 & 9,277 & 82 & 77.8 & 11,928 & 347.4 & 5,300 & 14,455 & & & \\
\hline 1976 & 11,533 & 90 & 87.4 & 13,198 & 106.9 & 5,069 & 13,692 & & & \\
\hline 1977 & 13,136 & 91 & 90.6 & 14,494 & 399.0 & 5,123 & 13,871 & & & \\
\hline 1978 & 13,411 & 92 & 91.0 & 14,731 & 423.1 & 5,198 & 14,119 & & & \\
\hline 1979 & 11,975 & 70 & 78.8 & 15,200 & 483.3 & 5,321 & 14,523 & & & \\
\hline 1980 & 9,083 & 59 & 62.0 & 14,647 & 689.3 & 5,646 & $15,5 \%$ & & & \\
\hline 1981 & 9,070 & 64 & 63.1 & 14,380 & 198.1 & 5,523 & 15,189 & & & \\
\hline 1982 & 8,766 & 55 & 57.5 & 15,234 & 323.1 & 5,510 & 15,147 & & & \\
\hline 1983 & 11,427 & $\pi$ & 69.2 & 16,522 & 159.4 & 5,320 & 14,520 & & & \\
\hline 1984 & 13,627 & 86 & $B 4.2$ & 16,181 & 497.7 & 5,450 & 14,949 & & & \\
\hline 1985 & 13,742 & 78 & 81.2 & 16,916 & 621.9 & 5,693 & 15,752 & & & \\
\hline 1986 & 13,913 & 81 & 79.6 & 17,477 & 973.9 & 6,270 & 17,653 & & & \\
\hline$\cdots$ & $=--$ & -- & $-\cdots$ & $\cdots \cdot$ & $-\cdot-$ & $\cdots$ & $\cdots-$ & $-\cdots$ & $\cdots-$ & $\cdots$ \\
\hline 1987 & 14,201 & 82 & 80.8 & 17,568 & 512.2 & 6,375 & $17, \infty 90$ & & & \\
\hline 1988 & 14,583 & 77 & 79.3 & 18,383 & 719.7 & 6,680 & 19,007 & 25.671 & 27,671 & 28,188 \\
\hline 1989 & & & & & 752.5 & 6,999 & 20,058 & 27,090 & 29,201 & 29.747 \\
\hline 1990 & & & & & $\pi / 2$ & 7,318 & 21,110 & 28,510 & 30,731 & 31,305 \\
\hline 1991 & & & & & 819.0 & 7,662 & 22,243 & 30,042 & 32,382 & 32,987 \\
\hline 1992 & & & & & 794.0 & 7,958 & 23,221 & 31,362 & 33,805 & 34,437 \\
\hline
\end{tabular}

Capacity-Capital stock Regression: Code 0 RSQ 0.410 Coefficients: Const -3025.9 stock 3.3

Ave. Capacity/Stock $(74-86): 2.7$ Ave. Weekly Hours (High) 106.2 Shift Factor 1.48 Ave Weekly Hours (Low) 104.7 Shift Factor 1.50 Note: Output, Investment, and Capital Stock extrapolated for 1987 and 1988.

\section{Automotive stampings}

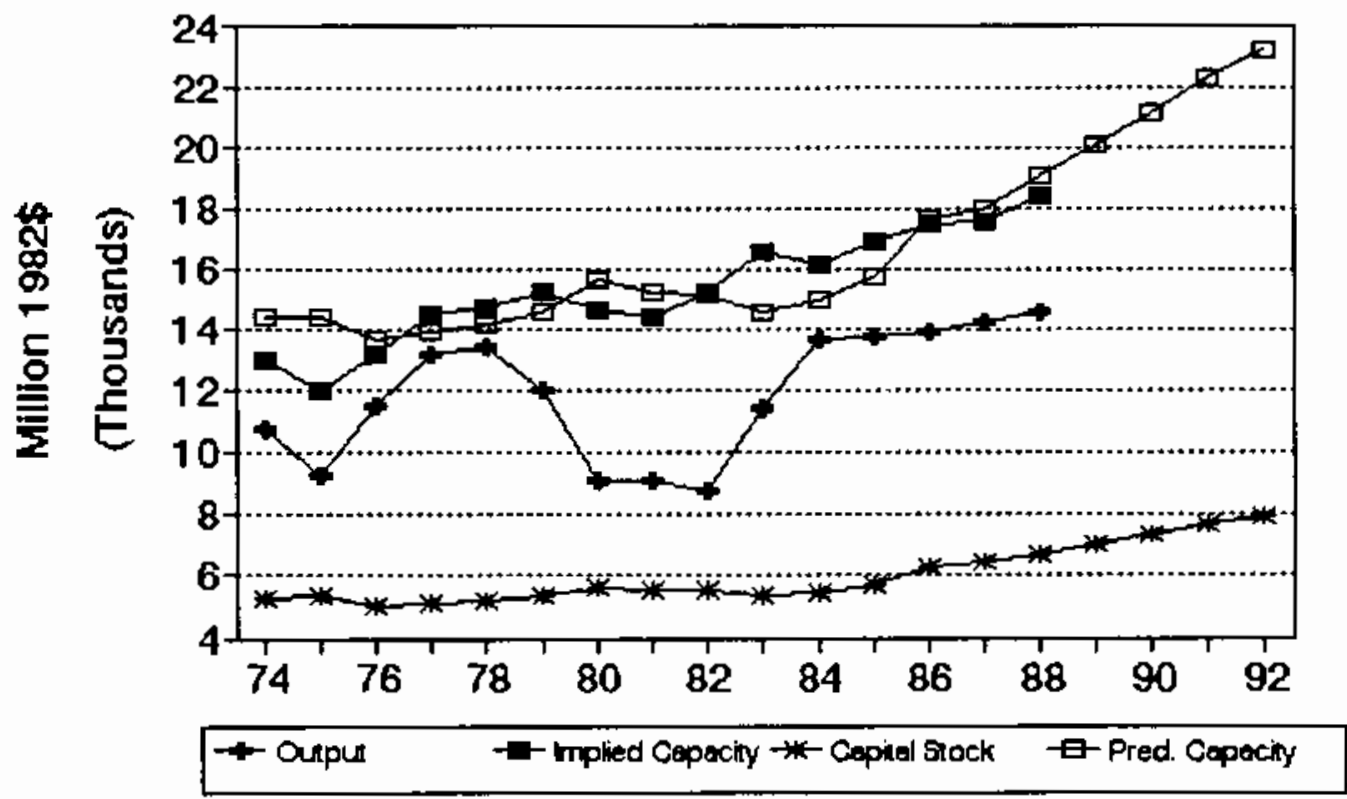




\section{NO. 301 SIC 3466 Crowng and closures}

MILLIONS OF 1982 OOLLARS (EXCL. CU RATES)

$\begin{array}{lccccccc} & 04 & \text { Amn. } & \text { Implied } & \text { Gross } & \text { Net } & \text { Pred. } & \ldots \ldots \ldots \\ \text { Gross } & \text { CU } & \text { CU } & \text { Prac. } & \text { In- } & \text { Cap. } & \text { Prac. } & \text { Emergency Capacity } \\ \text { Output Rate } & \text { Rete } & \text { Capac. } & \text { vest. } & \text { Stx. } & \text { Capec. } & 3 \text { mon. } 6 \text { mon. }>6 \text { mon. }\end{array}$

\begin{tabular}{|c|c|c|c|c|c|c|c|c|c|c|}
\hline 1974 & 837 & $\pi 7$ & 77.0 & 1,087 & 10.6 & 239 & 1,097 & & & \\
\hline 1975 & 790 & 80 & 78.9 & 1,001 & 27.1 & 245 & 1,110 & & & \\
\hline 1976 & $B 27$ & 70 & 73.8 & 1,122 & 11.5 & 236 & 1,089 & & & \\
\hline 1977 & $B 15$ & 66 & 67.5 & 1,207 & 35.4 & 248 & 1,116 & & & \\
\hline 1978 & $\pi 9$ & 62 & 63.5 & 1,227 & 21.6 & 247 & 1,113 & & & \\
\hline 1979 & $\sqrt{34}$ & 76 & 70.8 & 1,065 & 43.2 & 265 & 1,152 & & & \\
\hline 1980 & 755 & 83 & 80.4 & 939 & 2.3 & 245 & 1,108 & & & \\
\hline 1981 & 807 & 73 & 76.8 & 1,052 & 29.3 & 252 & 1,123 & & & \\
\hline 1982 & 823 & 70 & 71.1 & 1,157 & 15.9 & 246 & 1,110 & & & \\
\hline 1983 & 857 & 70 & 70.0 & 1,225 & 36.2 & 258 & 1,137 & & & \\
\hline 1984 & 756 & 87 & 80.6 & 937 & $(0.4)$ & 237 & 1,091 & & & \\
\hline 1985 & 816 & 68 & $\pi .1$ & 1,087 & 14.5 & 231 & 1,078 & & & \\
\hline 1988 & 881 & 63 & 64.9 & 1,327 & 32.8 & 243 & 1,105 & & & \\
\hline-- & -- & $-\cdot$ & - - - & 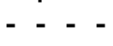 & $-\cdots$ & -- & $\therefore$ & -- & -- & $-\cdots$ \\
\hline 1987 & 86: & 57 & 59.3 & 1,453 & 21.9 & 244 & 1,107 & & & \\
\hline 1988 & 861 & 67 & 63.3 & 1,361 & 21.9 & 245 & 1,109 & 1,243 & 1,524 & 1,528 \\
\hline 1989 & & & & & 22.0 & 246 & 1,110 & 1,245 & 1,526 & 1,530 \\
\hline 1990 & & & & & 22.1 & 246 & 1,112 & 1,247 & 1,529 & 1,533 \\
\hline 1991 & & & & & 22.1 & 247 & 1,114 & 1,249 & 1,531 & 1,535 \\
\hline 1992 & & & & & 22.2 & 248 & 1,116 & 1,251 & 1.533 & 1,537 \\
\hline
\end{tabular}

Capacity-Capital Stock Regression: Code 0 RSQ 0.030

Coefficients: Const 588.7 stock 2.1 Ave. Capacity/stock (74-86): 4.5 Ave. Weekly Hours (High) 114.4 Shift factor 1.38 Ave Heekly Hours (LOW) 105.9 Shift Fector 1.49 Note: Dutput, Investment, and Capital stock extrapolated for 1987 and 1988.

\section{Crowns and closures}

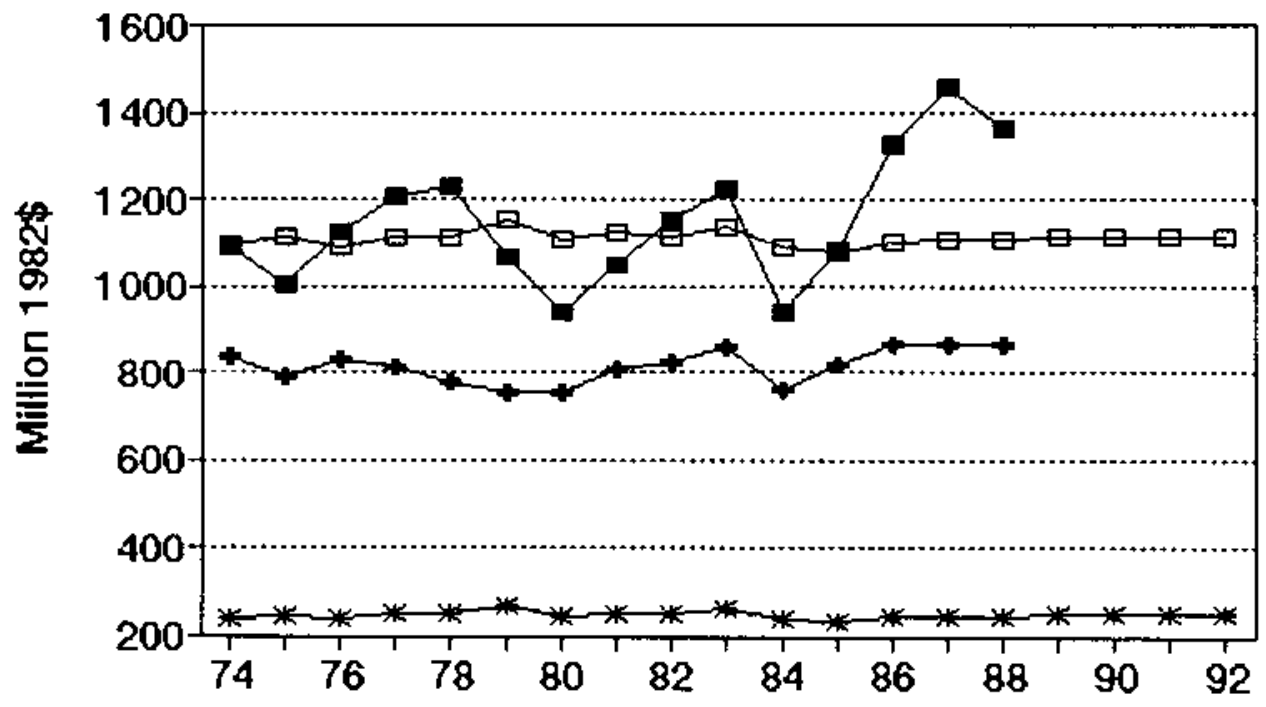

- Ouput $\rightarrow$ - Implied Cepacity - Capial Stock 다 Pred. Capacity 
No. 302 SIC 3469 Metal stampings, nec

MILLIOWS OF 1982 DOLLARS (EXCL. CU RATES)

$\begin{array}{lccccccc} & \text { Q4 } & \text { Ann. Implied Gross } & \text { Net } & \text { Pred. } & -\ldots & - \\ \text { Gro6s } & \text { CU } & \text { CU } & \text { Prac. } & \text { In. } & \text { Cap. } & \text { Prac. } & \text { Emergency Capacity } \\ \text { Dutput } & \text { Rate } & \text { Rate } & \text { Capac. } & \text { vest. } & \text { Stk. } & \text { Capac. } & 3 \text { mon. } 6 \text { mon. }>6 \text { mon. }\end{array}$

\begin{tabular}{|c|c|c|c|c|c|c|c|c|c|c|}
\hline 1974 & 6,976 & 83 & 85.1 & 8,201 & 82.2 & 1,730 & 8,921 & & & \\
\hline 1975 & 5,390 & 65 & 68.4 & 7,881 & 111.5 & 1,707 & 8,736 & & & \\
\hline 1976 & 6,489 & 66 & 65.9 & 9,840 & 185.5 & 1,746 & 9,046 & & & \\
\hline 1977 & 7,348 & 70 & 68.5 & 10,731 & 318.0 & 1,897 & 10,246 & & & \\
\hline 1978 & 7,601 & $\pi$ & 72.7 & 10,456 & 223.7 & 1,944 & 10,627 & & & \\
\hline 1979 & 8,219 & 67 & 70.4 & 11,669 & 228.1 & 1,990 & 10,989 & & & \\
\hline 1980 & 7,835 & 56 & 59.2 & 13,244 & 283.4 & 2,080 & 11,706 & & & \\
\hline 1981 & 7,880 & 56 & 56.8 & 13,886 & 279.4 & 2,156 & 12,309 & & & \\
\hline 1982 & 6,523 & 54 & 54.2 & 12,037 & 278.5 & 2,219 & 12,817 & & & \\
\hline 1983 & 6,795 & 69 & 62.8 & 10,821 & 147.7 & 2,156 & 12,312 & & & \\
\hline 1984 & 7,742 & 68 & 69.1 & 11,209 & 195.6 & 2,150 & 12,262 & & & \\
\hline 985 & 7,119 & 70 & 69.4 & 10,254 & 248.3 & 2,198 & 10,156 & & & \\
\hline 1986 & 6,712 & 63 & 65.4 & 10,259 & 224.7 & 2,223 & 10,357 & & & \\
\hline$-\cdot-$ & $--\cdot-$ & - & -- & $-\cdot-$ & $\because \cdot$ & $\cdots$ & $\cdots \cdot$ & --- & ---- & --- \\
\hline 1987 & 6,811 & $\pi$ & 71.1 & 9,580 & 220.7 & 2,243 & 10,516 & & & \\
\hline 1988 & 6,943 & 73 & 74.9 & 9,272 & 248.0 & 2,289 & 10,877 & 12,183 & 16,033 & 16,088 \\
\hline 1989 & & & & & 252.3 & 2,334 & 11,241 & 12,589 & 16,568 & 16,625 \\
\hline 1990 & & & & & 255.0 & 2,378 & 11,593 & 12,984 & 17,087 & 17,146 \\
\hline 1991 & & & & & 261.0 & 2,425 & 11,961 & 13,396 & 17,630 & 17,690 \\
\hline 1992 & & & & & 257.7 & 2,463 & 12,270 & 13,742 & 18,085 & 18,147 \\
\hline
\end{tabular}

Capacity-Capitat Stock Regression: Code -1 RSO 0.670

Coefficients: Const -4865.0 stock 8.0 D(85-86) -2492.1 Ave. Capacity/Stock (74-86): 5.4 Ave. Weekly Hours (High) 106.5 Shift Factor 1.48 Ave Heekly Hours (Low) 102.0 Shift factor 1.54 Note: Output, Investment, and Capital stock extrapolated for 1987 and 1988.

\section{Metal stampings, nec}

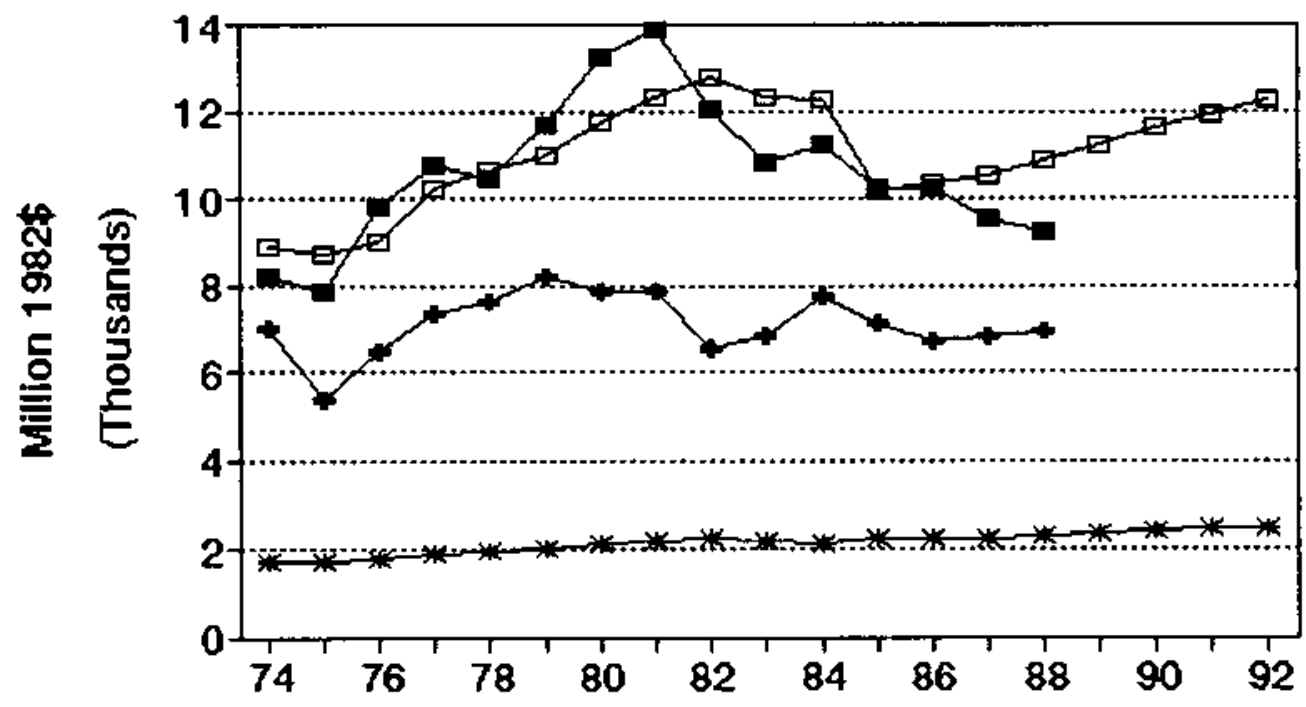

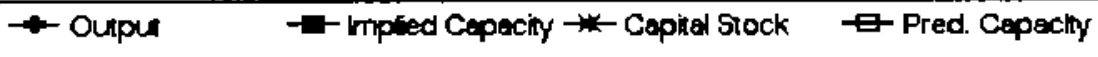




\section{No. 303 sIc 3471 Plating and polishing}

MILLIONS OF 1982 DOLLARS (EXCL. CU RATES)

$\begin{array}{lcccclcc} & \text { Q4 } & \text { Ann. Implied Gross } & \text { Net } & \text { Pred. } & \ldots \ldots \ldots \\ \text { Gross } & \text { CU } & \text { CU } & \text { Prac. } & \text { In- } & \text { Cap. } & \text { Prac. } & \text { Emergency Capacity } \\ \text { Output Rate } & \text { Rate } & \text { Capac. vest. } & \text { Stk. } & \text { Capac. } & 3 \text { mon. } 6 \text { mon. }>6 \text { mon. }\end{array}$

\begin{tabular}{|c|c|c|c|c|c|c|c|c|c|c|}
\hline 1974 & 2,447 & $7 B$ & 78.6 & 3,115 & (36.1) & 788 & 3,348 & & & \\
\hline 1975 & 2,253 & 82 & 79.6 & 2,832 & 91.4 & 817 & 3,559 & & & \\
\hline 1976 & 2,601 & $\infty$ & 72.2 & 3,605 & 92.4 & 843 & 3,746 & & & \\
\hline 1977 & 2,803 & 73 & 70.4 & 3,984 & 96.8 & 869 & 3,936 & & & \\
\hline 1978 & 3,104 & 7 & 74.1 & 4,188 & 108.2 & 905 & 4,196 & & & \\
\hline 1979 & 3,142 & 71 & 72.6 & 4,325 & 137.5 & 964 & 4,625 & & & \\
\hline 1980 & 3,064 & 60 & 63.8 & 4,801 & $(25.3)$ & 870 & 3,939 & & & \\
\hline 1981 & 2,773 & 53 & 55.8 & 4,968 & 114.4 & 907 & 4,213 & & & \\
\hline 1982 & 2,758 & 54 & 53.5 & $5,15 B$ & 181.0 & 999 & 4,876 & & & \\
\hline 1983 & 2,813 & 75 & 66.9 & 4,204 & 135.7 & 1,035 & 5,137 & & & \\
\hline 1984 & 3,254 & 63 & 67.7 & 4,806 & 80.0 & 1,022 & 5,046 & & & \\
\hline 1985 & 3,239 & 59 & 60.6 & 5,350 & 80.7 & 1,012 & 4,973 & & & \\
\hline 1986 & 3,136 & 59 & 59.0 & 5,319 & 103.6 & 1,024 & 5,061 & & & \\
\hline-- & $--\cdot$ & -- & $-\cdot$ & $\cdots$ & $\cdots$ & $\cdots$ & $-\cdots$ & -- & $\cdots$ & $\cdots-$ \\
\hline 1987 & 3,199 & 64 & 62.0 & 5,163 & 114.5 & 1,047 & 5,226 & & & \\
\hline 1988 & 3,283 & 61 & 62.2 & 5,277 & 117.4 & 1,071 & 5,398 & 5,636 & 7,365 & 7,390 \\
\hline 1989 & & & & & 120.3 & 1,095 & 5,575 & 5,821 & 7,607 & 7,632 \\
\hline 1990 & & & & & 123.1 & 1,121 & 5,757 & 6,012 & 7,856 & 7,882 \\
\hline 1991 & & & & & 126.0 & 1,146 & 5,945 & 6,207 & 8,111 & 8,138 \\
\hline 1992 & & & & & 128.9 & 1,173 & 6,136 & 6,407 & 8,372 & 8,400 \\
\hline
\end{tabular}

Capacity-Capital Stock Regression: Code 0 RSO $0.5 \%$

Coefficients: Const -2366.3 stock 7.3

Ave. Capacity/Stock $(74-86): 4.7$ Ave. Weekly Hours (High) 115.3 Shift Factor 1.37 Ave Weekly Hours (Low) 113.4 Shift Factor 1.39 Note: Output, Investment, and Cepital stock extrapolated for 1987 and 1988.

\section{Plating and polishing}

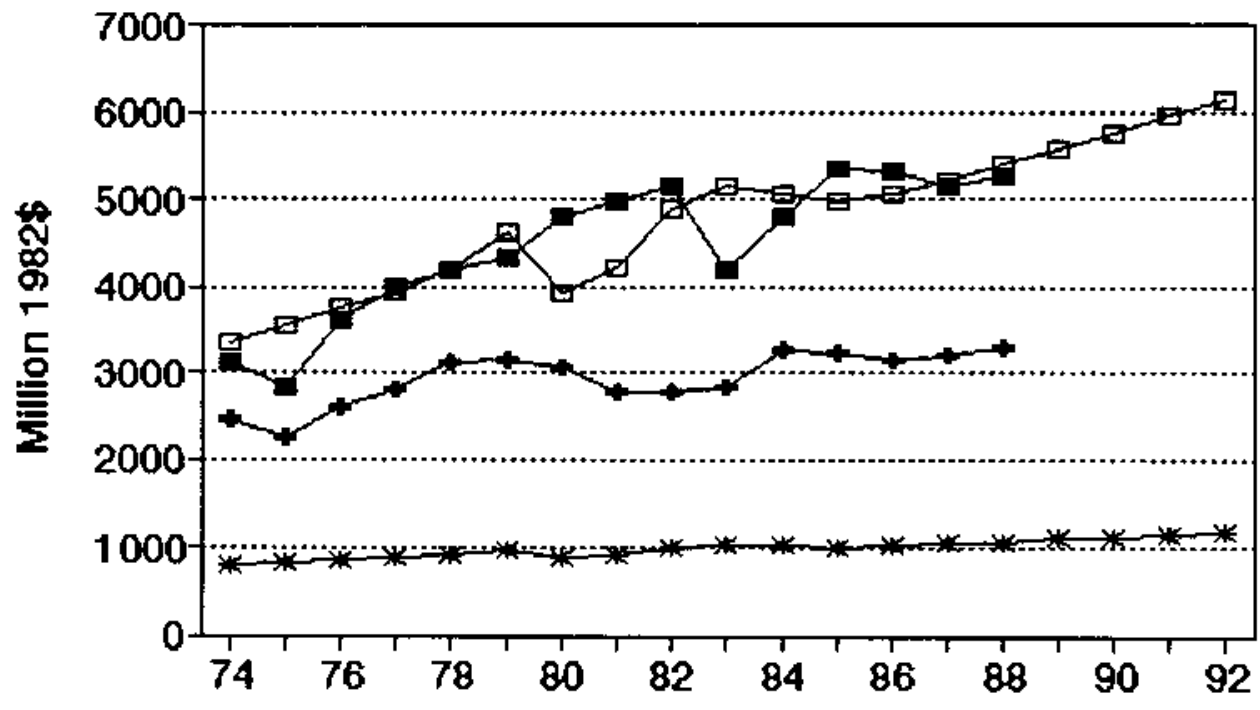

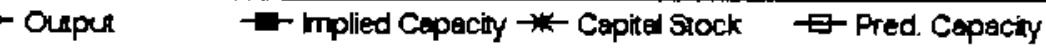




\section{No. 304 sIC 3479 ketal coating and allied services}

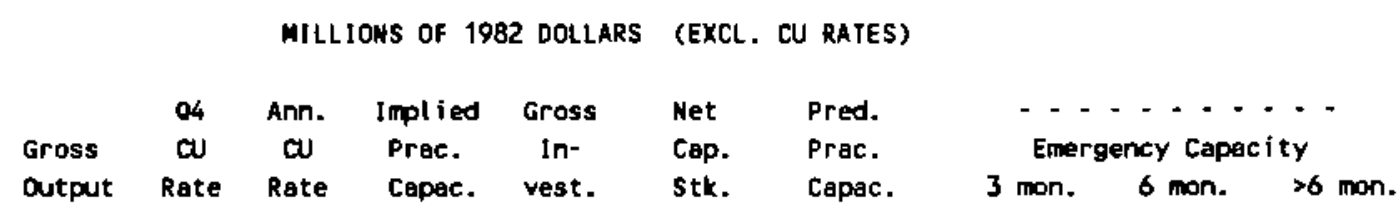

\begin{tabular}{|c|c|c|c|c|c|c|c|c|c|c|}
\hline 1974 & 1,664 & 65 & 67.4 & 2,469 & 101.0 & 544 & 2,067 & & & \\
\hline 1975 & 1,722 & 72 & 65.1 & 2,644 & 57.9 & 566 & 2,315 & & & \\
\hline 1976 & 1,919 & 69 & 70.8 & 2,710 & 61.9 & 589 & 2,563 & & & \\
\hline 1977 & 2,117 & 62 & 64.7 & 3,270 & 142.6 & 684 & 3,605 & & & \\
\hline 1978 & 2,366 & 71 & 67.0 & 3,530 & 40.6 & 678 & 3,536 & & & \\
\hline 1979 & 2,641 & 86 & 81.1 & 3,256 & 149.9 & 774 & 4,592 & & & \\
\hline 1980 & 2,624 & 51 & 62.3 & 4,210 & 85.9 & 800 & $4,8 \pi 5$ & & & \\
\hline 1981 & 2,741 & 38 & 43.7 & 6,276 & 150.5 & 888 & 5,840 & & & \\
\hline 1982 & 2,433 & 37 & 36.9 & 6,594 & 93.0 & 913 & 6,116 & & & \\
\hline 1983 & 2,277 & 33 & 34.4 & 6,628 & 74.6 & 918 & 6,176 & & & \\
\hline 1984 & 2,798 & 54 & 46.5 & 6,023 & 14.6 & 868 & 5,625 & & & \\
\hline 1985 & 2,938 & 43 & 47.3 & 6,208 & 176.6 & 973 & 6,778 & & & \\
\hline 1986 & 3,106 & 39 & 40.3 & 7.703 & 135.8 & 1,033 & 7,436 & & & \\
\hline $\begin{array}{l}\cdots \\
1987\end{array}$ & 3,218 & 33 & $\begin{array}{l}\cdots \\
36.8\end{array}$ & $\begin{array}{l}\cdots \\
9.249\end{array}$ & $\begin{array}{l}-- \\
103.3\end{array}$ & $\begin{array}{l}\cdots \\
1,061\end{array}$ & 7,738 & -- & $-\cdot \cdot$ & $\cdot \cdot \cdot$ \\
\hline 1988 & 3,370 & 34 & 33.9 & 9,955 & 132.2 & 1,115 & 8,334 & 7,633 & 10,765 & 10,809 \\
\hline 1989 & & & & & 136.7 & 1,170 & 8,936 & 8,184 & 11,543 & 11,591 \\
\hline 1990 & & & & & 139.6 & 1,224 & 9,527 & 8,725 & 12,305 & 12,356 \\
\hline 1991 & & & & & 146.0 & 1,280 & 10,143 & 9,289 & 13,101 & 13,155 \\
\hline 1992 & & & & & 142.5 & 1,329 & 10,676 & 9,778 & 13,790 & 13,847 \\
\hline
\end{tabular}

Capacity-Capital Stock Regression: Code 0 Rso 0.910 Coefficients: Const $\mathbf{- 3 8 9 3 . 9}$ stock 11.0

Ave. Capacity/Stock $(74-86): 6.0$ Ave. Weekly Hours (High) 121.9 Shift Factor 1.30 Ave Heekly Hours (LOH) 111.1 Shift Factor 1.42 Mote: Output, Imvestment, and Capital stock extrapolated for 1987 and 1988.

\section{Metal coating and allied services}

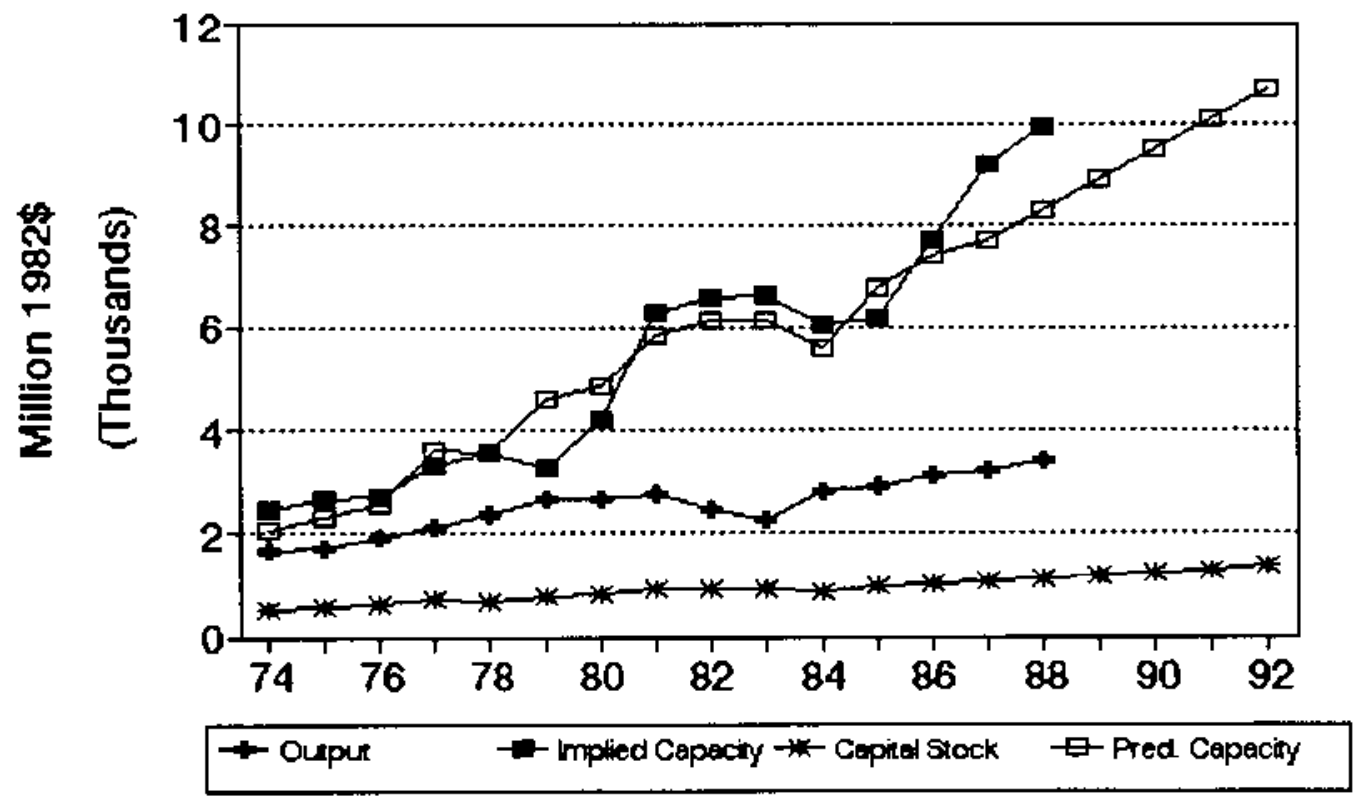




\section{No. 305 sIC 3482 gnall arms ammunition}

MILLIONS OF 1982 DOLLARS (EXCL. CU RATES)

$\begin{array}{lccccccc} & 04 & \text { Am. Implied Gross } & \text { Net } & \text { Pred. } & \ldots \ldots \ldots \\ \text { Gross } & \text { CU } & \text { dU } & \text { Prac. } & \text { In- } & \text { Cap. } & \text { Prac. } & \text { Emergency Capacity } \\ \text { Output Rate } & \text { Rate } & \text { Capac. } & \text { vest. } & \text { Stk. } & \text { Capac. } & 3 \text { mon. } 6 \text { mon. }>6 \text { mon. }\end{array}$

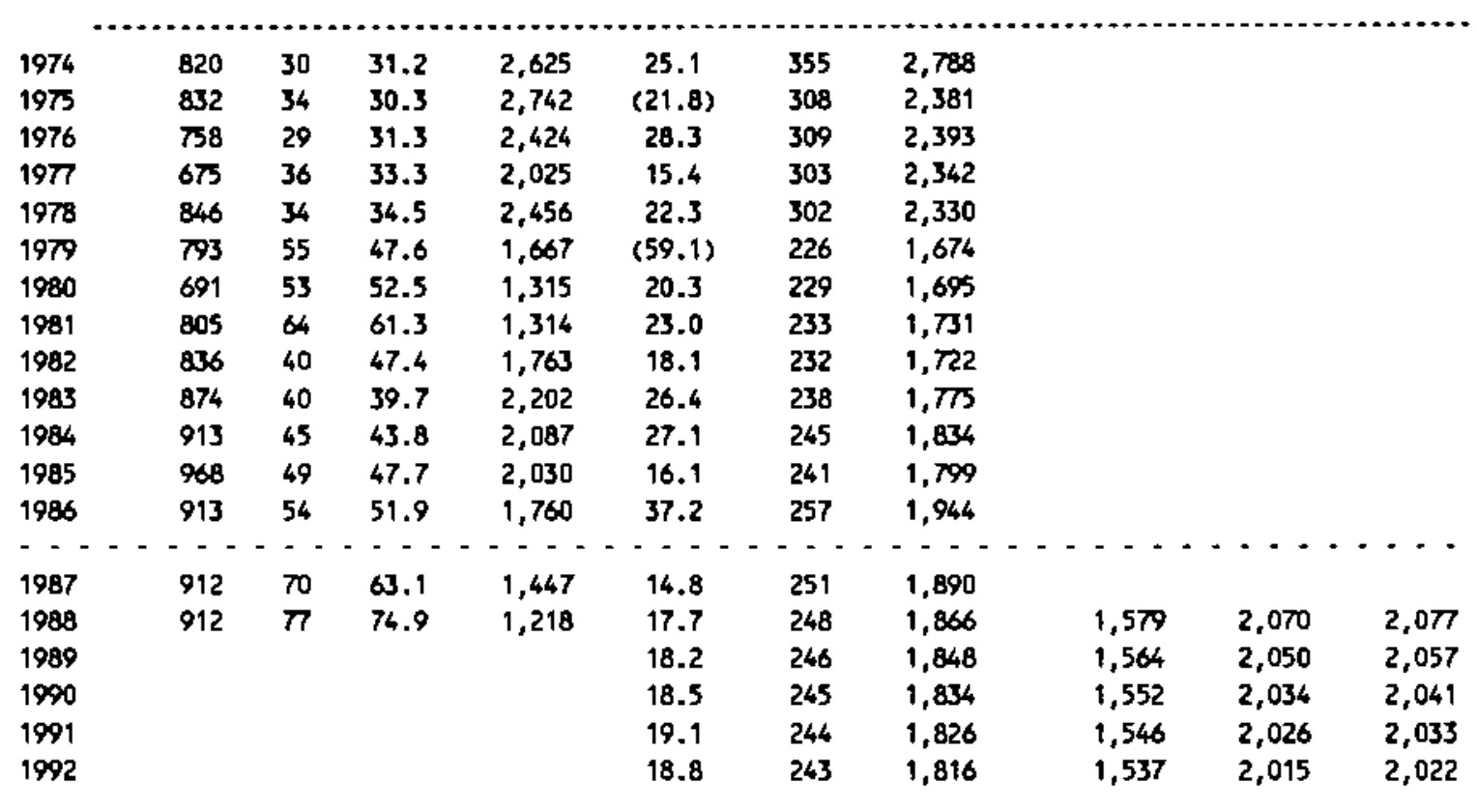

Capacity-Capital Stock Regression: Code 1 RSO 0.630

Coefficients: Const -280.9 Stock 8.6

Ave, Capacity/Stock $(74-86) ; 7.6$ Ave. Heekly Hours (High) 143.0 Shift Factor 1.11 Ave Weekly Hours (Low) 134.5 Shift factor 1.18 Note: Output, Investment, and Capital stock extrapolated for 1987 and 1988.

\section{Small arms ammunition}
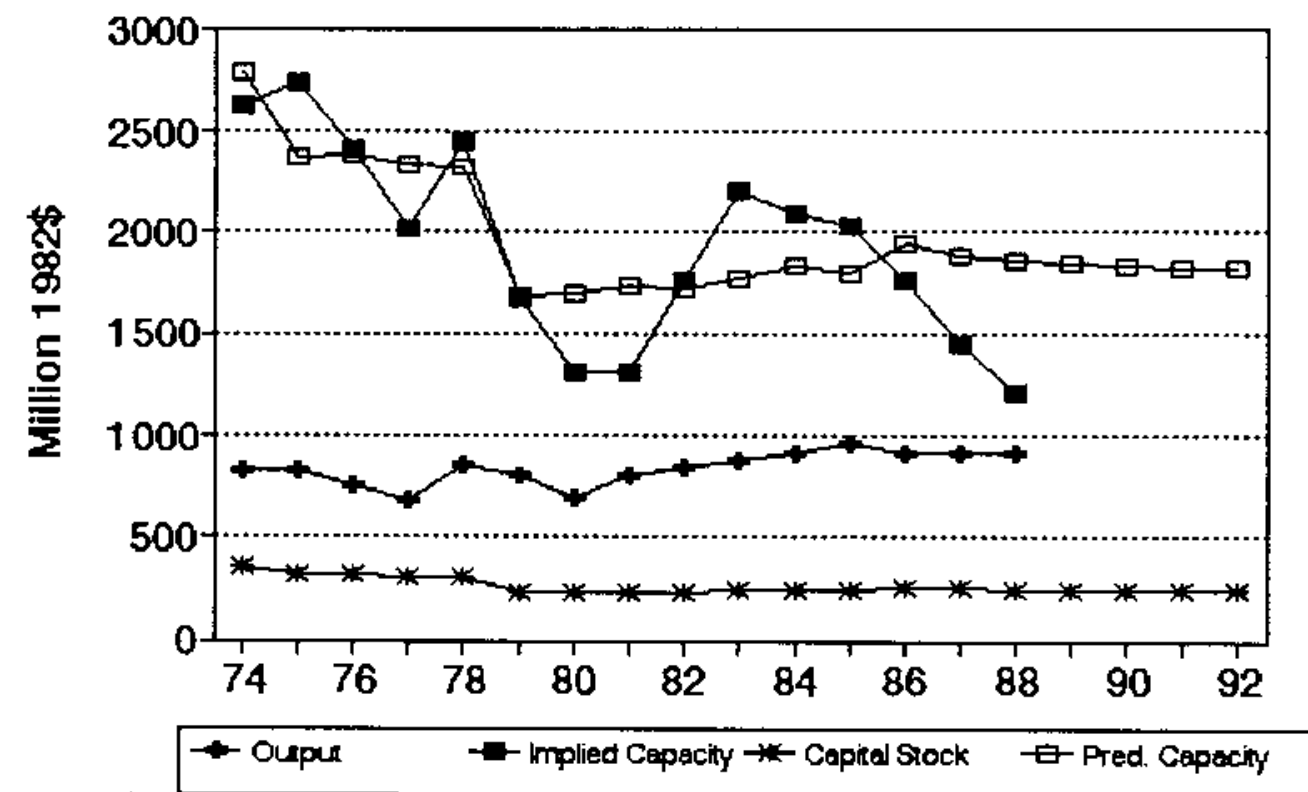
No. 306 sIC 3483 Amunition, except for small arms, nec MILLIONS OF 1982 DOLLARS (EXCL. CU RATES)

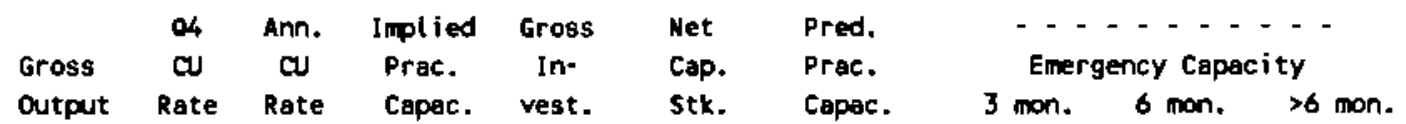

\begin{tabular}{|c|c|c|c|c|c|c|c|c|c|c|}
\hline 1974 & 2,422 & 47 & 51.2 & 4,730 & $(30.4)$ & 341 & 7,004 & & & \\
\hline 1875 & 2,931 & 27 & 27.7 & 10,573 & $(106.5)$ & 222 & 5,991 & & & \\
\hline 1976 & 2,509 & 33 & 31.4 & 7,984 & $(19.3)$ & 191 & 5,709 & & & \\
\hline 1977 & 1,125 & 35 & 34.3 & 3,277 & $(27.0)$ & 155 & 5,384 & & & \\
\hline 1978 & 1,260 & 42 & 38.7 & 3,259 & 16.5 & 161 & 5,441 & & & \\
\hline 1979 & 1,483 & 48 & 46.6 & 3,180 & 23.4 & 172 & 5,536 & & & \\
\hline 1980 & 1,781 & 29 & 34.0 & 5,235 & 9.8 & 168 & 5,503 & & & \\
\hline 1981 & 1,470 & 27 & 29.1 & 5,050 & 44.9 & 196 & 5,760 & & & \\
\hline 1982 & 1,760 & 22 & 23.7 & 7,430 & 65.2 & 241 & 6,170 & & & \\
\hline 1983 & 2,087 & 21 & 21.7 & 9,634 & 25.7 & 246 & 6,213 & & & \\
\hline 1984 & 2,347 & 46 & 37.1 & 6,333 & 81.1 & 304 & 6,740 & & & \\
\hline 1985 & 2,866 & 50 & 48.9 & 5,860 & 72.0 & 350 & 7,158 & & & \\
\hline 1986 & 3,831 & 52 & 50.7 & 7,552 & 54.0 & $3 \pi$ & 7,409 & & & \\
\hline$\cdot \cdot$ & $\cdots \cdot$ & $\because$ & $\cdots$ & $\cdots$ & $\cdots \cdot$ & $\cdots$ & $\cdots \cdot$ & $=\cdot \cdot \cdot$ & $=\cdot$ & $-\cdot-$ \\
\hline 1987 & 3,777 & 55 & 52.3 & 7,223 & 17.9 & 368 & 7,323 & & & \\
\hline 1988 & 3,709 & 58 & 57.8 & 6,421 & 23.4 & 364 & 7,292 & 8,938 & 12,780 & 12,834 \\
\hline 1989 & & & & & 24.2 & 362 & 7,272 & 8,913 & 12,744 & 12,799 \\
\hline 1990 & & & & & 24.8 & 361 & 7,258 & 8,896 & 12,720 & 12,775 \\
\hline 1991 & & & & & 26.0 & 360 & 7,257 & 8,894 & 12,718 & $12, \pi 72$ \\
\hline 1992 & & & & & 25.3 & 360 & 7,269 & 8,885 & 12,704 & 12,759 \\
\hline
\end{tabular}

Capacity-Capital Stock Regression: Code 0 RSa 0.090 Coefficients: Const 3967.1 stock 9.1

Ave. Capacity/Stack $(74-86): 25.6$ Ave. Weekly Hours (High) 89.5 Shift Factor 1.76 Ave Heekly Hours (LOW) 81.9 Shift factor 1.92 Note: Output, Investment, and Capital stock extrapolated for 1987 and 1988.

\section{Ammunition, except for small arms,}
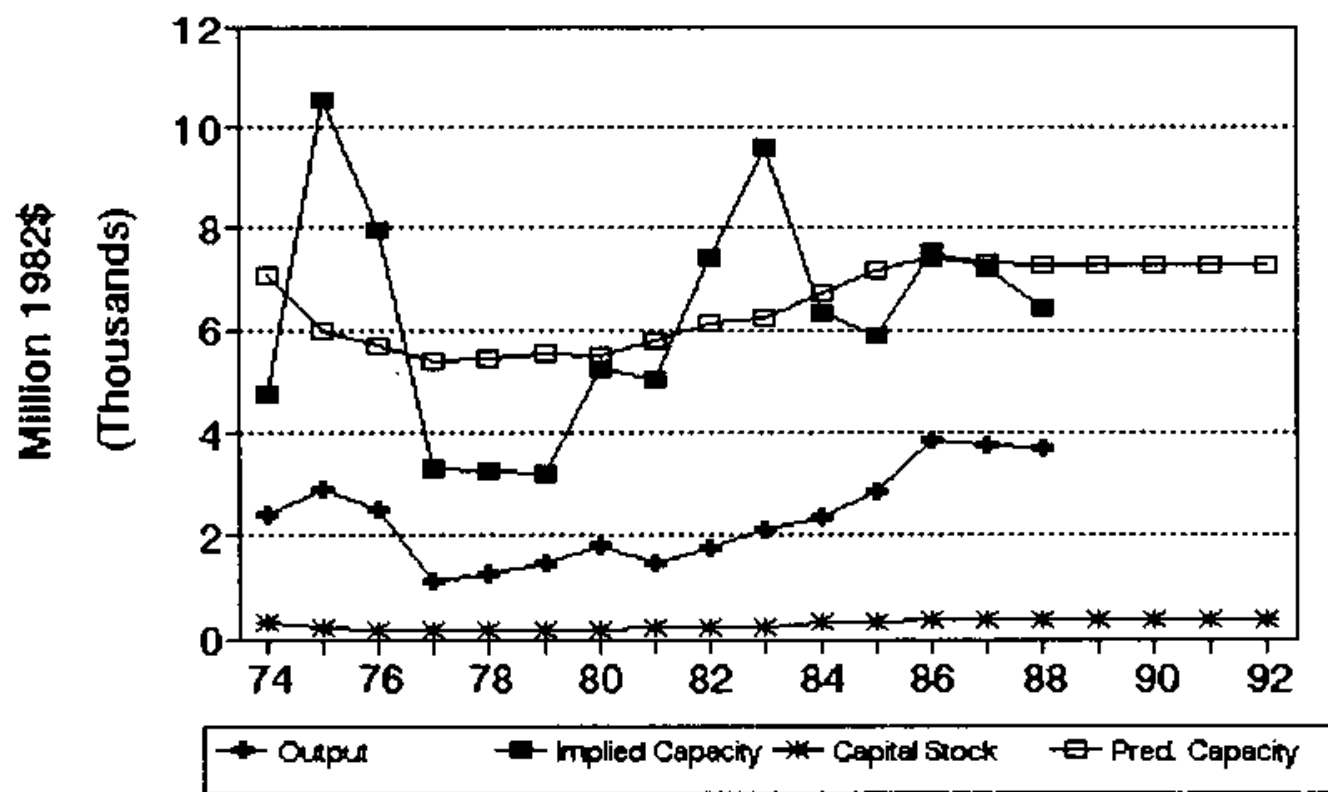
No. 307 SIC 3484 small armB

MILLIONS OF 1982 DOLLARS (EXCL. CU RATES)

$\begin{array}{lccccccc} & \text { Q4 } & \text { Ann. } & \text { Implied Gross } & \text { Net } & \text { Pred. } & \ldots \ldots \ldots \\ \text { Gross } & \text { CU } & \text { CU } & \text { Prac. } & \text { In- } & \text { Cap. } & \text { Prac. } & \text { Emergency Capacity } \\ \text { Output } & \text { Rate } & \text { Rate } & \text { Capoc. } & \text { vest. } & \text { Stk. } & \text { Capac. } & 3 \text { mon. } 6 \text { mon. }>6 \text { mon. }\end{array}$

\begin{tabular}{|c|c|c|c|c|c|c|c|c|c|c|}
\hline 1974 & 1,014 & 79 & $B 0.7$ & 1,257 & 8.4 & 236 & 1,533 & & & \\
\hline 1975 & 1,152 & 74 & 73.1 & 1,576 & 45.5 & 260 & 1,667 & & & \\
\hline 1976 & 1,338 & 66 & 69.4 & 1,929 & 30.8 & 269 & 1,715 & & & \\
\hline 1977 & 1,162 & 57 & 60.4 & 1,922 & 26.2 & 272 & 1,730 & & & \\
\hline 1978 & 1,274 & 57 & 56.7 & 2,246 & 43.1 & 291 & 1,834 & & & \\
\hline 1979 & 1,290 & $\pi$ & 68.6 & 1,879 & 27.6 & 295 & 1,854 & & & \\
\hline 1980 & 1,326 & 70 & 71.0 & 1,869 & 48.3 & 318 & 1,978 & & & \\
\hline 1981 & 1,286 & 64 & 67.0 & 1,920 & 0.5 & 295 & 1.853 & & & \\
\hline 1982 & 1,108 & 47 & 52.6 & 2,106 & 39.6 & 309 & 1,931 & & & \\
\hline 1983 & 839 & 48 & 47.4 & 1,771 & 39.1 & 321 & 1,995 & & & \\
\hline 1984 & 1,027 & $\Delta 4$ & 58.3 & 1,761 & 1.5 & 296 & 1,859 & & & \\
\hline 1985 & 976 & 56 & 59.1 & 1,651 & 33.1 & 301 & 1,887 & & & \\
\hline 1986 & 1,084 & 59 & 57.7 & 1,878 & 37.0 & 309 & 1,929 & & & \\
\hline-- & $-\cdots$ & 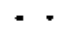 & $\cdots-$ & $---\cdot$ & $* \cdot$ & $\cdots-$ & --- & $=$ & -- & $-\cdot$ \\
\hline 1987 & 1,093 & 65 & 62.3 & 1,755 & 27.8 & 308 & 1,923 & & & \\
\hline 1988 & 1,105 & 73 & 70.2 & 1,573 & 27.5 & 306 & 1,916 & 2,000 & 2,658 & 2,667 \\
\hline 1989 & & & & & 27.3 & 305 & 1,908 & 1,991 & 2,647 & 2,656 \\
\hline 1990 & & & & & 27.0 & 303 & 1,899 & 1,982 & 2,634 & 2,644 \\
\hline 1991 & & & & & 26.7 & 302 & 1,890 & 1,972 & 2,621 & 2,630 \\
\hline 1992 & & & & & 26.4 & 300 & 1,879 & 1,962 & 2,607 & 2,616 \\
\hline
\end{tabular}

Capacity-Capital stock Regression: Code O RSO 0.300

Coefficients: Const 256.3 stock 5.4

Aye. Capacity/Stock $(74-86): 6.3$ Ave. Weekly Hours (High) 113.3 Shift factor 1.39 Ave Heekly Hours (Low) 110.6 Shift factor 1.42 Note: Output, Investment, and Capital Stock extrapolated for 1987 and 1988.

\section{Small arms}

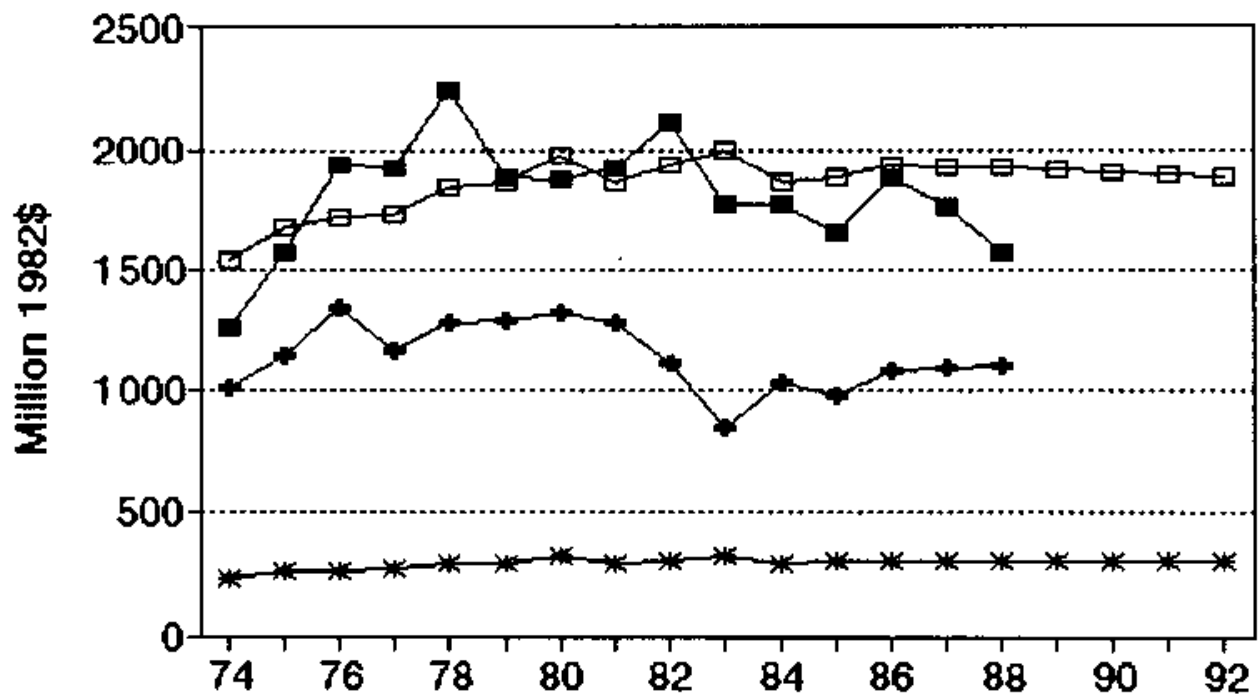

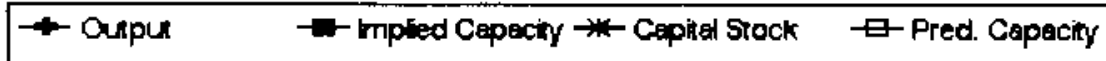


No. 308 sIC 3489 Ordnance and accessories, nec

MILLIONS OF 1982 DOLLARS (EXCL. CU RATES)

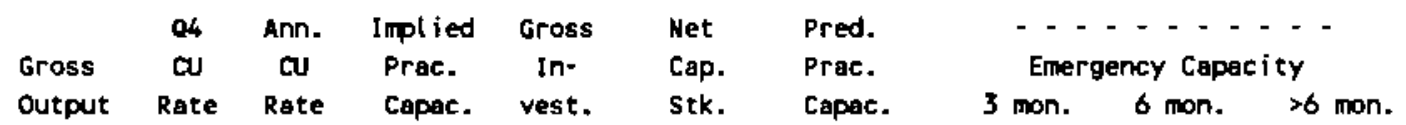

\begin{tabular}{|c|c|c|c|c|c|c|c|c|c|c|}
\hline 1974 & 1,096 & 35 & 35.0 & 3,132 & 31.1 & 173 & 2,656 & & & \\
\hline 1975 & 982 & 48 & 43.1 & 2,276 & 43.2 & 192 & 2,732 & & & \\
\hline 1976 & 1,030 & 36 & 40.5 & 2,543 & (78.2) & 101 & 3,291 & & & \\
\hline 1977 & 1,405 & 32 & 33.5 & 4,193 & 12.0 & 100 & 3,284 & & & \\
\hline 1978 & 1,427 & 42 & 38.3 & 3,730 & 20.5 & 106 & 3,318 & & & \\
\hline 1979 & 1,435 & 50 & 47.0 & 3,053 & 37.1 & 126 & 3,420 & & & \\
\hline 1980 & 1,618 & 42 & 45.0 & 3,595 & 23.7 & 132 & 3,449 & & & \\
\hline 1981 & 1,499 & 56 & 50.8 & 2,954 & 25.4 & 139 & 3,484 & & & \\
\hline 1982 & 1,402 & 51 & 52.9 & 2,651 & 6.1 & 129 & 3,434 & & & \\
\hline 1983 & 1,578 & 54 & 52.9 & 2,984 & $(21.6)$ & 96 & 3,264 & & & \\
\hline 1984 & 2,025 & 56 & 55.3 & 3,664 & 35.1 & 118 & 3,379 & & & \\
\hline 1985 & 2,310 & $55 *$ & 55.2 & 4,183 & 42.3 & 146 & 3,524 & & & \\
\hline 1986 & 2,143 & 55 & 54.9 & 3,902 & 29.7 & 162 & 3,604 & & & \\
\hline-- & $-\cdot-$ & -- & -- & 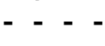 & $--\cdot$ & -- & $-\cdots$ & -- & -- & $-\cdot-$ \\
\hline 1987 & 2,188 & $57 *$ & 56.4 & 3,879 & 22.3 & 169 & 3,642 & & & \\
\hline 1988 & 2,247 & 55 & 55.8 & 4,025 & 48.4 & 202 & 3,811 & $3, \infty \infty$ & 5,286 & 5,305 \\
\hline 1989 & & & & & 52.5 & 236 & 3,986 & 4,183 & 5,529 & 5,548 \\
\hline 1990 & & & & & 55.2 & 269 & 4,158 & 4,363 & 5,768 & 5,788 \\
\hline 1991 & & & & & 60.9 & 305 & 4,344 & 4,559 & 6,026 & 6,047 \\
\hline 1992 & & & & & 57.8 & 335 & 4,497 & 4,719 & 6,238 & 6,259 \\
\hline
\end{tabular}

Capacity-Capital Stock Regression: Code - 1 RSO 0.200

Coefficients: Const 1763.4 stock $5.2 \quad$ D(76-86) 1007.5

Ave. Cepecity/Stock $(74-86): 24.9$ Ave. Weekly Hours (High) 113.3 Shift Factor 1.39 Ave Weekly Hours (Low) 110.6 Shift Factor 1.42 * Imputed Mote: Output, Imestment, and Capital Stock extrapolated for 1987 and 1988.

\section{Ordnance and accessories, nec}

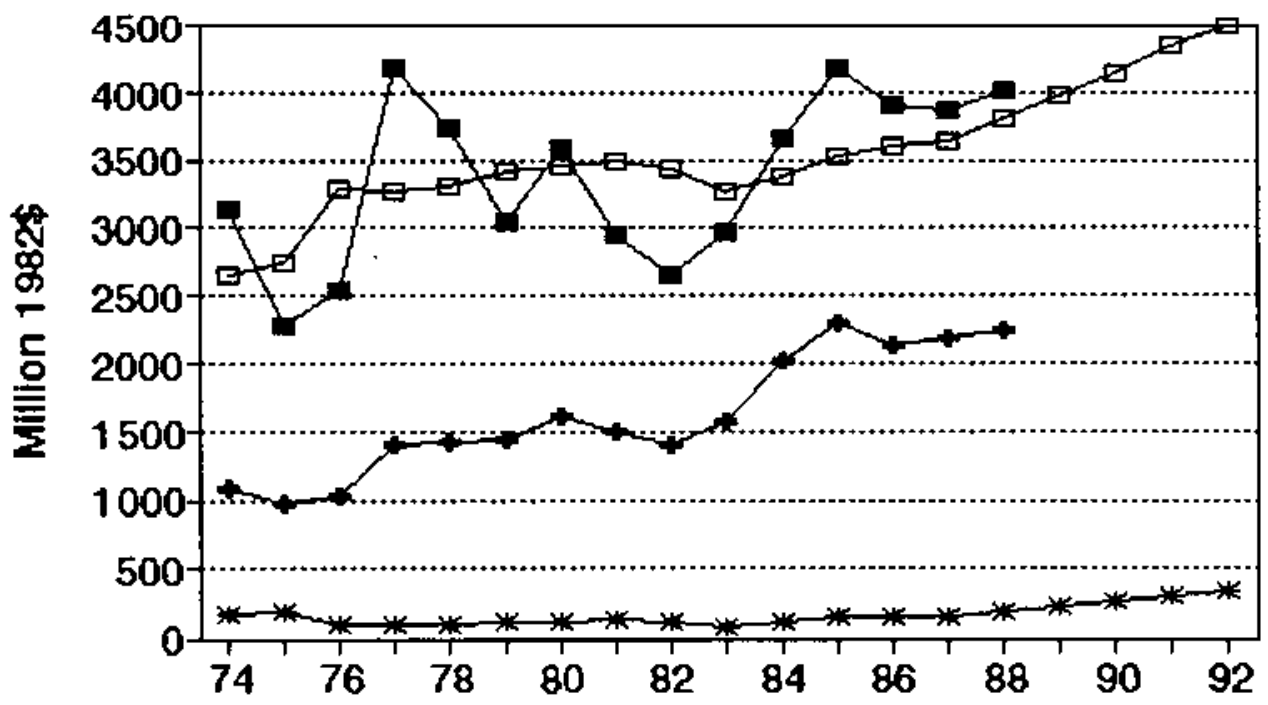

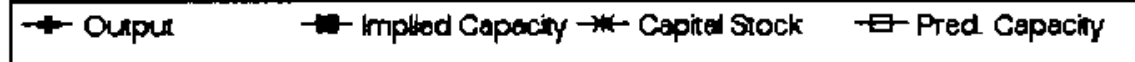




\section{No. 309 sIC 3493 steel springs, except wire}

\section{MILLIONS OF 1982 DOLLARS (EXCL. CU RATES)}

$\begin{array}{lccccccc} & \text { Q } & \text { Am. Implied Gross } & \text { Net } & \text { Pred. } & -\ldots \\ \text { Gross } & \text { a } & \text { CU } & \text { Prac. } & \text { In- } & \text { Cap. } & \text { Prac. } & \text { Emergency Capacity } \\ \text { Output } & \text { Rate } & \text { Rate } & \text { Capac. } & \text { vest. } & \text { Stk. } & \text { Capac. } & 3 \text { mon. } 6 \text { mon. }>6 \text { mon. }\end{array}$

\begin{tabular}{|c|c|c|c|c|c|c|c|c|c|c|}
\hline 1974 & 823 & 41 & 42.3 & 1,948 & 14.2 & 181 & 1,594 & & & \\
\hline 1975 & 593 & 53 & 46.2 & 1,282 & 44.5 & 213 & 1,635 & & & \\
\hline 1976 & 663 & 71 & 64.4 & 1,030 & 17.1 & 217 & 834 & & & \\
\hline 1977 & 659 & 74 & 7.9 & 904 & 2.3 & 207 & 822 & & & \\
\hline 1978 & 671 & 72 & 72.3 & 929 & 14.3 & 210 & 825 & & & \\
\hline 1979 & 660 & 67 & 69.4 & 951 & 7.9 & 205 & 819 & & & \\
\hline 1980 & 487 & 62 & 62.7 & 776 & to. 2 & 203 & 816 & & & \\
\hline 1981 & 484 & 62 & 63.0 & 768 & 19.2 & 208 & 823 & & & \\
\hline 1982 & 414 & 55 & 56.8 & 729 & 7.6 & 201 & 814 & & & \\
\hline 1983 & 423 & 62 & 58.9 & 719 & 7.2 & 194 & 804 & & & \\
\hline 1984 & 439 & 63 & 63.4 & 692 & 4.9 & 183 & 791 & & & \\
\hline 1985 & 435 & 64 & 63.8 & 682 & 4.7 & 174 & 778 & & & \\
\hline 1986 & 409 & 53 & 56.9 & 719 & 10.6 & 170 & $\pi 73$ & & & \\
\hline-- & $-\cdots$ & $\because$ & $\cdots$ & $-\cdots$ & $\cdots$ & $\because$ & $\cdots$ & -- & $-\cdots$ & $-\cdots$ \\
\hline 1987 & 407 & 64 & 59.2 & 688 & 13.9 & 169 & 772 & & & \\
\hline 1988 & 404 & 67 & 6.2 & 610 & 16.2 & 171 & $\pi / 5$ & 892 & 1,250 & 1,255 \\
\hline 1989 & & & & & 16.5 & 173 & $\pi 7$ & 895 & 1,254 & 1,259 \\
\hline 1990 & & & & & 16.8 & 175 & 780 & 898 & 1,259 & 1.264 \\
\hline 1991 & & & & & 17.3 & 178 & 783 & 902 & 1,264 & 1,269 \\
\hline 1992 & & & & & 17.0 & 180 & 786 & 905 & 1,268 & 1,273 \\
\hline
\end{tabular}

Capacity-Capital Stock Regression: Code -1 RSO 0.750

Coefficients: Const 1357.4 stock $1.3 \quad 0(76-86) \quad-806.4$

Ave. Capacity/Stock $(74-86): 4.7$ Ave. Weekly Hours (High) 97.2 Shift Factor 1.62 Ave Weekly Hours (Low) 90.7 Shift Factor 1.74 Hote: Qutput, Investment, and Capital Stock extrapolated for 1987 and 1988.

\section{Steel springs, except wire}

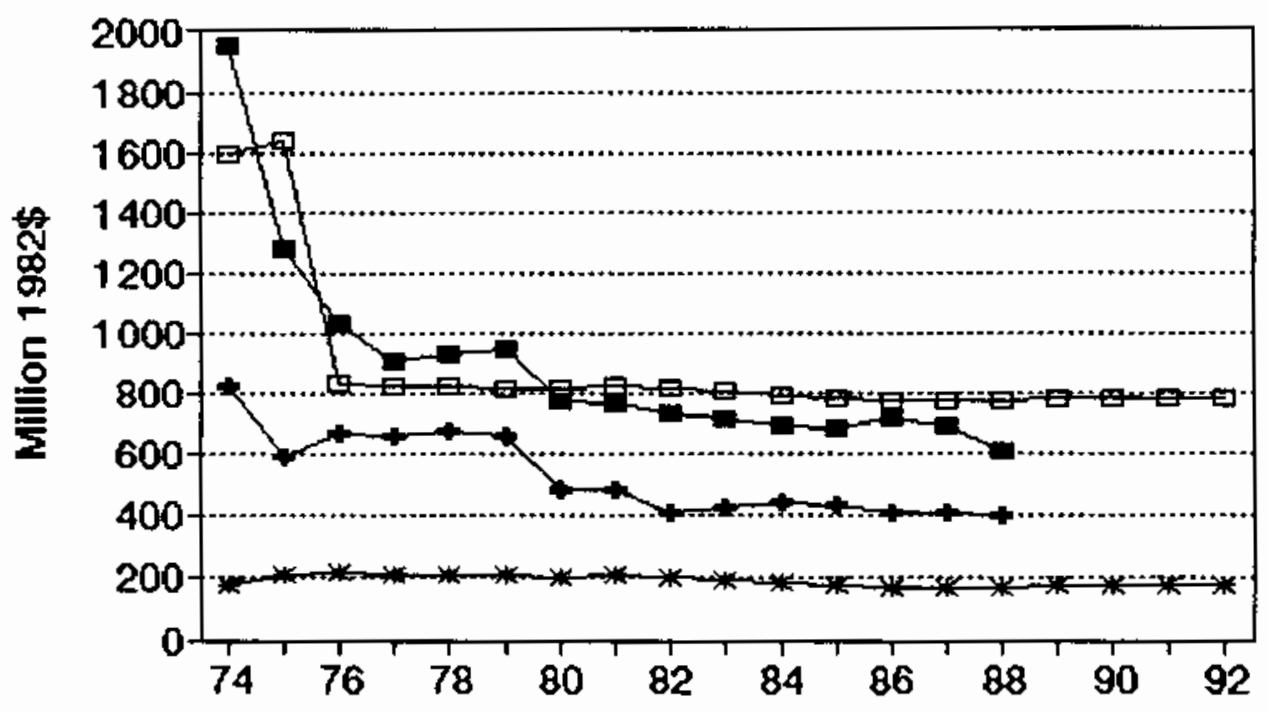

$\rightarrow$ - Ouput $\quad-$ - mplied Cepecity $\rightarrow$ - Cepitel Stock $\quad \square$-Pred Capacity


No. 310 sIC 3494 Valved and pipe fittings

MILLIONS OF 1982 DOLLARS (EXCL. CU RATES)

$\begin{array}{lccccccc} & \text { Q4 } & \text { Am. } & \text { Implied Gross } & \text { Met } & \text { Pred. } & \ldots \ldots \ldots \\ \text { Gross } & \text { CU } & \text { CU } & \text { Prac. } & \text { In- } & \text { Cap. } & \text { Prac. } & \text { Emergency Capacity } \\ \text { Output Rate } & \text { Rate } & \text { Capac. } & \text { vest. } & \text { Stk. } & \text { Capac. } & 3 \text { mon. } 6 \text { mon. }>6 \text { mon. }\end{array}$

\begin{tabular}{|c|c|c|c|c|c|c|c|c|c|c|}
\hline 1974 & 8,281 & 80 & 83.0 & 9,975 & 293.4 & 1,957 & 11,652 & & & \\
\hline 1975 & 7,435 & 41 & 51.0 & 14,586 & 292.8 & 2,099 & 12,237 & & & \\
\hline 1976 & 7,567 & 66 & 56.6 & 13,369 & 261.0 & 2,201 & 12,655 & & & \\
\hline 1977 & 8,626 & 72 & 69.7 & 12,373 & 295.9 & 2,327 & 13,174 & & & \\
\hline 1978 & 8,986 & 71 & 70.8 & 12,693 & 310.7 & 2,457 & 13,709 & & & \\
\hline 1979 & 9,648 & 67 & 69.1 & 13,958 & 281.3 & 2,550 & 14,092 & & & \\
\hline 1980 & 9,442 & 62 & 62.5 & 15,114 & 285.0 & 2,638 & 14,454 & & & \\
\hline 1981 & 9,682 & 62 & 63.3 & 15,303 & 349.2 & 2,779 & 15,034 & & & \\
\hline 1982 & 9,208 & 53 & 55.4 & 16,631 & 454.7 & 3,002 & 15,049 & & & \\
\hline 1983 & 8,039 & 55 & 53.8 & 14,937 & 189.7 & 2,954 & 15,753 & & & \\
\hline 1984 & 8,631 & 56 & 56.5 & 15,279 & 138.0 & 2,856 & 15,350 & & & \\
\hline 1985 & 8,487 & 54 & 55.0 & 15,442 & 202.3 & 2,821 & 15,208 & & & \\
\hline 1986 & 8,011 & 55 & 54.4 & 14,732 & 223.4 & 2,802 & 15,126 & & & \\
\hline - - - & $-\cdots$ & $\because$ & $\because \cdots$ & - - - & -- & $\because \cdots$ & $-\cdots$ & $-\ldots$ & --- & $-\cdots$ \\
\hline 1987 & 8,079 & 60 & 57.4 & 14,086 & -242.5 & 2,805 & 15,140 & & & \\
\hline 1988 & 8,169 & 67 & 64.8 & 12,614 & 238.8 & 2,804 & 15,137 & 17,218 & 23,868 & 23,962 \\
\hline 1989 & & & & & 235.2 & 2,800 & 15,120 & 17,199 & 23,840 & 23,935 \\
\hline 1990 & & & & & 231.6 & 2,793 & 15,089 & 17,164 & 23,792 & 23,886 \\
\hline 1991 & & & & & 227.9 & 2,782 & 15,046 & 17,115 & 23,724 & 23,818 \\
\hline 1992 & & & & & 224.3 & 2,769 & 14,992 & 17,053 & 23,638 & 23,732 \\
\hline
\end{tabular}

Capacity-Capital Stock Regression: Code 0 RSO 0.650 Coefficients: Const 3604.4 stock 4.1

Ave. Capacity/Stock $(74-86): 5.5$ Ave. Heekly Hours (High) 9.5 Shift factor 1.58 Ave Veekly Hours (Low) 92.8 Shift Factor 1.70 Wote: Output, Investment, and Capital Stock extrapolated for 1987 and 1988.

\section{Valves and pipe fittings}

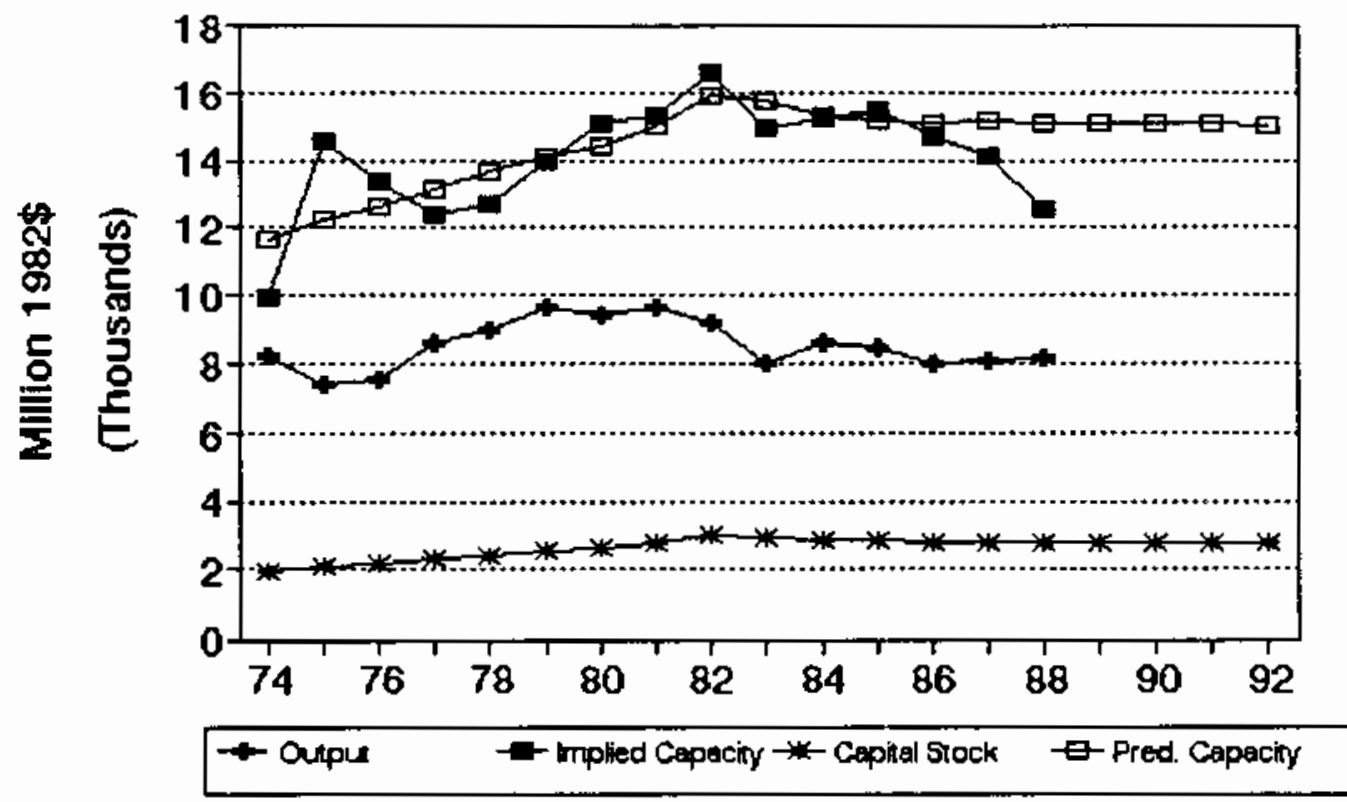


No. 311 sIC 3395 Wire springs

MILLIONS DF 1982 DOLLARS (EXCL. CU RATES)

$\begin{array}{lccccccc} & \text { Q4 } & \text { Ann. Implied } & \text { Gross } & \text { Net } & \text { Pred. } & \ldots \ldots \ldots \\ \text { Gross } & \text { CU } & \text { CU } & \text { Prac. } & \text { In- } & \text { Cap. } & \text { Prac. } & \text { Emergency Capacity } \\ \text { Output Rate } & \text { Rate } & \text { Capac. } & \text { vest. } & \text { Stk. } & \text { Capac. } & 3 \text { mon. } 6 \text { mon. }>6 \text { mon. }\end{array}$

\begin{tabular}{|c|c|c|c|c|c|c|c|c|c|c|}
\hline 1974 & 1,508 & 71 & 7.5 & 2,080 & 56.7 & 330 & 2,161 & & & \\
\hline 1975 & 1,225 & 66 & 65.3 & 1,876 & $(1.9)$ & 302 & 2,097 & & & \\
\hline 1976 & 1,381 & 67 & 66.9 & 2,065 & 13.5 & 290 & 2,068 & & & \\
\hline 1977 & 1,621 & 77 & 73.2 & 2,215 & 40.2 & 303 & 2,098 & & & \\
\hline 1978 & 1,665 & 62 & 67.3 & 2,473 & 40.8 & 316 & 2,128 & & & \\
\hline 1979 & 1,432 & 57 & 59.2 & 2,419 & 47.9 & 334 & 2,169 & & & \\
\hline 1980 & 1,167 & 67 & 62.5 & $1,8 \infty 7$ & 38.0 & 341 & 2,185 & & & \\
\hline 1981 & 1,056 & 50 & 57.0 & 1,853 & 1.7 & 313 & 2,121 & & & \\
\hline 1982 & 1,104 & 42 & 44.5 & 2,484 & 79.6 & 357 & 2,223 & & & \\
\hline 1983 & 1,271 & 61 & 53.4 & 2,382 & 79.2 & 397 & 2,315 & & & \\
\hline 1984 & 1,435 & 70 & 67.1 & 2,139 & 83.8 & 441 & 2,417 & & & \\
\hline 1985 & 1,532 & 61 & 64.5 & 2,374 & 35.4 & 439 & 2,413 & & & \\
\hline 1986 & 1,511 & 55 & 57.1 & 2,647 & 64.8 & 466 & $2,4 \pi 5$ & & & \\
\hline-- & --- & - & -- & --- & $\cdots$ & -- & $\therefore-$ & -- & $\cdots$ & $=\cdot$ \\
\hline 1987 & 1,528 & 55 & 54.6 & 2,800 & 70.8 & 498 & 2,549 & & & \\
\hline 1988 & $1,55 t$ & 57 & 56.5 & 2,746 & 74.6 & 531 & 2,626 & 2,841 & 3,788 & 3,802 \\
\hline 1989 & & & & & 78.3 & 566 & 2,705 & 2,926 & 3,902 & 3,916 \\
\hline 1990 & & & & & 82.1 & 601 & 2,786 & 3,014 & 4,019 & 4,034 \\
\hline 1991 & & & & & 85.9 & 637 & 2,869 & 3,104 & 4,139 & 4,154 \\
\hline 1992 & & & & & 89.7 & 673 & 2,954 & 3,196 & 4,262 & 4,277 \\
\hline
\end{tabular}

Capecity-Capital stock Regression: Code 0 RSQ 0.270

Coefficients: Const 1398.4 stock 2.3

Ave. Capacity/Stock $(74 \cdot 86): 6.2$ Ave. Heekly Hours (High) 108.8 Shift factor 1.45 Ave Ueekly Hours (Low) 107.5 Shift Factor 1.47 Note: Output, Investment, and Capital Stock extrapolated for 1987 and 1988.

3395 Wire springs

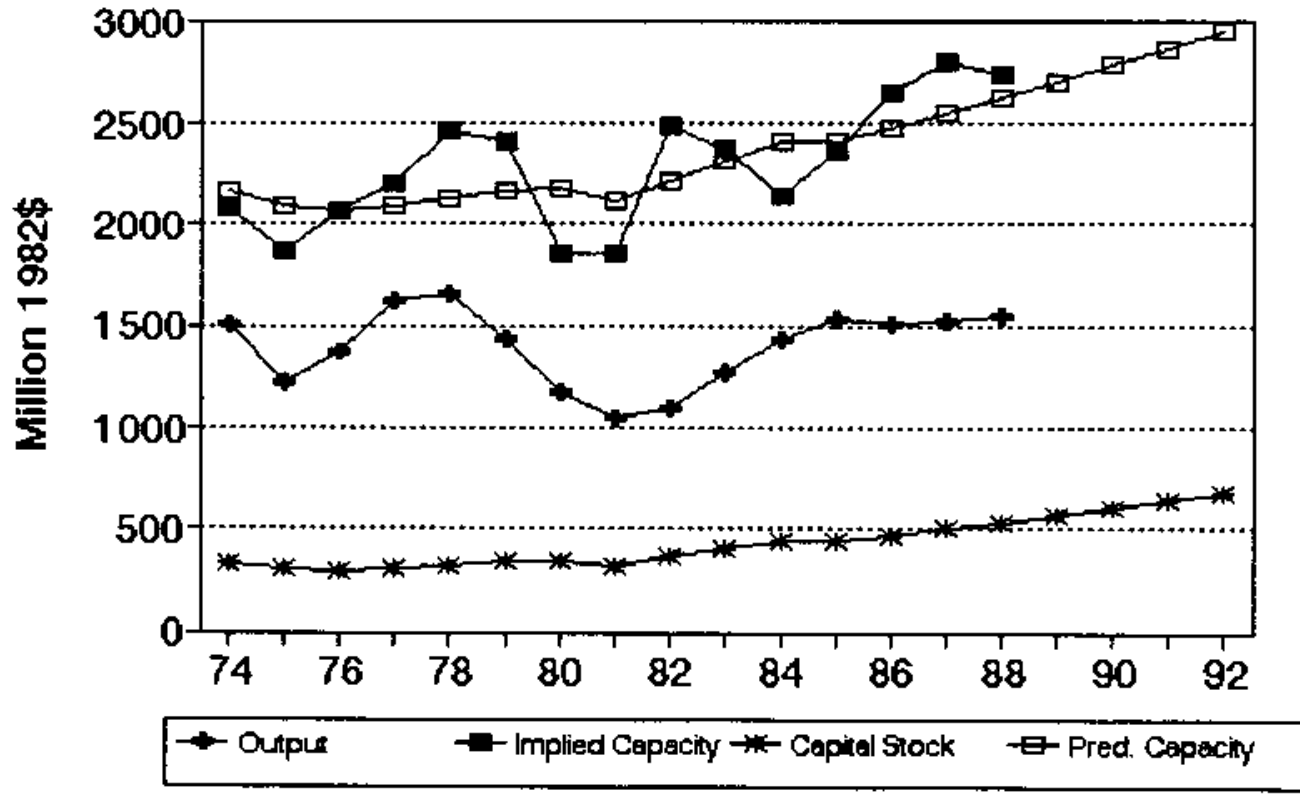


No. 312 SIC 3496 Niscellaneous fabricated wire products MILLIONS OF 1982 DOLLARS (EXCL. CU RATES)

$\begin{array}{lccccccc} & 04 & \text { Anm. } & \text { Implied Gross } & \text { Net } & \text { Pred. } & \ldots . . . . . . . \\ \text { Gross } & \text { CU } & \text { CU } & \text { Prac. } & \text { In- } & \text { Cap. } & \text { Prac. } & \text { Energency Capacity } \\ \text { Output Rate } & \text { Rate } & \text { Capac. } & \text { vest. } & \text { Stk. } & \text { Capec. } & 3 \text { mon. } 6 \text { mon. }>6 \text { mon. }\end{array}$

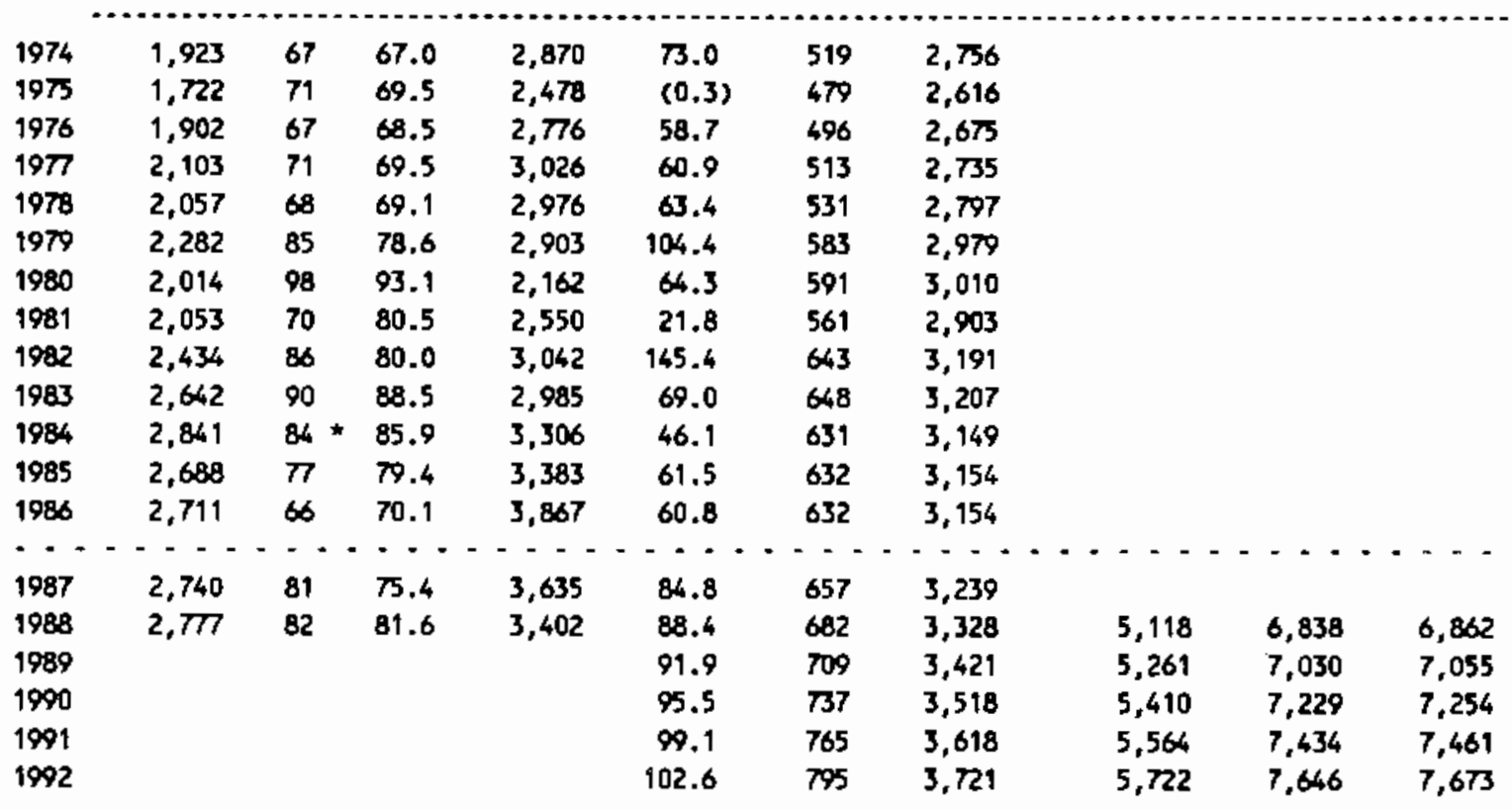

Capacity-Capital Stock Regression: Code 1 RSQ 0.250

Coefficients: Const 941.6 stock 3.5

Ave. Capecity/stock $(74-86): 5.1$

Ave. Weekly Hours (High) 76.4 Shift Factor 2.06 Ave Weekly Hours (LOW) 65.9 Shift Factor 2.39

- Inputed Note: Output, Investment, and Capital Stock extrapolated for 1987 and 1988.

\section{Miscellaneous fabricated wire prod}

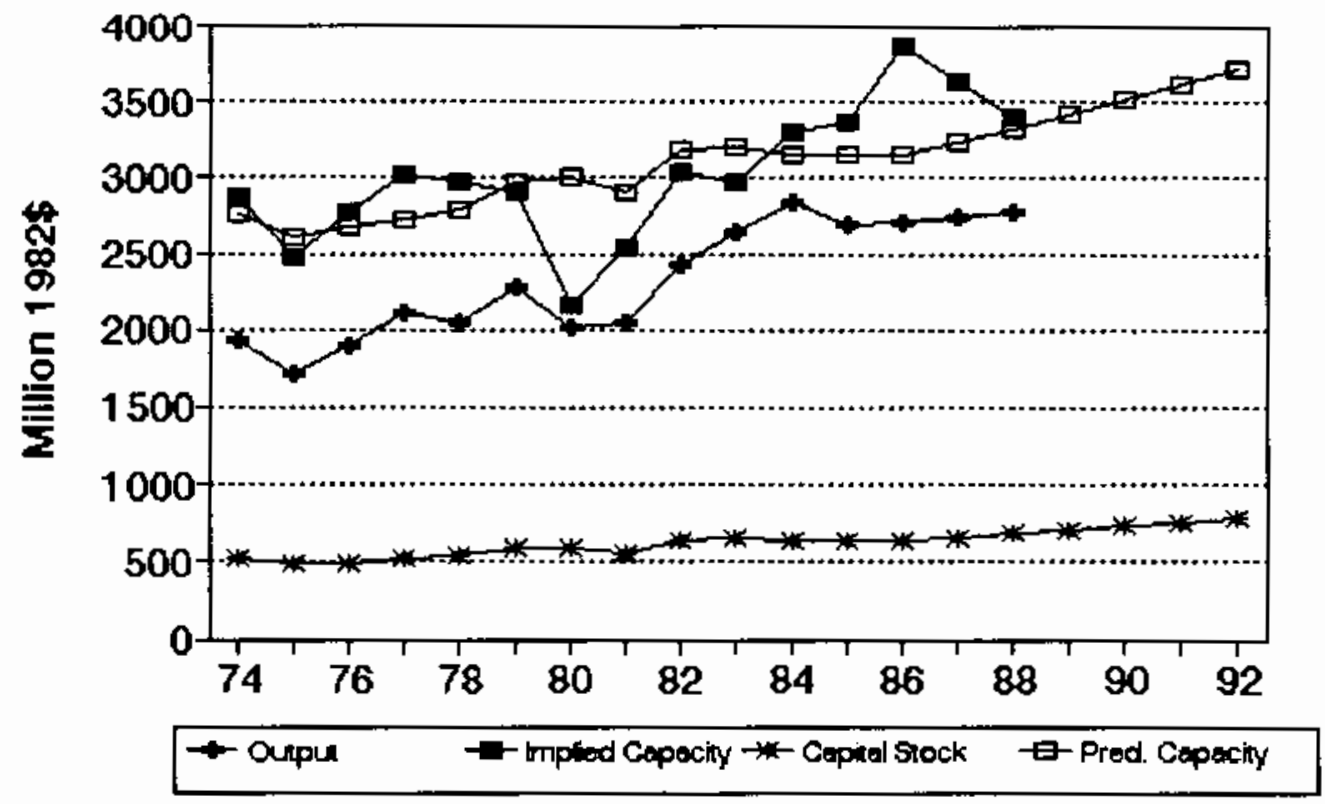




\section{No. 313 sIC 3497 Metal foil and leaf}

MILLIONS OF 1982 DOLLARS (EXCL. CU RATES)

$\begin{array}{lccccccc} & \alpha 4 & \text { Am. } & \text { Implied } & \text { Gross } & \text { Net } & \text { Pred. } & \ldots . . . \\ \text { Gross } & \text { a } & \text { CU } & \text { Prac. } & \text { In- } & \text { Cap. } & \text { Prac. } & \text { Emergency Capacity } \\ \text { Output } & \text { Rate } & \text { Rate } & \text { Capac. } & \text { vest. } & \text { Stk. } & \text { Capac. } & 3 \text { mon. } 6 \text { mon. }>6 \text { mon. }\end{array}$

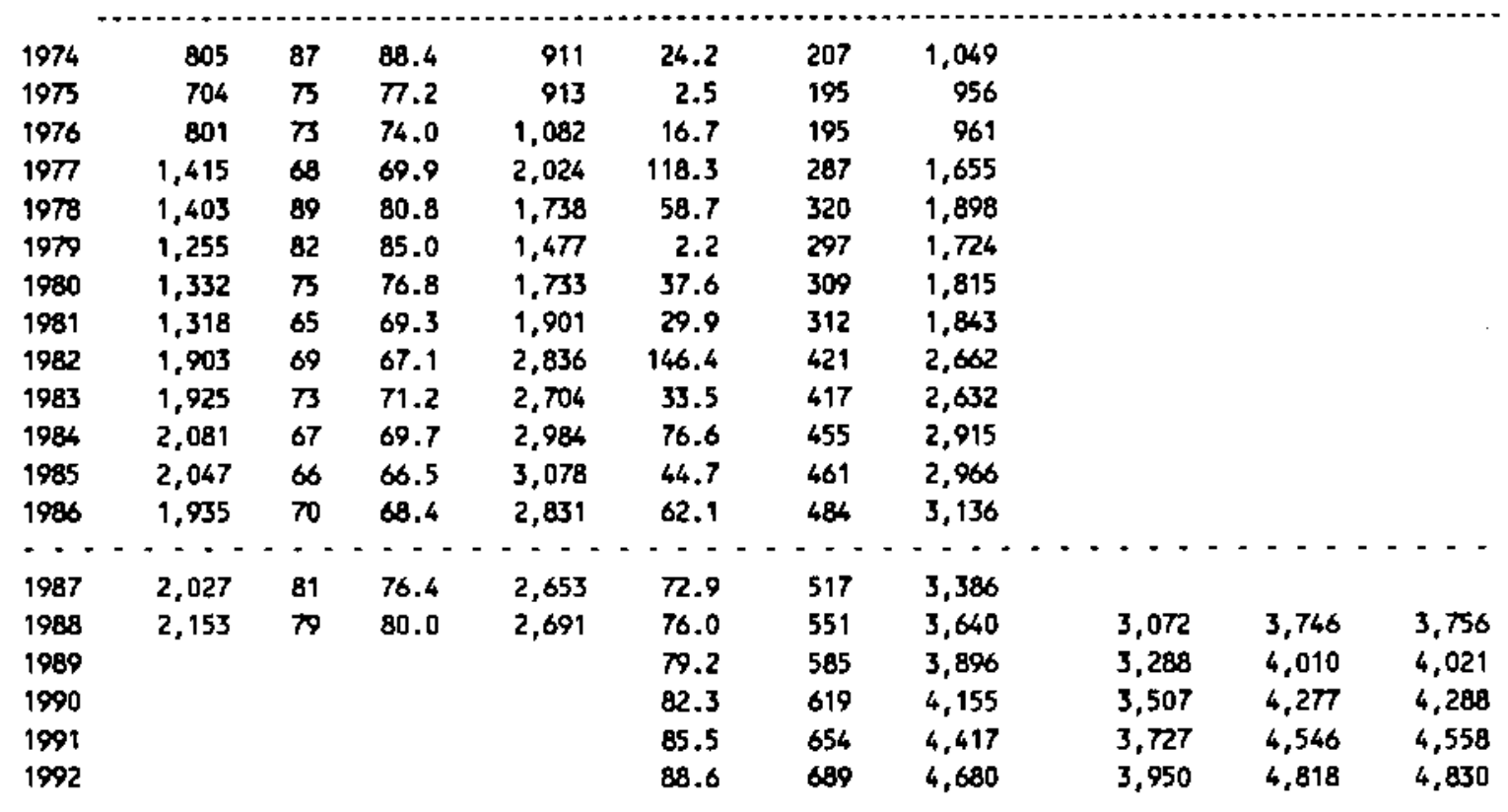

Capacity-Capital stock Regression: Code 0 RSO 0.950

Coefficients: Const -510.1 stock 7.5

Ave. Capacity/stock $(74-86): 6.0$ Ave. Ueekly Hours (High) 154.8 shift Factor 1.03 Ave Yeekly Hours (Low) 151.2 Shift Factor 1.05 Mote: Output, Investment, and Capital stock extrapolated for 1987 and 1988.

\section{Metal foil and leaf}

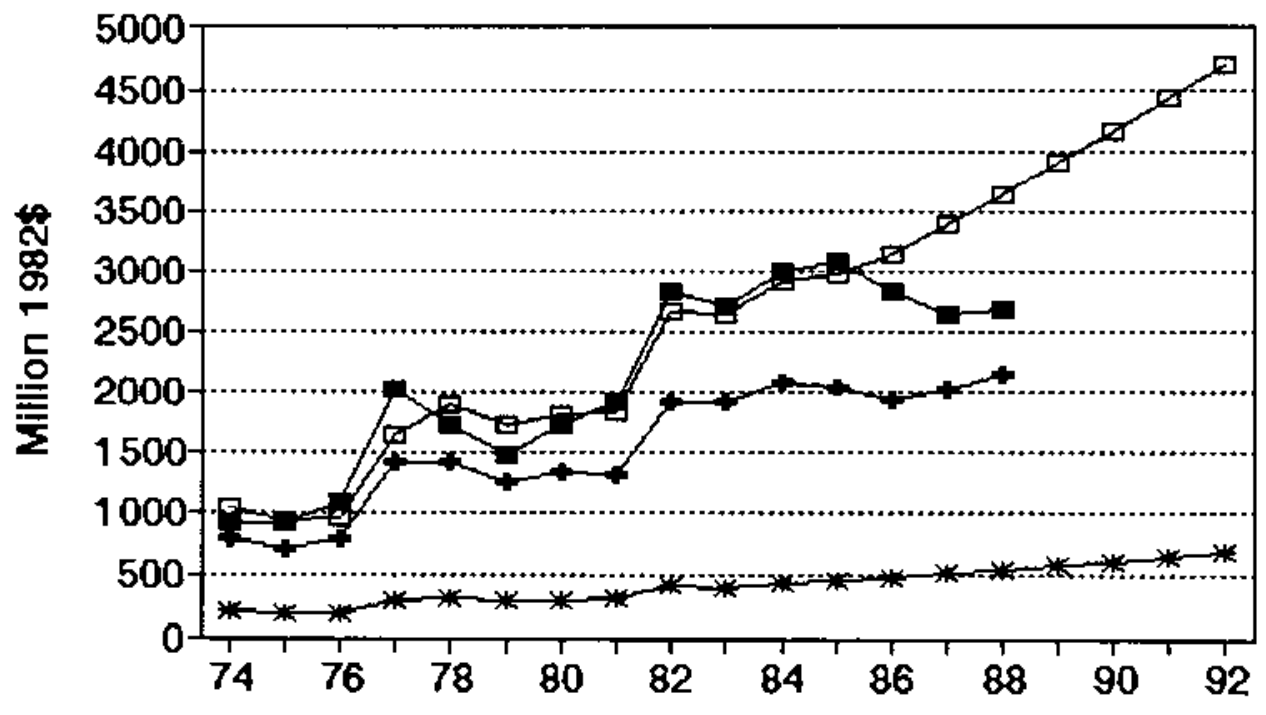

- Oupur $\rightarrow$ - mplied Cepaciny $\rightarrow$ Capite Stock $\rightarrow$ Pred. Cepaciny 
No. 314 SIC 3498 Fabricated pipe and fittings

MILLIONS OF 1982 DOLLARS (EXCL. CU RATES)

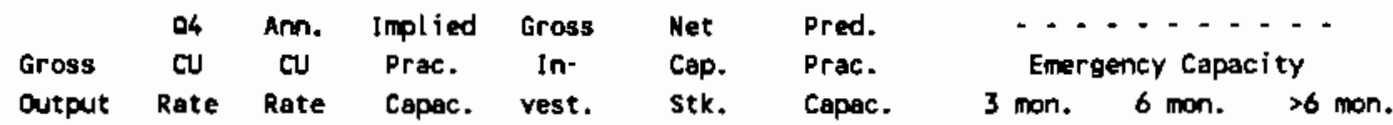

\begin{tabular}{|c|c|c|c|c|c|c|c|c|c|c|}
\hline 1974 & 2,461 & 77 & 77.6 & 3,172 & 57.6 & 300 & 3,618 & & & \\
\hline 1975 & 2,284 & 73 & 73.5 & 3,106 & 80.5 & 358 & 3,739 & & & \\
\hline 1976 & 2,615 & 47 & 56.9 & 4,593 & 96.9 & 429 & 3,887 & & & \\
\hline 1977 & 2,969 & 60 & 55.1 & 5,390 & 110.4 & 507 & 4,051 & & & \\
\hline 1978 & 3,144 & 82 & 73.6 & 4,272 & 64.6 & 538 & 4,116 & & & \\
\hline 1979 & 3,110 & $\pi$ & 70.5 & 4,064 & 93.4 & 593 & 4,230 & & & \\
\hline 1980 & 3,013 & 69 & 70.2 & 4,295 & 95.0 & 646 & 4,340 & & & \\
\hline 1981 & 3,090 & 71 & 70.5 & 4,394 & 95.0 & 696 & 4,446 & & & \\
\hline 1982 & 3,232 & 59 & 63.2 & 5,113 & 162.0 & 807 & 4,677 & & & \\
\hline 1983 & 2,691 & 60 & 59.5 & 4,522 & 76.7 & 824 & 4,713 & & & \\
\hline 1984 & 2,532 & 58 & 58.9 & 4,298 & (37.6) & 730 & 4,517 & & & \\
\hline 1985 & 2,264 & 56 & 56.8 & 3,987 & (19.7) & 659 & 4,368 & & & \\
\hline 1986 & 1,954 & 48 & 51.0 & 3,836 & 40.2 & 645 & 4,339 & & & \\
\hline$\cdots$ & $-\cdot-$ & $-\cdots$ & -- & $\cdots$ & $\cdots$ & -- & $\therefore-$ & $\cdots$ & -- & - \\
\hline 1987 & 1,974 & 55 & 52.2 & 3,779 & 42.6 & 635 & 4,318 & & & \\
\hline 1988 & $1, \infty 9$ & $57 \star$ & 56.4 & 3,544 & 39.3 & 623 & 4,292 & 5,096 & 7,372 & 7,404 \\
\hline 1989 & & & & & 36.1 & 608 & 4,262 & 5,060 & 7,319 & 7,351 \\
\hline 1990 & & & & & 32.8 & 591 & 4,227 & 5,019 & 7.259 & 7,291 \\
\hline 1991 & & & & & 29.5 & 573 & 4,188 & 4,973 & 7,193 & 7,224 \\
\hline 1992 & & & & & 26.3 & 553 & 4,146 & 4,922 & 7,120 & 7,151 \\
\hline
\end{tabular}

Capacity-Capital stock Regression: Code 1 RSO 0.280

Coefficients: Const 2990.4 stock 2.1

Ave. Capacity/Stock (74-86): 7.1 Ave. Heekly Hours (High) 91.3 Shift Factor 1.73 Ave Weekly Hours (Low) 83.2 Shift Factor 1.89 * Imputed Mote: Output, Investment, and Capital Stock extrapolated for 1987 and 1988.

\section{Fabricated pipe and fittings}

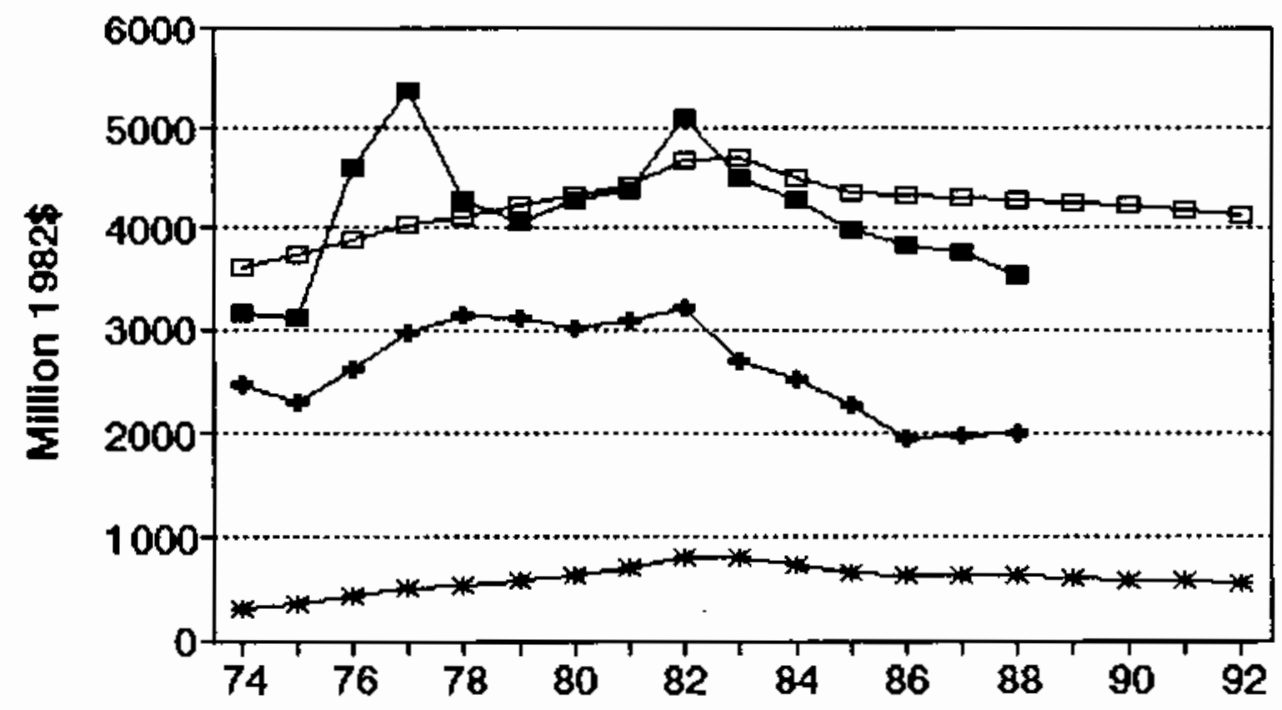

$\rightarrow$ - Qutpus $\rightarrow-$ mplied Cepeciny $\rightarrow-$ Cepited Stock $\square$ Pred. Capacity 
No. 315 SIC 3499 Fabricated metal products, nec MILLIONS OF 1982 DOLLARS (EXCL. CU RATES)

$\begin{array}{lccccccc} & \text { Q4 } & \text { Ann. Implied Gross } & \text { Ket } & \text { Pred. } & -\ldots \ldots \ldots \\ \text { Gross } & \text { CU } & \text { CU } & \text { Prac. } & \text { In- } & \text { Cap. } & \text { Prac. } & \text { Emergency Capacity } \\ \text { Output } & \text { Rate } & \text { Rate } & \text { Capac. } & \text { vest. } & \text { Stk. } & \text { Capac. } & 3 \text { mon. } 6 \text { mon. }\end{array}$

\begin{tabular}{|c|c|c|c|c|c|c|c|c|c|c|}
\hline 1974 & 5,560 & 67 & 69.2 & 8,037 & 157.7 & 1,021 & 8,390 & & & \\
\hline 1975 & 5,033 & 50 & 52.8 & 9,528 & 169.1 & 1,111 & 8,274 & & & \\
\hline 1976 & 5,325 & 67 & 60.8 & 8,765 & 159.0 & 1,185 & 8,170 & & & \\
\hline 1977 & 5,410 & 69 & 68.3 & 7,927 & 101.2 & 1,200 & 8,150 & & & \\
\hline 1978 & 5,665 & 70 & 69.1 & 8,197 & 111.3 & 1,220 & 8,121 & & & \\
\hline 1979 & 6,069 & $\pi$ & 73.7 & 8,233 & 251.2 & 1,371 & 7,912 & & & \\
\hline 1980 & 5,775 & 70 & 70.5 & 8,192 & 143.9 & 1,412 & 7,855 & & & \\
\hline 1981 & 5,503 & 60 & 64.8 & 8,488 & 72.4 & 1,382 & 7,897 & & & \\
\hline 1982 & 4,365 & 58 & 58.0 & 7,522 & 56.5 & 1,335 & 7,962 & & & \\
\hline 1983 & 4,321 & 52 & 54.0 & 7,908 & $(5.8)$ & 1,229 & 8,109 & & & \\
\hline 1984 & 4,903 & 76 & 67.5 & 7,263 & 87.2 & 1,218 & 8,125 & & & \\
\hline 1985 & 4,843 & 6 & 70.0 & 6,919 & 174.3 & 1,286 & 8,030 & & & \\
\hline 1986 & 5,230 & 66 & 65.7 & 7,955 & 115.9 & 1,294 & 8,020 & & & \\
\hline-- & $-\cdots$ & - & $-\cdot$ & $\cdots$ & $\cdots$ & $\cdots$ & $\cdots$ & --- & --- & $-\cdot$ \\
\hline 1987 & 5,286 & 71 & 68.3 & 7,736 & 122.3 & 1,308 & 7,999 & & & \\
\hline 1988 & 5,360 & 64 & 67.1 & 7,987 & 151.7 & 1,351 & 7,940 & $11,36 t$ & 15,769 & 15,832 \\
\hline 1989 & & & & & 156.3 & 1,395 & 7,879 & 11,273 & 15,648 & 15,711 \\
\hline 1990 & & & & & 159.3 & 1,438 & 7,819 & 11,187 & 15,529 & 15,591 \\
\hline 1991 & & & & & 165.8 & 1,484 & 7,755 & 11,096 & 15,402 & 15,463 \\
\hline 1992 & & & & & 162.2 & 1,523 & 7,701 & 11,019 & 15,295 & 15,356 \\
\hline
\end{tabular}

Capacity-Capital Stock Regression: Code 2 RSO 0.060

Coefficients: Const 9818.7 Stock $-1.4 \quad$ Ave. Capacity/Stock $(74-86): 6.5$ Ave. Weekly Hours (High) 79.0 Shift Factor 1.99 Ave Weekly Hours (Low) 73.7 Shift Factór 2.14 Mote: Output, Investment, and Capital Stock extrapolated for 1987 and 1988.

\section{Fabricated metal products, nec}
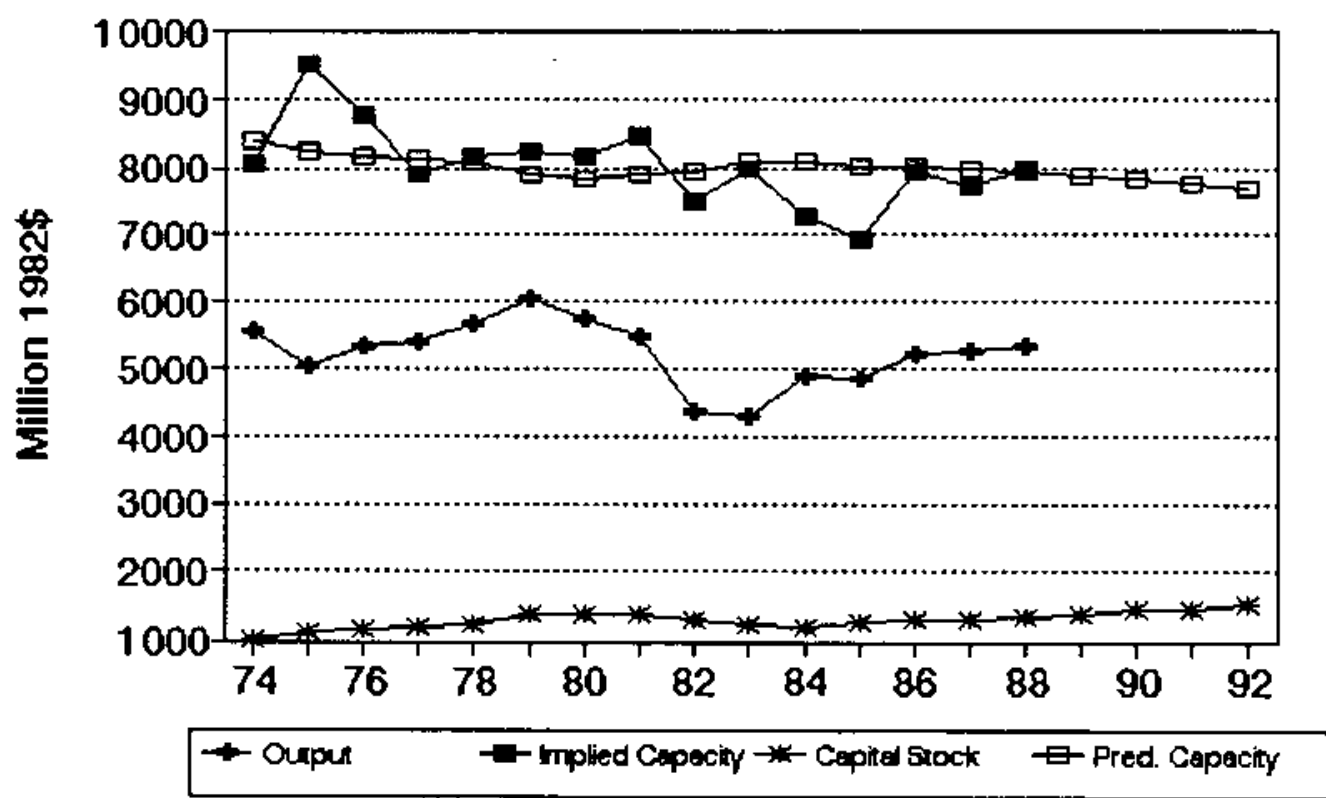


\section{No. 316 sIC 3511 Turbines and turbine generator sets}

MILLLIONS OF 1982 DOLLARS (EXCL. CU RATES)

$\begin{array}{lcccclcc} & 04 & \text { Am. Implied Gross } & \text { Net } & \text { Pred. } & \ldots \\ \text { Gross } & \text { CU } & \text { CU } & \text { Prac. } & \text { In- } & \text { Cap. } & \text { Prac. } & \text { Emergency Capacity } \\ \text { Qutput Rate } & \text { Rate } & \text { Capac. } & \text { vest. } & \text { Stk. } & \text { Capac. } & 3 \text { mon. } 6 \text { mon. }>6 \text { mon. }\end{array}$

\begin{tabular}{|c|c|c|c|c|c|c|c|c|c|c|}
\hline 1974 & 5,913 & 87 & 88.8 & 6,657 & 142.3 & 1,660 & 6,023 & & & \\
\hline 1975 & 4,501 & 78 & 78.4 & 5,743 & 119.6 & 1,677 & 6,042 & & & \\
\hline 1976 & 4.772 & 74 & 75.3 & 6,340 & 164.8 & 1,716 & 6,127 & & & \\
\hline 1977 & 4,298 & 84 & 80.3 & 5,355 & 12.7 & 1,598 & 5,866 & & & \\
\hline 1978 & 3,863 & 83 & 83.0 & 4,653 & 90.0 & 1,547 & 5,753 & & & \\
\hline 1979 & 4,279 & 68 & 74.6 & 5,738 & 137.0 & 1,532 & 5,721 & & & \\
\hline 1980 & 4,100 & 65 & 65.6 & 6,253 & 131.5 & 1,504 & 5,659 & & & \\
\hline 1981 & 3,886 & 74 & 71.6 & 5,425 & 109.0 & 1,444 & 5,527 & & & \\
\hline 1982 & 4,142 & 65 & 67.6 & 6,127 & 158.0 & 1,429 & 5,493 & & & \\
\hline $19 a 3$ & 2,873 & 55 & 58.2 & 4,939 & 45.3 & 1,310 & 5,232 & & & \\
\hline 1984 & 3,378 & 63 & 61.1 & 5,528 & 218.8 & 1,355 & 5,330 & & & \\
\hline 1985 & 3,236 & 61 & 61.8 & 5,234 & 16.6 & 1,212 & 5,016 & & & \\
\hline 1986 & 2,858 & 62 & 61.6 & 4,643 & 74.2 & 1,135 & 4,846 & & & \\
\hline $\begin{array}{l}\cdots \\
1987\end{array}$ & $\begin{array}{l}\cdots \\
2,890\end{array}$ & 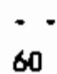 & 60.5 & $\begin{array}{l}-7.74 \\
4.774\end{array}$ & $\begin{array}{l}--- \\
118.1\end{array}$ & . & $\cdots$ & $\cdots$ & -- & $-\cdots$ \\
\hline 1988 & 2,932 & 56 & 57.9 & 5,061 & 158.8 & 1,116 & 4,803 & 4,445 & 5,556 & 5,572 \\
\hline 1989 & & & & & 178.8 & 1,147 & 4,872 & 4,508 & 5,636 & 5,652 \\
\hline 1990 & & & & & 178.8 & 1,174 & 4,932 & 4,564 & 5,705 & 5,721 \\
\hline 1991 & & & & & 171.3 & 1,190 & 4,967 & 4,596 & 5,746 & 5,762 \\
\hline 1992 & & & & & 178.8 & 1,212 & 5,014 & 4,640 & 5,800 & 5,817 \\
\hline
\end{tabular}

Capacity-Capital Stock Regression: Code 0 RSO 0.390

Coefficients: Const 2342.3 stock 2.2

Ave. Capacity/Stock $(74-86): 3.8$ Ave. Weekly Hours (High) 136.9 Shift Factor 1.16 Ave Heekly Hours (LOW) 136.0 Shift factor 1.17 Note: Output, Investment, and Capital stock extrapolated for 1987 and 1988.

\section{Turbines and turbine generator set}

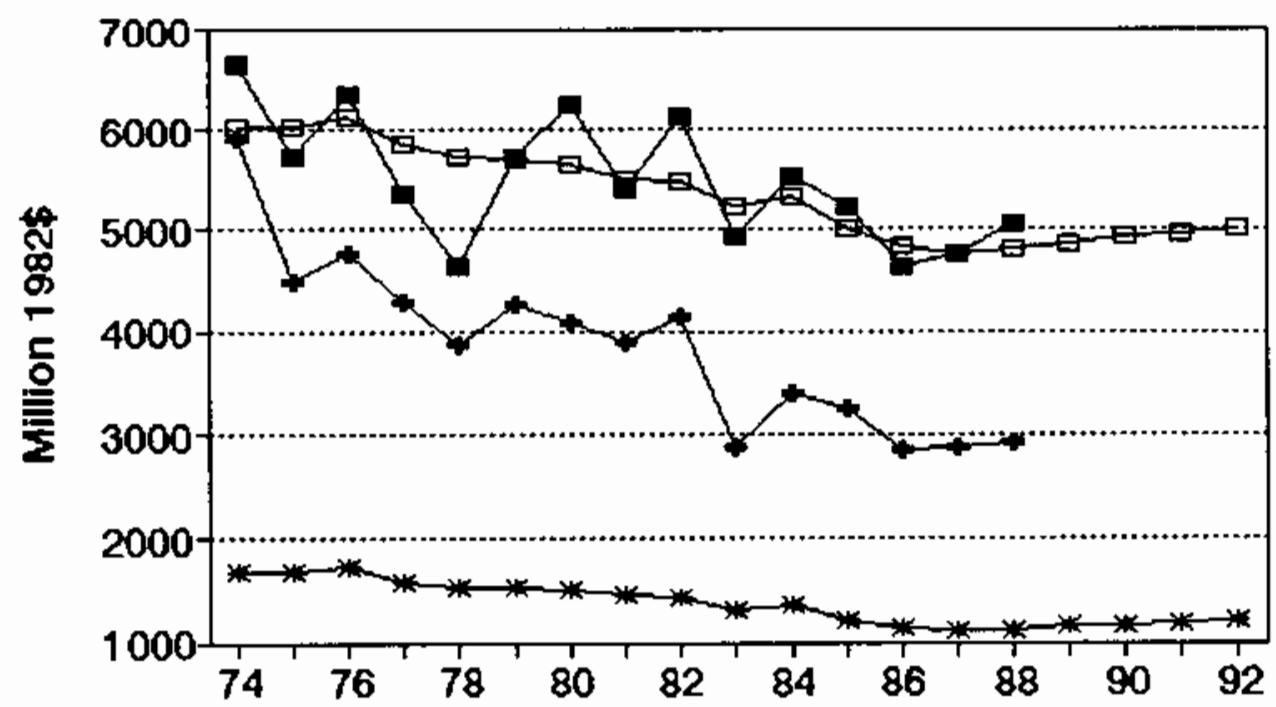

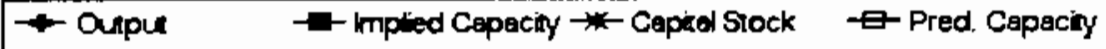


No. 317 sIc 3519 Internal combubtion engines, nec

MILLIONS OF 1982 DOLLARS (EXCL. CU RATES)

$\begin{array}{lccccccc} & 04 & \text { Ann. Implied Gross } & \text { Net } & \text { Pred. } & \ldots \ldots \ldots \\ \text { Gross } & \text { CU } & \text { CU } & \text { Prac. } & \text { In- } & \text { Cap. } & \text { Prac. } & \text { Emergency Capacity } \\ \text { Output Rate } & \text { Rate } & \text { Capac. } & \text { vest. } & \text { Stk. } & \text { Capac. } & 3 \text { mon. } 6 \text { mon. }>6 \text { mon. }\end{array}$

\begin{tabular}{|c|c|c|c|c|c|c|c|c|c|c|}
\hline 1974 & 11,067 & 76 & 79.6 & 13,898 & 391.5 & 2,245 & 15,408 & & & \\
\hline 1975 & 9,804 & 63 & 61.9 & 15,828 & 360.3 & 2,453 & 15,808 & & & \\
\hline 1976 & 11,113 & 74 & 69.2 & 16,072 & 363.8 & 2,645 & 16,178 & & & \\
\hline 1977 & 13,103 & 79 & 77.4 & 16,939 & 467.3 & 2,921 & 16,711 & & & \\
\hline 1978 & 14,815 & 78 & 77.7 & 19,076 & 330.4 & 3,046 & 16,951 & & & \\
\hline 1979 & 14,030 & 76 & 79.1 & 17,735 & 419.5 & 3,246 & 17,337 & & & \\
\hline 1980 & 12,386 & 67 & 68.9 & 17,973 & 317.4 & 3,325 & 17,490 & & & \\
\hline 1981 & 12,436 & 71 & 72.0 & 17,282 & 677.2 & 3,737 & 18,283 & & & \\
\hline 1982 & 9,372 & 38 & 48.0 & 19,545 & 498.5 & 3,952 & 18,698 & & & \\
\hline 1983 & 8,536 & 57 & 47.9 & 17,805 & 390.0 & 4,030 & 18,848 & & & \\
\hline 1984 & 11,575 & 61 & 62.7 & 18,457 & 358.3 & 4,058 & 18,902 & & & \\
\hline 1985 & 10,419 & 55 & 57.4 & 18,150 & 337.5 & 4,049 & 18,885 & & & \\
\hline 1988 & 10,106 & 50 & 51.7 & 19,551 & 324.2 & 4,011 & 18,812 & & & \\
\hline--- & --- & -- & -- & --- & -- & 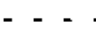 & --- & $-\cdot-$ & $\cdot \cdot \cdot=$ & $\cdot \cdot-$ \\
\hline 1987 & 10,235 & 55 & 52.6 & 19,457 & 404.8 & 4,063 & 18,912 & & & \\
\hline 1988 & 10,404 & 62 & 60.3 & 17,256 & 525.0 & 4,231 & 19,236 & 20,634 & 28,150 & 28,257 \\
\hline 1989 & & & & & 584.3 & 4,443 & 19,645 & 21,073 & 28,749 & 28,858 \\
\hline 1990 & & & & & 584.3 & 4,637 & 20,018 & 21,473 & 29,295 & 29,407 \\
\hline 1991 & & & & & 561.9 & 4,791 & 20,316 & 21,792 & 29,730 & 29,844 \\
\hline 1992 & & & & & 584.3 & 4,954 & 20,630 & 22,129 & 30,190 & 30,305 \\
\hline
\end{tabular}

Capacity-Capital stock Regression: Code 0 RSD 0.630

Coefficients: Const 11080.3 stock 1.8

Ave. Capacity/Stock $(74-86): 5.2$

Ave. Weekly Hours (High) 107.2 Shift Factor 1.47 Ave weekly Hours (LOW) 103.1 Shift Factor 1.53 Note: Output, Investment, and Capital Stock extrapolated for 1987 and 1988.

\section{Internal combustion engines, nec}

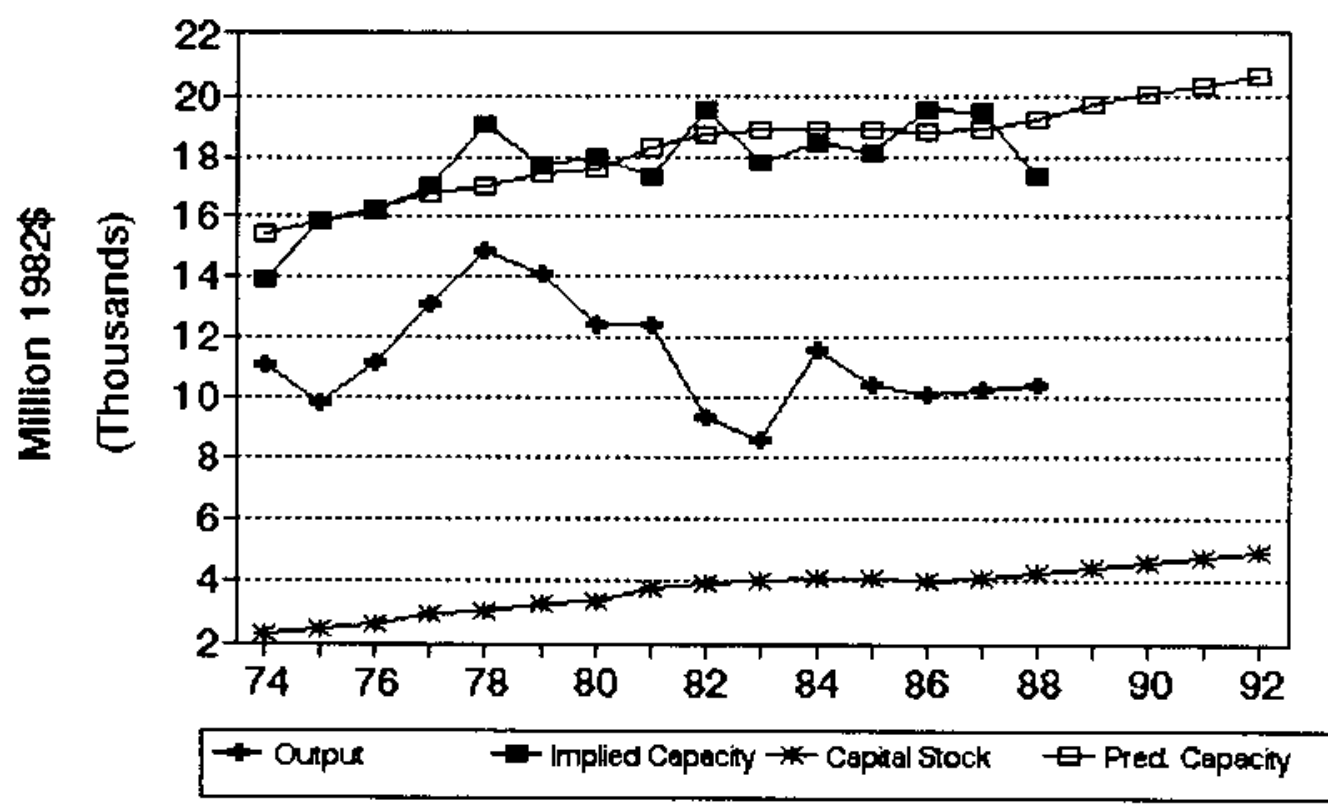


No. 318 BIC 3523 Fare anchinery and equipment

MILLIONS OF 1982 DOLLARS (EXCL. CU RATES)

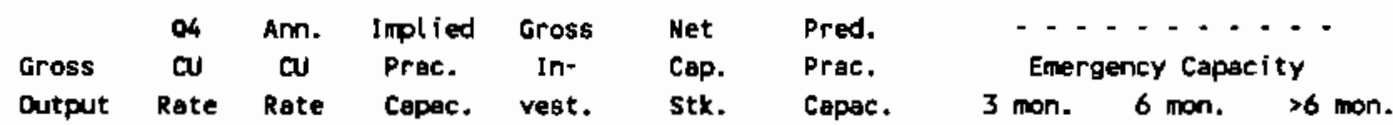

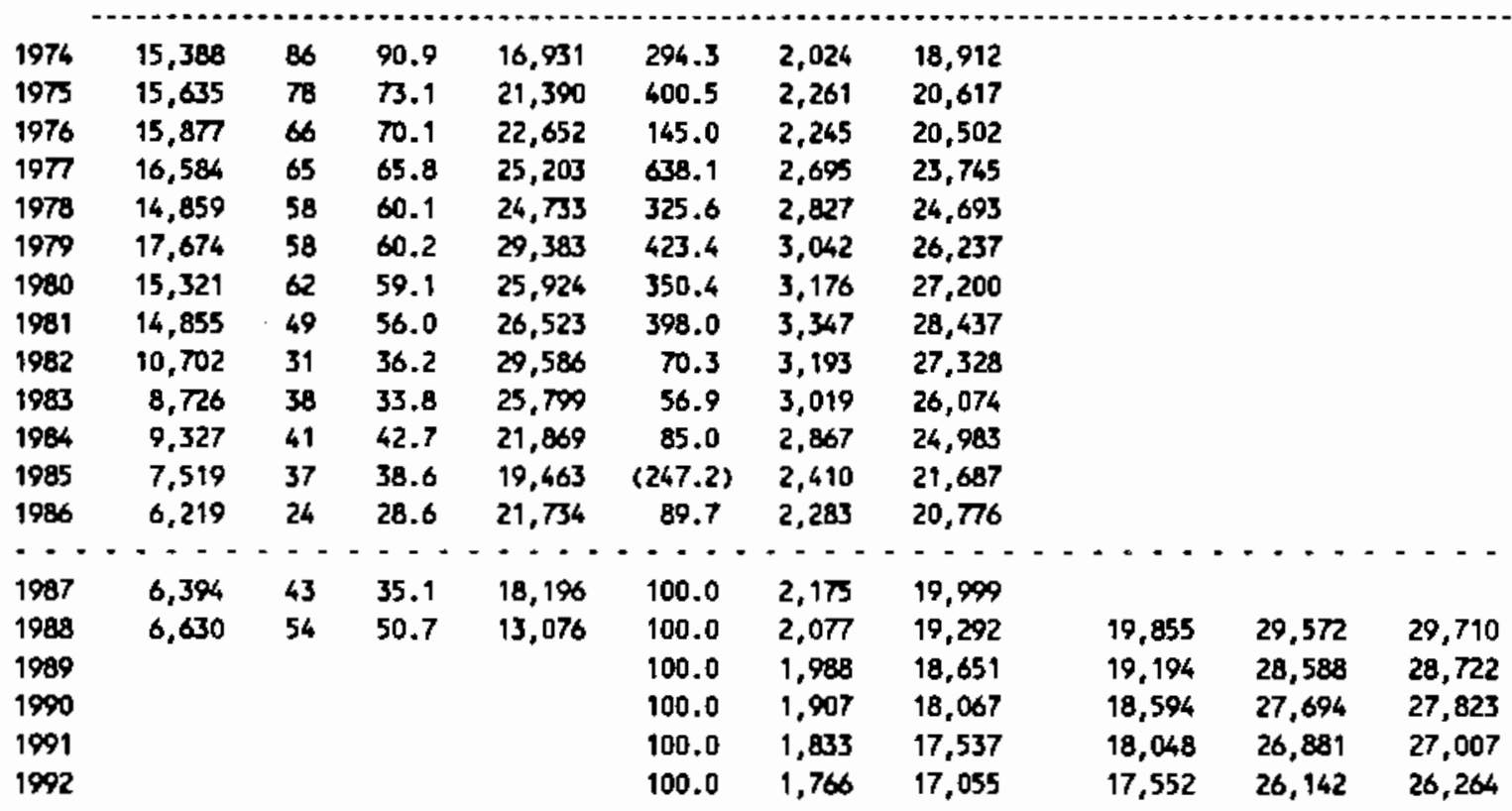

Capacity-Capital Stock Regression: Code 0 RSa 0.710

Coefficients: Const 4344.9 stock 7.2

Ave. Capacity/stock $(74-86): 8.8$

Ave, Yeekly Hours (High) 102.3 Shift Factor 1.54 Ave Veekly Hours (Lou) 98.9 Shift factor 1.59

Note: Output, Imestment, and Capital stock extrapolated for 1987 and 1988.

\section{Farm machinery and equipment}

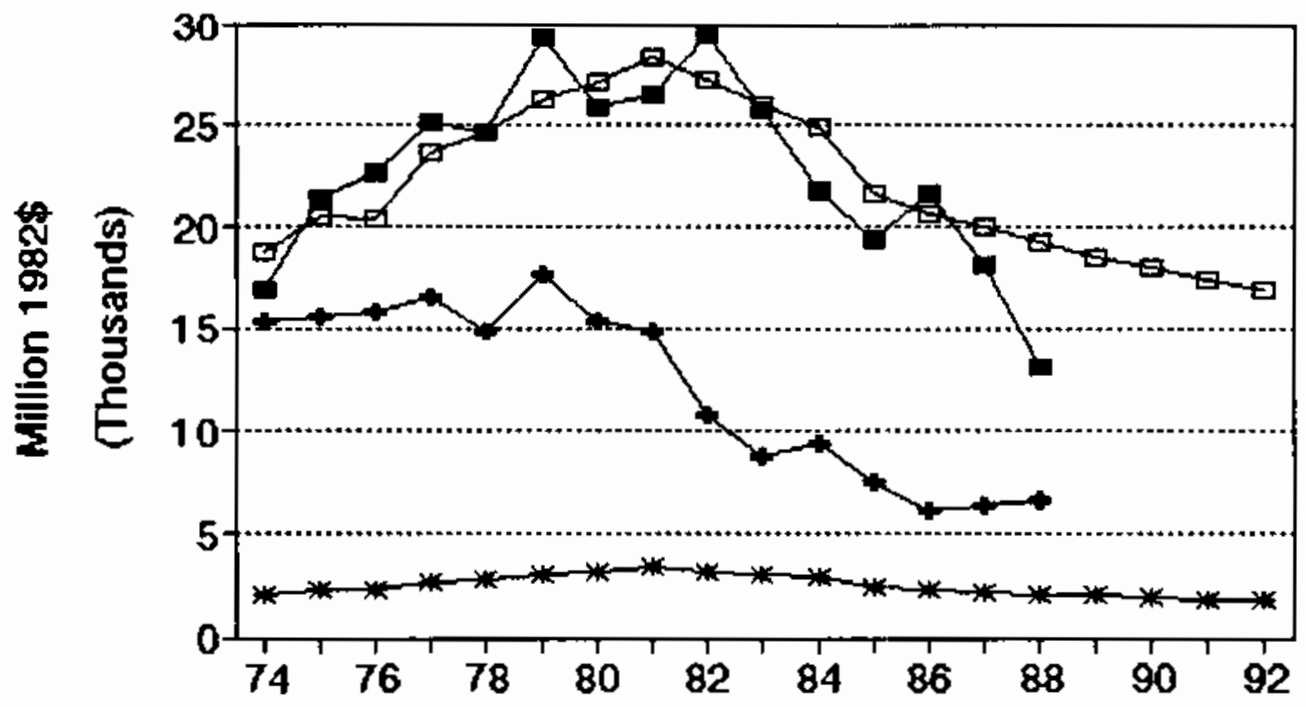

- Tupur $\quad-$ - Implied Capecin $\rightarrow$ - Cepite Srock $\quad \square$-Pred. Capaciny 
No. 319 sIC 3524 Lawn and garden equipment

MILLIONS OF 1982 DOLLARS (EXCL. CU RATES)

$\begin{array}{lccccccc} & Q 4 & \text { Ann. } & \text { Implied Gross } & \text { Net } & \text { Pred. } & \ldots \ldots \ldots \\ \text { Gross } & \text { CU } & \text { CU } & \text { Prac. } & \text { In- } & \text { Cap. } & \text { Prac. } & \text { Emergency Capacity } \\ \text { Output } & \text { Rate } & \text { Rate } & \text { Capac. } & \text { vest. } & \text { Stk. } & \text { Capac. } & 3 \text { mon. } 6 \text { mon. }>6 \text { mon. }\end{array}$

\begin{tabular}{|c|c|c|c|c|c|c|c|c|c|c|}
\hline 1974 & 2,987 & $\pi$ & 73.3 & 4,076 & 51.7 & 392 & 3,860 & & & \\
\hline 1975 & 2,068 & 60 & 62.4 & 3,315 & $(9.1)$ & 366 & 3,647 & & & \\
\hline 1976 & 2,139 & 64 & 62.3 & 3,433 & 22.2 & 370 & 3,680 & & & \\
\hline 1977 & 2,244 & 59 & 61.0 & 3,681 & 40.9 & 390 & 3,844 & & & \\
\hline 1978 & 2,653 & 76 & 69.3 & 3,829 & 13.6 & 381 & 3,774 & & & \\
\hline 1979 & 3,287 & 71 & 73.7 & 4,461 & 50.3 & 407 & 3,987 & & & \\
\hline 1980 & 2,546 & 52 & 58.7 & 4,341 & 56.2 & 435 & 4,221 & & & \\
\hline 1981 & 2,083 & 55 & 54.5 & 3,821 & 30.7 & 434 & 4,217 & & & \\
\hline 1982 & 2,355 & 52 & 52.7 & 4,471 & 46.5 & 446 & 4,320 & & & \\
\hline 1983 & 2,443 & 65 & 59.5 & 4,108 & $(26.1)$ & 390 & 3,845 & & & \\
\hline 1984 & 3,065 & 68 & 68.0 & 4,510 & 88.8 & 442 & 4,280 & & & \\
\hline 1985 & 2,987 & $\pi$ & 70.6 & 4,231 & 64.8 & 467 & 4,494 & & & \\
\hline 1986 & 3,191 & $\pi$ & 7.6 & 4,398 & 42.4 & 469 & 4,508 & & & \\
\hline$\cdots$ & $\therefore-$ & - & - - & $-\cdots$ & --- & - & $\therefore$ & $\cdots$ & --- & $-\cdots$ \\
\hline 1987 & 3,195 & 78 & $\pi .9$ & 4,211 & 52.1 & 481 & 4,609 & & & \\
\hline 1988 & 3,199 & 60 & 67.3 & 4,756 & 54.3 & 494 & 4,719 & 5,392 & 7,004 & 7,027 \\
\hline 1989 & & & & & 56.5 & 508 & 4,839 & 5,529 & 7,181 & 7,205 \\
\hline 1990 & & & & & 58.7 & 523 & 4,967 & $5,6 \pi \mathrm{s}$ & 7,371 & 7,395 \\
\hline 1991 & & & & & 60.9 & 539 & 5,102 & 5,830 & 7,572 & 7,597 \\
\hline 1992 & & & & & 63.1 & 556 & 5,244 & 5,992 & 7,783 & 7,808 \\
\hline
\end{tabular}

Capecity-Capital Stock Regression: Code 0 RSQ 0.560

Coefficients: Const 571.5 stock 8.4

Ave. Capacity/Stock $(74-86): 9.8$ Ave. Heekly Hours (High) 105.7 Shift Factor 1.49 Ave Ueekly Hours (Low) 101.5 Shift Factor 1.55 Note: Output, Investment, and Capital Stock extrapolated for 1987 and 1988.

\section{Lawn and garden equipment}

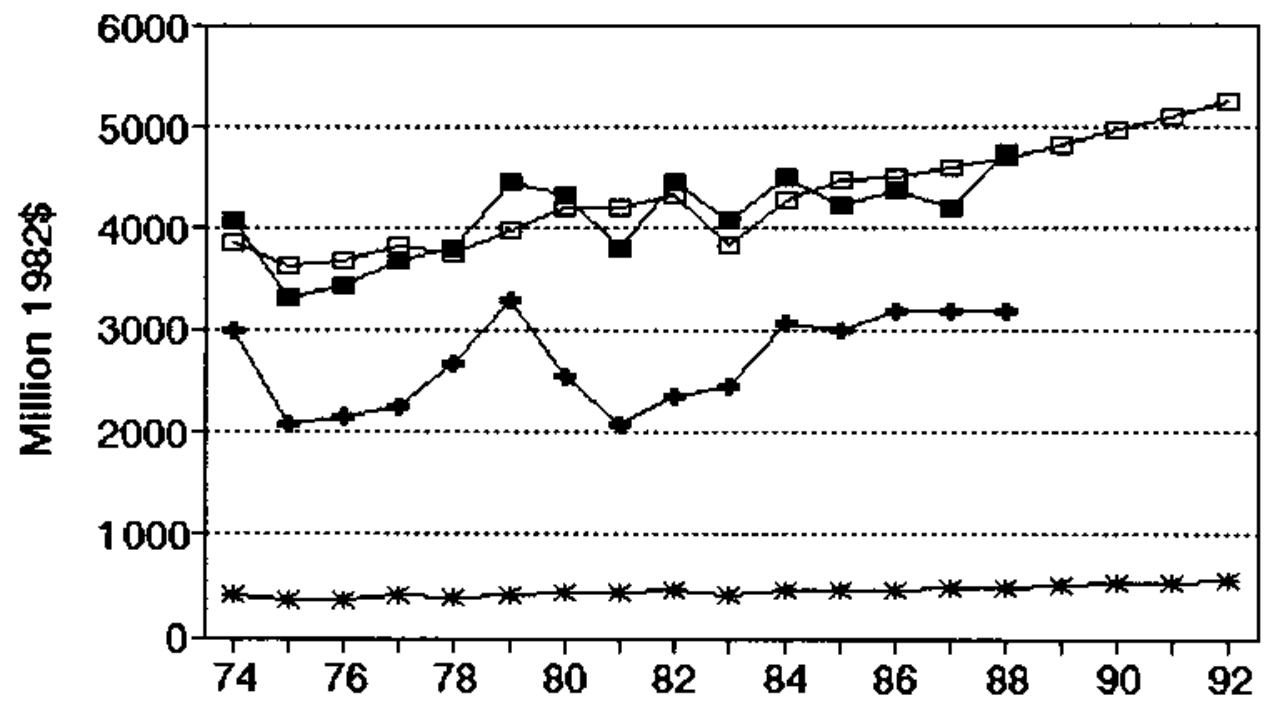

- Oupur $\rightarrow-$ mplied Cepeciny -*-Cepitel Srock $\quad$ - Pred. Capacity 
MO. 320 GIC 3531 Construction machinery

MILLIONS OF 1982 DOLLARS (EXCL. CU RATES)

$\begin{array}{lccccccc} & 04 & \text { Ann. } & \text { Implied } & \text { Gross } & \text { Het } & \text { Pred. } & -\ldots . . . \\ \text { Gross } & \text { CU } & \text { CU } & \text { Prac. } & \text { In- } & \text { Cap. } & \text { Prac. } & \text { Emergency Capacity } \\ \text { Qutput } & \text { Rate } & \text { Rate } & \text { Capac. } & \text { vest. } & \text { Stk. } & \text { Capac. } & 3 \text { mon. } 6 \text { mon. }>6 \text { mon. }\end{array}$

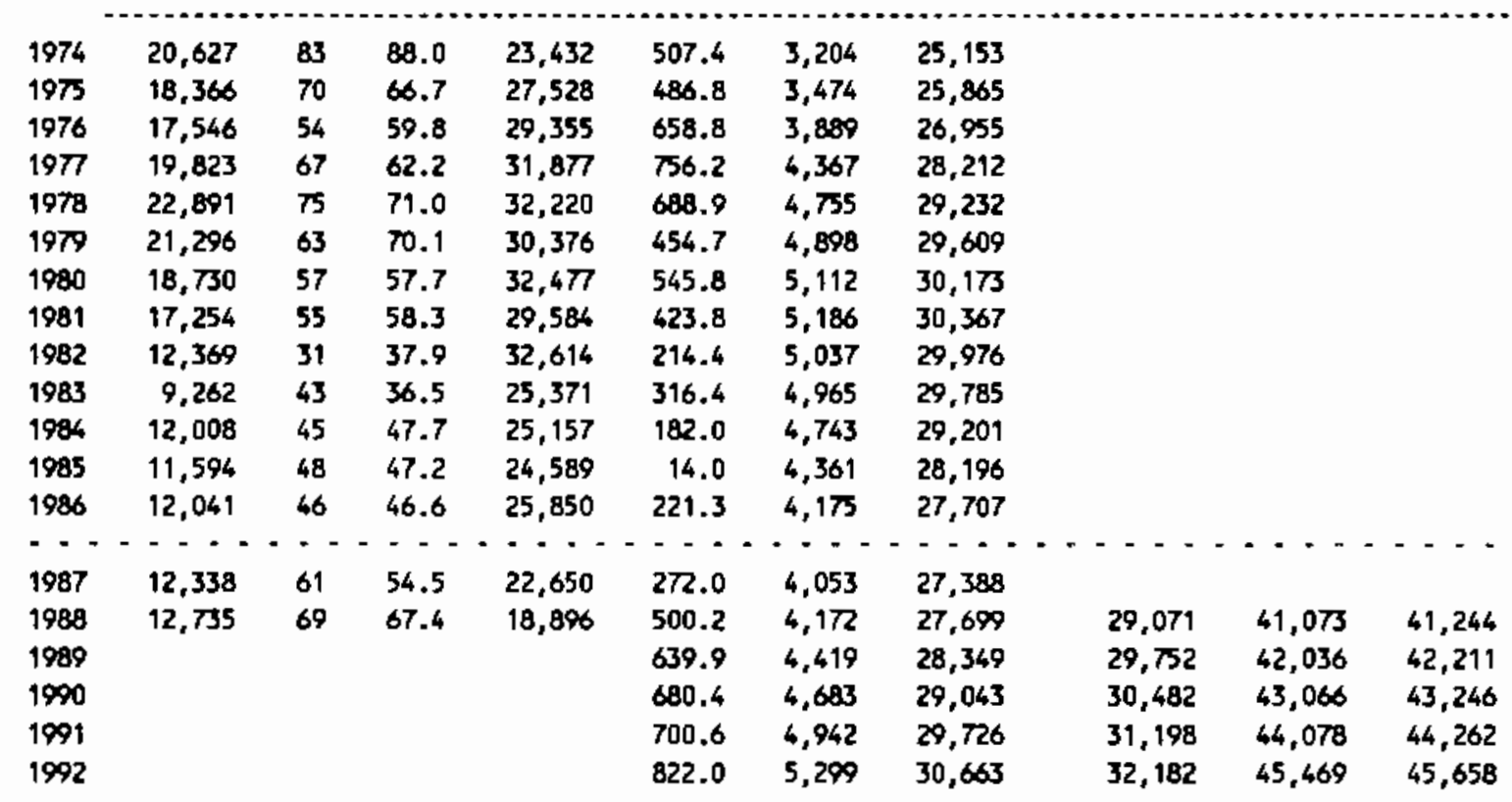

Capecity-Capital stock Regression: Code 1 RSQ 0.250

Coefficients: Const 16725.7 Stock 2.6

Ave. Capacity/Stock $(74-86): 6.4$ Ave. Weekly Hours (High) 105.8 shift Factor 1.49 Ave Heekly Hours (Low) 103.4 shift Factor 1.52 Note: Output, Investment, and Capital Stock extrapolated for 1987 and 1988.

\section{Construction machinery}

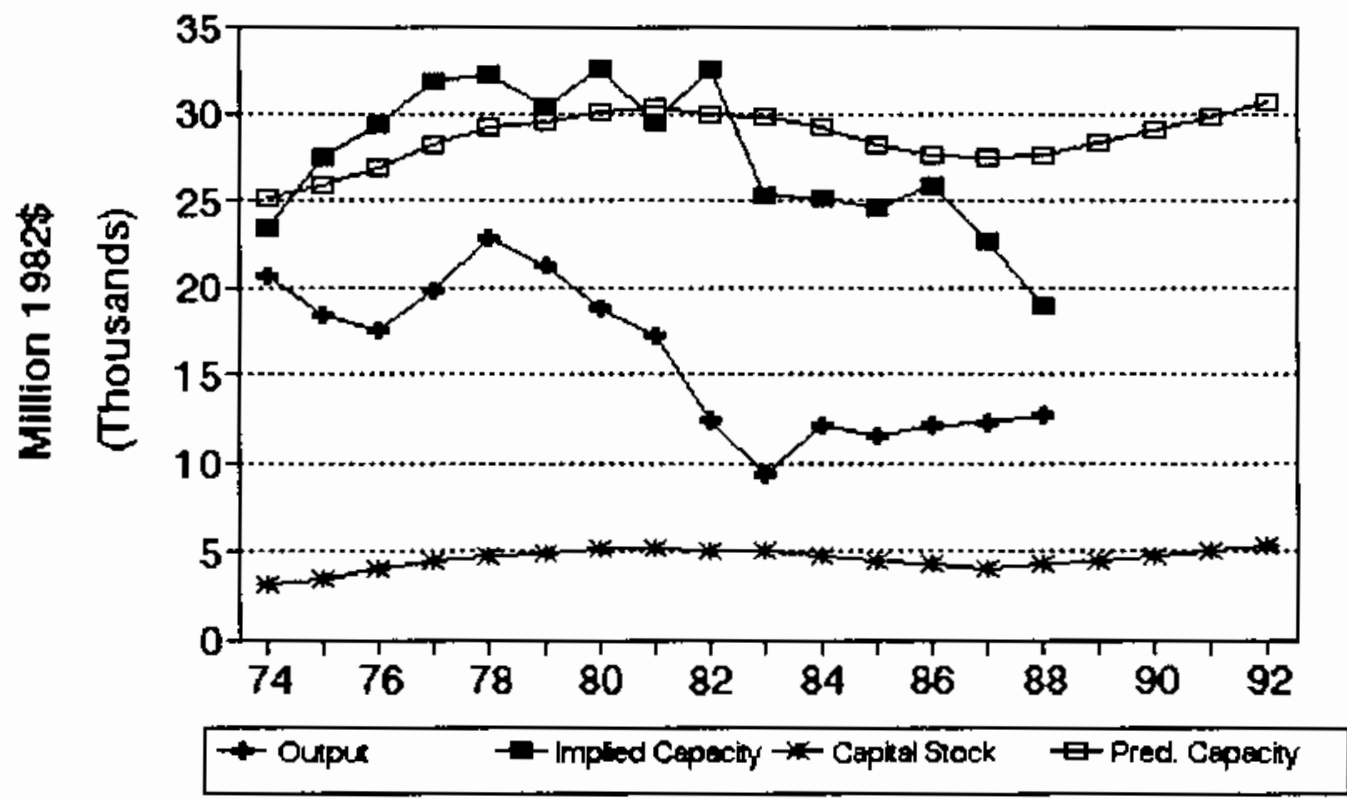




\section{No. 321 sIC 3532 wining machinery}

MILLIONS OF 1982 DOLLARS (EXCL. CU RATES)

$\begin{array}{lccccccc} & \text { Q4 } & \text { Ann. } & \text { Implied } & \text { Gross } & \text { Net } & \text { Pred. } & \ldots . . \\ \text { Gross } & \text { CU } & \text { CU } & \text { Prac. } & \text { In- } & \text { Cap. } & \text { Prac. } & \text { Emergency Capacity } \\ \text { Output } & \text { Rate } & \text { Rate } & \text { Capac. } & \text { vest. } & \text { Stk. } & \text { Capec. } & 3 \text { mon. } 6 \text { mon. }>6 \text { mon. }\end{array}$

\begin{tabular}{|c|c|c|c|c|c|c|c|c|c|c|}
\hline 1974 & 3,071 & 80 & 83.7 & 3,671 & 39.6 & 383 & 3,408 & & & \\
\hline 1975 & 3,452 & 81 & 74.7 & 4,621 & 137.9 & 491 & 3,570 & & & \\
\hline 1976 & 3,272 & 78 & 78.6 & 4,164 & 52.4 & 512 & 3,603 & & & \\
\hline 1977 & 3,131 & 80 & 79.5 & 3,937 & 69.9 & 549 & 3,658 & & & \\
\hline 1978 & 2,865 & $\pi$ & 76.3 & 3,757 & 69.6 & 585 & 3,711 & & & \\
\hline 1979 & 2,812 & 76 & 77.9 & 3,611 & 57.8 & 605 & 3,742 & & & \\
\hline 1980 & 2,867 & 70 & 70.8 & 4,047 & 90.9 & 656 & 3,818 & & & \\
\hline 1981 & 2,730 & 78 & 77.6 & 3,520 & 25.7 & 641 & 3,796 & & & \\
\hline 1982 & 2,022 & 42 & 52.9 & 3,820 & 27.7 & 627 & $3, \pi 5$ & & & \\
\hline 1983 & 1,403 & 31 & 34.4 & 4,085 & 4.0 & 588 & 3,717 & & & \\
\hline 1984 & 1,572 & 59 & 50.1 & 3,138 & $(11.2)$ & 535 & 3,637 & & & \\
\hline 1985 & 1,534 & 61 & 60.5 & 2,537 & 13.8 & 506 & 3,593 & & & \\
\hline 1986 & 1,423 & 49 & 53.3 & 2,673 & 18.4 & 479 & 3,553 & & & \\
\hline$\cdots$ & $\cdots \cdot-$ & - & $\cdots$ & $\cdots$ & $-\cdots$ & -- & $\therefore-$ & $=\cdot$ & $+\cdot$ & -- \\
\hline 1987 & 1,456 & 55 & 52.2 & 2,787 & 3.4 & 440 & 3,494 & & & \\
\hline 1988 & 1,500 & 56 & 56.6 & 2,652 & $(2.2)$ & 398 & 3,431 & 3,784 & 5,463 & 5,487 \\
\hline 1989 & & & & & $(7.8)$ & 355 & 3,366 & 3.712 & 5,359 & 5,382 \\
\hline 1990 & & & & & $(13.4)$ & 310 & 3,298 & 3,637 & 5,251 & 5,274 \\
\hline 1991 & & & & & (19.1) & 263 & 3,228 & 3,560 & 5,139 & 5,161 \\
\hline 1992 & & & & & $(24.7)$ & 215 & 3,155 & 3,480 & 5,023 & 5,045 \\
\hline
\end{tabular}

Capacity-Capital Stock Regression: code 1 RSO 0.040

Coefficients: Const 2832.0 stock 1.5

Ave. Capacity/Stock $(74-86): 6.6$ Ave. Weekly Hours (High) 98.5 Shift Factor 1.60 Ave Heekly Hours (Lou) 90.1 Shift factor 1.75 Note: Output, Investment, and Capital Stock extrapolated for 1987 and 1988.

\section{Mining machinery}

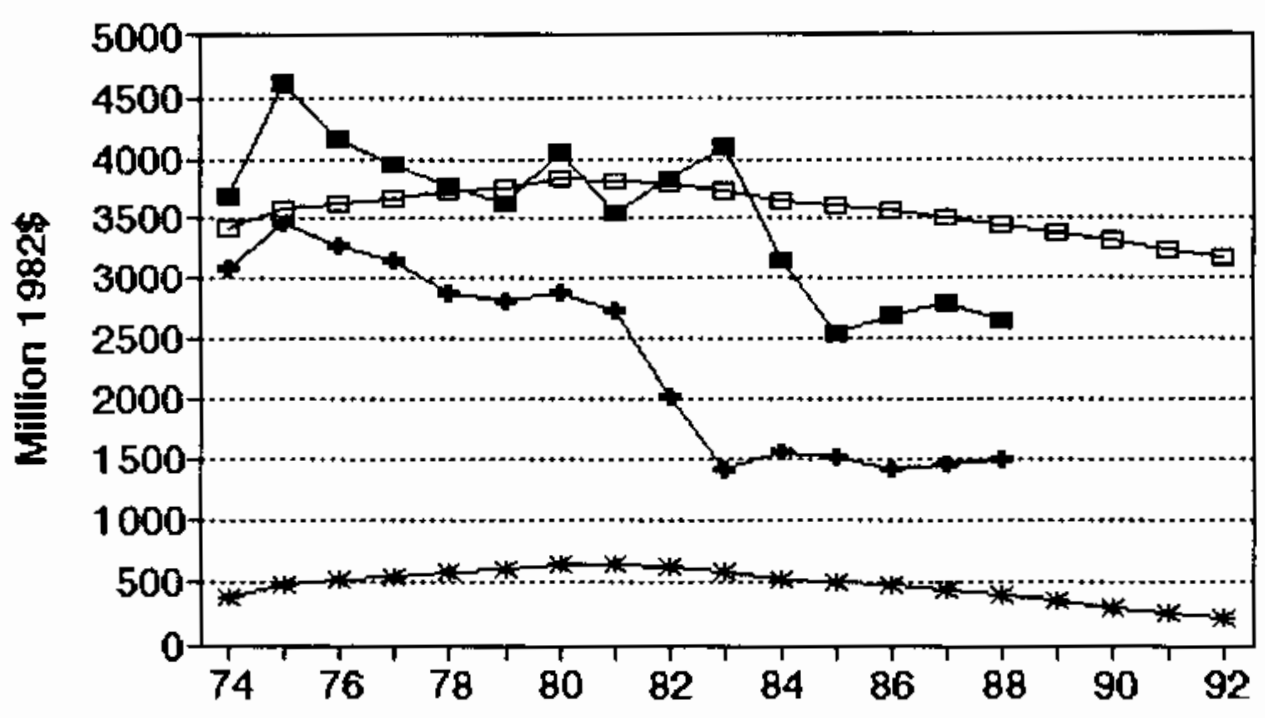

$\rightarrow$ - Oupur $\quad-\quad$ - inplied Ceapacity $\rightarrow$ Cepital Stock $\quad \boxminus$ Pred. Cepacity 


\section{Ho. 322 sIC 3533 Oil field machinery}

HILLIONS OF 1982 DOLLARS (EXCL. CU RATES)

$\begin{array}{lccccccc} & Q 4 & \text { Ann. } & \text { Implied Gross } & \text { Net } & \text { Pred. } & -\ldots \ldots \ldots \\ \text { Gross } & \alpha & \text { CU } & \text { Prac. } & \text { In- } & \text { Cap. } & \text { Prac. } & \text { Emergency Capacity } \\ \text { Output } & \text { Rate } & \text { Rate } & \text { Capac. } & \text { vest. } & \text { Stk. } & \text { Capac. } & 3 \text { mon. } 6 \text { mon. }>6 \text { mon. }\end{array}$

\begin{tabular}{|c|c|c|c|c|c|c|c|c|c|c|}
\hline 1974 & 6,462 & 76 & 79.7 & 8,113 & 326.9 & 1,192 & 8,125 & & & \\
\hline 1975 & 7,016 & 76 & 70.1 & 10,007 & 185.2 & 1,312 & 8,559 & & & \\
\hline 1976 & 6,765 & $\pi$ & 74.8 & 9,044 & 298.2 & 1,536 & 9,370 & & & \\
\hline 1977 & 7,306 & $\pi$ & 74.1 & 9,857 & 214.0 & 1,673 & 9,867 & & & \\
\hline 1978 & 8,873 & 81 & 7.2 & 11,498 & 349.5 & 1,931 & 10,802 & & & \\
\hline 1979 & 9.238 & 88 & 88.1 & 10,491 & 312.2 & 2,145 & 11,576 & & & \\
\hline 1980 & 10,545 & 84 & 83.8 & 12,588 & 379.3 & 2,412 & 12,545 & & & \\
\hline 1981 & 13,891 & 84 & 86.9 & 15,979 & 628.5 & 2,910 & 14,348 & & & \\
\hline 1982 & 11,960 & 52 & 61.5 & 19,461 & 856.3 & 3,604 & 16,860 & & & \\
\hline 1983 & 6.198 & 35 & 40.5 & 15,302 & 51.4 & 3,475 & 16,393 & & & \\
\hline 1984 & 5,519 & 48 & 45.1 & 12,237 & $(365.8)$ & 2,944 & 14,470 & & & \\
\hline 1985 & 5,341 & 42 & 44.4 & 12,040 & (186.1) & 2,594 & 13,204 & & & \\
\hline 1986 & 3,411 & 20 & 27.9 & 12,209 & 40.2 & 2,457 & 12.707 & & & \\
\hline - - - & $\because \cdots$ & -- & $-\cdots$ & $\therefore$ & $\begin{array}{l}-\cdot-\cdot- \\
775\end{array}$ & - 516 & 12071 & $-\cdots$ & $\cdots--$ & $-\cdots$ \\
\hline $\begin{array}{l}7987 \\
1988\end{array}$ & $\begin{array}{l}3,507 \\
3,622\end{array}$ & $\begin{array}{l}31 \\
35\end{array}$ & $\begin{array}{l}26.5 \\
34.0\end{array}$ & $\begin{array}{l}13,225 \\
10,647\end{array}$ & $\begin{array}{l}225.2 \\
232.6\end{array}$ & $\begin{array}{l}2,516 \\
2,570\end{array}$ & $\begin{array}{l}12,921 \\
13,148\end{array}$ & 11,514 & 17,312 & 17,394 \\
\hline 1989 & & & & & 242.6 & 2,647 & 13,395 & 11,730 & 17,637 & 17,721 \\
\hline 1990 & & & & & 243.3 & 2,711 & 13,628 & 11,934 & 17,944 & 18,030 \\
\hline 1991 & & & & & 244.7 & 2,773 & 13,850 & 12,129 & 18,237 & 18,324 \\
\hline 1992 & & & & & 237.6 & 2,823 & 14,032 & 12,288 & 18,476 & 18,565 \\
\hline
\end{tabular}

Capacity-Capital stock Regression: Code 0 \&SO 0.820 Coefficients: Const 3807.1 stock 3.6 Ave. Capacity/stock $(74-86): 5.3$ Ave. Weekly Hours (High) 119.4 Shift Factor 1.32 Ave Heekly Hours (Low) 111.4 Shift Factor 1.41 Note: Output, Investment, and Capital Stock extrapolated for 1987 and 1988.

\section{Oil field machinery}

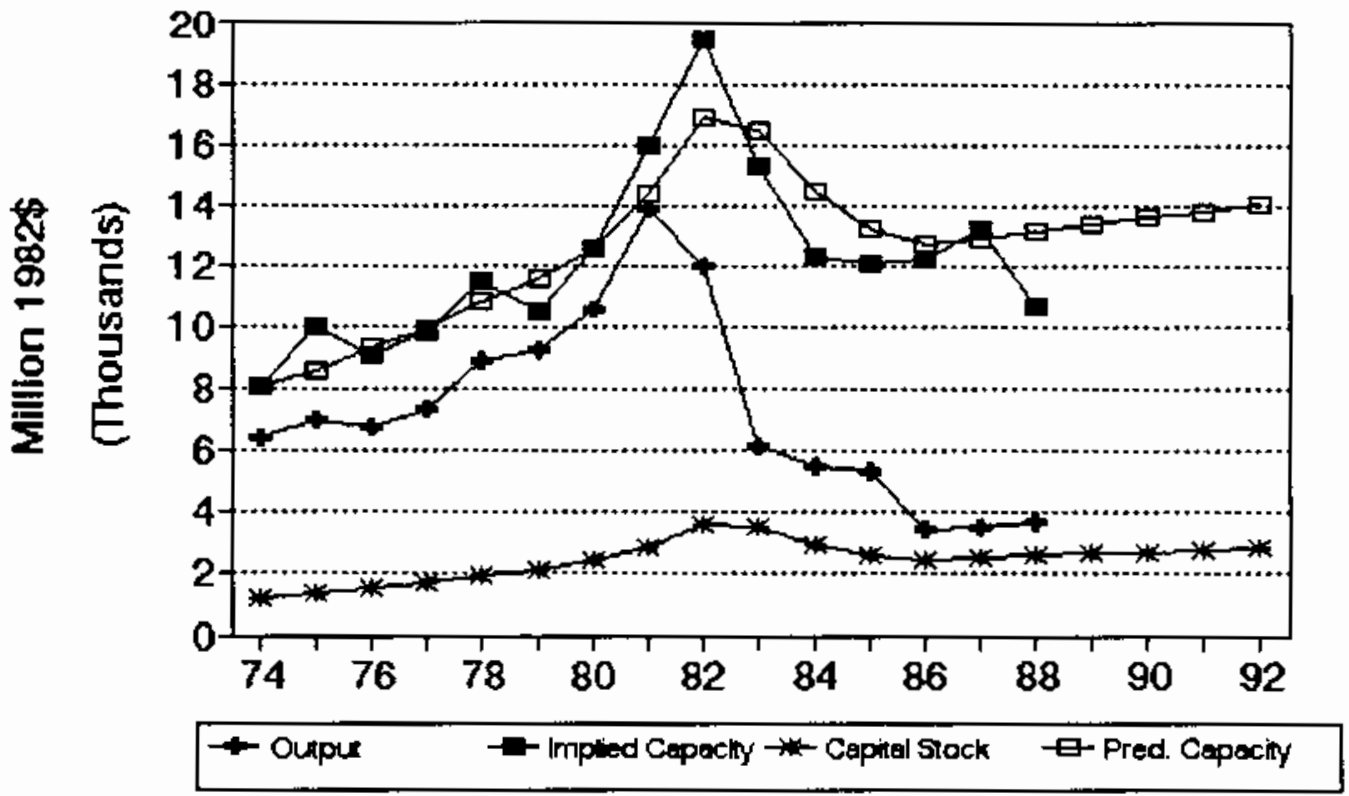


No. 323 gIC 3534 Elevators and moving stairways

MILLIONS OF 1982 DOLLARS (EXCL. CU RATES)

$\begin{array}{lccccccc} & \text { Q4 } & \text { Ann. } & \text { Implied Gross } & \text { Net } & \text { Pred. } & \ldots \ldots \ldots \\ \text { Gross } & \text { CU } & \text { CU } & \text { Prac. } & \text { In- } & \text { Cap. } & \text { Prac. } & \text { Energency Capacity } \\ \text { Output } & \text { Rate } & \text { Rate } & \text { Capac. } & \text { vest. } & \text { Stk. } & \text { Capac. } & 3 \text { mon. } 6 \text { mon. }>6 \text { mon. }\end{array}$

\begin{tabular}{|c|c|c|c|c|c|c|c|c|c|c|}
\hline 1974 & 1,035 & 80 & 82.8 & 1,250 & 22.3 & 151 & 1,350 & & & \\
\hline 1975 & 672 & 73 & 71.1 & 945 & 18.0 & 158 & 1,501 & & & \\
\hline 1976 & 591 & 60 & 64.7 & 914 & 1.1 & 148 & 1,266 & & & \\
\hline 1977 & 711 & 65 & 63.2 & 1,124 & 17.0 & 152 & 1,373 & & & \\
\hline 1978 & 744 & 63 & 63.3 & 1,175 & 8.7 & 148 & 1,277 & & & \\
\hline 1979 & 882 & 66 & 66.3 & 1,330 & $(1.6)$ & 135 & 976 & & & \\
\hline 1980 & 803 & 79 & 73.2 & 1,098 & 29.4 & 152 & 1,366 & & & \\
\hline 1981 & 914 & 61 & 69.2 & 1,320 & 15.2 & 155 & 1,439 & & & \\
\hline 1982 & 1,178 & 41 & 47.2 & 2,497 & 31.2 & 173 & 1,848 & & & \\
\hline 1983 & 1,146 & $52 *$ & 46.9 & 2,443 & $(9.2)$ & 152 & 1,373 & & & \\
\hline 1984 & 1,192 & 76 & 69.0 & 1,729 & 27.6 & 167 & 1,706 & & & \\
\hline 1985 & 1,204 & 78 & 77.4 & 1,555 & 17.2 & 171 & 1,788 & & & \\
\hline 1986 & 1,222 & 70 & 72.8 & 1,678 & 14.7 & 171 & 1,797 & & & \\
\hline$-\cdots$ & $-\cdots$ & $\cdots$ & $\cdots$ & $\therefore-$ & $--\cdot$ & -- & $-\cdots$ & $-\cdot--$ & --- & --- \\
\hline 1987 & 1,202 & 74 & 72.0 & 1,670 & 14.1 & 171 & 1,800 & & & \\
\hline 1988 & 1,177 & 76 & 76.1 & 1,547 & 21.7 & 179 & 1,976 & 3,000 & 4,339 & 4,358 \\
\hline 1989 & & & & & 31.9 & 196 & 2,370 & 3,598 & 5,205 & 5,227 \\
\hline 1990 & & & & & 32.6 & 213 & 2,748 & 4,173 & 6,035 & 6,062 \\
\hline 1991 & & & & & 34.0 & 229 & 3,128 & 4,750 & 6,871 & 6,901 \\
\hline 1992 & & & & & 26.8 & 237 & 3,313 & 5,030 & 7,276 & 7,308 \\
\hline
\end{tabular}

Capecity-Capital Stock Regression: Code 0 RSO 0.250

Coefficients: Const -2106.7 stock 22.8

Ave. Capacity/Stock $(74-86): 9.4$ Ave. Heekly Hours (High) 71.4 Shift Factor 2.21 Ave Weekly Hours (Low) 66.8 Shift Factor 2.36 * Imputed Hote: Output, Investment, and Capital Stock extrapolated for 1987 and 1988.

\section{Elevators and moving stairways}

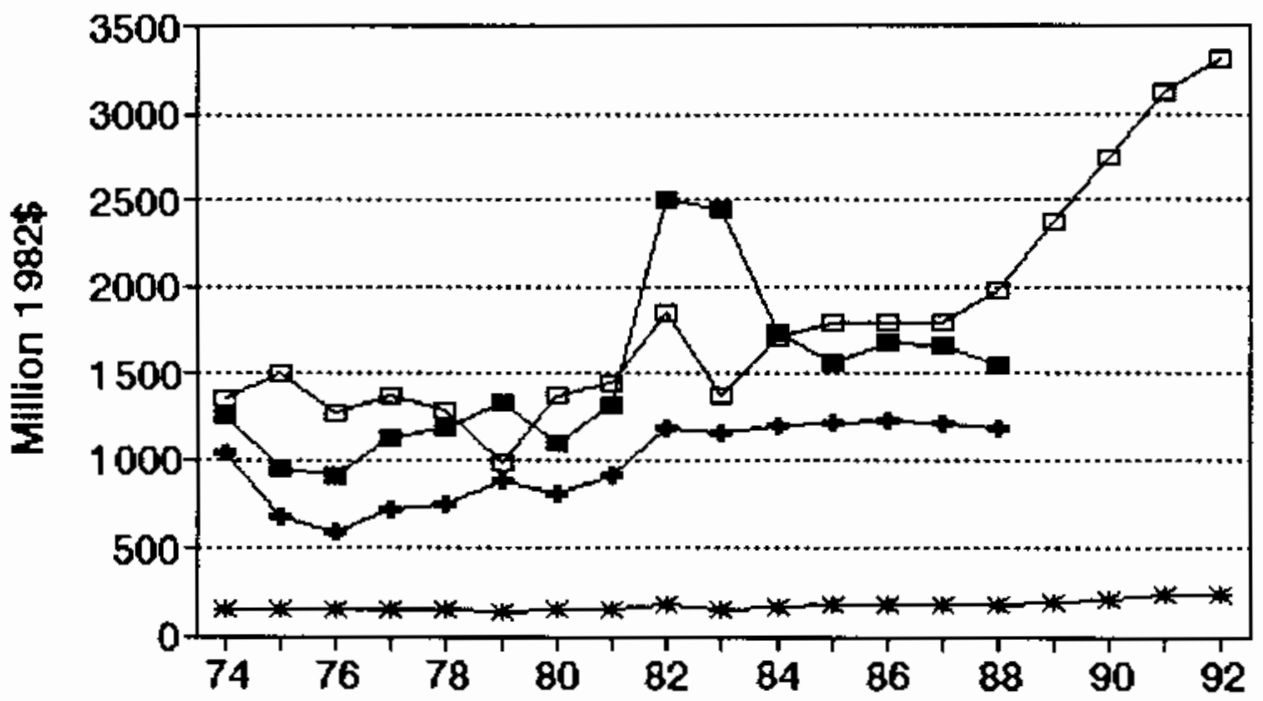

$\rightarrow$ Outpu $\rightarrow-$ Impled Capaciy $\rightarrow$ - Cepital Stock $\quad$ - Pred. Capacity 
No. 324 SIC 3535 Conveyors and conveying equipment.

MILLIONS OF 1982 DOLLARS (EXCL. CU RATES)

$\begin{array}{lccccccc} & \text { a4 } & \text { Ann. } & \text { Implied } & \text { Gross } & \text { Net } & \text { Pred. } & \ldots \ldots \ldots \ldots \\ \text { Gross } & \text { CU } & \text { CU } & \text { Prac. } & \text { In- } & \text { Cap. } & \text { Prac. } & \text { Energency Capacity } \\ \text { Output } & \text { Rate } & \text { Rate } & \text { Capac. } & \text { vest. } & \text { Stk. } & \text { Capac. } & 3 \text { mon. } 6 \text { mon. }\end{array}$

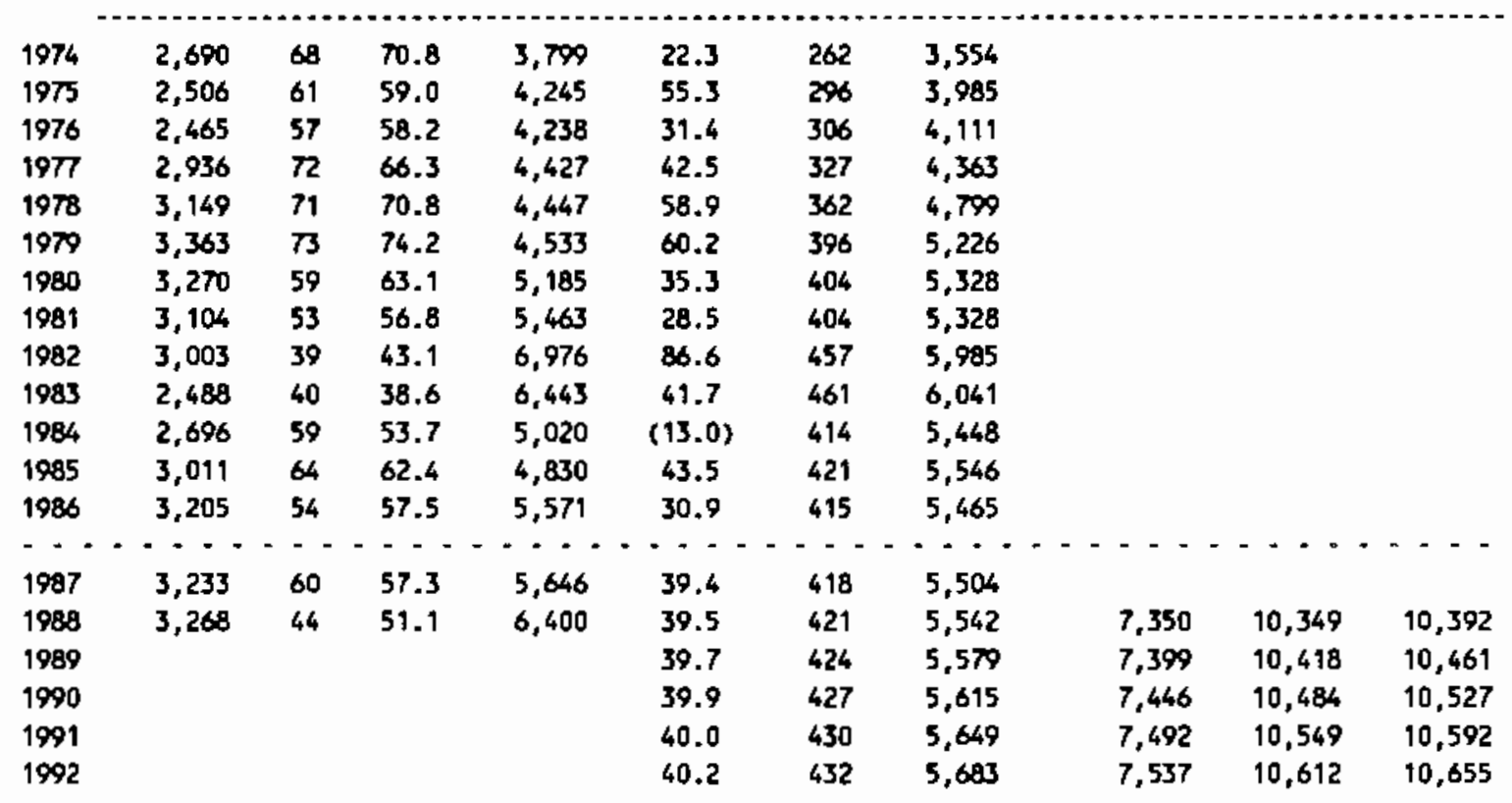

Capecity-Cepital Stock Regression: Code 0 RSO 0.740

Coefficients: Const 286.4 Stock 12.5 Ave. Capacity/Stock (74-86): 13.2 Ave. Weekly Hours (High) 84.0 Shift Factor 1.88 Ave Weekly Hours (Low) 79.1 Shift Factor 1.99 Note: Output, Investment, and Capital Stock extrapolated for 1987 and 1988.

\section{Conveyors and conveying equipment}

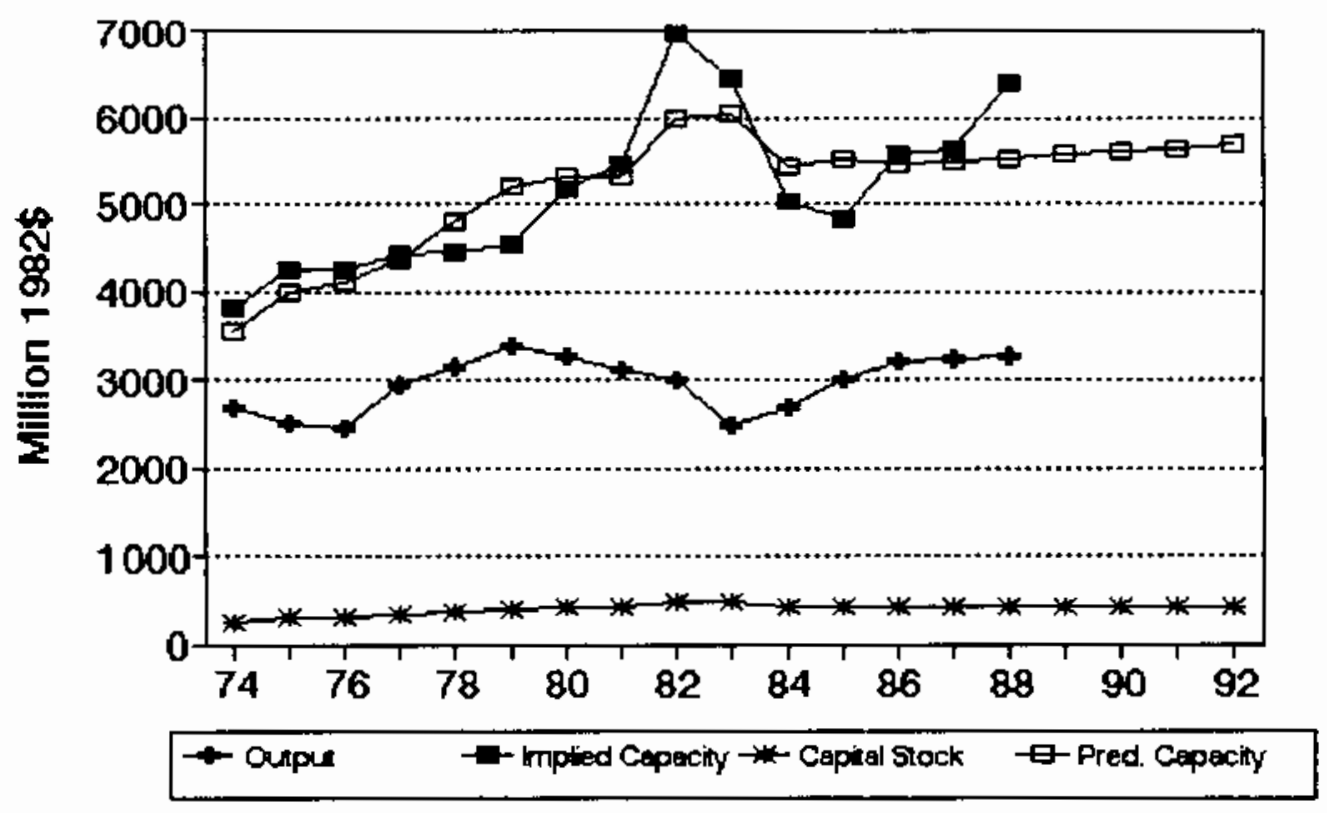


Ho. 325 sIc 3536 Hoist, cranes, and monorailg

MILLIONS OF 1982 DOLLARS (EXCL. CU RATES)

$\begin{array}{lccccccc} & 04 & \text { Ann. } & \text { Implied Gross } & \text { Net } & \text { Pred. } & \ldots \ldots \ldots \\ \text { Gross } & \text { CU } & \text { CU } & \text { Prac. } & \text { In- } & \text { Cap. } & \text { Prac. } & \text { Emergency Capacity } \\ \text { Output } & \text { Rate } & \text { Rate } & \text { Cepac. vest. } & 5 \text { tk. } & \text { Capac. } & 3 \text { mon. } 6 \text { mon. }\end{array}$

\begin{tabular}{|c|c|c|c|c|c|c|c|c|c|c|}
\hline 1974 & 1,521 & 79 & 82.1 & 1,852 & 4.4 & 228 & 2,066 & & & \\
\hline 1975 & 1,354 & 79 & 73.9 & 1,031 & 14.3 & 227 & 2,066 & & & \\
\hline 1976 & 1,388 & 69 & 72.4 & 1,917 & 20,1 & 232 & 2,076 & & & \\
\hline 1977 & 1,214 & 63 & 65.6 & 1,853 & $(9.0)$ & 208 & 2,023 & & & \\
\hline 1978 & 1,358 & 73 & 68.6 & 1,979 & 27.3 & 220 & 2,048 & & & \\
\hline 1979 & 1,386 & 72 & 74.2 & 1,868 & (13.2) & 192 & 1,986 & & & \\
\hline 1980 & 1,453 & 63 & 65.3 & 2,227 & 24.6 & 201 & 2,005 & & & \\
\hline 1981 & 1,561 & 56 & 60.2 & 2,594 & 62.2 & 242 & 2,099 & & & \\
\hline 1982 & 1,053 & 38 & 43.4 & 2,425 & 13.5 & 234 & 2,081 & & & \\
\hline 1983 & 795 & 52 & 45.4 & 1,750 & 3.2 & 215 & 2,038 & & & \\
\hline 1984 & 822 & 28 & 39.3 & 2,091 & $(44.8)$ & 154 & 1,900 & & & \\
\hline 1985 & 904 & $30 *$ & 29.3 & 3,085 & 22.1 & 158 & 3,106 & & & \\
\hline 1986 & 849 & $26 *$ & 27.4 & 3,095 & 4.4 & 144 & 3,074 & & & \\
\hline$\ddot{*}=$ & $\cdots$ & $\cdots$ & $-\cdot-$ & - - - - & $\cdots$ & $\because \cdot$ & $\because-$ & $-=$ & $\cdots$ & -- \\
\hline 1987 & 862 & $28 *$ & 27.3 & 3,159 & 10.8 & 138 & 1,863 & & & \\
\hline 1988 & 880 & $22 \star$ & 25.0 & 3,522 & 12.5 & 134 & 1,854 & 1,438 & 1,848 & 1,854 \\
\hline 1989 & & & & & 14.8 & 132 & 1,851 & 1,436 & 1,845 & 1,851 \\
\hline 1990 & & & & & 15.0 & 131 & 1,849 & 1,434 & 1,843 & 1,849 \\
\hline 1991 & & & & & 15.3 & 131 & 1,848 & 1,433 & 1,842 & 1,848 \\
\hline 1992 & & & & & 13.7 & 129 & 1,843 & 1,430 & 1,837 & 1,843 \\
\hline
\end{tabular}

Capacity-Capital Stock Regression: Code -1 RSO 0.730

Coefficients: Const 2748.5 stock $2.3 \mathrm{D}(74 \cdot 84)-1196.5$ Ave. Capacity/Stack (74-86): 10.8 Ave. Weekly Hours (High) 1984.0 Shift Factor 1.00 Ave Heekly Hours (Low) 1984.0 Shift Factor 1.0 - Imputed Note: Output, Investment, and Capital 5tock extrapolated for 1987 and 1988.

3536 Hoist, cranes, and monorails

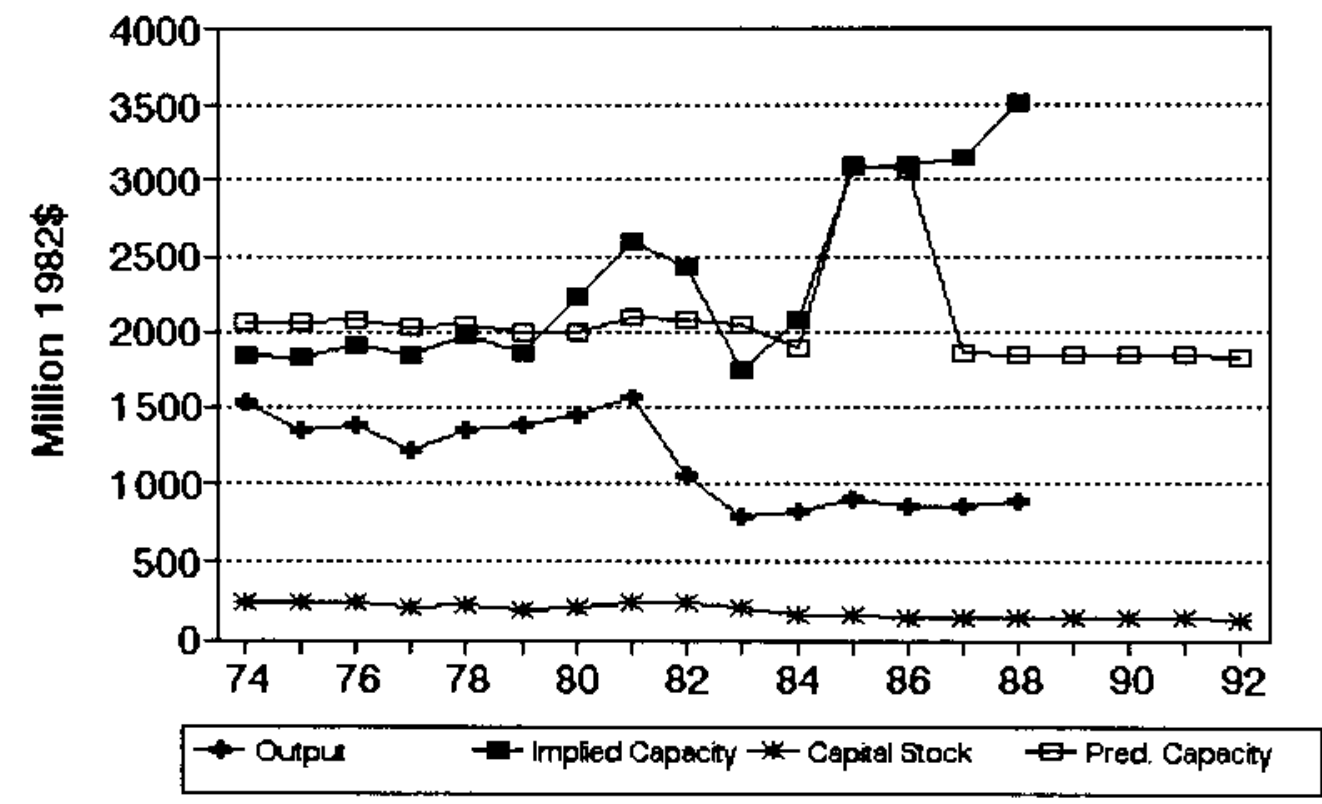




\section{No. 326 sIC 3537 Industrial trucks and tractors}

Milliows of 1982 dollars (EXCL. CU RATES)

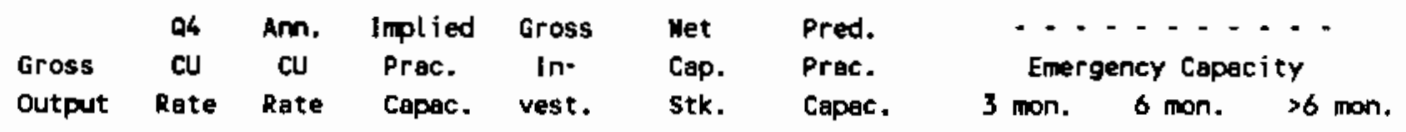

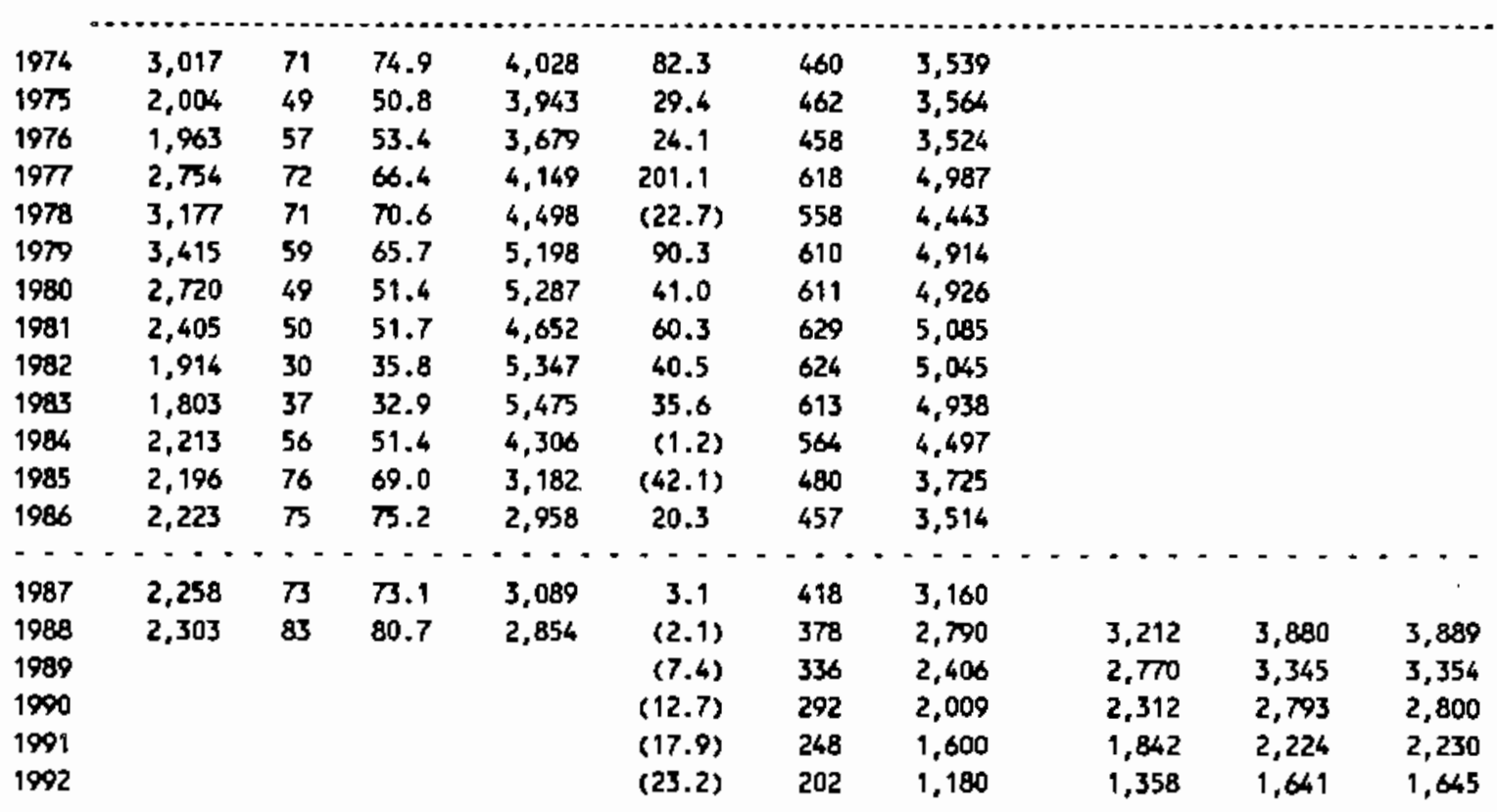

Capacity-Capital Stock Regression: Code 1 RSQ 0.690 Coefficients: Const -665.4 stock 9.2

Ave. Capecity/stock $(74-86): 7.9$ Ave. Weekly Hours (High) 113.1 Shift Factor 1.39 Ave Heekly Hours (Low) 112.0 Shift Factor 1.41 Note: Output, Imvestment, and Capital Stock extrapolated for 1987 and 1988.

\section{Industrial trucks and tractors}

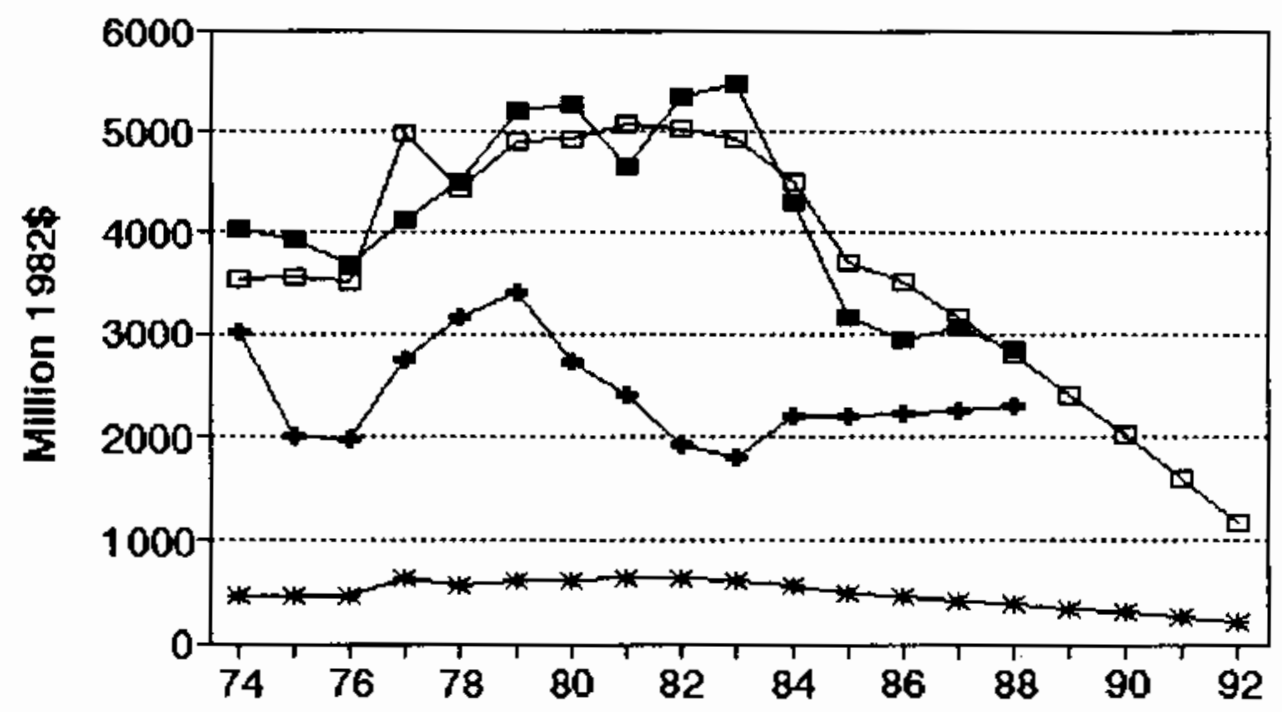

- Oupur $\quad-$ mplied Capeciny $\rightarrow$ - Cepitel Stock $\square$ - Pred Capaciny 


\section{No. 327 sIC 3541 Machine tools, netal cutting types}

MILLIOWS OF 1982 DOLLARS (EXCL. CU RATES)

$\begin{array}{lccccccc} & \text { O4 } & \text { Amn. Implied Gross } & \text { Net } & \text { Pred. } & -\ldots . \ldots \\ \text { Gross } & \text { CU } & \text { CU } & \text { Prac. } & \text { In- } & \text { Cap. } & \text { Prac. } & \text { Emergency Capacity } \\ \text { Output } & \text { Rate } & \text { Rate } & \text { Capac. } & \text { vest. } & \text { Stk. } & \text { Capac. } & 3 \text { mon. } 6 \text { mon. }>6 \text { mon. }\end{array}$

\begin{tabular}{|c|c|c|c|c|c|c|c|c|c|c|}
\hline 1974 & 5,929 & 65 & 67.7 & 8,757 & 157.5 & 1,334 & 7,648 & & & \\
\hline 1975 & 5,310 & 72 & 65.0 & 8,166 & 119.6 & 1,337 & 7,668 & & & \\
\hline 1976 & 4,658 & 66 & 67.9 & 6,863 & 138.7 & 1,354 & 7,770 & & & \\
\hline 1977 & 5,036 & 67 & 66.8 & 7,536 & 82.1 & 1,317 & 7,546 & & & \\
\hline 1978 & 5,722 & 70 & 68.3 & 8,381 & 114.7 & 1,311 & 7,513 & & & \\
\hline 1979 & 6,084 & 70 & 71.9 & 8,466 & 281.1 & $\uparrow, 459$ & 8,389 & & & \\
\hline 1980 & 6,127 & 70 & 68.8 & 8,905 & 136.0 & 1,464 & 8,423 & & & \\
\hline 1981 & 6,257 & 66 & 69.5 & 9,010 & 181.1 & 1,518 & 8,743 & & & \\
\hline 1982 & 4,272 & 41 & 48.6 & 8,792 & 97.1 & 1,490 & 8,577 & & & \\
\hline 1983 & 2,685 & 46 & 42.9 & 6,256 & 31.8 & 1,403 & 8,057 & & & \\
\hline 1984 & 3,006 & 43 & 46.3 & 6,496 & 9.5 & 1,302 & 7,457 & & & \\
\hline 1985 & 3,190 & 46 & 45.0 & 7,084 & 44.3 & 1,239 & 7,085 & & & \\
\hline 1986 & 2,991 & 42 & 43.4 & 6,896 & 46.4 & 1,180 & 6,730 & & & \\
\hline 1987 & 2,985 & 50 & $\begin{array}{l}-\cdots \\
46.6\end{array}$ & 6,413 & $\begin{array}{l}\cdots \\
109.5\end{array}$ & $\cdots$ & 6,764 & $\cdots$ & --- &.-- \\
\hline 1988 & 2,978 & 63 & 58.8 & 5,069 & 142.7 & 1,223 & 6,991 & 6,824 & 9,649 & 9,690 \\
\hline 1989 & & & & & 187.2 & 1,303 & 7,463 & 7,285 & 10,301 & 10,344 \\
\hline 1990 & & & & & 190.4 & 1,378 & 7,913 & 7,724 & 10,922 & 10,967 \\
\hline 1991 & & & & & 196.7 & 1,454 & 8,360 & 8,160 & 11,539 & 11,587 \\
\hline 1992 & & & & & 165.1 & 1,491 & 8,580 & 8,375 & 11,843 & 11,893 \\
\hline
\end{tabular}

Capacity-Capital Stock Regression: Code 0 RSQ 0.360

Coefficients: Const -281.3 stock 6.0

Ave. Capacity/Stock $(74-86): 5.7$ Ave. Heekly Hours (High) 113.8 Shift Factor 1.39 Ave Heekly Hours (LOW) 110.1 Shift Factor 1.43 Note: Output, Investment, and Capital stock extrapolated for 1987 and 1988.

3541 Machine tools, metal cutting types

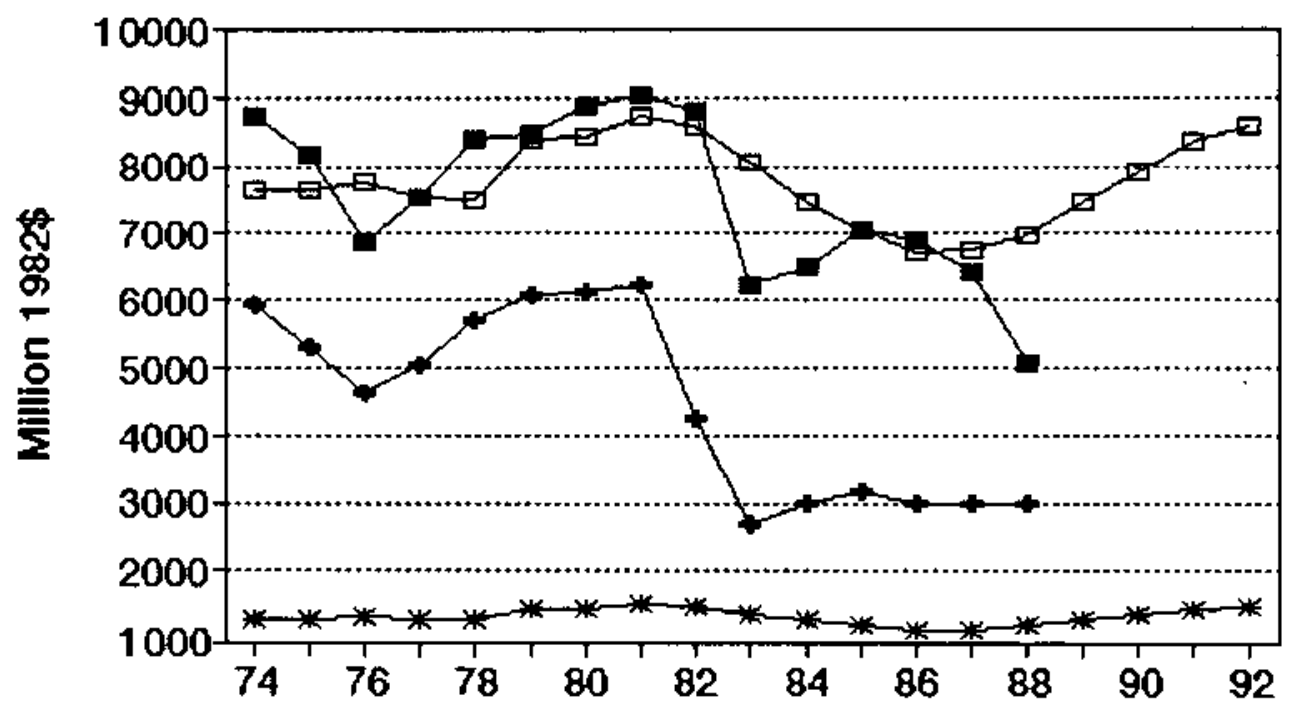

$\rightarrow$ - Oupur $\rightarrow-$ Implied Ceapacity $\rightarrow$ Cepitel Sock $\square$ - Pred. Capacity 
No. 328 sIc 3542 Machine tools, metal forming types

MILLIONS OF 1982 DOLLARS (EXCL. CU RATES)

$\begin{array}{lccccccc} & Q 4 & \text { Ann. } & \text { Implied } & \text { Gross } & \text { Net } & \text { Pred. } & \ldots \\ \text { Gross } & \text { CU } & \text { CU } & \text { Prac. } & \text { In- } & \text { Cap. } & \text { Prac. } & \text { Emergency Capacity } \\ \text { Output } & \text { Rate } & \text { Rate } & \text { Capac. } & \text { vest. } & 5 t k . & \text { Capac. } & 3 \text { mon. } 6 \text { mon. }>6 \text { mon. }\end{array}$

\begin{tabular}{|c|c|c|c|c|c|c|c|c|c|c|}
\hline 1974 & 2,906 & 66 & 68.7 & 4,232 & 66.9 & 700 & 3,155 & & & \\
\hline 1975 & 2,321 & 74 & 66.7 & 3,481 & 13.1 & 665 & 2,924 & & & \\
\hline 1976 & 2,032 & 71 & 71.7 & 2,834 & 5.1 & 624 & 2,649 & & & \\
\hline 1977 & 2,028 & 72 & 71.8 & 2,823 & 66.6 & 640 & 2,755 & & & \\
\hline 1978 & 2,175 & 75 & 73.3 & 2,969 & 72.9 & 660 & 2,886 & & & \\
\hline 1979 & 2,257 & 72 & $\pi .0$ & 3,009 & 95.4 & 698 & 3,139 & & & \\
\hline 1980 & 2,056 & 75 & 72.7 & 2,830 & 64.4 & 705 & 3,181 & & & \\
\hline 1981 & 1,757 & 66 & 71.3 & 2,465 & 32.4 & 684 & 3,050 & & & \\
\hline 1982 & 1,436 & 45 & 51.3 & 2,802 & 106.0 & 729 & 3,351 & & & \\
\hline 1983 & 1,081 & 38 & 39.8 & 2,719 & (57.7) & 621 & 2,627 & & & \\
\hline 1984 & 1,269 & 71 & 60.3 & 2,104 & $(14.5)$ & 561 & 2,227 & & & \\
\hline 1985 & 1,465 & 69 & 69.9 & 2,095 & 64.3 & 577 & 2,336 & & & \\
\hline 1986 & 1,372 & 61 & 63.8 & 2,151 & 30.1 & 560 & 2,222 & & & \\
\hline$\cdots$ & $\cdots--$ & $-\cdot$ & $\because \cdot$ & $-\cdots$ & $\cdots$ & $\cdots$ & $\cdots-$ & -- & $\cdots$ & $-\cdots$ \\
\hline 1987 & 1,353 & 80 & 72.1 & 1,876 & 42.5 & 555 & 2,193 & & & \\
\hline 1988 & 1,328 & 81 & 81.8 & 1.624 & 73.9 & 583 & 2,375 & 2,442 & 3,261 & 3,272 \\
\hline 1989 & & & & & 116.0 & 650 & 2,822 & 2,903 & 3,875 & 3,889 \\
\hline 1990 & & & & & 119.0 & 715 & 3,252 & 3,345 & 4,466 & 4,482 \\
\hline 1991 & & & & & 125.0 & 780 & 3,686 & 3,791 & 5,061 & 5,079 \\
\hline 1992 & & & & & 95.1 & 810 & 3,885 & 3,995 & 5,334 & 5,353 \\
\hline
\end{tabular}

Capacity-Capital Stock Regression: Code 0 RSO 0.420 Coefficients: Const -1506.9 stock 6.7

Ave. Capacity/Stock $(74-86): 4.3$ Ave. Ueekly Hours (High) 114.5 Shift Factor 1.38 Ave Ueekly Hours (Low) 102.5 Shift Factor 1.54 Note: Output, Investment, and Capital stock extrapolated for 1987 and 1988.

\section{Machine tools, metal forming types}

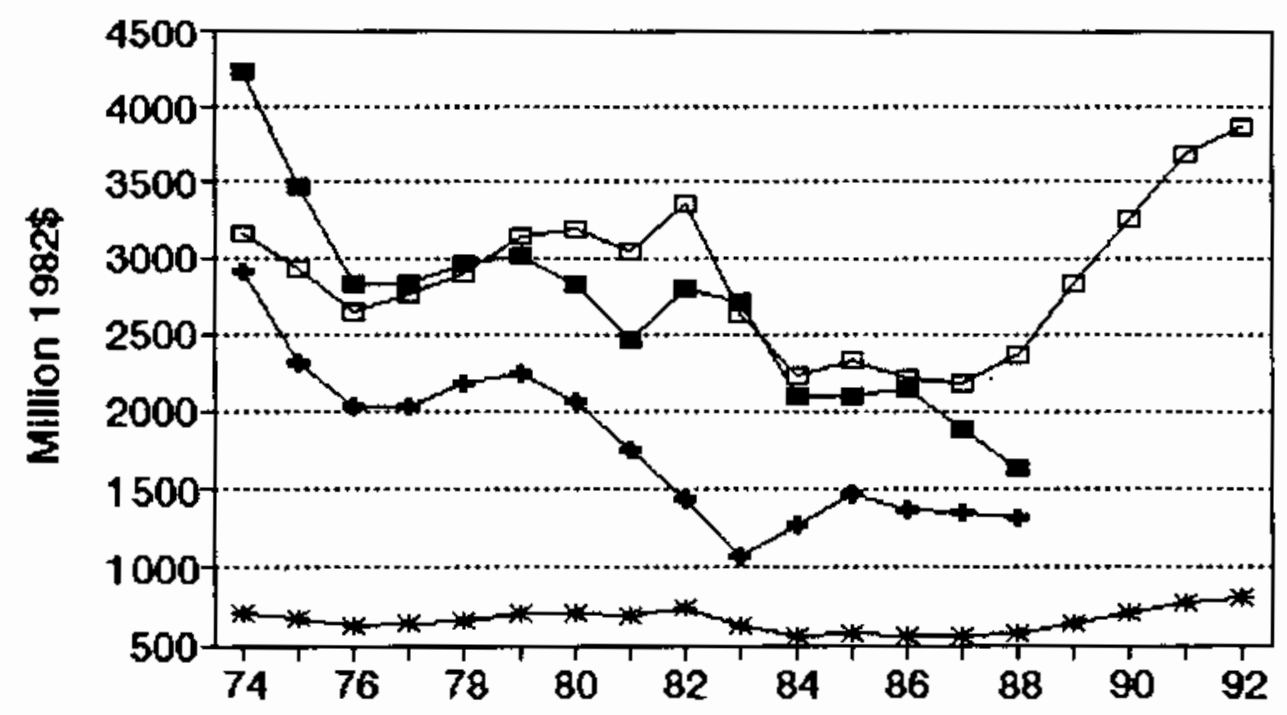

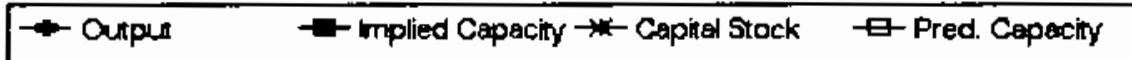


No. 329 SIC 3544 special dies, tools, jigs and fixtures MILLIONS OF 1982 DOLLARS (EXCL. CU RATES)

$\begin{array}{lccccccc} & 04 & \text { Ann. } & \text { Implied Gross } & \text { Net } & \text { Pred. } & \ldots \ldots \ldots \\ \text { Gross } & \text { CU } & \text { CU } & \text { Prac. } & \text { In- } & \text { Cap. } & \text { Prac. } & \text { Emergency Capacity } \\ \text { Output Rate } & \text { Rate } & \text { Capac. } & \text { vest. } & \text { Stk. } & \text { Capac. } & 3 \text { mon. } 6 \text { mon. }>6 \text { mon. }\end{array}$

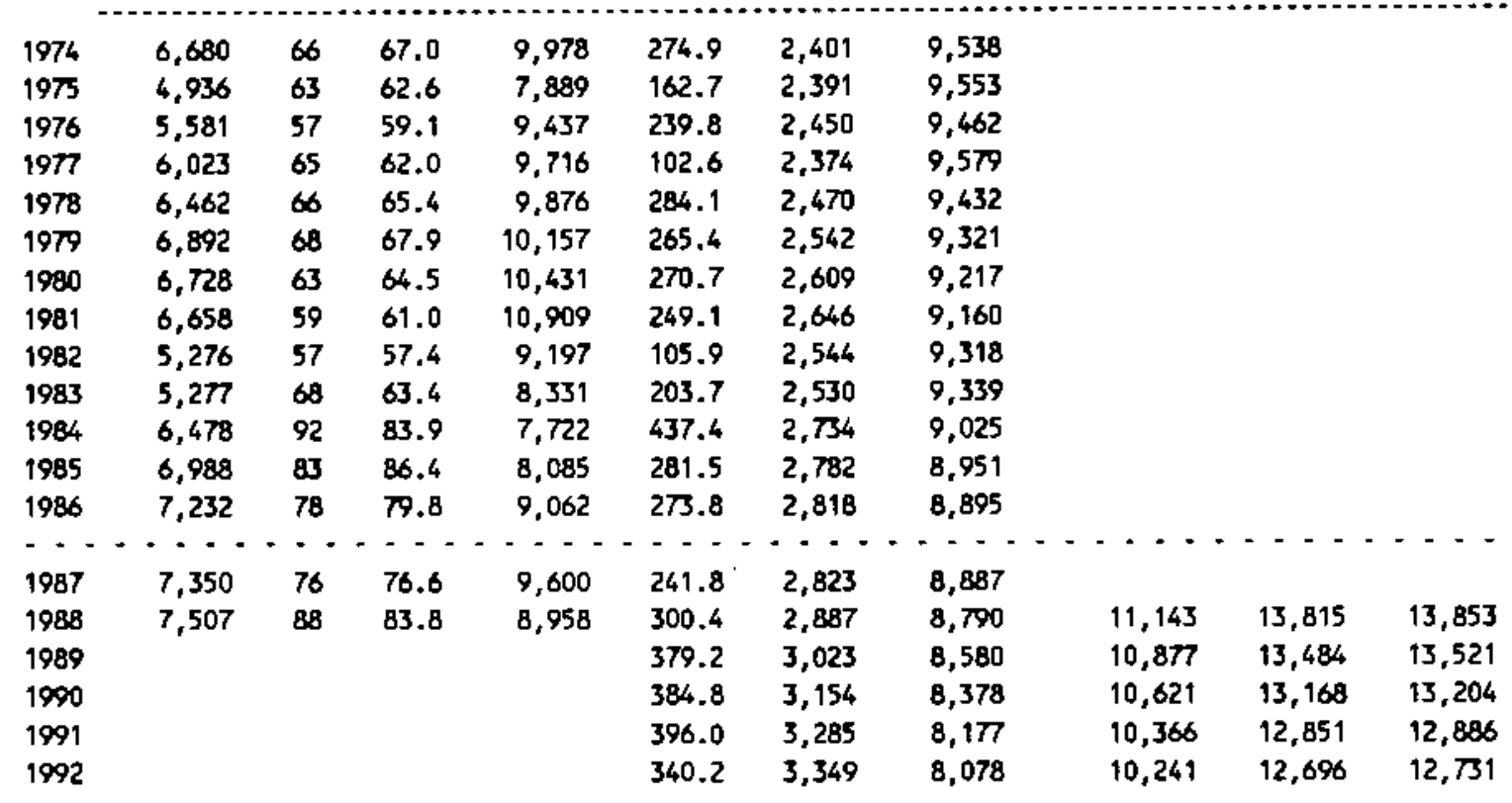

Capacity-Capital stock Regression: Code 0 RSD 0.050 Coefficients: Const 13234.7 stock -1.5

Ave. Capacity/Stock $(74-86): 3.6$ Ave. Hekkly Hours (High) 99.9 Shift Factor 1.58 Ave Weekly Hours (Low) 94.6 Shift Factor 1.66 Note: Output, Investment, and Capital Stock extrapolated for 1987 and 1988.

3544 Special dies, tools, jigs and fixt

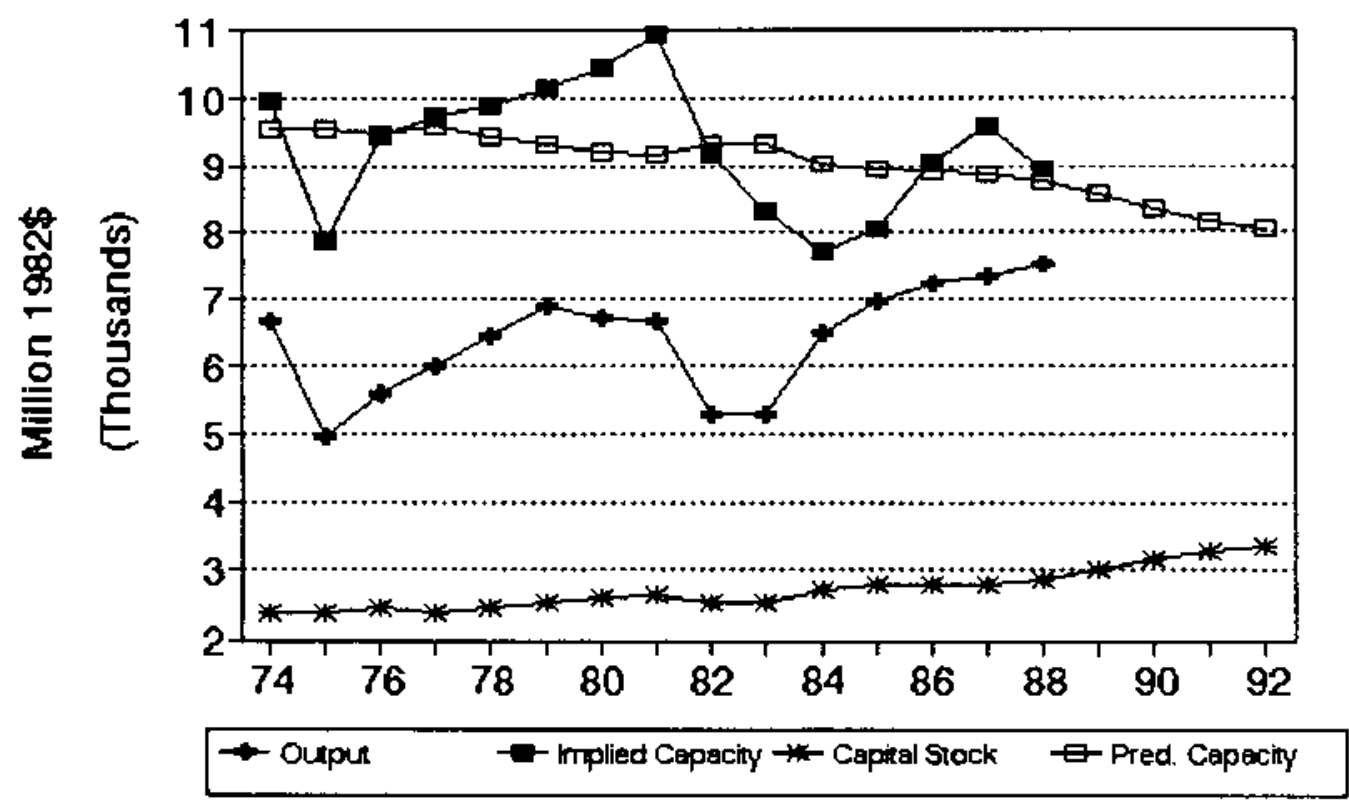




\section{No. 330 sic 3545 Machine tool accessories}

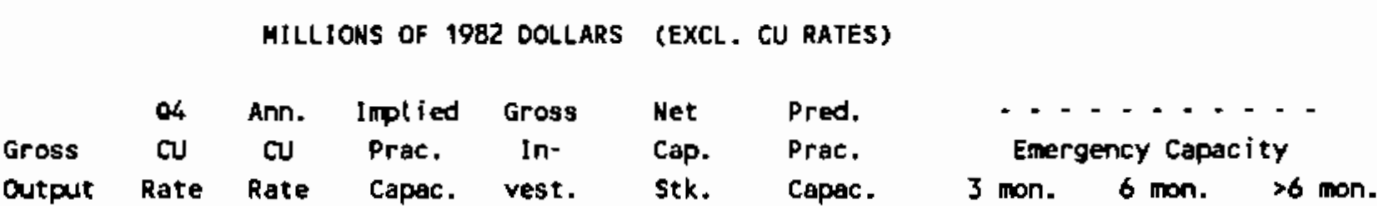

\begin{tabular}{|c|c|c|c|c|c|c|c|c|c|c|}
\hline 1974 & 3,897 & 83 & 86.1 & 4,524 & 161.1 & 1,038 & 4,908 & & & \\
\hline $19 \pi$ & 3,301 & 67 & 67.9 & 4,865 & 97.7 & 1,050 & 4,928 & & & \\
\hline 1976 & 3,324 & 74 & 70.9 & 4,692 & 45.4 & 1,011 & 4,863 & & & \\
\hline 197 & 3,661 & 82 & 79.1 & 4,628 & 149.7 & 1,071 & 4,964 & & & \\
\hline 1978 & 4,133 & 76 & 7.7 & 5,317 & 136.5 & 1,116 & 5,038 & & & \\
\hline 1979 & 4,348 & 77 & 78.5 & 5,540 & 70.7 & 1,099 & 5,010 & & & \\
\hline 1980 & 4,327 & 72 & 72.7 & 5,951 & 166.0 & 1,173 & 5,134 & & & \\
\hline 1981 & 4,339 & 72 & 73.9 & 5,872 & 216.9 & 1,293 & 5,335 & & & \\
\hline 1982 & 3,178 & 49 & 56.0 & 5,678 & 105.8 & 1,302 & 5,349 & & & \\
\hline 1983 & 2,568 & 55 & 51.5 & 4,989 & 58.4 & 1,264 & 5,286 & & & \\
\hline 1984 & 3,322 & 71 & 67.2 & 4,940 & 113.8 & $t, 283$ & 5,318 & & & \\
\hline 1985 & 3,273 & 65 & 67.4 & 4,857 & 105.2 & 1,294 & 5,336 & & & \\
\hline 1986 & 3,182 & 64 & 64.2 & 4,953 & 98.1 & 1,294 & 5,337 & & & \\
\hline $\begin{array}{l}-\cdots \\
1987\end{array}$ & 3,226 & $\dot{0}$ & $\begin{array}{l}-\cdots \\
58.1\end{array}$ & $\begin{array}{l}\cdots, 552 \\
5,5\end{array}$ & $\begin{array}{l}-\cdots \\
117.3\end{array}$ & 1,316 & $\begin{array}{c}\cdots, 373 \\
5,373\end{array}$ & $\cdots$ & $\cdots$ & $-\cdots$ \\
\hline 1988 & 3,283 & 65 & 61.9 & 5,304 & 155.1 & 1,373 & 5,469 & 7,099 & 9,902 & 9,942 \\
\hline 1989 & & & & & 205.9 & 1,477 & 5,643 & 7,325 & 10,217 & 10,259 \\
\hline 1990 & & & & & 209.5 & 1.577 & 5,810 & 7,542 & 10,520 & 10,562 \\
\hline 1991 & & & & & 216.7 & 1,676 & 5,977 & 7.758 & 10,822 & 10,856 \\
\hline 1992 & & & & & 180.7 & 1,733 & 6,071 & 7,880 & $10, \infty 93$ & 11,037 \\
\hline
\end{tabular}

Capacity-Capital Stock Regression: Code 1 RSO 0.160 Coefficients: Const 3470.2 stock 1.7

Ave. Capseity/Stock $(74-86): 4.4$ Ave. Weekly hours (High) 86.6 Shift Factor 1.82 Ave Heekly Hours (Low) 81.1 Shift factor 1.94 Note: Output, Investment, and Capital stock extrapolated for 1987 and 1988.

\section{Machine tool accessories}

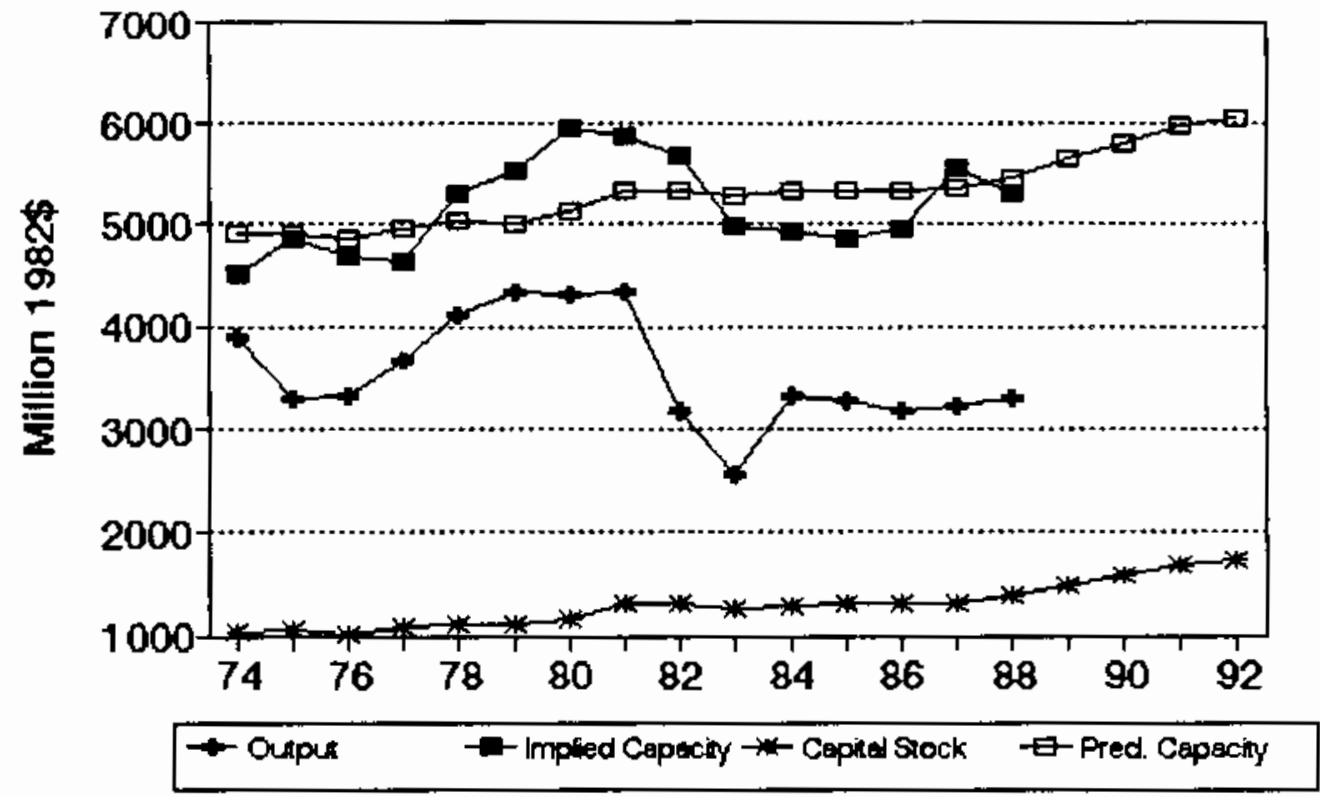


No. 331 SIC 3546 Power driven hand tools

MILLIONS OF 1982 DOLLARS (EXCL. CU RATES)

$\begin{array}{lccccccc} & \text { Q4 } & \text { Ann. Implied Gross } & \text { Net } & \text { Pred. } & \ldots \ldots \ldots \\ \text { Gross } & \text { CU } & \text { CU } & \text { Prac. } & \text { In- } & \text { Cap. } & \text { Prac. } & \text { Emergency Capacity } \\ \text { Output } & \text { Rate } & \text { Rate } & \text { Capac. } & \text { vest. } & \text { Stk. } & \text { Capac. } & 3 \text { mon. } 6 \text { mon. }>6 \text { mon. }\end{array}$

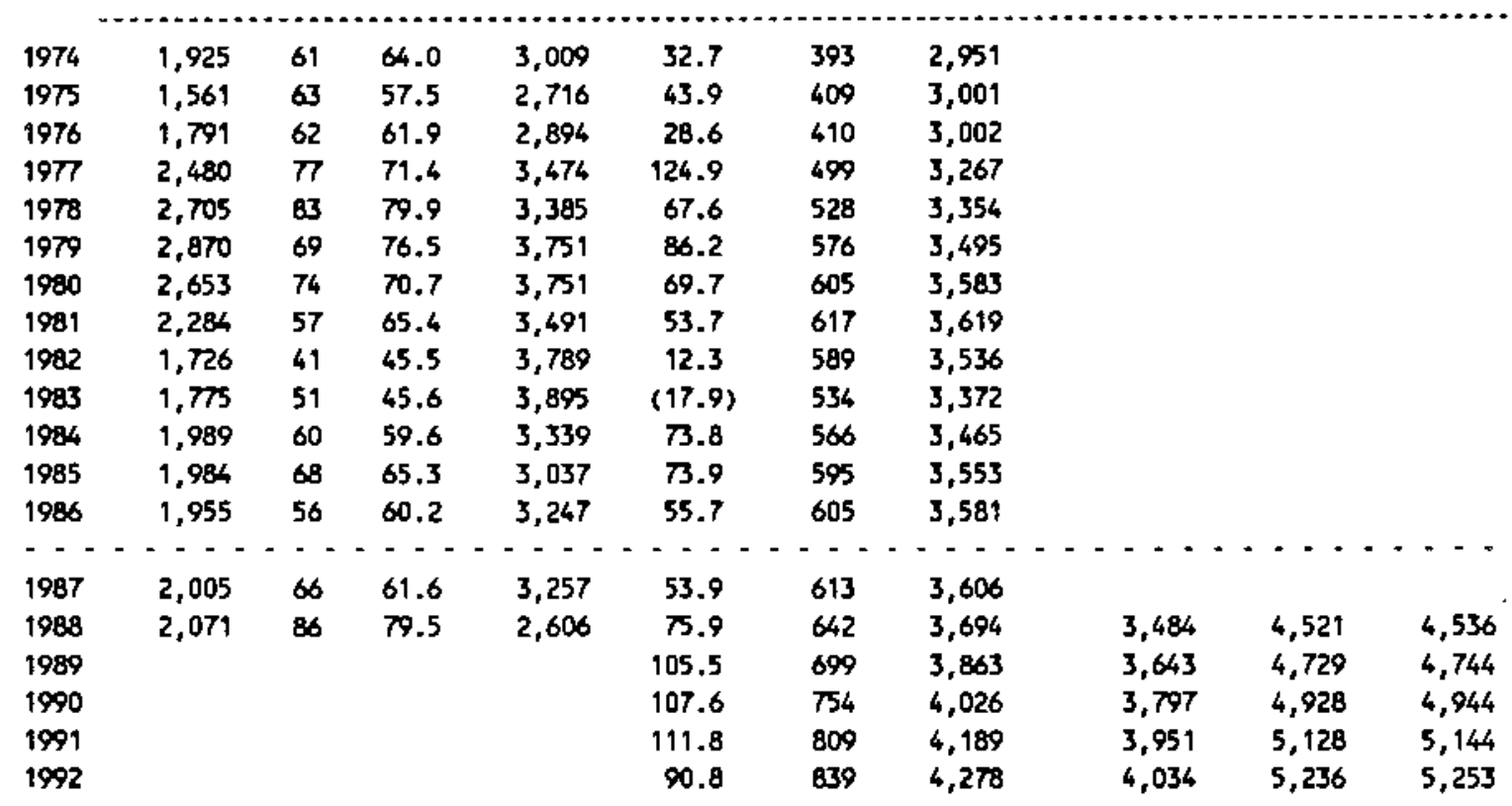

Capacity-Capital Stock Regression: Code 1 RSO 0.410

Coefficients: Const 1784.3 stock 3.0

Ave. Capacity/Stock $(74-86): 6.3$ Ave. Weekly Hours (High) 129.1 Shift Factor 1.23 Ave Weekly Hours (Low) 128.8 Shift factor 1.23 Note: Output, Investment, and Capital Stock extrapolated for 1997 and 1988.

3546 Power driven hand tools

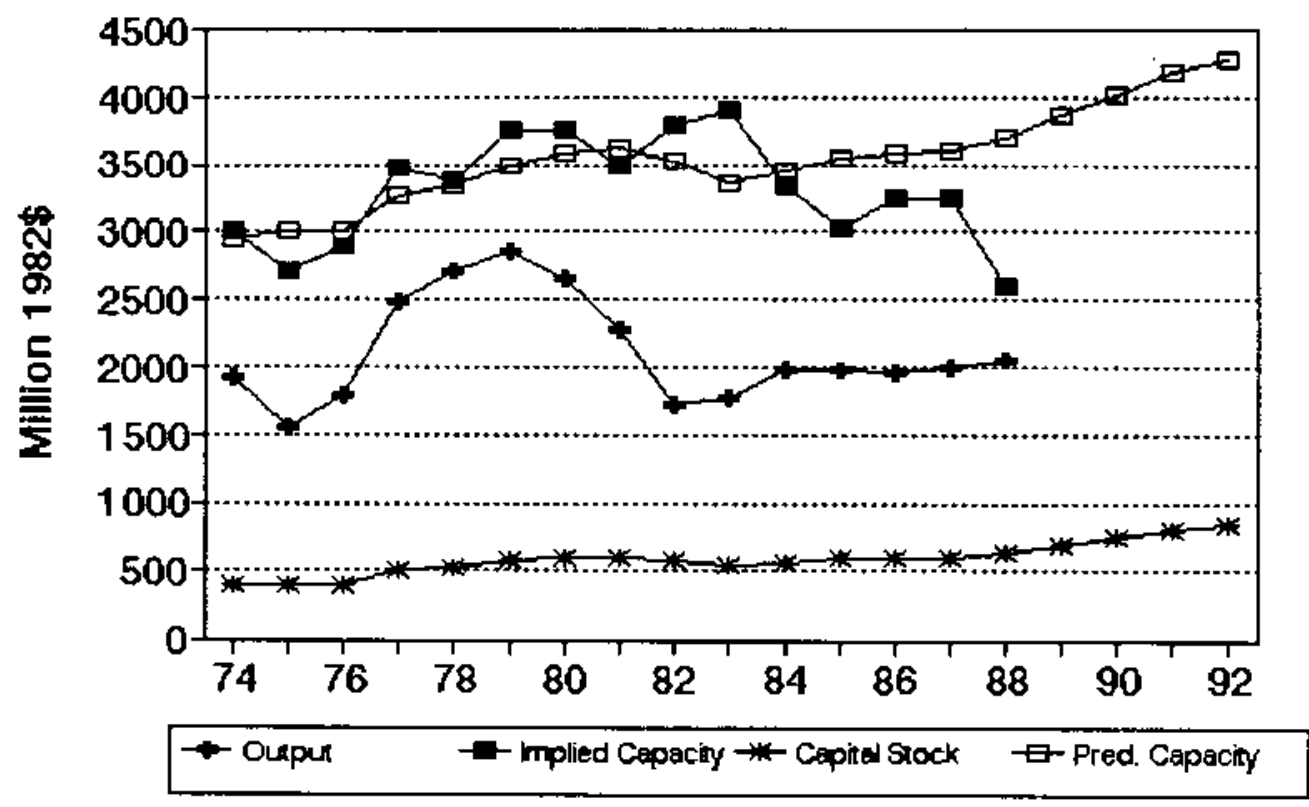




\section{No. 332 sIC 3547 Rolling mill aachinery}

\section{MILLIONS OF 1982 DOLLARS (EXCL. CU RATES)}

$\begin{array}{lccccccc} & \text { Q4 } & \text { Ann. Implied Gross } & \text { Net } & \text { Pred. } & \ldots \ldots \\ \text { Gross } & \text { CU } & \text { CU } & \text { Prac. } & \text { In- } & \text { Cap. } & \text { Prac. } & \text { Emergency Capecity } \\ \text { Output Rate } & \text { Rate } & \text { Capac. } & \text { vest. } & \text { Stk. } & \text { Capac. } & 3 \text { mon. } 6 \text { mon. }>6 \text { mon. }\end{array}$

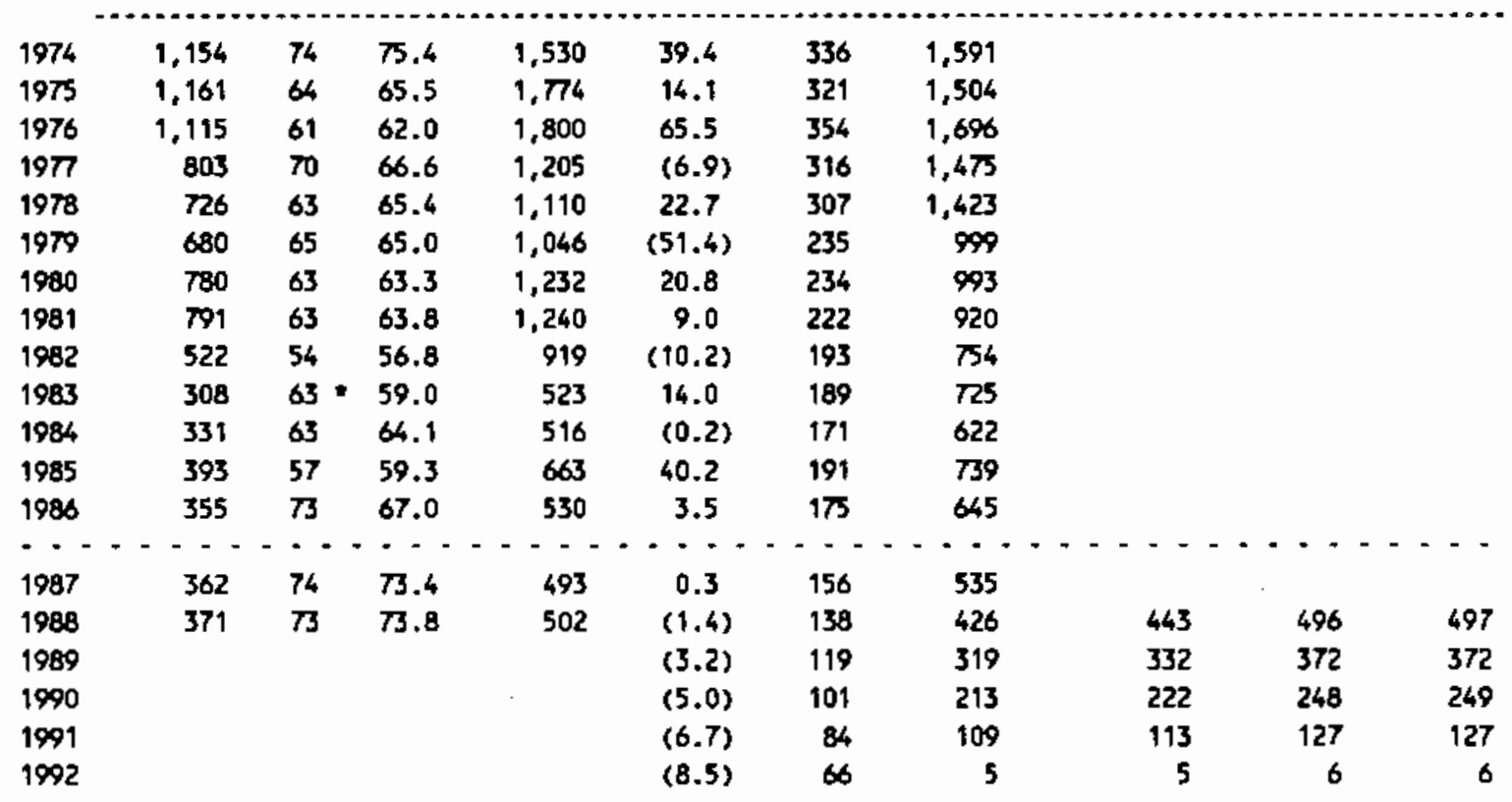

Capacity-Capital Stock Regression: Code 0 RSO 0.780

Coefficients: Const -382.6 Stock 5.9

Ave. Capecity/Stock $(74-86): 4.3$ Ave. Heekly Hours (High) 136.1 Shift Factor 1.17 Ave Heekly Hours (Low) 136.1 Shift Factor 1.17 - Imputed Note: Output, Investment, and Capital Stock extrapolated for 1987 and 1988.

3547 Rolling mill machinery

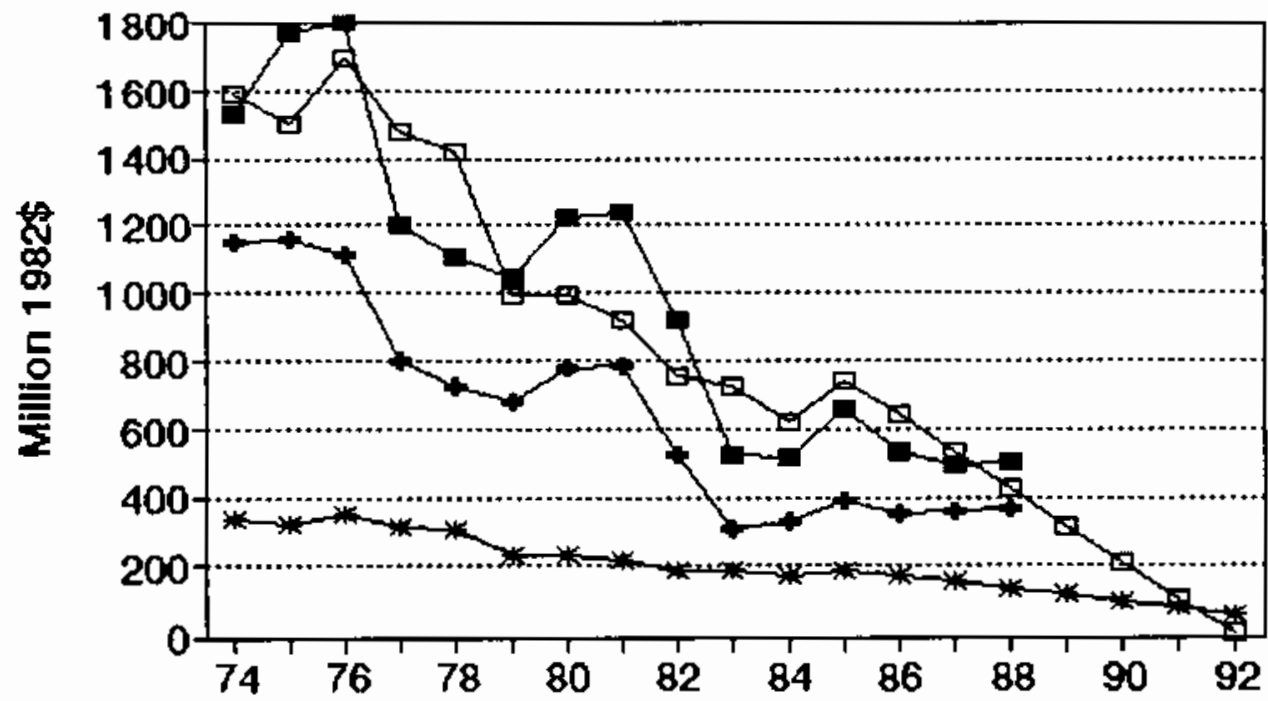

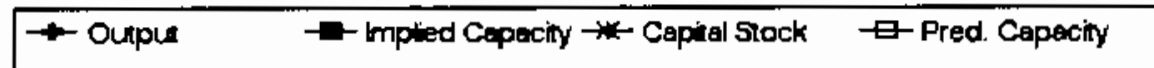




\section{No. 333 sIC 3549 Motal working machinery, nec}

MILLIONS OF 1982 DOLLARS (EXCL. CU RATES)

$\begin{array}{lcccclcc} & 04 & \text { Ann. Implied Gross } & \text { Net } & \text { Pred. } & \ldots & -\ldots \ldots \\ \text { Gross } & \text { CU } & \text { CU } & \text { Prac. } & \text { In- } & \text { Cap. } & \text { Prac. } & \text { Emergency Capacity } \\ \text { Output } & \text { Rate } & \text { Rate } & \text { Capac. } & \text { vest. } & \text { \$tk. } & \text { Capac. } & 3 \text { mon. } 6 \text { mon. }>6 \text { mon. }\end{array}$

\begin{tabular}{|c|c|c|c|c|c|c|c|c|c|c|}
\hline 1974 & 1,197 & 57 & 59.1 & 2,026 & 1.2 & 203 & 1,760 & & & \\
\hline 1975 & 997 & 51 & 49.9 & 1,999 & 23.7 & 213 & 1,812 & & & \\
\hline 1976 & 1,019 & 81 & 69.0 & 1,477 & 28.8 & 227 & 1,873 & & & \\
\hline 1977 & $\imath, 523$ & 78 & 79.4 & 1,918 & 76.7 & 284 & 2,129 & & & \\
\hline 1978 & 1,600 & 71 & 73.2 & 2,187 & 53.7 & 316 & 2,271 & & & \\
\hline 1979 & 1,797 & 70 & 72.0 & 2,496 & 85.0 & $3 \pi 5$ & 2,536 & & & \\
\hline 1980 & 1,833 & 68 & 67.7 & 2,706 & 100.7 & 448 & 2,865 & & & \\
\hline 1981 & 1,787 & 54 & 60.6 & 2,947 & 40.6 & 461 & 2,921 & & & \\
\hline 1982 & 1,466 & 45 & 47.4 & 3,092 & $(40.5)$ & 396 & 2,631 & & & \\
\hline 1983 & 1,288 & 53 & 48.8 & 2,638 & 59.3 & 427 & 2,769 & & & \\
\hline 1984 & 1,698 & 67 & 63.8 & 2,661 & 2.2 & 402 & 2,657 & & & \\
\hline 1985 & 1,687 & 69 & 68.4 & 2,466 & $(10,8)$ & 366 & 2,496 & & & \\
\hline 1986 & 1,565 & 56 & 60.7 & 2,581 & 21.0 & 360 & 2,470 & & & \\
\hline$-\cdots$ & $\cdots$ & $\cdots$ & -- & $-\cdots$ & $-\cdots$ & -- & $\cdots$ & $\cdots$ & $\cdots$ & $\cdots$ \\
\hline 1987 & 1,612 & 69 & 63.6 & 2,536 & 20.6 & 355 & 2,447 & & & \\
\hline 1988 & 1,675 & 73 & 73.6 & 2,277 & 18.9 & 349 & 2,417 & 2,468 & 3,050 & 3,058 \\
\hline 1989 & & & & & 17.2 & 341 & 2,382 & 2,432 & 3,006 & 3,014 \\
\hline 1990 & & & & & 15.5 & 332 & 2,342 & 2,391 & 2,955 & 2,963 \\
\hline 1991 & & & & & 13.8 & 322 & 2.298 & 2,345 & 2,899 & 2,906 \\
\hline 1992 & & & & & 12.0 & 311 & 2,248 & 2,295 & 2,836 & 2,846 \\
\hline
\end{tabular}

Capacity-Capital Stack Regression: Code 0 RSO 0.760 Coefficients: Const 857.3 Stock 4.5

Ave. Capacity/stock $(74-86): 7.0$ Ave. Heekly Hours (High) 125.1 Shift factor 1.26 Ave Weekly Hours (Low) 112.6 Shift Factor 1.40 Note: Output, Investment, and Capital stock extrapolated for 1987 and 1988.

\section{Metal working machinery, nec}

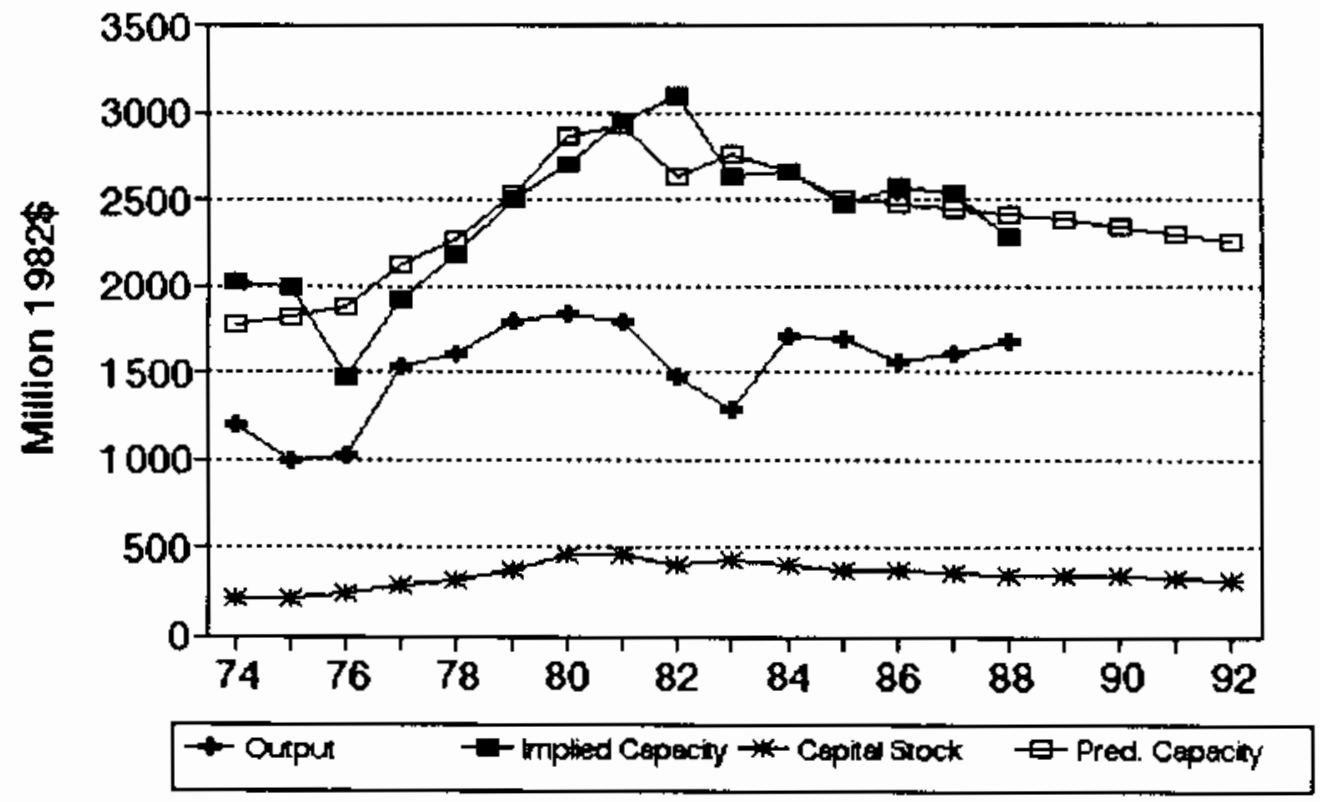




\section{No. 334 sIC 3551 Food products aachinery}

MILLIONS OF 1982 DOLLARS (EXCL. CU RATES)

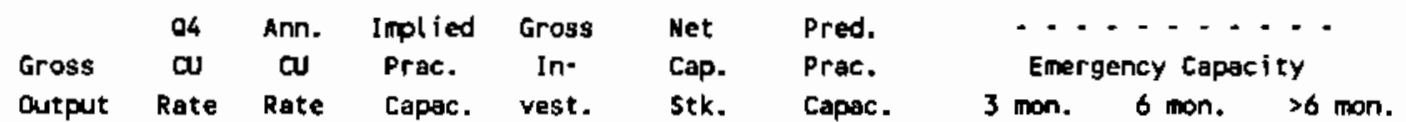

\begin{tabular}{|c|c|c|c|c|c|c|c|c|c|c|}
\hline 1974 & 3,170 & 74 & 74.5 & 4,254 & 129.6 & 592 & 4,606 & & & \\
\hline 1975 & 2,714 & 61 & 65.0 & 4,174 & 27.5 & 580 & 4,494 & & & \\
\hline 1976 & 2,569 & 68 & 65.3 & 3,934 & 32.6 & 572 & 4,384 & & & \\
\hline 1977 & 2,893 & 65 & 66.2 & 4,374 & 75.9 & 605 & 4,273 & & & \\
\hline 1978 & 3,221 & 65 & 64.9 & 4,962 & 110.0 & 667 & 4,163 & & & \\
\hline 1979 & 2,976 & $\infty$ & 65.9 & 4,515 & $(47.8)$ & 577 & 4,053 & & & \\
\hline 1980 & 2,826 & 62 & 63.3 & 4,462 & 39.9 & 574 & 3,943 & & & \\
\hline 1981 & 2,491 & 65 & 64.2 & 3,883 & 50.1 & 578 & 3,833 & & & \\
\hline 1992 & 2,348 & 65 & 64.8 & 3,623 & 48.8 & 578 & 3,722 & & & \\
\hline 1983 & 2,205 & 68 & 66.6 & 3,309 & 45.2 & 573 & 3,612 & & & \\
\hline 1984 & 2,200 & 63 & 65.3 & 3,370 & 186.9 & 694 & 3,502 & & & \\
\hline 1985 & 2,028 & 61 & 61.8 & 3,284 & 29.8 & 660 & 3,392 & & & \\
\hline 1986 & 1,977 & 65 & 63.5 & 3,114 & 48.3 & 644 & 3,282 & & & \\
\hline$-\cdot$ & $\cdots-$ & - & $\cdots$ & $\cdots$ & $\cdots$ & $\cdots=$ & $-\cdots$ & $\cdots$ & $\because \cdot$ & $--\cdot$ \\
\hline 1987 & 2.018 & $\pi$ & 71.1 & 2,836 & 73.3 & 654 & 3.172 & & & \\
\hline 1988 & 2,073 & 68 & 70.8 & 2,928 & 114.7 & 704 & 3,061 & 4,416 & 6,138 & 6,163 \\
\hline 1989 & & & & & 148.0 & 782 & 2,951 & 4,257 & 5,917 & 5,961 \\
\hline 1990 & & & & & 123.2 & 828 & 2,841 & 4,098 & 5,696 & 5,719 \\
\hline 1991 & & & & & 133.1 & 880 & 2,731 & 3,939 & 5,675 & 5,497 \\
\hline 1992 & & & & & 98.4 & 891 & 2,621 & 3,780 & 5,254 & 5,275 \\
\hline
\end{tabular}

Capacity-Capital Stock Regression: Code -2 RSO 0.560 Coefficients: Const 4714.0 Time -110.2

Ave. Capocity/Stock $(74-86): 6.5$ Ave. Weekly Hours (High) 78.2 Shift Factor 2.01 Ave Weekly Hours (Low) 74.9 Shift factor 2.10 Wote: Output, Investment, and Capital Stock extrapolated for 1987 and 1988.

\section{Food products machinery}

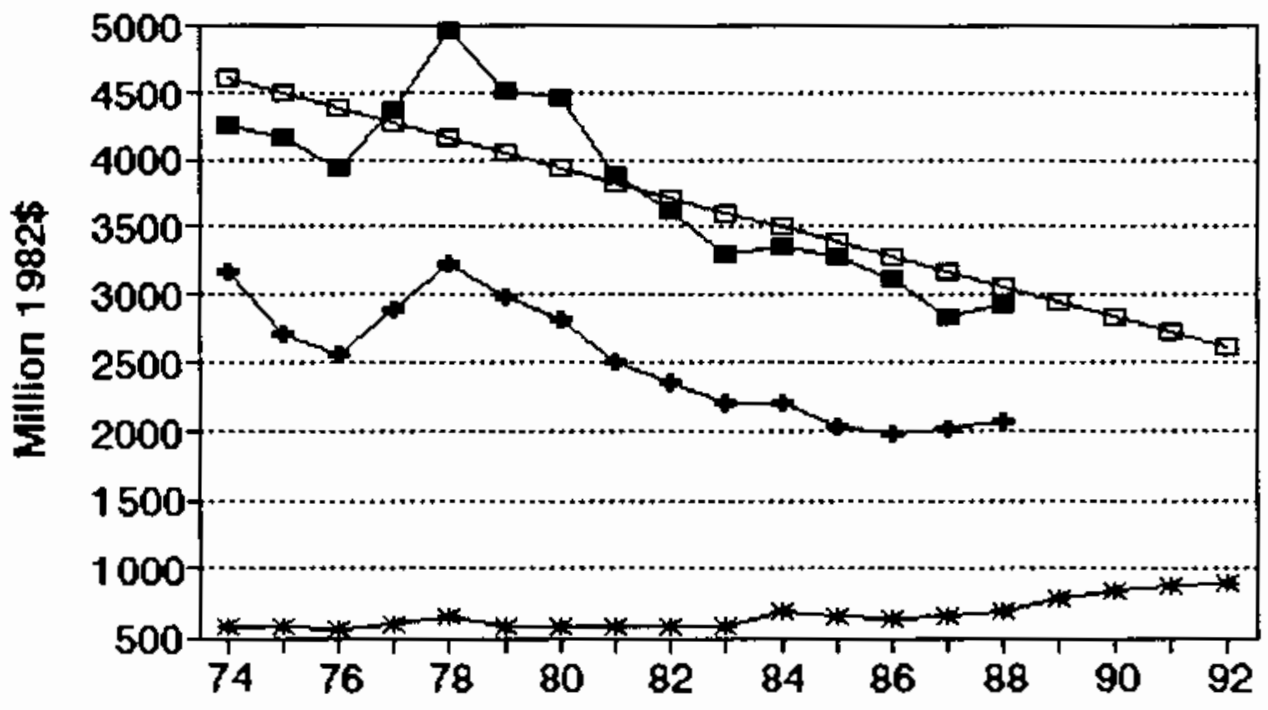

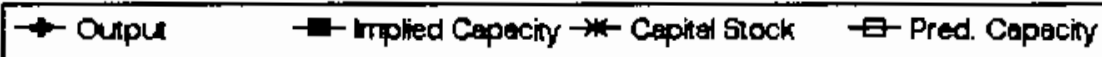


No. 335 sIc 3552 Textile machinery

MILLIONS OF 1982 DOLLARS (EXCL. CU RATES)

$\begin{array}{lccccccc} & \text { Q4 } & \text { Ann. Implied } & \text { Gross } & \text { Net } & \text { Pred. } & \ldots \ldots \ldots \\ \text { Gross } & \text { CU } & \text { CU } & \text { Prac. } & \text { In- } & \text { Cap. } & \text { Prac. } & \text { Emergency Capacity } \\ \text { Output } & \text { Rate } & \text { Rate } & \text { Capac. } & \text { vest. } & \text { Stk. } & \text { Capac. } & 3 \text { mon. } 6 \text { mon. }>6 \text { mon. }\end{array}$

\begin{tabular}{|c|c|c|c|c|c|c|c|c|c|c|}
\hline 1974 & 2,152 & 59 & 59.1 & 3,640 & $(0.8)$ & 563 & 3,338 & & & \\
\hline 1975 & 1,612 & 64 & 61.9 & 2,603 & 22.5 & 542 & 3,020 & & & \\
\hline 1976 & 1,632 & 48 & 54.0 & 3,022 & 41.5 & 539 & 2,974 & & & \\
\hline 1977 & 1,406 & 58 & 54.3 & 2,591 & $(0.6)$ & 496 & 2,322 & & & \\
\hline 1978 & 1,409 & 61 & 59.9 & 2,354 & 18.2 & $4 \pi 5$ & 1,995 & & & \\
\hline 1979 & 1,392 & 92 & 80.5 & 1,730 & 28.5 & 464 & 1,817 & & & \\
\hline 1980 & 1,408 & 84 & 86.9 & 1,620 & 52.5 & 473 & 1,958 & & & \\
\hline 1981 & 1,326 & 93 & 89.7 & 1,477 & 33.0 & 462 & 1,701 & & & \\
\hline 1982 & 1,085 & 79 & 84.2 & 1,289 & 17.4 & 438 & 1,418 & & & \\
\hline 1983 & 967 & 60 & 67.1 & 1,441 & 22.6 & 419 & 1,133 & & & \\
\hline 1984 & 1,061 & 58 & 58.9 & 1,803 & $(8.3)$ & 373 & 1,729 & & & \\
\hline 1985 & 900 & $65 *$ & 62.6 & 1,438 & 29.2 & 363 & 1,587 & & & \\
\hline 1986 & 1,002 & $80 \div$ & 74.8 & 1,341 & 16.7 & 343 & 1,266 & & & \\
\hline$-\overline{-}$ & $-\cdot-$ & 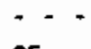 & $\cdots$ & $\cdots$ & $\cdots \cdot$ & $\cdots$ & $\cdots$ & $\cdots \cdot$ & $\cdots$ & $\cdots \cdot$ \\
\hline 1987 & 1,011 & 95 & 89.5 & 1,130 & 28.3 & 334 & 1,133 & & & \\
\hline 1988 & 1,022 & 86 & 89.4 & 1,143 & 37.8 & 336 & 1,160 & 1,363 & 1,613 & 1,617 \\
\hline 1989 & & & & & 45.4 & 345 & 1,301 & 1,529 & 1,809 & 1,813 \\
\hline 1990 & & & & & 39.7 & 347 & 1,339 & 1,574 & 1,863 & 1,867 \\
\hline 1991 & & & & & 42.0 & 352 & 1,409 & 1,655 & 1,559 & 1,964 \\
\hline 1992 & & & & & 34.0 & 348 & 1,348 & 1,585 & 1,876 & 1,880 \\
\hline
\end{tabular}

Capacity-Capital stock Regression: Code -1 RSO 0.880

Coefficients: Const -5295.2 Stock $15.4 \mathrm{D}(84-86) 1305.5$ Ave. Capacity/Stock $(74-86): 4.4$ Ave. Heekly Hours (High) 113.1 Shift factor 1.39 Ave Heekly Hours (LOW) 101.4 Shift Factor 1.55 * Imputed Note: Output, Imvestment, and Capital stock extrapolated for 1987 and 1988.

\section{Textile machinery}

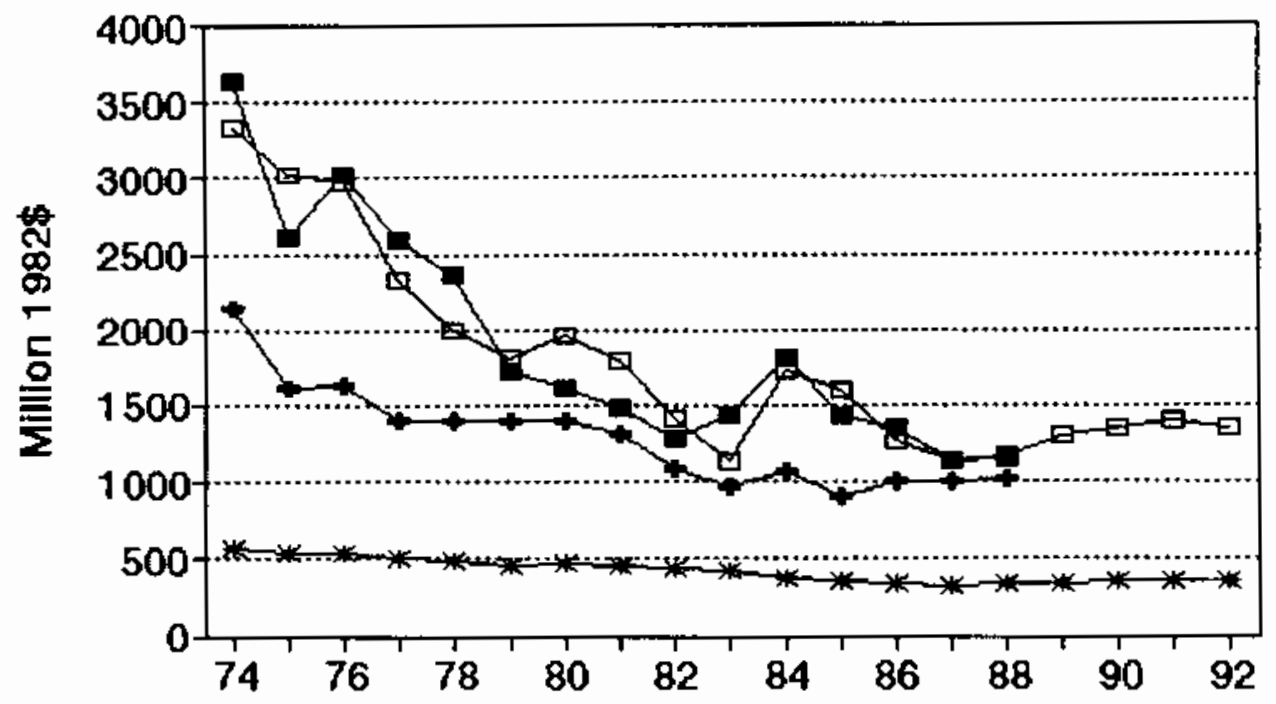

- Ourpu $\quad \rightarrow$-Impled Cepecity $\rightarrow$ - Capial Stock $\quad$ - Pred. Cepacity




\section{No. 336 SIC 3553 Woodworking machinery}

MILLIONS OF 1982 DOLLARS (EXCL. CU RATES)

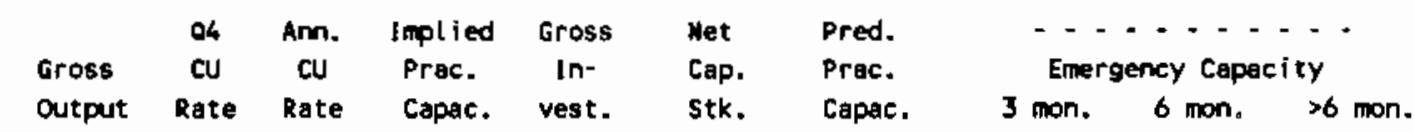

\begin{tabular}{|c|c|c|c|c|c|c|c|c|c|c|}
\hline 1974 & 1,572 & 46 & 46.1 & 3,411 & 28.2 & 195 & 1,593 & & & \\
\hline 1975 & 1,377 & 81 & 67.7 & 2,033 & 62.1 & 239 & 1,892 & & & \\
\hline 1976 & 1,360 & 69 & 73.5 & 1,850 & 42.7 & 262 & 2,051 & & & \\
\hline 1977 & 855 & 78 & 74.6 & 1,146 & (72.1) & 177 & 1,472 & & & \\
\hline 1978 & 968 & 73 & 74.9 & 1,294 & 20.5 & 184 & 1,515 & & & \\
\hline 1979 & 961 & 69 & 70.6 & 1,362 & 34.5 & 202 & 1,638 & & & \\
\hline 1980 & 848 & 68 & 68.3 & 1,241 & 12.6 & 198 & 1,613 & & & \\
\hline 1981 & 881 & 62 & 64.3 & 1,370 & 2.6 & 184 & 1,520 & & & \\
\hline 1982 & 711 & 56 & 58.2 & 1,222 & 20.2 & 187 & 1,540 & & & \\
\hline 1983 & 743 & 64 & 60.9 & 1,219 & (1.5) & 169 & 1,415 & & & \\
\hline 1984 & 785 & $65 *$ & 04.4 & 1,218 & (27.3) & 129 & 1,143 & & & \\
\hline 1985 & 696 & 65 & 64.8 & 1,073 & 2.8 & 119 & 1,076 & & & \\
\hline 1986 & 727 & 72 & 69.4 & 1,048 & 3.9 & 111 & 1,018 & & & \\
\hline$\cdots$ & $-\cdots$ & $\cdot-$ & $\cdots$ & $\therefore-$ & $\cdots$ & $-\cdots$ & $\because-$ & $\cdot--$ & $\cdots$ & $-\cdots$ \\
\hline 1987 & 735 & 70 & 70.7 & 1,039 & 12.9 & 111 & 1,024 & & & \\
\hline 1988 & 745 & $74 *$ & 72.3 & 1,031 & 21.2 & 120 & 1,085 & 1,106 & 1,371 & 1,375 \\
\hline 1989 & & & & & 27.8 & 135 & 1,185 & 1,208 & 1,497 & 1,502 \\
\hline 1990 & & & & & 22.8 & 143 & 1,240 & 1,264 & 1,567 & 1,572 \\
\hline 1991 & & & & & 24.8 & 152 & 1,303 & 1,328 & 1,647 & 1,651 \\
\hline 1992 & & & & & 17.9 & 154 & 1,312 & 1,337 & 1,658 & 1,662 \\
\hline
\end{tabular}

Capacity-Capital Stock Regression: Code 0 RSO 0.210 Coefficients: Const 264.5 Stock 6.8

Ave. Capacity/Stock $(74-86): 8.3$ Ave. Heekly Hours (High) 124.8 Shift Factor 1.27 Ave Heekly Hours (Low) 124.3 Shift Factor 1.27 - Imputed Mote: Output, Investment, and Capital Stock extrapolated for 1987 and 1988.

3553 Woodworking machinery

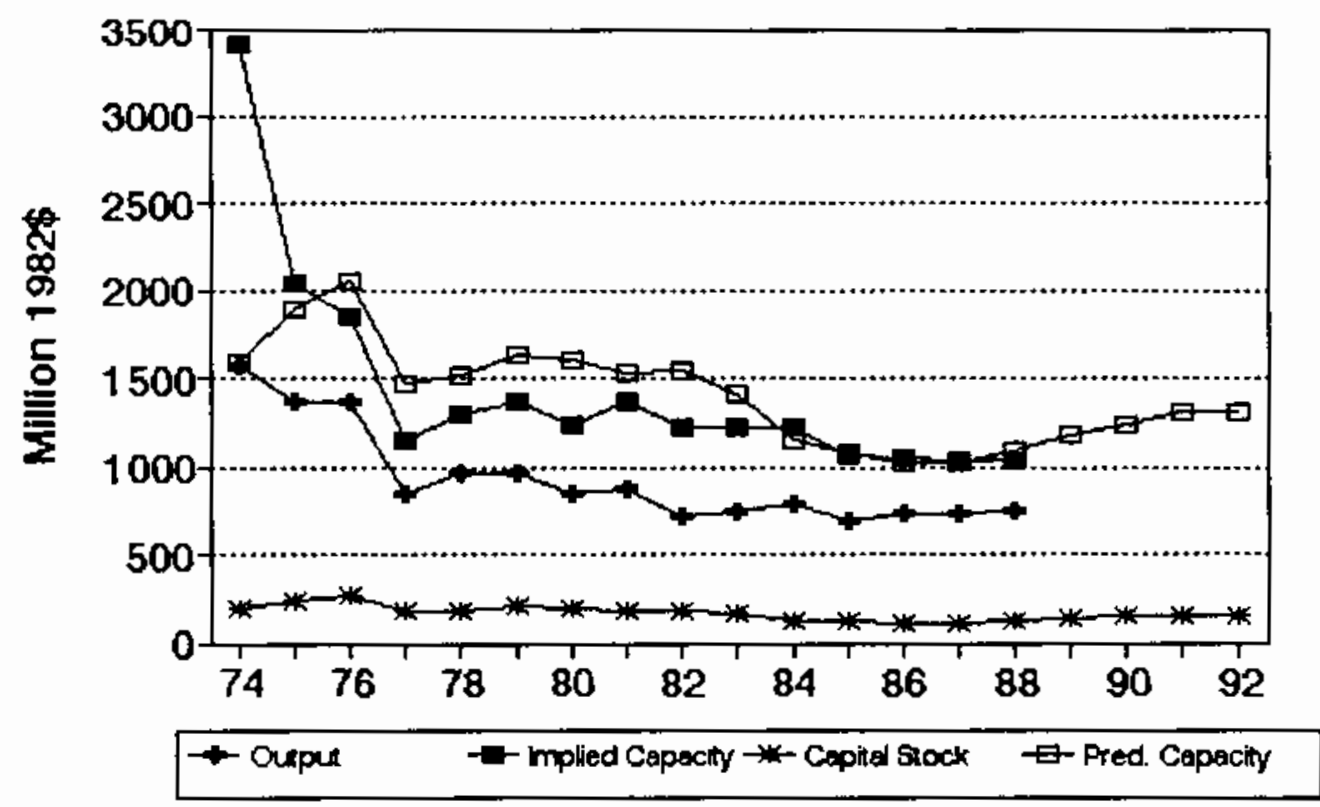


No. 337 SIC 3554 Paper industries machinery

MILLIONS OF 1982 DOLLARS (EXCL. CU RATES)

$\begin{array}{lccccccc} & \text { Q6 } & \text { Ann. Implied Gross } & \text { Net } & \text { Pred. } & \ldots \ldots \ldots \\ \text { Gross } & \text { CU } & \text { CU } & \text { Prac. } & \text { In- } & \text { Cap. } & \text { Prac. } & \text { Emergency Capacity } \\ \text { Output } & \text { Rate } & \text { Rate } & \text { Capac. } & \text { vest. } & \text { Stk. } & \text { Capac. } & 3 \text { mon. } 6 \text { mon. }>6 \text { mon. }\end{array}$

\begin{tabular}{|c|c|c|c|c|c|c|c|c|c|c|}
\hline 1974 & 1,814 & 96 & 99.0 & 1,833 & 43.8 & 313 & 2,097 & & & \\
\hline 1975 & 1,590 & 85 & 84.2 & 1,889 & 5.9 & 294 & 2,074 & & & \\
\hline 1976 & 1,603 & 77 & 79.7 & 2,012 & 28.8 & 297 & 2,078 & & & \\
\hline 1977 & 1,398 & 56 & 64.3 & 2,175 & 5.1 & 278 & 2,055 & & & \\
\hline 1978 & 1,505 & 69 & 63.6 & 2,365 & 35.1 & 288 & 2,066 & & & \\
\hline 1979 & 1,690 & 81 & 78.1 & 2,166 & 56.3 & 316 & 2,100 & & & \\
\hline 1980 & 1,578 & 82 & 80.6 & 1,957 & 48.5 & 337 & 2,124 & & & \\
\hline 1981 & 1,425 & 65 & 72.8 & 1,958 & 51.8 & 360 & 2,151 & & & \\
\hline 1982 & 1,385 & 69 & 66.9 & 2,071 & 49.6 & 377 & 2,171 & & & \\
\hline 1983 & 1,058 & 41 & 50.9 & 2,078 & 21.9 & 366 & 2,158 & & & \\
\hline 1984 & 1,196 & 57 & 52.3 & 2,287 & 34.1 & 366 & 2,158 & & & \\
\hline 1985 & 1,305 & 55 & 55.9 & 2,336 & 39.0 & 370 & 2,163 & & & \\
\hline 1986 & 1,348 & 56 & 55.5 & 2,426 & 31.0 & 366 & 2,159 & & & \\
\hline$\because \cdot$ & $\cdots \cdot \cdot$ & $\because-$ & -- & $-\cdots$ & $-\cdots$ & $\cdots-$ & --- & $-\cdots$ & $\cdots$ & -- \\
\hline 1987 & 1,360 & 77 & 68.5 & 1,984 & 35.5 & 367 & 2,160 & & & \\
\hline 1988 & 1,377 & 81 & 80.3 & 1,714 & 42.5 & 375 & 2,169 & 2,145 & 2,709 & 2,718 \\
\hline 1989 & & & & & 48.2 & 387 & 2,184 & 2,160 & 2,728 & 2,736 \\
\hline 1990 & & & & & 44.0 & 395 & 2,192 & 2,168 & 2,739 & 2,747 \\
\hline 1991 & & & & & 45.7 & 403 & 2,202 & 2,178 & 2,731 & 2,759 \\
\hline 1992 & & & & & 39.8 & 405 & 2,204 & 2,180 & $2, \sqrt{34}$ & 2,762 \\
\hline
\end{tabular}

Capacity-Capital stock Regression: Code 1 RSO 0.050

Coefficients: Const 1728.0 stock 1.2 Ave. Capacity/Stock $(74-86): 6.4$ Ave. Weekly Hours (High) 126.3 Shift factor 1.25 Ave Heekly Hours (LOW) 116.1 Shift factor 1.36 Note: Output, Imvestment, and Capital Stock extrapolated for 1987 and 1988.

\section{Paper industries machinery}

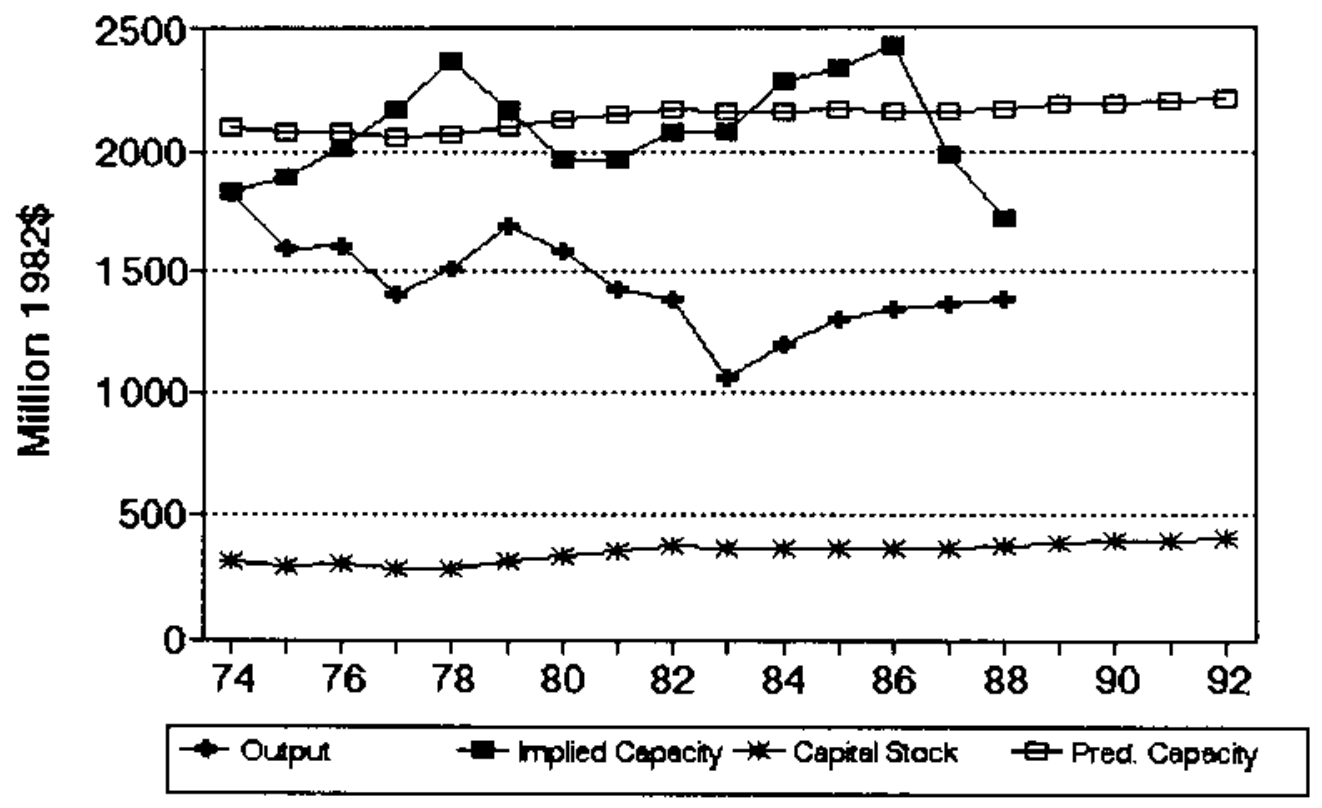


No. 338 gIC 3555 Printing trades machinery

MILLIONS OF 1982 DOLLARS (EXCL. CU RATES)

$\begin{array}{lccccccc} & \text { Q4 } & \text { Ann. Implied Gross } & \text { Net } & \text { Pred. } & -\ldots \ldots \\ \text { Gross } & \text { CU } & \text { CU } & \text { Prac. } & \text { In- } & \text { Cap. } & \text { Prac. } & \text { Emergency Capacity } \\ \text { Output } & \text { Rate } & \text { Rate } & \text { Capac. vest. } & \text { Stk. } & \text { Capac. } & 3 \text { mon. } 6 \text { mon. }>6 \text { mon. }\end{array}$

\begin{tabular}{|c|c|c|c|c|c|c|c|c|c|c|}
\hline 1974 & 1,963 & 70 & 71.2 & $2, \pi 56$ & 20.2 & 453 & 2,944 & & & \\
\hline 1975 & 1,657 & 63 & 63.6 & 2,606 & 25.6 & 445 & 2,916 & & & \\
\hline 1976 & 1,651 & 49 & 54.2 & 3,049 & 9.3 & 422 & 2,839 & & & \\
\hline 1977 & 2,000 & 7 & 66.4 & 3,014 & 95.6 & 479 & 3,029 & & & \\
\hline 1978 & 2,195 & 65 & 69.3 & 3,167 & 87.6 & 527 & 3,188 & & & \\
\hline 1979 & 2,346 & 69 & 68.3 & 3,437 & 45.9 & 535 & 3,214 & & & \\
\hline 1980 & 2,485 & $\infty 8$ & 67.9 & 3,660 & 115.5 & 610 & 3,461 & & & \\
\hline 1981 & 2,497 & 70 & 70.0 & 3,565 & 88.5 & 656 & 3,613 & & & \\
\hline 1982 & 2,282 & 57 & 61.2 & 3,730 & 50.5 & 662 & 3,633 & & & \\
\hline 1993 & 2,386 & 68 & 63.2 & $3,7 \pi$ & $(7.0)$ & 612 & 3,470 & & & \\
\hline 1984 & 2,419 & 68 & 69.1 & 3,499 & 78.2 & 645 & 3,579 & & & \\
\hline 1985 & 2,624 & 72 & 70.6 & 3,718 & 80.8 & 679 & 3,690 & & & \\
\hline 1986 & 2,491 & 77 & $\pi 5.1$ & 3,318 & 58.3 & $6 B 7$ & 3,716 & & & \\
\hline$\cdots$ & $\cdots-$ & - & $-\cdot-$ & $\cdots$ & $\cdots$ & $\cdots$ & $-\cdots$ & -- & . - & $\cdot$ \\
\hline 1987 & 2,526 & 53 & 61.9 & 4,070 & 65.8 & 703 & 3,772 & & & \\
\hline 1988 & 2,573 & 80 & 70.1 & 3,673 & 90.1 & 743 & 3,903 & 4,357 & 5,668 & $5,6 \mathrm{~B} 7$ \\
\hline 1989 & & & & & 109.5 & 800 & 4,090 & 4,566 & 5,940 & 5,959 \\
\hline 1990 & & & & & 95.0 & 837 & 4,215 & 4,706 & 6,122 & 6,142 \\
\hline 1991 & & & & & 100.8 & 878 & 4,351 & 4,857 & 6,318 & 6,339 \\
\hline 1992 & & & & & 80.5 & 896 & 4,410 & 4,923 & 6,404 & 6,425 \\
\hline
\end{tabular}

Capacity-Capital Stock Regression: Code 0 RSQ 0.700

Coefficients: Const $\mathbf{1 4 4 3 . 2}$ stock

Ave. Capacity/Stack ( $74-86$ ): 5.8

Ave. Heekly Hours (High) 108.1 Shift Factor 1.46 Ave Heekly Hours (Low) 102.7 Shift Factor 1.53 Note: Output, Investment, and Capital Stock extropolated for 1987 and 1988.

\section{Printing trades machinery}

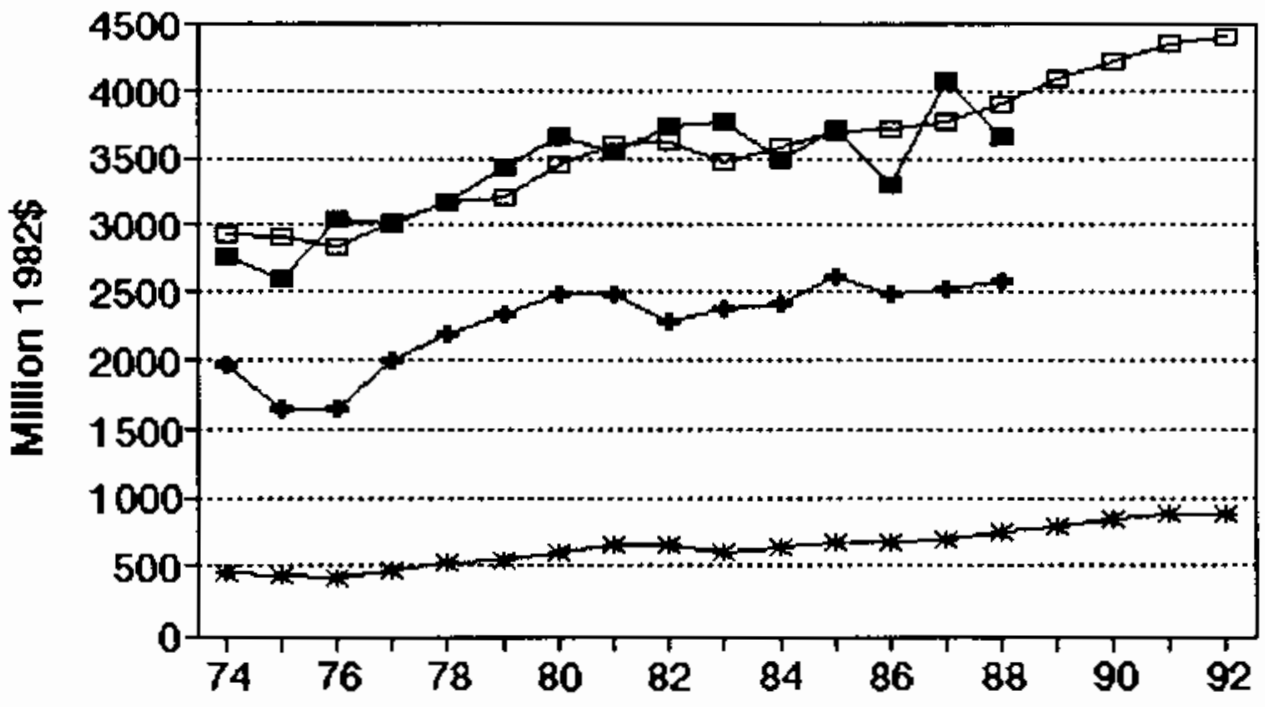

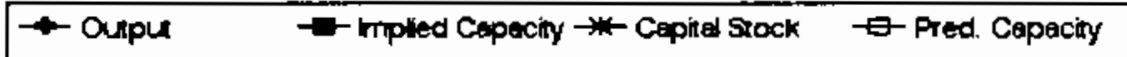


No. 339 sIc 3559 special industry machinery, nec

MILLIONS OF 1982 DOLLARS (EXCL. CU RATES)

$\begin{array}{lccccccc} & \text { Q4 } & \text { Ann. } & \text { Implied Gross } & \text { Net } & \text { Pred. } & \ldots \ldots \ldots \\ \text { Gross } & \text { CU } & \text { CU } & \text { Prac. } & \text { In- } & \text { Cap. } & \text { Prac. } & \text { Emergency Capocity } \\ \text { Output Rate } & \text { Rate } & \text { Capac. } & \text { vest. } & \text { Stk. } & \text { Capac. } & 3 \text { mon. } 6 \text { mon. }>6 \text { mon. }\end{array}$

\begin{tabular}{|c|c|c|c|c|c|c|c|c|c|c|}
\hline 1974 & 8,731 & 66 & 68.5 & 12,743 & 190.1 & 1,379 & 10,707 & & & \\
\hline 1975 & 7,467 & 64 & 60.7 & 12,312 & 85.1 & 1,373 & 10,586 & & & \\
\hline 1976 & 7,496 & 61 & 61.8 & 12,134 & 116.6 & 1,393 & 10,964 & & & \\
\hline 1977 & 6,020 & 65 & 63.6 & 9,462 & (30.1) & 1,272 & 8,698 & & & \\
\hline 1978 & 6,129 & 69 & 67.0 & 9,155 & 188.8 & 1,363 & 10,400 & & & \\
\hline 1979 & 6,070 & 74 & 73.9 & 8,214 & 36.2 & 1,304 & 9,294 & & & \\
\hline 1980 & 5,760 & 59 & 63.5 & 9,067 & 111.6 & 1,315 & 9,499 & & & \\
\hline 1981 & 5,325 & 58 & 59.9 & 8,887 & 125.4 & 1,334 & 9,862 & & & \\
\hline 1982 & 5,360 & 49 & 51.4 & 10,439 & 164.7 & 1,385 & 10,818 & & & \\
\hline 1983 & 4,772 & 50 & 48.5 & 9,843 & 94.8 & 1,361 & 10,366 & & & \\
\hline 1984 & 5,711 & 49 & 51.5 & 11,098 & 190.8 & 1,427 & 11,606 & & & \\
\hline 1985 & 5,743 & 40 & 43.4 & 13,224 & 210.1 & 1,505 & 13,067 & & & \\
\hline 1986 & 5,442 & 45 & 43.1 & 12,633 & 149.1 & 1,519 & 13,340 & & & \\
\hline$-\cdots$ & $-\cdot-$ & - & -- & --- & $\cdots$ & $\therefore-$ & -- & $---\cdot$ & --- & --- \\
\hline 1987 & 5,495 & 53 & 49.6 & 11,086 & 149.8 & 1,535 & 13,634 & & & \\
\hline 1988 & 5,565 & 66 & 61.7 & 9,014 & 220.5 & 1,620 & 15,231 & 16,898 & 25,226 & 25,344 \\
\hline 1989 & & & & & 277.2 & $1, \pi 54$ & 17,755 & 19,698 & 29,406 & 29,544 \\
\hline 1990 & & & & & 234.9 & 1,834 & 19,260 & 21,368 & 31,898 & 32,048 \\
\hline 1991 & & & & & 251.8 & 1,924 & 20,950 & 23,243 & 34,698 & 34,861 \\
\hline 1992 & & & & & 192.6 & 1,946 & 21,377 & 23,717 & 35,404 & 35,571 \\
\hline
\end{tabular}

Capecity-Capital Stock Regression: Code 0 RSO 0.610

Coefficients: Const -15229.7 Stock 18.8

Ave. Capecity/Stock $(74-86): 7.8$

Ave. Heekly Hours (High) 94.6 Shift Factor 1.66 Ave Heekly Hours (Low) 81.9 Shift factor 1.92 Note: Output, Investment, and Capital Stock extrapolated for 1987 and 1988.

3559 Special industry machinery, nec

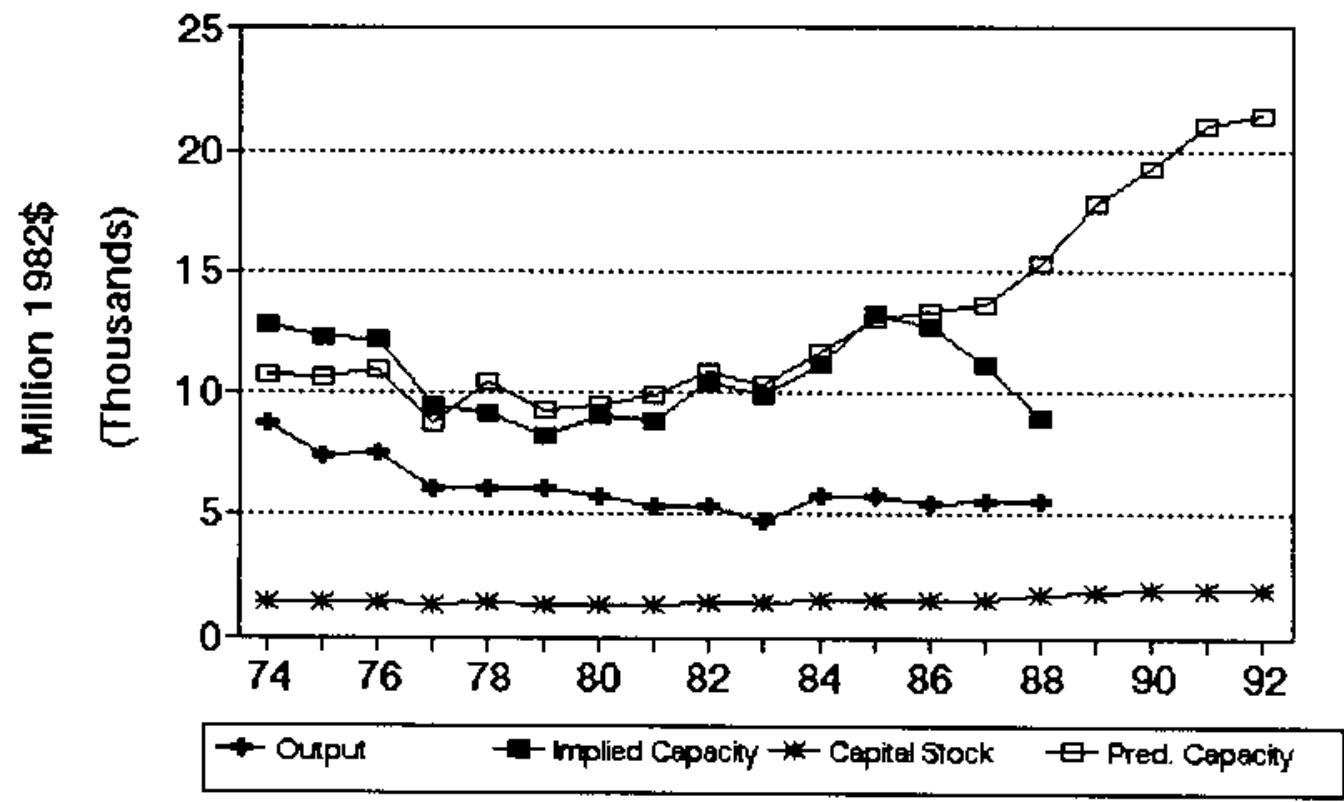


No. 340 sIc 3561 Pumps and pumping equipment

MILLIONS OF 1982 DOLLARS (EXCL. CU RATES)

$\begin{array}{lccccccc} & \text { 04 } & \text { Ann. Inplied Gross } & \text { Net } & \text { Pred. } & \ldots & \ldots \\ \text { Gross } & \text { CU } & \text { CU } & \text { Prac. } & \text { In- } & \text { Cap. } & \text { Prac. } & \text { Energency Capacity } \\ \text { Output } & \text { Rate } & \text { Rate } & \text { Capac. } & \text { vest. } & \text { Stk. } & \text { Capac. } & 3 \text { mon. } 6 \text { mon. }>6 \text { mon. }\end{array}$

\begin{tabular}{|c|c|c|c|c|c|c|c|c|c|c|}
\hline 1974 & 5,720 & 71 & 72.6 & 7,876 & 197.9 & 1,151 & 8,502 & & & \\
\hline 1975 & 5,625 & 64 & 64.0 & $8,7 \%$ & 208.6 & 1,277 & 8,694 & & & \\
\hline 1976 & 5,938 & 74 & 69.9 & 8,492 & 184.5 & 1,374 & 8,841 & & & \\
\hline 1977 & 6,191 & 54 & 61.8 & 10,023 & 96.3 & 1,381 & 8.852 & & & \\
\hline 1978 & 6,570 & 80 & 69.8 & 9,417 & 205.9 & 1,491 & 9,019 & & & \\
\hline 1979 & 6,897 & 78 & 79.9 & 8,634 & 207.5 & 1,593 & 9,174 & & & \\
\hline 1980 & 6,912 & 72 & 73.6 & 9,398 & 227.6 & 1,705 & 9,345 & & & \\
\hline 1981 & 7,018 & 70 & 71.8 & 9,774 & 219.9 & 1,801 & 9,490 & & & \\
\hline 1982 & 6,202 & 57 & 61.0 & 10,165 & 258.7 & 1,920 & 9,671 & & & \\
\hline 1983 & 4,979 & 65 & 61.2 & 8,141 & 37.9 & 1,819 & 9,517 & & & \\
\hline 1984 & 5,761 & 56 & 60.8 & 9,470 & 152.9 & 1,825 & 9,527 & & & \\
\hline 1985 & 5,483 & 59 & 58.0 & 9,458 & 89.5 & 1,768 & 9,440 & & & \\
\hline 1986 & 5,355 & 52 & 54.5 & 9,821 & 123.4 & 1,738 & 9,394 & & & \\
\hline $\begin{array}{l}--- \\
1987\end{array}$ & $\cdots$ & $\begin{array}{l}-- \\
57\end{array}$ & $\begin{array}{l}\cdots \\
54.9\end{array}$ & 9,939 & . & $\begin{array}{l}-\cdot-\cdot- \\
1,742\end{array}$ & 9,400 & $-\cdot \cdot=$ & $-\cdot-$ & $\cdots-$ \\
\hline 1988 & 5,583 & 59 & 58.7 & 9,516 & 237.4 & 1,830 & 9,535 & 10,732 & 14,515 & 14,569 \\
\hline 1989 & & & & & 274.7 & 1,948 & 9,714 & 10,934 & 14,788 & 14,843 \\
\hline 1990 & & & & & 315.9 & 2,098 & 9,941 & 11,189 & 15,133 & 15,189 \\
\hline 1991 & & & & & 352.6 & 2,271 & 10,203 & 11,485 & 15,533 & 15,591 \\
\hline 1992 & & & & & 320.5 & 2,397 & 10,395 & 11,700 & 15,825 & 15,884 \\
\hline
\end{tabular}

Capacity-Capital stock Regression: Code 0 RSO 0.260

Coefficients: Const o7st.6 stock 1.5

Ave. Capacity/stock $(74-86): 5.7$ Ave. Weekly Hours (High) 103.1 Shift Factor 1.53 Ave Weekly Hours (Low) 98.3 Shift factor 1.60 Note: Output, Imvestment, and Capital Stock extrapolated for 1987 and 1988.

\section{Pumps and pumping equipment}

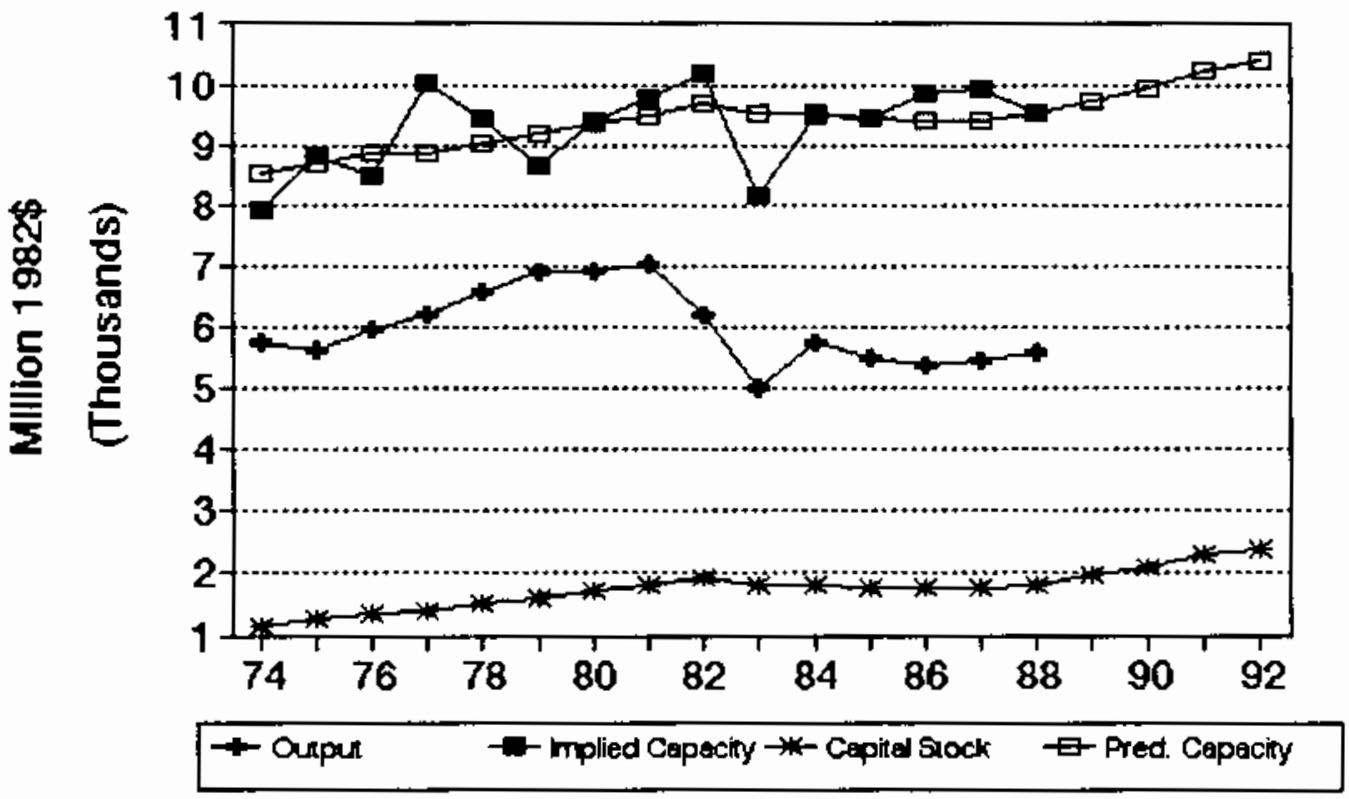




\section{No. 341 sIC 3562 Ball and roller bearings}

MILLIONS OF 1982 DOLLARS (EXCL. CU RATES)

$\begin{array}{lccccccc} & \text { Q4 } & \text { Ann. } & \text { Implied Gross } & \text { Het } & \text { Pred. } & -\ldots \ldots \ldots \\ \text { Gross } & \text { CU } & \text { CU } & \text { Prac. } & \text { In- } & \text { Cap. } & \text { Prac. } & \text { Emergency Capacity } \\ \text { Output } & \text { Rate } & \text { Rate } & \text { Capac. } & \text { vest. } & \text { Stk. } & \text { Capac. } & 3 \text { mon. } 6 \text { mon. }>6 \text { mon. }\end{array}$

\begin{tabular}{|c|c|c|c|c|c|c|c|c|c|c|}
\hline 1974 & $4,87 t$ & 84 & 87.9 & 5,539 & 221.7 & 1,818 & 5,535 & & & \\
\hline 1975 & 4.110 & 72 & 70.1 & 5,865 & 153.8 & 1,822 & 5,540 & & & \\
\hline 1976 & 4,250 & 79 & $\pi .7$ & 5,615 & 168.9 & 1,834 & 5,556 & & & \\
\hline 1977 & 4,442 & 78 & 78.7 & 5,642 & 225.9 & 1,896 & 5,638 & & & \\
\hline 1978 & 4,710 & 87 & 82.8 & 5,692 & 151.2 & 1,882 & 5,619 & & & \\
\hline 1979 & 4,923 & 84 & 87.7 & 5,614 & 271.9 & 1,976 & 5.743 & & & \\
\hline 1980 & 4,319 & 75 & 76.8 & 5,624 & 305.6 & 2,100 & 5,907 & & & \\
\hline 1981 & 4,286 & 62 & 69.0 & 6,212 & 283.8 & 2,190 & 6,027 & & & \\
\hline 1982 & 3,150 & 45 & 49.8 & 6,322 & 140.9 & 2,140 & 5,960 & & & \\
\hline 1983 & 2,935 & 62 & 53.6 & 5,474 & 70.1 & 2,023 & 5,806 & & & \\
\hline 1984 & 3,756 & 65 & 67.3 & 5,583 & 67.4 & 1,910 & 5,657 & & & \\
\hline 1985 & 3,435 & 62 & 63.3 & 5,425 & 176.8 & 1,903 & 5,667 & & & \\
\hline 1986 & 3,310 & 57 & 58.7 & 5.640 & 156.7 & 1,875 & 5,610 & & & \\
\hline$\cdots$ & --- & $\cdots$ & $\cdots$ & --- & $\cdots$ & $\cdots$ & $\cdots \cdot$ & $-\cdot$ & $\cdots$ & $-\cdots$ \\
\hline 1987 & 3,393 & 67 & 62.6 & 5,423 & 170.8 & 1,863 & 5,594 & & & \\
\hline 1988 & 3,505 & 74 & 72.5 & 4,836 & 259.9 & 1,941 & 5,697 & 5,355 & 6,715 & 6,734 \\
\hline 1989 & & & & & 299.1 & 2,050 & 5,842 & 5,491 & 6,885 & 6,905 \\
\hline 1990 & & & & & 342.5 & 2,193 & 6,030 & 5,668 & 7,107 & 7,127 \\
\hline 1991 & & & & & 381.0 & 2,360 & 6,250 & 5,875 & 7,366 & 7,388 \\
\hline 1992 & & & & & 347.3 & 2,476 & 6,404 & 6,020 & 7,548 & 7,570 \\
\hline
\end{tabular}

Capacity-Capital Stock Regression: Code 0 RSO 0.380 Coefficients: Const 3136.3 stock 1.3

Ave. Capacity/stock $(74-86): 2.9$ Ave. Weekly Hours (High) 134.2 Shift Factor 1.18 Ave Heekly Hours (LOW) 130.8 Shift factor 1.21 Wote: Output, Investment, and Capital Stock extrapolated for 1987 and 1988.

\section{Ball and roller bearings}

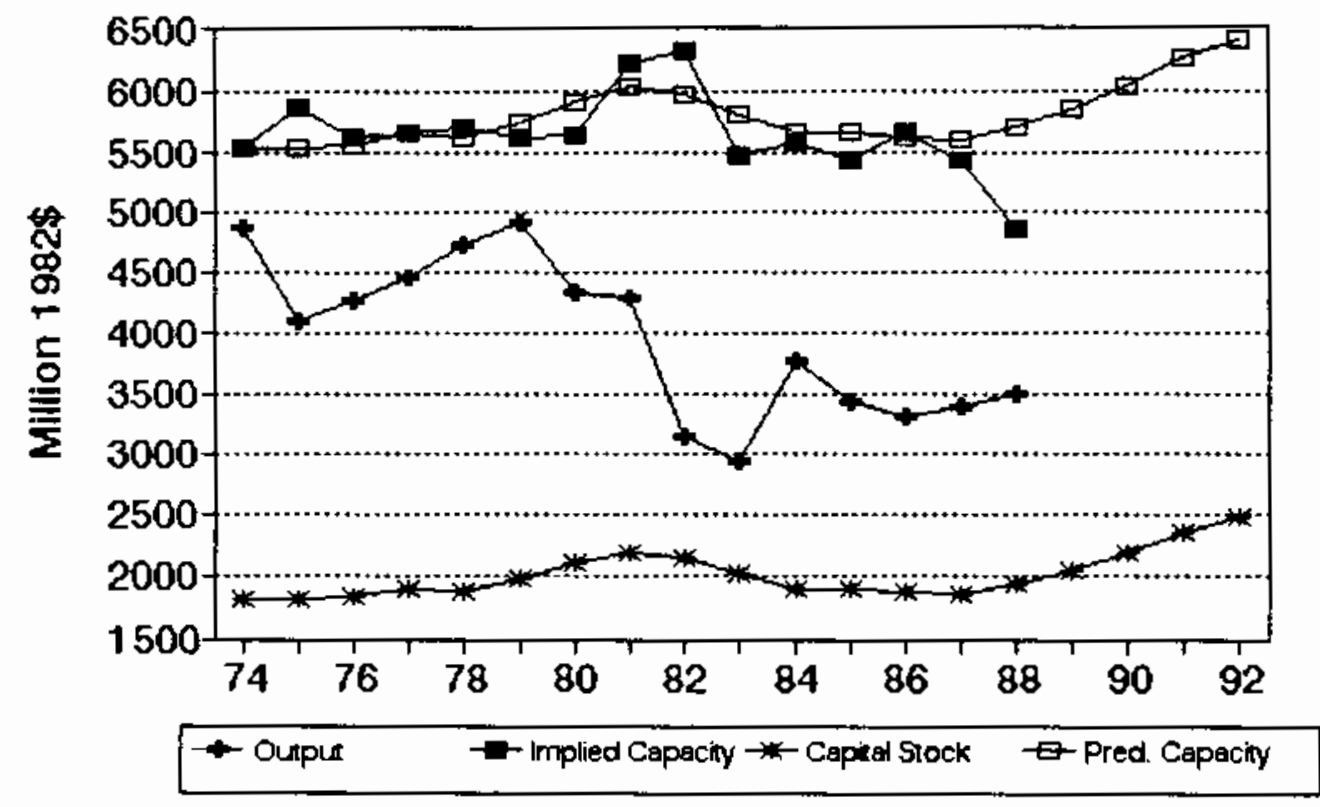


No. 342 SIC 3563 Air and gas compressors

MILLIONS DF 1982 DOLLARS (EXCL. CU RATES)

$\begin{array}{lccccccc} & \text { Q4 } & \text { Ann. Implied Gross } & \text { Net } & \text { Pred. } & -\ldots \ldots \\ \text { Gross } & \text { CU } & \text { CU } & \text { Prac. } & \text { In. } & \text { Cap. } & \text { Prac. } & \text { Emergency Capacity } \\ \text { Output } & \text { Rate } & \text { Rate } & \text { Capac. } & \text { vest. } & \text { Stk. } & \text { Capac. } & 3 \text { mon. } 6 \text { mon. }>6 \text { mon. }\end{array}$

\begin{tabular}{|c|c|c|c|c|c|c|c|c|c|c|}
\hline 1974 & 2,849 & 64 & 86.6 & 4,279 & 126.5 & 495 & 3,950 & & & \\
\hline 1975 & 2,425 & 64 & 59.8 & 4,055 & 21.4 & 479 & 3.870 & & & \\
\hline 1976 & 2,710 & 67 & 65.4 & 4,143 & 70.8 & 511 & 4,029 & & & \\
\hline 1977 & 3,058 & 77 & 73.3 & 4.172 & 137.6 & 604 & 4,489 & & & \\
\hline 1978 & 3,257 & 72 & 73.3 & 4,441 & 81.1 & 640 & 4,670 & & & \\
\hline 1979 & 3,524 & 85 & 82.3 & 4,284 & 40.3 & 637 & 4,654 & & & \\
\hline 1980 & 3,353 & 76 & 78.0 & 4,299 & 102.0 & 693 & 4,930 & & & \\
\hline 1981 & 3,403 & 67 & 72.3 & 4,707 & 63.2 & 711 & 5,018 & & & \\
\hline 1982 & 3,256 & 49 & 54.3 & 6,001 & 119.7 & 780 & 5,358 & & & \\
\hline 1983 & 2,638 & 42 & 43.7 & 6,044 & 27.2 & 757 & 5,245 & & & \\
\hline 1984 & 3,054 & 54 & 51.4 & 5,944 & 175.0 & 873 & 5,821 & & & \\
\hline 1985 & 2,912 & 49 & 51.0 & 5,711 & 56.2 & 870 & 5,806 & & & \\
\hline 1986 & 2,714 & 49 & 48.9 & 5,550 & 60.4 & $8 \times 7$ & 5,791 & & & \\
\hline :- - & 2,779 & $\begin{array}{l}-- \\
58\end{array}$ & $\begin{array}{l}\cdots \\
54.1\end{array}$ & 5,135 & $\begin{array}{r}-- \\
77.1\end{array}$ & $\begin{array}{c}\ldots 83 \\
883\end{array}$ & 5,870 & $-\cdots$ & $--\cdot-$ & $-\cdot-$ \\
\hline 1988 & 2,867 & 54 & 56.4 & 5,083 & 89.3 & 910 & 6,003 & 6,449 & 9,081 & 9.119 \\
\hline 1989 & & & & & 94.7 & 941 & 6,154 & 6,611 & 9,309 & 9,348 \\
\hline 1990 & & & & & 100.7 & 973 & 6,323 & 6,793 & 9,566 & 9,605 \\
\hline 1991 & & & & & 106.0 & 1,012 & 6,507 & 6,991 & 9,844 & 9,884 \\
\hline 1992 & & & & & 101.3 & 1,042 & 6.655 & 7,150 & 10,068 & 10,109 \\
\hline
\end{tabular}

Capacity-Capital stock Regression: Code 0 RSO 0.740

Coefficients: Const 1504.3 stock 4.9

Ave. Capacity/Stock $(74-\infty 6): 7.1$

Ave. Weekly Hours (High) 103.7 Shift Factor 1.52 Ave Heekly Hours (Low) 98.0 Shift Factor 1.61 Note: Output, Investment, and Capital stock extrapolated for 1987 and 1988.

\section{Air and gas compressors}

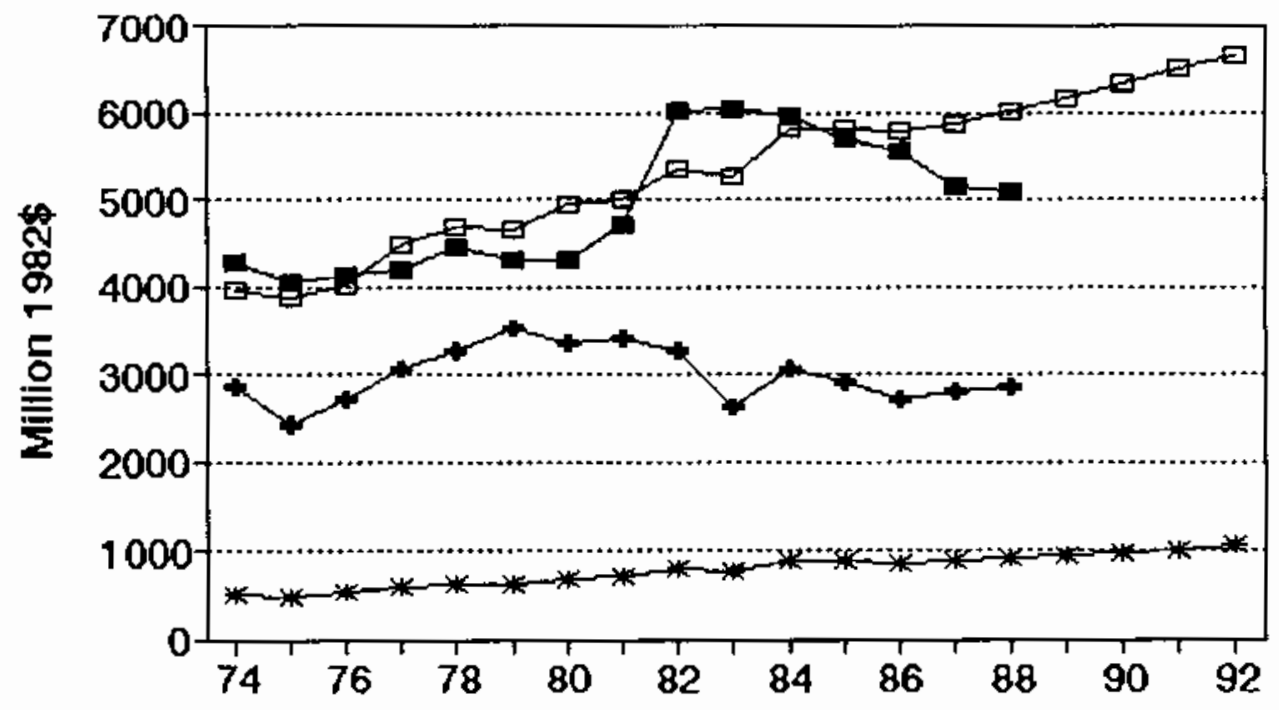

- Ouput $\rightarrow-$ Implied Capacin $\rightarrow$ - Cepitel Scack $\square$ Pred Capaciny




\section{No. 343 sIC 3564 Blowers and fans}

\section{MILLIONS OF 1982 DOLLARS (EXCL. CU RATES)}

$\begin{array}{lccccccc} & \text { Q4 } & \text { Amn. Implied } & \text { Gross } & \text { Net } & \text { Pred. } & -\ldots \ldots \ldots \\ \text { Gross } & \text { CU } & \text { CU } & \text { Prac. } & \text { In- } & \text { Cap. } & \text { Prac. } & \text { Emergency Capacity } \\ \text { Output } & \text { Rate } & \text { Rate } & \text { Capac. } & \text { vest. } & \text { StK. } & \text { Capac. } & 3 \text { mon. } 6 \text { mon. }>6 \text { mon. }\end{array}$

\begin{tabular}{|c|c|c|c|c|c|c|c|c|c|c|}
\hline 1974 & 2,182 & 83 & 84.8 & 2,574 & 16.8 & 324 & 3,033 & & & \\
\hline 1975 & 2,116 & 74 & 74.5 & 2,841 & 81.1 & 379 & 3,315 & & & \\
\hline 1976 & 2,219 & 73 & 73.1 & 3,033 & 14.1 & 368 & 3,258 & & & \\
\hline 1977 & 2,085 & 68 & 70.0 & 2,979 & 23.0 & 366 & 3,248 & & & \\
\hline 1978 & 2,078 & 68 & 67.7 & 3,070 & 20.4 & 362 & 3,225 & & & \\
\hline 1979 & 2,202 & 56 & 61.3 & 3,593 & 50.3 & 384 & 3,340 & & & \\
\hline 1980 & 2,158 & 43 & 47.4 & 4,551 & 26.0 & 382 & 3,328 & & & \\
\hline 1981 & 2,115 & 54 & 50.6 & 4,178 & 33.5 & 386 & 3,347 & & & \\
\hline 1982 & 2,227 & 52 & 52.3 & 4,262 & 97.3 & 447 & 3,658 & & & \\
\hline 1983 & 2,039 & 59 & 55.7 & 3,662 & 19.4 & 430 & 3,571 & & & \\
\hline 1984 & 2,030 & 68 & 65.8 & 3,084 & 48.5 & 440 & 3,624 & & & \\
\hline 1985 & 2,007 & 61 & 63.7 & 3,152 & 36.7 & 438 & 3,611 & & & \\
\hline 1986 & 1,982 & 61 & 60.9 & 3,253 & 52.9 & 450 & 3,675 & & & \\
\hline$-\cdot$ & $-\cdot-$ & -- & $-\cdot$ & $\cdots$ & $\cdots$ & $\cdots$ & $\cdots$ & $-\cdot$ & $\cdots$ & $\cdot-$ \\
\hline 1987 & 1,996 & 65 & 63.2 & 3,156 & 38.6 & 448 & 3,666 & & & \\
\hline 1988 & 2,013 & 73 & 70.4 & 2,860 & 48.2 & 456 & 3,707 & 3,606 & 4,776 & 4,793 \\
\hline 1989 & & & & & 52.4 & 468 & 3,765 & 3,663 & 4.851 & 4,868 \\
\hline 1990 & & & & & 57.0 & 483 & 3,842 & 3,737 & 4,950 & 4,967 \\
\hline 1991 & & & & & 61.2 & 501 & 3,933 & 3,826 & 5,067 & 5,085 \\
\hline 1992 & & & & & 57.6 & 513 & 3,997 & 3,888 & 5,150 & 5,168 \\
\hline
\end{tabular}

Capacity-Capital Stock Regression: Code 0 RSa 0.110

Coefficients: Const 1384.9 Stock 5.1

Ave. Capacity/Stock $(74-86): 8.6$ Ave. Heekly Hours (High) 122.3 Shift factor 1.29 Ave Heekly Hours (Lou) 109.1 Shift Factor 1.44 Note: Output, Investment, and Capitat Stock extrapolated for 1987 and 1988.

\section{Blowers and fans}

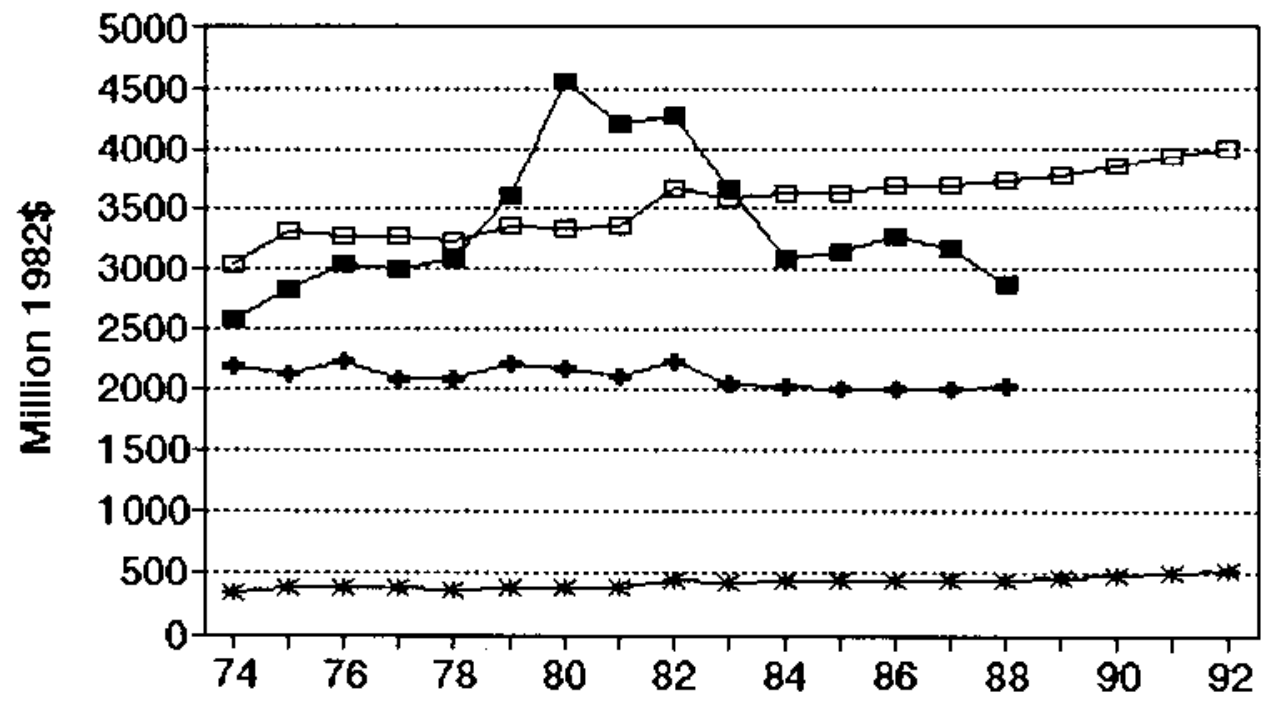

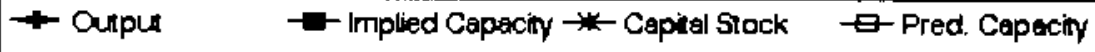


No. 344 SIC 3565 Industrial patterns

MILLIONS OF 1982 DOLLARS (EXCL. CU RATES)

$\begin{array}{lccccccc} & \text { Q4 } & \text { Ann. Imptied Gross } & \text { Net } & \text { Pred. } & \ldots \ldots \ldots \\ \text { Gross } & \text { CU } & \text { CU } & \text { Prac. } & \text { In- } & \text { Cap. } & \text { Prac. } & \text { Emergency Capecity } \\ \text { Output } & \text { Rate } & \text { Rate } & \text { Capac. } & \text { vest. } & \text { Stk. } & \text { Capec. } & 3 \text { mon. } 6 \text { mon. }>6 \text { mon. }\end{array}$

\begin{tabular}{|c|c|c|c|c|c|c|c|c|c|c|}
\hline 1974 & 643 & 66 & 66.0 & 974 & 15.0 & 129 & 880 & & & \\
\hline $19 \pi$ & 479 & 65 & 65.4 & 733 & 32.8 & 151 & 858 & & & \\
\hline 1976 & 447 & 69 & 67.5 & 663 & 19.2 & 158 & 837 & & & \\
\hline 1977 & 497 & 67 & 67.8 & 734 & (17.7) & 130 & 816 & & & \\
\hline 1978 & 484 & 42 & 51.4 & 942 & 8.2 & 128 & 795 & & & \\
\hline 1979 & 518 & 61 & 53.9 & 961 & $(9.5)$ & 110 & 774 & & & \\
\hline 1980 & 485 & 63 & 62.3 & 779 & 25.2 & 125 & 752 & & & \\
\hline 1981 & 478 & 63 & 63.0 & 759 & 28.7 & 140 & 731 & & & \\
\hline 1982 & 446 & 49 & 54.3 & 823 & 19.4 & 145 & 710 & & & \\
\hline 1983 & 411 & 76 & 65.9 & 624 & 17.4 & 148 & 689 & & & \\
\hline 1984 & 453 & 87 & 82.9 & 546 & $(32.0)$ & 106 & 667 & & & \\
\hline 1985 & 505 & 90 & 88.9 & 568 & 31.2 & 125 & 646 & & & \\
\hline 1986 & 552 & 77 & 81.9 & 674 & 11.2 & 124 & 625 & & & \\
\hline-- & ---- & - & $=$ & $=\cdot$ &.-- & -- & $\cdots$ & $\cdots$ & $\cdots$ & -- \\
\hline 1987 & 557 & 70 & 72.6 & 768 & 9.8 & 121 & 604 & & & \\
\hline$\$ 988$ & 565 & 77 & 74.4 & 759 & 19.2 & 128 & 583 & 914 & 1,171 & 1,175 \\
\hline 1989 & & & & & 23.4 & 139 & 561 & 881 & 1,129 & 1,132 \\
\hline 1990 & & & & & 28.0 & 153 & 540 & 848 & 1,086 & 1,090 \\
\hline 1991 & & & & & 32.1 & 170 & 519 & 815 & 1,044 & 1,047 \\
\hline 1992 & & & & & 28.5 & 182 & 498 & 781 & 1,001 & 1,004 \\
\hline
\end{tabular}

Capacity-Capital Stock Regression: Code -2 RSO 0.340

Coefficients: Const 900.8 Time -21.2

Ave. Capacity/Stock $(74-86): 5.7$

Ave. Heekly Hours (High) 78.1 Shift Factor 2.02 Ave Heekly Hours (LOW) 69.2 Shift Factor 2.28 Note: Output, Investment, and Capital Stock extrapolated for 1987 and 1988.

\section{Industrial patterns}

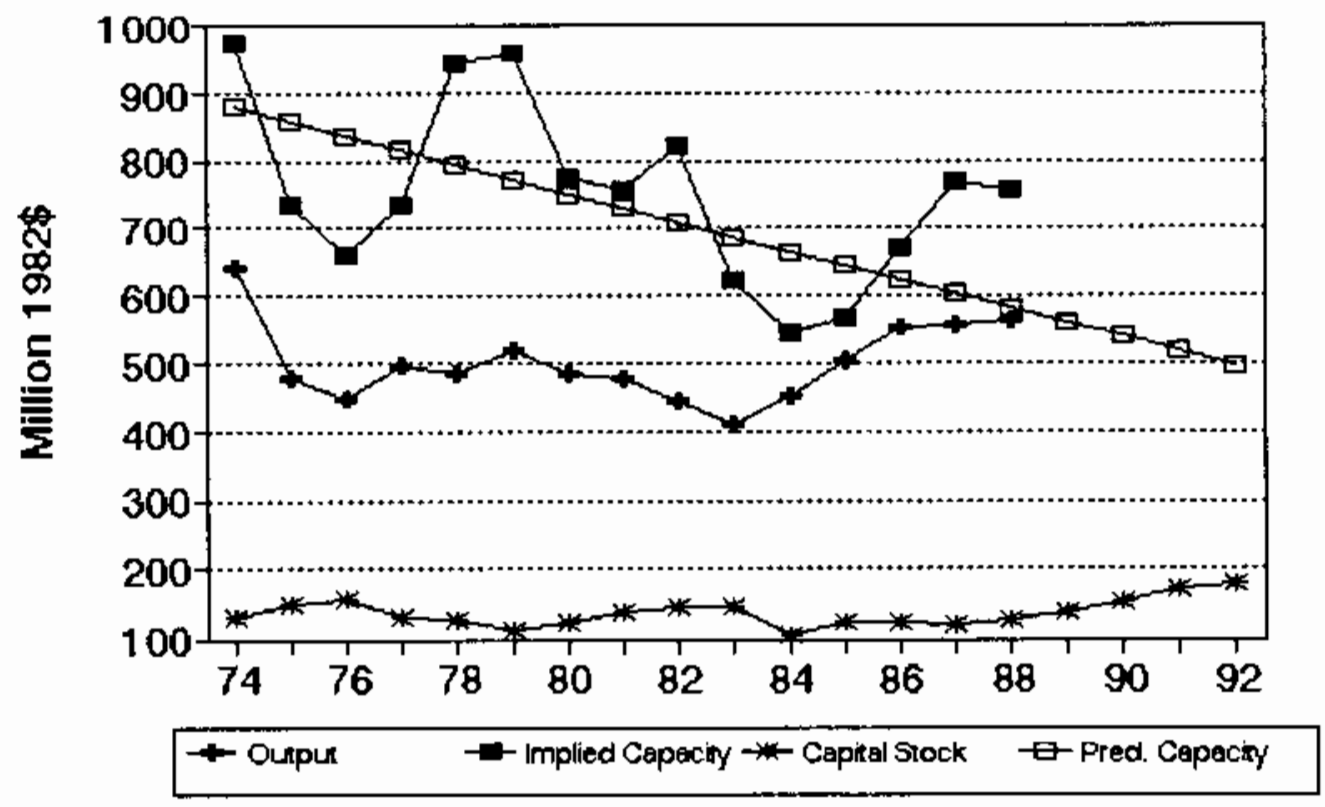


No. 345 SIC 3566 speed changers, drives, and gears MILLIONS OF 1982 DOLLARS (EXCL. CU RATES)

$\begin{array}{lccccccc} & \text { Q4 } & \text { Ann. Implied Gross } & \text { Het } & \text { Pred. } & \ldots & \ldots \\ \text { Gross } & \text { CU } & \text { CU } & \text { Prac. } & \text { In- } & \text { Cap. } & \text { Prac. } & \text { Emergency Capacity } \\ \text { Output } & \text { Rate } & \text { Rate } & \text { Capac. } & \text { vest. } & \text { Stk. } & \text { Capac. } & 3 \text { mon. } 6 \text { mon. }\end{array}$

\begin{tabular}{|c|c|c|c|c|c|c|c|c|c|c|}
\hline 1974 & 2,182 & 78 & 80.8 & 2,701 & 59.1 & 637 & 3,165 & & & \\
\hline 1975 & 2,182 & 69 & 67.8 & 3,219 & 132.2 & 711 & 3,125 & & & \\
\hline 1976 & 2,066 & 61 & 63.7 & 3,243 & 87.7 & 741 & 3,085 & & & \\
\hline 1977 & 1,978 & 69 & 6.1 & 2,995 & 112.6 & 791 & 3,045 & & & \\
\hline 1978 & 2,001 & 76 & 72.8 & 2,749 & 82.2 & 812 & 3,005 & & & \\
\hline 1979 & 2,228 & 61 & 68.1 & 3,271 & 48.0 & 802 & 2,965 & & & \\
\hline 1980 & 2,055 & 63 & 61.4 & 3,350 & 93.7 & 036 & 2,925 & & & \\
\hline 1981 & 1,904 & 63 & 64.6 & 2,949 & 85.8 & 860 & 2,886 & & & \\
\hline 1982 & 1,636 & 49 & 53.1 & 3,083 & 102.2 & 897 & 2,846 & & & \\
\hline 1983 & 1,332 & 56 & 52.1 & 2,555 & 85.2 & 913 & 2,806 & & & \\
\hline 1984 & 1,540 & 50 & 54.4 & 2,829 & 43.1 & 898 & 2,766 & & & \\
\hline 1985 & 1,427 & 50 & so.1 & 2,848 & 64.3 & 881 & 2,726 & & & \\
\hline 1986 & 1,387 & 69 & 61.9 & 2,240 & 67.0 & 874 & 2,606 & & & \\
\hline$-\cdot-$ & $\cdots$ & -- & $-\cdot$ & $\cdots$ & -- & $\cdots$ & $\cdots \cdot$ & --- & $=\cdot$ & $-\cdots$ \\
\hline 1987 & 1.421 & 57 & 61.3 & 2,319 & 79.5 & 882 & 2,647 & & & \\
\hline 1988 & 1,466 & 82 & 73.1 & 2,004 & 89.9 & 899 & 2,607 & 2,471 & 3,209 & 3,219 \\
\hline 1989 & & & & & 94.5 & 919 & 2,567 & 2,433 & 3,160 & 3,170 \\
\hline 1990 & & & & & 99.6 & 943 & 2,527 & 2,395 & 3,111 & 3,121 \\
\hline 1991 & & & & & 104.1 & 969 & 2,487 & 2,357 & 3,062 & 3,072 \\
\hline 1992 & & & & & 100.1 & 989 & 2,447 & 2,320 & 3,013 & 3,023 \\
\hline
\end{tabular}

Capacity-Capital Stock Regression: Code -2 RSQ 0.240

Coefficients: Const 3204.3 Time -39.8

Ave. Capecity/Stock $(74-86): 3.6$ Ave. Weekly Hours (High) 128.3 Shift Factor 1.24 Ave Heekly Hours (Low) 120.6 Shift Factor 1.31 Note: Output, Investment, and Capital Stock extrapolated for 1987 and 1988.

\section{Speed changers, drives, and gears}

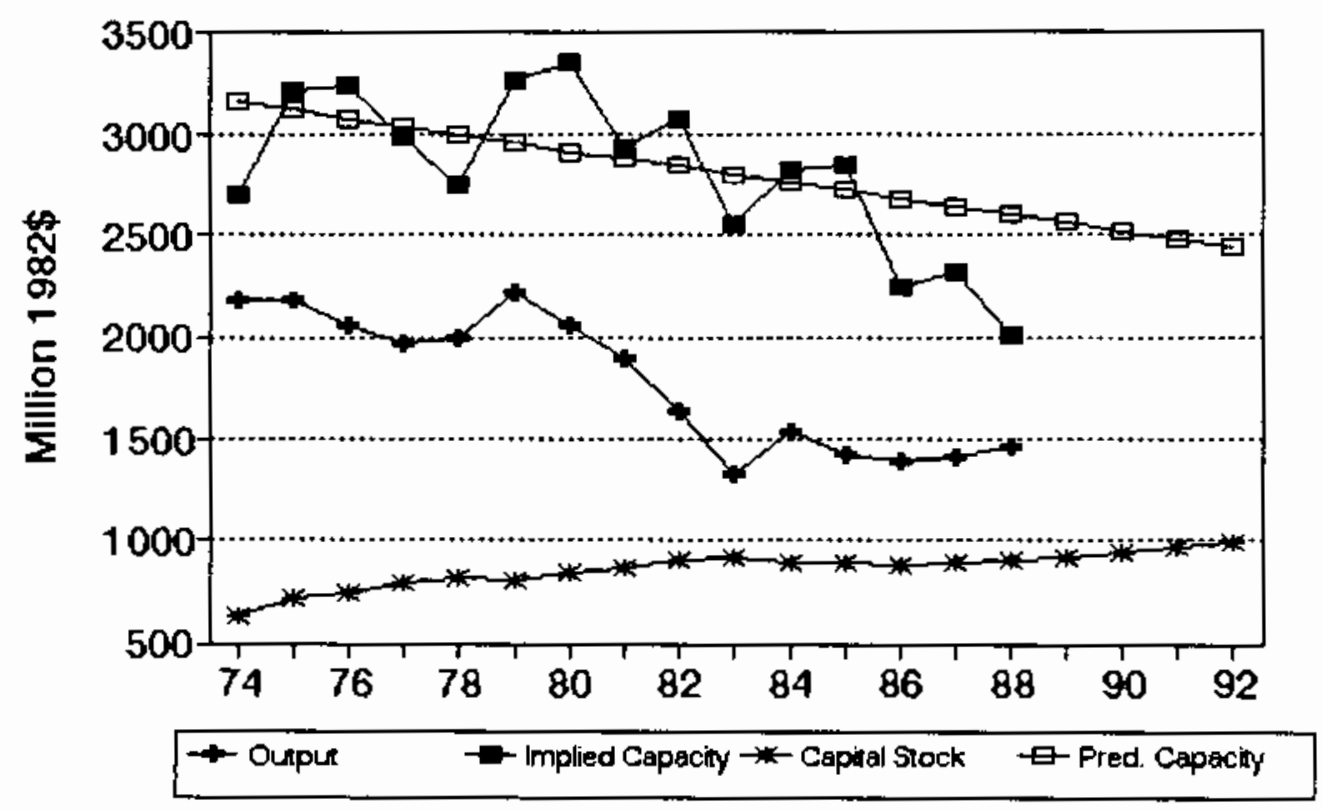




\section{No. 346 sIC 3567 Industrial furnaces and ovens}

MILLIONS OF 1982 DOLLARS (EXCL. CU RATES)

$\begin{array}{lccccccc} & \text { Q4 } & \text { Ann. Inplied Gross } & \text { Het } & \text { Pred. } & \ldots \ldots \ldots \\ \text { Gross } & \text { CU } & \text { CU } & \text { Prac. } & \text { In- } & \text { Cap. } & \text { Prac. } & \text { Emergency Capacity } \\ \text { Qutput Rate } & \text { Rate } & \text { Capac. } & \text { vest. } & \text { Stk. } & \text { Capac. } & 3 \text { mon. } 6 \text { mon. }>6 \text { mon. }\end{array}$

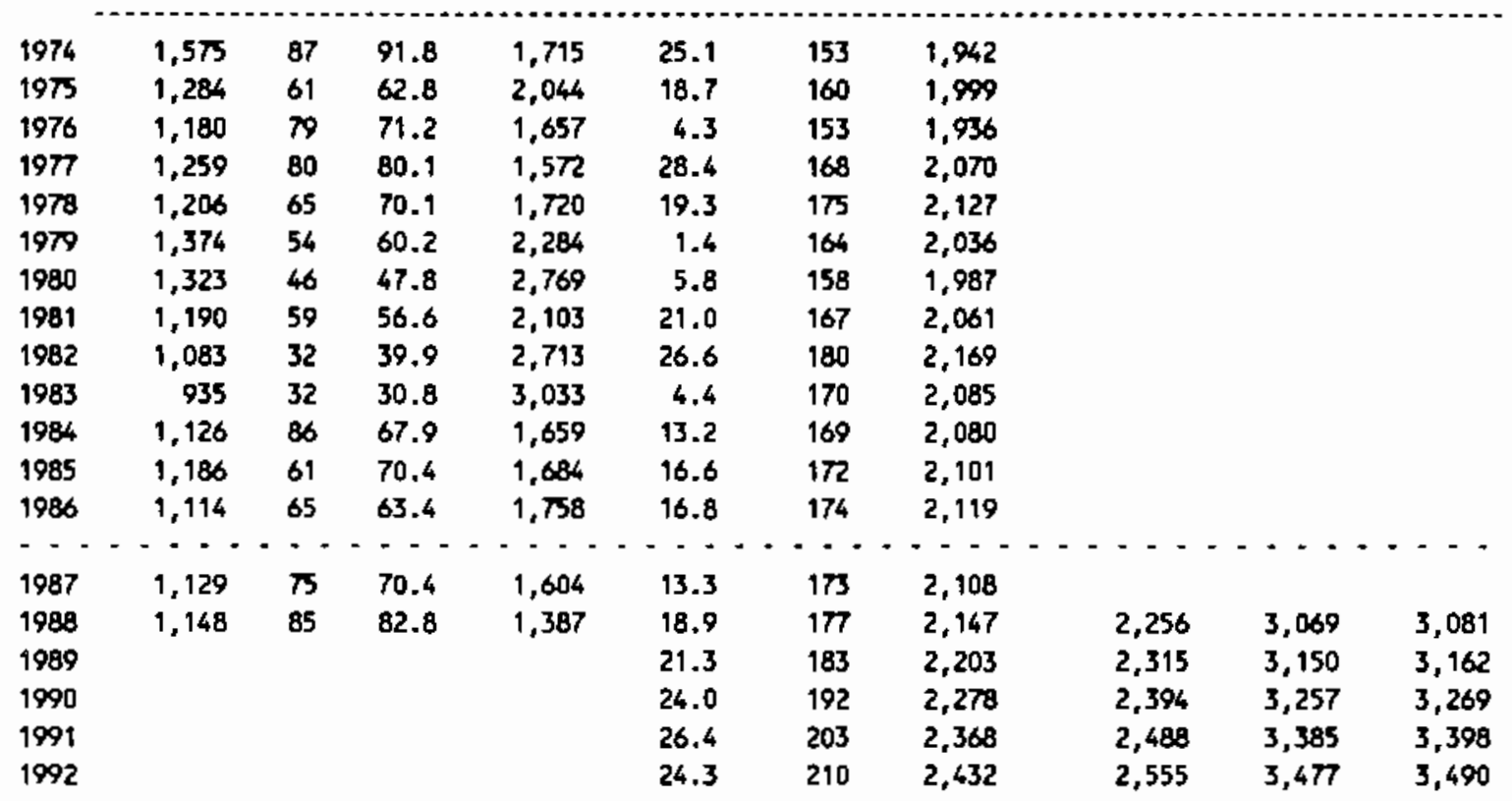

Capecity-Capital stock Regression: Code 1 RSQ 0.020

Coefficients: Const 617.2 Stock 8.7

Ave. Capacity/Stock $(74-86): 12.4$ Ave. Weekly Hours (High) 109.7 Shift Factor 1.43 Ave Heekly Hours (LOW) 103.8 Shift factor 1.52 Note: Output, Imvestment, and Capital Stock extrapolated for 1987 and 1988.

3567 Industrial furnaces and ovens

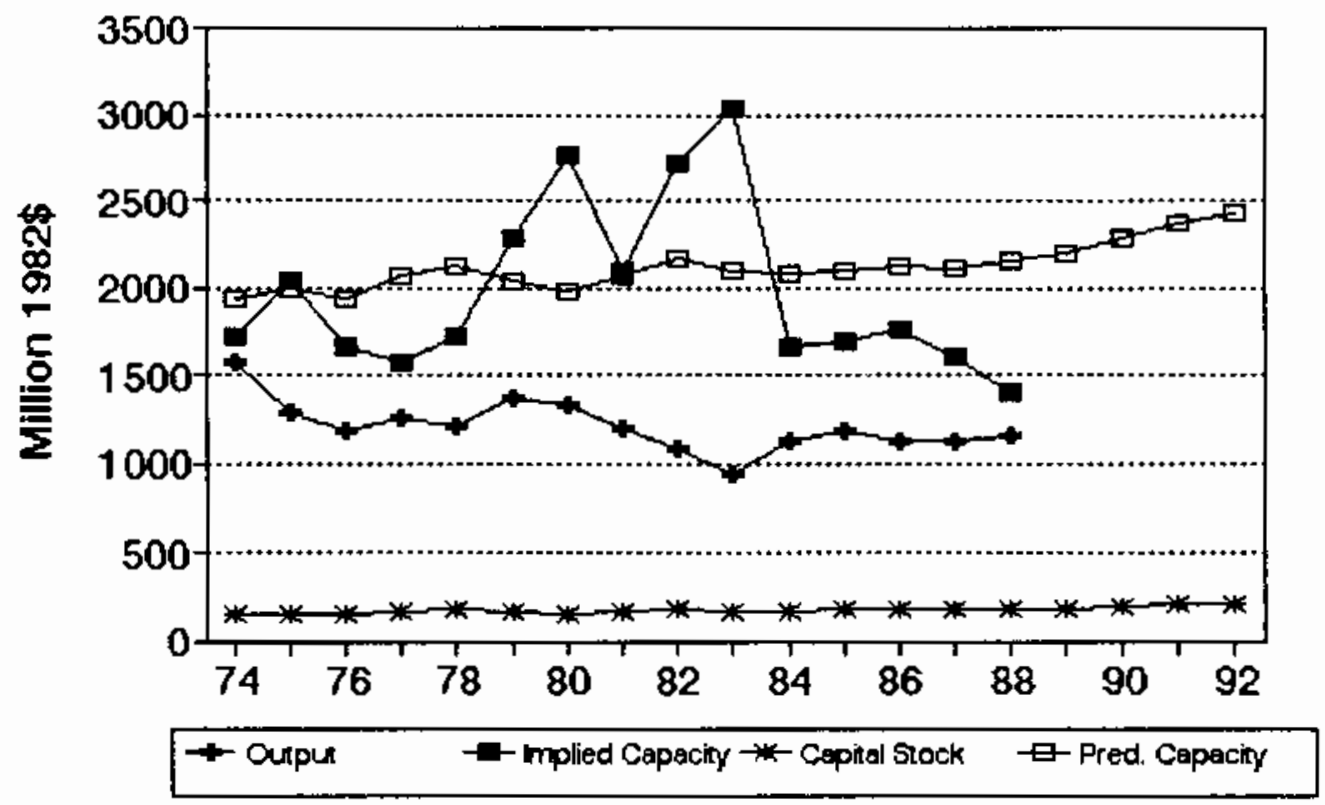


yo. 347 src 3568 Power trangmission equipinent, nec

MILLIONS OF 1982 DOLLARS (EXCL. CU RATES)

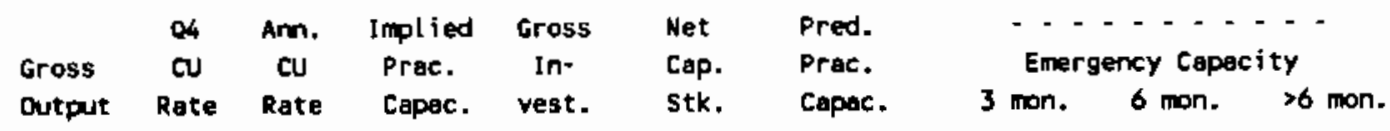

\begin{tabular}{|c|c|c|c|c|c|c|c|c|c|c|}
\hline 1974 & 2,480 & 81 & 84.4 & 2,937 & 59.7 & 660 & 3,139 & & & \\
\hline 1975 & 2,356 & $\infty$ & 67.3 & 3,503 & 126.5 & 728 & 3,471 & & & \\
\hline 1976 & 2,143 & 68 & 67.5 & 3,174 & 47.4 & 716 & 3,413 & & & \\
\hline 1977 & 2,583 & $\pi$ & 71.3 & 3,623 & 80.1 & 735 & 3,507 & & & \\
\hline 1978 & 2,630 & 73 & 7.4 & 3,633 & 138.5 & 805 & 3,854 & & & \\
\hline 1979 & 2,750 & 68 & 71.8 & 3,832 & 44.7 & 784 & 3,749 & & & \\
\hline 1980 & 2,456 & 61 & 62.5 & 3,931 & 92.4 & 811 & 3,884 & & & \\
\hline 1981 & 2,309 & $\infty$ & 67.4 & 3,425 & $(10.5)$ & 741 & 3,539 & & & \\
\hline 1982 & 1,942 & 46 & 52.5 & 3,697 & 19.0 & 704 & 3,357 & & & \\
\hline 1983 & 1,654 & 49 & 46.6 & 3,552 & 69.9 & 715 & 3,408 & & & \\
\hline 1984 & 2,313 & 54 & 54.5 & 4.247 & 262.7 & 900 & 4,318 & & & \\
\hline 1985 & 2,225 & 47 & 49.7 & $4,4 \pi 5$ & 52.0 & 876 & 4,200 & & & \\
\hline 1986 & 2,080 & 56 & 52.6 & 3,956 & 66.5 & 865 & 4,146 & & & \\
\hline$\cdot \cdot$ & --- & -- & $\cdots \cdot$ & --- & $\cdots$ & $\cdots$ & --- & -- & $-\cdots$ & -- \\
\hline 1987 & 2,129 & 73 & 65.9 & 3,231 & 80.1 & 868 & 4,164 & & & \\
\hline 1988 & 2,194 & 70 & 72.3 & 3,035 & 79.6 & 871 & 4,178 & 4.155 & 5,595 & 5,616 \\
\hline 1989 & & & & & 79.0 & 873 & 4,189 & 4,165 & 5,609 & 5,630 \\
\hline 1990 & & & & & 78.4 & 875 & 4,195 & 4,172 & 5,618 & 5,638 \\
\hline 1991 & & & & & 77.9 & 875 & 4,199 & 4,175 & 5,622 & 5,643 \\
\hline 1992 & & & & & 77.3 & 875 & 4,199 & 4,175 & 5,622 & 5,643 \\
\hline
\end{tabular}

Capecity-Capital stock Regression: Code 0 RSQ 0.790

Coefficients: Const -107.8 stock 4.9

Ave. Capacity/Stock $(74-86): 4.8$ Ave. Weekly Hours (High) 117.4 Shift Factor 1.34 Ave Weekly Hours (LOW) 113.6 Shift Factor 1.39 Wote: Output, Investment, and Capital Stock extrapolated for 1987 and 1988.

\section{Power transmission equipment, nec}

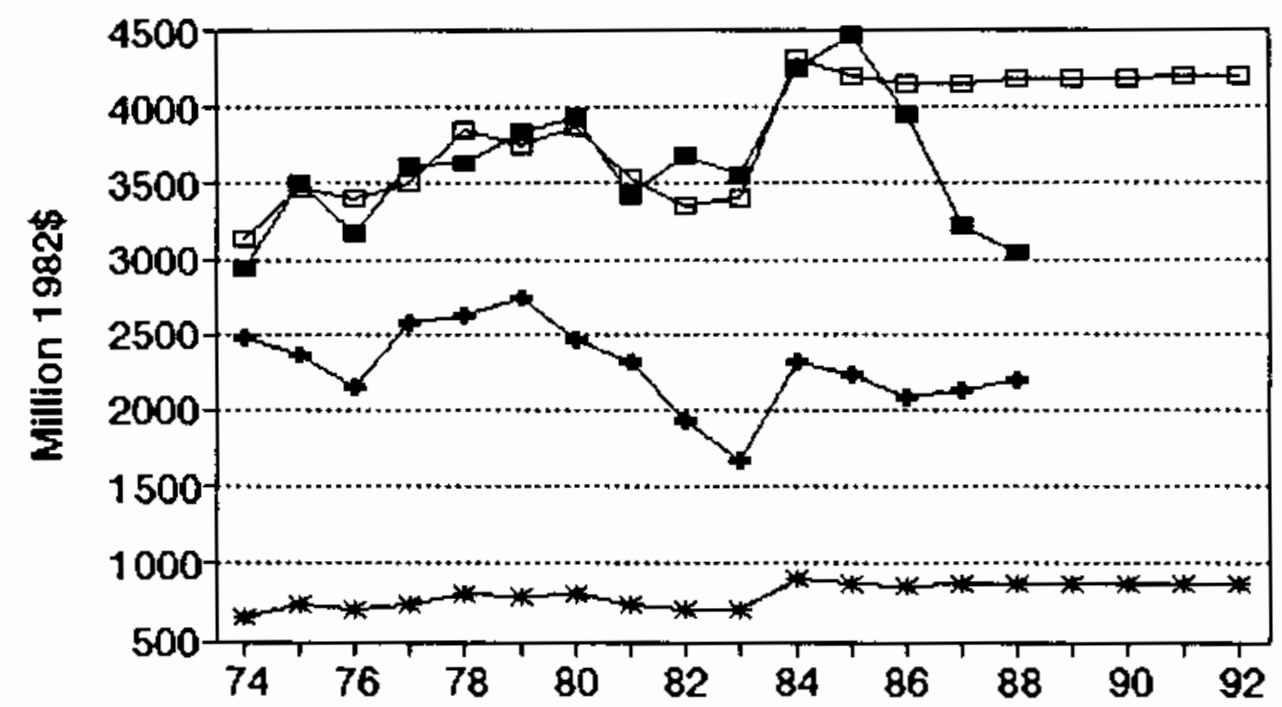

- Ouput $\rightarrow-$ mplied Cepeciny $\rightarrow$ - Cepitel Stock $\square-$ Pred. Capecity 
Ho. 348 SIC 3569 General industrial machinery, nec

MILLIONS OF 1982 DOLLARS (EXCL, CU RATES)

$\begin{array}{lccccccc} & 04 & \text { Ann. Implied Gross } & \text { Net } & \text { Pred. } & \ldots \ldots \\ \text { Gross } & \text { CU } & \text { CU } & \text { Prac. } & \text { In- } & \text { Cop. } & \text { Prac. } & \text { Emergency Capacity } \\ \text { Output Rate } & \text { Rate } & \text { Capac. } & \text { vest. } & \text { Stk. } & \text { Capac. } & 3 \text { mon. } 6 \text { mon. }>6 \text { mon. }\end{array}$

\begin{tabular}{|c|c|c|c|c|c|c|c|c|c|c|}
\hline 1974 & 3,170 & 34 & 34.0 & 9,323 & 21.8 & 489 & 6.771 & & & \\
\hline 1973 & 2,649 & 51 & 44.6 & 5,936 & 93.2 & 535 & 6,825 & & & \\
\hline 1976 & 2,927 & 56 & 54.1 & 5,408 & 76.3 & 562 & 6,855 & & & \\
\hline 1977 & 4,237 & 62 & 59.8 & 7,091 & 246.9 & 746 & 7,068 & & & \\
\hline 1978 & 4,319 & 59 & 60.1 & 7,183 & 95.9 & 780 & 7,106 & & & \\
\hline 1979 & 4,421 & 59 & 59.0 & 7,492 & 112.9 & 827 & 7,161 & & & \\
\hline 1980 & 4,314 & $\pi$ & 70.3 & 6,140 & 97.5 & $8 \infty$ & 7,199 & & & \\
\hline 1981 & 4,511 & 71 & 73.3 & 6,159 & 130.7 & 923 & 7,272 & & & \\
\hline 1982 & 4,652 & 64 & 66.6 & 6,983 & 166.6 & 1,015 & 7,37 & & & \\
\hline 1983 & 4,199 & 61 & 62.1 & 6,759 & 49.9 & 989 & 7,347 & & & \\
\hline 1984 & 4,579 & 57 & 58.5 & 7,827 & 109.2 & 1,020 & 7,383 & & & \\
\hline 1985 & 4,848 & 56 & 56.4 & 8,599 & 94.3 & 1,034 & 7,400 & & & \\
\hline 1986 & 4,646 & 56 & 56.0 & 8,297 & 111.4 & 1.062 & 7,431 & & & \\
\hline$\cdot-$ & $-\cdots$ & 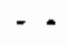 & $\cdot-$ & $\cdots-$ & $\cdots$ & $\cdots$ & $\cdots$ & $\cdots-$ & $-\cdot-$ & $\cdots-$ \\
\hline 1987 & 4,716 & 52 & 53.5 & 8,816 & 95.3 & 1,074 & 7,446 & & & \\
\hline 1988 & 4,809 & 70 & 63.3 & 7,604 & 135.4 & 1,126 & 7,505 & 9.106 & 12,263 & 12,308 \\
\hline 1989 & & & & & 153.0 & 1,191 & 7.580 & 9.197 & 12,386 & 12,431 \\
\hline 1990 & & & & & 172.5 & 1,270 & 7.672 & 9,309 & 12,536 & 12,582 \\
\hline 1991 & & & & & 189.9 & 1,361 & 7,776 & 9,435 & 12,707 & 12,753 \\
\hline 1992 & & & & & 174.7 & 1,429 & 7,855 & 9,531 & 12,835 & $12,8 B 2$ \\
\hline
\end{tabular}

Capacity-Capital stock Regression: Code 1 RSO 0.040

Coefficients: Const 6207.8 Stock 1.2

Ave. Capacity/Stock $(74-86): 8.6$ Ave. Weekly Hours (High) 96.0 Shift Factor 1.64 Ave Weekly Hours (LOW) 86.7 Shift Factor 1.82 Note: Output, Investment, and Capital Stock extrapolated for 1987 and 1988.

\section{General industrial machinery, nec}
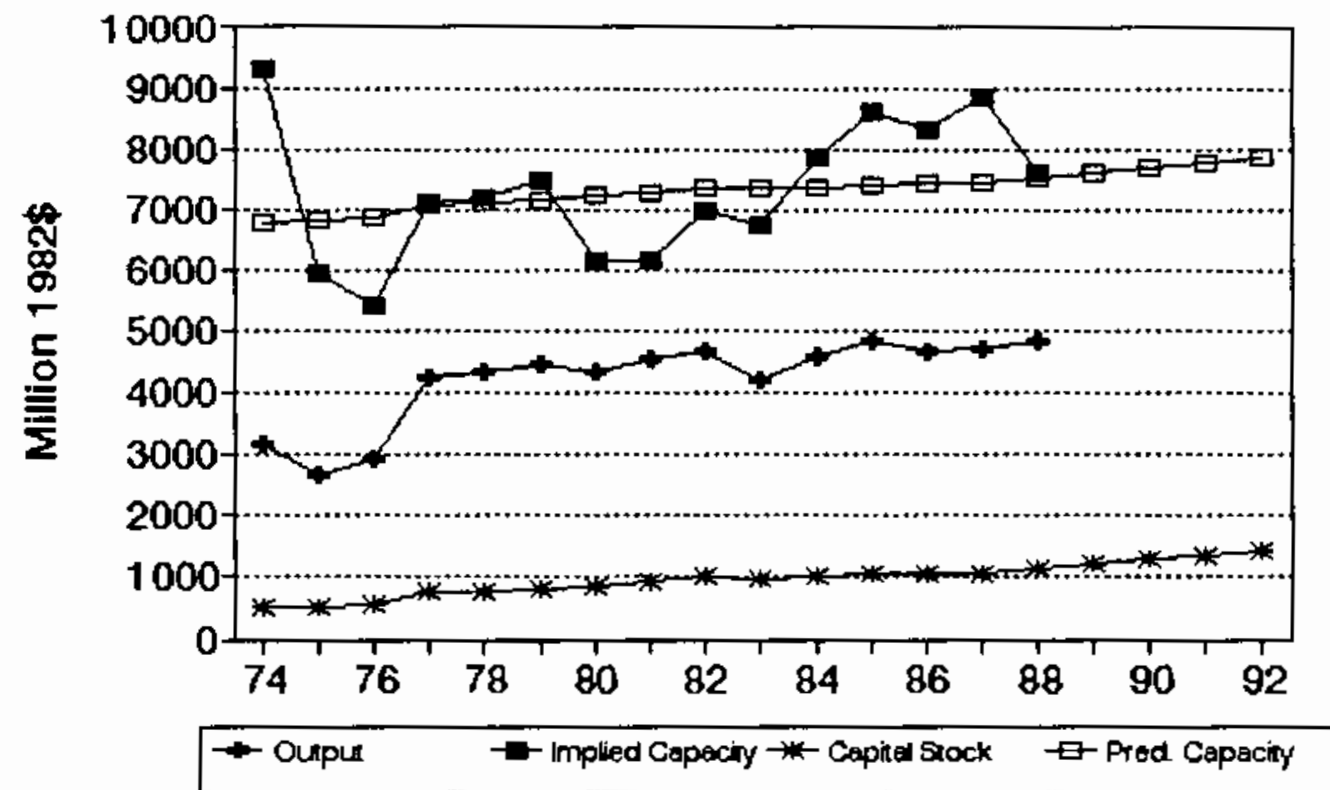


\section{No. 349 sIC 3573 Electronic computing equipment}

MILLIOAS OF 1982 DOLLARS (EXCL. CU RATES)

$\begin{array}{lccccccc} & \text { Q4 } & \text { Am. Implied Gross } & \text { Net } & \text { Pred. } & -\ldots & \ldots \ldots \ldots \\ \text { Gross } & \text { CU } & \text { CU } & \text { Prac. } & \text { In- } & \text { Cap. } & \text { Prac. } & \text { Emergency Capecity } \\ \text { Output } & \text { Rate } & \text { Rate } & \text { Capac. } & \text { vest. } & \text { Stk. } & \text { Capac. } & 3 \text { mon. } 6 \text { mon. }>6 \text { mon. }\end{array}$

\begin{tabular}{|c|c|c|c|c|c|c|c|c|c|c|}
\hline 1974 & 3,267 & 70 & 71.1 & 4,597 & 377.7 & 2,487 & $(7,434)$ & & & \\
\hline 1975 & 2,748 & 60 & 62.0 & 4,435 & 255.7 & 2,559 & $(6,178)$ & & & \\
\hline 1976 & 4,429 & 64 & 62.3 & 7,105 & 160.8 & 2,529 & $(6,698)$ & & & \\
\hline 1977 & 7,162 & 66 & 65.3 & 10,971 & 758.8 & 3,062 & 2,573 & & & \\
\hline 1978 & 10,625 & 75 & 71.4 & 14,889 & 937.8 & 3,742 & 14,399 & & & \\
\hline 1979 & 15,703 & 74 & 75.1 & 20,912 & $1,016.7$ & 4,470 & 27,068 & & & \\
\hline 1980 & 23,403 & 72 & 72.3 & 32,366 & $1,233.5$ & 5,381 & 42,914 & & & \\
\hline 1981 & 30,171 & 74 & 74.0 & 40,787 & 1.427 .7 & 6,443 & 61,375 & & & \\
\hline 1982 & 37,349 & 63 & 66.5 & 56,143 & $1,341.7$ & 7,367 & 77,447 & & & \\
\hline 1983 & 56,903 & 74 & 69.3 & 79,276 & $1,806.4$ & 8,676 & 100,225 & & & \\
\hline 1984 & 84,974 & 71 & 73.2 & 116,125 & $1,889.7$ & 9,593 & 123,138 & & & \\
\hline 1985 & 107,770 & 65 & 67.3 & 160,144 & $2,099.9$ & 11,429 & 148,105 & & & \\
\hline 1986 & 122,489 & 60 & 61.8 & 198,171 & $2,006.7$ & 12,629 & 168,987 & & & \\
\hline$=-$ & $-\cdots$ & $\cdots$ & $\therefore \cdot$ & $\cdots \cdot$ & --- & --- & ---- & ---- & ---- & $-\cdot-$ \\
\hline 1987 & 129,201 & 65 & 62.9 & 205,301 & $1,504.5$ & 13,364 & 181,759 & & & \\
\hline 1988 & 138,425 & 68 & 67.2 & 206,054 & $2,301.9$ & 14,851 & 207,623 & 239,225 & 313,285 & 314,341 \\
\hline 1989 & & & & & $3,260.4$ & 17,205 & 248,579 & 286,416 & 37,085 & 376,349 \\
\hline 1990 & & & & & $3,510.2$ & 19,666 & 291,383 & 335,735 & 439,673 & 441,154 \\
\hline 1991 & & & & & $3,433.3$ & 21,900 & 330,240 & 300,506 & 498,305 & 499,984 \\
\hline 1992 & & & & & $3,125.9$ & 23,691 & 361,379 & 416,385 & 545,291 & 547,128 \\
\hline
\end{tabular}

Capecity-Capital Stock Regression: Code 0 RSO 0.940

Coefficients: Const -50682.9 stock 17.4

Ave. Capacity/Stock $(74-86): 9.2$ Ave. Weekly Hours (High) 104.0 Shift Factor 1.51 Ave Weekly Hours (Low) 101.1 Shift Factor 1.56 Note: Output, Investment, and Capital Stock extrapolated for 1987 and 1988.

3573 Electronic computing equipment

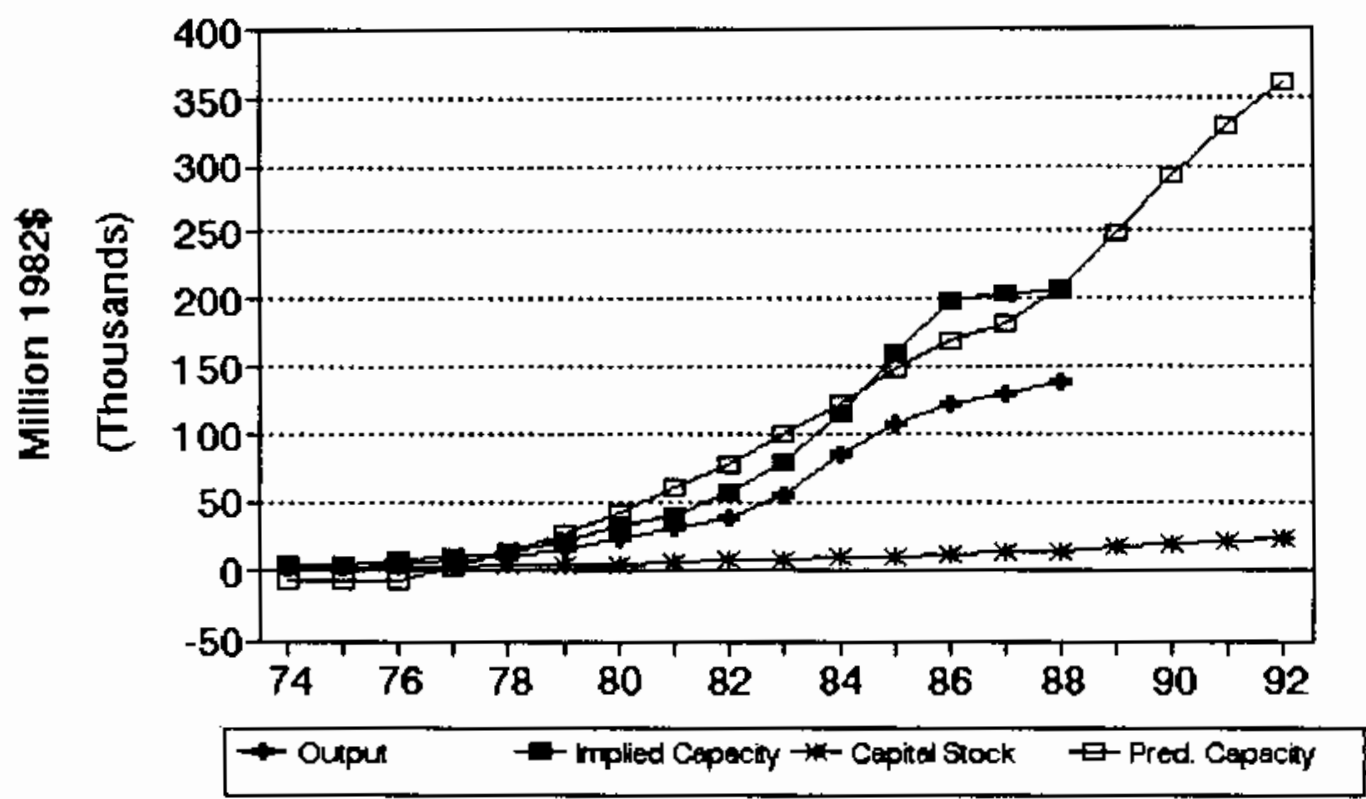




\section{No. 350 SIC 3574 Calculating and accounting achines}

HILLIONS OF 1982 DOLLARS (EXCL. CU RATES)

$\begin{array}{lccccccc} & 04 & \text { Ann. Implied } & \text { Gross } & \text { Net } & \text { Pred. } & \ldots & \ldots \\ \text { Gross } & \text { CU } & \text { CU } & \text { Prac. } & \text { In* } & \text { Cap. } & \text { Prac. } & \text { Emergency Capacity } \\ \text { Output } & \text { Rete } & \text { Rate } & \text { Capac. } & \text { vest. } & \text { Stk. } & \text { Capac. } & 3 \text { mon. } 6 \text { mon. }>6 \text { mon. }\end{array}$

\begin{tabular}{|c|c|c|c|c|c|c|c|c|c|c|}
\hline 1974 & 660 & 56 & 57.2 & 1,155 & 61.2 & 264 & 1,527 & & & \\
\hline 1975 & 513 & 40 & 44.0 & 1,166 & 14.0 & 242 & 1,603 & & & \\
\hline 1976 & 806 & 42 & 41.1 & 1,861 & 47.3 & 250 & 1,574 & & & \\
\hline 1977 & 640 & 47 & 45.1 & 1,417 & $(20.3)$ & 199 & 1,752 & & & \\
\hline 1978 & 946 & 70 & 61.0 & 1,552 & 20.6 & 190 & 1,783 & & & \\
\hline 1979 & 1,191 & 68 & 69.7 & 1,710 & 87.9 & 242 & 1.602 & & & \\
\hline 1980 & 1,434 & 74 & 71.2 & 2,014 & 46.5 & 253 & 1,564 & & & \\
\hline 1981 & 1,192 & 65 & 69.3 & 1,720 & $(65.6)$ & 165 & 1,871 & & & \\
\hline 1982 & 1,470 & 53 & 56.8 & 2,590 & $(6.4)$ & 139 & 1,959 & & & \\
\hline 1983 & 1,331 & 56 & 54.2 & 2,454 & 24.5 & 145 & 1,938 & & & \\
\hline 1984 & 1,188 & 49 & 52.8 & 2,252 & 1.2 & 130 & 1,990 & & & \\
\hline 1985 & 903 & $69 *$ & 56.7 & 1,592 & 2.6 & 117 & 2,035 & & & \\
\hline 1986 & 1,071 & 66 & 64.1 & 1,671 & 9.1 & 111 & 2,057 & & & \\
\hline$\cdot-$ & $-\cdots$ & -- & $=-$ & $\cdots \cdot$ & $\cdots$ & $-\cdots$ & $--\cdot$ & $\cdots--\cdots$ & --- & $--\cdots$ \\
\hline 1987 & 1,087 & 51 & 56.5 & 1,924 & (7.1) & 89 & 2,133 & & & \\
\hline 1988 & 1,109 & 53 & 52.6 & 2,108 & $(10.6)$ & 67 & 2,212 & 3,127 & 4,611 & 4,632 \\
\hline 1989 & & & & & $(14.0)$ & 44 & 2,292 & 3,240 & 4,777 & 4.799 \\
\hline 1990 & & & & & (17.5) & 20 & 2,373 & 3,355 & 4,946 & 4,969 \\
\hline 1991 & & & & & $(20.9)$ & (4) & 2,455 & 3,471 & 5,117 & 5,141 \\
\hline 1992 & & & & & $(24.4)$ & (28) & 2,538 & 3,589 & 5,291 & 5,315 \\
\hline
\end{tabular}

Capecity-Capital stock Regression: Code 1 RSO 0.190

Coefficients: Const 2442.7 Stock -3.5

Ave. Capacity/Stock $(74-86): 9.5$

Ave. Weekly Hours (High) 73.2 shift Factor 2.09 Ave Weekly Hours (Low) 63.9 Shift Factor 2.47

- Imputed Mote: Output, Investment, and Capital Stock extrapolated for 1987 and 1988.

\section{Calculating and accounting machine}

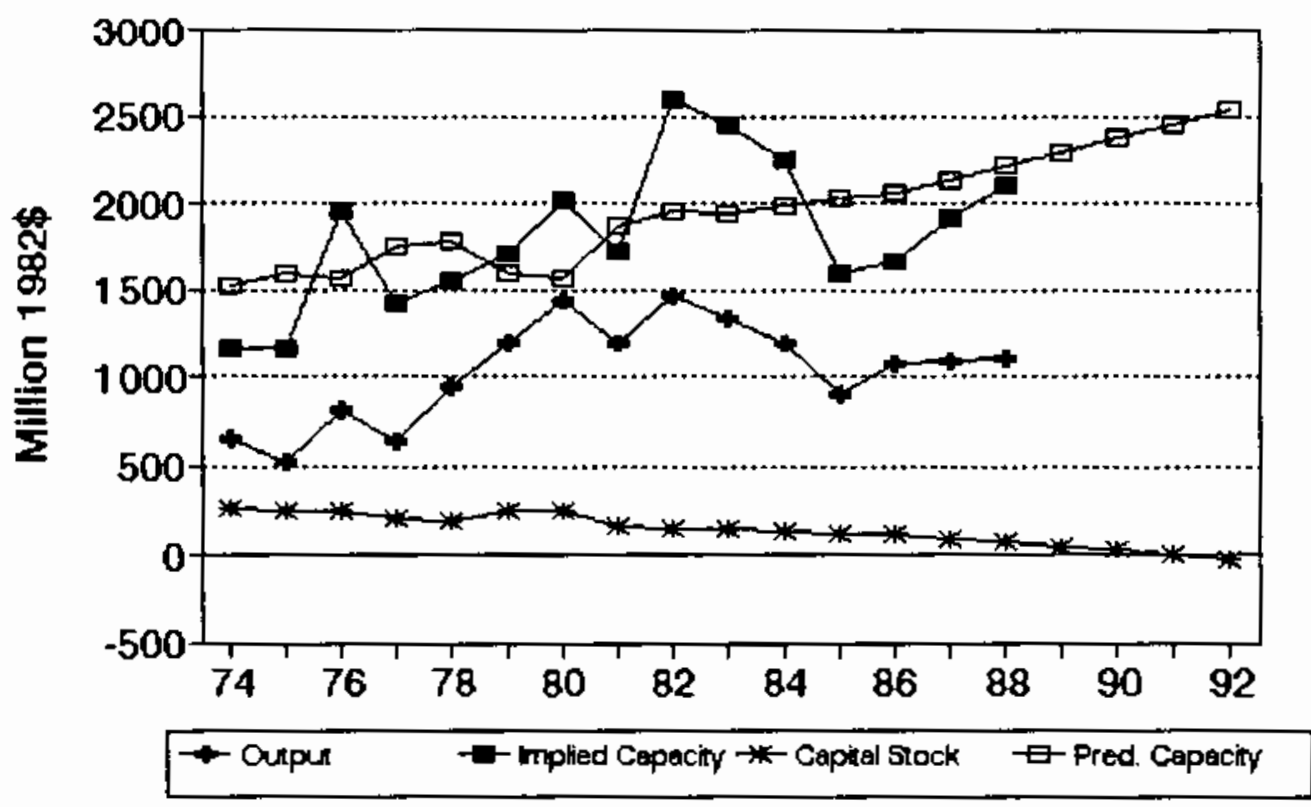




\section{No. 351 sIC 3576 scales and balances, except laboratory}

MILLIONS OF 1982 DOLLARS (EXCL. CU RATES)

$\begin{array}{lccccccc} & Q 4 & \text { Ann. Implied } & \text { Gross } & \text { Net } & \text { Pred. } & \ldots \ldots \ldots \\ \text { Gross } & \text { CU } & \text { CU } & \text { Prac. } & \text { In- } & \text { Cap. } & \text { Prac. } & \text { Emergency Capacity } \\ \text { Output } & \text { Rate } & \text { Rate } & \text { Capac. } & \text { vest. } & \text { Stk. } & \text { Capac. } & 3 \text { mon. } 6 \text { mon. }\end{array}$

\begin{tabular}{|c|c|c|c|c|c|c|c|c|c|c|}
\hline 1974 & 485 & 84 & 88.1 & 550 & 1.7 & 54 & 461 & & & \\
\hline 1975 & 494 & 64 & 64.7 & 763 & 17.0 & 63 & 689 & & & \\
\hline 1976 & 535 & 67 & 65.3 & $B 19$ & 9.9 & 67 & 769 & & & \\
\hline 1977 & 487 & 78 & 74.0 & 659 & 4.9 & 66 & 749 & & & \\
\hline 1978 & 540 & 71 & 73.0 & 739 & 8.3 & 68 & 794 & & & \\
\hline 1979 & 581 & 78 & 77.8 & 747 & 5.2 & 68 & $77 a$ & & & \\
\hline 1980 & 550 & 56 & 62.8 & 877 & 17.3 & 78 & 983 & & & \\
\hline 1981 & 470 & 53 & 56.0 & 838 & $(2.5)$ & 69 & 809 & & & \\
\hline 1982 & 514 & 43 & 45.6 & 1,126 & 27.4 & 89 & 1,189 & & & \\
\hline 1983 & 557 & 32 & 35.3 & 1,579 & 12.7 & 93 & 1,271 & & & \\
\hline 1984 & 544 & 73 & 59.4 & 916 & 0.4 & 85 & 1,121 & & & \\
\hline 1985 & 557 & 80 & 77.7 & 717 & . 13.4 & 90 & 709 & & & \\
\hline 1986 & 596 & $77 \pm$ & 77.9 & 765 & 12.4 & 93 & $\pi 3$ & & & \\
\hline-- & $\cdots$ & $\cdots$ & $\cdots$ & $-\cdots$ & $\cdots$ & -- & $\cdots$ & -1 & $-\cdots$ & $\cdots$ \\
\hline 1987 & 599 & 80 & 78.1 & 766 & 10.2 & 94 & 795 & & & \\
\hline 1988 & 603 & $81 \star$ & $B 2.0$ & 736 & 11.1 & 96 & 833 & 1,250 & 1,725 & 1,732 \\
\hline 1989 & & & & & 12.3 & 99 & 891 & 1,337 & 1,845 & 1,852 \\
\hline 1990 & & & & & 12.6 & 102 & 949 & 1,424 & 1,966 & 1,973 \\
\hline 1991 & & & & & 12.5 & 105 & 1,000 & 1,500 & 2,071 & 2,079 \\
\hline 1992 & & & & & 12.2 & 107 & 1,038 & 1,558 & 2,150 & 2,159 \\
\hline
\end{tabular}

Capacity-Capital Stock Regression: Code -1 RSQ 0.770

Coefficients: Const -538.9 Stock 19.5 D(85-B6) -504.0 Ave. Capacity/Stock (74-86): 11.3 Ave. Weekly Hours (High) 75.8 Shift Fector 2.08 Ave Weekly Hours (LOW) 69.5 Shift Factor 2.27 * Imputed Note: Output, Investment, and Capital Stock extrapolated for 1987 and 1988.

3576 Scales and balances, except labora

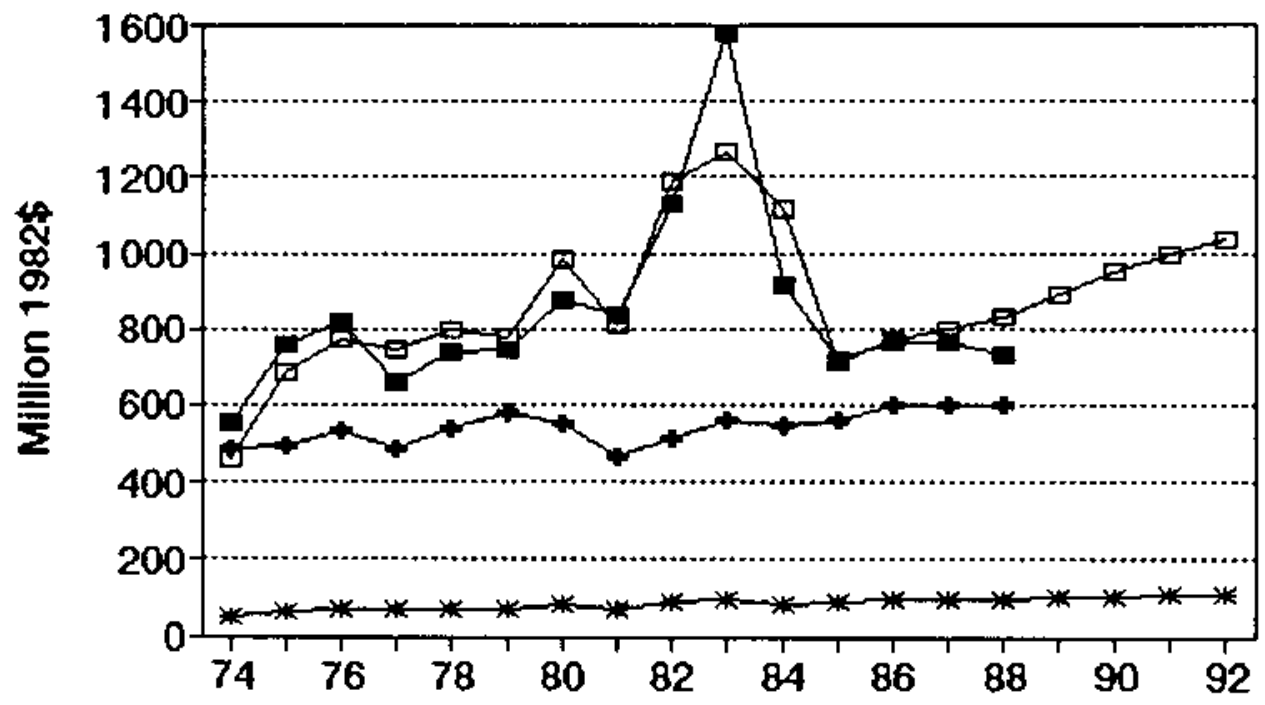

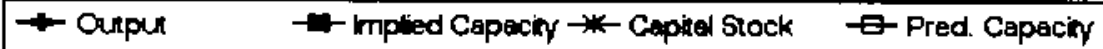




\section{No. 353 SIC 3581 Autonatic merchandising machines}

MILLIONS OF 1982 DOLLARS (EXCL. CU RATES)

\begin{tabular}{|c|c|c|c|c|c|c|}
\hline & $\begin{array}{l}04 \\
a v\end{array}$ & $\mathrm{Am}$. & $\begin{array}{l}\text { Implied } \\
\text { Prac. }\end{array}$ & $\begin{array}{c}\text { Gross } \\
\text { In- }\end{array}$ & $\begin{array}{l}\text { Net } \\
\text { Cap. }\end{array}$ & $\begin{array}{l}\text { Pred. } \\
\text { Prac. }\end{array}$ \\
\hline itput & Rate & Rate & Capac. & vest. & stk. & Capac. \\
\hline
\end{tabular}

\begin{tabular}{|c|c|c|c|c|c|c|c|c|c|c|}
\hline 1974 & 631 & 64 & 65.8 & 959 & 20.2 & 107 & 844 & & & \\
\hline 1975 & 366 & 74 & 67.3 & 543 & 20.5 & 117 & 891 & & & \\
\hline 1976 & 494 & 64 & 67.5 & 732 & 6.7 & 114 & 876 & & & \\
\hline 1977 & 583 & $71 *$ & 68.1 & 857 & 9.7 & 113 & 874 & & & \\
\hline 1978 & 630 & 81 & 76.6 & 823 & 19.1 & 122 & 912 & & & \\
\hline 1979 & 621 & 75 & 78.6 & 790 & 10.5 & 122 & 912 & & & \\
\hline 1980 & 568 & 69 & 70.4 & 807 & 20.4 & 131 & 953 & & & \\
\hline 1981 & 609 & 63 & 66.4 & 917 & 1.1 & 121 & 910 & & & \\
\hline 1982 & 479 & 57 & 58.4 & 819 & 3.0 & 114 & 879 & & & \\
\hline 1983 & 589 & 51 & 52.5 & 1,122 & 20.6 & 125 & 927 & & & \\
\hline 1984 & 594 & 47 & 50.0 & 1,189 & 5.8 & 121 & 911 & & & \\
\hline 1985 & 640 & 65 & 58.4 & 1,095 & 7.0 & 119 & 899 & & & \\
\hline 1986 & 635 & 59 & 61.1 & 1,038 & 10.5 & 119 & 902 & & & \\
\hline-- & $-\cdots$ & $\cdots$ & $\cdots$ & $\cdots \cdot$ & $\cdots$ & $\cdots$ & $\cdots \cdot$ & $\cdots$ & $\cdots \cdot$ & $\cdots$ \\
\hline 1987 & 636 & 70 & 65.5 & 972 & 11.3 & $12 t$ & 909 & & & \\
\hline 1988 & 638 & 75 & 73.8 & 865 & 11.5 & 123 & 917 & 935 & 1,154 & 1,157 \\
\hline 1989 & & & & & 11.6 & 124 & 924 & 943 & 1,163 & 1,167 \\
\hline 1990 & & & & & 11.8 & 126 & 932 & 951 & 1,173 & 1,177 \\
\hline 1991 & & & & & 12.0 & 128 & 940 & 959 & 1,183 & 1,187 \\
\hline 1992 & & & & & 12.2 & 130 & 949 & 968 & 1,194 & 1,197 \\
\hline
\end{tabular}

Capacity-Capitat Stock Regression: Code 1 RSO 0.020

Coefficients: Const 351.4 stock 4.6

Ave. Capacity/Stock (74-86): 7.6 Ave. Weekly Hours (High) 125.5 Shift Factor 1.26 Ave Weekly Hours (Low) 116.1 Shift Factor 1.36

* Imputed Note: Output, Investment, and Capital stock extrapolated for 1987 and 1988.

\section{Automatic merchandising machines}

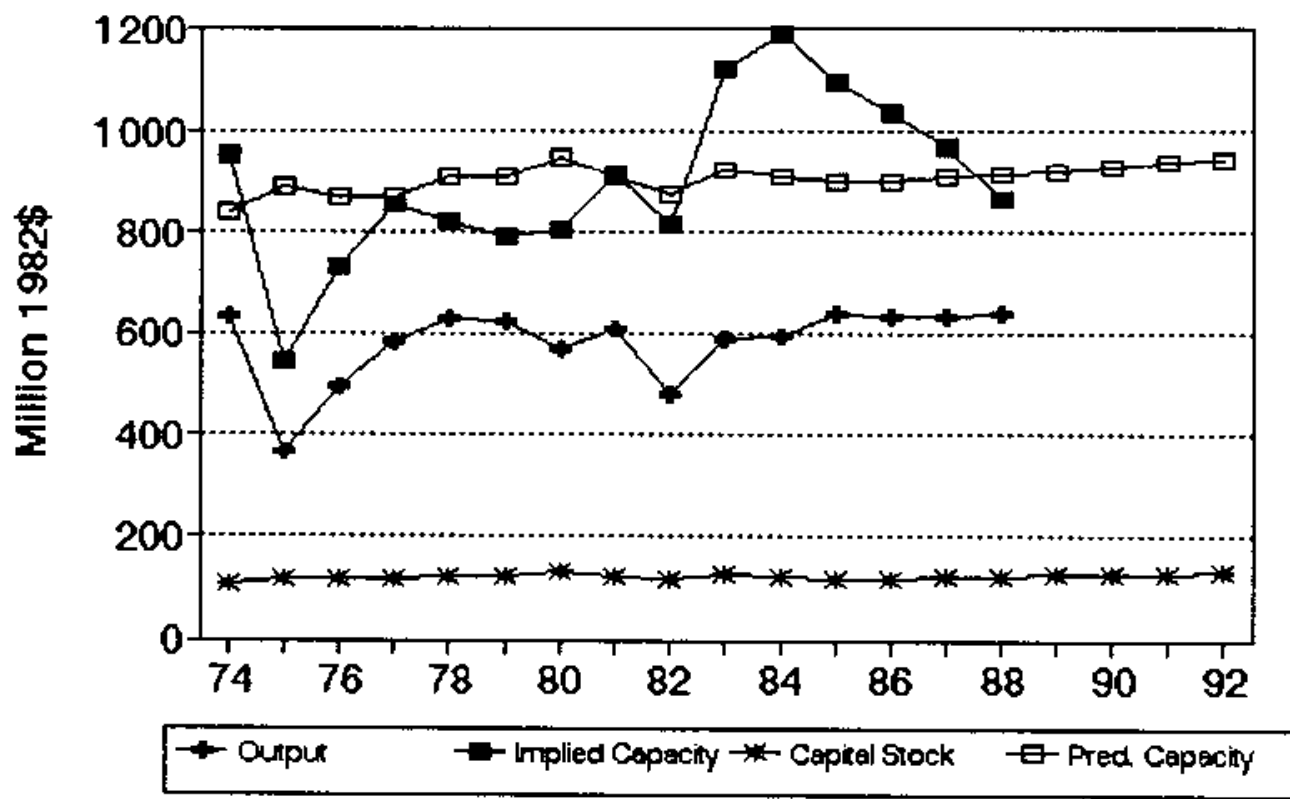




\section{No. 354 SIC 3582 comercial laundry equipent}

HILLIONS OF 1982 DOLLARS (EXCL. CU RATES)

$\begin{array}{lccccccc} & \text { O4 Ann. Implied Gross } & \text { Net } & \text { Pred. } & \ldots \ldots \ldots \ldots \\ \text { Gross } & \text { CU } & \text { CU } & \text { Prac. } & \text { In- } & \text { Cap. } & \text { Prac. } & \text { Emergency Capacity } \\ \text { Output } & \text { Rate } & \text { Rate } & \text { Capac. } & \text { vest. } & \text { Stk. } & \text { Capac. } & 3 \text { mon. } 6 \text { mon. }>6 \text { mon. }\end{array}$

\begin{tabular}{|c|c|c|c|c|c|c|c|c|c|c|}
\hline 1974 & 315 & 53 & 53.0 & 595 & 6.1 & 55 & 576 & & & \\
\hline 1975 & 232 & 53 & 53.0 & 438 & $(2.4)$ & 48 & 573 & & & \\
\hline 1976 & 255 & 53 & 53.0 & 481 & 3.5 & 46 & 570 & & & \\
\hline 1977 & 302 & 53 & 53.0 & 569 & $(1.9)$ & 41 & 568 & & & \\
\hline 1978 & 330 & 48 & 49.9 & 661 & 9.0 & 45 & 565 & & & \\
\hline 1979 & 359 & 53 & 51.1 & 702 & 7.4 & 49 & 562 & & & \\
\hline 1980 & 340 & 56 & 54.9 & 619 & $(1.0)$ & 45 & 559 & & & \\
\hline 1981 & 333 & 58 & 57.3 & 582 & $(0.9)$ & 41 & 556 & & & \\
\hline 1982 & 332 & 60 & 59.3 & 560 & 2.8 & 41 & 554 & & & \\
\hline 1983 & 329 & 62 & 61.3 & 537 & 9.9 & 47 & 551 & & & \\
\hline 1984 & 323 & 64 & 63.3 & 511 & 9.9 & 53 & 548 & & & \\
\hline 1985 & 343 & 64 & 64.0 & 537 & 5.9 & 55 & 545 & & & \\
\hline 1986 & 342 & 76 & 71.5 & 479 & 5.5 & 56 & 543 & & & \\
\hline $\begin{array}{l}\cdots \\
1987\end{array}$ & 344 & 80 & $\begin{array}{l}-- \\
78.5\end{array}$ & $\begin{array}{c}\cdots 38 \\
438\end{array}$ & $\begin{array}{r}-7 \\
5.6\end{array}$ & 58 & $\begin{array}{c}-- \\
540\end{array}$ & - & $\cdots$ & -- \\
\hline 1988 & 345 & 54 & 63.8 & 541 & 7.8 & 61 & 537 & 752 & 1,052 & 1,056 \\
\hline 1989 & & & & & 8.9 & 66 & 534 & 748 & 1,046 & 1,050 \\
\hline 1990 & & & & & 8.5 & 69 & 532 & 745 & 1,041 & 1,045 \\
\hline 1991 & & & & & 8.7 & 7 & 529 & 741 & 1,035 & 1,039 \\
\hline 1992 & & & & & 7.3 & 75 & 526 & 737 & 1,030 & 1,034 \\
\hline
\end{tabular}

Capacity-Capital Stock Regression: Code - 2 RSO 0.020 Coefficients: Const 578.6 Time -2.8

Ave. Cepacity/stock (74-86): 11.7 Ave. Weekly Hours (High) 80.1 Shift Factor 1.97 Ave Weekly Hours (LOW) 73.6 Shift Factor 2.14 Note: Output, Investment, and Capital Stock extrapolated for 1987 and 1988.

\section{Commercial laundry equipment}

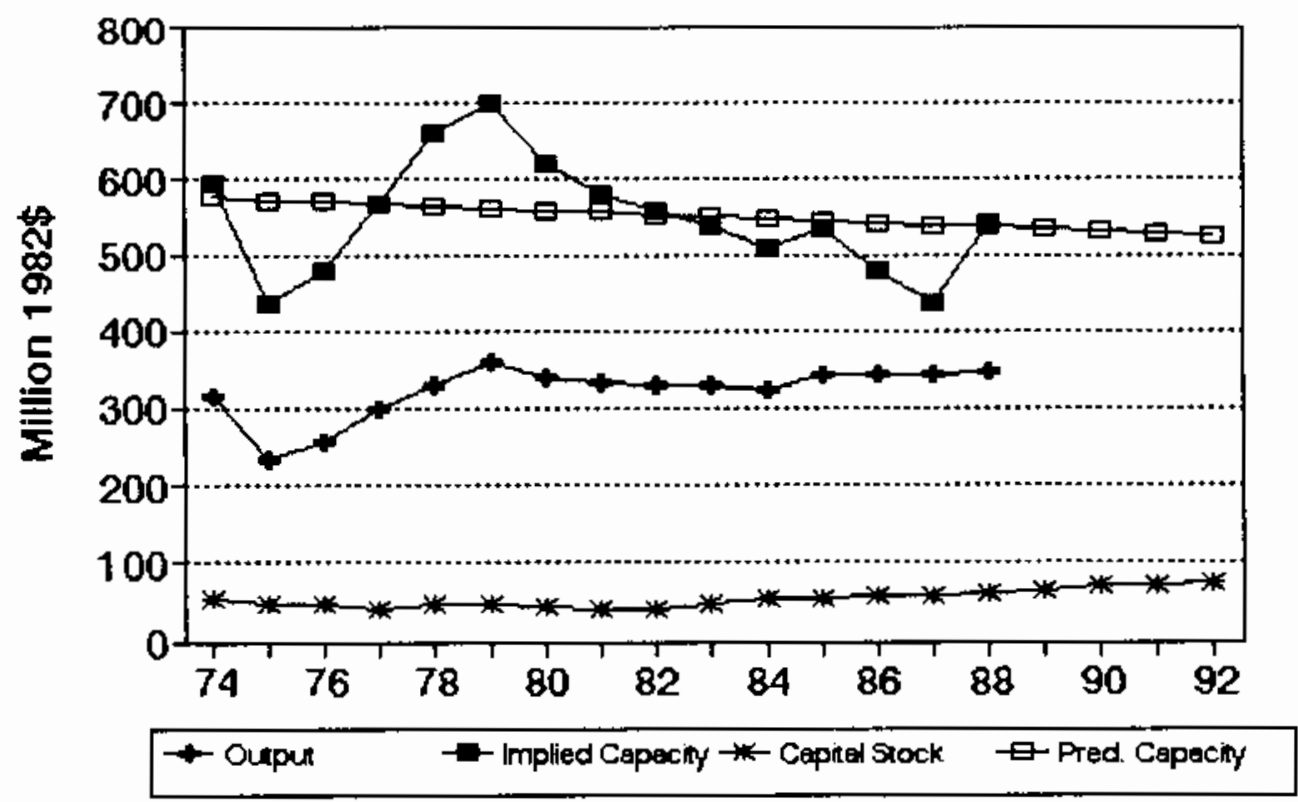




\section{No. 355 sIc 3585 Refrigeration and heating equipment}

MILLIONS OF 1982 DOLLARS (EXCL. CU RATES)

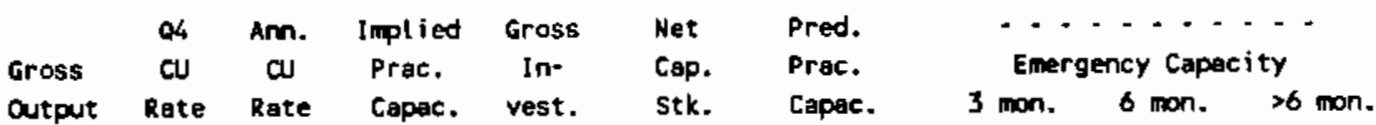

\begin{tabular}{|c|c|c|c|c|c|c|c|c|c|c|}
\hline 1974 & 13,718 & 62 & 62.6 & 21,917 & 360.3 & 2,950 & 20,130 & & & \\
\hline $19 \pi$ & 9,629 & 59 & 59.2 & 16,278 & $(47.5)$ & 2,746 & 18,526 & & & \\
\hline 1976 & 12,628 & 69 & 65.1 & 19,300 & 203.0 & $2,7 \pi 8$ & 18,781 & & & \\
\hline 1977 & 13,404 & 71 & 70.3 & 19,075 & 269.1 & 2,864 & 19,454 & & & \\
\hline 1978 & 15,048 & 71 & 70.9 & 21,236 & 309.3 & 2,976 & 20,326 & & & \\
\hline 1979 & 15,226 & 64 & 67.0 & 22,721 & 324.0 & 3,082 & 21,174 & & & \\
\hline 1980 & 12,651 & 55 & 58.1 & 21,738 & 291.6 & 3,143 & 21.655 & & & \\
\hline 1981 & 13,746 & 60 & 58.5 & 23,502 & 283.7 & 3,187 & 22,003 & & & \\
\hline 1982 & 12,517 & 57 & 57.9 & 21,635 & 202.1 & 3,161 & 21,639 & & & \\
\hline 1983 & 12,898 & 69 & 64.2 & 20,106 & 179.0 & 3,061 & 21,010 & & & \\
\hline 1984 & 15,170 & 71 & 70.8 & 21,417 & 334.0 & 3,128 & 21,534 & & & \\
\hline 1985 & 14,790 & 68 & 69.2 & 21,386 & 312.3 & 3,167 & 21,840 & & & \\
\hline 1986 & 14.474 & 73 & 71.1 & 20,358 & 391.1 & 3,277 & 22,707 & & & \\
\hline$\because-$ & $-\cdots$ & $\because$ & $-\cdots$ & $\cdots$ & $-\cdots$ & $\cdots-$ & $\cdots$ & $\cdots \cdot$ & $---\cdot$ & - - - \\
\hline 1987 & 16,519 & $\pi$ & 76.1 & 19,588 & 337.9 & 3,332 & 23,148 & & & \\
\hline 1988 & 14,578 & 73 & 76.0 & 19,707 & 467.7 & 3,513 & 24,573 & 28,965 & 39,535 & 39,685 \\
\hline 1989 & & & & & 533.6 & 3,744 & 26,394 & 31,111 & 42,465 & 42,626 \\
\hline 1990 & & & & & 506.5 & 3,928 & 27,846 & 32,823 & 44,801 & 44,972 \\
\hline 1991 & & & & & 520.0 & 4,110 & 29,280 & 36,512 & 47,107 & 47,286 \\
\hline 1992 & & & & & 439.3 & 4.196 & 29,953 & 35,307 & 48,191 & 48,373 \\
\hline
\end{tabular}

Capacity-Capital Stock Regression: Code 0 RSO 0.490 Coefficients: Const -3123.1 stock 7.9

Ave. Capacity/stock $(74-86): 6.9$ Ave. Weekly Hours (High) 97.5 Shift Factor 1.62 Ave Veekly Hours (Low) 95.9 Shift Factor 1.64 Wote: Output, Investment, and Capital Stock extropolated for 1987 and 1988.

\section{Refrigeration and heating equipmen}

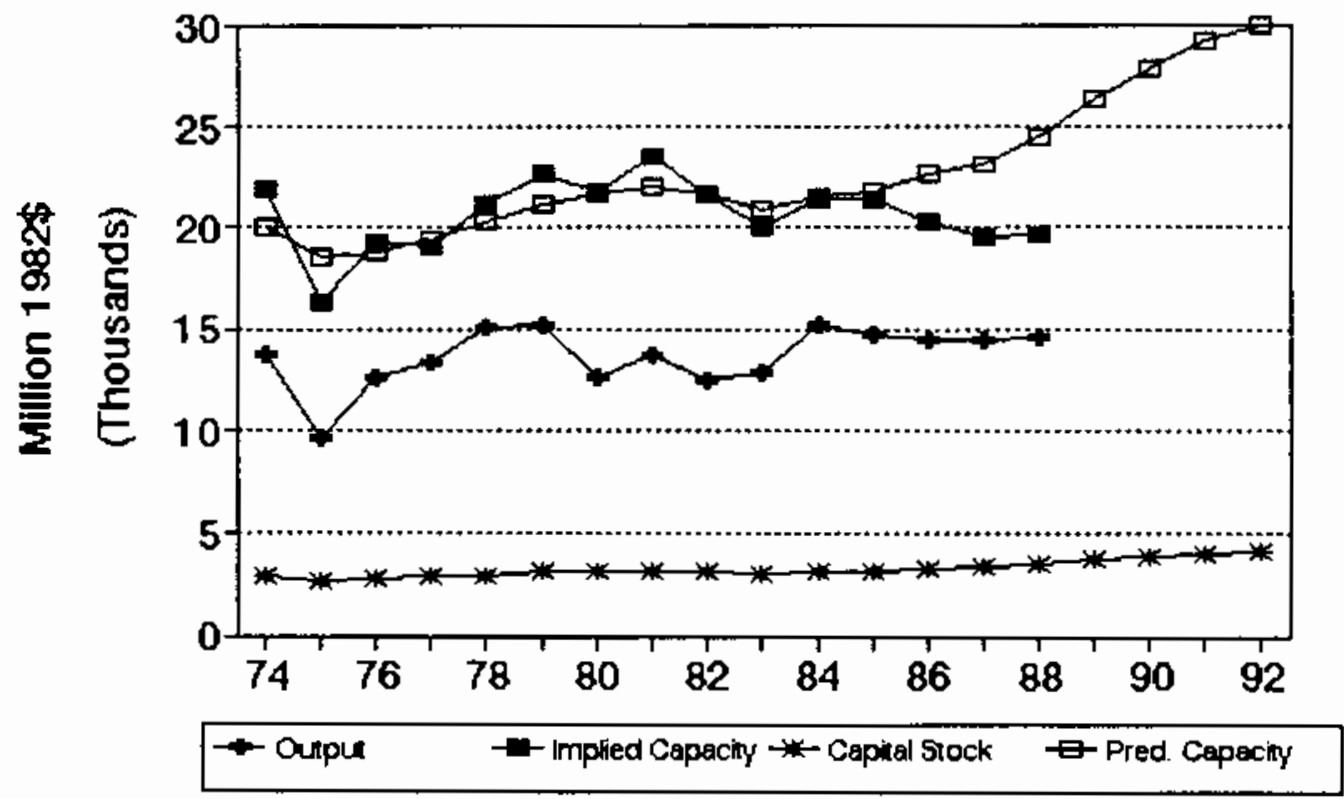


No. 356 sIC 3586 Measuring and dispensing pumps

MILLIONS OF 1982 DOLLARS (EXCL. CU RATES)

$\begin{array}{lccccccc} & 04 & \text { Ann. } & \text { Implied Gross } & \text { Net } & \text { Pred. } & \ldots \\ \text { Gross } & \text { CU } & \text { CU } & \text { Prac. } & \text { In- } & \text { Cap. } & \text { Prac. } & \text { Emergency Capacity } \\ \text { Output } & \text { Rate } & \text { Rate } & \text { Capac. } & \text { vest. } & \text { Stk. } & \text { Capac. } & 3 \text { mon. } 6 \text { mon. }>6 \text { mon. }\end{array}$

\begin{tabular}{|c|c|c|c|c|c|c|c|c|c|c|}
\hline 1974 & 680 & 83 & 85.3 & 797 & 27.5 & 116 & 935 & & & \\
\hline 1975 & 608 & 69 & 70.5 & 862 & 13.1 & 117 & 953 & & & \\
\hline 1976 & 612 & 61 & 63.8 & 960 & 8.8 & 116 & 938 & & & \\
\hline 1977 & 611 & 71 & 67.3 & 909 & $(3.0)$ & 104 & 830 & & & \\
\hline 1978 & 586 & 83 & 78.0 & 751 & 0.2 & 96 & 763 & & & \\
\hline 1979 & 631 & 73 & 78.1 & 809 & 12.5 & 101 & 804 & & & \\
\hline 1980 & 659 & $69 *$ & 69.5 & 948 & 15.1 & 108 & 866 & & & \\
\hline 1981 & 590 & $67 *$ & 68.6 & 860 & $(0.6)$ & 100 & 794 & & & \\
\hline 1982 & 699 & $64 *$ & 64.2 & 1,089 & 50.0 & 139 & 1,142 & & & \\
\hline 1983 & 657 & 66 & 64.2 & 1,023 & 11.6 & 139 & 1,146 & & & \\
\hline 1984 & 877 & $67 *$ & 68.3 & 1,285 & 19.0 & 146 & 1,209 & & & \\
\hline 1985 & 803 & 60 & 62.6 & 1,283 & 22.0 & 156 & 1,301 & & & \\
\hline 1986 & 762 & 47 & 51.7 & 1,473 & 19.7 & 164 & 1,367 & & & \\
\hline$-\cdots$ & $\cdots \cdot \cdot$ & - - - & --- & $-\cdots$ & $\cdots \cdot$ & $-\cdots$ & $-\cdots$ & $\cdot \cdot \cdot$ & $-\cdot-$ & --- \\
\hline 1987 & 764 & 63 & 56.6 & 1,350 & 17.4 & 169 & 1,412 & & & \\
\hline 1988 & 767 & $62 *$ & 62.7 & 1,223 & 22.3 & 178 & 1,498 & 1.685 & 2,377 & 2,387 \\
\hline 1989 & & & & & 24.8 & 189 & 1,590 & 1,810 & 2,538 & 2,549 \\
\hline 1990 & & & & & 23.8 & 199 & 1,684 & 1,906 & 2,673 & 2,684 \\
\hline 1991 & & & & & 24.3 & 208 & 1,766 & 2,000 & 2,804 & 2,816 \\
\hline 1992 & & & & & 21.2 & 214 & 1,816 & 2,055 & 2,882 & 2,894 \\
\hline
\end{tabular}

Capecity-Capital stock Regression: Code 0 RSO 0.860

Coefficients: Const -101.9 stock 9.0

Ave. Capacity/Stock $(74-86): 8.2$ Ave. Heekly Hours (High) 98.8 Shift Factor 1.59 Ave Heekly Hours (Low) 87.0 Shift Factor 1.81

* Imputed Note: Output, Imvestment, and Capital Stock extrapolated for 1987 and 1988.

\section{Measuring and dispensing pumps}

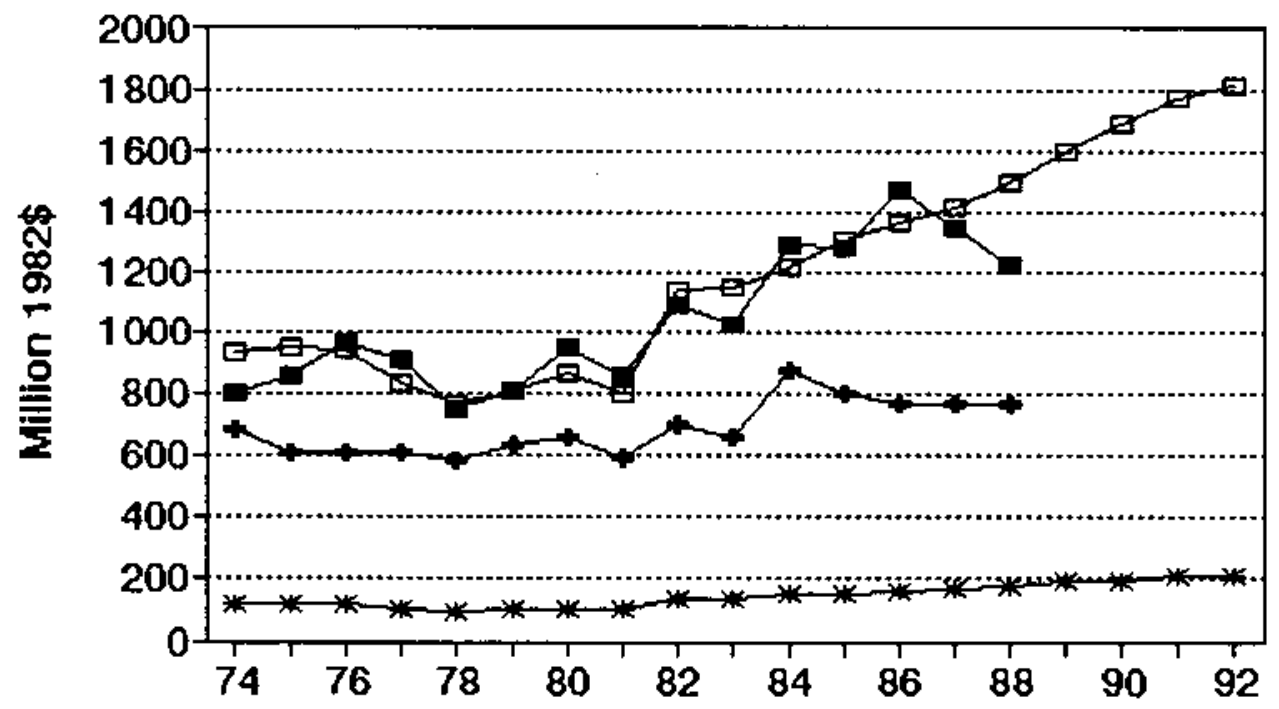

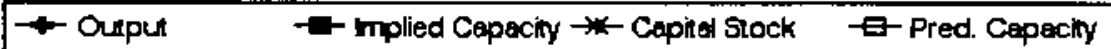




\section{No. 357 sIC 3589 Service industry achinery, nec}

MILLIONS OF 1982 DOLLARS (EXCL. CU RATES)

$\begin{array}{lccccccc} & \text { Q4 Am. Implied Gross } & \text { Net } & \text { Pred. } & \ldots \ldots \ldots & \ldots . . \\ \text { Gross } & \text { CU } & \text { CU } & \text { Prac. } & \text { In+ } & \text { Cap. } & \text { Prac. } & \text { Emergency Capacity } \\ \text { Output Rate } & \text { Rate } & \text { Capac. } & \text { vest. } & \text { Stk. } & \text { Capac. } & 3 \text { mon. } 6 \text { mon. }>6 \text { mon. }\end{array}$

\begin{tabular}{|c|c|c|c|c|c|c|c|c|c|c|}
\hline 1976 & 1,641 & 53 & 53.6 & 3,059 & 37.2 & 233 & 2,735 & & & \\
\hline 1975 & 1,523 & 67 & 60.7 & 2,509 & $(6.7)$ & 213 & 2,501 & & & \\
\hline 1976 & 1,767 & 73 & 70.6 & 2,503 & 18.4 & 217 & 2,568 & & & \\
\hline 1977 & 2,482 & 71 & 71.8 & 3,457 & 110.7 & 307 & 3,566 & & & \\
\hline 1978 & 2,526 & 76 & 73.9 & 3,417 & 27.8 & 314 & 3,649 & & & \\
\hline 1979 & 2,620 & 53 & 62.1 & 4,221 & 94.5 & 384 & 4,430 & & & \\
\hline 1980 & 2,711 & 48 & 49.6 & 5,462 & 76.4 & 432 & 4,981 & & & \\
\hline 1981 & 2,735 & 48 & 48.4 & 5,655 & 51.7 & 455 & 5,233 & & & \\
\hline 1982 & 2,583 & 47 & 47.1 & 5,483 & $(7.0)$ & 420 & 4,845 & & & \\
\hline 1983 & 2,444 & 58 & 53.5 & 4,569 & 32.7 & 424 & 4,887 & & & \\
\hline 1984 & 2,741 & 70 & 66.1 & 4,145 & 41.7 & 436 & 5,026 & & & \\
\hline 1985 & 2,874 & 61 & 64.4 & 4,462 & 26.3 & 432 & 4,978 & & & \\
\hline 1986 & 2,956 & 49 & 53.6 & 5,531 & 42.7 & 442 & 5,094 & & & \\
\hline$\cdots$ & $\cdots$ & & $\because \cdot$ & $\cdots$ & $\cdots$ & $\cdots$ & $\cdots$ & $\cdots-$ & $-\cdots$ & $-\cdot-$ \\
\hline 1997 & 2,982 & 55 & 52.6 & 5,667 & 41.0 & 451 & 5,197 & & & \\
\hline 1988 & 3,016 & 51 & 52.7 & 5,721 & 43.7 & 463 & 5,323 & 5,195 & 6,795 & 6,818 \\
\hline 1989 & & & & & 45.0 & 474 & 5,455 & 5,324 & 6,964 & 6,988 \\
\hline 1990 & & & & & 44.5 & 485 & 5,571 & 5,437 & 7,113 & 7.137 \\
\hline 1991 & & & & & 44.8 & 495 & 5,682 & 5,546 & 7,255 & 7,279 \\
\hline 1992 & & & & & 43.1 & 502 & 5,767 & 5,628 & 7,362 & 7,387 \\
\hline
\end{tabular}

Capacity-Capital Stock Regression: Code 1 RSO 0.850 Coefficients: Const 106.2 stock 11.3

Ave. Capacity/Stock $(74-86): 11.6$ Ave. Weekly Hours (High) 123.5 Shift Factor 1.28 Ave Weekly Hours (Low) 119.1 Shift Factor 1.33 Note: Output, Investment, and Capital Stock extrapolated for 1987 and 1988.

\section{Service industry machinery, nec}

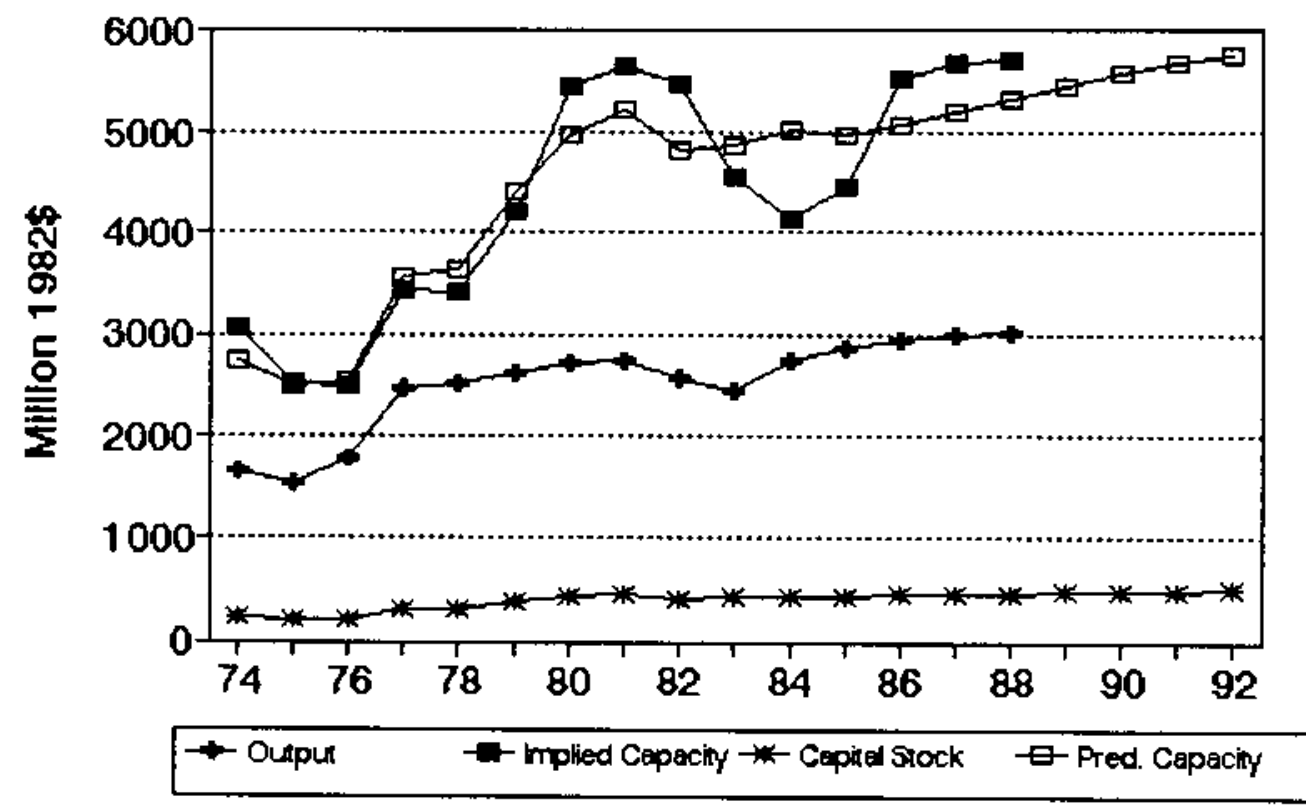


NO. 358 SIC 3592 Carburetors, pistons, rings, valves

MILLIONS OF 1982 DOLLARS (EXCL. CU RATES)

$\begin{array}{lccccccc} & 04 & \text { Amn. } & \text { Implied } & \text { Gross } & \text { Net } & \text { Pred. } & \ldots \ldots \\ \text { Gross } & \text { CU } & \text { CU } & \text { Prac. } & \text { In- } & \text { Cap. } & \text { Prac. } & \text { Emergency Capacity } \\ \text { Output } & \text { Rate } & \text { Rate } & \text { Capac. } & \text { vest. } & \text { Stk. } & \text { Capac. } & 3 \text { mon. } 6 \text { mon. }>6 \text { mon. }\end{array}$

\begin{tabular}{|c|c|c|c|c|c|c|c|c|c|c|}
\hline 1974 & 2,496 & 74 & 7.1 & 3,322 & 73.2 & 732 & 3,349 & & & \\
\hline 1975 & 2,045 & 71 & 70.3 & 2,910 & 73.2 & 755 & 3,370 & & & \\
\hline 1976 & 2,477 & 65 & 67.1 & 3,691 & 46.9 & 749 & 3,365 & & & \\
\hline 1977 & 2,529 & 69 & 67.5 & 3,745 & 68.4 & 766 & 3,379 & & & \\
\hline 1978 & 2,441 & $\pi$ & 72.5 & 3,367 & 114.7 & 824 & 3,435 & & & \\
\hline 1979 & 2,688 & 78 & 77.6 & 3,466 & 144.4 & 908 & 3,514 & & & \\
\hline 1980 & 2,265 & 63 & 68.2 & 3,323 & 121.4 & 964 & 3,567 & & & \\
\hline 1981 & 2,342 & 70 & 68.0 & 3,442 & 49.6 & $\$ 52$ & 3,556 & & & \\
\hline 1982 & 2,202 & 52 & 58.1 & 3,789 & 259.9 & 1,131 & 3,724 & & & \\
\hline 1983 & 2,375 & $\pi$ & 65.7 & 3,613 & 31.0 & 1,081 & 3,677 & & & \\
\hline 1984 & 2,965 & 74 & 75.4 & 3,932 & 108.5 & 1,104 & 3,699 & & & \\
\hline 1985 & 2,826 & 72 & 72.8 & 3,881 & 105.5 & 1,125 & 3,718 & & & \\
\hline 1986 & 2,597 & 69 & 70.1 & 3,706 & 210.5 & 1,245 & 3,832 & & & \\
\hline$\cdots$ & $\cdots \cdot$ & -- & -- & $\cdots$ & $\cdots$ & $\cdots$ & $-\cdot-$ & $-\cdot$ & $\cdot \cdot$ & -- \\
\hline 1987 & 2,600 & 71 & 70.1 & 3,712 & 117.5 & 1,271 & 3,856 & & & \\
\hline 1988 & 2,605 & 58 & 63.3 & 4,119 & 117.8 & 1,295 & 3,878 & 4,090 & 5,182 & 5,197 \\
\hline 1989 & & & & & 117.9 & 1,318 & 3,900 & 4.113 & 5,210 & 5,225 \\
\hline 1990 & & & & & 118.1 & 1,339 & 3,919 & 4,133 & 5,236 & 5,252 \\
\hline 1991 & & & & & 118.2 & 1,358 & 3,938 & 4.153 & 5,261 & 5,276 \\
\hline 1992 & & & & & $\uparrow 18.1$ & 1,376 & 3.955 & 4,171 & 5,283 & 5,299 \\
\hline
\end{tabular}

Capacity-Capitat Stock Regression: Code 0 RSO 0.340

Coefficients: Const 2660.5 stock 0.9

Ave. Capacity/stock $(74-86): 3.7$ Ave. Heekly Hours (High) 117.9 Shift factor 1.34 Ave Heekly Hours (Low) 112.0 Shift Factor 1.41 Note: Output, Investment, and Capital stock extrapolated for 1987 and 1988.

\section{Carburetors, pistons, rings, valve}

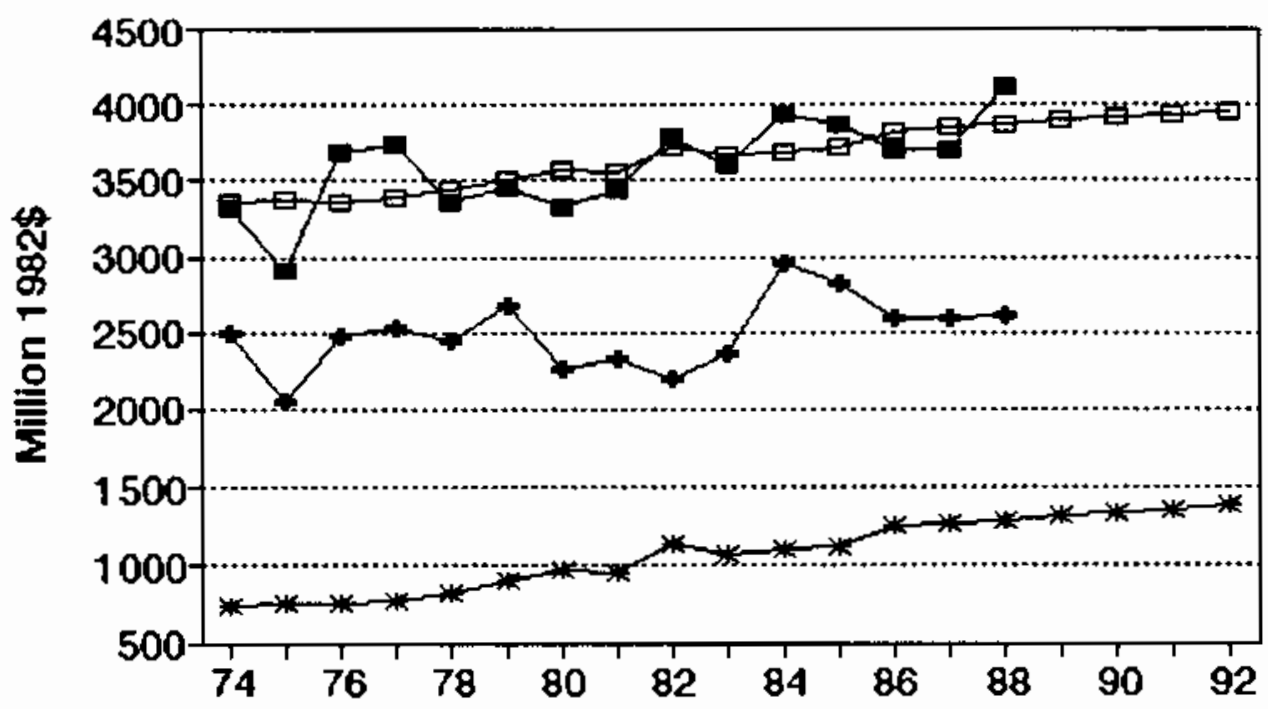

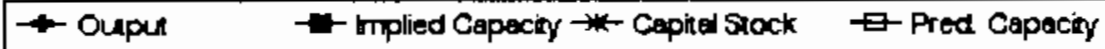


No. 359 SIC 3599 Machinery, except electrical nec MILLIONS OF 1982 DOLLARS (EXCL. CU RATES)

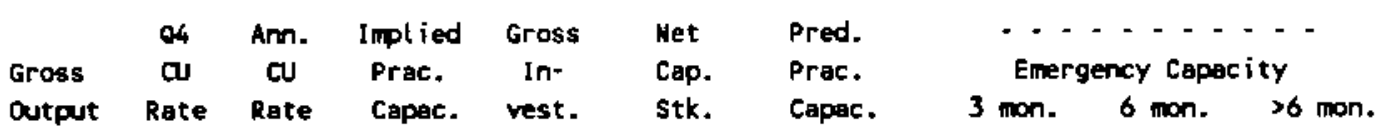

\begin{tabular}{|c|c|c|c|c|c|c|c|c|c|c|}
\hline 1974 & 10,360 & $\pi$ & 72.9 & 14,219 & 389.0 & 3,202 & 14,759 & & & \\
\hline 1975 & 9,526 & 67 & 67.5 & 14,123 & 516.7 & 3,500 & 15,450 & & & \\
\hline 1976 & 9,372 & 62 & 63.8 & 14,696 & 96.0 & 3,378 & 15,168 & & & \\
\hline 197 & 10,247 & 64 & 63.3 & 16,195 & 659.9 & 3,789 & 16,120 & & & \\
\hline 1978 & 11,429 & 66 & 65.1 & 17,560 & 665.9 & 4,186 & 17,042 & & & \\
\hline 1979 & 13,047 & 68 & 67.8 & 19,257 & 673.8 & 4,580 & 17,956 & & & \\
\hline 1980 & 13,426 & 56 & 60.2 & 22,306 & 665.3 & 4,944 & 18,790 & & & \\
\hline 1981 & 13,892 & 72 & 66.5 & 20,880 & 625.8 & 5,250 & 19,511 & & & \\
\hline 1982 & 12,278 & 55 & 60.9 & 20,171 & 827.3 & 5,716 & 20,590 & & & \\
\hline 1983 & 12,001 & 73 & 65.8 & 18,247 & 483.0 & 5,813 & 20,816 & & & \\
\hline 1984 & 12,323 & 70 & 71.9 & 17,136 & 5.9 & 5,448 & 19,970 & & & \\
\hline 1985 & 13,716 & 63 & 65.7 & 20,891 & $5+3.7$ & 5,574 & 20,260 & & & \\
\hline 1986 & 13,592 & 65 & 64.2 & 21,166 & 476.9 & 5,636 & 20,405 & & & \\
\hline$\cdots$ & $-\cdots$ & - & - - & --- & $-\ldots$ & $\therefore$ & $\because \cdots$ & $=\cdot \cdot$ & $-\cdot-$ & --- \\
\hline 1987 & 13,679 & 73 & 69.8 & 19,591 & 437.2 & 5,669 & 20,483 & & & \\
\hline 1988 & 13,794 & 71 & 72.0 & 19,151 & 726.4 & 5,990 & 21,226 & 27,272 & 37,174 & 37,315 \\
\hline 1989 & & & & & 853.6 & 6,414 & 22,211 & 28,537 & 38,899 & 39,047 \\
\hline 1990 & & & & & 994.3 & 6,949 & 23,452 & 30,132 & 41,072 & 41,228 \\
\hline 1991 & & & & & $1,119.4$ & 7,571 & 24,894 & 31,995 & 43,598 & 43,764 \\
\hline 1992 & & & & & $1,010.0$ & 8,039 & 25,979 & 33,379 & 45,499 & 45,671 \\
\hline
\end{tabular}

Capacity-Capital stock Regression: Code 0 RSO 0.640 Coefficients: Const 7330.1 stock 2.3

Ave. Capacity/Stock $(74-86): 3.9$ Ave. Weekly Hours (High) 89.6 Shift Factor 1.76 Ave Weekly Hours (Low) 82.6 Shift factor 1.91 Note: Output, Investment, and Capital Stock extrapoloted for 1987 and 1988.

\section{Machinery, except electrical nec}

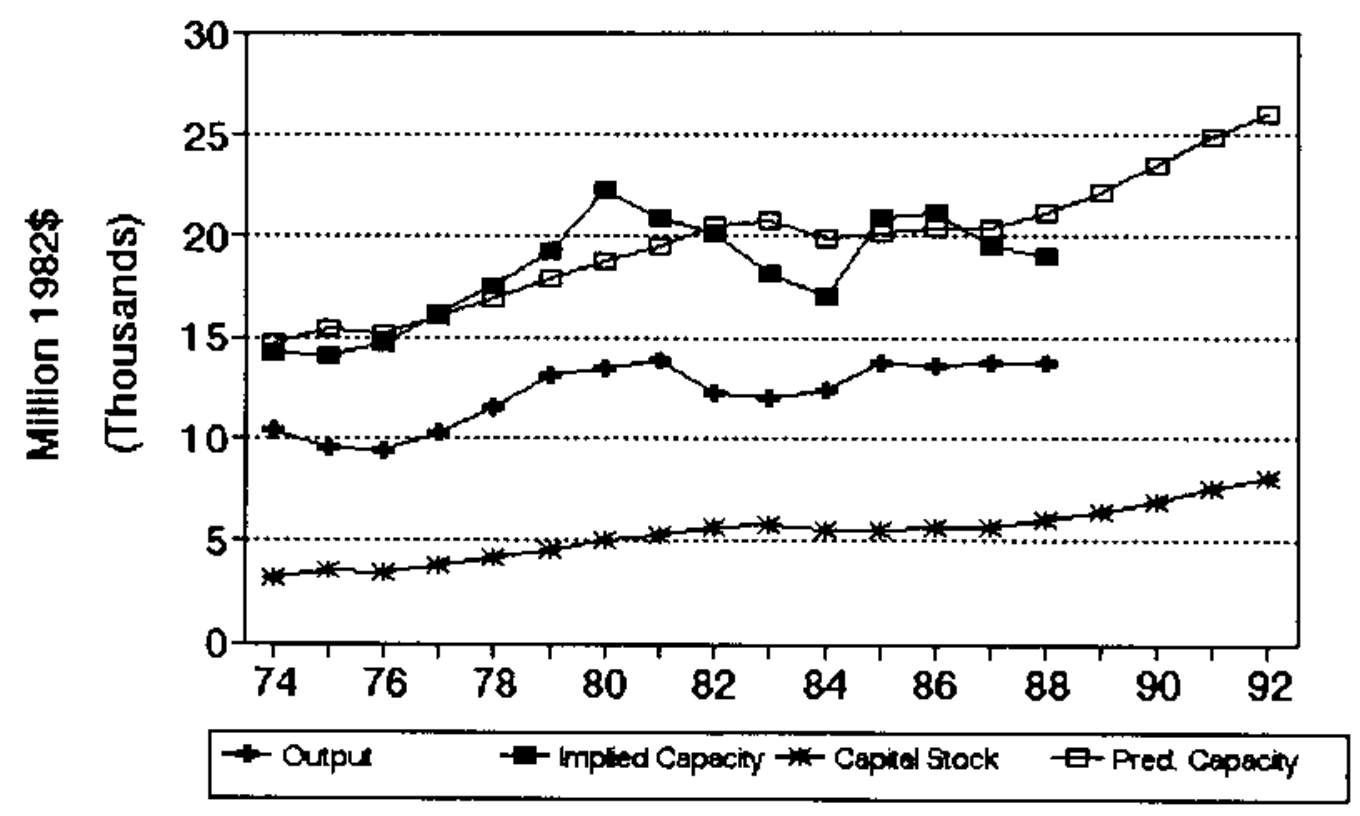


NO. 360 SIC 3612 Trans formers

MILLIONS OF 1982 DOLLARS (EXCL. CU RATES)

$\begin{array}{lccccccc} & \text { Q4 } & \text { Ann. Implied Gross } & \text { Net } & \text { Pred. } & -\ldots \ldots \\ \text { Gross } & \text { CU } & \text { du } & \text { Prac. } & \text { In- } & \text { Cap. } & \text { Prac. } & \text { Emergency Capacity } \\ \text { Output } & \text { Rate } & \text { Rate } & \text { Capac. } & \text { vest. } & \text { Stk. } & \text { Capsc. } & 3 \text { mon. } 6 \text { mon. }>6 \text { mon. }\end{array}$

\begin{tabular}{|c|c|c|c|c|c|c|c|c|c|c|}
\hline 1974 & 3,811 & $\pi$ & 80.2 & $4, \pi 54$ & 72.5 & 702 & 4,425 & & & \\
\hline 1975 & 2,877 & 61 & 64.1 & 4,487 & 63.2 & 705 & 4,447 & & & \\
\hline 1976 & 2,853 & $\pi$ & 69.7 & 4,097 & 15.5 & 662 & 4,156 & & & \\
\hline 1977 & 3,322 & 76 & 75.6 & 4,393 & 135.4 & 733 & 4,631 & & & \\
\hline 1978 & 3,472 & $\pi$ & 75.0 & 4,629 & 42.9 & 712 & 4,489 & & & \\
\hline 1979 & 3,658 & 76 & $\pi .5$ & 4,843 & 122.9 & 765 & 4,843 & & & \\
\hline 1980 & 3,553 & 66 & 69.4 & 5,123 & 111.6 & 802 & 5,097 & & & \\
\hline 1981 & 3,440 & 63 & 64.4 & 5,338 & 100.8 & 826 & 5,257 & & & \\
\hline 1982 & 2,950 & 52 & 55.7 & 5,300 & 79.5 & 828 & 5,267 & & & \\
\hline 1983 & 2,735 & 52 & 51.6 & 5,340 & 68.8 & 818 & 5,198 & & & \\
\hline 1984 & 3,088 & 63 & 50.1 & 5,223 & 23.9 & 768 & 4,867 & & & \\
\hline 1985 & 3,041 & 71 & 67.7 & 4,489 & 79.3 & $\pi 3$ & 4,899 & & & \\
\hline 1986 & 3,017 & 69 & 69.2 & 4,358 & 58.9 & $\pi 57$ & 4,794 & & & \\
\hline - & 3,063 & $\therefore$ & $\ddot{72.5}$ & 4,226 & 73.4 & 757 & $\begin{array}{l}\cdots-\cdot \\
4,792\end{array}$ & $-\cdots$ & $-\cdots$ & -- \\
\hline 1988 & 3,125 & 80 & 78.3 & 3,993 & $\pi .3$ & 758 & 4,802 & 5,324 & 6,940 & 6,963 \\
\hline 1989 & & & & & 76.9 & 761 & 4,822 & 5,346 & 6,969 & 6,992 \\
\hline 1990 & & & & & $\pi .2$ & 764 & 4.842 & 5,368 & 6,998 & 7,021 \\
\hline 1991 & & & & & 78.1 & 768 & 4,866 & 5,395 & 7,033 & 7,056 \\
\hline 1992 & & & & & 7.2 & 770 & 4,882 & 5,412 & 7,055 & 7,079 \\
\hline
\end{tabular}

Capacity-Capital Stock Regression: Code 1 RSO 0.680

Coefficients: Const -280.1 stock 6.7

Ave. Capocity/Stock $(74-86): 6.3$

Ave. Heekly Hours (High) 108.6 Shift Factor 1.45 Ave Heekly Hours (Low) 104.0 Shift Factor 1.51 Note: Output, Investment, and Capital Stock extrapolated for 1987 and 1988.

\section{Transformers}

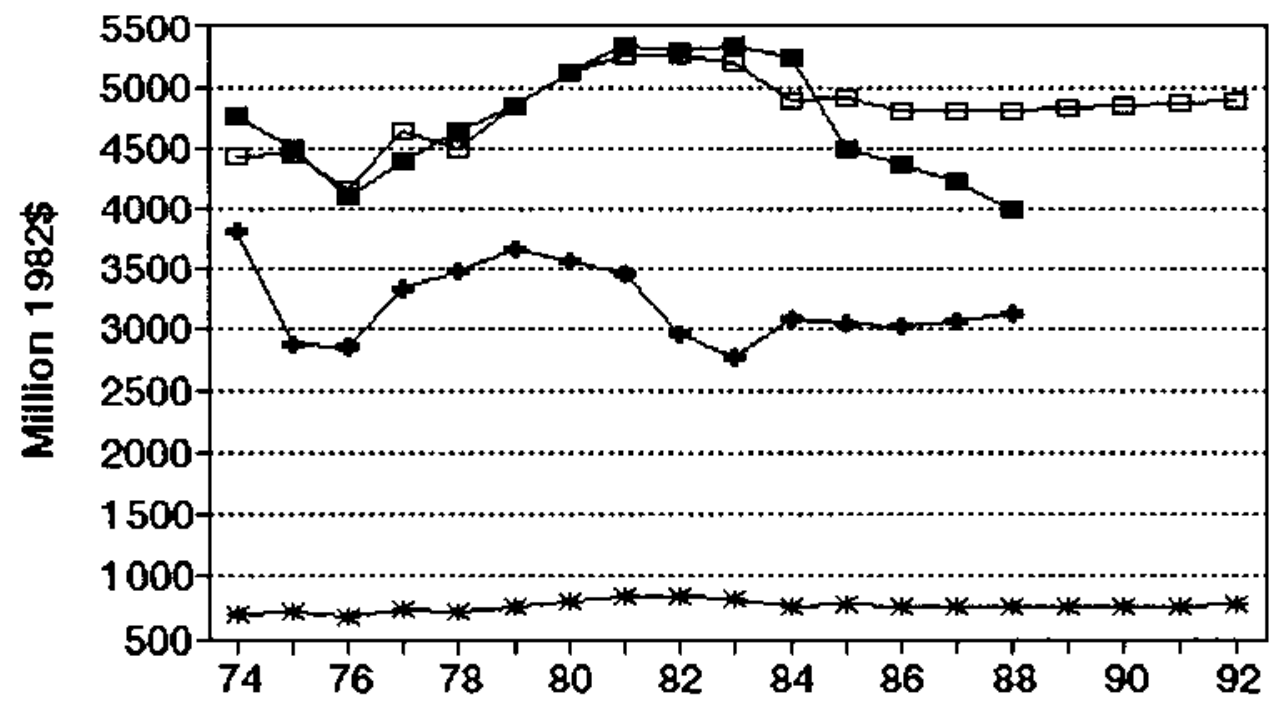

- Oupur $\quad-$ Implied Capeciny - + - Capited Stock $\quad \square$ Pred Capaciny 


\section{No. 361 sIC 3613 Switchgear and switchboard apparatus}

MILLIONS OF 1982 DOLLARS (EXCL. CU RATES)

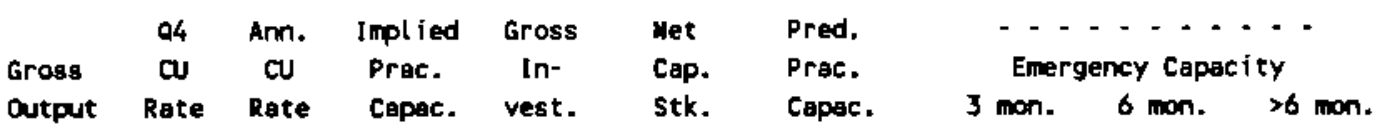

\begin{tabular}{|c|c|c|c|c|c|c|c|c|c|c|}
\hline 1974 & 5,327 & 73 & 73.8 & 7,219 & 124.9 & 801 & 7,121 & & & \\
\hline $19 \pi$ & 4,415 & 74 & 7.9 & 6,058 & 72.1 & 809 & 7,139 & & & \\
\hline 1976 & 4,636 & 67 & 69.7 & 6,656 & 82.2 & 826 & 7,176 & & & \\
\hline 1977 & 5,460 & 72 & 70.1 & 7,789 & 137.6 & 893 & 7,324 & & & \\
\hline $197 \mathrm{~B}$ & 5,853 & 63 & 66.3 & 8,829 & 127.8 & 946 & 7,441 & & & \\
\hline 1979 & 5,996 & 73 & 69.2 & 8,663 & 88.9 & 962 & 7,477 & & & \\
\hline 1980 & 5,729 & $\pi$ & $\pi .4$ & 7,598 & 108.2 & 997 & 7,555 & & & \\
\hline 1981 & 5,426 & 80 & 79.0 & 6,870 & 116.5 & 1,038 & 7,645 & & & \\
\hline 1982 & 5,214 & 63 & 69.2 & 7,536 & 145.6 & 1,104 & 7,790 & & & \\
\hline 1983 & 5,031 & 65 & 64.1 & 7,849 & 77.1 & 1,100 & 7,781 & & & \\
\hline 1984 & 5,395 & 73 & 70.1 & 7,698 & 61.5 & 1,082 & 7,742 & & & \\
\hline 1985 & 5,115 & 65 & 67.9 & 7,537 & 65.0 & 1,068 & 7,712 & & & \\
\hline 1986 & 4,989 & 69 & 67.4 & 7,405 & 122.6 & 1,109 & 7,801 & & & \\
\hline$-\cdots$ & --- & - & $\ldots$ & $\therefore$ & -- & $\therefore-$ & -- & -- & -5 & $-\cdot-$ \\
\hline 1987 & 5,053 & 68 & 68.3 & 7,396 & 94.2 & 1,121 & 7,828 & & & \\
\hline 1988 & 5,137 & 76 & 73.0 & 7.034 & 93.1 & 1,131 & 7,851 & 8,712 & 11,338 & 11,373 \\
\hline 1989 & & & & & 92.0 & 1,140 & 7,869 & 8,732 & 11,365 & 11,402 \\
\hline 1990 & & & & & 91.0 & 1,146 & 7,884 & 8,749 & 11,386 & 11,424 \\
\hline 1991 & & & & & 89.9 & 1,151 & 7,896 & 8,762 & 11,403 & 11,441 \\
\hline 1992 & & & & & 88.8 & 1,155 & 7,904 & 8,771 & 11,415 & 11,452 \\
\hline
\end{tabular}

Capacity-Capital Stock Regression: Code 0 RSO 0.120 Coefficients: Const 5353.6 stock 2.2

Ave. Capacity/Stock $(74-86): 7.7$ Ave. Heekly Hours (High) 108.7 Shift Factor 1.45 Ave Weekly Hours (Low) 105.6 Shift factor 1.49 Note: Output, Imestment, and Capital Stock extrapolated for 1987 and 1988.

\section{Switchgear and switchboard apparat}

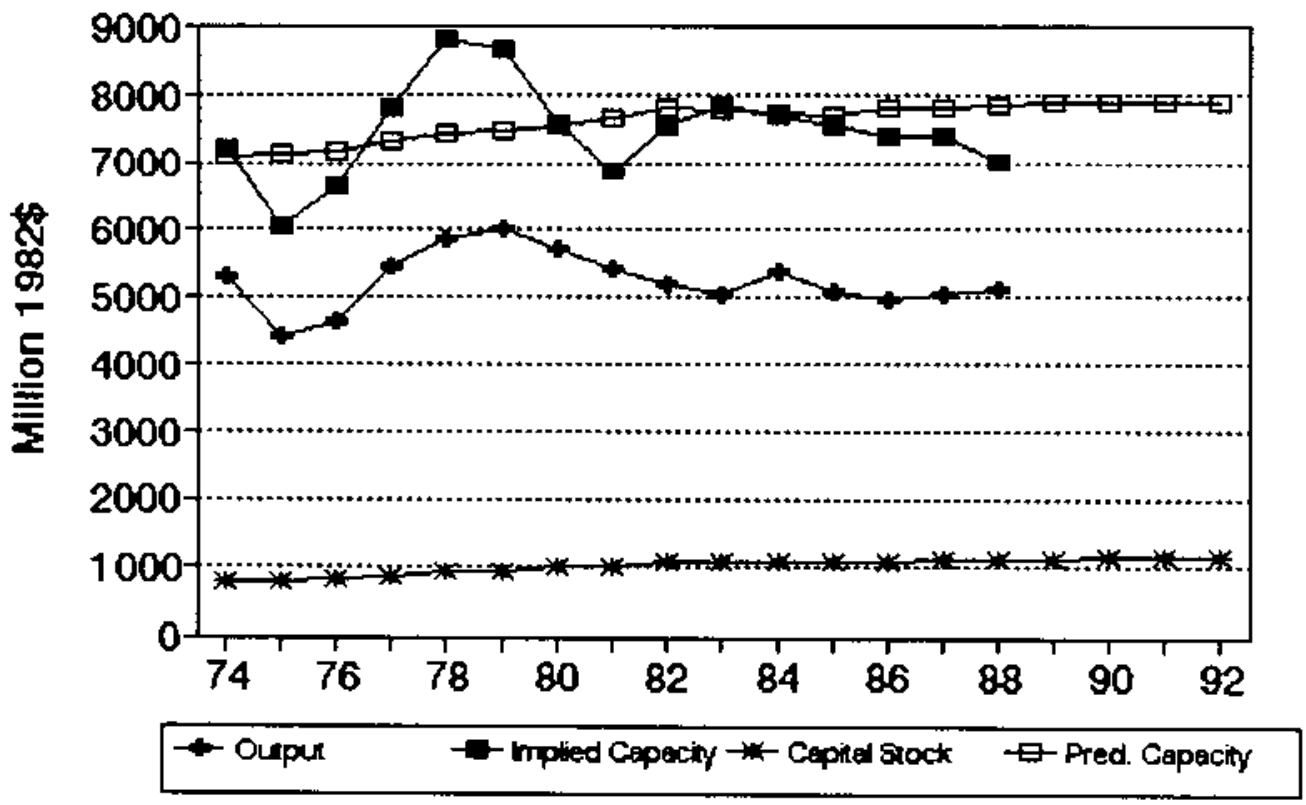


No. 362 sIC 3621 Notors and generators

MILLIONS OF 1982 DOLLARS (EXCL. CU RATES)

$\begin{array}{lccccccc} & \text { Q4 } & \text { Ann. } & \text { Implied Gross } & \text { Net } & \text { Pred. } & \ldots \\ \text { Gross } & \text { CU } & \text { CU } & \text { Prac. } & \text { In- } & \text { Cap. } & \text { Prac. } & \text { Emergency Capacity } \\ \text { Output } & \text { Rate } & \text { Rate } & \text { Capac. } & \text { vest. } & \text { Stk. } & \text { Capac. } & 3 \text { mon. } 6 \text { mon. }>6 \text { mon. }\end{array}$

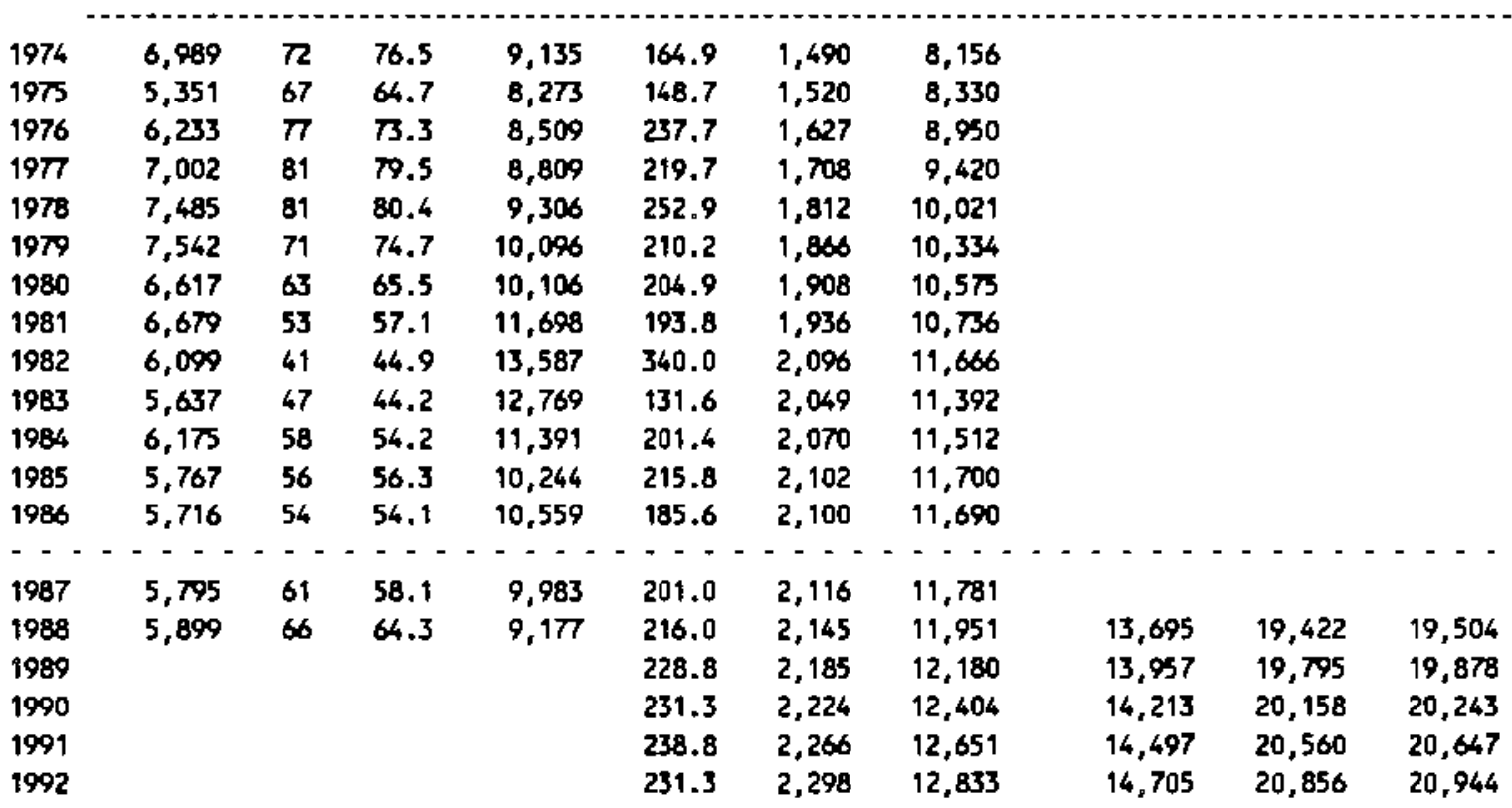

Capecity-Capital stock Regression: Code 1 Rso 0.620

Coefficients: Const -471.1 stock 5.8

Ave. Capacity/Stock $(74-86): 5.5$

Ave. Weekly Hours (High) 96.5 Shift Factor 1.63 Ave Weekly Hours (Low) 92.7 Shift Factor 1.70

Hote: Output, Investment, and Capital stock extrapolated for 1987 and 1988.

\section{Motors and generators}

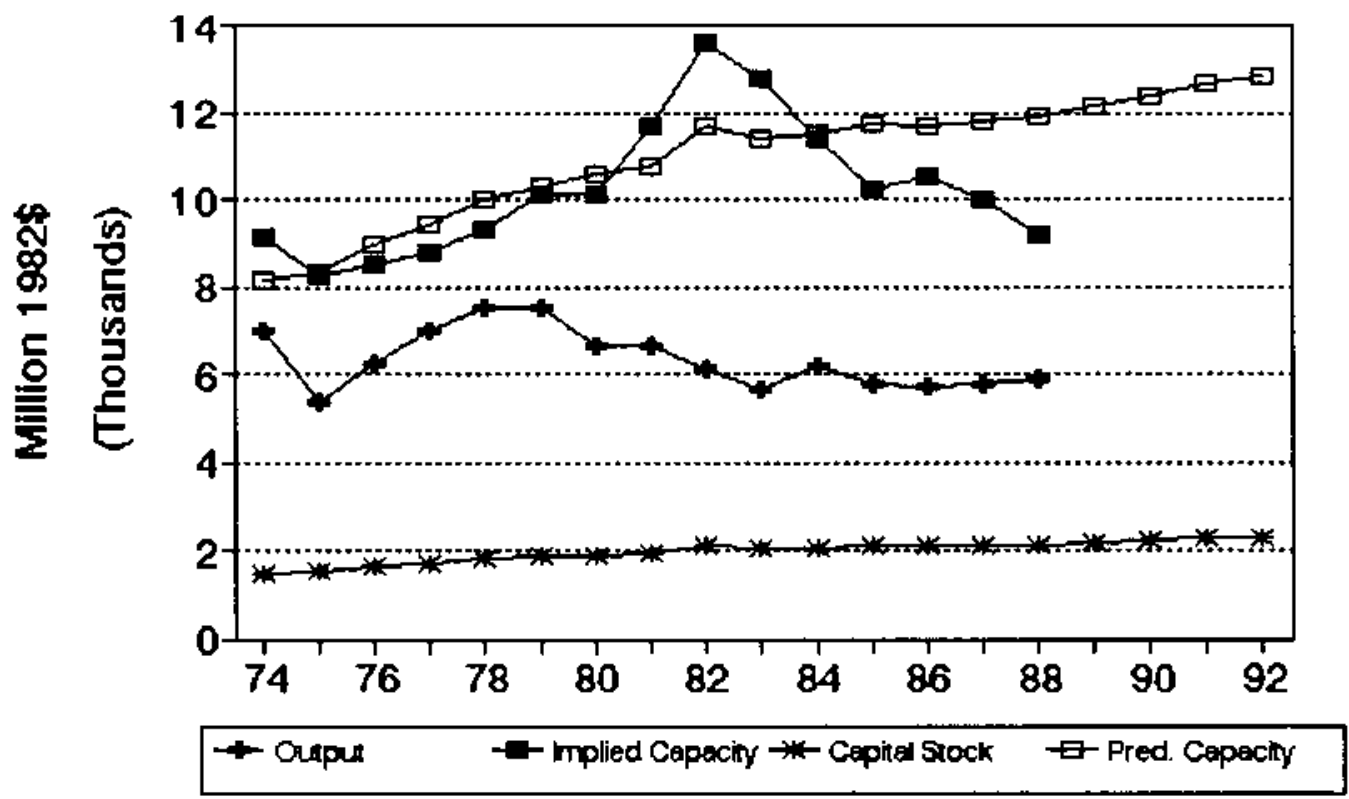




\section{Ho. 363 sIC 3622 Industrial controlls}

MILLIONS OF 1982 DOLLARS (EXCL. CU RATES)

$\begin{array}{lccccccc} & \text { Q4 } & \text { Ann. Implied Gross } & \text { Net } & \text { Pred. } & \ldots \ldots \ldots \\ \text { Gross } & \text { dU } & \text { CU } & \text { Prac. } & \text { In- } & \text { Cap. } & \text { Prac. } & \text { Emergency Capacity } \\ \text { Output } & \text { Rate } & \text { Rate } & \text { Capac. } & \text { vest. } & \text { Stk. } & \text { Capac. } & 3 \text { mon. } 6 \text { mon. }>6 \text { mon. }\end{array}$

\begin{tabular}{|c|c|c|c|c|c|c|c|c|c|c|}
\hline 1974 & 4,340 & 62 & 63.0 & 6,887 & 78.6 & 616 & 6,522 & & & \\
\hline 1975 & 3,723 & 58 & 58.6 & 6,357 & 45.6 & 616 & 6,530 & & & \\
\hline 1976 & 3,848 & 66 & 63.0 & 6,110 & 31.2 & 603 & 6,467 & & & \\
\hline 1977 & 3,923 & 60 & 62.3 & 6,301 & 41.4 & 599 & 6,468 & & & \\
\hline 1978 & 4,490 & 61 & 60.5 & 7,422 & 103.9 & 655 & 6,726 & & & \\
\hline 1979 & 4,611 & 68 & 65.3 & 7,059 & 56.3 & 662 & 6,762 & & & \\
\hline 1980 & 4,401 & 58 & 61.6 & 7,146 & 137.9 & 746 & 7,181 & & & \\
\hline 1981 & 4,574 & 65 & 62.5 & 7,315 & 163.0 & 850 & 7,699 & & & \\
\hline 1982 & 4,327 & 56 & 59.2 & 7,311 & 82.3 & 872 & 7.810 & & & \\
\hline 1983 & 3,968 & 57 & 56.4 & 7,032 & 50.2 & 861 & 7,751 & & & \\
\hline 1984 & 4,971 & 52 & 54.0 & 9,205 & 184.8 & 976 & 8,325 & & & \\
\hline 1985 & 4,946 & 61 & 57.5 & 8,598 & 127.6 & 1,030 & 8,594 & & & \\
\hline 1986 & 5,065 & 55 & 57.1 & 8,873 & 119.4 & 1,071 & 8,800 & & & \\
\hline$-\cdot-$ & $\cdots \cdot$ & $\because$ & -- & $--\cdot$ & $\cdots$ & $\cdots-$ & $-\cdots$ & $\cdots-$ & 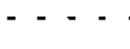 & $\cdots \cdot$ \\
\hline 1987 & 5,143 & 46 & 49.3 & 10,428 & 81.3 & 1,075 & 8,819 & & & \\
\hline 1988 & 5,246 & 51 & 49.2 & 10,673 & 122.1 & 1,119 & 9,041 & 11,176 & 15,533 & 15,595 \\
\hline 1989 & & & & & 156.9 & 1,195 & 9,420 & 11,645 & 16,185 & 16,249 \\
\hline 1990 & & & & & 163.7 & 1,273 & 9,806 & 12,122 & 16,848 & 16,915 \\
\hline 1991 & & & & & 184.3 & 1,365 & 10,267 & 12,692 & 17,639 & 17,710 \\
\hline 1992 & & & & & 163.7 & 1,430 & 10,591 & 13,093 & 18,197 & 18,270 \\
\hline
\end{tabular}

Capacity-Capital Stock Regression: code 0 RSO 0.770

Coefficients: Const 3459.0 stock 5.0

Ave. Capacity/Stock (74-86): 9.4 Ave. Heekly Hours (High) 91.3 Shift Factor 1.73 Ave Heekly Hours (Low) 86.7 Shift Factor 1.82 Note: Output, Investment, and Capital Stock extrapolated for 1987 and 1988.

3622 Industrial controlls

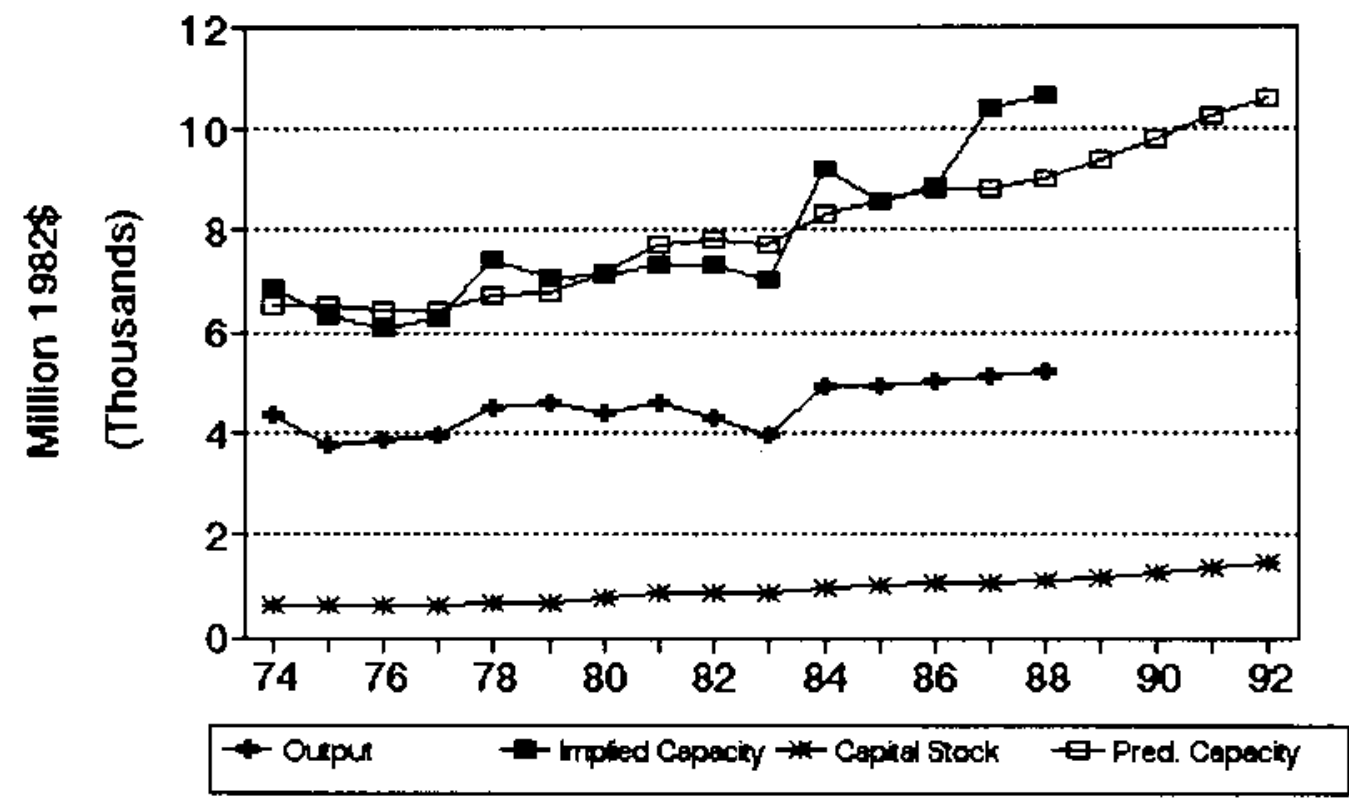




\section{No. 364 SIC 3623 Welding apparatus, electric}

MILLIONS OF 1982 DOLLARS (EXCL. CU RATES)

$\begin{array}{lccccccc} & 04 & \text { Ann. Implied Gross } & \text { Net } & \text { Pred. } & \ldots . \ldots . . . \\ \text { Gross } & \text { CU } & \text { CU } & \text { Prac. } & \text { In- } & \text { Cap. } & \text { Prac. } & \text { Emergency Capacity } \\ \text { Output } & \text { Rete } & \text { Rate } & \text { Capec. } & \text { vest. } & \text { Stk. } & \text { Capac. } & 3 \text { mon. } 6 \text { mon. }>6 \text { mon. }\end{array}$

\begin{tabular}{|c|c|c|c|c|c|c|c|c|c|c|}
\hline 1974 & 1,797 & 50 & 55.3 & 3,252 & 21.3 & 236 & 3,188 & & & \\
\hline 1975 & 1,473 & 43 & 40.7 & 3,618 & 20.8 & 238 & 3,133 & & & \\
\hline 1976 & 1,472 & 55 & 50.5 & 2,916 & 24.3 & 242 & 3,078 & & & \\
\hline 1977 & 1,678 & 57 & 56.3 & 2,978 & 47.8 & 268 & 3,023 & & & \\
\hline 1978 & 1,795 & 57 & 56.4 & 3,183 & 34.6 & 281 & 2,968 & & & \\
\hline 1979 & 1,834 & 79 & 70.4 & 2,606 & 52.8 & 310 & 2,913 & & & \\
\hline 1980 & 1,683 & 69 & 71.9 & 2,342 & 24.8 & 311 & 2,858 & & & \\
\hline 1981 & 1,853 & 64 & 66.7 & 2,776 & 54.2 & 341 & 2,803 & & & \\
\hline 1982 & 1,366 & 44 & 50.3 & 2,718 & 45.0 & 359 & 2,748 & & & \\
\hline 1983 & 1,147 & 49 & 46.3 & 2,478 & 8.0 & 341 & 2,693 & & & \\
\hline 1984 & 1,435 & 58 & 55.2 & 2,598 & 13.2 & 328 & 2,638 & & & \\
\hline 1985 & 1,493 & 46 & 49.6 & 3,009 & 18.9 & 321 & 2,583 & & & \\
\hline 1986 & 1,374 & 56 & 51.3 & 2,679 & 21.9 & 315 & 2,528 & & & \\
\hline$\cdot-$ & $\cdots \cdot$ & $\cdots$ & $\cdots$ &.-- & -- & $-\cdot-$ & $\cdots$ & $\cdots$ & $\because \cdot$ & $\cdots$ \\
\hline 1987 & 1,405 & 57 & 56.1 & 2,503 & 26.3 & 315 & 2,473 & & & \\
\hline 1988 & 1,447 & 66 & 62.9 & 2,303 & 26.0 & 316 & 2,418 & 2,171 & 2,702 & 2,710 \\
\hline 1989 & & & & & 25.6 & 313 & 2,362 & 2,121 & 2,641 & 2,648 \\
\hline 1990 & & & & & 25.2 & 312 & 2,307 & 2,072 & 2,579 & 2,587 \\
\hline 1991 & & & & & 24.8 & 310 & 2,252 & 2,023 & 2,518 & 2,525 \\
\hline 1992 & & & & & 24.5 & 309 & 2,197 & 1,973 & 2,456 & 2,463 \\
\hline
\end{tabular}

Capacity-Capital Stock Regression: Code -2 RSO 0.370

Coefficients: Const 3243.1 Time -55.0

Ave. Capacity/Stock $(74-86): 9.5$ Ave. Weekly Hours (High) 141.9 Shift Factor 1.12 Ave Ueekly Hours (Low) 129.6 Shift factor 1.22 Note: Output, Investment, and Capital Stock extrapolated for 1987 and 1988.

\section{Welding apparatus, electric}

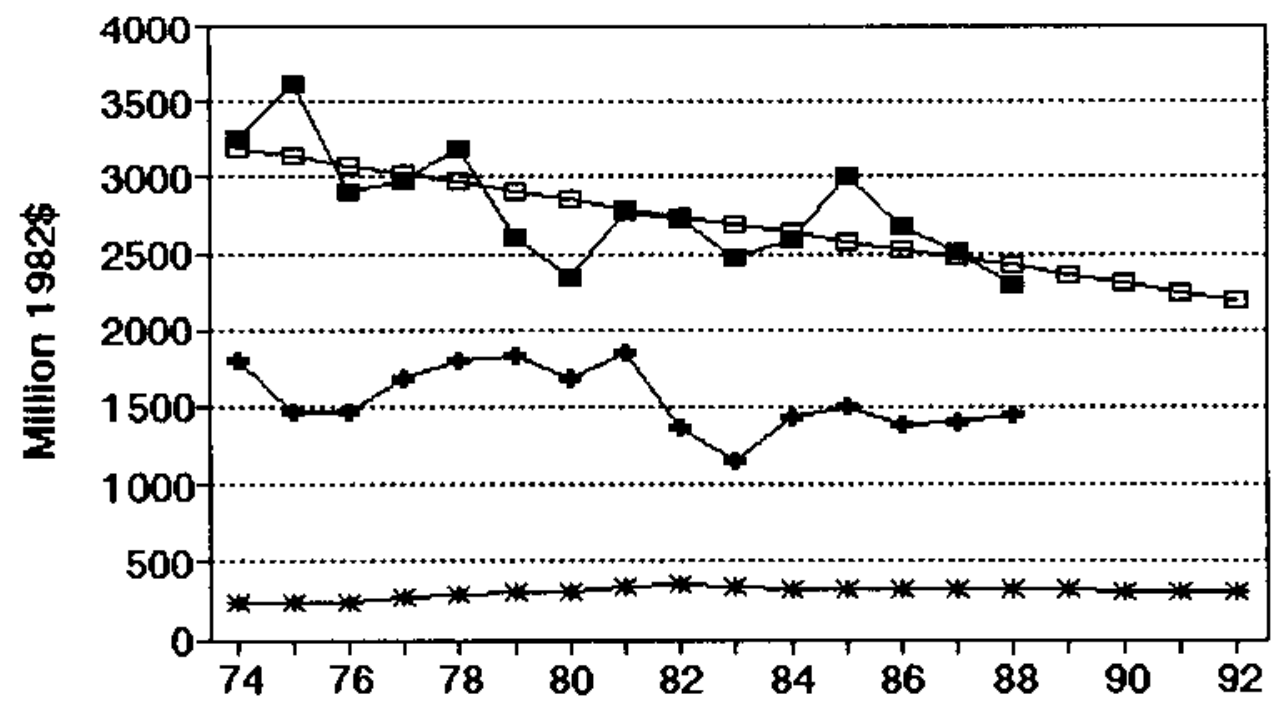

\begin{tabular}{|c|c|}
\hline- arpur & $\rightarrow-$ mplied Cepecin $\rightarrow \cdots$ Capial Stock \\
\hline
\end{tabular}


No. 365 SIC 3624 carbon and graphite product:

MILLIONS OF 1982 DOLLARS (EXCL. CU RATES)

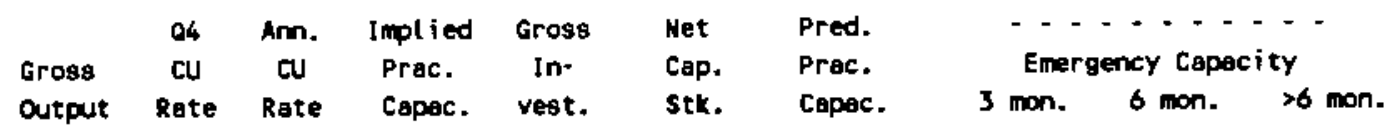

\begin{tabular}{|c|c|c|c|c|c|c|c|c|c|c|}
\hline 1974 & 1,453 & 65 & 72.3 & 2,010 & 86.5 & 526 & 1,953 & & & \\
\hline 1975 & 1,105 & 54 & 51.4 & 2,150 & 117.0 & 598 & 1,904 & & & \\
\hline 1976 & 1,305 & 71 & 64.6 & 2,020 & 93.0 & 644 & 2,020 & & & \\
\hline 1977 & 1,249 & 71 & 71.2 & 1,755 & 0.7 & 601 & 1,995 & & & \\
\hline 1978 & 1,379 & 85 & 78.7 & 1,731 & 44.6 & 599 & 1,995 & & & \\
\hline 1979 & 1,671 & 81 & 82.4 & 2.029 & 45.8 & 597 & 1,993 & & & \\
\hline 1980 & 1,464 & $\infty 6$ & 70.7 & 2,071 & 84.1 & 633 & 2,013 & & & \\
\hline 1981 & 1,602 & 68 & 68.4 & 2,346 & 189.3 & 769 & 2,091 & & & \\
\hline 1982 & 1,078 & 41 & 49.5 & 2,177 & 221.0 & 928 & 2,182 & & & \\
\hline 1983 & 955 & 49 & 45.0 & 2,121 & 134.8 & 998 & 2,221 & & & \\
\hline 1984 & 1,284 & 63 & 58.4 & 2,201 & 20.9 & 953 & 2,196 & & & \\
\hline 1985 & 1,065 & 52 & 55.2 & 1,929 & $(50.7)$ & 842 & 2,132 & & & \\
\hline 1986 & 1,180 & 51 & 50.3 & 2,344 & 39.8 & 818 & 2,119 & & & \\
\hline$\therefore-$ & $\cdots$ & $\because$ & $\because-$ & $\cdots$ & $\cdots$ & - - & - . - & $-\cdots$ & $\cdots$ & $-\cdots$ \\
\hline 1987 & 1,207 & 65 & 59.1 & 2,042 & 70.9 & 827 & 2,126 & & & \\
\hline 1988 & 1,244 & 76 & 72.2 & 1,723 & 73.5 & 838 & 2,130 & 2,063 & 2,665 & 2,674 \\
\hline 1989 & & & & & 75.8 & 851 & 2,138 & 2,070 & 2,674 & 2,683 \\
\hline 1990 & & & & & 76.2 & 863 & 2,145 & 2,077 & 2,683 & 2,691 \\
\hline 1991 & & & & & 77.5 & 876 & 2,152 & 2,084 & 2,692 & 2,700 \\
\hline 1992 & & & & & 76.2 & 286 & 2,158 & 2,089 & 2,699 & 2,708 \\
\hline
\end{tabular}

Capacity-Capitat Stock Regression: Code 1 RSQ 0.240 Coefficients: Const 1653.5 stock 0.6

Ave. Capecity/Stock $(74-86): 2.8$ Ave. Heekly Hours (High) 126.1 Shift Factor 1.25 Ave Weekly Hours (Low) 121.7 Shift Factor 1.30 Note: Output, Imvestment, and Capital Stock extrapolated for 1987 and 1988.

\section{Carbon and graphite products}

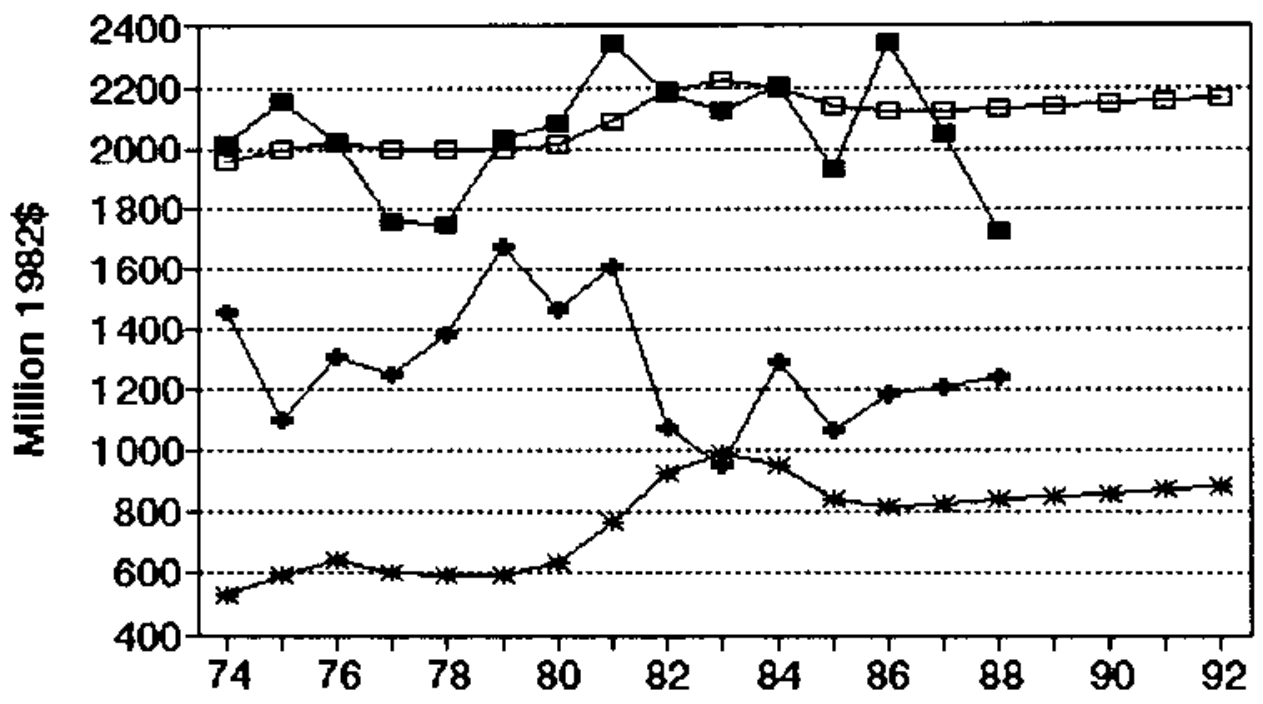

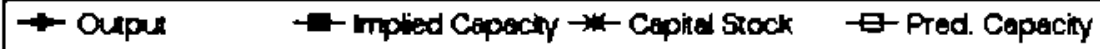


NO. 366 sIC 3629 Electrical industrial apparatus, nec

MILLIONS OF 1982 DOLLARS (EXCL. CU RATES)

$\begin{array}{lccccccc} & \text { Q4 Ann. Implied Gross } & \text { Net } & \text { Pred. } & - & - \\ \text { Gross } & \text { du } & \text { du } & \text { Prac. } & \text { In- } & \text { Cap. } & \text { Prac. } & \text { Emergency Capacity } \\ \text { Output Rete } & \text { Rate } & \text { Capac. } & \text { vest. } & \text { Stk. } & \text { Capac. } & 3 \text { mon. } 6 \text { mon. }>6 \text { mon. }\end{array}$

\begin{tabular}{|c|c|c|c|c|c|c|c|c|c|c|}
\hline 1974 & 1,394 & 73 & 7.4 & 1,801 & 54.5 & 238 & 1,609 & & & \\
\hline $19 \pi$ & 1,011 & 50 & 54.7 & 1,849 & 14.7 & 235 & 1,596 & & & \\
\hline 1976 & 1,144 & 68 & 61.1 & 1,874 & 34.2 & 251 & 1,682 & & & \\
\hline 1977 & 958 & $\pi$ & 73.5 & 1,302 & 9.3 & 243 & 1,637 & & & \\
\hline 1978 & 1,059 & 84 & 80.8 & 1,311 & 48.6 & 271 & 1,789 & & & \\
\hline 1979 & 1,142 & 60 & 74.6 & 1,531 & 19.1 & 270 & 1,784 & & & \\
\hline 1980 & 1,244 & 67 & 67.3 & 1,849 & 34.8 & 285 & 1,864 & & & \\
\hline 1981 & 1,242 & 66 & 66.9 & 1,856 & 37.9 & 301 & 1,950 & & & \\
\hline 1982 & 1,094 & 58 & 60.4 & 1,812 & 20.8 & 300 & 1,943 & & & \\
\hline 1983 & 1,137 & 60 & 58.6 & 1,940 & $(0.4)$ & 279 & 1,831 & & & \\
\hline 1984 & 1,372 & 63 & 62.3 & 2,202 & 35.8 & 292 & 1,902 & & & \\
\hline 1985 & 1,373 & 65 & 63.8 & 2,152 & 39.5 & 307 & 1,982 & & & \\
\hline 1986 & 1,339 & 66 & 64.9 & 2,062 & 23.4 & 305 & 1,972 & & & \\
\hline $\begin{array}{l}\because 987 \\
1987\end{array}$ & $\cdots$ & 63 & 63.8 & $\begin{array}{l}-\cdots \\
2,120\end{array}$ & - - & $\ddot{308}$ & $\cdots$ & $\cdots$ & -- & -- \\
\hline 1988 & 1,372 & 70 & 67.5 & 2,032 & 29.3 & 312 & 2,007 & 2,418 & 3,186 & 3,197 \\
\hline 1989 & & & & & 31.1 & 318 & 2,037 & 2,455 & 3,235 & 3,246 \\
\hline 1990 & & & & & 34.5 & 323 & 2,067 & 2,490 & 3,282 & 3,293 \\
\hline 1991 & & & & & 32.5 & 329 & 2,100 & 2,530 & 3,334 & 3,346 \\
\hline 1992 & & & & & 31.5 & 334 & 2,125 & 2,560 & 3,374 & 3,385 \\
\hline
\end{tabular}

Capacity-Capital Stock Regression: Code 1 RSQ 0.250

Coefficients: Const 339,0 stock 5.4

Ave. Capacity/Stock $(74-86): 6.6$

Ave. Weekly Hours (High) 98.9 Shift Factor 1.59 Ave Heekly Hours (Low) 96.3 Shift factor 1.63 Note: Output, Investment, and Capital Stock extrapolated for 1987 and 1988.

\section{Electrical industrial apparatus, $n$}

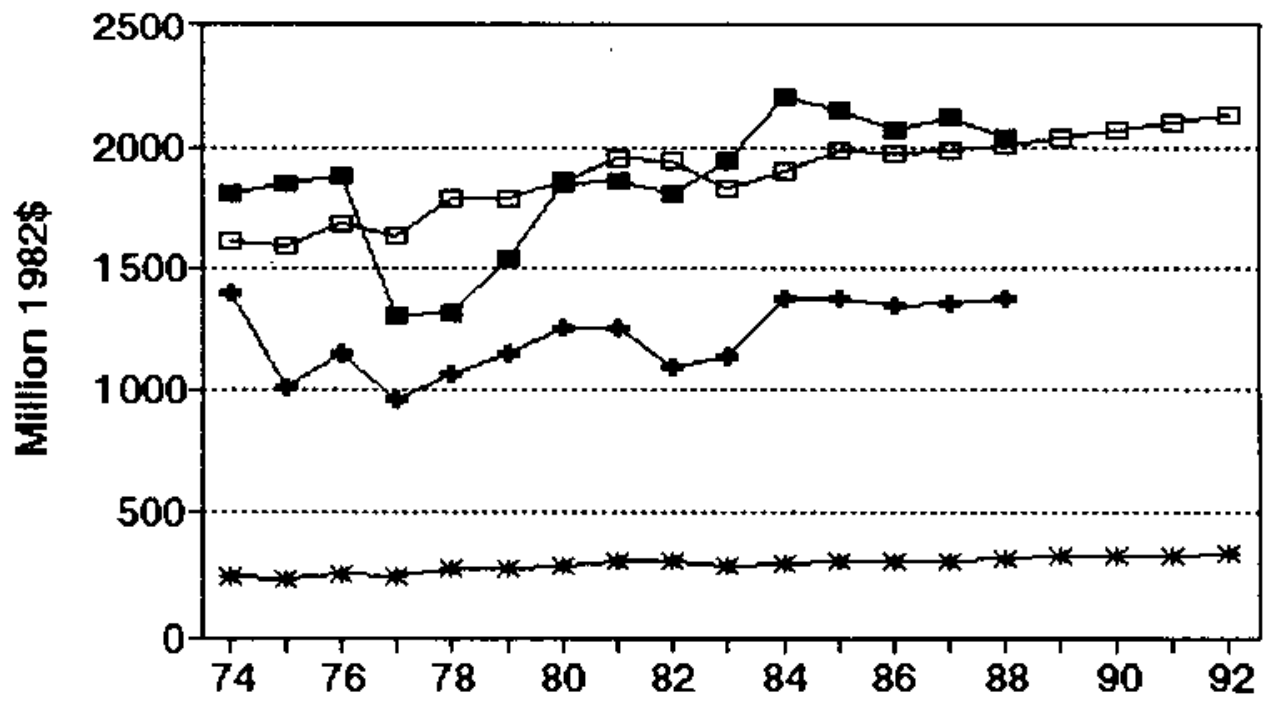

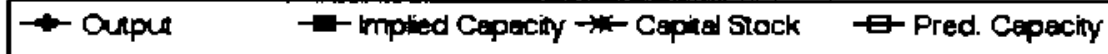




\section{MO. 367 sIc 3631 Eousehold cooking}

\section{MILLIOWS OF 1982 DOLLARS (EXCL. CU RATES)}

$\begin{array}{lccccccc} & \text { a4 } & \text { Am. } & \text { Implied Gross } & \text { Net } & \text { Pred. } & \ldots \ldots \ldots \\ \text { Gross } & \text { CU } & \text { CU } & \text { Prac. } & \text { In. } & \text { Cap. } & \text { Prac. } & \text { Emergency Capacity } \\ \text { Output } & \text { Rate } & \text { Rate } & \text { Capac. } & \text { vest. } & \text { Stk. } & \text { Capac. } & 3 \text { mon. } 6 \text { mon. }>6 \text { mon. }\end{array}$

\begin{tabular}{|c|c|c|c|c|c|c|c|c|c|c|}
\hline 1974 & $1, \pi 7$ & 58 & 60.7 & 2,848 & 32.4 & 323 & 2,633 & & & \\
\hline 1975 & 1,609 & 63 & 58.6 & 2,745 & 68.2 & 366 & 3,201 & & & \\
\hline 1976 & 2,113 & 58 & 60.0 & 3,519 & 33.4 & 373 & 3,291 & & & \\
\hline 1977 & 2,294 & 76 & 67.9 & 3,380 & 31.9 & 378 & 3,349 & & & \\
\hline 1978 & 2,468 & 68 & 69.9 & 3,531 & 51.6 & 399 & 3,627 & & & \\
\hline 1979 & 2,863 & 67 & 67.3 & 4,254 & 94.1 & 456 & 4,386 & & & \\
\hline 1980 & 2,715 & 60 & 62.3 & 4,361 & 34.8 & 454 & 4,358 & & & \\
\hline 1981 & 2,093 & 54 & 56.5 & 4,765 & 62.8 & 478 & 4,670 & & & \\
\hline 1982 & 2,424 & 47 & 49.2 & 4,924 & 54.9 & 491 & 4,840 & & & \\
\hline 1983 & 2,933 & 61 & 55.1 & 5,327 & 62.3 & 510 & 5,090 & & & \\
\hline 1984 & 3,381 & 61 & 61.4 & 5,510 & 68.7 & 533 & 5,403 & & & \\
\hline 1985 & 2,848 & 56 & 57.5 & 4,955 & 20.2 & 511 & 5,101 & & & \\
\hline 1986 & 3,150 & 62 & 59.3 & 5,315 & 74.0 & 540 & 5,487 & & & \\
\hline-- & $\cdots$ & $\because$ & $\because \cdot$ & $\cdots \cdot$ & --- & -- & $\because \cdot$ & 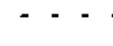 & --- & $\cdots$ \\
\hline 1987 & 3,190 & 60 & 60.5 & 5,270 & 61.1 & 556 & 5,697 & & & \\
\hline 1988 & 3,243 & 71 & 67.0 & 4,841 & 62.2 & $5 \pi$ & 5,905 & 6,196 & 8,366 & 8,397 \\
\hline 1989 & & & & & 63.4 & 587 & 6,111 & 6,412 & 8,657 & 8,689 \\
\hline 1990 & & & & & 64.5 & 603 & 6,314 & 6,625 & 8,945 & 8,978 \\
\hline 1991 & & & & & 65.7 & 618 & 6,515 & 6,836 & 9.230 & 9,264 \\
\hline 1992 & & & & & 66.8 & 633 & 6,714 & 7,045 & 9,513 & 9,548 \\
\hline
\end{tabular}

Capacity-Capital stock Regression: Code 0 RSO 0.960

Coefficients: Const -1625.0 Stock 13.2

Ave, Capacity/Stock $(74-86): 9.5$ Ave. Weekly Hours (High) t to.8 Shift Factor 1.42 Ave Heekly Hours (Low) 103.3 Shift Factor 1.52 Wote: Output, Investment, and Capital Stock extrapolated for 1987 and 1988.

\section{Household cooking}

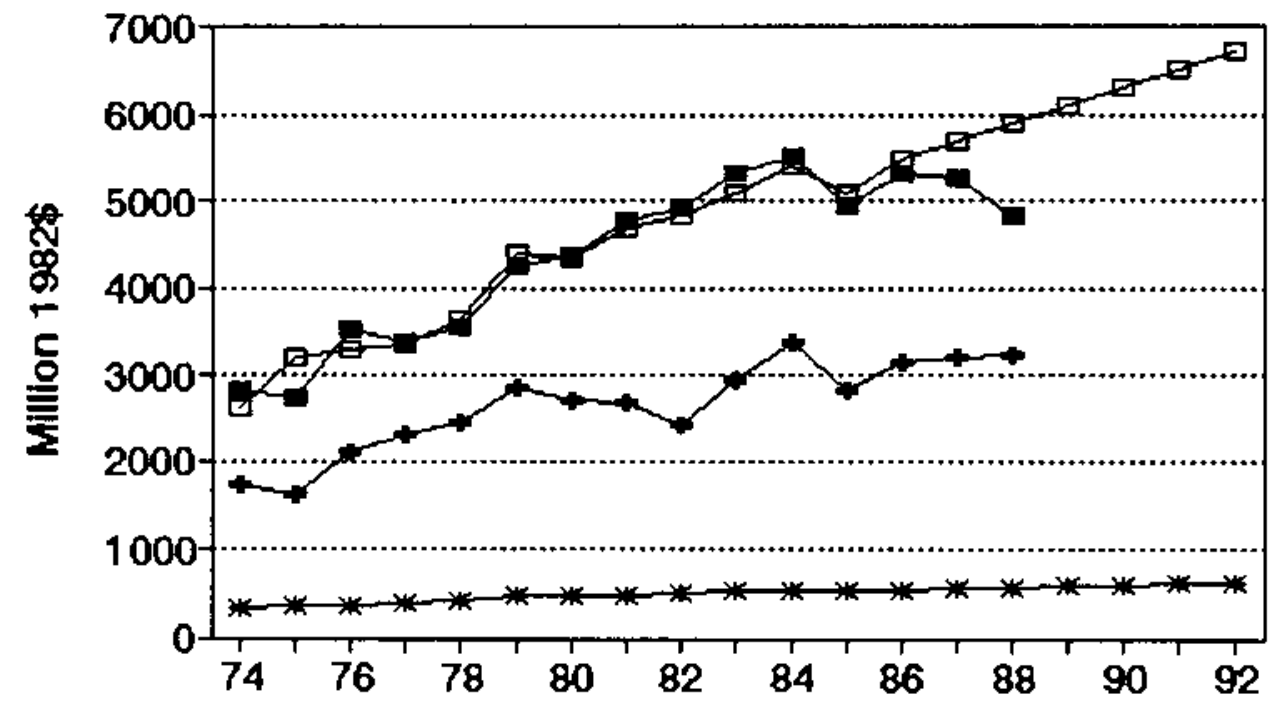

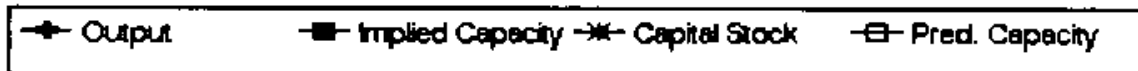


No. 368 SIC 3632 Household refrigerators and freezers

MILLIONS OF 1982 DOLLARS (EXCL, CU RATES)

$\begin{array}{lccccccc} & \text { Q4 } & \text { Am. } & \text { Implied Gross } & \text { Net } & \text { Pred. } & -\ldots \ldots \\ \text { Gross } & \text { CU } & \text { CU } & \text { Prac. } & \text { In- } & \text { Cap. } & \text { Prac. } & \text { Emergency Capacity } \\ \text { Output } & \text { Rete } & \text { Rate } & \text { Capac. } & \text { vest. } & \text { Stk. } & \text { Capec. } & 3 \text { mon. } 6 \text { mon. }>6 \text { mon. }\end{array}$

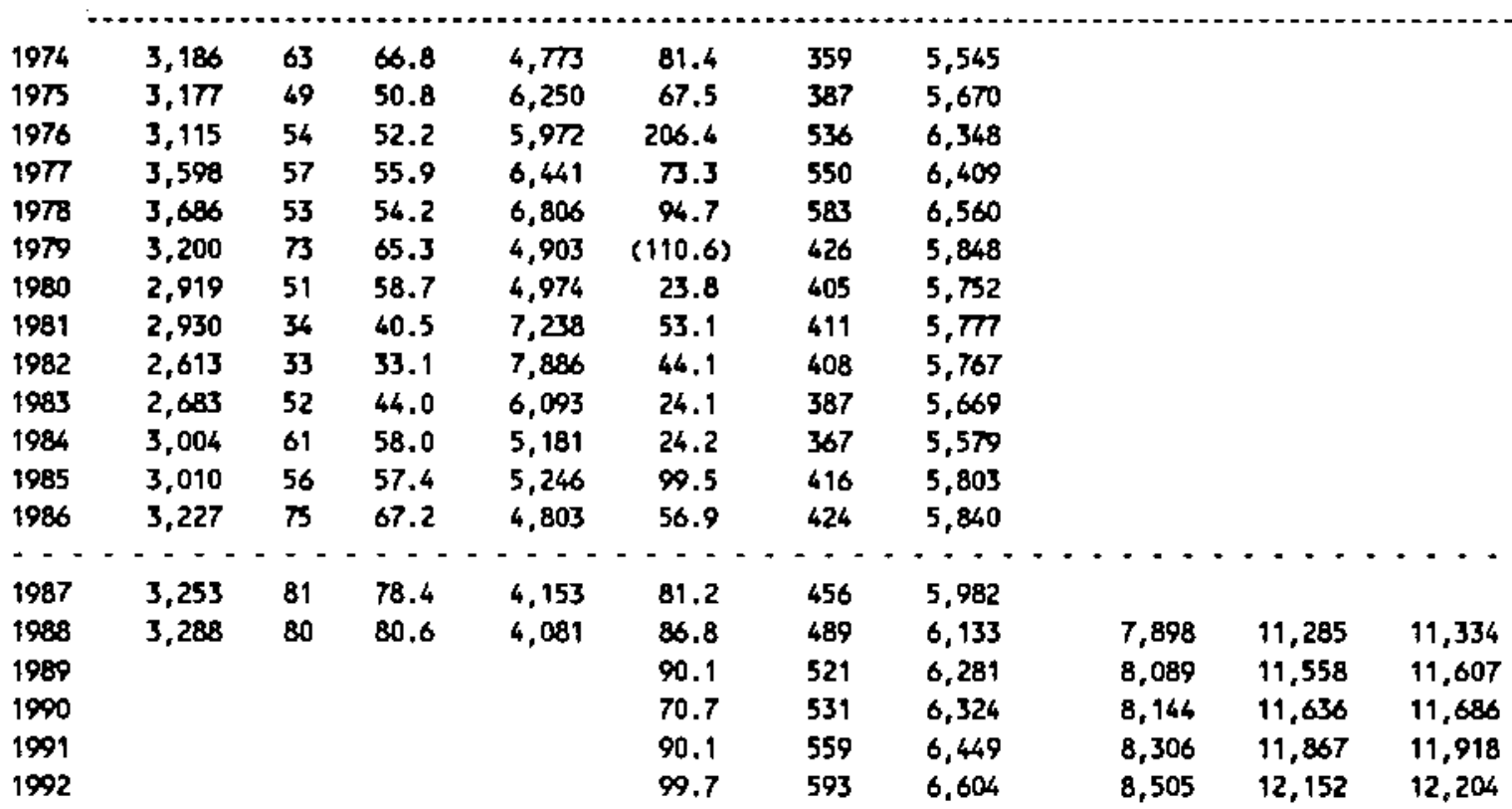

Capacity-Capital Stock Regression: Code 1 RSO 0.110 Coefficients: Const 3914.3 stock 4.5

Ave. Capacity/Stock (74-86): 13.5 Ave. Weekly Hours (High) 85.3 Shift Factor 1.85 Ave Weekly Hours (Low) 82.0 Shift Factor 1.92 Note: Output, Investment, and Capital Stock extrapolated for 1987 and 1988.

\section{Household refrigerators and freeze}

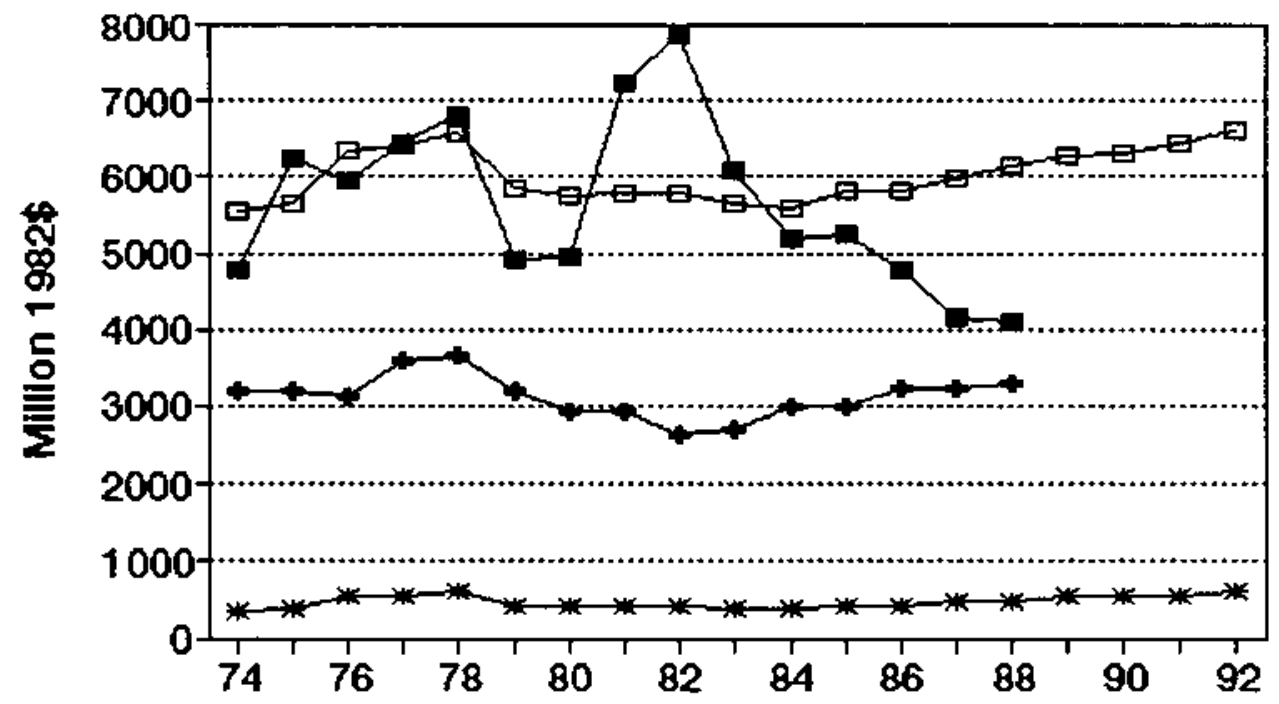

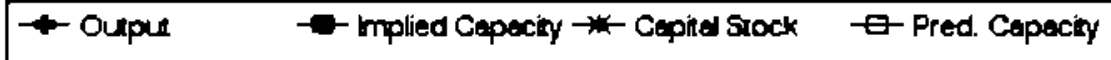




\section{No. 369 BIC 3633 Bousehold laundry equipent}

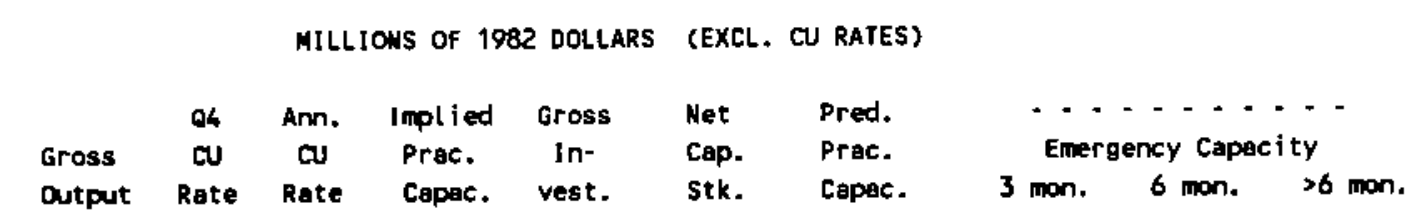

\begin{tabular}{|c|c|c|c|c|c|c|c|c|c|c|}
\hline 1974 & 2,443 & 50 & 51.2 & 4,775 & 79.5 & 464 & 3,875 & & & \\
\hline $19 \pi$ & 2,054 & 64 & 57.6 & 3,564 & 24.1 & 453 & 3,767 & & & \\
\hline 1976 & 2,237 & 58 & 60.3 & 3,708 & 66.6 & 482 & 4,049 & & & \\
\hline 1977 & 2,458 & 68 & 64.2 & 3,829 & 11.3 & 455 & 3,789 & & & \\
\hline 1978 & 2,544 & 59 & 62.2 & 4,089 & 32.1 & 448 & 3,722 & & & \\
\hline 1979 & 2,761 & 79 & 71.4 & 3,868 & 50.0 & 456 & 3,799 & & & \\
\hline 1980 & 2,441 & 79 & 78.8 & 3,099 & 33.5 & 447 & 3,711 & & & \\
\hline 1981 & 2,418 & 60 & 67.2 & 3,597 & 41.1 & 444 & 3,680 & & & \\
\hline 1982 & 2,208 & 63 & 61.7 & 3,578 & 11.8 & 413 & 3,378 & & & \\
\hline 1983 & 2,353 & 83 & 75.0 & 3,137 & 3.0 & 376 & 3,020 & & & \\
\hline 1984 & 2,625 & 82 & 82.6 & 3,177 & 61.9 & 397 & 3,221 & & & \\
\hline 1985 & 2,711 & 85 & 83.6 & 3,242 & 64.4 & 419 & 3,439 & & & \\
\hline 1986 & 2,944 & 91 & 88.4 & 3,331 & 52.7 & 430 & 3,545 & & & \\
\hline$\because \cdot$ & $=-$ & - & $\because \cdot$ & $\because-1$ & $\because-$ & $\therefore-$ & $\cdots$ & --- & $\cdots$ & $-\cdot-$ \\
\hline 1987 & 2,978 & 91 & 90.8 & 3,279 & 68.3 & 456 & 3,797 & & & \\
\hline 1988 & 3,024 & 85 & 87.4 & 3,462 & 74.0 & 485 & 4,079 & 4,687 & 5,909 & 5,927 \\
\hline 1989 & & & & & 7.3 & 514 & 4,365 & 5,017 & 6,324 & 6,343 \\
\hline 1990 & & & & & 57.8 & 521 & 4,433 & 5,095 & 6,423 & 6,442 \\
\hline 1991 & & & & & 7.3 & 547 & 4,685 & 5,384 & 6,787 & 6,807 \\
\hline 1992 & & & & & 87.0 & 580 & 5,006 & 5,753 & 7,253 & 7,274 \\
\hline
\end{tabular}

Capacity-Capital Stock Regression: Code 1 RSO 0.370

Coefficients: const -656.4 Stock 9.8

Ave. Capacity/Stock $(74-86): 8.3$ Ave. Heekly hours (High) 108.4 Shift Factor 1.45 Ave Weekly Hours (LOW) 106.9 Shift Factor 1.47 Mote: Output, Investment, and Capital stack extrapolated for 1987 and 1988.

\section{Household laundry equipment}

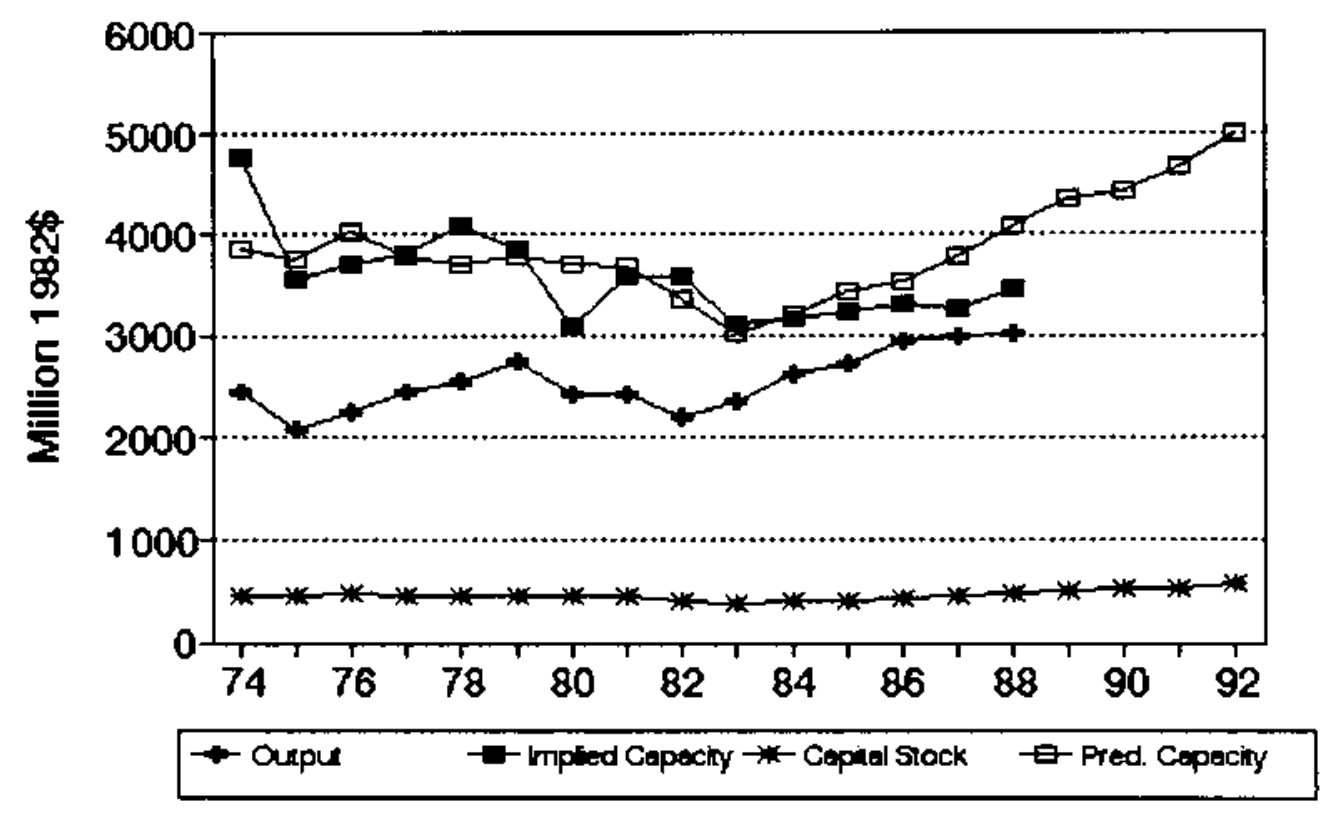


Ho. 370 sIC 3634 Electric housewares and fans

MILLIONS OF 1982 DOLLARS (EXCL. CU RATES)

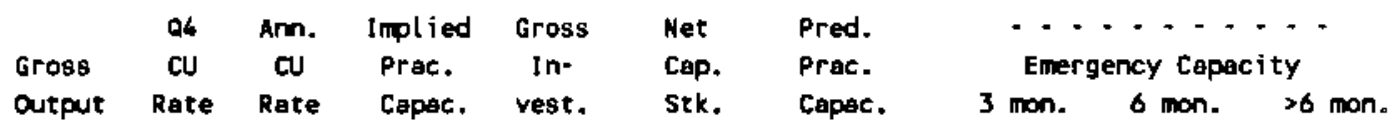

\begin{tabular}{|c|c|c|c|c|c|c|c|c|c|c|}
\hline 1974 & 3,072 & 65 & 67.0 & 4,582 & 100.0 & 558 & 4,187 & & & \\
\hline 1975 & 2,731 & 71 & 6.8 & 4,086 & 44.4 & 559 & 4,272 & & & \\
\hline 1976 & 3,131 & 76 & 74.1 & 4,223 & 24.6 & 540 & 4,357 & & & \\
\hline 1977 & 3,499 & 80 & 78.5 & 4,459 & 71.6 & 566 & 4,461 & & & \\
\hline 1978 & 3,348 & $\pi$ & 77.8 & 4,302 & 65.2 & 583 & 4,526 & & & \\
\hline 1979 & 3,478 & 7 & 76.9 & 4,521 & 46.2 & 581 & 4,611 & & & \\
\hline 1980 & 3,674 & 74 & 74.8 & 4,910 & 63.7 & 594 & 4,696 & & & \\
\hline 1981 & 3,237 & $\infty 6$ & 69.2 & 4,676 & 59.5 & 602 & 4,781 & & & \\
\hline 1982 & 3,141 & 64 & 64.5 & 4,873 & 62.5 & 610 & 4,866 & & & \\
\hline 1983 & 3,164 & 67 & 65.4 & 4,834 & $\uparrow 7.9$ & 576 & 4,951 & & & \\
\hline 1984 & 3,093 & 55 & 59.8 & 5,171 & $(38.0)$ & 494 & 5,035 & & & \\
\hline 1985 & 3,014 & 58 & 56.7 & 5,319 & 34.3 & 484 & 5,120 & & & \\
\hline 1986 & 2,937 & 58 & 57.7 & 5,093 & 37.7 & $4 \pi$ & 5,205 & & & \\
\hline 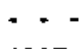 & --- & - & -- & -- & --- & $\because-$ & $-\cdots$ & $-\cdots$ & -- & -- \\
\hline 1987 & 2,967 & 67 & 63.4 & 4,677 & 19.2 & 452 & 5,290 & & & \\
\hline 1988 & 3,006 & 67 & 67.1 & 4,481 & 15.8 & 426 & 5,375 & 6,144 & 8,449 & 8,482 \\
\hline 1989 & & & & & 12.4 & 39 & 5,460 & 6,241 & 8,582 & 8,615 \\
\hline 1990 & & & & & 9.0 & 371 & 5,545 & 6,338 & 8,715 & 8,749 \\
\hline 1991 & & & & & 5.6 & 342 & 5,629 & 6,435 & 8,849 & 8,883 \\
\hline 1992 & & & & & 2.2 & 313 & 5,714 & 6,532 & 8,982 & 9,017 \\
\hline
\end{tabular}

Capacity-Capital stock Regression: Code -2 RSO 0.760

Coefficients: Const 4102.0 Time 84.9

Ave. Capseity/stock (74-86): 8.5 Ave. Weekly Hours (High) $\$ 9.8$ Shift factor 1.58 Ave Weekly Hours (Low) 90.5 Shift Factor 1.74 Note: Output, Imvestment, and Capital stock extrapolated for 1987 and 1988.

\section{Electric housewares and fans}

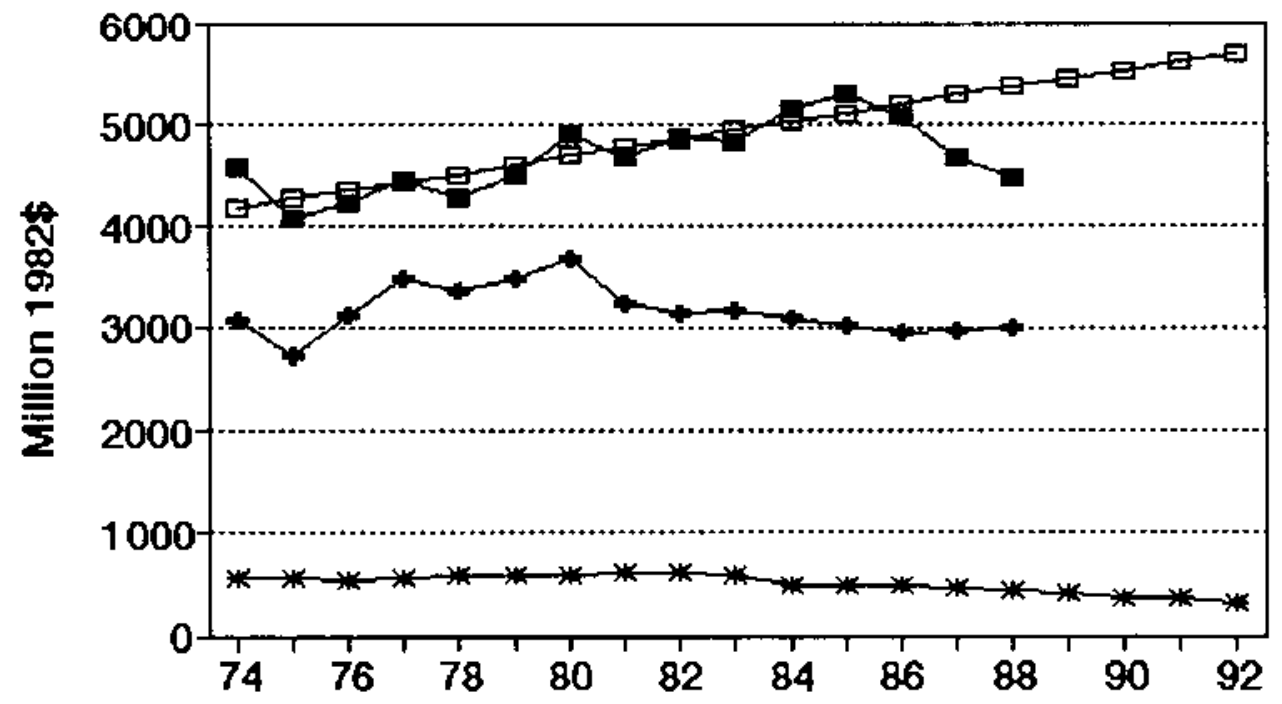

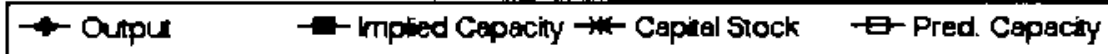




\section{No. 371 SIC 3635 Bousehold vacuum cleaners}

\section{MILLIONS OF 1982 DOLLARS (EXCL. CU RATES)}

\begin{tabular}{|c|c|c|c|c|c|c|c|}
\hline & $\begin{array}{l}\alpha \\
\mathrm{CU}\end{array}$ & $\stackrel{\text { Ann. }}{\mathrm{CU}}$ & $\begin{array}{l}\text { Implied } \\
\text { Prac. }\end{array}$ & $\begin{array}{l}\text { Gross } \\
\text { In. }\end{array}$ & $\begin{array}{l}\text { Net } \\
\text { Cap. }\end{array}$ & $\begin{array}{l}\text { Pred. } \\
\text { Prac. }\end{array}$ & $\begin{array}{l}---\cdots \\
\text { Energency Capacity }\end{array}$ \\
\hline & Rate & Rate & Canac. & vest. & Stk. & Capec. & \\
\hline
\end{tabular}

\begin{tabular}{|c|c|c|c|c|c|c|c|c|c|c|}
\hline 1974 & 983 & 71 & 71.0 & 1,385 & 27.8 & 207 & 1,502 & & & \\
\hline $197 \pi$ & 672 & 71 & 71.0 & 946 & $(1.6)$ & 193 & 1,181 & & & \\
\hline 1976 & 816 & 77 & 74.8 & 1,092 & 22.4 & 202 & 1,387 & & & \\
\hline 1977 & 814 & 70 & 72.6 & 1,121 & 3.4 & 193 & 1,165 & & & \\
\hline 1978 & 829 & 57 & 61.9 & 1,340 & 19.2 & 197 & 1,270 & & & \\
\hline 1979 & 928 & 55 & 55.8 & 1,665 & 26.4 & 207 & 1,504 & & & \\
\hline 1980 & 786 & 53 & 53.8 & 1,463 & 17.4 & 208 & 1,515 & & & \\
\hline 1981 & 793 & 52 & 52.4 & 1,513 & 14.4 & 205 & 1,449 & & & \\
\hline 1982 & 802 & 41 & 45.1 & 1,778 & 14.6 & 202 & 1,369 & & & \\
\hline 1983 & 948 & 54 & 49.1 & 1,930 & 41.7 & 222 & 1,834 & & & \\
\hline 1984 & 1,316 & 58 & 56.5 & 2,329 & 37.3 & 237 & 2,173 & & & \\
\hline 1985 & 1,207 & 55 & 56.1 & 2,151 & 16.7 & 232 & 2,060 & & & \\
\hline 1986 & 1,251 & 68 & 63.1 & 1,982 & 31.7 & 242 & 2,285 & & & \\
\hline$\because \cdot$ & & -- & $\cdots$ & $\cdots$ & $-\cdot-$ & $\because$ & $\cdots$ & $\cdots$ & $-\cdots$ & $=-$ \\
\hline 1987 & 1,284 & 64 & 65.5 & 1,961 & 30.0 & 249 & 2,463 & & & \\
\hline 1988 & 1,329 & 62 & 62.8 & 2,118 & 31.7 & 258 & 2,664 & 3,027 & 4,275 & 4,292 \\
\hline 1989 & & & & & 32.7 & 267 & 2,871 & 3,261 & 4,606 & 4,625 \\
\hline 1990 & & & & & 26.7 & 269 & 2,921 & 3,318 & 4,685 & 4,705 \\
\hline 1991 & & & & & 32.7 & 277 & 3,103 & 3,525 & 4,979 & $4, \infty$ \\
\hline 1992 & & & & & 35.7 & 288 & 3,338 & 3,792 & 5,355 & 5,377 \\
\hline
\end{tabular}

Capacity-Capital Stock Regression: Code 0 RSO 0.770

Coefficients: Const $\mathbf{- 3 2 4 5 . 6}$ Stock 22.9

Ave. Capacity/Stock $(74-86): 7.5$ Ave. Heekly Hours (High) 97.8 Shift Factor 1.61 Ave Heekly Hours (LOW) 85.5 Shift Factor 1.84 Mote: Output, Investment, and Capital Stock extrapolated for 1987 and 1988.

3635 Household vacuum cleaners

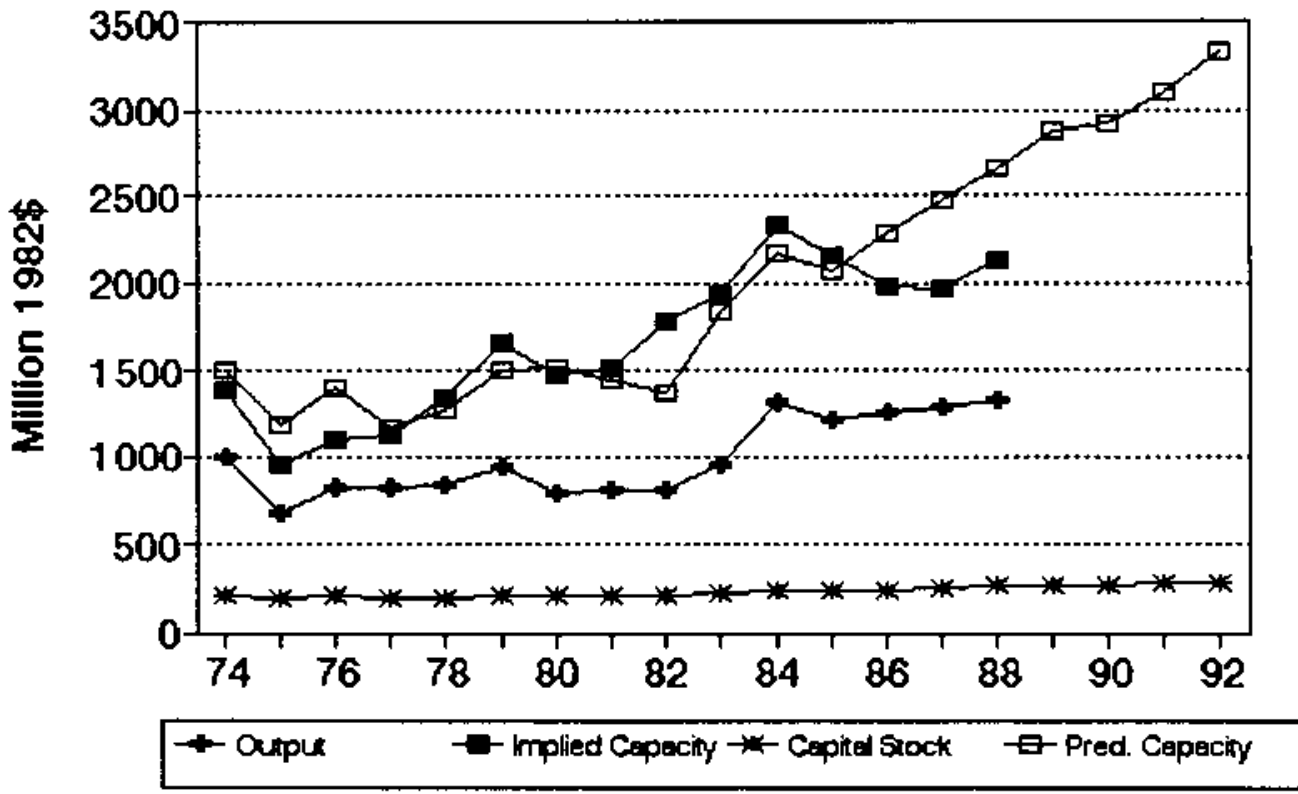


No. 372 sIC 3636 sewing machines

\section{MILLIONS OF 1982 DOLLARS (EXCL. CU RATES)}

$\begin{array}{lccccccc} & 04 & \text { Ann. Implied } & \text { Gross } & \text { Net } & \text { Pred. } & \ldots \ldots \ldots \\ \text { Gross } & \text { CU } & \text { CU } & \text { Prac. } & \text { In- } & \text { Cap. } & \text { Prac. } & \text { Emergency Capacity } \\ \text { Output Rate } & \text { Rate } & \text { Capac. } & \text { vest. } & \text { Stk. } & \text { Capac. } & 3 \text { mon. } 6 \text { mon. }\end{array}$

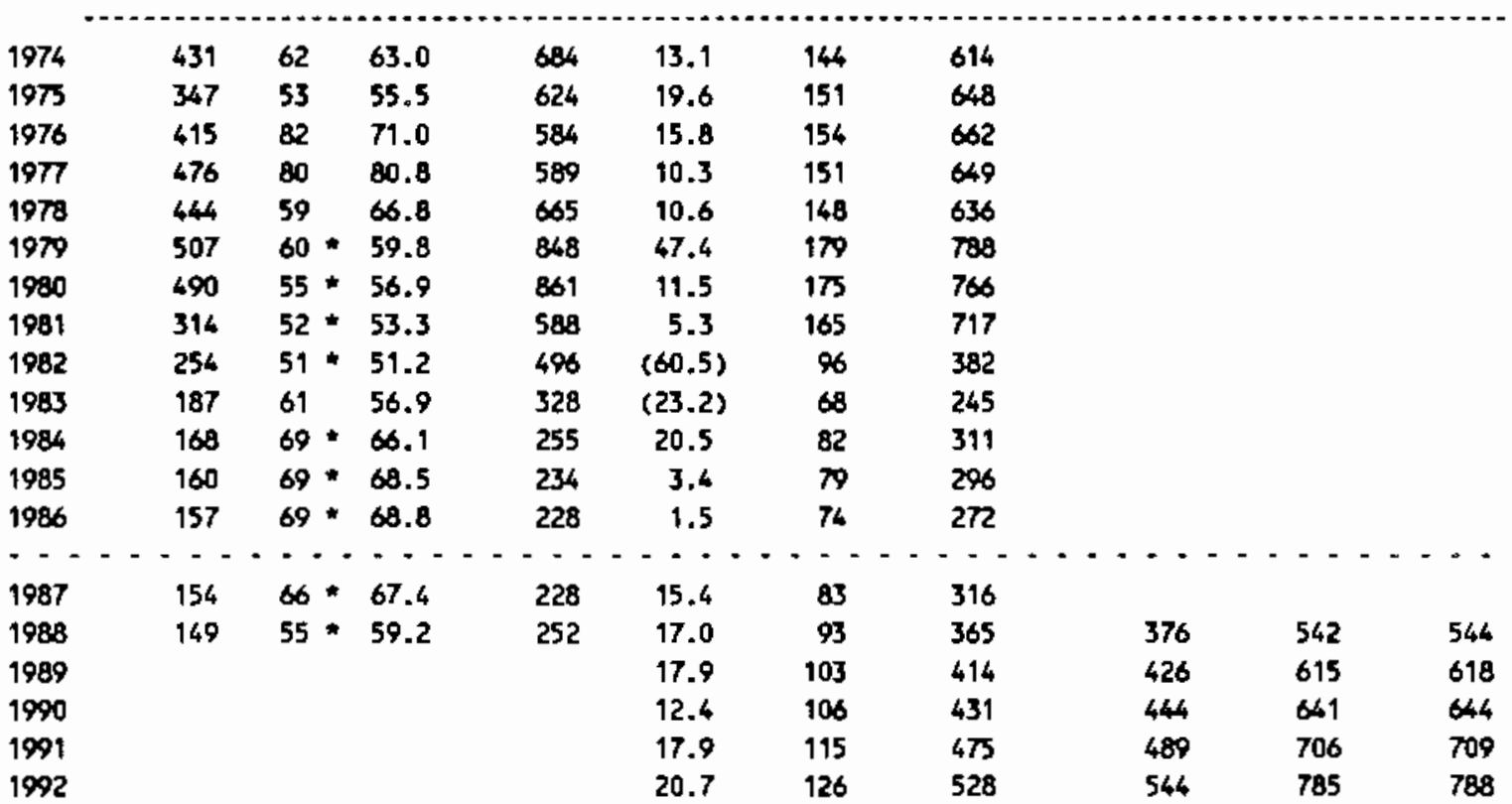

Capecity-Capital Stock Regression: Code 1 RSQ 0.870

Coefficients: Const -89.9 stock $4.9 \quad$ Ave. Capecity/Stock $(74-86): 4.2$ Ave. Weekly Hours (High) 105.5 Shift Factor 1.49 Ave Yeekly Hours (LOw) 100.0 Shift Factor 1.58 - Imputed Note: Output, Imvestment, and Capital Stock extrapolated for 1987 and 1988.

\section{Sewing machines}

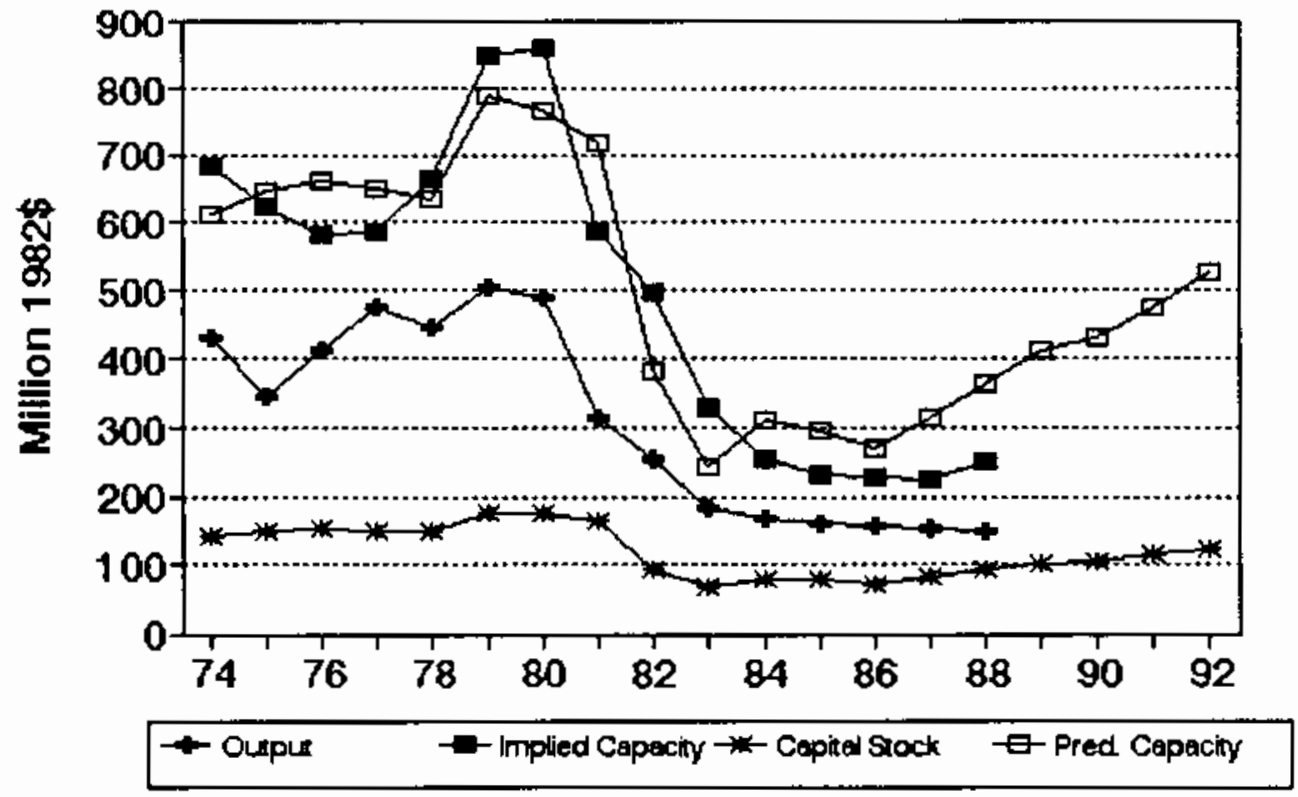


No. 373 sIC 3639 Household appliances, nec

HILLIONS OF 1982 DOLLARS (EXCL. CU RATES)

$\begin{array}{lccccccc} & 04 & \text { Ann. Implied Gross } & \text { Net } & \text { Pred. } & \ldots & \ldots \\ \text { Gross } & \text { CU } & \text { aU } & \text { Prac. } & \text { In- } & \text { Cap. } & \text { Prac. } & \text { Emergency Capacity } \\ \text { Output } & \text { Rate } & \text { Rate } & \text { Capac. } & \text { vest. } & \text { Stk. } & \text { Capac. } & 3 \text { mon. } 6 \text { mon. }>6 \text { mon. }\end{array}$

\begin{tabular}{|c|c|c|c|c|c|c|c|c|c|c|}
\hline 1974 & 1,460 & 68 & 71.9 & 2,031 & 5.6 & 233 & 2,115 & & & \\
\hline 1975 & 1,222 & 64 & 61.9 & 1,973 & 27.5 & 241 & 2,153 & & & \\
\hline 1976 & 1,435 & 66 & 65.4 & 2,196 & 11.6 & 232 & 2,110 & & & \\
\hline 1977 & 1,635 & 83 & 76.4 & 2,139 & 39.6 & 250 & 2,197 & & & \\
\hline 1978 & 1,669 & 80 & 80.6 & 2,070 & 23.6 & 251 & 2,203 & & & \\
\hline 1979 & 1,809 & 80 & 79.9 & 2,265 & 42.4 & 269 & 2,291 & & & \\
\hline 1980 & 1,580 & 63 & 68.8 & 2,296 & 27.4 & 271 & 2,301 & & & \\
\hline 1981 & 1,570 & 53 & 57.1 & 2,751 & 51.6 & 295 & 2,418 & & & \\
\hline 1982 & 1,452 & 47 & 48.8 & 2,976 & 47.8 & 313 & 2,509 & & & \\
\hline 1983 & 1,816 & 69 & 59.7 & 3,040 & 51.9 & 333 & 2,608 & & & \\
\hline 1984 & 2,032 & 88 & 81.3 & 2,409 & 21.6 & 324 & 2,566 & & & \\
\hline 1985 & 1,966 & 87 & B6. $B$ & 2,267 & 52.4 & 347 & 2,676 & & & \\
\hline 1986 & 2,046 & 89 & 87.4 & 2,342 & 35.6 & 352 & 2,700 & & & \\
\hline-- & & $\cdots$ & $\cdots$ & --- & $\cdots$ & $\cdots$ & $--\cdot$ & $\cdots$ & $-\cdots$ & $\cdots$ \\
\hline 1987 & 2,091 & 82 & 84.3 & 2,481 & 39.6 & 361 & 2,744 & & & \\
\hline 1988 & 2,149 & 54 & 64.7 & 3,320 & 40.5 & 370 & 2,789 & 3,304 & 4,152 & 4,164 \\
\hline 1989 & & & & & 41.0 & 378 & 2,832 & 3,355 & 4,216 & 4,229 \\
\hline 1990 & & & & & 38.1 & 383 & 2,857 & 3,385 & 4,254 & 4,266 \\
\hline 1991 & & & & & 41.0 & 391 & 2,895 & 3,429 & 4,309 & 4,322 \\
\hline 1992 & & & & & 42.4 & 399 & 2,936 & 3,478 & 4,371 & 4,383 \\
\hline
\end{tabular}

Capacity-Capital Stock Regression: Code 1 RSQ 0.390

Coefficients: Const 959.6 Stock $\mathbf{5 . 0}$

Ave. Capacity/Stock (74-86): 8.3

Ave. Weekly Hours (High) 105.5 Shift Factor 1.49 Ave Heekly Hours (LOW) 100.0 Shift Factor 1.58 Mote: Output, Investment, and Capital Stock extrapolated for 1987 and 1988.

\section{Household appliances, nec}

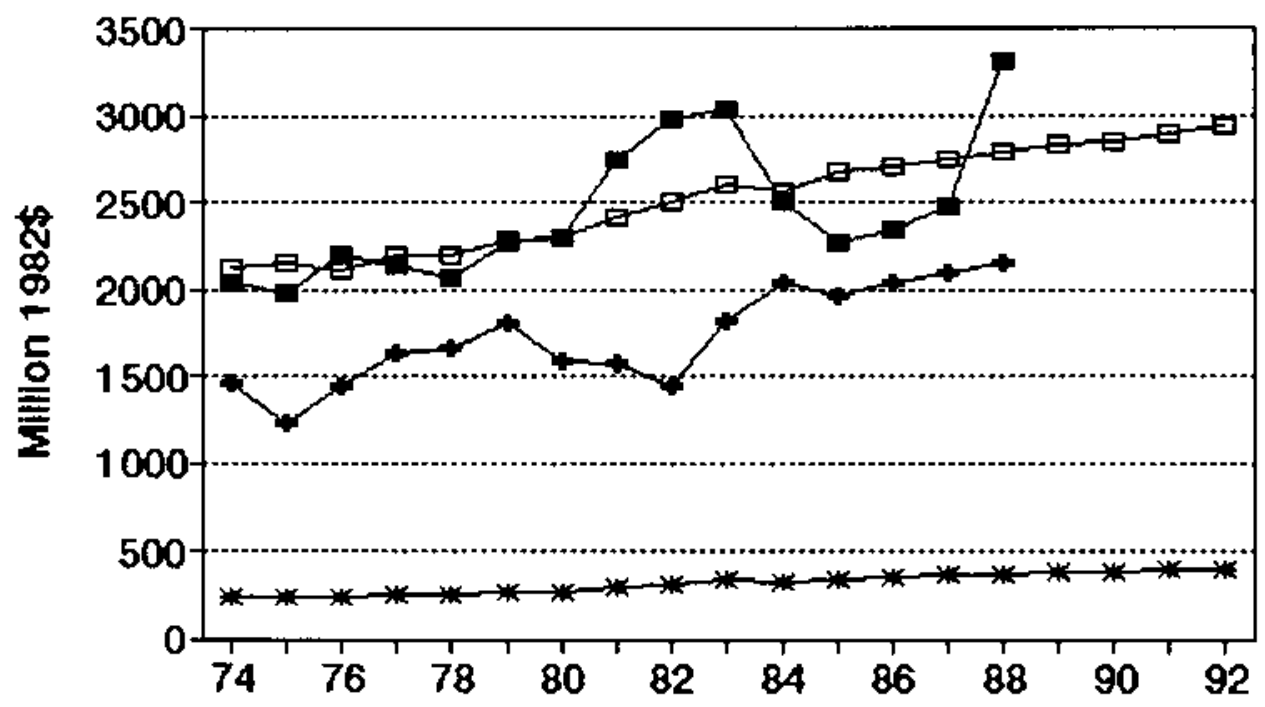

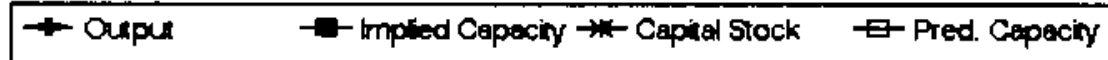


No. 374 SIC 3641 Electric lamps

MILLIONS OF 1982 DOLLARS (EXCL. CU RATES)

$\begin{array}{lcccclcc} & \alpha 4 & \text { Ann. Implied Gross } & \text { Het } & \text { Pred. } & -\ldots & \ldots \\ \text { Gross } & \text { aU } & \text { CU } & \text { Prac. } & \text { In- } & \text { Cap. } & \text { Prac. } & \text { Emergency Capacity } \\ \text { Output } & \text { Rate } & \text { Rate } & \text { Capac. } & \text { vest. } & \text { Stk. } & \text { Capac. } & 3 \text { mon. } 6 \text { mon. }>6 \text { mon. }\end{array}$

\begin{tabular}{|c|c|c|c|c|c|c|c|c|c|c|}
\hline 1974 & 2,557 & 71 & 72.5 & 3,529 & 90.6 & 803 & 3,480 & & & \\
\hline 1975 & 2,135 & 68 & 67.8 & 3,150 & 67.7 & 815 & 3,518 & & & \\
\hline 1976 & 2,418 & $\pi$ & 71.1 & 3,399 & 64.1 & 819 & 3,555 & & & \\
\hline 1977 & 2,507 & 70 & 71.1 & 3,524 & 75.4 & 829 & 3,592 & & & \\
\hline 1978 & 2,593 & 80 & 76.0 & 3,610 & 71.5 & 833 & 3,630 & & & \\
\hline 1979 & 2,662 & 63 & 69.4 & 3,837 & 83.7 & 847 & 3,667 & & & \\
\hline 1980 & 2,283 & 55 & 57.8 & 3,948 & $\pi .2$ & 849 & 3,705 & & & \\
\hline 1981 & 2,135 & 56 & 55.8 & 3,027 & 60.9 & 837 & 3,742 & & & \\
\hline 1982 & 2,117 & 44 & 48.3 & 4,387 & 87.7 & 848 & 3,780 & & & \\
\hline 1983 & 2,195 & 51 & 48.1 & 4,561 & $(32.0)$ & 748 & 3,817 & & & \\
\hline 1984 & 2,259 & 63 & 58.6 & 3,854 & 36.0 & 722 & 3,855 & & & \\
\hline 1985 & 2,403 & 71 & 67.9 & 3,541 & 137.1 & 792 & 3,892 & & & \\
\hline 1986 & 2,240 & 70 & 70.1 & 3,194 & 115.3 & 840 & 3,929 & & & \\
\hline$\therefore-$ & $--\cdot-$ & -- & $\cdots$ & $\because-$ & -- & $-\cdots$ & 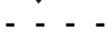 & $-\cdots$ & $-\cdots$ & $\cdot-\cdot$ \\
\hline 1987 & 2,238 & 71 & 70.5 & 3,175 & 85.6 & 856 & 3,967 & & & \\
\hline 1988 & 2,237 & 71 & 71.1 & 3,148 & 98.2 & 884 & 4,004 & 4,287 & 5,703 & 5,814 \\
\hline 1989 & & & & & 91.8 & 903 & 4,042 & 4,327 & 5,847 & 5,869 \\
\hline 1990 & & & & & 93.9 & 922 & 4,079 & 4,367 & 5,901 & 5,923 \\
\hline 1991 & & & & & 98.1 & 944 & 4,117 & 4,407 & 5,955 & 5,977 \\
\hline 1992 & & & & & 106.4 & 973 & 4,154 & 4,447 & 6,010 & 6,032 \\
\hline
\end{tabular}

Capecity-Capital Stock Regression: Code -2 RSO 0.120 coefficients: Const 3442.6 Time $\mathbf{3 7 . 5}$

Ave. Capacity/Stock $(74-86): 4.6$ Ave. Heekly Hours (High) 108.5 Shift Factor 1.45 Ave Veekly Hours (Low) 103.5 Shift Factor 1.52 Note: Output, Investment, and Capital stock extrapoleted for 1987 and 1988.

\section{Electric lamps}

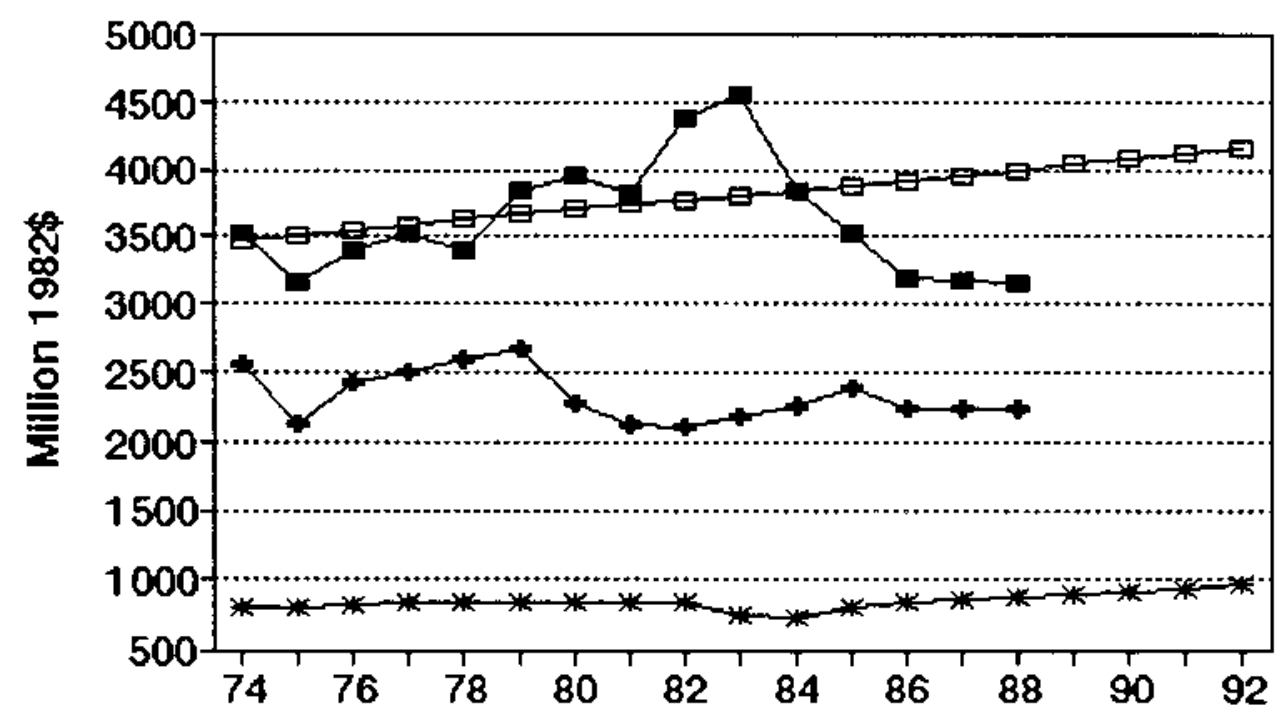

$\rightarrow-$ Ouput $\rightarrow$-mplied Capacity $\rightarrow$ Cepited Stock $\quad$ - Pred Capaciny 
No. 375 src 3643 Current-carrying wiring devices

MILLIONS OF 1982 DOLLARS (EXCL. CU RATES)

$\begin{array}{lccccccc} & \alpha 4 & \text { Am. } & \text { Implied Gross } & \text { Net } & \text { Pred. } & \ldots \\ \text { Gross } & \text { CU } & \text { CU } & \text { Prac. } & \text { In- } & \text { Cap. } & \text { Prac. } & \text { Emergency Capecity } \\ \text { OUtput } & \text { Rate } & \text { Rate } & \text { Capac. } & \text { vest. } & \text { Stk. } & \text { Capac. } & 3 \text { mon. } 6 \text { mon. }\end{array}$

\begin{tabular}{|c|c|c|c|c|c|c|c|c|c|c|}
\hline 1974 & 3,716 & 52 & 55.0 & 6,751 & 127.4 & 700 & 4,787 & & & \\
\hline 1975 & 2,207 & 56 & 51.6 & 4,276 & $(39.4)$ & 620 & 4,355 & & & \\
\hline 1976 & 2,958 & 68 & 63.4 & 4,663 & 44.7 & 623 & 4,368 & & & \\
\hline 1977 & 2,942 & 78 & 74.2 & 3,967 & 58.5 & 637 & 4,447 & & & \\
\hline 1978 & 2,880 & 73 & 74.4 & 3,871 & 11.5 & 606 & 4,280 & & & \\
\hline 1979 & 3,419 & 72 & 72.5 & 4,730 & 137.8 & 695 & 4,761 & & & \\
\hline 1980 & 3,178 & 72 & 71.5 & 4,663 & 91.4 & 735 & 4,976 & & & \\
\hline 1981 & 3,065 & 68 & 70.0 & 4,379 & 71.1 & 752 & 5,069 & & & \\
\hline 1982 & 2,509 & 58 & 61.1 & 4,106 & 75.6 & 770 & 5,168 & & & \\
\hline 1983 & 2,838 & 65 & 61.6 & 4,606 & 61.2 & 772 & 5,17 & & & \\
\hline 1984 & 3,199 & 55 & 59.3 & 5,397 & 43.6 & 757 & 5,093 & & & \\
\hline 1985 & 3,185 & 53 & 53.3 & 5,972 & 82.4 & $m 7$ & 5,204 & & & \\
\hline 1986 & 3,155 & 57 & 54.9 & 5,745 & 66.6 & 780 & 5,221 & & & \\
\hline$-\cdots$ & $-\cdots$ & -- & $\cdots$ & $\cdots$ & $\cdots \cdot$ & $-\cdots$ & $\cdots$ & $\cdots$ & $\cdot-$ & --- \\
\hline 1987 & 3,195 & 72 & 66.0 & 4,840 & 86.4 & 804 & 5,349 & & & \\
\hline 1988 & 3,246 & 64 & 67.2 & 4,831 & 100.9 & 840 & 5,545 & 5,570 & 7,278 & 7,303 \\
\hline 1989 & & & & & 93.5 & 866 & 5,685 & 5,711 & 7,462 & 7,487 \\
\hline 1990 & & & & & 95.9 & 892 & 5,827 & 5,854 & 7,648 & 7,674 \\
\hline 1991 & & & & & 100.8 & 921 & 5,984 & 6,011 & 7,854 & 7,880 \\
\hline 1992 & & & & & 110.5 & 957 & 6,180 & 6,209 & 8,112 & 8,140 \\
\hline
\end{tabular}

Capecity-Capital Stock Regression: Code 0 RSQ 0.170

Coefficients: Const 994.7 stock 5.4

Ave. Capacity/Stock $(74-86): 6.8$

Ave. Weekly Hours (High) 120.0 Shift Factor 1.32 Ave Heekly Hours (LOW) 115.8 Shift Factor 1.36 Note: Dutput, Investment, and Capital stock extrapolated for 1987 and 1988.

3643 Current-carrying wiring devices

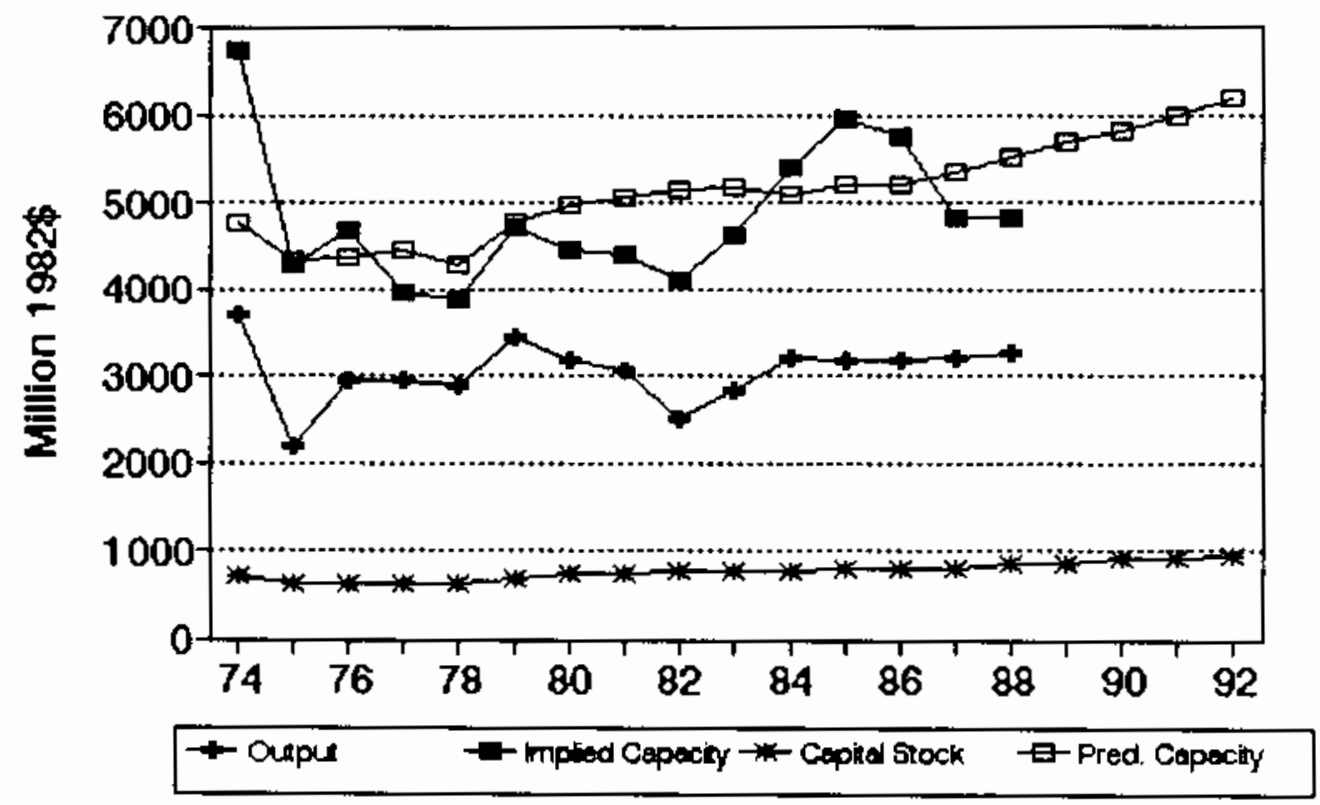




\section{No. 376 sIC 3644 Noncurrent-carrying wiring devices}

MILLIONS OF 1982 DOLLARS (EXCL. CU RATES)

$\begin{array}{lcccclcc} & \text { Q4 } & \text { Ann. Implied Gross } & \text { Net } & \text { Pred. } & -\ldots \ldots \ldots \\ \text { Gross } & \text { CU } & \text { CU } & \text { Prac. } & \text { In- } & \text { Cap. } & \text { Prec. } & \text { Emergency Capacity } \\ \text { Output } & \text { Rate } & \text { Rate } & \text { Capac. } & \text { vest. } & \text { Stk. } & \text { Capac. } & 3 \text { mon. } 6 \text { mon. }>6 \text { mon. }\end{array}$

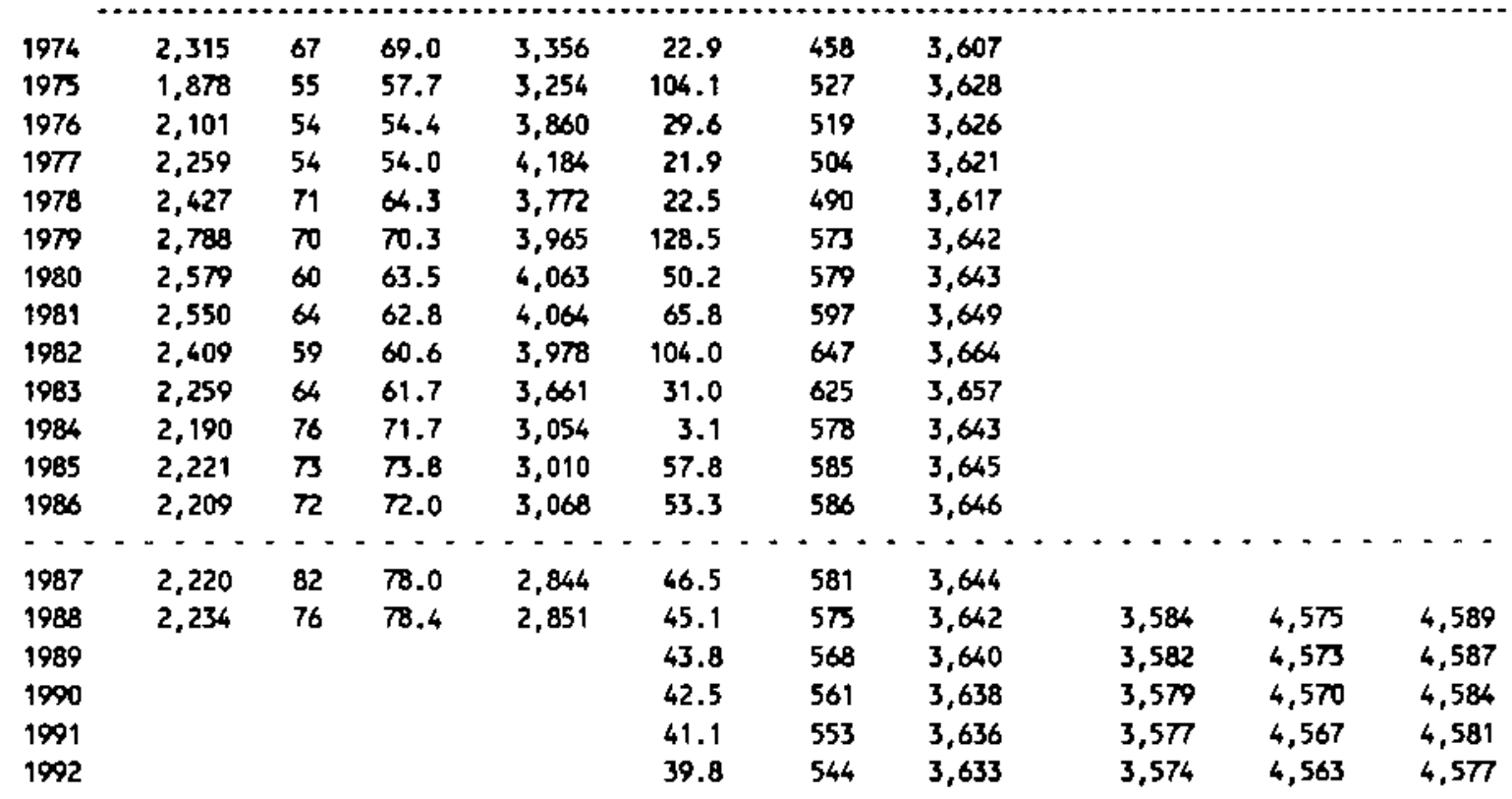

Capecity-Capital Stock Regression: Code 0 RSO 0.000 Coefficients: Const 3470.7 stock 0.3

Ave. Capacity/Stock $(74-86): 6.5$ Ave. Heekly Hours (High) 125.6 Shift Factor 1.26 Ave Weekly Hours (Low) 121.8 Shift Factor 1.30 Note: Qutput, Investment, and Capital Stock extrapolated for 1987 and 1988.

\section{Noncurrent-carrying wiring devices}

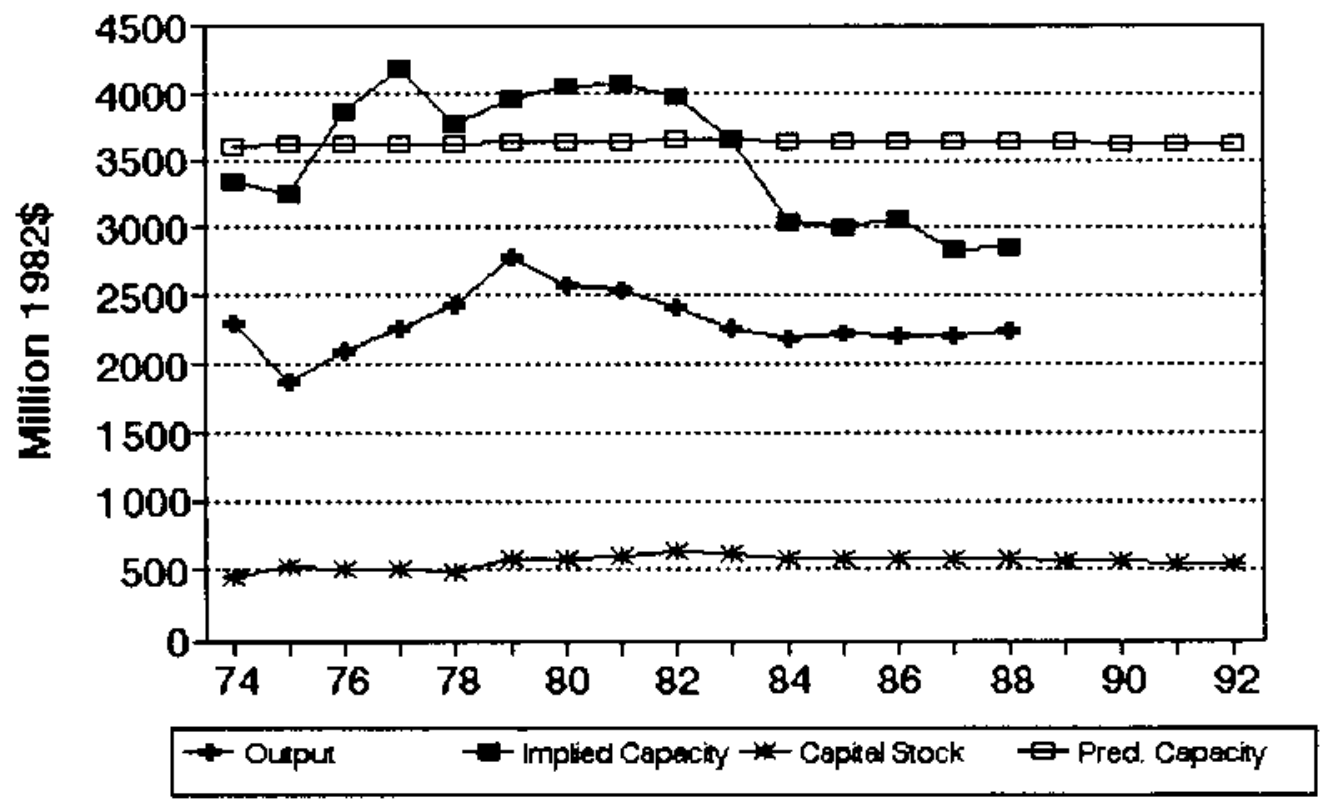




\section{Ho. 377 SIC 3645 Residential lighting fixtures}

MILLIONS OF 1982 DOLLARS (EXCL. CU RATES)

$\begin{array}{lccccccc} & 04 & \text { Ann. Implied } & \text { Gross } & \text { Net } & \text { Pred. } & \ldots & \ldots \\ \text { Gross } & \text { CU } & \text { CJ } & \text { Prac. } & \text { In. } & \text { Cap. } & \text { Prac. } & \text { Emergency Capacity } \\ \text { Output } & \text { Rate } & \text { Rate } & \text { Capac. } & \text { vest. } & \text { Stk. } & \text { Capac. } & 3 \text { mon. } 6 \text { mon. }>6 \text { mon. }\end{array}$

\begin{tabular}{|c|c|c|c|c|c|c|c|c|c|c|}
\hline 1974 & 1,374 & $\pi$ & 77.2 & 1,780 & (83.8) & 141 & 2,279 & & & \\
\hline 1975 & 1,165 & 70 & 69.9 & 1,667 & 14.7 & 146 & 2,338 & & & \\
\hline 1976 & 1,394 & $\infty 6$ & 67.6 & 2,063 & 0.5 & 143 & 2,302 & & & \\
\hline 1977 & 1,512 & 73 & 70.3 & 2,150 & 19.0 & 151 & 2,395 & & & \\
\hline 1978 & 1,570 & $\sqrt{5}$ & 74.0 & 2,122 & 11.1 & 151 & 2,395 & & & \\
\hline 1979 & 1,464 & 55 & 62.5 & 2,342 & 4.1 & 144 & 2,317 & & & \\
\hline 1980 & 1,354 & 53 & 53.6 & 2,528 & 3.2 & 137 & 2,231 & & & \\
\hline 1981 & 1,291 & 41 & 45.6 & 2,832 & 13.8 & 139 & 2,255 & & & \\
\hline 1982 & 1,367 & 40 & 40.2 & 3,399 & 43.9 & 167 & 2,573 & & & \\
\hline 1983 & 1,499 & 51 & 46.5 & 3,222 & 2.2 & 154 & 2,429 & & & \\
\hline 1984 & 1,501 & 68 & 61.8 & 2,430 & 38.9 & 176 & 2,674 & & & \\
\hline 1985 & 1,447 & $6 \infty$ & 60.5 & 2,176 & 15.7 & 175 & 2,662 & & & \\
\hline 1986 & 1,453 & 44 & 52.0 & 2,795 & 16.1 & 174 & 2,655 & & & \\
\hline-- & --- & $-\cdot-$ & $\cdots$ & $\cdots$ & $\cdots$ & $-\cdots$ & $\cdots$ & $\cdots$ & $-\cdots$ & $-\cdots$ \\
\hline 1987 & 1,449 & 46 * & 45.1 & 3,211 & 31.5 & 189 & 2,823 & & & \\
\hline 1988 & 1,444 & 36 & 39.8 & 3,628 & 34.5 & 205 & 3,008 & 3,745 & 5,436 & 5,460 \\
\hline 1989 & & & & & 37.5 & 223 & 3,210 & 3,997 & 5,801 & 5,827 \\
\hline 1990 & & & & & 40.5 & 242 & 3,427 & 4,260 & 6,193 & 6,220 \\
\hline 1991 & & & & & 43.5 & 263 & 3,657 & 4,553 & 6,608 & 6,637 \\
\hline 1992 & & & & & 46.6 & 284 & 3,899 & 4,854 & 7,046 & 7,077 \\
\hline
\end{tabular}

Capecity-Capital stock Regression: Code 0 RSO 0.100 Coefficients: Const 679.6 stock 11.3

Ave. Capecity/Stock $(74-86): 15.8$ Ave. Weekly Hours (High) 86.8 Shift Factor 1.82 Ave Weekly Hours (Lou) 82.2 Shift Factor 1.92 * Imputed Note: Output, Investment, and Capital stock extrapolated for 1987 and 1988.

\section{Residential lighting fixtures}

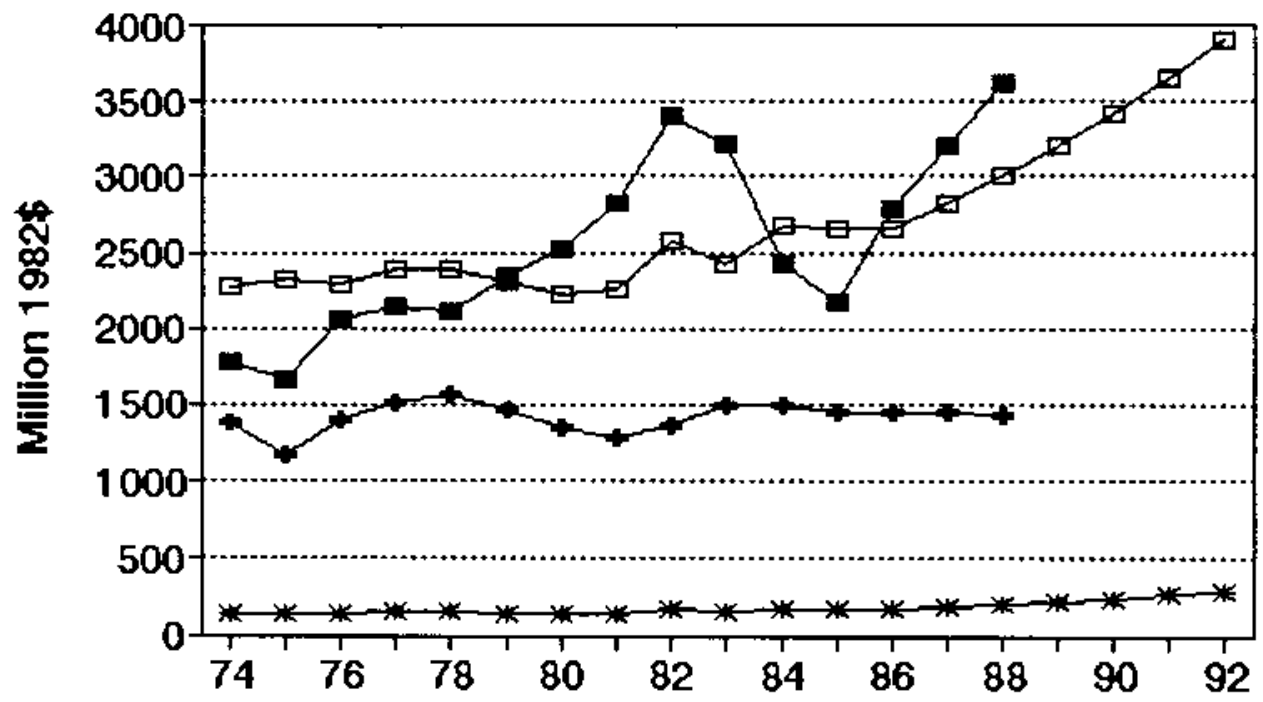

- Oupu $\rightarrow$-Implied Capecity $\rightarrow-$ Cepital Stock $\quad$-Pred Cepecity 


\section{No. 378 sIC 3646 Commexcial lighting fixtures}

MILLIONS OF 1982 DOLLARS (EXCL. CU RATES)

$\begin{array}{lccccccc} & 04 & \text { Ann. } & \text { Implied Gross } & \text { Net } & \text { Pred. } & \ldots \ldots \ldots \\ \text { Gross } & \text { CU } & \text { CU } & \text { Prac. } & \text { In- } & \text { Cap. } & \text { Prac. } & \text { Emergency Capacity } \\ \text { Output } & \text { Rate } & \text { Rate } & \text { Capac. } & \text { vest. } & \text { Stk. } & \text { Capac. } & 3 \text { mon. } 6 \text { mon. }>6 \text { mon. }\end{array}$

\begin{tabular}{|c|c|c|c|c|c|c|c|c|c|c|}
\hline 1974 & 1,493 & $5 t$ & 52.8 & 2,830 & $(7.8)$ & 233 & 2,309 & & & \\
\hline 1975 & 1,237 & 60 & 55.0 & 2,250 & 22.2 & 239 & 2,379 & & & \\
\hline 1976 & 1,667 & 58 & 58.8 & 2,494 & 37.8 & 258 & 2,605 & & & \\
\hline 1977 & 1,640 & 72 & 66.6 & 2,161 & (6.3) & 234 & 2,316 & & & \\
\hline 1978 & 1,565 & 63 & 66.1 & 2,366 & 11.4 & 228 & 2,237 & & & \\
\hline 1979 & 1,748 & 86 & 77.2 & 2,264 & 18.4 & 227 & 2,235 & & & \\
\hline 1980 & 1,728 & 7 & 80.0 & 2,160 & 24.8 & 232 & 2,296 & & & \\
\hline 1981 & 1,702 & 74 & 75.4 & 2,256 & 20.2 & 232 & 2,296 & & & \\
\hline 1982 & 1,713 & 74 & 73.7 & 2,326 & 66.1 & 273 & 2,790 & & & \\
\hline 1983 & 1,779 & 71 & 71.7 & 2,482 & 32.5 & 280 & 2,868 & & & \\
\hline 1984 & 1,928 & 63 & 66.3 & 2,906 & 18.3 & 274 & 2,797 & & & \\
\hline 1985 & 2,151 & 65 & 64.0 & 3,362 & 37.5 & 287 & 2,954 & & & \\
\hline 1986 & 2,366 & 76 & 71.5 & 3,311 & 35.5 & 298 & 3,085 & & & \\
\hline$\cdots$ & $\cdots \cdot$ & 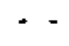 & -- & $\therefore-$ & --- & $-\cdots$ & $\cdots \cdot$ & $\cdots \cdot \cdots$ & $-\cdot-$ & --- \\
\hline 1987 & 2,402 & 74 & 74.5 & 3,222 & 37.4 & 310 & 3,235 & & & \\
\hline 1988 & 2,450 & 66 & 69.1 & 3,544 & 39.0 & 323 & 3,391 & 4,377 & 5,793 & 5,813 \\
\hline 1989 & & & & & 40.7 & 337 & 3,554 & 4,587 & 6,071 & 6,092 \\
\hline 1990 & & & & & 42.3 & 351 & 3,723 & 4,805 & 6,360 & 6,382 \\
\hline 1991 & & & & & 43.9 & 365 & 3,898 & 5,031 & 6,658 & 6,681 \\
\hline 1992 & & & & & 45.6 & 380 & 4,078 & 5,262 & 6,965 & 6,989 \\
\hline
\end{tabular}

Capacity-Capital Stock Regression: Code 0 RSa 0.540

Coefficients: Const $\mathbf{- 5 0 7 . 2}$ stock 12.1

Ave. Capacity/stock (74-86): 10.1 Ave. Weekly Hours (High) 91.9 Shift Factor 1.71 Ave Heekly Hours (LOw) 86.2 Shift Factor 1.83 Note: Output, Investment, and Capital Stock extrapolated for 1987 and 1988.

3646 Commercial lighting fixtures

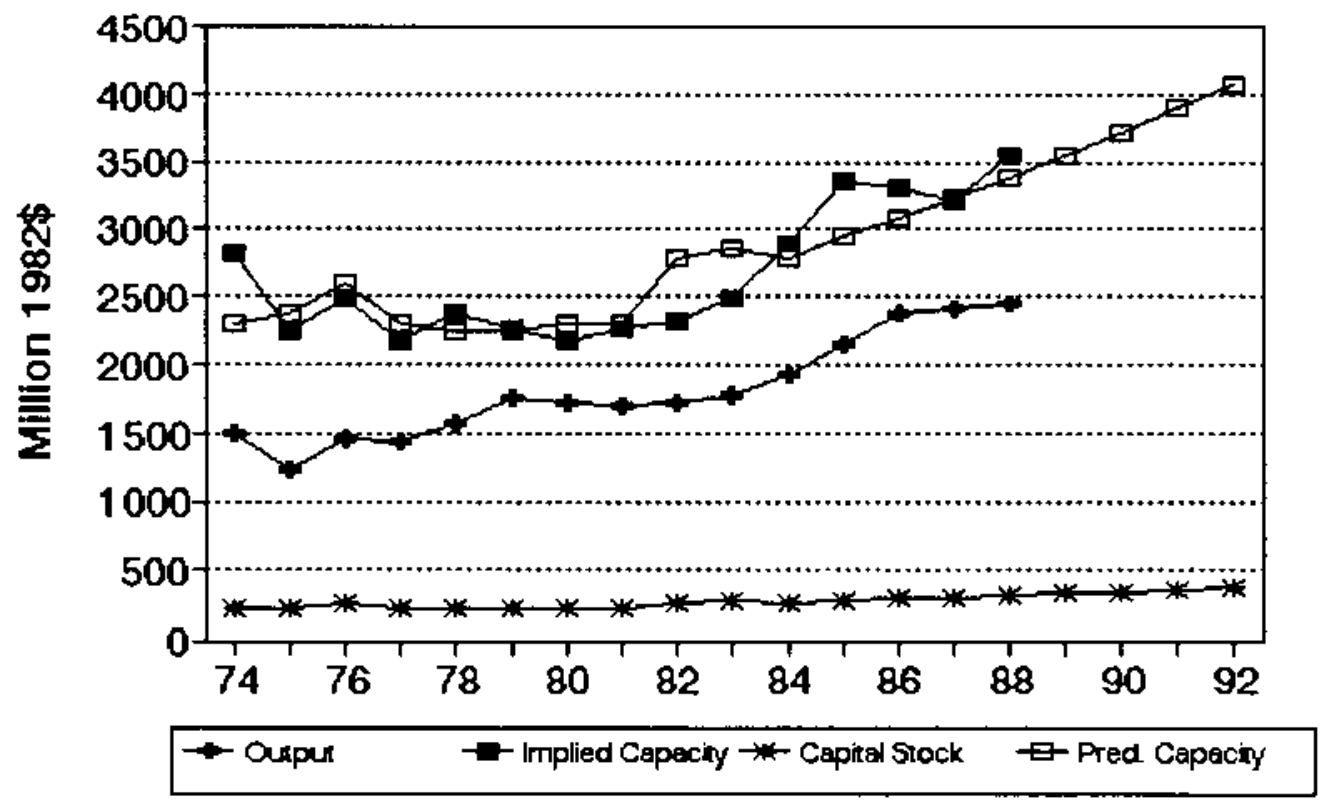




\section{No. 379 sIC 3647 Vehicular lighting equiprent}

MILLIONS OF 1982 DOLLARS (EXCL. CU RATES)

$\begin{array}{lccccccc} & \text { Q4 } & \text { Ann. Implied } & \text { Gross } & \text { Net } & \text { Pred. } & -\ldots \ldots \ldots \\ \text { Gross } & \text { CU } & \text { CU } & \text { Prac. } & \text { In- } & \text { Cap. } & \text { Prac. } & \text { Emergency Capacity } \\ \text { Output Rate } & \text { Rate } & \text { Capac. } & \text { vest. } & \text { Stk. } & \text { Capac. } & 3 \text { mon. } 6 \text { mon. }>6 \text { mon. }\end{array}$

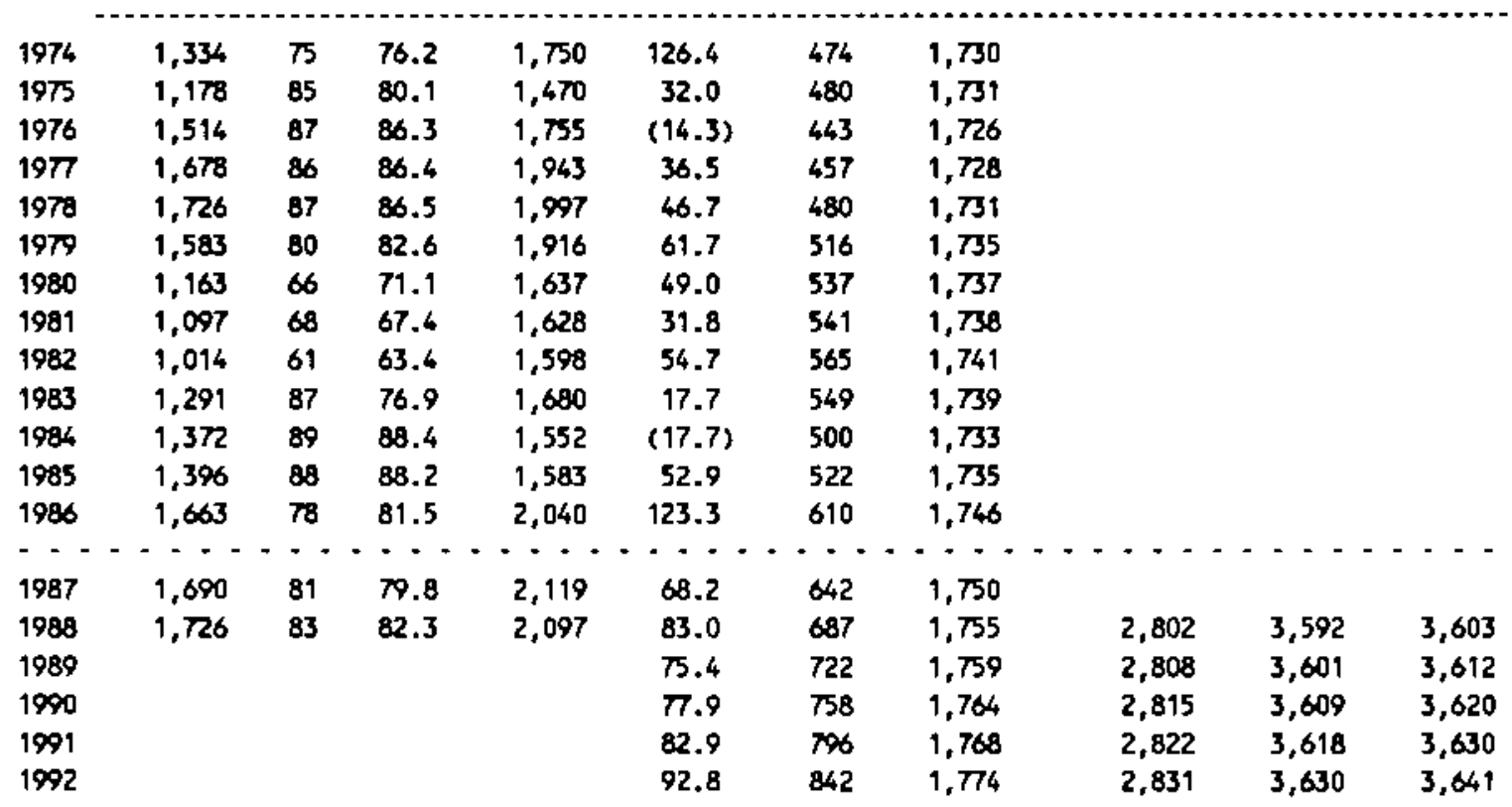

Capacity-Capital Stock Regression: Code 1 RSQ 0.000

Coefficients: Const 1673.5 stock 0.1

Ave. Capacity/Stock $(74-86): 3.4$ Ave. Heekly Hours (High) 76.7 Shift Factor 2.05 Ave Ueekly Hours (Low) $\mathbf{2 . 6}$ Shift Factor 2.17 Note: Output, Investment, and Capital Stock extrapolated for 1987 and 1988.

\section{Vehicular lighting equipment}

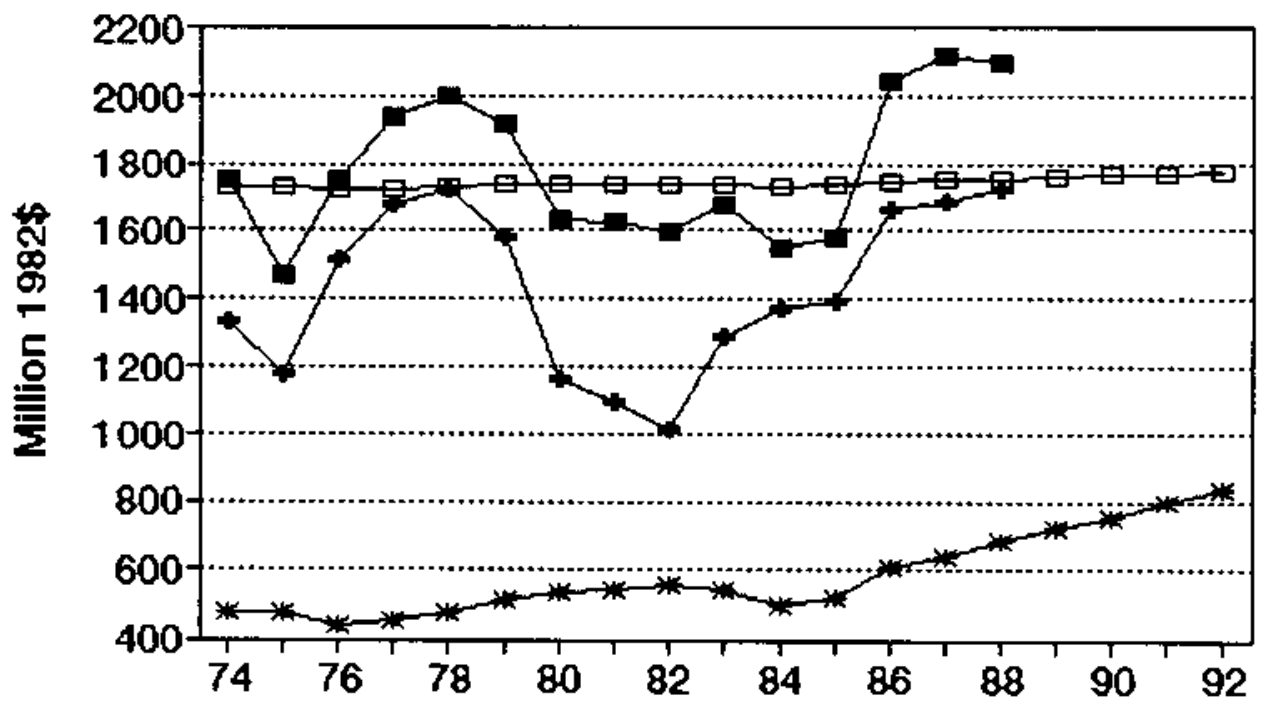

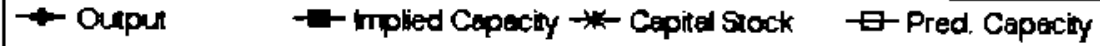


No. 380 sIC 3648 Lighting equipment, nec

MILLIONS OF 1982 DOLLARS (EXCL. CU RATES)

$\begin{array}{lccccccc} & 04 & \text { Ann. } & \text { Implied } & \text { Gross } & \text { Net } & \text { Pred. } & \ldots \\ \text { Gross } & \text { CU } & \text { CU } & \text { Prac. } & \text { In- } & \text { Cap. } & \text { Prac. } & \text { Emergency Capacity } \\ \text { Output } & \text { Rate } & \text { Rate } & \text { Capac. } & \text { vest. } & \text { Stk. } & \text { Capac. } & 3 \text { mon. } 6 \text { mon. }>6 \text { mon. }\end{array}$

\begin{tabular}{|c|c|c|c|c|c|c|c|c|c|c|}
\hline 1974 & 1,040 & 49 & 51.3 & 2,030 & 14.4 & 215 & 1,679 & & & \\
\hline 1975 & 809 & 48 & 46.3 & 1,747 & 2.3 & 206 & 1,579 & & & \\
\hline 1976 & 702 & 65 & 58.5 & 1,201 & $(26.8)$ & 170 & 1,190 & & & \\
\hline 1977 & 1,085 & 88 & 79.2 & 1,371 & 69.1 & 225 & 1,786 & & & \\
\hline 1978 & 1,113 & 77 & 80.7 & 1,379 & 12.3 & 224 & 1,770 & & & \\
\hline 1979 & 1,224 & 56 & 63.9 & 1,916 & 47.5 & 255 & 2,101 & & & \\
\hline 1980 & 1,255 & 48 & 50.7 & 2,477 & 30.9 & 267 & 2,234 & & & \\
\hline 1981 & 1,254 & 48 & 48.3 & 2,597 & 24.3 & 272 & 2,285 & & & \\
\hline 1982 & 1,010 & 52 & 50.3 & 2,008 & $(13.4)$ & 241 & 1,951 & & & \\
\hline 1983 & 979 & 54 & 52.8 & 1,855 & 7.9 & 231 & 1,842 & & & \\
\hline 1984 & 1,212 & $58 *$ & 56.8 & 2,133 & 32.6 & 244 & 1,982 & & & \\
\hline 1985 & 1,302 & 64 & 61.5 & 2,118 & 55.3 & 277 & 2,340 & & & \\
\hline 1986 & 1,363 & 60 & 61.0 & 2,235 & 21.7 & 273 & 2,326 & & & \\
\hline $\begin{array}{l}\cdots \\
1987\end{array}$ & $\cdots$ & $\begin{array}{l}\cdots \\
71\end{array}$ & 66.6 & 2,079 & $\begin{array}{l}--- \\
33.2\end{array}$ & 286 & $\begin{array}{l}\cdots \\
2,442\end{array}$ & $-\cdots$ & -- & -- \\
\hline 1988 & 1,412 & 68 & 69.3 & 2,038 & 42.1 & 305 & 2,645 & 3,652 & 5,298 & 5,321 \\
\hline 1989 & & & & & 37.6 & 318 & 2,782 & 3,841 & 5,572 & 5,597 \\
\hline 1990 & & & & & 39.1 & 331 & 2,924 & 4,037 & 5,856 & 5,882 \\
\hline 1991 & & & & & 42.1 & 346 & 3,086 & 4,261 & 6,182 & 6,209 \\
\hline 1992 & & & & & 48.0 & 366 & 3,300 & 4.556 & 6,609 & 6,639 \\
\hline
\end{tabular}

Capacity-Capital Stock Regression: Code 0 RSO 0.640

Coefficients: Const -646.6 stock 10.8

Ave. Capacity/Stock $(74-86): 8.1$

Ave. Weekly Hours (High) 78.3 Shift Factor 2.01 Ave Weekly Hours (Low) 70.3 Shift Factor 2.24

* Imputed Hote: Output, Investment, and Capital Stock extrapolated for 1987 and 1988.

\section{Lighting equipment, nec}

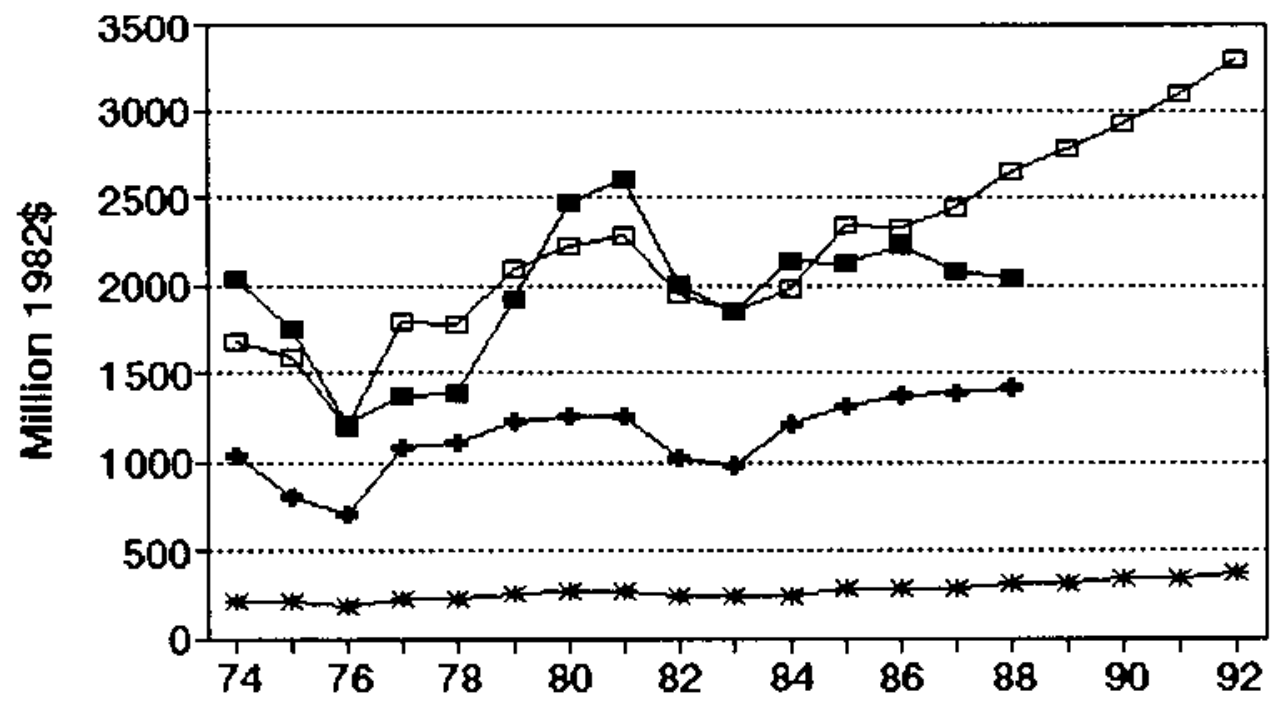

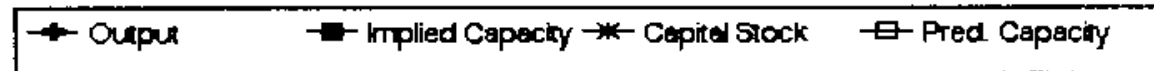




\section{No. 381 sIc 3651 Radio and TV receiving sets}

MILLIONS OF 1982 DOLLARS (EXCL. CU RATES)

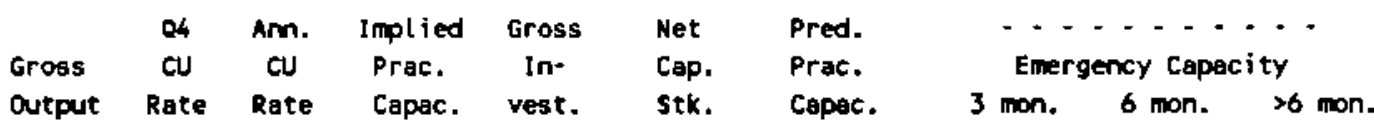

\begin{tabular}{|c|c|c|c|c|c|c|c|c|c|c|}
\hline 1974 & 4,778 & 63 & 64.7 & 7,384 & 91.3 & 625 & 7,646 & & & \\
\hline 1975 & 4,173 & 52 & 54.6 & 7,647 & 63.7 & 637 & 7,781 & & & \\
\hline 1976 & 5,047 & 57 & 55.1 & 9,158 & 65.3 & 649 & 7,920 & & & \\
\hline 1977 & 6,043 & 67 & 63.2 & 9,564 & 132.3 & 722 & 8,736 & & & \\
\hline 1978 & 6,770 & 65 & 65.5 & 10,330 & 147.3 & 803 & 9,649 & & & \\
\hline 1979 & 6,625 & 58 & 60.6 & 10,932 & 155.5 & 888 & 10.613 & & & \\
\hline 1980 & 6,760 & 66 & 62.8 & 10,760 & 124.7 & 939 & 11,193 & & & \\
\hline 1981 & 7,011 & 70 & 68.6 & 10,196 & 135.1 & 998 & 11,850 & & & \\
\hline 1982 & 5,863 & 59 & 62.8 & 9,340 & $(0.7)$ & 925 & 11,029 & & & \\
\hline 1983 & 6,846 & 74 & 67.9 & 10,086 & $\pi .9$ & 925 & 11,032 & & & . \\
\hline 1984 & 8,885 & $\pi$ & 74.9 & 11,865 & 196.8 & 1,042 & 12,351 & & & \\
\hline 1985 & 9,683 & $\pi$ & 72.8 & 13,295 & 164.3 & 1,122 & 13,245 & & & \\
\hline 1986 & 10,704 & 61 & 64.8 & 16,516 & 159.5 & 1,191 & 14,027 & & & \\
\hline-- & & $\cdots$ & $\cdots$ & $\cdots \cdot$ & $\cdots$ & $\cdots$ & $\cdots \cdot$ & $\cdots \cdot \cdots$ & $\cdots \cdot$ & $=-\cdot$ \\
\hline 1987 & 10,974 & 67 & 64.6 & 16,988 & 183.8 & 1,284 & 15,082 & & & \\
\hline 1988 & 11,334 & 67 & 67.1 & 16,897 & 153.3 & 1,340 & 15,713 & 20,289 & 26,869 & 26,963 \\
\hline 1989 & & & & & 137.8 & 1,377 & 16,121 & 20,817 & 27,568 & 27,664 \\
\hline 1990 & & & & & 106.0 & 1,378 & 16,141 & 20,842 & 27,601 & 27,697 \\
\hline 1991 & & & & & 84.9 & 1,359 & 15,920 & 20,557 & 27,223 & 27,318 \\
\hline 1992 & & & & & 74.3 & 1,330 & 15,596 & 20,139 & 26,670 & 26,764 \\
\hline
\end{tabular}

Capecity-Capital stock Regression: Code 0 2S0 0.770

Coefficients: Const 602.1 stock 11.3

Ave. Capecity/Stock (74-86): 12.0

Ave. Weekly Hours (High) 91.8 Shift Factor 1.72 Ave Weekly Hours (Low) 89.3 Shift factor 1.76 Note: Output, Investment, and Capital Stock extrapolated for 1987 and 1988.

\section{Radio and TV receiving sets}

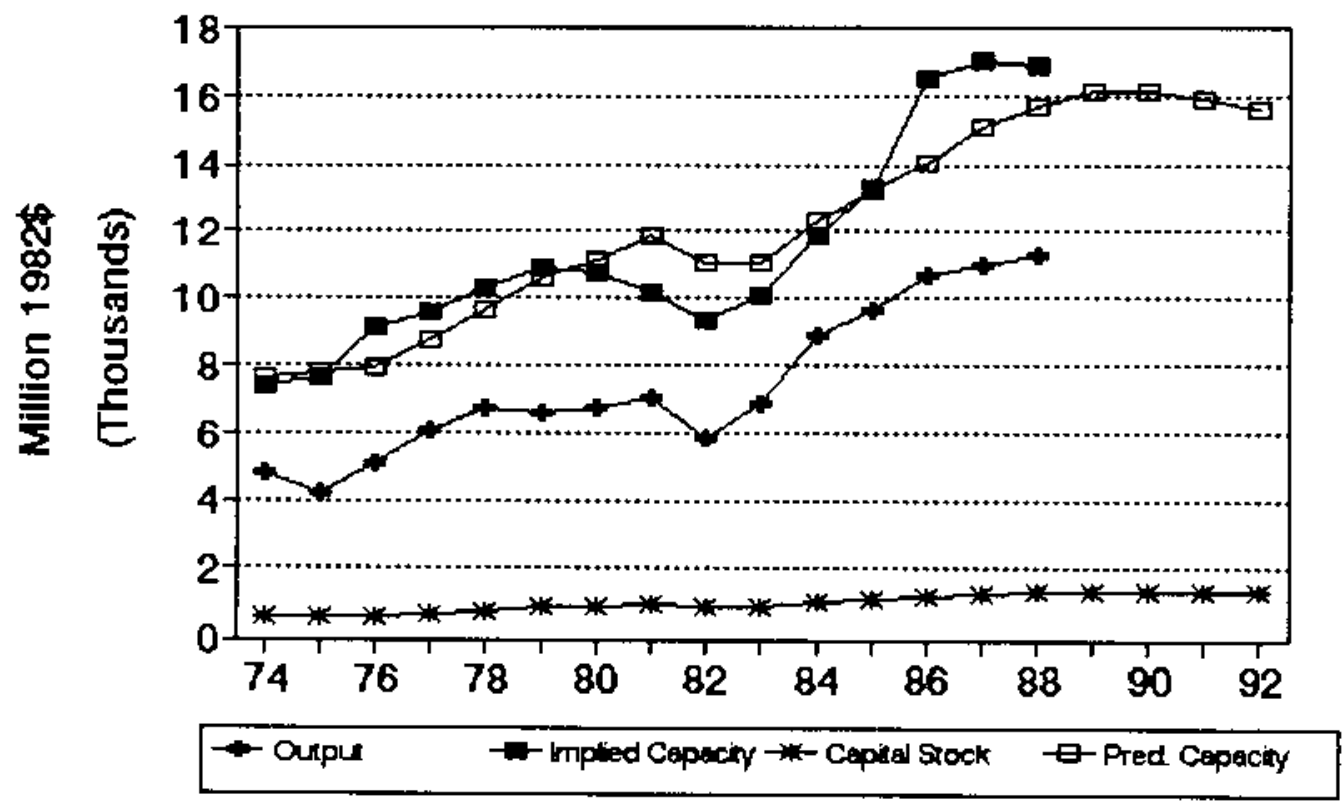


No. 382 SIC 3652 Phonograph recorda

HILLIONS OF 1982 DOLLARS (EXCL. CU RATES)

$\begin{array}{lccccccc} & \text { Q4 } & \text { Amn. Implied Gross } & \text { Net } & \text { Pred. } & -\ldots \ldots \ldots \\ \text { Gross } & \text { CU } & \text { CU } & \text { Prac. } & \text { In- } & \text { Cap. } & \text { Prac. } & \text { Emergency Capacity } \\ \text { Output } & \text { Rate } & \text { Rate } & \text { Capac. } & \text { yest. } & \text { Stk. } & \text { Capac. } & 3 \text { mon. } 6 \text { mon. }>6 \text { mon. }\end{array}$

\begin{tabular}{|c|c|c|c|c|c|c|c|c|c|c|}
\hline 1974 & 960 & 84 & 84.9 & 1,131 & 23.1 & 233 & 1,450 & & & \\
\hline 1975 & 969 & 83 & 82.6 & 1,174 & 6.2 & 225 & 1,346 & & & \\
\hline 1976 & 1,104 & 72 & 76.2 & 1,450 & 7.9 & 219 & 1,266 & & & \\
\hline 1977 & 1,715 & 109 & 95.0 & 1.805 & 45.5 & 248 & 1,639 & & & \\
\hline 1978 & 2,059 & 95 & 100.0 & 2,059 & 62.0 & 292 & 2,202 & & & \\
\hline 1979 & 1,862 & $\pi$ & 82.5 & 2,257 & 88.0 & 357 & 3,031 & & & \\
\hline 1980 & 1,954 & 59 & 64.9 & 3,011 & 36.9 & 369 & 3,191 & & & \\
\hline 1981 & 1,781 & 53 & 55.3 & 3,220 & $(17.7)$ & 329 & 2,670 & & & \\
\hline 1982 & 1,736 & 86 & 73.6 & 2,357 & 29.7 & 334 & 2,745 & & & \\
\hline 1983 & 2,105 & 94 & 90.8 & 2,319 & $(4.5)$ & 306 & 2,387 & & & \\
\hline 1984 & 1,667 & 86 & 89.1 & 1,870 & 36.4 & 317 & 2,516 & & & \\
\hline 1985 & 2,451 & 54 & 65.8 & 3,725 & 30.8 & 320 & 2,561 & & & \\
\hline 1986 & 2,509 & $81 *$ & 70.8 & 3,542 & 55.5 & 347 & 2,905 & & & \\
\hline 1987 & $\begin{array}{l}-\cdots \\
2,554\end{array}$ & $74 *$ & $\begin{array}{l}\cdots \\
76.9\end{array}$ & $\cdots$ & 46.8 & 365 & $\cdots$ & -- & -- & $\cdots$ \\
\hline 1988 & 2,614 & 83 & 79.7 & 3,278 & 39.6 & 374 & 3,247 & 2,977 & 3,243 & 3,247 \\
\hline 1989 & & & & & 36.0 & 378 & 3,306 & 3,031 & 3,303 & 3,306 \\
\hline 1990 & & & & & 28.5 & 375 & 3,265 & 2,994 & 3,262 & 3,265 \\
\hline 1991 & & & & & 23.5 & 367 & 3,164 & 2,901 & 3,160 & 3,164 \\
\hline 1992 & & & & & 21.0 & 357 & 3,039 & 2,786 & 3,036 & 3,039 \\
\hline
\end{tabular}

Capecity-Capital stock Regression: Code 1 RSO 0.600

Coefficients: Const -1526.0 stock 12.8

Ave. Capacity/Stock $(74-86): 7.7$ Ave. Weekly Hours (High) 1985.0 Shift factor 1.00 Ave Weekly Hours (Low) 1985.0 Shift factor 1.0 - Imputed Wote: Cutput, Imvestment, and Capital Stock extrapolated for 1987 and 1988.

\section{Phonograph records}

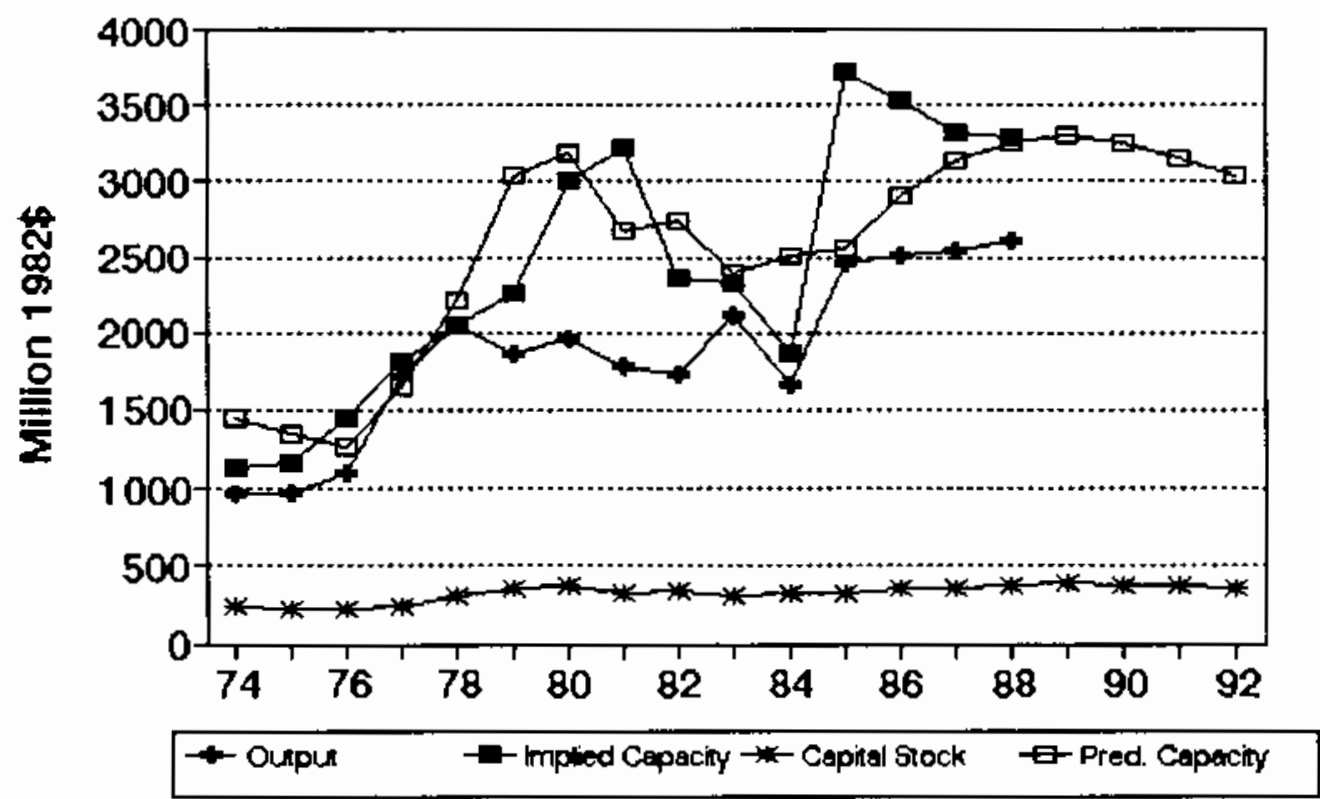


Ho. 383 sIC 3661 Telephone and tolegraph apparatus

MILLIOWS OF 1982 DOLLARS (EXCL. CU RATES)

$\begin{array}{lccccccc} & \text { Q4 } & \text { Amn. Implied Gross } & \text { Net } & \text { Pred. } & \ldots \ldots \ldots \ldots \\ \text { Gross } & \text { CU } & \text { CU } & \text { Prac. } & \text { In- } & \text { Cap. } & \text { Prac. } & \text { Emergency Capacity } \\ \text { Output } & \text { Rate } & \text { Rate } & \text { Capac. } & \text { vest. } & \text { Stk. } & \text { Capac. } & 3 \text { mon. } 6 \text { mon. }>6 \text { mon. }\end{array}$

\begin{tabular}{|c|c|c|c|c|c|c|c|c|c|c|}
\hline 1974 & 10,190 & 76 & 79.6 & 12,805 & 312.3 & 2,540 & 13,199 & & & \\
\hline 1975 & 7,370 & 62 & 61.4 & 12,012 & 173.5 & 2,533 & 13,112 & & & \\
\hline 1976 & 7,093 & 67 & 65.3 & 10,868 & $(84.2)$ & 2,283 & 10,101 & & & \\
\hline 1977 & 9,583 & 83 & 76.8 & 12,476 & 336.6 & 2,438 & 11,965 & & & \\
\hline 1978 & 10,319 & 77 & 78.6 & 13,122 & 316.4 & 2,559 & 13,625 & & & \\
\hline 1979 & 12,744 & 90 & 84.9 & 15,017 & 420.8 & 2,769 & 15,958 & & & \\
\hline 1980 & 13,521 & 77 & 81.1 & 16,676 & 461.7 & 2,987 & 18,581 & & & \\
\hline 1981 & 13,628 & 67 & 71.3 & 19,109 & 320.2 & 3,077 & 19,663 & & & \\
\hline 1982 & 13,585 & 59 & 61.3 & 22,161 & 217.8 & 3,065 & 19,521 & & & \\
\hline 1983 & 13,302 & 60 & 58.9 & 22,593 & 361.0 & 3,187 & 20,987 & & & \\
\hline 1984 & 15,655 & $\infty$ & 64.3 & 24,345 & 440.7 & 3,383 & 23,354 & & & \\
\hline 1985 & 16,153 & 62 & 62.9 & 25,689 & 245.9 & 3,382 & 23,345 & & & \\
\hline 1986 & 13,921 & 68 & 64.9 & 21,458 & 408.2 & 3,530 & 25,118 & & & \\
\hline$\cdots$ & $\cdot-\cdot$ & -- & -- & $-\cdots$ & $\cdots \cdot$ & 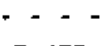 & $-\cdot-$ & $\cdots$ & --- & $\cdots$ \\
\hline 1987 & 14,098 & 61 & 63.3 & 22,281 & 364.4 & 3,635 & 26,386 & & & \\
\hline 1988 & 14,332 & 56 & 58.1 & 24,675 & 378.8 & 3,747 & 27,734 & 27,955 & 37,964 & 38,107 \\
\hline 1989 & & & & & 372.8 & 3,844 & 28,911 & 29,141 & 39,575 & 39,723 \\
\hline 1990 & & & & & 351.8 & 3,914 & 29,748 & 29,984 & 40,720 & 40,873 \\
\hline 1991 & & & & & 398.3 & 4,025 & 31,083 & 31,330 & 42,548 & 42,708 \\
\hline 1992 & & & & & 350.4 & 4,080 & 31,744 & 31,997 & 43,453 & 43,617 \\
\hline
\end{tabular}

Capacity-Capital stock Regression: Code 1 RSQ 0.890

Coefficients: Const -17394.3 stock 12.1

Ave. Capecity/Stock $(74-86): 6.1$ Ave. Weekly Hours (High) 114.8 Shift Factor 1.37 Ave Yeekly Hours (Low) 112.1 Shift Factor 1.41 Note: Output, Investment, and Capital Stock extrapolated for 1987 and 1988.

3661 Telephone and telegraph apparatus

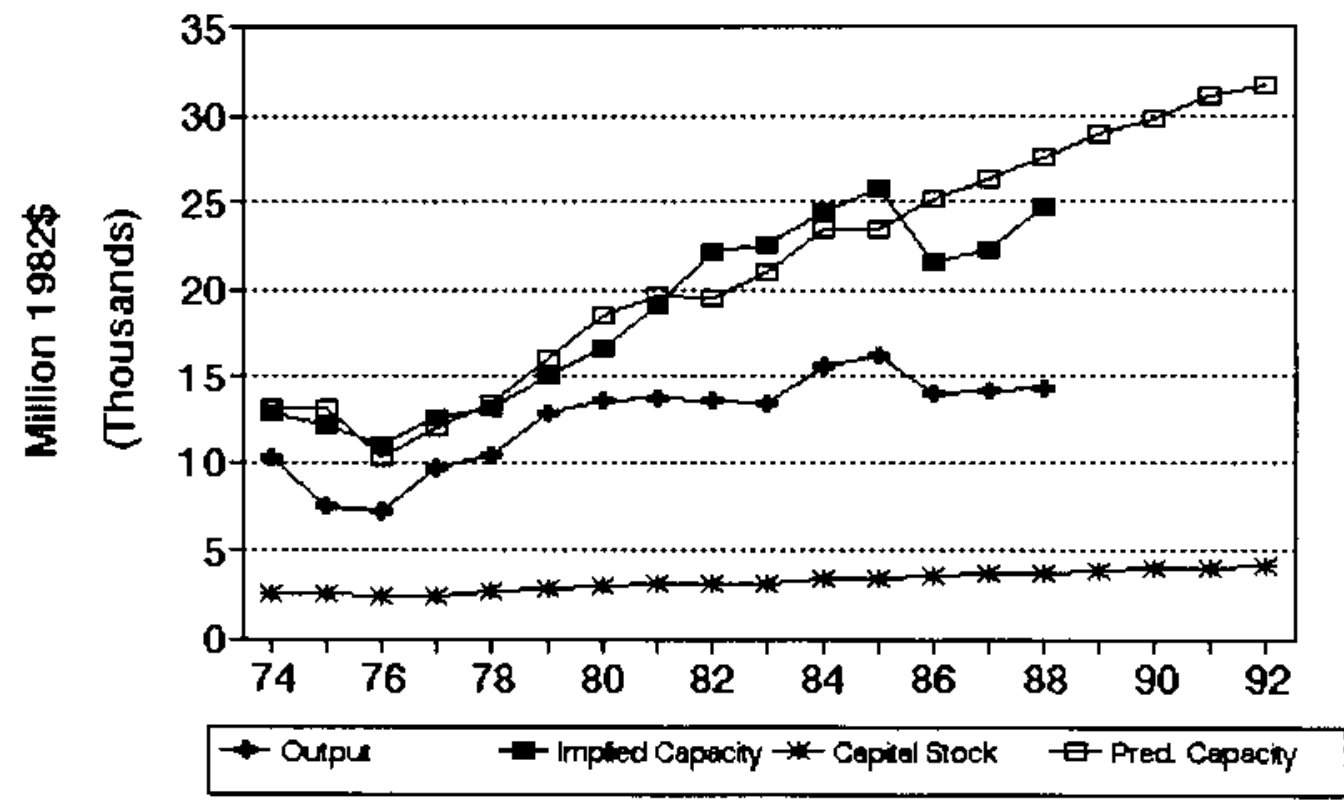


No. 384 sIC 3662 Radio and TV communication equipment

MILLIONS OF 1982 DOLLARS (EXCL. CU RATES)

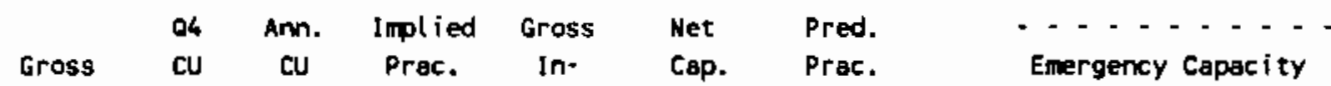

Output Rate Rate Capac. vest. StK. Capac. 3 mon. 6 mon. $>6$ mon.

\begin{tabular}{|c|c|c|c|c|c|c|c|c|c|c|}
\hline 1976 & 15,882 & 54 & 54.8 & 28,991 & 91.3 & 2,807 & 32,213 & & & \\
\hline 1973 & 16,646 & 53 & 52.7 & 31,614 & 430.5 & 2,989 & 33,052 & & & \\
\hline 1976 & 17,548 & 56 & 56.9 & 31,979 & 371.9 & 3,100 & 33,565 & & & \\
\hline 1977 & 19,406 & 61 & 59.1 & 32,834 & 563.0 & 3,382 & 34,867 & & & \\
\hline 1978 & 22,050 & 66 & 64.0 & 34,653 & 576.3 & 3,665 & 36,169 & & & \\
\hline 1979 & 25,472 & 64 & 64.7 & 39,355 & 756.2 & 4,103 & 38,195 & & & \\
\hline 1980 & 28,948 & 66 & 65.1 & 44,445 & 756.9 & 4,530 & 40,161 & & & \\
\hline 1981 & 30,227 & 69 & 68.0 & 44,445 & 809.8 & 4.991 & 42,290 & & & \\
\hline 1982 & 33,579 & 67 & 67.6 & 49,678 & $1,332.7$ & 5,929 & 46,619 & & & \\
\hline 1983 & 34,439 & 63 & 64.3 & 53,538 & 1.275 .6 & $6, \pi 75$ & 50,525 & & & \\
\hline 1984 & 38,700 & 70 & 67.5 & 57,346 & $1,561.4$ & 7,871 & 55,581 & & & \\
\hline 1985 & 44,309 & 74 & 72.4 & 61,230 & $1,975.0$ & 9,322 & 62,281 & & & \\
\hline 1986 & 46,759 & 74 & 73.8 & 63,350 & $1,774.3$ & 10,506 & 67,743 & & & \\
\hline$-\cdots$ & $-\cdots$ & $\because$ & $\cdots$ & $-\cdot-$ & $\cdots$ & $\cdots \cdot$ & $-\cdots$ & $-\cdots \cdot$ & $-\cdot-$ & $---\cdot$ \\
\hline 1987 & 47,731 & 71 & 72.0 & 66,255 & 735.7 & 10,651 & 68,413 & & & \\
\hline 1988 & 49,024 & 72 & 71.7 & 68,403 & $1,262.3$ & 11,315 & 71,477 & 86,259 & 113,616 & 114,006 \\
\hline 1989 & & & & & $1,711.8$ & 12,391 & 76.443 & 92,251 & 121,510 & 121,927 \\
\hline 1990 & & & & & $1,800.3$ & 13,495 & 81,539 & 98,401 & 129,609 & 130,054 \\
\hline 1991 & & & & & $2,066.1$ & 14,803 & 87,575 & 105,685 & 139,204 & 139,681 \\
\hline 1992 & & & & & $1,800.3$ & 15,771 & 92,045 & 111,080 & 146,309 & 146,811 \\
\hline
\end{tabular}

Capacity-Capital Stock Regression: Code 0 RSO 0.950

Coefficients: Const 19256.5 5tack 4.6

Ave. Capacity/Stock $(74-86): 8.2$

Ave. Weekly Hours (High) 98.7 Shift Factor 1.60 Ave Weekly Hours (LOH) 97.1 Shift Factor 1.62

Note: Output, Imvestment, and Capital stock extrapolated for 1987 and 1988.

3662 Radio and TV communication equipme

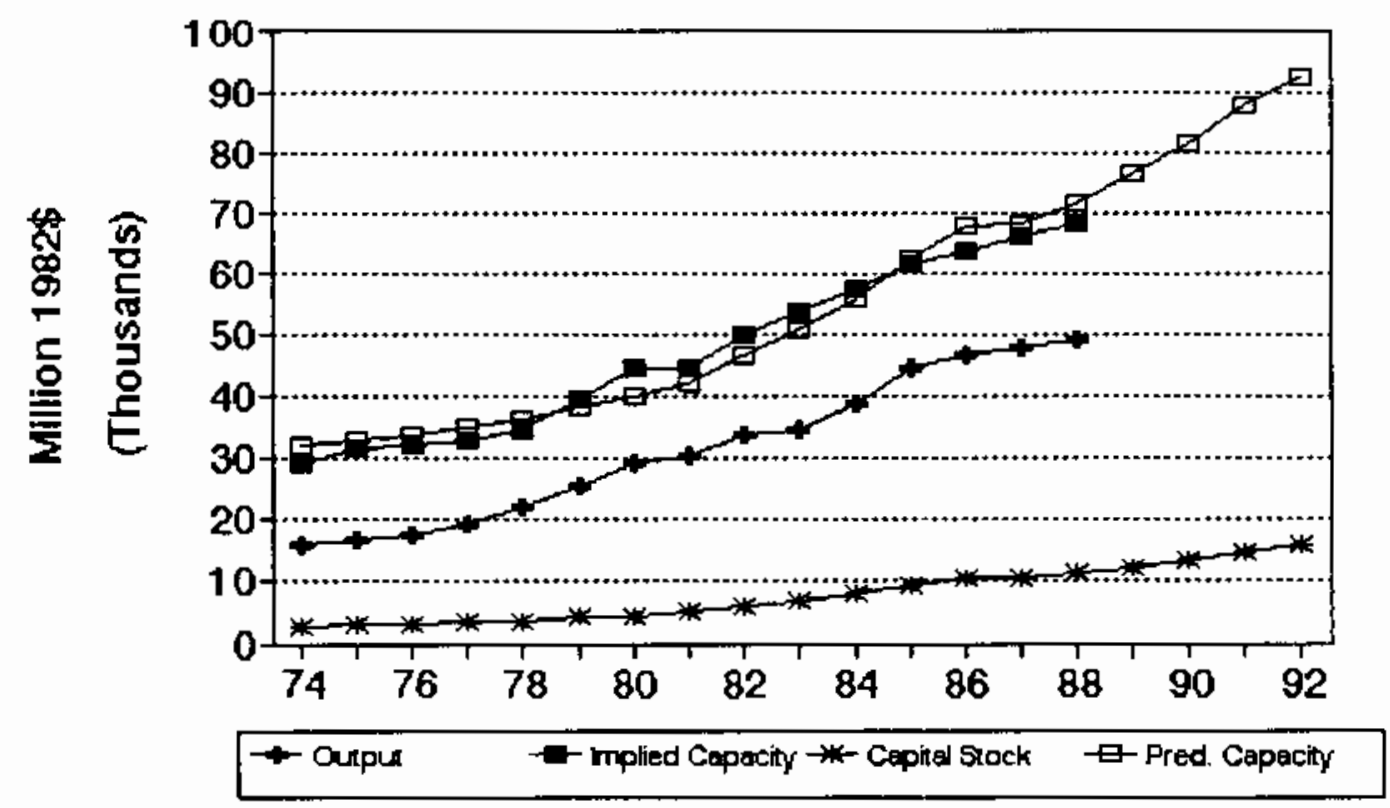


No. 385 sIC 3671 Electron tubes, all types

MILLIONS OF 1982 DOLLARS (EXCL. CU RATES)

\begin{tabular}{|c|c|c|c|c|c|c|c|}
\hline & Q4 & An. & $\begin{array}{l}\text { Implied } \\
\text { Prac. }\end{array}$ & $\begin{array}{l}\text { Gross } \\
\text { In- }\end{array}$ & $\begin{array}{l}\text { Net } \\
\text { Cap. }\end{array}$ & $\begin{array}{l}\text { Pred. } \\
\text { Prac. }\end{array}$ & Emergency Capacity \\
\hline & Rate & Rate & Capac. & yest. & stk. & Canac. & \\
\hline
\end{tabular}

\begin{tabular}{|c|c|c|c|c|c|c|c|c|c|c|}
\hline 1974 & 2,399 & 30 & 30.1 & 7,959 & 60.9 & 917 & 5,165 & & & \\
\hline 1975 & 2,157 & 72 & 56.1 & 3,844 & 81.5 & 912 & 5,125 & & & \\
\hline 1976 & 2,375 & 71 & 71.4 & 3,327 & 57.6 & 882 & 4,894 & & & \\
\hline 1977 & 2,523 & 69 & 69.8 & 3,617 & (5.5) & 794 & 4,223 & & & \\
\hline 1978 & 2,500 & $\pi$ & 72.7 & 3,439 & 98.9 & 806 & 4,315 & & & \\
\hline 1979 & 2,535 & 69 & 71.2 & 3,559 & 35.9 & 759 & 3,954 & & & \\
\hline 1980 & 5,133 & 68 & 68.3 & 7,511 & 56.6 & 734 & 3,765 & & & \\
\hline 1981 & 2,267 & 69 & 68.7 & 3,302 & 6.3 & 668 & 3,257 & & & \\
\hline 1982 & 2,340 & 71 & 70.2 & 3,333 & 97.7 & 691 & 3,428 & & & \\
\hline 1983 & 2,360 & 84 & 79.0 & 2,987 & 22.8 & 645 & 3,083 & & & \\
\hline 1984 & 2,340 & 82 & 82.8 & 2,826 & 84.9 & 665 & 3,229 & & & \\
\hline 1985 & 2,331 & 75 & 77.6 & 3,005 & 111.6 & 711 & 3,587 & & & \\
\hline 1986 & 2,434 & 84 & 80.6 & 3,021 & 79.7 & 727 & 3,704 & & & \\
\hline$-\cdots$ & $\cdots$ & $\cdots$ & $\cdots$ & $\cdots$ & $\cdots$ & $\cdots$ & $\cdots$ & $\cdots \cdot$ & $-\cdots$ & $-\cdot-$ \\
\hline 1987 & 2,450 & 95 & 90.8 & 2,697 & 66.2 & 727 & 3,711 & & & \\
\hline 1988 & 2,471 & 95 & 95.0 & 2,601 & 66.2 & 728 & 3,716 & 4,085 & 5,271 & 5,288 \\
\hline 1989 & & & & & 66.2 & 729 & 3,722 & 4,091 & 5,279 & 5,296 \\
\hline 1990 & & & & & 66.2 & 729 & 3,726 & 4,097 & 5,286 & 5,303 \\
\hline 1991 & & & & & 66.2 & 730 & 3,731 & 4,102 & 5,292 & 5,309 \\
\hline 1992 & & & & & 66.2 & 731 & 3,735 & 4,106 & 5,298 & 5,315 \\
\hline
\end{tabular}

Capacity-Capital Stock Regression: Code 0 RSQ 0.180

Coefficients: Const -1864.4 stock 7.7

Ave. Capacity/Stock $(74-86): 5.2$

Ave. Weekly Hours (High) 110.7 Shift Factor 1.42 Ave Heekly Hours (LOW) 101.6 Shift Factor 1.55 Note: Output, Investment, and Capital stock extrapolated for 1987 and 1988.

3671 Electron tubes, all types

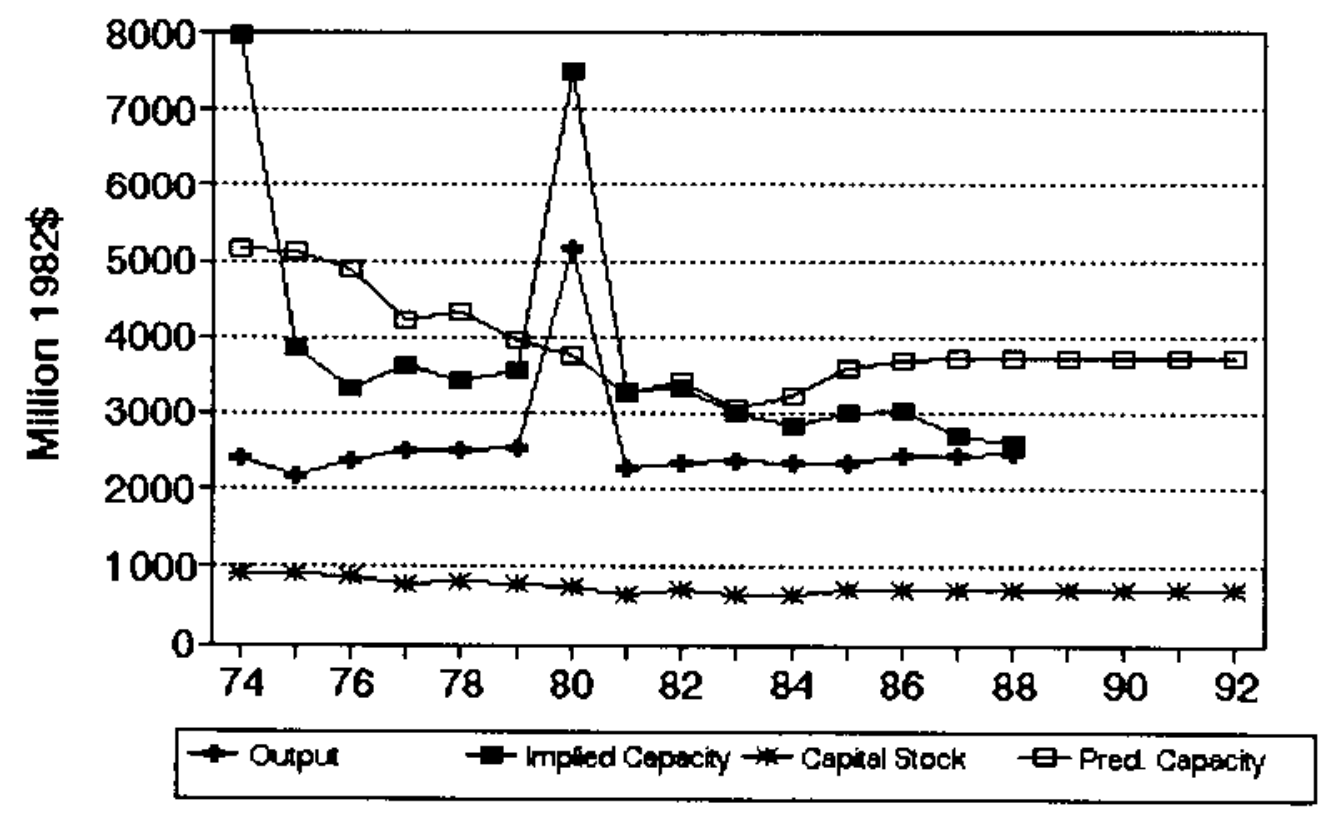




\section{No. 386 SIC 3674 semiconductors and related devices}

MILLIONS OF 1982 DOLLARS (EXCL. CU RATES)

$\begin{array}{lccccccc} & \text { Q4 } & \text { Am. Implied } & \text { Gross } & \text { Net } & \text { Pred. } & \ldots & \ldots \\ \text { Gross } & \text { CU } & \text { CU } & \text { Prac. } & \text { In- } & \text { Cap. } & \text { Prac. } & \text { Emergency Capacity } \\ \text { Output } & \text { Rate } & \text { Rate } & \text { Capac. } & \text { vest. } & \text { Stk. } & \text { Capac. } & 3 \text { mon. } 6 \text { mon. }>6 \text { mon. }\end{array}$

\begin{tabular}{|c|c|c|c|c|c|c|c|c|c|c|}
\hline 1974 & 3,569 & 64 & 67.8 & 5,264 & 748.2 & 2,983 & 5,594 & & & \\
\hline 1975 & 2,349 & 63 & 59.8 & 3,926 & 215.9 & 3,035 & 5,725 & & & \\
\hline 1976 & 3,485 & 63 & 63.2 & 5,517 & 213.4 & 3,080 & 5,840 & & & \\
\hline 1977 & 4,524 & 74 & 69.8 & 6,484 & 455.1 & 3,345 & 6,514 & & & \\
\hline 1978 & 6,111 & 82 & 78.4 & 7,793 & 627.1 & 3,761 & 7,569 & & & \\
\hline 1979 & 8,252 & 83 & 82.5 & 10,004 & 803.2 & 4,322 & 8,995 & & & \\
\hline 1980 & $9, \pi 73$ & 82 & 81.8 & 11,943 & $1,426,4$ & 5,467 & 11,901 & & & \\
\hline 1981 & 11,067 & 76 & 78.8 & 14,045 & $1,116.7$ & 6,254 & 13,900 & & & \\
\hline 1982 & 12,632 & 72 & 72.9 & 17,324 & $1,118,0$ & 6,998 & 15,788 & & & \\
\hline 1983 & 13,768 & $\pi$ & 73.1 & 18,845 & $1,275.1$ & 7,851 & 17,955 & & & \\
\hline 1984 & 17,489 & 76 & 76.2 & 22,952 & $1,982.3$ & 9,356 & $21, \pi 7$ & & & \\
\hline 1985 & 15,595 & 62 & 66.5 & 23,436 & $1,457.3$ & 10,283 & 24,130 & & & \\
\hline 1986 & 15,321 & 65 & 63.2 & 24,241 & $1,378.6$ & 11,054 & 26,086 & & & \\
\hline$\cdots$ & $--\cdot$ & - & -- & $-\cdot-$ & --- & $-\cdot-$ & $\cdots$ & $=--$ & --- & -- \\
\hline 1987 & 16,481 & 71 & 68.4 & 24,097 & 810.9 & 11,275 & 26,649 & & & \\
\hline 1988 & 18,107 & 71 & 71.2 & 25,438 & $1,326.2$ & 12,000 & 28,491 & 30,081 & 38,878 & 39,004 \\
\hline 1989 & & & & & $1,766.1$ & 13,127 & $3 t, 351$ & 33,101 & 42,781 & 42,919 \\
\hline 1990 & & & & & $1,852.7$ & 14,280 & 34,279 & 36,192 & 46,777 & 46.927 \\
\hline 1991 & & & & & $2,112.8$ & 15,632 & 37.711 & 39.815 & 51,460 & 51,626 \\
\hline 1992 & & & & & $1,852.7$ & 16,651 & 40,299 & 42,549 & 54,992 & 55,170 \\
\hline
\end{tabular}

Capacity-Capital Stock Regression: Code 0 RSO 0.980 Coefficients: Const -1979.8 Stock 2.5

Ave. Capacity/Stock $(74-86): 2.2$ Ave. Heekly Hours (High) 115.2 Shift Factor 1.37 Ave Heekly Hours (Low) 109.5 Shift Factor 1.44 Note: Output, Investment, and Capital Stock extrapolated for 1987 and 1988.

\section{Semiconductors and related devices}

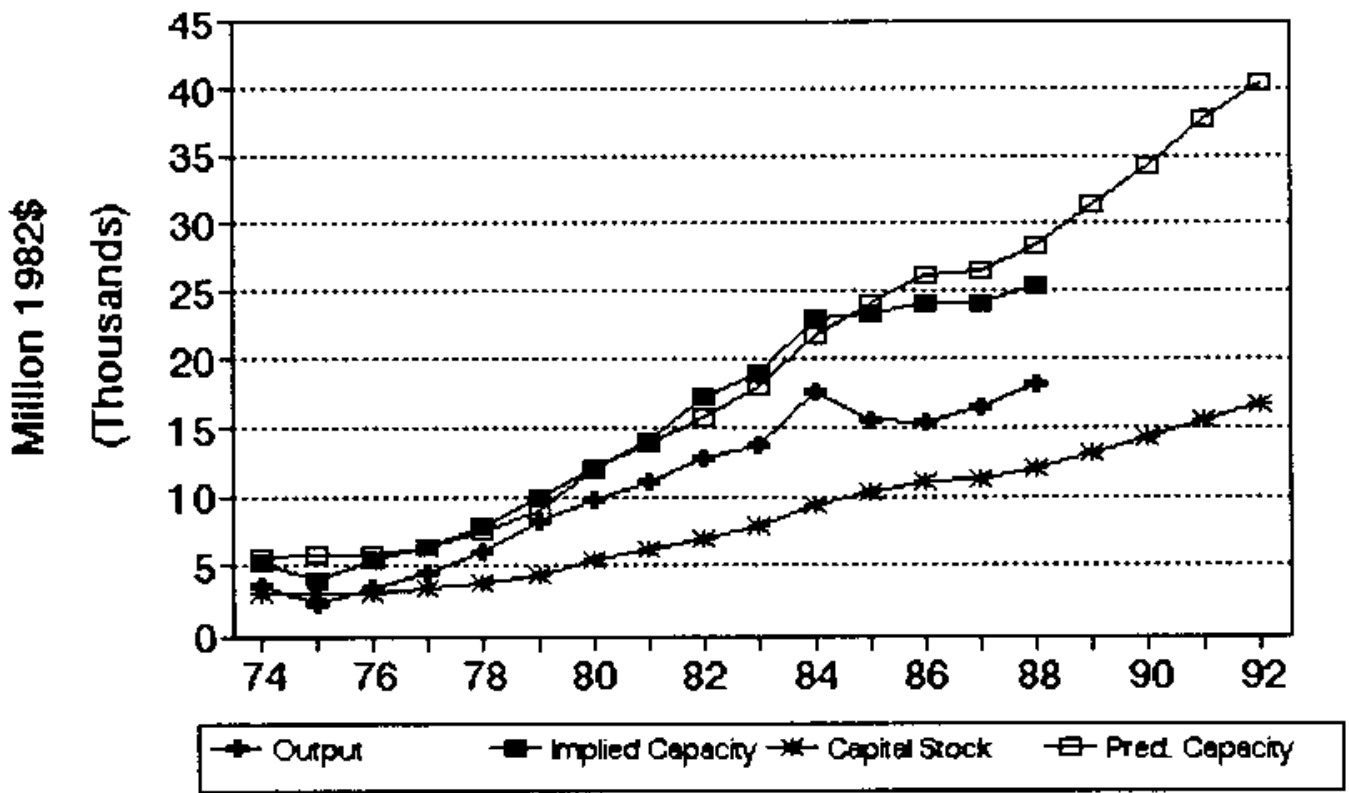


No. 387 sIC 3675 Blectronic capacitor:

MILLIONS OF 1982 DOLLARS (EXCL. CU RATES)

$\begin{array}{lcccclcc} & \text { Q4 } & \text { Amn. Implied } & \text { Gross } & \text { Net } & \text { Pred. } & \ldots \ldots \ldots \\ \text { Gross } & \text { CU } & \text { CU } & \text { Prac. } & \text { In- } & \text { Cap. } & \text { Prac. } & \text { Emergency Capacity } \\ \text { Qutput Rate } & \text { Rate } & \text { Capac. } & \text { vest. } & \text { Stk. } & \text { Capac. } & 3 \text { mon. } 6 \text { mon. }>6 \text { mon. }\end{array}$

\begin{tabular}{|c|c|c|c|c|c|c|c|c|c|c|}
\hline 1974 & 1,194 & 63 & 67.5 & 1,768 & 61.0 & 319 & 2,055 & & & \\
\hline 1975 & 849 & 39 & 43.9 & 1,932 & 0.8 & 299 & 2,049 & & & \\
\hline 1976 & 1,066 & 46 & 42.2 & 2,529 & 27.5 & 304 & 2,051 & & & \\
\hline 1977 & 1,194 & 68 & 58.7 & 2,035 & 39.0 & 319 & 2,055 & & & \\
\hline 1978 & 1,275 & 37 & 48.5 & 2,631 & 36.2 & 330 & 2,059 & & & \\
\hline 1979 & 1,368 & 80 & 63.4 & 2,157 & 10.6 & 317 & 2,054 & & & \\
\hline 1980 & 1,225 & $\pi$ & 7.5 & 1,584 & 65.8 & 356 & 2,067 & & & \\
\hline 1981 & 1,126 & 73 & 7.2 & 1,498 & 7.7 & 401 & 2,081 & & & \\
\hline 1982 & 1,218 & 56 & 61.4 & 1,982 & 61.5 & 430 & 2,091 & & & \\
\hline 1983 & 1,364 & 64 & 60.1 & 2,269 & 68.1 & 463 & 2,101 & & & \\
\hline 1984 & 1,778 & 73 & 70.2 & 2,534 & 112.4 & 536 & 2,124 & & & \\
\hline 1985 & 1,408 & 66 & 67.9 & 2,073 & 108.8 & 603 & 2,146 & & & \\
\hline 1986 & 1,300 & 61 & 62.1 & 2,095 & 56.7 & 615 & 2,149 & & & \\
\hline$-\cdots$ & & $\because$ & $\cdots$ & $-\cdot-$ & -- & $\cdots$ & $\cdots$ & $\cdot \cdot \cdot$ & --- & -- \\
\hline 1987 & 1,320 & 67 & 64.3 & 2,052 & 46.0 & 617 & 2,150 & & & \\
\hline 1988 & 1,348 & 73 & 71.0 & 1,900 & 79.6 & 652 & 2,161 & 2,161 & 2,738 & 2,767 \\
\hline 1989 & & & & & 108.2 & 714 & 2,181 & 2,180 & 2,783 & 2,792 \\
\hline 1990 & & & & & 113.9 & $\pi$ & 2,201 & 2,200 & 2,809 & 2,817 \\
\hline 1991 & & & & & 130.8 & 852 & 2,225 & 2,224 & 2,839 & 2,848 \\
\hline 1992 & & & & & 113.9 & 905 & 2,242 & 2,241 & 2,861 & 2,870 \\
\hline
\end{tabular}

Capecity-Capital Stock Regression: Code 0 RSO 0.010

Coefficients: Const 1953.7 stock 0.3

Ave. Capecity/Stock $(74-86): 5.1$ Ave. Weekly Hours (High) 123.6 Shift Factor 1.28 Ave Weekly Hours (Low) 116.2 Shift factor 1.36 Note: Output, Investment, and Capital Stock extrapolated for 1987 and 1988.

\section{Electronic capacitors}

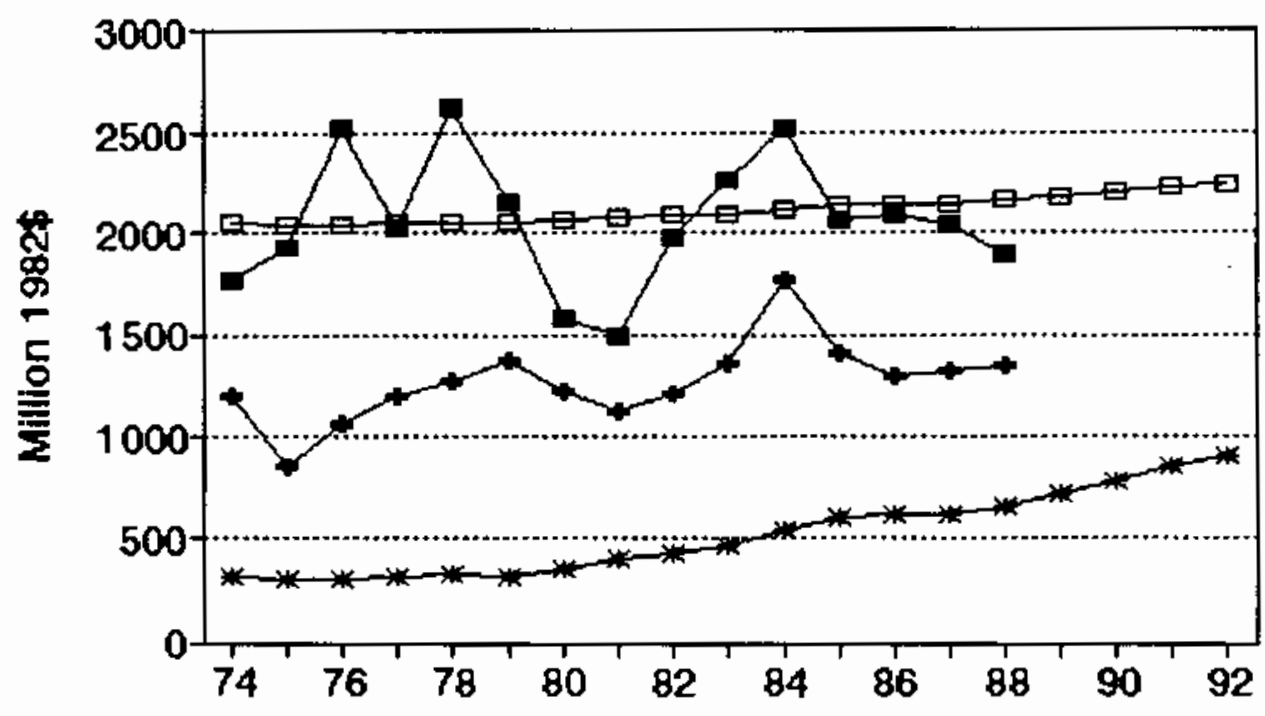

$\rightarrow$ - Oupu $\quad-$ - mplied Cepeciny to- Cepirel Siock E-Pred. Cepecin 


\section{No. 388 sIC 3676 Electronic resistors}

\section{MILLIONS OF 1982 DOLLARS (EXCL. CU RATES)}

$\begin{array}{lccccccc} & 04 & \text { Ann. Implied Gross } & \text { Net } & \text { Pred. } & \ldots \ldots . . . \\ \text { Gross } & \text { CU } & \text { CU } & \text { Prac. } & \text { In- } & \text { Cap. } & \text { Prac. } & \text { Emergency Capacity } \\ \text { Output Rate } & \text { Rate } & \text { Capac. } & \text { vest. } & \text { Stk. } & \text { Capac. } & 3 \text { mon. } 6 \text { mon. }>6 \text { mon. }\end{array}$

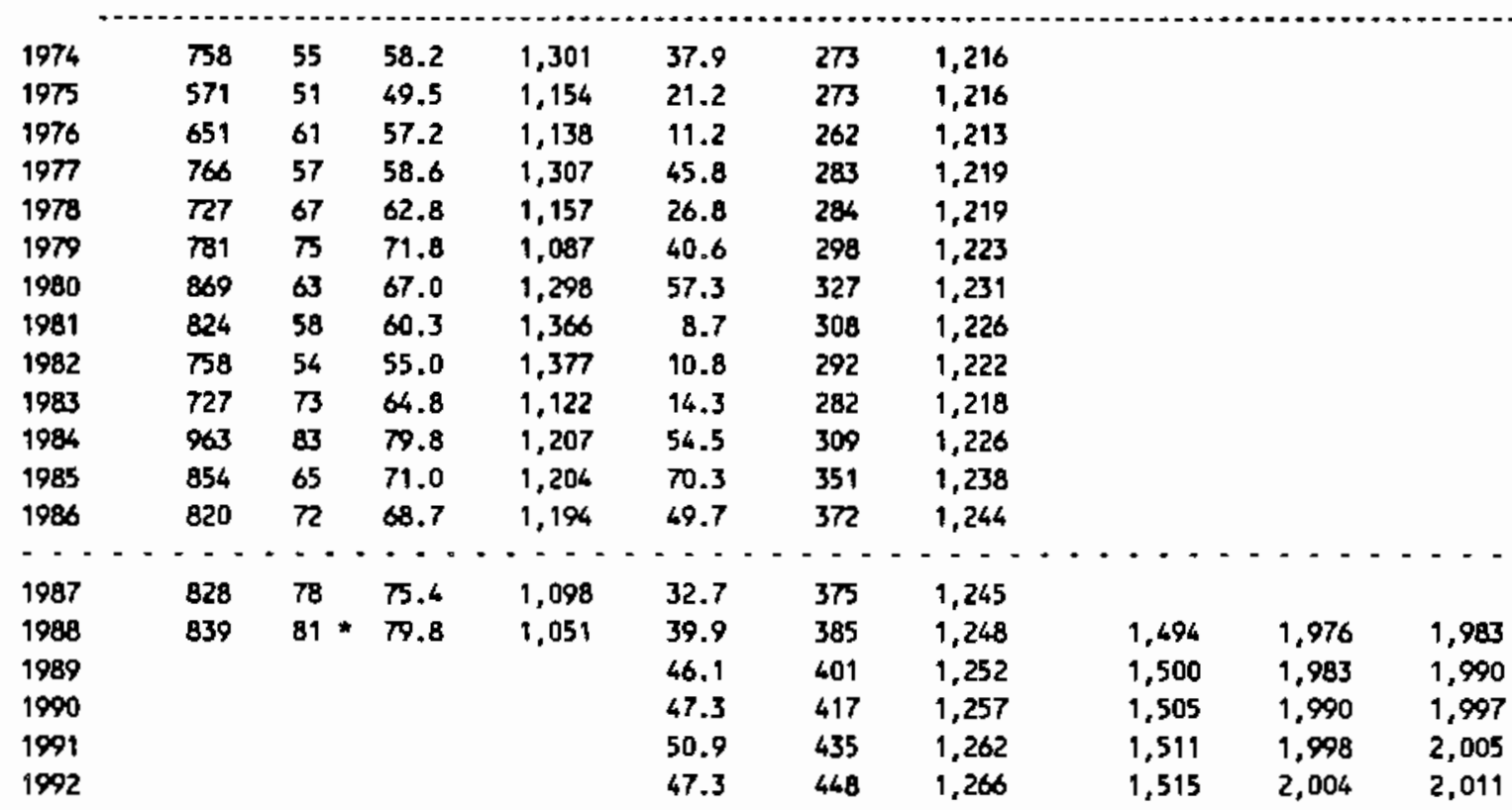

Capacity-Capital Stock Regression: Cade 0 RSO 0.010 Coefficients: Const 1138.7 stock 0.3 Ave. Weekly Hours (High) 99.1 Shift Factor 1.59 Ave Heekly Hours (LOW) 95.7 Shift Fector 1.65 * Imputed Note: Output, Investment, and Capital Stock extrapolated for 1987 and 1988.

\section{Electronic resistors}

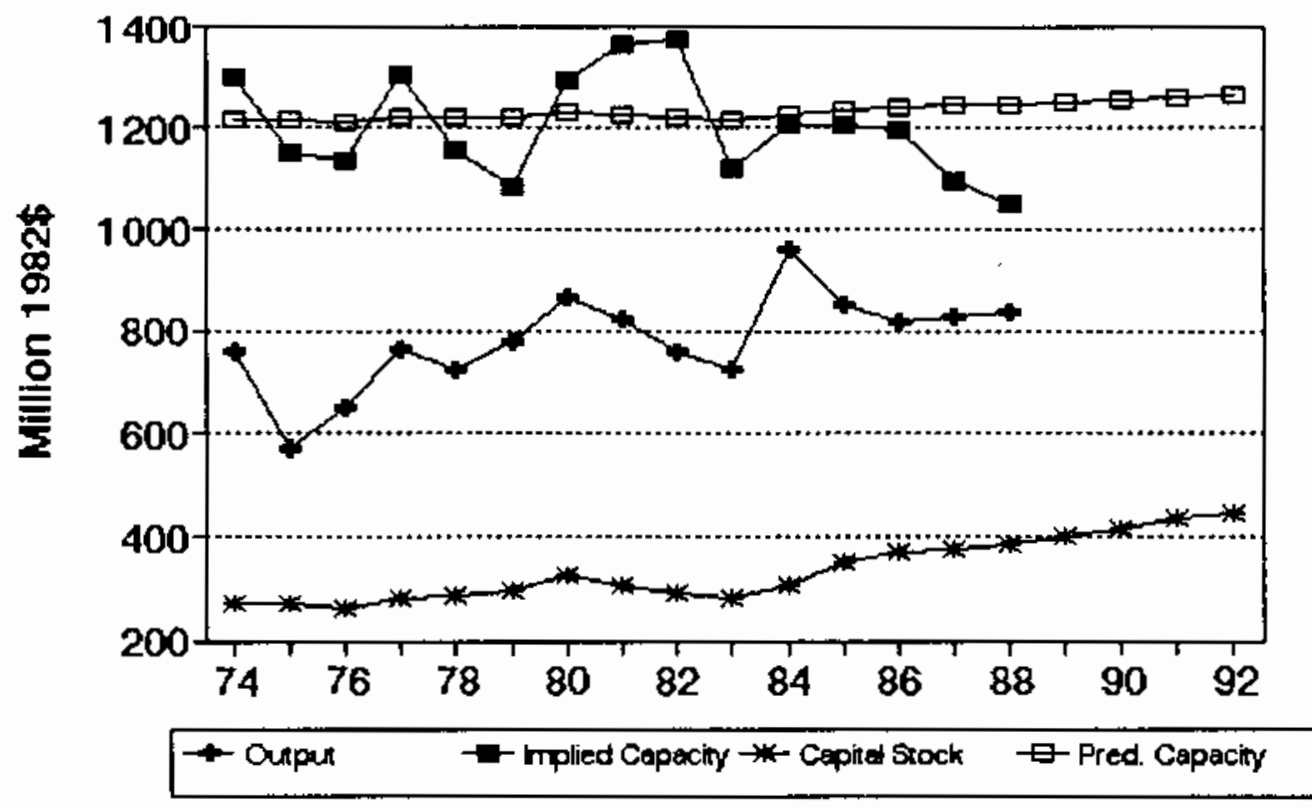




\title{
No. 389 sIC 3677 Electronic coils and transformers
}

\author{
MILLIOWS OF 1982 DOLLARS (EXCL. CU RATES)
}

$\begin{array}{lccccccc} & 04 & \text { Am. Implied Gross } & \text { Net } & \text { Pred. } & \ldots \ldots \ldots \\ \text { Gross } & \text { CU } & \text { CU } & \text { Prac. } & \text { In- } & \text { Cap. } & \text { Prac. } & \text { Emergency Capacity } \\ \text { Output } & \text { Rate } & \text { Rate } & \text { Capac. } & \text { vest. } & \text { stk. } & \text { Capac. } & 3 \text { mon. } 6 \text { mon. }>6 \text { mon. }\end{array}$

\begin{tabular}{|c|c|c|c|c|c|c|c|c|c|c|}
\hline 1974 & 894 & 80 & 84.7 & 1,055 & $(51.3)$ & 106 & 915 & & & \\
\hline 1975 & 667 & 71 & 70.0 & 953 & 18.5 & 115 & 1,027 & & & \\
\hline 1976 & 719 & 81 & 77.3 & 931 & $(8.6)$ & 99 & 837 & & & \\
\hline 1977 & 767 & 73 & 76.1 & 1,008 & 25.0 & 114 & 1,005 & & & \\
\hline 1978 & 788 & 79 & 76.2 & 1,034 & 21.3 & 123 & 1,114 & & & \\
\hline 1979 & 886 & 81 & 80.1 & 1,106 & 13.5 & 124 & 1,127 & & & \\
\hline 1980 & 845 & 80 & 79.8 & 1,058 & 18.1 & 129 & 1,184 & & & \\
\hline 1981 & 845 & 69 & 73.6 & 1,148 & 24.7 & 139 & 1,300 & & & \\
\hline 1982 & 883 & 46 & 53.7 & 1,644 & 29.3 & 153 & 1,657 & & & \\
\hline 1983 & 1,027 & 56 & 51.5 & 1,994 & 64.5 & 197 & 1,967 & & & \\
\hline 1984 & 1,056 & 82 & 7.6 & 1,455 & $(23.8)$ & 158 & 1,345 & & & \\
\hline 1985 & 1,059 & $\pi$ & 77.0 & 1,376 & 21.3 & 162 & 1,400 & & & \\
\hline 1986 & 863 & 65 & 68.0 & 1,268 & 12.2 & 158 & 1,356 & & & \\
\hline & $--\cdot-$ & - & -- & $-\cdots$ & -- & -- & $\cdots$ & $\cdots-$ & -- & -- \\
\hline 1987 & 866 & 77 & $\pi .1$ & 1,202 & 11.7 & 154 & 1,303 & & & \\
\hline 1988 & 871 & 80 & 79.1 & 1,102 & 13.8 & 152 & 1,281 & 1,976 & 2,787 & 2,790 \\
\hline 1989 & & & & & 15.5 & 152 & 1,281 & 1,977 & 2,788 & 2,800 \\
\hline 1990 & & & & & 15.9 & 153 & 1,286 & 1,983 & 2,798 & 2,810 \\
\hline 1991 & & & & & 16.9 & 154 & 1,302 & 2,008 & 2,833 & 2,845 \\
\hline 1992 & & & & & 15.9 & 154 & 1,304 & 2,012 & 2,838 & 2,850 \\
\hline
\end{tabular}

Capacity-Capital Stock Regression: Cade -1 RSO 0.880

Coefficients: Const -295.9 stock 11.5 D(84-86) $-165.6 \quad$ Ave. Capacity/Stock $(74-86): 9.0$ Ave. Weekly Hours (High) 72.1 Shift Factor 2.18 Ave Ueekly Hours (Low) 43.5 Shift Factor 3.62 Note: Output, Investment, and Capital stock extrapolated for 1987 and 1988.

\section{Electronic coils and transformers}

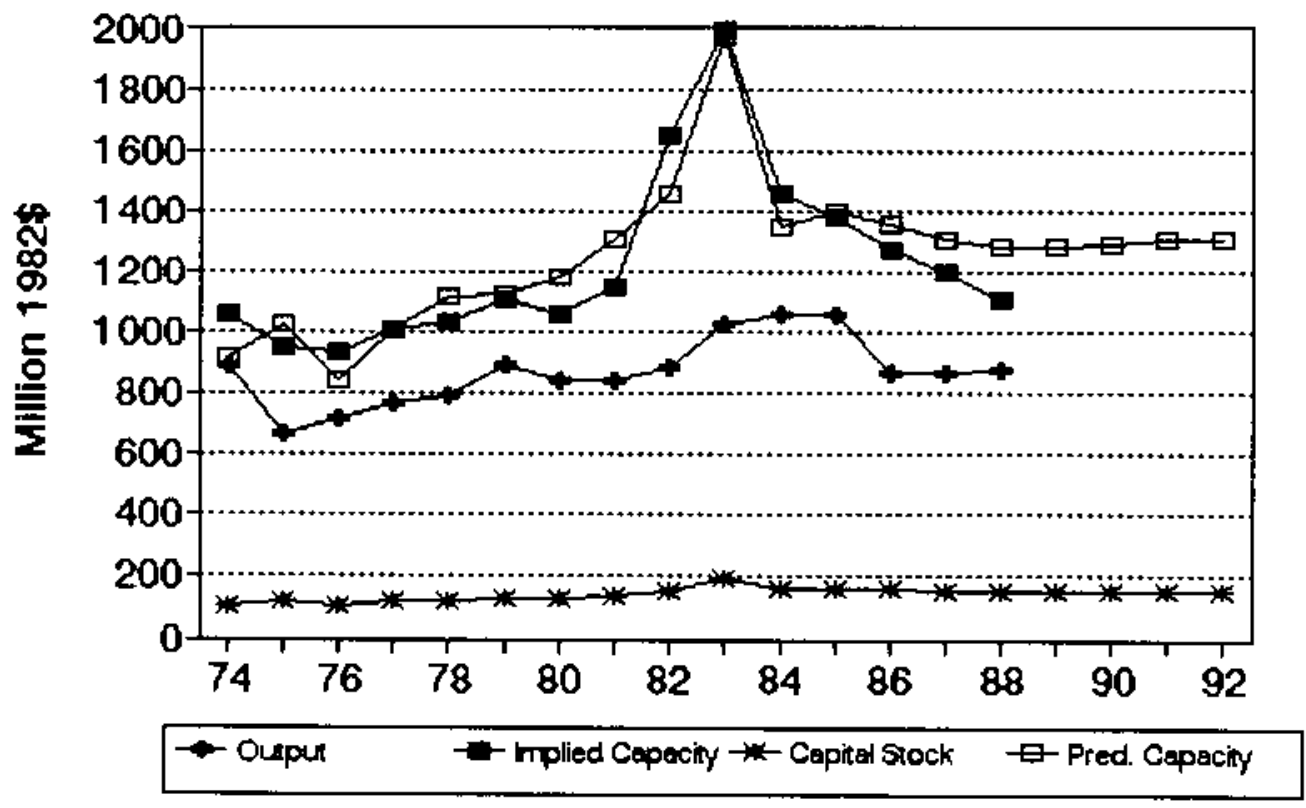




\section{No. 390 SIC 3678 Electronic connectors}

MILLIONS OF 1982 DOLLARS (EXCL. CU RATES)

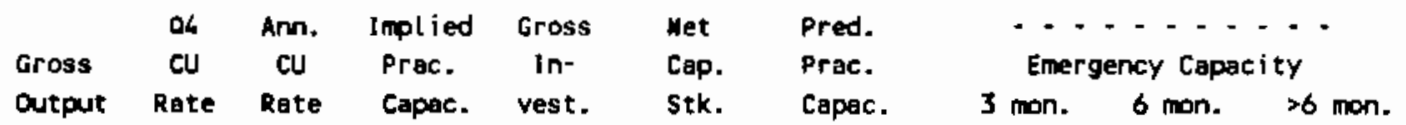

\begin{tabular}{|c|c|c|c|c|c|c|c|c|c|c|}
\hline 1974 & 1,350 & 49 & 52.6 & 2,567 & 69.4 & 302 & 1,927 & & & \\
\hline 1975 & 1,181 & 65 & 55.5 & 2,129 & 60.4 & 339 & 2,109 & & & \\
\hline 1976 & 1,306 & 59 & 61.7 & 2,119 & 37.3 & 350 & 2,164 & & & \\
\hline 1977 & 1,597 & 82 & 73.1 & 2,186 & 96.2 & 414 & 2,485 & & & \\
\hline 1978 & 1,919 & 87 & 84.4 & 2,273 & 91.9 & 469 & 2,759 & & & \\
\hline 1979 & 2,286 & $\pi$ & 80.7 & 2,833 & 50.5 & 483 & 2,827 & & & \\
\hline 1980 & 2,425 & $\pi$ & 72.0 & 3,369 & 113.9 & 559 & 3,206 & & & \\
\hline 1981 & 2,327 & 68 & 69.4 & 3,353 & 104.9 & 623 & 3,524 & & & \\
\hline 1982 & 2,625 & 62 & 63.6 & 4,127 & 196.3 & 770 & 4,258 & & & \\
\hline 1983 & 2,840 & 73 & 67.8 & 4,191 & 66.8 & 788 & 4,344 & & & \\
\hline 1984 & 3,365 & 60 & 65.6 & 5,127 & 125.1 & 861 & 4,711 & & & \\
\hline 1985 & 2,854 & 51 & 53.7 & 5,311 & 186.3 & 990 & 5,351 & & & \\
\hline 1986 & 3,014 & 56 & 53.4 & 5,641 & 106.3 & 1,032 & 5,561 & & & \\
\hline$\cdots-$ & $-\cdots$ & 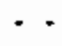 & $-\cdot-$ & $\cdots$ & -- & $\cdots$ & $\therefore-$ & $-\cdot$ & $-\cdot \cdot$ & $-\quad-$ \\
\hline 1987 & 3,118 & 57 & 56.3 & 5,539 & 83.5 & 1,054 & 5,668 & & & \\
\hline 1988 & 3,258 & 70 & 65.3 & 4,990 & 127.6 & 1,118 & 5,987 & 6,067 & 8,131 & 8,161 \\
\hline 1989 & & & & & 165.3 & 1,236 & 6,475 & 6,562 & 8,794 & 8,826 \\
\hline 1990 & & & & & 172.7 & 1,315 & 6,971 & 7,064 & 9.467 & 9,502 \\
\hline 1991 & & & & & 195.0 & 1,431 & 7,548 & 7,648 & 10,250 & 10,287 \\
\hline 1992 & & & & & 172.7 & 1,518 & 7,979 & 8,085 & 10,836 & 10,875 \\
\hline
\end{tabular}

Capacity-Capital Stock Regression: Code 0 RSQ 0.950 Coefficients: Const 421.5 Stock 5.0 Ave. Weekly Hours (High) 115.8 Shift Factor 1.36 Ave Weekly Hours (Low) 112.6 Shift Factor 1.40 Hote: Output, Imvestment, and Capital Stock extrapolated for 1987 and 1988.

\section{Electronic connectors}

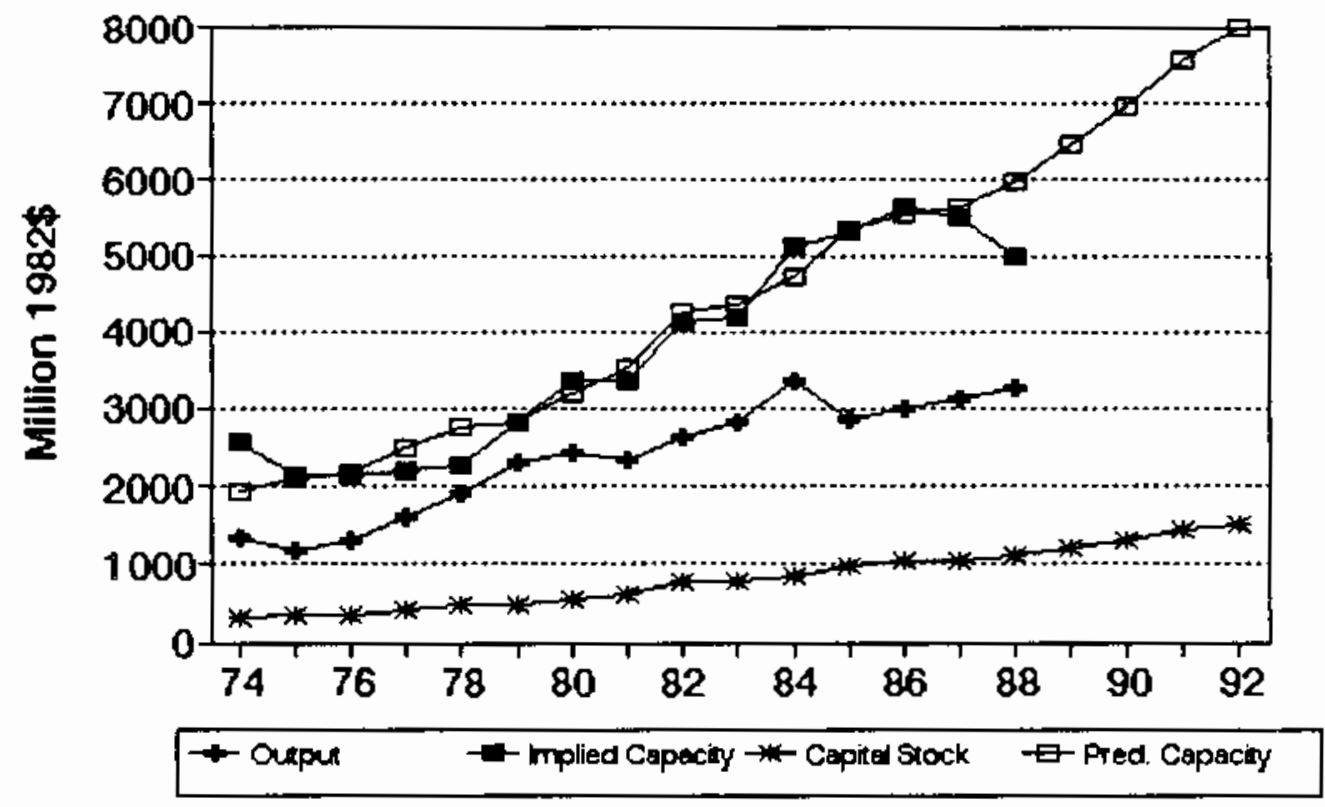


No. 391 SIC 3679 Blectronic components, nec

MILLIONS OF 1982 DOLLARS (EXCL. CU RATES)

$\begin{array}{lccccccc} & \text { a4 } & \text { Ann. } & \text { Implied Gross } & \text { Net } & \text { Pred. } & \ldots \ldots \ldots \\ \text { Gross } & \text { CU } & \text { CU } & \text { Prac. } & \text { In- } & \text { Cap. } & \text { Prac. } & \text { Emergenty Capacity } \\ \text { Output } & \text { Rate } & \text { Rate } & \text { Capac. } & \text { vest. } & \text { Stk. } & \text { Capac. } & 3 \text { mon. } 6 \text { mon. }>6 \text { mon. }\end{array}$

\begin{tabular}{|c|c|c|c|c|c|c|c|c|c|c|}
\hline 1974 & 5,390 & 76 & 79.1 & 6,819 & $(65.8)$ & 1,468 & 8,622 & & & \\
\hline 1975 & $5,7 \pi$ & 67 & 67.6 & 8,547 & 301.8 & 1,656 & 9,537 & & & \\
\hline 1976 & 7,098 & 68 & 67.7 & 10,486 & 239.3 & 1,767 & 10,073 & & & \\
\hline 1977 & 9,066 & 70 & 69.2 & 13,005 & 437.3 & 2,047 & 11.436 & & & \\
\hline 1978 & 8,700 & 69 & 69.1 & 12,600 & 345.5 & 2,220 & 12,280 & & & \\
\hline 1979 & 10,932 & 82 & 77.0 & 14,202 & 467.6 & 2,503 & 13,659 & & & \\
\hline 1980 & 11,528 & 78 & 79.1 & 14,574 & 577.0 & 2,876 & 15,471 & & & \\
\hline 1981 & 12,225 & $\pi$ & 74.6 & 16,390 & 447.0 & 3,106 & 16,590 & & & \\
\hline 1982 & 14,573 & $\infty$ & 67.8 & 21,493 & 688.9 & 3,560 & 18,801 & & & \\
\hline 1983 & 13,894 & $\pi$ & 72.2 & 19,250 & 415.1 & 3,729 & 19,622 & & & \\
\hline 1984 & 17,902 & 73 & 74.9 & 23,897 & $1,147.5$ & 4,591 & 23,821 & & & \\
\hline 1985 & 15,814 & 60 & 64.4 & 24,559 & 503.4 & 4,802 & 24,846 & & & \\
\hline 1986 & 14,959 & 60 & 59.6 & 25,111 & 612.9 & 5,093 & 26,263 & & & \\
\hline-- & --- & -- & $-\cdots$ & $\cdots$ & 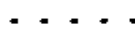 & $\cdots$ & --- & ---- & $=\cdot$ & $-\cdot-$ \\
\hline 1987 & 15,454 & 66 & 63.5 & 24,328 & 387.1 & 5,164 & 26,607 & & & \\
\hline 1988 & 16,120 & 72 & 69.9 & $23,0 \pi 5$ & 619.9 & 5,463 & 28,064 & 29,670 & 39,625 & 39,767 \\
\hline 1989 & & & & & 818.6 & 5,943 & 30,397 & 32,136 & 42,919 & 43,073 \\
\hline 1990 & & & & & 857.8 & 6,431 & $32, \pi 7$ & 34,652 & 46,279 & 46,444 \\
\hline 1991 & & & & & 975.3 & 7,007 & 35,580 & 37,615 & 50,237 & 50,416 \\
\hline 1992 & & & & & 857.8 & 7,430 & 37,637 & 39,790 & 53,141 & 53,332 \\
\hline
\end{tabular}

Capacity-Capital Stock Regression: Code 0 RSQ 0.960

Coefficients: Const 1473.5 stock 4.9

Ave. Capacity/Stock $(74-86): 5.4$ Ave. Heekly Hours (High) 111.2 Shift Factor 1.42 Ave Heekly Hours (LOW) 110.4 Shift Factor 1.43 Note: Output, Investment, and Capital Stock extrapolated for 1987 and 1988.

\section{Electronic components, nec}

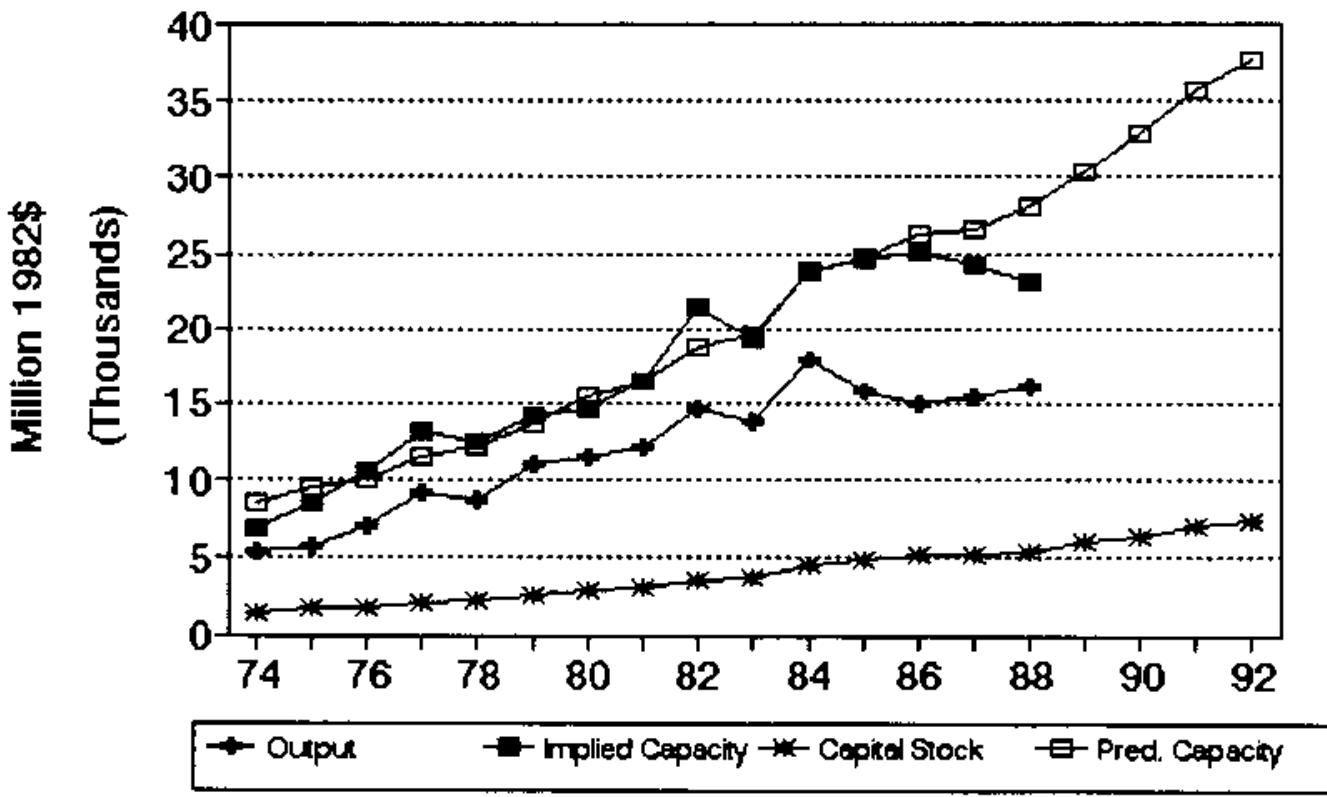


No. 392 sIc 3691 storage batteries

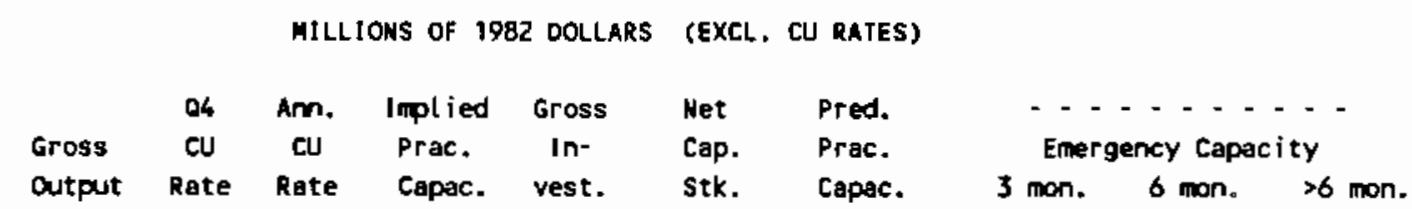

\begin{tabular}{|c|c|c|c|c|c|c|c|c|c|c|}
\hline 1974 & 2,241 & 89 & 89.9 & 2,493 & 78.7 & 455 & 2,304 & & & \\
\hline 1975 & 1,991 & 76 & 80.1 & 2,486 & 110.9 & 535 & 2,588 & & & \\
\hline 1976 & 2,318 & 87 & 82.9 & 2,790 & 92.7 & 594 & 2,801 & & & \\
\hline 1977 & 2,785 & 90 & 86.9 & 3,134 & 147.3 & 704 & 3,191 & & & \\
\hline 1978 & 2,932 & 80 & B3.7 & 3,505 & 83.3 & 748 & 3,347 & & & \\
\hline 1979 & 2,827 & 77 & 78.1 & 3,619 & 69.3 & 774 & 3,442 & & & \\
\hline 1980 & 2,522 & 83 & 80.7 & 3,127 & 51.8 & 780 & 3,461 & & & \\
\hline 1981 & 2,520 & 83 & 83.1 & 3,032 & 55.6 & 786 & 3,483 & & & \\
\hline 1982 & 2,404 & 72 & 76.0 & 3,164 & 72.6 & 804 & 3,548 & & & \\
\hline 1983 & 2,730 & 74 & 7.1 & 3,735 & 68.1 & 812 & 3,577 & & & \\
\hline 1984 & 3,238 & 83 & 79.7 & 4,062 & 114.8 & 860 & 3,748 & & & \\
\hline 1985 & 3,109 & 85 & 84.1 & 3,696 & 47.3 & 839 & 3,673 & & & \\
\hline 1986 & 3,302 & 82 & 83.0 & 3,979 & 70.5 & 837 & 3,660 & & & \\
\hline - - - & - - - - & -- & $\because-$ & $\cdots \cdot$ & $\because-$ & $-\cdot$ & --- & $\cdots$ & $\cdots \cdot-$ & --- \\
\hline 1987 & 3,359 & 79 & 80.1 & 4,196 & 67.9 & 834 & 3,656 & & & \\
\hline 1988 & 3,435 & 79 & 79.0 & 4,346 & 66.4 & 831 & 3,643 & 3,925 & 4,286 & 4.291 \\
\hline 1989 & & & & & 64.8 & 825 & 3,624 & 3,906 & 4,265 & 4,270 \\
\hline 1990 & & & & & 63.3 & 819 & 3,602 & 3,882 & 4,238 & 4,244 \\
\hline 1991 & & & & & 61.7 & 812 & 3,576 & 3,854 & 4,208 & 4,213 \\
\hline 1992 & & & & & 60.2 & 804 & 3,547 & 3,823 & 4,174 & 4,179 \\
\hline
\end{tabular}

Cepacity-Capital Stock Regression: Code 0 RSQ 0.760 Coefficients: Const 684.7 Stock 3.6

Ave. Capacity/Stock $(74-86): 4.5$ Ave. Weekly Hours (High) 134.7 Shift Factor 1.18 Ave veekly Hours (Low) 113.1 Shift Factor 1.39 Note: Output, Investment, and Capital Stock extrepolated for 1987 and 1988.

3691 Storage batteries

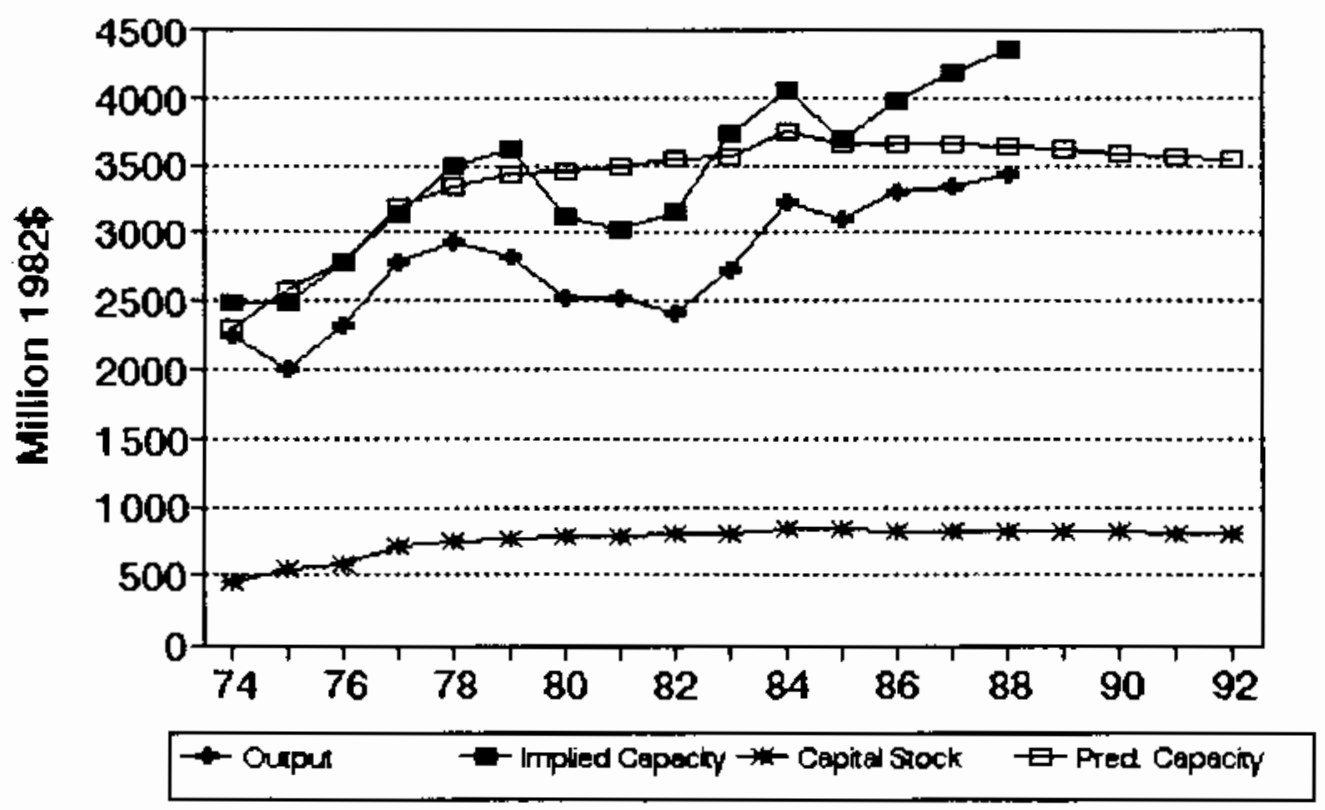




\section{No. 393 SIC 3692 Primary batteries, dry and wot}

MILLIONS OF 1982 DOLLARS (EXCL. CU RATES)

$\begin{array}{lccccccc} & \text { Q } & \text { Ann. Implied } & \text { Gross } & \text { Net } & \text { Pred. } & \ldots \ldots \ldots \\ \text { Gross } & \text { CU } & \text { CU } & \text { Prac. } & \text { In- } & \text { Cap. } & \text { Prac. } & \text { Emergency Capacity } \\ \text { Output Rate } & \text { Rate } & \text { Capac. } & \text { vest. } & \text { Stk. } & \text { Capac. } & 3 \text { mon. } 6 \text { mon. }>6 \text { mon. }\end{array}$

\begin{tabular}{|c|c|c|c|c|c|c|c|c|c|c|}
\hline 1974 & 641 & 86 & 86.0 & 746 & 25.9 & 184 & 716 & & & \\
\hline 1975 & 600 & 70 & 76.0 & 789 & 19.9 & 192 & 778 & & & \\
\hline 1976 & 792 & $n$ & 74.4 & 1,065 & 22.3 & 201 & 858 & & & \\
\hline 1977 & 761 & 80 & 78.9 & 965 & 44.0 & 231 & 1,114 & & & \\
\hline 1978 & 914 & 67 & 71.9 & 1,271 & 73.0 & 288 & 1,598 & & & \\
\hline 1979 & 981 & 55 & 59.5 & 1,649 & 26.2 & 297 & 1,683 & & & \\
\hline 1980 & 1,046 & 53 & 53.8 & 1,946 & 42.0 & 322 & 1,895 & & & \\
\hline 1981 & 1,132 & 51 & 51.8 & 2,187 & 46.1 & 349 & 2,124 & & & \\
\hline 1982 & 1,088 & 43 & 46.0 & 2,365 & 47.7 & 374 & 2,341 & & & \\
\hline 1983 & 1,126 & 47 & 45.5 & 2,475 & 17.3 & 367 & 2,281 & & & \\
\hline 1984 & 1,302 & 51 & 49.5 & 2,631 & 40.9 & 382 & 2,403 & & & \\
\hline 1985 & 1,365 & 62 & 57.9 & 2,359 & 26.2 & 381 & 2,396 & & & \\
\hline 1986 & 1,409 & $64 *$ & 63.4 & 2,224 & 39.8 & 391 & 2,486 & & & \\
\hline-- & $-\cdots$ & $-\cdots$ & $\cdots$ & $\cdots$ & $\cdots$ & $\cdots$ & $\cdots-$ & -- & $-\cdots$ & $\cdots$ \\
\hline 1987 & 1,445 & 65 & 64.7 & 2,253 & 33.7 & 396 & 2,530 & & & \\
\hline 1988 & 1,492 & 71 & 68.8 & 2,170 & 37.2 & 405 & 2,602 & 2,657 & 3,513 & 3,525 \\
\hline 1989 & & & & & 40.2 & 416 & 2,693 & $2, \pi 51$ & 3,637 & 3,650 \\
\hline 1990 & & & & & 40.8 & 426 & 2,784 & 2,843 & 3,759 & 3,772 \\
\hline 1991 & & & & & 42.6 & 438 & 2,882 & 2,944 & 3,892 & 3,906 \\
\hline 1992 & & & & & 40.8 & 447 & 2,959 & 3,022 & 3,995 & 4,009 \\
\hline
\end{tabular}

Capacity-Capital Stock Regression: Code 0 RSO 0.940

Coefficients: Const -862.4 Stock 8.6

Ave. Capacity/Stock $(74-86): 5.7$

Ave. Ueekly Hours (High) 116.5 Shift Factor 1.36 Ave Heekly Hours (Low) 115.4 Shift Factor 1.37 * Imputed Note: Output, Investment, and Capital Stock extrapolated for 1987 and 1988.

\section{Primary batteries, dry and wet}

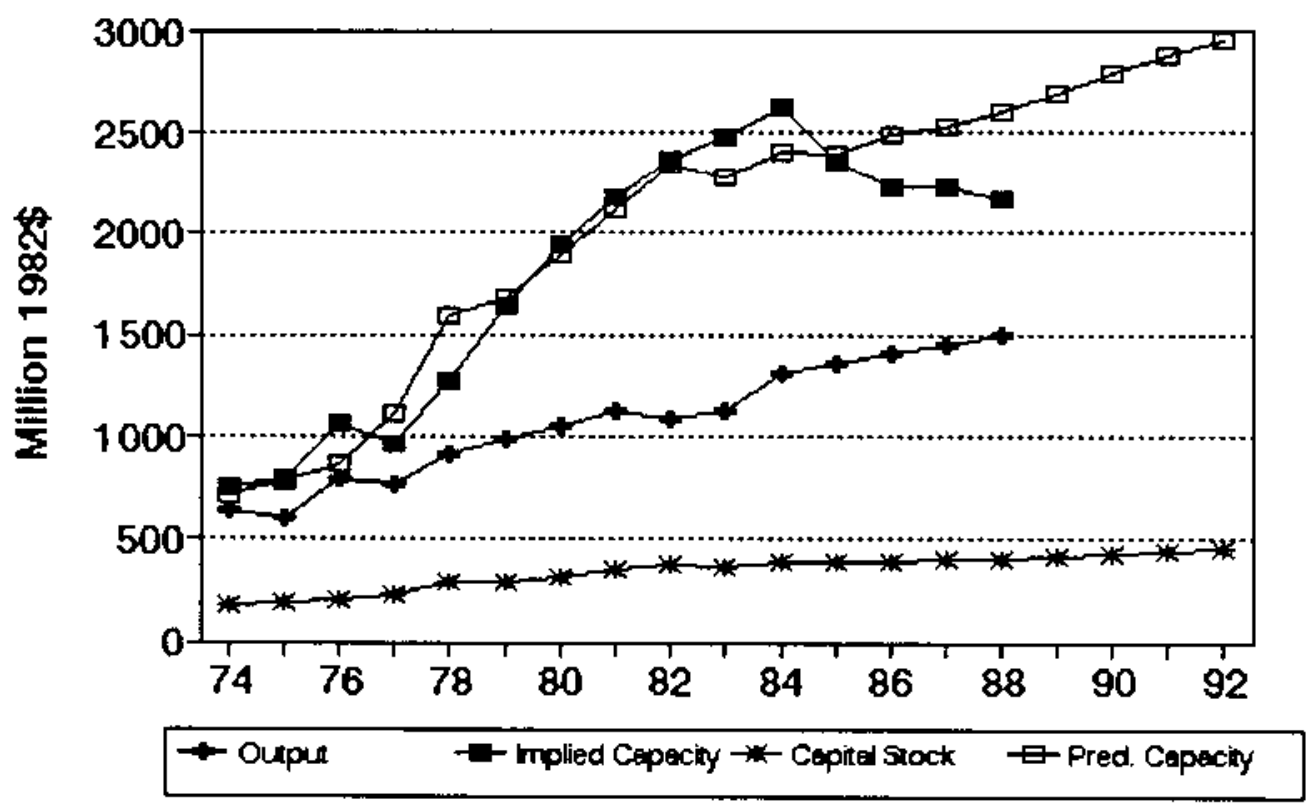


No. 394 sIC 3693 X-ray apparatus and tubes

MILLIONS OF 1982 DOLLARS (EXCL. CU RATES)

$\begin{array}{lccccccc} & \text { Q4 } & \text { Ann. } & \text { Implied Gross } & \text { Net } & \text { Pred. } & \ldots \ldots \ldots \ldots \\ \text { Gross } & \text { CU } & \text { CU } & \text { Prac. } & \text { In- } & \text { Cap. } & \text { Prac. } & \text { Emergency Capacity } \\ \text { Output } & \text { Rate } & \text { Rate } & \text { Capac. } & \text { vest. } & \text { Stk. } & \text { Capac. } & 3 \text { mon. } 6 \text { mon. }\end{array}$

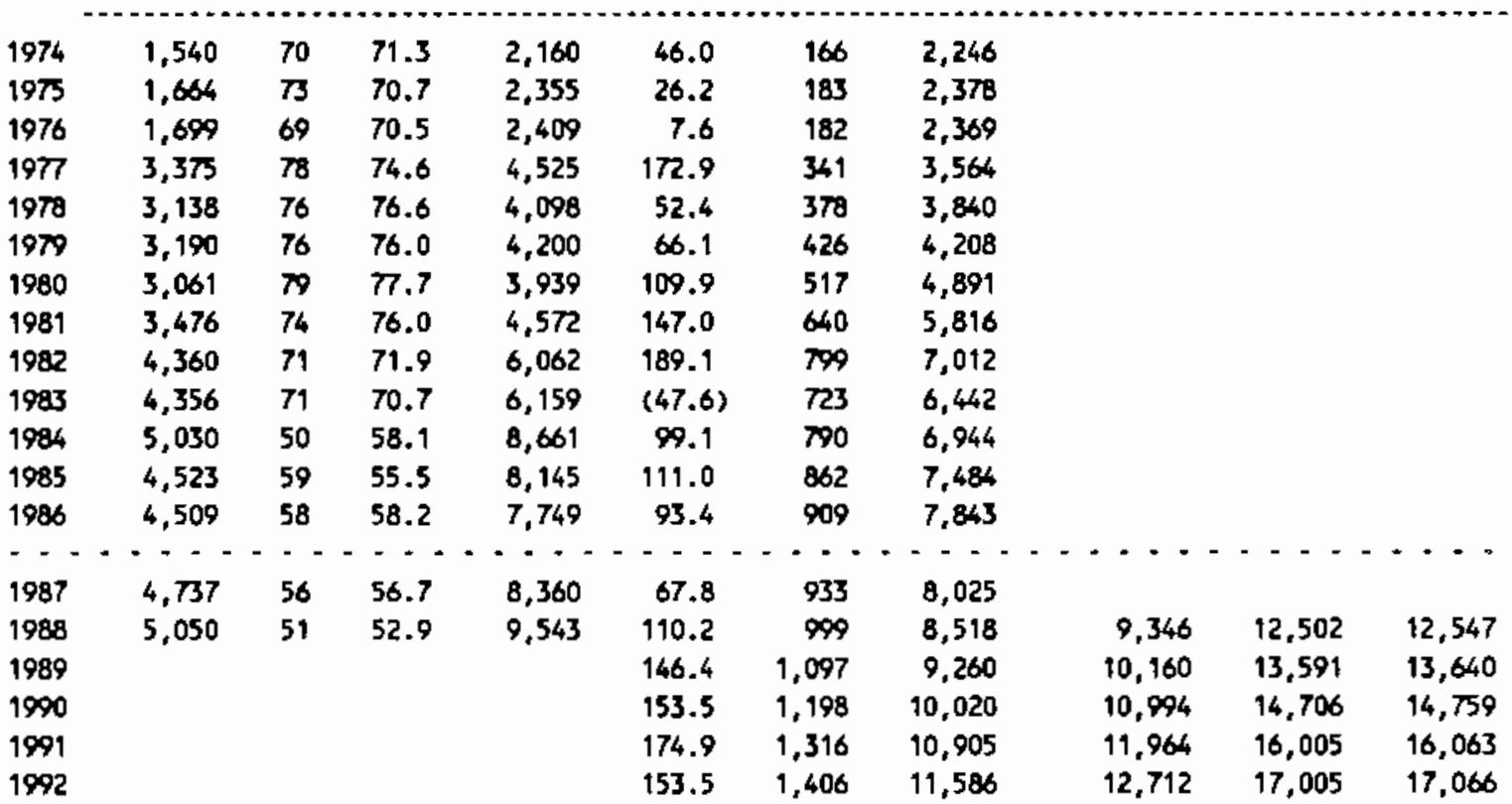

Capacity-Capital Stock Regression: Code 0 RSQ 0.870

Coefficients: Const $\$ 6.3$ stock 7.5

Ave. Capacity/Stock $(74-86): 9.4$

Ave. Weekly Hours (High) 106.9 Shift factor 1.47 Ave Weekly Hours (Low) 100.8 Shift Factor 1.56 Note: Output, Imvestment, and Capital Stock extrapolated for 1987 and 1988.

\section{X-ray apparatus and tubes}

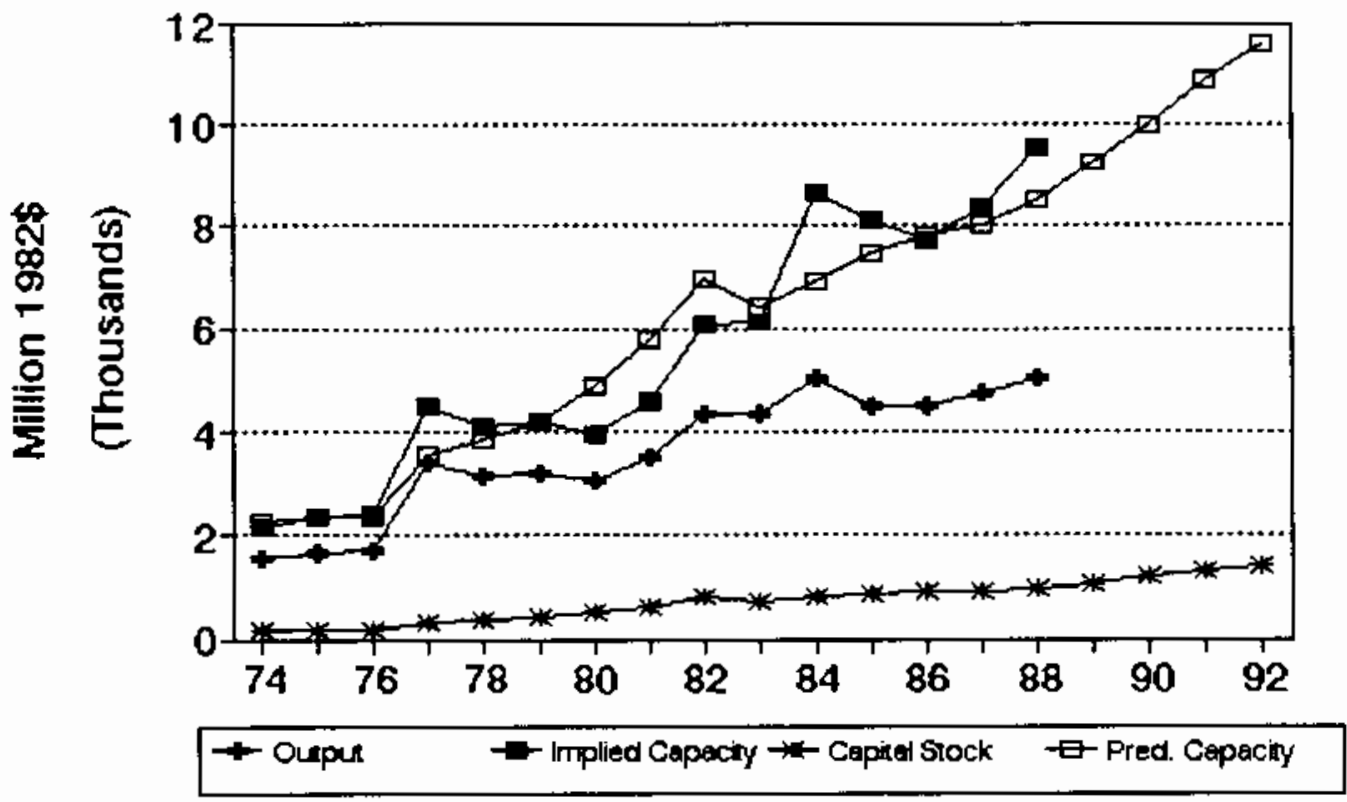




\section{No. 395 SIC 3694 Engine electrical equipment}

MILLIONS OF 1982 DOLLARS (EXCL. CU RATES)

$\begin{array}{lccccccc} & 04 & \text { Ann. Implied Gross } & \text { Het } & \text { Pred. } & \ldots \ldots \ldots \\ \text { Gross } & \text { CU } & \text { CU } & \text { Prac. } & \text { In- } & \text { Cap. } & \text { Prac. } & \text { Emergency Capacity } \\ \text { Output } & \text { Rate } & \text { Rate } & \text { Capac. } & \text { vest. } & \text { Stk. } & \text { Capac. } & 3 \text { mon. } 6 \text { mon. }>6 \text { mon. }\end{array}$

\begin{tabular}{|c|c|c|c|c|c|c|c|c|c|c|}
\hline 1974 & 5,079 & $\pi$ & 76.6 & 6,633 & 254.5 & 1,228 & 6,293 & & & \\
\hline 1975 & 4,198 & 86 & 80.4 & 5,221 & 98.8 & 1,249 & 6,331 & & & \\
\hline 1976 & 5,135 & 96 & 92.2 & 5,569 & 117.1 & 1,284 & 6,393 & & & \\
\hline 1977 & 5,805 & 96 & 96.0 & 6,047 & 240.2 & 1,433 & 6,661 & & & \\
\hline 1978 & 6,015 & 90 & 92.0 & 6,537 & 57.7 & 1,405 & 6,610 & & & \\
\hline 1979 & 5,503 & 82 & 85.0 & 6,476 & 126.8 & 1,441 & 6,675 & & & \\
\hline 1980 & 4,307 & 60 & 68.0 & 6,333 & 114.8 & 1,459 & 6,708 & & & \\
\hline 1981 & 4,382 & 57 & 58.3 & 7,522 & 93.2 & 1,453 & 6,697 & & & \\
\hline 1982 & 3,366 & 56 & 56.2 & 5,989 & $(204.5)$ & 1,168 & 6,184 & & & \\
\hline 1983 & 4,134 & 66 & 61.9 & 6,677 & 119.3 & 1,197 & 6,237 & & & \\
\hline 1984 & 5,859 & 74 & 71.2 & 8,233 & 221.6 & 1,315 & 6,449 & & & \\
\hline 1985 & 5,644 & $\pi$ & 74.4 & 7,585 & 148.3 & 1,355 & 7,512 & & & \\
\hline 1986 & 5,674 & $\pi$ & 76.0 & 7,469 & 130.0 & 1,373 & 7,543 & & & \\
\hline 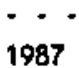 & 5,688 & 78 & $\begin{array}{l}\cdots \\
7.5\end{array}$ & 7340 & 81.2 & $\cdots$ & $7 .-$ & --- & $\cdots \cdot \cdot$ & --- \\
\hline 1988 & 5,705 & 73 & $\pi .0$ & 7.612 & 76.3 & 1,311 & 7,431 & 8.735 & 10,996 & 11,028 \\
\hline 1989 & & & & & 71.4 & 1,276 & 7,369 & 8,682 & 10,904 & 10,935 \\
\hline 1990 & & & & & 66.4 & 1,239 & 7,303 & 8,604 & 10,806 & 10,838 \\
\hline 1991 & & & & & 61.5 & 1,201 & 7,234 & 8,522 & 10,703 & 10,735 \\
\hline 1992 & & & & & 56.6 & 1,160 & 7,161 & 8,437 & 10,596 & 10,627 \\
\hline
\end{tabular}

Capecity-Capital Stock Regression: Code -1 RSQ 0.250

Coefficients: Const 4087.6 stock $1.8 \quad D(85-86) \quad 990.3$

Ave. Capacity/Stock $(76-86): 5.0$ Ave. Weekly Hours (High) 106.1 Shift factor 1.48 Ave Heekly Hours (LOW) 103.0 Shift factor 1.53 Note: Output, Investment, and Capital stock extrapolated for 1987 and 1988.

\section{Engine electrical equipment}

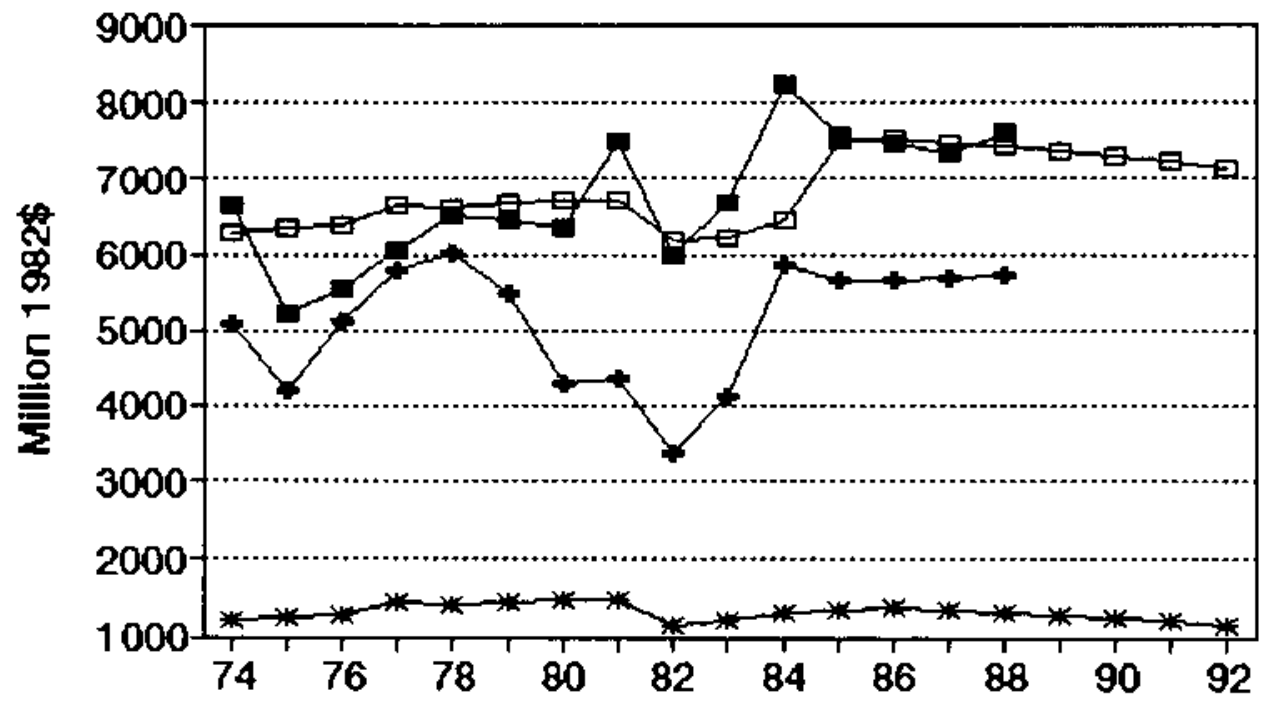


No. 396 sIC 3699 Electrical equipment and supplies, nec MILLIONS OF 1982 DOLLARS (EXCL. CU RATES)

$\begin{array}{lccccccc} & \text { Q4 } & \text { Ann. } & \text { Implied Gross } & \text { Net } & \text { Pred. } & \ldots \ldots \ldots \\ \text { Gross } & \text { CU } & \text { CU } & \text { Prac. } & \text { In- } & \text { Cap. } & \text { Prac. } & \text { Emergency Capacity } \\ \text { Output } & \text { Rate } & \text { Rate } & \text { Capac. } & \text { vest. } & \text { Stk. } & \text { Capac. } & 3 \text { mon. } 6 \text { mon. }>6 \text { mon. }\end{array}$

\begin{tabular}{|c|c|c|c|c|c|c|c|c|c|c|}
\hline 1974 & 1,068 & 63 & 65.4 & 1,633 & $(5.9)$ & 275 & 1,689 & & & \\
\hline 1975 & 748 & 66 & 62.7 & 1,194 & $(8.8)$ & 251 & 1,446 & & & \\
\hline 1976 & 945 & 71 & 69.1 & 1,367 & 2.2 & 238 & 1,310 & & & \\
\hline 1977 & 1,089 & 73 & 72.2 & 1,507 & 65.8 & 283 & 1,761 & & & \\
\hline 1978 & 1,161 & $\pi 3$ & 72.7 & 1,598 & 30.2 & 290 & 1,838 & & & \\
\hline 1979 & 1,346 & 69 & 70.4 & 1,911 & 18.8 & 285 & 1,790 & & & \\
\hline 1980 & 1,164 & 56 & 60.5 & 1,922 & 5.4 & 268 & 1.610 & & & \\
\hline 1981 & 1,169 & 61 & 59.5 & 1.966 & 38.8 & 281 & 1,748 & & & \\
\hline 1982 & 1,309 & 47 & 51.8 & 2,527 & 56.6 & 310 & 2,036 & & & \\
\hline 1983 & 1,386 & 94 & 75.2 & 1,842 & 20.3 & 302 & 1,962 & & & \\
\hline 1984 & 1,660 & 84 & 88.2 & 1,881 & 48.5 & 321 & 2,156 & & & \\
\hline 1985 & 1,638 & 72 & 76.0 & 2,155 & $(0.6)$ & 295 & 2,345 & & & \\
\hline 1986 & 1,737 & 66 & 67.8 & 2,562 & 29.2 & 297 & 2,372 & & & \\
\hline$\because-$ & ---- & - & - - - & $-\cdots$ & $\cdots$ & $\cdots$ & $-\cdots$ & ----- & $\cdots \cdot$ & $-\cdots$ \\
\hline 1987 & 1,763 & $\pi$ & 71.4 & 2,469 & 24.4 & 295 & 2,351 & & & \\
\hline 1988 & 1,797 & 67 & 70.1 & 2,562 & 33.3 & 302 & 2,422 & 4,619 & 6,692 & 6,721 \\
\hline 1989 & & & & & 40.9 & 316 & 2,564 & 4,889 & 7,084 & 7,115 \\
\hline 1990 & & & & & 42.4 & 330 & 2,708 & 5,164 & 7,483 & 7,516 \\
\hline 1991 & & & & & 46.9 & 348 & 2,886 & 5,502 & 7,972 & 8,008 \\
\hline 1992 & & & & & 42.4 & 359 & 3,001 & 5,723 & 8,292 & 8,329 \\
\hline
\end{tabular}

Capacity-Capital stock Regression: Code - 1 RSQ 0.620

Coefficients: Const -1104.8 stock 10.2 D(85-86) 460.9 Ave. Capacity/Stock $(74-86): 6.5$ Ave. Weekly Hours (High) 56.8 Shift Factor 2.78 Ave Weekly Hours (Low) 52.8 Shift Factor 2.99 Wote: Output, Investment, and Capital stock extrapolated for 1987 and 1988.

\section{Electrical equipment and supplies,}

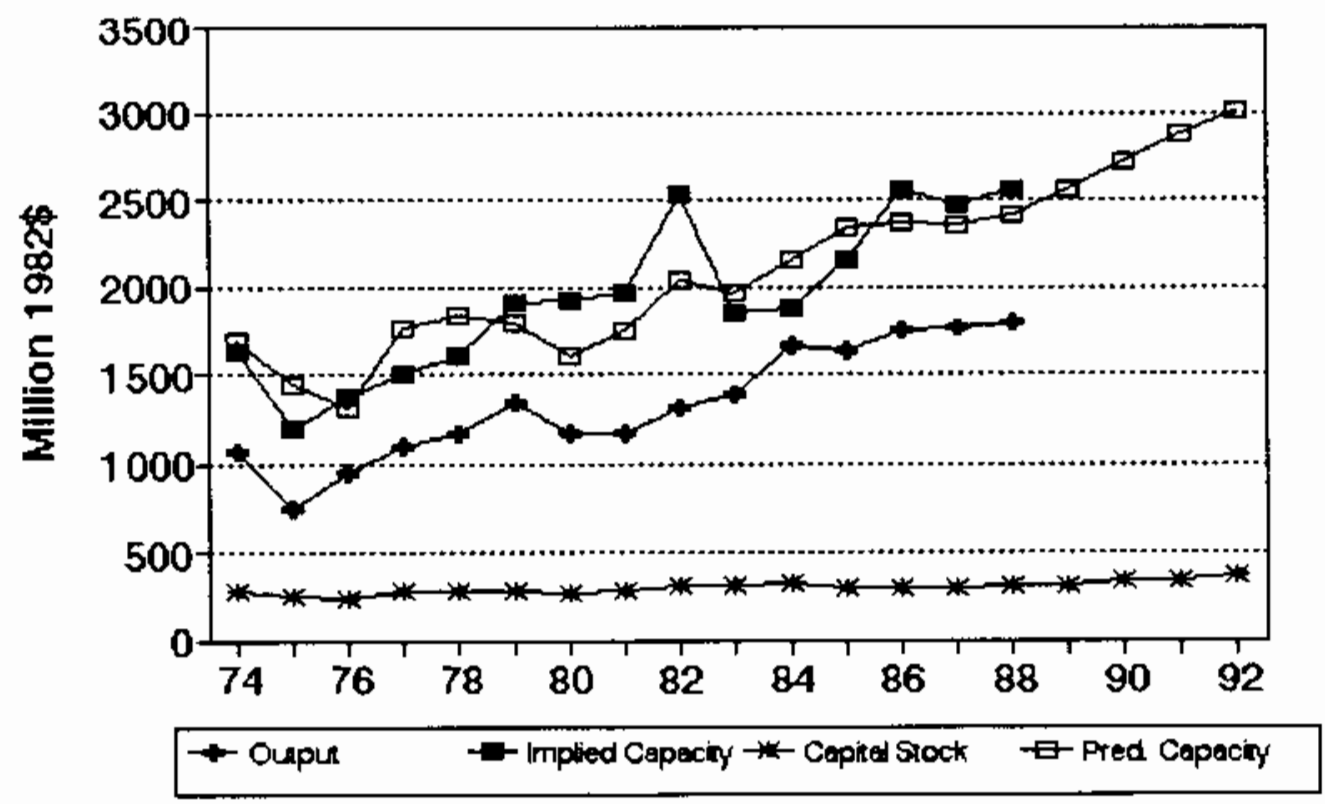




\section{No. 397 sIc 3711 wotor vehicles and car bodie:}

MILLIOWS OF 1982 DOLLARS (EXCL. CU RATES)

$\begin{array}{lccccccc} & 04 & \text { Ann. Implied Gross } & \text { Net } & \text { Pred. } & \ldots & \ldots \ldots \\ \text { Gross } & \text { CU } & \text { CU } & \text { Prac. } & \text { In- } & \text { Cap. } & \text { Prac. } & \text { Emergency Capacity } \\ \text { Output } & \text { Rate } & \text { Rate } & \text { Capac. } & \text { vest. } & \text { Stk. } & \text { Capac. } & 3 \text { mon. } 6 \text { mon. }>6 \text { mon. }\end{array}$

\begin{tabular}{|c|c|c|c|c|c|c|c|c|c|c|}
\hline 1974 & 79,410 & 68 & 70.1 & 113,284 & 631.5 & 7,703 & 114,964 & & & \\
\hline 1975 & 74,822 & 76 & 65.3 & 114,591 & $1,395.4$ & 8,495 & 117,843 & & & \\
\hline 1976 & 97.828 & 85 & 84.4 & 115,901 & 972.9 & 8,894 & 119,294 & & & \\
\hline 1977 & 111,815 & 90 & 90.8 & 123,184 & $1,219.1$ & 9,561 & 121,720 & & & \\
\hline 1978 & 116,086 & 96 & 91.8 & 126,528 & $1,288.8$ & 10,298 & 124,404 & & & \\
\hline 1979 & 107,165 & 74 & 84.9 & 126,269 & $1,117.7$ & 10,852 & 126,416 & & & \\
\hline 1980 & 75,929 & 50 & 54.3 & 139,764 & 771.4 & 11,057 & 127,162 & & & \\
\hline 1981 & 77,608 & 52 & 58.5 & 132,776 & $3,214.2$ & 13,620 & 136,487 & & & \\
\hline 1982 & 70,950 & 49 & 53.5 & 132,518 & 254.4 & 13,194 & 134,936 & & & \\
\hline 1983 & 94,097 & 74 & 69.2 & 135,950 & 745.3 & 13.183 & 134,896 & & & \\
\hline 1984 & 112,309 & 85 & 83.3 & 134.859 & $1,762.7$ & 14,095 & 138,214 & & & \\
\hline 1985 & 114,085 & 78 & 85.1 & 134,017 & 982.9 & 14,178 & 138,517 & & & \\
\hline 1986 & 112,916 & 76 & 77.5 & 145,736 & 1.515 .4 & 14,730 & 140,523 & & & \\
\hline-- &.--- & $\because$ & $-\cdot$ & $-\cdot-\cdot$ & $\cdot \cdot \cdot$ & --- & $-\cdot \cdot-$ & $-\cdots$ & --- & $\cdots$ \\
\hline 1987 & 115,131 & 80 & 76.6 & 150,359 & $1,146.1$ & 14,927 & 141,239 & & & \\
\hline 1988 & 118,073 & 83 & 78.2 & 150,948 & $1,268.6$ & 15,233 & 142,355 & 191,695 & 245,787 & 246,558 \\
\hline 1989 & & & & & $1,276.3$ & 15,528 & 143,426 & 193,138 & 247.638 & 248,414 \\
\hline 1990 & & & & & $1,396,1$ & 15,923 & 144,865 & 195,075 & 250,121 & 250,905 \\
\hline 1991 & & & & & $1,460.6$ & 16,358 & 146,445 & 197,203 & 252,849 & 253,642 \\
\hline 1992 & & & & & $1,511.2$ & 16,815 & 148,107 & 199,442 & 255,720 & 256,522 \\
\hline
\end{tabular}

Capacity-Capital Stock Regression: Code 0 RSO 0.780 Coefficients: Const 86941.9 Stock 3.6

Ave, Capacity/Stock (74-86): 11.2 Ave. Weekly Hours (High) 91.0 Shift Factor 1.73 Ave Heekly Hours (Low) 87.9 Shift Factor 1.79 Wote: Output, Investment, and Capital stock extrapolated for 1987 and 1988.

\section{Motor vehicles and car bodies}

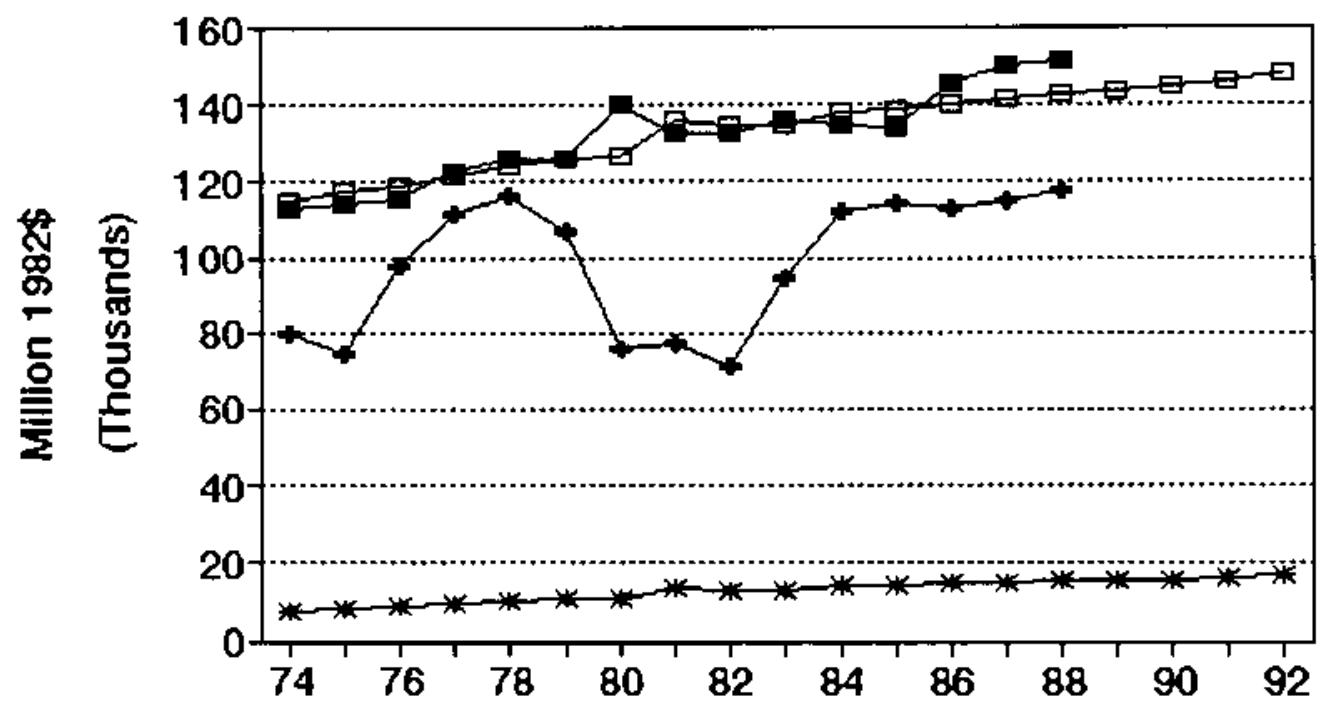

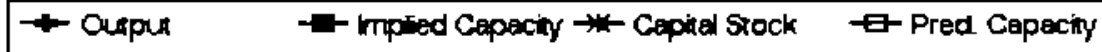


No. 398 sIC 3713 Truck and bus bodies

MILLIONS OF 1982 DOLLARS (EXCL. CU RATES)

$\begin{array}{lccccccc} & 04 & \text { Ann. Implied Gross } & \text { Net } & \text { Pred. } & \ldots \ldots . . . . . . \\ \text { Gross } & \text { CU } & \text { CU } & \text { Prac. } & \text { In. } & \text { Cap. } & \text { Prac. } & \text { Emergency Capacity } \\ \text { Output Rate } & \text { Rate } & \text { Capac. } & \text { vest. } & \text { Stk. } & \text { Capac. } & 3 \text { mon. } 6 \text { mon. }>6 \text { mon. }\end{array}$

\begin{tabular}{|c|c|c|c|c|c|c|c|c|c|c|}
\hline 1974 & 2,948 & 67 & 47.1 & 6,254 & $(4.3)$ & 353 & 6,878 & & & \\
\hline 1975 & 3,192 & 52 & 49.6 & 6,435 & 46.4 & 371 & $6,9 \pi$ & & & \\
\hline 1976 & 4,088 & 50 & 50.9 & 8,026 & 32.5 & 375 & 6,998 & & & \\
\hline 1977 & 5,710 & 56 & 53.9 & 10,581 & 71.6 & 418 & 7,221 & & & \\
\hline 1978 & 6,306 & 64 & 60.9 & 10,361 & 26.1 & 416 & 7,211 & & & \\
\hline 1979 & 4,405 & 87 & 78.7 & 5,597 & 8.7 & 398 & 7,119 & & & \\
\hline 1980 & 3,541 & 74 & 78.4 & 4,519 & 37.5 & 408 & 7,171 & & & \\
\hline 1981 & 3,679 & 58 & 64.5 & 5,704 & 52.5 & 430 & 7,288 & & & \\
\hline 1982 & 3,428 & 47 & 51.3 & 6.679 & 48.2 & 447 & 7,376 & & & \\
\hline 1983 & 4.792 & 58 & 54.1 & 8,857 & 32.3 & 448 & 7,381 & & & \\
\hline 1984 & 5,110 & 73 & 67.6 & 7,563 & 25.2 & 443 & 7,356 & & & \\
\hline 1985 & 5,142 & 80 & 7.8 & 6,611 & 38.1 & 451 & 7,394 & & & \\
\hline 1986 & 5,141 & 74 & 76.3 & 6,737 & 65.2 & 484 & 7,568 & & & \\
\hline$\because-$ & $-\cdot-$ & - & $-\cdots$ & $\cdots$ & $\cdots$ & -- & $\cdots$ & $\cdot \cdot$ & --- & --- \\
\hline 1987 & 5,214 & 84 & 80.1 & 6,512 & 36.5 & 487 & 7,589 & & & \\
\hline 1988 & 5,310 & 89 & 86.8 & 6,121 & 37.4 & 492 & 7,613 & 9,617 & 12,782 & 12,827 \\
\hline 1989 & & & & & 37.5 & 496 & 7,635 & 9,646 & 12,820 & 12,865 \\
\hline 1990 & & & & & 38.4 & 501 & 7,661 & 9.679 & 12,864 & 12,909 \\
\hline 1991 & & & & & 38.9 & 506 & 7,688 & 9,713 & 12,909 & 12,954 \\
\hline 1992 & & & & & 39.3 & 511 & 7.715 & 9.747 & 12,954 & 13,000 \\
\hline
\end{tabular}

Capacity-Capital Stock Regression: Code $\uparrow$ RSO 0.010

Coefficients: Const 5019.0 stock 5.3

Ave. Capacity/stock $(74-86): 17.3$

Ave. Weekly Hours (High) 93.4 Shift Factor 1.68 Ave Weekly Hours (Low) 84.9 Shift Factor 1.86 Wote: Output, Investment, and Capital Stock extrepolated for 1987 and 1988.

\section{Truck and bus bodies}

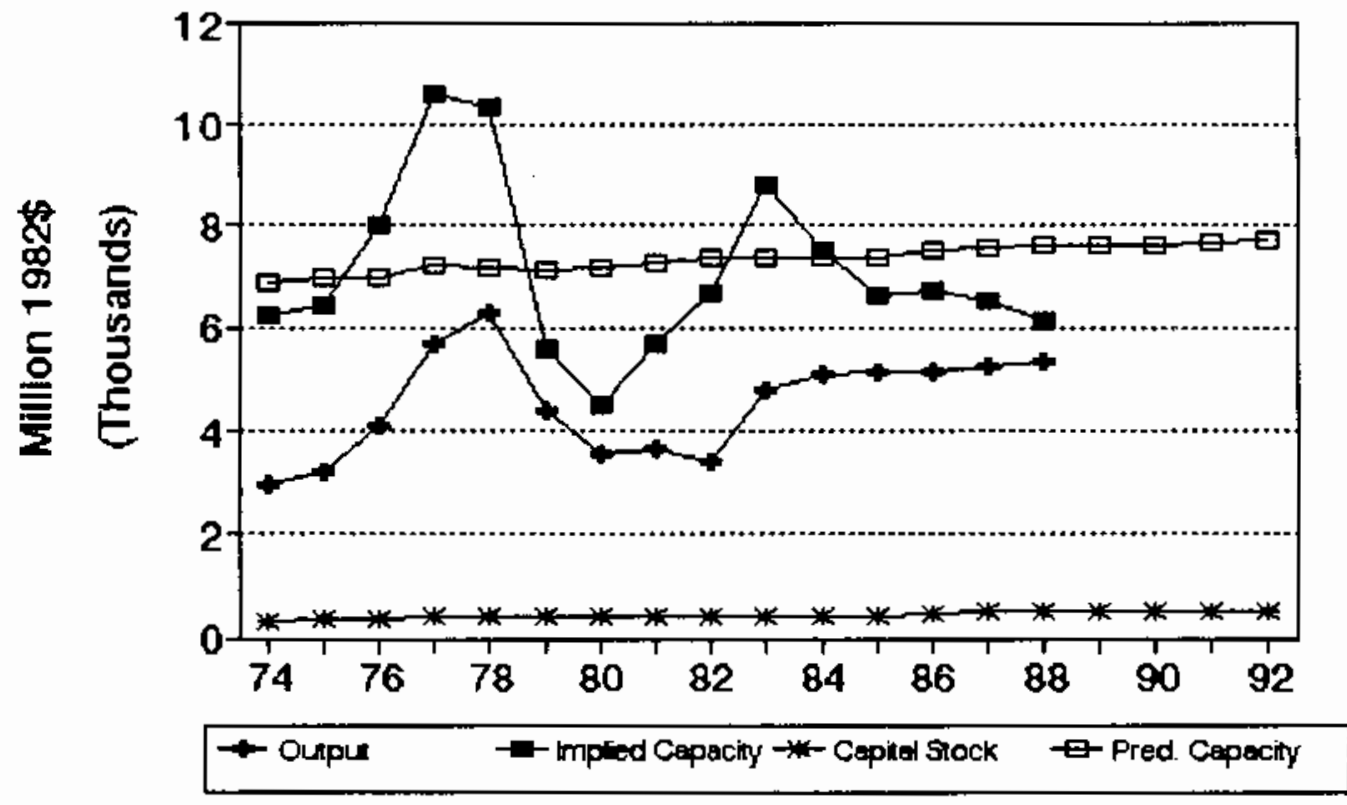


No. 399 sIC 3714 notor vehicle parta and accesaories

MILLIONS OF 1982 DOLLARS (EXCL. CU RATES)

$\begin{array}{lccccccc} & \text { Q4 } & \text { Amn. Implied Gross } & \text { Net } & \text { Pred. } & \ldots \ldots \ldots \ldots \\ \text { Gross } & \text { CU } & \text { CU } & \text { Prac. } & \text { In- } & \text { Cap. } & \text { Prac. } & \text { Emergency Capacity } \\ \text { Output Rate } & \text { Rate } & \text { Capac. } & \text { vest. } & \text { Stk. } & \text { Capac. } & 3 \text { mon. } 6 \text { mon. }>6 \text { mon. }\end{array}$

\begin{tabular}{|c|c|c|c|c|c|c|c|c|c|c|}
\hline 1974 & 54,534 & 69 & 71.0 & 76,855 & 733.2 & 13,116 & 73,108 & & & \\
\hline 1975 & 45,289 & 78 & 67.4 & 67,178 & $3,083.3$ & 15,220 & 72,841 & & & \\
\hline 1976 & 57,349 & 84 & 84.4 & 67,983 & 674.3 & 14,918 & 72,575 & & & \\
\hline 197 & 64,445 & 89 & 89.5 & 71,972 & $2,381.3$ & 16,276 & 72,308 & & & \\
\hline 1978 & 67,537 & 91 & 88.4 & 76,377 & $2,337.4$ & 17,561 & 72,041 & & & \\
\hline 1979 & 60,944 & 70 & 80.1 & 76,100 & $2,390.5$ & 18,845 & 71,774 & & & \\
\hline 1980 & 44,122 & 51 & 54.0 & 81,725 & $2,717.2$ & 20,369 & 71,508 & & & \\
\hline 1981 & 40,431 & 55 & 60.2 & 67,187 & $2,248.2$ & 21,372 & 71,241 & & & \\
\hline 1982 & 35,911 & 50 & 54.9 & 65,384 & 1.472 .5 & 21,485 & 70,974 & & & \\
\hline 1983 & 43,975 & 70 & 66.6 & 65,987 & 153.6 & 20,250 & 70,707 & & & \\
\hline 1984 & 52,034 & 76 & 75.8 & 68,657 & 917.7 & 19,735 & 70,441 & & & \\
\hline 1985 & 56,308 & 76 & 79.8 & 70,541 & $2,388.0$ & 20,591 & 70,174 & & & \\
\hline 1986 & 55,536 & 74 & 75.4 & 73,653 & $1,769.7$ & 20,740 & 69,907 & & & \\
\hline$-\cdots$ & $-\cdots$ & $\cdot$ & -- & $\cdots \cdot$ & --- & $\cdots \cdot$ & $\cdot \cdot$ & ---- & 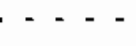 & $-\cdot-\cdot$ \\
\hline 1987 & 56,488 & 74 & 72.3 & 78,108 & $1,687.3$ & 20,845 & 69,640 & & & \\
\hline 1988 & 57,749 & 80 & 74.5 & 77,471 & $1,819.5$ & 21,075 & 69,374 & 87,664 & 109,644 & 109,957 \\
\hline 1989 & & & & & $1,827.9$ & 21,295 & 69,107 & 87,327 & 109,222 & 109,534 \\
\hline 1990 & & & & & $1,957.1$ & 21,628 & 68,840 & 86,990 & 108,801 & 109,112 \\
\hline 1991 & & & & & $2,026.7$ & 22,005 & 68,573 & 86,653 & 108,379 & 108,689 \\
\hline 1992 & & & & & $2,081,4$ & 22,408 & 68,307 & $B 6,316$ & 107,958 & 108,266 \\
\hline
\end{tabular}

Capacity-Capital Stock Regression: Code -2 RSO 0.040

Coefficients: Const 73374.8 Time -266.8

Ave. Capacity/Stock $(74-86): 3.9$

Ave. Heekly Hours (High) 99.4 Shift Factor 1.59 Ave Heekly Hours (Low) 97.2 Shift Factor 1.62 Note: Output, Imestment, and Capital stock extrapolated for 1987 and 1988.

\section{Motor vehicle parts and accessorie}

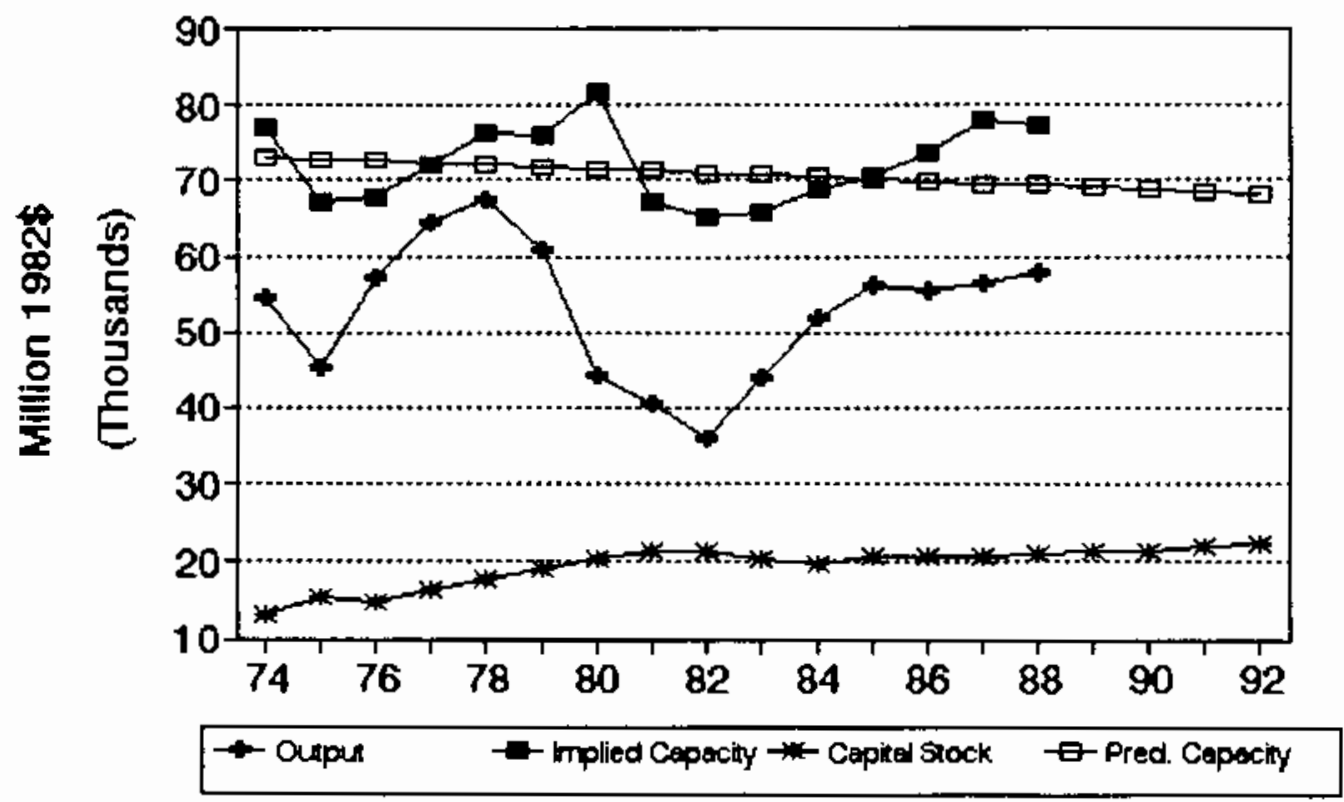




\section{No. 400 sIC 3715 Truck trailers}

MILLIONS OF 1982 DOLLARS (EXCL. CU RATES)

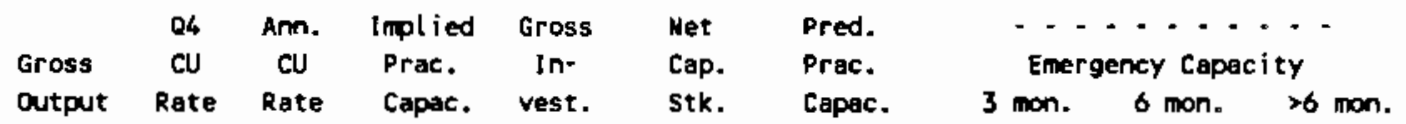

\begin{tabular}{|c|c|c|c|c|c|c|c|c|c|c|}
\hline 1974 & 3,413 & 70 & 71.8 & 4.756 & 53.7 & 298 & 4,087 & & & \\
\hline 1975 & 1,649 & 44 & 48.4 & 3,409 & 35.4 & 312 & 4,184 & & & \\
\hline 1976 & 2,218 & 65 & 58.5 & 3,794 & 1.6 & 292 & 4,046 & & & \\
\hline 1977 & 3,100 & 75 & 72.9 & 4,250 & 51.8 & 321 & 4,248 & & & \\
\hline 1978 & 3,706 & $B 0$ & 76.7 & 4,830 & 55.0 & 351 & 4,461 & & & \\
\hline 1979 & 4,153 & 67 & 73.8 & 5,627 & 41.1 & 365 & 4,565 & & & \\
\hline 1980 & 2,914 & 64 & 61.2 & 4,759 & 32.3 & 371 & 4,604 & & & \\
\hline 1984 & 2,314 & 53 & 62.5 & 3,704 & 34.2 & 378 & 4,656 & & & \\
\hline 1982 & 1,820 & 36 & 44.2 & 4,115 & $(15.5)$ & 338 & 4,372 & & & \\
\hline 1983 & 2,162 & 71 & 59.7 & 3,624 & 23.9 & 336 & 4,360 & & & \\
\hline 1984 & 3,189 & 58 & 64.5 & 4,943 & 17.6 & 328 & 4,301 & & & \\
\hline 1985 & 2,742 & 62 & 63.2 & 4,342 & 52.4 & 352 & 4,471 & & & \\
\hline 1986 & 2,565 & 51 & 55.5 & 4,619 & 21.9 & 345 & 4,419 & & & \\
\hline - - - & 2,595 & 62 & $\because \ddot{56.8}$ & $\begin{array}{l}\cdots \\
4,571\end{array}$ & $\begin{array}{l}--- \\
30.8\end{array}$ & $\begin{array}{l}-- \\
347\end{array}$ & $\because \cdots$ & - - - & $-\cdot$ & $\cdots$ \\
\hline 1988 & 2,635 & 70 & 64.5 & 4,082 & 33.3 & 352 & 4,467 & 5,195 & 7,076 & 7,103 \\
\hline 1989 & & & & & 33.5 & 356 & 4,498 & 5,231 & 7,125 & 7,152 \\
\hline 1990 & & & & & 35.9 & 362 & 4,544 & 5,284 & 7,197 & 7,224 \\
\hline 1991 & & & & & 37.2 & 370 & 4,595 & 5,343 & 7,278 & 7,306 \\
\hline 1992 & & & & & 38.2 & 377 & 4,649 & 5,406 & 7,364 & 7,392 \\
\hline
\end{tabular}

Capacity-Capital Stock Regression: Code 0 RSQ 0.090 Coefficients: Const 1971.5 stock 7.1

Ave. Cepecity/stock $(74-86): 12.9$ Ave. Ueekly Hours (High) 99.1 Shift Factor 1.59 Ave Heekly Hours (LOW) 93.4 Shift Factor 1.69 Note: Output, Investment, and Capital stock extrapolated for 1987 and 1988.

\section{Truck trailers}

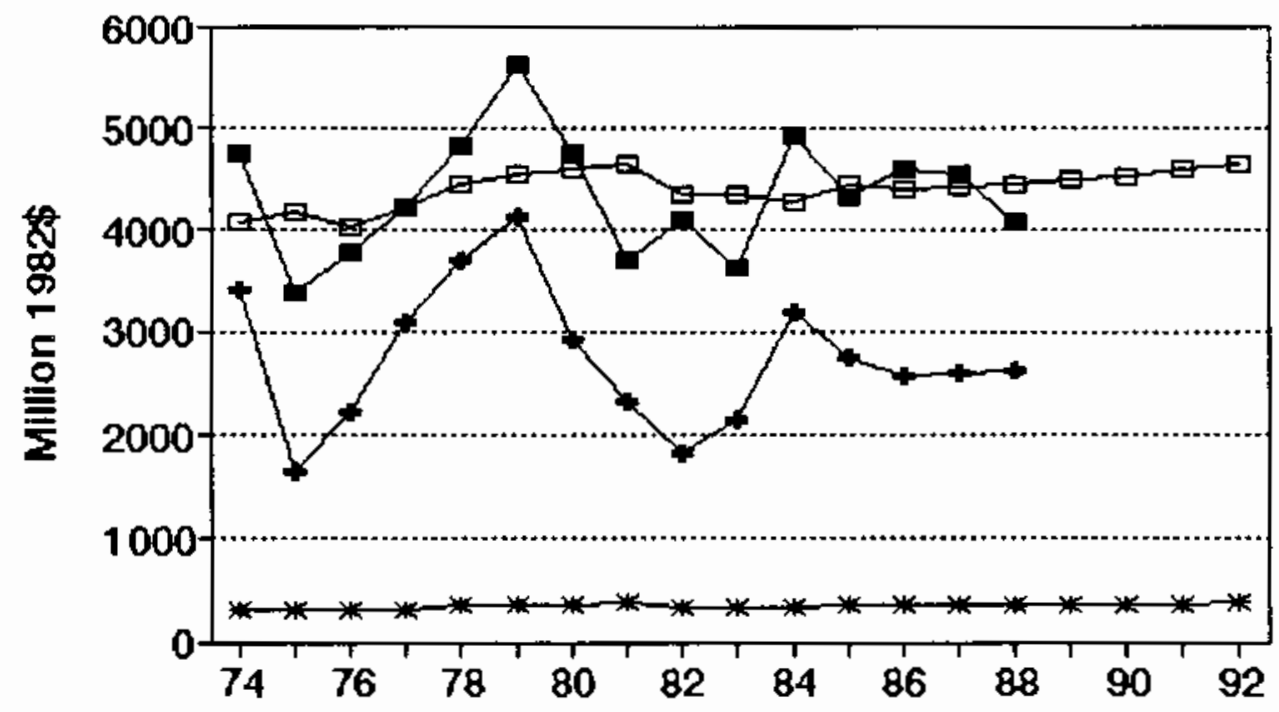

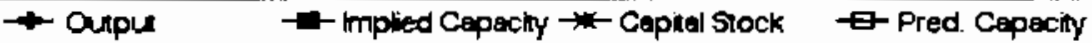




\section{yo. 401 SIC 3721 Aircraft}

MILLIONS OF 1982 DOLLARS (EXCL, CU RATES)

$\begin{array}{lccccccc} & \text { Q4 } & \text { Ann. } & \text { Implied Gross } & \text { Net } & \text { Pred. } & \ldots \ldots \\ \text { Gross } & \text { CU } & \text { CU } & \text { Prac. } & \text { In- } & \text { Cap. } & \text { Prac. } & \text { Emergency Capacity } \\ \text { Output } & \text { Rate } & \text { Rate } & \text { Capac. } & \text { vest. } & \text { Stk. } & \text { Capac. } & 3 \text { mon. } 6 \text { mon. }>6 \text { mon. }\end{array}$

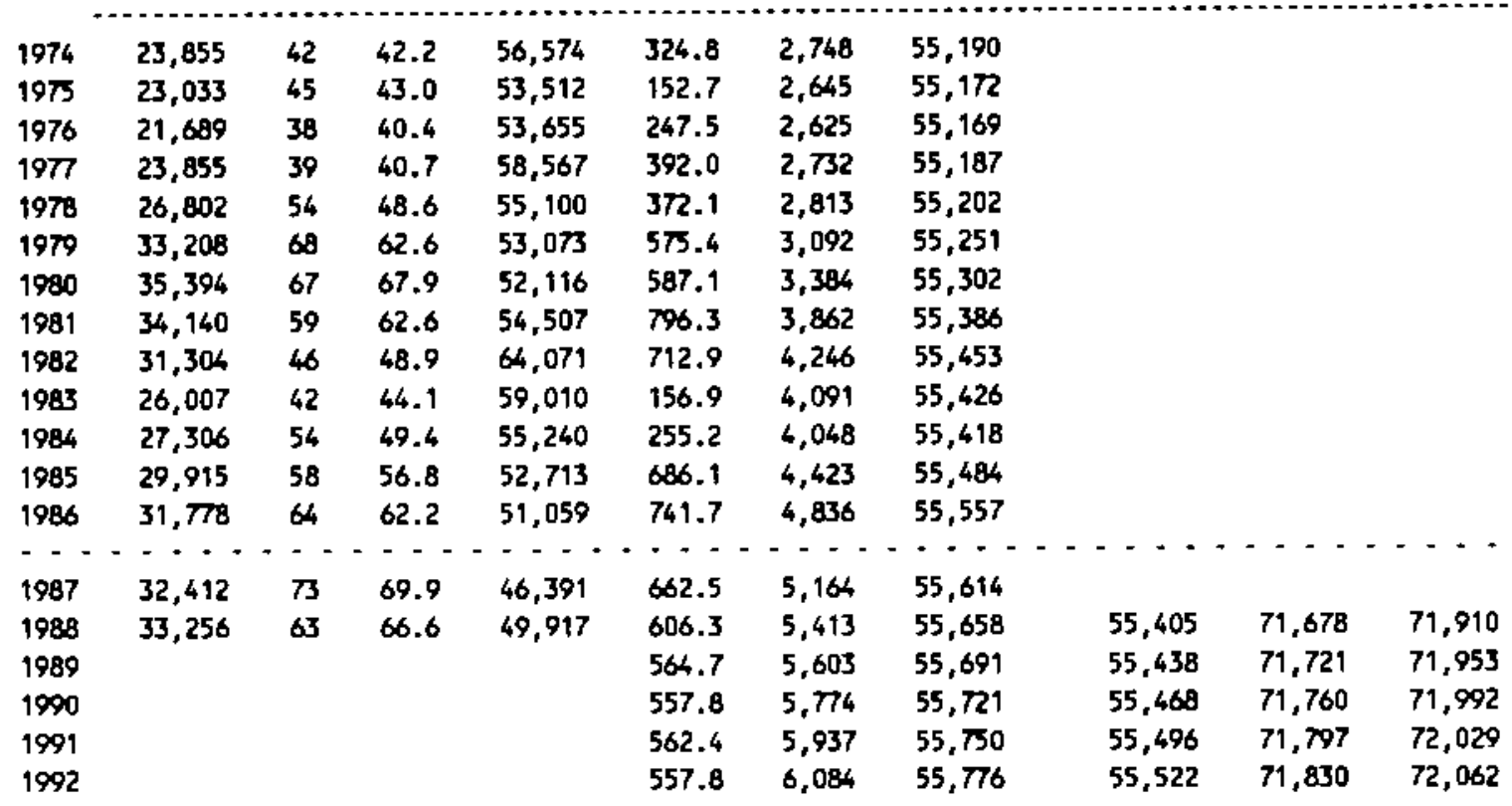

Capacity-Capital Stock Regression: Code 1 RSQ 0.000 Coefficients: Const 54707.6 Stock 0.2 Ave. Weekly Hours (High) 122.3 Shift Factor 1.29 Ave Heekly Hours (LOW) 121.2 Shift Factor 1.30 Mote: Output, Investment, and Capital Stock extrapolated for 1987 and 1988.

\section{Aircraft}

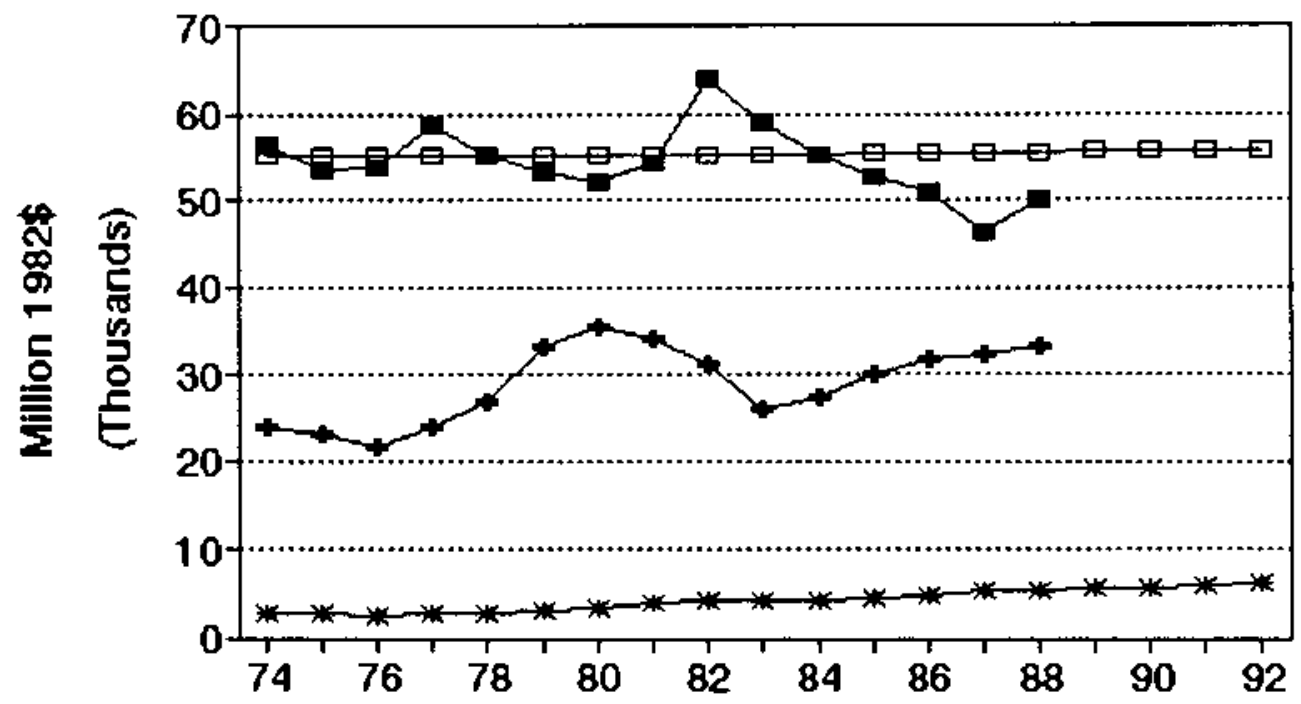

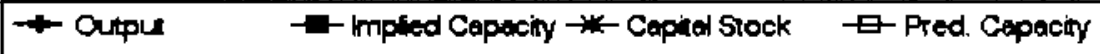


No. 402 sIC 3724 Aircraft engines and engine parts

MILLIOWS OF 1982 DOLLARS (EXCL. CU RATES)

$\begin{array}{lccccccc} & \text { QG } & \text { Ann. Implied Gross } & \text { Net } & \text { Pred. } & \ldots \ldots & \ldots \\ \text { Gross } & \text { CU } & \text { CU } & \text { Prac. } & \text { In- } & \text { Cap. } & \text { Prac. } & \text { Emergency Capacity } \\ \text { Output } & \text { Rate } & \text { Rate } & \text { Capac. } & \text { vest. } & \text { Stk. } & \text { Capac. } & 3 \text { mon. } 6 \text { mon. }>6 \text { mon. }\end{array}$

\begin{tabular}{|c|c|c|c|c|c|c|c|c|c|c|}
\hline 1974 & 12,025 & 59 & 59.1 & 20,353 & 265.8 & 2,496 & 19,138 & & & \\
\hline 1975 & 10,715 & 80 & 59.2 & 18,098 & 168.3 & 2,458 & 19,065 & & & \\
\hline 1976 & 10,181 & 56 & 57.4 & 17,732 & 177.7 & 2,436 & 19,021 & & & \\
\hline 1977 & 11,187 & 59 & 58.8 & 19,028 & 265.1 & 2,493 & 19,132 & & & \\
\hline 1978 & 12,246 & 60 & 59.8 & 20,480 & 325.0 & 2,604 & 19,351 & & & \\
\hline 1979 & 14.255 & 80 & 72.3 & 19,728 & 383.9 & 2,772 & 19,679 & & & \\
\hline 1980 & 16,108 & $\pi$ & 73.9 & 21,234 & 500.7 & 3,048 & 20,220 & & & \\
\hline 1981 & 15,068 & 69 & 70.8 & 21,296 & 653.8 & 3,454 & 21,016 & & & \\
\hline 1982 & 14,075 & 65 & 65.6 & 21,442 & 443.3 & 3,635 & 21,369 & & & \\
\hline 1983 & 12,936 & 58 & 60.9 & 21,242 & 410.4 & 3,774 & 29,642 & & & \\
\hline 1984 & 12,535 & 66 & 63.0 & 19,900 & 284.7 & 3,798 & 21,689 & & & \\
\hline 1985 & 14,483 & 63 & 66.2 & 22,552 & 667.9 & 4,186 & 22,449 & & & \\
\hline 1986 & 17,208 & 76 & 71.4 & 24,118 & 803.9 & 4,687 & 23,431 & & & \\
\hline-- & $-\cdots$ & $\cdot-$ & -- & $-\cdot \cdot$ & -- & $\cdots$ & $\cdots$ & $--\cdots$ & --- & . - \\
\hline 1987 & 17,543 & 65 & 69.2 & 25,350 & 665.0 & 5,044 & 24,130 & & & \\
\hline 1988 & 17,989 & 66 & 66.3 & 27,958 & 597.3 & 5,310 & 24,651 & 24,984 & 30,124 & 30,198 \\
\hline 1989 & & & & & 547.1 & 5,508 & 25,039 & 25,378 & 30,599 & 30,673 \\
\hline 1990 & & & & & 538.7 & 5,685 & 25,385 & 25,729 & 31,022 & 31,097 \\
\hline 1991 & & & & & 544.3 & 5,855 & 25,720 & 26,068 & 31,430 & 31,507 \\
\hline 1992 & & & & & 538.7 & 6,009 & 26,021 & 26,373 & 31,799 & 31,876 \\
\hline
\end{tabular}

Capecity-Capital Stock Regression: Code 0 RSO 0.720 Coefficients: Const 14249.9 stock 2.0

Ave. Capacity/Stock $(74-86): 6.4$ Ave. Ueekty Hours (High) 129.4 Shift Factor 1.23 Ave Ueekly Hours (Low) 127.1 Shift Factor 1.25 Note: Output, Investment, and Capital stock extrapolated for 1987 and 1988.

\section{Aircraft engines and engine parts}

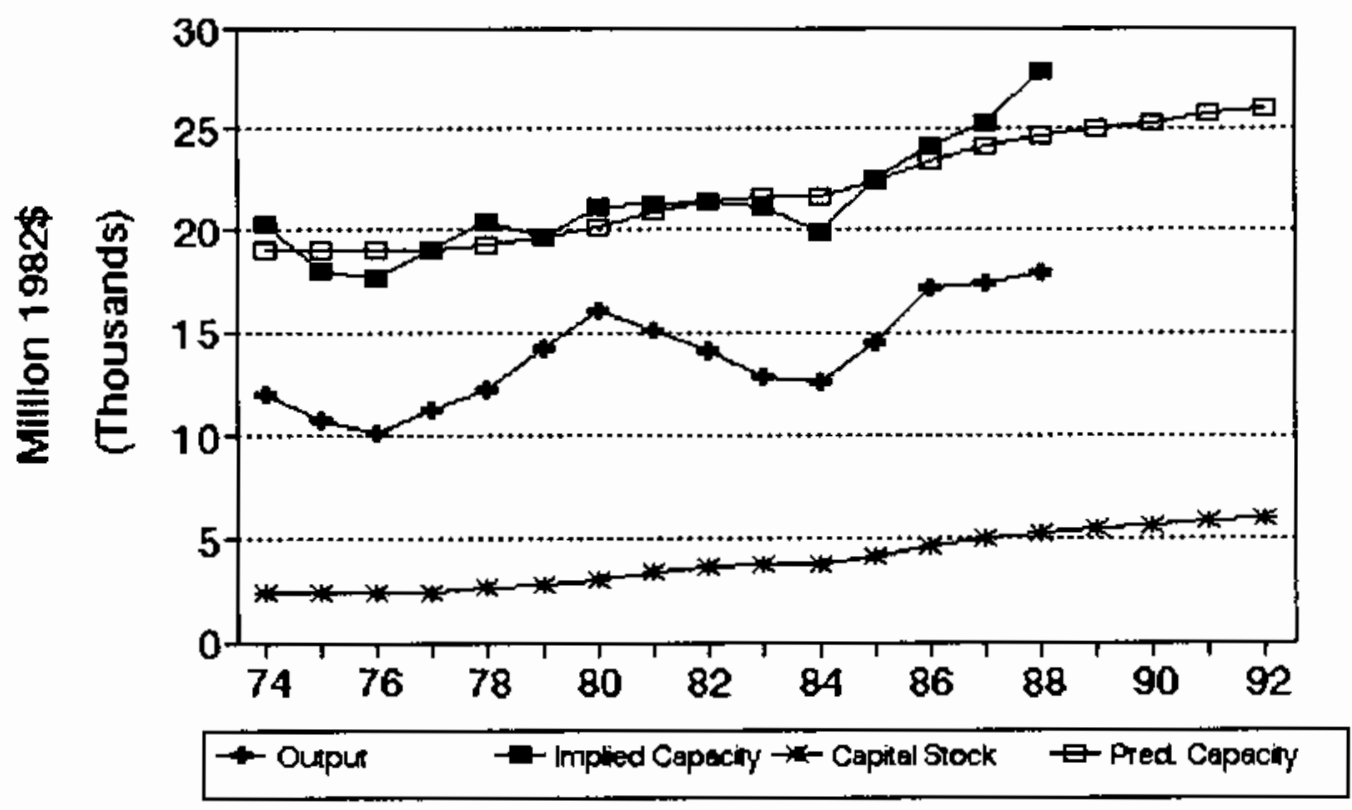




\section{No. 403 sIC 3728 Aircraft equipment, nec}

MILLIONS OF 1982 DOLLARS (EXCL. CU RATES)

$\begin{array}{lccccccc} & \text { Q4 } & \text { Arm. Implied Gross } & \text { Het } & \text { Pred. } & \ldots & \ldots \\ \text { Gross } & \text { CU } & \text { CU } & \text { Prac. } & \text { In- } & \text { Cap. } & \text { Prac. } & \text { Emergency Capacity } \\ \text { Output Rate } & \text { Rate } & \text { Capac. } & \text { vest. } & \text { Stk. } & \text { Capac. } & 3 \text { mon. } 6 \text { mon. } 26 \text { mon. }\end{array}$

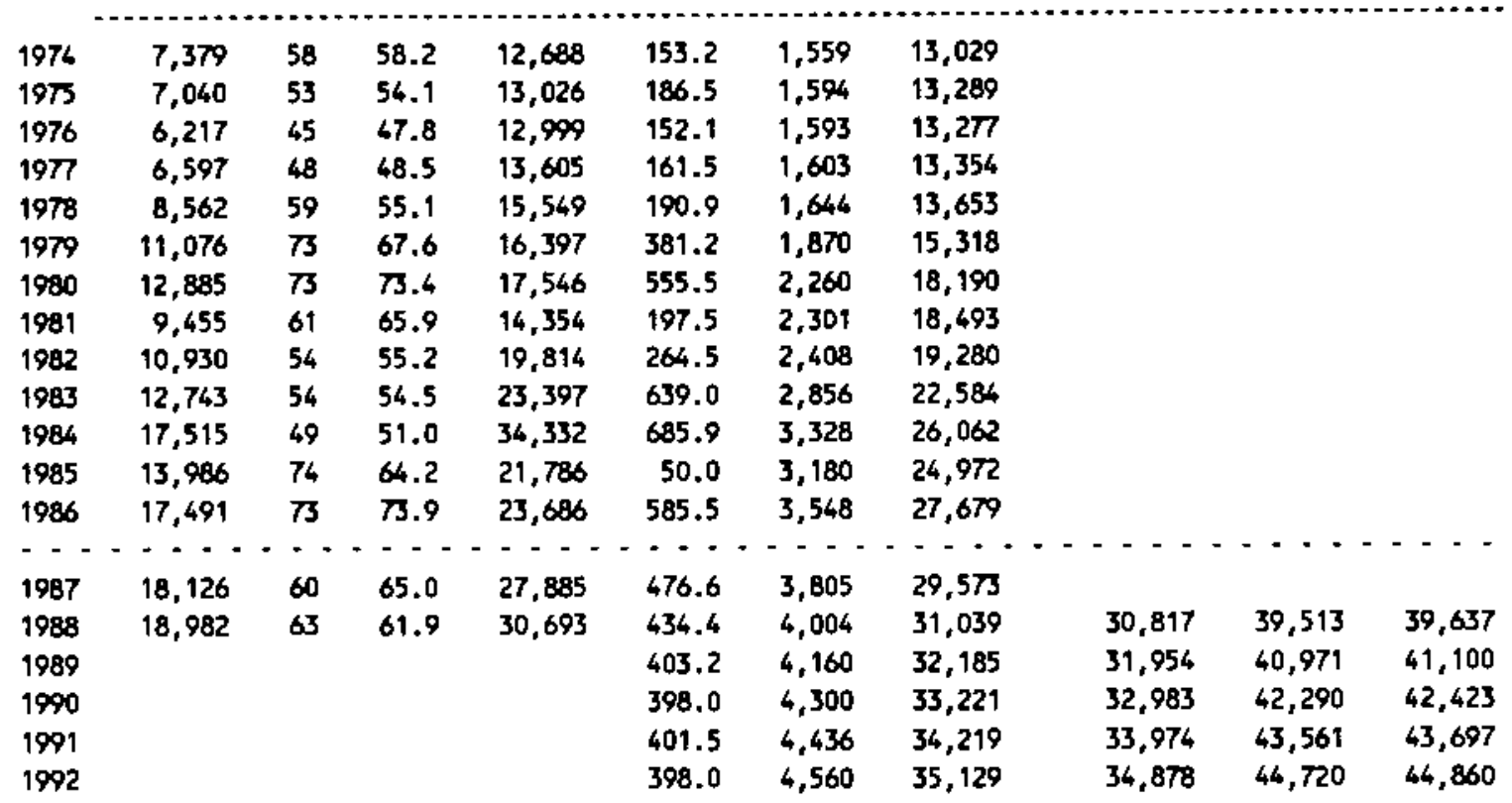

Capecity-Capital Stock Regression: Code 1 RSQ 0.750

Coefficients: Const 1548.0 stock 7.4

Ave. Capacity/Stock $(74-86): 8.0$ Ave. Weekly Hours (High) 123.9 Shift Factor 1.28 Ave Weekly Hours (Low) 120.8 Shift Factor 1.31 Note: Output, Investment, and Copital Stock extrapolated for 1987 and 1988.

\section{Aircraft equipment, nec}

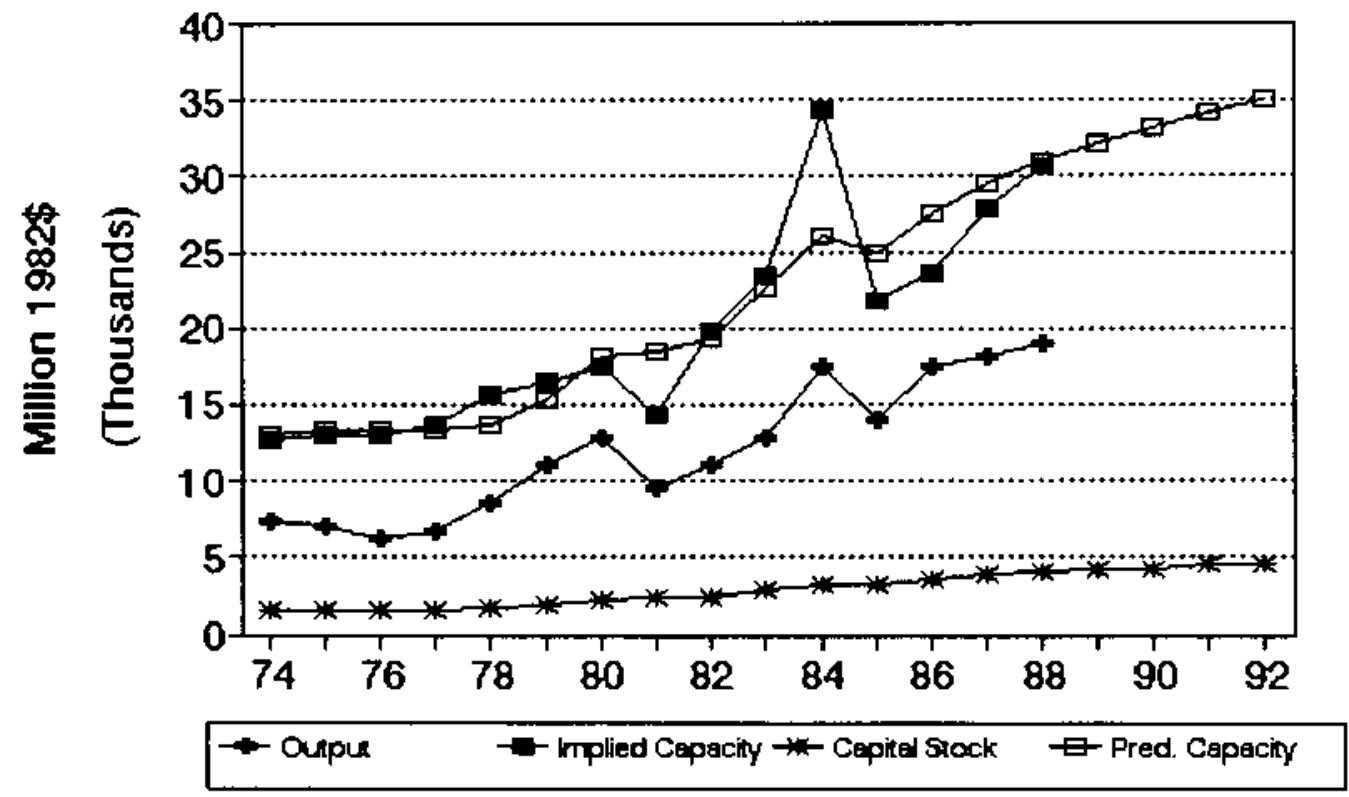




\section{No. 404 gIC 3731 Ship building and repairing}

MILLIOKS OF 1982 DOLLARS (EXCL. CU RATES)

$\begin{array}{lccccccc} & \text { Q4 } & \text { Ann. } & \text { Implied Gross } & \text { Net } & \text { Pred. } & \ldots \\ \text { Gross } & \text { CU } & \text { CU } & \text { Prac. } & \text { In- } & \text { Cap. } & \text { Prac. } & \text { Emergency Capecity } \\ \text { Output } & \text { Rate } & \text { Rate } & \text { Capec. } & \text { vest. } & \text { Stk. } & \text { Capec. } & 3 \text { mon. } 6 \text { mon. }>6 \text { mon. }\end{array}$

\begin{tabular}{|c|c|c|c|c|c|c|c|c|c|c|}
\hline 1974 & 9,081 & 66 & 66.0 & 13,759 & 331.2 & 1,814 & 13,590 & & & \\
\hline 1975 & 9,754 & 70 & 68.5 & 14,239 & 333.5 & 2,042 & 14,784 & & & \\
\hline 1976 & 9,352 & 65 & 66.9 & 13,984 & 419.4 & 2,347 & 16,382 & & & \\
\hline 1977 & 9,575 & $\pi$ & 69.4 & 13,802 & 32.8 & 2,258 & 15,914 & & & \\
\hline 1978 & 9,832 & 58 & 63.3 & 15,545 & 44.6 & 2,180 & 15,508 & & & \\
\hline 1979 & 9,740 & 65 & 62.4 & 15,616 & 220.2 & 2,263 & 15,942 & & & \\
\hline 1980 & $10, \pi 27$ & 66 & 65.6 & 16,346 & 146.0 & 2,261 & 15,931 & & & \\
\hline 1981 & 11,440 & 64 & 64.8 & 17,668 & 321.7 & 2,416 & 16,747 & & & \\
\hline 1982 & 10,592 & 53 & 57.1 & 18,541 & 42.5 & 2,297 & 16,118 & & & \\
\hline 1983 & 8,908 & 55 & 54.3 & 16,419 & 191.1 & 2,311 & 16,196 & & & \\
\hline 1984 & 9,082 & 53 & 53.8 & 16,897 & 71.7 & 2,207 & 15,649 & & & \\
\hline 1985 & 8,188 & 58 & 56.1 & 14,589 & 40.6 & 2,074 & 14,950 & & & \\
\hline 1986 & 7,675 & 48 & 51.8 & 14.830 & 97.6 & 1,992 & 14,524 & & & \\
\hline $\begin{array}{l}\cdots \\
1987\end{array}$ & 7,841 & 48 & $\begin{array}{l}\cdots \\
48.0\end{array}$ & $\cdots \cdots$ & $\begin{array}{l}--- \\
120.6\end{array}$ & $\cdots$ & 14,249 & $-\cdots$ & 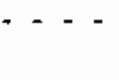 & $-\cdot-$ \\
\hline 1988 & 8,061 & 50 & 49.3 & 16,368 & 226.4 & 1,998 & 14,553 & 13,674 & 17,798 & 17,857 \\
\hline 1989 & & & & & 242.7 & 2,067 & 14,916 & 14,015 & 18,241 & 18,302 \\
\hline 1990 & & & & & 338.9 & 2,227 & 15,752 & 14,800 & 19,264 & 19,327 \\
\hline 1991 & & & & & 326.9 & 2,360 & 16,452 & 15,458 & 20,120 & 20,186 \\
\hline 1992 & & & & & 338.9 & 2,494 & 17,154 & 16,118 & 20,979 & 21,048 \\
\hline
\end{tabular}

Capecity-Capital Stock Regression: Code 0 RSQ 0.320

Coefficients: Const 4077.6 stock 5.2

Ave. Capecity/5tock (74-86): 7.1 Ave. Heekly Hours (High) 129.1 Shift Factor 1.23 Ave Weekly Hours (Low) 126.5 Shift factor 1.25 Mote: Output, Investment, and Capital Stock extrapolated for 1987 and 1988.

\section{Ship building and repairing}

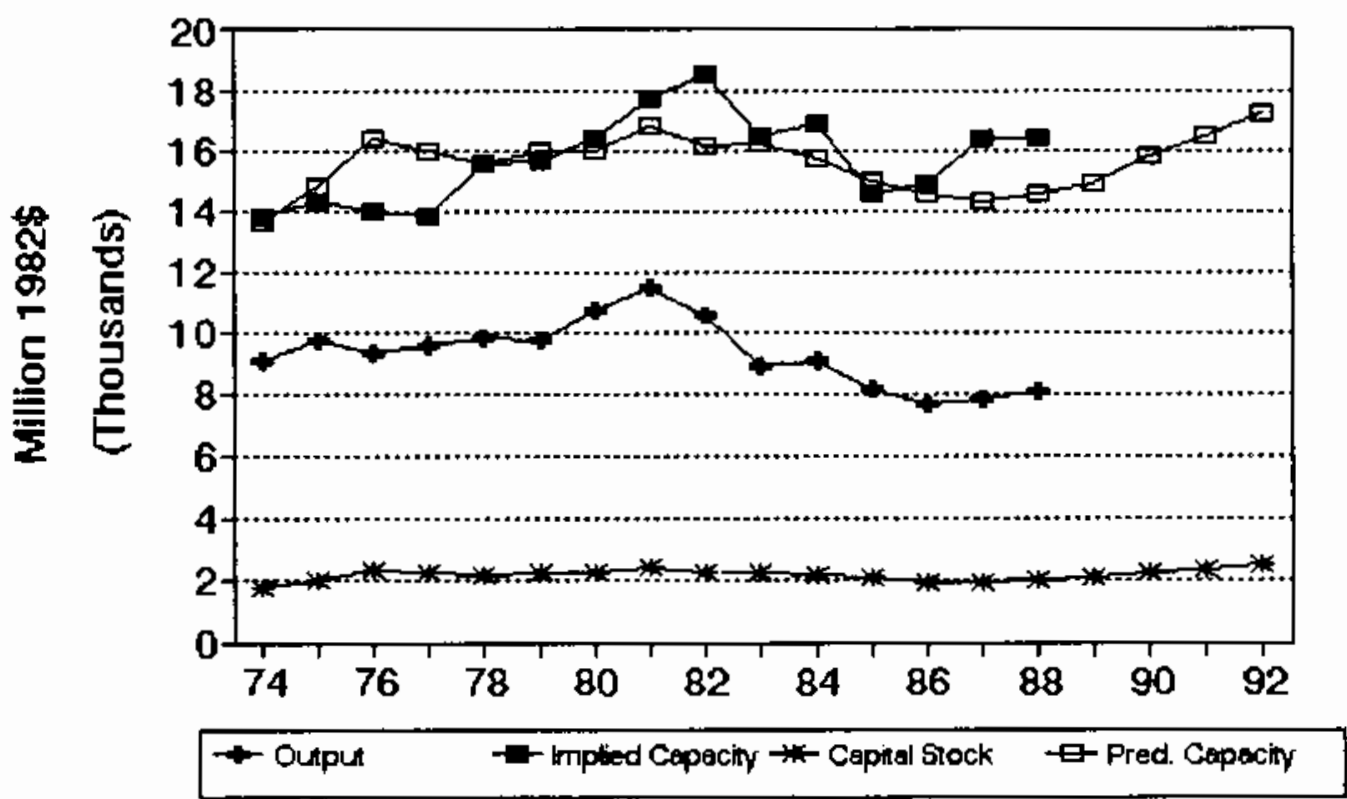




\section{No. 405 grc 3732 boat building and repairing}

MILLIONS OF 1982 DOLLARS (EXCL. CU RATES)

$\begin{array}{lccccccc} & \text { Q4 Am. Implied Gross } & \text { Net } & \text { Pred. } & \ldots \ldots \ldots \ldots \\ \text { Gross } & \text { CU } & \text { Cu } & \text { Prac. } & \text { In- } & \text { Cap. } & \text { Prac. } & \text { Emergency Capacity } \\ \text { Qutput Rate } & \text { Rate } & \text { Capac. } & \text { vest. } & \text { Stk. } & \text { Capac. } & 3 \text { mon. } 6 \text { mon. }>6 \text { mon. }\end{array}$

\begin{tabular}{|c|c|c|c|c|c|c|c|c|c|c|}
\hline 1974 & 1,857 & 49 & 50.7 & 3,663 & $(39.7)$ & 203 & 3,913 & & & \\
\hline 1975 & 2,333 & 62 & 50.6 & 4,607 & 37.9 & 223 & 4,118 & & & \\
\hline 1976 & 2,668 & 58 & 62.0 & 4,304 & 9.5 & 216 & 4,043 & & & \\
\hline 1977 & 2,801 & 69 & 66.7 & 4,197 & 36.8 & 236 & 4,253 & & & \\
\hline 1978 & 2,867 & 67 & 65.7 & 4,364 & 52.8 & 270 & 4,592 & & & \\
\hline 1979 & 2,656 & 65 & 68.2 & 3,892 & 48.6 & 297 & 4,871 & & & \\
\hline 1980 & 2,312 & 40 & 44.5 & 5,196 & 34.5 & 310 & 4,997 & & & \\
\hline 1981 & 2,367 & 45 & 45.2 & 5,235 & 39.6 & 325 & 5,158 & & & \\
\hline 1982 & 2,386 & 31 & 37.8 & 6,307 & 90.6 & 386 & 5,776 & & & \\
\hline 1983 & 2,540 & 40 & 37.4 & 6,790 & 35.6 & 389 & 5,810 & & & \\
\hline 1984 & 3,206 & 56 & 50.9 & 6,298 & 15.2 & 374 & 5,656 & & & \\
\hline 1985 & 3,156 & 62 & 60.8 & 5,188 & 45.2 & 387 & 5,789 & & & \\
\hline 1986 & 3,478 & 76 & 71.1 & 4,890 & 49.9 & 404 & 5,956 & & & \\
\hline - - - & $\cdots \cdots$ & $-\overline{-}$ & $-\cdots$ & $\because--$ & 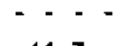 & $\because-$ & - - - - & $-\cdots$ & $\cdots$ & $\cdots$ \\
\hline 1987 & 3,504 & 65 & 69.3 & 5,058 & 61.3 & 432 & 6,239 & & & \\
\hline 1988 & 3,538 & 64 & 63.4 & 5,584 & 65.1 & 461 & 6,537 & 6,283 & 8,299 & 8,328 \\
\hline 1989 & & & & & 68.8 & 491 & 6,848 & 6,582 & 8,695 & 8,725 \\
\hline 1990 & & & & & 72.6 & 523 & 7,172 & 6,894 & 9,106 & 9,138 \\
\hline 1991 & & & & & 76.4 & 556 & 7,508 & 7,216 & 9,532 & 9,565 \\
\hline 1992 & & & & & 80.2 & 590 & 7,854 & 7,549 & 9.972 & 10,006 \\
\hline
\end{tabular}

Capacity-Capital stock Regression: Code 1 RSO 0.600

Coefficients: Const 1848.2 stock 10.2

Ave. Capacity/Stock $(74-86): 16.2$ Ave. Ueekly Hours (High) 124.2 Shift Factor 1.27 Ave Weekly Hours (Low) 101.3 Shift Factor 1.55 - Imputed Mote: Output, Investment, and Capital stock extrapolated for 1987 and 1988.

\section{Boat building and repairing}

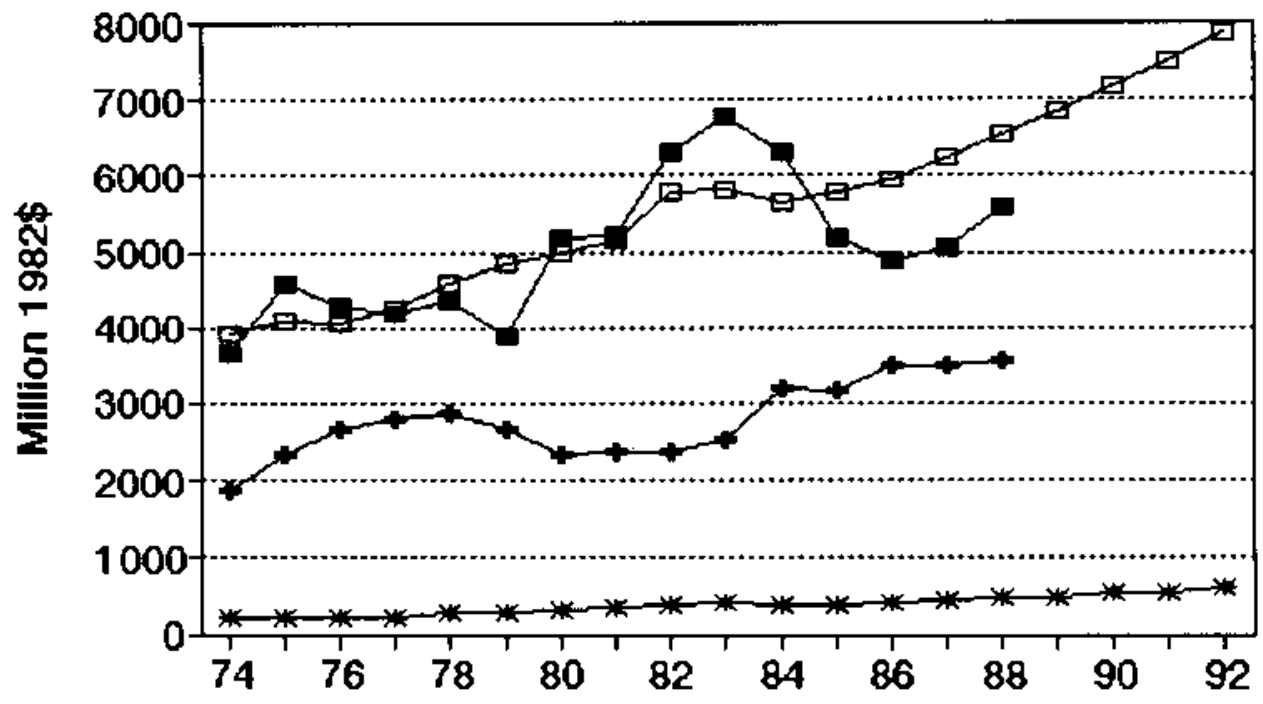

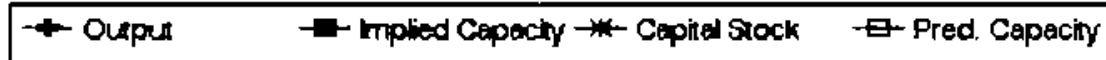




\section{No. 406 sIC 3743 Railroad equipent}

MILLIONS OF 1982 DOLLARS (EXCL. CU RATES)

$\begin{array}{lccccccc} & 04 & \text { Amn. } & \text { Implied } & \text { Gross } & \text { Net } & \text { Pred. } & -\ldots \\ \text { Gross } & \text { CU } & \text { CU } & \text { Prac. } & \text { In- } & \text { Cap. } & \text { Prac. } & \text { Emergency Capacity } \\ \text { Output Rate } & \text { Rate } & \text { Capac. } & \text { vest. } & \text { Stk. } & \text { Capac. } & 3 \text { mon. } 6 \text { mon. }>6 \text { mon. }\end{array}$

\begin{tabular}{|c|c|c|c|c|c|c|c|c|c|c|}
\hline 1974 & 7,577 & 80 & 81.9 & 9,255 & 75.2 & 990 & 7,146 & & & \\
\hline 1975 & 7,053 & 78 & 72.2 & 9,763 & 111.9 & 1,026 & 7,543 & & & \\
\hline 1976 & 5,640 & 64 & 71.4 & 7,904 & 84.5 & 1,033 & 7,619 & & & \\
\hline 1977 & 6,401 & 82 & 76.7 & 8,348 & 145.7 & 1,094 & 8,307 & & & \\
\hline 1978 & 7,655 & 89 & 84.5 & 9.055 & 139.3 & 1,147 & 8,893 & & & \\
\hline 1978 & 9,828 & 83 & 87.1 & 11,288 & 166.0 & 1,222 & 9,732 & & & \\
\hline 1980 & 8,238 & 69 & 69.7 & 11,813 & 107.8 & 1,237 & 9,903 & & & \\
\hline 1981 & 5,330 & 47 & 56.4 & 9,446 & 65.3 & 1,211 & 9,604 & & & \\
\hline 1982 & 3,372 & 29 & 36.6 & 9,203 & 78.2 & 1,195 & 9,425 & & & \\
\hline 1983 & 2,070 & 26 & 27.8 & 7,456 & 91.1 & 1,190 & 9,374 & & & \\
\hline 1984 & 2,855 & 47 & 39.6 & 7,216 & 23.2 & 1,121 & 8,606 & & & \\
\hline 1985 & 2,441 & 41 & 43.8 & 5,569 & 53.5 & 1,081 & 8,153 & & & \\
\hline 1986 & 2,212 & 39 & 39.9 & 5,552 & 42.6 & 1,028 & 7,561 & & & \\
\hline$-\cdots$ & $\cdots \cdots$ & - & -- & $\cdot \cdot \cdot$ & -- & $-\cdots$ & $\cdots$ & $\cdots$ & --- & $\cdots$ \\
\hline 1987 & 2,235 & 64 & 54.4 & 4,110 & 35.8 & 971 & 6,927 & & & \\
\hline 1988 & 2,266 & 55 & 57.7 & 3,929 & 48.8 & 932 & 6,496 & 7,418 & 11,204 & 11,258 \\
\hline 1989 & & & & & 37.2 & 885 & 5,974 & 6,822 & 10,303 & 10,353 \\
\hline 1990 & & & & & 77.1 & 883 & 5,945 & 6,788 & 10,252 & 10,302 \\
\hline 1991 & & & & & 57.1 & 860 & 5,695 & 6,503 & 9,822 & 9,869 \\
\hline 1992 & & & & & 67.1 & 850 & 5,570 & 6,371 & 9,622 & 9,668 \\
\hline
\end{tabular}

Capecity-Capital Stock Regression: Code 1 RSQ 0.250

Coefficients: Const -3909.6 stock 11.2

Ave. Capacity/stock $(74-86): 7.7$ Ave. Heekly Hours (High) 90.9 Shift Factor 1.73 Ave Weekly Hours (Low) 88.5 Shift Factor 1.78 Note: Qutput, Investment, and Capital Stock extrapolsted for 1987 and 1988.

\section{Railroad equipment}

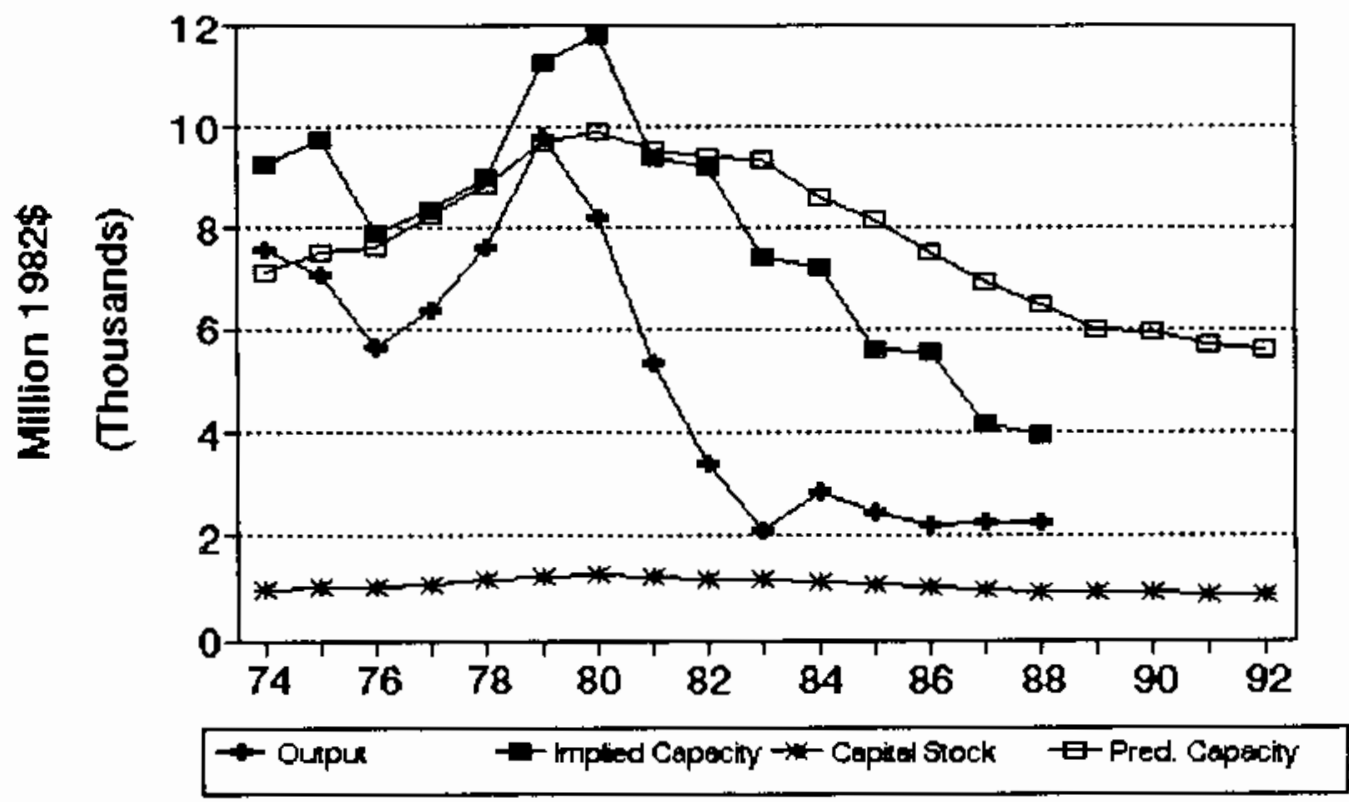


40. 408 sIC 3761 Guided missiles and space rehicles

MILLIONS OF 1982 DOLLARS (EXCL. CU RATES)

$\begin{array}{lccccccc} & \text { Q4 } & \text { Ann. Implied Gross } & \text { Net } & \text { Pred. } & \ldots \ldots . . . \\ \text { Gross } & \text { CU } & \text { CU } & \text { Prac. } & \text { In- } & \text { Cap. } & \text { Prac. } & \text { Emergency Capacity } \\ \text { Output } & \text { Rate } & \text { Rate } & \text { Capac. } & \text { vest. } & \text { Stk. } & \text { Capac. } & \text { I mon. } 6 \text { mon. }>6 \text { mon. }\end{array}$

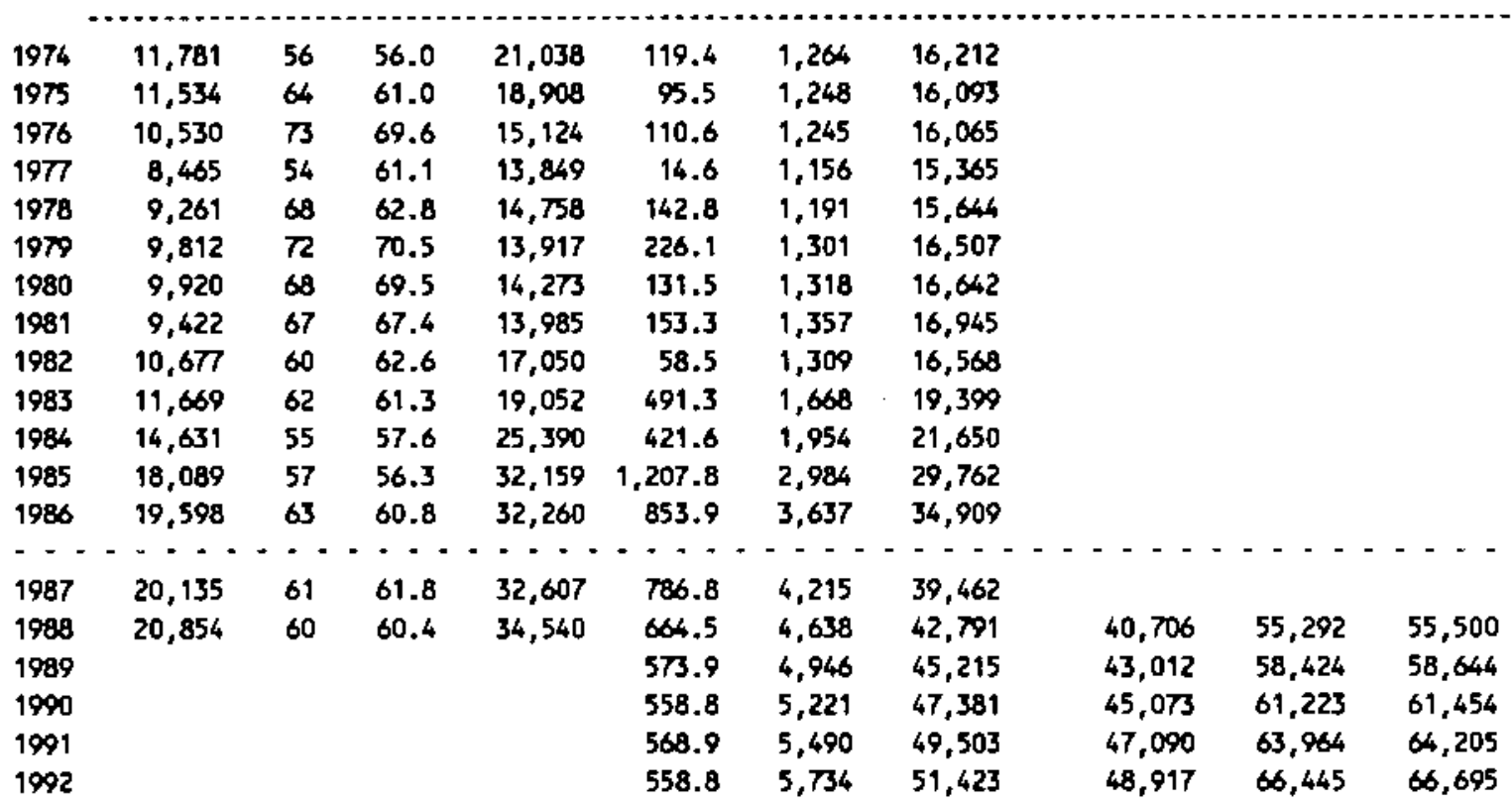

Capacity-Capital stock Regression: Code 0 RSO 0.840

Coefficients: Const 6258.9 stock 7.9

Ave. Capacity/Stock $(74-86): 11.6$ Ave. Heekly Hours (High) 121.9 Shift Factor 1.30 Ave Weekly Hours (LOW) 113.1 Shift Factor 1.39 Note: Output, Investment, and Capital Stock extrapolated for 1987 and 1988.

\section{Guided missiles and space vehicles}

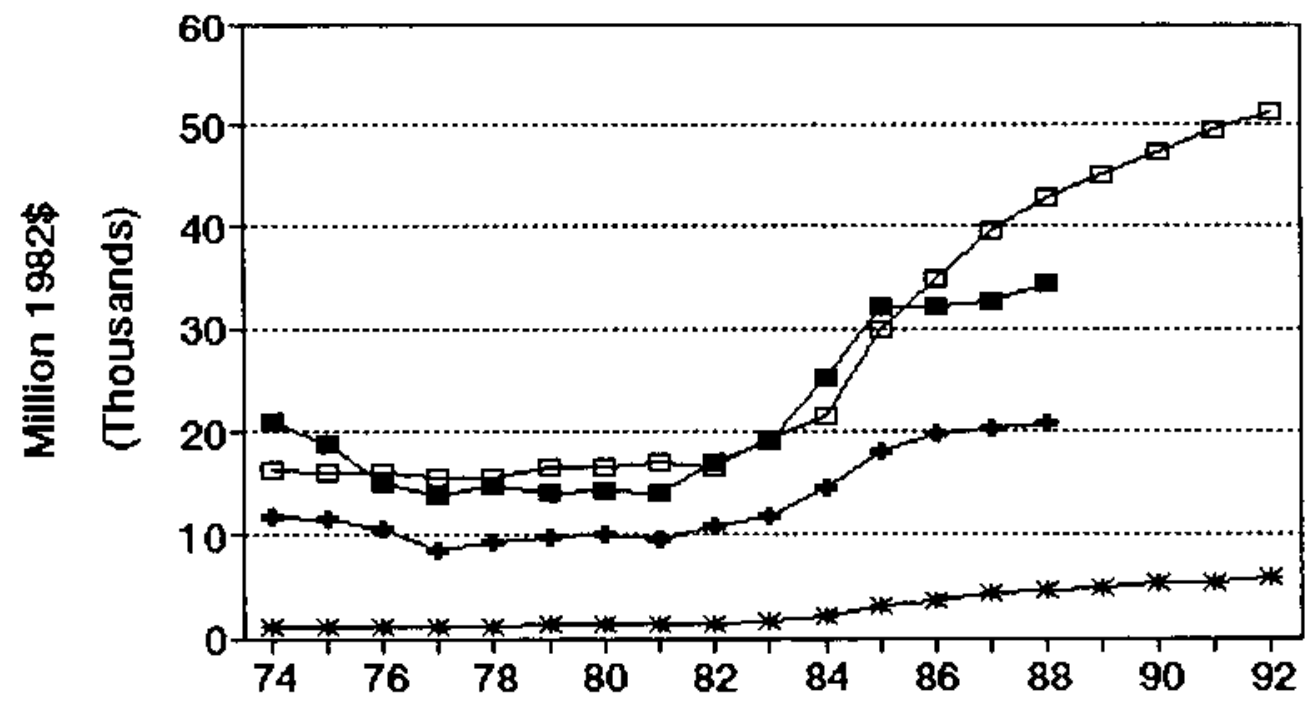

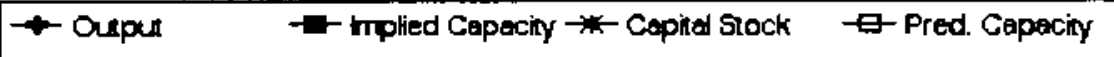




\section{No. 409 SIC 3764 space propulsion unita and parts}

MILLIONS OF 1982 DOLLARS (EXCL. CU RATES)

$\begin{array}{lccccccc} & Q 4 & \text { Amn. Implied } & \text { Gross } & \text { Het } & \text { Pred. } & \ldots & \ldots \ldots \\ \text { Gross } & \text { d } & \text { CU } & \text { Prac. } & \text { In- } & \text { Cap. } & \text { Prac. } & \text { Emergency Capacity } \\ \text { Output } & \text { Rate } & \text { Rate } & \text { Capac. } & \text { vest. } & \text { Stk. } & \text { Capac. } & 3 \text { mon. } 6 \text { mon. }>6 \text { mon. }\end{array}$

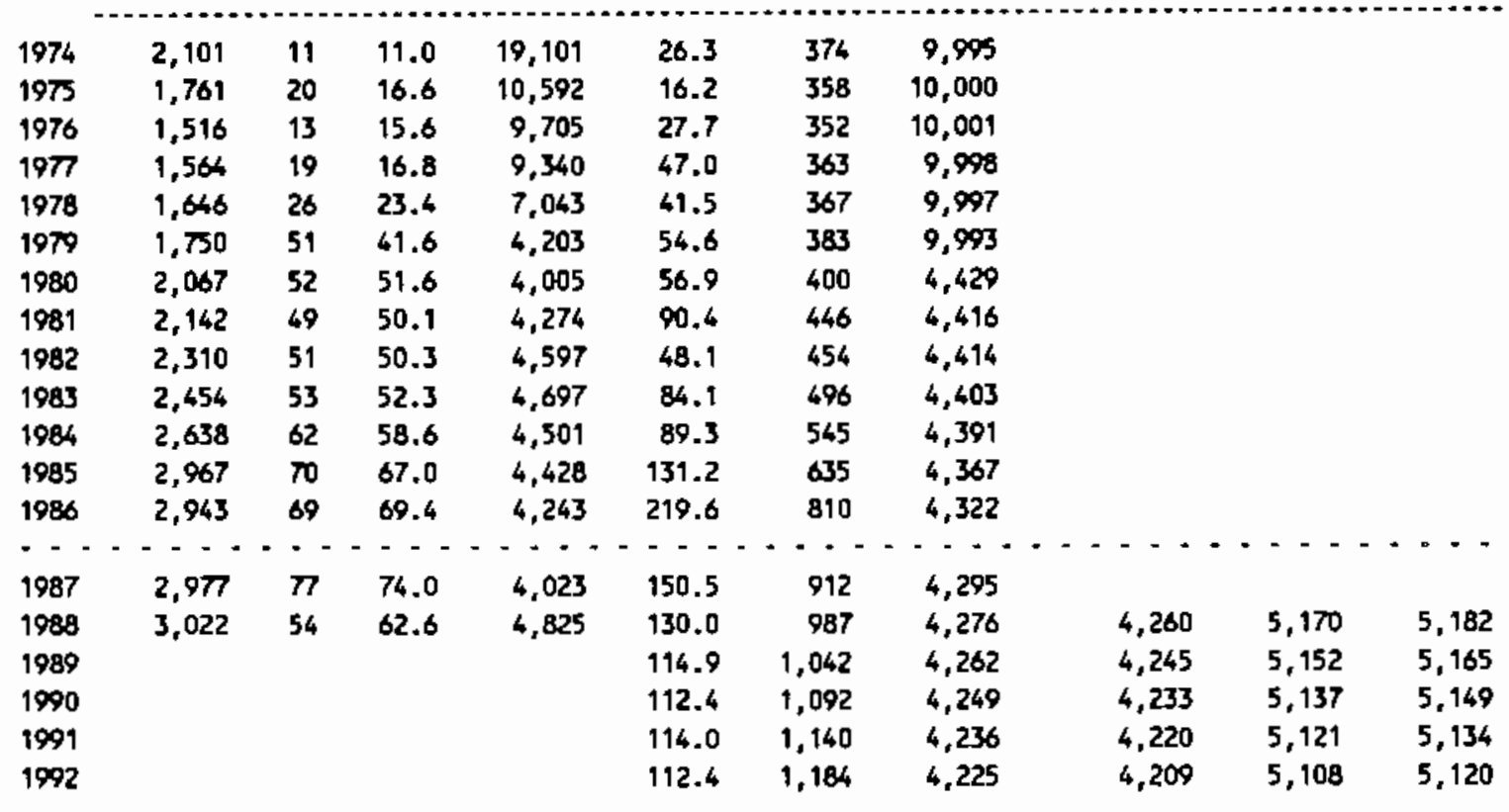

Capacity-Capital Stock Regression: Cade -1 RSQ 0.440

Coefficients: Const 10092.3 stock $-0.3 \quad 0(80-86) \quad-5560.1$ Ave, Capacity/Stock $(74-86): 15.2$ Ave. Heekly Hours (High) 130.9 shift Factor 1.21 Ave Heekly Hours (LOW) 124.7 Shift Factor 1.27 Note: Output, Investment, and Capital Stock extrapolated for 1987 and 1988.

\section{Space propulsion units and parts}

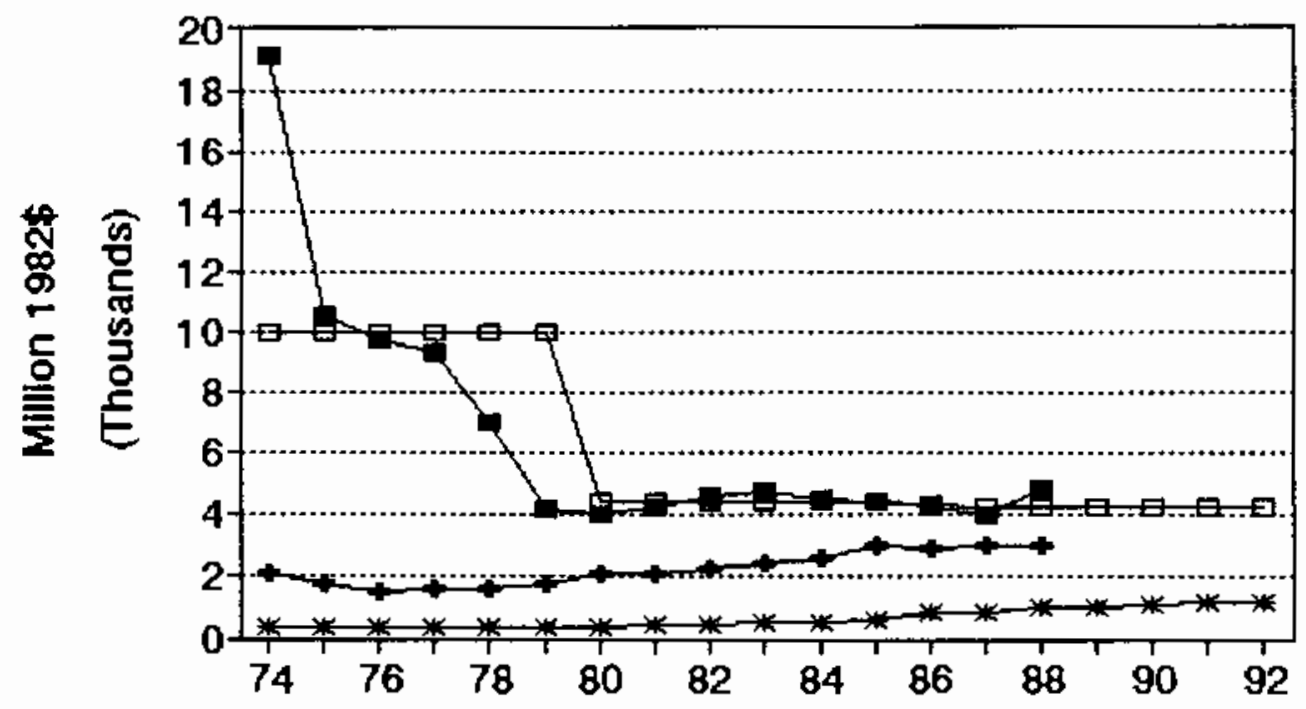

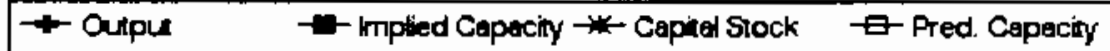


No. 410 SIC 3769 space vehicle equipment, nec

MILLIONS OF 1982 DOLLARS (EXCL. CU RATES)

$\begin{array}{lccccccc} & \text { Q4 } & \text { Ann. } & \text { Implied Gross } & \text { Net } & \text { Pred. } & -\ldots . \ldots \\ \text { Gross } & \text { CU } & \text { CU } & \text { Prac. } & \text { In- } & \text { Cap. } & \text { Prac. } & \text { Emergency Capacity } \\ \text { Output } & \text { Rate } & \text { Rate } & \text { Capac. } & \text { vest. } & \text { Stk. } & \text { Capac. } & 3 \text { mon. } 6 \text { mon. }>6 \text { mon. }\end{array}$

\begin{tabular}{|c|c|c|c|c|c|c|c|c|c|c|}
\hline 1974 & 1,319 & 68 & 68.6 & 1,922 & (8.5) & 271 & 3,220 & & & \\
\hline 1975 & 1,213 & 53 & 56.7 & 2,139 & $(10.6)$ & 235 & 2,718 & & & \\
\hline 1976 & 1,336 & 50 & 51.7 & 2,586 & 24.6 & 234 & 2,695 & & & \\
\hline 1977 & $4 \pi$ & 51 & 51.1 & 931 & $(103.5)$ & 118 & 1,067 & & & \\
\hline 1978 & 557 & 51 & 50.6 & 1,101 & 14.5 & 120 & 1,086 & & & \\
\hline 1979 & 621 & 50 & 50.7 & 1,224 & 16.9 & 122 & 1,122 & & & \\
\hline 1980 & 654 & 49 & 48.3 & 1,352 & 15.8 & 122 & 1,123 & & & \\
\hline 1981 & 1,754 & 63 & 58.3 & 3,006 & 123.7 & 216 & 2,444 & & & \\
\hline 1982 & 1,977 & 47 & 53.5 & 3,698 & 98.7 & 279 & 3,327 & & & \\
\hline 1983 & 2,241 & 47 & 47.2 & 4,750 & 97.7 & 337 & 4,156 & & & \\
\hline 1984 & 3,292 & 64 & 57.9 & 5,685 & 130.9 & 428 & 5,438 & & & \\
\hline 1985 & 2,973 & 67 & 66.2 & 4,495 & 71.7 & 460 & 3,328 & & & \\
\hline 1986 & 1,874 & 71 & 69.6 & 2,693 & 77.1 & 498 & 3,860 & & & \\
\hline-- & --- & -- & -- & $-\cdots$ & $-\cdots$ & $\cdots$ & $\cdots \cdot$ & $\cdots$ & -- & -- \\
\hline 1987 & 1,930 & 77 & 74.7 & 2,585 & 111.1 & 568 & 4,846 & & & \\
\hline 1988 & 2,006 & 69 & 71.7 & 2,800 & 93.1 & 614 & 5,497 & 4,816 & 6,881 & 6,910 \\
\hline 1989 & & & & & 79.8 & 643 & 5,908 & 5,176 & 7,304 & 7,426 \\
\hline 1990 & & & & & 77.6 & 668 & 6,253 & 5,478 & 7,826 & 7,860 \\
\hline 1991 & & & & & 79.1 & 692 & 6,590 & 5,774 & 8,248 & 8,284 \\
\hline 1992 & & & & & 77.6 & 712 & 6,879 & 6,026 & 8,610 & 8,647 \\
\hline
\end{tabular}

Capacity-Capital Stock Regression: Code -1 RSO 0.800

Coefficients: Const -601.8 stock $14.1 \quad \mathrm{D}(85-86) \quad-2562.7 \quad$ Ave. Capacity/Stock $(74-86): 10.3$ Ave. Weekly Hours (High) 125.9 Shift Factor 1.26 Ave Weekly Hours (Low) 115.5 Shift Factor 1.37 Mote: Output, Investment, and Capital stock extrapolated for 1987 and 1988.

\section{Space vehicle equipment, nec}

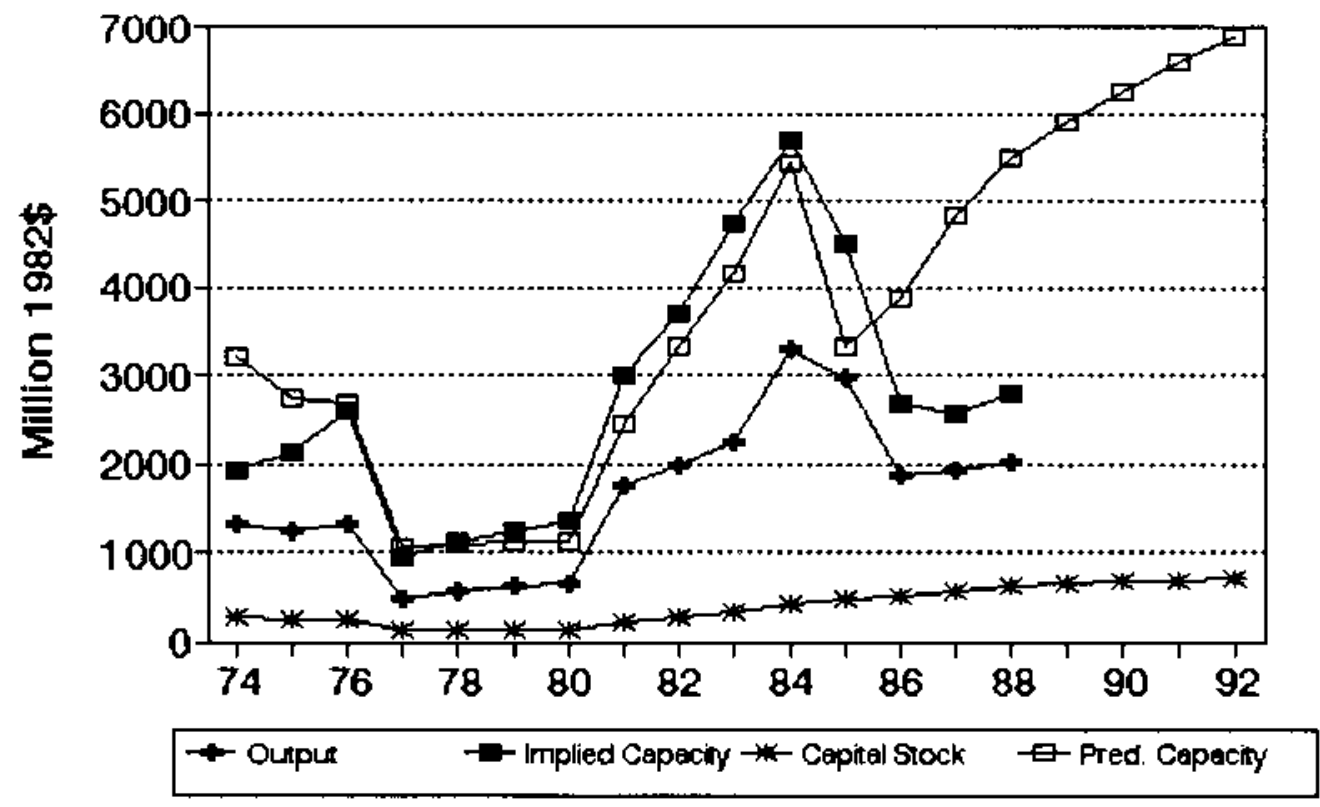




\section{No. 411 SIC 3792 Travel trailers and campers}

MILLIONS OF 1982 DOLLARS (EXCL. CU RATES)

$\begin{array}{lccccccc} & 04 & \text { Ann. Implied } & \text { Gross } & \text { Net } & \text { Pred. } & \ldots \ldots \ldots & \ldots \\ \text { Gross } & \text { CU } & \text { CU } & \text { Prac. } & \text { In- } & \text { Cap. } & \text { Prac. } & \text { Emergency Capacity } \\ \text { Output } & \text { Rate } & \text { Rate } & \text { Capac. } & \text { vest. } & \text { Stk. } & \text { Capac. } & 3 \text { mon. } 6 \text { mon. }>6 \text { mon. }\end{array}$

\begin{tabular}{|c|c|c|c|c|c|c|c|c|c|c|}
\hline 1974 & 1,428 & 57 & 59.5 & 2,401 & $(41,1)$ & 109 & 3,061 & & & \\
\hline 1975 & 1,803 & 70 & 55.7 & 3,237 & 11.5 & 116 & 3,096 & & & \\
\hline 1976 & 2,275 & 69 & 73.0 & 3,118 & 4.4 & 115 & 3,090 & & & \\
\hline 1977 & 2,239 & 71 & 73.0 & 3,069 & 34.0 & 141 & 3,238 & & & \\
\hline 1978 & 2,328 & 72 & 69.0 & 3,372 & 22.8 & 155 & 3,313 & & & \\
\hline 1979 & 1,364 & 29 & 45.1 & 3,023 & $(2.2)$ & 144 & 3,250 & & & \\
\hline 1980 & 1,010 & 31 & 26.7 & 3,787 & $(1.3)$ & 133 & 3,191 & & & \\
\hline 1981 & 1,149 & 35 & 35.8 & 3,209 & $(2.5)$ & 121 & 3,125 & & & \\
\hline 1982 & 1,258 & 42 & 42.2 & 2,978 & 6.0 & 117 & 3,102 & & & \\
\hline 1983 & 1,602 & 51 & 49.9 & 3,214 & 6.9 & 113 & 3,082 & & & \\
\hline 1984 & 1,270 & 43 & 47.3 & 2,685 & $(20.9)$ & 85 & 2,924 & & & \\
\hline 1985 & 1,272 & 41 & 42.7 & 2,970 & 8.9 & 85 & 2,927 & & & \\
\hline 1986 & 1,288 & 39 & 39.9 & 3,226 & 2.8 & 80 & 2,897 & & & \\
\hline-- & & $\cdots$ & 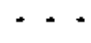 & $\cdots \cdot$ & $\cdots \cdot$ & $-\cdot-$ & --- & $----\cdot$ & $\cdot \cdot \cdot \cdot$ & - - - \\
\hline 1987 & 1,283 & $21 *$ & 27.5 & 4,660 & (8.2) & 64 & 2,807 & & & \\
\hline 1988 & 1,277 & 77 & 54.1 & 2,360 & $(6,1)$ & 51 & 2,738 & 3,712 & 5,502 & 5,527 \\
\hline 1989 & & & & & $(8.0)$ & 38 & 2,665 & 3,614 & 5,356 & 5,381 \\
\hline 1990 & & & & & $(1.7)$ & 32 & 2,634 & $3,5 \mathrm{T2}$ & 5,294 & 5,319 \\
\hline 1991 & & & & & $(4.8)$ & 24 & 2,590 & 3,512 & 5,204 & 5,228 \\
\hline 1992 & & & & & $(3.3)$ & 19 & 2,558 & 3,469 & 5,141 & 5,164 \\
\hline
\end{tabular}

Capacity-Capital Stock Regression: Code 0 RSO 0.150

Coefficients: Const 2454.9 Stock 5.5

Ave. Capacity/stock $(74-86): 26.6$ Ave. Weekly Hours (High) 78.0 Shift Factor 2.02 Ave Heekly Hours (Low) 78.0 Shift Factor 2.02 - imputed Note: Output, Investment, and Capital Stock extrapolated for 1987 and 1988.

\section{Travel trailers and campers}
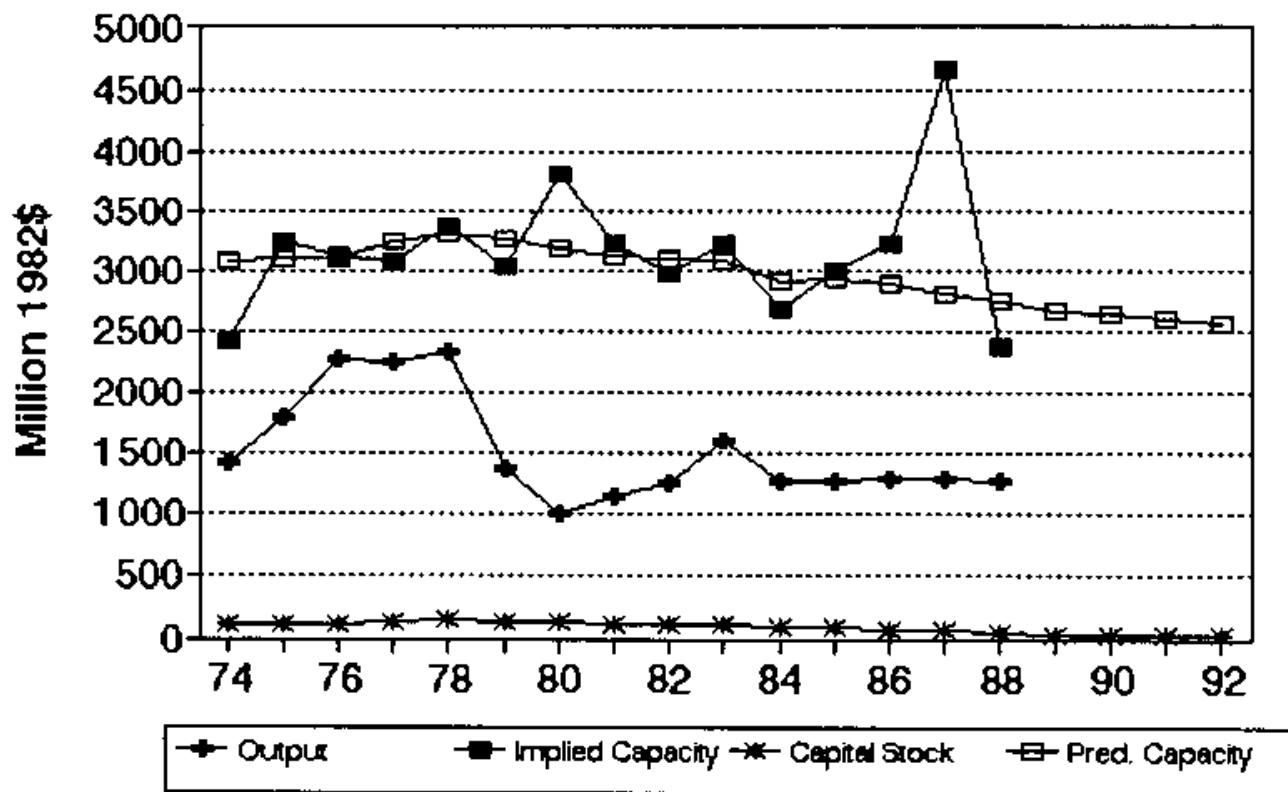


\section{No. 412 SIC 3795 Tanks and tank conponents}

MILLIONS OF 1982 DOLLARS (EXCL. CU RATES)

$\begin{array}{lccccccc} & \text { Q4 } & \text { Ann. } & \text { Implied Gross } & \text { Net } & \text { Pred. } & \ldots \ldots \ldots \\ \text { Gross } & \text { CU } & \text { CU } & \text { Prac. } & \text { In- } & \text { Cap. } & \text { Prac. } & \text { Emergency Capacity } \\ \text { Output } & \text { Rate } & \text { Rate } & \text { Capac. } & \text { vest. } & \text { Stk. } & \text { Capac. } & 3 \text { mon. } 6 \text { mon. }>6 \text { mon. }\end{array}$

\begin{tabular}{|c|c|c|c|c|c|c|c|c|c|c|}
\hline 1974 & 930 & $60 *$ & 60.4 & 1,540 & 29.9 & 69 & 2,347 & & & \\
\hline 1975 & 1,192 & 50 & 53.9 & 2,211 & 9.0 & 70 & 2,357 & & & \\
\hline 1976 & 1,519 & 50 & 50.0 & 3,038 & 12.5 & $\pi$ & 2,414 & & & \\
\hline 1977 & 1,848 & 51 & 50.6 & 3,650 & 38.0 & 103 & 2,770 & & & \\
\hline 1978 & 1,757 & 63 & 58.5 & 3,004 & 13.1 & 107 & 2,821 & & & \\
\hline $197 \%$ & 1,698 & 65 & 64.3 & 2,643 & 43.0 & 140 & 3,236 & & & \\
\hline 1980 & 1,890 & 53 & 57.5 & 3,286 & 16.2 & 148 & 3,334 & & & \\
\hline 1981 & 2,094 & 67 & 61.8 & 3,391 & 31.2 & 170 & 3,613 & & & \\
\hline 1982 & 2,717 & 55 & 59.5 & 4,567 & 81.6 & 242 & 4,520 & & & \\
\hline 1983 & 3,521 & 58 & 56.9 & 6,191 & 81.5 & 309 & 5,361 & & & \\
\hline 1984 & 3,722 & $63 \star$ & 61.0 & 6,107 & 19.3 & 315 & 5,433 & & & \\
\hline 1985 & 3,631 & $67 *$ & 65.6 & 5,532 & 0.9 & 300 & 5,248 & & & \\
\hline 1986 & 2,572 & 72 & 70.3 & 3,660 & 27.9 & 310 & 5,366 & & & \\
\hline $\begin{array}{l}--\cdot \\
1987\end{array}$ & 2,664 & $\because 7$ & $\ddot{37.6}$ & 7,080 & 20.2 & 313 & 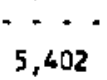 & $-\cdots$ & -- & -- \\
\hline 1988 & 2,788 & 26 & 22.6 & 12,324 & 22.3 & 318 & 5,463 & 4,813 & 6,300 & 6,321 \\
\hline 1989 & & & & & 20.4 & 320 & 5,497 & 4,842 & 6,339 & 6,360 \\
\hline 1990 & & & & & 26.9 & 329 & 5,610 & 4,942 & 6,469 & 6,491 \\
\hline 1991 & & & & & 23.7 & 335 & 5,676 & 5,000 & 6,545 & 6,567 \\
\hline 1992 & & & & & 25.3 & 341 & 5,759 & 5,073 & 6,641 & 6,663 \\
\hline
\end{tabular}

Capacity-Capital Stock Regression: Code 0 RSO 0.750

Coefficients: Const 1478.1 Stock 12.6

Ave. Capacity/Stock $(74-86): 20.7$ Ave, Heekly Hours (High) 137.3 Shift Factor 1.16 Ave Heekly Hours (Low) 115.1 Shift factor 1.37 * Imputed Note: Output, Investment, and Capital stock extrapolated for 1987 and 1988.

\section{Tanks and tank components}

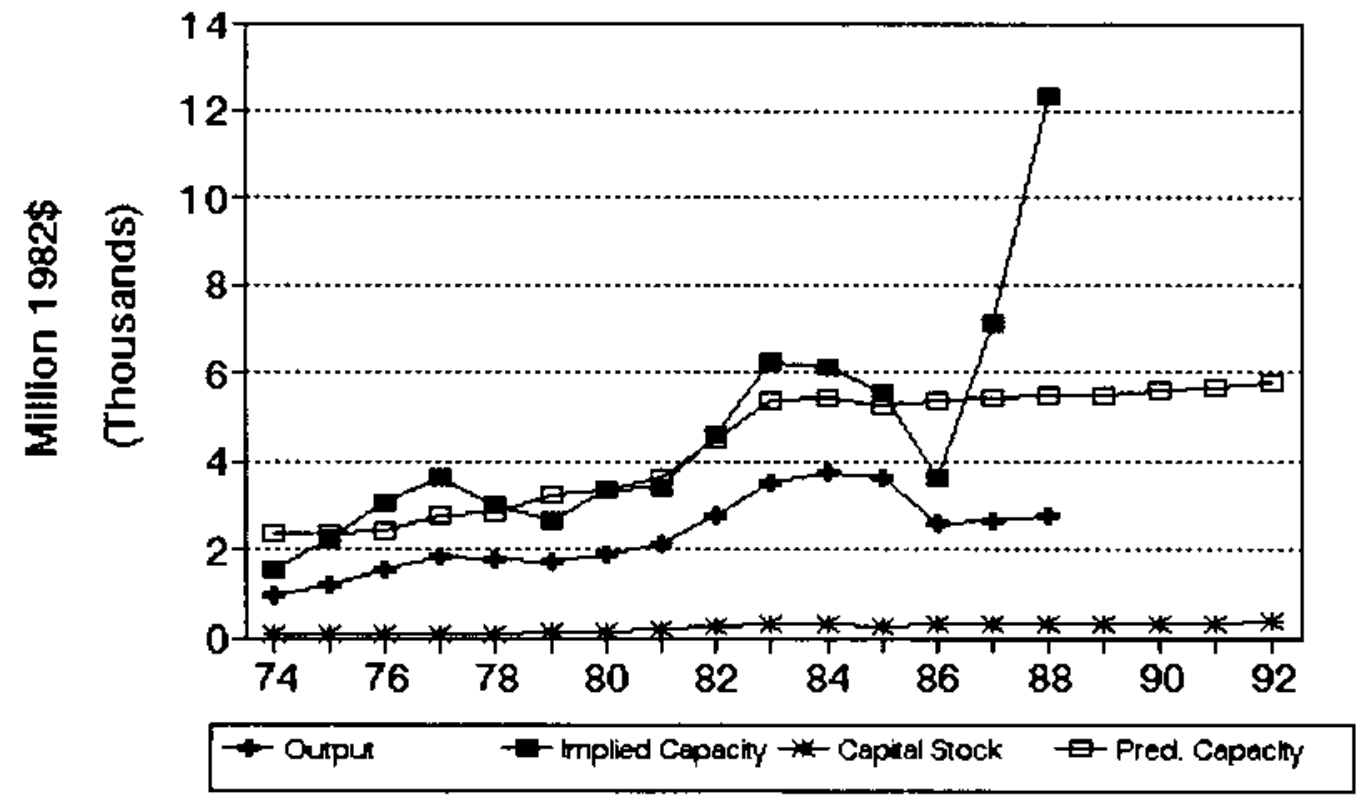




\section{No. 413 sIC 3799 Transportation equipient, nec}

MILLIONS OF 1982 DOLLARS (EXCL. CU RATES)

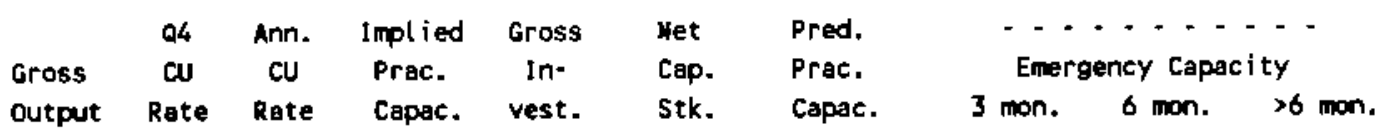

\begin{tabular}{|c|c|c|c|c|c|c|c|c|c|c|}
\hline 1974 & 1,412 & 54 & 54.5 & 2,592 & 53.0 & 220 & 2,823 & & & \\
\hline 1975 & 1,268 & 20 & 31.5 & 4,030 & 31.5 & 241 & 3,125 & & & \\
\hline 1976 & 1,421 & 63 & 47.1 & 3,015 & 2.4 & 232 & 2,990 & & & \\
\hline 1977 & 871 & 62 & 62.9 & 1,384 & $(27.9)$ & 194 & 2,453 & & & \\
\hline 1978 & 943 & 54 & 56.5 & 1,670 & 2.2 & 187 & 2,340 & & & \\
\hline 1979 & 847 & 40 & 45.4 & 1,865 & $(37.2)$ & 142 & 1,694 & & & \\
\hline 1980 & 683 & 32 & 34.3 & $1, \$ 94$ & $(2.6)$ & 132 & 1,553 & & & \\
\hline 1981 & 660 & 51 & 44.3 & 1,489 & 1.8 & 127 & 1,474 & & & \\
\hline 1982 & 933 & 50 & 50.8 & 1,835 & 53.1 & 167 & 2,061 & & & \\
\hline 1983 & 1,094 & $49 *$ & 49.8 & 2,197 & 29.9 & 182 & 2,273 & & & \\
\hline 1984 & 1,196 & 49 & 49.2 & 2,429 & 7.1 & 174 & 2,161 & & & \\
\hline 1985 & 1,159 & $48 *$ & 48.6 & 2,385 & 17.7 & 177 & 2,202 & & & \\
\hline 1986 & 1,226 & $47 *$ & 47.8 & 2,568 & 21.7 & 184 & 2,295 & & & \\
\hline 1987 & 1,244 & $16 *$ & $\begin{array}{l}-\cdot- \\
27.8\end{array}$ & 4,471 & 10.3 & $\because 78$ & 2, 221 & $\cdot \cdot$ & $=-$ & --- \\
\hline 1988 & 1,266 & $30 *$ & 26.5 & 5,175 & 9.9 & 173 & 2,147 & 3,025 & 4,317 & 4,336 \\
\hline 1989 & & & & & 9.4 & 168 & 2,073 & 2,920 & 4,168 & 4,185 \\
\hline 1990 & & & & & 8.9 & 163 & $1, \$ 98$ & 2,815 & 4,017 & 4,035 \\
\hline 1991 & & & & & 8.5 & 158 & 1,923 & 2,709 & 3,866 & 3,883 \\
\hline 1992 & & & & & 8.0 & 153 & 1,848 & 2,603 & 3,715 & 3.731 \\
\hline
\end{tabular}

Capacity-Capital Stock Regression: Code 1 RSO 0.520

Coefficients: Const -369.5 Stock 14.5 Ave. Capacity/Stock $(76-86): 12.5$ Ave. Heekly Hours (High) 78.0 Shift factor 2.02 Ave Weekly Hours (Low) 78.0 Shift Factor 2.02 * Imputed Note: Output, Investment, and Capital Stock extrapolated for 1987 and 1988.

\section{Transportation equipment, nec}
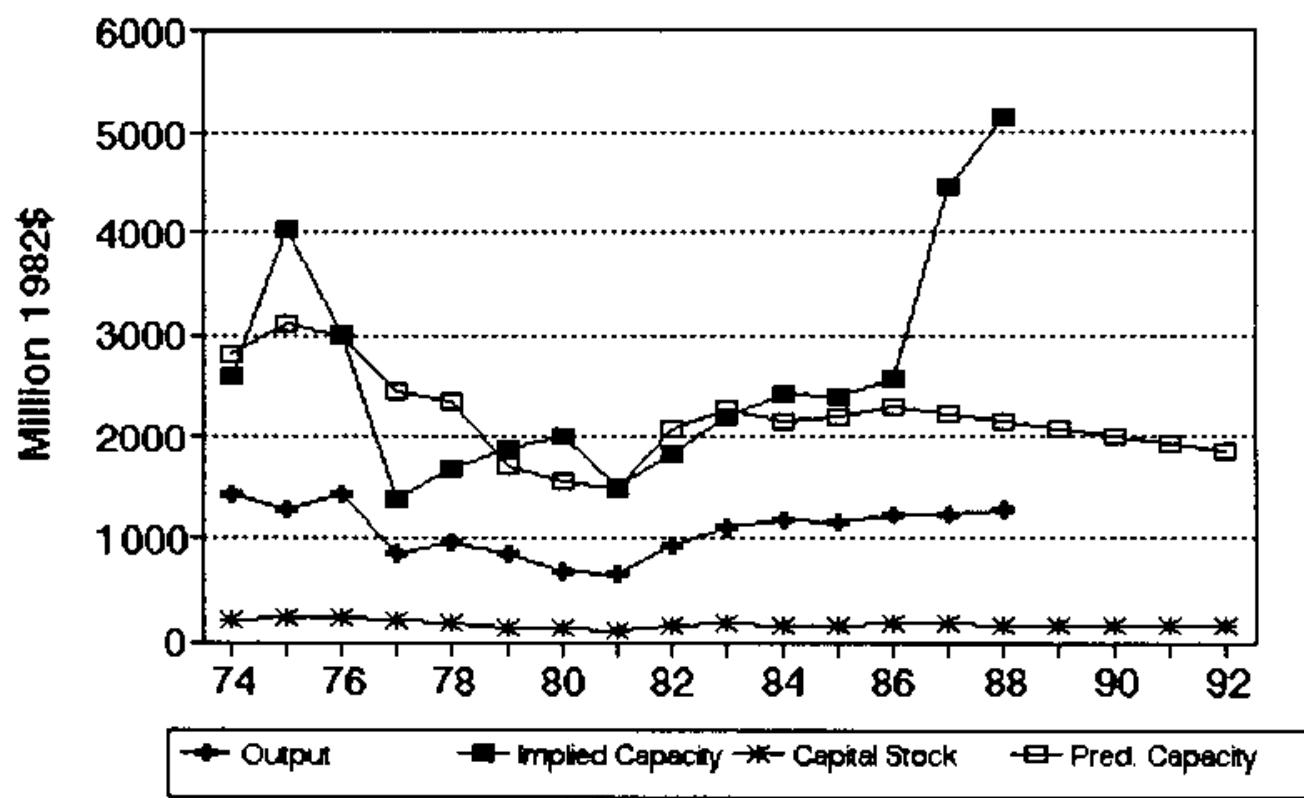
No. 414 SIC 3811 Engineering and scientific instrunents MiLlions of 1982 dOLLARS (EXCL. CU RATES)

$\begin{array}{lccccccc} & 04 & \text { Ann. } & \text { Imptied } & \text { Gross } & \text { Net } & \text { Pred. } & -\ldots \\ \text { Gross } & \text { CU } & \text { CU } & \text { Prec. } & \text { In- } & \text { Cap. } & \text { Prac. } & \text { Emergency Capacity } \\ \text { Output } & \text { Rate } & \text { Rate } & \text { Capac. } & \text { vest. } & \text { Stk. } & \text { Capac. } & 3 \text { mon. } 6 \text { mon. }>6 \text { mon. }\end{array}$

\begin{tabular}{|c|c|c|c|c|c|c|c|c|c|c|}
\hline 1974 & 2,907 & 72 & 75.7 & 3,839 & 60.4 & 451 & 4,145 & & & \\
\hline 1975 & 2,741 & 63 & 62.7 & 4,371 & 44.9 & 457 & 4,176 & & & \\
\hline 1976 & 2,810 & 70 & 67.1 & 4,188 & 48.0 & 466 & 4,221 & & & \\
\hline 1977 & 2,816 & 70 & 70.2 & 4,014 & 46.1 & 474 & 4,262 & & & \\
\hline 1978 & 3,077 & 73 & 72.2 & 4,261 & 95.6 & 529 & 4,534 & & & \\
\hline 1979 & 2,887 & 71 & 72.1 & 4,003 & 45.3 & 537 & 4,569 & & & \\
\hline 1980 & 3,260 & 60 & 63.7 & 5,120 & 57.3 & 557 & 4,668 & & & \\
\hline 1981 & 3,055 & 62 & 61.7 & 4,955 & 59.5 & 579 & 4,779 & & & \\
\hline 1982 & 3,155 & $5 i$ & 56.4 & 5,595 & 65.4 & 606 & 4,912 & & & \\
\hline 1983 & 2,996 & 55 & 52.5 & 5,708 & 97.8 & 661 & 5,183 & & & \\
\hline 1984 & 3,244 & 68 & 64.9 & 4,999 & 18.0 & 638 & 5,067 & & & \\
\hline 1985 & 3,731 & 67 & 67.6 & 5,523 & 155.5 & 746 & 5,601 & & & \\
\hline 1986 & 3,613 & 63 & 64.7 & 5,585 & 141.1 & 835 & 6,042 & & & \\
\hline$-\cdots$ & & -- & -- & $-\cdot-$ & -- & $-\cdots$ & $\cdots$ & $-\cdots$ & $\cdot \cdot \cdot$ & $\therefore-$ \\
\hline 1987 & 3,645 & $\infty$ & 64.8 & 5,623 & 90.3 & 873 & 6,229 & & & \\
\hline 1988 & 3,687 & 64 & 64.6 & 5,711 & 104.4 & 922 & 6,474 & 8,196 & 11,508 & 11,555 \\
\hline 1989 & & & & & 114.1 & 979 & 6,751 & 8,547 & 12,001 & 12,051 \\
\hline 1990 & & & & & 119.6 & 1,037 & 7,038 & 8,910 & 12,512 & 12,563 \\
\hline 1991 & & & & & 129.7 & 1,101 & 7,357 & 9,314 & 13,078 & 13,132 \\
\hline 1992 & & & & & 120.5 & 1,153 & 7,611 & 9.635 & 13,529 & 13,585 \\
\hline
\end{tabular}

Capacity-Capital Stock Regression: Code 0 RSO 0.690

Coefficients: Const 1920.4 stock 4.9

Ave. Capacity/Stock $(74-86): 8.3$ Ave. Ueekly Hours (High) 88.2 Shift Factor 1.78 Ave Heekly Hours (Low) 79.6 Shift Factor 1.98 Hote: Output, Investment, and Capital Stock extrapolated for 1987 and 1988.

\section{Engineering and scientific instrum}

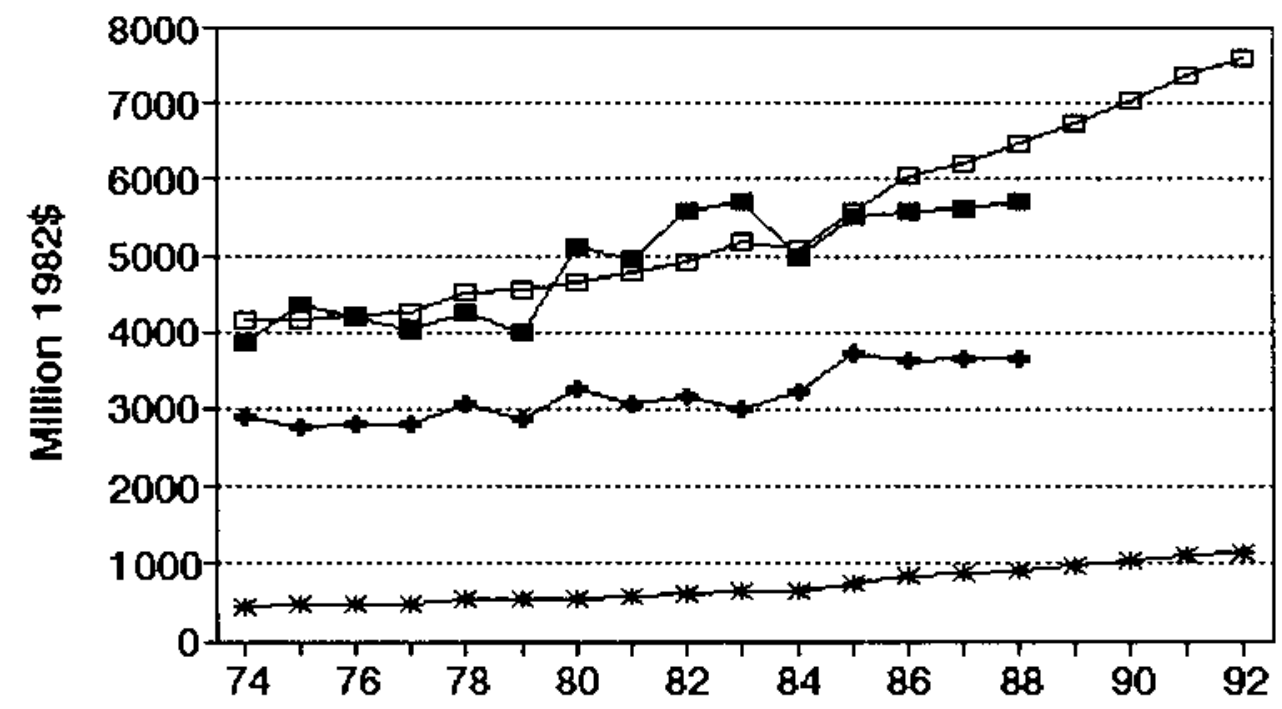

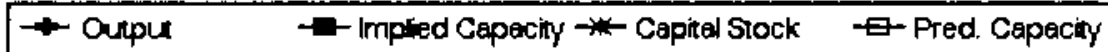




\section{No. 415 SIC 3822 Enpironmental controls}

MILLIDNS DF 1982 DOLLARS (EXCL. CU RATES)

$\begin{array}{lccccccc} & 04 & \text { Ann. } & \text { Implied } & \text { Gross } & \text { Net } & \text { Pred. } & \ldots \ldots \ldots \ldots \\ \text { Gross } & \text { du } & \text { CU } & \text { Prac. } & \text { In- } & \text { Cap. } & \text { Prac. } & \text { Emergency Capacity } \\ \text { Output } & \text { Rate } & \text { Rate } & \text { Capac. } & \text { vest. } & \text { Stk. } & \text { Capac. } & 3 \text { mon. } 6 \text { mon. }>6 \text { mon. }\end{array}$

\begin{tabular}{|c|c|c|c|c|c|c|c|c|c|c|}
\hline 1974 & 1,605 & 71 & 73.3 & 2,132 & 65.1 & 284 & 2,186 & & & \\
\hline 1973 & 1,201 & 72 & 67.3 & 1,785 & 9.1 & 268 & 2,123 & & & \\
\hline 1976 & 1,576 & 67 & 68.7 & 2,295 & 11.6 & 254 & 2,072 & & & \\
\hline 1977 & 1,941 & 88 & 80.1 & 2,422 & 78.0 & 304 & 2,260 & & & \\
\hline 1978 & 1,967 & 89 & 89.1 & 2,206 & 44.8 & 320 & 2,321 & & & \\
\hline 1979 & 1.662 & 79 & 83.2 & 1,997 & $(14.2)$ & $2 B t$ & 2,173 & & & \\
\hline 1980 & 1,771 & 71 & 73.4 & 2,411 & 49.3 & 305 & 2,266 & & & \\
\hline 1981 & 1.670 & 60 & 64.6 & 2,587 & 53.7 & 332 & 2,368 & & & \\
\hline 1982 & 1,552 & 60 & 61.8 & 2,509 & 44.4 & 349 & 2,432 & & & \\
\hline 1983 & 1,660 & 67 & 62.9 & 2,637 & 18.1 & 341 & 2,400 & & & \\
\hline 1984 & 1,810 & 79 & 77.0 & 2,351 & 28.1 & 341 & 2,403 & & & \\
\hline 1985 & 1,768 & 73 & 73.5 & 2,343 & 53.7 & 366 & 2,497 & & & \\
\hline 1986 & 1,699 & 72 & 72.7 & 2,337 & 34.0 & 370 & 2,511 & & & \\
\hline-- & $--\cdot$ & - & $\cdots$ & $\cdots$ & $-\cdots$ & $\cdot \cdot$ & $\cdots \cdot$ & $-\cdot$ & -- & -- \\
\hline 1987 & 1,709 & 74 & 73.2 & 2,335 & 41.8 & 382 & 2,556 & & & \\
\hline 1988 & 1,723 & 70 & 71.3 & 2,417 & 45.3 & 397 & 2,612 & 2,678 & 3,380 & 3,390 \\
\hline 1989 & & & & & 47.8 & 412 & 2,672 & 2,740 & 3,458 & 3,468 \\
\hline 1990 & & & & & 49.2 & 428 & 2,732 & 2,802 & 3,536 & 3,547 \\
\hline 1991 & & & & & 51.7 & 446 & 2,798 & 2,869 & 3,621 & 3,631 \\
\hline 1992 & & & & & 49.4 & 459 & 2,849 & 2,921 & 3.687 & 3,698 \\
\hline
\end{tabular}

Capacity-Capital Stock Regression: Code 1 RSa 0.360 Coefficients: Const 1110.3 Stock 3.8 Ave. Weekly Hours (High) 121.8 Shift Factor 1.30 Ave Heekly Hours (Low) 117.6 Shift Factor 1.34 Mote: Output, Investment, and Capital Stock extrapolated for 1987 and 1988.

\section{Environmental controls}

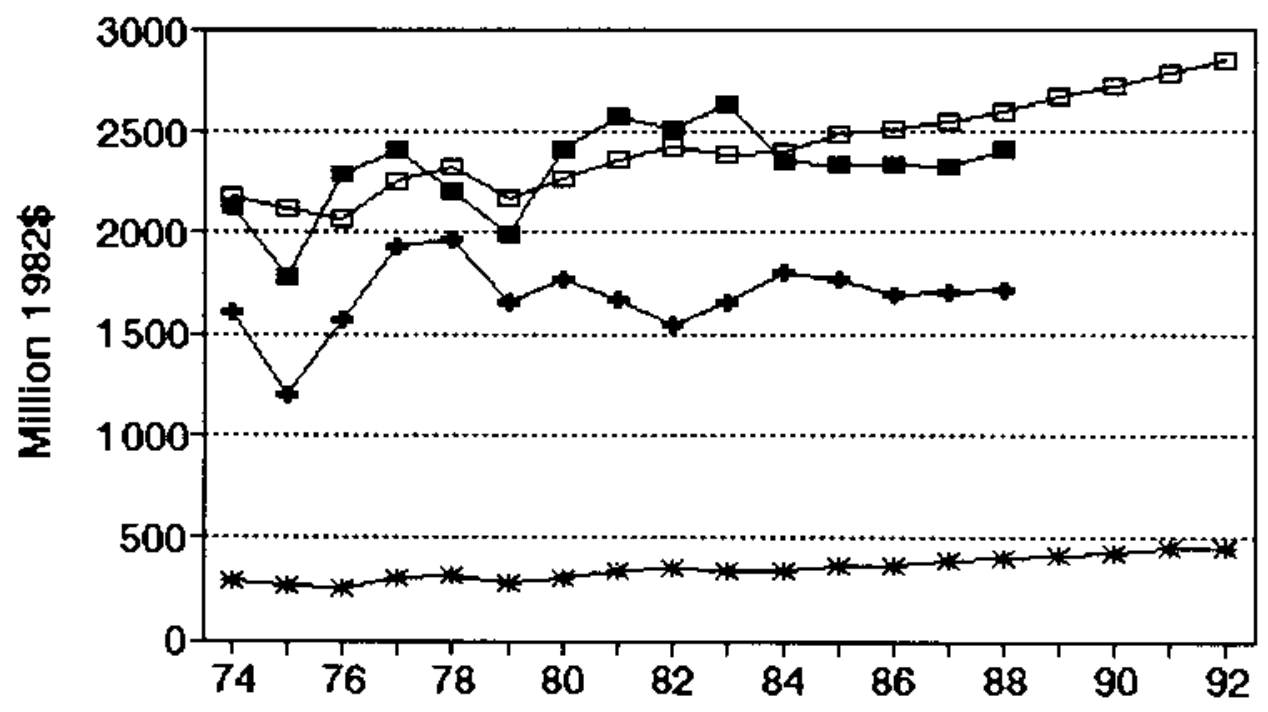

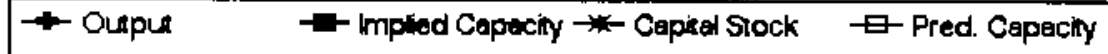


No. 416 SIC 3823 Process control ingtrunents

MILLIONS OF 1982 DOLLARS (EXCL. CU RATES)

$\begin{array}{lccccccc} & \text { Q4 } & \text { Ann. } & \text { Implied } & \text { Gross } & \text { Net } & \text { Pred. } & \ldots \\ \text { Gross } & \text { CU } & \text { CU } & \text { Prac. } & \text { In- } & \text { Cap. } & \text { Prac. } & \text { Emergency Capacity } \\ \text { Output } & \text { Rate } & \text { Rate } & \text { Capac. } & \text { vest. } & \text { Stk. } & \text { Capac. } & 3 \text { mon. } 6 \text { mon. }>6 \text { mon. }\end{array}$

\begin{tabular}{|c|c|c|c|c|c|c|c|c|c|c|}
\hline 1974 & 2,662 & 80 & 88.2 & 3,017 & 29.0 & 400 & 3,820 & & & \\
\hline 1975 & 2,368 & 77 & 69.7 & 3,398 & 16.4 & 384 & 3,699 & & & \\
\hline 1976 & 2,471 & 70 & 72.4 & 3,414 & 11.5 & 365 & 3,551 & & & \\
\hline 1977 & 3,740 & 74 & 72.8 & 5,136 & 124.4 & 450 & 4,210 & & & \\
\hline 1978 & 3,327 & 77 & 76.6 & 4,343 & 61.0 & 472 & 4,378 & & & \\
\hline 1979 & 3,677 & $\pi$ & 79.0 & 4,652 & 73.2 & 505 & 4,632 & & & \\
\hline 1980 & 4,044 & 78 & 77.4 & 5,223 & 72.6 & 537 & 4,877 & & & \\
\hline 1981 & 4,067 & 70 & 73.9 & 5,506 & 60.7 & 576 & 5,177 & & & \\
\hline 1982 & 4,116 & 58 & 65.7 & 6,265 & 170.5 & 697 & 6,110 & & & \\
\hline 1983 & 3,537 & 56 & 54.5 & 6,486 & 91.8 & 737 & 6,418 & & & \\
\hline 1984 & 3,706 & 54 & 58.3 & 6,355 & 140.9 & 822 & 7,070 & & & \\
\hline 1985 & 3,939 & 52 & 53.1 & 7.423 & 95.1 & 859 & 7,355 & & & \\
\hline 1986 & 3,833 & 47 & 49.2 & 7,789 & 108.6 & 905 & 7,710 & & & \\
\hline - : & $\cdots$ & $\because 4$ & $\because 4$ & $\because \cdots$ & $\ddot{103.9}$ & 948 & 8,034 & $-\cdot-$ & $--\cdot$ & $\cdots$ \\
\hline 1988 & 3,999 & 49 & 47.2 & 8,476 & 119.9 & 1,003 & 8,459 & 11,304 & 16,672 & 16,748 \\
\hline 1989 & & & & & 130.9 & 1,065 & 8,939 & 11,946 & 17,618 & 17,699 \\
\hline 1990 & & & & & 137.1 & 1,130 & 9,434 & 12,608 & 18,594 & 18,680 \\
\hline 1991 & & & & & 148.5 & 1,201 & 9,984 & 13,342 & 19,677 & 19,768 \\
\hline 1992 & & & & & 138.1 & 1,257 & 10,416 & 13,919 & 20,529 & 20,623 \\
\hline
\end{tabular}

Capacity-Capital stock Regression: Code 0 RSQ 0.910 Coefficients: Const 744.8 stock 7.7

Ave. Capacity/Stock $(74-86): 8.9$ Ave. Weekly Hours (High) 79.6 Shift Factor 1.98 Ave Ueekly Hours (Low) 75.7 Shift Factor 2.08 Note: Output, Investment, and Capital Stock extrapolated for 1987 and 1988.

\section{Process control instruments}

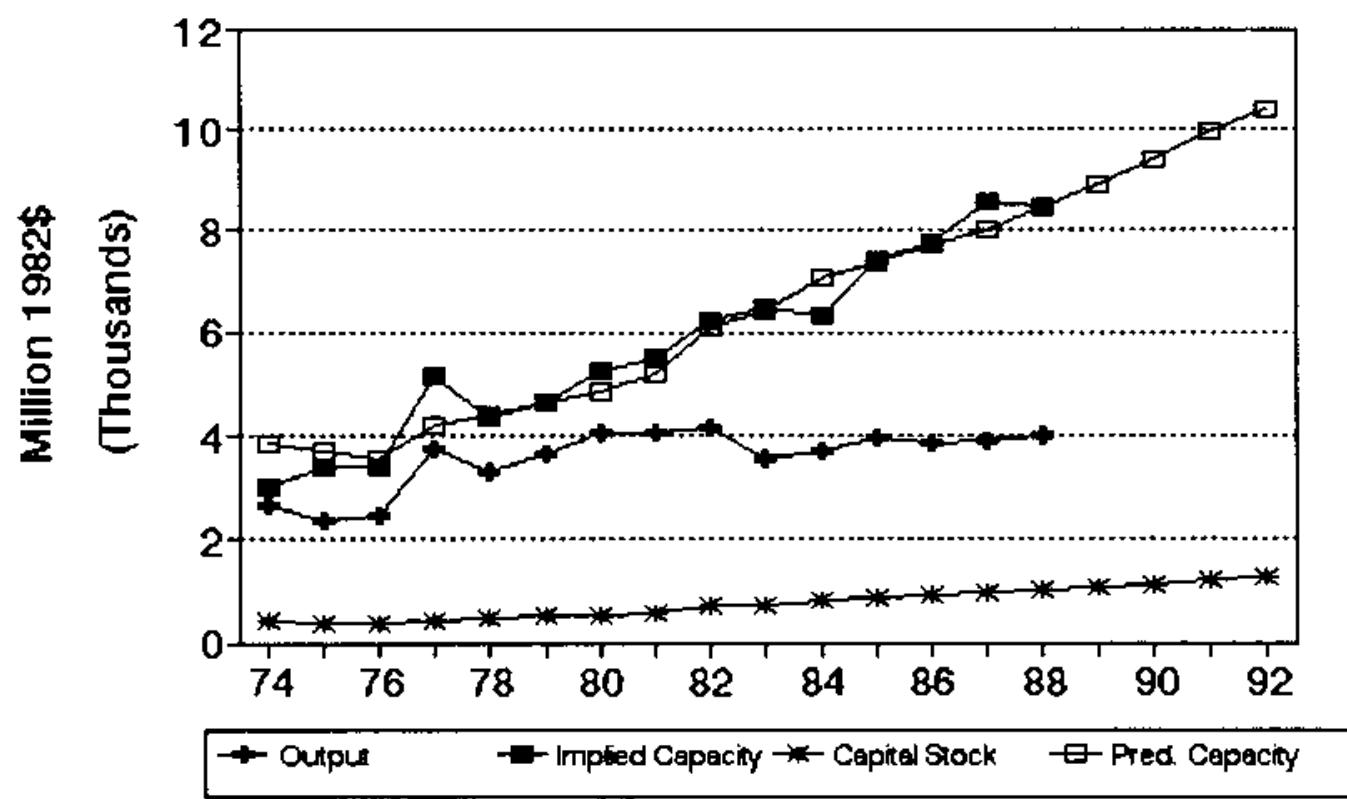




\section{No. 417 sIC 3824 Fluid meters and counting devices}

MILLIONS OF 1982 DOLLARS (EXCL. CU RATES)

$\begin{array}{lccccccc} & 04 & \text { Ann. } & \text { Implied Gross } & \text { Net } & \text { Pred. } & \ldots \\ \text { Gross } & \text { CU } & \text { CU } & \text { Prac. } & \text { In- } & \text { Cep. } & \text { Prac. } & \text { Emergency Capacity } \\ \text { Output } & \text { Rate } & \text { Rate } & \text { Capac. } & \text { vest. } & \text { Stk. } & \text { Capac. } & 3 \text { mon. } 6 \text { mon. }>6 \text { mon. }\end{array}$

\begin{tabular}{|c|c|c|c|c|c|c|c|c|c|c|}
\hline 1974 & 768 & 82 & 65.7 & 1,170 & 39.4 & 183 & 1,220 & & & \\
\hline 1975 & 727 & 68 & 61.9 & 1,174 & 49.6 & 213 & 1,124 & & & \\
\hline 1976 & 764 & 80 & 75.1 & 1,018 & 18.3 & 212 & 1,192 & & & \\
\hline 1977 & 913 & 93 & 88.2 & 1,034 & 39.4 & 231 & 1,130 & & & \\
\hline 1978 & 999 & 85 & 88.6 & 1,128 & 27.6 & 239 & 1,105 & & & \\
\hline 1979 & 1,059 & 83 & 84.3 & 1,257 & 26.8 & 246 & 1,080 & & & \\
\hline 1980 & 989 & 80 & 80.5 & 1,229 & 12.7 & 242 & 1,095 & & & \\
\hline 1981 & 947 & 70 & 74.3 & 1,276 & 33.7 & 258 & 1,043 & & & \\
\hline 1982 & 702 & 51 & 59.5 & 1.179 & 1.3 & 243 & 1,090 & & & \\
\hline 1983 & 645 & 62 & 56.6 & 1,140 & 10.6 & 258 & 1,108 & & & \\
\hline 1984 & 766 & 92 & 83.1 & 921 & 38.2 & 258 & 1,042 & & & \\
\hline 1985 & 773 & 94 & 93.6 & 826 & 33.3 & 273 & 993 & & & \\
\hline 1986 & 755 & 87 & 89.9 & 839 & 27.2 & 281 & 968 & & & \\
\hline$\cdots$ & $\cdots \cdot \cdot$ & - - - & $\cdots$ & $\therefore-$ & -- & $\cdots$ & $\cdots \cdot$ & -- & --- & $\cdots$ \\
\hline 1987 & 761 & 87 & 86.9 & 875 & 28.0 & 290 & 940 & & & \\
\hline 1988 & 768 & $88 \div$ & 87.0 & 893 & 28.2 & 298 & 912 & 1,082 & 1,313 & 1,316 \\
\hline 1989 & & & & & 28.3 & 306 & 886 & 1,051 & 1,276 & 1,279 \\
\hline 1990 & & & & & 28.3 & 316 & 862 & 1,023 & 1,241 & 1,244 \\
\hline 1991 & & & & & 28.4 & 321 & 839 & 995 & 1,208 & 1,211 \\
\hline 1992 & & & & & 28.3 & 327 & 818 & 970 & 1,177 & 1,180 \\
\hline
\end{tabular}

Capecity-Capital Stock Regression: Code -1 RSO 0.220

Coefficients: Const 1815.3 stock -3.2 D(76-86) 63.8

Ave. Capacity/5tock $(74-86): 4.6$ Ave. Weekly Hours (High) 109.2 Shift factor 1.44 Ave Weekly Hours (Low) 104.4 Shift Factor 1.51 * Imputed Note: Output, Investment, and Capital Stock extrapolated for 1987 and 1988.

\section{Fluid meters and counting devices}

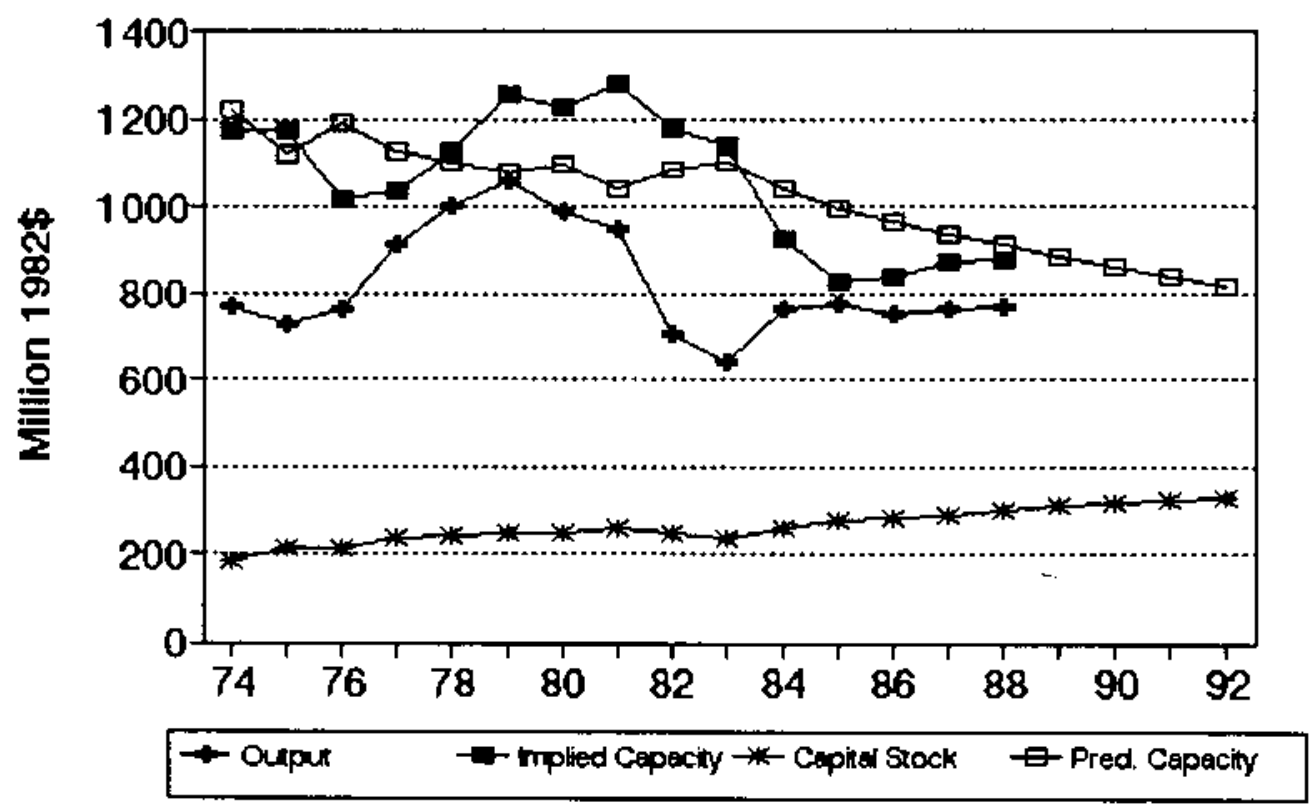


No. 418 SIC 3825 Instruments to measure electricity

MILLIONS OF 1982 DOLLARS (EXCL. CU RATES)

$\begin{array}{lccccccc} & \text { Q4 } & \text { Ann. } & \text { Implied Gross } & \text { Net } & \text { Pred. } & \ldots . . . . . . \\ \text { Gross } & C U & \text { CU } & \text { Prac. } & \text { In- } & \text { Cap. } & \text { Prac. } & \text { Emergency Capacity } \\ \text { Output } & \text { Rate } & \text { Rate } & \text { Capac. } & \text { vest. } & \text { Stk. } & \text { Capac. } & 3 \text { mon. } 6 \text { mon. }>6 \text { mon. }\end{array}$

\begin{tabular}{|c|c|c|c|c|c|c|c|c|c|c|}
\hline 1974 & 4,458 & 72 & 73.4 & 6,077 & 55.4 & 559 & 7,172 & & & \\
\hline 1975 & 6,212 & 59 & 62.6 & 6,731 & 49.7 & 568 & 7,194 & & & \\
\hline 1976 & 4,421 & 67 & 63.9 & 6,919 & 61.9 & 586 & 7,241 & & & \\
\hline 1977 & 5,131 & 62 & 63.9 & 8,025 & 106.9 & 645 & 7,394 & & & \\
\hline 1978 & 5,110 & $\infty 6$ & 64.6 & 7,909 & 124.5 & 718 & 7,581 & & & \\
\hline 1979 & 6,047 & 74 & 71.2 & 8,500 & 166.3 & 827 & 7,864 & & & \\
\hline 1980 & 7,004 & 78 & 76.3 & 9,177 & 229.3 & 991 & 8,288 & & & \\
\hline 1981 & 6,574 & 74 & 75.7 & 8,687 & 200.0 & 1,121 & 8,622 & & & \\
\hline 1982 & 6,150 & 68 & 70.8 & 8,683 & 226.5 & 1,268 & 9,002 & & & \\
\hline 1983 & 6,328 & 71 & 69.4 & 9,116 & 178.6 & 1,362 & 9,247 & & & \\
\hline 1984 & 7,677 & 72 & 72.4 & 10,602 & 326.8 & 1,596 & 9,851 & & & \\
\hline 1985 & 7,356 & 69 & 70.2 & 10,481 & 90.7 & 1,592 & 9.841 & & & \\
\hline 1986 & 6,255 & 77 & 74.1 & 8,442 & 185.2 & 1,673 & 10,050 & & & \\
\hline$\because-{ }^{-1087}$ & $-\cdots$ & -- & -7 & $-\cdots-$ & $\begin{array}{l}-- \\
1740\end{array}$ & - - - & --- & $--\cdot-$ & $--\cdot-$ & $-\cdot-$ \\
\hline $\begin{array}{l}198 \% \\
1988\end{array}$ & $\begin{array}{l}0,581 \\
6,562\end{array}$ & $\begin{array}{l}63 \\
69\end{array}$ & $\begin{array}{l}08.3 \\
66.6\end{array}$ & $\begin{array}{l}9,359 \\
9,847\end{array}$ & $\begin{array}{l}114.0 \\
193.2\end{array}$ & $\begin{array}{l}1,140 \\
1,833\end{array}$ & $\begin{array}{l}10,237 \\
10,462\end{array}$ & 12,441 & 16,761 & 16,822 \\
\hline 1989 & & & & & 206.4 & 1,928 & 10,707 & 12,733 & 17,154 & 17,217 \\
\hline 1990 & & & & & 213.9 & 2,026 & 10,956 & 13,030 & 17,554 & 17,618 \\
\hline 1991 & & & & & 227.6 & 2,129 & 11,226 & 13,351 & 17,986 & 18,052 \\
\hline 1992 & & & & & 215.1 & 2,214 & 11,448 & 13,614 & 18,341 & 18,408 \\
\hline
\end{tabular}

Capacity-Capital Stock Regression: Code 1 RSO 0.680

Coefficients: Const 5727.0 stock 2.6

Ave. Capacity/Stock $(74-86): 8.1$ Ave. Weekly Hours (High) 98.0 Shift factor 1.61 Ave Weekly Hours (Low) 94.0 Shift Factor 1.67 Note: Output, Imvestment, and Capital Stock extrapolated for 1987 and 1988.

\section{Instruments to measure electricity}

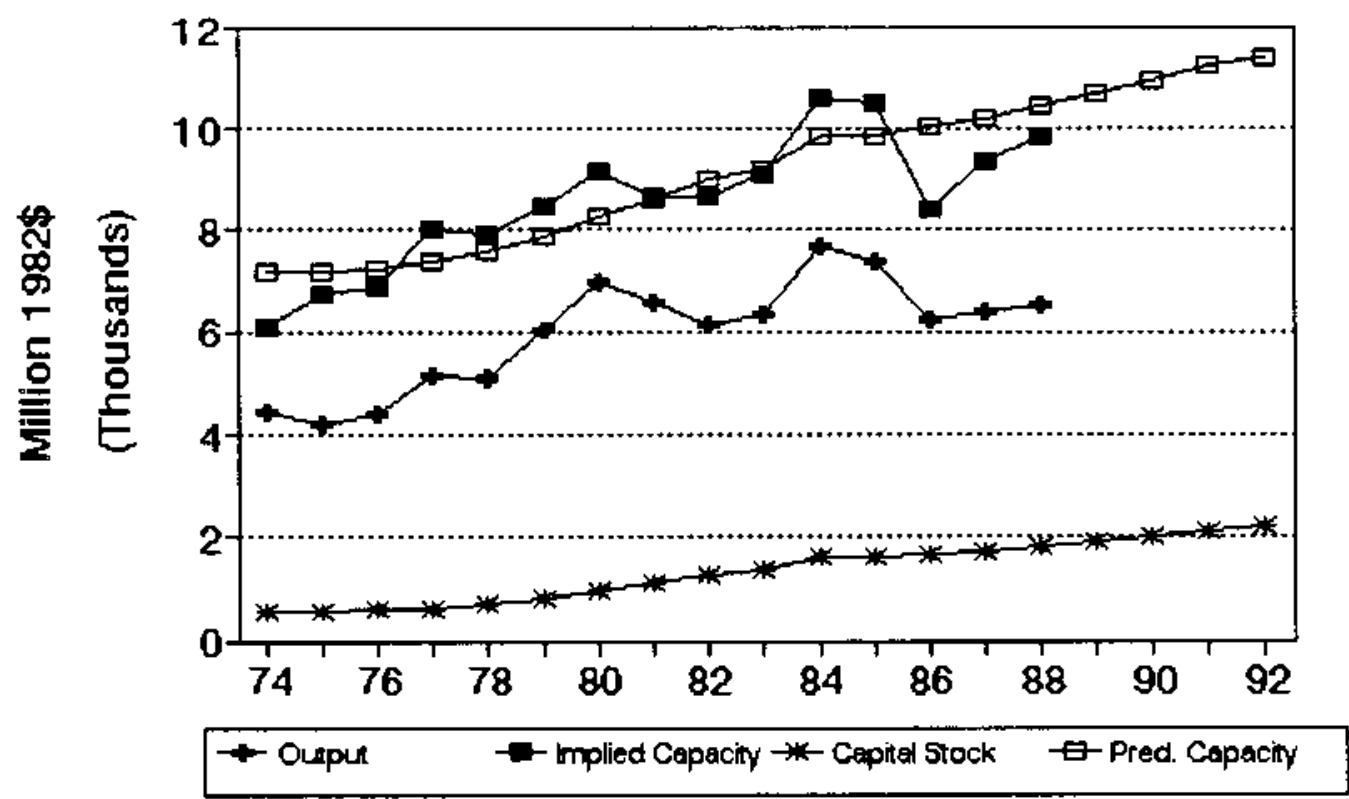


No. 419 sIC 3829 veasuring and controlling devices, nec HILLIONS OF 1982 DOLLARS (EXCL. CU RATES)

\begin{tabular}{|c|c|c|c|c|c|c|c|}
\hline & $\begin{array}{l}04 \\
\text { CU }\end{array}$ & Ann. & $\begin{array}{l}\text { Implied } \\
\text { Prac. }\end{array}$ & $\begin{array}{c}\text { Gross } \\
\text { In- }\end{array}$ & $\begin{array}{l}\text { Net } \\
\text { Cap. }\end{array}$ & $\begin{array}{l}\text { Pred. } \\
\text { Prac. }\end{array}$ & $\begin{array}{l}- \\
\text { Emergency Capaci ty }\end{array}$ \\
\hline & Rate & Rate & Capac. & vest. & stk. & Capac. & \\
\hline
\end{tabular}

\begin{tabular}{|c|c|c|c|c|c|c|c|c|c|c|}
\hline 1974 & 1,368 & 68 & 76.2 & $1, \pi 95$ & $(5.9)$ & 242 & 1,890 & & & \\
\hline 1975 & 1,186 & 56 & 52.3 & 2,260 & 5.2 & 230 & 1,757 & & & \\
\hline 1976 & 1,241 & 53 & 53.9 & 2,302 & 61.6 & 271 & 2,207 & & & \\
\hline 1977 & 1,625 & 85 & 72.8 & 2,231 & 44.4 & 293 & 2,456 & & & \\
\hline 1978 & 1,785 & 63 & 72.5 & 2,464 & 48.7 & 317 & 2,729 & & & \\
\hline 1979 & 1,804 & 71 & 68.8 & 2,622 & 48.6 & 342 & 3,001 & & & \\
\hline 1980 & 1,979 & 69 & 68.8 & 2,877 & 58.0 & 374 & 3,358 & & & \\
\hline 1981 & 2,049 & $\infty$ & 68.1 & 3,009 & $(0.5)$ & 351 & 3,100 & & & \\
\hline 1982 & 2,351 & 53 & 61.4 & 3,827 & 120.6 & 441 & 4,110 & & & \\
\hline 1983 & 2,213 & 52 & 49.9 & 4,433 & 72.3 & 480 & 4,537 & & & \\
\hline 1984 & 2,103 & 59 & 60.4 & 3,482 & $(18.8)$ & 431 & 3,992 & & & \\
\hline 1985 & 2,117 & 48 & 52.3 & 4,045 & 0.0 & 403 & 3,678 & & & \\
\hline 1986 & 2,323 & 40 & 43.3 & 5,365 & 50.7 & 423 & 3,906 & & & \\
\hline-- & $--\cdot-$ & -- & -- & $\cdots$ & $\cdots$ & $\cdots$ & $-\cdots$ & $-\cdot-\cdot$ & $\cdot \cdot-$ & --- \\
\hline 1987 & 2,364 & 53 & 48.0 & 4,928 & 38.1 & 431 & 3,996 & & & \\
\hline 1988 & 2,418 & 49 & 50.3 & 4,805 & 37.9 & 438 & 4,076 & 5,131 & 7,125 & 7,153 \\
\hline 1989 & & & & & 37.7 & 445 & 4,149 & 5,224 & 7,253 & 7,282 \\
\hline 1990 & & & & & 37.5 & 451 & 4,215 & 5,307 & 7,368 & 7,398 \\
\hline 1991 & & & & & 37.3 & 456 & 4,274 & 5,381 & 7,471 & 7,501 \\
\hline 1992 & & & & & 37.1 & 461 & 4,326 & 5,447 & 7,562 & 7,593 \\
\hline
\end{tabular}

Capacity-Capital Stock Regression: Code 1 RSO 0.740

Coefficients: Const -806.4 Stock 11.1

Ave. Capacity/Stock $(74-86): 8.9$ Ave. Weekly Hours (High) 89.7 Shift Factor 1.75 Ave Weekly Hours (Low) 85.1 Shift Factor 1.85 Note: Output, Investment, and Cepital Stock extrapolated for 1987 and 1988.

\section{Measuring and controlling devices,}

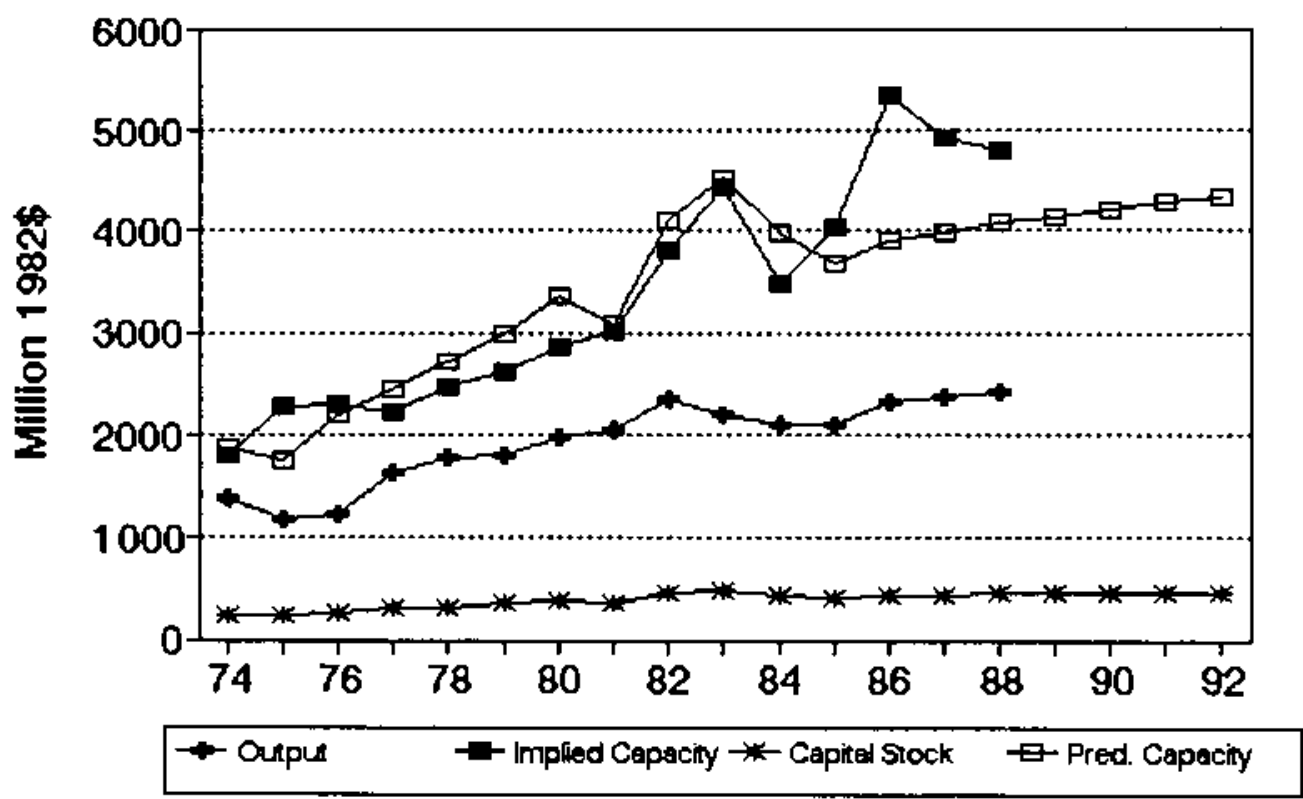




\section{No. 420 gIC 3832 Optical instruments and lenses}

MILLIONS OF 1982 dOLLARS (EXCL. CU RATES)

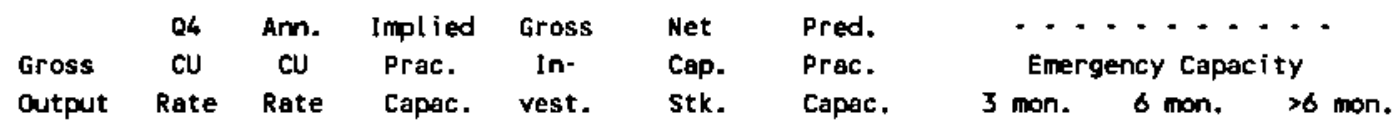

\begin{tabular}{|c|c|c|c|c|c|c|c|c|c|c|}
\hline 1974 & 1,034 & 35 & 37.0 & 2,793 & 30.5 & 225 & 2,059 & & & \\
\hline 1975 & 1,135 & 42 & 37.2 & 3,052 & 34.2 & 238 & 2,178 & & & \\
\hline 1976 & 1,305 & 65 & 55.9 & 2,334 & 65.2 & 277 & 2,552 & & & \\
\hline 1977 & 1,697 & 81 & 75.1 & $2,2 \measuredangle 2$ & 72.8 & 322 & 2,977 & & & \\
\hline 1978 & 2,232 & 71 & $\pi .3$ & 2,965 & 43.0 & 339 & 3,133 & & & \\
\hline 1979 & 2,578 & 63 & 66.4 & 3,884 & 109.7 & 419 & 3,890 & & & \\
\hline 1980 & 2,970 & 63 & 62.6 & 4,748 & 105.8 & 494 & 4,590 & & & \\
\hline 1981 & 3,173 & 72 & 69.2 & 4,588 & 68.9 & 532 & 4.964 & & & \\
\hline 1982 & 3,909 & 68 & 71.5 & 5,468 & 161.2 & 658 & 6,153 & & & \\
\hline 1983 & 4,105 & 74 & 70.2 & 5,846 & 120.4 & 739 & 6,924 & & & \\
\hline 1984 & 5,195 & 59 & 67.2 & 7,736 & 115.8 & 814 & 7,630 & & & \\
\hline 1985 & 5,517 & $59 *$ & 59.0 & 9,350 & 131.9 & 900 & 8,446 & & & \\
\hline 1986 & 5,463 & $58 *$ & 58.7 & 9,310 & 90.4 & 940 & 8,830 & & & \\
\hline$-\cdots$ & $-\ldots$ & -- & -- & --- & $-\cdots$ & $-\cdots$ & 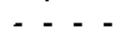 & $--\cdot-$ & $--\cdot-$ & -- \\
\hline 1987 & 5,714 & 58 & 58.1 & 9,839 & 103.1 & 995 & 9,343 & & & \\
\hline 1988 & 6,055 & 65 & 62.1 & 9.755 & 118.5 & 1,062 & 9,977 & 12,818 & 17,787 & 17,858 \\
\hline 1989 & & & & & 129.2 & 1,136 & 10,679 & 13,720 & 19,039 & 19,115 \\
\hline 1990 & & & & & 135.3 & 1,212 & 11,401 & 14,649 & 20,327 & 20,408 \\
\hline 1991 & & & & & 146.4 & 1,295 & 12,192 & 15,064 & 21,737 & 21,823 \\
\hline 1992 & & & & & 136.3 & 1,365 & 12,845 & 16,504 & 22,902 & 22,993 \\
\hline
\end{tabular}

Cepecity-Capital stock Regression: Code 0 RSO 0.940

Coefficients: Const -75.0 stock 9.5

Ave. Capacity/Stock $(74-86): 9.3$ Ave. Weekly Hours (High) 88.0 Shift factor 1.79 Ave Weekly Hours (Low) 85.1 Shift Factor 1.85 - Imputed Note: Output, Investment, and Capital Stock extrapolated for 1987 and 1988.

3832 Optical instruments and lenses
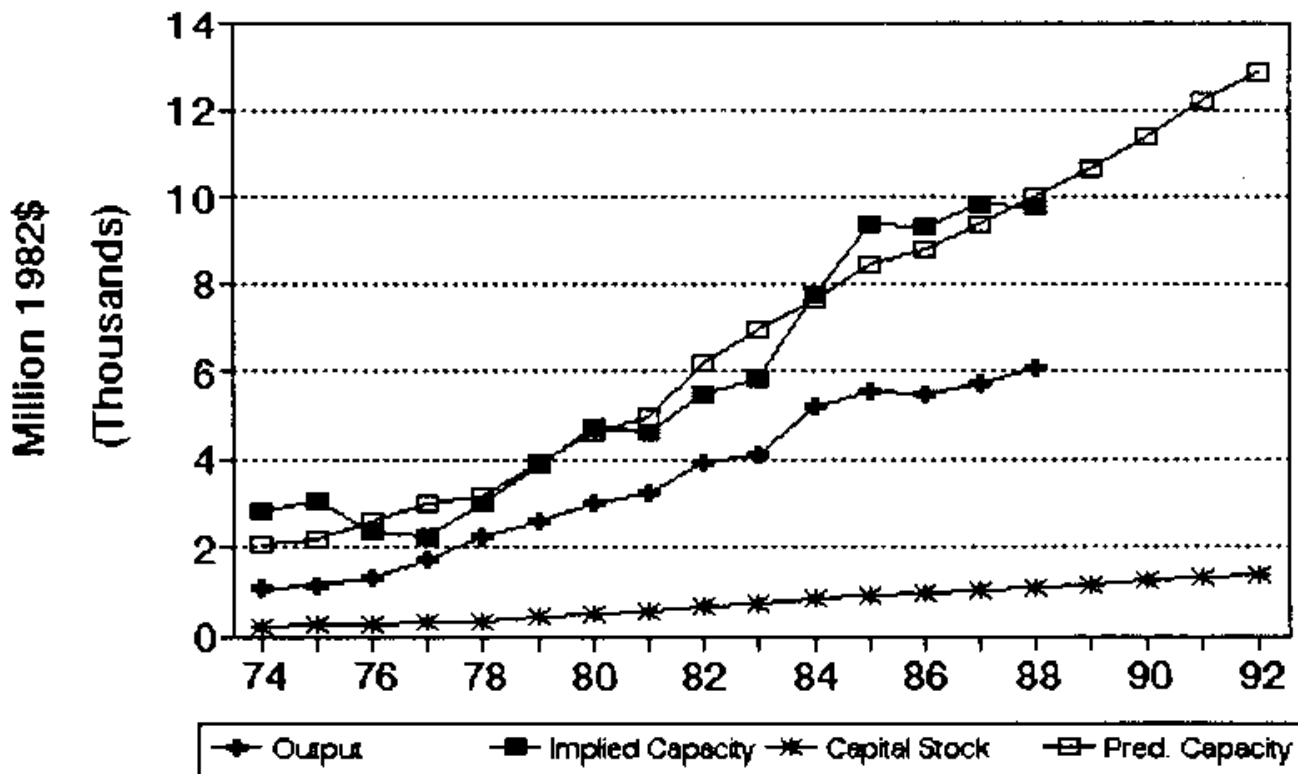
No. 421 sIC 3841 surgical and medical instruments MILLIONS OF 1982 DOLLARS (EXCL. CU RATES)

$\begin{array}{lccccccc} & 04 & \text { Ann. Implied Gross } & \text { Net } & \text { Pred. } & \ldots \ldots \ldots \\ \text { Gross } & \text { CU } & \text { CU } & \text { Prac. } & \text { In- } & \text { Cap. } & \text { Prac. } & \text { Emergency Capacity } \\ \text { Output } & \text { Rate } & \text { Rate } & \text { Capac. } & \text { vest. } & \text { Stk. } & \text { Capac. } & 3 \text { mon. } 6 \text { mon. }>6 \text { mon. }\end{array}$

\begin{tabular}{|c|c|c|c|c|c|c|c|c|c|c|}
\hline 1974 & 2,593 & 74 & 74.0 & 3,504 & 70.3 & 449 & 3,230 & & & \\
\hline 1975 & 2,535 & 80 & 7.8 & 3,260 & 124.9 & 546 & 3,737 & & & \\
\hline 1976 & 2,865 & 73 & 75.6 & 3,789 & 66.5 & 580 & 3,915 & & & \\
\hline 1977 & 2,680 & 84 & 79.9 & 3,355 & (17.1) & 533 & 3,669 & & & \\
\hline 1978 & 2,842 & 77 & 79.6 & 3,569 & 73.8 & 572 & 3,873 & & & \\
\hline 1979 & 2,950 & 49 & 50.5 & 4,057 & 155.5 & 686 & 4,471 & & & \\
\hline 1980 & 3,241 & 58 & 54.6 & 5,932 & 137.7 & 778 & 4,952 & & & \\
\hline 1981 & 3,403 & 67 & 63.6 & 5,340 & 80.2 & 810 & 5,120 & & & \\
\hline 1982 & 4,209 & 81 & 75.8 & 5,556 & 179.0 & 934 & 5,768 & & & \\
\hline 1983 & 4,168 & 70 & 74.1 & 5,623 & 172.0 & 1,043 & 6,336 & & & \\
\hline 1984 & 4,411 & 68 & 68.8 & 6,416 & 172.1 & 1,145 & 6,870 & & & \\
\hline 1985 & 4,819 & 57 & 61.1 & 7,883 & 112.3 & 1,182 & 7,066 & & & \\
\hline 1986 & 5,025 & 76 * & 68.8 & 7,300 & 162.7 & 1,263 & 7,485 & & & \\
\hline$\cdots$ & $-\cdots$ & $\cdots$ & $\cdots$ & $-\cdot-$ & $-\cdots$ & $-\cdots$ & $-\cdots$ & $\cdots$ & $\cdot \cdot \cdot$ & $\cdots$ \\
\hline 1987 & 5,128 & 80 & 78.5 & 0.535 & 131.0 & 1,312 & 7,746 & & & \\
\hline 1988 & 5,266 & 73 & 73.6 & 6,964 & 148.1 & 1,376 & 8,079 & 8,049 & 10,094 & 10,123 \\
\hline 1989 & & & & & 159.9 & 1,447 & 8,452 & 8,421 & 10,560 & 10,591 \\
\hline 1990 & & & & & 166.6 & 1,521 & 8,836 & 8,804 & 11,040 & 11,072 \\
\hline 1991 & & & & & 178.8 & 1,602 & 9.260 & 9,226 & 11,569 & 11,603 \\
\hline 1992 & & & & & 167.7 & 1,666 & 9,508 & 9,563 & 11,992 & 12,026 \\
\hline
\end{tabular}

Capacity-Capital Stock Regression: Code 0 RSO 0.890

Coefficients: Const 882.0 stock 5.2

Ave. Capacity/Stock $(74-86): 6.3$ Ave. Heekly Hours (High) 126.4 Shift factor 1.25 Ave Heekly Hours (LOW) 118.8 Shift Factor 1.33 * Imputed Note: Output, Investment, and Capital Stock extrapolated for 1987 and 1988.

\section{Surgical and medical instruments}

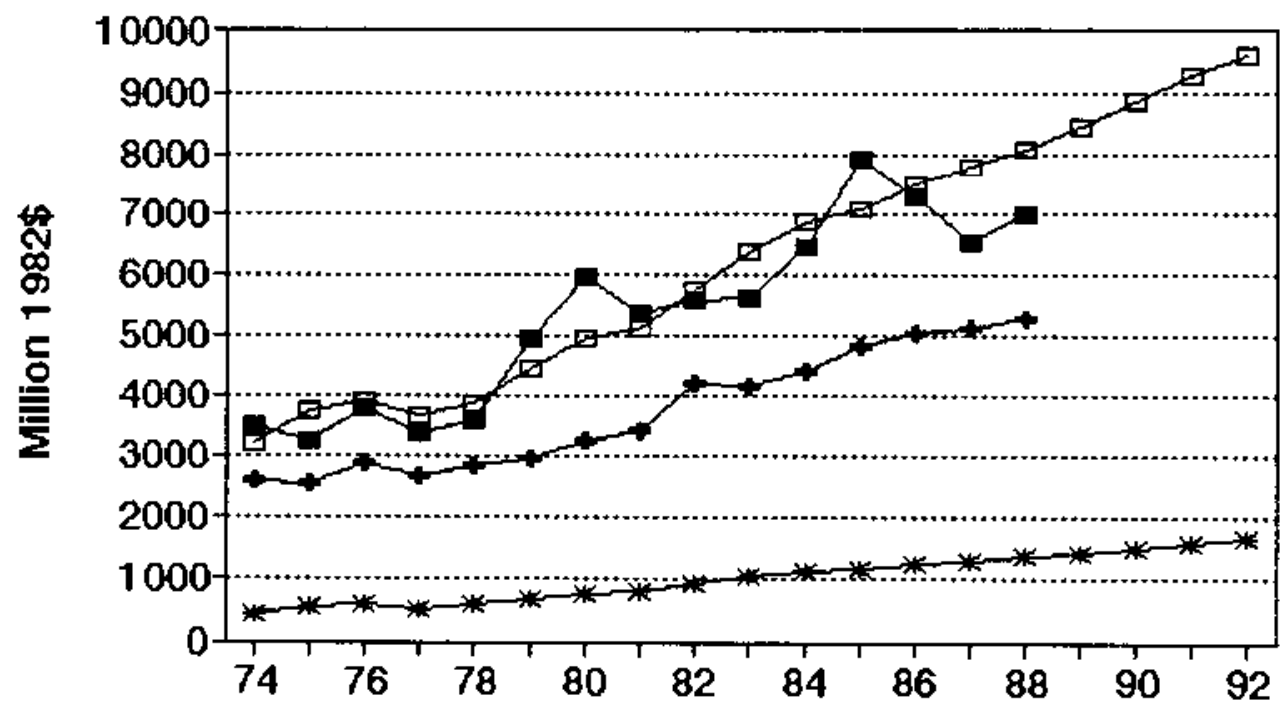

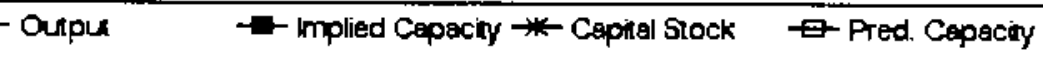




\section{No. 422 gIC 3842 Surgical appliances and supplies}

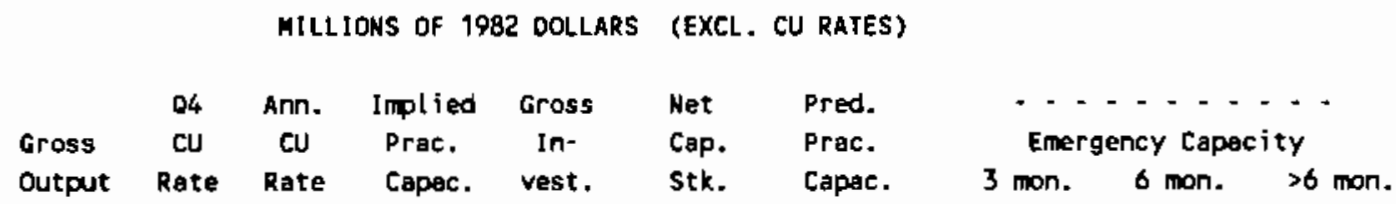

\begin{tabular}{|c|c|c|c|c|c|c|c|c|c|c|}
\hline 1974 & 2,835 & 73 & 73.9 & 3,834 & 77.8 & 473 & 3,993 & & & \\
\hline 1973 & 3,205 & 64 & 66.5 & 4,822 & 147.5 & 578 & 4,754 & & & \\
\hline 1976 & 3,123 & $\infty 6$ & 65.2 & 4,791 & 67.1 & 600 & 4,919 & & & \\
\hline 1977 & 3,348 & 59 & 61.7 & 5,429 & 67.1 & 621 & 5,066 & & & \\
\hline 1978 & 3,713 & 74 & 68.4 & 5,426 & 80.2 & 652 & 5,294 & & & \\
\hline 1979 & 4,089 & 84 & 80.4 & 5,088 & 76.2 & 679 & 5,485 & & & \\
\hline 1980 & 4,104 & 83 & 83.2 & 4,931 & 97.3 & 724 & 5,812 & & & \\
\hline 1981 & 4,731 & $\pi$ & 79.4 & 5,985 & 110.6 & 780 & 6,222 & & & \\
\hline 1982 & 5,857 & 76 & 76.8 & 7,624 & 185.4 & 905 & 7,122 & & & \\
\hline 1983 & 5,898 & 68 & 70.7 & 8,344 & 206.0 & 1,040 & 8,103 & & & \\
\hline 1984 & 6,623 & 58 & 62.3 & 10,640 & 169.5 & 1,138 & 8,809 & & & \\
\hline 1985 & 7,048 & $\pi$ & 70.0 & 10,074 & 249.7 & 1,309 & 10,050 & & & \\
\hline 1986 & 7,179 & $\pi$ & 75.8 & 9,471 & 195.1 & 1,417 & 10,831 & & & \\
\hline$\cdots$ & $-\cdots$ & $\cdots$ & $\cdots$ & $\cdots$ & $\cdots$ & $\cdots$ & $\cdots$ & $-\cdots$ & $\cdots-$ & $--\cdot$ \\
\hline 1987 & 7,327 & 76 & $\pi 5.6$ & 9,690 & 177.3 & 1,508 & 11,499 & & & \\
\hline 1988 & 7,522 & 67 & 70.3 & 10,696 & 212.3 & 1,629 & 12,365 & 13,549 & 17,978 & 18,041 \\
\hline 1989 & & & & & 236.5 & 1,766 & 13,361 & 14,641 & 19,425 & 19,494 \\
\hline 1990 & & & & & 250.1 & 1,909 & 14,395 & 15,774 & 20,929 & 21,003 \\
\hline 1991 & & & & & 275.2 & 2,068 & 15,548 & 17,037 & 22,606 & 22,685 \\
\hline 1992 & & & & & 252.4 & 2,195 & 16,466 & 18,043 & 23,940 & 24,024 \\
\hline
\end{tabular}

Capacity-Capital Stock Regression: Code 0 RSO 0.890 Coefficients: Const 571.1 stock 7.2

Ave. Copecity/Stock $(74-86): 7.9$ Ave. Weekly Hours (High) 107.9 Shift Factor 1.46 Ave Veekly Hours (Lou) 107.5 Shift Factor 1.47 Note: Output, Investment, and Capital Stock extrapolated for 1987 and 1988.

\section{Surgical appliances and supplies}

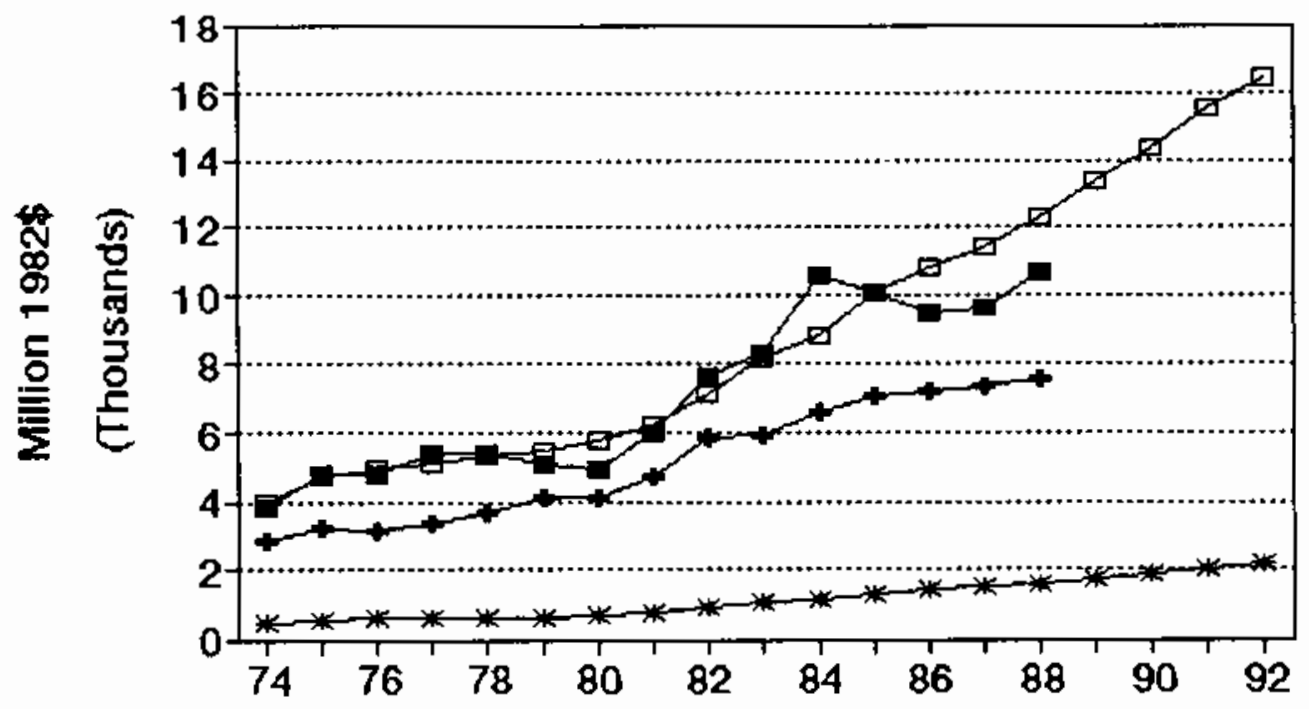

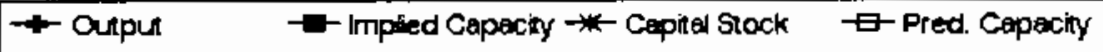


No. 423 sIC 3843 Dental equipment and supplies

MILLIONS OF 1982 DOLLARS (EXCL. CU RATES)

$\begin{array}{lccccccc} & \text { Q4 } & \text { Ann. Implied Gross } & \text { Net } & \text { Pred. } & \ldots & \ldots \\ \text { Gross } & \text { CU } & \text { CU } & \text { Prac. } & \text { In- } & \text { Cap. } & \text { Prac. } & \text { Emergency Capacity } \\ \text { Output } & \text { Rate } & \text { Rate } & \text { Capec. } & \text { vest. } & \text { Stk. } & \text { Capac. } & 3 \text { mon. } 6 \text { mon. }>6 \text { mon. }\end{array}$

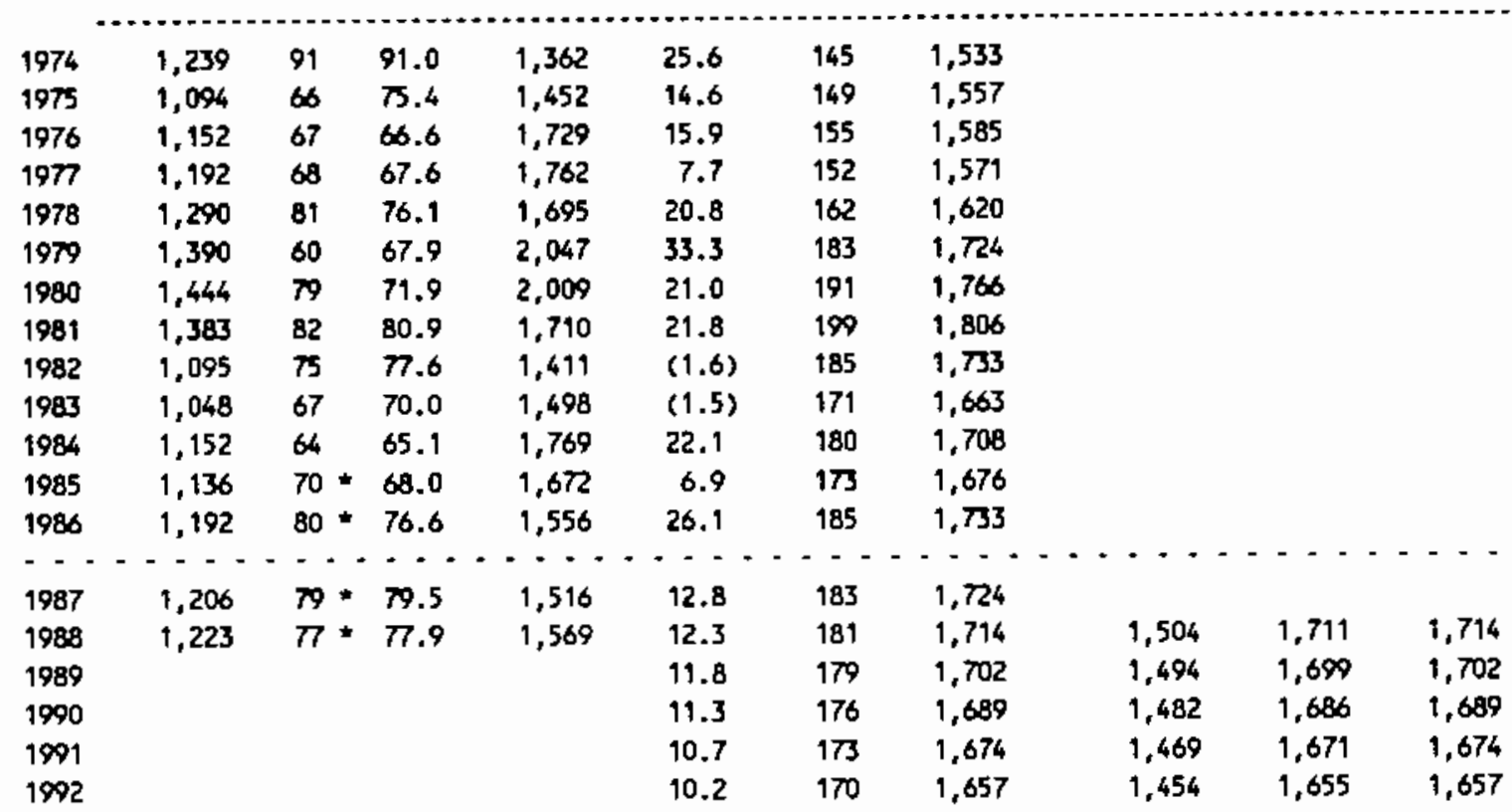

Capacity-Capital Stock Regression: Code 0 RSO 0.170 Coefficients: Const 809.1 Stock 5.0

Ave. Capecity/Stock $(74-86): 9.7$ Ave. Weekly Hours (High) 1984.0 Shift Factor 1.00 Ave Heekly Hours (Low) 1984.0 Shift Factor 1.0 * Imputed Note: Output, Investment, and Capital stock extrapolated for 1987 and 1988.

\section{Dental equipment and supplies}

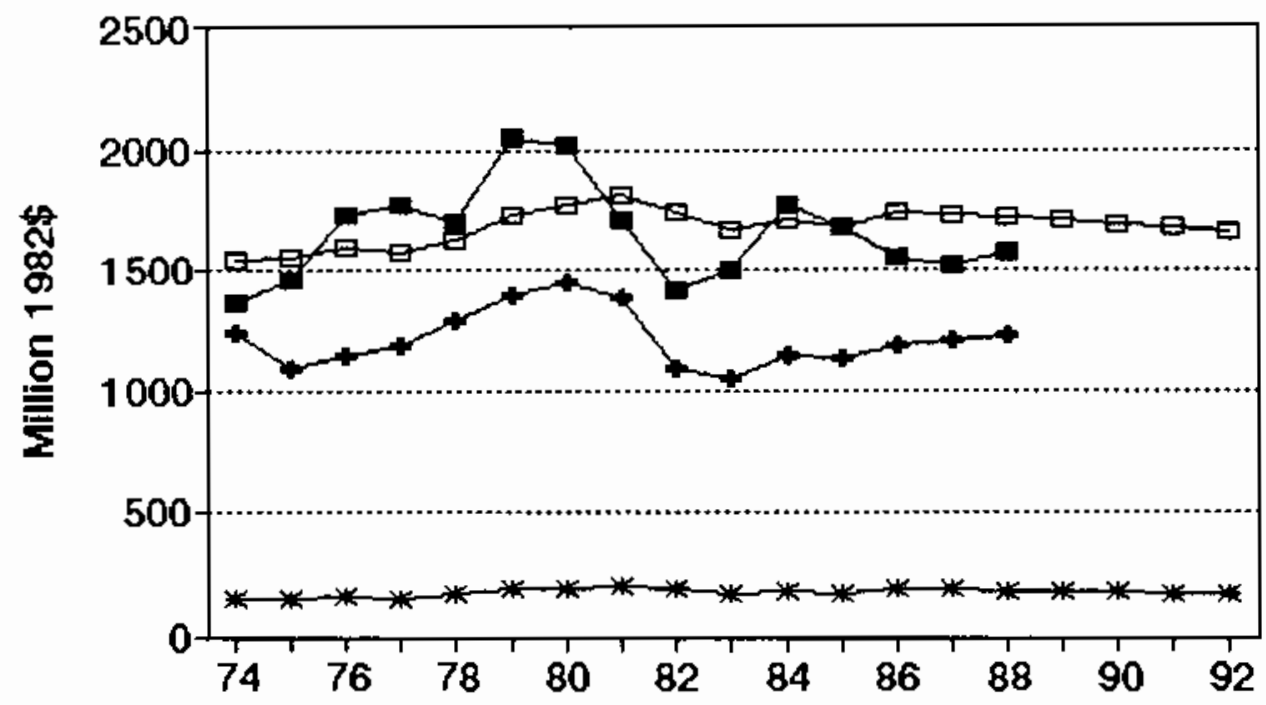

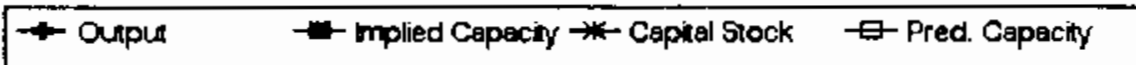




\section{No. 424 SIC 3851 Opthalmic goods}

MILLIONS OF 1982 DOLLARS (EXCL. CU RATES)

$\begin{array}{lccccccc} & \text { Q4 } & \text { Ann. Implied Gross } & \text { Net } & \text { Pred. } & \ldots \ldots \\ \text { Gross } & \text { CU } & \text { CU } & \text { Prac. } & \text { In- } & \text { Cap. } & \text { Prac. } & \text { Emergency Capacity } \\ \text { Output } & \text { Rate } & \text { Rate } & \text { Capac. } & \text { vest. } & \text { Stk. } & \text { Capac. } & 3 \text { mon. } 6 \text { mon. } 26 \text { mon. }\end{array}$

\begin{tabular}{|c|c|c|c|c|c|c|c|c|c|c|}
\hline 1974 & 1,140 & 28 & 28.0 & 4,071 & 12.7 & 303 & 3,460 & & & \\
\hline 1975 & 998 & 35 & 32.4 & 3,082 & 43.7 & 320 & 3,428 & & & \\
\hline 1976 & 1,142 & 37 & 36.3 & 3,150 & 32.5 & 326 & 3,417 & & & \\
\hline 1977 & 1,302 & 38 & 37.6 & 3,461 & 51.5 & 349 & 3,374 & & & \\
\hline 1978 & 1,412 & 47 & 43.6 & 3,236 & 57.4 & 376 & 3,322 & & & \\
\hline 1979 & 1,245 & 80 & 67.6 & 1,842 & 18.1 & 364 & 1,881 & & & \\
\hline 1980 & 1,267 & 78 & 78.8 & 1,609 & 39.8 & 372 & 1,866 & & & \\
\hline 1981 & 1,246 & 54 & 63.0 & 1,977 & 24.8 & 365 & 1,880 & & & \\
\hline 1982 & 1,285 & 71 & 64.6 & 1,989 & 17.8 & 352 & 1,904 & & & \\
\hline 1983 & 1,297 & 73 & 72.3 & 1,795 & 53.5 & 371 & 1,867 & & & \\
\hline 1984 & 1,317 & $\pi *$ & 74.5 & 1,768 & 56.8 & 394 & 1,825 & & & \\
\hline 1985 & 1,363 & $71 \star$ & 72.5 & 1,880 & 46.3 & 406 & 1,802 & & & \\
\hline 1986 & 1,344 & 73 & 72.2 & 1,862 & 91.0 & 461 & 1,696 & & & \\
\hline-- & $-\cdot-$ & $\cdots$ & $\cdots$ & $\cdot \cdot \cdot$ & $\cdots$ & -- & $-\cdots$ & $-\cdots$ & $\cdots$ & -- \\
\hline 1987 & 1,354 & $77 *$ & 75.3 & 1,798 & 46.1 & 470 & 1,679 & & & \\
\hline 1988 & 1,366 & $\pi$ & 75.6 & 1,806 & 50.5 & 483 & 1,655 & 1,953 & 2,385 & 2,391 \\
\hline 1989 & & & & & 53.5 & 498 & 1,627 & 1,920 & 2,345 & 2,351 \\
\hline 1990 & & & & & 55.3 & 513 & 1,598 & 1,886 & 2,303 & 2,309 \\
\hline 1991 & & & & & 58.4 & 530 & 1,566 & 1,848 & 2,256 & 2,262 \\
\hline 1992 & & & & & 55.5 & 543 & 1,541 & 1,819 & 2,221 & 2,227 \\
\hline
\end{tabular}

Capacity-Capital Stock Regression: Code -1 RSO 0.910

Coefficients: Const 4036.8 Stock $-1.9 \mathrm{D}(79-86)-1463.6$ Ave. Capacity/Stock (74-86): 6.7 Ave. Weekly Hours (High) 109.0 shift Factor 1.45 Ave Weekly Hours (LOW) 99.0 Shift factor 1.59 - Imputed Note: Output, Investment, and Cepital stock extrapolated for 1987 and 1988.

3851 Opthalmic goods

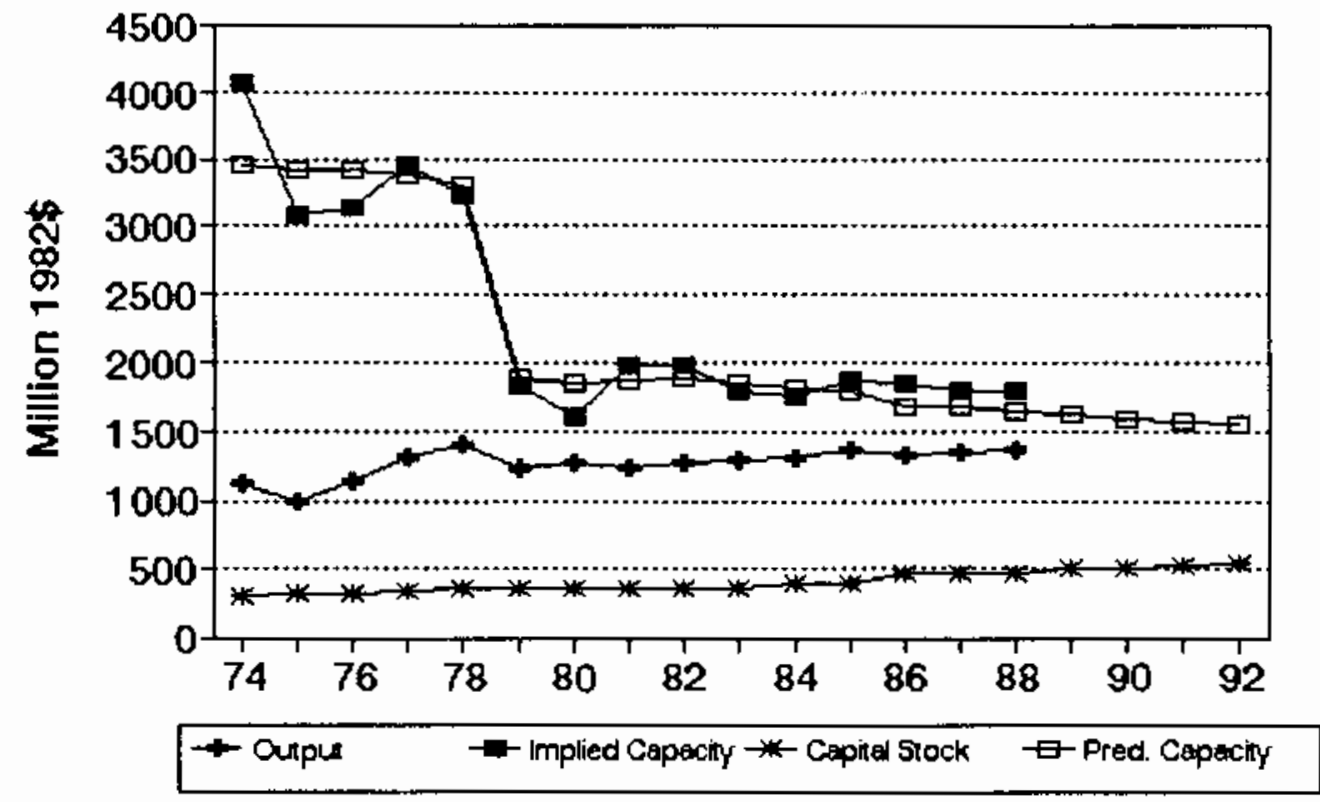




\section{No. 425 SIC 3861 Photographic equipment and supplies}

MILLIONS OF 1982 DOLLARS (EXCL. CU RATES)

$\begin{array}{lccccccc} & 04 & \text { Amn. } & \text { Implied } & \text { Gross } & \text { Het } & \text { Pred. } & \ldots \\ \text { Gross } & \text { CU } & \text { CU } & \text { Prac. } & \text { In- } & \text { Cap. } & \text { Prac. } & \text { Emergency Capacity } \\ \text { Output } & \text { Rate } & \text { Rate } & \text { Capac. } & \text { vest. } & \text { Stk. } & \text { Capac. } & 3 \text { mon. } 6 \text { mon. }>6 \text { mon. }\end{array}$

\begin{tabular}{|c|c|c|c|c|c|c|c|c|c|c|}
\hline 1974 & 13,149 & 77 & 81.1 & 16,206 & 529.1 & 3,742 & 17,906 & & & \\
\hline 1975 & 11,858 & 64 & 64.8 & 18,288 & 417.5 & 3,912 & 18,316 & & & \\
\hline 1976 & 13,539 & 66 & 65.0 & 20,826 & 680.4 & 4,306 & 19,268 & & & \\
\hline 1977 & 14,415 & 69 & 68.0 & 21,194 & $4 \pi 5.0$ & 4,466 & 19,657 & & & \\
\hline 1978 & 16,109 & 85 & 79.3 & 20,314 & 451.0 & 4,583 & 19,938 & & & \\
\hline 1979 & 17,744 & 87 & 86.7 & 20,459 & 499.5 & 4,731 & 20,297 & & & \\
\hline 1980 & 16,499 & 82 & 83.3 & 19,806 & 595.4 & 4,957 & 20,844 & & & \\
\hline 1981 & 17,042 & 77 & 79.4 & 21,467 & 712.7 & 5,284 & 21,633 & & & \\
\hline 1982 & 17,209 & 76 & 78.4 & 21,941 & 746.1 & 5,618 & 22,440 & & & \\
\hline 1983 & 17,104 & $\pi$ & 73.9 & 23,142 & 691.7 & 5,863 & 23,033 & & & \\
\hline 1984 & 17,605 & 77 & 78.7 & 22,374 & 574.9 & 5,979 & 23,313 & & & \\
\hline 1985 & 17,219 & 71 & 73.4 & 23,454 & 775.9 & 6,277 & 24,034 & & & \\
\hline 1986 & 18,368 & 71 & 71.3 & 25,778 & 727.4 & 6,499 & 24,569 & & & \\
\hline$\because$ & --- & -- & & $-\cdots$ & $\cdots$ & $-\cdots$ & --- & $\cdot \cdot \cdot-$ & --- & $-\cdots$ \\
\hline 1987 & 18,643 & 77 & 74.7 & 24,962 & 675.5 & 6.674 & 24.993 & & & \\
\hline 1988 & 19,006 & 77 & 76.7 & 24,767 & 734.8 & 6,895 & 25,528 & 28,565 & 35,588 & 35,688 \\
\hline 1989 & & & & & 775.9 & 7,140 & 26,120 & 29,228 & 36,414 & 36,516 \\
\hline 1990 & & & & & 799.1 & 7,390 & 26,724 & 29,903 & 37,255 & 37,360 \\
\hline 1991 & & & & & 841.7 & 7,663 & 27,383 & 30,641 & 38,175 & 38,282 \\
\hline 1992 & & & & & 803.0 & 7,876 & 27,899 & 31,218 & 38,893 & 39,003 \\
\hline
\end{tabular}

Capacity-Capital Stock Regression: Code 0 RSO 0.830

Coefficients: Const 8860.2 stock 2.4

Ave. Capecity/5tock $(74-86): 4.2$

Ave. Heekly Hours (High) 112.8 Shift factor 1.40 Ave Heekly Hours (Low) 109.5 Shift Factor 1.44 Note: Output, Investment, and Capital Stock extrapolated for 1987 and 1988.

\section{Photographic equipment and supplie}

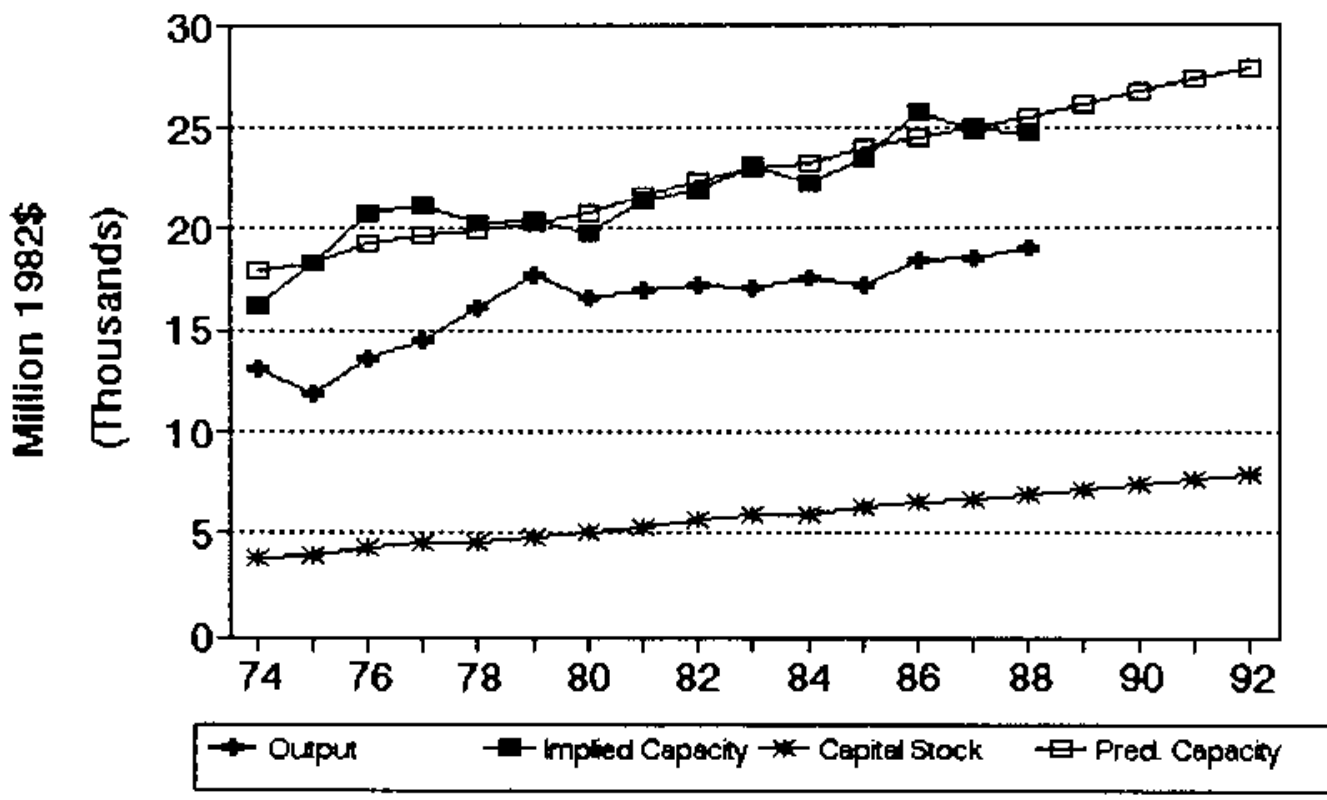


No. 426 SIC 3873 Watches, clocks, and watchcases

MILLIONS OF 1982 DOLLARS (EXCL. CU RATES)

$\begin{array}{lcccclcc} & Q 4 & \text { Ann. Implied Gross } & \text { Net } & \text { Pred. } & \ldots \ldots \\ \text { Grass } & \text { CU } & \text { CU } & \text { Prac. } & \text { In- } & \text { Cap. } & \text { Prac. } & \text { Emergency Capacity } \\ \text { Output Rete } & \text { Rate } & \text { Capac. } & \text { vest. } & \text { Stk. } & \text { Capac. } & 3 \text { mon. } 6 \text { mon. }>6 \text { mon. }\end{array}$

\begin{tabular}{|c|c|c|c|c|c|c|c|c|c|c|}
\hline 1974 & 1,734 & 71 & 74.5 & 2,329 & 23.8 & 230 & 3,030 & & & \\
\hline 1975 & 1,603 & 58 & 59.5 & 2,693 & 34.2 & 244 & 3,290 & & & \\
\hline 1976 & 2,061 & 51 & 53.5 & 3,851 & 24.9 & 249 & 3,374 & & & \\
\hline 1977 & 2,105 & 51 & 51.1 & 4,118 & 27.5 & 255 & 3,479 & & & \\
\hline 1978 & 2,322 & 52 & 51.9 & 4,477 & 71.4 & 301 & 4,301 & & & \\
\hline 1979 & 1,801 & 67 & 61.7 & 2,919 & $(62.2)$ & 221 & 2,873 & & & \\
\hline 1980 & 1,588 & 74 & 71.0 & 2,238 & B.3 & 211 & 2,706 & & & \\
\hline 1981 & 1,391 & 63 & 67.5 & 2,061 & $(1.0)$ & 194 & 2,394 & & & \\
\hline 1982 & 1,163 & 47 & 54.1 & 2,152 & $(8.4)$ & 171 & 1,981 & & & \\
\hline 1983 & 969 & 38 & 40.7 & 2,382 & 5.6 & 161 & 1,818 & & & \\
\hline 1984 & 1,031 & 64 & 55.5 & 1.859 & 2.4 & 150 & 1,606 & & & \\
\hline 1985 & 830 & $\pi$ & 69.9 & 1,187 & 8.9 & 144 & 1,510 & & & \\
\hline 1986 & 1,040 & 58 & 63.7 & 1,632 & 15.8 & 146 & 1,536 & & & \\
\hline$\cdots$ & $--\cdot$ & -- & $\cdots$ & $\cdots-$ & $-\cdots$ & $\cdots$ & $\cdots$ & $-\cdots$ & $\cdots$ & $\cdots$ \\
\hline 1987 & 1,029 & 60 & 59.2 & 1,737 & 8.0 & 139 & 1,423 & & & \\
\hline 1988 & 1,014 & $62 *$ & 60.9 & 1,066 & 8.0 & 134 & 1,321 & 1,458 & 1,785 & 1,790 \\
\hline 1989 & & & & & 8.0 & 128 & 1,229 & 1,357 & 1,660 & 1,665 \\
\hline 1990 & & & & & 8.0 & 124 & 1,145 & 1,265 & 1,548 & 1,552 \\
\hline 1991 & & & & & 8.0 & 119 & 1,071 & 1,182 & 1,447 & 1,451 \\
\hline 1992 & & & & & 8.0 & 116 & 1,003 & 1,108 & 1,356 & 1,359 \\
\hline
\end{tabular}

Capacity-Capital Stock Regression: Code 0 RSO 0.800

Coefficients: Const -1055.5 stock 17.8

Ave. Capacity/Stock $(74-86): 12.7$ Ave. Weekly Hours (High) 116.5 Shift factor 1.36 Ave Weekly Hours (Low) 82.3 Shift factor 1.91 * Imputed Note: Output, Investment, and Capitel stock extrapoleted for 1987 and 1988.

\section{Watches, clocks, and watchcases}

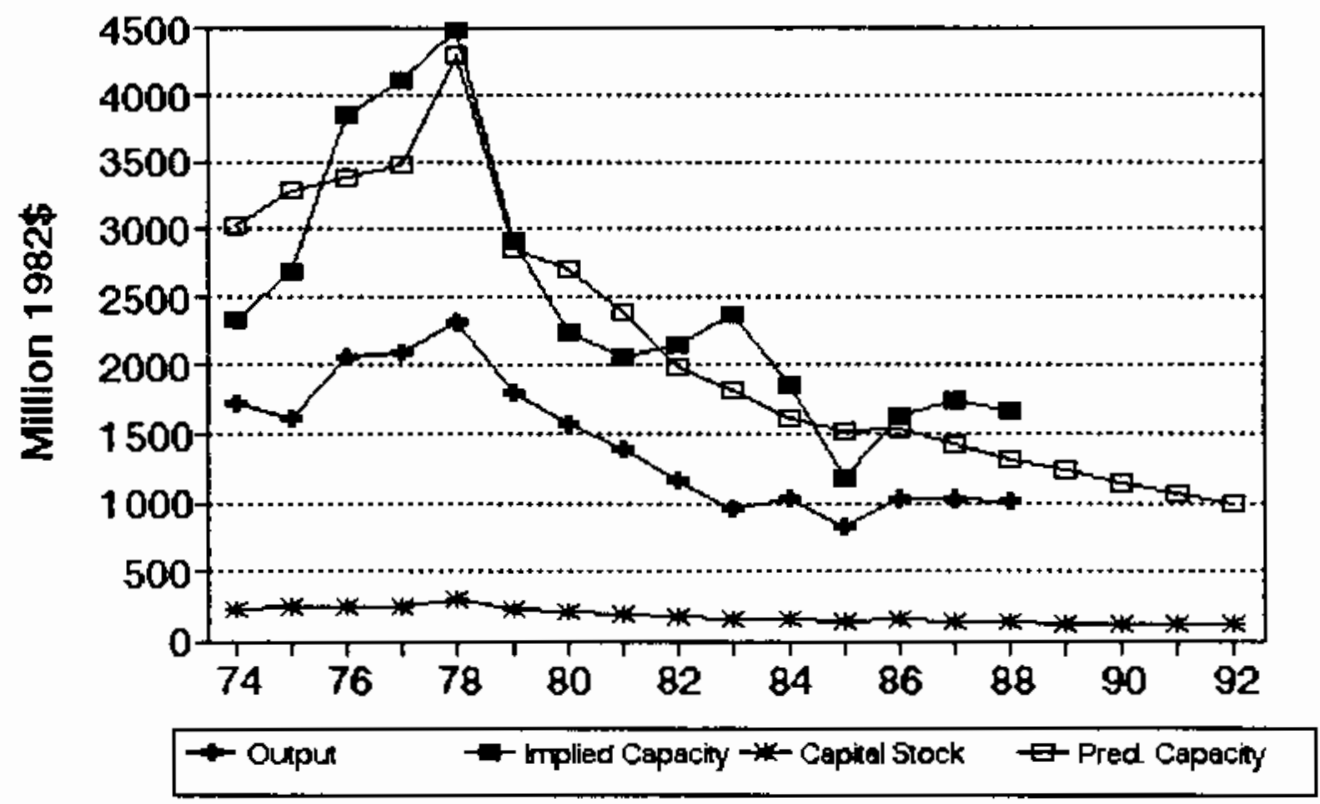




\section{No. 427 SIC 3911 Jewelry, precious metal}

MILLIONS OF 1982 DOLLARS (EXCL. CU RATES)

$\begin{array}{lccccccc} & \text { O4 } & \text { Amn. Implied Gross } & \text { Net } & \text { Pred. } & \ldots \ldots \ldots \\ \text { Gross } & \text { CU } & \text { CU } & \text { Prac. } & \text { In- } & \text { Cap. } & \text { Prac. } & \text { Emergency Capacity } \\ \text { Output Rate } & \text { Rate } & \text { Capac. } & \text { vest. } & \text { Stk. } & \text { Capac. } & 3 \text { mon. } 6 \text { mon. }\end{array}$

\begin{tabular}{|c|c|c|c|c|c|c|c|c|c|c|}
\hline 1974 & 4,261 & 90 & 90.0 & 4,734 & 5.8 & 452 & 5,479 & & & \\
\hline 1975 & 4.181 & $\pi$ & 80.6 & 5,186 & 88.5 & 508 & 5,738 & & & \\
\hline 1976 & 5,446 & 75 & 75.0 & 7,262 & 81.8 & 560 & 5,982 & & & \\
\hline 1977 & 5,680 & 88 & Q3.1 & 6,833 & $(184.4)$ & 358 & 5,039 & & & \\
\hline 1978 & 4,609 & 86 & 86.8 & 5,312 & 10.5 & 352 & 5,010 & & & \\
\hline 1979 & 3,610 & 92 & 89.8 & 4,023 & 19.9 & 355 & 5,023 & & & \\
\hline 1980 & 2,258 & 68 & 77.0 & 2,933 & 12.1 & 350 & 5,000 & & & \\
\hline 1981 & $2, \pi 1$ & 64 & 65.5 & 4,200 & 16.4 & 349 & 4,994 & & & \\
\hline 1982 & 3,083 & 57 & 59.6 & 5,171 & 15.4 & 346 & 4,982 & & & \\
\hline 1983 & 2,810 & 87 & 75.8 & 3,709 & 12.0 & 339 & 4,950 & & & \\
\hline 1984 & 3,070 & 56 & 67.6 & 4,539 & $(0.7)$ & 321 & 4,863 & & & \\
\hline 1985 & 3,212 & 51 & 52.9 & 6,075 & 2.7 & 306 & 4,794 & & & \\
\hline 1986 & 3,277 & 48 & 49.1 & 6,671 & 17.7 & 306 & 4,794 & & & \\
\hline $\begin{array}{l}--- \\
1987\end{array}$ & 3,249 & $\begin{array}{l}\cdots \\
44\end{array}$ & $\because \cdots$ & $\cdots$ & $\begin{array}{l}-- \\
(0.3)\end{array}$ & $\begin{array}{l}\cdots \\
288\end{array}$ & $\begin{array}{l}\cdots, 709 \\
4,70\end{array}$ & $-\cdots$ & -- & $-\cdots$ \\
\hline 1988 & 3,213 & 44 & 44.0 & 7,301 & (1.7) & 270 & 4,624 & 4,795 & 5,957 & 5,974 \\
\hline 1989 & & & & & (3.1) & 251 & 4,536 & 4,704 & 5,845 & 5,861 \\
\hline 1990 & & & & & $(4.4)$ & 232 & 4,448 & 4,612 & 5,731 & 5,747 \\
\hline 1991 & & & & & (5.8) & 213 & 4,358 & 4,519 & 5,615 & 5,630 \\
\hline 1992 & & & & & $(7.2)$ & 193 & 4,267 & 4,425 & 5,497 & 5,513 \\
\hline
\end{tabular}

Capacity-Capital Stock Regression: Code 1 RSO 0.080

Coefficients: Const 3360.7 stock 4.7

Ave. Capacity/Stock (74-86): 13.6 Ave. Heekly Hours (High) 122.3 Shift Factor 1.29 Ave Veekly Hours (Low) 118.1 Shift Factor 1.34 Note: Output, Imvestment, and Capital stock extrepolated for 1987 and 1988.

\section{Jewelry, precious metal}

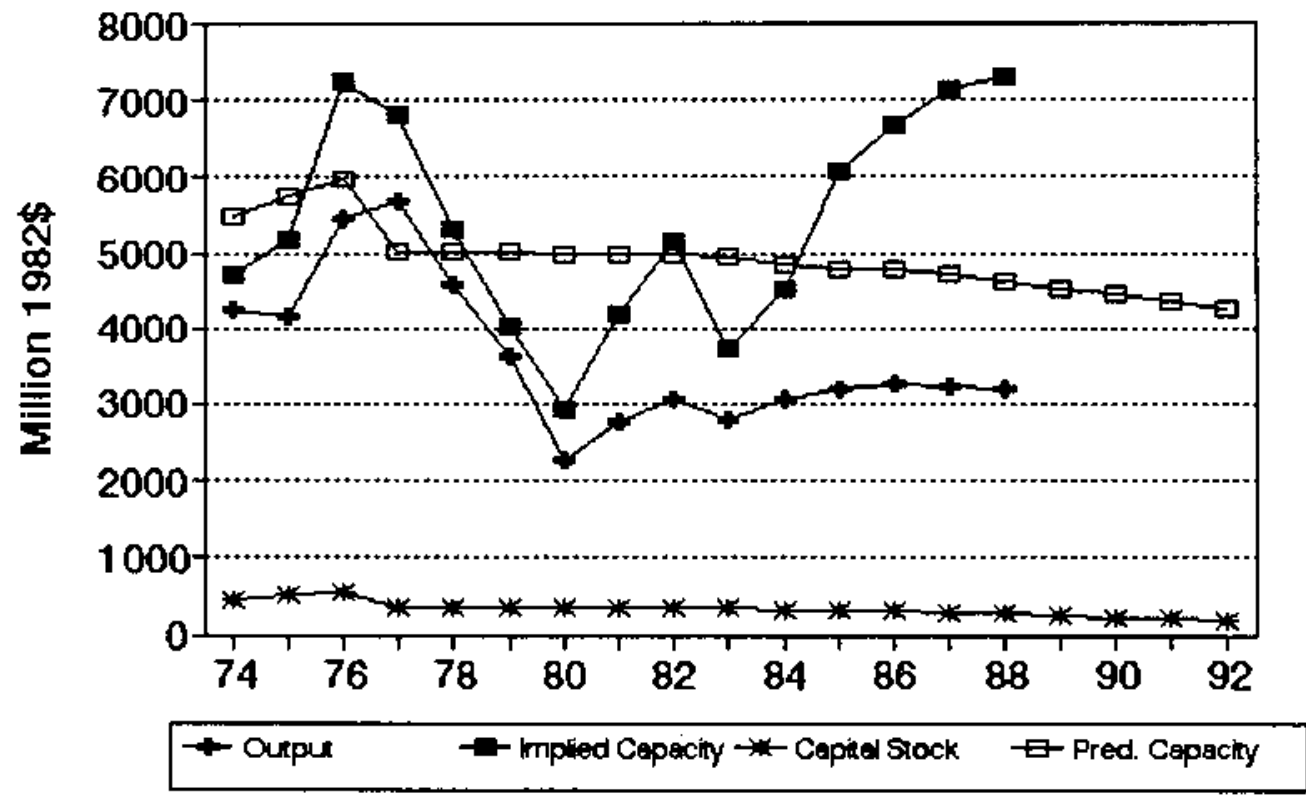




\section{No. 428 sIC 3914 Silverware and plated ware}

MILLIONS OF 1992 DOLLARS (EXCL. CU RATES)

$\begin{array}{lccccccc} & 04 & \text { Ann. Implied Gross } & \text { Net } & \text { Pred. } & -\ldots-\ldots \\ \text { Gross } & \text { CU } & \text { CU } & \text { Prac. } & \text { In- } & \text { Cap. } & \text { Prac. } & \text { Emergency Capecity } \\ \text { Output Rate } & \text { Rate } & \text { Capac. } & \text { vest. } & \text { Stk. } & \text { Capec. } & 3 \text { mon. } 6 \text { mon. }>6 \text { mon. }\end{array}$

\begin{tabular}{|c|c|c|c|c|c|c|c|c|c|c|}
\hline 1974 & 695 & 34 & 47.8 & 1,454 & 0.8 & 167 & 1,435 & & & \\
\hline 1975 & 599 & 30 & 26.8 & 2,240 & 17.7 & 171 & 1,481 & & & \\
\hline 1976 & 684 & 74 & 55.9 & 1,223 & 1.0 & 159 & 1,357 & & & \\
\hline 1977 & 764 & 59 & 68.3 & 1,120 & 24.9 & 169 & 1,461 & & & \\
\hline 1978 & 780 & 71 & 65.9 & 1,184 & 7.3 & 162 & 1,384 & & & \\
\hline 1979 & 645 & $44^{*}$ & 53.0 & 1,219 & 10.3 & 157 & 1,336 & & & \\
\hline 1980 & 394 & $36 *$ & 35.9 & 1,096 & $(0.3)$ & 143 & 1,188 & & & \\
\hline 1981 & 533 & $52 *$ & 52.8 & 1,009 & 20.4 & 149 & 1,250 & & & \\
\hline 1982 & 597 & 30 & 37.1 & 1,612 & 9.2 & 144 & 1,194 & & & \\
\hline 1983 & 436 & $51 *$ & 46.6 & 935 & $(3.6)$ & 127 & 1,017 & & & \\
\hline 1984 & 524 & 52 & 55.4 & 946 & 27.1 & 138 & 1,138 & & & \\
\hline 1985 & 561 & 59 & 56.5 & 993 & $(9.3)$ & 116 & 902 & & & \\
\hline 1986 & 508 & 51 & 54.0 & 941 & 6.0 & 109 & 830 & & & \\
\hline$\cdots$ & $\cdots$ & $\cdots$ & $\because$ & $\cdots$ & $\cdots$ & $\cdots$ & $\cdots$ & $\cdots$ & $\because \cdot$ & $=-$ \\
\hline 1987 & 500 & 82 & 68.8 & 727 & 9.8 & 106 & 799 & & & \\
\hline 1988 & 489 & 80 & 80.9 & 605 & 11.5 & 305 & 790 & 1,568 & 2,360 & 2,371 \\
\hline 1989 & & & & & 14.4 & 107 & 813 & 1,613 & 2,428 & 2,439 \\
\hline 1990 & & & & & 12.7 & 107 & 815 & 1,616 & 2,432 & 2,444 \\
\hline 1991 & & & & & 13.7 & 109 & 827 & 1,639 & 2,468 & 2,479 \\
\hline 1992 & & & & & 12.4 & 108 & 824 & 1.634 & 2,460 & 2,472 \\
\hline
\end{tabular}

Capecity-Capital stock Regression: Code 1 Rso 0.330 Coefficients: Const $\mathbf{- 3 0 8 . 1}$ stock 10.5

Ave. Capacity/Stock $(74-86): 8.4$ Ave, Heekly Hours (High) 52.5 Shift Factor 3.00 Ave Ueekly Hours (Lou) 47.4 Shift Factor 3.32 - Imputed Note: Output, Investment, and Cepital Stock extrapolated for 1987 and 1988.

\section{Silverware and plated ware}

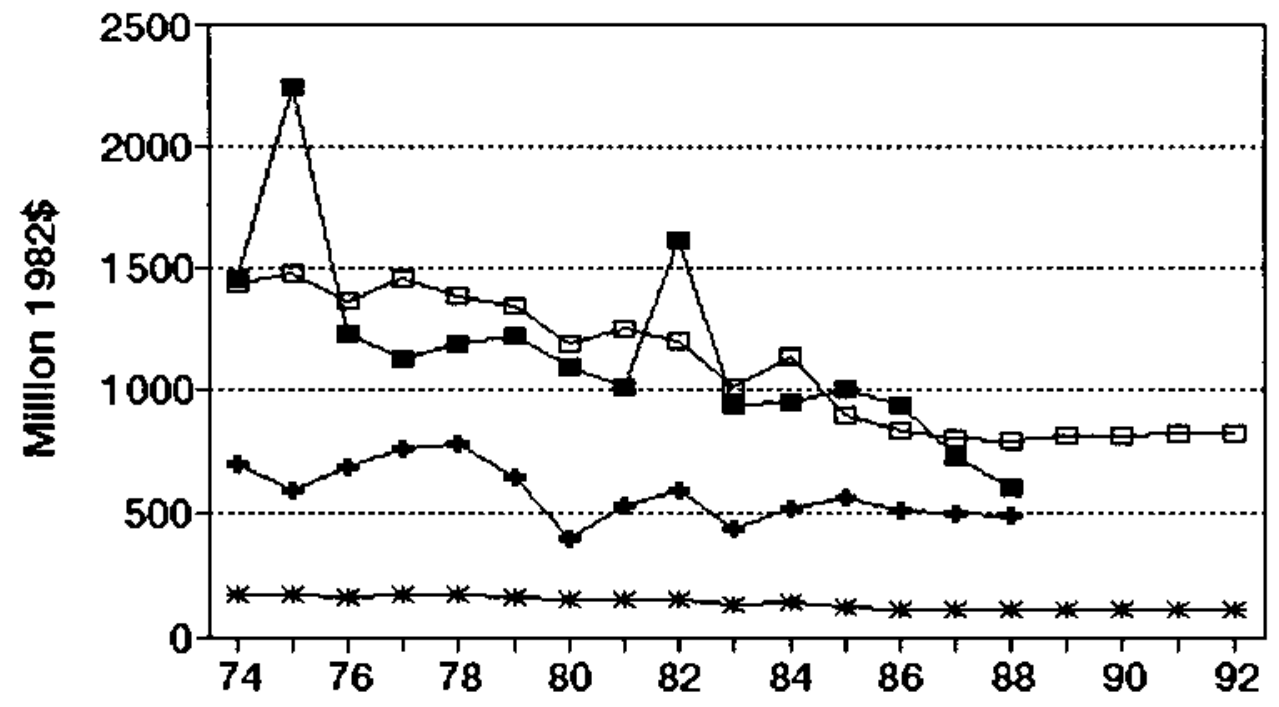

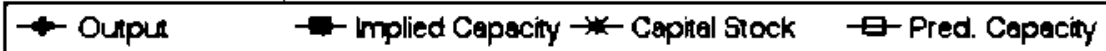


No. 429 sIC 3915 Jewelers' materiale and lapidary work MILLIONS OF 1982 DOLLARS (EXCL. CU RATES)

$\begin{array}{lccccccc} & 04 & \text { Ann. } & \text { Implied Gross } & \text { Net } & \text { Pred. } & \ldots \ldots \\ \text { Gross } & \text { CU } & \text { CU } & \text { Prac. } & \text { In- } & \text { Cap. } & \text { Prac. } & \text { Emergency Capacity } \\ \text { Output } & \text { Rate } & \text { Rate } & \text { Capac. } & \text { vest. } & \text { Stk. } & \text { Capac. } & 3 \text { mon. } 6 \text { mon. }>6 \text { mon. }\end{array}$

\begin{tabular}{|c|c|c|c|c|c|c|c|c|c|c|}
\hline 1974 & 717 & 62 & 81.1 & $8 B 4$ & $(4.9)$ & 85 & 1,268 & & & \\
\hline 1975 & 587 & 59 & 53.8 & 1,092 & 2.2 & 82 & 1,293 & & & \\
\hline 1976 & 776 & 74 & 68.4 & 1,134 & 18.1 & 94 & 1,200 & & & \\
\hline 1977 & 1,001 & 81 & 81.4 & 1,230 & $(0.4)$ & 89 & 1,248 & & & \\
\hline 1978 & 1,051 & 85 & 82.9 & 1,268 & 12.5 & 94 & 1,205 & & & \\
\hline 1979 & 1,084 & $\infty 6$ & 71.8 & 1,510 & $(0.9)$ & 87 & 1,258 & & & \\
\hline 1980 & 1,155 & 60 & 58.7 & 1,969 & 4.6 & 85 & 1,269 & & & \\
\hline 1981 & $\$ 54$ & 72 & 74.5 & 1,281 & 1.0 & 80 & 1,306 & & & \\
\hline 1982 & 735 & 23 & 40.1 & 1,895 & (11.1) & 65 & 1,424 & & & \\
\hline 1993 & 760 & 72 & 54.3 & 1,400 & 9.9 & 69 & 1,390 & & & \\
\hline 1984 & 659 & 7 & 76.9 & 857 & 7.4 & 71 & 1,379 & & & \\
\hline 1985 & 814 & $74 *$ & 73.5 & 1,107 & 17.6 & 82 & 1,293 & & & \\
\hline 1986 & 906 & 74 & 73.7 & 1,229 & 4.0 & 79 & 1,313 & & & \\
\hline-- & $-\cdots$ & -- & $-\cdots$ & $\cdots \cdot$ & $-\cdots$ & - - & $\ldots$ & $\cdots \cdot-\cdot$ & --- & 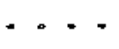 \\
\hline 1987 & 949 & 93 & 84.1 & 1,129 & 4.6 & 78 & 1,327 & & & \\
\hline 1989 & 1,009 & 93. & 92.9 & 1,086 & 6.7 & 78 & 1,324 & 3,417 & 5,187 & 5,212 \\
\hline 1989 & & & & & 10.1 & 82 & 1,295 & 3,343 & 5,074 & 5,099 \\
\hline 1990 & & & & & 8.0 & 83 & 1,284 & 3,315 & 5,032 & 5,057 \\
\hline 1991 & & & & & 9.2 & 86 & 1,265 & 3,267 & 4,958 & 4,982 \\
\hline 1992 & & & & & 7.7 & 87 & 1,259 & 3,251 & 4,934 & 4,958 \\
\hline
\end{tabular}

Capacity-Capital Stock Regression: Code 2 RSO 0.040

Coefficients: Const 1917.4 Stock $-7.6 \quad$ Ave. Capacity/Stock (74-86): 15.9 Ave. Weekly Hours (High) 40.0 Shift factor 3.94 Ave Heexly Hours (LOW) 40.0 Shift Factor 3.94

* Imputed Note: Output, Investment, and Capital stock extrapolated for 1987 and 1988.

\section{Jewelers' materials and lapidary $w$}

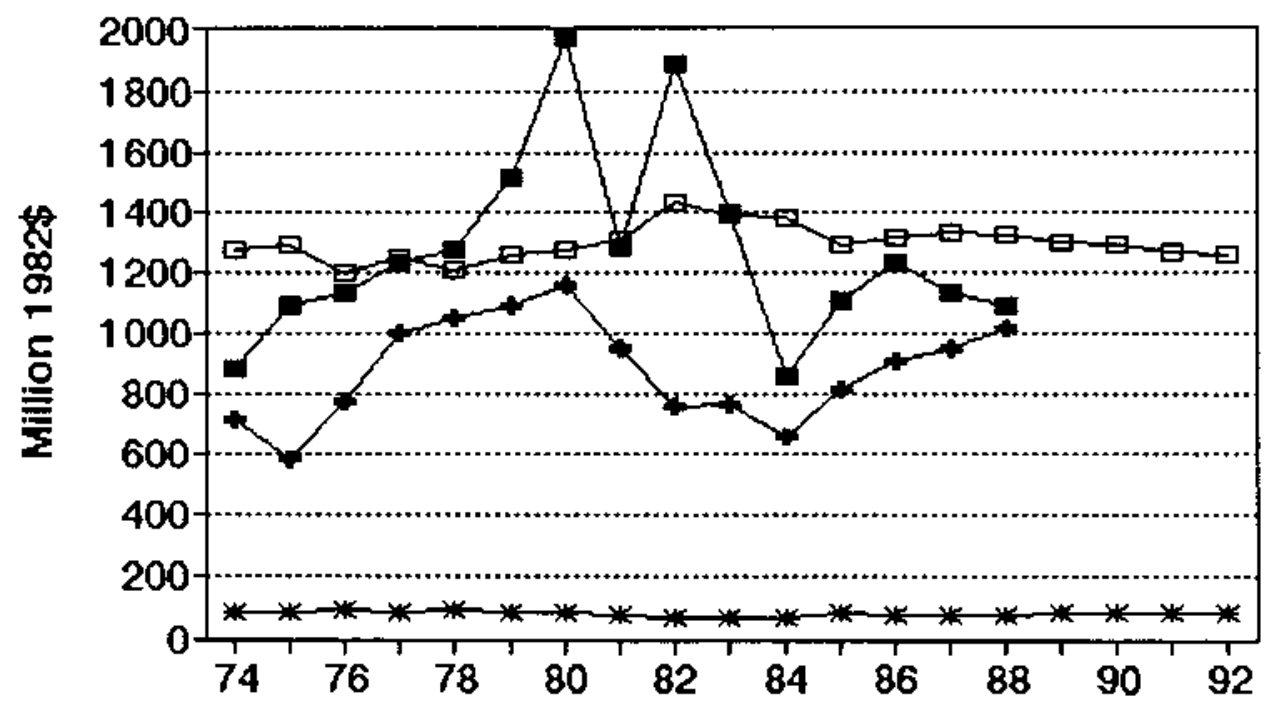

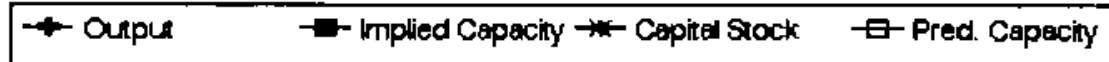




\section{No. 430 sIC 3931 Musical ingtruments}

MILLIONS OF 1982 DOLLARS (EXCL. CU RATES)

$\begin{array}{lccccccc} & 04 & \text { Ann. Implied Gross } & \text { Net } & \text { Pred. } & \ldots & \ldots . . \\ \text { Gross } & \text { CU } & \text { CU } & \text { Prac. } & \text { In- } & \text { Cap. } & \text { Prac. } & \text { Emergency Capacity } \\ \text { Output } & \text { Rate } & \text { Rate } & \text { Capac. } & \text { vest. } & \text { 5tk. } & \text { Capac. } & 3 \text { mon. } 6 \text { mon. }>6 \text { mon. }\end{array}$

\begin{tabular}{|c|c|c|c|c|c|c|c|c|c|c|}
\hline 1974 & 1,379 & 73 & 73.0 & 1,889 & 16.1 & 157 & 2,057 & & & \\
\hline 1975 & 1,160 & 63 & 66.8 & 1,731 & 21.3 & 165 & 2,013 & & & \\
\hline 1976 & 1,449 & 57 & 59.3 & 2,445 & 22.2 & 173 & 1,969 & & & \\
\hline $19 \pi 7$ & 1,313 & 62 & 60.1 & 2,184 & 19.7 & 177 & 1,924 & & & \\
\hline 1978 & 1,421 & 71 & 67.6 & 2,101 & 29.2 & 191 & 1,880 & & & \\
\hline 1979 & 1,263 & 90 & 82.9 & 1,524 & 33.7 & 207 & 1,836 & & & \\
\hline 1980 & 1,240 & 60 & 71.3 & 1,741 & 52.6 & 242 & 1,792 & & & \\
\hline 1981 & 1,217 & 74 & 68.8 & $1, \pi 71$ & 3.2 & 227 & 1,747 & & & \\
\hline 1982 & 898 & 64 & 67.8 & 1,325 & $(22.9)$ & 190 & 1,703 & & & \\
\hline 1983 & 903 & 53 & 57.1 & 1,581 & 20.6 & 195 & 1,659 & & & \\
\hline 1984 & 763 & 41 & 45.5 & 1,676 & (13.7) & 168 & 1,616 & & & \\
\hline 1985 & 650 & 41 & 41.0 & 1,586 & $(5.5)$ & 150 & 1,570 & & & \\
\hline 1986 & 700 & $41 *$ & 40.8 & 1,716 & 6.2 & 143 & 1,526 & & & \\
\hline$\cdots$ & - - - - & -- & -- & --- &..- & $\because$ & $\cdots$ & -- & -- & $-\cdots$ \\
\hline 1987 & 707 & 44 & 42.8 & 1,653 & 13.4 & 145 & 1,482 & & & \\
\hline 1988 & 715 & $48 *$ & 46.7 & 1,532 & 16.5 & 149 & 1,437 & 1,715 & 2,450 & 2,461 \\
\hline 1989 & & & & & 21.6 & 157 & 1,303 & 1,663 & 2,375 & 2,385 \\
\hline 1990 & & & & & 18.5 & 162 & 1,349 & 1,610 & 2,299 & 2,309 \\
\hline 1991 & & & & & 20.3 & 169 & 1,305 & 1,557 & 2,224 & 2,233 \\
\hline 1992 & & & & & 18.1 & 172 & 1,260 & 1,504 & 2,148 & 2,158 \\
\hline
\end{tabular}

Capacity-Capital Stock Regression: Code -2 RSQ 0.330 Coefficients: Const 2101.3 Time $\mathbf{- 4 4 . 3}$ Ave. Heekly Hours (High) 92.0 Shift Factor 1.74 Ave Heekty Hour - Imputed Note: Output, Investment, and Capital Stock extrapolated for 1987 and 1988.
Ave, Capacity/Stock $(74-86): 9.8$ rs (Low) 92.0 Shift factor 1.71 3931 Musical instruments

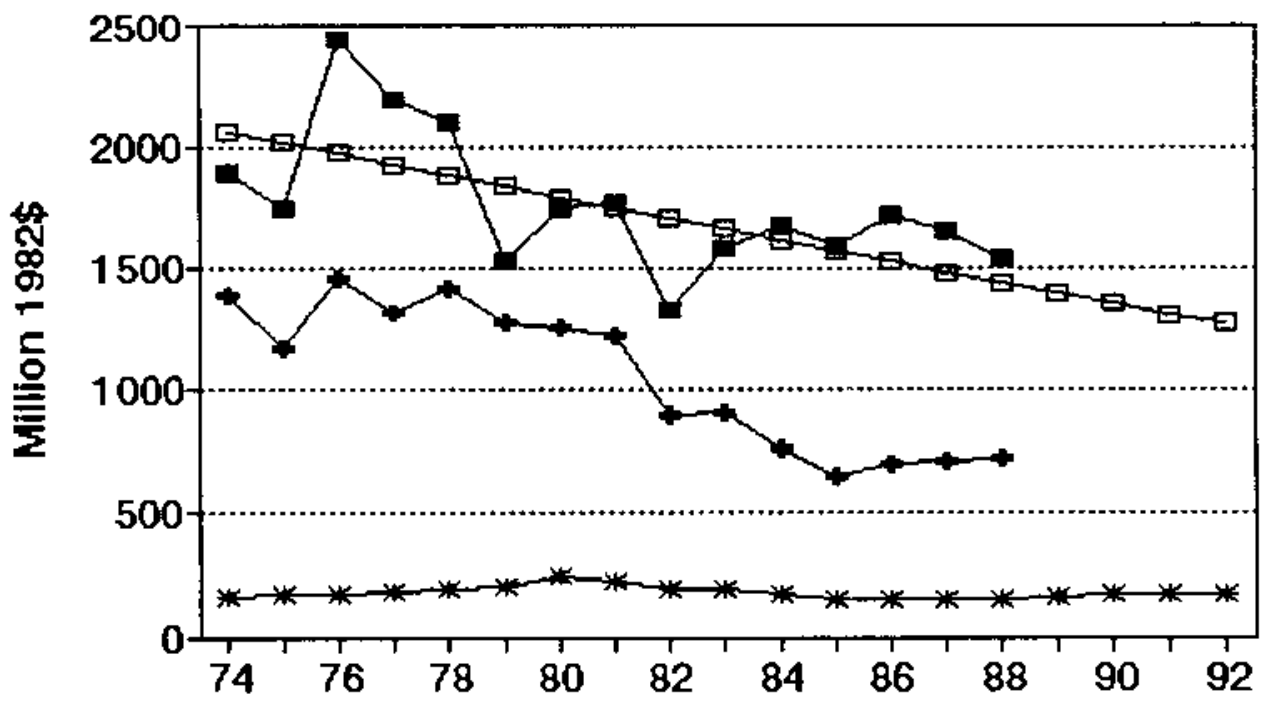


No. 431 sIC 3942 Dol1s

MILLLIONS OF 1982 DOLLARS (EXCL. CU RATES)

$\begin{array}{lccccccc} & \text { Q4 } & \text { Am. } & \text { Implied Gross } & \text { Net } & \text { Pred. } & \ldots . . . . . . \\ \text { Gross } & \text { CU } & \text { CU } & \text { Prac. } & \text { In- } & \text { Cap. } & \text { Prac. } & \text { Emergency Capacity } \\ \text { Output Rate } & \text { Rate } & \text { Capac. } & \text { vest. } & \text { Stk. } & \text { Capac. } & 3 \text { mon. } 6 \text { mon. }>6 \text { mon. }\end{array}$

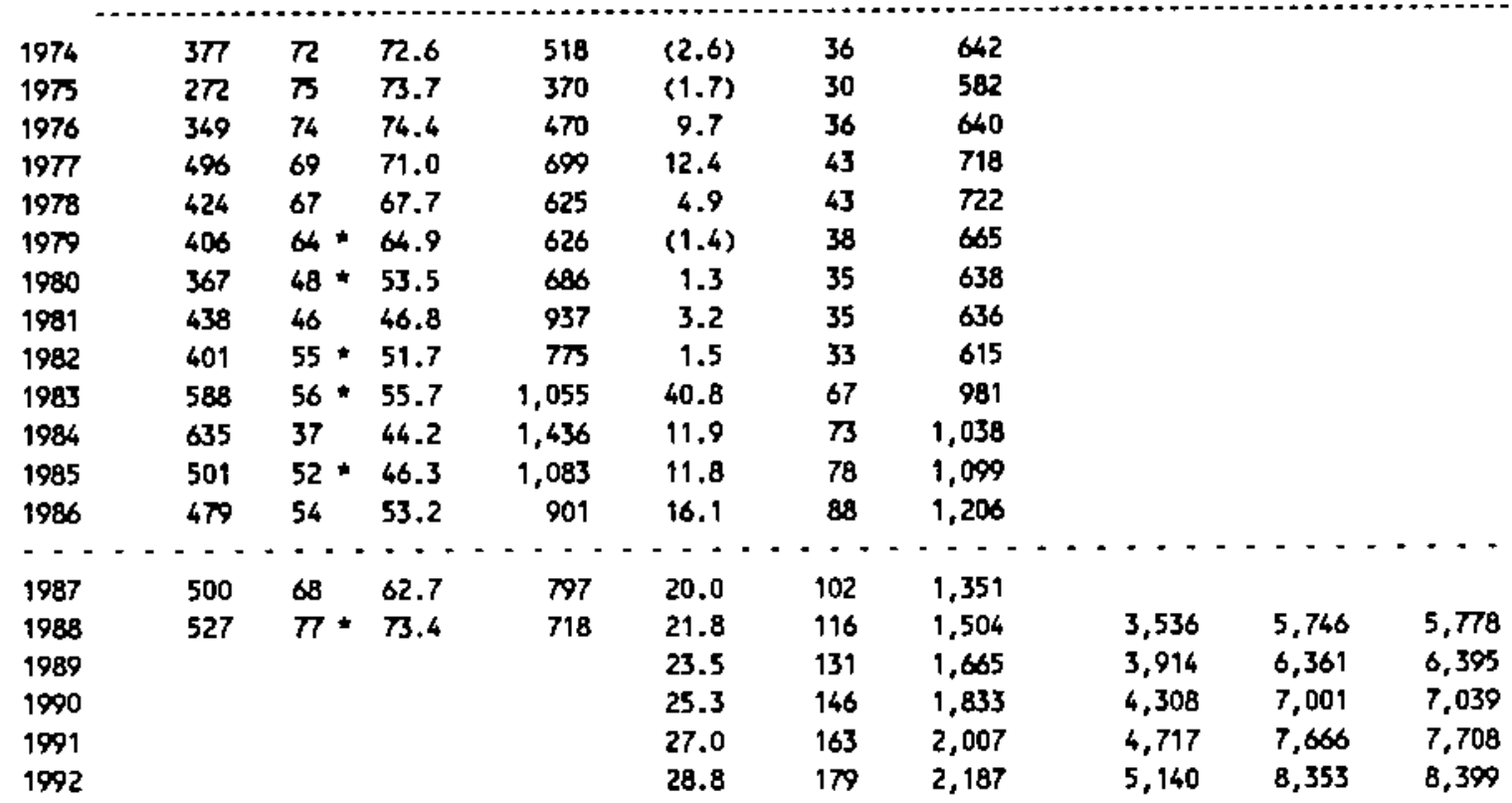

Capacity-Capital Stock Regression: Code O RSO 0.540

Coefficients: Const 258.0 stock 10.8

Ave. Capacity/Stock $(74-86): 16.0$ Ave. Weekly Hours (High) 41.0 Shift Factor 3.84 Ave Weekly Hours (Low) 40.5 Shift factor 3.89 - Imputed Note: Output, Investment, and Capital Stock extrapolated for 1987 and 1988.

3942 Dolls

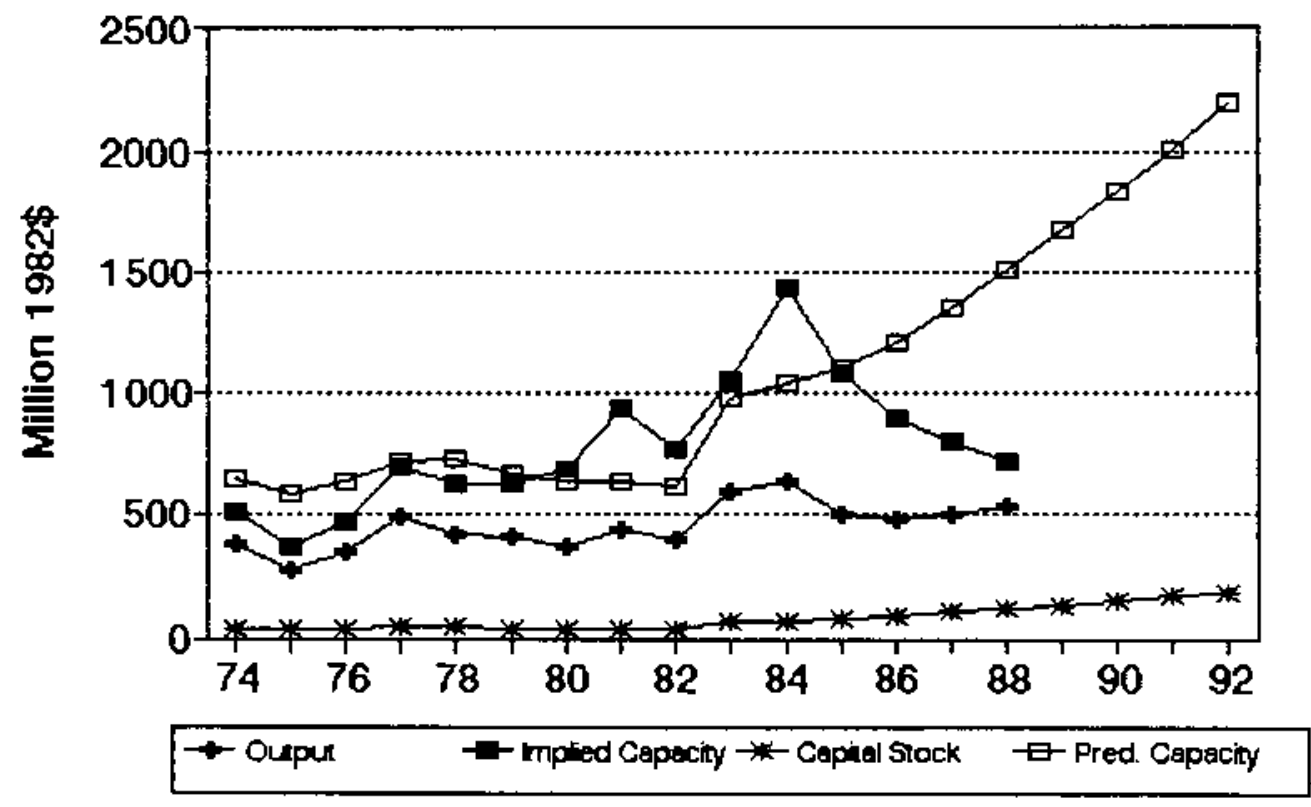


No. 432 sIC 3944 Ganes, toyg, and chtldrens vehicles

MILLIONS OF 1982 DOLLARS (EXCL. CU RATES)

$\begin{array}{lccccccc} & 04 & \text { Ann. } & \text { Implied Gross } & \text { Het } & \text { Pred. } & \ldots \ldots \ldots \\ \text { Gross } & \text { CU } & \text { CU } & \text { Prac. } & \text { In- } & \text { Cap. } & \text { Prac. } & \text { Emergency Capacity } \\ \text { Dutput } & \text { Rate } & \text { Rate } & \text { Capac. } & \text { vest. } & \text { Stk. } & \text { Capac. } & 3 \text { mon. } 6 \text { mon. }>6 \text { mon. }\end{array}$

\begin{tabular}{|c|c|c|c|c|c|c|c|c|c|c|}
\hline 1974 & 3,354 & 61 & 68.8 & 4,875 & 29.4 & 722 & 6,346 & & & \\
\hline 1975 & 2,892 & 67 & 62.0 & 4,662 & 37.0 & 707 & 6,167 & & & \\
\hline 1976 & 3,299 & 76 & 72.6 & 4,544 & 7.4 & 665 & 5,668 & & & \\
\hline 1977 & 4,029 & 65 & 70.5 & 5,711 & 84.5 & 695 & 6,026 & & & \\
\hline 1978 & 3,981 & 80 & 74.1 & 5,372 & 49.4 & 689 & 5,954 & & & \\
\hline 1979 & 4,376 & 65 & 70.0 & 6,251 & 122.9 & 749 & 6,666 & & & \\
\hline 1980 & 4,255 & 53 & 55.8 & 7,622 & 92.0 & $\pi 5$ & 6,977 & & & \\
\hline 1981 & 4,893 & 60 & 59.7 & 8,197 & 40.7 & 751 & 6,695 & & & \\
\hline 1982 & 4,412 & 52 & 54.7 & 8,070 & 71.7 & 756 & 6,752 & & & \\
\hline 1983 & 3,469 & 53 & 54.2 & 6,395 & $(14.5)$ & 680 & 5,851 & & & \\
\hline 1984 & 3,950 & 73 & 67.1 & 5,884 & (38.4) & 587 & 4,746 & & & \\
\hline 1985 & 3,122 & 76 & 74.9 & 4,169 & $(4,5)$ & 532 & 4,088 & & & \\
\hline 1986 & 3,022 & 68 & 71.0 & 4,258 & 49.5 & 531 & 4,073 & & & \\
\hline 1987 & $\begin{array}{c}-\cdots \\
3,079\end{array}$ & 70 & $\begin{array}{l}\cdots \\
68.5\end{array}$ & $\begin{array}{l}\cdots \\
4,498\end{array}$ & $\cdots$ & $\begin{array}{l}--- \\
526\end{array}$ & $\begin{array}{l}\cdots, 014 \\
4,\end{array}$ & $\cdot \cdot$ & -- & -- \\
\hline 1988 & 3,155 & 78 & $\pi .0$ & 4,205 & 52.5 & 528 & 4,041 & 5,091 & 6,514 & 6,534 \\
\hline 1989 & & & & & 63.5 & 541 & 4,196 & 5,287 & 6,764 & 6,785 \\
\hline 1990 & & & & & 56.8 & 546 & 4,257 & 5,364 & 6,862 & 6,884 \\
\hline 1991 & & & & & 60.7 & 555 & 4,358 & 5,490 & 7,024 & 7,046 \\
\hline 1992 & & & & & 55.9 & 557 & 4,392 & 5,533 & 7,079 & 7,101 \\
\hline
\end{tabular}

Capacity-Capital stock Regression: Code 0 RSQ 0.480

Coefficients: Const -2240.1 stock 11.9

Ave. Capacity/Stock $(74-86): 8.6$ Ave. Weekly Hours (High) 97.6 Shift factor 1.62 Ave Weekly Hours (Low) 94.3 Shift factor 1.67 Note: Dutput, Investment, and Capital stock extrapoloted for 1987 and 1988.

\section{Games, toys, and childrens vehicle}

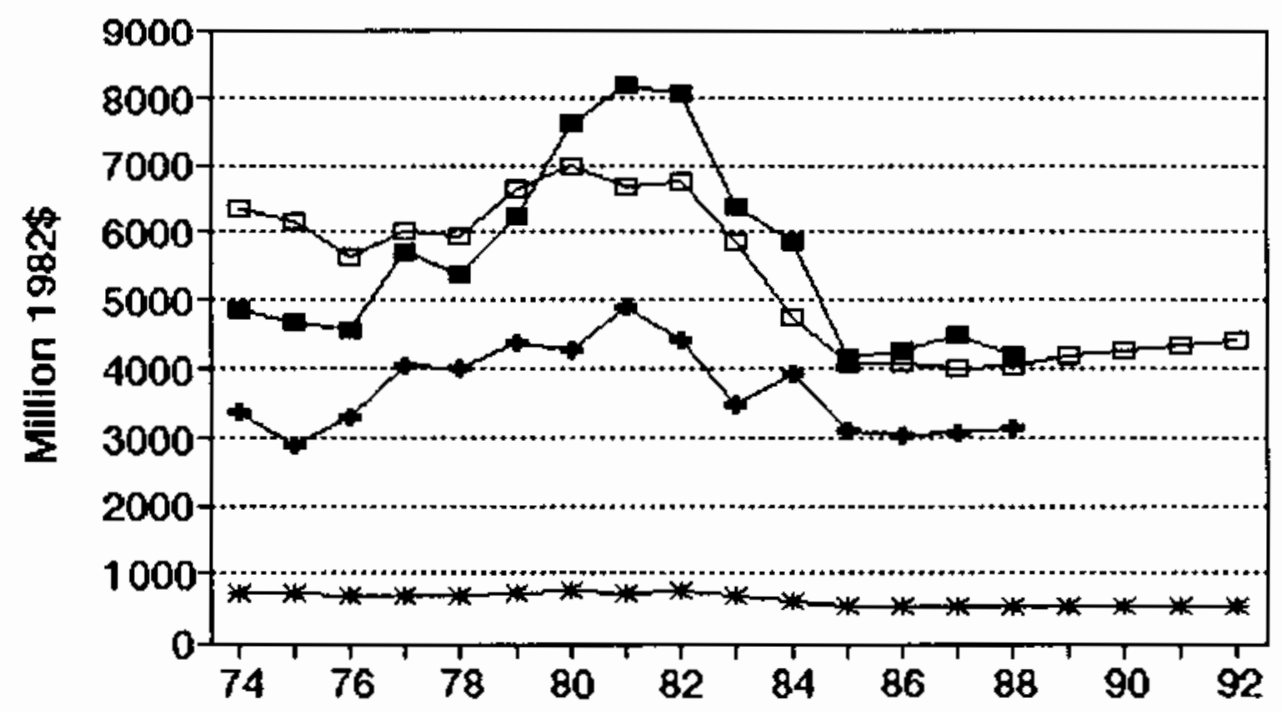

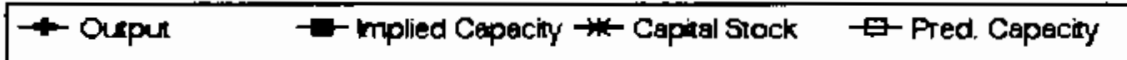


No. 433 sIC 3949 sporting and athletic goods, nec MILLIONS OF 1982 DOLLARS (EXCL. CU RATES)

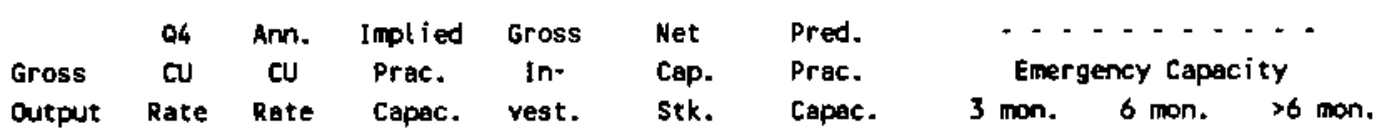

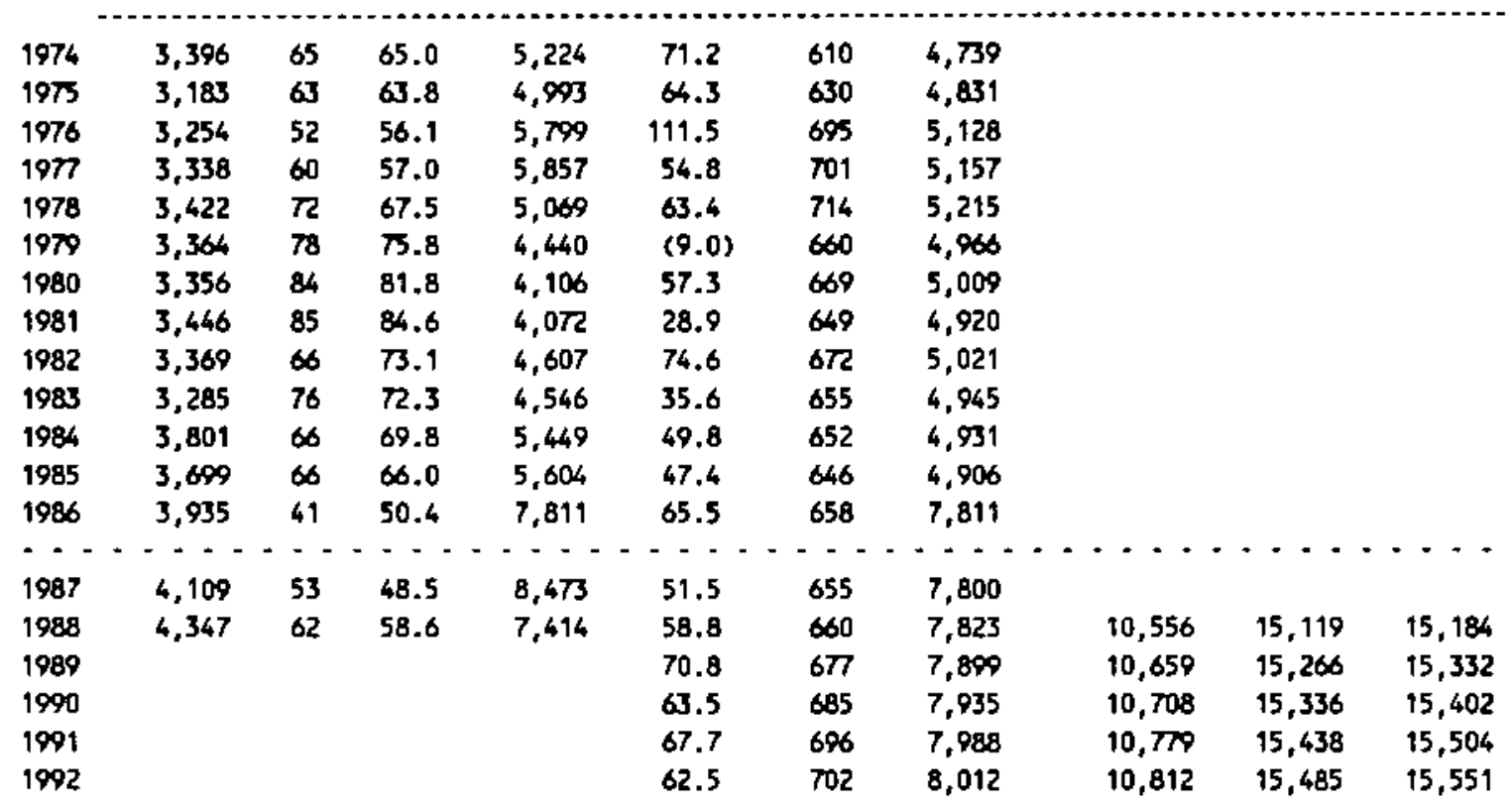

Capacity-Capital stock Regression: Code -1 RSO 0.650

Coefficients: Const 1948.7 stock $4.6 \quad$ D(86-86) 2853.4

Ave. Capacity/Stock $(74-86): 7.8$ Ave. Weekly Hours (High) 81.1 Shift Factor 1.94 Ave Neekly Hours (Low) 72.7 Shift Factor 2.17 Note: Output, Investment, and Capital stock extrapolated for 1987 and 1988.

\section{Sporting and athletic goods, nec}

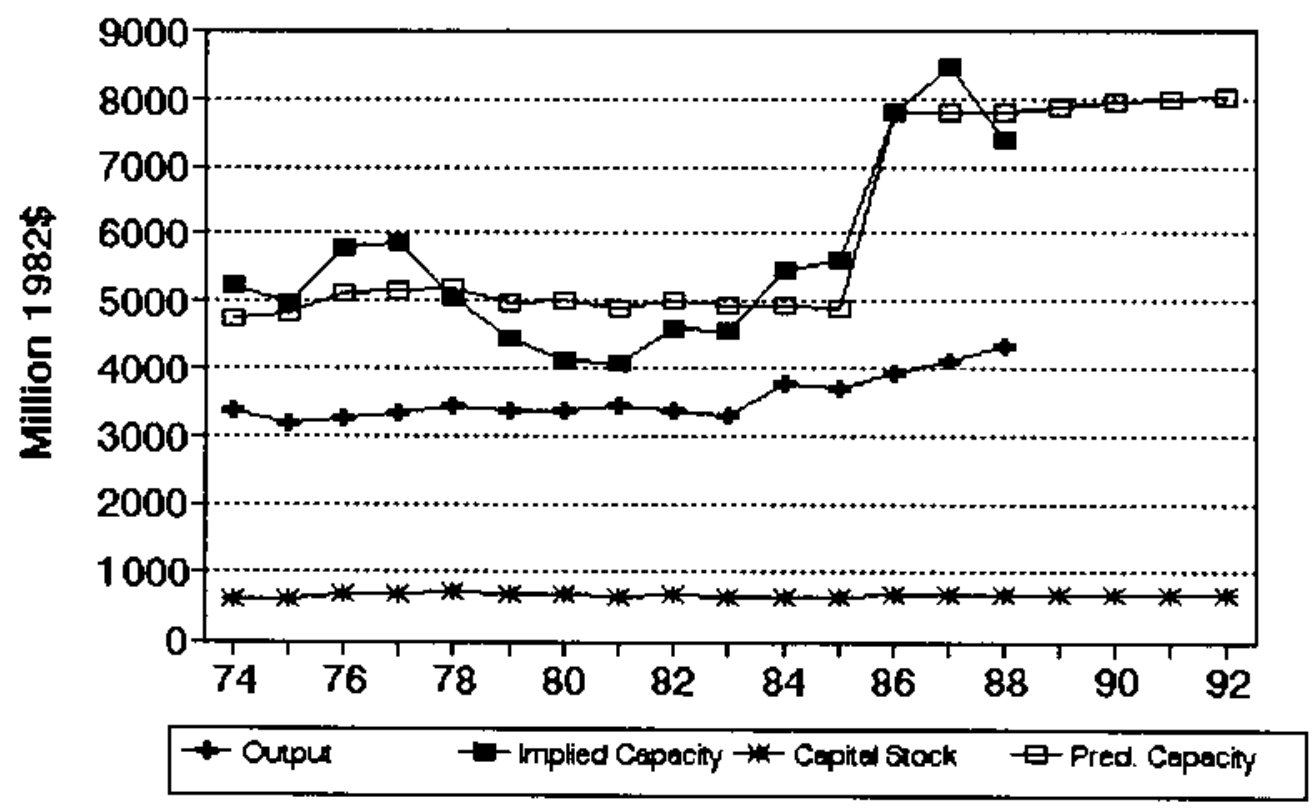




\section{NO. 434 SIC 3951 Pens and mechanical pencils}

MILLIONS OF 1982 DOLLARS (EXCL. CU RATES)

$\begin{array}{lccccccc} & \text { o4 } & \text { Ann. Implied Gross } & \text { Het } & \text { Pred. } & -\ldots \\ \text { Gross } & \text { CU } & \text { CU } & \text { Prac. } & \text { In- } & \text { Cap. } & \text { Prac. } & \text { Emergency Capacity } \\ \text { Output } & \text { Rate } & \text { Rate } & \text { Capac. } & \text { vest. } & \text { Stk. } & \text { Capac. } & 3 \text { mon. } 6 \text { mon. }>6 \text { mon. }\end{array}$

\begin{tabular}{|c|c|c|c|c|c|c|c|c|c|c|}
\hline 1974 & 602 & 90 & 90.0 & 669 & 31.6 & 150 & 685 & & & \\
\hline 1975 & 621 & 82 & 85.0 & 731 & 37.7 & 174 & 795 & & & \\
\hline 1976 & 722 & 81 & 81.4 & 887 & 35.6 & 194 & 905 & & & \\
\hline 1977 & 946 & 87 & 84.8 & 1,117 & 57.3 & 233 & 1,121 & & & \\
\hline 1978 & 998 & 89 & 88.3 & 1,131 & 43.3 & 257 & 1,256 & & & \\
\hline 1979 & 1,064 & 67 & 75.3 & 1,414 & 43.3 & 281 & 1,388 & & & \\
\hline 1980 & 1,033 & 62 & 63.9 & 1,616 & 34.2 & 295 & 1,464 & & & \\
\hline 1981 & 1,076 & 83 & 7.1 & 1,432 & 33.5 & 306 & 1,527 & & & \\
\hline 1982 & 788 & $B 2$ & $B 2.4$ & 957 & $(49.1)$ & 240 & 1,162 & & & \\
\hline 1983 & 868 & 60 & 68.3 & 1,271 & $(4.9)$ & 219 & 1,044 & & & \\
\hline 1984 & 915 & 85 & 75.6 & 1,210 & 25.6 & 226 & 1,082 & & & \\
\hline 1985 & 741 & 61 & 70.0 & 1,058 & 10.6 & 217 & 1,037 & & & \\
\hline 1986 & 729 & 87 & 77.3 & 944 & 11.3 & 200 & 992 & & & \\
\hline-- & $-\cdot-\cdot \cdot$ & $\because$ & $\cdots$ & $-\cdots$ & $-\cdots$ & $-\cdots$ & $\cdots$ & $=-$ & -- & $\cdot \cdot$ \\
\hline 1987 & 763 & 83 & 84.5 & 903 & 22.1 & 213 & 1,011 & & & \\
\hline 1988 & 810 & 71 & $\pi .5$ & 1,072 & 31.7 & 226 & 1,081 & 1,633 & 2,238 & 2,246 \\
\hline 1989 & & & & & 47.6 & 253 & 1,232 & 1,861 & 2,550 & 2,560 \\
\hline 1990 & & & & & 38.0 & 268 & 1,316 & 1,988 & 2,725 & 2,735 \\
\hline 1991 & & & & & 43.4 & 288 & 1,423 & 2,150 & 2,946 & 2,958 \\
\hline 1992 & & & & & 36.6 & 298 & 1,483 & 2,240 & 3,070 & 3,082 \\
\hline
\end{tabular}

Capecity-Capital Stock Regression: Code 0 RSO 0.820

Coefficients: Const -161.6 Stock 5.5

Ave. Capecity/Stock $(74-86): 4.8$

Ave. Weekly Hours (High) 75.8 Shift Factor 2.08 Ave Heekly Hours (Low) 66.0 shift Factor 2.38 Note: Output, Investment, and Capital Stock extrapolated for 1987 and 1988.

\section{Pens and mechanical pencils}

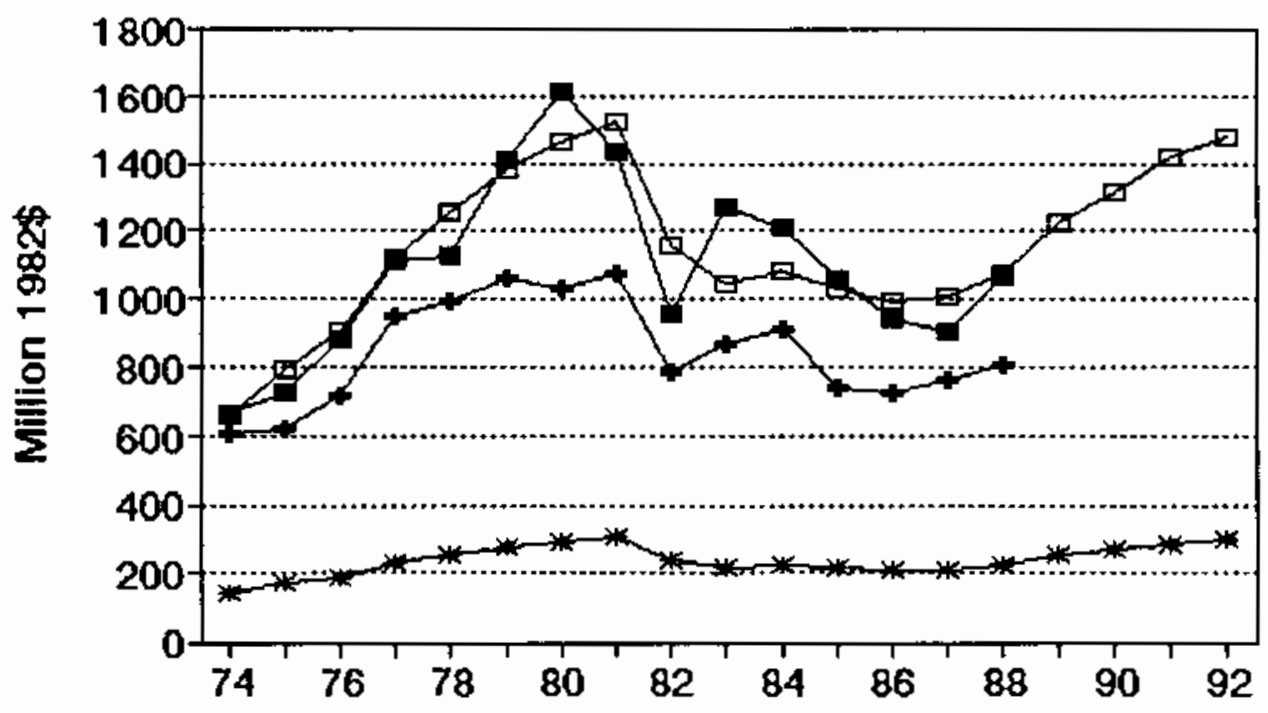

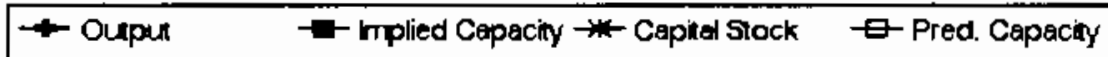




\section{xo. 435 sIC 3952 Lead pencils and art goods}

MILLIONS OF 1982 DOLLARS (EXCL. CU RATES)

$\begin{array}{lccccccc} & \text { Q4 } & \text { Ann. Implied Gross } & \text { Net } & \text { Pred. } & \ldots & \ldots \ldots \ldots \\ \text { Gross } & \text { CU } & \text { CU } & \text { Prac. } & \text { In- } & \text { Cap. } & \text { Prac. } & \text { Emergency Capacity } \\ \text { Output Rete } & \text { Rate } & \text { Capac. } & \text { vest. } & \text { Stk. } & \text { Capac. } & 3 \text { mon. } 6 \text { mon. }>6 \text { mon. }\end{array}$

\begin{tabular}{|c|c|c|c|c|c|c|c|c|c|c|}
\hline 1974 & 425 & 50 & 55.2 & 770 & $(0.5)$ & 73 & 737 & & & \\
\hline 1975 & 399 & 74 & 63.0 & 633 & 7.1 & 74 & 741 & & & \\
\hline 1976 & 449 & 78 & 76.5 & 587 & 12.6 & 80 & 759 & & & \\
\hline 1977 & 439 & 51 & 62.3 & 705 & 6.6 & 81 & 761 & & & \\
\hline 1978 & 462 & 54 & 52.7 & 877 & 0.8 & 76 & 746 & & & \\
\hline 1979 & 442 & $53 *$ & 53.2 & 830 & 10.1 & 80 & 737 & & & \\
\hline 1980 & 383 & $50 *$ & 50.2 & 764 & 9.2 & 82 & 764 & & & \\
\hline 1981 & 413 & $49 *$ & 50.8 & 814 & 4.7 & 80 & 757 & & & \\
\hline 1982 & 454 & $46 *$ & 47.1 & 965 & 21.4 & 93 & 794 & & & \\
\hline 1983 & 479 & 49 & 49.2 & 974 & 11.1 & 95 & 801 & & & \\
\hline 1984 & 449 & $61 \star$ & 57.4 & 781 & $(5.3)$ & 82 & 764 & & & \\
\hline 1985 & 458 & 74 & 69.0 & 684 & 20.2 & 94 & 797 & & & \\
\hline 1986 & 480 & 81 & 78.2 & 613 & 9.7 & 95 & 800 & & & \\
\hline$\because \cdot$ & $=-$ & $-\cdots$ & -- & $\cdots$ & $\because-$ & - & $=-\cdot$ & $-\quad-$ & --- & $\cdots$ \\
\hline 1987 & 486 & 59 & 66.5 & 730 & 12.3 & 98 & 810 & & & \\
\hline 1988 & 493 & 57 & 57.8 & 853 & 12.9 & 102 & 821 & 1,274 & 1,847 & 1,856 \\
\hline 1989 & & & & & 13.5 & 106 & 832 & 1,292 & 1,873 & 1,882 \\
\hline 1990 & & & & & 14.1 & 111 & 844 & 1,310 & 1,900 & 1,909 \\
\hline 1991 & & & & & 14.7 & $\$ 15$ & 857 & 1,330 & 1,929 & 1,937 \\
\hline 1992 & & & & & 15.2 & 120 & 870 & 1,350 & 1,958 & 1,967 \\
\hline
\end{tabular}

Capacity-Capital Stock Regression: Code 1 RSO 0.030

Coefficients: Const 532.3 stock 2.8

Ave. Capacity/Stock $(74-86): 9.2$ Ave. Weekly Hours (High) 69.7 Shift Factor 2.26 Ave Neekly Hours (Low) 69.7 Shift Factor 2.26

* Imputed Note: Output, Investment, and Capital stock extrapolated for 1987 and 1988.

\section{Lead pencils and art goods}

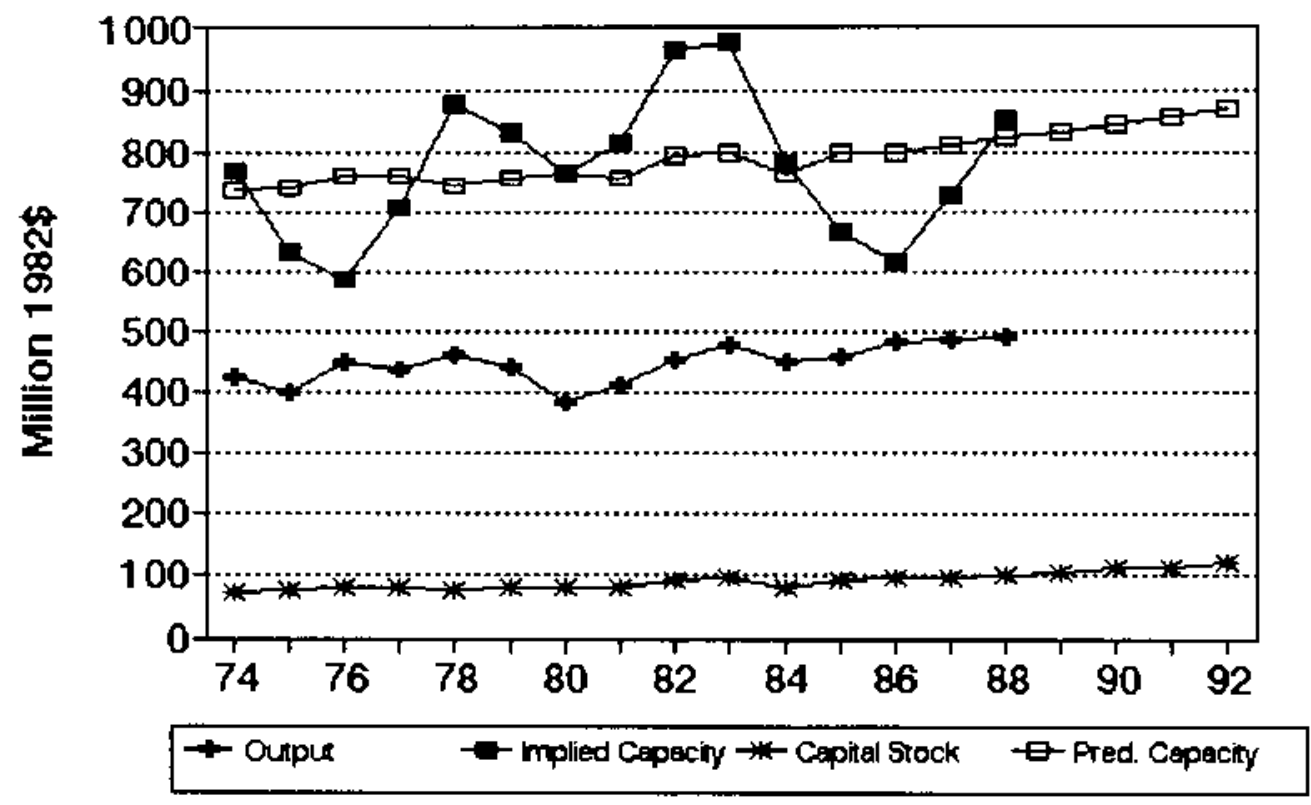


No. 436 SIC 3953 Marking devices

MILLIONS OF 1982 DOLLARS (EXCL. CU RATES)

$\begin{array}{lccccccc} & \text { Q4 } & \text { Ann. Implied Gross } & \text { Het } & \text { Pred. } & \ldots \ldots \ldots \\ \text { Gross } & \text { CU } & \text { CU } & \text { Prac. } & \text { In- } & \text { Cap. } & \text { Prac. } & \text { Emergency Capacity } \\ \text { Qutput } & \text { Rate } & \text { Rate } & \text { Capac. vest. } & \text { Stk. } & \text { Capac. } & 3 \text { mon. } 6 \text { mon. }>6 \text { mon. }\end{array}$

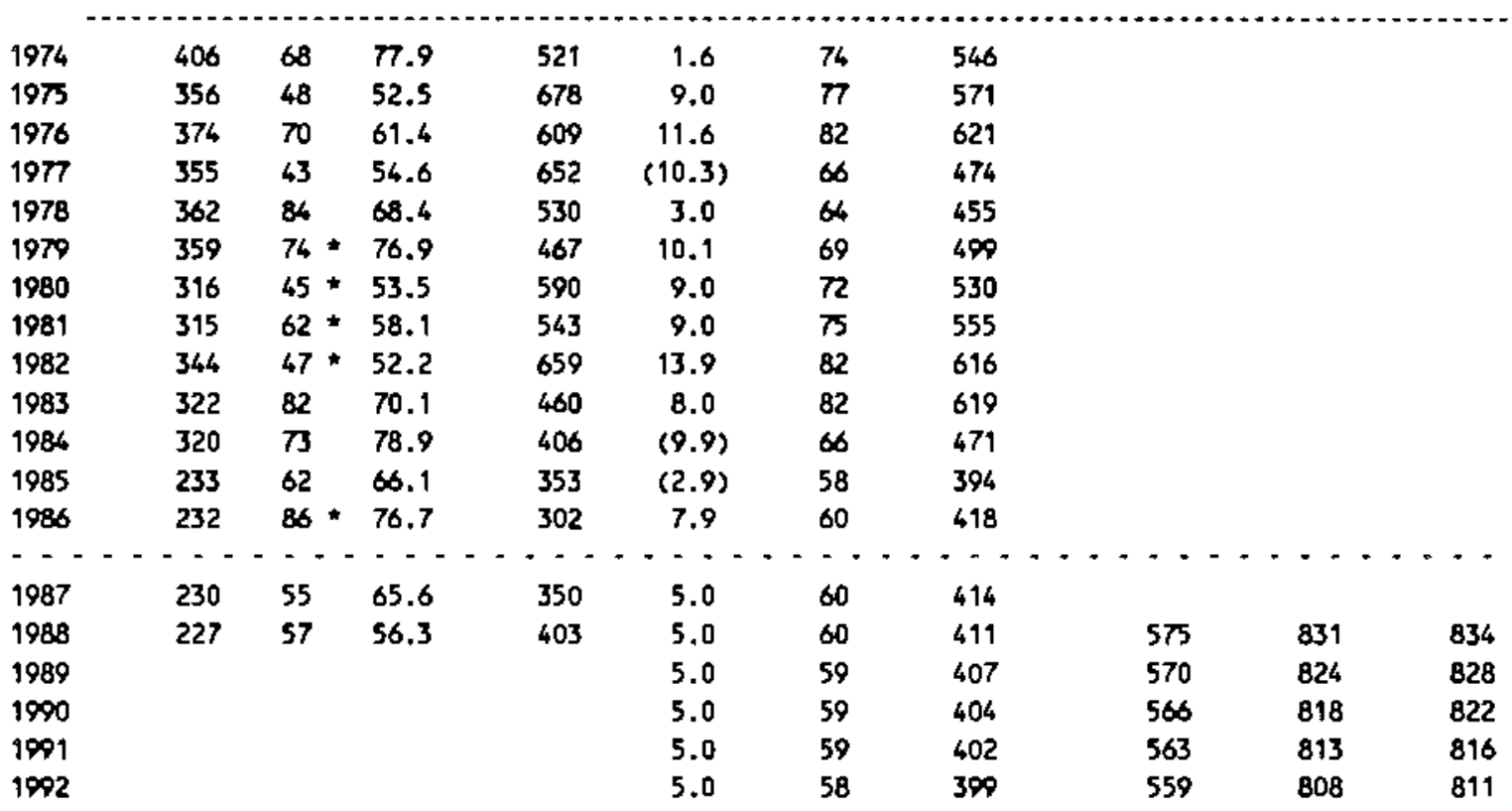

Capacity-Capital Stock Regression: Code 1 RSa 0.410

Coefficients: Const -149.7 stock 9.4

Ave. Capacity/stock $(74-86): 7.3$

Ave. Ueekly Hours (High) 77.5 Shift Factor 2.03 Ave Ueekly Hours (LOw) 72.4 Shift Factor 2.18

- Imputed Note: Output, Investment, and Capital Stock extrapolated for 1987 and 1988.

\section{Marking devices}

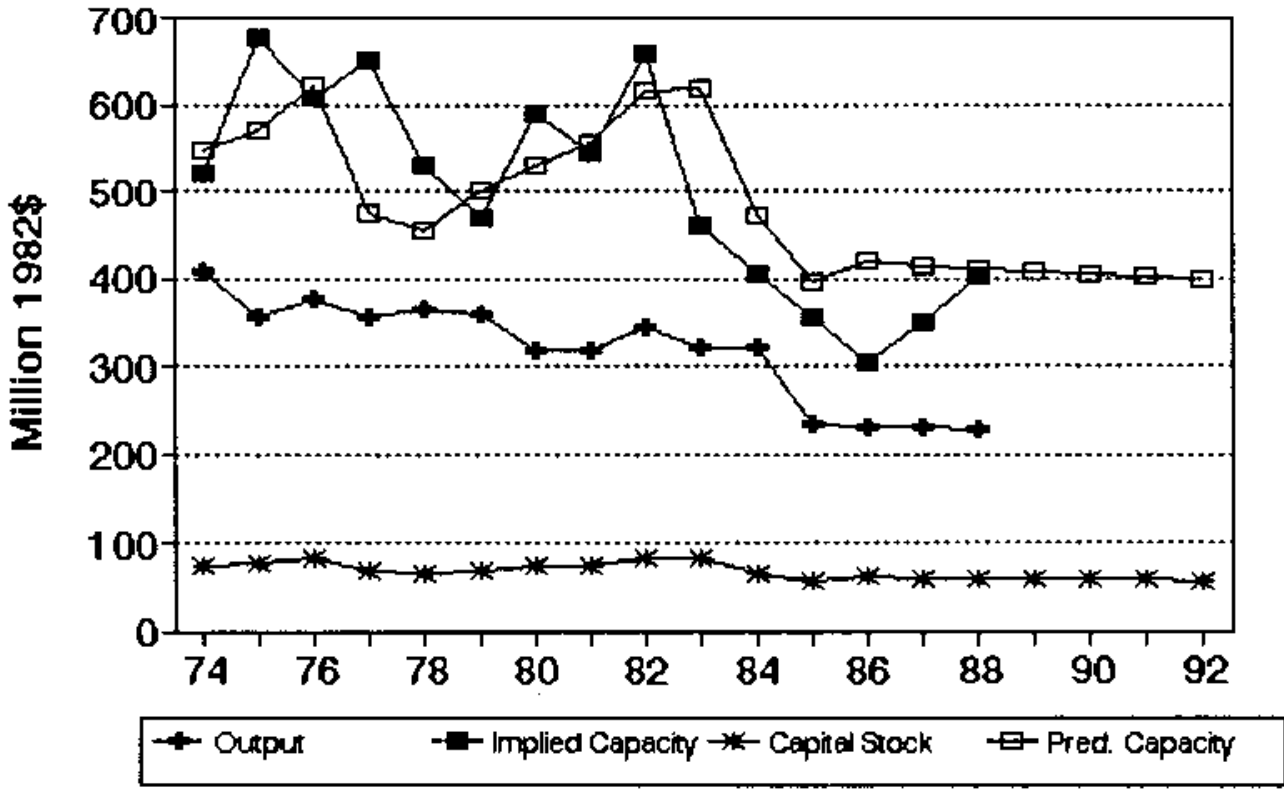


No. 437 sIC 3955 Carbon paper and inked ribbons

MILLIONS OF 1982 DOLLARS (EXCL. CU RATES)

$\begin{array}{lccccccc} & 04 & \text { Ann. } & \text { inplied Gross } & \text { Net } & \text { Pred. } & -\ldots \\ \text { Gross } & \text { CU } & \text { CU } & \text { Prac. } & \text { In- } & \text { Cap. } & \text { Prac. } & \text { Emergency Capacity } \\ \text { Output } & \text { Rate } & \text { Rate } & \text { Capac. } & \text { vest. } & \text { Stk. } & \text { Capac. } & 3 \text { mon. } 6 \text { mon. }>6 \text { mon. }\end{array}$

\begin{tabular}{|c|c|c|c|c|c|c|c|c|c|c|}
\hline 1974 & 511 & $42 *$ & 48.6 & 1,051 & 28.1 & 84 & 1,172 & & & \\
\hline 1975 & 406 & $38 *$ & 37.7 & 1,078 & 2.8 & 80 & 1,156 & & & \\
\hline 1976 & 422 & $44 *$ & 41.9 & 1,007 & $(8.1)$ & 67 & 1,097 & & & \\
\hline 1977 & 513 & $38 *$ & 41.0 & 1,251 & 12.8 & 73 & 1,124 & & & \\
\hline 1978 & 524 & $48 *$ & 43.9 & 1,193 & 13.2 & 79 & 1,149 & & & \\
\hline 1979 & 534 & $45 *$ & 45.5 & 1,173 & 17.4 & 88 & 1,188 & & & \\
\hline 1980 & 626 & 37 & 38.6 & 1,623 & 32.0 & 110 & 1,283 & & & \\
\hline 1981 & 660 & $56 *$ & 51.6 & 1,277 & 6.0 & 107 & 1,269 & & & \\
\hline 1982 & 795 & 65 & 61.4 & 1,295 & 36.9 & 133 & 1,381 & & & \\
\hline 1983 & 879 & 73 & 72.4 & 1,215 & 1.6 & 124 & 1,343 & & & \\
\hline 1984 & 589 & 58 & 65.9 & 894 & 3.0 & 118 & 930 & & & \\
\hline 1985 & 589 & 67 & 63.7 & 926 & 8.0 & 116 & 924 & & & \\
\hline 1986 & 656 & 68 & 67.5 & 971 & 12.5 & 119 & 937 & & & \\
\hline-- & ---- & -- & -- & $-\cdots$ & $-\cdots$ & $-\cdots$ & $\cdots-$ & $-\cdots$ & $\cdots$ & $\cdot \cdot$ \\
\hline 1987 & 672 & 74 & 70.8 & 949 & 13.6 & 124 & 955 & & & \\
\hline 1988 & 693 & $n$ & 72.8 & 852 & 13.8 & 128 & 972 & 1,057 & 1,324 & 1,320 \\
\hline 1989 & & & & & 14.1 & 131 & 989 & 1,075 & 1,346 & 1,350 \\
\hline 1990 & & & & & 14.3 & 135 & 1,005 & 1,092 & 1,369 & 1,372 \\
\hline 1991 & & & & & 14.5 & 139 & 1,021 & 1,110 & 1,390 & 1,394 \\
\hline 1992 & & & & & 14.7 & 143 & 1,036 & 1,127 & 1,411 & 1,415 \\
\hline
\end{tabular}

Capacity-Capital Stock Regression: Code -1 RSO 0.580

Coefficients: Const 811.7 stock $4.3 \quad D(84-86) \quad-386.1$

Ave. Capecity/5tock (74-86): 11.5 Ave. Weekly Hours (High) 115.5 Shift Factor 1.37 Ave Weekly Hours (Low) 115.5 Shift Factor 1.37 - Imputed Note: Output, Investment, and Capital Stock extrapolated for 1987 and 1988.

3955 Carbon paper and inked ribbons

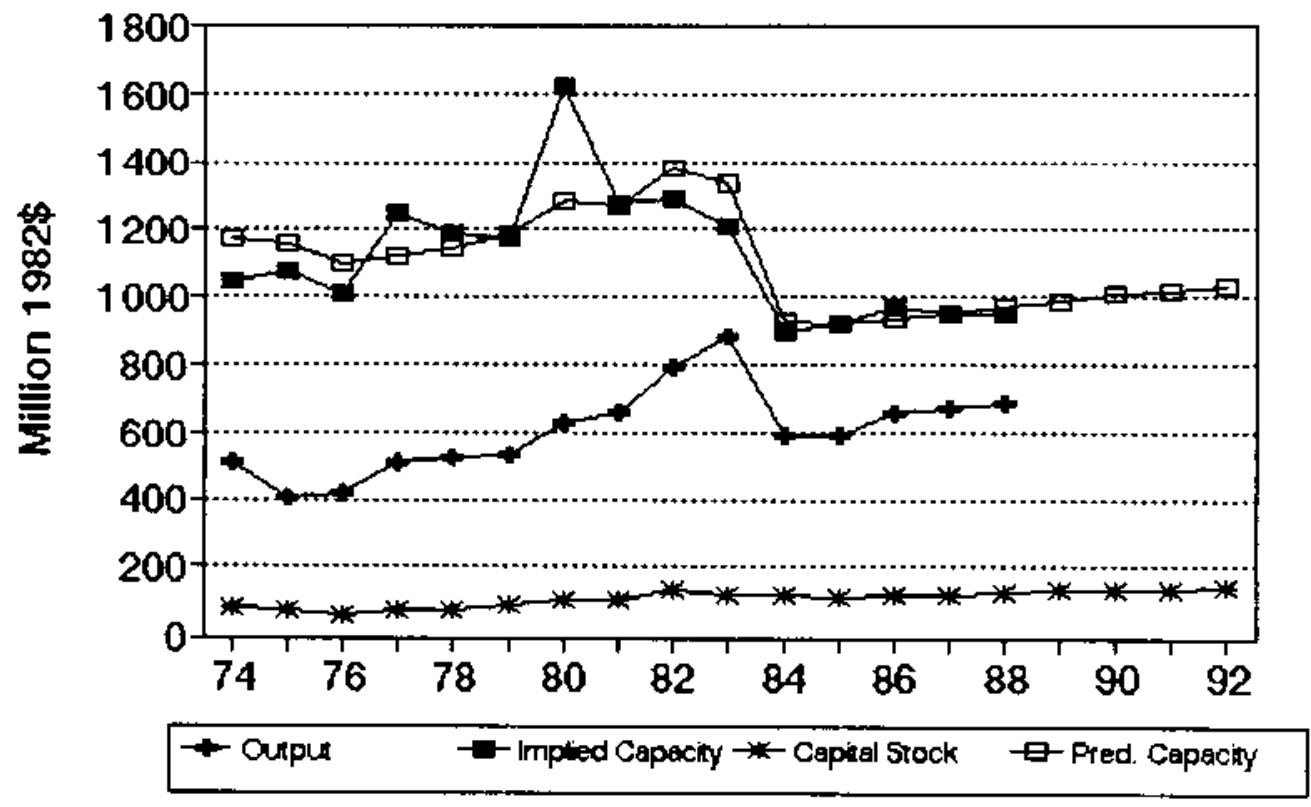


No, 438 sIc 3961 costume jewelry

MILLIONS OF 1982 DOLLAR5 (EXCL. CU RATES)

$\begin{array}{lccccccc} & \text { Q4 } & \text { Anm. Implied } & \text { Gross } & \text { Net } & \text { Pred. } & \ldots \ldots \ldots \\ \text { Gross } & \text { CU } & \text { CU } & \text { Prac. } & \text { In- } & \text { Cap. } & \text { Prac. } & \text { Emergency Capacity } \\ \text { Output } & \text { Rate } & \text { Rate } & \text { Capac. } & \text { vest. } & 5 \text { 5tk. } & \text { Capac. } & \end{array}$

\begin{tabular}{|c|c|c|c|c|c|c|c|c|c|c|}
\hline 1974 & 776 & 66 & 72.7 & 1,067 & $(10.8)$ & 93 & 1,113 & & & \\
\hline $19 \sqrt{5}$ & 1,009 & 79 & 71.7 & 1,407 & 9.4 & 95 & 1,139 & & & \\
\hline 1976 & 904 & 92 & 87.0 & 1,039 & 23.5 & 111 & 1,328 & & & \\
\hline 1977 & 1,052 & 72 & 80.9 & 1,301 & 8.8 & 111 & 1,338 & & & \\
\hline 1978 & 1,070 & 70 & 70.5 & 1,517 & 13.8 & 117 & 1,405 & & & \\
\hline 1979 & 1,038 & 79 & $\pi .1$ & 1,381 & 21.3 & 129 & 1,546 & & & \\
\hline 1980 & 1,133 & 88 & 82.9 & 1,367 & 19.7 & 138 & 1,653 & & & \\
\hline 1981 & 1,051 & $56 *$ & 69.6 & 1,511 & 1.9 & 129 & 1,550 & & & \\
\hline 1982 & 1,034 & 49 & 51.3 & 2,017 & 27.0 & 144 & 1,725 & & & \\
\hline 1983 & 1,189 & 68 & 61.9 & 1,922 & 27.2 & 157 & 1,886 & & & \\
\hline 1984 & 1,115 & 70 & 70.8 & 1.574 & $(10.6)$ & 134 & 1,612 & & & \\
\hline 1985 & 1,119 & 59 & 63.1 & 1,773 & 7.8 & 130 & 1,566 & & & \\
\hline 1986 & 1,111 & 78 & 70.7 & 1,572 & 13.5 & 132 & 1,585 & & & \\
\hline$\cdots$ & & & & & & & & & & \\
\hline 1987 & 1,139 & $85 *$ & 81.4 & 1,399 & 15.3 & 135 & 1,626 & & & \\
\hline 1988 & 1,176 & 79 & 81.2 & 1,468 & 15.9 & 139 & 1,671 & 2,568 & 3,591 & 3,605 \\
\hline 1989 & & & & & 16.5 & 143 & 1,718 & 2,641 & 3,693 & 3,708 \\
\hline 1990 & & & & & 17.0 & 147 & 1,768 & 2,718 & 3,801 & 3,816 \\
\hline 1991 & & & & & 17.6 & 152 & 1,821 & 2,790 & 3,914 & 3,929 \\
\hline 1992 & & & & & 18.2 & 156 & 1,876 & 2,883 & 4,031 & 4,048 \\
\hline
\end{tabular}

Capacity-Capital Stock Regression: Code 0 RSQ 0.590

Coefficients: Const -0.1 stock 12.0

Ave. Capacity/Stock $(74-86): 12.0$ Ave. Weekly Hours (High) 73.0 Shift Factor 2.16 Ave Heekly Hours (LOW) 73.0 5hift Factor 2.16

- Imputed Note: Output, Investment, and Capital stock extrapolated for 1987 and 1988.

3961 Costume jewelry
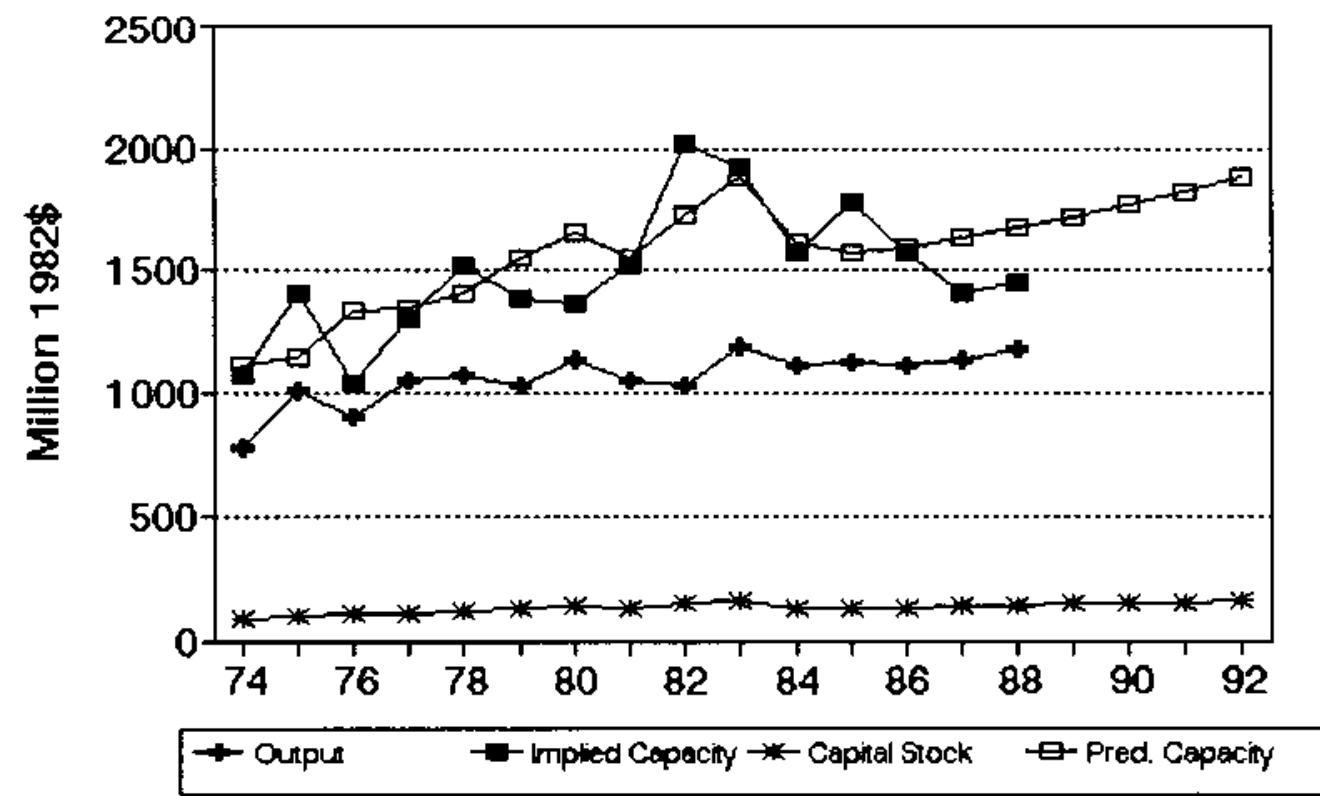


\section{No. 439 SIC 3962 Artificial flowers}

MILLIONS OF 1982 DOLLARS (EXCL. CU RATES)

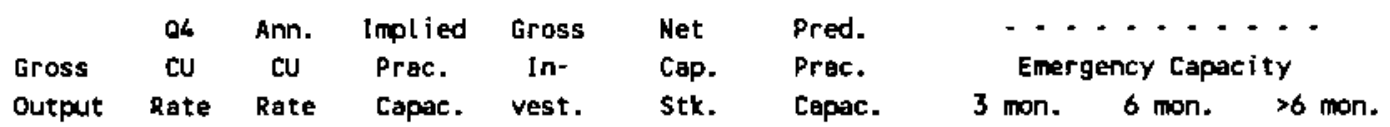

\begin{tabular}{|c|c|c|c|c|c|c|c|c|c|c|}
\hline 1974 & 250 & $80 *$ & 84.3 & 296 & 10.1 & 43 & 348 & & & \\
\hline 1975 & 211 & $82 *$ & 79.8 & 265 & (2.1) & 38 & 338 & & & \\
\hline 1976 & 227 & 85 & 84.2 & 270 & 3.0 & 38 & 339 & & & \\
\hline 1977 & 259 & $83 \star$ & 84.4 & 307 & $(4.8)$ & 31 & 325 & & & \\
\hline 1978 & 325 & $82 *$ & 82.2 & 395 & 6.6 & 35 & 332 & & & \\
\hline 1978 & 305 & $83 *$ & 82.2 & 371 & 12.6 & 44 & 350 & & & \\
\hline 1980 & 273 & $78 *$ & 79.0 & 345 & $(0.6)$ & 40 & 343 & & & \\
\hline 1981 & 277 & 72 & 75.5 & 367 & 2.0 & 39 & 340 & & & \\
\hline 1982 & 266 & $73 *$ & 72.5 & 367 & 6.3 & 42 & 346 & & & \\
\hline 1983 & 280 & 77 & 76.6 & 365 & 1.6 & 40 & 342 & & & \\
\hline 1984 & 296 & 79 & 79.3 & 373 & $(0.8)$ & 36 & 335 & & & \\
\hline 1985 & 302 & 83 & 81.5 & 371 & 12.2 & 45 & 353 & & & \\
\hline 1986 & 303 & $83 *$ & 83.2 & 364 & 10.6 & 52 & 366 & & & \\
\hline$-\quad-$ & $-\cdots$ & -- & $-\cdot$ & $\cdots$ & $\cdots$ & - & $\cdots$ & $-\quad-$ & -- & - \\
\hline 1987 & 312 & 85 & 83.9 & 372 & 6.9 & 55 & 372 & & & \\
\hline 1988 & 325 & 68 * & 86.9 & 374 & 7.2 & 58 & 378 & 499 & 627 & 629 \\
\hline 1989 & & & & & 7.6 & 62 & 385 & 508 & 638 & 640 \\
\hline 1990 & & & & & 7.9 & 65 & 392 & 517 & 649 & 651 \\
\hline 1991 & & & & & 8.3 & 69 & 399 & 526 & 661 & 662 \\
\hline 1992 & & & & & 8.6 & 72 & 406 & 535 & 672 & 674 \\
\hline
\end{tabular}

Cepacity-Capital stock Regression: Code 0 RSA 0.060

Coefficients: Const 264.9 stock 2.0

Ave. Capacity/Stock $(74-86): 8.6$ Ave. Weekly Hours (High) 94.8 Shift Factor 1.66 Ave Weekly Hours (Low) 94.8 Shift factor 1.66 * Imputed Note: Output, Investment, and Capital Stock extrapolated for 1987 and 1988.

3962 Artificial flowers

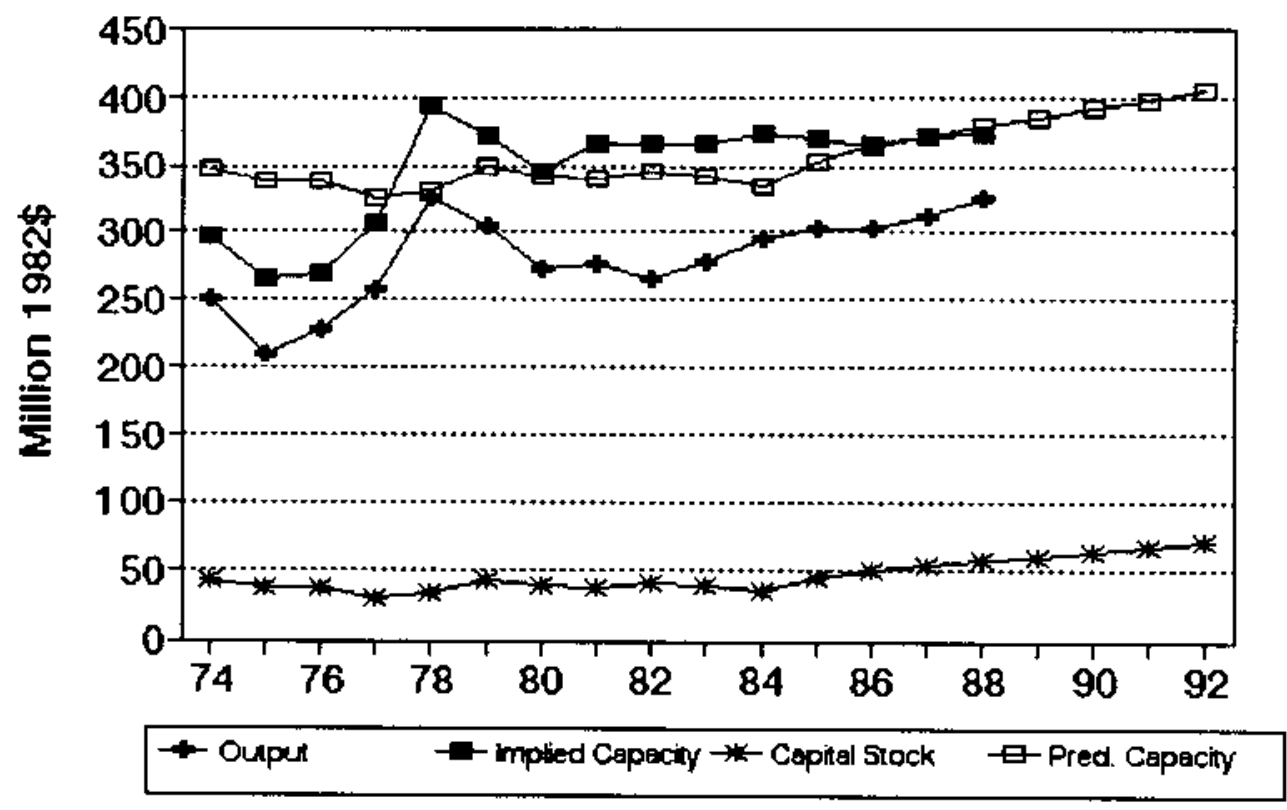




\section{No. 440 SIC 3963 Buttons}

MILLIONS OF 1982 DOLLARS (EXCL, CU RATES)

$\begin{array}{lccccccc} & \text { Q4 } & \text { Ann. Implied Gross } & \text { Net } & \text { Pred. } & \ldots . . . . . \\ \text { Gross } & \text { CU } & \text { CU } & \text { Prac. } & \text { In- } & \text { Cap. } & \text { Prac. } & \text { Emergency Capacity } \\ \text { Output } & \text { Rete } & \text { Rate } & \text { Capac. } & \text { vest. } & \text { Stk. } & \text { Capac. } & 3 \text { mon. } 6 \text { mon. }>6 \text { mon. }\end{array}$

\begin{tabular}{|c|c|c|c|c|c|c|c|c|c|c|}
\hline 1974 & 128 & $47 *$ & 59.7 & 214 & 2.5 & 39 & 264 & & & \\
\hline 1973 & 127 & $51 *$ & 45.7 & 278 & 5.2 & 41 & 259 & & & \\
\hline 1976 & 128 & $58 *$ & 55.9 & 229 & $(1.3)$ & 36 & 270 & & & \\
\hline 1977 & 125 & $53 *$ & 56.7 & 221 & 7.7 & 40 & 260 & & & \\
\hline 1978 & 114 & $52 *$ & 51.9 & 221 & 6.0 & 42 & 256 & & & \\
\hline 1979 & 115 & $53 *$ & 51.6 & 222 & 5.1 & 43 & 254 & & & \\
\hline 1980 & 104 & $45 *$ & 45.2 & 230 & $(2.2)$ & 36 & 270 & & & \\
\hline 1981 & 119 & $32 *$ & 39.1 & 304 & 4.6 & 37 & 269 & & & \\
\hline 1982 & 122 & $34 *$ & 33.0 & 371 & 14.7 & 45 & 247 & & & \\
\hline 1983 & 140 & 42 & 41.4 & 338 & $(20.8)$ & 22 & 307 & & & \\
\hline 1984 & 160 & 58 & 54.3 & 294 & 17.1 & 34 & 273 & & & \\
\hline 1985 & 171 & $59 *$ & 58.5 & 292 & 0.0 & 30 & 286 & & & \\
\hline 1986 & 172 & 59 * & 58.6 & 294 & 1.9 & 28 & 291 & & & \\
\hline $\begin{array}{l}\cdots= \\
1987\end{array}$ & $\begin{array}{r}\cdots \\
\\
170\end{array}$ & * & $\ddot{59.0}$ & 289 & $\begin{array}{l}\cdots \\
3.0\end{array}$ & 27 & 294 & -- & - & - \\
\hline 1988 & 168 & $67 *$ & 64.9 & 259 & 4.0 & 27 & 293 & 340 & 467 & 468 \\
\hline 1989 & & & & & 5.8 & 29 & 288 & 334 & 459 & 461 \\
\hline 1990 & & & & & 4.7 & 30 & 287 & 333 & 456 & 458 \\
\hline 1991 & & & & & 5.3 & 31 & 284 & 329 & 452 & 453 \\
\hline 1992 & & & & & 4.6 & 31 & 283 & 329 & 451 & 453 \\
\hline
\end{tabular}

Capecity-Capital stock Regression: Code 2 RSD 0.110 Coefficients: Const 363.2 Stock -2.6 Ave. Weekly Hours (High) 98.6 Shift Factor 1.60 Ave Heekly Hours (LOW) 98.6 Shift Factor 1.60 * Imputed Note: Dutput, Investment, and Cepital stock extrapolated for 1987 and 1988.

\section{Buttons}

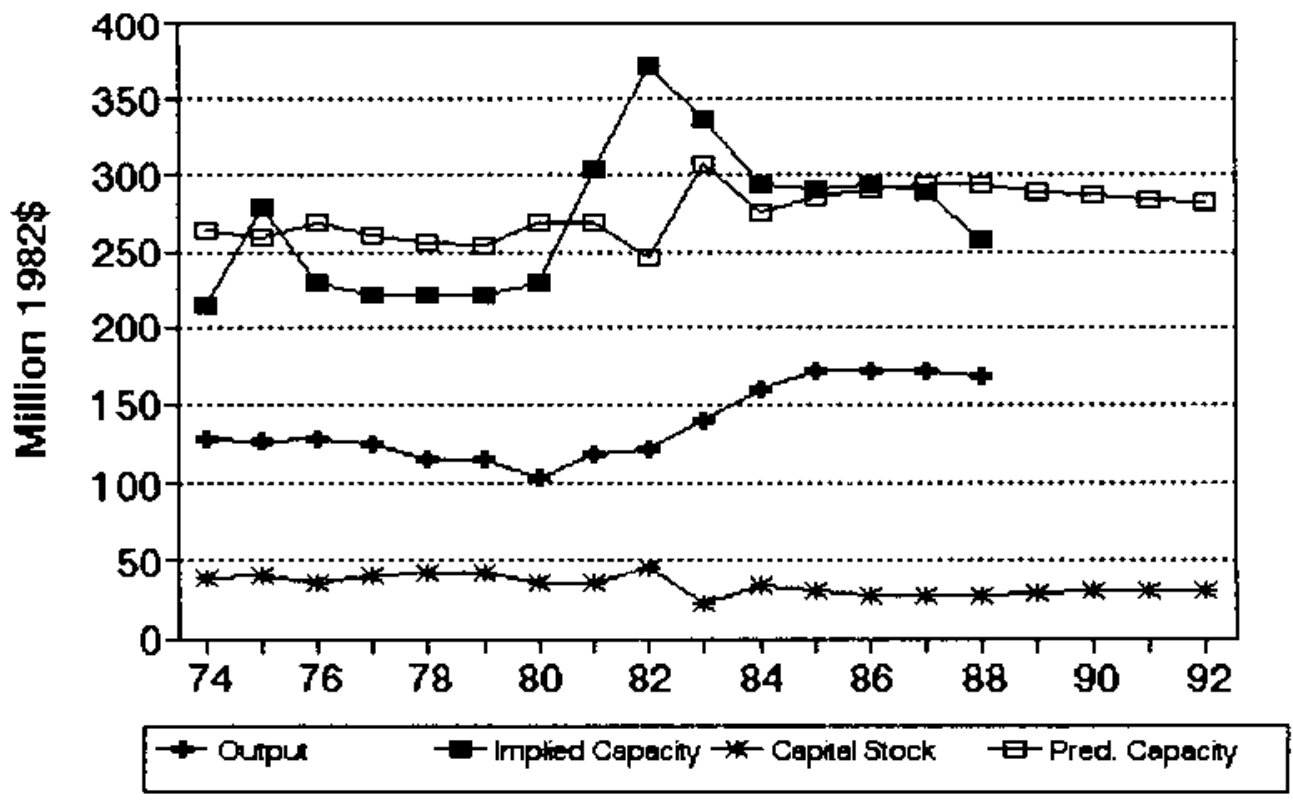




\title{
No. 441 SIC 3964 Needles, pins, and fasteners
}

\author{
MILLIONS OF 1982 DOLLARS (EXCL. CU RATES)
}

$\begin{array}{lccccccc} & 04 & \text { Arn, } & \text { Implied } & \text { Gross } & \text { Net } & \text { Pred. } & -\ldots \ldots . . . \\ \text { Gross } & \text { CU } & \text { CU } & \text { Prac. } & \text { In- } & \text { Cap. } & \text { Prac. } & \text { Emergency Capacity } \\ \text { Output } & \text { Rate } & \text { Rate } & \text { Capac. } & \text { vest. } & \text { Stk. } & \text { Capac. } & 3 \text { mon. } 6 \text { mon. }>6 \text { mon. }\end{array}$

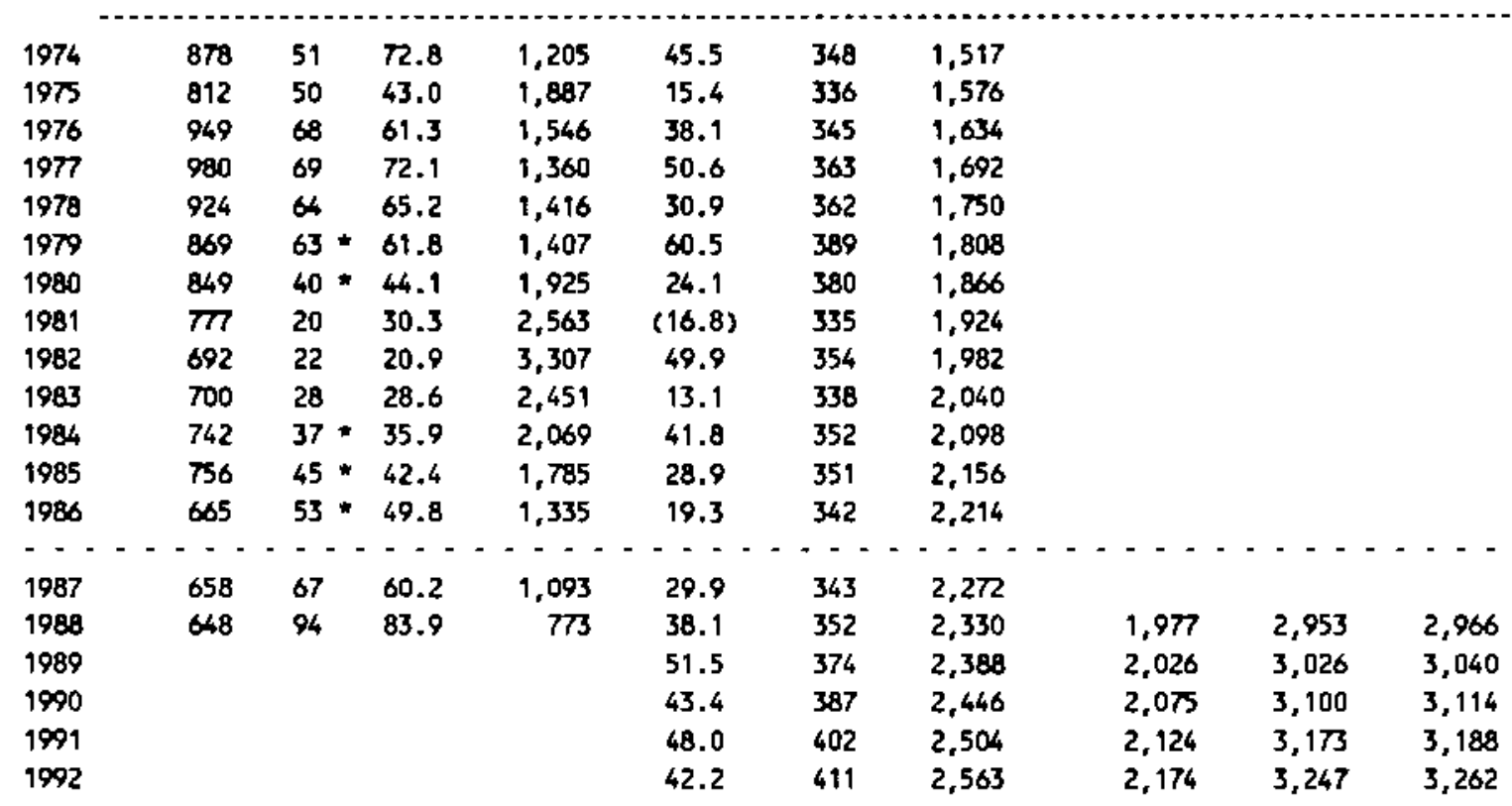

Capacity-Capital Stock Regression: Code -2 RSO 0.140 Coefficients: Const 1459.4 Time 58.1

Ave. Capecity/Stock $(74-86): 5.3$ Ave. Weekly Hours (High) 124.3 Shift Factor 1.27 Ave Weekly Hours (Low) 68.4 Shift Factor 2.30 * Imputed Note: Output, Investment, and Cepital Stock extrapolated for 1987 and 1988.

3964 Needles, pins, and fasteners

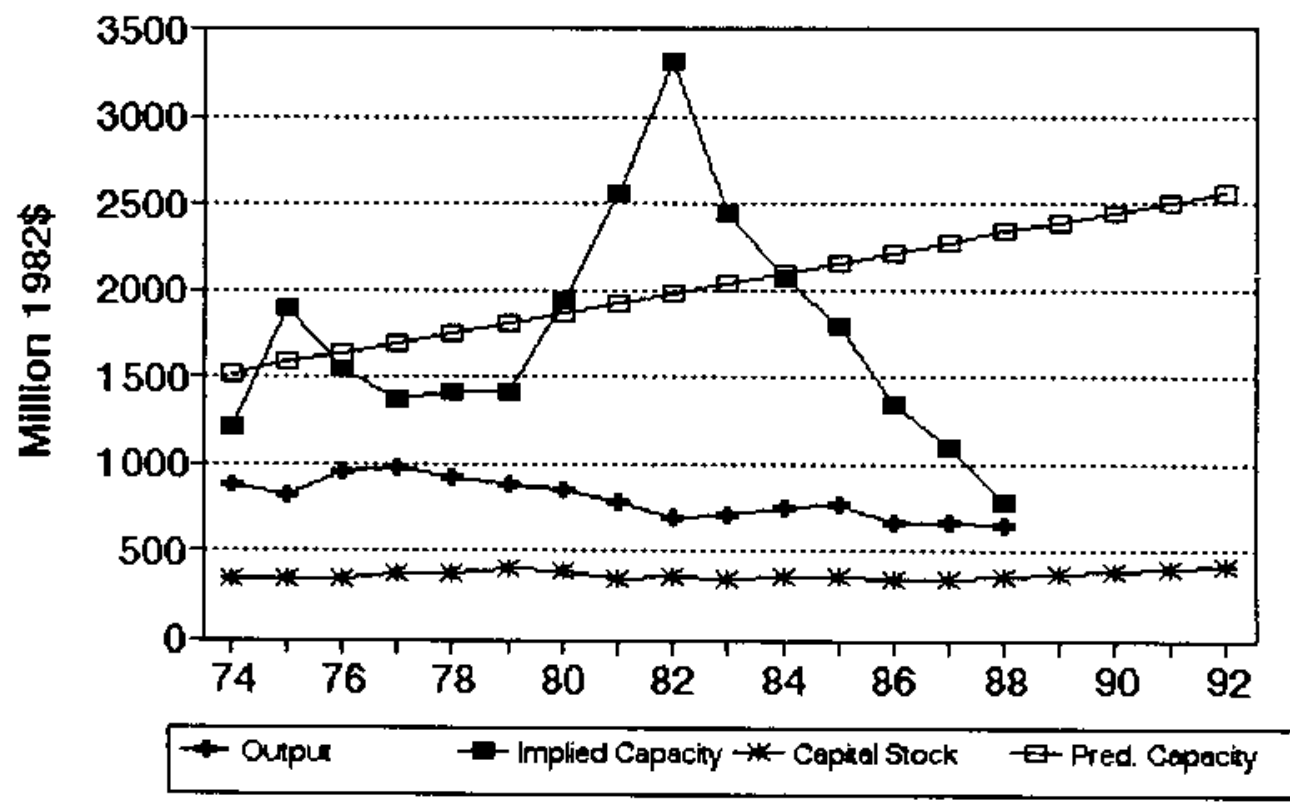




\section{No. 442 sIC 3991 Brooms and brushes}

MILLIONS OF 1982 DOLLARS (EXCL. CU RATES)

$\begin{array}{lccccccc} & \text { Q4 } & \text { Ann. Implied Gross } & \text { Het } & \text { Pred. } & \ldots . . . . . \\ \text { Gross } & \text { CU } & \text { CU } & \text { Prac. } & \text { In- } & \text { Cap. } & \text { Prac. } & \text { Emergency Capacity } \\ \text { Output } & \text { Rate } & \text { Rate } & \text { Capac. } & \text { vest. } & \text { Stk. } & \text { Capac. } & 3 \text { mon. } 6 \text { mon. }>6 \text { mon. }\end{array}$

\begin{tabular}{|c|c|c|c|c|c|c|c|c|c|c|}
\hline 1974 & 906 & 72 & 72.0 & 1,259 & $(t 1.1)$ & 194 & 1,123 & & & \\
\hline 1975 & 782 & 79 & 76.4 & 1,023 & 3.9 & 184 & 1,210 & & & \\
\hline 1976 & 845 & 85 & 83.4 & 1,013 & 20.2 & 189 & 1,164 & & & \\
\hline 1977 & 986 & 86 & 86.0 & 1,147 & 16.8 & 190 & 1,154 & & & \\
\hline 1978 & 961 & 68 & 74.8 & 1,286 & 15.4 & 189 & 1,166 & & & \\
\hline 1979 & 936 & 88 & 80.5 & 1,162 & 28.0 & 199 & 1,075 & & & \\
\hline 1980 & 878 & $\pi$ & 79.9 & 1,099 & 14.9 & 196 & 1,107 & & & \\
\hline 1981 & 851 & 64 & 68.1 & 1,269 & $(1.7)$ & 17 & 1,275 & & & \\
\hline 1982 & 816 & 46 & 52.8 & 1,548 & $(3.2)$ & 158 & 1,443 & & & \\
\hline 1983 & 864 & 57 & 52.9 & 1,634 & 21.7 & 163 & 1,399 & & & \\
\hline 1984 & 847 & 69 & 64.5 & 1,313 & 21.1 & 166 & 1,371 & & & \\
\hline 1985 & 744 & 61 & 64.0 & 1,162 & 19.7 & 168 & 1,354 & & & \\
\hline 1988 & 836 & 64 & 62.9 & 1,329 & 14.4 & 165 & 1,383 & & & \\
\hline$\because 9$ & $\cdots=$ & 56 & $\begin{array}{l}= \\
59.0\end{array}$ & $\because \cdots$ & $\cdots$ & $\begin{array}{l}--- \\
160\end{array}$ & $\cdots$ & $\cdots$ & -- & -- \\
\hline 1988 & 843 & 45 & 49.1 & 1,717 & 15.6 & 159 & 1,436 & 1,817 & 2,532 & 2,542 \\
\hline 1989 & & & & & 19.9 & 162 & 1,410 & 1,783 & 2,485 & 2,495 \\
\hline 1990 & & & & & 17.3 & 162 & 1,409 & 1,782 & 2,484 & 2,494 \\
\hline 1991 & & & & & 18.8 & 163 & 1,395 & 1,765 & 2,460 & 2,470 \\
\hline 1992 & & & & & 16.9 & 163 & 1,400 & 1,771 & 2,467 & 2,477 \\
\hline
\end{tabular}

Capecity-Capital stock Regression: Code 2 RSA 0.490

Coefficients: Const 2844.0 stock -8.9

Ave. Capacity/Stock $(74-86): 6.9$ Ave. Heekly Hours (High) 89.0 Shift Factor 1.77 Ave Weekly Hours (Low) 67.0 Shift factor 2.35 Note: Output, Investment, and Capital stock extrapolated for 1987 and 1988.

\section{Brooms and brushes}

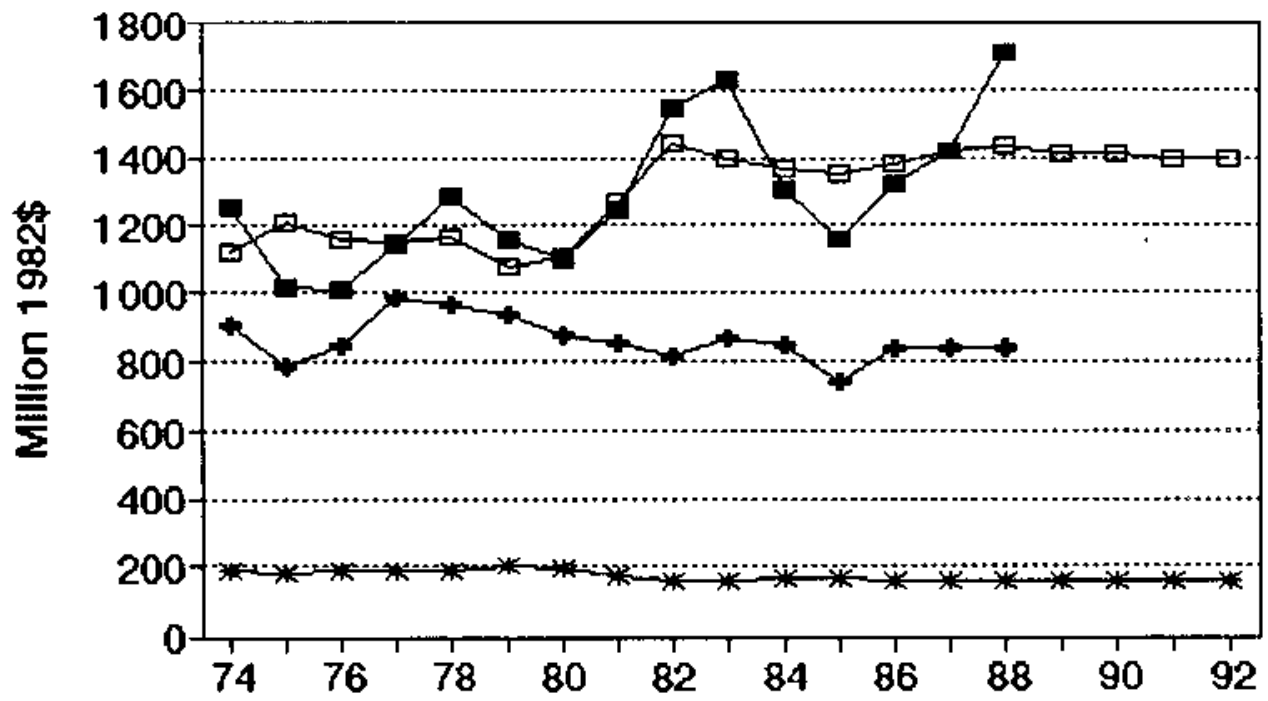

+-Oupur te- Implied Capecity - - Capital Stock E-Pred. Capacity 


\section{xo. 443 sIC 3993 Signs and advertising displays}

HILLIONS OF 1982 DOLLARS (EXCL. CU RATES)

$\begin{array}{lccccccc} & 04 & \text { Ann. } & \text { Implied } & \text { Gross } & \text { Net } & \text { Pred. } & \ldots \\ \text { Gross } & \text { CU } & \text { CU } & \text { Prac. } & \text { In. } & \text { Cap. } & \text { Prac. } & \text { Emergency Capacity } \\ \text { Output Rate } & \text { Rate } & \text { Capac. } & \text { vest. } & \text { Stk. } & \text { Capac. } & 3 \text { mon. } 6 \text { mon. }>6 \text { mon. }\end{array}$

\begin{tabular}{|c|c|c|c|c|c|c|c|c|c|c|}
\hline 1974 & 2,703 & 72 & 72.0 & 3,734 & (32.2) & 359 & 3,741 & & & \\
\hline $19 \pi$ & 2,157 & $61 *$ & 65.0 & 3,319 & 28.2 & 358 & 3,743 & & & \\
\hline 1976 & 2,188 & 65 & 63.4 & 3,450 & 75.7 & 403 & 3,638 & & & \\
\hline 1977 & 2,804 & 76 & 71.9 & 3,901 & 53.2 & 422 & 3,593 & & & \\
\hline 1978 & 2,660 & 68 & 71.0 & 3,746 & 37.1 & 424 & 3,587 & & & \\
\hline 1979 & 2,990 & 89 & 81.1 & 3,685 & 67.5 & 456 & 3,517 & & & \\
\hline 1980 & 2,777 & 84 & 85.9 & 3,233 & 59.7 & 473 & 3,473 & & & \\
\hline 1981 & 2,690 & 86 & 85.3 & 3,156 & 27.3 & 460 & 3,502 & & & \\
\hline 1982 & 2,697 & 70 & 76.0 & 3,548 & 48.8 & 468 & 3,483 & & & \\
\hline 1983 & 2,689 & 85 & 79.4 & 3,387 & 15.7 & 444 & 3,540 & & & \\
\hline 1984 & 3,172 & 68 & 74.4 & 4,264 & $(2.4)$ & 406 & 3,629 & & & \\
\hline 1985 & 3,274 & 70 & 69.3 & 4,727 & 59.4 & 428 & 4,737 & & & \\
\hline 1986 & 3,239 & 68 & 68.8 & 4,711 & 53.5 & 444 & 4,701 & & & \\
\hline 1987 & $\cdots$ & 53 & - & 5.566 & 36.6 & $\because 4$ & $\because .706$ & $\cdots-$ & $-\cdot-$ & $-\cdots$ \\
\hline 1988 & 3,294 & 60 & 57.4 & 5,741 & 49.1 & 445 & 4,699 & 5,114 & 6,452 & 6,471 \\
\hline 1989 & & & & & 48.5 & 455 & 4,676 & 5,089 & 6,419 & 6,438 \\
\hline 1990 & & & & & 44.0 & 459 & 4,665 & 5,077 & 6,404 & 6,423 \\
\hline 1991 & & & & & 46.6 & 466 & 4,649 & 5,059 & 6,382 & 6,401 \\
\hline 1992 & & & & & 43.4 & 469 & 4,642 & 5,052 & 6,373 & 6,302 \\
\hline
\end{tabular}

Capacity-Capital stock Regression: Code - 1 RSQ 0.700

Coefficients: Const 4584.2 stock -2.4 D(85-86) 1160.3 Ave. Capacity/Stock $(74-86): 8.8$ Ave. Weekly Hours (High) 114.5 Shift Factor 1.38 Ave Weekly Hours (LOW) 108.8 Shift Factor 1.45 - Imputed Note: Output, Investment, and Cepital Stock extrapolated for 1987 and 1988.

\section{Signs and advertising displays}

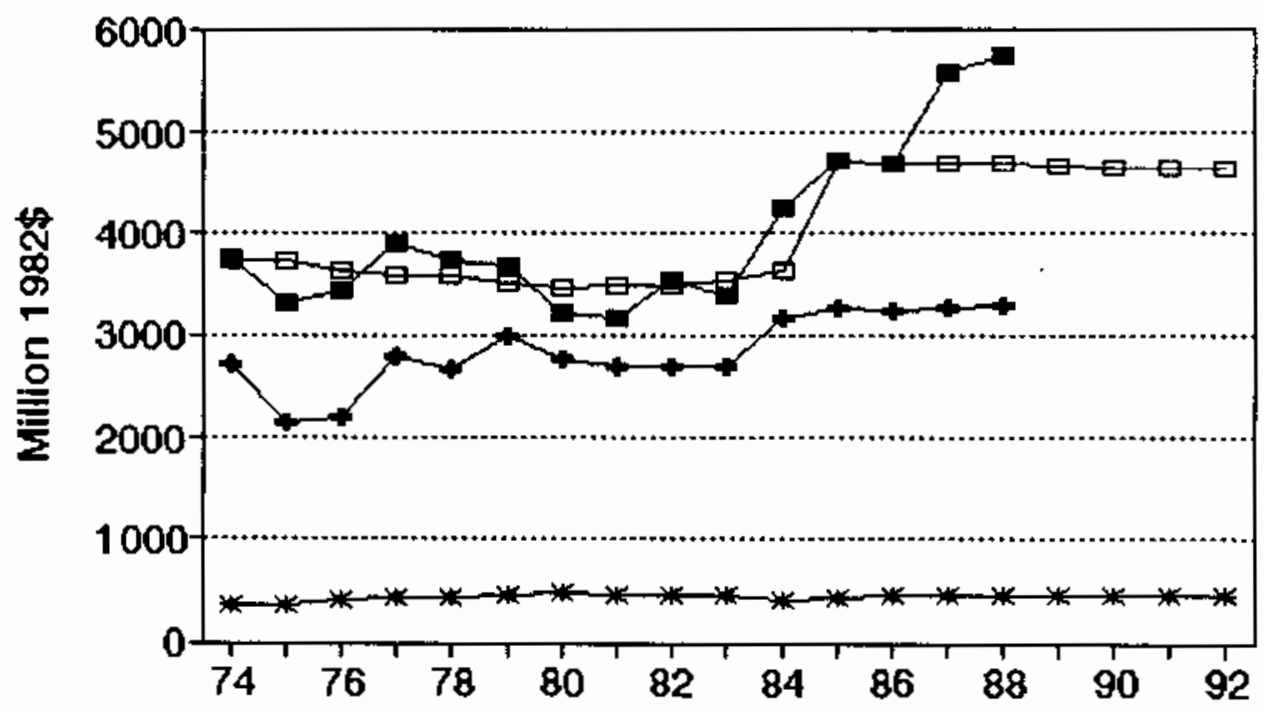

$\rightarrow$ Oupur - Implied Cepeciny - - Cepinet Stock $\quad \square-$ Pred Capacity 


\section{No. 444 SIC 3995 Burial caskets}

MILLIONS OF 1982 DOLLARS (EXCL. CU RATES)

$\begin{array}{lccccccc} & 04 & \text { Ann. } & \text { Implied Gross } & \text { Ret } & \text { Pred. } & \ldots \ldots \ldots \\ \text { Gross } & \text { CU } & \text { CU } & \text { Prac. } & \text { In* } & \text { Cap. } & \text { Prac. } & \text { Emergency Capacity } \\ \text { Output } & \text { Rate } & \text { Rate } & \text { Capac. } & \text { vest. } & \text { Stk. } & \text { Capac. } & 3 \text { mon. } 6 \text { mon. }>6 \text { mon. }\end{array}$

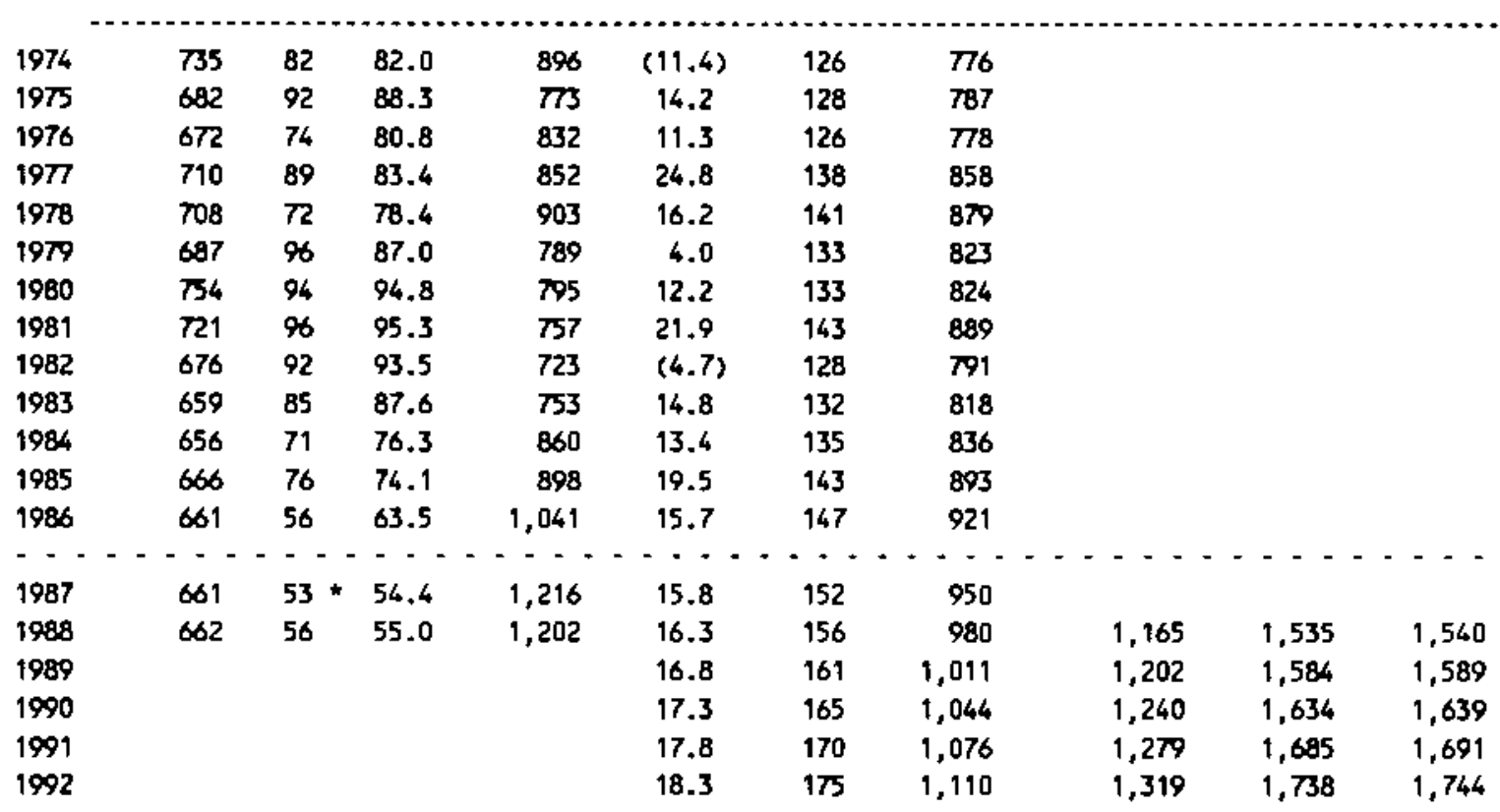

Capacity-Capital Stock Regression: Code 0 RSQ 0.310

Coefficients: Const -81.6 stock $6.8 \quad$ Ave. Capacity/Stock (74-86): 6.2 Ave. Weekly Hours (High) 100.2 Shift factor 1.57 Ave Neekly Hours (Low) 90.6 Shift factor 1.74 * Imputed Note: Output, Investment, and Capital Stock extrapolated for 1987 and 1988.

\section{Burial caskets}

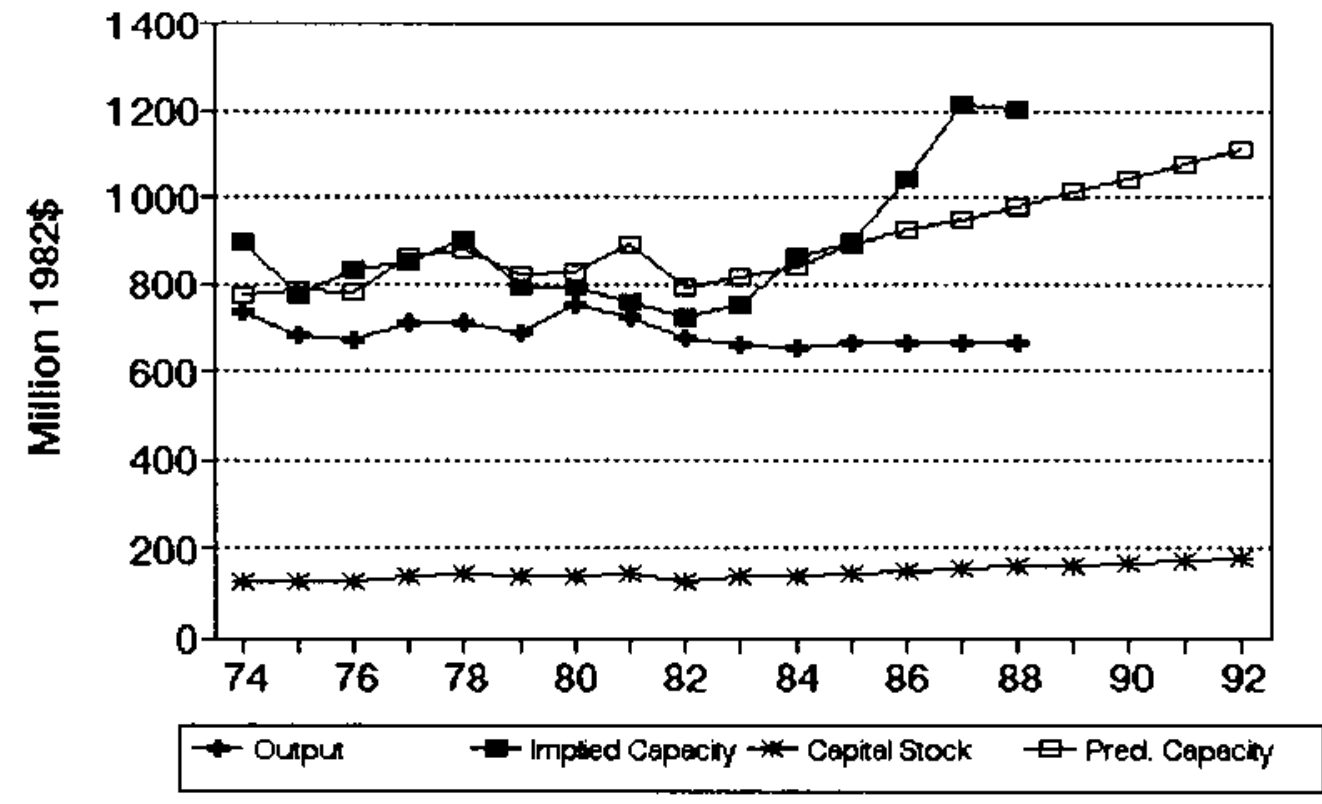




\section{No. 445 SIC 3996 Hard surface floor coverings}

MILLIONS OF 1982 DOLLARS (EXCL. CU RATES)

$\begin{array}{llllllll} & 04 & \text { Ann. } & \text { Implifed Gross } & \text { Ket } & \text { Pred. } & \ldots \\ \text { Gross } & \text { CU } & \text { CU } & \text { Prac. } & \text { In- } & \text { Cap. } & \text { Prac. } & \text { Emergency Capacity } \\ \text { Output } & \text { Rate } & \text { Rate } & \text { Capec. } & \text { vest. } & \text { Stk. } & \text { Capac. } & 3 \text { mon. } 6 \text { mon. }>6 \text { mon. }\end{array}$

\begin{tabular}{|c|c|c|c|c|c|c|c|c|c|c|}
\hline 1974 & 662 & $81 *$ & 90.8 & 729 & 35.0 & 264 & 914 & & & \\
\hline 1975 & 667 & $7 *$ & $\pi .3$ & 885 & 26.6 & 266 & 921 & & & \\
\hline 1976 & 758 & $81 \div$ & 79.6 & 952 & 28.5 & 272 & 943 & & & \\
\hline 1977 & 859 & $81 *$ & 82.6 & 1,043 & 41.0 & 287 & 1,006 & & & \\
\hline 1978 & 682 & $85 *$ & 83.4 & 1,058 & 36.8 & 295 & 1,037 & & & \\
\hline 1979 & 874 & 89 & 86.9 & 1,006 & 40.6 & 307 & 1,087 & & & \\
\hline 1980 & 666 & 74 & 77.4 & 860 & 13.5 & 292 & 1,027 & & & \\
\hline 1981 & 670 & 54 & 63.6 & 1,053 & 18.3 & 282 & 984 & & & \\
\hline 1982 & 633 & 63 & 59.4 & 1,066 & 11.4 & 265 & 917 & & & \\
\hline 1983 & 841 & 72 & 70.6 & 1,195 & 60.5 & 294 & 1,035 & & & \\
\hline 1984 & 944 & 72 & 74.0 & 1,277 & 72.2 & 333 & 1,196 & & & \\
\hline 1985 & 900 & 76 & 74.5 & 1,208 & 40.8 & 341 & 1,226 & & & \\
\hline 1986 & 1,001 & 81 & 79.0 & 1,267 & 52.7 & 360 & 1,305 & & & \\
\hline$-\cdot-$ & $-\cdots$ & - - - & -- &.--1 & $\cdots$ & 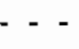 & $-\cdots$ & $\cdots$ & $\cdots$ & $-\cdots$ \\
\hline 1987 & 1,042 & 90 & 85.7 & 1,216 & 51.6 & 378 & 1,376 & & & \\
\hline 1988 & 1,099 & 87 & 88.2 & 1,246 & 53.9 & 396 & 1,449 & 1,433 & 1,679 & 1,683 \\
\hline 1989 & & & & & 56.2 & 414 & 1,525 & 1,508 & 1,767 & 1,771 \\
\hline 1990 & & & & & 58.4 & 433 & 1,603 & 1,585 & 1,857 & 1,861 \\
\hline 1991 & & & & & 60.7 & 453 & 1,683 & 1,664 & 1,949 & 1,953 \\
\hline 1992 & & & & & 63.0 & 473 & 1,764 & 1,744 & 2,044 & 2,048 \\
\hline
\end{tabular}

Capacity-Capital stock Regression: Cade 0 RSO 0.590

Coefficients: Const -166.6 Stock 4.1

Ave. Capecity/Stock $(74-86): 3.5$ Ave. Weekly Hours (High) 136.8 Shift Factor 1.16 Ave Weekly Hours (Low) 134.4 Shift Factor 1.18 * Imputed Note: Output, Investment, and Capital Stock extrapolated for 1987 and 1988.

\section{Hard surface floor coverings}

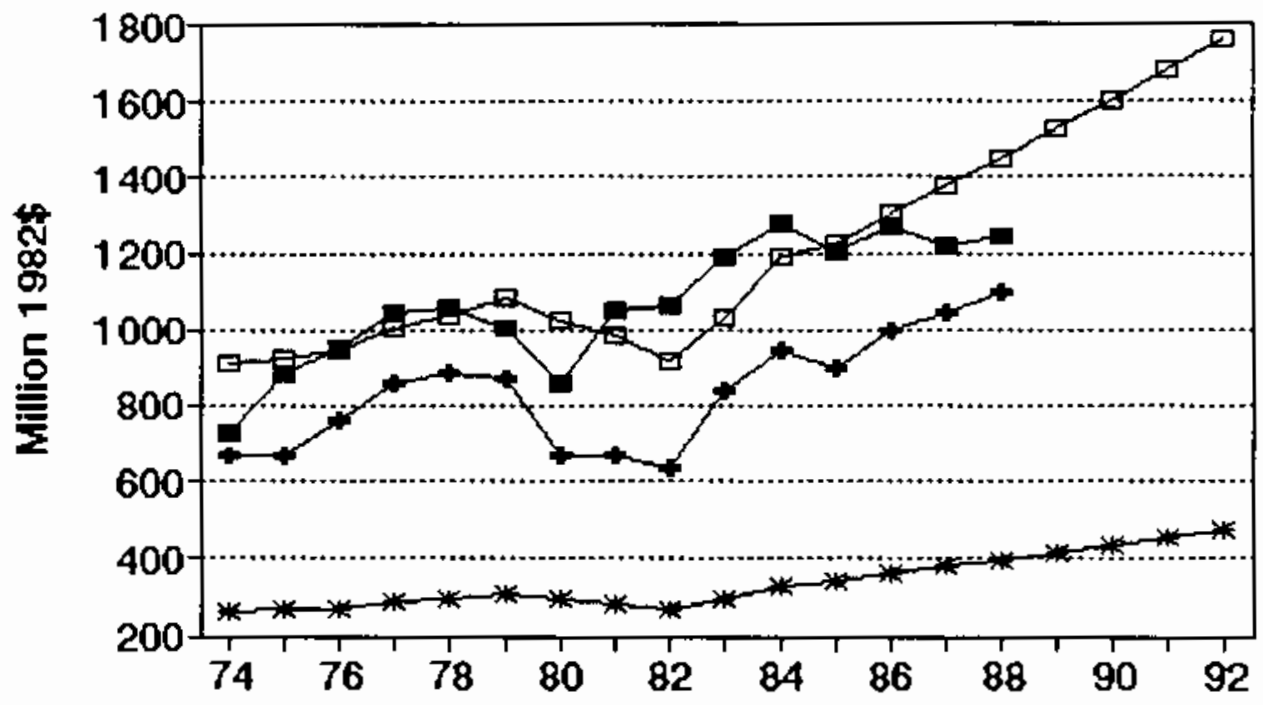

$$
\text { +- Ourpu } \rightarrow \text {-Impled Capaciny } \rightarrow \text { - Capinal Stock \#-Pred Capacisy }
$$


NO. 446 SIC 3999 Manufacturing industries, nec

MILLIONS OF 1982 DOLLARS (EXCL. CU RATES)

$\begin{array}{lccccccc} & \text { Q4 } & \text { Ann. } & \text { Implied Gross } & \text { Net } & \text { Pred. } & \ldots \ldots \ldots \\ \text { Gross } & \text { CU } & \text { CU } & \text { Prac. } & \text { In- } & \text { Cap. } & \text { Prac. } & \text { Emergency Capacity } \\ \text { Output Rate } & \text { Rate } & \text { Capec. } & \text { vest. } & \text { Stk. } & \text { Capac. } & 3 \text { mon. } 6 \text { mon. }>6 \text { mon. }\end{array}$

\begin{tabular}{|c|c|c|c|c|c|c|c|c|c|c|}
\hline 1974 & 3,728 & 61 & 63.3 & 5,890 & 61.3 & 573 & 5,508 & & & \\
\hline 1975 & 3,841 & 55 & 56.5 & 6,797 & 68.1 & 599 & 5,723 & & & \\
\hline 1976 & 4,055 & 65 & 61.2 & 6,627 & 95.4 & 653 & 6,164 & & & \\
\hline 1977 & 4,509 & 67 & 66.6 & 6,765 & 134.7 & 740 & 6.884 & & & \\
\hline 1978 & 4,510 & 65 & 65.7 & 6,868 & 66.9 & 758 & 7,032 & & & \\
\hline 1979 & 3,920 & 77 & 72.3 & 5,421 & $(69.2)$ & 648 & 6.130 & & & \\
\hline 1980 & 3,723 & 49 & 58.9 & 6,323 & 42.7 & 649 & 6,136 & & & \\
\hline 1981 & 3,931 & 71 & 63.5 & 6,187 & 27.4 & 636 & 6,026 & & & \\
\hline 1982 & 4,170 & 56 & 61.5 & 6.779 & 54.5 & 646 & 6,111 & & & \\
\hline 1983 & 3,510 & 69 & 64.5 & 5,438 & 35.4 & 635 & 6,020 & & & \\
\hline 1984 & 3,236 & 62 & 65.2 & 4,960 & $(1.6)$ & 589 & 5,646 & & & \\
\hline 1985 & 2,864 & 55 & 57.6 & 4,971 & $(0.5)$ & 547 & 5,295 & & & \\
\hline 1988 & 2,994 & 62 & 59.3 & 5,047 & 58.8 & 559 & 5,397 & & & \\
\hline$\therefore$ & $\therefore-$ & $\cdots$ & $\cdot-$ & $-\cdots$ & $-\cdots$ & $\cdots$ & $\cdots$ & --- & --- & -- \\
\hline 1987 & 3,057 & 55 & 57.4 & 5,328 & 44.3 & 559 & 5,393 & & & \\
\hline 1988 & 3,142 & 71 & 65.0 & 4,833 & 61.3 & 575 & 5,530 & 8,214 & 11,941 & 11,994 \\
\hline 1989 & & & & & 89.4 & 619 & 5,885 & 8,742 & 12,708 & 12,765 \\
\hline 1990 & & & & & 72.3 & 641 & 6,072 & 9,020 & 13,113 & 13,171 \\
\hline 1991 & & & & & 82.1 & 672 & 6,324 & 9,395 & 13,657 & 13,718 \\
\hline 1992 & & & & & 69.9 & 688 & 6,456 & 9,591 & 13,942 & 14,004 \\
\hline
\end{tabular}

Capacity-Capital Stock Regression: Code 0 RSO 0.470

Coefficients: Const 807.4 Stack 8.2

Ave. Capacity/Stock $(74-86): 9.5$

Ave. Weekly Hours (High) 72.6 Shift factor 2.17 Ave Ueekly Hours (Low) 69.0 Shift factor 2.28 Note: Output, Investment, and Capital Stock extrapolated for 1987 and 1988.

\section{Manufacturing industries, nec}

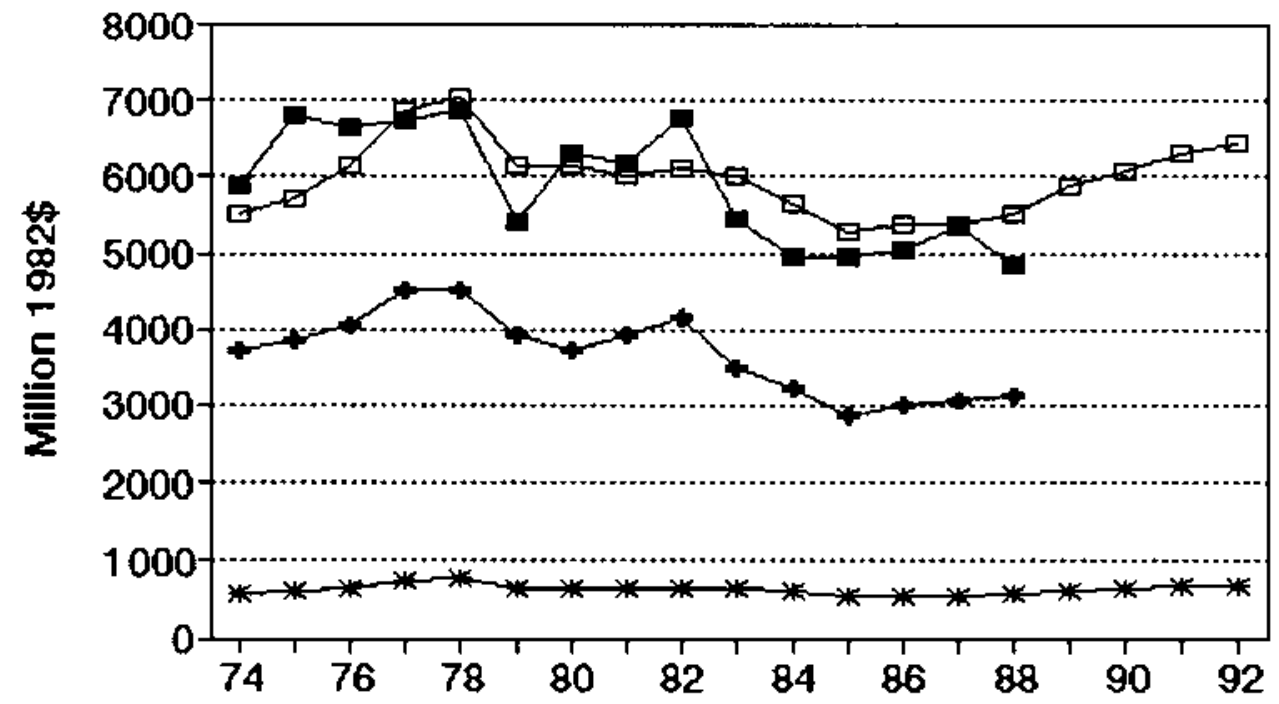

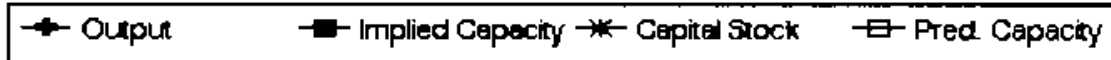


PNL-7661 Vol. 2

UC-000

\section{DISTRIBUTION}

No. of

Copies

OFFSITE

2 DOE/Office of Scientific and Technical Information

20 L. Salkin Federal Emergency Management Agency

500 C Street, S.W.

Washington, D.C. 20472

D. Serot

$D / E / S$ Research 2000 Logston Blvd.

Richland, WA 99352

M. Ke]logg

ERCE

621 S.W. Alder St.

Suite 520

Portland, OR 97205
No. of

Copies

ONSITE

DOE Richland Operations office

R. Goranson

13 Pacific Northwest Laboratory

D. B. Betzer (7)

Publishing Coordination

Technical Report Files (5) 
